Comparative Effectiveness Review

Number 210

\title{
Breastfeeding \\ Programs and \\ Policies, Breastfeeding \\ Uptake, and Maternal \\ Health Outcomes in \\ Developed Countries
}

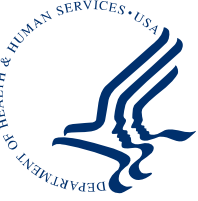




\section{Comparative Effectiveness Review}

Number 210

\section{Breastfeeding Programs and Policies, Breastfeeding Uptake, and Maternal Health Outcomes in Developed Countries}

\section{Prepared for:}

Agency for Healthcare Research and Quality

U.S. Department of Health and Human Services

5600 Fishers Lane

Rockville, MD 20857

www.ahrq.gov

Contract No. 290-2015-00011-I

\section{Prepared by:}

RTI International-University of North Carolina at Chapel Hill Evidence-based Practice Center Research Triangle Park, NC

\section{Investigators:}

Cynthia Feltner, M.D., M.P.H.

Rachel Palmieri Weber, Ph.D.

Alison Stuebe, M.D., M.Sc.

Catherine A. Grodensky, M.P.H.

Colin Orr, M.D.

Meera Viswanathan, Ph.D.

\section{AHRQ Publication No. 18-EHC014-EF}

July 2018 


\section{Key Messages}

\section{Purpose of Review}

To summarize the effectiveness of community, workplace, and health care system-based programs and policies aimed at supporting and promoting breastfeeding, and to determine the association between breastfeeding and maternal health

\section{Key Messages}

- Baby-Friendly Hospital Initiative (BFHI) is associated with improved rates of breastfeeding initiation and duration.

- Health care staff education combined with postpartum home visits may be effective for increasing breastfeeding duration.

- Health care staff education alone (with no additional breastfeeding support services) may not be effective for increasing breastfeeding initiation rates.

- For women enrolled in the WIC Program, peer-support interventions offered by WIC agencies may improve rates of breastfeeding initiation and duration.

- Breastfeeding is associated with reduced maternal risk of breast and ovarian cancer, hypertension, and type 2 diabetes.

- Workplace, school-based, and community-based interventions and underlying socioeconomic factors need further research. 
This report was funded by the Office on Women's Health at the Office of the Assistant Secretary for Health (OASH) and the Centers for Disease Control and Prevention (CDC) through an interagency agreement with the Agency for Healthcare Research and Quality (AHRQ) and is based on research conducted by the RTI International-University of North Carolina Evidencebased Practice Center (EPC) under contract to AHRQ, Rockville, MD (Contract No. 290-201500011-I). The findings and conclusions in this document are those of the authors, who are responsible for its contents; the findings and conclusions do not necessarily represent the views of AHRQ. Therefore, no statement in this report should be construed as an official position of AHRQ or of the U.S. Department of Health and Human Services.

None of the investigators have any affiliations or financial involvement that conflicts with the material presented in this report.

The information in this report is intended to help health care decisionmakers—patients and clinicians, health system leaders, and policymakers, among others-make well-informed decisions and thereby improve the quality of health care services. This report is not intended to be a substitute for the application of clinical judgment. Anyone who makes decisions concerning the provision of clinical care should consider this report in the same way as any medical reference and in conjunction with all other pertinent information, i.e., in the context of available resources and circumstances presented by individual patients.

This report is made available to the public under the terms of a licensing agreement between the author and the Agency for Healthcare Research and Quality. This report may be used and reprinted without permission except those copyrighted materials that are clearly noted in the report. Further reproduction of those copyrighted materials is prohibited without the express permission of copyright holders.

AHRQ or U.S. Department of Health and Human Services endorsement of any derivative products that may be developed from this report, such as clinical practice guidelines, other quality enhancement tools, or reimbursement or coverage policies, may not be stated or implied.

This report may periodically be assessed for the currency of conclusions. If an assessment is done, the resulting surveillance report describing the methodology and findings will be found on the Effective Health Care Program Web site at www.effectivehealthcare.ahrq.gov. Search on the title of the report.

Persons using assistive technology may not be able to fully access information in this report. For assistance contact EPC@ahrq.gov.

Suggested citation: Feltner C, Weber RP, Stuebe A, Grodensky CA, Orr C, Viswanathan M. Breastfeeding Programs and Policies, Breastfeeding Uptake, and Maternal Health Outcomes in Developed Countries. Comparative Effectiveness Review No. 210. (Prepared by the RTI International-University of North Carolina at Chapel Hill Evidence-based Practice Center under Contract No. 290-2015-00011-I.) AHRQ Publication No. 18-EHC014-EF. Rockville, MD: Agency for Healthcare Research and Quality; July 2018. Posted final reports are located on the Effective Health Care Program search page. DOI: https://doi.org/10.23970/AHRQEPCCER210. 


\section{Preface}

The Agency for Healthcare Research and Quality (AHRQ), through its Evidence-based Practice Centers (EPCs), sponsors the development of evidence reports and technology assessments to assist public- and private-sector organizations in their efforts to improve the quality of health care in the United States.

The Office on Women's Health at the Office of the Assistant Secretary for Health (OASH) and the Centers for Disease Control and Prevention (CDC) requested this report from the EPC Program at AHRQ. AHRQ assigned this report to the following EPC: RTI InternationalUniversity of North Carolina at Chapel Hill Evidence-based Practice Center (Contract No. 2902015-00011-I).

The reports and assessments provide organizations with comprehensive, evidence-based information on common medical conditions and new health care technologies and strategies. They also identify research gaps in the selected scientific area, identify methodological and scientific weaknesses, suggest research needs, and move the field forward through an unbiased, evidence-based assessment of the available literature. The EPCs systematically review the relevant scientific literature on topics assigned to them by AHRQ and conduct additional analyses when appropriate prior to developing their reports and assessments.

To bring the broadest range of experts into the development of evidence reports and health technology assessments, AHRQ encourages the EPCs to form partnerships and enter into collaborations with other medical and research organizations. The EPCs work with these partner organizations to ensure that the evidence reports and technology assessments they produce will become building blocks for health care quality improvement projects throughout the Nation. The reports undergo peer review and public comment prior to their release as a final report.

AHRQ expects that the EPC evidence reports and technology assessments, when appropriate, will inform individual health plans, providers, and purchasers as well as the health care system as a whole by providing important information to help improve health care quality.

If you have comments on this evidence report, they may be sent by mail to the Task Order Officer named below at: Agency for Healthcare Research and Quality, 5600 Fishers Lane, Rockville, MD 20857, or by email to epc@ahrq.hhs.gov.

Gopal Khanna, M.B.A.

Director

Agency for Healthcare Research and Quality

Stephanie Chang, M.D., M.P.H.

Director

Evidence-based Practice Center Program

Center for Evidence and Practice Improvement Agency for Healthcare Research and Quality
Arlene S. Bierman, M.D., M.S.

Director

Center for Evidence and Practice

Improvement

Agency for Healthcare Research and Quality

Suchitra Iyer, Ph.D.

Task Order Officer

Center for Evidence and Practice

Improvement

Agency for Healthcare Research and Quality 
Ruowei (Rosie) Li, M.D., Ph.D.

Senior Epidemiologist

Division of Nutrition, Physical Activity, and Obesity

Centers for Disease Control and Prevention
Ursuline Singleton, M.P.H., R.D.

Public Health Advisor

Office on Women's Health

Office of the Assistant Secretary for Health 


\section{Acknowledgments}

The authors gratefully acknowledge the following individuals for their contributions to this project and deeply appreciate their considerable support, commitment, and contributions: Suchitra Iyer, Ph.D., our AHRQ Task Order Officer ; Carrie Patnode, Ph.D., M.P.H., our Associate Editor; RTI International-University of North Carolina at Chapel Hill EPC staff: Carol Woodell, B.S.P.H.; Christiane Voisin, M.S.L.S.; Laurie Leadbetter, M.S.L.S; Carla Bann, Ph.D.; Sharon Barrell, M.A.; Loraine Monroe; Jennifer Cook Middleton, Ph.D.; Charlotte E. Randolph, B.A.; Christine Jackson, M.P.H.; and Cassandra J. Barnhart, M.P.H., ACRP-CP. 


\section{Technical Expert Panel}

In designing the study questions and methodology at the outset of this report, the EPC consulted several technical and content experts. Broad expertise and perspectives were sought. Divergent and conflicted opinions are common and perceived as healthy scientific discourse that results in a thoughtful, relevant systematic review. Therefore, in the end, study questions, design, methodologic approaches, and/or conclusions do not necessarily represent the views of individual technical and content experts.

Technical Experts must disclose any financial conflicts of interest greater than \$5,000 and any other relevant business or professional conflicts of interest. Because of their unique clinical or content expertise, individuals with potential conflicts may be retained. The TOO and the EPC work to balance, manage, or mitigate any potential conflicts of interest identified.

The Technical Experts who participated in developing this report are as follows:

Cathy Carothers, B.L.A., IBCLC, RLC Every Mother, Inc.

Greenville, MS

Susan M. Gross, Ph.D., M.P.H., R.D., L.D.N.* Department of Population, Family and Reproductive Health

Johns Hopkins Bloomberg School of Public Health Baltimore, MD

Mona Liza Hamlin, M.S.N., R.N., IBCLC U.S. Breastfeeding Committee

Chicago, IL

Lauren Hanley, M.D., IBCLC

American Congress of Obstetricians and Gynecologists

Boston, MA

Summer Sherburne Hawkins, Ph.D., M.S.* Boston College School of Social Work Chestnut Hill, MA

Joan Meek, M.D., M.S.

Florida State University College of Medicine Tallahassee, FL

*Provided input on draft report.
Tonse Raju, M.D., D.C.H. Pregnancy and Perinatology Branch

National Institute of Child Health and Human

Development

Bethesda, MD

Julie Reeder, Ph.D., M.P.H., M.S., C.H.E.S.* State of Oregon WIC Program

Portland, OR

Megan Renner, B.A.

U.S. Breastfeeding Committee

Chicago, IL

Kelley S. Scanlon, Ph.D., R.D.*

U.S. Department of Agriculture

Washington, D.C.

Eleanor Bimla Schwarz, M.D., M.S.* University of California at Davis School of Medicine Sacramento, CA

Irene Zoppi, R.N., M.S.N., IBCLC Medela

Hanover, MA 


\section{Peer Reviewers}

Prior to publication of the final evidence report, EPCs sought input from independent Peer Reviewers without financial conflicts of interest. However, the conclusions and synthesis of the scientific literature presented in this report do not necessarily represent the views of individual reviewers.

Peer Reviewers must disclose any financial conflicts of interest greater than $\$ 5,000$ and any other relevant business or professional conflicts of interest. Because of their unique clinical or content expertise, individuals with potential nonfinancial conflicts may be retained. The TOO and the EPC work to balance, manage, or mitigate any potential nonfinancial conflicts of interest identified.

The Peer Reviewers are as follows:

Valerie Flaherman, M.D.

University of California at San Francisco

School of Medicine

San Francisco, CA

Laurence Grummer-Strawn, Ph.D.

World Health Organization

Geneva, Switzerland
Laurie Nommsen-Rivers, Ph.D., R.D., IBCLC

University of Cincinnati

Cincinnati, $\mathrm{OH}$

Leanne Redman, Ph.D.

Pennington Biomedical Research Center

Baton Rouge, LA 


\section{Breastfeeding Programs and Policies, Breastfeeding Uptake, and Maternal Health Outcomes in Developed Countries}

\section{Structured Abstract}

Objectives. To summarize the effectiveness of community, workplace, and health care systembased programs and policies aimed at supporting and promoting breastfeeding and determine the association between breastfeeding and maternal health.

Data sources. We searched PubMed ${ }^{\circledR} / \mathrm{MEDLINE}^{\circledR}$, the Cochrane Library, and CINAHL ${ }^{\circledR}$ from January 1, 1980, to October 12, 2017, for studies relevant to the effectiveness of health care system-based, workplace, and community breastfeeding programs and policies. For evidence on breastfeeding and maternal health, we updated the 2007 Agency for Healthcare Research and Quality report on this topic and searched the same databases from November 1, 2005, to October 12, 2017. For studies of breastfeeding programs and policies, trials, systematic reviews, and observational studies with a control group were eligible; we excluded primary care-based programs delivered as part of routine care. For studies related to breastfeeding and maternal health, we included systematic reviews, case-control studies, and cohort studies.

Review methods. Pairs of reviewers independently selected, extracted data from, and rated the risk of bias of relevant studies; they graded the strength of evidence (SOE) using established criteria. We synthesized all evidence qualitatively.

Results. We included 128 studies (137 publications) and 10 systematic reviews. Of these, 40 individual studies were relevant to the effectiveness of breastfeeding programs or policies, and the remainder were relevant to one or more maternal health outcomes. Based on evidence from one large randomized controlled trial (RCT) (Promotion of Breastfeeding Intervention Trial [PROBIT], $\mathrm{N}=17,046)$ enrolling mothers who intended to breastfeed and nine cohort studies $(1,227,182$ women), we graded the SOE for the Baby-Friendly Hospital Initiative (BFHI) as moderate for improving rates of breastfeeding duration. Evidence from eight cohort studies of BFHI (135,983 women) also demonstrates improved rates of breastfeeding initiation (low SOE). Low SOE ( $k=4$ studies; 1,532 women) supports the conclusion that health care education or training of staff alone (without additional breastfeeding support services) does not improve breastfeeding initiation rates. Women, Infants and Children (WIC, a Federal supplemental nutrition program) interventions that focus on peer support are effective in improving rates of breastfeeding initiation and duration (low SOE). We found limited evidence for other (community-based) interventions and no comparative studies on workplace or school-based interventions or harms associated with interventions.

For maternal health outcomes, low SOE supports the conclusion that ever breastfeeding or breastfeeding for longer durations may be associated with lower rates of breast cancer, epithelial ovarian cancer, hypertension, and type 2 diabetes, but not fractures. Because of heterogeneity and inconsistent results, we found insufficient evidence on whether breastfeeding is associated with postpartum depression, cardiovascular disease, or postpartum weight change. 
Conclusions. The body of evidence for breastfeeding programs and policies was diverse in terms of interventions and settings. Current evidence supports the benefit of BFHI for improving rates of breastfeeding initiation and duration; however, evidence from one large RCT (PROBIT) has limited applicability, and observational studies do not clearly establish the magnitude of benefit. For women enrolled in WIC, low SOE supports peer-support interventions for improving breastfeeding outcomes. The identified associations between breastfeeding and improved maternal health outcomes are supported by evidence from observational studies, which cannot determine cause-and-effect relationships. 


\section{Contents}

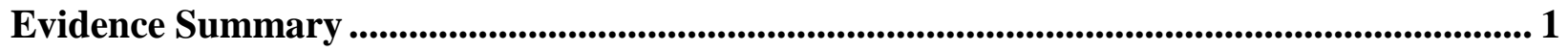

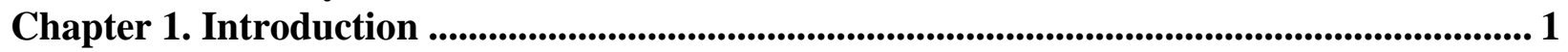

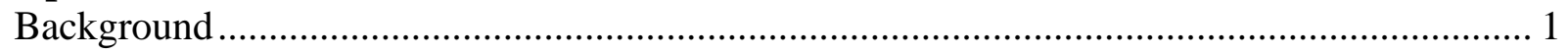

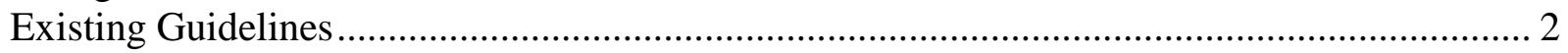

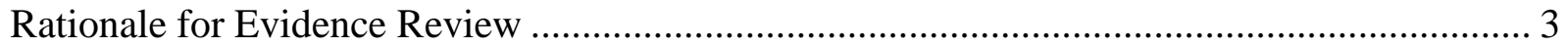

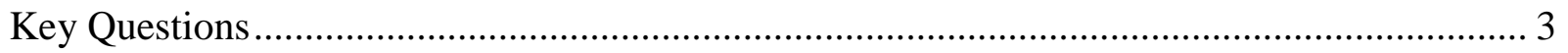

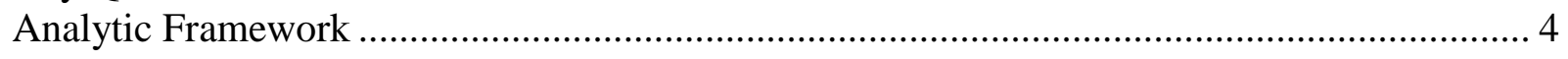

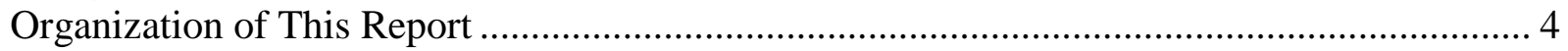

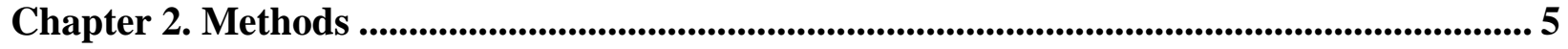

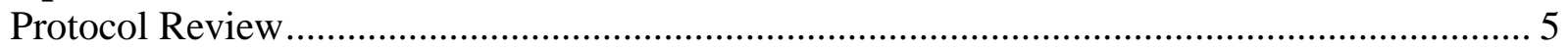

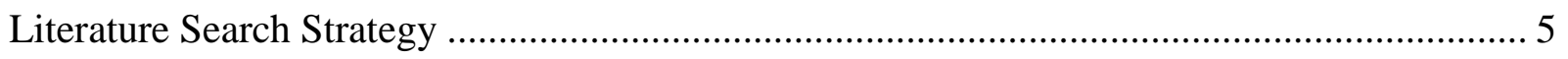

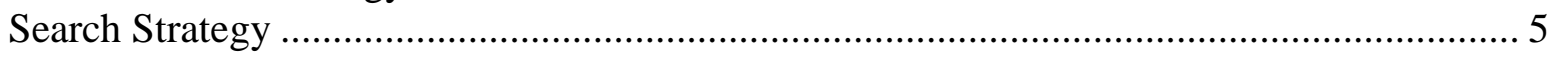

Inclusion and Exclusion Criteria.......................................................................... 5

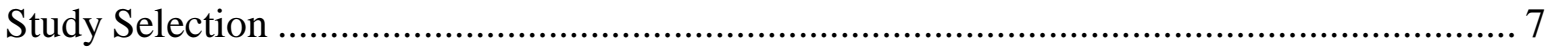

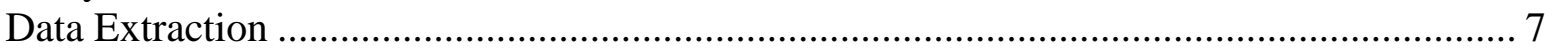

Risk of Bias Assessment of Individual Studies .............................................................. 8

Risk of Bias Assessment of Systematic Reviews ........................................................ 8

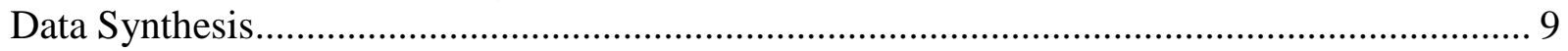

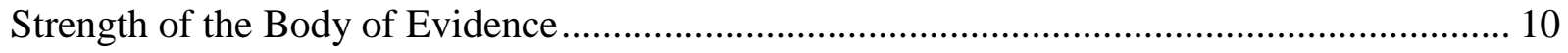

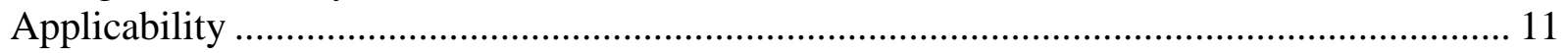

Peer Review and Public Commentary ...................................................................... 11

Chapter 3. Results ..................................................................................................................... 12

Results of Literature Search and Screening ................................................................... 12

Effectiveness and Harms of Breastfeeding Programs and Policies ...................................... 12

Key Points: Baby-Friendly Hospital Initiative ........................................................ 12

Key Points: Non-BFHI Health Care System-Based Interventions................................... 12

Key Points: Women, Infants and Children-Based Interventions .................................... 13

Key Points: Community-Based Interventions .......................................................... 13

Baby-Friendly Hospital Initiative Interventions ........................................................... 13

Other (Non-BFHI) Health Care System-Based Interventions........................................... 24

Women, Infants and Children-Based Interventions .................................................. 41

Community-Based Interventions ........................................................................ 51

Effectiveness and Harms of Breastfeeding Programs and Policies for Subpopulations

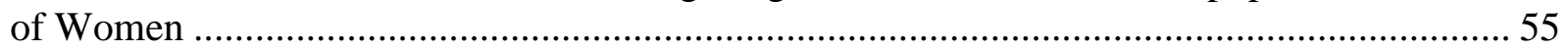

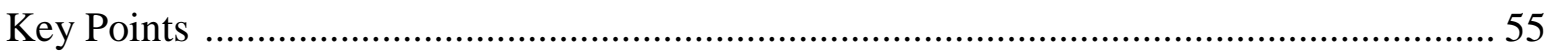

Characteristics and Results ..................................................................................... 58

Effect of Intervention Characteristics on Breastfeeding Outcomes...................................... 58

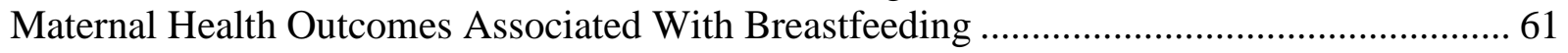

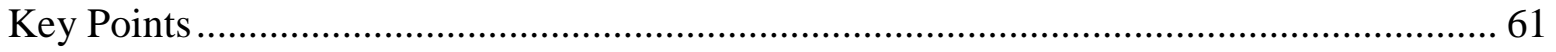

Breast Cancer and Ovarian Cancer ........................................................................... 62

Cardiovascular Disease and Hypertension..................................................................... 84

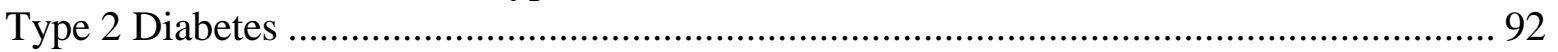

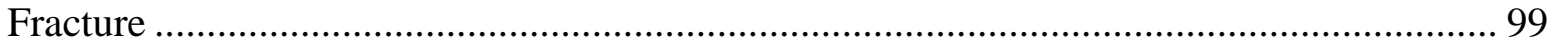

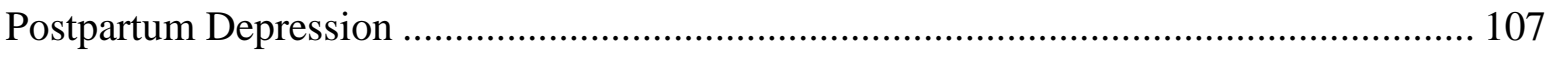


Return to Prepregnancy Weight or Postpartum Weight Change .................................... 120

Chapter 4. Discussion ................................................................................................................... 132

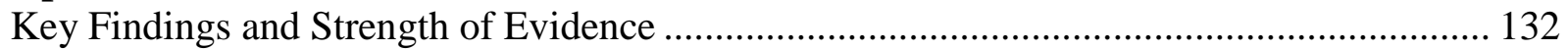

Effectiveness and Harms of Breastfeeding Programs and Policies .................................... 132

Baby-Friendly Hospital Initiative Interventions ....................................................... 132

Other (Non-BFHI) Health Care System-Based Interventions........................................ 133

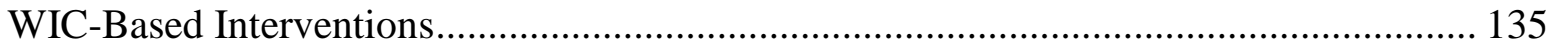

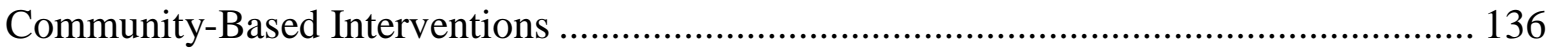

Effectiveness and Harms of Breastfeeding Programs and Policies for Subpopulations

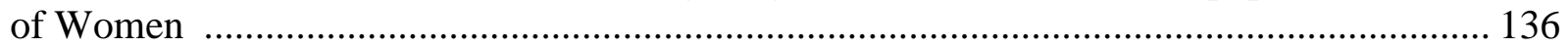

Effect of Intervention Characteristics on Breastfeeding Outcomes.................................... 137

Maternal Health Outcomes Associated With Breastfeeding ............................................. 137

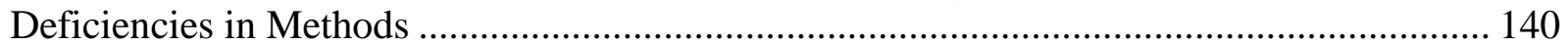

Findings in Relation to What Is Already Known ............................................................ 140

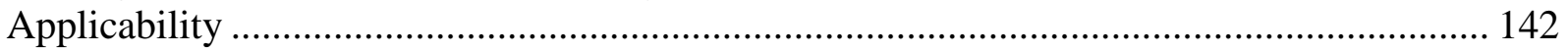

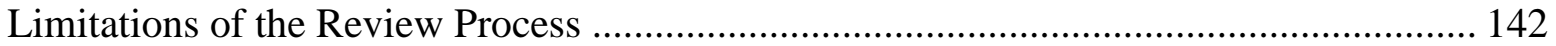

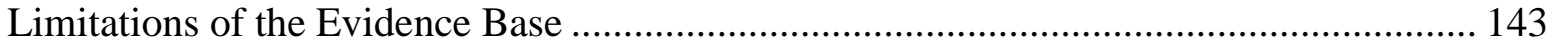

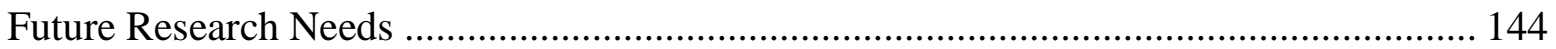

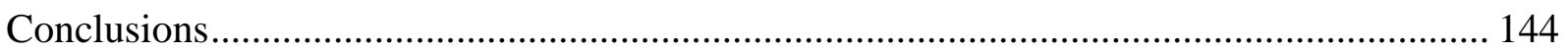

References..................................................................................................................................... 146

Tables

Table A. Baby-Friendly Hospital Initiative’s 10 steps to successful breastfeeding .................ES-2

Table B. Summary of key findings and strength of evidence: Studies assessing BFHI............ES-7

Table C. Summary of key findings and strength of evidence: Non-BFHI health care

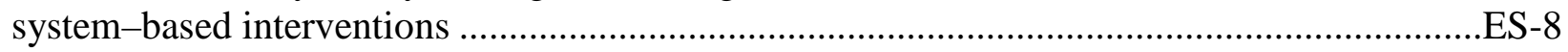

Table D. Summary of key findings and strength of evidence: WIC-based interventions .......ES-10

Table E. Summary of key findings and strength of evidence: Community-based interventions.

Table F. Summary of key findings and strength of evidence: KQ 1 studies reporting on subgroups

Table G. Summary of key findings and strength of evidence: Maternal health outcomes ......ES-13

Table 1. Baby-Friendly Hospital Initiative's 10 steps to successful breastfeeding........................2

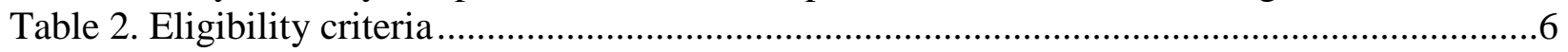

Table 3. Definitions of the grades of overall strength of evidence .........................................10

Table 4. Characteristics of included studies assessing BFHI interventions.................................15

Table 5. BFHI studies reporting on breastfeeding initiation.................................................18

Table 6. BFHI studies reporting on breastfeeding duration and exclusivity ..............................21

Table 7. Characteristics of studies evaluating non-BFHI health care system-based

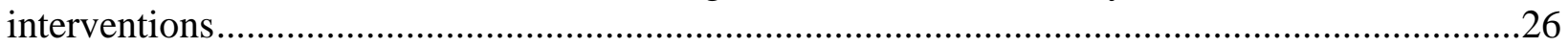

Table 8. Non-BFHI health care system-based interventions reporting on breastfeeding

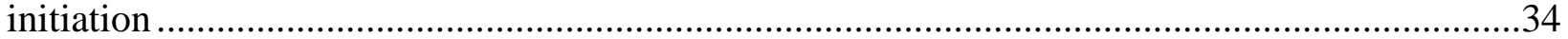

Table 9. Non-BFHI health care system-based interventions reporting on breastfeeding

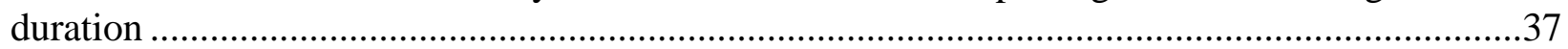

Table 10. Characteristics of studies assessing WIC-based interventions ..................................43

Table 11. WIC interventions reporting on breastfeeding initiation and duration .........................47

Table 12. Characteristics of studies assessing community-based interventions...........................52 
Table 13. Results of studies assessing community-based interventions ........................................56

Table 14. KQ 1 studies reporting on eligible subgroups ..........................................................59

Table 15. Breastfeeding and overall breast cancer: Summary of published systematic

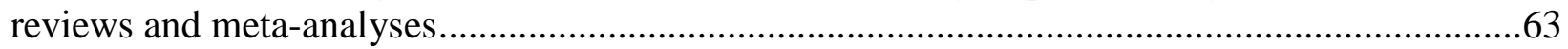

Table 16. Ever breastfeeding and overall breast cancer .......................................................67

Table 17. Duration of breastfeeding and breast cancer.................................................................68

Table 18. Breastfeeding and breast cancer in situ.......................................................................71

Table 19. Breastfeeding and tumor subtypes of breast cancer defined by hormone

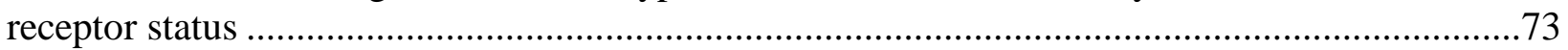

Table 20. Breastfeeding and ovarian cancer: Summary of published systematic reviews .............78

Table 21. Breastfeeding and ovarian cancer: Summary of individual studies..................................80

Table 22. Breastfeeding and CVD or hypertension: Summary of individual studies....................85

Table 23. Breastfeeding and type 2 diabetes: Summary of published systematic review ..............93

Table 24. Breastfeeding and type 2 diabetes: Summary of individual studies ...............................94

Table 25. Breastfeeding and fractures: Summary of individual studies ......................................100

Table 26. Breastfeeding and postpartum depression: Summary of published systematic

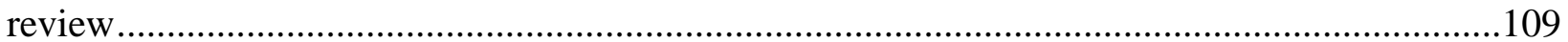

Table 27. Breastfeeding and postpartum depression: Summary of individual studies ................111

Table 28. Breastfeeding and postpartum weight change: Summary of individual studies...........122

Table 29. Summary of key findings and strength of evidence: Studies assessing BFHI .............133

Table 30. Summary of key findings and strength of evidence: Non-BFHI health care system-based interventions ......................................................................................................134

Table 31. Summary of key findings and strength of evidence: WIC-based interventions ...........135

Table 32. Summary of key findings and strength of evidence: Community-based

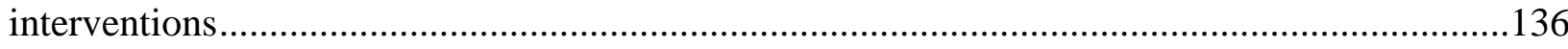

Table 33. Summary of key findings and strength of evidence: KQ 1 studies reporting on subgroups

Table 34. Summary of key findings and strength of evidence: Maternal health outcomes.........138 


\section{Figures}

Figure A. Analytic framework for breastfeeding programs and policies, breastfeeding uptake, and maternal health outcomes in developed countries.

Figure 1. Analytic framework for breastfeeding programs and policies, breastfeeding uptake, and maternal health outcomes in developed countries.

\section{Appendixes}

Appendix A. Literature Search Strategies and Yields

Appendix B. PRISMA

Appendix C. Relevance and Risk of Bias Assessments

Appendix D. Strength of Evidence Tables

Appendix E. Excluded Studies

Appendix F. Breast Cancer Evidence Tables

Appendix G. References for All Appendixes 


\section{Evidence Summary}

\section{Background}

In reproductive physiology, lactation follows pregnancy; a growing body of evidence supports the association between breastfeeding and better health outcomes for both infants and mothers. ${ }^{1-3}$ A 2007 Agency for Healthcare Research and Quality (AHRQ) review by Ip and colleagues concluded that breastfeeding was associated with reduced maternal type 2 diabetes, breast cancer and ovarian cancer, but not fractures. ${ }^{2}$ For other outcomes (e.g., postpartum depression), the authors concluded that the relationship between breastfeeding and maternal health was unclear. Since 2007, several new studies have reported on maternal outcomes not addressed in the 2007 AHRQ review, including hypertension, rates of myocardial infarction, and other cardiovascular outcomes. ${ }^{4-7}$

In 2014, an estimated 82.5 percent of infants born in the United States were breastfed at birth, meeting Healthy People 2020 targets for the percentage of infants who are ever breastfed (81.9\%). However, rates of breastfeeding duration fell short of Healthy People 2020 targets. In 2014, only 55.3 percent of women breastfed at 6 months and 33.7 percent at 12 months ${ }^{8}$ (falling short of the 2020 targets of 66.6 and 34.1 percent, respectively, for 6 and 12 months). ${ }^{9}$ Rates of exclusive breastfeeding through 3 and 6 months were 46.6 and 24.9 percent, respectively; these measures are close to Health People 2020 targets (46.2 and $25.5 \%$, respectively). ${ }^{8}$ Women would prefer to breastfeed longer: in a national survey, 45 percent of U.S. women who initiated breastfeeding reported early, undesired weaning. ${ }^{10}$ Despite rising breastfeeding initiation and duration rates in the United States, racial and ethnic differences persist. From 2000 to 2014, the percentage of women who initiated breastfeeding went up from 47.4 to 68.0 percent for blacks, 71.8 to 85.7 percent for whites, and 77.6 to 84.8 percent for Hispanics. ${ }^{11,12}$

In addition to setting targets for breastfeeding initiation rates and duration of breastfeeding, other Healthy People 2020 objectives related to breastfeeding include (1) increasing the proportion of live births that occur in facilities that provide recommended care for lactating mothers and their babies and (2) increasing the proportion of employers that have worksite lactation support programs. ${ }^{9}$ These community, workplace, and health care system-based programs and policies may be promising strategies to support initiation and increase duration of breastfeeding.

Health care system-based interventions may include maternity staff education or the BabyFriendly Hospital Initiative (BFHI). The BFHI is a global program sponsored by the World Health Organization (WHO) and United Nations Children's Fund to encourage and recognize hospitals and birth centers that create an environment supporting breastfeeding. In each country, a BFHI Coordination Group is charged with designating facilities as Baby-Friendly; ${ }^{13}$ there are likely country-specific differences in the process for determining final accreditation (or certification) status. As a result, details of implementation vary from country to country. The Baby-Friendly USA “Ten Steps to Successful Breastfeeding” for hospitals and birthing facilities are listed in Table A. Insurance coverage for lactation support is another strategy that may enable women to achieve their breastfeeding goals. Costs associated with breastfeeding support (e.g., comprehensive lactation support and counseling, breastfeeding equipment) are currently covered by health insurance marketplace plans and private nongrandfathered health plans under the 2010 Patient Protection and Affordable Care Act. ${ }^{14}$ It is not clear whether certain lactation benefit packages (e.g., type of breastfeeding supplies offered, number of visits provided, or qualifications of intervention delivery personnel) are more or less effective than others in 
increasing breastfeeding initiation and duration. In addition, a key program relevant to breastfeeding is the Special Supplemental Nutrition Program for Women, Infants and Children (WIC), which serves 53 percent of infants born in the United States. ${ }^{15}$ Because WIC reaches more than half of U.S. infants, its programs have considerable influence on population health.

Although there is broad appeal and interest in workplace interventions to increase duration and exclusivity of breastfeeding, their effectiveness and harms are uncertain. ${ }^{16}$

Table A. Baby-Friendly Hospital Initiative's 10 steps to successful breastfeeding ${ }^{a}$

1. Have a written breastfeeding policy that is routinely communicated to all health care staff.

2. Train all health care staff in skills necessary to implement this policy.

3. Inform all pregnant women about the benefits and management of breastfeeding.

4. Help mothers initiate breastfeeding within 1 hour of birth.

5. Show mothers how to breastfeed and how to maintain lactation even if they should be separated from their infants.

6. Give infants no food or drink other than breast milk, unless medically indicated.

7. Practice rooming in-allow mothers and infants to remain together 24 hours a day.

8. Encourage breastfeeding on demand.

9. Give no pacifiers or artificial nipples to breastfeeding infants.

10. Foster the establishment of breastfeeding support groups and refer mothers to them on discharge from the hospital or birth center.

${ }^{a}$ Baby-Friendly USA "Ten Steps to Successful Breastfeeding"17

\section{Existing Guidelines}

Multiple clinical guidelines and health-related organizations recommend exclusive breastfeeding up to (or around) 6 months, including the American Academy of Pediatrics, ${ }^{18}$ the American Congress of Obstetrics and Gynecology, ${ }^{19}$ the WHO, ${ }^{20,}{ }^{21}$ and others. ${ }^{22,}{ }^{23}$ These organizations recommend continued breastfeeding through the first year of life and beyond; the WHO recommends continued breastfeeding through the second year of life and beyond. ${ }^{24}$

\section{Rationale for Evidence Review}

The purpose of this review is to develop an evidence report that summarizes the effectiveness of community, workplace, and health care system-based programs and policies aimed at supporting and promoting breastfeeding. Such knowledge is needed to inform allocation of resources to enable more women to achieve their infant feeding goals. The U.S. Preventive Services Task Force (USPSTF) recommends providing interventions during pregnancy and after birth to support breastfeeding as part of routine primary care (B recommendation). ${ }^{25}$ To avoid duplication, this review will not address the effectiveness of individual-level primary care interventions to support breastfeeding covered in the recent systematic review to support the USPSTF recommendation. ${ }^{26}$

In addition, this review will address the association between breastfeeding and maternal health. Substantial time has elapsed since the last AHRQ review on this topic in 2007, and the body of literature focused on the maternal health benefits of breastfeeding has grown. ${ }^{1,27-29}$ This review will conduct a partial update of the 2007 AHRQ review focused on the relationship between breastfeeding and various maternal health outcomes. This review will inform the extent to which breastfeeding may be an effective primary prevention strategy for improving women's health. 


\section{Key Questions}

\section{Key Question 1:}

1a. What are the effectiveness and harms of programs and policies on initiation, duration, and exclusivity of breastfeeding?

1b. To what extent do the effectiveness and harms of programs and policies on initiation, duration, and exclusivity of breastfeeding differ for subpopulations of women defined by sociodemographic factors (e.g., age, race, ethnicity, socioeconomic status)?

1c. To what extent do intervention-related characteristics (e.g., type of breast pump provided-manual or electric; delivery personnel) influence the initiation, duration, and exclusivity of breastfeeding?

\section{Key Question 2:}

2a. What are the comparative benefits and harms for maternal health outcomes among women who breastfeed for different intensities and durations?

2b. To what extent do benefits and harms for maternal health outcomes differ for subpopulations of women defined by age, race, ethnicity, and comorbidity?

\section{Analytic Framework}

We developed an analytic framework to guide the systematic review process (Figure A). The analytic framework illustrates the population, interventions, outcomes, and adverse effects that guided our literature search and synthesis. 
Figure A. Analytic framework for breastfeeding programs and policies, breastfeeding uptake, and maternal health outcomes in developed countries

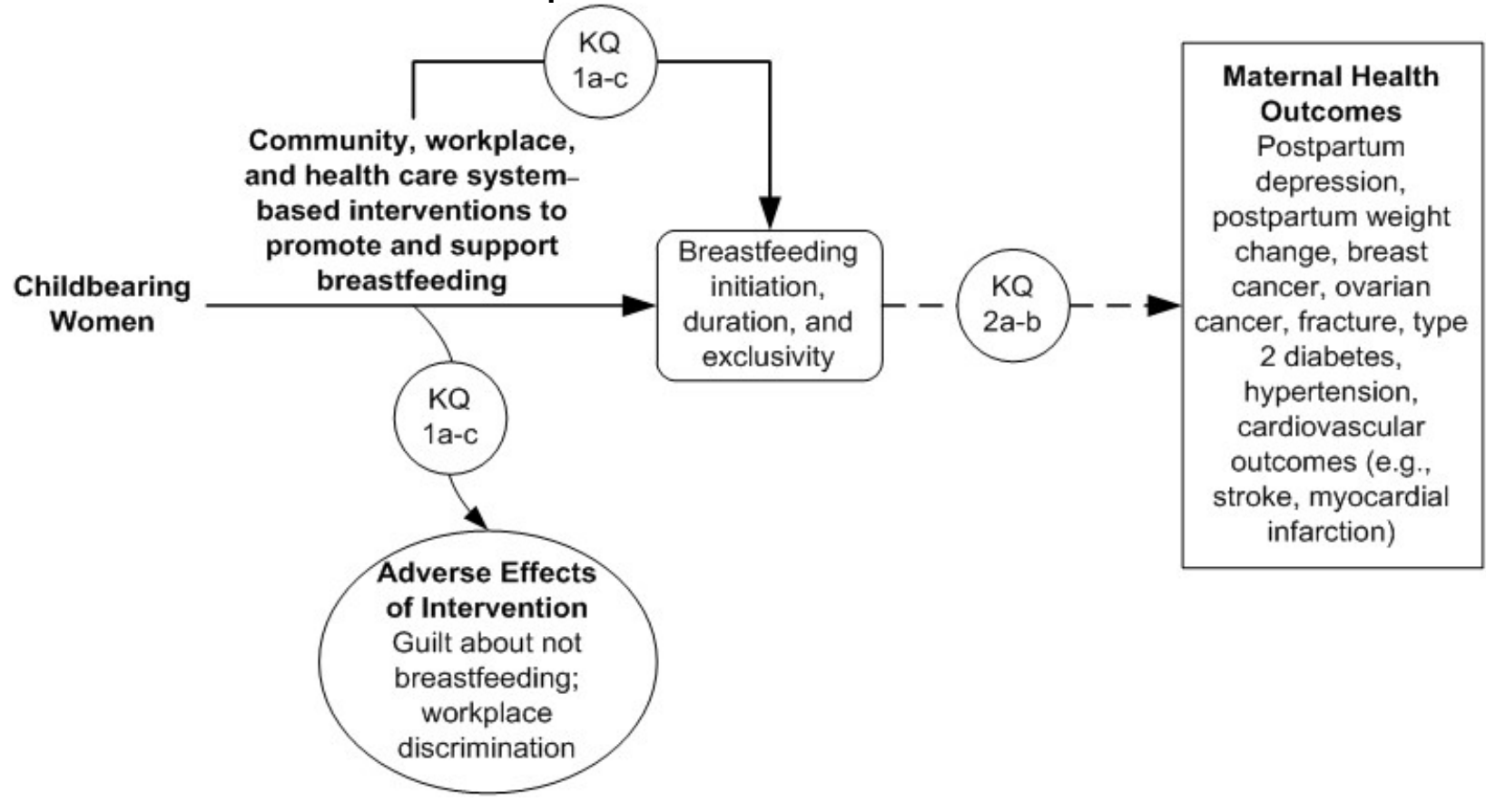

$\mathrm{KQ}=$ Key Question .

\section{Methods}

The initial Key Questions (KQs) were provided by AHRQ and developed in collaboration with partners from the Centers for Disease Control and Prevention (CDC) and National Institutes of Health Office of Women's Health. The Evidence-based Practice Center further refined the KQs. We sought input from a Technical Expert Panel on the final research protocol, which was posted on the AHRQ Web site on March 20, 2017, at https://effectivehealthcare.ahrq.gov/topics/breastfeeding/research-protocol/; our PROSPERO registration number is CRD42017079125.

\section{Literature Search Strategy}

\section{Search Strategy}

For KQ 1, we searched PubMed/MEDLINE, the Cochrane Library, and CINAHL from January 1, 1980, to October 12, 2017, to ensure that evidence is applicable to current breastfeeding policies and practices. For KQ 2, we searched PubMed/MEDLINE, the Cochrane Library, and CINAHL from November 1, 2005 (6 months prior to the search date of the 2007 AHRQ review searches) to October 12, 2017. A full description of the search strategy is provided in the methods section of the full report.

\section{Inclusion and Exclusion Criteria}

Interventions of interest for KQ 1 included any community, workplace, or health care system-based interventions aimed at promoting and supporting breastfeeding. Included studies 
for KQ 1 had to have a concurrent control group or (for single-group pre-post studies) include multiple pre- and post-measures of breastfeeding rates. For KQ1, we included studies conducted in countries categorized as "very high" and "high" human development index per the United Nations Development Programme. ${ }^{30}$

Eligibility criteria for KQ 2 were based on criteria used in the 2007 AHRQ review by Ip and colleagues for maternal health outcomes (postpartum depression, postpartum weight change, breast cancer, ovarian cancer, osteoporotic fracture, and type 2 diabetes). For this update, we also included hypertension and cardiovascular disease. Eligible studies compared groups of women exposed to breastfeeding with those who did not breastfeed (or breastfed for shorter duration and/or less intensity). To maintain consistency with the 2007 review, we limited to studies enrolling women from countries categorized as "very high" human development index per the United Nations Development Programme. ${ }^{30}$ A detailed search strategy is provided in the methods section of the full report.

\section{Risk of Bias Assessment of Individual Studies}

We adapted existing tools (ROBINS- $\mathrm{I}^{31}$ for observational studies, and the Cochrane tool ${ }^{32}$ for trials) and used predefined criteria based on the AHRQ Methods Guide for Effectiveness and Comparative Effectiveness Reviews. Criterion details are included in the full report, including Appendix C. ${ }^{33}$

\section{Risk of Bias Assessment of Systematic Reviews}

We assessed the relevance of systematic reviews published within the past 5 years using predefined criteria. For reviews determined to be relevant, we rated the risk of bias (ROB) as low, unclear, or high ROB using the ROBIS tool. ${ }^{34}$ Appendix $\mathrm{C}$ of the full report lists the specific questions used for evaluating the ROB of all relevant reviews.

\section{Data Synthesis}

For those KQ 2 outcomes for which we included a recent published systematic review rated low or unclear ROB, we first described the results of the review and then summarized data from primary studies published after the latest search date of those reviews. We included systematic reviews for some outcomes (breast cancer, ovarian cancer, and type 2 diabetes) that had conducted meta-analyses. If individual studies identified in our database searches were generally consistent with the pooled results reported by existing systematic reviews, we did not conduct new meta-analyses.

\section{Strength of the Body of Evidence}

We graded the strength of evidence (SOE) based on guidance established for the Evidencebased Practice Center Program. ${ }^{35}$ This approach incorporates five key domains: study limitations (aggregate ROB), consistency, directness, precision, and reporting bias.

\section{Applicability}

We assessed applicability following guidance from the Methods Guide for Effectiveness and Comparative Effectiveness Reviews. ${ }^{36}$ For individual studies, we examined conditions that may limit applicability of evidence such as race or ethnicity of enrolled populations, setting of 
enrolled populations, geographic setting, time period of enrollment, and availability of health insurance and other health-related employment benefits.

\section{Peer Review and Public Commentary}

This report was posted for public comment and peer reviewed. We addressed all comments in the final report, making revisions as needed; a disposition of comments report will be publicly posted 3 months after release of the final report.

\section{Results of Literature Searches}

Searches of all sources identified a total of 11,006 potentially relevant citations. We included 128 unique individual studies (described in 137 publications) and 10 systematic reviews. Of these, 40 individual studies (from 44 publications) were relevant to KQ 1, and 88 individual studies (from 93 publications) and 10 systematic reviews were relevant to KQ 2. Of the KQ 2 included studies, 18 were studies from a prior 2007 AHRQ review addressing the maternal health benefits of breastfeeding. ${ }^{2}$ The remaining 34 studies from the 2007 review were included in at least 1 of our 10 systematic reviews or superseded by a new included study. Appendix B in the full report provides a complete list of articles excluded at the full-text screening stage, with reasons for exclusion.

\section{Effectiveness and Harms of Breastfeeding Programs and Policies}

The 40 studies that met our inclusion criteria evaluated a range of strategies to improve rates of breastfeeding initiation and duration. No included studies assessed the benefit of workplace interventions or the potential harms of interventions. To aid in synthesizing results of similar studies, we categorized interventions primarily based on intervention type: BFHI, other (nonBFHI) health care system-based interventions (e.g., residency curriculum related to breastfeeding), WIC-based interventions, and community-based interventions (not primarily delivered as part of the health care system). In addition to categorizing interventions by intervention type, we also summarized results for breastfeeding initiation and duration separately when we had similar studies reporting on multiple breastfeeding outcome types. Below, we provide a summary of our main conclusions related to the effectiveness of programs and policies for improving rates of breastfeeding initiation and duration organized by intervention type.

\section{BFHI Interventions}

Twelve included studies (described in 13 publications) assessed the effectiveness of BFHI interventions. ${ }^{37-50}$ Studies were conducted in diverse country settings including the United States (2 studies); ${ }^{39,40}$ Taiwan (2 studies); ${ }^{46,50}$ and one each in the Republic of Belarus, ${ }^{37}$ Hong Kong, ${ }^{41}$

Czech Republic, ${ }^{42}$ Russia, ${ }^{51}$ Brazil, ${ }^{44}$ Croatia, ${ }^{45}$ Brazil, ${ }^{49}$ United Kingdom (multiple regions), ${ }^{47}$ and Scotland. ${ }^{52}$ Table B presents key findings and SOE related to the benefit of BFHI interventions. Overall, the evidence supports the effectiveness of BFHI for improving rates of breastfeeding initiation and duration. 
Table B. Summary of key findings and strength of evidence: Studies assessing BFHI

\begin{tabular}{|c|c|c|c|}
\hline $\begin{array}{l}\text { Breastfeeding Outcome } \\
\text { Intervention Versus } \\
\text { Comparator }\end{array}$ & $\begin{array}{l}\mathbf{N} \\
\text { Studies; } \\
\mathrm{N} \\
\text { Subjects } \\
\text { Study } \\
\text { Limitation } \\
\mathrm{S}\end{array}$ & Outcome and Results & $\begin{array}{l}\text { Strength } \\
\text { of } \\
\text { Evidence }\end{array}$ \\
\hline $\begin{array}{l}\text { Initiation } \\
\text { BFHI certified/accredited } \\
\text { vs. no BFHI status }\end{array}$ & $\begin{array}{l}9 \\
\text { cohorts; } 39 \\
40,42,43,46-50 \\
1,227,532 \\
\text { Medium }\end{array}$ & $\begin{array}{l}\text { Any BF initiation }(\mathrm{k}=6) \text { : higher rates of BF at discharge among } \\
\text { BFHI-accredited hospitals than control hospitals (by } 0.5 \% \text { to } \\
10 \% \text {; differences between groups were not statistically } \\
\text { significant in } 4 \text { studies } \\
\text { Exclusive BF initiation }(\mathrm{k}=5) \text { : significantly higher rates of } \\
\text { exclusive BF at discharge among BFHI-accredited hospitals than } \\
\text { control hospitals; magnitude varied, ranging from } 3 \text { to } 56 \%\end{array}$ & $\begin{array}{l}\text { Low for } \\
\text { benefit } \\
\text { (consisten } \\
\text { t, } \\
\text { imprecise) }\end{array}$ \\
\hline $\begin{array}{l}\text { Duration } \\
\text { BFHI vs. no BFHI } \\
\text { intervention (evidence } \\
\text { from RCTs) }\end{array}$ & $\begin{array}{l}1 \mathrm{RCT} ; 37 \\
38 \text { 17,046 }\end{array}$ & $\begin{array}{l}\text { One RCT found significantly higher rates of exclusive BF among } \\
\text { women at BFHI hospitals at } 3 \text { mos ( } 43 \% \text { vs. } 6 \% ; p<0.001) \text { and } 6 \\
\text { mos postpartum ( } 7.9 \% \text { vs. } 0.6 \% ; p=0.01) \text {, and lower odds of } \\
\text { weaning (from any BF) at } 3,6,9 \text {, and } 12 \text { mos postpartum than } \\
\text { women in control hospitals }\end{array}$ & $\begin{array}{l}\text { Moderate } \\
\text { for benefit } \\
\text { (consisten } \\
\text { t, } \\
\text { imprecise) }\end{array}$ \\
\hline $\begin{array}{l}\text { Duration } \\
\text { BFHI certified/accredited } \\
\text { vs. no BFHI status } \\
\text { (evidence from } \\
\text { observational studies) }\end{array}$ & $\begin{array}{l}8 \text { cohorts; } \\
39-41,43,46,47 \\
49,50 \\
136,983 \\
\text { Medium }\end{array}$ & $\begin{array}{l}\text { Any BF duration ( } k=8 \text { cohort studies): higher rates of BF } 1 \text { to } 12 \\
\text { mos postpartum among women at BFHI hospitals (by } \\
\text { approximately } 0.6 \% \text { to } 15 \% \text { ) than women at control hospitals; } \\
\text { one study found slightly higher BF rates at } 1 \text { mo among women } \\
\text { in control hospitals than BFHI hospitals (by } 0.4 \% \text { to } 7 \% \text { ) } \\
\text { Exclusive BF duration ( } k=5 \text { cohort studies): higher rates of } \\
\text { exclusive BF over } 1 \text { to } 2 \text { mos among infants born in BFHI } \\
\text { hospitals than control hospitals (by approximately } 4 \% \text { to } 25 \% \text { ) }\end{array}$ & \\
\hline $\begin{array}{l}\text { Breastfeeding Outcome } \\
\text { Intervention Versus. } \\
\text { Comparator }\end{array}$ & $\begin{array}{l}\text { N Studies; } \\
\text { N } \\
\text { Subjects } \\
\text { Study } \\
\text { Limitations }\end{array}$ & Outcome and Results & $\begin{array}{l}\text { Strength } \\
\text { of } \\
\text { Evidence }\end{array}$ \\
\hline $\begin{array}{l}\text { Duration } \\
\text { Six or more BFHI steps } \\
\text { vs. fewer than six steps }\end{array}$ & $\begin{array}{l}1 \text { cohort; } \\
1,417 \\
\text { Medium }\end{array}$ & $\begin{array}{l}\text { Significantly higher odds of weaning at or before } 8 \text { wks } \\
\text { postpartum among women giving birth in hospitals practicing } \leq \\
\text { four BFHI steps than women giving birth in hospitals practicing } \\
\text { six BFHI steps (ORs ranged from } 2.08 \text { and } 3.13 \text { ); no difference } \\
\text { between women exposed to five vs. six steps }\end{array}$ & $\begin{array}{l}\text { Low for } \\
\text { benefit } \\
\text { (consisten } \\
\mathrm{t}^{\mathrm{a}} \text { precise) }\end{array}$ \\
\hline
\end{tabular}

a Although only one study compared groups of women based on number of BFHI steps practiced by hospitals, we considered evidence on duration from studies comparing BFHI implementation (or accreditation) with nonaccredited hospitals. As shown in the table, we concluded that moderate SOE supports the effectiveness of BFHI for improving breastfeeding duration.

$\mathrm{BF}=$ breastfeeding; $\mathrm{BFHI}=$ Baby-Friendly Hospital Initiative; $\mathrm{HV}=$ home visits; $\mathrm{k}=$ number of studies; $\mathrm{N}=$ number; OR $=$ odds ratio; $\mathrm{RCT}=$ randomized controlled trial; $\mathrm{SOE}=$ strength of evidence.

For breastfeeding initiation, evidence from nine cohort studies (1,227,532 women) comparing women giving birth in BFHI-certified (or accredited) hospitals with noncertified hospitals supports the effectiveness of BFHI (low SOE). Although the included studies consistently found higher rates of initiation at accredited hospitals, results were imprecise and the magnitude of benefit varied by breastfeeding measure and country setting (Table B).

Based on evidence from one large RCT (Promotion of Breastfeeding Intervention Trial [PROBIT], N=17,046) and five cohort studies (62,834 women), we concluded that BFHI increases rates of breastfeeding duration through 12 months postpartum (moderate SOE). In the PROBIT trial, women in the intervention group had significantly higher rates of exclusive breastfeeding and lower rates of weaning across various multiple time points (1 to 12 months postpartum). Although the eight observational studies were mostly consistent in finding benefit 
for BFHI, results were imprecise, and the magnitude of benefit varied by breastfeeding measure and country setting. One cohort study $(\mathrm{N}=1,417)$ compared rates of breastfeeding at 6 months among women discharged from hospitals that differed in the number of BFHI steps implemented; low SOE supports the conclusion that implementation of four or more BFHI steps is associated with lower rates of weaning than implementation of fewer than four steps.

\section{Other (Non-BFHI) Health Care System-Based Interventions}

Fifteen studies (described in 16 publications) assessed the effectiveness of other (non-BFHI) health care system-based interventions. ${ }^{44,}{ }^{53-67}$ Studies were conducted in diverse country settings including the United States (3 studies), ${ }^{59,66,68}$ Canada (1 study), ${ }^{64}$ Sri Lanka (1 study), ${ }^{61}$ Brazil (2 studies), ${ }^{65,69}$ China (1 study), ${ }^{58}$ and various European countries (6 studies). ${ }^{53,56,57,60,62,63}$ Studies assessed a variety of intervention types; the majority focused on health care provider education or training related to breastfeeding, with or without additional services offered (e.g., breastfeeding groups, home visits). Table C presents key findings and SOE conclusions. Overall, the evidence supports the effectiveness of three intervention types for improving the duration of exclusive breastfeeding: modified BFHI policy implementation in outpatient setting (e.g., development of a breastfeeding policy, staff training, outcome assessment, and quality improvement initiatives), continuous nursing care during the perinatal period (the same nurse provides routine perinatal care to the mother and infant), and health care provider education combined with a series of home visits (low SOE). In addition, the evidence suggests that health care provider education and training alone (without additional breastfeeding support services) are not effective in improving rates of breastfeeding initiation (low SOE). As a result of methodological limitations and imprecise and inconsistent findings, we rated the SOE as insufficient for other intervention types.

Table C. Summary of key findings and strength of evidence: Non-BFHI health care system-based interventions

\begin{tabular}{|c|c|c|c|}
\hline $\begin{array}{l}\text { Breastfeeding Outcome } \\
\text { Intervention Versus } \\
\text { Comparator }\end{array}$ & $\begin{array}{l}\text { N Studies; } \mathbf{N} \\
\text { Subjects } \\
\text { Study } \\
\text { Limitations }\end{array}$ & Outcome and Results & Strength of Evidence \\
\hline $\begin{array}{l}\text { Initiation } \\
\text { Education/staff training } \\
\text { related to BF alone vs. } \\
\text { usual practice }\end{array}$ & $\begin{array}{l}4 \text { (2 RCTs, }^{55,56,61} \\
\left.2 \text { NRCTs }^{59,64}\right) \\
1,532^{a} \\
\text { Medium }\end{array}$ & $\begin{array}{l}\text { No significant difference between } \\
\text { intervention and control groups in } \\
\text { rates of any or exclusive BF } \\
\text { initiation }\end{array}$ & $\begin{array}{l}\text { Low for no benefit } \\
\text { (consistent, imprecise) }\end{array}$ \\
\hline $\begin{array}{l}\text { Initiation } \\
\text { Education and staff } \\
\text { training plus additional } \\
\text { individual services vs. } \\
\text { usual care }\end{array}$ & $\begin{array}{l}4\left(2 \text { RCTs, }{ }^{60,63} 1\right. \\
\text { NRCT }^{57} 1 \text { pre- } \\
\left.\text { post study }{ }^{53}\right) ; \\
34,018 \\
\text { Medium }\end{array}$ & $\begin{array}{l}\text { Inconsistent findings across four } \\
\text { studies assessing heterogeneous } \\
\text { interventions }\end{array}$ & $\begin{array}{l}\text { Insufficient (inconsistent, } \\
\text { imprecise) }\end{array}$ \\
\hline $\begin{array}{l}\text { Duration } \\
\text { Education and staff } \\
\text { training related to BF } \\
\text { only vs. usual practice }\end{array}$ & $\begin{array}{l}3 \text { (2 RCTs, }^{55,56,65} \\
\left.1 \text { NRCT }^{59}\right) \\
1,526^{a} \\
\text { Medium }\end{array}$ & $\begin{array}{l}\text { Inconsistent findings across three } \\
\text { studies for duration of any and } \\
\text { exclusive BF }\end{array}$ & $\begin{array}{l}\text { Insufficient (inconsistent, } \\
\text { imprecise) }\end{array}$ \\
\hline
\end{tabular}




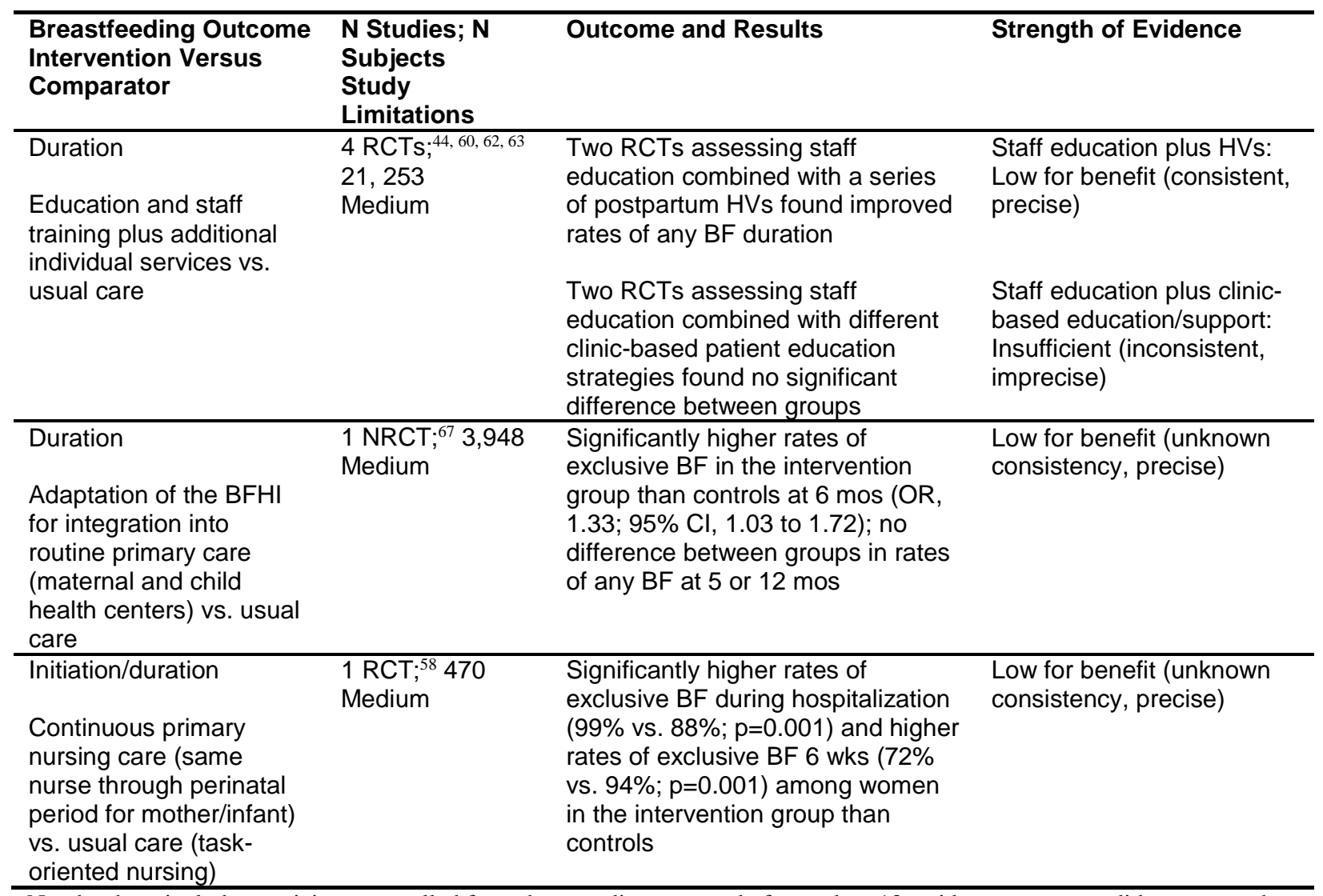

${ }^{a}$ Number here includes participants enrolled from three studies; one study focused on 13 residency programs did not report the number of women included in analyses of breastfeeding outcomes. ${ }^{59}$

$\mathrm{BF}$ = breastfeeding; BFHI = Baby-Friendly Hospital Initiative; $\mathrm{CI}=$ confidence interval; $\mathrm{HV}=$ home visit; $\mathrm{N}=$ number; $\mathrm{NRCT}=$ non-randomized controlled trial; OR = odds ratio; RCT = randomized controlled trial.

\section{WIC-Based Interventions}

Eight included studies assessed changes in breastfeeding rates associated with a WIC program or policy. ${ }^{70-77}$ Although all studies were set in the United States, they included women from diverse States. Included studies assessed heterogeneous interventions and policies; key findings and SOE assessments are shown in Table D. Overall, low SOE supports the effectiveness of WIC-based peer-support programs for improving rates of any breastfeeding initiation and duration from 6 weeks to 6 months postpartum. We found insufficient evidence (primarily because of unknown consistency and imprecision) to make a conclusion on the benefit of other WIC programs or policies for improving breastfeeding outcomes, including policy changes related to WIC food packages, provision of different types of breast pumps (electric vs. manual), tailored counseling, cash incentives, and peer-support programs targeted at fathers. 
Table D. Summary of key findings and strength of evidence: WIC-based interventions

\begin{tabular}{|c|c|c|c|}
\hline $\begin{array}{l}\text { Breastfeeding Outcome } \\
\text { Intervention Versus } \\
\text { Comparator }\end{array}$ & $\begin{array}{l}\text { N Studies; N } \\
\text { Subjects } \\
\text { Study } \\
\text { Limitations }\end{array}$ & Outcome and Results & $\begin{array}{l}\text { Strength } \\
\text { of } \\
\text { Evidence }\end{array}$ \\
\hline $\begin{array}{l}\text { Initiation/duration } \\
\text { Mother peer support vs. } \\
\text { control }\end{array}$ & $\begin{array}{l}3\left(1 \mathrm{RCT},{ }^{73} 1\right. \\
\text { NRCT, }^{74} 1 \\
\left.\text { cohort }^{75}\right) ; 2,480 \\
\text { Medium }\end{array}$ & $\begin{array}{l}\text { Two studies of in-person peer support resulted in } \\
\text { significantly higher rates of BF initiation and } \\
\text { increased BF duration; one telephone-based } \\
\text { peer-support study found significantly higher } \\
\text { rates of any BF at } 3 \text { and } 6 \text { mos than controls }\end{array}$ & $\begin{array}{l}\text { Low for } \\
\text { benefit } \\
\text { (consisten } \\
\text { t, precise) }\end{array}$ \\
\hline $\begin{array}{l}\text { Initiation/duration } \\
\text { BF rates post- } 2007 \text { policy } \\
\text { revising the WIC food } \\
\text { package vs. pre-policy } \\
\text { implementation }\end{array}$ & $\begin{array}{l}1 \text { (3 pop. } \\
\text { cohorts); } 71 \\
\text { PRAMS } \\
\text { (127,477) } \\
\text { NIS (73,991) } \\
\text { PedNSS (744 } \\
\text { infants): } 744 \\
\text { High }\end{array}$ & $\begin{array}{l}\text { No association between the policy change and } \\
\text { rates of BF;a BF rates increased overall with no } \\
\text { difference between women receiving WIC } \\
\text { benefits and similar groups of women not } \\
\text { receiving WIC benefits }\end{array}$ & $\begin{array}{l}\text { Insufficient } \\
\text { (high } \\
\text { ROB, } \\
\text { unknown } \\
\text { consistenc } \\
\text { y, } \\
\text { imprecise) }\end{array}$ \\
\hline $\begin{array}{l}\text { Duration } \\
\text { Provision of electric breast } \\
\text { pump vs. manual pump }\end{array}$ & $\begin{array}{l}1 \mathrm{RCT} ;{ }^{70} 280 \\
\text { Medium }\end{array}$ & $\begin{array}{l}\text { No difference in BF duration among women } \\
\text { assigned to an electric vs. manual breast pump; } \\
\text { median duration of BF was } 12 \text { vs. } 11 \text { mos, } \\
\text { respectively (HR,1.13; } 95 \% \mathrm{Cl}, 0.79 \text { to } 1.50)\end{array}$ & $\begin{array}{l}\text { Insufficient } \\
\text { (unknown } \\
\text { consistency } \\
\text {, imprecise) }\end{array}$ \\
\hline $\begin{array}{l}\text { Initiation/duration } \\
\text { Peer-support program for } \\
\text { fathers (in addition to } \\
\text { mother peer support) vs. } \\
\text { peer support for mothers } \\
\text { alone }\end{array}$ & $\begin{array}{l}1 \text { NRCT; } 72200 \\
\text { Medium }\end{array}$ & $\begin{array}{l}\text { Mothers in the intervention group had slightly } \\
\text { higher rate of any BF at } 6 \text { mos than controls } \\
(63 \% \text { vs. } 55 \%) \text { that was not statistically } \\
\text { significant }(p=0.20)\end{array}$ & $\begin{array}{l}\text { Insufficient } \\
\text { (unknown } \\
\text { consistency } \\
\text {, imprecise) }\end{array}$ \\
\hline $\begin{array}{l}\text { Duration } \\
\text { Cash incentives vs. usual } \\
\text { WIC services }\end{array}$ & $\begin{array}{l}1 \mathrm{RCT} ;{ }^{77} 36 \\
\text { Medium }\end{array}$ & $\begin{array}{l}\text { BF rates in the intervention group were } \\
\text { significantly higher than controls at } 1,3 \text {, and } 6 \\
\text { months ( } 89 \% \text { vs. } 44 \%, 89 \% \text { vs. } 17 \% \text {, and } 72 \% \\
\text { vs. } 0 \% \text {, respectively) }\end{array}$ & $\begin{array}{l}\text { Insufficient } \\
\text { (unknown } \\
\text { consistency } \\
\text {; precise) }\end{array}$ \\
\hline $\begin{array}{l}\text { Duration } \\
\text { Tailored BF counseling and } \\
\text { support based on BAPT } \\
\text { survey }\end{array}$ & $\begin{array}{l}1 \text { cohort; }{ }^{76} 826 \\
\text { High }\end{array}$ & $\begin{array}{l}\text { Significantly higher rates of exclusive BF in the } \\
\text { intervention group at } 7 \text { and } 30 \text { days than } \\
\text { controls; no difference between groups at } 2 \text { mos }\end{array}$ & $\begin{array}{l}\text { Insufficient } \\
\text { (high ROB, } \\
\text { unknown } \\
\text { consistency } \\
\text {, imprecise) }\end{array}$ \\
\hline
\end{tabular}

a All three databases measured rates of "ever-breastfeeding"; in addition, PRAMS measured rates of breastfeeding for at least 4 weeks, NIS measured rates of breastfeeding for at least 3 months, and PedNSS measured rates of breastfeeding for at least 1 month. Conclusions were consistent across the different measures.

BAPT = Breastfeeding Attrition Prediction Tool; BF = breastfeeding; $\mathrm{CI}$ = confidence interval; $\mathrm{HR}=$ hazard ratio; $\mathrm{N}=$ number; NIS = National Immunization Survey; NRCT = non-randomized controlled trial; PedNSS = Pediatric Nutrition Surveillance System; PRAMS = Pregnancy Risk Assessment Monitoring System; RCT = randomized controlled trial; ROB= risk of bias; WIC = Special Supplemental Nutrition Program for Women, Infants and Children.

\section{Community-Based Interventions}

Five included studies (described in 7 publications) assessed the effectiveness of a community-based intervention; ${ }^{78-84}$ key findings and SOE assessments are shown in Table E. Studies were conducted in diverse country settings including one each in Italy, ${ }^{79}$ Australia, ${ }^{80}$ Mexico, ${ }^{82}$ Chile, ${ }^{84}$ and Canada. ${ }^{83}$ No studies assessed the same intervention type, which limited our ability to make conclusions on the SOE for most intervention types. Low SOE supports the benefit of community-based interventions that provide mothers with peer-support (via home visits). In addition, access to a community-based breastfeeding drop-in center among women receiving early home-based breastfeeding support does not increase breastfeeding duration (low SOE). 
Table E. Summary of key findings and strength of evidence: Community-based interventions

\begin{tabular}{|c|c|c|c|}
\hline $\begin{array}{l}\text { Breastfeeding Outcome } \\
\text { Intervention Versus } \\
\text { Comparator }\end{array}$ & $\begin{array}{l}\text { N Studies; N } \\
\text { Subjects } \\
\text { Study } \\
\text { Limitations }\end{array}$ & Outcome and Results & $\begin{array}{l}\text { Strength of } \\
\text { Evidence }\end{array}$ \\
\hline Initiation/duration & $\begin{array}{l}1 \mathrm{NRCT} ;{ }^{78,79} \\
5,094\end{array}$ & $\begin{array}{l}\text { No significant difference in rates of exclusive BF at } \\
\text { discharge, } 3 \text { and } 6 \text { mos, or rates of any BF at } 5 \text { and }\end{array}$ & $\begin{array}{l}\text { Insufficient } \\
\text { (unknown }\end{array}$ \\
\hline $\begin{array}{l}\text { Community-based policy aimed } \\
\text { at promoting BF in nonhospital- } \\
\text { based health and community } \\
\text { centers vs. no intervention }\end{array}$ & Medium & 12 mos between groups & $\begin{array}{l}\text { consistency, } \\
\text { imprecise) }\end{array}$ \\
\hline Duration & $\begin{array}{l}\text { 1 RCT; } 80,81 \\
9,675\end{array}$ & $\begin{array}{l}\text { No difference between groups in rates of any BF at } \\
3,4 \text {, or } 5 \text { mos. }\end{array}$ & $\begin{array}{l}\text { Low for no } \\
\text { benefit }\end{array}$ \\
\hline $\begin{array}{l}\text { Access to community-based BF } \\
\text { drop-in centers (plus early BF } \\
\text { support) vs. early BF support } \\
\text { alone vs. usual care }\end{array}$ & Low & & $\begin{array}{l}\text { (unknown } \\
\text { consistency, } \\
\text { precise) }\end{array}$ \\
\hline Duration & $\begin{array}{l}1 \mathrm{RCT} ;{ }^{82} \\
130\end{array}$ & $\begin{array}{l}\text { Significantly higher rates of exclusive BF at } 3 \text { mos } \\
\text { among intervention groups ( } 50 \% \text { to } 67 \% \text { ) than }\end{array}$ & $\begin{array}{l}\text { Low for benefit } \\
\text { (unknown }\end{array}$ \\
\hline $\begin{array}{l}\text { Community-based peer support } \\
\text { vs. usual care }\end{array}$ & Low & $\begin{array}{l}\text { control group }(12 \%), p<0.001 \text {; rates of any BF were } \\
\text { significantly longer in intervention groups } \\
\text { (combined) than in the control group at } 3 \text { mos (but } \\
\text { not } 6 \text { mos) }\end{array}$ & $\begin{array}{l}\text { consistency, } \\
\text { precise) }\end{array}$ \\
\hline $\begin{array}{l}\text { Duration } \\
\text { Peer-led BF support class vs. } \\
\text { Nurse-led BF support class }\end{array}$ & $\begin{array}{l}1 \text { cohort; }^{83} \\
109 \\
\text { High }\end{array}$ & $\begin{array}{l}\text { No significant difference between groups in rates of } \\
\text { any BF at } 1 \text { and } 6 \text { mos postpartum }\end{array}$ & $\begin{array}{l}\text { Insufficient } \\
\text { (high ROB, } \\
\text { unknown } \\
\text { consistency, } \\
\text { imprecise) }\end{array}$ \\
\hline $\begin{array}{l}\text { Duration } \\
\text { Integrated postpartum program } \\
\text { (BF education and support, } \\
\text { maternal/infant health care) vs. } \\
\text { usual care }\end{array}$ & $\begin{array}{l}1 \text { NRCT; } 84 \\
392 \\
\text { High }\end{array}$ & $\begin{array}{l}\text { Significantly higher rates of exclusive BF at } 6 \text { mos } \\
\text { among the intervention group than control group } \\
(74 \% \text { vs. } 10 \% ; p=0.001)\end{array}$ & $\begin{array}{l}\text { Insufficient } \\
\text { (high ROB, } \\
\text { unknown } \\
\text { consistency, } \\
\text { precise) }\end{array}$ \\
\hline
\end{tabular}

\section{Effectiveness and Harms of Breastfeeding Programs and Policies for Subpopulations of Women}

Few studies reported on subgroups of women. Of the four included studies reporting on subgroups of women, two focused on BFHI and reported on differences by education status, ${ }^{39,40}$ one focused on a WIC peer-support intervention and reported on subgroups by language spoken (Spanish only vs. English), ${ }^{73}$ and one prospective cohort study assessed a tailored breastfeeding counseling intervention. ${ }^{76}$ Table F shows our key findings and SOE related to subgroups of women. Low SOE supports the conclusion that BFHI effectiveness may vary among women who differ by education status. For WIC interventions, we found insufficient evidence to make a conclusion on whether benefit of telephone peer support varies by subgroups of women based on language spoken (Spanish only vs. English) or whether benefit of tailored breastfeeding counseling intervention varies by race/ethnicity, primarily because of unknown consistency (and inconsistency across time points) and imprecision. 
Table F. Summary of key findings and strength of evidence: KQ 1 studies reporting on subgroups

\begin{tabular}{|c|c|c|c|}
\hline $\begin{array}{l}\text { Breastfeeding Outcome } \\
\text { Intervention Versus } \\
\text { Comparator }\end{array}$ & $\begin{array}{l}\text { N Studies; } \\
\text { N Subjects } \\
\text { Study } \\
\text { Limitations }\end{array}$ & Outcome and Results & $\begin{array}{l}\text { Strength of } \\
\text { Evidence }\end{array}$ \\
\hline $\begin{array}{l}\text { Initiation (subgroups: } \\
\text { education status) } \\
\text { BFHI certified/accredited vs. } \\
\text { no BFHI status }\end{array}$ & $\begin{array}{l}2 \text { cohort; } 39,40 \\
27,341 \\
\text { Medium }\end{array}$ & $\begin{array}{l}\text { Higher rates of BF initiation found among women with } \\
\text { lower education ( } \leq 12 \text { yrs) at BFHI hospitals compared } \\
\text { with control hospitals, but no difference in rates among } \\
\text { women with higher education ( } \geq 13 \text { yrs) }\end{array}$ & $\begin{array}{l}\text { Low } \\
\text { (consistent, } \\
\text { imprecise) }\end{array}$ \\
\hline $\begin{array}{l}\text { Duration (subgroups: } \\
\text { education status) } \\
\text { BFHI certified/accredited vs. } \\
\text { no BFHI status }\end{array}$ & $\begin{array}{l}2 \text { cohort; } 39,40 \\
27,341 \\
\text { Medium }\end{array}$ & Two studies found mixed results. & $\begin{array}{l}\text { Insufficient } \\
\text { (inconsistent, } \\
\text { imprecise) }\end{array}$ \\
\hline $\begin{array}{l}\text { Initiation/duration } \\
\text { (subgroups: language } \\
\text { spoken) } \\
\text { Mother peer support vs. } \\
\text { control }\end{array}$ & $\begin{array}{l}1 \mathrm{RCT} ;{ }^{73} \\
1948 \\
\text { Medium }\end{array}$ & $\begin{array}{l}\text { One RCT of telephone peer support found mixed } \\
\text { results for subgroups of women defined by language } \\
\text { (English-speaking vs. Spanish-speaking only) }\end{array}$ & $\begin{array}{l}\text { Insufficient } \\
\text { (unknown } \\
\text { consistency, } \\
\text { imprecise) }\end{array}$ \\
\hline $\begin{array}{l}\text { Duration (subgroups: } \\
\text { race/ethnicity) } \\
\text { Tailored BF counseling and } \\
\text { support based on BAPT } \\
\text { survey }\end{array}$ & $\begin{array}{l}1 \text { cohort; } \\
826 \\
\text { High }\end{array}$ & $\begin{array}{l}\text { Significantly higher rates of exclusive BF among non- } \\
\text { Hispanic black and Hispanic women in the intervention } \\
\text { group than controls at } 1 \text { and } 2 \text { mos; no significant } \\
\text { difference in exclusive BF rates among white women at } \\
\text { any time point }\end{array}$ & $\begin{array}{l}\text { Insufficient } \\
\text { (high ROB, } \\
\text { unknown } \\
\text { consistency, } \\
\text { precise) }\end{array}$ \\
\hline
\end{tabular}

\section{Effect of Intervention Characteristics on Breastfeeding Outcomes}

This KQ focused on the extent to which intervention-related characteristics (e.g., type of breast pump provided-manual or electric, delivery personnel) influence the initiation, duration, and exclusivity of breastfeeding. We found no evidence to address this KQ.

\section{Maternal Health Outcomes Associated With Breastfeeding}

Table G summarizes our key findings related to KQ 2, including evidence for subpopulations of women, by outcome. Low SOE supports the conclusion that ever breastfeeding, as well as longer durations of breastfeeding, may be associated with a reduced risk of developing (any) breast cancer, luminal breast cancer, or triple-negative breast cancer. Despite a large body of observational evidence, study and participant characteristics and methodological limitations did not explain the significant heterogeneity of results. Low SOE supports the association between ever breastfeeding, as well as longer versus shorter durations of breastfeeding, and a reduced risk of developing epithelial ovarian cancer. The body of evidence is relatively large and includes one systematic review of 41 studies and 8 additional studies (39,618 women); however, we rated SOE as low because the results included significant heterogeneity not explained by study and participant characteristics and methodological limitations.

Table G. Summary of key findings and strength of evidence: Maternal health outcomes

\begin{tabular}{llll}
\hline $\begin{array}{l}\text { Maternal Health } \\
\text { Outcome }\end{array}$ & $\begin{array}{l}\text { N Studies; N Subjects } \\
\text { Study Limitations }\end{array}$ & Outcome and Results ${ }^{\mathrm{a}}$ & $\begin{array}{l}\text { Strength of } \\
\text { Evidence }\end{array}$ \\
\hline Breast cancer & $\begin{array}{l}\text { 1 SR of } 98 \text { cohort/case- } \\
\text { control studies; }{ }^{1} \mathrm{NR}^{\mathrm{b}}\end{array}$ & $\begin{array}{l}\text { Consistent association in one SR (98 } \\
\text { observational studies) between ever BF and }\end{array}$ & $\begin{array}{l}\text { Low for } \\
\text { beneficial }\end{array}$ \\
\hline
\end{tabular}




\begin{tabular}{|c|c|c|c|}
\hline $\begin{array}{l}\text { Maternal Health } \\
\text { Outcome }\end{array}$ & $\begin{array}{l}\text { N Studies; N Subjects } \\
\text { Study Limitations }\end{array}$ & Outcome and Results ${ }^{a}$ & $\begin{array}{l}\text { Strength of } \\
\text { Evidence }\end{array}$ \\
\hline & $\begin{array}{l}19 \text { cohort/case-control } \\
\text { studies; } 85-103256,891 \\
\text { women } \\
\text { Medium }\end{array}$ & $\begin{array}{l}\text { lower rates of breast cancer compared with } \\
\text { never BF (pooled OR } 0.78,95 \% \mathrm{Cl} 0.74 \text { to } \\
0.82 \text { ); longer durations of BF was also } \\
\text { associated with significantly lower rates of } \\
\text { breast cancer than never BF. Results of } \\
\text { individual studies were generally consistent in } \\
\text { direction of effect (although results were } \\
\text { imprecise); magnitude varied significantly across } \\
\text { all studies and pooled results were associated } \\
\text { with significant heterogeneity, only partially } \\
\text { explained by subgroup analyses. }\end{array}$ & $\begin{array}{l}\text { association } \\
\text { (consistent, } \\
\text { imprecise) }\end{array}$ \\
\hline $\begin{array}{l}\text { Breast cancer: } \\
\text { BRCA1/2 carriers }\end{array}$ & $\begin{array}{l}1 \text { case-control study; }{ }^{104} \\
5,708 \text { women } \\
\text { Medium }\end{array}$ & $\begin{array}{l}\text { Unclear association between BF and breast } \\
\text { cancer among } B R C A \text { carriers. }\end{array}$ & $\begin{array}{l}\text { Insufficient } \\
\text { (unknown } \\
\text { consistency, } \\
\text { imprecise) }\end{array}$ \\
\hline $\begin{array}{l}\text { Breast cancer: In } \\
\text { situ }\end{array}$ & $\begin{array}{l}3 \text { cohort/case-control } \\
\text { studies; } 96,99,10567,234 \\
\text { women } \\
\text { Medium }\end{array}$ & $\begin{array}{l}\text { Unclear association between BF and breast } \\
\text { cancer in situ. }\end{array}$ & $\begin{array}{l}\text { Insufficient } \\
\text { (inconsistent, } \\
\text { imprecise) }\end{array}$ \\
\hline $\begin{array}{l}\text { Breast cancer: } \\
\text { Hormone receptor } \\
\text { subtypes }\end{array}$ & $\begin{array}{l}1 \text { SR of } 11 \text { cohort/case- } \\
\text { control studies; } 106 \\
169,879 \text { women for } \\
\text { luminal, } 14,266 \text { women } \\
\text { for HER2, and } 176,430 \\
\text { women for triple- } \\
\text { negative analyses } \\
7 \text { cohort/case-control } \\
\text { studies; } 91,95,102,107-110 \\
592,558 \text { women } \\
\text { Medium }\end{array}$ & $\begin{array}{l}\text { Consistent association between ever BF or } \\
\text { longer duration of BF and lower rates of luminal } \\
\text { and triple negative breast cancer (although } \\
\text { magnitude of association varies); for HER2, } \\
\text { pooled estimates show unclear association } \\
\text { between BF and lower rates of breast cancer } \\
\text { (results are imprecise and pooled estimate is not } \\
\text { statistically significant). }\end{array}$ & $\begin{array}{l}\text { Low for } \\
\text { beneficial } \\
\text { association } \\
\text { (luminal, } \\
\text { triple- } \\
\text { negative; } \\
\text { consistent, } \\
\text { imprecise); } \\
\text { insufficient } \\
\text { (HER2, } \\
\text { inconsistent, } \\
\text { imprecise) }\end{array}$ \\
\hline $\begin{array}{l}\text { Breast cancer: } \\
\text { Mortality }\end{array}$ & $\begin{array}{l}1 \text { cohort study; } 111 \\
250,470 \text { parous women } \\
\text { Medium }\end{array}$ & $\begin{array}{l}\text { Unclear association; one study found no } \\
\text { significant association between } \mathrm{BF} \text { and breast } \\
\text { cancer mortality (HR,1.01; } 95 \% \mathrm{Cl}, 0.79 \text { to } 1.29 \text { ). }\end{array}$ & $\begin{array}{l}\text { Insufficient } \\
\text { (unknown } \\
\text { consistency, } \\
\text { imprecise) }\end{array}$ \\
\hline Ovarian cancer & $\begin{array}{l}1 \text { SR of } 41 \text { cohort/case- } \\
\text { control studies; }{ }^{1} \mathrm{NR}^{\mathrm{c}} \\
9 \text { cohort/case-control } \\
\text { studies; }{ }^{112-121} 42,611 \\
\text { women } \\
\text { Medium }\end{array}$ & $\begin{array}{l}\text { Consistent association between ever BF and } \\
\text { longer durations of BF and lower risk of ovarian } \\
\text { cancer; magnitude of association varies across } \\
\text { studies by BF exposure definition. }\end{array}$ & $\begin{array}{l}\text { Moderate for } \\
\text { beneficial } \\
\text { association } \\
\text { (inconsistent, } \\
\text { precise) }\end{array}$ \\
\hline
\end{tabular}




\begin{tabular}{|c|c|c|c|}
\hline Intervention type & $\begin{array}{l}\text { N Studies; N Subjects } \\
\text { Study Limitations }\end{array}$ & Outcome and Results & $\begin{array}{l}\text { Strength of } \\
\text { Evidence }\end{array}$ \\
\hline Hypertension & $\begin{array}{l}5 \text { cohort studies; } 4,5,122- \\
124441,989 \text { women } \\
\text { Medium }\end{array}$ & $\begin{array}{l}\text { Consistent association between longer duration } \\
\text { of BF (>6-12 mos) and lower rates of HTN; } \\
\text { magnitude of association varies by BF exposure } \\
\text { comparisons and study design. }\end{array}$ & $\begin{array}{l}\text { Low for } \\
\text { beneficial } \\
\text { association } \\
\text { (consistent, } \\
\text { imprecise) }\end{array}$ \\
\hline CVD & $\begin{array}{l}3 \text { cohort studies; } ;, 6,125 \\
301,989 \text { women } \\
\text { Medium }\end{array}$ & $\begin{array}{l}\text { Unclear association between BF and CVD; three } \\
\text { studies conclude an association between longer } \\
\text { BF duration and lower CVD rates, each using a } \\
\text { different composite outcome. Magnitude of } \\
\text { association varies by exposure comparisons, } \\
\text { age at cohort enrolment, and study design. }\end{array}$ & $\begin{array}{l}\text { Insufficient } \\
\text { (unknown } \\
\text { consistency, } \\
\text { imprecise) }\end{array}$ \\
\hline CVD mortality & $\begin{array}{l}1 \text { cohort study; } \\
15,000 \text { women } \\
\text { Medium }\end{array}$ & $\begin{array}{l}\text { Unclear association between BF and CVD } \\
\text { mortality. One study found mixed results: parous } \\
\text { women } \leq 65 \text { yrs at enrollment who had never BF } \\
\text { had higher CVD mortality over } 14 \text { yrs of followup } \\
\text { than women who BF } \geq 24 \text { mos (HR } 2.77 ; 95 \% \mathrm{Cl} \text {, } \\
1.28 \text { to } 5.99 \text { ). No clear associations were } \\
\text { observed among women } \leq 65 \text { yrs at enrollment. }\end{array}$ & $\begin{array}{l}\text { Insufficient } \\
\text { (unknown } \\
\text { consistency, } \\
\text { imprecise) }\end{array}$ \\
\hline Type 2 diabetes & $\begin{array}{l}1 \text { SR of } 6 \text { cohort } \\
\text { studies; } 127273,961 \\
\text { women } \\
5 \text { cohort studies; }{ }^{4,128-132} \\
325,815 \text { women } \\
\text { Medium }\end{array}$ & $\begin{array}{l}\text { Consistent association between ever BF and } \\
\text { longer durations of BF and lower rates of type } 2 \\
\text { diabetes (among women with and without } \\
\text { gestational diabetes); magnitude of association } \\
\text { varies by BF exposure duration and study } \\
\text { design. }\end{array}$ & $\begin{array}{l}\text { Low for } \\
\text { beneficial } \\
\text { association } \\
\text { (consistent, } \\
\text { imprecise) }\end{array}$ \\
\hline Fractures & $\begin{array}{l}11 \text { cohort/case-control } \\
\text { studies):133-143 } 101,726 \\
\text { women } \\
\text { Medium }\end{array}$ & $\begin{array}{l}\text { Consistent lack of association between BF and } \\
\text { fractures. Magnitude varies by exposure and } \\
\text { outcome measure, but only } 1 \text { high ROB study } \\
\text { reported statistically significant differences. }\end{array}$ & $\begin{array}{l}\text { Low for no } \\
\text { association } \\
\text { (consistent, } \\
\text { imprecise) }\end{array}$ \\
\hline $\begin{array}{l}\text { Postpartum } \\
\text { depression }\end{array}$ & $\begin{array}{l}1 \text { SR of } 48 \text { cohort } \\
\text { studies; } 14471,245 \\
\text { women } \\
14 \text { cohort studies; }{ }^{145-158} \\
39,372 \text { women } \\
\text { Medium }\end{array}$ & $\begin{array}{l}\text { Unclear association between BF and postpartum } \\
\text { depression. Magnitude of association and } \\
\text { direction of effect unclear; studies are } \\
\text { heterogeneous in design and results } \\
\text { inconsistent. }\end{array}$ & $\begin{array}{l}\text { Insufficient } \\
\text { (unknown } \\
\text { consistency, } \\
\text { imprecise) }\end{array}$ \\
\hline $\begin{array}{l}\text { Postpartum weight } \\
\text { change }\end{array}$ & $\begin{array}{l}16 \text { cohort studies; } ;^{159-177} \\
47,655 \text { women } \\
\text { Medium }\end{array}$ & $\begin{array}{l}\text { Unclear association between BF and postpartum } \\
\text { weight change. Magnitude of postpartum weight } \\
\text { change varies by BF exposure and outcome } \\
\text { measure. }\end{array}$ & $\begin{array}{l}\text { Insufficient } \\
\text { (inconsistent, } \\
\text { imprecise) }\end{array}$ \\
\hline
\end{tabular}

${ }^{a}$ We marked outcomes as indirect for long-term maternal health outcomes primarily due to uncertainty of the relative contribution of breastfeeding to risk (given that many other potential factors also contribute to outcomes such as hypertension, fracture, and breast cancer); for short-term maternal health outcomes (e.g., postpartum depression) there is uncertainty in the direction of effect between breastfeeding and health outcomes.

b Per authors, there were 52 studies with $>1,500$ women, 31 studies with 500-1,499 women, and 15 studies with <500 women. Exact number of participants is unclear.

${ }^{c}$ Per authors, there were 22 studies with $>1,500$ women, 12 studies with 500-1,499 women, and 7 studies with $<500$ women. Exact number of participants is unclear.

$\mathrm{BF}$ = breastfeeding; $\mathrm{CI}$ = confidence interval; CVD = cardiovascular disease; HER2 = human epidermal growth factor receptor 2; $\mathrm{HR}=$ hazard ratio; HTN = hypertension; $\mathrm{N}$ = number; $\mathrm{NR}=$ not reported; $\mathrm{ROB}=$ risk of bias; $\mathrm{SR}=$ systematic review .

For both hypertension and type 2 diabetes, studies varied in terms of outcomes and case definition; however, evidence was consistent in finding an association between longer duration of breastfeeding and lower rates of hypertension and type 2 diabetes (low SOE for both outcomes). 
Eleven studies reported on the association between breastfeeding using different measures (e.g., ever versus never and duration per child) and hip, vertebral, and forearm fracture risk. Apart from two studies (rated high ROB), no study reported a statistically significant association between breastfeeding and fracture. We rated the SOE as low for no association.

Because of significant heterogeneity in study design, breastfeeding exposure definitions, outcomes, and inconsistency in results, we found insufficient evidence on whether breastfeeding is associated with postpartum depression or postpartum weight change. For postpartum depression, current evidence does not establish the direction of relationship between breastfeeding and higher or lower rates of postpartum depression.

\section{Discussion and Findings in Context}

For KQ 1, our findings related to the benefit of BFHI for improving breastfeeding initiation and duration support continued efforts to implement this policy. Because of heterogeneity in study design, country setting, and outcome measures, we were not able to pool results. The absolute difference in rates of breastfeeding initiation and duration vary by setting and are likely influenced by a range of factors, such as intervention fidelity, social factors, and others. Although our scope is narrower (in terms of eligible country setting and study design), our conclusions are consistent with a recent narrative review ${ }^{178}$ focused on BFHI; the authors concluded that adherence to the BFHI Ten Steps has a positive influence on breastfeeding outcomes. In terms of other health care interventions, staff training alone (without other breastfeeding support components) did not lead to improved breastfeeding outcomes. However, health care interventions that pair staff education with other services, such as a series of home visits, lead to improved rates of exclusive breastfeeding duration. For workplace interventions, we looked for both trials and observational studies with a control group and still found no eligible studies; the absence of eligible evidence precludes us from commenting on the effectiveness of workplace breastfeeding interventions. In 2012 the Affordable Care Act required large employers to provide reasonable break time and a private place for expressing breastmilk, and mandated insurance coverage of lactation support services and equipment without costsharing for new health insurance policies. Without adequate time to express breastmilk in the workplace, working mothers would face significant barriers to breastfeeding. Future studies (as noted below) could address whether certain workplace interventions are more effective than others in improving breastfeeding duration among working mothers.

For other intervention types, our results show that WIC programs providing in-person or telephone peer support improve breastfeeding outcomes. We also identified evidence on a range of other WIC programs (e.g., cash incentives, provision of different types of breast pumps, and changes in food package policies); however, primarily as a result of unknown consistency and imprecision, we had insufficient evidence to make a conclusion regarding the benefit of these interventions. We identified no eligible studies assessing workplace breastfeeding interventions; other reviews have highlighted the lack of controlled trials of workplace interventions for promoting breastfeeding in employed women. ${ }^{16}$

Our conclusions related to the maternal benefits of breastfeeding (KQ 2) suggest that breastfeeding is associated with lower rates of breast cancer, ovarian cancer, hypertension, and type 2 diabetes. The potential to improve maternal health could be highlighted as a rationale for improving rates of breastfeeding by health care and public health practitioners. For cardiometabolic outcomes, it has been hypothesized that lactation "resets" maternal metabolism after pregnancy, thereby reducing cardiovascular disease risk. ${ }^{179}$ Our conclusions related to 
hypertension and type 2 diabetes support this hypothesis. Results of our current review are, in general, consistent with those in previous reviews with respect to conclusions about the limitations of the evidence base. As was the case in 2007, we are not able to make a conclusion about the association between breastfeeding and postpartum weight change or postpartum depression (because of study limitations and imprecise and inconsistent results). For this review, we added two additional maternal health outcomes: hypertension and cardiovascular disease. We concluded that low SOE supports the association between breastfeeding and reduced hypertension; however, primarily because of heterogeneity in outcome measures and study limitations, we concluded that evidence was insufficient to reach a conclusion about cardiovascular disease.

\section{Limitations of the Review Process}

For KQ 1, we looked for and included a broad range of interventions to promote and support breastfeeding. At the same time, we specifically excluded primary care-relevant interventions delivered to individual women (to avoid duplicating a recent review conducted for the USPSTF). ${ }^{26}$ The studies that met our inclusion criteria assessed a variety of different intervention types. As a result of the inclusion criteria we used, we may have excluded some interventions that could be considered system level or community based. The breadth of our eligibility criteria was also a limitation in terms of evidence synthesis; included studies may have been categorized in different ways. We chose to focus on intervention type and setting because these may be important factors for decisionmakers who plan to implement breastfeeding programs and policies. For KQ 2, we chose to include recent, relevant systematic reviews in our evidence synthesis. Although including these reviews may improve efficiency, this approach has limitations. Some included systematic reviews do not fully report details related to methods (particularly ROB assessment). Because KQ 2 was an update of the 2007 Ip review, we limited our search to very high-income countries; as a result, a secondary analysis of maternal obesity and hypertension from the PROBIT study was excluded from the KQ 2 review.

\section{Limitations of the Evidence Base}

For KQ 1, we found no evidence on certain types of interventions (e.g., workplace and school-based interventions), limited evidence for subgroups of women, and no included studies reported on potential harms of interventions. Studies used various definitions of breastfeeding initiation and exclusivity, which may limit the comparability of findings. In addition, because of heterogeneity across studies, we were not able to assess whether certain characteristics of interventions have a greater influence on breastfeeding initiation, duration, and exclusivity. We were also not able to determine whether heterogeneity within some categories of interventions such as BFHI is due to study design, differences in outcome measures, or country setting (since variation exists across all these factors). Factors most likely to limit the applicability of the evidence include country setting, community breastfeeding rates, variation in usual maternity care practices (including other policies and practices to support breastfeeding), and potentially socioeconomic factors.

For KQ 2, although we found a large volume of evidence supporting the association between breastfeeding and improved maternal health, methodological limitations specific to observational study designs limit the ability to determine the magnitude of effect that lactation has on maternal health outcomes. Although a growing literature documents protective associations between lifetime lactation and improved maternal health, these findings do not establish that 
breastfeeding prevents poor maternal health. Several other factors may be at work. First, women in very high income countries who choose to and successfully breastfeed are typically better educated, wealthier, and more likely to engage in other beneficial health behaviors. ${ }^{180,181}$ Moreover, it is plausible that, rather than breastfeeding preventing poor maternal health, poor maternal health may prevent breastfeeding. One limitation of the evidence is related to time frame of enrollment. Many observational studies (including data from Women's Health Initiative participants $^{4}$ ) enrolled women who breastfed decades ago. In 1970, only 26.5 percent of women initiated breastfeeding ${ }^{182}$ compared with more than 80 percent of women today. Because of these secular changes, confounders of the association between breastfeeding and maternal health have changed over time, and evidence on the association between breastfeeding from older cohorts of women may or may not reflect the strength of association for women currently breastfeeding. Women who chose to breastfeed when breastfeeding rates in the United States were lower could be different in ways that affect risk of adverse maternal health outcomes.

\section{Future Research Needs}

For KQ 1, future research should assess the benefit of workplace, school-based, and other community-based interventions for improving rates of breastfeeding. Authors of future studies should more clearly describe characteristics of usual care and what other breastfeeding support services are available. For studies conducted in the United States, future research should address whether certain interventions are more effective for groups of women who differ by socioeconomic factors in order to assess the consistency of current evidence suggesting a difference by education status. In addition, studies are needed to compare types of support, such as manual versus electric pumps or interventions delivered by International Board Certified Lactation Consultants versus Certified Lactation Consultants, to tailor support to the needs of each woman. Study designs with a concurrent control group (e.g., trials or prospective cohort studies) would be helpful in reducing bias and informing the benefit of breastfeeding programs or policies implemented in a wide range of settings, particularly workplace programs.

For KQ 2, observational studies will likely remain the major source of evidence on the association between breastfeeding and maternal health. Use of standardized breastfeeding definitions and clear reporting of how participants were selected could help minimize bias. In terms of analyses, authors should adequately address known confounders, such as breastfeeding intention, birth complications, diet, physical activity, tobacco use, mental health, and social support, and they should clearly report a rationale for why certain factors were chosen. Further studies might also consider the extent to which adverse lactation outcomes, like adverse pregnancy outcomes, ${ }^{183}$ may be a window to maternal health.

More generally, standardized definitions of breastfeeding, as well as consistent methods of collecting these data, are needed to facilitate future systematic reviews and meta-analyses.

\section{Conclusions}

The body of evidence for breastfeeding programs and policies was diverse in terms of interventions and settings. Current evidence supports the effectiveness of BFHI for improving rates of breastfeeding initiation and duration; however, evidence from one large RCT (PROBIT) has limited applicability, and observational studies do not clearly establish the magnitude of benefit. For U.S. women enrolled in WIC, peer-support interventions have low SOE for improving breastfeeding outcomes. The identified associations between breastfeeding and 
improved maternal health outcomes are supported by evidence from observational studies, which cannot determine cause and effect relationships. 


\section{References}

1. Chowdhury R, Sinha B, Sankar MJ, et al.

Breastfeeding and maternal health outcomes: a systematic review and metaanalysis. Acta Paediatr. 2015

Dec;104(467):96-113. doi:

10.1111/apa.13102. PMID: 26172878.

2. Ip S, Chung M, Raman G, et al. Breastfeeding and maternal and infant health outcomes in developed countries. Evid Rep Technol Assess (Full Rep). 2007 Apr(153):1-186. PMID: 17764214.

3. Victora CG, Bahl R, Barros AJ, et al. Breastfeeding in the 21st century: epidemiology, mechanisms, and lifelong effect. Lancet. 2016 Jan 30;387(10017):47590. doi: 10.1016/s0140-6736(15)01024-7. PMID: 26869575.

4. Schwarz EB, Ray RM, Stuebe AM, et al. Duration of lactation and risk factors for maternal cardiovascular disease. Obstet Gynecol. 2009 May;113(5):974-82. doi: 10.1097/01.AOG.0000346884.67796.ca. PMID: 19384111.

5. Stuebe AM, Schwarz EB, Grewen K, et al. Duration of lactation and incidence of maternal hypertension: a longitudinal cohort study. Am J Epidemiol. 2011 Nov 15;174(10):1147-58. doi: 10.1093/aje/kwr227. PMID: 21997568.

6. Stuebe AM, Michels KB, Willett WC, et al. Duration of lactation and incidence of myocardial infarction in middle to late adulthood. Am J Obstet Gynecol. 2009 Feb;200(2):138.e1-8. doi: 10.1016/j.ajog.2008.10.001. PMID: 19110223.

7. Schwarz EB, McClure CK, Tepper PG, et al. Lactation and maternal measures of subclinical cardiovascular disease. Obstet Gynecol. 2010 Jan;115(1):41-8. doi: 10.1097/AOG.0b013e3181c5512a. PMID: 20027032.

8. Centers for Disease Control and Prevention. Breastfeeding report cards. Atlanta, GA: Centers for Disease Control and Prevention; 2017. http://www.cdc.gov/breastfeeding/data/repor tcard.htm. Accessed on November 282017.
9. Office of Disease Prevention and Health Promotion. Maternal, Infant, and Child Health. Washington, DC: Office of Disease Prevention and Health Promotion; 2016. https://www.healthypeople.gov/2020/topicsobjectives/topic/maternal-infant-and-childhealth/objectives. Accessed on September 8 2016.

10. Stuebe AM, Horton BJ, Chetwynd E, et al. Prevalence and risk factors for early, undesired weaning attributed to lactation dysfunction. J Womens Health (Larchmt). 2014 May;23(5):404-12. doi: 10.1089/jwh.2013.4506. PMID: 24655291.

11. Centers for Disease Control and Prevention. Progress in increasing breastfeeding and reducing racial/ethnic differences_-United States, 2000-2008 Births. Atlanta, GA: Centers for Disease Control and Prevention; 2013.

www.cdc.gov/breastfeeding/resources/breast feeding-trends.htm. Accessed on January 3 2017.

12. Centers for Disease Control and Prevention. Breastfeeding among U.S. Children Born 2002-2012, CDC National Immunization Surveys. Atlanta, GA: Centers for Disease Control and Prevention; 2016.

www.cdc.gov/breastfeeding/data/nis_data/in dex.htm.

13. World Health Organization. BFHI Section 1. Background and Implementation. Sweden:

World Health Organization, UNICEF; 2009. http://www.who.int/nutrition/publications/in fantfeeding/bfhi_trainingcourse_s1/en/. Accessed on February 192018.

14. Health Resources and Services Administration. Women's Preventive Services Guidelines. Rockville, MD: Health Resources and Services Administration; 2016. www.hrsa.gov/womensguidelines/. Accessed on January 32017.

15. U.S. Department of Agriculture. Women, Infants and Children (WIC). About WICWIC at a Glance. Washington, DC: U.S. Department of Agriculture; 2015. https://www.fns.usda.gov/wic/about-wicwic-glance. Accessed on February 212018. 
16. Abdulwadud OA, Snow ME. Interventions in the workplace to support breastfeeding for women in employment. Cochrane Database Syst Rev. 2012 Oct 17;10:Cd006177. doi: 10.1002/14651858.CD006177.pub3. PMID: 23076920.

17. Baby-Friendly USA. Baby-Friendly Hospital Initiative. The Ten Steps to Successful Breastfeeding. Albany, NY: Baby-Friendly USA; 2010. www.babyfriendlyusa.org/about-us/babyfriendly-hospital-initiative/the-ten-steps. Accessed on January 32017.

18. American Academy of Pediatrics. Breastfeeding and the use of human milk. Pediatrics. 2012 Mar;129(3):e827-41. doi: 10.1542/peds.2011-3552. PMID: 22371471.

19. American College of Obstetricians and Gynecologists. Optimizing support for breastfeeding as part of obstetric practice. Committee Opinion No. 658. Obstet Gynecol Annu. 2016;127:e86-92.

20. World Health Organization. International Code of Marketing Breast-milk Substitutes. Geneva: World Health Organization; 1981.

21. World Health Organization. Health topics. Breastfeeding. Switzerland: World Health Organization; 2017. Accessed on January 3 2017.

22. World Health Organization, UNICEF. Protecting, promoting and supporting breastfeeding: the special role of maternity services. Geneva: World Health Organization; 1989.

23. American Academy of Family Physicians. Breastfeeding (policy statement): American Academy of Family Physicians [serial on the Internet]; 2012.

24. World Health Organization. Nutrition. Exclusive breastfeeding. Geneva, Switzerland: World Health Organization; n.d.

http://www.who.int/nutrition/topics/exclusiv e breastfeeding/en/. Accessed on November 282017.
25. Bibbins-Domingo K, Grossman DC, Curry SJ, et al. Primary care interventions to support breastfeeding: US Preventive Services Task Force recommendation statement. JAMA. 2016 Oct 25;316(16):1688-93. doi: 10.1001/jama.2016.14697. PMID: 27784102.

26. Patnode CD, Henninger ML, Senger CA, et al. Primary care interventions to support breastfeeding: updated evidence report and systematic review for the US Preventive Services Task Force. JAMA. 2016 Oct 25;316(16):1694-705. doi: 10.1001/jama.2016.8882. PMID: 27784101.

27. Wang L, Li J, Shi Z. Association between breastfeeding and endometrial cancer risk: evidence from a systematic review and meta-analysis. Nutrients. $2015 \mathrm{Jul}$ 14;7(7):5697-711. doi: 10.3390/nu7075248. PMID: 26184301.

28. Chen H, Wang J, Zhou W, et al. Breastfeeding and risk of rheumatoid arthritis: a systematic review and metaanalysis. J Rheumatol. 2015 Sep;42(9):1563-9. doi: 10.3899/jrheum.150195. PMID: 26178286.

29. Zhou Y, Chen J, Li Q, et al. Association between breastfeeding and breast cancer risk: evidence from a meta-analysis. Breastfeed Med. 2015 Apr;10(3):175-82. doi: 10.1089/bfm.2014.0141. PMID: 25785349.

30. Programme UND. Human Development Report 2015: Work for Human Development. Table 1: Human Development Index and its components. United Nations Development Programme; n.d. New York, NY: 2015. http://hdr.undp.org/en/composite/HDI

31. Sterne JA, Hernan MA, Reeves BC, et al. ROBINS-I: a tool for assessing risk of bias in non-randomised studies of interventions. BMJ. 2016 Oct 12;355:i4919. doi: 10.1136/bmj.i4919. PMID: 27733354.

32. Higgins JPT, Green S. Cochrane Handbook for Systematic Reviews of Interventions. The Cochrane Collaboration.; 2011. www.handbook.cochrane.org. Accessed on January 102017. 
33. Agency for Healthcare Research and Quality. Methods Guide for Effectiveness and Comparative Effectiveness Reviews. AHRQ Publication No. 10(14)-EHC063-EF. Rockville, MD: Agency for Healthcare Research and Quality; January 2014. www.effectivehealthcare.ahrq.gov/methodsg uide.cfm

34. Whiting P, Savovic J, Higgins JP, et al. ROBIS: A new tool to assess risk of bias in systematic reviews was developed. J Clin Epidemiol. 2016 Jan;69:225-34. doi: 10.1016/j.jclinepi.2015.06.005. PMID: 26092286.

35. Berkman ND, Lohr KN, Ansari MT, et al. Grading the strength of a body of evidence when assessing health care interventions: an EPC update. J Clin Epidemiol. 2015 Nov;68(11):1312-24. doi: 10.1016/j.jclinepi.2014.11.023. PMID: 25721570 .

36. Atkins D, Chang SM, Gartlehner G, et al. Assessing applicability when comparing medical interventions: AHRQ and the Effective Health Care Program. J Clin Epidemiol. 2011 Nov;64(11):1198-207. doi: 10.1016/j.jclinepi.2010.11.021. PMID: 21463926.

37. Kramer MS, Chalmers B, Hodnett ED, et al. Promotion of Breastfeeding Intervention Trial (PROBIT): a randomized trial in the Republic of Belarus. JAMA. 2001 Jan 2431;285(4):413-20. PMID: 11242425.

38. Yang S, Platt RW, Dahhou M, et al. Do population-based interventions widen or narrow socioeconomic inequalities? The case of breastfeeding promotion. Int $\mathrm{J}$ Epidemiol. 2014 Aug;43(4):1284-92. doi: 10.1093/ije/dyu051. PMID: 24639438.

39. Hawkins SS, Stern AD, Baum CF, et al. Evaluating the impact of the Baby-Friendly Hospital Initiative on breast-feeding rates: a multi-state analysis. Public Health Nutr. 2015 Feb;18(2):189-97. doi: 10.1017/s1368980014000238. PMID: 24625787.
40. Hawkins SS, Stern AD, Baum CF, et al. Compliance with the Baby-Friendly Hospital Initiative and impact on breastfeeding rates. Arch Dis Child Fetal Neonatal Ed. 2014 Mar;99(2):F138-43. doi: 10.1136/archdischild-2013-304842. PMID: 24277661.

41. Tarrant M, Wu KM, Fong DY, et al. Impact of baby-friendly hospital practices on breastfeeding in Hong Kong. Birth. 2011 Sep;38(3):238-45. doi: 10.1111/j.1523536X.2011.00483.x. PMID: 21884232.

42. Mydlilova A, Sipek A, Vignerova J. Breastfeeding rates in baby-friendly and non-baby-friendly hospitals in the Czech Republic from 2000 to 2006. J Hum Lact. 2009 Feb;25(1):73-8. doi: 10.1177/0890334408325820. PMID: 19020285.

43. Abolyan LV. The breastfeeding support and promotion in Baby-Friendly Maternity Hospitals and Not-as-Yet Baby-Friendly Hospitals in Russia. Breastfeed Med. 2006 Summer;1(2):71-8. doi: 10.1089/bfm.2006.1.71. PMID: 17661566.

44. Coutinho SB, de Lira PI, de Carvalho Lima $\mathrm{M}$, et al. Comparison of the effect of two systems for the promotion of exclusive breastfeeding. Lancet. 2005 Sep 2430;366(9491):1094-100. doi: 10.1016/s0140-6736(05)67421-1. PMID: 16182897.

45. Bosnjak AP, Batinica M, Hegedus-Jungvirth $\mathrm{M}$, et al. The effect of baby friendly hospital initiative and postnatal support on breastfeeding rates--Croatian experience. Coll Antropol. 2004 Jun;28(1):235-43. PMID: 15636080.

46. Gau ML. Evaluation of a lactation intervention program to encourage breastfeeding: a longitudinal study. Int J Nurs Stud. 2004 May;41(4):425-35. doi: 10.1016/j.ijnurstu.2003.11.002. PMID: 15050853.

47. Bartington S, Griffiths LJ, Tate AR, et al. Are breastfeeding rates higher among mothers delivering in Baby Friendly accredited maternity units in the UK? Int $\mathrm{J}$ Epidemiol. 2006 Oct;35(5):1178-86. doi: 10.1093/ije/dyl155. PMID: 16926214. 
48. Broadfoot M, Britten J, Tappin DM, et al. The Baby Friendly Hospital Initiative and breast feeding rates in Scotland. Arch Dis Child Fetal Neonatal Ed. 2005

Mar;90(2):F114-6. doi:

10.1136/adc.2003.041558. PMID: 15724033 .

49. Venancio SI, Saldiva SR, Escuder MM, et al. The Baby-Friendly Hospital Initiative shows positive effects on breastfeeding indicators in Brazil. J Epidemiol Community Health. 2012 Oct;66(10):914-8. doi: 10.1136/jech-2011-200332. PMID: 22080818 .

50. Weng DR, Hsu CS, Gau ML, et al. Analysis of the outcomes at baby-friendly hospitals: appraisal in Taiwan. Kaohsiung J Med Sci. 2003 Jan;19(1):19-28. doi: 10.1016/s1607551x(09)70443-7. PMID: 12693722.

51. Yotebieng M, Labbok M, Soeters HM, et al. Ten Steps to Successful Breastfeeding programme to promote early initiation and exclusive breastfeeding in DR Congo: a cluster-randomised controlled trial. Lancet Glob Health. 2015 Sep;3(9):e546-55. doi: 10.1016/s2214-109x(15)00012-1. PMID: 26246225.

52. Osband YB, Altman RL, Patrick PA, et al. Breastfeeding education and support services offered to pediatric residents in the US. Acad Pediatr. 2011 Jan-Feb;11(1):75-9. doi: 10.1016/j.acap.2010.11.002. PMID: 21272827.

53. Wagner CL, Hulsey TC, Southgate WM, et al. Breastfeeding rates at an urban medical university after initiation of an educational program. South Med J. 2002 Aug;95(8):90913. PMID: 12190230.

54. Nilsson IMS, Strandberg-Larsen K, Knight $\mathrm{CH}$, et al. Focused breastfeeding counselling improves short- and long-term success in an early-discharge setting: A clusterrandomized study. Matern Child Nutr. 2017;13(4):n/a-n/a. doi: 10.1111/mcn.12432. PMID: 125350139 Language: English. Entry Date: 20171006. Revision Date: 20171006. Publication Type: Article. Journal Subset: Allied Health.
55. Ekstrom A, Abrahamsson H, Eriksson RM, et al. Women's use of nipple shields-their influence on breastfeeding duration after a process-oriented education for health professionals. Breastfeed Med. 2014 Nov;9(9):458-66. doi: 10.1089/bfm.2014.0026. PMID: 25188544.

56. Ekstrom A, Kylberg E, Nissen E. A processoriented breastfeeding training program for healthcare professionals to promote breastfeeding: an intervention study. Breastfeed Med. 2012 Apr;7(2):85-92. doi: 10.1089/bfm.2010.0084. PMID: 22168946.

57. Hannula LS, Kaunonen ME, Puukka PJ. A study to promote breast feeding in the Helsinki Metropolitan area in Finland. Midwifery. 2014 Jun;30(6):696-704. doi: 10.1016/j.midw.2013.10.005. PMID: 24210842

58. Wan $\mathrm{H}, \mathrm{Hu} \mathrm{S}$, Thobaben M, et al. Continuous primary nursing care increases satisfaction with nursing care and reduces postpartum problems for hospitalized pregnant women. Contemp Nurse. 2011 Feb;37(2):149-59. doi: 10.5172/conu.2011.37.2.149. PMID: 21692586 .

59. Feldman-Winter L, Barone L, Milcarek B, et al. Residency curriculum improves breastfeeding care. Pediatrics. 2010 Aug;126(2):289-97. doi: 10.1542/peds.2009-3250. PMID: 20603262.

60. Hoddinott P, Britten J, Prescott GJ, et al. Effectiveness of policy to provide breastfeeding groups (BIG) for pregnant and breastfeeding mothers in primary care: cluster randomised controlled trial. BMJ. 2009 Jan 30;338:a3026. doi: 10.1136/bmj.a3026. PMID: 19181729.

61. Senarath U, Fernando DN, Rodrigo I. Effect of training for care providers on practice of essential newborn care in hospitals in Sri Lanka. J Obstet Gynecol Neonatal Nurs. 2007 Nov-Dec;36(6):531-41. doi: 10.1111/j.1552-6909.2007.00183.x. PMID: 17973696.

62. Kronborg H, Vaeth M, Olsen J, et al. Effect of early postnatal breastfeeding support: a cluster-randomized community based trial. Acta Paediatr. 2007 Jul;96(7):1064-70. doi: 10.1111/j.1651-2227.2007.00341.x. PMID: 17524018. 
63. Kools EJ, Thijs C, Kester AD, et al. A breast-feeding promotion and support program a randomized trial in The Netherlands. Prev Med. 2005 Jan;40(1):6070. doi: 10.1016/j.ypmed.2004.05.013. PMID: 15530582.

64. Martens PJ. Does breastfeeding education affect nursing staff beliefs, exclusive breastfeeding rates, and Baby-Friendly Hospital Initiative compliance? The experience of a small, rural Canadian hospital. J Hum Lact. 2000 Nov;16(4):30918. PMID: 11155609.

65. Taddei JA, Westphal MF, Venancio S, et al. Breastfeeding training for health professionals and resultant changes in breastfeeding duration. Sao Paulo Med J. 2000 Nov 09;118(6):185-91. PMID: 11120550.

66. Madden JM, Soumerai SB, Lieu TA, et al. Effects on breastfeeding of changes in maternity length-of-stay policy in a large health maintenance organization. Pediatrics. 2003 Mar;111(3):519-24. PMID: 12612230.

67. Bærug A, Langsrud Ø, Løland BF, et al. Effectiveness of baby-friendly community health services on exclusive breastfeeding and maternal satisfaction: a pragmatic trial. Matern Child Nutr. 2016;12(3):428-39. doi: 10.1111/mcn.12273. PMID: 116415662.

68. Walsh A, Moseley J, Jackson W. The effects of an infant-feeding classroom activity on the breast-feeding knowledge and intentions of adolescents. J Sch Nurs. 2008

Jun;24(3):164-9. doi: 10.1622/10598405(2008)024[0164:teoaic]2.0.co;2. PMID: 18557676.

69. Barcellos NT, Fuchs SC, Mondini LG, et al. Human T lymphotropic virus type I/II infection: prevalence and risk factors in individuals testing for HIV in counseling centers from Southern Brazil. Sex Transm Dis. 2006 May;33(5):302-6. doi: 10.1097/01.olq.0000194598.47821.b6. PMID: 16505751.

70. Hayes DK, Prince CB, Espinueva V, et al. Comparison of manual and electric breast pumps among WIC women returning to work or school in Hawaii. Breastfeed Med. 2008 Mar;3(1):3-10. doi: 10.1089/bfm.2007.0022. PMID: 18333763.
71. Joyce T, Reeder J. Changes in breastfeeding among WIC participants following implementation of the new food package. Matern Child Health J. 2015 Apr;19(4):86876. doi: 10.1007/s10995-014-1588-7. PMID: 25095768.

72. Lovera D, Sanderson M, Bogle ML, et al. Evaluation of a breastfeeding peer support program for fathers of Hispanic participants in a Texas special supplemental nutrition program for women, infants, and children. J Am Diet Assoc. 2010 Nov;110(11):1696702. doi: 10.1016/j.jada.2010.08.001. PMID: 21034883.

73. Reeder JA, Joyce T, Sibley K, et al. Telephone peer counseling of breastfeeding among WIC participants: a randomized controlled trial. Pediatrics. 2014 Sep;134(3):e700-9. doi: 10.1542/peds.20134146. PMID: 25092936.

74. Schafer E, Vogel MK, Viegas S, et al. Volunteer peer counselors increase breastfeeding duration among rural lowincome women. Birth. 1998 Jun;25(2):1016. PMID: 9668744.

75. Shaw E, Kaczorowski J. The effect of a peer counseling program on breastfeeding initiation and longevity in a low-income rural population. J Hum Lact. 1999 Mar;15(1):19-25. PMID: 10578771.

76. Edmunds LS, Lee FF, Eldridge JD, et al. Outcome evaluation of the You Can Do It Initiative to Promote Exclusive Breastfeeding Among Women Enrolled in the New York State WIC Program by Race/Ethnicity. J Nutr Educ Behav. 2017;49:S162-S8.e1. doi: 10.1016/j.jneb.2017.05.350. PMID: 123721522.

77. Washio Y, Humphreys M, Colchado E, et al. Incentive-based intervention to maintain breastfeeding among low-income Puerto Rican mothers. Pediatrics. 2017 Mar;139(3). doi: 10.1542/peds.2016-3119. PMID: 28167511.

78. Macaluso A, Bettinelli ME, Chapin EM, et al. A controlled study on baby-friendly communities in Italy: methods and baseline data. Breastfeed Med. 2013 Apr;8:198-204. doi: 10.1089/bfm.2012.0130. PMID: 23398142. 
79. Cattaneo A, Bettinelli M, Chapin E, et al. Effectiveness of the baby friendly community initiative in Italy: a nonrandomised controlled study. BMJ Open. 2016;6(5). doi: 10.1136/bmjopen-2015010232. PMID: CN-01159959.

80. McLachlan HL, Forster DA, Amir LH, et al. Supporting breastfeeding In Local Communities (SILC) in Victoria, Australia: a cluster randomised controlled trial. BMJ Open. 2016 Feb 01;6(2):e008292. doi: 10.1136/bmjopen-2015-008292. PMID: 26832427.

81. Cramer RL, McLachlan HL, Shafiei T, et al. Implementation and evaluation of community-based drop-in centres for breastfeeding support in Victoria, Australia. International Breastfeeding Journal. 2017;12(46).

82. Morrow AL, Guerrero ML, Shults J, et al. Efficacy of home-based peer counselling to promote exclusive breastfeeding: a randomised controlled trial. Lancet. 1999 Apr 10;353(9160):1226-31. doi: 10.1016/s0140-6736(98)08037-4. PMID: 10217083.

83. Rempel LA, Moore KC. Peer-led prenatal breast-feeding education: a viable alternative to nurse-led education. Midwifery. 2012 Feb;28(1):73-9. doi: 10.1016/j.midw.2010.11.005. PMID: 21236530 .

84. Alvarado R, Zepeda A, Rivero S, et al. Integrated maternal and infant health care in the postpartum period in a poor neighborhood in Santiago, Chile. Stud Fam Plann. 1999 Jun;30(2):133-41. PMID: 16617547.

85. Al-Amri FA, Saeedi MY, Al-Tahan FM, et al. Breast cancer correlates in a cohort of breast screening program participants in Riyadh, KSA. J Egypt Natl Canc Inst. 2015 Jun;27(2):77-82. doi: 10.1016/j.jnci.2015.04.002. PMID: 25935858.
86. Al-Qutub ST, Al-Raddadi RM, Bin Sadiq $\mathrm{BM}$, et al. Potential breast cancer risk factors among Saudi women aged 19-50 years in Jeddah: a case-control study. J Egypt Public Health Assoc. 2013 Dec;88(3):165-70. doi: 10.1097/01.EPX.0000435728.60811.bd. PMID: 24374950.

87. Beaber EF, Holt VL, Malone KE, et al. Reproductive factors, age at maximum height, and risk of three histologic types of breast cancer. Cancer Epidemiol Biomarkers Prev. 2008 Dec;17(12):3427-34. doi: 10.1158/1055-9965.epi-08-0641. PMID: 19064558.

88. Castello A, Martin M, Ruiz A, et al. Lower breast cancer risk among women following the World Cancer Research Fund and American Institute for Cancer Research Lifestyle Recommendations: EpiGEICAM Case-Control Study. PLoS One. 2015;10(5):e0126096. doi: 10.1371/journal.pone.0126096. PMID: 25978407.

89. Dalamaga M, Karmaniolas K, Papadavid E, et al. Elevated serum visfatin/nicotinamide phosphoribosyl-transferase levels are associated with risk of postmenopausal breast cancer independently from adiponectin, leptin, and anthropometric and metabolic parameters. Menopause. 2011 Nov;18(11):1198-204. doi: 10.1097/gme.0b013e31821e21f5. PMID: 21712732.

90. Hadji P, Gottschalk M, Ziller V, et al. Bone mass and the risk of breast cancer: the influence of cumulative exposure to oestrogen and reproductive correlates. Results of the Marburg breast cancer and osteoporosis trial (MABOT). Maturitas. 2007 Mar 20;56(3):312-21. doi: 10.1016/j.maturitas.2006.09.005. PMID: 17049767.

91. Holm J, Eriksson L, Ploner A, et al. Assessment of Breast Cancer Risk Factors Reveals Subtype Heterogeneity. Cancer Res. 2017 Jul 01;77(13):3708-17. doi: 10.1158/0008-5472.can-16-2574. PMID: 28512241. 
92. Kruk J. Association between vegetable, fruit and carbohydrate intake and breast cancer risk in relation to physical activity. Asian Pac J Cancer Prev. 2014;15(11):4429-36. PMID: 24969864.

93. Lee E, Ma H, McKean-Cowdin R, et al. Effect of reproductive factors and oral contraceptives on breast cancer risk in BRCA1/2 mutation carriers and noncarriers: results from a population-based study. Cancer Epidemiol Biomarkers Prev. 2008 Nov;17(11):3170-8. doi: 10.1158/10559965.epi-08-0396. PMID: 18990759.

94. Lumachi F, Frigo AC, Basso U, et al. Estrogen therapy and risk of breast cancer in postmenopausal women: a case-control study and results of a multivariate analysis. Menopause. 2010 May-Jun;17(3):524-8. doi: 10.1097/gme.0b013e3181ca0c74. PMID: 20130492.

95. Ma H, Bernstein L, Ross RK, et al. Hormone-related risk factors for breast cancer in women under age 50 years by estrogen and progesterone receptor status: results from a case-control and a case-case comparison. Breast Cancer Res. 2006;8(4):R39. doi: 10.1186/bcr1514. PMID: 16846528.

96. Phillips LS, Millikan RC, Schroeder JC, et al. Reproductive and hormonal risk factors for ductal carcinoma in situ of the breast. Cancer Epidemiol Biomarkers Prev. 2009 May;18(5):1507-14. doi: 10.1158/10559965.epi-08-0967. PMID: 19423528.

97. Pieta B, Opala T, Wilczak M, et al. Past obstetric history and risk of malignant breast neoplasms. Eur J Gynaecol Oncol. 2008;29(4):374-7. PMID: 18714573.

98. Press DJ, Pharoah P. Risk factors for breast cancer: a reanalysis of two case-control studies from 1926 and 1931. Epidemiology. 2010 Jul;21(4):566-72. doi: 10.1097/EDE.0b013e3181e08eb3. PMID: 20498604.

99. Ruszczyk M, Zirpoli G, Kumar S, et al. Breast cancer risk factor associations differ for pure versus invasive carcinoma with an in situ component in case-control and casecase analyses. Cancer Causes Control. 2016 Feb;27(2):183-98. doi: 10.1007/s10552-0150696-z. PMID: 26621543.
100. Stendell-Hollis NR, Thompson PA, Thomson CA, et al. Investigating the association of lactation history and postmenopausal breast cancer risk in the Women's Health Initiative. Nutr Cancer. 2013;65(7):969-81. doi: 10.1080/01635581.2013.815787. PMID: 24127779.

101. Sugawara Y, Kakizaki M, Nagai M, et al. Lactation pattern and the risk for hormonerelated female cancer in Japan: the Ohsaki Cohort Study. Eur J Cancer Prev. 2013 Mar;22(2):187-92. doi: 10.1097/CEJ.0b013e3283564610. PMID: 23358107.

102. Tamimi RM, Spiegelman D, Smith-Warner SA, et al. Population Attributable Risk of Modifiable and Nonmodifiable Breast Cancer Risk Factors in Postmenopausal Breast Cancer. Am J Epidemiol. 2016 Dec 15;184(12):884-93. doi: 10.1093/aje/kww145. PMID: 27923781.

103. Ge I, Rudolph A, Shivappa N, et al. Dietary inflammation potential and postmenopausal breast cancer risk in a German case-control study. Breast. 2015 Aug;24(4):491-6. doi: 10.1016/j.breast.2015.04.012. PMID: 25987487.

104. Kotsopoulos J, Lubinski J, Lynch HT, et al. Oophorectomy after menopause and the risk of breast cancer in BRCA1 and BRCA2 mutation carriers. Cancer Epidemiol Biomarkers Prev. 2012 Jul;21(7):1089-96. doi: 10.1158/1055-9965.epi-12-0201. PMID: 22564871.

105. Kabat GC, Kim MY, Woods NF, et al. Reproductive and menstrual factors and risk of ductal carcinoma in situ of the breast in a cohort of postmenopausal women. Cancer Causes Control. 2011 Oct;22(10):1415-24. doi: 10.1007/s10552-011-9814-8. PMID: 21750889.

106. Lambertini M, Santoro L, Del Mastro L, et al. Reproductive behaviors and risk of developing breast cancer according to tumor subtype: a systematic review and metaanalysis of epidemiological studies. Cancer Treat Rev. 2016 Sep;49:65-76. doi: 10.1016/j.ctrv.2016.07.006. PMID: 27529149. 
107. Atkinson RL, El-Zein R, Valero V, et al. Epidemiological risk factors associated with inflammatory breast cancer subtypes.

Cancer Causes Control. 2016

Mar;27(3):359-66. doi: 10.1007/s10552-

015-0712-3. PMID: 26797453.

108. Ma H, Ursin G, Xu X, et al. Reproductive factors and the risk of triple-negative breast cancer in white women and AfricanAmerican women: a pooled analysis. Breast Cancer Res. 2017 Jan 13;19(1):6. doi: 10.1186/s13058-016-0799-9. PMID: 28086982.

109. Ritte R, Tikk K, Lukanova A, et al. Reproductive factors and risk of hormone receptor positive and negative breast cancer: a cohort study. BMC Cancer. 2013 Dec 09;13:584. doi: 10.1186/1471-2407-13-584. PMID: 24321460.

110. Warner ET, Tamimi RM, Boggs DA, et al. Estrogen receptor positive tumors: do reproductive factors explain differences in incidence between black and white women? Cancer Causes Control. 2013 Apr;24(4):731-9. doi: 10.1007/s10552-0130153-9. PMID: 23380944.

111. Merritt MA, Riboli E, Murphy N, et al. Reproductive factors and risk of mortality in the European Prospective Investigation into Cancer and Nutrition; a cohort study. BMC Med. 2015 Oct 30;13:252. doi: 10.1186/s12916-015-0484-3. PMID: 26515238.

112. Cook LS, Pestak CR, Leung AC, et al. Combined oral contraceptive use before the first birth and epithelial ovarian cancer risk. Br J Cancer. 2017 Jan 17;116(2):265-9. doi: 10.1038/bjc.2016.400. PMID: 27959890.

113. Gay GM, Lim JS, Chay WY, et al. Reproductive factors, adiposity, breastfeeding and their associations with ovarian cancer in an Asian cohort. Cancer Causes Control. 2015 Nov;26(11):1561-73. doi: 10.1007/s10552-015-0649-6. PMID: 26342607.

114. Gierach GL, Modugno F, Ness RB. Gender of offspring and maternal ovarian cancer risk. Gynecol Oncol. 2006 Jun;101(3):47680. doi: 10.1016/j.ygyno.2005.11.008. PMID: 16364411.
115. John EM, Whittemore AS, Harris R, et al. Characteristics relating to ovarian cancer risk: collaborative analysis of seven U.S. case-control studies. Epithelial ovarian cancer in black women. Collaborative Ovarian Cancer Group. J Natl Cancer Inst. 1993 Jan 20;85(2):142-7. PMID: 8418303.

116. Jordan SJ, Green AC, Whiteman DC, et al. Serous ovarian, fallopian tube and primary peritoneal cancers: a comparative epidemiological analysis. Int J Cancer. 2008 Apr 01;122(7):1598-603. doi: 10.1002/ijc.23287. PMID: 18058817.

117. Nagle CM, Olsen CM, Webb PM, et al. Endometrioid and clear cell ovarian cancers: a comparative analysis of risk factors. Eur $\mathrm{J}$ Cancer. 2008 Nov;44(16):2477-84. doi: 10.1016/j.ejca.2008.07.009. PMID: 18707869.

118. Kotsopoulos J, Lubinski J, Gronwald J, et al. Factors influencing ovulation and the risk of ovarian cancer in BRCA1 and BRCA2 mutation carriers. Int J Cancer. 2015 Sep 01;137(5):1136-46. doi: 10.1002/ijc.29386. PMID: 25482078.

119. Risch HA, Marrett LD, Howe GR. Parity, contraception, infertility, and the risk of epithelial ovarian cancer. Am J Epidemiol. 1994 Oct 01;140(7):585-97. PMID: 7942759 .

120. West RO. Epidemiologic study of malignancies of the ovaries. Cancer. 1966 Jul;19(7):1001-7. PMID: 5939299.

121. Wynder EL, Dodo H, Barber HR. Epidemiology of cancer of the ovary. Cancer. 1969 Feb;23(2):352-70. PMID: 5764976.

122. Lee SY, Kim MT, Jee SH, et al. Does longterm lactation protect premenopausal women against hypertension risk? A Korean women's cohort study. Prev Med. 2005 Aug;41(2):433-8. doi: 10.1016/j.ypmed.2004.11.025. PMID: 15917038.

123. Lupton SJ, Chiu CL, Lujic S, et al. Association between parity and breastfeeding with maternal high blood pressure. Am J Obstet Gynecol. 2013 Jun;208(6):454.e1-7. doi: 10.1016/j.ajog.2013.02.014. PMID: 23395924. 
124. Aboud FE, Moore AC, Akhter S.

Effectiveness of a community-based responsive feeding programme in rural Bangladesh: a cluster randomized field trial. Matern Child Nutr. 2008 Oct;4(4):275-86. doi: 10.1111/j.1740-8709.2008.00146.x. PMID: 18811792.

125. Parikh NI, Jeppson RP, Berger JS, et al. Reproductive risk factors and coronary heart disease in the Women's Health Initiative Observational Study. Circulation. 2016 May 31;133(22):2149-58. doi:

10.1161/circulationaha.115.017854. PMID: 27143682.

126. Natland Fagerhaug T, Forsmo S, Jacobsen $\mathrm{GW}$, et al. A prospective population-based cohort study of lactation and cardiovascular disease mortality: the HUNT study. BMC Public Health. 2013 Nov 13;13:1070. doi: 10.1186/1471-2458-13-1070. PMID: 24219620.

127. Aune D, Norat T, Romundstad P, et al. Breastfeeding and the maternal risk of type 2 diabetes: a systematic review and doseresponse meta-analysis of cohort studies. Nutr Metab Cardiovasc Dis. 2014 Feb;24(2):107-15. doi: 10.1016/j.numecd.2013.10.028. PMID: 24439841.

128. Chamberlain CR, Oldenburg B, Wilson AN, et al. Type 2 diabetes after gestational diabetes: greater than fourfold risk among Indigenous compared with non-Indigenous Australian women. Diabetes Metab Res Rev. 2016 Feb;32(2):217-27. doi: 10.1002/dmrr.2715. PMID: 26385131.

129. Gunderson EP, Hurston SR, Ning X, et al. Lactation and progression to Type 2 Diabetes Mellitus after Gestational Diabetes Mellitus: a prospective cohort study. Ann Intern Med. 2015 Dec 15;163(12):889-98. doi: 10.7326/m15-0807. PMID: 26595611.

130. Martens PJ, Shafer LA, Dean HJ, et al. Breastfeeding initiation associated with reduced incidence of diabetes in mothers and offspring. Obstet Gynecol. 2016

Nov;128(5):1095-104. doi: 10.1097/aog.0000000000001689. PMID: 27741196.
131. Zong G, Grandjean P, Wang X, et al. Lactation history, serum concentrations of persistent organic pollutants, and maternal risk of diabetes. Environ Res. 2016 Oct;150:282-8. doi: 10.1016/j.envres.2016.06.023. PMID: 27336232.

132. Gunderson EP, Hedderson MM, Chiang V, et al. Lactation intensity and postpartum maternal glucose tolerance and insulin resistance in women with recent GDM: the SWIFT cohort. Diabetes Care. 2012 Jan;35(1):50-6. doi: 10.2337/dc11-1409. PMID: 22011407.

133. Alderman BW, Weiss NS, Daling JR, et al. Reproductive history and postmenopausal risk of hip and forearm fracture. Am J Epidemiol. 1986 Aug;124(2):262-7. PMID: 3728442.

134. Chan HH, Lau EM, Woo J, et al. Dietary calcium intake, physical activity and the risk of vertebral fracture in Chinese. Osteoporos Int. 1996;6(3):228-32. PMID: 8783297.

135. Hoffman S, Grisso JA, Kelsey JL, et al. Parity, lactation and hip fracture. Osteoporos Int. 1993 Jul;3(4):171-6. PMID: 8338971.

136. Cumming RG, Klineberg RJ. Breastfeeding and other reproductive factors and the risk of hip fractures in elderly women. Int J Epidemiol. 1993 Aug;22(4):684-91. PMID: 8225744.

137. Kreiger N, Kelsey JL, Holford TR, et al. An epidemiologic study of hip fracture in postmenopausal women. Am J Epidemiol. 1982 Jul;116(1):141-8. PMID: 7102649.

138. Michaelsson K, Baron JA, Farahmand BY, et al. Influence of parity and lactation on hip fracture risk. Am J Epidemiol. 2001 Jun 15;153(12):1166-72. PMID: 11415951.

139. Mori T, Ishii S, Greendale GA, et al. Parity, lactation, bone strength, and 16-year fracture risk in adult women: findings from the Study of Women's Health Across the Nation (SWAN). Bone. 2015 Apr;73:160-6. doi: 10.1016/j.bone.2014.12.013. PMID: 25528102. 
140. Hwang IR, Choi YK, Lee WK, et al. Association between prolonged breastfeeding and bone mineral density and osteoporosis in postmenopausal women: KNHANES 2010-2011. Osteoporos Int. 2016 Jan;27(1):257-65. doi: 10.1007/s00198-015-3292-x. PMID: 26373982.

141. Lambrinoudaki I, Flokatoula M, Armeni E, et al. Vertebral fracture prevalence among Greek healthy middle-aged postmenopausal women: association with demographics, anthropometric parameters, and bone mineral density. Spine J. 2015 Jan 01;15(1):86-94. doi: 10.1016/j.spinee.2014.07.021. PMID: 25106754 .

142. Naves M, Diaz-Lopez JB, Gomez C, et al. Determinants of incidence of osteoporotic fractures in the female Spanish population older than 50. Osteoporos Int. 2005 Dec;16(12):2013-7. doi: 10.1007/s00198005-1983-4. PMID: 16091836.

143. Crandall CJ, Liu J, Cauley J, et al. Associations of parity, breastfeeding, and fractures in the Women's Health Observational Study. Obstet Gynecol. 2017 Jul;130(1):171-80. doi: 10.1097/aog.0000000000002096. PMID: 28594759.

144. Dias CC, Figueiredo B. Breastfeeding and depression: a systematic review of the literature. J Affect Disord. 2015 Jan 15;171:142-54. doi: 10.1016/j.jad.2014.09.022. PMID: 25305429.

145. Ahn S, Corwin EJ. The association between breastfeeding, the stress response, inflammation, and postpartum depression during the postpartum period: prospective cohort study. Int J Nurs Stud. 2015 Oct;52(10):1582-90. doi: 10.1016/j.ijnurstu.2015.05.017. PMID: 26143358.

146. Banks E, Killpack S, Furman L. Lowincome inner-city fathers and breastfeeding-where's the program for us? Breastfeed Med. 2013 Dec;8(6):507-8. doi: 10.1089/bfm.2012.0147. PMID: 23560474.
147. Chojenta CL, Lucke JC, Forder PM, et al. Maternal health factors as risks for postnatal depression: a prospective longitudinal study. PLoS One. 2016;11(1):e0147246. doi: 10.1371/journal.pone.0147246. PMID: 26785131.

148. Davey HL, Tough SC, Adair CE, et al. Risk factors for sub-clinical and major postpartum depression among a community cohort of Canadian women. Matern Child Health J. 2011 Oct;15(7):866-75. doi: 10.1007/s10995-008-0314-8. PMID: 18256913.

149. Fiala A, Svancara J, Klanova J, et al. Sociodemographic and delivery risk factors for developing postpartum depression in a sample of 3233 mothers from the Czech ELSPAC study. BMC Psychiatry. 2017 Mar 21;17(1):104. doi: 10.1186/s12888-0171261-y. PMID: 28327118.

150. Hamdan A, Tamim H. The relationship between postpartum depression and breastfeeding. Int J Psychiatry Med. 2012;43(3):243-59. PMID: 22978082.

151. Iliadis SI, Comasco E, Sylven S, et al. Prenatal and postpartum evening salivary cortisol levels in association with peripartum depressive symptoms. PLoS One. 2015;10(8):e0135471. doi: 10.1371/journal.pone.0135471. PMID: 26322643.

152. Lau Y, Chan KS. Perinatal depressive symptoms, sociodemographic correlates, and breast-feeding among Chinese women. J Perinat Neonatal Nurs. 2009 OctDec;23(4):335-45. doi: 10.1097/JPN.0b013e3181bbbea9. PMID: 19915417.

153. Maimburg RD, Vaeth M. Postpartum depression among first-time mothers results from a parallel randomised trial. Sex Reprod Healthc. 2015 Jun;6(2):95-100. doi: 10.1016/j.srhc.2015.01.003. PMID: 25998877.

154. McCoy SJ, Beal JM, Saunders B, et al. Risk factors for postpartum depression: a retrospective investigation. J Reprod Med. 2008 Mar;53(3):166-70. PMID: 18441719. 
155. Reifsnider E, Flowers J, Todd M, et al. The relationship among breastfeeding, postpartum depression, and postpartum weight in Mexican American Women. J Obstet Gynecol Neonatal Nurs. 2016;45(6):760-71. doi: 10.1016/j.jogn.2016.05.009. PMID: 119339849.

156. Sword W, Landy CK, Thabane L, et al. Is mode of delivery associated with postpartum depression at 6 weeks: a prospective cohort study. BJOG. 2011 Jul;118(8):966-77. doi: 10.1111/j.1471-0528.2011.02950.x. PMID: 21489126.

157. Trivino-Juarez JM, Nieto-Pereda B, Romero-Ayuso D, et al. Quality of life of mothers at the sixth week and sixth month post partum and type of infant feeding. Midwifery. 2016 Mar;34:230-8. doi: 10.1016/j.midw.2015.11.003. PMID: 26621376.

158. Warner R, Appleby L, Whitton A, et al. Demographic and obstetric risk factors for postnatal psychiatric morbidity. Br J Psychiatry. 1996 May;168(5):607-11. PMID: 8733800.

159. Brewer MM, Bates MR, Vannoy LP. Postpartum changes in maternal weight and body fat depots in lactating vs nonlactating women. Am J Clin Nutr. 1989 Feb;49(2):259-65. PMID: 2916446.

160. Endres LK, Straub H, McKinney C, et al. Postpartum weight retention risk factors and relationship to obesity at 1 year. Obstet Gynecol. 2015 Jan;125(1):144-52. doi: 10.1097/aog.0000000000000565. PMID: 25560116.

161. Haiek LN, Kramer MS, Ciampi A, et al. Postpartum weight loss and infant feeding. J Am Board Fam Pract. 2001 MarApr;14(2):85-94. PMID: 11314929.

162. Janney CA, Zhang D, Sowers M. Lactation and weight retention. Am J Clin Nutr. 1997 Nov;66(5):1116-24. PMID: 9356528.

163. Lyu LC, Lo CC, Chen HF, et al. A prospective study of dietary intakes and influential factors from pregnancy to postpartum on maternal weight retention in Taipei, Taiwan. Br J Nutr. 2009

Dec;102(12):1828-37. doi: 10.1017/s0007114509991243. PMID: 19678967.
164. Ng SK, Cameron CM, Hills AP, et al. Socioeconomic disparities in prepregnancy BMI and impact on maternal and neonatal outcomes and postpartum weight retention: the EFHL longitudinal birth cohort study. BMC Pregnancy Childbirth. 2014 Sep 08;14:314. doi: 10.1186/1471-2393-14-314. PMID: 25201481.

165. Ohlin A, Rossner S. Maternal body weight development after pregnancy. Int J Obes. 1990 Feb;14(2):159-73. PMID: 2341224.

166. Ohlin A, Rossner S. Factors related to body weight changes during and after pregnancy: the Stockholm Pregnancy and Weight Development Study. Obes Res. 1996 May;4(3):271-6. PMID: 8732961.

167. Linne Y, Dye L, Barkeling B, et al. Weight development over time in parous women-the SPAWN study--15 years follow-up. Int J Obes Relat Metab Disord. 2003

Dec;27(12):1516-22. doi: 10.1038/sj.ijo.0802441. PMID: 14634683.

168. Olson CM, Strawderman MS, Hinton PS, et al. Gestational weight gain and postpartum behaviors associated with weight change from early pregnancy to 1 y postpartum. Int J Obes Relat Metab Disord. 2003

Jan;27(1):117-27. doi: 10.1038/sj.ijo.0802156. PMID: 12532163.

169. Onyango AW, Nommsen-Rivers L, Siyam A, et al. Post-partum weight change patterns in the WHO Multicentre Growth Reference Study. Matern Child Nutr. 2011 Jul;7(3):228-40. doi: 10.1111/j.17408709.2010.00295.x. PMID: 21338469.

170. Ostbye T, Krause KM, Swamy GK, et al. Effect of breastfeeding on weight retention from one pregnancy to the next: results from the North Carolina WIC program. Prev Med. 2010 Nov;51(5):368-72. doi: 10.1016/j.ypmed.2010.07.017. PMID: 20655944.

171. Palmer JR, Kipping-Ruane K, Wise LA, et al. Lactation in relation to long-term maternal weight gain in African-American Women. Am J Epidemiol. 2015 Jun 15;181(12):932-9. doi: 10.1093/aje/kwv027. PMID: 25944887. 
172. Sichieri R, Field AE, Rich-Edwards J, et al. Prospective assessment of exclusive breastfeeding in relation to weight change in women. Int J Obes Relat Metab Disord. 2003 Jul;27(7):815-20. doi: 10.1038/sj.ijo.0802285. PMID: 12821967.

173. Stuebe AM, Kleinman K, Gillman MW, et al. Duration of lactation and maternal metabolism at 3 years postpartum. $\mathrm{J}$ Womens Health (Larchmt). 2010

May;19(5):941-50. doi:

10.1089/jwh.2009.1660. PMID: 20459331.

174. Straub H, Simon C, Plunkett B, et al. Evidence for a complex relationship among weight retention, cortisol and breastfeeding in postpartum women. Matern Child Health J. 2016;20(7):1375-83. doi: 10.1007/s10995016-1934-z. PMID: 116170909.

175. Walker L, Freeland-Graves JH, Milani T, et al. Weight and behavioral and psychosocial factors among ethnically diverse, lowincome women after childbirth: II. Trends and correlates. Women Health. 2004;40(2):19-34. doi: 10.1300/J013v40n02_02. PMID: 15778136.

176. Jarlenski MP, Bennett WL, Bleich SN, et al. Effects of breastfeeding on postpartum weight loss among U.S. women. Prev Med. 2014 Dec;69:146-50. doi:

10.1016/j.ypmed.2014.09.018. PMID: 25284261.

177. Sharma AJ, Dee DL, Harden SM. Adherence to breastfeeding guidelines and maternal weight 6 years after delivery. Pediatrics. 2014 Sep;134 Suppl 1:S42-9. doi: 10.1542/peds.2014-0646H. PMID: 25183755.

178. Perez-Escamilla R, Martinez JL, SeguraPerez S. Impact of the Baby-friendly Hospital Initiative on breastfeeding and child health outcomes: a systematic review. Matern Child Nutr. 2016 Jul;12(3):402-17. doi: 10.1111/mcn.12294. PMID: 26924775.

179. Stuebe AM, Rich-Edwards JW. The reset hypothesis: lactation and maternal metabolism. Am J Perinatol. 2009 Jan;26(1):81-8. doi: 10.1055/s-00281103034. PMID: 19031350.
180. Beck LF, Morrow B, Lipscomb LE, et al. Prevalence of selected maternal behaviors and experiences, Pregnancy Risk Assessment Monitoring System (PRAMS), 1999. MMWR Surveill Summ. 2002;51(2):1-27.

181. Pesa JA, Shelton MM. Health-enhancing behaviors correlated with breastfeeding among a national sample of mothers. Public Health Nurs. 1999;16(2):120-4.

182. Grummer-Strawn LM, Shealy KR. Progress in protecting, promoting, and supporting breastfeeding: 1984-2009. Breastfeed Med. 2009 Oct;4 Suppl 1:S31-9. doi: 10.1089/bfm.2009.0049. PMID: 19827921.

183. Saade GR. Pregnancy as a window to future health. Obstet Gynecol. 2009

Nov;114(5):958-60. doi: 10.1097/AOG.0b013e3181bf5588. PMID: WOS:000271293500001. 


\section{Chapter 1. Introduction}

\section{Background}

In reproductive physiology, lactation follows pregnancy; evidence supports the association between breastfeeding and better health outcomes for both infants and mothers. ${ }^{1-3}$ A 2007 Agency for Healthcare Research and Quality (AHRQ) review by Ip and colleagues concluded that breastfeeding was associated with reduced maternal type 2 diabetes, breast cancer, and ovarian cancer, but not fracture. ${ }^{2}$ For other outcomes (e.g., postpartum depression), the authors concluded that the relationship between breastfeeding and maternal health was unclear. Since 2007, several new studies have reported on maternal outcomes not addressed in the 2007 AHRQ review, including hypertension, rates of myocardial infarction, and other cardiovascular outcomes. $^{4-7}$

Breastfeeding initiation rates are increasing, and 2014 estimates for the percentage of infants breastfed at birth in the United States $(82.5 \%)^{8}$ met the Healthy People 2020 target for the percentage of infants ever breastfed (81.9\%). ${ }^{9}$ However, rates of breastfeeding duration did not meet Healthy People 2020 targets. In 2014, only 55.3 percent were breastfed at 6 months and 33.7 percent at 12 months, $^{8}$ falling short of Healthy People 2020 targets of 66.6 and 34.1 percent, respectively, for 6 and 12 months. ${ }^{9}$ Rates of exclusive breastfeeding through 3 and 6 months were 46.6 and 24.9 percent, respectively; these measures are close to Health People 2020 targets (46.2 and $25.5 \%$, respectively). ${ }^{8}$ Women would prefer to breastfeed longer: in a national survey, 45 percent of U.S. women who initiated breastfeeding reported early, undesired weaning. ${ }^{10}$ Despite rising breastfeeding initiation (starting) and duration (continuing) rates in the United States, racial and ethnic differences persist. From 2000 to 2014, the percentage of women who initiated breastfeeding went up from 47.4 percent to 68.0 percent for blacks, 71.8 percent to 85.7 percent for whites, and 77.6 percent to 84.8 percent for Hispanics. ${ }^{11,12}$ Sociodemographic factors associated with an increased likelihood of breastfeeding initiation and continuation include older maternal age, being married, Asian or white race, Hispanic ethnicity, higher maternal education, and access to private insurance. ${ }^{12-15}$

In addition to setting targets for breastfeeding initiation rates and duration of breastfeeding, other Healthy People 2020 objectives related to breastfeeding include (1) increasing the proportion of live births that occur in facilities that provide recommended care for lactating mothers and their babies and (2) increasing the proportion of employers that have worksite lactation support programs. ${ }^{9}$ These community, workplace, and health care system-based programs and policies may be promising strategies to support initiation and increase duration of breastfeeding.

Health care system-based interventions may include maternity staff education or the BabyFriendly Hospital Initiative (BFHI). The BFHI is a global program sponsored by the World Health Organization and United Nations Children's Fund to encourage and recognize hospitals and birth centers that create an environment that supports breastfeeding; the "Ten Steps to Successful Breastfeeding” are listed in Table 1. In each country, a BFHI Coordination Group is charged with designating facilities as Baby-Friendly; ${ }^{16}$ there are likely country-specific differences in the process for determining final accreditation (or certification) status. As a result, details of implementation vary from country to country. For U.S. hospitals, Baby-Friendly accreditation is awarded to facilities that successfully implement the 10 steps and the International Code of Marketing of Breast-Milk Substitutes ${ }^{17}$ and pass an intensive site visit. ${ }^{18}$ Site visits and certification are adjudicated by Baby-Friendly USA, a 501c3 nonprofit 
organization. ${ }^{19}$ In addition to certification by Baby-Friendly USA, State departments of public health have encouraged implementation of the Ten Steps through local programs such as the Texas Ten Step Program, ${ }^{20}$ the North Carolina Maternity Center Breastfeeding-Friendly Designation Program, ${ }^{21}$ and others. On a national level, the Centers for Disease Control and Prevention has audited maternity care practices during the past 10 years with a biennial maternity care practice survey of all U.S. facilities where births occur, results of which are distributed to each facility. ${ }^{22}$

Table 1. Baby-Friendly Hospital Initiative's 10 steps to successful breastfeeding ${ }^{a}$

1. Have a written breastfeeding policy that is routinely communicated to all health care staff.

2. Train all health care staff in skills necessary to implement this policy.

3. Inform all pregnant women about the benefits and management of breastfeeding.

4. Help mothers initiate breastfeeding within 1 hour of birth.

5. Show mothers how to breastfeed, and how to maintain lactation even if they should be separated from their infants.

6. Give infants no food or drink other than breast milk, unless medically indicated.

7. Practice rooming in-allow mothers and infants to remain together 24 hours a day.

8. Encourage breastfeeding on demand.

9. Give no pacifiers or artificial nipples to breastfeeding infants.

10. Foster the establishment of breastfeeding support groups and refer mothers to them on discharge from the hospital or birth center.

a Baby-Friendly USA “Ten Steps to Successful Breastfeeding”23

Insurance coverage for lactation support is another strategy that may enable women to achieve their breastfeeding goals. Costs associated with breastfeeding support (e.g., comprehensive lactation support and counseling and breastfeeding equipment) are currently covered by health insurance marketplace plans and private nongrandfathered health plans under the 2010 Patient Protection and Affordable Care Act. ${ }^{24}$ It is not clear whether certain lactation benefit packages (e.g., type of breastfeeding supplies offered, number of visits provided, or qualifications of intervention delivery personnel) are more or less effective than others in increasing breastfeeding initiation and duration. In addition, a key system-based program relevant to breastfeeding is the Special Supplemental Nutrition Program for Women, Infants and Children (WIC), which serves 53 percent of infants born in the United States. ${ }^{25}$ Because WIC reaches more than half of U.S. infants, its programs have considerable impact on population health.

There is broad appeal and interest in workplace interventions to increase duration and exclusivity of breastfeeding. If they are not supported by their employers, working mothers may have difficulty expressing and storing milk and thus may not be able to maintain breastfeeding. At the same time, there is limited evidence assessing these interventions, ${ }^{26}$ and the benefits and harms associated with workplace breastfeeding interventions are uncertain. The 2010 Patient Protection and Affordable Care Act included a provision aimed at workplace breastfeeding policies by amending Section 7 of the Fair Labor Standards Act to require employers to provide reasonable break time and a private space (other than a bathroom) for breastfeeding women to express breast milk for at least 1 year after childbirth. ${ }^{27}$

\section{Existing Guidelines}

Multiple clinical guidelines and health-related organizations recommend exclusive breastfeeding up to (or around) 6 months, including the American Academy of Pediatrics, ${ }^{28}$ the American Congress of Obstetrics and Gynecology, ${ }^{29}$ World Health Organization, ${ }^{17,}{ }^{30}$ and 
others. ${ }^{31,32}$ These organizations recommend continued breastfeeding through the first year of life and beyond; the WHO recommends continued breastfeeding through the second year of life and beyond.

\section{Rationale for Evidence Review}

Programs and policies to support breastfeeding are quite diverse and often complex. ${ }^{33-35}$ The purpose of this review is to develop an evidence report that summarizes the effectiveness of community, workplace, and health care system-based programs and policies aimed at supporting and promoting breastfeeding. This review will describe the effectiveness of programs or policies in supporting breastfeeding and whether effectiveness varies for subgroups of women defined by important sociodemographic factors (e.g., maternal age, education, and income; family and social support). The U.S. Preventive Services Task Force (USPSTF) recommends providing interventions during pregnancy and after birth to support breastfeeding as part of routine primary care (B recommendation). ${ }^{36}$ To avoid duplication, this review will not address the effectiveness of individual-level primary care interventions to support breastfeeding covered in the recent systematic review to support the USPSTF recommendation. ${ }^{37}$

In addition, this review will address the association between breastfeeding and maternal health. Substantial time has elapsed since the last AHRQ review on this topic in 2007, and the body of literature focused on the maternal health benefits of breastfeeding has grown. ${ }^{1,38-40}$ This review will conduct a partial update of the 2007 AHRQ review focused on the relationship between breastfeeding and various maternal health outcomes.

\section{Key Questions}

\section{Key Question 1:}

1a. What are the effectiveness and harms of programs and policies on initiation, duration, and exclusivity of breastfeeding?

1b. To what extent do the effectiveness and harms of programs and policies on initiation, duration, and exclusivity of breastfeeding differ for subpopulations of women defined by sociodemographic factors (e.g., age, race, ethnicity, socioeconomic status)?

1c. To what extent do intervention-related characteristics (e.g., type of breast pump provided-manual or electric; delivery personnel) influence the initiation, duration, and exclusivity of breastfeeding?

\section{Key Question 2:}

2a. What are the comparative benefits and harms for maternal health outcomes among women who breastfeed for different intensities and durations?

2b. To what extent do benefits and harms for maternal health outcomes differ for subpopulations of women defined by age, race, ethnicity, and comorbidity? 


\section{Analytic Framework}

We developed an analytic framework to guide the systematic review process (Figure 1). The analytic framework illustrates the population, interventions, outcomes, and adverse effects that guided our literature search and synthesis.

Figure 1. Analytic framework for breastfeeding programs and policies, breastfeeding uptake, and maternal health outcomes in developed countries

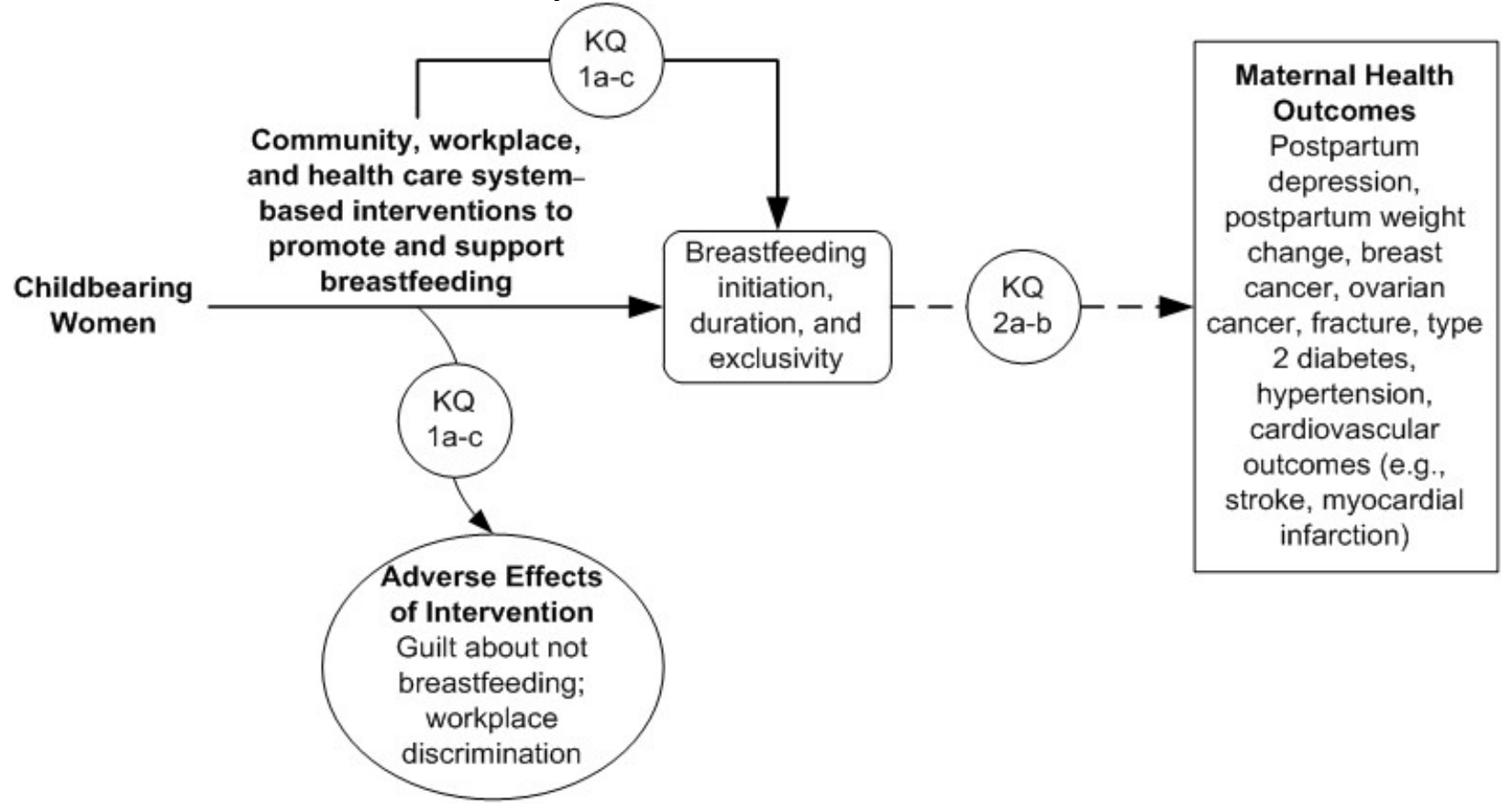

KQ=Key Question.

\section{Organization of This Report}

The remainder of the review describes our methods in detail and presents the results of our synthesis of the literature by Key Question (KQ), with summary tables and the strength of evidence (SOE) grades for intervention types (KQ 1) or eligible maternal health outcomes (KQ 2). The discussion section offers our conclusions, summarizes our findings, and provides other information relevant to interpreting this work for practice and future research.

Appendix A contains the exact search strings we used in our literature searches. Appendix B contains the PRISMA and provides a summary of evidence and search selection. Appendix C provides the specific questions used for evaluating the risk of bias of all included studies and documents risk of bias ratings for each study and explains the rationale for high or medium ratings. Appendix $\mathrm{C}$ also provides questions used for evaluating risk of bias and relevance of included systematic reviews and documents relevance and risk of bias ratings for each review. Appendix D presents information about our grading of the strength of the various bodies of evidence (tables for individual domain assessments and overall SOE grades for each intervention type or outcome). Appendix E lists studies excluded at the stage of reviewing full-text articles with reasons for exclusion. Appendix F contains the breast cancer evidence tables. Appendix G lists references cited across all appendixes. 


\section{Chapter 2. Methods}

\section{Protocol Review}

The initial Key Questions (KQs) were provided by Agency for Healthcare Research and Quality (AHRQ) and developed in collaboration with partners from the Centers for Disease Control and Prevention and National Institutes of Health Office of Women's Health. The RTI International-University of North Carolina at Chapel Hill (RTI-UNC) Evidence-based Practice Center further refined the KQs. We sought guidance from a Technical Expert Panel on the final research protocol, which was posted on the AHRQ Web site on March 20, 2017, at https://effectivehealthcare.ahrq.gov/topics/breastfeeding/research-protocol/; our PROSPERO registration number is CRD42017079125.

\section{Literature Search Strategy}

\section{Search Strategy}

We conducted two separate search strategies, one for KQ 1 and a second for KQ 2. Appendix A presents the full search strategies for each KQ. For KQ 1, we searched PubMed/MEDLINE, the Cochrane Library, and CINAHL from January 1, 1980, to October 12, 2017. Its start date (January 1, 1980) was chosen to ensure that evidence is applicable to current breastfeeding policies and practices. For KQ 2, we searched PubMed/MEDLINE, the Cochrane Library, and CINAHL from November 1, 2005 (6 months prior to the search date of the 2007 AHRQ review searches) to October 12, 2017.

For both searches, we used either Medical Subject Headings or major headings as search terms when available or key words when appropriate, focusing on terms to describe the relevant population and interventions of interest. We reviewed our search strategy with the Technical Expert Panel and incorporated their input into our search strategy. An experienced information scientist (a librarian at the Evidence-based Practice Center) conducted the searches. The literature search will be updated concurrent with the peer review process.

We searched for unpublished studies relevant to this review using ClinicalTrials.gov. On our behalf, the AHRQ Scientific Resource Center solicited scientific information packages via Federal Register notices or informational requests.

We also manually searched reference lists of pertinent reviews and included trials and background articles on this topic to look for any relevant citations that our searches might have missed. We imported all citations into an EndNote® X7.5 electronic database.

\section{Inclusion and Exclusion Criteria}

We developed eligibility (inclusion and exclusion) criteria with respect to PICOTS (populations, interventions, comparators, outcomes, time frames, settings), study designs, and study durations for each KQ (Table 2). Both KQs focused on populations of childbearing women (adults and adolescents). 
Table 2. Eligibility criteria

\section{PICOTS Inclusion}

Population KQs 1 and 2: Childbearing women and adolescents ${ }^{\mathrm{a}}$; we will also search for evidence on subgroups of women defined by age, race, ethnicity, comorbidity, and socioeconomic status (including insurance status and payer type).

Intervention/ KQ 1: Community, workplace, and health care system-based Exposure interventions aimed at promoting and supporting BF, including the following: health plan benefits; State and Federal policies or programs (e.g., WIC programs); workplace and school-based programs; BFHI implementation, including full or partial implementation (defined as three or more steps)

$\mathrm{KQ}$ 2: Exposure to $\mathrm{BF}^{\mathrm{b}}$

Comparator KQ 1: No intervention (or usual practice); comparisons of two interventions that differ in content or intensity KQ 2: No BF; shorter duration (e.g., BF for 1 month vs. 12 months) and/or less intensive BF (e.g., exclusive BF vs. mixed feeding or formula feeding)

Outcomes $\quad \mathrm{KQ}$ 1: Rates of BF initiation, duration, and exclusivity of BF; harms of interventions (e.g., guilt about not BF, workplace discrimination, and other reported harms)

KQ 2: Postpartum depression (any measure), postpartum weight change, ${ }^{c}$ breast cancer, ovarian cancer, osteoporotic fracture, ${ }^{\mathrm{d}}$ type 2 diabetes, hypertension, cardiovascular outcomes (e.g., stroke, myocardial infarction, cardiovascular disease specific mortality, and composite outcomes)

\section{Country \\ setting \\ Study \\ designs}

Publication

language

KQ 1 and 2: Studies conducted in a developed country ("very high" [KQs 1 and 2] e and "high" [KQ 1] human development index per the United Nations Development Programme $)^{41}$

\section{KQ 1: RCTs; CCTs; prospective cohort studies with concurrent} control groups; systematic reviews; for studies assessing policy or system-level interventions, pre-post studies with repeated outcome measures before and after the intervention are also eligible

KQ 2: RCTs, CCTs, cohort studies, ${ }^{f}$ case-control studies, systematic reviews

${ }^{a}$ Childbearin KQs 1 and 2: English

\section{Exclusion}

KQs 1 and 2: Men; nulliparous women; children

KQ 1: Interventions delivered in primary care settings as part of pre- or postnatal care; interventions specific to $\mathrm{NICU}$ care

KQ 2: All other exposures

KQs 1 and 2: All other comparisons; no comparisons

KQ 1: Any other outcome not specified, including compliance with policies or practices and attitudes toward BF and complications of lactation KQ 2: Any other outcome not specified, including complications of lactation (e.g., mastitis) and other maternal outcomes (e.g., lung cancer) KQs 1 and 2: Studies conducted in other countries

KQs 1 and 2: All other designs targeted toward the woman's partner or family.

${ }^{b}$ This includes women who breastfeed their infant at the breast and/or express milk.

c The prior 2007 review on this topic for AHRQ²restricted results to prospective cohort studies reporting on women for whom the exclusivity or amount of breastfeeding was known. Studies reporting on weight change needed to control for gestational weight gain or prepregnancy weight and have at least 3 months of followup. All included studies for the update met these criteria. ${ }^{\mathrm{d}}$ We excluded studies with surrogate measures of fracture (e.g., fracture risk score or index) or bone turnover markers or with measures of osteoporosis only.

${ }^{\text {e }}$ The United Nations does not recognize Taiwan (i.e., Republic of China) as a sovereign state and did not include it in the 2015 Human Development Index report. However, Taiwan's government calculated its human development index to be 0.882, based on 2014 data and using the same methodology as the United Nations. This human development index value would place Taiwan among countries in the "very high" human development category, so it will be included in this report. ${ }^{42}$

${ }_{\mathrm{f}}^{\mathrm{f}}$ For all KQ 2 outcomes, we included cohort studies that report on the incidence of eligible health outcomes prospectively regardless of whether women were classified into categories based on breastfeeding exposure prospectively (i.e., at study enrollment) or retrospectively. Additionally, for long-term outcomes for which no prospective studies of outcomes exist, we included cohort studies that collected information on exposure and outcomes at a single time point (retrospectively). Such studies provide evidence on associations rather than on causal relationships.

AHRQ = Agency for Healthcare Research and Quality; BF = breastfeeding; BFHI = Baby-Friendly Hospital Initiative; CCT = controlled clinical trial; KQ = Key Question; NICU = neonatal intensive care unit; PICOTS = population, intervention/exposure, comparator, outcomes, time frames, country settings, study design; RCT = randomized controlled trial; WIC = Special Supplemental Nutrition Program for Women, Infants and Children. 
The focus of KQ 1 is on providing an overall synthesis of community, workplace, or health care system-based interventions aimed at promoting and supporting breastfeeding. We specifically excluded studies assessing individual-level primary care interventions to support and promote breastfeeding to avoid duplicating a recent review conducted for the U.S. Preventive Services Task Force. ${ }^{37}$ For KQ 1, we included studies conducted in countries categorized as "very high" and "high" on the human development index per the United Nations Development Programme, given the relatively small body of literature on the Baby-Friendly Hospital Initiative and other community-based breastfeeding interventions. ${ }^{43}$ Eligible outcomes include rates of breastfeeding initiation, duration and exclusivity of breastfeeding (based on any definition, as described by study authors), and potential harms of interventions (e.g., guilt about not breastfeeding, workplace discrimination).

Our eligibility criteria for KQ 2 are based on those used in the 2007 AHRQ review by Ip and colleagues for maternal health outcomes. Eligible studies are those that compare groups of women exposed to breastfeeding with those who did not breastfeed (or breastfed for shorter duration and/or less intensity). For this update, we also expanded to include additional outcomes including hypertension and cardiovascular disease (e.g., stroke and myocardial infarction). For outcomes relevant to osteoporotic fracture, we limited to studies reporting on fractures and excluded intermediate outcomes such as bone mineral density.

\section{Study Selection}

Two members of the research team independently reviewed all titles and abstracts of individual studies and published systematic reviews (identified through searches) for eligibility against our inclusion and exclusion criteria (Table 2). We retrieved any publications marked for inclusion by either reviewer for evaluation of the full text. For titles and abstracts that lacked adequate information to determine inclusion or exclusion, we retrieved the full text for review. Then, two investigators independently reviewed the full text to determine final inclusion or exclusion. The reviewers resolved any disagreements by discussion and consensus or by consulting a third member of the review team.

All results in both review stages were tracked in an EndNote database. We recorded the principal reason that each excluded full-text publication did not satisfy the eligibility criteria (see Appendix B).

\section{Data Extraction}

For studies that met our inclusion criteria, we designed and used structured data extraction forms to gather pertinent information from each article, including characteristics of study populations, settings, interventions, comparators, study designs, methods, and results. One investigator extracted the relevant data from each included article; all data abstractions were reviewed for completeness and accuracy by a second member of the team. We recorded intention-to-treat results if available. For KQ 2, we abstracted results relevant to the association between breastfeeding and health outcomes that were adjusted for potential confounders rather than unadjusted results (when both were provided). All data abstraction was performed using Microsoft Excel ${ }^{\circledR}$ software. 


\section{Risk of Bias Assessment of Individual Studies}

To assess the risk of bias (ROB) (i.e., internal validity) of individual studies, we adapted existing tools (ROBINS-I ${ }^{44}$ for observational studies and the Cochrane tool $^{45}$ for trials) and used predefined criteria based on the AHRQ Methods Guide for Effectiveness and Comparative Effectiveness Reviews. These criteria included questions to assess selection bias, confounding, performance bias, detection bias, and attrition bias; concepts covered include those about adequacy of randomization, similarity of groups at baseline, masking, attrition, whether intention-to-treat analysis was used, method of handling missing data, validity and reliability of outcome measures, and treatment fidelity. ${ }^{46}$ Appendix $C$ lists the specific questions used for evaluating the ROB of all included studies. It also includes a table showing the responses to these questions and $\mathrm{ROB}$ ratings for each study and explains the rationale for all ratings that were either high or medium. When tools use the term "some concerns" for final ROB assessments in Appendix B, we changed this to "medium" for consistency in our summary tables and text; a final rating of "medium" or "some concerns" refers to the same level of concern regarding ROB.

In general terms, results from a low ROB study are considered to be valid. A study with medium (i.e., some concerns in Appendix C) ROB is susceptible to some risk of bias but probably not enough to invalidate its results. A study assessed as high ROB has significant ROB (e.g., stemming from serious errors in design, conduct, or analysis) that may invalidate its results. To assess publication bias, we looked for evidence of unpublished literature through searches of gray literature (clinicaltrials.gov).

We evaluated the ROB from selection, confounding, measurement of exposure, missing data, measurement of outcomes, and reporting. Based on multiple signaling questions, we rated the ROB for each domain as high, some concerns, or low and provided justification for the rating. Studies with a high ROB in any domain received an overall high ROB rating. Studies with one or more domains with some concerns received an overall rating of medium ROB (i.e., some concerns in Appendix C). Studies with low ROB in all domains received an overall low ROB rating. We describe the results of all included studies regardless of the ROB rating. ROB for individual studies influences the aggregate ROB rating for the overall strength of evidence (SOE) for each outcome.

Two independent reviewers assessed ROB for each study. Disagreements between the two reviewers were resolved by discussion and consensus or by consulting a third member of the team.

\section{Risk of Bias Assessment of Systematic Reviews}

For both KQs, we capitalized on the availability of existing systematic reviews and metaanalyses; these were captured in our database searches and identified during the literature review. One systematic review was identified for KQ 1 but was rated as not relevant, primarily due to ineligible country settings and study designs of the included studies (Appendix Table C-1). For KQ 2, we assessed the relevance of recent systematic reviews (published within the past 5 years) using predefined criteria. Appendix Table C-17 shows our assessment of each potentially relevant review identified during our database searches. For reviews determined to be relevant, we rated the ROB as low, unclear, or high using the ROBIS tool; ${ }^{47}$ we excluded reviews rated as high ROB. Appendix $\mathrm{C}$ lists the specific questions used for evaluating the ROB of all relevant reviews. 


\section{Data Synthesis}

We summarized all included studies in narrative form and in summary tables that tabulate the important features of the study populations, design, intervention, outcomes, and results for KQ 1 and KQ 2.

KQ 2 is a partial update of the 2007 AHRQ review by Ip and colleagues; we synthesized evidence from that review with newly identified evidence and did not report results from that review separately from the body of literature published since. As noted above, we included other recent (published within the past 5 years) relevant systematic reviews rated low or unclear ROB using the ROBIS tool ${ }^{47}$ for KQ 2. Conclusions from systematic reviews rated high ROB may not be valid because of bias stemming from uncertain study eligibility criteria, lack of dual-review during identification and selection of studies, and other factors. For those KQ 2 outcomes for which we included a recent published systematic review, we first describe the results of the review and then summarize data from primary studies not included in the review (i.e., those published after the latest search date of those reviews or identified by searching reference lists of relevant articles).

When recent, relevant existing systematic reviews were identified for a particular outcome, we assessed whether newly identified primary studies were likely to change judgments about conclusions made in existing reviews using an SOE framework (i.e., assessment of study limitations, consistency, precision, directness, and reporting bias). If we deemed their results likely to change the conclusions, we considered conducting a new quantitative synthesis if appropriate (i.e., if conclusions made in existing reviews are based on a pooled analysis of studies). If the new studies were consistent with prior syntheses and would not change the conclusion of the review, we presented the results of the existing review along with an updated qualitative synthesis including the newly identified studies and an explanation of how they are consistent with the prior findings. We conducted a new SOE for each outcome and did not use SOE grading from existing reviews.

We considered performing meta-analyses when we had at least three unique studies of low or medium risk of bias that we deemed sufficiently similar (in population, interventions, comparators, and outcomes), because of the potential biases of meta-analyses that include a small number of studies. ${ }^{48}$ We planned to consider heterogeneity carefully before calculating a pooled summary estimate in a meta-analysis, we carefully considered the heterogeneity across studies. As described above, in cases where we identified a recent eligible meta-analysis for an eligible outcome, we assessed whether to update the analysis by considering how the results of recently published primary studies would change the conclusions of the meta-analyses using a SOE framework. For KQ 1, we did not conduct meta-analyses of studies assessing the benefit of interventions to improve rates of breastfeeding initiation and duration because we identified few studies that were similar in population, setting, intervention type and design. For KQ 2, the size of the literature and heterogeneity varied across outcomes. For postpartum depression and cardiovascular disease, we did not conduct meta-analyses primarily due to significant heterogeneity in terms of study designs and outcome measures. For type 2 diabetes, breast and ovarian cancer, we included recent existing systematic reviews with meta-analyses; individual studies identified in our database searches were generally consistent with the pooled results reported by existing systematic reviews and so we did conduct new meta-analyses for these outcomes.

We synthesized and described results of all studies regardless of the risk of bias rating. When possible, we describe whether results of studies rated high risk of bias differ from those rated low 
or medium risk of bias (narratively, or by noting whether existing systematic reviews or metaanalyses found inconsistent results for studies that varied by risk of bias).

\section{Strength of the Body of Evidence}

We graded the SOE of the accumulated evidence on a given issue to answer the specific KQs on the benefits and harms of the interventions in this review; we used the guidance established for the Evidence-based Practice Center Program. ${ }^{49}$ Developed to grade the overall strength of a body of evidence, this approach now incorporates five key domains: ROB (including study design and aggregate ROB); consistency, directness, and precision of the evidence; and reporting bias. It also considers other optional domains that may be relevant for some scenarios, such as plausible confounding that would decrease the observed effect and strength of association (i.e., magnitude of effect) or factors that would increase the strength of association (i.e., dose-response effect).

Table 3 describes the grades of evidence that can be assigned. Grades reflect the strength of the body of evidence to answer the KQs on the comparative effectiveness, efficacy, and harms of the interventions in this review and on associations between breastfeeding and health outcomes. Two reviewers assessed each domain for each key outcome, and differences were resolved by consensus. For each assessment, one of the two reviewers was always an experienced Evidenced-based Practice Center investigator. We graded the SOE for all included outcomes.

Table 3. Definitions of the grades of overall strength of evidence

\begin{tabular}{ll}
\hline Grade & Definition \\
\hline High & $\begin{array}{l}\text { We are very confident that the estimate of effect lies close to the true effect for this } \\
\text { outcome. The body of evidence has few or no deficiencies. We believe that the findings are stable } \\
\text { (i.e., another study would not change the conclusions). }\end{array}$ \\
\hline Moderate & $\begin{array}{l}\text { We are moderately confident that the estimate of effect lies close to the true effect for this } \\
\text { outcome. The body of evidence has some deficiencies. We believe that the findings are likely to } \\
\text { be stable, but some doubt remains. }\end{array}$ \\
\hline Low & $\begin{array}{l}\text { We have limited confidence that the estimate of effect lies close to the true effect for this } \\
\text { outcome. The body of evidence has major or numerous deficiencies (or both). We believe that } \\
\text { additional evidence is needed before concluding either that the findings are stable or that the } \\
\text { estimate of effect is close to the true effect. }\end{array}$ \\
\hline Insufficient & $\begin{array}{l}\text { We have no evidence, we are unable to estimate an effect, or we have no confidence in the } \\
\text { estimate of effect for this outcome. No evidence is available or the body of evidence has } \\
\text { unacceptable deficiencies, precluding reaching a conclusion. }\end{array}$ \\
\hline Source: Berkman et al. ${ }^{49}$
\end{tabular}

An unfavorable assessment for any one of the five key domains (e.g., inconsistency, indirectness, imprecision, high or medium aggregate ROB, or evidence of reporting bias) typically resulted in downgrading from high to moderate SOE. Two unfavorable assessments typically resulted in downgrading to low SOE. When only one study reported an outcome of interest (with unknown consistency and imprecision arising from nonsignificant results or wide confidence intervals spanning the null), we usually graded the SOE as insufficient. Insufficient ratings also applied to evidence bases with multiple studies with inconsistent results. When aggregate ROB was high or uncertain, we usually graded the SOE as low or insufficient (depending on responses to other key domains). Appendix D presents tables showing our assessments for each domain and the resulting SOE grades for outcomes organized by KQ and then intervention type (for KQ 1) and outcome category (for KQ 2). 


\section{Applicability}

We assessed applicability following guidance from the Methods Guide for Effectiveness and Comparative Effectiveness Reviews. ${ }^{50}$ For individual studies, we examined conditions that may limit applicability based on the PICOTS structure. Some factors identified a priori that may limit the applicability of evidence include the following: race or ethnicity of enrolled populations, setting of enrolled populations, geographic setting, and availability of health insurance and other health-related employment benefits. We also paid close attention to secular trends when interpreting the evidence. Such trends are of concern in that breastfeeding initiation rates in the United States have changed dramatically in the past 40 years, from a nadir of less than 25 percent in $1971^{51}$ to more than 80 percent in $2014 .^{8}$ This is important because the time period between exposure to breastfeeding and some outcomes of interest (e.g., cancer, cardiovascular disease) may be decades, and secular trends in social determinants of infant feeding may confound observed associations. Findings linking breastfeeding to maternal health among women feeding their infants decades ago may not be generalizable to contemporary women.

\section{Peer Review and Public Commentary}

This report was posted for public comment and was peer reviewed. We addressed all comments in the final report, making revisions as needed; a disposition of comments report will be publicly posted 3 months after release of the final report. 


\section{Chapter 3. Results}

\section{Results of Literature Search and Screening}

Searches of all sources identified a total of 11,006 potentially relevant citations. We included 128 unique individual studies (described in 137 publications) and 10 systematic reviews. Of these, 40 individual studies (from 44 publications) were relevant to Key Question (KQ) 1, and 88 individual studies (from 93 publications) and 10 systematic reviews were relevant to KQ 2. Of the KQ 2 included studies, 18 were studies from a prior 2007 Agency for Healthcare Research and Quality (AHRQ) review addressing the maternal health benefits of breastfeeding. ${ }^{2}$ The remaining 34 studies from the 2007 review were included in at least one 1 of our 10 systematic reviews or superseded by a new included study. Appendix E provides a complete list of articles excluded at the full-text screening stage, with reasons for exclusion.

\section{Effectiveness and Harms of Breastfeeding Programs and Policies}

To aid in synthesizing results of similar studies, we categorized interventions primarily based on intervention type: Baby-Friendly Hospital Initiative (BFHI), other (non-BFHI) health care system-based interventions (e.g., residency curriculum related to breastfeeding), Special Supplemental Nutrition Program for Women, Infants and Children (WIC)-based interventions, and community-based interventions (not primarily delivered as part of the health care system). Key points and strength of evidence (SOE) assessments are summarized below by intervention category.

\section{Key Points: Baby-Friendly Hospital Initiative}

- Twelve studies set in diverse country settings assessed the effectiveness of BFHI interventions; no study reported on potential harms associated with the intervention.

- Based on results from nine cohort studies (1,227,532 women), rates of any and exclusive breastfeeding at discharge is higher among women giving birth in BFHI-certified facilities than control facilities (low SOE).

- Based on evidence from one large randomized controlled trial (RCT) (Promotion of Breastfeeding Intervention Trial [PROBIT], N=17,046) and eight cohort studies $(136,983$ women), we concluded that BFHI is associated with increased breastfeeding duration (moderate SOE).

- Low SOE supports the conclusion that implementation of four or more BFHI steps is associated with lower rates of weaning than implementation of fewer than four steps (1 cohort study; 1,417 women).

\section{Key Points: Non-BFHI Health Care System-Based Interventions}

- Fifteen studies assessed the effectiveness of other (non-BFHI) health care system-based interventions. Included studies assessed heterogeneous interventions; no study reported on potential harms associated with the interventions.

- Overall, low SOE evidence supports the effectiveness of three intervention types for improving the duration of exclusive breastfeeding: modified BFHI policy implementation in outpatient setting (e.g., development of a breastfeeding policy, staff training, outcome 
assessment, and quality improvement initiatives), continuous nursing care during the perinatal period (the same nurse provides routine perinatal care to the mother and infant), and health care provider education combined with a series of home visits.

- Interventions assessing health care provider education and training alone (without additional breastfeeding support services) are not effective in improving rates of breastfeeding initiation (low SOE); 4 studies (1,532 women) found no significant difference between groups.

\section{Key Points: Women, Infants and Children-Based Interventions}

- Eight included studies assessed a WIC program or policy; studies evaluated heterogeneous interventions. Although all studies were set in the United States, they included women from diverse States. None reported on potential harms.

- Based on 4 studies (2,480 women), low SOE supports the effectiveness of WIC-based peer-support programs for improving rates of any breastfeeding initiation (exclusive or nonexclusive) and breastfeeding duration.

- We found insufficient evidence (primarily because of unknown consistency and imprecision) to make a conclusion on the benefit of other WIC programs or policies for improving breastfeeding outcomes, including policy changes related to WIC food packages, provision of different types of breast pumps (electric vs. manual), tailored counseling, cash incentives, and peer-support programs targeted at fathers.

\section{Key Points: Community-Based Interventions}

- Five studies assessed the effectiveness of community-based interventions (not associated with the health care system or delivered as part of routine maternity care). Interventions were heterogeneous; we found no eligible studies evaluating workplace breastfeeding programs or policies.

- Low SOE supports the benefit of community-based interventions that provide mothers with peer support (via home visits) for improving rates of breastfeeding duration.

- Based on evidence from one large RCT, access to a community-based breastfeeding drop-in center among women receiving early home-based breastfeeding support does not increase breastfeeding duration (low SOE).

- We found insufficient evidence to draw a conclusion on the effectiveness of other community-based interventions, primarily because of unknown consistency, imprecision, and study limitations.

\section{Baby-Friendly Hospital Initiative Interventions}

\section{Characteristics}

Twelve included studies (described in 13 publications) assessed the effectiveness of BFHI interventions (Table 4). ${ }^{52-64}$ All focused on postpartum women enrolled from hospital wards or birth facilities soon after delivery. Studies were conducted in diverse country settings including the United States (two studies); ${ }^{44}{ }^{55}$ Taiwan (two studies); ${ }^{60,64}$ and one each in the Republic of Belarus, ${ }^{52}$ Hong Kong, ${ }^{56}$ Czech Republic, ${ }^{57}$ Russia, ${ }^{65}$ Croatia, ${ }^{59}$ Brazil, ${ }^{63}$ United Kingdom (multiple regions), ${ }^{61}$ and Scotland. ${ }^{66}$ All studies focused on multiple hospitals ( $>4$ ) or clusters of 
hospitals. The majority of studies focused on women giving birth between 2000 and 2009 (Table 4); two enrolled women in the late 1990s. ${ }^{52,62}$

One included study was an RCT, ${ }^{2} 10$ were prospective cohort studies, ${ }^{54-58,60-62,64,67}$ and 1 was a single-group pre-post study. ${ }^{59}$ The RCT (PROBIT) ${ }^{52}$ was a cluster-randomized trial of $\mathrm{BFHI}$ (34 hospitals were randomized to BFHI or routine practice). The majority of observational studies compared cohorts of women giving birth in hospitals that had implemented BFHI with women giving birth in hospitals that had not implemented BFHI (or were not accredited). Most assessed the implementation of all 10 steps; one study set in Hong Kong compared breastfeeding initiation rates at hospitals that had implemented at least 6 steps with those that had implemented fewer than 6 steps ${ }^{56}$ For observational studies comparing outcomes among women giving birth in BFHI-certified hospitals versus control hospitals, studies differed in the extent to which they described whether control hospitals had implemented any BFHI steps or whether they were planning to become certified. For example, one study compared outcomes among women giving birth in fully accredited hospitals (those that had implemented all 10 steps, had completed an annual audit of compliance and progress monitoring visits, and had completed a reassessment 24 months after the initial award), certified hospitals (those that adopted a policy covering all 10 steps and had an action planning visit from United Nations Children's Fund (UNICEF) UK Baby Friendly but were not yet fully accredited), and control hospitals. ${ }^{62}$

In terms of population characteristics, seven studies reported on maternal age and generally enrolled women in their 20s and 30s. ${ }^{52,54,56,58,60,61,63}$ Three studies (set in the United States and United Kingdom) reported on race; the percentage of nonwhite participants enrolled ranged from 3 to 47 percent. $^{54,55,61}$ In the six studies reporting on the percentage of enrolled women who were primiparous, the range was 38 to 67 percent. ${ }^{54,56,58,60,61,63}$ One study (PROBIT) reported on the percentage of women who previously breastfed a child for 3 months or longer (25\%), ${ }^{52}$ and one reported on the percentage of included women who had previously breastfed for any duration (34\%). ${ }^{56}$ Six studies reported on the rate of cesarean delivery among enrolled participants; rates ranged from 10 to 48 percent. $^{52,56,58,60,61,63}$

Included studies varied in terms of whether they had other specific inclusion criteria for mothers or hospitals or clusters (Table 4). Notably, the PROBIT RCT included only mothers who initiated breastfeeding, ${ }^{52}$ and another study recruited only women who were willing to breastfeed or experienced in breastfeeding. ${ }^{60}$ Three included studies were limited to women giving birth to healthy singletons. ${ }^{52,56,61}$

The majority (9 studies) were rated medium risk of bias (ROB) and three were rated high ROB. ${ }^{59,60,64}$ Common methodological limitations included selection bias, high or unclear rates of attrition, and baseline differences between groups that were not accounted for in analyses. 
Table 4. Characteristics of included studies assessing BFHI interventions

\begin{tabular}{|c|c|c|c|c|c|c|c|c|c|}
\hline $\begin{array}{l}\text { First Author, } \\
\text { Year } \\
\text { ROB }\end{array}$ & $\begin{array}{l}\text { Study Design } \\
\text { N Sites } \\
\text { N Participants }\end{array}$ & $\begin{array}{l}\text { Country } \\
\text { Year(s) of } \\
\text { Enrollment }\end{array}$ & Inclusion Criteria & $\begin{array}{l}\text { Intervention } \\
\text { Description }\end{array}$ & Comparator(s) & $\begin{array}{l}\text { Age, } \\
\text { Mean } \\
\text { (SD) }\end{array}$ & $\begin{array}{l}\% \\
\text { Non- } \\
\text { white }\end{array}$ & $\begin{array}{l}\text { \% Primi- } \\
\text { parous } \\
\% \\
\text { Previously } \\
\text { BF }\end{array}$ & $\begin{array}{l}\% \\
\text { Cesarean } \\
\text { Birth }\end{array}$ \\
\hline $\begin{array}{l}\text { Abolyan, } \\
2006^{58} \\
\text { Medium }\end{array}$ & $\begin{array}{l}\text { Prospective } \\
\text { cohort } \\
8 \text { hospitals } \\
741 \text { women }\end{array}$ & $\begin{array}{l}\text { Russia } \\
2004\end{array}$ & $\begin{array}{l}\text { Mothers with no } \\
\text { mental illness who } \\
\text { had healthy } \\
\text { infants (Apgar }>7 \text {, } \\
\text { weight }>2,500 \mathrm{~g} \text { ) }\end{array}$ & $\begin{array}{l}\text { Mothers giving } \\
\text { birth in } \\
\text { hospitals with } \\
\text { BFHI } \\
\text { certification }\end{array}$ & $\begin{array}{l}\text { Mothers giving } \\
\text { birth in "not-as } \\
\text { yet Baby- } \\
\text { Friendly"a } \\
\text { hospitals }\end{array}$ & 26 & NR & $\begin{array}{l}\text { 63-67 } \\
\text { NR }\end{array}$ & $15-16$ \\
\hline $\begin{array}{l}\text { Bartington, } \\
2006^{61} \\
\text { Medium }\end{array}$ & $\begin{array}{l}\text { Prospective } \\
\text { cohort } \\
496 \text { maternity } \\
\text { units; } 17,359 \\
\text { mother-infant } \\
\text { parts }\end{array}$ & $\begin{array}{l}\text { United } \\
\text { Kingdom } \\
\text { 2000-2002 }\end{array}$ & $\begin{array}{l}\text { Women with } \\
\text { singleton live } \\
\text { births }\end{array}$ & $\begin{array}{l}\text { Birth in a } \\
\text { BFHI- } \\
\text { accredited } \\
\text { hospital or } \\
\text { certified unit }\end{array}$ & $\begin{array}{l}\text { Birth in a } \\
\text { hospital that } \\
\text { was not BFHI } \\
\text { accredited or } \\
\text { certified }\end{array}$ & $\begin{array}{l}\text { Median } \\
\text { age: } 28-29\end{array}$ & $6-13$ & $\begin{array}{l}\text { 43-44 } \\
\text { NR }\end{array}$ & $22-23$ \\
\hline $\begin{array}{l}\text { Bosnjak, } \\
2004^{59} \\
\text { High }\end{array}$ & $\begin{array}{l}\text { Single-group, pre- } \\
\text { post study } \\
14 \text { primary care } \\
\text { sites } \\
7,414 \text { infants }\end{array}$ & $\begin{array}{l}\text { Croatia } \\
1990-2000\end{array}$ & NR & $\begin{array}{l}\text { Implementa- } \\
\text { tion of BFHI } \\
\text { (1994-1998) } \\
\text { and, later, } \\
\text { establishment } \\
\text { of community- } \\
\text { based BF } \\
\text { support groups } \\
\text { (1999-2000) }\end{array}$ & $\begin{array}{l}\text { Period before } \\
\text { intervention } \\
\text { implementation } \\
(1990-1993)\end{array}$ & NR & NR & $\begin{array}{l}\text { NR } \\
\text { NR }\end{array}$ & NR \\
\hline $\begin{array}{l}\text { Broadfoot, } \\
2005^{62} \\
\text { Medium }\end{array}$ & $\begin{array}{l}\text { Prospective } \\
\text { cohort } \\
33 \text { maternity units } \\
445,623 \text { births }\end{array}$ & $\begin{array}{l}\text { Scotland } \\
1995-2002\end{array}$ & $\begin{array}{l}\text { Maternity units: } \\
\text { those with >50 } \\
\text { births/year }\end{array}$ & $\begin{array}{l}\text { Hospitals that } \\
\text { held a UNICEF } \\
\text { U.K. BFHI } \\
\text { standard } \\
\text { award }\end{array}$ & $\begin{array}{l}\text { Hospitals } \\
\text { without BFHI } \\
\text { accreditation }\end{array}$ & NR & NR & $\begin{array}{l}\text { NR } \\
\text { NR }\end{array}$ & NR \\
\hline $\begin{array}{l}\text { Gau, 2004 } \\
\text { High }\end{array}$ & $\begin{array}{l}\text { Prospective } \\
\text { cohort } \\
12 \text { hospitals } \\
4,614 \text { mothers }\end{array}$ & $\begin{array}{l}\text { Taiwan } \\
2000-2002\end{array}$ & $\begin{array}{l}\text { Women giving } \\
\text { birth at } \\
\text { participating } \\
\text { hospitals }\end{array}$ & $\begin{array}{l}\text { Hospital } \\
\text { lactation } \\
\text { intervention } \\
\text { focused on } \\
\text { BFHI initiation } \\
\text { and } \\
\text { accreditation }\end{array}$ & $\begin{array}{l}\text { Control } \\
\text { hospitals not } \\
\text { participating in } \\
\text { intervention } \\
\text { (and pre-post } \\
\text { comparisons) }\end{array}$ & $30-31$ & NR & $\begin{array}{l}\text { 49-53 } \\
\text { NR }\end{array}$ & $24-30$ \\
\hline
\end{tabular}




\begin{tabular}{|c|c|c|c|c|c|c|c|c|c|}
\hline $\begin{array}{l}\text { Author, Year } \\
\text { ROB }\end{array}$ & $\begin{array}{l}\text { Study Design } \\
\text { N Sites } \\
\text { N Participants }\end{array}$ & $\begin{array}{l}\text { Country } \\
\text { Year(s) of } \\
\text { Enrollment }\end{array}$ & Inclusion Criteria & $\begin{array}{l}\text { Intervention } \\
\text { Description }\end{array}$ & Comparator(s) & $\begin{array}{l}\text { Age, } \\
\text { Mean } \\
\text { (SD) }\end{array}$ & $\begin{array}{l}\% \\
\text { Non- } \\
\text { white }\end{array}$ & $\begin{array}{l}\% \text { Primi- } \\
\text { parous } \\
\% \\
\text { Previously } \\
\text { BF }\end{array}$ & $\begin{array}{l}\% \\
\text { Cesarean } \\
\text { Birth }\end{array}$ \\
\hline $\begin{array}{l}\text { Hawkins, } \\
2014^{54} \\
\text { Medium }\end{array}$ & $\begin{array}{l}\text { Prospective } \\
\text { cohort } \\
32 \text { birth facilities } \\
\text { in } 5 \text { U.S. States } \\
25,327 \text { women }\end{array}$ & $\begin{array}{l}\text { United States } \\
1999-2009\end{array}$ & $\begin{array}{l}\text { Facilities: datab }^{\text {b }} \\
\text { from } 5 \text { States } \\
\text { participating in } \\
\text { PRAMS } \\
\text { monitoring } \\
\text { (1999-2009) } \\
\text { Mothers giving } \\
\text { birth at facilities } \\
\text { with } \geq 100 \text { births } \\
\text { during study } \\
\text { period who had } \\
\text { information on BF } \\
\text { initiation }\end{array}$ & $\begin{array}{l}\text { Mothers giving } \\
\text { birth in a BFHI- } \\
\text { accredited } \\
\text { birth } \\
\text { facility/hospital }\end{array}$ & $\begin{array}{l}\text { Mothers giving } \\
\text { birth in a } \\
\text { nonaccredited } \\
\text { birth } \\
\text { facility/hospital }\end{array}$ & $\begin{array}{l}\text { \% by age } \\
\text { category: } \\
\leq 19: 9 \\
\text { 20-24: } 24 \\
\text { 25-29: } 29 \\
\text { 30-34: } 24 \\
\geq 35: 15\end{array}$ & 49 & $\begin{array}{l}42 \\
\text { NR }\end{array}$ & NR \\
\hline $\begin{array}{l}\text { Hawkins, } \\
2014^{55} \\
\text { Medium }\end{array}$ & $\begin{array}{l}\text { Prospective } \\
\text { cohort } \\
10 \text { birth facilities } \\
\text { in } 1 \text { U.S. State } \\
\text { (Maine) } \\
2,014 \text { women }\end{array}$ & $\begin{array}{l}\text { United States } \\
2004-2008\end{array}$ & $\begin{array}{l}\text { Mothers giving } \\
\text { birth in Maine at a } \\
\text { hospital where at } \\
\text { least } 100 \text { births } \\
\text { occur }\end{array}$ & $\begin{array}{l}\text { Mothers giving } \\
\text { birth in an } \\
\text { accredited or } \\
\text { becoming } \\
\text { accredited } \\
\text { BFHI facility }\end{array}$ & $\begin{array}{l}\text { Mothers giving } \\
\text { birth in a facility } \\
\text { that is not BFHI } \\
\text { accredited }\end{array}$ & NR & $\begin{array}{l}\leq 7 \% \\
\text { across } \\
\text { all } \\
\text { facili- } \\
\text { ties }\end{array}$ & $\begin{array}{l}\mathrm{NR} \\
\mathrm{NR}\end{array}$ & NR \\
\hline $\begin{array}{l}\text { Kramer, } \\
2001^{52} \text {; Yang, } \\
2014^{53} \\
\text { (PROBIT) } \\
\text { Medium }\end{array}$ & $\begin{array}{l}\text { Cluster RCT } \\
31 \text { maternity } \\
\text { hospitals/ } \\
\text { polyclinics } \\
17,046 \\
\text { mother/infant } \\
\text { pairs }\end{array}$ & $\begin{array}{l}\text { Republic of } \\
\text { Belarus } \\
1996-1997\end{array}$ & $\begin{array}{l}\text { Mothers with } \\
\text { healthy singleton } \\
\text { infants ( } \geq 37 \text { wks, } \\
\text { birth weight } \\
\geq 2,500 \mathrm{~g} \text { ) who } \\
\text { initiated BF during } \\
\text { their postpartum } \\
\text { stay }\end{array}$ & $\begin{array}{l}\text { Maternity care } \\
\text { in a BFHI } \\
\text { facility, } \\
\text { included staff } \\
\text { training (18 } \\
\text { hours) and } \\
\text { implementa- } \\
\text { tion of all } 10 \\
\text { steps }\end{array}$ & $\begin{array}{l}\text { Standard } \\
\text { maternity care } \\
\text { (non-BFHI- } \\
\text { accredited } \\
\text { facilities) }\end{array}$ & $\begin{array}{l}\% \text { by age } \\
\text { category: } \\
<20: 14 \\
20-34: \\
81-83 \\
\geq 35: 4\end{array}$ & NR & $\begin{array}{l}\text { \% with no } \\
\text { other } \\
\text { children in } \\
\text { household: } \\
56-60 \\
\% \text { BF } \\
\text { previous } \\
\text { child } \geq 3 \\
\text { mos: } 24-25\end{array}$ & $11-13$ \\
\hline
\end{tabular}




\begin{tabular}{|c|c|c|c|c|c|c|c|c|c|}
\hline $\begin{array}{l}\text { Author, Year } \\
\text { ROB }\end{array}$ & $\begin{array}{l}\text { Study Design } \\
\text { N Sites } \\
\text { N Participants }\end{array}$ & $\begin{array}{l}\text { Country } \\
\text { Year(s) of } \\
\text { Enrollment }\end{array}$ & Inclusion Criteria & $\begin{array}{l}\text { Intervention } \\
\text { Description }\end{array}$ & Comparator(s) & $\begin{array}{l}\text { Age, } \\
\text { Mean } \\
\text { (SD) }\end{array}$ & $\begin{array}{l}\% \\
\text { Non- } \\
\text { white }\end{array}$ & $\begin{array}{l}\text { \% Primi- } \\
\text { parous } \\
\% \\
\text { Previously } \\
\text { BF } \\
\end{array}$ & $\begin{array}{l}\% \\
\text { Cesarean } \\
\text { Birth }\end{array}$ \\
\hline $\begin{array}{l}\text { Mydlilova, } \\
2009^{57} \\
\text { Medium }\end{array}$ & $\begin{array}{l}\text { Prospective } \\
\text { cohort } \\
112 \text { hospitals } \\
660,355 \text { births }\end{array}$ & $\begin{array}{l}\text { Czech } \\
\text { Republic } \\
2000-2006\end{array}$ & $\begin{array}{l}\text { Mothers giving } \\
\text { birth in a maternity } \\
\text { hospital with a } \\
\text { living infant at } \\
\text { discharge }\end{array}$ & $\begin{array}{l}\text { Mothers giving } \\
\text { birth in a BFHI- } \\
\text { accredited } \\
\text { hospital }\end{array}$ & $\begin{array}{l}\text { Mothers giving } \\
\text { birth in a non- } \\
\text { BFHI- } \\
\text { accredited } \\
\text { hospital }\end{array}$ & NR & NR & $\begin{array}{l}\text { NR } \\
\text { NR }\end{array}$ & NR \\
\hline $\begin{array}{l}\text { Tarrant, } \\
2011^{56} \\
\text { Medium }\end{array}$ & $\begin{array}{l}\text { Prospective } \\
\text { cohort } \\
4 \text { hospitals } \\
1,417 \\
\text { mother/infant } \\
\text { pairs }\end{array}$ & $\begin{array}{l}\text { Hong Kong } \\
2006-2007\end{array}$ & $\begin{array}{l}\text { Healthy mothers } \\
\text { intending to BF } \\
\text { with healthy } \\
\text { singleton births } \\
\text { ( } \geq 37 \text { wks, } \geq 2,500 \\
\text { g) }\end{array}$ & $\begin{array}{l}\text { Completion of } \\
\text { steps } 6 \text { out of } \\
6 \text { steps }(4,6 \text {, } \\
7,8,9,10)\end{array}$ & $\begin{array}{l}\text { Completion of } \\
<6 \text { of } 6 \text { of the } \\
\text { identified BFHI } \\
\text { steps }\end{array}$ & $\begin{array}{l}\% \text { by age } \\
\text { category: } \\
\text { 18-24: } 7 \\
\text { 25-29: } 23 \\
30-34: 46 \\
\geq 35: 24\end{array}$ & NA & $\begin{array}{l}59 \\
34\end{array}$ & 12 \\
\hline $\begin{array}{l}\text { Venancio, } \\
2011^{63} \\
\text { Medium }\end{array}$ & $\begin{array}{l}\text { Prospective } \\
\text { cohort } \\
64 \text { municipalities } \\
65,936 \text { infants }\end{array}$ & $\begin{array}{l}\text { Brazil } \\
2008\end{array}$ & $\begin{array}{l}\text { Participants of a } \\
2008 \text { nationwide } \\
\text { survey related to } \\
\text { child health }\end{array}$ & $\begin{array}{l}\text { Birth in a } \\
\text { BFHI- } \\
\text { accredited } \\
\text { facility }\end{array}$ & $\begin{array}{l}\text { Birth in a } \\
\text { hospital that } \\
\text { was not BFHI } \\
\text { accredited }\end{array}$ & $\begin{array}{l}\text { \% by age } \\
\text { category: } \\
<20: 16 \\
\geq 20: 84\end{array}$ & NR & $\begin{array}{l}50 \\
N R\end{array}$ & 48 \\
\hline $\begin{array}{l}\text { Weng, } 2003^{64} \\
\text { High }\end{array}$ & $\begin{array}{l}\text { Prospective } \\
\text { cohort } \\
56 \text { hospitals } \\
7,563 \text { women }\end{array}$ & $\begin{array}{l}\text { Taiwan } \\
\text { 2000-2001 }\end{array}$ & $\begin{array}{l}\text { Women giving } \\
\text { birth in hospitals } \\
\text { registered for } \\
\text { BFHI appraisal }\end{array}$ & $\begin{array}{l}\text { Hospitals that } \\
\text { passed BFHI } \\
\text { appraisal }\end{array}$ & $\begin{array}{l}\text { Hospitals that } \\
\text { did not pass } \\
\text { BFHI appraisal }\end{array}$ & $\mathrm{NR}^{\mathrm{c}}$ & NR & $\begin{array}{l}\text { NR } \\
\text { NR }\end{array}$ & NR \\
\hline $\begin{array}{l}\text { a Per authors, the " } \\
\text { obstetrical service } \\
\text { b Per authors, data } \\
1999 \text { through 200s } \\
\text { BFHI accreditatiol } \\
\text { c Characteristics o } \\
\text { the appraisal in the }\end{array}$ & $\begin{array}{l}\text { ot-as-yet BFHI" mat } \\
\text { number of deliveries } \\
\text { rom Alaska, Maine, } \\
\text { when PRAMS data } \\
\text { mothers were not rep } \\
\text { northern region than }\end{array}$ & $\begin{array}{l}\text { braska, Ohio, at } \\
\text { e collected, and } \\
\text { ed; there were } \\
\text { er regions of T }\end{array}$ & $\begin{array}{l}\text { State released hospita } \\
\text { ataphic differences in } \\
\text { an, which may be due } \\
\text { atington were used }\end{array}$ & $\begin{array}{l}\text { ecause these States } \\
\text { identifiers. The yea } \\
\text { e percentage of hos } \\
\text { more resources or }\end{array}$ & $\begin{array}{l}\text { hospitals of the ex } \\
\text { had at least one bir } \\
\text { rs of PRAMS data } \\
\text { pitals that passed th } \\
\text { earlier adoption of }\end{array}$ & $\begin{array}{l}\text { erimental gro } \\
\text { facility that } \\
\text { aried across S } \\
\text { BFHI apprai } \\
\text { FHI practices }\end{array}$ & $\begin{array}{l}\text { in terms } \\
\text { eived BF } \\
\text { es as did } \\
\text {. Per autl }\end{array}$ & $\begin{array}{l}\text { quality indice } \\
\text { I accreditation } \\
\text { e timing of hos } \\
\text { rs, more hospit }\end{array}$ & $\begin{array}{l}\text { of } \\
\text { uring } \\
\text { itals’ } \\
\text { ls passed }\end{array}$ \\
\hline
\end{tabular}




\section{Results}

\section{Breastfeeding Initiation}

Nine studies reported on rates of breastfeeding initiation at hospitals that were BFHI accredited (or certified) compared with nonaccredited hospitals (Table 5). ${ }^{54,55,57,58,60-64}$ Six of these reported on rates of any breastfeeding initiation (exclusive or nonexclusive) at hospital discharge ${ }^{54,55,60-62,64}$ overall, rates of breastfeeding initiation were higher at BFHI hospitals than control hospitals, but the magnitude of benefit varied across studies. Two studies (by the same author) compared hospitals in the United States enrolling women from similar time periods (1999-2009); one evaluated data from five U.S. States ${ }^{54}$ and the other focused on hospitals in one State (Maine) ${ }^{55}$ In both studies, estimated rates of initiation were approximately 2.4 to 7.0 percent higher in BFHI-accredited facilities than nonaccredited facilities; however, the differences were not statistically significant in either study (Table 5). ${ }^{54}$, 55 Similarly, two studies set in the United Kingdom (one of which was limited to Scotland ${ }^{62}$ ) found higher rates of breastfeeding initiation in BFHI-accredited hospitals than nonaccredited hospitals (ranging from 0.5 to $7.4 \%$ ); however, the differences were not statistically significant (Table 5). ${ }^{61,62}$ Finally, two studies set in Taiwan found significantly higher rates of in-hospital breastfeeding at BFHI hospitals compared with control hospitals. ${ }^{60,64}$ One of these compared hospitals that passed with those that did not pass BFHI appraisal and found initiation rates of 88.1 percent and 78.1 percent respectively $(\mathrm{p}<0.001){ }^{64}$ The second measured breastfeeding rates over a four year period (19992002), trends in breastfeeding rates were significantly higher at BFHI certified hospitals than controls $(\mathrm{p}<0.001) .^{60}$

Five studies set in various country settings (Czech Republic, ${ }^{57}$ Russia, ${ }^{58}$ Taiwan, ${ }^{60,64}$ and Brazil $^{63}$ ) found significantly higher rates of exclusive breastfeeding initiation at discharge in BFHI-certified or -accredited hospitals than control hospitals (Table 5). The absolute difference in breastfeeding initiation between BFHI hospitals and control hospitals varies widely. In three studies, the difference between groups ranged from approximately 3 to 8 percent. ${ }^{57,63,64}$ Two studies found a larger difference between groups; one study set in Russia found a 56 percent higher rate of exclusive breastfeeding among mothers giving birth in BFHI- and non-BFHIcertified hospitals in a single year (2004), and the second (set in Taiwan) found a 23 to 27 percent higher rate of exclusive breastfeeding initiation in BFHI hospitals than controls over a 4year period. ${ }^{60}$

Table 5. BFHI studies reporting on breastfeeding initiation

\begin{tabular}{llll}
\hline $\begin{array}{l}\text { First } \\
\text { Author, } \\
\text { Year }\end{array}$ & Intervention & Study Design & \\
& Comparison & N Sites & Breastfeeding Initiation \\
ROB & & N Participants & \\
\hline Abolyan, & G1: Mothers giving birth in & Prospective & Exclusive BF at discharge, \% (N) \\
$2006^{58}$ & BFHI-certified hospitals & cohort & G1: 88.9 (383) \\
& & & G2: $32.6(358)$ \\
Medium & G2: Mothers giving birth in & 741 women & p<0.0001 \\
& "not-yet Baby-Friendly" & & \\
& hospitals & 8 sites & \\
\hline
\end{tabular}




\begin{tabular}{|c|c|c|c|}
\hline $\begin{array}{l}\text { First } \\
\text { Author, } \\
\text { Year } \\
\text { ROB }\end{array}$ & $\begin{array}{l}\text { Intervention } \\
\text { Comparison }\end{array}$ & $\begin{array}{l}\text { Study Design } \\
\text { N Sites } \\
\text { N Participants }\end{array}$ & Breastfeeding Initiation \\
\hline $\begin{array}{l}\text { Bartington, } \\
2006^{61}\end{array}$ & $\begin{array}{l}\text { G1: Birth in BFHI-accredited } \\
\text { maternity unit }\end{array}$ & $\begin{array}{l}\text { Prospective } \\
\text { cohort }\end{array}$ & $\begin{array}{l}\text { BF initiation (full sample), } \% \\
\text { G1: } 71.3 \\
\text { G2: } 70.8\end{array}$ \\
\hline Medium & $\begin{array}{l}\text { G2: Birth in BFHI-certified } \\
\text { maternity unit } \\
\text { G3: Birth in maternity unit } \\
\text { with neither award }\end{array}$ & $\begin{array}{l}496 \text { maternity } \\
\text { units } \\
17,359 \text { women }\end{array}$ & $\begin{array}{l}\text { G3: } 69.5 \\
\text { RR }^{a}(G 1 \text { vs. G3): } 1.10 \text { (1.05 to } 1.15) \\
\left.\text { RR }^{a} \text { (G2 vs. G3): } 1.02 \text { (0.99 to } 1.05\right)\end{array}$ \\
\hline $\begin{array}{l}\text { Broadfoot, } \\
2005^{62}\end{array}$ & $\begin{array}{l}\text { G1: Birth in BFHI- } \\
\text { accredited }^{\mathrm{b}} \text { maternity unit }\end{array}$ & $\begin{array}{l}\text { Prospective } \\
\text { cohort }\end{array}$ & $\begin{array}{l}\text { Initiation of any BF at } 7 \text { days (\%) } \\
\text { G1: } 49.4 \\
\text { G2: } 43.4\end{array}$ \\
\hline Medium & $\begin{array}{l}\text { G2: Birth in BFHI-certified } \\
\text { unit } \\
\text { G3: Birth in maternity unit } \\
\text { with neither award }\end{array}$ & $\begin{array}{l}33 \text { sites } \\
445,623 \text { births }\end{array}$ & $\begin{array}{l}\text { G3: } 42.0 \\
\text { OR }^{c}(G 1 \text { vs. G3); } 1.28 \text { (1.24 to } 1.31) \\
\text { OR }^{c}(G 2 \text { vs. G3): } 1.04 \text { (1.02 to } 1.06)\end{array}$ \\
\hline $\begin{array}{l}\text { Gau, } 2004^{60} \\
\text { High }\end{array}$ & $\begin{array}{l}\text { G1: Mothers giving birth in } \\
\text { BFHI-certified hospitals } \\
\text { G2: Mothers giving birth in } \\
\text { control hospitals }\end{array}$ & $\begin{array}{l}\text { Prospective } \\
\text { cohort } \\
12 \text { sites } \\
4,614 \text { mothers }\end{array}$ & $\begin{array}{l}\text { Rates of exclusive BF during hospitalization by year, } \\
\text { G1 vs. G2, \% (SD) } \\
\text { 1999: } 0.30(0.24) \text { vs. } 0.24(0.006) \\
\text { 2000: } 0.34(0.24) \text { vs. } 0.22(0.007) \\
\text { 2001: } 0.46(0.21) \text { vs. } 0.23(0.05) \\
\text { 2002: } 0.50(0.16) \text { vs. } 0.23(0.25) \\
\text { p<0.001 for all years } \\
\text { Total BF rate (any BF): } \\
\text { 1999: } 0.92(0.03) \text { vs. } 0.82(0.08) \\
\text { 2000: } 0.94(0.03) \text { vs. } 0.82(0.04) \\
\text { 2001: } 0.95(0.03) \text { vs. } 0.94(0.07) \\
\text { 2002: } 0.95(0.04) \text { vs. } 0.95(0.02) \\
\text { p<0.001 for all years }\end{array}$ \\
\hline $\begin{array}{l}\text { Hawkins, } \\
2014^{54} \\
\text { Medium }\end{array}$ & $\begin{array}{l}\text { G1: Mothers giving birth in a } \\
\text { BFHI-accredited facility } \\
\text { G2: Mothers giving birth in a } \\
\text { nonaccredited facility }\end{array}$ & $\begin{array}{l}\text { Prospective } \\
\text { cohort } \\
32 \text { birth } \\
\text { facilities in } 5 \\
\text { U.S. States } \\
25,327 \text { women }\end{array}$ & 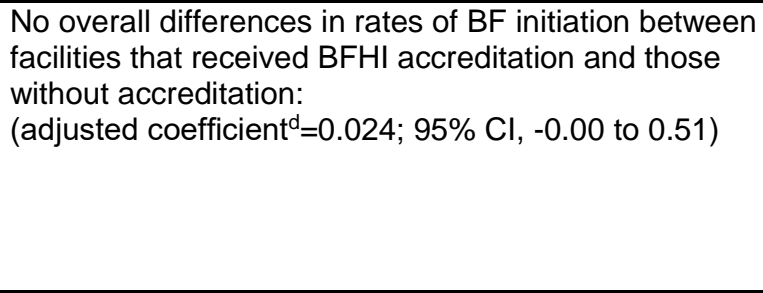 \\
\hline $\begin{array}{l}\text { Hawkins, } \\
2014^{55}\end{array}$ & $\begin{array}{l}\text { G1: Mothers giving birth in a } \\
\text { BFHI-accredited facility }\end{array}$ & $\begin{array}{l}\text { Prospective } \\
\text { cohort }\end{array}$ & $\begin{array}{l}\text { No effect of BFHI on BF initiation between facilities } \\
\text { that were accredited and those that were } \\
\text { nonaccredited: }\end{array}$ \\
\hline Medium & $\begin{array}{l}\text { G2: Mothers giving birth in a } \\
\text { nonaccredited facility }\end{array}$ & $\begin{array}{l}10 \text { birth } \\
\text { facilities in } \\
1 \text { U.S. State } \\
\text { (Maine) } \\
\text { 2,014 women }\end{array}$ & $\begin{array}{l}\text { (adjusted coefficient }{ }^{d}=00.070 ; 95 \% \mathrm{Cl},-0.04 \text { to } 0.18 \text {; } \\
p=0.2)\end{array}$ \\
\hline
\end{tabular}




\begin{tabular}{|c|c|c|c|}
\hline $\begin{array}{l}\text { First } \\
\text { Author, } \\
\text { Year } \\
\text { ROB } \\
\end{array}$ & $\begin{array}{l}\text { Intervention } \\
\text { Comparison }\end{array}$ & $\begin{array}{l}\text { Study Design } \\
\text { N Sites } \\
\text { N Participants }\end{array}$ & Breastfeeding Initiation \\
\hline $\begin{array}{l}\text { Mydlilova, } \\
2009^{57}\end{array}$ & $\begin{array}{l}\text { G1: Mothers giving birth in a } \\
\text { BFHI-accredited hospital }\end{array}$ & $\begin{array}{l}\text { Prospective } \\
\text { cohort }\end{array}$ & $\begin{array}{l}\text { Risk of not being exclusively BF was higher in non- } \\
\text { BFHI than in BFHI hospitals (by year): } \\
\text { Year: RR }(95 \% \mathrm{Cl})\end{array}$ \\
\hline Medium & $\begin{array}{l}\text { G2: Mothers giving birth in a } \\
\text { non-BFHI hospital }\end{array}$ & $\begin{array}{l}112 \text { hospitals } \\
660,355 \\
\text { women }\end{array}$ & $\begin{array}{l}\text { 2000: } 1.09 \text { (1.08 to } 1.10) \\
\text { 2004: } 1.54 \text { (1.48 to } 1.61) \\
\text { 2006: } 1.32 \text { (1.27 to } 1.37) \\
\text { Avg. rate of exclusively BF infants for } 2000-2006(\%) \text { : } \\
\text { G1: } 90.32 \\
\text { G2: } 87.53 \\
\text { p<0.001 }\end{array}$ \\
\hline $\begin{array}{l}\text { Venancio, } \\
2011^{63}\end{array}$ & $\begin{array}{l}\text { G1: Birth in BFHI-accredited } \\
\text { facility }\end{array}$ & $\begin{array}{l}\text { Prospective } \\
\text { cohort }\end{array}$ & 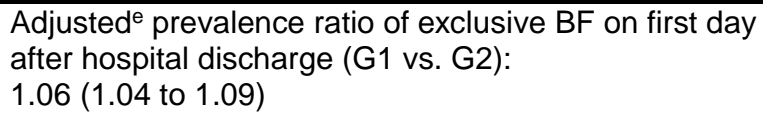 \\
\hline Medium & $\begin{array}{l}\text { G2: Birth in a nonaccredited } \\
\text { facility }\end{array}$ & $\begin{array}{l}64 \\
\text { municipalities } \\
65,936 \text { infants }\end{array}$ & $\begin{array}{l}\text { Unadjusted prevalence of exclusive BF on first day } \\
\text { after hospital discharge: } \\
\text { G1: } 87.2 \\
\text { G2: } 82.3 \\
p=0.0001\end{array}$ \\
\hline $\begin{array}{l}\text { Weng, } \\
2003^{64}\end{array}$ & $\begin{array}{l}\text { G1: Births in hospitals that } \\
\text { passed BFHI appraisal }\end{array}$ & $\begin{array}{l}\text { Prospective } \\
\text { cohort }\end{array}$ & $\begin{array}{l}\text { In-hospital exclusive BF rate, weighted \% (SD) } \\
\text { G1: } 31.43(22.40) \\
\text { G2: } 23.18(24.65)\end{array}$ \\
\hline High & $\begin{array}{l}\text { G2: Births in hospitals that } \\
\text { passed BFHI appraisal }\end{array}$ & $\begin{array}{l}56 \text { hospitals } \\
7,563 \text { women }\end{array}$ & $\begin{array}{l}\mathrm{p}<0.01 \\
\text { In-hospital BF rate (exclusive and nonexclusive), } \\
\text { weighted \% (SD) } \\
\text { G1: } 88.08(15.91) \\
\text { G2: } 78.11(14.62) \\
p<0.001\end{array}$ \\
\hline
\end{tabular}

a RRs adjusted for ward type, socioeconomic status, ethnicity, academic qualifications, maternal age, parity, and lone parent status.

${ }^{\mathrm{b}}$ Fully-accredited hospitals had implemented all Ten Steps, completed an annual audit of compliance and progress monitoring visits, and completed a reassessment 24 months after the initial award. Certified hospitals where those that adopted a policy covering all Ten Steps, had an action planning visit from UNICEF UK Baby Friendly, but were not yet fully accredited. c OR adjusted for Carstairs deprivation category, mother's age, number of births in hospital, and year of birth.

${ }^{\mathrm{d}}$ All models include a birth fixed effect and an interaction between year and whether a birth facility received accreditation.

e Adjusted for infant age (at time of survey), low birth weight, mode of delivery, firstborn, maternal age, and maternal education.

Avg.= average; $\mathrm{BF}=$ breastfeeding; $\mathrm{BFHI}=$ Baby-Friendly Hospital Initiative; $\mathrm{CI}$ = confidence interval; $\mathrm{G}=$ group; $\mathrm{N}=$ number; $\mathrm{NR}$ = not reported; $\mathrm{OR}$ = odds ratio; $\mathrm{ROB}$ = risk of bias; $\mathrm{RR}=$ risk ratio; $\mathrm{SD}$ = standard deviation; U.K. = United Kingdom.

\section{Breastfeeding Duration}

Ten studies reported on breastfeeding duration (Table 6); nine compared women giving birth in BFHI-certified or -accredited hospitals and control hospitals, 52, 54, 58-61, 63, 64, 68 and one study compared hospitals based on the number of BFHI steps implemented. ${ }^{56}$

Of the nine studies comparing hospitals with and without a BFHI status, one was an RCT (PROBIT) ${ }^{69}$ and eight were observational studies (one single group pre-post study, ${ }^{59}$ and the rest were prospective cohort studies). In the PROBIT RCT, women giving birth at intervention hospitals had higher rates of exclusive breastfeeding at 3 months postpartum (43\% vs. 6\%; $\mathrm{p}<0.001), 6$ months postpartum (7.9\% vs. $0.6 \%$; $\mathrm{p}=0.01)$, and lower odds of having been weaned (from any breastfeeding) at $3,6,9$, and 12 months (Table 6). ${ }^{52}$ 
Findings from eight observational studies reporting on breastfeeding duration were mostly consistent in finding benefit; however, results were often imprecise and varied by setting and outcome timing. ${ }^{54,58-61,63,64,68}$ Two studies set in the United States found slightly higher rates of any breastfeeding and exclusive breastfeeding for at least 4 weeks at BFHI than control hospitals (by approximately 0.5 to $6.8 \%$ ); however, differences between groups were not statistically significant. ${ }^{54,55}$ One study evaluating Scottish hospitals found no significant difference between women giving birth in BFHI-accredited hospitals, and non-accredited hospitals in rates of any breastfeeding at 1 month; rates were slightly lower at BFHI-accredited hospitals (44.6\%) than hospitals that were certified (i.e., had adopted a BFHI policy but were not yet audited for compliance) or had neither status (49.9\% vs. $49.5 \%$, respectively) ${ }^{61}$ A study set in Russia found higher rates of any breastfeeding at 6 to 12 months among women giving birth in BFHI hospitals than non-BFHI-certified hospitals; however, authors did not clearly report the sample size or whether differences are statistically significant (percentage change from baseline: 10.5 vs. 3.7; pvalue not reported). ${ }^{58}$ Four studies set in Taiwan, ${ }^{60,64}$ Croatia, ${ }^{59}$ and the Czech Republic found significantly higher rates of any or exclusive breastfeeding over 1 to 12 months of followup. Studies reported different outcome measures, which limits the ability to compare the magnitude of differences between groups.

Table 6. BFHI studies reporting on breastfeeding duration and exclusivity

\begin{tabular}{|c|c|c|c|c|}
\hline $\begin{array}{l}\text { First } \\
\text { Author, } \\
\text { Year } \\
\text { ROB }\end{array}$ & $\begin{array}{l}\text { Intervention } \\
\text { Comparison }\end{array}$ & $\begin{array}{l}\text { Study Design } \\
\text { N Sites } \\
\text { N Participants }\end{array}$ & $\begin{array}{l}\text { Breastfeeding Duration (Any, } \\
\text { Nonexclusive Plus Exclusive) }\end{array}$ & $\begin{array}{l}\text { Exclusive Breastfeeding } \\
\text { Duration }\end{array}$ \\
\hline $\begin{array}{l}\text { Abolyan, } \\
2006^{58} \\
\text { Medium }\end{array}$ & $\begin{array}{l}\text { G1: Birth in } \\
\text { BFHI- certified } \\
\text { hospitals } \\
\text { G2: Birth at } \\
\text { "not-yet Baby- } \\
\text { Friendly" } \\
\text { hospitals }\end{array}$ & $\begin{array}{l}\text { Prospective cohort } \\
741 \text { women } \\
\text { (survey); } \mathrm{N} \text { for BF } \\
\text { rates at } 6-12 \\
\text { months NR } \\
8 \text { sites }\end{array}$ & $\begin{array}{l}\text { Increase in BF rates (\%) at } 6-12 \\
\text { mos among infants born during } \\
\text { implementation of BFHI } \\
\text { Baseline }(1999-2003): \\
\text { G1: } 20.7 \text { to } 31.2(+10.5 \%) \\
\text { G2: } 15.6 \text { to } 19.3(+3.7 \%)\end{array}$ & NR \\
\hline $\begin{array}{l}\text { Bartington } \\
2006^{61} \\
\text { Medium }\end{array}$ & $\begin{array}{l}\text { G1: Birth in a } \\
\text { BFHI- } \\
\text { accredited } \\
\text { maternity unita } \\
\text { G2: Birth in } \\
\text { BFHI-certified } \\
\text { unit }^{\text {a }} \\
\text { G3: Birth in } \\
\text { unit with } \\
\text { neither award }\end{array}$ & $\begin{array}{l}\text { Prospective cohort } \\
496 \text { maternity } \\
\text { units } \\
17,359 \text { women }\end{array}$ & $\begin{array}{l}\text { Any BF at } 1 \text { mo, } \% \\
\text { G1: } 44.6 \\
\text { G2: } 49.9 \\
\text { G3: } 49.5 \\
\text { RR (G1 vs. G3): } 0.96 \text { (0.84 to } \\
\text { 1.09) } \\
\text { RR (G2 vs. G3): } 0.96 \text { (0.91 to } \\
1.01)\end{array}$ & NR \\
\hline
\end{tabular}




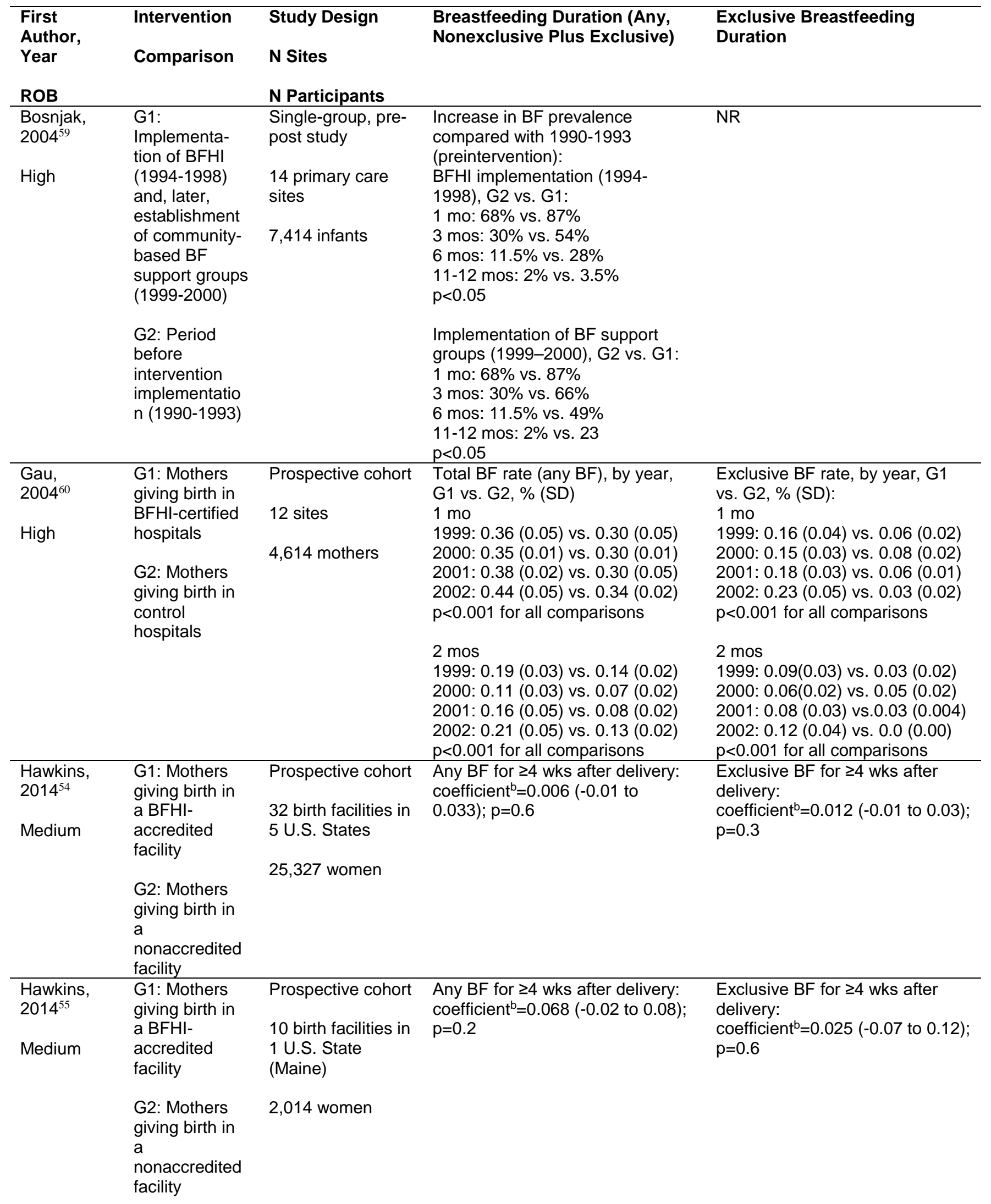




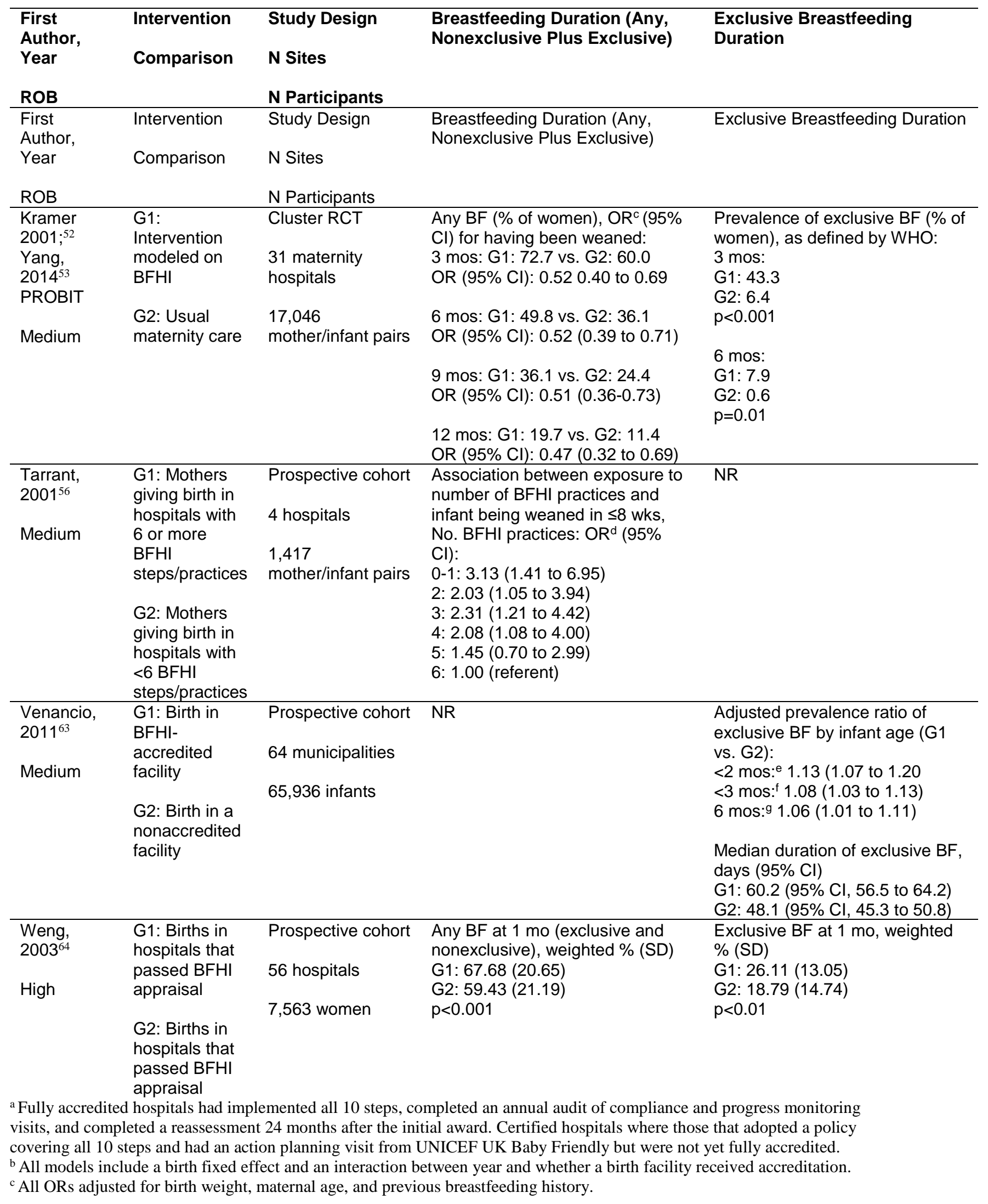


${ }^{\mathrm{d}}$ Adjusted for Baby-Friendly hospital practices, maternal age, maternal education, household income, emergency cesarean section, return to work, whether mother was breastfed as a child, mother's previous breastfeeding experience, and husband's feeding preference.

e Adjusted for infant age, low birth weight, maternal age, maternal education.

${ }^{\mathrm{f}}$ Adjusted for infant age, gender, maternal age, and maternal education

g Adjusted for infant age, gender, low birth weight, mode of delivery, firstborn, maternal age, maternal education, and maternal employment.

$\mathrm{BF}=$ breastfeeding; $\mathrm{BFHI}=$ Baby-Friendly Hospital Initiative; $\mathrm{CI}=$ confidence interval; $\mathrm{G}=$ group; $\mathrm{N}=$ number; $\mathrm{NR}=$ not reported; OR = odds ratio; PROBIT = Promotion of Breastfeeding Intervention Trial; RCT = randomized controlled trial; ROB = risk of bias; $\mathrm{RR}=$ risk ratio; $\mathrm{SD}=$ standard deviation; wks = weeks; $\mathrm{WHO}=$ World Health Organization.

One study set in Hong Kong assessed breastfeeding rates by degree of BFHI implementation. ${ }^{56}$ Women giving birth in hospitals practicing four or fewer BFHI steps had higher odds of weaning at or before 8 weeks postpartum compared with women giving birth in facilities practicing six BFHI steps (Table 6). ${ }^{56}$

\section{Other (Non-BFHI) Health Care System-Based Interventions}

\section{Characteristics}

Fifteen included studies (described in 16 publications) assessed the effectiveness of other health care system-based interventions (non-BFHI interventions) (Table 7). ${ }^{68,70-84}$ All enrolled women from routine maternity care settings (prenatal clinics, maternity hospitals, or postpartum care settings). Studies were conducted in diverse country settings including the United States (3 studies), ${ }^{73,80,82}$ Canada (1 study), ${ }^{78}$ Sri Lanka (1 study), ${ }^{75}$ Brazil (2 studies), ${ }^{79},{ }^{85}$ China (1 study), ${ }^{72}$ and various European countries (6 studies). ${ }^{68,71,74,76,77,83}$ Most were RCTs, 4 were nonrandomized controlled trials, ${ }^{68,73,78,81}$ and two were single-group pre-post studies. ${ }^{80,82}$ Most studies enrolled women prior to 2009; the majority (10 studies) enrolled women during the 2000s, and 5 studies enrolled women during the 1990s. ${ }^{78-80,82}$

In terms of population characteristics, two studies were limited to primiparous women only, ${ }^{71,72}$ and others included primiparous or multiparous women. Of the 13 studies enrolling both primiparous and multiparous women, 3 reported on the percentage of women who had previously breastfed (39\% to $60 \%$, across groups). ${ }^{68,71,75,76,81,83,84}$ Three studies did not report on the age of enrolled mothers; ${ }^{73,76,78,82}$ across the remaining studies, the mean age of women in the study samples ranged from approximately 27 to 34 years. Five studies reported on cesarean births, with rates ranging from 0 to 29 percent; ${ }^{68,71,78,83,84} 1$ study excluded mothers who had a cesarean delivery. ${ }^{80}$

Studies assessed the effectiveness of diverse interventions. Five assessed the effectiveness of education and training for health care professionals only (with no mention of additional individual-level services offered to women). ${ }^{71,73,75,78,79}$ Of these, one targeted training to midwives and postnatal nurses in Swedish municipalities, ${ }^{71}$ one focused on U.S. residency programs (pediatrics, OBGYN, and family medicine), ${ }^{73}$ and three focused on hospital staff (one focused on nursing staff only, ${ }^{78}$ and two included nurses, midwives, and physicians ${ }^{75,79}$ ). Length of training and content varied across studies (Table 7).

Seven studies assessed a multicomponent health system intervention that combined education (or skills training) to providers with expanded breastfeeding support services for mothers (or families). ${ }^{68,74,76,77,82-84}$ The intensity of training and range of providers included in staff training differed across studies (Table 7). Several studies included expanded patient education activities delivered in diverse health care settings, including hospitals or maternity units, ${ }^{82}$ prenatal classes, ${ }^{82}$ Web sites, ${ }^{68}$ and outpatient settings. ${ }^{77}$ One intervention targeted women who were 
expected to be discharged early and provided a multicomponent breastfeeding intervention during the hospital stay (emphasizing extended skin-to-skin contact, breastfeeding at least 8 
Table 7. Characteristics of studies evaluating non-BFHI health care system-based interventions

\begin{tabular}{|c|c|c|c|c|c|c|c|c|c|}
\hline $\begin{array}{l}\text { First Author, } \\
\text { Year } \\
\text { ROB }\end{array}$ & $\begin{array}{l}\text { Study Design } \\
\text { N Sites } \\
\text { N Participants }\end{array}$ & $\begin{array}{l}\text { Country } \\
\text { Year(s) of } \\
\text { Enrollment }\end{array}$ & $\begin{array}{l}\text { Inclusion } \\
\text { Criteria }\end{array}$ & $\begin{array}{l}\text { Intervention } \\
\text { Description }\end{array}$ & Comparator(s) & $\begin{array}{l}\text { Age, } \\
\text { Mean } \\
\text { (SD) }\end{array}$ & $\begin{array}{l}\% \\
\text { Non- } \\
\text { white }\end{array}$ & $\begin{array}{l}\text { \% } \\
\text { Primiparous } \\
\% \text { Previously } \\
\text { BF }\end{array}$ & $\begin{array}{l}\text { \% } \\
\text { Cesarean } \\
\text { Birth }\end{array}$ \\
\hline $\begin{array}{l}\text { Baerug, } \\
2016^{81} \\
\text { Medium }\end{array}$ & $\begin{array}{l}\text { NRCT } \\
54 \text { municipalities } \\
3,948 \text { mothers }\end{array}$ & $\begin{array}{l}\text { Norway } \\
\text { 2009-2012 }\end{array}$ & $\begin{array}{l}\text { Women with a } \\
\text { singleton birth } \\
>2,000 \mathrm{~g}\end{array}$ & $\begin{array}{l}\text { Implementing } \\
\text { the BFHI in } \\
\text { routine } \\
\text { antenatal and } \\
\text { child care } \\
\text { services at the } \\
\text { community level } \\
\text { (staff training } \\
\text { and } \\
\text { demonstration } \\
\text { of practical } \\
\text { skills) }\end{array}$ & $\begin{array}{l}\text { Routine } \\
\text { antenatal and } \\
\text { preventive health } \\
\text { care }^{\mathrm{a}}\end{array}$ & $\begin{array}{l}\text { \% by } \\
\text { age } \\
\text { categor } \\
\text { y: } \\
16-24: \\
15 \\
25-29 \text { : } \\
34 \\
30-34: \\
32 \\
35-44: \\
19\end{array}$ & NR & $\begin{array}{l}44 \\
N R\end{array}$ & NR \\
\hline $\begin{array}{l}\text { Coutinho, } \\
2005^{84} \\
\text { Low }\end{array}$ & $\begin{array}{l}\text { RCT } \\
2 \text { hospitals } \\
350 \text { women }\end{array}$ & $\begin{array}{l}\text { Brazil } \\
2001\end{array}$ & $\begin{array}{l}\text { Healthy } \\
\text { mothers with } \\
\text { singleton } \\
\text { births }\end{array}$ & $\begin{array}{l}\text { Staff training } \\
\text { (modeled after } \\
\text { BFHI training); } \\
\text { all mothers } \\
\text { assigned } 10 \\
\text { postnatal HVs } \\
\text { specific to BF } \\
\text { support }\end{array}$ & $\begin{array}{l}\text { Staff training } \\
\text { alone (with no } \\
\text { added HV) }\end{array}$ & $\begin{array}{l}\% \text { by } \\
\text { age } \\
\text { group: } \\
<20: 33 \\
\geq 20: 67\end{array}$ & NR & $\begin{array}{l}38 \\
N R\end{array}$ & 29 \\
\hline $\begin{array}{l}\text { Ekstrom, } \\
2014^{70} ; \\
\text { Ekstrom, } \\
2012^{71} \\
\text { Medium }\end{array}$ & $\begin{array}{l}\text { Cluster RCT } \\
10 \text { municipalities } \\
540 \text { women ( } 480 \\
\text { analyzed) }\end{array}$ & $\begin{array}{l}\text { Sweden } \\
2000-2002\end{array}$ & $\begin{array}{l}\text { Primiparous } \\
\text { healthy } \\
\text { mothers of } \\
\text { singleton full- } \\
\text { term infants } \\
\text { receiving care } \\
\text { from health } \\
\text { professionals } \\
\text { in the } \\
\text { intervention or } \\
\text { control } \\
\text { municipalities }\end{array}$ & $\begin{array}{l}\text { Process- } \\
\text { oriented training } \\
\text { in supportive BF } \\
\text { counseling for } \\
\text { midwives and } \\
\text { postnatal nurses } \\
\text { (7 days of } \\
\text { lectures) }\end{array}$ & $\begin{array}{l}\text { Usual care, } \\
\text { women were } \\
\text { sampled from } \\
\text { clusters not } \\
\text { receiving the } \\
\text { intervention at } \\
\text { two time points, } \\
\text { before the } \\
\text { intervention and } \\
\text { at the same time } \\
\text { as the } \\
\text { intervention } \\
\text { sample }\end{array}$ & 27 (NR) & NR & $\begin{array}{l}100 \\
N A\end{array}$ & $14-18$ \\
\hline
\end{tabular}




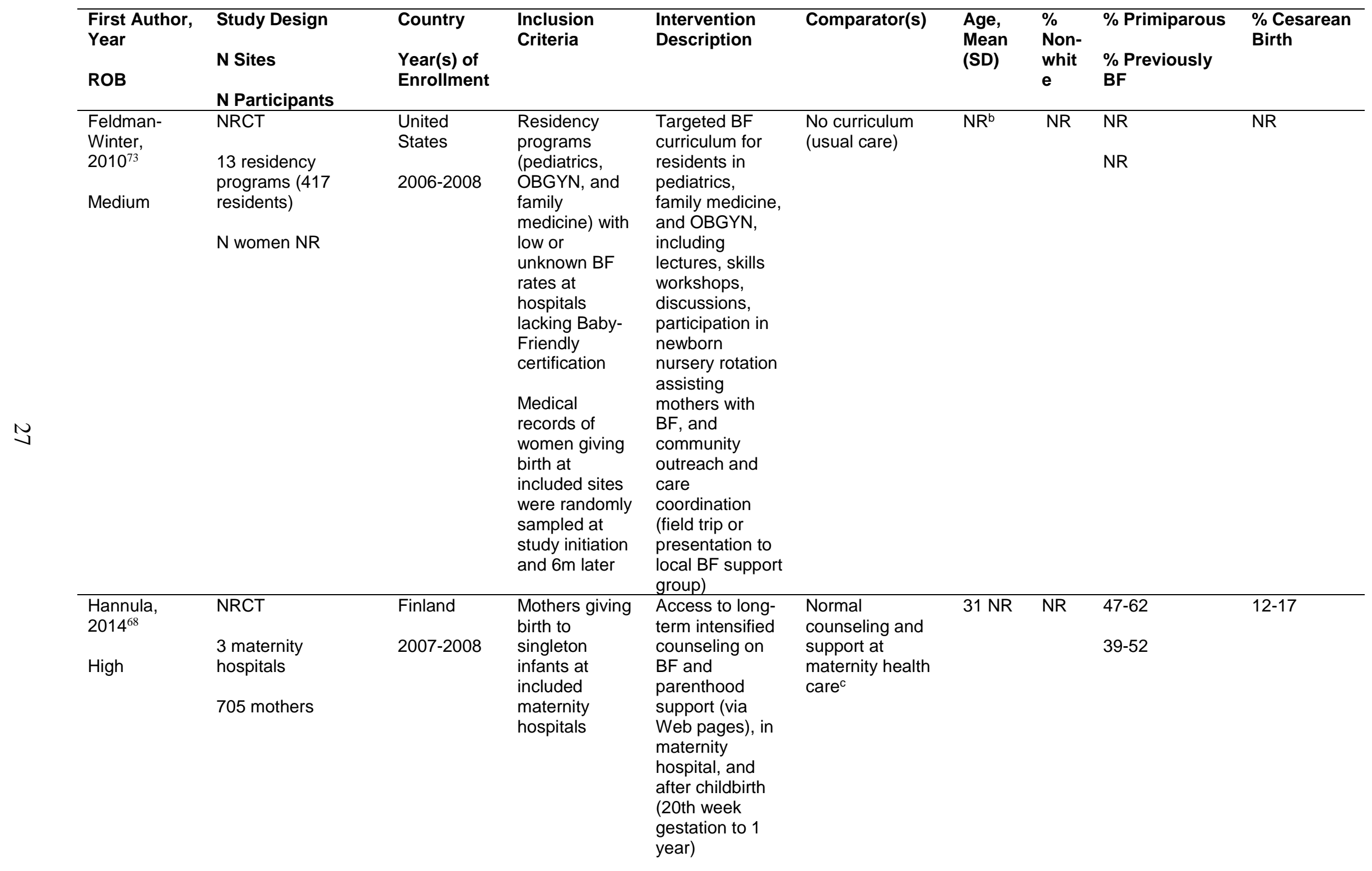




\begin{tabular}{|c|c|c|c|c|c|c|c|c|c|}
\hline $\begin{array}{l}\text { First Author, } \\
\text { Year } \\
\text { ROB }\end{array}$ & $\begin{array}{l}\text { Study Design } \\
\text { N Sites } \\
\text { N Participants }\end{array}$ & $\begin{array}{l}\text { Country } \\
\text { Year(s) of } \\
\text { Enrollment }\end{array}$ & $\begin{array}{l}\text { Inclusion } \\
\text { Criteria }\end{array}$ & $\begin{array}{l}\text { Intervention } \\
\text { Description }\end{array}$ & Comparator(s) & $\begin{array}{l}\text { Age, } \\
\text { Mean } \\
\text { (SD) }\end{array}$ & $\begin{array}{l}\% \\
\text { Non- } \\
\text { whit } \\
\text { e }\end{array}$ & $\begin{array}{l}\text { \% Primiparous } \\
\text { \% Previously } \\
\text { BF }\end{array}$ & $\begin{array}{l}\text { \% Cesarean } \\
\text { Birth }\end{array}$ \\
\hline $\begin{array}{l}\text { Hoddinott, } \\
2009^{74} \\
\text { Medium }\end{array}$ & $\begin{array}{l}\text { Cluster RCT } \\
14 \text { general practices } \\
\text { ('localities') } \\
18,603\end{array}$ & $\begin{array}{l}\text { Scotland } \\
2002\end{array}$ & $\begin{array}{l}\text { Clusters: } \\
\text { general } \\
\text { practices that } \\
\text { routinely } \\
\text { collected BF } \\
\text { data through a } \\
\text { national } \\
\text { surveillance } \\
\text { program } \\
\text { Women: no } \\
\text { specific criteria } \\
\text { other than } \\
\text { women } \\
\text { attending } \\
\text { participating } \\
\text { practices }\end{array}$ & $\begin{array}{l}\text { Adoption of a } \\
\text { BF group policy: } \\
\text { localities were } \\
\text { asked to double } \\
\text { the amount of } \\
\text { BF group } \\
\text { activity, set up a } \\
\text { minimum of } 2 \\
\text { new groups and } \\
\text { ensure all } \\
\text { centers had } \\
\text { access to a } \\
\text { group; individual } \\
\text { BF groups were } \\
\text { to be offered } \\
\text { weekly to } \\
\text { women only and } \\
\text { at least } 50 \% \text { of } \\
\text { the group } \\
\text { meeting was to } \\
\text { be social and } \\
\text { interactive }\end{array}$ & $\begin{array}{l}\text { Practices without } \\
\text { a new BF group } \\
\text { policy }^{d}\end{array}$ & $28-29$ & NR & $\begin{array}{l}\mathrm{NR} \\
\mathrm{NR}\end{array}$ & NR \\
\hline $\begin{array}{l}\text { Kools, } 2005^{77} \\
\text { Medium }\end{array}$ & $\begin{array}{l}\text { Cluster RCT } \\
10 \text { maternity and } \\
\text { child health centers } \\
781 \text { mothers }\end{array}$ & $\begin{array}{l}\text { Netherlands } \\
2000-2002\end{array}$ & $\begin{array}{l}\text { Women who } \\
\text { gave birth to } \\
\text { infants }>2,000 \\
g\end{array}$ & $\begin{array}{l}\text { Health care } \\
\text { provider training } \\
\text { to deliver a BF } \\
\text { promotion and } \\
\text { support program } \\
\text { (health } \\
\text { counseling, } \\
\text { early signaling } \\
\text { of BF problems, } \\
\text { continuity of } \\
\text { care, and free } \\
\text { access to } \\
\text { lactation } \\
\text { consultancy) }\end{array}$ & Usual care & $\begin{array}{l}\text { NR; } \% \\
\text { by age } \\
\text { group } \\
<25 \\
\text { yrs: } \\
8-10 \\
25 \text { to } \\
30 \text { yrs: } \\
44-45 \\
\geqq 31 \\
\text { yrs: } \\
46-47\end{array}$ & NR & $\begin{array}{l}55-56 \\
\text { NR (66-71\% } \\
\text { intended to BF) }\end{array}$ & NR \\
\hline
\end{tabular}




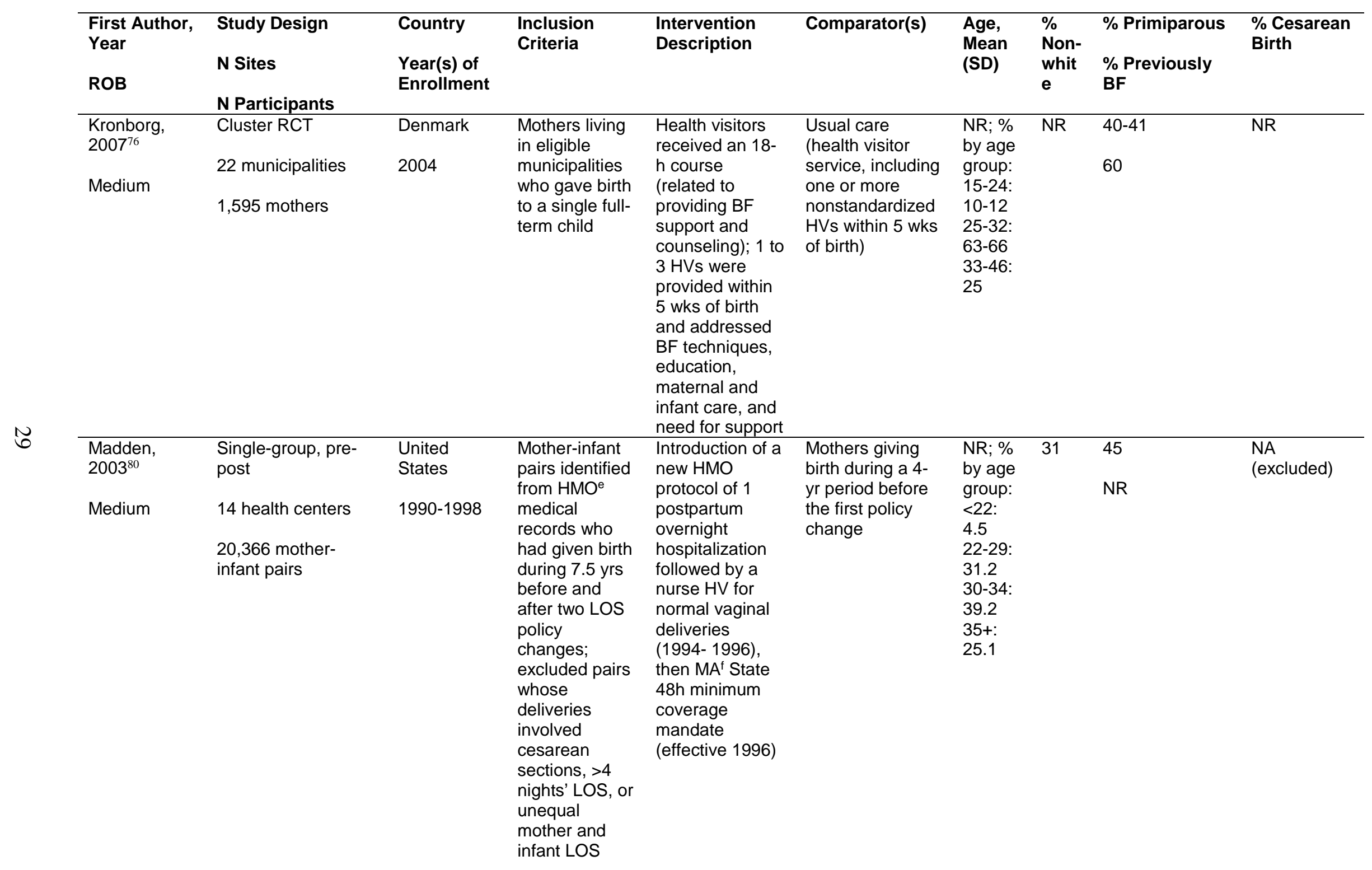




\begin{tabular}{|c|c|c|c|c|c|c|c|c|c|}
\hline $\begin{array}{l}\text { First Author, } \\
\text { Year } \\
\text { ROB }\end{array}$ & $\begin{array}{l}\text { Study Design } \\
\text { N Sites } \\
\text { N Participants }\end{array}$ & $\begin{array}{l}\text { Country } \\
\text { Year(s) of } \\
\text { Enrollment }\end{array}$ & $\begin{array}{l}\text { Inclusion } \\
\text { Criteria }\end{array}$ & $\begin{array}{l}\text { Intervention } \\
\text { Description }\end{array}$ & Comparator(s) & $\begin{array}{l}\text { Age, } \\
\text { Mean } \\
\text { (SD) }\end{array}$ & $\begin{array}{l}\% \\
\text { Non- } \\
\text { white }\end{array}$ & $\begin{array}{l}\text { \% Primiparous } \\
\text { \% Previously } \\
\text { BF }\end{array}$ & $\begin{array}{l}\text { \% Cesarean } \\
\text { Birth }\end{array}$ \\
\hline $\begin{array}{l}\text { Martens, } \\
2000^{78} \\
\text { High }\end{array}$ & $\begin{array}{l}\text { NRCT } \\
2 \text { rural hospitals } \\
75 \text { births }\end{array}$ & $\begin{array}{l}\text { Canada } \\
1997\end{array}$ & $\begin{array}{l}\text { Mothers giving } \\
\text { birth at } \\
\text { participating } \\
\text { hospitals }\end{array}$ & $\begin{array}{l}\text { Mandated 1.5-h } \\
\text { hospital staff BF } \\
\text { education } \\
\text { session for all } \\
\text { nursing staff; } \\
\text { optional tutorial } \\
\text { related to BF } \\
\text { education, } \\
\text { support and } \\
\text { hospital policy } \\
\text { completed } \\
\text { individually }\end{array}$ & Usual care & NR & $\begin{array}{l}\text { NR; } \\
27 \text { to } \\
76 \% \\
\text { were } \\
\text { First } \\
\text { Natio } \\
\text { ns } \\
\text { client } \\
\text { s }\end{array}$ & $\begin{array}{l}9.8-23.5 \\
\text { NR }\end{array}$ & 0 \\
\hline $\begin{array}{l}\text { Nilsson, } \\
2017^{83} \\
\text { Medium }\end{array}$ & $\begin{array}{l}\text { Cluster RCT } \\
9 \text { maternity settings } \\
3,541 \text { women }\end{array}$ & $\begin{array}{l}\text { Denmark } \\
\text { 2013-2014 }\end{array}$ & $\begin{array}{l}\text { Women } \\
\text { expecting a } \\
\text { single infant, } \\
\text { intending to } \\
\text { BF and } \\
\text { expected to be } \\
\text { discharged } \\
\text { within } 50 \mathrm{hrs} \\
\text { postpartum }\end{array}$ & $\begin{array}{l}\text { Multicomponent } \\
\text { BF program } \\
\text { emphasizing } \\
\text { extended skin- } \\
\text { to-skin contact, } \\
\text { BF at least } 8 \\
\text { times per day, } \\
\text { BF position } \\
\text { guidance, and } \\
\text { acknowledgeme } \\
\text { nt of equal roles } \\
\text { between } \\
\text { parents }\end{array}$ & Usual care & $30(5)$ & NR & $\begin{array}{l}40 \\
N R\end{array}$ & $13-16$ \\
\hline
\end{tabular}




\begin{tabular}{|c|c|c|c|c|c|c|c|c|c|}
\hline $\begin{array}{l}\text { First Author, } \\
\text { Year } \\
\text { ROB }\end{array}$ & $\begin{array}{l}\text { Study Design } \\
\text { N Sites } \\
\text { N Participants }\end{array}$ & $\begin{array}{l}\text { Country } \\
\text { Year(s) of } \\
\text { Enrollment }\end{array}$ & $\begin{array}{l}\text { Inclusion } \\
\text { Criteria }\end{array}$ & $\begin{array}{l}\text { Intervention } \\
\text { Description }\end{array}$ & Comparator(s) & $\begin{array}{l}\text { Age, } \\
\text { Mean } \\
(\mathrm{SD})\end{array}$ & $\begin{array}{l}\% \\
\text { Non- } \\
\text { white }\end{array}$ & $\begin{array}{l}\text { \% Primiparous } \\
\text { \% Previously } \\
\text { BF }\end{array}$ & $\begin{array}{l}\text { \% Cesarean } \\
\text { Birth }\end{array}$ \\
\hline $\begin{array}{l}\text { Senarath, } \\
2007^{75} \\
\text { High }\end{array}$ & $\begin{array}{l}\text { Cluster RCT } \\
5 \text { hospitals } \\
892 \text { women }\end{array}$ & $\begin{array}{l}\text { Sri Lanka } \\
\text { 2003-2004 }\end{array}$ & $\begin{array}{l}\text { Healthy } \\
\text { mother- } \\
\text { newborn pairs } \\
\text { (singleton } \\
\text { births only) } \\
\text { who received } \\
\text { care from the } \\
5 \text { study } \\
\text { hospitals }\end{array}$ & $\begin{array}{l}\text { Training } \\
\text { program (4 } \\
\text { days) for } \\
\text { hospital } \\
\text { obstetric service } \\
\text { staff (midwives, } \\
\text { nurses, and } \\
\text { doctors) to } \\
\text { increase } \\
\text { knowledge and } \\
\text { essential } \\
\text { newborn care } \\
\text { skills; } \\
\text { approximately } 5 \\
\text { h spent on BF } \\
\text { topics }\end{array}$ & $\begin{array}{l}\text { Usual care (no } \\
\text { additional } \\
\text { training) }\end{array}$ & $\begin{array}{l}\text { NR; } \% \\
\text { by age } \\
\text { group: } \\
\leq 19: 8- \\
11 \\
20-34 \text { : } \\
80-87 \\
\geqq 35 \text { : } \\
5-9\end{array}$ & $\begin{array}{l}100 \\
\text { (Sri } \\
\text { Lanka } \\
\mathrm{n} \\
\text { ethnic } \\
\text { i-ties) }\end{array}$ & $\begin{array}{l}36-46 \\
\text { NR }\end{array}$ & NR \\
\hline
\end{tabular}




\begin{tabular}{|c|c|c|c|c|c|c|c|c|c|}
\hline $\begin{array}{l}\text { First Author, } \\
\text { Year } \\
\text { ROB } \\
\end{array}$ & $\begin{array}{l}\text { Study Design } \\
\text { N Sites } \\
\text { N Participants }\end{array}$ & $\begin{array}{l}\text { Country } \\
\text { Year(s) of } \\
\text { Enrollment }\end{array}$ & $\begin{array}{l}\text { Inclusion } \\
\text { Criteria }\end{array}$ & $\begin{array}{l}\text { Intervention } \\
\text { Description }\end{array}$ & Comparator(s) & $\begin{array}{l}\text { Age, } \\
\text { Mean } \\
\text { (SD) }\end{array}$ & $\begin{array}{l}\% \\
\text { Non- } \\
\text { white }\end{array}$ & $\begin{array}{l}\% \\
\text { Primiparous } \\
\% \text { Previously } \\
\text { BF }\end{array}$ & $\begin{array}{l}\text { \% } \\
\text { Cesarean } \\
\text { Birth }\end{array}$ \\
\hline $\begin{array}{l}\text { Taddei, } \\
2000^{79} \\
\text { Medium }\end{array}$ & $\begin{array}{l}\text { Cluster RCT } \\
8 \text { hospitals } \\
970 \text { infants }\end{array}$ & $\begin{array}{l}\text { Brazil } \\
1992\end{array}$ & $\begin{array}{l}\text { Hospitals } \\
\text { located near } \\
\text { Sao Paulo, } \\
\text { Brazil with at } \\
\text { least } 2 \text { births } \\
\text { per day in the } \\
\text { maternity } \\
\text { ward; no } \\
\text { previous } \\
\text { exposure to a } \\
\text { similar } \\
\text { intervention } \\
\end{array}$ & $\begin{array}{l}\text { 3-wk intensive } \\
\text { BF promotion } \\
\text { program } \\
\text { designed to } \\
\text { train } \\
\text { multidisciplinary } \\
\text { teams in } \\
\text { lactation } \\
\text { management } \\
\text { (pediatricians, } \\
\text { obstetricians, } \\
\text { and nurses) } \\
\end{array}$ & $\begin{array}{l}\text { No exposure to } \\
\text { staff training }{ }^{g}\end{array}$ & $26-29$ & $\mathrm{NR}$ & $\begin{array}{l}34-37 \\
\text { NR (90-96\% } \\
\text { intended to BF) }\end{array}$ & $\overline{N R}$ \\
\hline $\begin{array}{l}\text { Wagner, } \\
2002^{82} \\
\text { Medium }\end{array}$ & $\begin{array}{l}\text { Single group, pre- } \\
\text { post } \\
1 \text { health care } \\
\text { system } \\
13,039 \text { infants }\end{array}$ & $\begin{array}{l}\text { United } \\
\text { States } \\
1993-1999\end{array}$ & $\begin{array}{l}\text { All mothers } \\
\text { and their } \\
\text { infants } \\
\text { admitted to the } \\
\text { Medical } \\
\text { University of } \\
\text { South Carolina } \\
\text { 1993-1999 }\end{array}$ & $\begin{array}{l}\text { Mandated BF } \\
\text { education for all } \\
\text { medical and } \\
\text { nursing staff } \\
\text { (including } \\
\text { residents) } \\
\text { emphasizing the } \\
\text { benefits of BF } \\
\text { for mothers and } \\
\text { infants offered } \\
\text { in a range of } \\
\text { forums } \text { patient }^{\mathrm{h}} \text { patient } \\
\text { education } \\
\text { offered via } \\
\text { prenatal classes } \\
\text { and postnatal } \\
\text { care }\end{array}$ & $\begin{array}{l}\text { Usual care (pre- } \\
\text { intervention) }\end{array}$ & NR & 69 & $\begin{array}{l}\mathrm{NR} \\
\mathrm{NR}\end{array}$ & NR \\
\hline
\end{tabular}




\begin{tabular}{|c|c|c|c|c|c|c|c|c|c|}
\hline $\begin{array}{l}\text { First Author, } \\
\text { Year }\end{array}$ & $\begin{array}{l}\text { Study Design } \\
\text { N Sites } \\
\text { N Participants }\end{array}$ & $\begin{array}{l}\text { Country } \\
\text { Year(s) of } \\
\text { Enrollment }\end{array}$ & $\begin{array}{l}\text { Inclusion } \\
\text { Criteria }\end{array}$ & $\begin{array}{l}\text { Intervention } \\
\text { Description }\end{array}$ & $\begin{array}{l}\text { Comparator( } \\
\text { s) }\end{array}$ & $\begin{array}{l}\text { Age, } \\
\text { Mean } \\
\text { (SD) }\end{array}$ & $\begin{array}{l}\% \\
\text { Non- } \\
\text { white }\end{array}$ & $\begin{array}{l}\text { \% } \\
\text { Primiparous } \\
\% \text { Previously } \\
\text { BF }\end{array}$ & $\begin{array}{l}\% \\
\text { Cesarean } \\
\text { Birth }\end{array}$ \\
\hline Wan, $2011^{72}$ & RCT & China & $\begin{array}{l}\text { Pregnant } \\
\text { women } \geq 20\end{array}$ & $\begin{array}{l}\text { Continuous } \\
\text { primary nursing }\end{array}$ & $\begin{array}{l}\text { Task-centered } \\
\text { nursing care: }\end{array}$ & 27-28 & NR & 100 & NR \\
\hline Medium & $\begin{array}{l}1 \text { hospital ( } 2 \\
\text { obstetric units) } \\
470 \text { pregnant } \\
\text { women }\end{array}$ & 2008-2009 & $\begin{array}{l}\text { yrs having } \\
\text { their first } \\
\text { delivery }\end{array}$ & $\begin{array}{l}\text { care: } \\
\text { individualized } \\
\text { continuous } \\
\text { holistic primary } \\
\text { nursing care } \\
\text { starting } 8 \text { wks } \\
\text { prior to delivery } \\
\text { (outpatient } \\
\text { setting), during } \\
\text { admission and } 2 \\
\text { wks post } \\
\text { discharge (to } \\
\text { mother and } \\
\text { infants) by the } \\
\text { same nurse }\end{array}$ & $\begin{array}{l}\text { women } \\
\text { received the } \\
\text { same care for } \\
\text { the same } \\
\text { duration, but } \\
\text { task centered } \\
\text { (not } \\
\text { individualized) } \\
\text {, and by } \\
\text { different } \\
\text { nurses who } \\
\text { carried out } \\
\text { different } \\
\text { services }\end{array}$ & & & NA & \\
\hline \multicolumn{10}{|c|}{$\begin{array}{l}\text { a Per authors, routine preventive health care for infants includes a home visit between } 0 \text { and } 2 \text { wks and consultations at } 6 \text { wks and at 3, 4, 5, 6, 7-8, 10, and } 11-12 \text { mos for } \\
\text { vaccination, anthropometric measurements, screening, and lactation counseling. }\end{array}$} \\
\hline \multicolumn{10}{|c|}{$\begin{array}{l}\text { b All characteristics provided in study are for the residents who were exposed to the educational curriculum, not for the women/infants whose BF rates were being measured. } \\
\text { c Described as personal advice from midwives and nurses according to the mother's wishes and a possibility to watch a BF video on the ward; no additional staff training and the } \\
\text { families did not have access to more individualized BF support. }\end{array}$} \\
\hline \multicolumn{10}{|c|}{$\begin{array}{l}\text { d At baseline, } 10 \text { BF groups were offered in the intervention and control localities. The number of BF groups increased to } 27 \text { in intervention localities and remained at } 10 \text { in control } \\
\text { localities. }\end{array}$} \\
\hline \multicolumn{10}{|c|}{ e The HMO included 14 health centers of the Harvard Vanguard Medical Associates in eastern Massachusetts. } \\
\hline \multirow{3}{*}{\multicolumn{10}{|c|}{$\begin{array}{l}{ }^{f} \text { The Massachusetts law established a "minimum stay" of } 48 \text { hours, with shorter stays permitted if a mother gave consent and was offered a home visit. A federal law effective } \\
\text { January } 1998 \text { extended the 48-hour coverage mandate to other States and health plans but did not change the policy environment for the study population. } \\
\text { g At the time of the study, few Brazilian hospitals had rooming-in, and approximately 30\% of all deliveries were via cesarean section. }\end{array}$}} \\
\hline & & & & & & & & & \\
\hline & & & & & & & & & \\
\hline \multicolumn{10}{|c|}{$\begin{array}{l}\text { h The learning forum included a yearly didactic conference for pediatric residents and twice yearly seminar for staff (pediatrics, obstetrics and nursing, bedside teaching, case } \\
\text { conferences journal club). }\end{array}$} \\
\hline
\end{tabular}


times per day, and education targeted at both parents). ${ }^{83}$ Three studies combined staff training with expanded access to breastfeeding groups ${ }^{74}$ or additional postpartum home visits. ${ }^{76,84}$

Three studies assessed changes in health service delivery or policies that relate to breastfeeding. One RCT compared two different types of perinatal nursing care: continuous primary nursing care (the same nurse provided care during prenatal appointments, during admission, and 2 weeks postdischarge to both mothers and infants) or usual task-centered nursing (women and infants received the same care, but different nurses carried out different tasks). ${ }^{72}$ The second study was a single group pre-post study assessing the effect of changes in maternity length of stay policies in one U.S. State (Massachusetts) among women enrolled in a large health maintenance organization. ${ }^{80}$ The third study assessed the benefit of implementing an adapted BFHI intervention in outpatient maternal and child health centers (e.g., developing a breastfeeding policy, staff training, outcome assessment, and quality improvement initiatives) ${ }^{81}$

The majority of studies (11) were rated as medium ROB, 1 was rated low ROB, ${ }^{84}$ and 3 were rated high ROB. ${ }^{68,75,78}$ Common sources of bias included selection bias, high attrition, and baseline differences between groups that were not controlled for in the analyses.

\section{Results}

\section{Breastfeeding Initiation}

Ten studies assessing non-BFHI health care system interventions reported on rates of breastfeeding initiation (Table 8). Studies generally measured breastfeeding initiation during a maternity stay or at discharge. Three studies assessed the initiation of any breastfeeding (exclusive and nonexclusive) only, ${ }^{71,74,80}$ two assessed the initiation of exclusive breastfeeding only, ${ }^{68,72}$ and the others reported on both measures.

Table 8. Non-BFHI health care system-based interventions reporting on breastfeeding initiation

\begin{tabular}{llll}
$\begin{array}{l}\text { First } \\
\begin{array}{l}\text { Author, } \\
\text { Year }\end{array}\end{array}$ & Intervention & $\begin{array}{l}\text { Study } \\
\text { Design }\end{array}$ & $\begin{array}{l}\text { Breastfeeding Initiation Outcome } \\
\text { Results }\end{array}$ \\
ROB & & N Sites & \\
& & N & \\
& & Participants & \\
\hline Ekstrom & G1: BF training & Cluster RCT & Assessed via questionnaire (3 days postpartum), \% \\
$2014^{70} ;$ & program for health & & initiated BF (N total): \\
Ekstrom & care professionals & 10 & G1: $100(172)$ \\
$2012^{71}$ & & municipalities & G2: $97(148)$ \\
& G2: Usual care (control & & G3: $97(160)$ \\
Medium & group A) & 480 women & G1 vs. G2: $p=0.863$ \\
& & & G1 vs. G3: $p=0.838$
\end{tabular}

G3: Usual care (control group B) 


\begin{tabular}{|c|c|c|c|}
\hline $\begin{array}{l}\text { First } \\
\text { Author, } \\
\text { Year } \\
\text { ROB }\end{array}$ & $\begin{array}{l}\text { Intervention } \\
\text { Comparison }\end{array}$ & $\begin{array}{l}\text { Study } \\
\text { Design } \\
\text { N Sites } \\
\text { N } \\
\text { Participants }\end{array}$ & $\begin{array}{l}\text { Breastfeeding Initiation Outcome } \\
\text { Results }\end{array}$ \\
\hline $\begin{array}{l}\text { Feldma } \\
\text { n- } \\
\text { Winter, } \\
2010^{73} \\
\text { Medium }\end{array}$ & $\begin{array}{l}\text { G1: Residency BF } \\
\text { curriculum } \\
\text { G2: Usual care }\end{array}$ & $\begin{array}{l}\text { NRCT } \\
13 \text { sites } \\
\text { (residency } \\
\text { programs) } \\
\text { N women NR }\end{array}$ & $\begin{array}{l}\text { Initiation of exclusive } \mathrm{BF}, \% \text { (n/N total): } \\
\text { G1 pre: } 15.5(78 / 504) \\
\text { G1 post: } 23.1(114 / 493) \\
\text { G1 pre-post \% change: } 7.5 ; p=0.002 \\
\text { G2 pre: } 27.5(193 / 701) \\
\text { G2 post: } 30.5(214 / 701) \\
\text { G2 pre-post \% change: } 3.0 ; p=0.239 \\
\text { Initiation of any BF (\% (n/N total): } \\
\text { G1 pre: } 76.0(383 / 504) \\
\text { G1 post: } 80.7(398 / 493) \\
\text { G1 pre-post } \% \text { change: } 4.7 ; p=0.071 \\
\text { G2 pre: } 64.8(454 / 701) \\
\text { G2 post: } 66.6(467 / 701) \\
\text { G2 pre-post } \% \text { change: } 1.8 ; p=0.500\end{array}$ \\
\hline $\begin{array}{l}\text { Hannula } \\
\text {, 2014 } \\
\text { High }\end{array}$ & $\begin{array}{l}\text { G1: Intensified } \\
\text { perinatal family BF } \\
\text { support } \\
\text { G2: Usual care }\end{array}$ & $\begin{array}{l}\text { NRCT } \\
3 \text { maternity } \\
\text { hospitals } \\
705 \text { mothers }\end{array}$ & $\begin{array}{l}\text { Exclusive BF at hospital discharge, \% }(\mathrm{N}) \text { : } \\
\text { G1: } 76(431) \\
\text { G2: } 66(274) \\
p=0.0099\end{array}$ \\
\hline $\begin{array}{l}\text { Hoddino } \\
\text { tt, } \\
2009^{74}\end{array}$ & $\begin{array}{l}\text { G1: Adoption of a } \\
\text { policy to provide BF } \\
\text { groups for pregnant } \\
\text { and BF women } \\
\text { G2: Usual care (no } \\
\text { new policy) }\end{array}$ & $\begin{array}{l}\text { Cluster RCT } \\
14 \text { general } \\
\text { practices } \\
\text { ("localities") } \\
18,603^{\mathrm{b}}\end{array}$ & $\begin{array}{l}\text { Initiation of any BF at birth, mean difference }(95 \% \mathrm{Cl}) \\
\text { between intervention and control groups at } \\
\text { postintervention (adj. for preintervention rate): } \\
-0.009(-0.045 \text { to } 0.027) ; p=0.58\end{array}$ \\
\hline $\begin{array}{l}\text { Kools, } \\
2005^{77} \\
\text { Medium }\end{array}$ & $\begin{array}{l}\text { G1: BF promotion and } \\
\text { support program } \\
\text { G2: Usual care }\end{array}$ & $\begin{array}{l}\text { Cluster RCT } \\
10 \text { maternity } \\
\text { and child } \\
\text { health centers } \\
781 \text { mothers }\end{array}$ & $\begin{array}{l}\text { Exclusive BF at birth, } \%(\mathrm{~N}): \\
\text { G1: } 61(371) \\
\text { G2: } 67(330) \\
\text { OR }(95 \% \mathrm{Cl}): 0.80(0.57 \text { to } 1.11) \\
\text { Total BF at birth (exclusive or nonexclusive), \% (N): } \\
\text { G1: } 68(371) \\
\text { G2: } 72(330) \\
\text { OR (95\% Cl): } 0.84(0.61 \text { to } 1.16)\end{array}$ \\
\hline $\begin{array}{l}\text { Madden } \\
, 2003^{80} \\
\text { Medium }\end{array}$ & $\begin{array}{l}\text { Changes in maternity } \\
\text { length of stay policy } \\
\text { (pre-post)c }\end{array}$ & $\begin{array}{l}\text { Single-group, } \\
\text { pre-post } \\
14 \text { health } \\
\text { centers } \\
20,366 \text { births }\end{array}$ & $\begin{array}{l}\text { BF initiation rates increased an estimated } 0.4 \% \text { per } \\
\text { quarter over time: } 70.1 \% \text { in the } 4 \text { th quarter of } 1990 \text { to } \\
81.9 \% \text { in the } 1 \text { st quarter of } 1998(\mathrm{p}<0.0001) \text {; no } \\
\text { statistically significant changes in BF rates were found at } \\
\text { either of the } 17 \text { policy intervention points. } \\
\text { Subgroups: no reductions in BF rates were associated } \\
\text { with the changes in LOS policies for the following } \\
\text { subgroups: maternal age, primiparous status, Medicaid } \\
\text { enrollment, living in a low-income neighborhood, black } \\
\text { race, Asian race, and Hispanic nonblack race. }\end{array}$ \\
\hline $\begin{array}{l}\text { Martens } \\
, 2000^{78}\end{array}$ & $\begin{array}{l}\text { G1: Nursing staff BF } \\
\text { education }\end{array}$ & $\begin{array}{l}\text { NRCT } \\
2 \text { rural }\end{array}$ & $\begin{array}{l}\text { Any BF initiation, } \%(N)^{d} \text { : } \\
\text { G1 pre: } 62(21) \\
\text { G1 post: } 65(20)\end{array}$ \\
\hline High & G2: Usual care & $\begin{array}{l}\text { hospitals } \\
75 \text { births }\end{array}$ & $\begin{array}{l}\text { G2 pre: } 74(74) \\
\text { G2 post: } 64(14) \\
p>0.05 \text { for pre-post change in both groups }\end{array}$ \\
\hline
\end{tabular}




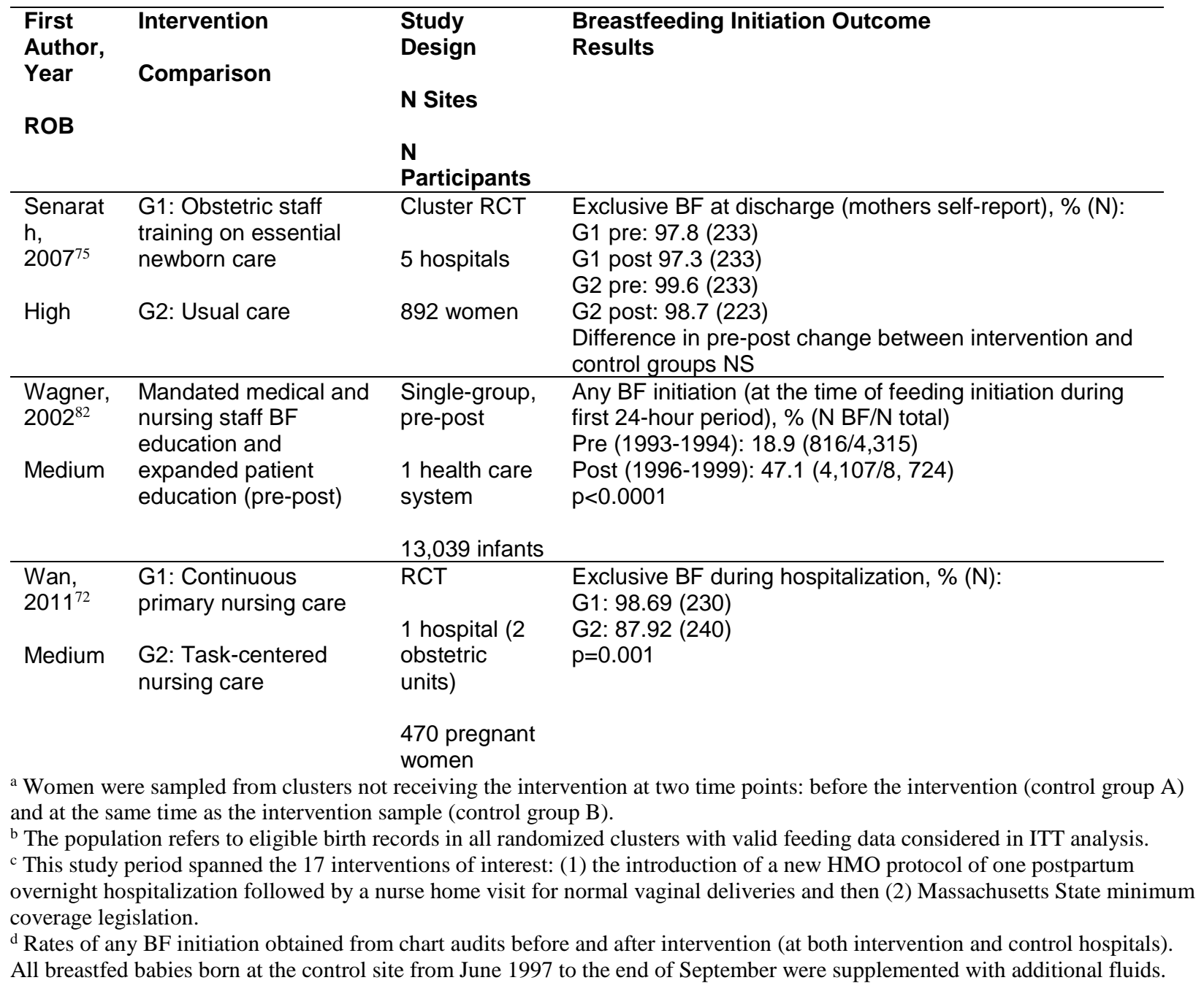

BF = breastfeeding; BFHI = Baby-Friendly Health Initiative; $\mathrm{CI}=$ confidence interval; $\mathrm{G}=$ group; HMO = health maintenance organization; ITT = intent-to-treat; LOS = length of stay; $\mathrm{N}$ = sample size; $\mathrm{NR}=$ not reported: $\mathrm{NRCT}=$ nonrandomized controlled trial; NS = not sufficient; RCT = randomized controlled trial; ROB = risk of bias.

Four studies assessing the effectiveness of education and training for health care professionals only (with no mention of additional individual-level services offered to women) reported on breastfeeding initiation; overall, studies found no difference between intervention and control groups. ${ }^{71,73,75,78}$ Two of these were RCTs enrolling populations with a high rate of breastfeeding initiation before and after implementation of the intervention, at both intervention and control sites (>97\%). ${ }^{71,75}$ One NRCT assessed the effectiveness of a residency breastfeeding curriculum; at intervention sites, initiation of exclusive breastfeeding increased (pre-post \% change, 7.5; $\mathrm{p}=0.002$ ), however, control sites also had an increase in breastfeeding initiation (pre-post \% change, 3.0; $\mathrm{p}=0.239$ ) and authors do not note whether differences between groups are statistically significant. ${ }^{73}$

Four studies assessed a health-system intervention that combined education (or skills training) to providers with expanded breastfeeding support services and reported on breastfeeding initiation rates. ${ }^{68,74,77,82}$ Of these studies, two did not find benefit; one was an RCT assessing adoption of a policy to provide increased access to breastfeeding groups (mean difference between groups in breastfeeding initiation rates, -0.009; 95\% confidence interval [CI], 
-0.045 to 0.027$),{ }^{74}$ and the other assessed a multicomponent breastfeeding promotion and support program and found no difference in initiation of any or exclusive breastfeeding at birth (Table 8). ${ }^{77}$ Two other studies found benefit. ${ }^{68,82}$ One found increased rates of exclusive breastfeeding initiation at hospitals implementing an intensified perinatal family breastfeeding support intervention compared with controls (75 vs. 66\%; $\mathrm{p}=0.0099)$, ${ }^{68}$ and the other found increased initiation of any breastfeeding after a mandated medical and nursing staff educational intervention paired with increased pre- and postnatal breastfeeding support (19\% vs. 48\%; $\mathrm{p}<0.0001){ }^{82}$

Two studies assessing changes in different health service delivery methods or policies that relate to breastfeeding reported on initiation rates with inconsistent results. ${ }^{72,80}$ One study was a single-group pre-post study assessing the relationship between changes in maternity length-ofstay policies among women belonging to one Massachusetts health maintenance organization (HMO); the study period spanned two separate interventions: (1) the introduction of a new protocol of one postpartum overnight hospitalization for women who had normal vaginal deliveries (followed by a nurse home visit) and (2) Massachusetts State law mandating minimum 48-hour coverage legislation. ${ }^{80}$ Breastfeeding initiation rates increased an estimated 0.4 percent per quarter over time with no statistically significant changes at either of the two policy intervention time points. ${ }^{80}$ The RCT comparing continuous primary nursing care with usual care (task oriented nursing) among Chinese women found higher rates of exclusive breastfeeding during hospitalization among the intervention group than in the controls (99\% vs. 88\%; $\mathrm{p}=0.001) .^{72}$

\section{Breastfeeding Duration}

Table 9 shows results of the 11 non-BFHI health care system intervention studies reporting on breastfeeding duration. Two measured duration of exclusive breastfeeding only, ${ }^{72,}{ }^{76} 2$ measured the duration of any breastfeeding only, ${ }^{74,80}$ and 5 measured both exclusive and nonexclusive breastfeeding duration.

Table 9. Non-BFHI health care system-based interventions reporting on breastfeeding duration

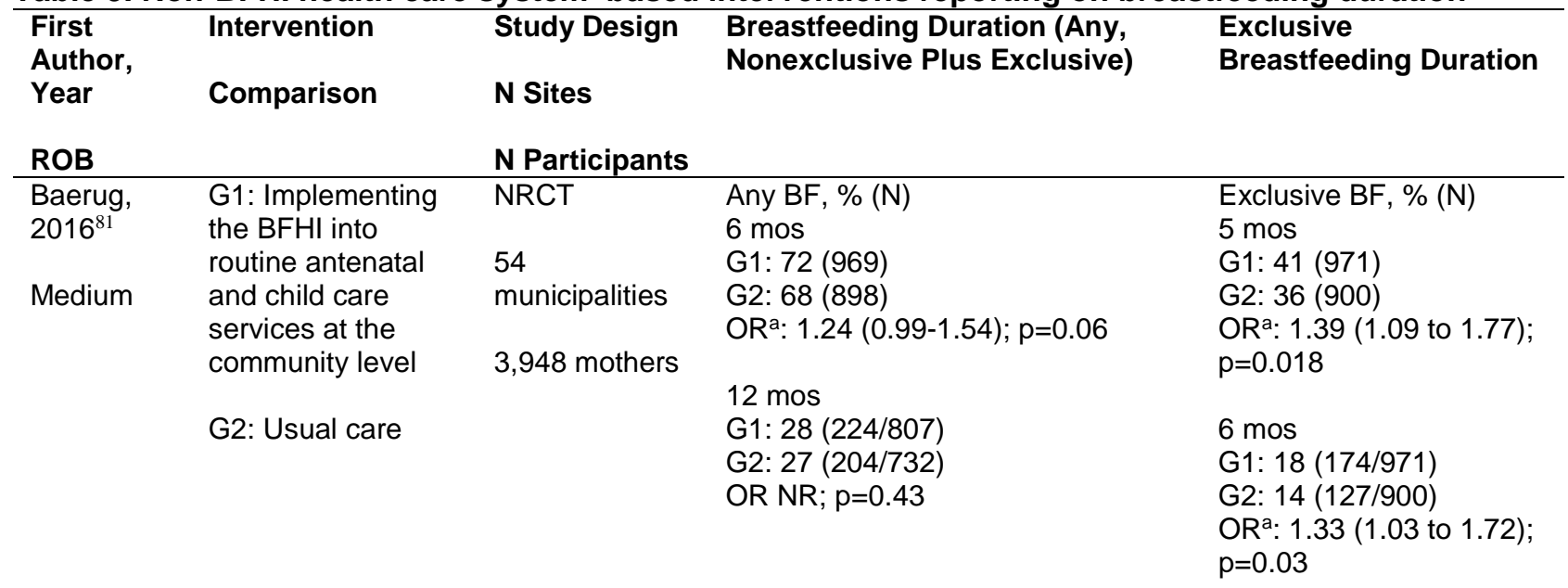




\begin{tabular}{|c|c|c|c|c|}
\hline $\begin{array}{l}\text { First } \\
\text { Author, } \\
\text { Year } \\
\text { ROB } \\
\end{array}$ & $\begin{array}{l}\text { Intervention } \\
\text { Comparison }\end{array}$ & $\begin{array}{l}\text { Study Design } \\
\text { N Sites } \\
\text { N Participants }\end{array}$ & $\begin{array}{l}\text { Breastfeeding Duration (Any, } \\
\text { Nonexclusive Plus Exclusive) }\end{array}$ & $\begin{array}{l}\text { Exclusive } \\
\text { Breastfeeding Duration }\end{array}$ \\
\hline $\begin{array}{l}\text { Coutinho, } \\
2005^{84} \\
\text { Low }\end{array}$ & $\begin{array}{l}\text { G1: BFHI staff } \\
\text { training }+10 \mathrm{HVs} \\
\text { providing BF } \\
\text { support and } \\
\text { promotion } \\
\text { G2: BFHI staff } \\
\text { training alone }\end{array}$ & $\begin{array}{l}\text { RCT } \\
2 \text { sites } \\
350 \text { women }\end{array}$ & $\begin{array}{l}\text { Mean rate of any BF 10-180 days } \\
\text { after discharge, } \%(\mathrm{~N}) \\
\text { G1: } 78(174) \\
\text { G2: } 62(175) \\
\text { p<0.001 }\end{array}$ & $\begin{array}{l}\text { Mean rate of exclusive } \\
\text { BF 10-180 days after } \\
\text { discharge, \% (N) } \\
\text { G1: } 45(174) \\
\text { G2: } 13(175) \\
\text { p<0.0001 }\end{array}$ \\
\hline $\begin{array}{l}\text { Ekstrom, } \\
2014^{70} \\
\text { Ekstrom, } \\
2012^{71} \\
\text { Medium }\end{array}$ & $\begin{array}{l}\text { G1: BF training } \\
\text { program for health } \\
\text { care professionals } \\
\text { G2: Usual care } \\
\text { (control group A)b } \\
\text { G3: Usual care } \\
\text { (control group B) }\end{array}$ & $\begin{array}{l}\text { Cluster RCT } \\
\text { Antenatal and } \\
\text { child health } \\
\text { centers in } 10 \\
\text { municipalities } \\
480 \text { women }\end{array}$ & $\begin{array}{l}\text { Total BF duration }{ }^{\mathrm{c}} \text { (exclusive plus } \\
\text { partial BF) assessed at } 9 \text { mos, } \\
\text { mean mos (SD) } \\
\text { G1: } 7.5(4.7) \\
\text { G2: } 7.1(4.6) \\
\text { G3: } 7.0(4.5) \\
\text { p=NS per authors }\end{array}$ & $\begin{array}{l}\text { Duration of exclusive }{ }^{\mathrm{C} F} \\
\text { (mos), mean (SD) } \\
\text { G1: } 3.9(2.2) \\
\text { G2: } 3.2(1.7) \\
\text { G3: } 3.5(2.0) \\
\text { G1 vs. G2= p=0.02 } \\
\text { G1 vs. G3= NS per } \\
\text { authors }\end{array}$ \\
\hline $\begin{array}{l}\text { Feldman- } \\
\text { Winter, } \\
2010^{73} \\
\text { Medium }\end{array}$ & $\begin{array}{l}\text { G1: Residency BF } \\
\text { curriculum } \\
\text { G2: Usual care }\end{array}$ & $\begin{array}{l}\text { NRCT } \\
13 \text { sites }^{d} \\
\text { (residency } \\
\text { programs) }\end{array}$ & $\begin{array}{l}\text { Any BF at } 6 \text { mos, } \%(\mathrm{~N}): \\
\text { G1 pre: } 25.3(300) \\
\text { G1 post: } 28.7(300) \\
\text { pre-post change: } 3.4 ; p=0.291 \\
\text { G2 pre: } 26.9(499) \\
\text { G2 post: } 25.3(550) \\
\text { pre-post } \% \text { change: }-1.6 ; p=0.574\end{array}$ & $\begin{array}{l}\text { Exclusive BF at } 6 \text { mos, } \\
\%(N) \text { : } \\
\text { G1 pre: } 2.3(300) \\
\text { G1 post: } 9.0(300) \\
\text { pre-post } \% \text { change: } 6.7 ; \\
\text { p=0.001 } \\
\text { G2 pre: } 11.6(499) \\
\text { G2 post: } 6.2(550) \\
\text { pre-post } \% \text { change: }-5.4 ; \\
\text { p=0.002 }\end{array}$ \\
\hline $\begin{array}{l}\text { Hoddinott, } \\
2009^{74}\end{array}$ & $\begin{array}{l}\text { G1: Adoption of a } \\
\text { policy to provide } \\
\text { BF groups for } \\
\text { pregnant and BF } \\
\text { women } \\
\text { G2: Usual care (no } \\
\text { new policy) }\end{array}$ & $\begin{array}{l}\text { Cluster RCT } \\
14 \text { general } \\
\text { practices } \\
\text { ("localities") } \\
18,603\end{array}$ & $\begin{array}{l}\text { Any BF at } 6-8 \text { wks postpartum, } \\
\text { mean difference ( } 95 \% \mathrm{Cl} \text { ) between } \\
\text { intervention and control groups at } \\
\text { postintervention (adj. for } \\
\text { preintervention rate): } \\
-0.017 \text { (-0.036 to } 0.002) ; p=0.08 \\
\text { Any BF at } 8-9 \text { mos postpartum, } \\
\text { mean difference ( } 95 \% \mathrm{Cl} \text { ) between } \\
\text { intervention and control groups at } \\
\text { postintervention (adj. for } \\
\text { preintervention rate): } \\
0.007 \text { (-0.056 to } 0.070) ; p=0.82\end{array}$ & NR \\
\hline $\begin{array}{l}\text { Kools, } \\
2005^{77} \\
\text { Medium }\end{array}$ & $\begin{array}{l}\text { G1: BF promotion } \\
\text { and support } \\
\text { program } \\
\text { G2: Usual care }\end{array}$ & $\begin{array}{l}\text { Cluster RCT } \\
10 \text { maternity } \\
\text { and child } \\
\text { health centers }\end{array}$ & $\begin{array}{l}\text { Any BF } 3 \text { mos postpartum, \% (N): } \\
\text { G1: } 32(371) \\
\text { G2: } 38(330) \\
\text { OR }(95 \% \mathrm{Cl}): \\
0.79(0.58 \text { to } 1.08)\end{array}$ & $\begin{array}{l}\text { Exclusive BF } 3 \text { mos } \\
\text { postpartum, \% (N): } \\
\text { G1: } 27(371) \\
\text { G2: } 32(330) \\
\text { OR }(95 \% \mathrm{Cl}): \\
0.79(0.57 \text { to } 1.10)\end{array}$ \\
\hline
\end{tabular}




\begin{tabular}{|c|c|c|c|c|}
\hline $\begin{array}{l}\text { First } \\
\text { Author, } \\
\text { Year } \\
\text { ROB }\end{array}$ & $\begin{array}{l}\text { Intervention } \\
\text { Comparison }\end{array}$ & $\begin{array}{l}\text { Study Design } \\
\text { N Sites } \\
\text { N Participants }\end{array}$ & $\begin{array}{l}\text { Breastfeeding Duration (Any, } \\
\text { Nonexclusive Plus Exclusive) }\end{array}$ & $\begin{array}{l}\text { Exclusive } \\
\text { Breastfeeding Duration }\end{array}$ \\
\hline $\begin{array}{l}\text { Kronborg, } \\
2007^{76} \\
\text { Medium }\end{array}$ & $\begin{array}{l}\text { G1: Health visitor } \\
\text { education and } \\
\text { delivery of BF } \\
\text { support } \\
\text { intervention (1-3 } \\
\text { HVs) } \\
\text { G2: Usual care }\end{array}$ & $\begin{array}{l}\text { Cluster RCT } \\
22 \\
\text { municipalities } \\
1,595 \text { mothers }\end{array}$ & NR & $\begin{array}{l}\text { Exclusive BF } 6 \text { mos } \\
\text { postpartum, \% (N): } \\
\text { G1: } 7.7(780) \\
\text { G2: } 4.9(815) \\
\text { Cessation rate of } \\
\text { exclusive BF at } 6 \text { mos } \\
\text { postpartum (intervention } \\
\text { vs. control): } \\
\text { HR }(95 \% \mathrm{Cl}) \\
0.86(0.75 \text { to } 0.99) \\
p=0.04\end{array}$ \\
\hline $\begin{array}{l}\text { Madden, } \\
2003^{80} \\
\text { Medium }\end{array}$ & $\begin{array}{l}\text { Changes in } \\
\text { maternity length-of- } \\
\text { stay policy (pre- } \\
\text { post) }\end{array}$ & $\begin{array}{l}\text { Single-group, } \\
\text { pre-post } \\
14 \text { health } \\
\text { centers } \\
20,366 \text { births }\end{array}$ & $\begin{array}{l}\text { Rates of BF continuation within } 90 \\
\text { days of birth among BF initiators } \\
\text { remained constant at an estimated } \\
72.6 \% \text { throughout the study period. }\end{array}$ & NR \\
\hline $\begin{array}{l}\text { Nilsson, } \\
2017^{83} \\
\text { Medium }\end{array}$ & $\begin{array}{l}\text { G1: } \\
\text { Multicomponent BF } \\
\text { intervention in } \\
\text { early-discharge } \\
\text { maternity settings } \\
\text { G2: Usual care }\end{array}$ & $\begin{array}{l}\text { Cluster RCT } \\
9 \text { maternity } \\
\text { settings } \\
\text { 3,541 women }\end{array}$ & NR & $\begin{array}{l}\text { Exclusive BF 5-7 days } \\
\text { postpartum, \%: } \\
\text { G1: } 82 \\
\text { G2: } 82 \\
\text { AOR (95\% CI) } \\
1.01(0.88 \text { to } 1.14) \\
\text { Exclusive BF } 1 \text { mo } \\
\text { postpartum, \%: } \\
\text { G1: } 74 \\
\text { G2: } 76 \\
\text { AOR }(95 \% \mathrm{Cl}) \\
0.87(0.64 \text { to } 1.18) \\
\text { Exclusive BF } 6 \text { mo } \\
\text { postpartum, } \%: \\
\text { G1: } 7 \\
\text { G2: } 5 \\
\text { AOR (95\% CI) } \\
\text { 1.36 (1.02 to } 1.81)\end{array}$ \\
\hline $\begin{array}{l}\text { Taddei, } \\
2000^{79} \\
\text { Medium }\end{array}$ & $\begin{array}{l}\text { G1: Intensive staff } \\
\text { BF training (3-wk } \\
\text { course) } \\
\text { G2: Usual care (no } \\
\text { staff training) }\end{array}$ & $\begin{array}{l}\text { Cluster RCT } \\
8 \text { hospitals } \\
970 \text { infants }\end{array}$ & $\begin{array}{l}\text { Full BF duration (days), }{ }^{\mathrm{e}} \text { measured } \\
\text { at } 6 \text { mos, adj. HR }(95 \% \mathrm{Cl} \text { ) of } \\
\text { before-after cohort: } \\
\mathrm{G} 1: 0.80 \text { (0.67 to } 0.97) \\
\mathrm{G} 2: 1.16(0.93 \text { to } 1.45) \\
\mathrm{p}=0.0019 \\
\\
\text { Total BF duration (age at which BF } \\
\text { was terminated, days), measured } \\
\text { at } 6 \text { mos, adjusted }{ }^{f} \mathrm{HR}(95 \% \mathrm{Cl}) \text { of } \\
\text { before-after cohort: } \\
\mathrm{G} 1: 1.01 \text { (0.83 to } 1.22) \\
\mathrm{G} 2: 1.55 \text { (1.23 to } 1.96) \\
\mathrm{p}=0.0019\end{array}$ & $\begin{array}{l}\text { Exclusive BF duration } \\
\text { (days), measured at } 6 \\
\text { mos, adjusted }^{f} \text { HR }(95 \% \\
\text { Cl) of before-after cohort: } \\
\text { G1: } 0.71(0.59 \text { to } 0.85) \\
\text { G2: } 0.98(0.79 \text { to } 1.22) \\
p=0.0020\end{array}$ \\
\hline
\end{tabular}




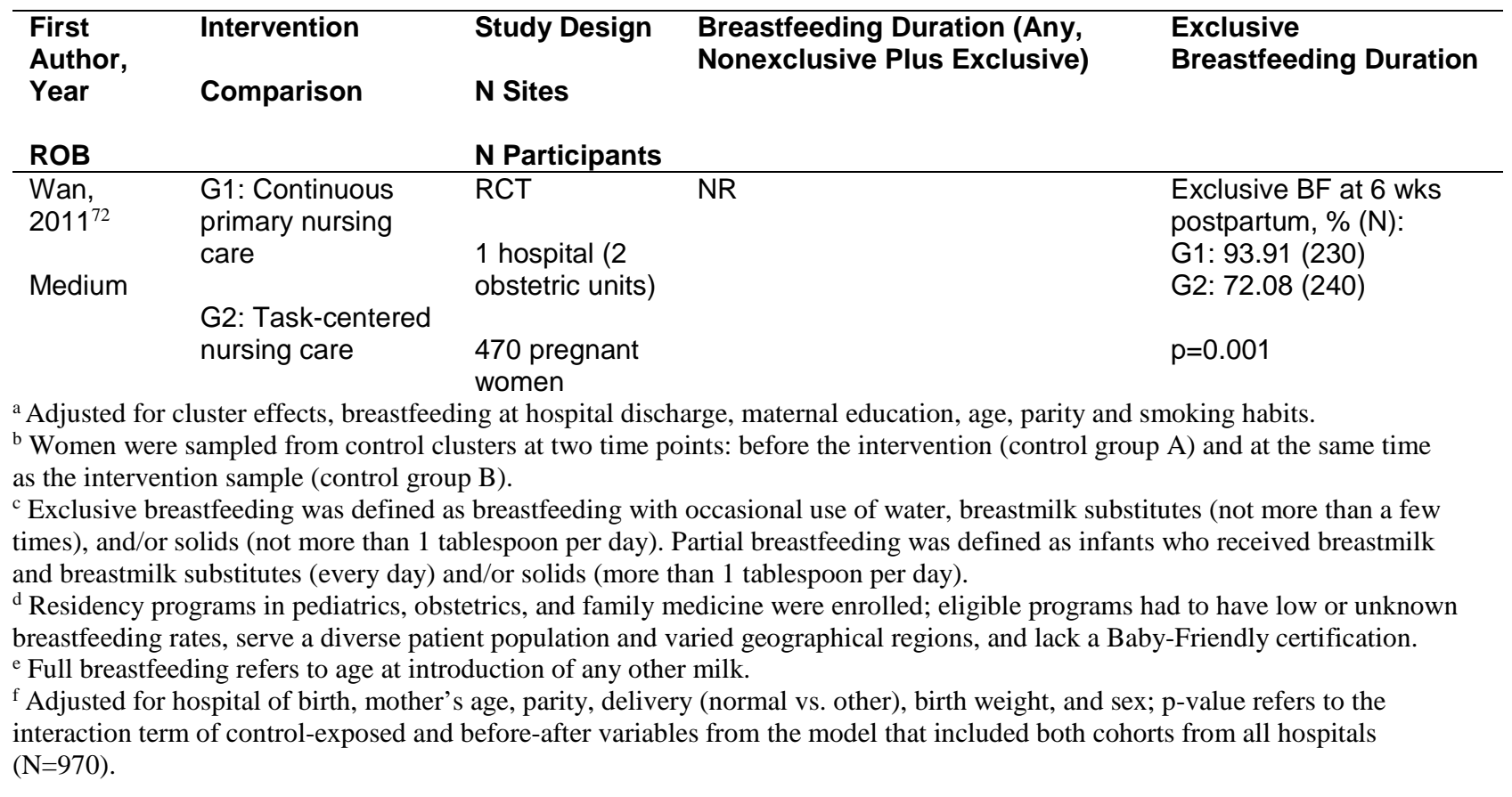

$\mathrm{AOR}=$ adjusted odds ratio; $\mathrm{BF}=$ breastfeeding; $\mathrm{BFHI}=$ Baby-Friendly Hospital Initiative; $\mathrm{CI}$ = confidence interval; $\mathrm{G}=$ group; $\mathrm{HR}=$ hazard ratio; $\mathrm{HV}=$ home visit; $\mathrm{N}=$ number; $\mathrm{NR}=$ not reported; NRCT = nonrandomized controlled trial; NS = not sufficient; $\mathrm{OR}=$ odds ratio; $\mathrm{RCT}=$ randomized controlled trial; $\mathrm{ROB}=$ risk of bias; $\mathrm{SD}=$ standard deviation; wks $=$ weeks.

Three studies assessed the effectiveness of an intervention providing education and training to health care professionals (with no additional individual-level services offered to women); the results did not consistently demonstrate benefit. ${ }^{71,73,79}$ In the cluster RCT comparing breastfeeding training to Swedish midwives and postnatal nurses, ${ }^{71}$ women in the intervention and control groups had similar durations of any breastfeeding (7.5, 7.1, and 7.0 months, respectively) and exclusive breastfeeding durations (3.9, 3.2, and 3.5 months, respectively) $;^{71}$ only one comparison was significant: mean exclusive BF duration among women in intervention municipalities versus women from control group municipalities measured before the start of the intervention (3.9 vs. 3.2 months; $\mathrm{p}=0.02$ ). The NRCT enrolling U.S. residency programs (pediatrics, OBGYN, and family medicine) reported on breastfeeding rates at 6 months following implementation of a breastfeeding residency curriculum; there was no difference in the pre-post change among intervention sites $(3.4 \%$; $=0.291)$ or control sites $(1.6 \%$; $\mathrm{p}=0.574)$ for rates of any breastfeeding at 6 months. ${ }^{73}$ For rates of exclusive breastfeeding at 6 months, intervention sites had improved rates $(6.7 \%$; $=0.001)$, while control sites had decreased rates $(-5.4 ; p=0.002)$ during the study period. ${ }^{73}$ Authors provide only p-values for pre-post comparisons and not sufficient data to calculate differences between intervention and control groups. The cluster RCT assessing staff education (nurses, midwives, and physicians) found lower rates of weaning for exclusive breastfeeding, full breastfeeding (age at introduction of another milk), and total (any) breastfeeding among women in the intervention group compared with controls (Table 9). ${ }^{79}$

Four studies assessed a health system intervention that combined education (or skills training) to providers with expanded individually delivered breastfeeding support services; results were mixed (two studies found benefit and two did not). ${ }^{74,76,77,84}$ At 3 months, the RCT set in The Netherlands found no difference in rates of any and exclusive breastfeeding between groups randomized to the health care provider training and support program (counseling, 
identification of breastfeeding problems, and free access to lactation consultants) and control sites (Table 9) ${ }^{77}$ One cluster RCT assessing a policy to provide breastfeeding groups found no difference in rates of any breastfeeding among intervention and control practices 6 to 8 weeks postpartum and 8 to 9 months postpartum. ${ }^{74}$ The two studies providing staff education along with expanded or new home visits found benefit. ${ }^{76,84}$ One evaluated breastfeeding education to health visitors plus expanded home visits (1 to 3 within 5 weeks of birth) to support breastfeeding; mothers in the intervention group had a lower cessation rate of any breastfeeding than controls (hazard ratio [HR], 0.86; 95\% CI, 0.75 to 0.99). ${ }^{76}$ The second was an RCT set in Brazil that compared staff training (modeled after BFHI training) plus 10 postpartum home visits providing breastfeeding support with staff training alone; women receiving home visits had higher rates of any breastfeeding over 6 months post-discharge ( $78 \%$ vs. $62 \%$; $\mathrm{p}=0.001)$ and higher rates of exclusive breastfeeding ( $45 \%$ vs. $13 \%$; $\mathrm{p}<0.001$ ) ${ }^{84}$

Three studies assessed changes in health service delivery or policies that relate to breastfeeding and reported on breastfeeding duration; the studies did not consistently demonstrate benefit. The RCT comparing continuous primary nursing care with usual care (taskoriented nursing) among Chinese women found higher rates of exclusive breastfeeding at 6 weeks postpartum among the intervention group than in the controls (94\% vs. $72 \%$; $\mathrm{p}=0.001) .{ }^{72}$ One nonrandomized trial set in Norway adapted BFHI for implementation into routine antenatal and child services; there was no difference between the intervention and control groups in rates of any breastfeeding at 6 months (OR, 1.24; 95\% CI, 0.99 to 1.54) or at 12 months. For rates of exclusive breastfeeding, the intervention group had a significantly higher rate than controls at 5 months and 6 months postpartum (OR, 1.33; 95\% CI, 1.03 to 1.72) ${ }^{81}$ The single-group pre-post study assessing the relationship between changes in maternity length-of-stay policies among women belonging to one Massachusetts HMO found that rates of breastfeeding 90 days after birth among initiators remained constant at an estimated 73 percent throughout the study period. ${ }^{80}$

\section{Women, Infants and Children-Based Interventions}

\section{Characteristics}

Eight included studies assessed changes in breastfeeding rates associated a Special Supplemental Nutrition Program for Women, Infants and Children (WIC) program or policy. ${ }^{86-93}$ Although all studies were set in the United States, they included women from diverse States (Table 10). Studies generally enrolled a population of women with mean ages in the 20s to 30s. Two studies focused on populations of women enrolled during the 1990s, ${ }^{90,91}$ and the other four enrolled women from 2002 to 2016.

Included studies assessed heterogeneous interventions and policies. One study used three separate U.S. population cohorts to assess breastfeeding rates before and after a 2007 policy revising the WIC food package (implemented by States in 2009): ${ }^{87}$ (1) the Pregnancy Risk Assessment Monitoring System (PRAMS), women from 19 States between 2004 to 2010; (2) the Pediatric Nutrition Surveillance System (PedNSS), women/infants from 16 States from 2007 to 2010; and (3) the National Immunization Survey (NIS), infants from all 50 States and the District of Columbia from 2004 to 2010. One goal of the food package revision was to provide a greater economic incentive for women to breastfeed (e.g., by providing mothers of infants exclusively breastfed with additional milk, cheese, eggs, canned fish, and whole wheat bread). ${ }^{87}$ 
The other seven studies assessed various individual-level interventions implemented in individual States or WIC agencies (Table 10). One RCT assessed whether an electric breast pump (vs. a manual pump) would increase breastfeeding duration in mothers returning to fulltime work or school. ${ }^{86}$ One RCT compared cash incentives contingent on demonstrating breastfeeding to research staff with usual WIC services among Puerto Rican women. ${ }^{93}$ One prospective cohort study compared use of the Breastfeeding Attrition Prediction Tool (BAPT) with usual WIC services. ${ }^{92}$ Four studies assessed a type of peer support intervention. One nonrandomized trial compared a Peer Dad program (plus Peer Support for Mothers) with Peer Support for Mothers alone among Hispanic participants at a single WIC agency in Texas. ${ }^{88}$ One RCT compared telephone peer counseling with standard care (no peer counseling) at four WIC agencies in Oregon. ${ }^{89}$ Two compared a peer counseling program with usual care (no peer counseling) at different agencies in Iowa (8 counties) ${ }^{90}$ and Tennessee (9 health departments). ${ }^{91}$

\section{Results}

Results of the eight included studies are presented in Table 11. Most studies reported on both breastfeeding duration and initiation, and we present results together below grouped by intervention type. Overall, peer-support interventions targeted to mothers were effective for improving breastfeeding rates. We found insufficient evidence (primarily due to unknown consistency and imprecision) to make a conclusion on the benefit of other WIC programs or policies for improving breastfeeding outcomes.

One study assessing breastfeeding rates in relationship to the 2007 Federal rule revising WIC food packages found no association between the policy change and rates of breastfeeding. ${ }^{87}$ Data from all three sources (PRAMS, PedNSS, and NIS) showed steady upward trends in rates of ever breastfed infants on WIC during the study period; in neither PRAMS nor NIS data were trends in breastfeeding after implementation of the new food package statistically different from trends in breastfeeding among low-income women not on WIC (Table 11). ${ }^{87}$ Similarly, there was no change in monthly breastfeeding rates by birth cohort associated with the new food package in the PedNSS data (pre- vs. postimplementation of the new policy). ${ }^{87}$

In the RCT $(\mathrm{N}=280)$ enrolling Hawaiian mothers on WIC who planned to return to work or school, there was no difference in breastfeeding duration among mothers assigned to an electric or manual breast pump (median duration of breastfeeding: 12 vs. 11 months, respectively; HR, 1.13; 95\% CI, 0.79 to 1.50). ${ }^{86}$ The RCT comparing monthly cash incentives for breastfeeding with usual WIC services among Puerto Rican women found significantly higher rates of any breastfeeding at 1, 3, and 6 months postpartum (Table 11); there was no difference between groups for self-reported exclusive breastfeeding. ${ }^{93}$ In one prospective cohort study, women who agreed to participate in a tailored breastfeeding counseling and support intervention (based on responses to the BAPT tool) had higher rates of exclusive breastfeeding at 1 week and 1 month postpartum than women who declined to participate (Table 11); there was no difference in rates of exclusive breastfeeding between groups at 2 months. 
Table 10. Characteristics of studies assessing WIC-based interventions

\begin{tabular}{|c|c|c|c|c|c|c|c|c|c|}
\hline $\begin{array}{l}\text { First Author, } \\
\text { Year } \\
\text { ROB }\end{array}$ & $\begin{array}{l}\text { Study Design } \\
\text { N Sites } \\
\text { N Participants }\end{array}$ & $\begin{array}{l}\text { Country } \\
\text { Year(s) of } \\
\text { Enrollment }\end{array}$ & $\begin{array}{l}\text { Inclusion } \\
\text { Criteria }\end{array}$ & $\begin{array}{l}\text { Intervention } \\
\text { Description }\end{array}$ & $\begin{array}{l}\text { Comparator( } \\
\text { s) }\end{array}$ & $\begin{array}{l}\text { Age, } \\
\text { Mean } \\
\text { (SD) }\end{array}$ & $\begin{array}{l}\text { \% Non- } \\
\text { white }\end{array}$ & $\begin{array}{l}\text { \% } \\
\text { Primiparo } \\
\text { us } \\
\% \\
\text { Previously } \\
\text { BF }\end{array}$ & $\begin{array}{l}\text { \% } \\
\text { Cesarean } \\
\text { Birth }\end{array}$ \\
\hline $\begin{array}{l}\text { Edmunds, } \\
2017^{92} \\
\text { High }\end{array}$ & $\begin{array}{l}\text { Prospective cohort } \\
12 \text { WIC clinics (NY) } \\
826\end{array}$ & $\begin{array}{l}\text { United States } \\
\text { 2013-2016 }\end{array}$ & $\begin{array}{l}\text { Women } \geq 18 \\
\text { yrs, enrolled in } \\
\text { WIC during the } \\
\text { first trimester } \\
\text { who intended to } \\
\text { BF or were } \\
\text { undecided }\end{array}$ & $\begin{array}{l}\text { Use of the } \\
\text { Breastfeeding } \\
\text { Attrition Prediction } \\
\text { Tool to inform } \\
\text { tailored BF } \\
\text { counseling } \\
\text { targeting attitudes, } \\
\text { social } \\
\text { /professional } \\
\text { support, and BF } \\
\text { confidence }\end{array}$ & $\begin{array}{l}\text { Women who } \\
\text { declined to } \\
\text { participate } \\
\text { and received } \\
\text { usual WIC } \\
\text { services }\end{array}$ & $29(6.0)$ & 89 & $\begin{array}{l}75 \\
\text { NR }\end{array}$ & NR \\
\hline $\begin{array}{l}\text { Hayes, } 2008^{86} \\
\text { Medium }\end{array}$ & $\begin{array}{l}\text { RCT } \\
13 \text { WIC sites } \\
\text { (Hawaii) } \\
280 \text { women }\end{array}$ & $\begin{array}{l}\text { United States } \\
2002-2003\end{array}$ & $\begin{array}{l}\text { Women who } \\
\text { planned to } \\
\text { return to work } \\
\text { or school full } \\
\text { time after } \\
\text { delivery and } \\
\text { had no medical } \\
\text { conditions that } \\
\text { prevented or } \\
\text { limited BF }\end{array}$ & $\begin{array}{l}\text { Provision of an } \\
\text { electric breast } \\
\text { pump (loaned) }\end{array}$ & $\begin{array}{l}\text { Provision of a } \\
\text { manual } \\
\text { breast pump } \\
\text { (loaned) }\end{array}$ & $\begin{array}{l}\text { NR: \% by } \\
\text { age } \\
\text { group: } \\
<20: 11- \\
18 \\
20-29: \\
64-59 \\
30-44: \\
18-30\end{array}$ & NR & $\begin{array}{l}50-55 \\
N R\end{array}$ & NR \\
\hline
\end{tabular}




\begin{tabular}{|c|c|c|c|c|c|c|c|c|c|}
\hline $\begin{array}{l}\text { First Author, } \\
\text { Year } \\
\text { ROB }\end{array}$ & $\begin{array}{l}\text { Study Design } \\
\text { N Sites } \\
\text { N Participants }\end{array}$ & $\begin{array}{l}\text { Country } \\
\text { Year(s) of } \\
\text { Enrollment }\end{array}$ & $\begin{array}{l}\text { Inclusion } \\
\text { Criteria }\end{array}$ & $\begin{array}{l}\text { Intervention } \\
\text { Description }\end{array}$ & Comparator(s) & $\begin{array}{l}\text { Age, } \\
\text { Mean } \\
\text { (SD) }\end{array}$ & $\begin{array}{l}\text { \% Non- } \\
\text { white }\end{array}$ & $\begin{array}{l}\% \\
\text { Primiparo } \\
\text { us } \\
\text { \%Previous } \\
\text { ly BF }\end{array}$ & $\begin{array}{l}\% \\
\text { Cesarean } \\
\text { Birth }\end{array}$ \\
\hline $\begin{array}{l}\text { Joyce, } 2015^{87} \\
\text { High }\end{array}$ & $\begin{array}{l}\text { Prospective cohort } \\
\text { NA (3 population } \\
\text { cohorts: PRAMS, } \\
\text { NIS, and PedNSS) } \\
\text { PRAMS (WIC): } \\
85,458 \\
\text { PRAMS (Non-WIC): } \\
42,019 \\
\text { NIS (WIC): } 62,289 \\
\text { NIS (Non-WIC): } \\
11,702 \\
\text { PedNSS (infants): } \\
744\end{array}$ & $\begin{array}{l}\text { United States } \\
2004-2010\end{array}$ & $\begin{array}{l}\text { PRAMS: WIC } \\
\text { participants and } \\
\text { women w/ low } \\
\text { SES }{ }^{a} \text { who did } \\
\text { not participate } \\
\text { in WIC } \\
\text { NIS: Children } \\
\text { who ever } \\
\text { received WIC } \\
\text { benefits and } \\
\text { children who did } \\
\text { not but had } \\
\text { family income } \\
\leq 250 \% \text { of the } \\
\text { Federal poverty } \\
\text { level } \\
\text { PedNSS }{ }^{\text {b }} \text { All } \\
\text { participants } \\
2007-2011 \text { (pre- } \\
\text { and post-policy } \\
\text { change) }\end{array}$ & $\begin{array}{l}\text { WIC food package } \\
\text { policy change } \\
\text { (2007 rule } \\
\text { implemented by } \\
\text { States in 2009): } \\
\text { vouchers available } \\
\text { to BF women with } \\
\text { monetary value } \\
\text { comparable to } \\
\text { vouchers received } \\
\text { by mothers not } \\
\text { exclusively BF; } \\
\text { restrictions on } \\
\text { issuance of } \\
\text { formula to BF } \\
\text { infants in first mo.; } \\
\text { infants exclusively } \\
\text { BF eligible to } \\
\text { receive extra } \\
\text { commercially } \\
\text { prepared fruits } \\
\text { and vegetables }\end{array}$ & $\begin{array}{l}\text { Mothers/infants } \\
\text { not receiving } \\
\text { WIC benefits } \\
\text { (PRAMS and } \\
\text { NIS); or pre- } \\
\text { implementation } \\
\text { of WIC policy } \\
\text { change } \\
\text { (PedNSS) }\end{array}$ & $\begin{array}{l}\text { PRAMS: } \\
\text { <20: } \\
6-17 \\
20-29: \\
61-62 \\
\geq 30: \\
21-31 \\
\\
\text { NIS: } \\
<20: 1-5 \\
20-29: \\
35-54 \\
\geq 30: \\
41-63 \\
\text { PedNSS: } \\
\text { NR }\end{array}$ & $\begin{array}{l}\text { PRAMS } \\
: 56 \\
\text { (WIC); } \\
34 \\
\text { (non- } \\
\text { WIC) } \\
\text { NIS:67 } \\
\text { (WIC } \\
\text { and } \\
\text { non- } \\
\text { WIC) } \\
\text { PedNS } \\
\text { S: NR }\end{array}$ & NR & NR \\
\hline $\begin{array}{l}\text { Lovera, } \\
2010^{88} \\
\text { Medium }\end{array}$ & $\begin{array}{l}\text { NRCT } \\
1 \text { WIC agency in } \\
\text { Texas } \\
200 \text { women }\end{array}$ & $\begin{array}{l}\text { United States } \\
2005-2006\end{array}$ & $\begin{array}{l}\text { Program } \\
\text { participation; } \\
\text { mother had } \\
\text { initiated BF of } \\
\text { the index child; } \\
\text { current WIC } \\
\text { program } \\
\text { participation; } \\
\text { Hispanic; } \geq 18 \\
\text { yrs }\end{array}$ & $\begin{array}{l}\text { Peer Dad } \\
\text { Program (plus } \\
\text { Peer Counseling } \\
\text { Program for } \\
\text { Mothers): } \\
\text { Peer Dad } \\
\text { volunteers acted } \\
\text { as role models } \\
\text { and provided } \\
\text { counseling and } \\
\text { classes pre- and } \\
\text { postnatally for } \\
\text { WIC father }\end{array}$ & $\begin{array}{l}\text { Peer counseling } \\
\text { program for } \\
\text { Mothers only: } \\
\text { Experienced BF } \\
\text { women serve as } \\
\text { role models }\end{array}$ & $\begin{array}{l}\text { NR; \% by } \\
\text { age } \\
\text { group, } \\
\text { Mother: } \\
20-35 \text { yrs: } \\
84-86 \% \\
36-50 \text { yrs: } \\
14-16 \% \\
\text { Father: } \\
20-35 \text { yrs: } \\
73-78 \% \\
36-55 \text { yrs: } \\
22-27 \%\end{array}$ & $\begin{array}{l}\text { NR } \\
(100 \% \\
\text { Hispani } \\
\text { c) }\end{array}$ & $\begin{array}{l}29-30 \\
72-78\end{array}$ & $46-54$ \\
\hline
\end{tabular}




\begin{tabular}{|c|c|c|c|c|c|c|c|c|c|}
\hline $\begin{array}{l}\text { First Author, } \\
\text { Year } \\
\text { ROB }\end{array}$ & $\begin{array}{l}\text { Study Design } \\
\text { N Sites } \\
\text { N Participants }\end{array}$ & $\begin{array}{l}\text { Country } \\
\text { Year(s) of } \\
\text { Enrollment }\end{array}$ & $\begin{array}{l}\text { Inclusion } \\
\text { Criteria }\end{array}$ & $\begin{array}{l}\text { Intervention } \\
\text { Description }\end{array}$ & Comparator(s) & $\begin{array}{l}\text { Age, } \\
\text { Mean } \\
\text { (SD) }\end{array}$ & $\begin{array}{l}\text { \% Non- } \\
\text { white }\end{array}$ & $\begin{array}{l}\% \\
\text { Primiparo } \\
\text { us } \\
\text { \%Previous } \\
\text { ly BF }\end{array}$ & $\begin{array}{l}\% \\
\text { Cesarean } \\
\text { Birth }\end{array}$ \\
\hline $\begin{array}{l}\text { First Author, } \\
\text { Year } \\
\text { ROB }\end{array}$ & $\begin{array}{l}\text { Study Design } \\
\text { N Sites } \\
\text { N Participants }\end{array}$ & $\begin{array}{l}\text { Country } \\
\text { Year(s) of } \\
\text { Enrollment }\end{array}$ & $\begin{array}{l}\text { Inclusion } \\
\text { Criteria }\end{array}$ & $\begin{array}{l}\text { Intervention } \\
\text { Description }\end{array}$ & Comparator(s) & $\begin{array}{l}\text { Age, } \\
\text { Mean } \\
(S D)\end{array}$ & $\begin{array}{l}\% \text { Non- } \\
\text { white }\end{array}$ & $\begin{array}{l}\text { \% } \\
\text { Primiparou } \\
\text { s } \\
\% \\
\text { Previously } \\
\text { BF }\end{array}$ & $\begin{array}{l}\text { \% } \\
\text { Cesarean } \\
\text { Birth }\end{array}$ \\
\hline $\begin{array}{l}\text { Reeder, } \\
2014^{89} \\
\text { Medium }\end{array}$ & $\begin{array}{l}\text { RCT } \\
4 \text { WIC agencies in } \\
\text { Oregon } \\
1,948 \text { women }\end{array}$ & $\begin{array}{l}\text { United States } \\
2005-2007\end{array}$ & $\begin{array}{l}\text { Women } \\
\text { attending a WIC } \\
\text { new pregnancy } \\
\text { appointment } \\
\text { who indicated } \\
\text { an intention to } \\
\text { BF or were } \\
\text { undecided } \\
\text { about BF }\end{array}$ & $\begin{array}{l}\text { Telephone peer } \\
\text { counseling } \\
\text { program: } \\
\text { delivered through } \\
\text { WIC agencies }^{c}\end{array}$ & $\begin{array}{l}\text { Standard WIC } \\
\text { BF } \\
\text { promotion and } \\
\text { support (no } \\
\text { contact with a } \\
\text { peer counselor) }\end{array}$ & 27 & $44-47$ & $\begin{array}{l}\text { NR } \\
\text { NR }\end{array}$ & $26-30$ \\
\hline $\begin{array}{l}\text { Schafer, } \\
1998^{90} \\
\text { High }\end{array}$ & $\begin{array}{l}\text { NRCT } \\
8 \text { counties (lowa) } \\
241 \text { women }\end{array}$ & $\begin{array}{l}\text { United States } \\
1994-1996\end{array}$ & $\begin{array}{l}\text { Rural, low- } \\
\text { income } \\
\text { pregnant and } \\
\text { postpartum } \\
\text { women who } \\
\text { qualified for } \\
\text { WIC }\end{array}$ & $\begin{array}{l}\text { Peer counseling } \\
\text { program: trained } \\
\text { volunteer peer } \\
\text { counselors from } \\
\text { the community } \\
\text { met one-on-one } \\
\text { with women and } \\
\text { presented lessons } \\
\text { on nutrition, BF } \\
\text { and provided } \\
\text { support }\end{array}$ & $\begin{array}{l}\text { Usual care } \\
\text { (6 counties } \\
\text { without peer } \\
\text { counseling } \\
\text { program) }\end{array}$ & $23-25$ & $0-19$ & $\begin{array}{l}\text { NR } \\
\text { NR }\end{array}$ & NR \\
\hline Shaw, 199991 & Prospective cohort & United States & $\begin{array}{l}\text { Pregnant } \\
\text { women who }\end{array}$ & $\begin{array}{l}\text { Peer counseling } \\
\text { program: trained }\end{array}$ & Usual care & $22-23$ & $16-29$ & $37-47$ & $21-24$ \\
\hline Medium & $\begin{array}{l}9 \text { health } \\
\text { departments } \\
\text { (Tennessee) } \\
291 \text { women }\end{array}$ & 1996-1997 & $\begin{array}{l}\text { had registered } \\
\text { antepartum for } \\
\text { WIC at } 1 \text { of } 9 \\
\text { health } \\
\text { departments in } \\
\text { West } \\
\text { Tennessee }\end{array}$ & $\begin{array}{l}\text { peer counselors } \\
\text { held individualized } \\
\text { sessions with } \\
\text { participants based } \\
\text { on need }\end{array}$ & & & & $21-35$ & \\
\hline
\end{tabular}




\begin{tabular}{|c|c|c|c|c|c|c|c|c|c|}
\hline $\begin{array}{l}\text { First Author, } \\
\text { Year } \\
\text { ROB }\end{array}$ & $\begin{array}{l}\text { Study Design } \\
\text { N Sites } \\
\text { N Participants }\end{array}$ & $\begin{array}{l}\text { Country } \\
\text { Year(s) of } \\
\text { Enrollment }\end{array}$ & $\begin{array}{l}\text { Inclusion } \\
\text { Criteria }\end{array}$ & $\begin{array}{l}\text { Intervention } \\
\text { Description }\end{array}$ & $\begin{array}{l}\text { Comparator( } \\
\text { s) }\end{array}$ & $\begin{array}{l}\text { Age, } \\
\text { Mean } \\
\text { (SD) }\end{array}$ & $\begin{array}{l}\text { \% Non- } \\
\text { white }\end{array}$ & $\begin{array}{l}\text { \% } \\
\text { Primiparo } \\
\text { us } \\
\% \\
\text { Previously } \\
\text { BF }\end{array}$ & $\begin{array}{l}\text { \% } \\
\text { Cesarean } \\
\text { Birth }\end{array}$ \\
\hline $\begin{array}{l}\text { Washio, } \\
2017^{93} \\
\text { Medium }\end{array}$ & $\begin{array}{l}\text { RCT } \\
2 \text { WIC offices } \\
\text { (Philadelphia, PA) } \\
36 \text { women }\end{array}$ & $\begin{array}{l}\text { United States } \\
2015-2016\end{array}$ & $\begin{array}{l}\text { Puerto Rican } \\
\text { women enrolled } \\
\text { in WIC who } \\
\text { initiated BF }\end{array}$ & $\begin{array}{l}\text { Cash incentive } \\
\text { contingent on } \\
\text { demonstrating BF } \\
\text { in front of } \\
\text { research staff } \\
\text { ( } \$ 20 \text { at end of } 1 \text { st } \\
\text { mo. and increase } \\
\text { by } \$ 10 \text { every mo. } \\
\text { until end of } 6 \text { mos) } \\
\text { in addition to } \\
\text { usual WIC } \\
\text { services) }\end{array}$ & $\begin{array}{l}\text { Usual WIC } \\
\text { services (on- } \\
\text { site lactation } \\
\text { consultant, } \\
\text { peer } \\
\text { counseling } \\
\text { and support } \\
\text { meetings, } \\
\text { free breast } \\
\text { pump, } \\
\text { enhanced } \\
\text { food } \\
\text { package) }\end{array}$ & $23-24(5)$ & 100 & $\begin{array}{l}39-44 \\
47-50\end{array}$ & NR \\
\hline
\end{tabular}

a Included women with a family income of less than $\$ 50,000$ per year or whose prenatal care was covered by Medicaid. breastfeeding for at least 1 month.

c Low-frequency (4 calls) protocol or high-frequency protocol (8 calls) but analyzed together (see comments).

$\mathrm{BF}$ = breastfeeding; $\mathrm{N}=$ number; NA = not applicable; NIS = National Immunization Survey; NR = not reported; NRCT = nonrandomized controlled trial; PedNSS = Pediatric

Nutrition Surveillance System; PRAMS = Pregnancy Risk Assessment Monitoring System; RCT = randomized controlled trial; ROB = risk of bias; SD = standard deviation; SES

= socioeconomic status; WIC $=$ Special Supplemental Nutrition Program for Women, Infants and Children. 
Table 11. WIC interventions reporting on breastfeeding initiation and duration

\begin{tabular}{|c|c|c|c|c|c|}
\hline $\begin{array}{l}\text { First } \\
\text { Author, } \\
\text { Year } \\
\text { ROB } \\
\end{array}$ & $\begin{array}{l}\text { Intervention } \\
\text { Comparison }\end{array}$ & $\begin{array}{l}\text { Study Design } \\
\text { N Sites } \\
\text { N Participants } \\
\end{array}$ & $\begin{array}{l}\text { Breastfeeding Initiation } \\
\text { Outcome Results }\end{array}$ & $\begin{array}{l}\text { Breastfeeding Duration (Any, } \\
\text { Nonexclusive Plus Exclusive) }\end{array}$ & $\begin{array}{l}\text { Exclusive } \\
\text { Breastfeeding Duration }\end{array}$ \\
\hline $\begin{array}{l}\text { Edmunds, } \\
2017^{92} \\
\text { High }\end{array}$ & $\begin{array}{l}\text { G1: Tailored BF counseling } \\
\text { and support based on } \\
\text { Breastfeeding Attrition } \\
\text { Prediction Tool } \\
\text { G2: Usual WIC BF and } \\
\text { nutritional counseling }\end{array}$ & $\begin{array}{l}\text { Prospective cohort } \\
12 \text { WIC clinics (NY) } \\
826\end{array}$ & NR & NR & $\begin{array}{l}\mathrm{AOR}^{\mathrm{a}}(95 \% \mathrm{Cl}) \text { exclusive } \\
\text { BF at } 7 \text { days: } \\
1.6 \text { (1.1 to } 2.5) ; p<0.05 \\
\text { AOR }(95 \% \mathrm{Cl}) \text { exclusive } \\
\text { BF at } 1 \mathrm{mo}: \\
1.6(1.0 \text { to } 2.5) ; p<0.05 \\
\text { AOR ( } 95 \% \mathrm{Cl}) \text { exclusive } \\
\text { BF at } 2 \text { mos: } \\
1.3(0.8 \text { to } 2.1) ; p=N S\end{array}$ \\
\hline $\begin{array}{l}\text { Hayes, } \\
2008^{86} \\
\text { Medium }\end{array}$ & $\begin{array}{l}\text { G1: Provision of an electric } \\
\text { breast pump (loaned) } \\
\text { G1: Provision of a manual } \\
\text { breast pump (loaned) }\end{array}$ & $\begin{array}{l}\text { RCT } \\
13 \text { WIC sites } \\
\text { (Hawaii) } \\
280 \text { women }\end{array}$ & NR & $\begin{array}{l}\text { \% of women }(\mathrm{N}) \text { who BF for at } \\
\text { least } 6 \text { mos: } \\
\text { G1: } 72.3(94 / 130) \\
\text { G2: } 76.8(76 / 88) \\
\text { Adjusted OR }(95 \% \mathrm{Cl}),{ }^{\text {b }} \text { electric } \\
\text { vs. manual: } \\
0.85 \text { (0.45 to } 1.60) \\
\text { Median duration }(95 \% \mathrm{Cl}) \text { of BF } \\
\text { (mos): } \\
\text { G1: } 12(8 \text { t162) } \\
\text { G2: } 11(9 \text { to } 14) \\
\text { Adjusted HR }(95 \% \mathrm{Cl})^{\mathrm{c}} \\
\text { 1.13 }(0.79 \text { to } 1.50)\end{array}$ & $\mathrm{NR}$ \\
\hline
\end{tabular}




\begin{tabular}{|c|c|c|c|c|c|}
\hline $\begin{array}{l}\text { First } \\
\text { Author, } \\
\text { Year } \\
\text { ROB }\end{array}$ & $\begin{array}{l}\text { Intervention } \\
\text { Comparison }\end{array}$ & $\begin{array}{l}\text { Study Design } \\
\text { N Sites } \\
\text { N Participants } \\
\end{array}$ & $\begin{array}{l}\text { Breastfeeding Initiation } \\
\text { Outcome Results }\end{array}$ & $\begin{array}{l}\text { Breastfeeding Duration (Any, } \\
\text { Nonexclusive Plus Exclusive) }\end{array}$ & $\begin{array}{l}\text { Exclusive } \\
\text { Breastfeeding Duration }\end{array}$ \\
\hline $\begin{array}{l}\text { Joyce, } \\
2015^{87} \\
\text { High }\end{array}$ & $\begin{array}{l}\text { G1: Pre-WIC policy change } \\
\text { G2: Post-WIC policy change }\end{array}$ & $\begin{array}{l}\text { Prospective cohort } \\
\text { NA (3 population } \\
\text { cohorts: PRAMS, } \\
\text { NIS, and PedNSS) } \\
\text { PRAMS (WIC): } \\
85,458 \\
\text { PRAMS (Non-WIC): } \\
42,019 \\
\text { NIS (WIC): } 62,289 \\
\text { NIS (Non-WIC): } \\
11,702 \\
\text { PedNSS (infants): } \\
744\end{array}$ & $\begin{array}{l}\text { Trends in rates of ever } \\
\text { BF } \\
\text { (2008 to 2010), Adjusted } \\
\text { difference }{ }^{d} \text { between WIC } \\
\text { and non-WIC groups: } \\
\text { PRAMS: } \\
1.2 \% \text {; p=NS } \\
\text { NIS: } \\
-1.3 \% \text {; } p=N S \\
\text { PedNSS: pre-post } \\
\text { difference in proportion of } \\
\text { children ever BF after the } \\
\text { new food package } \\
\text { (adjusted for trends in } \\
\text { BF) =-0.1\% }\end{array}$ & $\begin{array}{l}\text { Trends in BF for at least } 4 \text { wks } \\
\text { ( } 2008 \text { to } 2010 \text { ), adjusted } \\
\text { difference between WIC and non- } \\
\text { WIC groups, PRAMS: } 0.3 \% \text {; } \\
\text { p=NS } \\
\text { Trends in BF for at least } 3 \text { mos } \\
\text { (2008 to 2010), adjusted } \\
\text { difference between WIC and non- } \\
\text { WIC groups, NIS: } 0.4 \% \text {; } p=N S \\
\text { PedNSS: Pre-post difference in } \\
\text { proportion of children BF for } \geq 1 \\
\text { mo after the new food package } \\
\text { (adjusted for trends in BF) }=0.5 \%\end{array}$ & NR \\
\hline $\begin{array}{l}\text { Lovera, } \\
2010^{88} \\
\text { Medium }\end{array}$ & $\begin{array}{l}\text { G1: BF peer-support } \\
\text { program for fathers (in } \\
\text { addition to mother peer- } \\
\text { support program) } \\
\text { G2: Mother participation in } \\
\text { peer-support program only }\end{array}$ & $\begin{array}{l}\text { NRCT } \\
1 \text { WIC site (Texas) } \\
200 \text { women }\end{array}$ & $\begin{array}{l}\text { NA (only mothers who } \\
\text { initiated BF were eligible) }\end{array}$ & $\begin{array}{l}\%(\mathrm{~N}) \text { of women who BF for } 6 \\
\text { mos or longer: } \\
\text { G1: } 63.4(64 / 101) \\
\text { G2: } 54.5(54 / 99) \\
p=0.20 \\
\text { OR }(95 \% \mathrm{Cl}) \\
1.44(0.82 \text { to } 2.54)\end{array}$ & $\mathrm{NR}$ \\
\hline $\begin{array}{l}\text { Reeder, } \\
2014^{89} \\
\text { Medium }\end{array}$ & $\begin{array}{l}\text { G1: Telephone peer } \\
\text { counseling program } \\
\text { G2: Usual care }\end{array}$ & $\begin{array}{l}\text { RCT } \\
4 \text { WIC agencies in } \\
\text { Oregon } \\
1948 \text { women }\end{array}$ & $\begin{array}{l}\text { No association between } \\
\text { telephone peer } \\
\text { counseling and BF } \\
\text { initiation rates (per } \\
\text { authors, results not } \\
\text { shown) }\end{array}$ & $\begin{array}{l}\text { Adjusted }^{\mathrm{e}} \mathrm{RR}(95 \% \mathrm{Cl}) \text { of } \\
\text { nonexclusive BF (vs. } \\
\text { nontreatment group): } \\
1 \text { mo: } 1.19 \text { (1.10 to } 1.27) \\
3 \text { mos: } 1.22 \text { (1.10 to } 1.34) \\
6 \text { mos: } 1.18 \text { (1.03 to } 1.34)\end{array}$ & $\begin{array}{l}\text { Adjusted }^{\mathrm{e}} \mathrm{RR}(95 \% \mathrm{Cl}) \text { of } \\
\text { exclusive BF (vs. } \\
\text { nontreatment group): } \\
1 \text { mo: } 1.07 \text { (0.97 to } 1.18) \\
3 \text { mos: } 1.09 \text { ( } 0.95 \text { to } \\
1.24) \\
6 \text { mos: } 1.01 \text { (0.85 to } \\
1.20)\end{array}$ \\
\hline
\end{tabular}




\begin{tabular}{|c|c|c|c|c|c|}
\hline $\begin{array}{l}\text { Author, } \\
\text { Year } \\
\text { ROB }\end{array}$ & $\begin{array}{l}\text { Intervention } \\
\text { Comparison }\end{array}$ & $\begin{array}{l}\text { Study Design } \\
\text { N Sites } \\
\text { N Participants }\end{array}$ & $\begin{array}{l}\text { Breastfeeding Initiation } \\
\text { Outcome Results }\end{array}$ & $\begin{array}{l}\text { Breastfeeding Duration (Any, } \\
\text { Nonexclusive Plus Exclusive) }\end{array}$ & $\begin{array}{l}\text { Exclusive } \\
\text { Breastfeeding Duration }\end{array}$ \\
\hline $\begin{array}{l}\text { Schafer, } \\
1998^{90} \\
\text { High }\end{array}$ & $\begin{array}{l}\text { G1: WIC peer counseling } \\
\text { program } \\
\text { G2: Usual care }\end{array}$ & $\begin{array}{l}\text { NRCT } \\
8 \text { counties } \\
241 \text { women }\end{array}$ & $\begin{array}{l}\text { BF initiation, \% (N/N } \\
\text { total): } \\
\text { G1: } 82(59 / 72) \\
\text { G2: } 31(20 / 64)\end{array}$ & $\begin{array}{l}\text { Average BF duration (wks): } \\
\text { G1: } 5.7 \\
\text { G2: } 2.5 \\
\text { \% of women BF at } 12 \text { wks: } \\
\text { G1: } 43(31 / 72) \\
\text { G2: } 0(0 / 64) \\
\text { p<0.001 }\end{array}$ & NR \\
\hline $\begin{array}{l}\text { Shaw, } \\
\text { 199991 } \\
\text { Medium }\end{array}$ & $\begin{array}{l}\text { G1: WIC peer counseling } \\
\text { program } \\
\text { G2: Usual care }\end{array}$ & $\begin{array}{l}\text { Prospective cohort } \\
9 \text { health } \\
\text { departments } \\
\text { (Tennessee) } \\
291 \text { women }\end{array}$ & $\begin{array}{l}\text { BF initiation (any attempt } \\
\text { to BF), \% (N/N total): } \\
\text { G1: } 53(82 / 156) \\
\text { G2: } 33(45 / 135) \\
\text { p<0.01 } \\
\text { Adjusted OR (95\%): } \\
2.43 \text { (1.23 to } 4.67)\end{array}$ & $\begin{array}{l}\text { Any BF at } \geq 6 \text { wks, } \\
\% \text { (N/N total): } \\
\text { G1: } 26(41 / 156) \\
\text { G2: } 13(18 / 135) \\
\text { p<0.01 } \\
\text { Adjusted OR }(95 \% \mathrm{Cl}): \\
2.78(1.31 \text { to } 5.91)\end{array}$ & NR \\
\hline & & & $\begin{array}{l}\text { BF at hospital discharge, } \\
\% \text { (N/N total): } \\
\text { G1: } 44(69 / 156) \\
\text { G2: } 26(35 / 136) \\
\text { p<0.01 }\end{array}$ & & \\
\hline
\end{tabular}




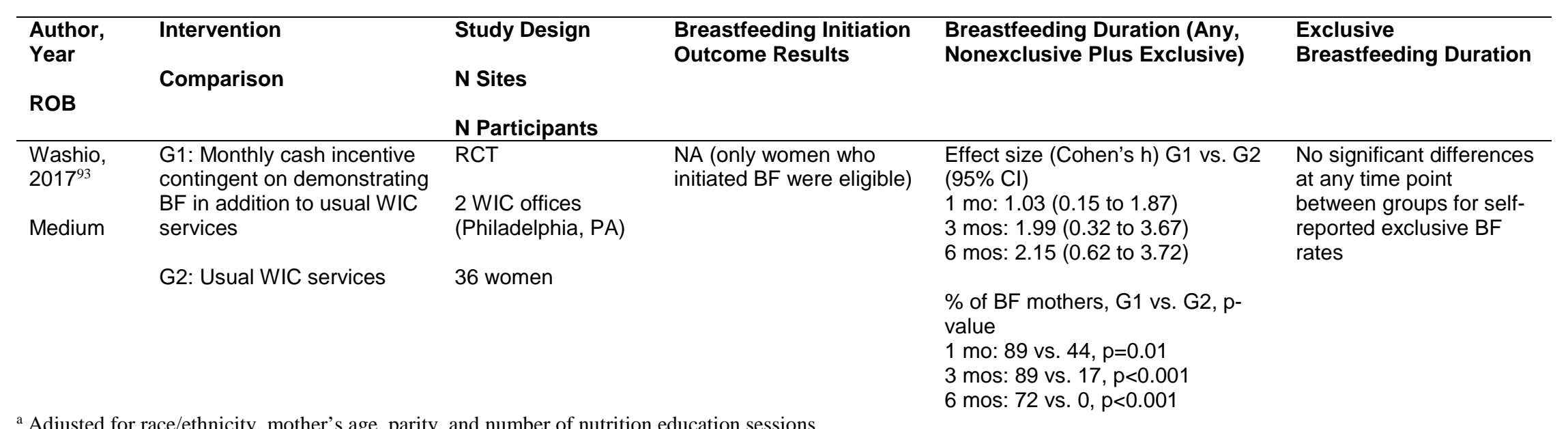

b Adjusted for mother's age, race/ethnicity, education, marital status, and parity.

c Adjusted for education, age, race/ethnicity, marital status, and parity.

d All models include State and year fixed effects. Additional covariates include indicators for age, race/ethnicity, parity, marital status, family income, mother's schooling, and whether prenatal care was paid for by Medicaid.

e Adjusted for age, education, race, language, marital status, month in pregnancy enrolled, family income, cesarean delivery, and LWA.

AOR = adjusted odds ratio; $\mathrm{BF}=$ breastfeeding; $\mathrm{CI}$ = confidence interval; $\mathrm{G}=$ group; $\mathrm{HR}=$ hazard ratio; $\mathrm{LWA}=$ local WIC agency; $\mathrm{N}=$ number; $\mathrm{NA}=$ not applicable; $\mathrm{NIS}=$ National Immunization Survey; NR = not reported; NRCT = nonrandomized controlled trial; NS = not significant; OR = odds ratio; PedNSS = Pediatric Nutrition Surveillance System; PRAMS = Pregnancy Risk Assessment Monitoring System; RCT = randomized controlled trial; ROB = risk of bias; RR = risk ratio; WIC = Special Supplemental Nutrition Program for Women, Infants and Children; wks = weeks. 
Four studies assessed a type of WIC peer-support program: three assessing peer support for mothers found benefit, and one (assessing added peer support for fathers) did not. Of the three studies assessing peer support for mothers, two compared in-person peer support to mothers with usual care. ${ }^{90,91}$ One was an NRCT assessing a volunteer peer counselor program at eight Iowa county WIC agencies; women in the intervention group had a higher rate of breastfeeding initiation than in the controls (82\% vs. 31\%, respectively) and a higher rate of breastfeeding at 12 weeks (43\% vs. $0 \%$; $\mathrm{p}<0.001) .{ }^{90}$ Similarly, the cohort study assessing a WIC peer-support program at nine Tennessee health departments found higher rates of breastfeeding at hospital discharge in the intervention group than in the controls ( $44 \%$ vs. $26 \%$ of women, $\mathrm{p}<0.01$ ) and higher rates of any breastfeeding at 6 weeks postpartum (26\% vs. $13 \%, \mathrm{p}<0.01) .{ }^{91}$ One RCT compared telephone-delivered breastfeeding support among women enrolled at four Oregon WIC agencies with usual care: there was no difference between groups in terms of breastfeeding initiation (per authors, data not provided) ${ }^{89}$ However, in the overall sample, rates of nonexclusive breastfeeding were greater in the intervention than controls at 3 months and 6 months (RR, 1.18; 95\% CI, 1.03 to 1.34$).{ }^{89}$ There was no significant difference in rates of exclusive breastfeeding in the overall sample at 3 and 6 months postpartum. ${ }^{89}$

The NRCT comparing a peer-support program for fathers (in addition to peer support for mothers) with peer support for mothers alone found a slightly higher rate of any breastfeeding at 6 months, but the difference was not statistically significant (63 vs. $55 \%$, respectively; $\mathrm{p}=0.20$ ). ${ }^{88}$

\section{Community-Based Interventions}

\section{Characteristics}

Five included studies (described in seven publications) assessed the effectiveness of a community-based intervention (Table 12). Studies were conducted in diverse country settings including one each in Italy, ${ }^{94}$ Australia, ${ }^{95}$ Mexico, ${ }^{96}$ Chile,,${ }^{97}$ and Canada. ${ }^{98}$ Two were cluster RCTs, ${ }^{95,} 96$ two were NRCTs, ${ }^{94,} 97$ and one was a prospective cohort study. ${ }^{98}$ Two studies enrolled women between 1991 and 1996, ${ }^{96,} 97$ and all others enrolled women after 2005.

In terms of populations, two studies enrolled a majority of primiparous women ${ }^{94,98}$ and three enrolled a minority of primiparous women. Rates of cesarean births ranged from 27 to 40 percent in three studies, and two did not report on the rate of cesarean birth. ${ }^{97,}{ }^{98}$ Most studies enrolled women living in a community in which the intervention was implemented; one Australian study targeted women who were considered at risk of early breastfeeding cessation (e.g., infant received formula in the early postpartum period or mother asked for help with breastfeeding). ${ }^{95}$

No studies assessed the same intervention type; details of intervention and comparators are shown in Table 12. Briefly, one Italian study assessed a community-based policy (Baby Friendly Community Initiative) aimed at promoting breastfeeding in nonhospital-based health and community centers (including workplaces and day care centers). ${ }^{94}$ The Australian cluster RCT evaluated an early home-based breastfeeding intervention in local health authorities with and without access to a community-based breastfeeding drop-in center. ${ }^{95}$ The cluster RCT set in Mexico compared community-based breastfeeding peer support to usual care (peer counselors were trained and supervised by staff of La Leche League of Mexico). ${ }^{96}$ The study set in Chile evaluated an integrated postpartum health care program that featured multiple components, including education, maternal and infant health care, support for the mother, and active participation of women from the community served. ${ }^{97}$ Finally, one prospective cohort study set in Canada compared peer-led breastfeeding education classes with standard nurse-led classes. ${ }^{98}$ 
Table 12. Characteristics of studies assessing community-based interventions

Study Design

Year

ROB

N Sites

N Participants

Year(s) of

Enrollment

Criteria

Description

N Participants

Alvarado, NRCT $\quad$ Chile

\begin{tabular}{lll}
\hline $\begin{array}{l}\text { Alvarado, } \\
1999^{97}\end{array}$ & NRCT & Chile \\
& $\begin{array}{l}\text { 2 neighborhoods with } \\
\text { High }\end{array}$ & 1991-1992 \\
& $\begin{array}{l}\text { extreme poverty in } \\
\text { Santiago, Chile }\end{array}$ &
\end{tabular}

Santiago, Chile

392 women

\begin{tabular}{ll}
\hline $\begin{array}{l}\text { Cattaneo, } \\
2016 ;{ }^{94}\end{array}$ & NRCT \\
$\begin{array}{l}\text { Macaluso, } \\
2013^{99}\end{array}$ & $\begin{array}{l}\text { 18 local health } \\
\text { authorities (9 regions } \\
\text { of Italy) }\end{array}$ \\
Medium &
\end{tabular}

5,094 mother/infant

pairs
Comparator(

s)

ator(

\section{n}

ne

(SD)

\% Non- \% \%

Primiparou Cesarea

s

n Birth

$\%$

Previously

BF

\begin{tabular}{|c|c|}
\hline $\begin{array}{l}\text { Pregnant } \\
\text { women living in } \\
\text { a defined area } \\
\text { close to the } \\
\text { nongovernment } \\
\text { organization } \\
\text { implementing } \\
\text { the intervention }\end{array}$ & $\begin{array}{l}\text { Community-based } \\
\text { integrated } \\
\text { postpartum health } \\
\text { care program }_{\text {focusing on BF }} \\
\text { education and } \\
\text { support, }^{\text {a and }} \\
\text { maternal/infant } \\
\text { health care }\end{array}$ \\
\hline $\begin{array}{l}\text { Mothers giving } \\
\text { birth to a } \\
\text { healthy infant in } \\
\text { hospitals within } \\
\text { eligible local } \\
\text { health } \\
\text { authorities }\end{array}$ & $\begin{array}{l}\text { Early } \\
\text { implementation of } \\
\text { BFCI, a policy } \\
\text { promoting BF in } \\
\text { nonhospital health } \\
\text { facilities, day care } \\
\text { centers, public } \\
\text { locations, and } \\
\text { businesses }\end{array}$ \\
\hline
\end{tabular}

Mothers in a

nearby

neighborhood

with similar

socioeconomi

C

characteristic

$\mathrm{s}$ attending a

public clinic

Later

32

NR; (96-

54-57

plementa

$\mathrm{n}$ of $\mathrm{BFCl}$

(NR)

$67 \%$

Italian)

34-46

NR

NR

2009-2012

businesses 


\begin{tabular}{|c|c|c|c|c|c|c|c|c|c|}
\hline $\begin{array}{l}\text { First Author, } \\
\text { Year } \\
\text { ROB }\end{array}$ & $\begin{array}{l}\text { Study Design } \\
\text { N Sites } \\
\text { N Participants }\end{array}$ & $\begin{array}{l}\text { Country } \\
\text { Year(s) of } \\
\text { Enrollment }\end{array}$ & $\begin{array}{l}\text { Inclusion } \\
\text { Criteria }\end{array}$ & $\begin{array}{l}\text { Intervention } \\
\text { Description }\end{array}$ & $\begin{array}{l}\text { Comparator( } \\
\text { s) }\end{array}$ & $\begin{array}{l}\text { Age, } \\
\text { Mea } \\
n \\
\text { (SD) }\end{array}$ & $\begin{array}{l}\text { \% Non- } \\
\text { white }\end{array}$ & $\begin{array}{l}\text { \% } \\
\text { Primiparou } \\
\text { s } \\
\% \\
\text { Previously } \\
\text { BF }\end{array}$ & $\begin{array}{l}\% \\
\text { Cesarea } \\
\text { n Birth }\end{array}$ \\
\hline $\begin{array}{l}\text { MacLachlan, } \\
2016: 95 \\
\text { Cramer, } \\
2017^{100} \\
\text { Medium }\end{array}$ & $\begin{array}{l}\text { Cluster RCT } \\
10 \text { LGAs, with } 99 \\
\text { maternal child centers } \\
9,675 \text { women }\end{array}$ & $\begin{array}{l}\text { Australia } \\
\text { 2012-2013 }\end{array}$ & $\begin{array}{l}\text { Clusters: LGAs } \\
\text { in Victoria, } \\
\text { Australia, with } \\
>450 \text { births per } \\
\text { year and lower } \\
\text { rates of any BF } \\
\text { at discharge } \\
\text { from hospital } \\
\text { than the Victoria } \\
\text { state average } \\
\text { Mothers: those } \\
\text { BF at } \\
\text { discharge; } \\
\text { mothers } \\
\text { considered at } \\
\text { risk of early BF } \\
\text { cessation were } \\
\text { targeted for } \\
\text { HVs }\end{array}$ & $\begin{array}{l}\text { Early home-based } \\
\text { nursing BF support } \\
\text { for women } \\
\text { identified as at risk } \\
\text { of BF cessation, } \\
\text { with or without } \\
\text { access to a } \\
\text { community-based } \\
\text { BF drop-in center } \\
\text { (offering women } \\
\text { the opportunity to } \\
\text { discuss BF } \\
\text { concerns with } \\
\text { nurse and/or } \\
\text { trained peer } \\
\text { supporter, meet } \\
\text { and learn from } \\
\text { other mothers, and } \\
\text { access other BF } \\
\text { support) }\end{array}$ & $\begin{array}{l}\text { Usual care: } \\
\text { hospital } \\
\text { midwife visit } \\
\text { 1-2 days after } \\
\text { discharge; } \\
\text { home visit 10- } \\
14 \text { days after } \\
\text { birth }\end{array}$ & $\begin{array}{l}31 \\
(N R)\end{array}$ & NR & $\begin{array}{l}\text { 39-41 } \\
\text { NR }\end{array}$ & $32-36$ \\
\hline
\end{tabular}




\begin{tabular}{|c|c|c|c|c|c|c|c|c|c|}
\hline $\begin{array}{l}\text { First Author, } \\
\text { Year } \\
\text { ROB }\end{array}$ & $\begin{array}{l}\text { Study Design } \\
\text { N Sites } \\
\text { N Participants }\end{array}$ & $\begin{array}{l}\text { Country } \\
\text { Year(s) of } \\
\text { Enrollment }\end{array}$ & $\begin{array}{l}\text { Inclusion } \\
\text { Criteria }\end{array}$ & $\begin{array}{l}\text { Intervention } \\
\text { Description }\end{array}$ & $\begin{array}{l}\text { Comparator( } \\
\text { s) }\end{array}$ & $\begin{array}{l}\text { Age, } \\
\text { Mea } \\
n \\
\text { (SD) }\end{array}$ & $\begin{array}{l}\text { \% Non- } \\
\text { white }\end{array}$ & $\begin{array}{l}\text { \% } \\
\text { Primiparou } \\
\text { s } \\
\% \\
\text { Previously } \\
\text { BF }\end{array}$ & $\begin{array}{l}\text { \% } \\
\text { Cesarea } \\
\text { n Birth }\end{array}$ \\
\hline $\begin{array}{l}\text { Morrow, } \\
1999^{96} \\
\text { Low }\end{array}$ & $\begin{array}{l}\text { Cluster RCT } \\
36 \text { clusters } \\
\text { (composed of } 2 \text { to } 4 \\
\text { city blocks) } \\
130 \text { women }\end{array}$ & $\begin{array}{l}\text { Mexico } \\
1995-1996\end{array}$ & $\begin{array}{l}\text { Pregnant } \\
\text { women residing } \\
\text { in the study } \\
\text { area who } \\
\text { agreed to } \\
\text { participate }\end{array}$ & $\begin{array}{l}\text { Community-based } \\
\text { BF peer counselor } \\
\text { support via home } \\
\text { visits ( } 3 \text { or } 6)\end{array}$ & $\begin{array}{l}\text { Standard care } \\
\text { (mothers with } \\
\text { lactation } \\
\text { problems } \\
\text { were referred } \\
\text { to their own } \\
\text { physicians) }\end{array}$ & NR & NR & $\begin{array}{l}21-30 \\
\text { NR }\end{array}$ & $23-40$ \\
\hline
\end{tabular}

\begin{tabular}{|c|c|c|c|c|c|c|c|c|c|}
\hline $\begin{array}{l}\text { Rempel, } \\
2012^{98} \\
\text { High }\end{array}$ & $\begin{array}{l}\text { Prospective cohort } \\
1 \text { hospital } \\
109 \text { women }\end{array}$ & $\begin{array}{l}\text { Canada } \\
\text { 2005-2006 }\end{array}$ & $\begin{array}{l}\text { NR; participants } \\
\text { sampled from } \\
\text { those who had } \\
\text { participated in } \\
\text { classes }\end{array}$ & $\begin{array}{l}\text { Peer-led BF class } \\
\text { ( } 2 \text { hours) providing } \\
\text { education and } \\
\text { support facilitated } \\
\text { by } 2 \text { volunteer } \\
\text { Breast-feeding } \\
\text { Buddies (who BF } \\
\text { for } 6 \text { mos and } \\
\text { completed } 18 \text { hours } \\
\text { of BF training) }\end{array}$ & $\begin{array}{l}\text { Standard } \\
\text { nurse-led } \\
\text { prenatal BF } \\
\text { class }\end{array}$ & $\begin{array}{l}28- \\
31\end{array}$ & NR & $\begin{array}{l}97 \\
2\end{array}$ & NR \\
\hline
\end{tabular}

a Exclusive BF on demand was promoted for the first 6 months postpartum, education focused on the nutritional and health benefits of BF.

${ }^{b}$ Women were considered at risk of early breastfeeding cessation if their infant received any formula (in addition to breast milk) in the early postpartum period and if a woman was distressed about breastfeeding or asked for help with breastfeeding when telephoned.

${ }^{\mathrm{c}}$ Peer counselors were trained and supervised by staff of La Leche League of Mexico and the physician study coordinator (trained in lactation management). The peer-counselor training consisted of 1 week of classes, 2 months in lactation clinics and with mother-to-mother support groups, and 1 day of observation and demonstration by visiting experts. The peer counselors practiced in a neighborhood near the study site for 6 months before the intervention trial, during which the content of messages and problem-solving skills were refined.

$\mathrm{BF}=$ breastfeeding; BFCI = Baby Friendly Community Initiative; HV = home visit; LGA = local governmental agency; $\mathrm{N}=$ number; $\mathrm{NR}=$ not reported; NRCT = nonrandomized controlled trial; RCT = randomized controlled trial; ROB = risk of bias; SD = standard deviation. 


\section{Results}

Results of studies are shown in Table 13. All studies reported on at least one measure of breastfeeding duration, and one also reported on rates of breastfeeding initiation. ${ }^{96}$ The studies generally did not demonstrate benefit, with the exception of one study that reported a benefit at 3 months but not at 6 months.

The study assessing a community-based policy aimed at promoting breastfeeding in nonhospital-based health and community centers ${ }^{94}$ found no differences in outcomes between intervention and control regions at any time point; rates of exclusive breastfeeding at discharge, 3 and 6 months, and of any breastfeeding at 5 and 12 months increased during the study period in both groups. ${ }^{94}$ The cluster RCT assessing an early home-based breastfeeding intervention in local health authorities with and without access to a community-based breastfeeding drop-in center $^{95}$ found no difference between groups in the rate of any breastfeeding at 4 months. ${ }^{95}$ The cluster RCT set in Mexico compared community-based breastfeeding peer support to usual care; ${ }^{96}$ at 3 months, more women in the intervention groups were exclusively breastfeeding than in the controls (67\% of the six-visit group, $50 \%$ of the three-visit group, vs. $12 \%$ of controls; $\mathrm{p}<0.001$ ), and rates of any breastfeeding were significantly longer in intervention groups (combined) than in control groups at 3 months, but not 6 months (Table 13). ${ }^{96}$ The multicomponent integrated postpartum program in Chile found a higher rate of full breastfeeding at 6 months in the intervention group than in the control group (74\% vs. 10\%; p=0.0001). ${ }^{97}$ Finally, in the prospective cohort study set in Canada comparing peer-led breastfeeding education classes with standard nurse-led classes, ${ }^{98}$ there was no difference between groups in rates of any breastfeeding at 1 and 6 months postpartum (Table 13).

\section{Effectiveness and Harms of Breastfeeding Programs and Policies for Subpopulations of Women}

\section{Key Points}

- Few eligible studies reported on subgroups of women.

- Low SOE supports the conclusion that BFHI effectiveness may vary among women who differ by education status; two cohort studies $(27,341)$ found higher rates of breastfeeding initiation among women with lower education ( $\leq 12$ years) at BFHI hospitals compared with control hospitals, but no difference in rates among women with higher education ( $\geq 13$ years).

- We found insufficient evidence to make a conclusion on whether the benefit of WIC peer support interventions varies by subgroups of women based on language spoken (Spanish only vs. English) or whether benefit of tailored breastfeeding counseling intervention varies by race/ethnicity, primarily because of unknown consistency and imprecision. 
Table 13. Results of studies assessing community-based interventions

\begin{tabular}{|c|c|c|c|c|c|}
\hline $\begin{array}{l}\text { First } \\
\text { Author, } \\
\text { Year } \\
\text { ROB }\end{array}$ & $\begin{array}{l}\text { Intervention } \\
\text { Comparison }\end{array}$ & $\begin{array}{l}\text { Study Design } \\
\text { N Sites } \\
\text { N Participants }\end{array}$ & $\begin{array}{l}\text { Breastfeeding } \\
\text { Initiation Outcome } \\
\text { Results }\end{array}$ & $\begin{array}{l}\text { Breastfeeding Duration (Any, } \\
\text { Nonexclusive Plus Exclusive) }\end{array}$ & $\begin{array}{l}\text { Exclusive Breastfeeding } \\
\text { Duration }\end{array}$ \\
\hline $\begin{array}{l}\text { Alvarado, } \\
1999^{97} \\
\text { High }\end{array}$ & $\begin{array}{l}\text { G1: Integrated } \\
\text { postpartum program } \\
\text { (BF education and } \\
\text { support, } \\
\text { maternal/infant } \\
\text { health care) } \\
\text { G2: Usual care at a } \\
\text { public clinic }\end{array}$ & $\begin{array}{l}\text { NRCT } \\
2 \\
\text { neighborhoods } \\
\text { with extreme } \\
\text { poverty in } \\
\text { Santiago, Chile } \\
392 \text { women }\end{array}$ & NR & $\begin{array}{l}\text { \% of women who weaned by } 6 \text { mos } \\
\text { postpartum: } \\
\text { G1: } 7 \\
\text { G2: } 50 \\
\text { p }=0.0001\end{array}$ & $\begin{array}{l}\text { \% of women exclusively }{ }^{a} \text { BF at } \\
6 \text { mos: } \\
\text { G1: } 74 \\
\text { G2: } 10 \\
p=0.0001\end{array}$ \\
\hline $\begin{array}{l}\text { Cattaneo, } \\
2016^{94} \\
\text { Macaluso, } \\
2013^{99} \\
\text { Medium }\end{array}$ & $\begin{array}{l}\text { G1: Early } \\
\text { implementation of } \\
\mathrm{BFCl} \\
\text { G2: Later } \\
\text { implementation of } \\
\mathrm{BFCl}\end{array}$ & $\begin{array}{l}\text { NRCT } \\
18 \text { local health } \\
\text { authorities ( } 9 \\
\text { regions of Italy) } \\
5,094 \text { mother/ } \\
\text { infant pairs }\end{array}$ & NR & $\begin{array}{l}\text { Any BF } 6 \text { mos, \% (data collected on } 3 \\
\text { separate cohorts of women): } \\
\text { G1: } \\
\text { Round 1: } 67.6 \\
\text { Round 2: } 66.7 \\
\text { Round 3: } 69.0 \\
\text { G2: } \\
\text { Round 1: } 62.4 \\
\text { Round 2: } 63.4 \\
\text { Round 3: } 65.4 \\
\text { Any BF, } 12 \text { mos, \%: } \\
\text { G1: } \\
\text { Round 1: } 32.4 \\
\text { Round 2: } 34.9 \\
\text { Round 3: } 36.2 \\
\text { G2: } \\
\text { Round 1: } 26.6 \\
\text { Round 2: } 30.8 \\
\text { Round 3: } 34.9\end{array}$ & $\begin{array}{l}\text { Exclusive BF } 3 \text { mos (24-hour } \\
\text { recall), \% (data collected on } 3 \\
\text { separate cohorts of women): } \\
\text { G1: } \\
\text { Round 1: } 58.1 \\
\text { Round 2: } 57.5 \\
\text { Round 3: } 62.3 \\
\text { G2: } \\
\text { Round 1: } 52.8 \\
\text { Round 2: } 53.6 \\
\text { Round 3: } 57.9 \\
\text { 6 mos (24-hour recall): } \\
\text { G1: } \\
\text { Round 1: } 9.0 \\
\text { Round 2: } 7.7 \\
\text { Round 3: } 7.6 \\
\text { G2: } \\
\text { Round 1: } 7.1 \\
\text { Round 2: } 8.4 \\
\text { Round 3: } 9.6\end{array}$ \\
\hline
\end{tabular}




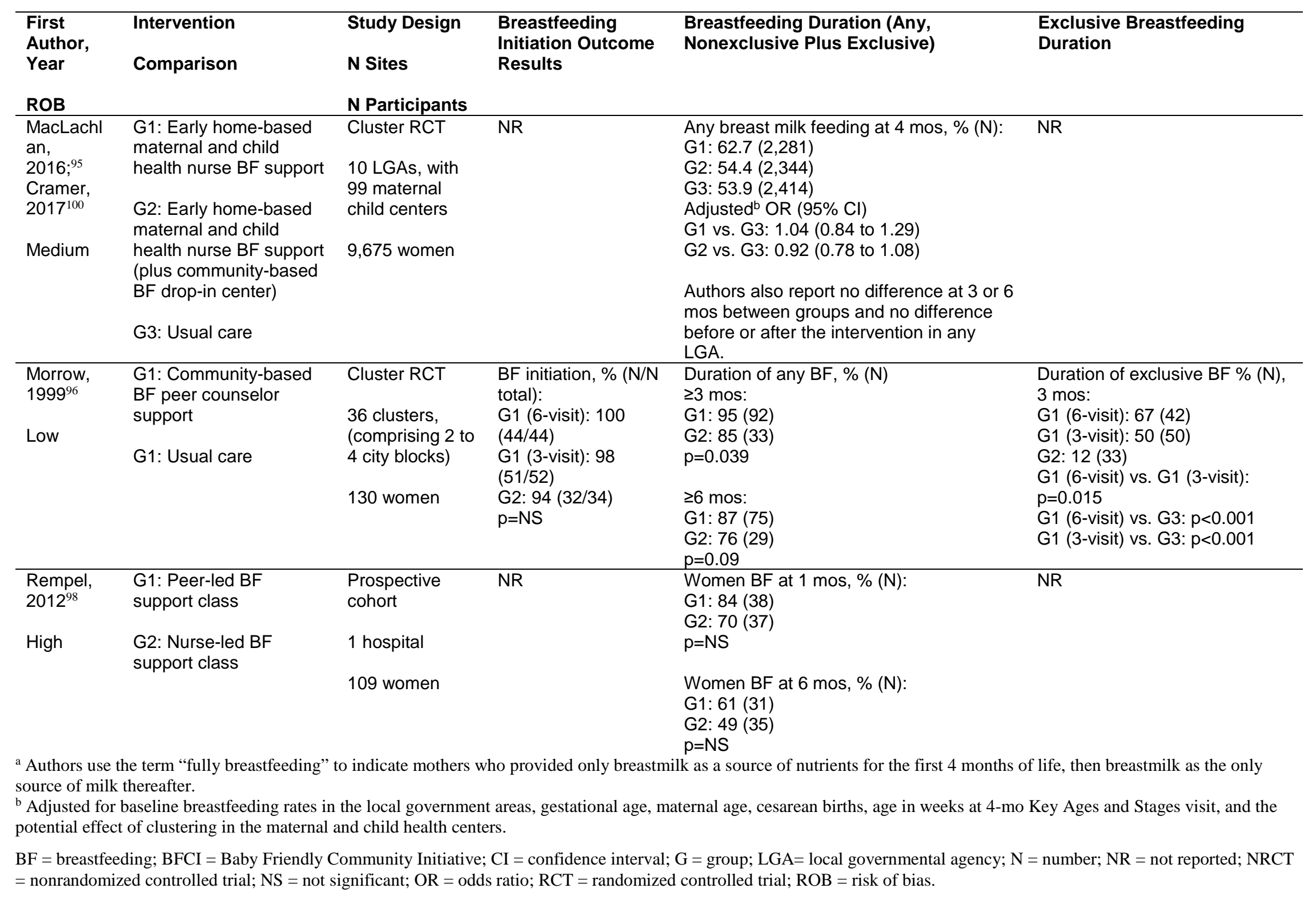




\section{Characteristics and Results}

Few eligible studies reported on subgroups of women; we found no studies reporting on subgroups defined by maternal age, comorbidity, and other factors. Of the four studies reporting on subgroups of women, two focused on BFHI and reported on differences by education status. ${ }^{54}$,

55 The other two focused on a WIC intervention; one peer-support intervention reported on subgroups by language spoken (Spanish only versus English), ${ }^{89}$ and one counseling intervention reported outcomes by race/ethnicity. ${ }^{92}$

The two studies assessing BFHI (by the same author) focused on hospitals in the United States enrolling women from similar time periods (1999-2009); one of these evaluated data from five U.S. States ${ }^{54}$ and the other focused on hospitals in one State (Maine). ${ }^{55}$ Characteristics are described above in KQ 1a and shown in Table 4. Neither study found a significant difference in breastfeeding initiation rates between facilities that received BFHI accreditation and nonaccredited facilities in the overall sample, but both offer some evidence of differences in effectiveness by education level, with benefits accruing to those with lower levels of education. ${ }^{54,55}$ Both studies found higher rates of breastfeeding initiation among women with lower education ( $\leq 12$ years) at BFHI facilities compared with nonaccredited facilities, but no difference in rates among women with higher education ( $\geq 13$ years). ${ }^{54,}{ }^{55}$ For breastfeeding duration, the study enrolling women from five U.S. States found increased rates of exclusive breastfeeding for 4 or more weeks among women with lower education in the intervention arm when compared with the control arm ( $\mathrm{p}=0.02)$, but no difference for women with higher education (Table 14); no difference was seen for rates of any breastfeeding by education status. ${ }^{54}$ The study evaluating women giving birth in Maine hospitals found no difference in rates of exclusive or any breastfeeding at 4 or more weeks postpartum among women who differed by education status. ${ }^{55}$

Two studies assessing a WIC intervention reported on subgroups relevant to race/ethnicity. ${ }^{89 \text {, }}$ 92 One RCT compared telephone peer counseling with standard care at four WIC agencies in Oregon; in the overall sample, the intervention group had significantly higher rates of any breastfeeding at 3 and 6 months than controls. In subgroups of women based on language (English speaking and Spanish speaking only), results were significant at 3 months for both groups, but for Spanish speaking only at 6 months (Table 14). There was no significant difference in rates of exclusive breastfeeding in the overall sample or by language at 3 or 6 months postpartum. ${ }^{89}$ The second WIC intervention reporting on subgroups was a prospective cohort study evaluating a tailored breastfeeding counseling and support intervention (based on responses to the BAPT tool). ${ }^{92}$ Women participating in the intervention had higher rates of exclusive breastfeeding at 1 week and 1 month postpartum than women who declined to participate; however, the benefit was significant only in black and Hispanic mothers at 1 month (but not white mothers). ${ }^{92}$

\section{Effect of Intervention Characteristics on Breastfeeding Outcomes}

This KQ focused on the extent to which intervention-related characteristics (e.g., type of breast pump provided-manual or electric, delivery personnel) influence the initiation, duration, and exclusivity of breastfeeding. We had insufficient evidence to address this KQ. As noted above (KQ 1a), few studies assessed similar interventions among similar groups of women (or settings). 
Table 14. KQ 1 studies reporting on eligible subgroups

\begin{tabular}{|c|c|c|c|c|}
\hline $\begin{array}{l}\text { First Author, } \\
\text { Year } \\
\text { ROB }\end{array}$ & $\begin{array}{l}\text { Intervention } \\
\text { Comparison }\end{array}$ & $\begin{array}{l}\text { Study Design } \\
\text { N Sites } \\
\text { N Participants }\end{array}$ & Breastfeeding Initiation & Breastfeeding Duration \\
\hline $\begin{array}{l}\text { Hawkins, } \\
2014^{54} \\
\text { Medium }\end{array}$ & $\begin{array}{l}\text { G1: Mothers } \\
\text { giving birth in a } \\
\text { BFHI-accredited } \\
\text { facility } \\
\text { G2: Mothers } \\
\text { giving birth in a } \\
\text { nonaccredited } \\
\text { facility }\end{array}$ & $\begin{array}{l}\text { Prospective cohort } \\
32 \text { birth facilities in } 5 \\
\text { U.S. States } \\
25,327 \text { women }\end{array}$ & $\begin{array}{l}\text { BF initiation increased by } 3.8 \% \text { among mothers } \\
\text { with less education }(\leq 12 \text { yrs) who delivered in } \\
\text { BFHI facilities }(p=0.05) \text {, but not among mothers } \\
\text { with more education ( } \geq 13 \text { yrs) }(p=0.9)\end{array}$ & $\begin{array}{l}\text { BF initiation increased exclusive BF for } \geq 4 \text { wks by } \\
4.5 \%(p=0.02) \text { among mothers with lower education } \\
\text { who delivered in BFHI facilities ( } p=0.1) \\
\text { No significant difference seen in subgroups based on } \\
\text { education for rates of any BF for } \geq 4 \text { wks }\end{array}$ \\
\hline $\begin{array}{l}\text { Hawkins, } \\
2014^{55} \\
\text { Medium }\end{array}$ & $\begin{array}{l}\text { G1: Mothers } \\
\text { giving birth in a } \\
\text { BFHI-accredited } \\
\text { facility } \\
\text { G2: Mothers } \\
\text { giving birth in a } \\
\text { nonaccredited } \\
\text { facility }\end{array}$ & $\begin{array}{l}\text { Prospective cohort } \\
10 \text { birth facilities in } 1 \\
\text { U.S. State (Maine) } \\
2,014 \text { women }\end{array}$ & $\begin{array}{l}\text { Among mothers with less education ( } \leq 12 \mathrm{yrs} \text { ), the } \\
\mathrm{BFHI} \text { increased } \mathrm{BF} \text { initiation by } 8.6 \% \text { (adjusted } \\
\text { coefficient, } 0.086 ; 95 \% \mathrm{Cl}, 0.01 \text { to } 0.16) \\
\text { No significant change in } \mathrm{BF} \text { initiation rates for } \\
\text { mothers with more education ( } \geq 13 \mathrm{yrs} \text { ); } \mathrm{p}=0.9\end{array}$ & $\begin{array}{l}\text { No significant change in exclusive BF for } \geq 4 \text { wks } \\
\text { among mothers with less education }(\leq 12 \text { yrs; } p=0.5 \text { ) } \\
\text { or more education ( } \geq 13 \text { yrs; } p=0.3 \text { ) } \\
\text { No significant change in any BF for } \geq 4 \text { wks among } \\
\text { mothers with less education ( } \leq 12 \text { yrs; } p=0.5 \text { ) or more } \\
\text { education ( } \geq 13 \text { yrs; } p=0.3 \text { ) }\end{array}$ \\
\hline $\begin{array}{l}\text { Edmunds, } \\
2017^{92} \\
\text { High }\end{array}$ & $\begin{array}{l}\text { G1: Tailored BF } \\
\text { counseling and } \\
\text { support based } \\
\text { on Breastfeeding } \\
\text { Attrition } \\
\text { Prediction Tool } \\
\text { G2: Usual WIC } \\
\text { BF and } \\
\text { nutritional } \\
\text { counseling }\end{array}$ & $\begin{array}{l}\text { Prospective cohort } \\
12 \text { WIC clinics (NY) } \\
826\end{array}$ & NR & $\begin{array}{l}\text { Results shown in figure only. } \\
\text { White women: No significant difference in exclusive } \\
\text { BF at } 7,30 \text {, or } 60 \text { days between groups. } \\
\text { Black women: No significant difference at } 7 \text { days; } \\
\text { higher rate of exclusive BF among intervention group } \\
\text { than control at } 30 \text { and } 60 \text { days ( } p=0.01 \text { and } p=0.02 \text {, } \\
\text { respectively) } \\
\text { Hispanic women: No significant difference at } 7 \text { days; } \\
\text { higher rate of exclusive BF among intervention group } \\
\text { than control at } 30 \text { and } 60 \text { days ( } p=0.4 \text { and } p=0.002 \text {, } \\
\text { respectively) }\end{array}$ \\
\hline
\end{tabular}




\begin{tabular}{|c|c|c|c|c|}
\hline $\begin{array}{l}\text { First Author, } \\
\text { Year } \\
\text { ROB }\end{array}$ & $\begin{array}{l}\text { Intervention } \\
\text { Comparison }\end{array}$ & $\begin{array}{l}\text { Study Design } \\
\text { N Sites } \\
\text { N Participants }\end{array}$ & Breastfeeding Initiation & Breastfeeding Duration \\
\hline $\begin{array}{l}\text { Reeder, } \\
2014^{89} \\
\text { Medium }\end{array}$ & $\begin{array}{l}\text { G1: Telephone } \\
\text { peer counseling } \\
\text { program } \\
\text { G2: Usual care }\end{array}$ & $\begin{array}{l}\text { RCT } \\
4 \text { WIC agencies in } \\
\text { Oregon } \\
1,948 \text { women }\end{array}$ & NR & $\begin{array}{l}\text { Adjusted }{ }^{a} \mathrm{RR}(95 \% \mathrm{Cl}) \text { of nonexclusive BF (vs. } \\
\text { nontreatment group): } \\
\text { Spanish only subgroup: } \\
1 \text { mo: } 1.16 \text { (1.06 to } 1.28) \\
3 \text { mos: } 1.29 \text { (1.13 to } 1.48) \\
6 \text { mos: } 1.29 \text { (1.10 to } 1.51) \\
\text { English only subgroup: } \\
1 \text { mo: } 1.22 \text { (1.09 to } 1.36) \\
3 \text { mos: } 1.19 \text { (1.02 to } 1.38) \\
6 \text { mos: } 1.10 \text { (0.88 to } 1.37) \\
\text { Adjusted }{ }^{\text {a }} \text { R (95\% Cl) of exclusive BF (vs. } \\
\text { nontreatment group): } \\
\text { Spanish-only subgroup: } \\
1 \text { mo: } 1.13 \text { (1.00 to } 1.29) \\
3 \text { mos: } 1.20 \text { (1.02 to } 142) \\
6 \text { mos: } 1.17 \text { (0.94 to } 1.44) \\
\text { English-only subgroup: } \\
1 \text { mo: } 1.04 \text { (0.90 to } 1.19) \\
3 \text { mos: } 1.02 \text { (0.84 to } 1.24) \\
6 \text { mos: } 0.91 \text { (0.70 to } 1.19)\end{array}$ \\
\hline
\end{tabular}

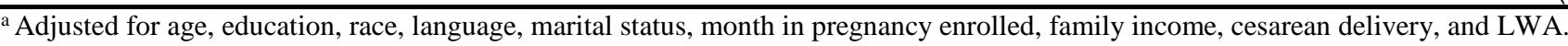

$\mathrm{BF}$ = breastfeeding; BFHI = Baby-Friendly Hospital Initiative; $\mathrm{CI}=$ confidence interval; $\mathrm{G}=$ group; $\mathrm{LWA}=$ local WIC agency; $\mathrm{N}=$ number; $\mathrm{NRCT}=$ nonrandomized controlled trial; NY = New York; ROB = risk of bias; RR = risk ratio; U.K. = United Kingdom; WIC = Special Supplemental Nutrition Program for Women, Infants and Children; wks = weeks. 


\section{Maternal Health Outcomes Associated With Breastfeeding}

\section{Key Points}

- Based on evidence from one systematic review (98 studies) and 19 individual studies (256,891 women), we concluded that ever breastfeeding, as well as longer durations of breastfeeding, is associated with lower rates of overall breast cancer (low SOE). Similarly, consistent evidence from one systematic review (11 studies) and 7 individual studies supports the association between ever breastfeeding and longer duration of breastfeeding and lower rates of breast cancer defined by two hormone receptor subtypes: luminal and triple-negative (low SOE).

- We rated the evidence as insufficient (primarily due to unknown consistency and imprecision) for the association between breastfeeding and subtypes of breast cancer defined by tumor behavior (i.e., in situ breast cancer), subgroups of women who are BRCA1/2 mutation carriers, HER2 hormone receptor subtype, and mortality due to breast cancer.

- Based on evidence from one systematic review (41 studies) and 9 individual studies (42,611 women), we concluded that ever breastfeeding, as well as longer durations of breastfeeding, is associated with reduced risk of developing epithelial ovarian cancer (moderate SOE).

- Based on consistent evidence from five cohort studies (441,989 women), we concluded that a longer duration of breastfeeding is associated with reduced risk of hypertension (low SOE). We found insufficient evidence for an association between breastfeeding and cardiovascular disease (CVD) because few studies assessed the same outcome among similar cohorts of women.

- Based on one systematic review (6 studies) and five individual studies (325,815 women) with consistent findings, we concluded that a longer duration of lifetime breastfeeding is associated with a reduced risk of developing type 2 diabetes (low SOE).

- Eleven studies (101,726 women) reported on the association between breastfeeding and hip, vertebral, and forearm fracture risk. Apart from one study of self-reported vertebral fractures (rated high ROB), no study reported a statistically significant association between breastfeeding and fracture. We rated the evidence as low for no association.

- For postpartum depression, we found insufficient evidence on whether breastfeeding is associated with postpartum depression. Studies were heterogeneous in design and varied in how breastfeeding exposure and depression outcomes were assessed and categorized; studies do not establish the direction of relationship between breastfeeding and depression.

- We rated the SOE on the association between breastfeeding and postpartum weight change as insufficient; results from 16 cohort studies are inconsistent and studies used heterogeneous measures of both exposure and outcomes limiting the ability to compare findings. 


\section{Breast Cancer and Ovarian Cancer}

\section{Background}

Breast cancer is the most frequently diagnosed cancer among women, with an estimated 266,120 new diagnoses in 2018; an estimated 40,920 women will die as a result of breast cancer in 2018, making it the second most deadly cancer diagnosis among women behind lung cancer. ${ }^{101}$ Ovarian cancer is substantially rarer than breast cancer, with an estimated 22,240 new diagnoses in 2018 but is the fifth most deadly cancer among women (estimated 14,070 deaths). ${ }^{101}$ Family history of breast or ovarian cancer, increased age, obesity, smoking, and postmenopausal hormone use are known or suspected risk factors of both breast and ovarian cancer. Reproductive factors that shorten a women's menstrual history, such as oral contraceptive use and parity, are associated with a decreased risk of both breast and ovarian cancer. Because breastfeeding is also associated with a shortened menstrual history, we examined the association between it and breast and ovarian cancer. ${ }^{102}$

\section{Methods}

We included systematic reviews published within the past 5 years that were rated low or unclear ROB. For breast cancer, we additionally included the three systematic reviews that were included in the 2007 report. $^{2}$ We (1) compared multiple systematic reviews to identify the extent of overlapping studies and homogeneity of results, (2) summarized all included reviews in tables, and (3) described the most recent or comprehensive systematic review in detail and considered it in the SOE assessment. We also included and summarize eligible primary studies not captured in the recent systematic reviews. In terms of outcomes, we included studies reporting on overall breast or ovarian cancer, as well as subtypes of cancer, defined by molecular or histopathologic features. Additionally, we included studies enrolling populations with BRCA1 and BRCA2 mutation carriers who are at increased risk of both breast and ovarian cancer.

\section{Breast Cancer: Systematic Reviews and Meta-Analyses}

\section{Overall Breast Cancer}

We included one recent systematic review on the relationship between breastfeeding and breast cancer. ${ }^{103}$ In addition, we briefly describe three other systematic reviews included in the 2007 AHRQ report ${ }^{2}$ since the overlap of included studies (described below) is not complete; the reviews were originally published in $2000^{104,105}$ and $2002^{106}$ These four reviews are summarized in Table $15 .{ }^{106}$

The recent systematic review (98 studies), rated unclear ROB, ${ }^{1}$ examined the association between ever breastfeeding, as well as duration of breastfeeding, and subsequent (overall) breast carcinoma; 73 percent of the studies were conducted in high-income countries (Table 15, Appendix Tables C-13 to C-18). The authors did not perform a formal ROB assessment of included articles but did assess 67 percent of the studies as adequate (i.e., had none or one of selection, measurement, or confounding bias or attrition of 20 percent as assessed by the review authors). They used random effects meta-analysis to estimate pooled ORs; when heterogeneity was high $\left(\mathrm{I}^{2}>60 \%\right.$ or $\mathrm{p}$ for heterogeneity $\left.<0.010\right)$, reasons for heterogeneity were explored with subgroup analyses and meta-regression for the main comparison of ever versus never breastfed. 
Table 15. Breastfeeding and overall breast cancer: Summary of published systematic reviews and meta-analyses

\begin{tabular}{|c|c|c|c|c|}
\hline $\begin{array}{l}\text { Author, Year } \\
\text { ROB }\end{array}$ & $\begin{array}{l}\text { Number of Studies } \\
\text { (Number of } \\
\text { Participants; } \\
\text { Number of Cases) } \\
\text { Included Study } \\
\text { Designs }\end{array}$ & $\begin{array}{l}\text { Overlap With } \\
\text { Other Published } \\
\text { SRs }\end{array}$ & $\begin{array}{l}\text { Search } \\
\text { Dates } \\
\text { Databases }\end{array}$ & Results \\
\hline $\begin{array}{l}\text { Bernier, } \\
2000^{104}\end{array}$ & $\begin{array}{l}23 \text { studies }(70,871 ; \\
25,871)\end{array}$ & $\begin{array}{l}\text { Included in the } 2007 \\
\text { AHRQ report } \\
19 \text { of } 23 \text { studies } \\
\text { included in } \\
\text { Chowdhury, } 2015^{1}\end{array}$ & $\begin{array}{l}\text { 1980-1998 } \\
\text { Medline, } \\
\text { Embase }\end{array}$ & $\begin{array}{l}\text { Total BF duration (mos) vs. never BF } \\
<6: \text { OR, } 1.00(95 \% \mathrm{Cl}, 0.86 \text { to } 1.16) \\
\text { 6-<12: OR, } 0.97(95 \% \mathrm{Cl}, 0.86 \text { to } 1.09) \\
\geq 12 \text { : OR, } 0.72(95 \% \mathrm{Cl}, 0.65 \text { to } 0.80)\end{array}$ \\
\hline $\begin{array}{l}\text { Chowdhury, } \\
2015^{1} \\
\text { Unclear }\end{array}$ & $\begin{array}{l}98 \text { studies (NR) } \\
\text { Cohort, case-control }\end{array}$ & $\begin{array}{l}34 \text { (35 articles) of } \\
98 \text { studies were } \\
\text { previously included } \\
\text { in at least one of the } \\
\text { SRs included in the } \\
2007 \text { AHRQ report }{ }^{2}\end{array}$ & $\begin{array}{l}\text { Inception } \\
\text { through } \\
\text { February } \\
2015 \\
\text { PubMed, } \\
\text { Cochrane, } \\
\text { CABI }\end{array}$ & $\begin{array}{l}\text { Ever vs. never } \mathrm{BF} \\
\text { OR, } 0.78(95 \% \mathrm{Cl}, 0.74 \text { to } 0.82), \mathrm{I}^{2}=72, \\
\mathrm{k}=98 \\
\text { Total } \mathrm{BF} \text { duration (mos) vs. never } \mathrm{BF} \\
\text { <6: OR, } 0.93(95 \% \mathrm{Cl}, 0.88 \text { to } 0.99), \mathrm{I}^{2}=59 \text {, } \\
\text { 39 studies } \\
6-12 \text { : OR, } 0.91(95 \% \mathrm{Cl}, 0.87 \text { to } 0.96), \\
\mathrm{I}^{2}=23,36 \text { studies } \\
>12: \text { OR, } 0.74(95 \% \mathrm{Cl}, 0.69 \text { to } 0.79), \\
\mathrm{I}^{2}=62,50 \text { studies }\end{array}$ \\
\hline $\begin{array}{l}\text { Collaborative } \\
\text { Group on } \\
\text { Hormonal } \\
\text { Factors in } \\
\text { Breast } \\
\text { Cancer, } \\
2002^{106} \\
\\
\text { Low }^{\text {a }}\end{array}$ & $\begin{array}{l}47 \text { studies }(147,275 ; \\
50,302) \\
\text { Cohort, case-control }\end{array}$ & $\begin{array}{l}\text { Included in the } 2007 \\
\text { AHRQ report }{ }^{2} \\
16 \text { ( } 17 \text { studies) of } \\
47 \text { studies included } \\
\text { in Chowdhury, } \\
2015^{1}\end{array}$ & $\begin{array}{l}1983-2001 \\
N A^{b}\end{array}$ & $\begin{array}{l}\text { Ever vs. never BF } \\
\text { RR=0.96 ( } 95 \% \text { SE: } 0.2), p=0.04 \\
\text { Reduction in relative risk of breast cancer } \\
\text { per } 12 \text { mos BF } \\
\text { 4.3\% ( } 99 \% \text { SE: } 0.8) \\
\text { Lifetime duration of BF (mos) vs. never BF } \\
\leq 6: \text { RR }=0.98 \text { ( } 99 \% \text { SE: } 0.017) \\
\text { 7-18: RR=0.94 (95\% SE: } 0.016) \\
\text { 19-30: RR=0.89 (95\% SE: } 0.025) \\
\text { 31-54: RR=0.88 (95\% SE: } 0.033) \\
\geq 55: R R=0.73(95 \% \text { SE: } 0.049)\end{array}$ \\
\hline $\begin{array}{l}\text { Lipworth, } \\
2000^{105} \\
\text { Medium }^{\mathrm{a}}\end{array}$ & $\begin{array}{l}27 \text { studies }(57,109 ; \\
19,482) \\
\text { Case-control }\end{array}$ & $\begin{array}{l}\text { Included in the } 2007 \\
\text { AHRQ report } \\
23 \text { of } 27 \text { studies } \\
\text { included in } \\
\text { Chowdhury, } 2015^{1}\end{array}$ & $\begin{array}{l}\text { 1966-1998 } \\
\text { Medline }\end{array}$ & $\begin{array}{l}\text { No details or quantitative results were } \\
\text { reported. Authors provided only a } \\
\text { qualitative synthesis: "The evidence with } \\
\text { respect to 'ever' breast-feeding remains } \\
\text { inconclusive, with results indicating either } \\
\text { no association or a rather weak protective } \\
\text { effect against breast cancer." } \\
\\
\text { "An inverse association between } \\
\text { increasing cumulative duration of } \\
\text { breastfeeding and breast cancer risk } \\
\text { among parous women has been reported } \\
\text { in some, but not all, studies." }\end{array}$ \\
\hline
\end{tabular}

${ }^{\text {a }}$ Risk of bias rating carried forward from the 2007 AHRQ report ${ }^{2}$

bAuthors pooled 45 studies published between 1983 and 2001 and two unpublished studies in a collaborative analysis. Authors did not describe how studies were identified and included in the collaborative analysis.

AHRQ = Agency for Healthcare Research and Quality; BF = breastfeeding; CABI = Centre for Agriculture and Biosciences International database; $\mathrm{CI}=$ confidence interval; $\mathrm{NA}$ = not applicable; $\mathrm{NR}$ = not reported; $\mathrm{OR}$ = odds ratio; ROB = risk of bias; $\mathrm{RR}=$ relative risk; $\mathrm{SE}=$ standard error; $\mathrm{SR}=$ systematic review. 
The authors found evidence of publication bias, as evidenced by (1) an asymmetrical funnel plot, (2) significant Egger's test $(\mathrm{p}<0.001)$, and (3) significant Begg's test $(\mathrm{p}<0.001)$; authors did not describe the potential impact of the publication bias.

Using data from all 98 included studies, the authors reported a pooled OR for ever versus never breastfeeding of 0.78 (95\% CI, 0.74 to 0.82 ). The substantial heterogeneity $\left(\mathrm{I}^{2}=72\right)$ of these results could not be completely explained in subgroup analyses. The reviewers found statistically significantly different results when studies were categorized according to number of participants (meta-regression p-value $=0.009$ ) and method of adjustment for parity (metaregression p-value $=0.037$ ); they continued, however, to find substantial residual heterogeneity within those subgroups ( $\mathrm{I}^{2}$ ranged from 23 to 77). Among 52 studies that included at least 1,500 participants, the pooled OR was 0.83 (95\% CI, 0.80 to $\left.0.88 ; \mathrm{I}^{2}=72\right)$; the association was stronger among 31 studies of 500 to 1,499 participants and 15 studies of fewer than 500 participants, but the CIs were wider (pooled OR, 0.74; 95\% CI, 0.66 to 0.83 ; $\mathrm{I}^{2}=67$ and pooled OR, 0.50; 95\% CI, 0.37 to $0.66 ; \mathrm{I}^{2}=59$, respectively). Among 60 studies that did not adjust for parity, the pooled OR was 0.73 (95\% CI, 0.68 to $\left.0.79 ; \mathrm{I}^{2}=77\right)$; the pooled ORs were attenuated among 19 studies that crudely adjusted for parity $\left(0.86\right.$; $95 \% \mathrm{CI}, 0.81$ to $\left.0.90 ; \mathrm{I}^{2}=23\right)$ and 19 studies that finely adjusted for parity (0.92; $95 \% \mathrm{CI}, 0.88$ to 0.96 ; $\left.\mathrm{I}^{2}=55\right)$.

Authors also conducted pooled analyses to evaluate the association between different total durations of breastfeeding (compared with women who never breastfed) and breast cancer among a subset of studies with duration data. They reported a numeric decrease in the pooled ORs with increasing duration of breastfeeding. For total breastfeeding duration of less than 6 months, the pooled OR was 0.93 (95\% CI, 0.88 to 0.99; $\left.\mathrm{I}^{2}=59\right)$; the pooled ORs for 6 to 12 months and greater than 12 months, respectively, were 0.91 (95\% CI, 0.87 to $0.96 ; \mathrm{I}^{2}=23$ ) and 0.74 (95\% CI, 0.69 to $0.79 ; \mathrm{I}^{2}=62$ ).

As described above, the 2007 AHRQ report ${ }^{2}$ included 2 systematic reviews ${ }^{104}$, 105 and 1 metaanalysis from the Collaborative Group on Hormonal Factors in Breast Cancer (CGHFBC) ${ }^{106}$ (Table 15). We found a substantial overlap of included studies (i.e., 80\%) among the 2 systematic reviews and the recent systematic review by Chowdhury et al. ${ }^{1}$ We found, however, less overlap between the CGHFBC meta-analysis of 47 studies ( 2 unpublished) ${ }^{106}$ and the recent meta-analysis; ${ }^{1}$ only 16 studies (17 articles) were included in both. ${ }^{1}$ Regardless of overlap (or lack thereof), all systematic reviews and meta-analyses included in the 2007 AHRQ report ${ }^{2}$ reported a nonsignificant reduced risk of breast cancer among women who had ever breastfed. The authors also reported varying degrees of an inverse association between increasing duration of breastfeeding and breast cancer risk. The results are generally consistent in direction and magnitude with pooled results reported by Chowdhury et al. ${ }^{1}$

\section{Tumor Subtypes of Breast Cancer Defined by Hormone Receptor Status}

One recent systematic review rated unclear ROB examined the association between ever breastfeeding and subsequent tumor subtypes of breast cancer, defined by hormone receptor status (Appendix Tables C-13 to C-18). ${ }^{107}$ They defined the breast cancer subtypes as (1) luminal (HR+ and HER2+/-), (2) HER2 (HR- and HER2+), or (3) triple negative (HR- and HER2-).

All 11 included studies, except for 2 case-control studies from China and Japan, were conducted in the United States; the studies were published between 2008 and 2014. The pooled OR for the association between ever breastfeeding and luminal breast cancer from 9 studies (169,870 women) was 0.77 (95\% CI, 0.66 to 0.88 ; $\left.\mathrm{I}^{2}=79\right)$. The pooled OR for the HER2 and triple-negative subtypes were 0.78 (95\% CI, 0.59 to 1.03; $\mathrm{I}^{2}=46$; 8 studies of 14,266 women) and 
0.79 (95\% CI, 0.6 to $0.94 ; \mathrm{I}^{2}=65 ; 11$ studies of 176,340 women), respectively. The authors reported that ORs were lower among case-control than among cohort or population-based casecontrol studies; there were only 2 case-control studies and 1 cohort study in the subgroup analysis compared to 6 population-based case-control studies.

\section{Breast Cancer: Individual Studies}

\section{Overall Breast Cancer}

\section{Characteristics}

Three cohort studies ${ }^{108-110}$ and 16 case-control studies, ${ }^{111-126}$ published since the 2007 AHRQ report $^{2}$ and not included in the recent systematic review by Chowdhury et al. ${ }^{1}$ evaluated the association between ever breastfeeding or duration of breastfeeding and overall breast cancer rates (Table 16, Table 17, Appendix Tables C-19 to C-23 and F1). One medium-rated ROB cohort study was conducted in Japan and compared mixed feeding (breastfeeding and formula feeding) and formula-only feeding with exclusive breastfeeding. ${ }^{109}$ Over 26,000 of these women in the Ohsaki National Health Insurance Cohort Study were followed for 11 years; 148 incident breast cancer cases were identified. ${ }^{109}$ Another medium-rated ROB cohort study combined the hormone trial and the observational study of the Women's Health Initiative (WHI) into one cohort for analysis; 24,095 women in the hormone trial and 45,263 women in the observational study were followed for a median duration of 7.9 years. The WHI evaluated the association between both ever breastfed and duration of breastfeeding and breast cancer, and it presented results for each group of women, defined by which WHI study they participated in and which hormone therapy group they were members of. ${ }^{108}$ Finally, the Nurses' Health cohort Study (NHS), also rated medium risk of bias, identified 8,421 cases of incident invasive breast cancer over 2,424,778 person-years; the authors did not evaluate duration of breastfeeding in their analyses. ${ }^{110}$ We rated eight of the case-control studies as medium ROB; the studies were conducted in the United States, ${ }^{113,}$ 119, 121, 122, 124, 125 Spain, ${ }^{114}$ Sweden, ${ }^{117}$ and Poland. ${ }^{123}$ The remaining case-control studies were rated high ROB and were conducted in the United Kingdom, ${ }^{124}$ Saudi Arabia, ${ }^{111,}{ }^{112}$ Germany, ${ }^{116,}{ }^{126}$ Greece, ${ }^{115}$ Italy, ${ }^{120}$ and Poland. ${ }^{118,}{ }^{124}$ For the most part, ever breastfeeding was not defined further with the exception of one study that described it as $\geq 1$ month of breastfeeding. ${ }^{113}$ In analyses that examined duration, never breastfed was the referent group, with a few exceptions. Some studies used durations up to 3 months ${ }^{124}$ and 12 months $^{112}$ as the referent group; one study in Spain actually grouped women into two categories where women reported whether they met a 2007 recommendation (from the World Cancer Research Fund and the American Institute of Cancer Research) of cumulatively breastfeeding for at least 6 months. ${ }^{114}$ In the Swedish study by Holm and colleagues, the referent group for the duration analysis was nulliparous women, which is slightly different from a referent group of parous women who have never breastfed. ${ }^{117}$ One medium ROB case-control study in the United States evaluated the risk of histologic subtypes of breast cancer that included ductal, lobular, and mixed ductal/lobular breast cancers; histologic subtype was determined by centralized pathology review for a vast majority of the cases. ${ }^{113}$ Finally, another medium ROB case-control study in the United States evaluated the risk of breast cancer among BRCA mutation carriers and noncarriers; the BRCA genes of only the cases were sequenced. ${ }^{119}$ 


\section{Results}

The WHI ${ }^{125}$ and $\mathrm{NHS}^{110}$ cohort studies reported no statistically significant association between ever breastfeeding and breast cancer, ${ }^{125}$ but in the Ohsaki National Health Insurance Cohort Study in Japan, investigators reported that women who only formula-fed their children had an 80 percent increased risk of breast cancer compared with women who exclusively breastfed their children (HR, 1.80; 95\% CI, 1.14 to 2.86). The hazard was numerically increased but not statistically significant, for women who both breast- and formula-fed their children. ${ }^{109}$ Beaber et al. ${ }^{13}$ reported a statistically significant decrease in risk of ductal breast cancer (OR, 0.7 ; $95 \%$ CI, 0.5 to 0.9 ) but not lobular or mixed ductal/lobular breast cancers, and two other studies found varying magnitudes in the statistically significant decrease of odds of breast cancer with ever breastfeeding. ${ }^{122,127}$ The Italian case-control study reported an 82 percent increased risk of breast cancer when comparing women who never breastfed with women who had ${ }^{120}$ and the Swedish case-control study reported a 59 percent increased risk for the same comparison. ${ }^{117}$ $\mathrm{T}^{128}$ wo other studies reported a higher prevalence of breastfeeding among controls than cases, but results were not significant. There was one study in Germany in which more cases than controls reported ever breastfeeding. ${ }^{116}$ Finally, Lee and colleagues reported that the prevalence of breastfeeding among controls and cases who were BRCA mutation carriers was 88 percent; the prevalence of breastfeeding among cases who were not BRCA carriers was 79 percent (Table 16, Appendix Table F1). ${ }^{119}$

Although the direction of effect is generally consistent with pooled results from systematic reviews, seven of 14 studies reported at least one significant inverse association between an increased duration of breastfeeding and breast cancer risk (Table 17, Appendix F1); however, confidence intervals were often wide and overlapping. Not all studies provided results from regression analyses and referent groups varied among the studies. Women in a small case-control study in Saudi Arabia were 44 percent less likely to develop breast cancer if they breastfed for $\geq 12$ months (compared with breastfeeding for $<12$ months), but results were unadjusted for any potential confounders ( $\mathrm{OR}=0.56$; $95 \% \mathrm{CI}, 0.35$ to 0.88$).{ }^{112}$ Two other case-control studies did report a significant trend between decreased risk of breast cancer and increased duration ${ }^{121}$ and with $\geq 12$ months of breastfeeding (but not 4 to 11 months). ${ }^{124}$ In the German study where more cases reported breastfeeding, multiple linear regression indicated that women with breast cancer had a significantly longer duration of breastfeeding, but specific details were not provided. ${ }^{116}$ Among cases who were not $B R C A$ carriers, there was a significant trend $(\mathrm{p}=0.002)$ of decreased breast cancer risk associated with increased duration of breastfeeding; there was no trend of decreased breast cancer risk with increased breastfeeding duration among cases who were $B R C A$ carriers $(\mathrm{p}=0.83) .{ }^{119}$ 
Table 16. Ever breastfeeding and overall breast cancer

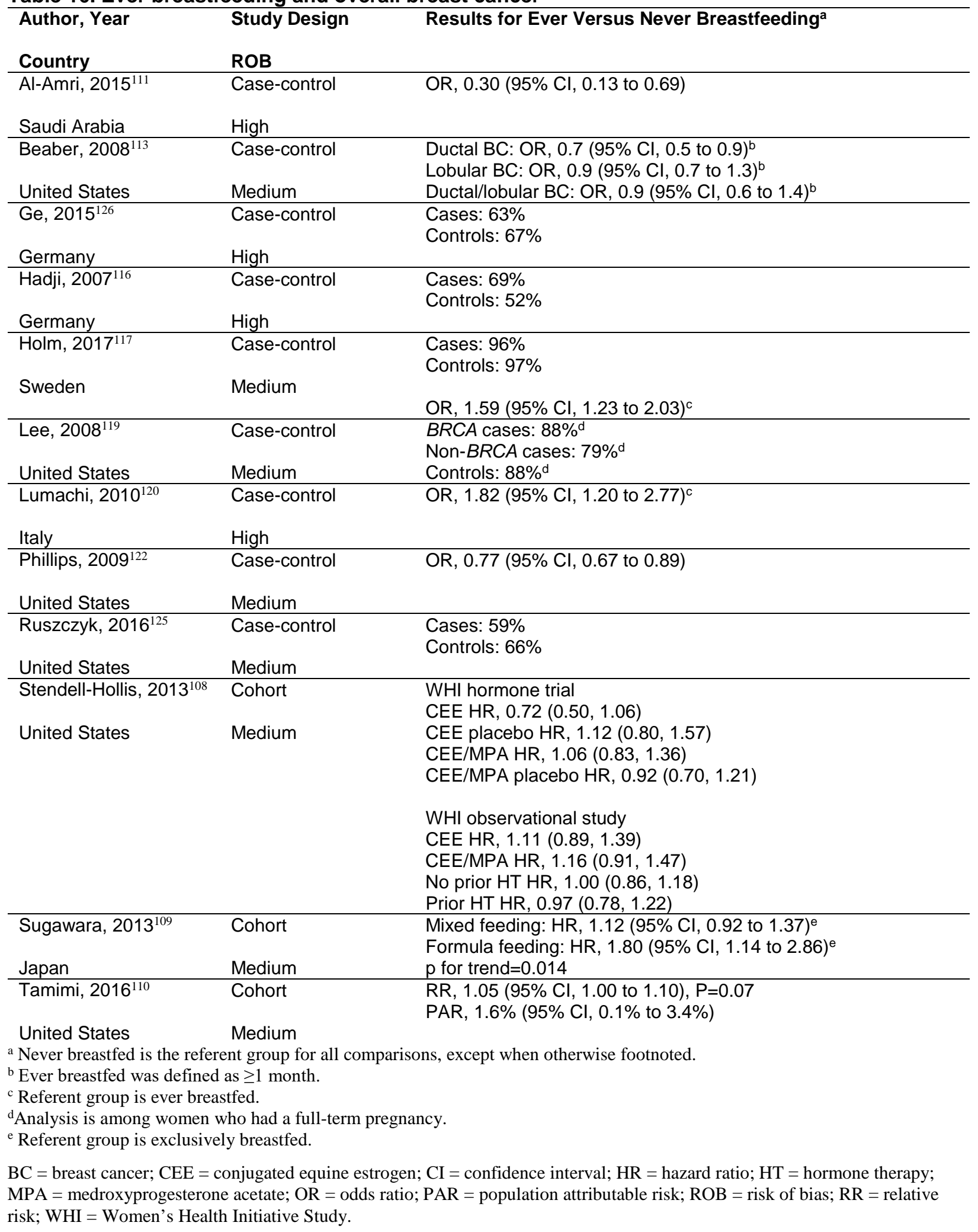


Table 17. Duration of breastfeeding and breast cancer

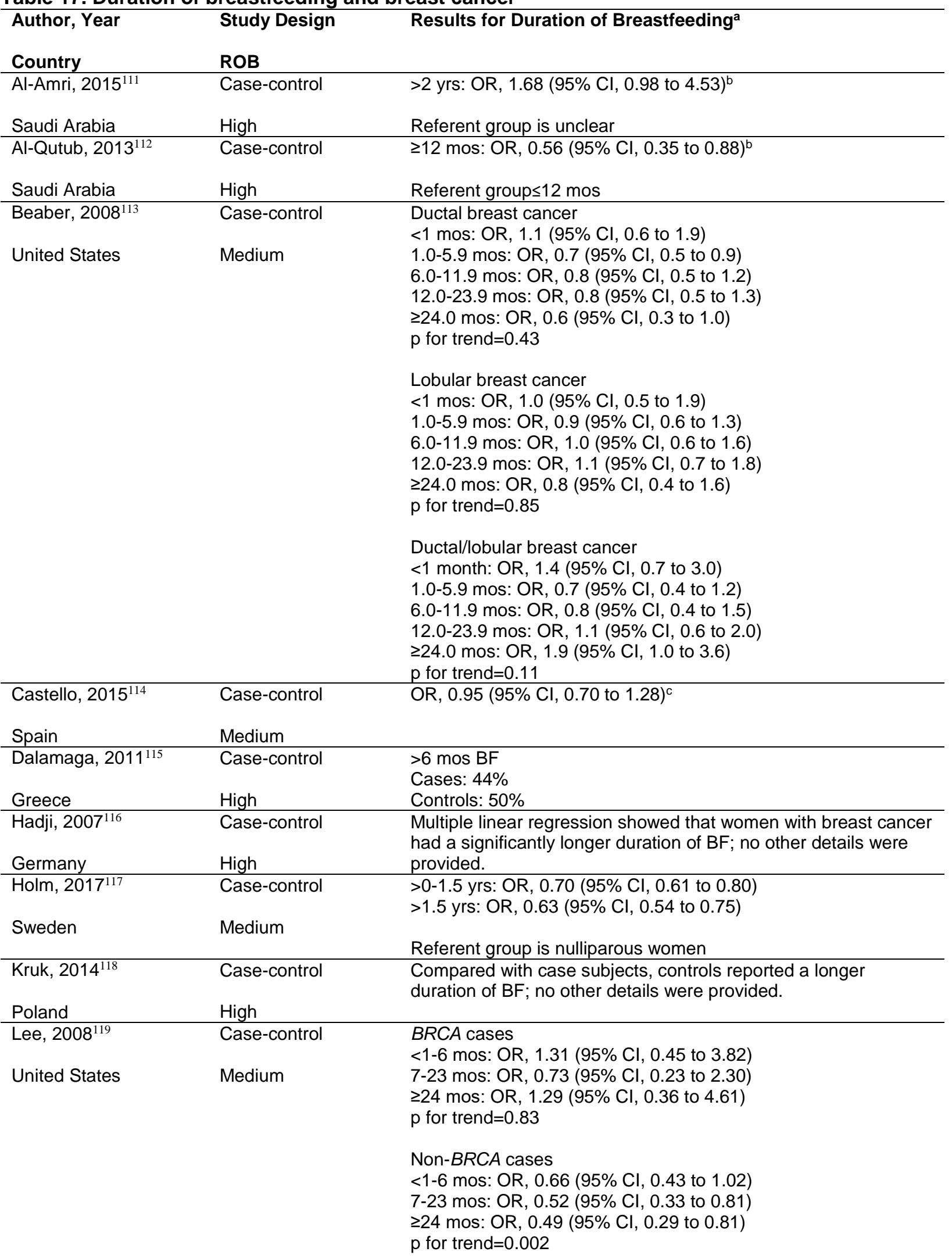




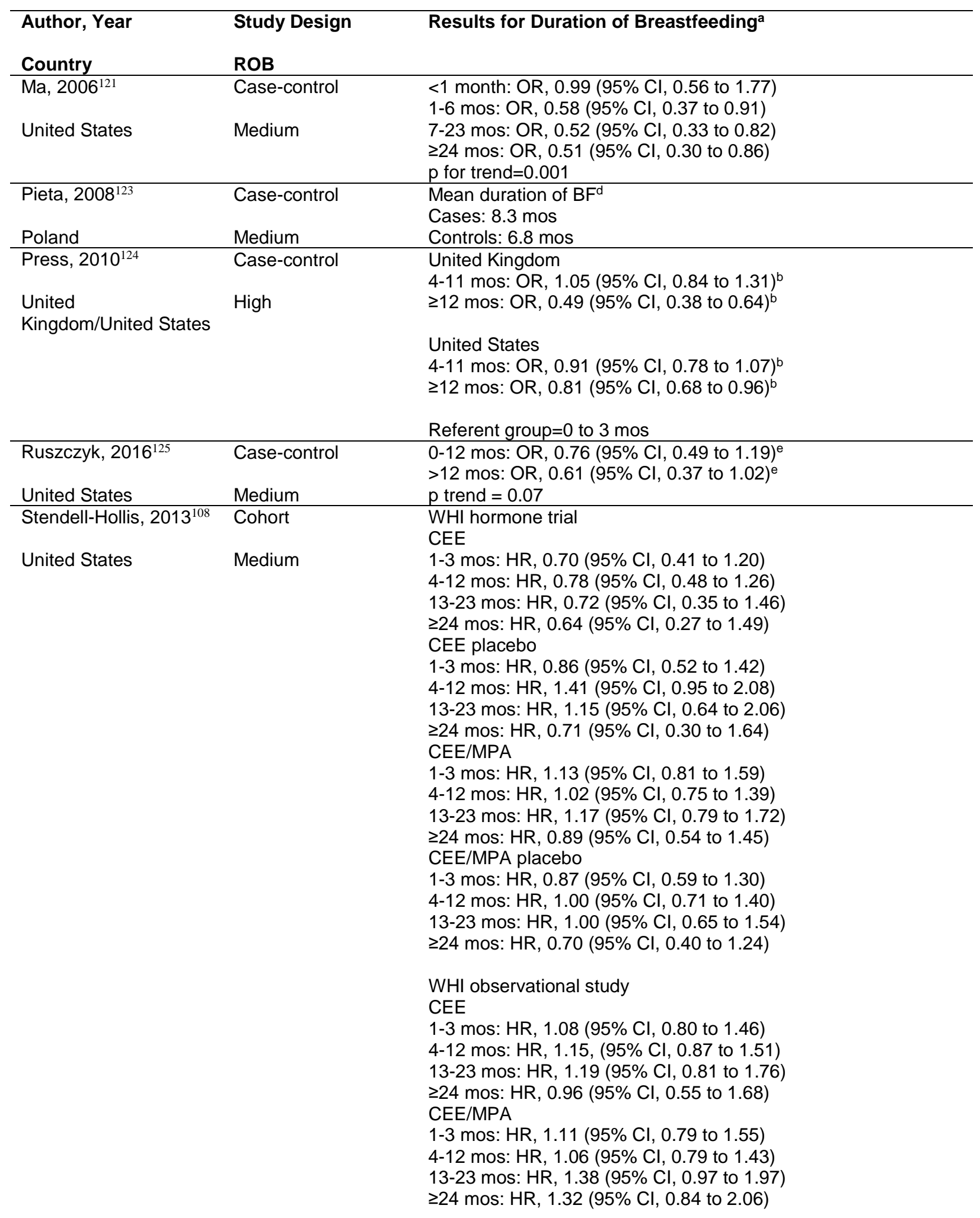


respectively. Among 714 BRCA2 carrier pairs, the ORs were 1.03, 1.04, 1.33, and 1.02 for the same duration categories, respectively; all 95 percent CIs included the null.

\section{Breast Cancer In Situ}

\section{Characteristics}

We included one cohort and two population-based case-controls studies, all rated medium ROB, that evaluated the association between breastfeeding and incident ductal carcinoma in situ (DCIS) $)^{122,132}$ or incident breast cancer defined by a mix of invasive ductal carcinoma and DCIS $^{125}$ confirmed by pathology (Table 18, Appendix Tables C-19 to C-23 and F1). The cohort study included 64,060 women originally enrolled in the WHI clinical trials that were followed for a median of 12 years. ${ }^{132}$ All studies were conducted in the United States, and the percentage of nonwhite participants ranged from 17 percent $^{132}$ to 63 percent. ${ }^{125}$ The Women's Circle of Health Study (WCHS) was a multicenter case-control study of breast cancer in 2,270 AfricanAmerican and European American women in metropolitan New York City and seven counties in New Jersey; the high percentage of African-American women in the study (56\% of cases and $63 \%$ of controls) was reported to be representative of the general community regarding education, income, marital status, and obesity status. ${ }^{125}$

Table 18. Breastfeeding and breast cancer in situ

\begin{tabular}{|c|c|c|c|c|}
\hline $\begin{array}{l}\text { Author, Year } \\
\text { Study } \\
\text { Design } \\
\text { ROB }\end{array}$ & $\begin{array}{l}\text { Description of Study }(\mathrm{N}) \\
\text { Description of Breast } \\
\text { Cancer Cases }(\mathrm{N})\end{array}$ & $\begin{array}{l}\text { Population } \\
\text { Characteristics }\end{array}$ & Results $^{a}$ & $\begin{array}{l}\text { Confounders } \\
\text { Adjusted for }\end{array}$ \\
\hline $\begin{array}{l}\text { Kabat, } \\
2011^{132} \\
\text { Cohort } \\
\text { Medium }\end{array}$ & $\begin{array}{l}\text { Cohort of women, } 50 \text { to } 79 \\
\text { years of age, originally } \\
\text { randomized in the WHI } \\
\text { clinical trial and followed for } \\
\text { a median of } 12 \text { yrs }(64,060) \\
\text { Incident diagnosis of DCIS, } \\
\text { verified by centralized } \\
\text { review of medical records } \\
\text { and pathology reports }(664)\end{array}$ & $\begin{array}{l}\text { Mean age (yrs) } \\
\text { Cases: } 62 \\
\text { Noncases: } 63 \\
\text { Nonwhite } \\
\text { Cases: } 17 \% \\
\text { Noncases: } 18 \% \\
\text { Parous } \\
\text { Cases: } 89 \% \\
\text { Noncases: } 89 \%\end{array}$ & $\begin{array}{l}\text { Ever BF } \\
\text { Cases: } 54 \% \\
\text { Noncases: } 52 \% \\
\text { Duration of } \mathrm{BF} \\
\text { 1-6 mos: } \mathrm{HR}, 1.05(95 \% \mathrm{Cl} \text {, } \\
0.86 \text { to } 1.28) \\
7-12 \text { mos: } \mathrm{HR}, 1.04(95 \% \\
\mathrm{Cl}, 0.80 \text { to } 1.36) \\
>12 \text { mos: } \mathrm{HR}, 1.01(95 \% \mathrm{Cl} \text {, } \\
0.80 \text { to } 1.29)\end{array}$ & $\begin{array}{l}\text { Age, } \\
\text { education, } \\
\text { hormone } \\
\text { therapy, family } \\
\text { history of } \\
\text { breast cancer, } \\
\text { history of } \\
\text { breast biopsy, } \\
\text { mammograms } \\
\text { in past } 2 \text { yrs, } \\
\text { age at } \\
\text { menarche, age } \\
\text { at menopause, } \\
\text { and parity }\end{array}$ \\
\hline $\begin{array}{l}\text { Phillips, } \\
2009^{122} \\
\text { Case-control } \\
\text { Medium }\end{array}$ & $\begin{array}{l}\text { Population-based case- } \\
\text { control study; cases were } \\
\text { rapidly ascertained and } \\
\text { controls were frequency } \\
\text { matched to cases on race } \\
\text { and age (904) } \\
\text { First diagnosis of DCIS, } \\
\text { pathology confirmed (446) }\end{array}$ & $\begin{array}{l}\text { Mean age (yrs) } \\
\text { Cases: } 55 \\
\text { Controls: } 55 \\
\text { Nonwhite } \\
\text { Cases: } 22 \% \\
\text { Controls: } 15 \% \\
\text { Parous } \\
\text { Cases: } 85 \% \\
\text { Controls: } 88 \%\end{array}$ & $\begin{array}{l}\text { Ever BF } \\
\text { DCIS } \\
\text { OR, } 1.02(95 \% \mathrm{Cl}, 0.78 \text { to } \\
1.34) \\
\text { High-grade DCIS } \\
\text { OR, } 0.82 \text { ( } 95 \% \mathrm{Cl}, 0.57 \text { to } \\
1.20) \\
\text { Medium/low-grade DCIS } \\
\text { OR, } 1.02(95 \% \mathrm{Cl}, 0.72 \text { to } \\
1.42)\end{array}$ & $\begin{array}{l}\text { Age, race, and } \\
\text { frequency } \\
\text { matching offset } \\
\text { terms }\end{array}$ \\
\hline
\end{tabular}




\begin{tabular}{|c|c|c|c|c|}
\hline $\begin{array}{l}\text { Author, Year } \\
\text { Study } \\
\text { Design } \\
\text { ROB }\end{array}$ & $\begin{array}{l}\text { Description of Study (N) } \\
\text { Description of Breast } \\
\text { Cancer Cases }(\mathrm{N})\end{array}$ & $\begin{array}{l}\text { Population } \\
\text { Characteristics }\end{array}$ & Results $^{a}$ & $\begin{array}{l}\text { Confounders } \\
\text { Adjusted for }\end{array}$ \\
\hline $\begin{array}{l}\text { a Compared to ne } \\
\text { b Among parous } \\
\text { c Study enrolled } 1 \\
\text { mixed cases are } \mathrm{r}\end{array}$ & $\begin{array}{l}\text { Population-based case- } \\
\text { control study of African- } \\
\text { American and white women, } \\
20 \text { to } 75 \text { yrs; cases were } \\
\text { rapidly ascertained through } \\
\text { hospitals and cancer } \\
\text { registries and controls were } \\
\text { frequency-matched to cases } \\
\text { by telephone prefixes } \\
(2,270)^{c} \\
\text { Histologically confirmed } \\
\text { incident mixed breast } \\
\text { cancer, defined by a mix of } \\
\text { IDC and DCIS (650) } \\
\text { breastfed, unless otherwise spe } \\
\text { men only. } \\
20 \text { controls, } 181 \text { pure IDC cases, } \\
\text { orted here. }\end{array}$ & $\begin{array}{l}\text { Mean age (yrs) } \\
\text { Cases: } 51 \\
\text { Controls: } 50 \\
\text { Nonwhite } \\
\text { Cases: } 56 \% \\
\text { Controls: } 63 \% \\
\text { Parous } \\
\text { Cases: } 77 \% \\
\text { Controls: } 78 \%\end{array}$ & $\begin{array}{l}\text { Ever BF } \\
\text { IDC/DCIS cases: } 67 \% \\
\text { Controls: } 66 \% \\
\text { Duration of BF } \\
\text { >0-12 mos: OR, } 1.15(95 \% \\
\text { Cl, } 0.88 \text { to } 1.50)^{\mathrm{b}} \\
>12 \text { mos: OR, } 0.94(95 \% \mathrm{Cl} \text {, } \\
0.70 \text { to } 1.27)^{\mathrm{b}}\end{array}$ & $\begin{array}{l}\text { Age, race, } \\
\text { birthplace, } \\
\text { family history, } \\
\text { composite } \\
\text { screening } \\
\text { score, } \\
\text { education, OC } \\
\text { use, age at } \\
\text { menarche, } \\
\text { parity, age at } \\
\text { first birth, and } \\
\text { menopausal } \\
\text { status }\end{array}$ \\
\hline
\end{tabular}

\section{Tumor Subtypes of Breast Cancer Defined by Hormone Receptor Status}

\section{Characteristics}

We included three medium ROB cohort studies ${ }^{110,133,134}$ and four case-control studies (three rated medium $\mathrm{ROB}^{117,121,135}$ and one rated high $\mathrm{ROB},{ }^{136}$ ) that evaluated the association between breastfeeding and breast cancer subtypes defined by hormone receptor status (i.e., estrogen receptor [ER], progesterone receptor [PR], and human epidermal growth factor receptor [HER2]) (Table 19, Appendix Tables C-19 to C-23 and F1). One study pooled data from two cohort studies [Black Women's Health Study (BWHS) and Nurses' Health Study II (NHSII)] and evaluated the association between duration of breastfeeding ( $<6$ months and $\geq 6$ months) and ER+ breast cancer; there were 140,194 women, with 1,506 confirmed-by-pathology ER+ breast cancer cases from over 1.5 million person-years of followup. ${ }^{134}$ The European Prospective Investigation into Cancer and Nutrition (EPIC) study followed women from 10 western European countries for a median of 11 years and evaluated the association between ever breastfeeding and duration of breastfeeding with ER+/PR+ and ER-/PR- breast cancer. ${ }^{133}$ The NHS, described above in the overall breast cancer section, determined the estrogen receptor status for 6,646 cases (79\% of the cases identified over 2.4 million person-years) and compared ever to never breastfeeding; a majority of the cases (81\%) were positive for the estrogen receptor. ${ }^{110}$ 
Table 19. Breastfeeding and tumor subtypes of breast cancer defined by hormone receptor status

\begin{tabular}{|c|c|c|c|c|}
\hline $\begin{array}{l}\text { Author, } \\
\text { Year } \\
\text { Study } \\
\text { Design } \\
\text { ROB }\end{array}$ & $\begin{array}{l}\text { Description of } \\
\text { Study (N) } \\
\text { Description of } \\
\text { Breast Cancer } \\
\text { Cases (N) }\end{array}$ & $\begin{array}{l}\text { Population } \\
\text { Characteristics }\end{array}$ & Results: Evera or Duration & $\begin{array}{l}\text { Confounders } \\
\text { Adjusted for }\end{array}$ \\
\hline $\begin{array}{l}\text { Atkinson, } \\
2016^{136} \\
\text { Case- } \\
\text { control } \\
\text { High }\end{array}$ & $\begin{array}{l}\text { Hospital-based } \\
\text { case-control study } \\
\text { in Texas cancer } \\
\text { center; controls } \\
\text { were undergoing } \\
\text { routine } \\
\text { mammography } \\
\text { screening (620) } \\
\text { Incident } \\
\text { inflammatory } \\
\text { breast cancer } \\
\text { (224) }\end{array}$ & $\begin{array}{l}\text { Mean age (yrs): } \\
\text { Cases: } 51 \\
\text { Controls: } 51 \\
\text { Nonwhite: } \\
\text { Cases: } 23 \% \\
\text { Controls: } 0 \%\end{array}$ & $\begin{array}{l}\text { Triple-negative IBC' } \\
\text { Ever BF': OR, } 0.30(95 \% \mathrm{Cl}, 0.15 \text { to } 0.62) \\
\text { HER2neu+ IBC } \\
\text { Ever BF': OR, } 1.01(95 \% \mathrm{Cl}, 0.55 \text { to } 1.87) \\
\text { Luminal IBCc } \\
\text { Ever BFd: OR, } 0.35(95 \% \mathrm{Cl}, 0.18 \text { to } 0.68)\end{array}$ & $\begin{array}{l}\text { Age at menarche, } \\
\text { menopausal status, } \\
\text { number of children, } \\
\text { age at first } \\
\text { pregnancy, BF } \\
\text { history, BMI, } \\
\text { smoking history, } \\
\text { breast cancer } \\
\text { family history }\end{array}$ \\
\hline $\begin{array}{l}\text { Holm, } \\
2017^{117} \\
\text { Case- } \\
\text { control } \\
\text { Medium }\end{array}$ & $\begin{array}{l}\text { Case-control } \\
\text { analysis of women } \\
\text { from two cohort } \\
\text { studies in } \\
\text { Sweden; controls } \\
\text { were frequency- } \\
\text { matched to cases } \\
\text { on age }(18,577) \\
\text { Primary invasive } \\
\text { breast cancer with } \\
\text { information on } \\
\text { immunohistochem } \\
\text { ical stains } \\
\text { diagnosed } 2005 \text { to } \\
2015 \text { (2,632) }\end{array}$ & $\begin{array}{l}\text { Mean age (SD), } \\
\text { range: } \\
\text { Cases: } 61 \\
(10.3), 27-88 \\
\text { Controls: } 58 \\
(9.7), 25-88\end{array}$ & 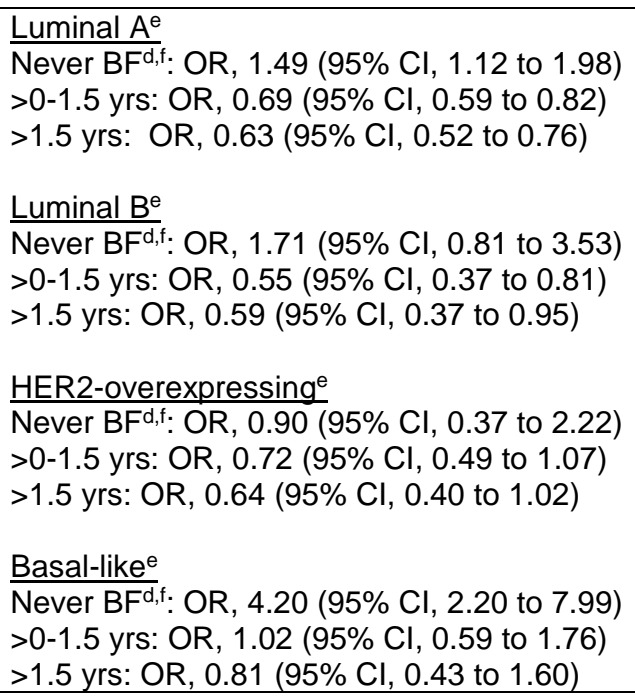 & $\begin{array}{l}\text { Country of birth, } \\
\text { age, education } \\
\text { level, parity, age at } \\
\text { first birth, BMl }\end{array}$ \\
\hline $\begin{array}{l}\text { Ma, } \\
2006121 \\
\text { Case- } \\
\text { control } \\
\text { Medium }\end{array}$ & $\begin{array}{l}\text { Population-based } \\
\text { case-control study } \\
\text { of cases identified } \\
\text { by the CSP and } \\
\text { neighborhood } \\
\text { controls matched } \\
\text { on age and race } \\
(2,238) \\
\text { First primary } \\
\text { invasive breast } \\
\text { cancer, } \\
\text { histologically } \\
\text { confirmed }(1,794)^{\mathrm{g}}\end{array}$ & $\begin{array}{l}\text { Mean age (yrs): } \\
\text { Cases: } 43 \\
\text { Controls: } 43 \\
\text { Nonwhite: } \\
\text { Cases: } 12 \% \\
\text { Controls: } 8 \%\end{array}$ & 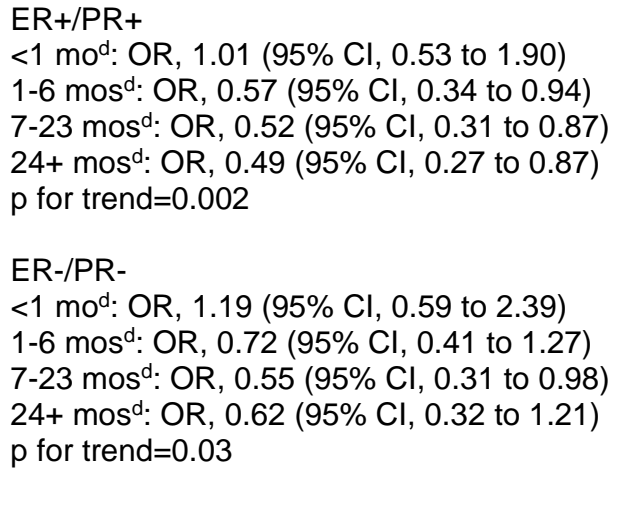 & $\begin{array}{l}\text { Race, age, } \\
\text { education, breast } \\
\text { cancer family } \\
\text { history, age at } \\
\text { menarche, full-term } \\
\text { pregnancies } \\
\text { (number and age } \\
\text { at), BMI, OC use, } \\
\text { alcohol use, } \\
\text { menopausal status, } \\
\text { and HRT use }\end{array}$ \\
\hline
\end{tabular}




\begin{tabular}{|c|c|c|c|c|}
\hline $\begin{array}{l}\text { Author, } \\
\text { Year } \\
\text { Study } \\
\text { Design } \\
\text { ROB }\end{array}$ & $\begin{array}{l}\text { Description of } \\
\text { Study (N) } \\
\text { Description of } \\
\text { Breast Cancer } \\
\text { Cases (N) }\end{array}$ & $\begin{array}{l}\text { Population } \\
\text { Characteristics }\end{array}$ & Results: Evera or Duration & $\begin{array}{l}\text { Confounders } \\
\text { Adjusted for }\end{array}$ \\
\hline $\begin{array}{l}\text { Ma, } \\
2017^{135} \\
\text { Case- } \\
\text { control } \\
\text { Medium }\end{array}$ & $\begin{array}{l}\text { Pooled analysis of } \\
\text { women from } 3 \\
\text { population-based } \\
\text { studies of breast } \\
\text { cancer, } \\
\text { predominantly in } \\
\text { Los Angeles; } \\
\text { controls }{ }^{\mathrm{h}} \text { were } \\
\text { frequency-match } \\
\text { to controls on age, } \\
\text { race, and } \\
\text { geographic area } \\
\text { of residence } \\
\text { (5,106) } \\
\text { Newly diagnosed } \\
\text { in situ and } \\
\text { invasive breast } \\
\text { cancer; some } \\
\text { were first primary } \\
\text { diagnoses and } \\
\text { were histologically } \\
\text { confirmed (2,658) }\end{array}$ & $\begin{array}{l}\text { Mean age (SD), } \\
\frac{\text { range }}{\text { Cases: } 47 \text { (8.1), }} \\
22-64 \\
\text { Controls: } 48 \\
(8.3), 24-64 \\
\text { African- } \\
\text { American Race } \\
\text { Cases: } 26 \% \\
\text { Controls: } 37 \%\end{array}$ & 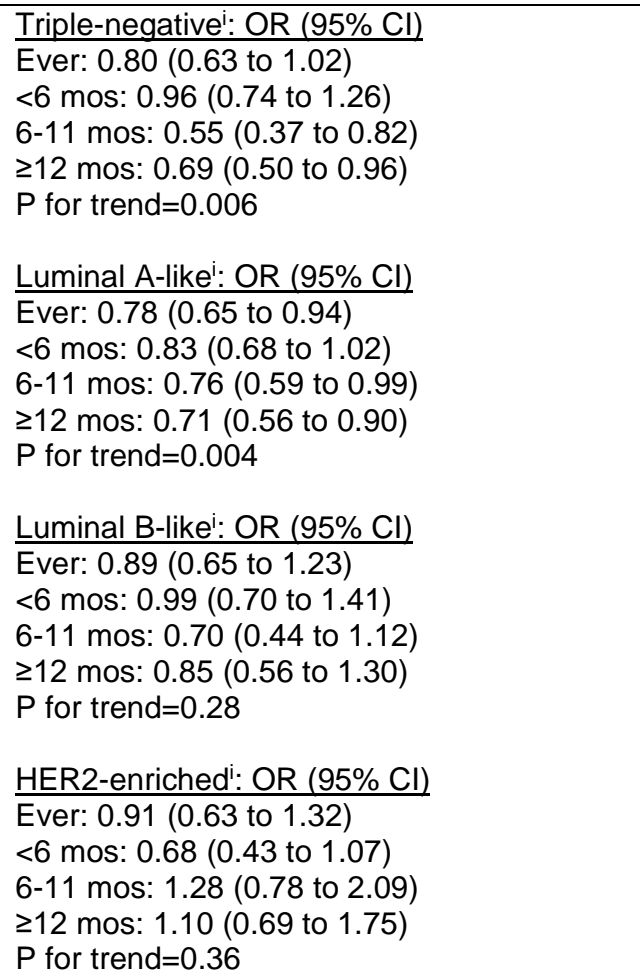 & $\begin{array}{l}\text { Sub-study (CARE, } \\
\text { BCIS, LIFE), study } \\
\text { site (Los Angeles, } \\
\text { Detroit), race, } \\
\text { reference age, } \\
\text { education, first- } \\
\text { degree breast } \\
\text { cancer family } \\
\text { history, BMI, } \\
\text { menopausal status, } \\
\text { hormone therapy } \\
\text { use, lifetime } \\
\text { recreational } \\
\text { physical activity, } \\
\text { alcohol intake, } \\
\text { smoking status, } \\
\text { age at menarche, } \\
\text { completed } \\
\text { pregnancies, oral } \\
\text { contraceptive use, } \\
\text { age at first } \\
\text { completed } \\
\text { pregnancy }\end{array}$ \\
\hline $\begin{array}{l}\text { Ritte, } \\
2013^{133} \\
\text { Cohort } \\
\text { Medium }\end{array}$ & $\begin{array}{l}\text { Cohort study of } \\
\text { women enrolled in } \\
\text { the EPIC study, } \\
\text { recruited from } 23 \\
\text { centers in } 10 \\
\text { western European } \\
\text { countries, and } \\
\text { followed for a } \\
\text { median of } 11 \text { yrs } \\
\text { (311,097) } \\
\text { First primary } \\
\text { invasive breast } \\
\text { cancer }(9,456)^{i}\end{array}$ & $\begin{array}{l}\text { Median age at } \\
\text { recruitment } \\
\text { (yrs): } 51 \\
\text { Ever BF: } 84 \% \\
\text { Median duration } \\
\text { of BF: } 6 \text { mos }\end{array}$ & 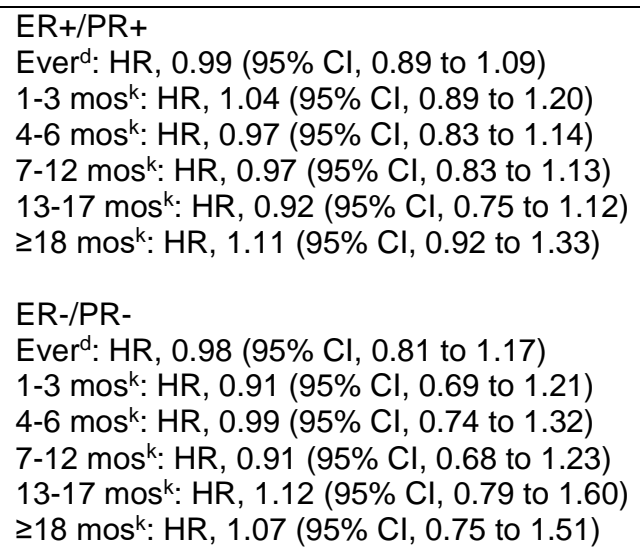 & $\begin{array}{l}\text { Age, EPIC center, } \\
\text { BMI, height, } \\
\text { menopausal status, } \\
\text { HRT use, physical } \\
\text { activity, smoking } \\
\text { status, alcohol use, } \\
\text { and education }\end{array}$ \\
\hline
\end{tabular}




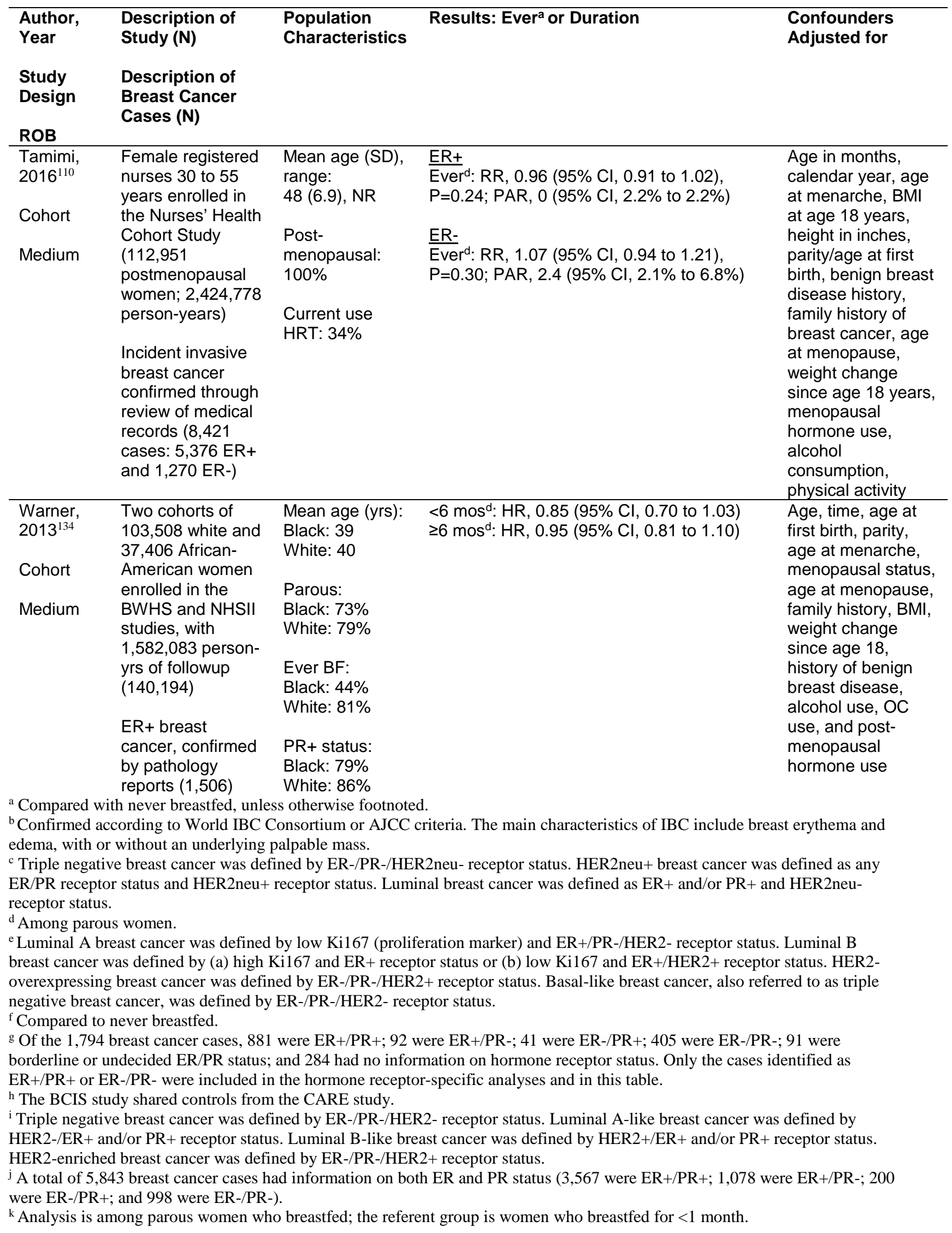


AJCC = American Joint Committee on Cancer; BF = breastfeeding; BMI = body mass index; BWHS = Black Women's Health Study; CI = confidence interval; CSP = LA Cancer Surveillance Program; EPIC = European Prospective Investigation into Cancer and Nutrition study; ER = estrogen receptor; HER2/HER2nue = human epidermal growth factor receptor 2; HR = hazard ratio; HRT $=$ hormone replacement therapy; $\mathrm{IBC}=$ inflammatory breast cancer; $\mathrm{N}=$ number; NHSII = Nurses’ Health Study II; $\mathrm{OC}=$ oral contraceptive; $\mathrm{OR}$ = odds ratio; $\mathrm{PAR}=$ population attributable risk; $\mathrm{PR}=$ progesterone receptor; $\mathrm{ROB}=$ risk of bias.

A population-based case-control study in Sweden, ${ }^{117}$ also described above in the overall breast cancer section, and a pooled analysis of three population-based case-control studies in the United States ${ }^{135}$ evaluated the association between both ever breastfeeding and duration of breastfeeding and breast cancer; breast cancer diagnoses in both studies were similarly defined as luminal, HER2, and triple negative (or basal-like) subtypes. A population-based case-control study of 2,238 women in Los Angeles County, California, included women with ER+/PR+ and ER-/PR- breast cancer but evaluated only duration of breastfeeding. ${ }^{121}$ Finally, a small hospitalbased case-control study, rated high risk of bias, at the MD Anderson Cancer Center in Texas evaluated the association between ever breastfeeding and inflammatory breast cancer further defined by hormone receptor status: (1) triple negative breast cancer (ER-/PR-/HER2neu-), (2) HER2neu breast cancer (any ER/PR status/HER2neu+), and (3) luminal breast cancer (ER+/any PR/HER2neu-). ${ }^{136}$ The mean age of women enrolled in these seven studies ranged from $39^{134}$ to 61 years. $^{117}$

\section{Results}

All three cohort studies reported no association between breastfeeding and breast cancer, regardless of the cancer subtype, with effect estimates close to and on both sides of the null. ${ }^{110 \text {, }}$ 133, 134 In a US-based case control study, ${ }^{133} \mathrm{Ma}$ and colleagues ${ }^{121}$ reported an inverse association between both ER+/PR+ and ER-/PR- breast cancer and increasing duration of breastfeeding (p for trend $=0.002$ and 0.03 , respectively). The ORs for $\geq 24$ months of breastfeeding were 0.49 (95\% CI, 0.27 to 0.87) and 0.62 (95\% CI, 0.32 to 1.21) for ER+/PR+ and ER-/PR- breast cancer, respectively. ${ }^{121}$ Results were mixed in three case-control studies that evaluated similarly defined luminal, HER2, and triple negative breast cancer cases. The Swedish study reported significantly decreased odds of luminal A, luminal B, and basal-like (i.e., triple negative), but not HER2 breast cancers; estimates for most comparisons were below the null. ${ }^{117}$ In the pooled analysis of three studies, there was a numerically decreased risk of all breast cancer subtypes with any breastfeeding. For triple negative and luminal A-like subtypes, there was a significant inverse association between increasing duration of breastfeeding and cancer risk; the p-values for trend were 0.006 and 0.004 , respectively. ${ }^{135}$ Finally, from a case-control study rated high risk of bias, Atkinson et al. ${ }^{136}$ reported a decreased risk of triple-negative (OR, 0.30; 95\% CI, 0.15 to 0.62 ) and luminal (OR, 0.35; 95\% CI, 0.18 to 0.68) but not HER2neu+ (OR, 1.01; 95\% CI, 0.55 to 1.87) breast cancers with ever breastfeeding. ${ }^{136}$

Two studies evaluated the relationship between breastfeeding and breast cancer risk among subgroups of women (Appendix Table F2). ${ }^{134,135}$ Both studies reported results stratified by race (white and black) and the pooled analysis of three case-control studies additionally evaluated age (20-44 years and 45-64 years of age). Ma and colleagues reported a significant inverse association between both triple negative and luminal A-like subtypes and any breastfeeding among African-American women (ORs of 0.67 and 0.78 , respectively) but not white women, though the odds of cancer were still numerically decreased; there was a similar trend of decreased cancer risk with increased duration of breastfeeding for African-American women but not white women. ${ }^{135}$ In a pooled analysis of two cohort studies, there was a nonsignificant decrease in the risk of ER+ breast cancer with both $<6$ months and $\geq 6$ months of 
breastfeeding. ${ }^{134}$ When the authors stratified by race, they found a nonsignificant increase in $\mathrm{ER}+$ breast cancer risk among black women, regardless of duration. For white women, the authors found an inverse association between $<6$ months breastfeeding and ER+ breast cancer (HR, $0.76 ; 95 \%$ CI, 0.60 to 0.98 ) but not $\geq 6$ months. Notably, although 81 percent of the white women in the two cohort studies reported ever breastfeeding, only 44 percent of the black women reported the same. ${ }^{134}$ In the pooled analysis of three case-control studies, there was a significant trend of decreased risk of triple negative breast cancer among younger women (20-44 years; $p$ for trend=0.02) but not older women (45-64 years; $p$ for trend=0.17). There was a significant trend of decreased luminal A-like breast cancer among the older women ( $\mathrm{p}$ for trend=0.03) but not the younger women ( $p$ for trend=0.0.12). All effect estimates were below the null and some confidence intervals were wide. ${ }^{135,136}$

\section{All-Cause and Breast Cancer-Specific Mortality}

\section{Characteristics}

We included one medium ROB study of women in the EPIC prospective cohort study that evaluated the association between breastfeeding and mortality (all cause and breast cancer specific) after a mean followup of 12.9 years (standard deviation=2.3 years) (Appendix Tables C-19 to C-23 and F1). ${ }^{137}$ The study included 322,972 (250,470 parous) women who were 25 to 70 years of age when they were recruited from 23 centers in 10 European countries between 1992 and 2000. Exposure and confounder data were collected at enrollment via questionnaires; mortality data were primarily ascertained from cancer registries, boards of health, and death indices.

\section{Results}

Among parous women, ever breastfeeding was associated with a decreased risk of all-cause mortality (fully adjusted HR, 0.92; 95\% CI, 0.87 to 0.97), but duration of breastfeeding was not associated with all-cause mortality ( $\mathrm{p}$ for trend=0.85). A total of 749 deaths were attributed to breast cancer; the confidence intervals for the association with ever breastfeeding spanned the null (fully adjusted HR, 1.01; 95\% CI, 0.79 to 1.29), and the study reported no statistically significant trend between duration of breastfeeding and death due to breast cancer ( $\mathrm{p}$ for trend $=0.35$ ).

\section{Ovarian Cancer: Systematic Reviews}

Four recent systematic reviews rated low ${ }^{138}$ or unclear $\mathrm{ROB}^{1,128,139}$ examined the association between breastfeeding and subsequent ovarian cancer (Table 20, Appendix Tables C-13 to C18). The number of studies included in the recent systematic reviews ranged from $15^{139}$ to $41{ }^{1}$ Most of the studies included in the systematic reviews were conducted in the United States, Europe, and Australia; seven studies were conducted in countries not categorized by the United Nations Development Programme ${ }^{41}$ as very high or high human development (Mexico, China, Thailand, Vietnam, and the Philippines) that contributed to one or more of the systematic reviews. All four systematic reviews evaluated the association between ovarian cancer and ever breastfeeding, and the duration of breastfeeding. Two reviews, both rated unclear ROB, performed a quality assessment of included articles according to the Newcastle-Ottawa Scale (NOS). In one review, approximately two-thirds of the studies were considered high quality but only 12 studies had a NOS score of 7 . The remaining studies had NOS scores ranging from 4 to

6. ${ }^{128}$ In the other review, only 4 of the 15 studies were considered high quality (i.e., NOS score $\geq$ 
“8”); the NOS scores for all studies ranged from "5" to "9."139The other two systematic reviews" 138 did not do a formal quality or ROB assessment but did perform a number of stratified analyses by factors related to potential biases (e.g., known confounders, aspects of study design).

Table 20. Breastfeeding and ovarian cancer: Summary of published systematic reviews

\begin{tabular}{|c|c|c|c|c|}
\hline $\begin{array}{l}\text { Author, } \\
\text { Year } \\
\text { ROB }\end{array}$ & $\begin{array}{l}\text { Number of } \\
\text { Studies } \\
\text { (Number of } \\
\text { Participants; } \\
\text { Number of } \\
\text { Cases) } \\
\text { Included Study } \\
\text { Designs }\end{array}$ & $\begin{array}{l}\text { Overlap with } \\
\text { Prior AHRQ } \\
\text { Report }^{2} \\
\text { Overlap with } \\
\text { Other Published } \\
\text { SRs }\end{array}$ & $\begin{array}{l}\text { Search Dates } \\
\text { Databases }\end{array}$ & Results \\
\hline $\begin{array}{l}\text { Chowdhury } \\
, 2015^{1} \\
\text { Unclear }\end{array}$ & $\begin{array}{l}41 \text { studies (NR) } \\
\text { Cohort, case- } \\
\text { control }\end{array}$ & $\begin{array}{l}9 \text { studies } \\
40 \text { of } 41 \text { studies } \\
\text { are included in } \\
\text { SRs by Ip, }{ }^{2} \mathrm{Li}^{128}, \\
\text { Luan, }^{138} \text { or } \\
\text { Sung }{ }^{139}\end{array}$ & $\begin{array}{l}\text { Inception } \\
\text { through } \\
\text { February } 2015 \\
\text { PubMed, } \\
\text { Cochrane, } \\
\text { CABI }\end{array}$ & $\begin{array}{l}\text { Ever vs. never BF } \\
\text { OR, } 0.70(95 \% \mathrm{Cl}, 0.64 \text { to } 0.77), \mathrm{I}^{2}=70,41 \\
\text { studies } \\
\text { Total BF duration (mos) vs. never BF } \\
\text { <6: OR,0.83 ( } 95 \% \mathrm{Cl}, 0.78 \text { to } 0.89), \mathrm{I}^{2}=3,20 \\
\text { studies } \\
\text { 6-12: OR, } 0.72(95 \% \mathrm{Cl}, 0.66 \text { to } 0.78), \mathrm{I}^{2}=22,19 \\
\text { studies } \\
\text { >12: OR, } 0.63(95 \% \mathrm{Cl}, 0.56 \text { to } 0.71), \mathrm{I}^{2}=52,29 \\
\text { studies }\end{array}$ \\
\hline $\begin{array}{l}\text { Li, } 2014^{128} \\
\text { Unclear }\end{array}$ & $\begin{array}{l}40 \text { studies } \\
(415,949 ; \\
17,139) \\
\text { Cohort, case- } \\
\text { control }\end{array}$ & $\begin{array}{l}9 \text { studies } \\
38 \text { of } 40 \text { studies } \\
\text { are included in } \\
\text { SRs by Ip, }{ }^{2} \\
\text { Chowdhury, }{ }^{1} \\
\text { Luan, }{ }^{138} \text { or Sung }{ }^{1,} \\
138,139\end{array}$ & $\begin{array}{l}\text { Inception } \\
\text { through March } \\
2013 \\
\text { PubMed, } \\
\text { Embase }\end{array}$ & $\begin{array}{l}\text { Ever vs. never BF } \\
\text { RR=0.70 ( } 95 \% \mathrm{Cl}, 0.64 \text { to } 0.76), \mathrm{I}^{2}=76,40 \\
\text { studies }^{\mathrm{a}} \\
\text { Total BF duration (mos) vs. never BF } \\
<6 \text { : RR, } 0.85(95 \% \mathrm{Cl}, 0.77 \text { to } 0.93), \mathrm{I}^{2}=38,16 \\
\text { studies } \\
\text { 6-12: RR, } 0.73(95 \% \mathrm{Cl}, 0.65 \text { to } 0.82), \mathrm{I}^{2}=37,15 \\
\text { studies } \\
>12: \mathrm{RR}, 0.64(95 \% \mathrm{Cl}, 0.56 \text { to } 0.73), \mathrm{I}^{2}=62,20 \\
\text { studies } \\
\text { Ptrend }=0.00,29 \text { studies }\end{array}$ \\
\hline $\begin{array}{l}\text { Luan, } \\
2013^{138} \\
\text { Low }\end{array}$ & $\begin{array}{l}35 \text { studies } \\
(720,617 \\
14,465) \\
\text { Cohort, case- } \\
\text { control }\end{array}$ & $\begin{array}{l}10 \text { studies } \\
\text { All } 35 \text { studies are } \\
\text { included in SRs } \\
\text { by Ip, }{ }^{2} \\
\text { Chowdhury, } \\
\mathrm{Li}^{1}{ }^{128} \text { or Sung } \\
139\end{array}$ & $\begin{array}{l}\text { Inception } \\
\text { through } \\
\text { December } \\
2012 \\
\text { PubMed }\end{array}$ & $\begin{array}{l}\text { Ever vs. never BF } \\
\text { RR, } 0.76(95 \% \mathrm{Cl}, 0.69 \text { to } 0.83), \mathrm{I}^{2}=55,32 \\
\text { studies } \\
\text { Total BF duration (per 5-month increase) } \\
\text { RR, } 0.92(95 \% \mathrm{Cl}, 0.90 \text { to } 0.95), \mathrm{I}^{2}=68,26 \\
\text { studies } \\
\text { Longest vs. shortest duration of } \mathrm{BF} \text { categories } \\
\text { RR, } 0.65(95 \% \mathrm{Cl}, 0.55 \text { to } 0.78), \mathrm{I}^{2}=64,26 \\
\text { studies }\end{array}$ \\
\hline $\begin{array}{l}\text { Sung, } \\
2016^{139}\end{array}$ & $\begin{array}{l}15 \text { studies } \\
(527,051 ; 7,639) \\
\text { Cohort, case- } \\
\text { control }\end{array}$ & $\begin{array}{l}5 \text { studies } \\
\text { All } 15 \text { studies are } \\
\text { included in SRs } \\
\text { by Ip, }{ }^{2} \text {, } \\
\text { Chowdhury, } \\
\mathrm{Li}^{1}{ }^{128} \text { or Luan } \\
138\end{array}$ & $\begin{array}{l}\text { Inception } \\
\text { through } \\
\text { December } \\
2015 \\
\text { PubMed, } \\
\text { Embase }\end{array}$ & $\begin{array}{l}\text { Total breastfeeding duration (mos) } \\
<6 \text { : RR, } 0.79(95 \% \mathrm{Cl}, 0.72 \text { to } 0.87), \mathrm{I}^{2}=25.5,15 \\
\text { studies } \\
6-12 \text { : } \mathrm{RR}, 0.72(95 \% \mathrm{Cl}, 0.64 \text { to } 0.81), \mathrm{I}^{2}=19.6 \text {, } \\
15 \text { studies } \\
\geq 13 \text { : } \mathrm{RR}, 0.67(95 \% \mathrm{Cl}, 0.56 \text { to } 0.79), \mathrm{I}^{2}=64.4 \text {, } \\
15 \text { studies }\end{array}$ \\
\hline
\end{tabular}

AHRQ = Agency for Healthcare Research and Quality; BF = breastfeeding; CABI = Centre for Agriculture and Biosciences International database; $\mathrm{CI}$ = confidence interval;; $\mathrm{NR}$ = not reported; $\mathrm{OR}$ = odds ratio; $\mathrm{ROB}$ = risk of bias; $\mathrm{RR}$ = relative risk; $\mathrm{SR}$ = systematic review. 
Because of substantial overlap among the four recent systematic reviews, results from pooled analyses were very similar (Table 20). As such, we describe in detail the most comprehensive systematic review by Chowdhury, published in 2015 and rated unclear ROB; the review included 41 studies and reported pooled results for both ever compared to never breastfeeding and different durations of breastfeeding. ${ }^{1}$

Chowdhury et al. ${ }^{1}$ included 5 cohort and 36 case-control studies in their systematic review; 9 of the 41 included studies were also included in the 2007 AHRQ review. ${ }^{2}$ The authors rated 27 (66\%) of the studies as being of adequate quality, defined as having only one or none of the following: selection bias, measurement bias, confounding bias, or attrition of 20 percent. They used random effects meta-analysis to estimate pooled ORs when heterogeneity was high $\left(\mathrm{I}^{2}>60 \%\right.$ or $\mathrm{p}$ for heterogeneity $<0.010$ ); reasons for heterogeneity were explored with subgroup analyses and meta-regression for the main comparison of ever versus never breastfed. Authors reported no evidence of publication bias using Begg's test, Egger's test, or funnel plot inspection.

Using data from all 41 included studies, the study reported a pooled OR for ever versus never breastfeeding of 0.70 (95\% CI, 0.64 to 0.77$)$; the substantial heterogeneity $\left(\mathrm{I}^{2}=70\right)$ of results could not be explained in subgroup analyses. The authors noted that among 35 studies in highincome countries, the association was significantly attenuated (pooled OR=0.74; 95\% CI, 0.68 to 0.80) when compared with 6 studies in lower mid-income countries (pooled OR=0.48; 95\% CI, 0.29 to 0.77 ), but the pooled estimates for two subgroups had substantial heterogeneity. The inverse association between ever breastfeeding and ovarian cancer was also attenuated but remained significant among cohort studies (pooled OR, 5 studies $=0.87 ; 95 \%$ CI, 0.78 to 0.98 ), studies that finely adjusted for parity (pooled OR, 16 studies $=0.80 ; 95 \% \mathrm{CI}, 0.75$ to 0.86 ), and studies that thoroughly adjusted for confounders (pooled OR, 14 studies $=0.76$; 95\% CI, 0.67 to 0.85) compared with case-control studies, studies with crude or no adjustment for parity, and studies with partial or no adjustment for confounders, respectively.

Authors also conducted pooled analyses to evaluate the association between different total durations of breastfeeding (compared with women who never breastfed) and ovarian cancer among a subset of studies with duration data. There was a numeric decrease in the pooled ORs with increasing duration of breastfeeding. For total breastfeeding duration of $<6$ months, the pooled OR was 0.83 (95\% CI, 0.78 to 0.89 ); the pooled ORs for 6 to 12 months and $>12$ months, respectively, were 0.72 (95\% CI, 0.66 to 0.78 ) and 0.63 (95\% CI, 0.56 to 0.71 ).

\section{Ovarian Cancer: Individual Studies}

\section{Characteristics}

We included four case-control studies ${ }^{140-143}$ of 2,131 women who were included in the 2007 AHRQ report by $\mathrm{Ip}^{2}$ but not included in subsequent systematic reviews (Table 21, Appendix Tables C-24 to C-28); these older studies were conducted as early as 1959 in the United States and Canada. One study was a pooled analysis of seven independent case-control studies ${ }^{140}$ among 465 black women. Three studies included cases with nonmalignant (i.e., low malignant potential or borderline) tumors, ${ }^{140,141,143}$ and one study enrolled women with benign ovarian tumors as controls. ${ }^{142}$ The two oldest studies, published in the 1960s, were rated high ROB by the authors of the 2007 AHRQ report. ${ }^{2}$ Two studies ${ }^{140,143}$ evaluated the association between ever breastfeeding and ovarian cancer, and three studies ${ }^{140-142}$ analyzed the association between different durations of breastfeeding and ovarian cancer. 
Table 21. Breastfeeding and ovarian cancer: Summary of individual studies

\begin{tabular}{|c|c|c|c|c|c|}
\hline $\begin{array}{l}\text { Author, Year } \\
\text { Study } \\
\text { Design } \\
\text { ROB }\end{array}$ & $\begin{array}{l}\text { Description of Study } \\
\text { (N) } \\
\text { Description of } \\
\text { Ovarian Cancer } \\
\text { Cases (N) }\end{array}$ & $\begin{array}{l}\text { Population } \\
\text { Characteristics }\end{array}$ & $\begin{array}{l}\text { Results: Ever } \\
\text { Breastfed }^{\mathrm{a}}\end{array}$ & $\begin{array}{l}\text { Results: } \\
\text { Duration of } \\
\text { Breastfeeding }^{a}\end{array}$ & $\begin{array}{l}\text { Confounders } \\
\text { Adjusted for }\end{array}$ \\
\hline $\begin{array}{l}\text { Cook, } 2017^{144} \\
\text { Case-control } \\
\text { Medium }\end{array}$ & $\begin{array}{l}\text { Population-based } \\
\text { case-control study in } \\
\text { British Columbia and } \\
\text { Calgary, Canada, with } \\
\text { controls frequency- } \\
\text { matched to cases on } \\
\text { age }(2,993) \\
\text { Women with } \\
\text { histologically } \\
\text { confirmed incident } \\
\text { epithelial ovarian } \\
\text { cancer (first primary) } \\
\text { and reported to cancer } \\
\text { registries }(2,139)\end{array}$ & $\begin{array}{l}\text { Mean age (yrs): } \\
50-69 \\
\text { Nonwhite: } 14 \% \\
\text { Post- } \\
\text { menopausal: } \\
75 \% \\
\text { Current or prior } \\
\text { use of HRT: } \\
27 \%\end{array}$ & $\begin{array}{l}\text { Cases: } 73 \% \\
\text { Controls: } 80 \%\end{array}$ & $\begin{array}{l}<10 \text { months } \\
\text { Cases: } 45 \% \\
\text { Controls: } 41 \% \\
\geq 10 \text { months } \\
\text { Cases: } 28 \% \\
\text { Controls: } 39 \%\end{array}$ & None \\
\hline $\begin{array}{l}\text { Gay, } 2015^{145} \\
\text { Cohort } \\
\text { Medium }\end{array}$ & $\begin{array}{l}\text { Women, } 50-64 \text { yrs of } \\
\text { age, enrolled in the } \\
\text { Singapore Breast } \\
\text { Cancer Project } \\
(28,107) \\
\text { Women diagnosed } \\
\text { with incident ovarian } \\
\text { cancer and reported to } \\
\text { the Singapore Cancer } \\
\text { Registry through } 2012 \\
(107)\end{array}$ & $\begin{array}{l}\text { Mean age (yrs): } \\
57 \\
\text { Postmenopausa } \\
\text { I: } 90 \% \\
\text { Current or prior } \\
\text { use of HRT: } \\
13 \%\end{array}$ & $\begin{array}{l}\mathrm{HR}=0.97(95 \% \\
\mathrm{Cl}, 0.63 \text { to } \\
1.51)^{\mathrm{b}}\end{array}$ & $\begin{array}{l}\text { s1 year } \\
\mathrm{HR}=1.09(95 \% \\
\mathrm{Cl}, 0.66 \text { to } 1.81)^{\mathrm{b}} \\
>1 \text { year } \\
\mathrm{HR}=1.20(95 \% \\
\mathrm{Cl}, 0.72 \text { to } 2.01)^{\mathrm{b}}\end{array}$ & $\begin{array}{l}\text { Age, housing } \\
\text { type, family } \\
\text { history of } \\
\text { breast } \\
\text { cancer, race, } \\
\text { BMI status, } \\
\text { smoking } \\
\text { status }\end{array}$ \\
\hline $\begin{array}{l}\text { Gierach, } \\
2006^{146} \\
\text { Case-control } \\
\text { Medium }\end{array}$ & $\begin{array}{l}\text { Population-based } \\
\text { case-control study in } \\
\text { the Delaware Valley, } \\
\text { USA, with controls } \\
\text { frequency-matched to } \\
\text { cases on age and } \\
\text { study area }(1,151) \\
\text { Incident epithelial } \\
\text { ovarian cancer, } \\
\text { confirmed by } \\
\text { pathologic review } \\
\text { (521) }\end{array}$ & $\begin{array}{l}\text { Mean age (yrs): } \\
40-59 \\
\text { Nonwhite: } 16 \%\end{array}$ & $\begin{array}{l}\text { Cases: } 44 \% \\
\text { Controls: } 51 \% \\
p=0.04\end{array}$ & NR & None \\
\hline $\begin{array}{l}\text { John, } 1993^{140} \\
\text { Case-control }\end{array}$ & $\begin{array}{l}\text { Pooled analysis of } 7 \\
\text { case-control studies } \\
\text { conducted in the USA } \\
\text { between } 1971 \text { and }\end{array}$ & $\begin{array}{l}\text { Mean age (yrs) } \\
\text { Invasive cases: } \\
53 \\
\text { Borderline }\end{array}$ & $\begin{array}{l}\text { OR=0.90 (95\% } \\
\mathrm{Cl}, 0.42 \text { to } \\
1.90)^{\mathrm{d}}\end{array}$ & $\begin{array}{l}1-5 \text { mos } \\
O R=1.00(95 \% \\
C l, 0.39 \text { to } 2.60)^{d}\end{array}$ & $\begin{array}{l}\text { Study, year of } \\
\text { birth, age, } \\
\text { parity }\end{array}$ \\
\hline Medium $^{c}$ & $\begin{array}{l}1986 \text { (465) } \\
\text { Epithelial ovarian } \\
\text { cancer ( } 72) \text {, ovarian } \\
\text { tumors of low } \\
\text { malignant potential } \\
\text { (borderline cases) } \\
\text { (35), and ovarian } \\
\text { tumors of unknown } \\
\text { behavior (3) }\end{array}$ & $\begin{array}{l}\text { cases: } 37 \\
\text { Controls: NR } \\
\text { Nonwhite: } 100 \%\end{array}$ & $\begin{array}{l}\text { Per month of } \\
\text { BF } \\
\text { OR=0.99 } \\
(p=0.57)^{d}\end{array}$ & $\begin{array}{l}\geq 6 \text { mos } \\
\text { OR=0.85 (95\% } \\
C l, 0.36 \text { to } 2.00)^{d}\end{array}$ & \\
\hline
\end{tabular}




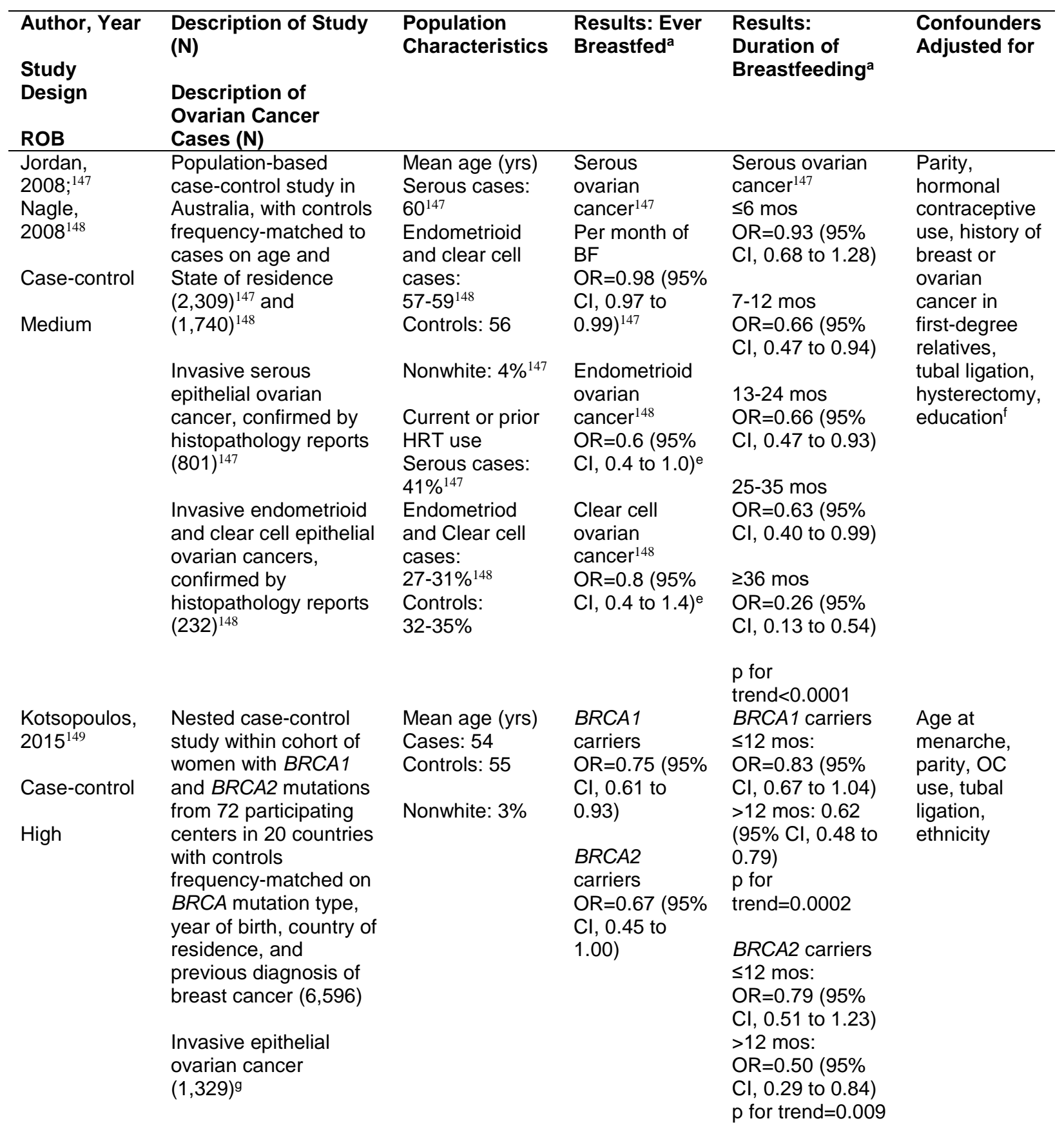




\begin{tabular}{|c|c|c|c|c|c|}
\hline $\begin{array}{l}\text { Author, Year } \\
\text { Study } \\
\text { Design } \\
\text { ROB }\end{array}$ & $\begin{array}{l}\text { Description of Study } \\
\text { (N) } \\
\text { Description of } \\
\text { Ovarian Cancer } \\
\text { Cases (N) }\end{array}$ & $\begin{array}{l}\text { Population } \\
\text { Characteristics }\end{array}$ & $\begin{array}{l}\text { Results: Ever } \\
\text { Breastfed }^{\text {a }}\end{array}$ & $\begin{array}{l}\text { Results: } \\
\text { Duration of } \\
\text { Breastfeeding }\end{array}$ & $\begin{array}{l}\text { Confounders } \\
\text { Adjusted for }\end{array}$ \\
\hline $\begin{array}{l}\text { Risch, } \\
1994^{141} \\
\text { Case-control } \\
\text { Medium }^{\mathrm{c}}\end{array}$ & $\begin{array}{l}\text { Population-based } \\
\text { case-control study } \\
\text { conducted in Canada } \\
\text { from } 1989 \text { to } 1992, \\
\text { with controls } \\
\text { frequency-matched to } \\
\text { cases by age }(1,014) \\
\text { Incident epithelial } \\
\text { ovarian cancer, based } \\
\text { on histology }(450)\end{array}$ & $\begin{array}{l}\text { Mean age (yrs) } \\
\text { Cases: } 57 \\
\text { Controls: } 58 \\
\text { Used } \\
\text { noncontraceptiv } \\
\text { e estrogens: } \\
18 \%\end{array}$ & NR & $\begin{array}{l}\text { Mean total } \\
\text { duration of } \\
\text { lactation (yrs) } \\
\text { Cases: } 0.51 \\
\text { Controls: } 0.65 \\
\text { OR=0.89 }(95 \% \\
\text { Cl, } 0.75 \text { to } 1.05)^{\mathrm{h}} \\
\text { Average duration } \\
\text { of lactation per } \\
\text { pregnancy (mos) } \\
\text { Cases: } 2.24 \\
\text { Controls: } 2.72 \\
\text { OR=0.87 }(95 \% \\
\text { Cl, } 0.76 \text { to } 0.99)^{\mathrm{h}}\end{array}$ & $\begin{array}{l}\text { Age, duration } \\
\text { of OC use, } \\
\text { number of } \\
\text { full-term } \\
\text { pregnancies }\end{array}$ \\
\hline $\begin{array}{l}\text { West, } 1966^{142} \\
\text { Case-control } \\
\text { High }^{c}\end{array}$ & $\begin{array}{l}\text { Hospital-based case- } \\
\text { control study where } \\
\text { ovarian cancer cases } \\
\text { and controls with } \\
\text { benign ovarian tumors, } \\
\text { matched to cases on } \\
\text { age, residence, and } \\
\text { date of surgery, were } \\
\text { ascertained from } 50 \\
\text { hospitals in the } \\
\text { Boston, MA, area } \\
\text { (194) } \\
\text { Malignant ovarian } \\
\text { cancer based on } \\
\text { pathology (97) }\end{array}$ & $\begin{array}{l}\text { Mean age (yrs) } \\
\text { Cases: } 45-64 \\
\text { Controls: } 40-59\end{array}$ & NR & $\begin{array}{l}\text { Duration of } \\
\text { lactation (mos) } \\
\text { Cases: } 6.6 \\
\text { Controls: } 5.8 \\
\text { p>0.03 }\end{array}$ & None \\
\hline $\begin{array}{l}\text { Wynder, } \\
1969^{143} \\
\text { Case-control }\end{array}$ & $\begin{array}{l}\text { Hospital-based case- } \\
\text { control study where } \\
\text { ovarian cancer cases } \\
\text { were matched to } \\
\text { controls on age (450) }\end{array}$ & $\begin{array}{l}\text { Mean age (yrs) } \\
\text { Cases: } 52 \\
\text { Controls: NR } \\
\text { Nonwhite }\end{array}$ & $\begin{array}{l}\text { No difference } \\
\text { between } \\
\text { cases and } \\
\text { controls for } \\
\text { never nursing }\end{array}$ & NR & None \\
\hline $\mathrm{High}^{\mathrm{c}}$ & $\begin{array}{l}\text { Malignant ovarian } \\
\text { cancer, based on } \\
\text { pathology; a vast } \\
\text { majority (89\%) were } \\
\text { epithelial (150) }\end{array}$ & $\begin{array}{l}\text { Cases: } 9 \% \\
\text { Controls: } 6 \%\end{array}$ & & & \\
\hline \multicolumn{6}{|c|}{$\begin{array}{l}\text { a Compared with never breastfed, unless otherwise specified. } \\
\text { b Among parous women only (25,975 women). }\end{array}$} \\
\hline \multicolumn{6}{|c|}{$\begin{array}{l}\text { c This study was included in the prior } 2007 \text { AHRQ review by Ip and colleagues. }{ }^{2} \text { We did not reassess ROB for individual studies } \\
\text { included in that review; this rating represents the decisions of the authors of that review. } \\
\text { d Among parous women only ( } 80 \text { cases, } 310 \text { controls). } \\
\text { e Among parous women only ( } 109 \text { endometrioid ovarian cancer cases, } 59 \text { clear cell ovarian cancer cases, } 1,328 \text { controls). } \\
\left.\text { f Jordan et al. (2008) })^{147} \text { adjusted for all confounders list; Nagle et al. (2008) }\right)^{148} \text { adjusted only for parity, hormonal contraceptive } \\
\text { use, and education. }\end{array}$} \\
\hline
\end{tabular}


$\mathrm{BF}=$ breastfeeding; $\mathrm{BMI}=$ body mass index; $\mathrm{CI}=$ confidence interval; $\mathrm{HR}=$ hazard ratio; $\mathrm{MA}=$ Massachusetts; $\mathrm{N}=$ number; $\mathrm{NR}=$ not reported; $\mathrm{OC}=$ oral contraceptive; $\mathrm{OR}=$ odds ratio; $\mathrm{ROB}=$ risk of bias.

We also included one cohort study and four case-control studies published after the most recent systematic review (Table 21, Appendix Tables C-24 to C-28). ${ }^{144-149}$ The cohort study followed 28,107 women, ages 50 to 64 years, participating in the Singapore Breast Cancer Project from 1994 to 1997; the Singapore Cancer Registry identified 107 ovarian cancer cases after an average 17 years of followup. The mean age of cases was 57 years and 90 percent of women were postmenopausal at the time of enrollment in the cohort. Information on breastfeeding was collected in 1994 to 1997; total duration of breastfeeding was calculated as the sum of breastfeeding duration for each child that was breastfed. More than 90 percent of women were parous at the time of enrollment, and 69 percent of them reported ever breastfeeding; the average total duration of breastfeeding was 1.88 years. ${ }^{145}$

Three recent, population-based case-control studies were conducted in the United States, ${ }^{146}$ Canada, ${ }^{144}$ and Australia. ${ }^{147,} 148$ The SHARE Study in the United States enrolled cases that were identified prior to surgery for ovarian cancer. ${ }^{146}$ Both the Australian Ovarian Cancer Study, ${ }^{147,} 148$ which contributed cases to two separate analyses, and the recent Canadian study, ${ }^{144}$ recruited incident cases that had been reported to population-based cancer registries; ${ }^{147,}{ }^{148}$ populationbased controls were recruited using random-digit dialing, Health Care Financing Administration or provincial health rosters, and electoral rolls; some controls from the Canadian study were also recruited from a mammography screening program. Information on parity, breastfeeding, and other risk factors for ovarian cancer was collected at enrollment during either an in-person interview $^{146}$ or questionnaire. ${ }^{144,147,148}$ The Australian Ovarian Cancer Study published two analyses, one including invasive serous ovarian cancer cases ${ }^{147}$ and the other including invasive endometrioid and clear cell ovarian cancer cases; ${ }^{148}$ both analyses compared the breastfeeding exposure of cases to that of 1,508 controls. We also included a high ROB case-control study, nested within a cohort of BRCA1 and BRCA2 mutation carriers, that recruited women from 72 participating centers across 20 countries; cases with self-reported invasive ovarian cancer $(n=1,329)$ were frequency-matched to women without ovarian cancer on BRCA mutation type, year of birth, and country of residence. For cases, the mean age at diagnosis was 50 years and the mean age at enrollment in the study was 54 years, indicating that a proportion of the ovarian cancer cases were enrolled with prevalent disease. ${ }^{149}$

With the exception of the SHARE, and recent Canadian ${ }^{144}$ studies, which only presented frequencies of ever breastfeeding or duration of breastfeeding among cases and controls only, ${ }^{146}$ the recent studies used regression analysis to estimate relative measures of association (i.e., hazard or odds ratios) for ever breastfeeding and different durations of breastfeeding, compared with never breastfeeding; regression models were adjusted for potential confounders including age, race/ethnicity, BMI, age at menarche, parity, oral contraceptive use, tubal ligation, hysterectomy, and family history of breast or ovarian cancer.

\section{Results}

The case-control studies, conducted across multiple countries and among women at both average and increased risk of ovarian cancer (i.e., BRCA1 and BRCA2 mutation carriers), generally reported an association between breastfeeding and reduced risk of ovarian cancer (Table 21). The SHARE Study reported that 51 percent of controls, compared with 44 percent of cases, reported ever breastfeeding; ${ }^{146}$ Prevalence of ever breastfeeding was high in the recent Canadian study overall and higher among controls (80\%) than among cases (73\%). ${ }^{144}$ ORs in the 
other studies ranged from 0.60 for endometrioid ovarian cancer (95\% CI, 0.4 to 1.0$)^{148}$ to 0.80 for clear cell ovarian cancer (95\% CI, 0.4 to 1.4$)^{148}$ to 0.90 (95\% CI, 0.42 to 1.90) for ovarian cancer among black women. ${ }^{140}$ A hospital-based case-control study, published in 1969, described no difference between groups with respect to ever breastfeeding but did not report specific frequencies. ${ }^{143}$ The cohort study in Singapore reported an adjusted HR of 0.97 (95\% CI, 0.63 to 1.51); the wide CI could be partially attributed to only 107 ovarian cancer cases being identified.

An association between various durations of breastfeeding and reduced rates of ovarian cancer was also reported across most studies. In a pooled analysis of black women from seven case-control studies, the OR for ovarian cancer among women who breastfed 6 months or more was significantly lower (0.85; 95\% CI, 0.36 to 2.00) but not for women who breastfed 1 to 5 months (OR, 1.00, 95\% CI, 0.39 to 2.60) when compared with no breastfeeding. ${ }^{140}$ A Canadian population-based study published in 1994 reported a higher mean total duration of breastfeeding and average duration of breastfeeding per pregnancy, respectively, among controls ( 0.65 years and 2.72 months) than among cases (0.51 years and 2.24 months); ${ }^{141}$ the more recent Canadian study reported that among women who breastfed, 49 percent of controls and 38 percent of cases breastfed for 10 or more months. ${ }^{144}$ Cases had a higher mean duration of breastfeeding (6.6 months) than controls with benign ovarian tumors (5.8 months) in a study published in 1966, but the difference was not statistically significant. ${ }^{142}$ The Australian Ovarian Cancer Study reported a significant trend $(\mathrm{p}<0.0001)$ of decreasing odds of invasive serous ovarian cancer with increasing duration of breastfeeding; for 6 or fewer months of breastfeeding, the reported OR was 0.93 (95\% CI, 0.68 to 1.28), and 0.26 for 3 or more years of breastfeeding, it was 0.26 (95\% CI, 0.13 to 0.54). ${ }^{147}$ One study reported similar results among BRCA1 and BRCA2 mutation carriers, ${ }^{148}$ with an OR per month of breastfeeding for invasive serous ovarian cancer of 0.98 (95\% CI, 0.97 to 0.99$).{ }^{147}$ The only study to report increased odds of ovarian cancer with increasing duration of breastfeeding was the cohort study in Singapore; because of the small number of cases, CIs were wide and overlapping, however, and included no difference. ${ }^{145}$

\section{Cardiovascular Disease and Hypertension}

\section{Background}

Physiologic changes associated with breastfeeding (e.g., increased plasma levels of oxytocin) have been associated with lower blood pressure. ${ }^{150}$ In addition, observational studies suggest that lactation has a beneficial effect on glucose and lipid metabolism that persists long after weaning. ${ }^{151}$ For these reasons, it is possible that breastfeeding leads to lower rates of CVD and hypertension in women. Commonly considered confounders in studies of the relationship between CVD and breastfeeding are diet, physical activity, cholesterol levels, and smoking status.

\section{Methods}

We included both case-control and cohort studies that examined the link between breastfeeding and incidence of CVD and hypertension. Although recent systematic reviews (published within the last 5 years) were eligible, we did not find one that was relevant to the scope of this review. We excluded metabolic markers associated with CVD (e.g., lipid levels and C-reactive protein) and measures of vascular characteristics (e.g., carotid artery intima media thickness) that could not be used to establish a diagnosis of CVD. For hypertension, we excluded changes in blood pressure levels that did not establish a diagnosis of hypertension. 


\section{Individual Studies}

\section{Characteristics}

Eight cohort studies were identified (Table 22); five reported on hypertension, ${ }^{4,5,152-154}$ three reported on composite measures of CVD (e.g., myocardial infarction [MI] or stroke), , 6, 155 and one reported on death from CVD. ${ }^{156}$ All studies were based on population cohorts. Four were set in the United States, ${ }^{4-6,155}$ two were set in Korea, ${ }^{152,154}$ and one each was set in Australia ${ }^{153}$ and Norway. ${ }^{156}$ Of the four studies set in the United States, two focused on women enrolled in the WHI, ${ }^{4,} 155$ one enrolled participants from the Nurses' Health Study I, ${ }^{6}$ and one enrolled participants from the Nurses' Health Study II. ${ }^{5}$ No study followed participants from the time of exposure; all assessed exposure based on self-report at cohort enrollment. The majority of studies enrolled women in their 20s or 30s through menopause; two studies (enrolling participants from the WHI) enrolled postmenopausal women only (mean ages 63 to 64 years). ${ }^{4,155}$ Most studies reported on smoking; rates ranged from 32 to 60 percent in six studies but were lower in the two studies enrolling Korean women ( $<1$ to $9 \%) .{ }^{152,154}$ All studies measured self-reported lifetime duration of lactation but used different categories (Table 22). Outcome ascertainment varied significantly and included self-report only, ${ }^{153}$ self-reported treatment or physical exam, ${ }^{154}$ biennial physical exams, ${ }^{152}$ and use of medical records or other means to confirm cardiovascular outcomes. 6,155

\section{Results: Hypertension}

Five studies reported on the association between breastfeeding duration and hypertension; all reported some statistically significant association (Table 22). ${ }^{4,5,152-154}$ Most studies (4) compared women by categories of breastfeeding duration (in months) with parous women who never breastfed; one study compared women who breastfed for less than 5 months with women who breastfed at least 6 months (or longer). ${ }^{154}$ Four studies found a consistent pattern of lower rates of hypertension across all categories of breastfeeding duration., 5, 152, 154 In one Australian cohort study, women who breastfed for longer than 6 months per lifetime (or greater than 3 months per child), on average, had significantly lower odds of having hypertension when compared with women who never breastfed (Table 22); however, results were only significant for women less than 64 years of age at cohort enrollment. ${ }^{153}$

Table 22. Breastfeeding and CVD or hypertension: Summary of individual studies

\begin{tabular}{|c|c|c|c|c|c|c|}
\hline $\begin{array}{l}\text { First } \\
\text { Author, } \\
\text { Year } \\
\text { ROB }\end{array}$ & $\begin{array}{l}\text { Study } \\
\text { Description } \\
\mathrm{N} \\
\text { Participants }\end{array}$ & Population & $\begin{array}{l}\text { Definition } \\
\text { of Exposure }\end{array}$ & $\begin{array}{l}\text { Outcome } \\
\text { Definition } \\
\text { Timing of } \\
\text { Followup }\end{array}$ & Results & $\begin{array}{l}\text { Confounders } \\
\text { Adjusted for }\end{array}$ \\
\hline $\begin{array}{l}\text { Choi, } \\
2017^{154} \\
\text { High }\end{array}$ & $\begin{array}{l}\text { Parous } \\
\text { women in } \\
\text { the } 2010- \\
2013 \text { Korean } \\
\text { National } \\
\text { Health and } \\
\text { Nutrition } \\
\text { Examination } \\
\text { Survey } \\
\text { (KNHANES) } \\
\text { ages 19-50 } \\
\text { yrs }\end{array}$ & $\begin{array}{l}\text { Mean age } \\
\text { (SD): } 40 \text { (NR) } \\
\text { \% nonwhite: } \\
\text { NR } \\
\text { \% current } \\
\text { smokers (by } \\
\text { BF duration): } \\
\leq 5 \text { mos: } 9 \\
6-11 \text { mos: } 5 \\
12-23 \text { mos: } 5 \\
24+\text { mos: } 3\end{array}$ & $\begin{array}{l}\text { Self-reported } \\
\text { lifetime BF } \\
\text { duration }\end{array}$ & $\begin{array}{l}\text { Elevated } \\
\text { BP } \\
\text { defined as } \\
\text { measured } \\
\mathrm{SBP} \geq 130 \\
\mathrm{mmHg} \text {, } \\
\mathrm{DBP} \geq 85 \\
\mathrm{mmHg} \text {, or } \\
\text { self- } \\
\text { reported } \\
\text { treatment } \\
\text { with HTN }\end{array}$ & $\begin{array}{l}\text { Adjusted OR }(95 \% \mathrm{Cl}) \text { of } \\
\text { elevated BP by } \mathrm{BF} \\
\text { duration: } \\
6-11 \text { mos vs. } \leq 5 \text { mos: } \\
0.67 \text { ( } 0.51 \text { to } 0.89) \\
12-23 \text { mos vs. } \leq 5 \text { mos: } \\
0.68 \text { (0.54 to } 0.86) \\
24+\text { mos vs. } \leq 5 \text { mos: } \\
0.82(0.65 \text { to } 1.03)\end{array}$ & $\begin{array}{l}\text { Age, BMI, } \\
\text { household } \\
\text { income, } \\
\text { education, } \\
\text { marriage status, } \\
\text { smoking status, } \\
\text { alcohol, physical } \\
\text { activity, age at } \\
\text { menarche, } \\
\text { menopause, } \\
\text { parity, and use of } \\
\text { OCs }\end{array}$ \\
\hline
\end{tabular}




\begin{tabular}{|c|c|c|c|c|c|c|}
\hline $\begin{array}{l}\text { First } \\
\text { Author, } \\
\text { Year } \\
\text { ROB }\end{array}$ & $\begin{array}{l}\text { Study } \\
\text { Description } \\
\text { N } \\
\text { Participants }\end{array}$ & Population & $\begin{array}{l}\text { Definition } \\
\text { of Exposure }\end{array}$ & $\begin{array}{l}\begin{array}{l}\text { Outcome } \\
\text { Definition }\end{array} \\
\text { Timing of } \\
\text { Followup }\end{array}$ & Results & $\begin{array}{l}\text { Confounders } \\
\text { Adjusted for }\end{array}$ \\
\hline & $\begin{array}{l}4,724 \\
\text { women }\end{array}$ & $\begin{array}{l}\text { \% with type } 2 \\
\text { DM (by BF } \\
\text { duration): } \\
\leq 5 \text { mos: } 2 \\
6-11 \text { mos: } 1 \\
\text { 12-23 mos: } 2 \\
24+\text { mos: } 2\end{array}$ & & $\begin{array}{l}\text { medicatio } \\
n \\
\text { NA: } \\
\text { Cross- } \\
\text { sectional } \\
\text { data from } \\
\text { cohort } \\
\text { study }\end{array}$ & $p$ for trend $=0.015$ & \\
\hline $\begin{array}{l}\text { First } \\
\text { Author, } \\
\text { Year } \\
\text { ROB }\end{array}$ & $\begin{array}{l}\text { Study } \\
\text { Description } \\
\mathrm{N} \\
\text { Participants }\end{array}$ & Population & $\begin{array}{l}\text { Definition of } \\
\text { Exposure }\end{array}$ & $\begin{array}{l}\text { Outcome } \\
\text { Definition } \\
\text { Timing of } \\
\text { Followup }\end{array}$ & Results & $\begin{array}{l}\text { Confounders } \\
\text { Adjusted for }\end{array}$ \\
\hline $\begin{array}{l}\text { Lee, } \\
2005^{152} \\
\text { Medium }\end{array}$ & $\begin{array}{l}\text { Women }>20 \\
\text { yrs through } \\
\text { menopause } \\
\text { without HTN } \\
\text { at baseline } \\
\text { enrolled in } \\
\text { the Korean } \\
\text { Women's } \\
\text { Cohort } \\
\text { Study } \\
177,749 \\
\text { (106,585 } \\
\text { ever } \\
\text { pregnant) }\end{array}$ & $\begin{array}{l}\text { Mean age } \\
\text { (SD): } 32.2 \\
(7.1) \\
\text { \% nonwhite: } \\
\text { NR } \\
\text { (presumably } \\
\text { all S. Korean) } \\
\text { \% current or } \\
\text { former } \\
\text { smokers: } 0.3 \\
\text { to } 0.9 \% \\
\text { (across BF } \\
\text { categories) }\end{array}$ & $\begin{array}{l}\text { Self-reported } \\
\text { history of BF } \\
\text { any or all } \\
\text { children } \\
\text { (lactation } \\
\text { records of } \\
\text { up to a } \\
\text { maximum of } \\
5 \text { children } \\
\text { per mother } \\
\text { were } \\
\text { recorded); } \\
\text { duration of } \\
\text { lactation was } \\
\text { defined by } \\
\text { total duration } \\
\text { for all } \\
\text { children }\end{array}$ & $\begin{array}{l}\text { SBP } \geq 140 \\
\mathrm{mmHg} \\
\mathrm{DBP} \geq 90 \\
\mathrm{mmHg} \text { or } \\
\text { current } \\
\text { use of } \\
\text { HTN } \\
\text { treatment } \\
\text { (determin } \\
\text { e during } \\
\text { biennial } \\
\text { physical } \\
\text { exam) } \\
6 \text { yrs (BP } \\
\text { measured } \\
\text { twice) }\end{array}$ & $\begin{array}{l}\text { RR }(95 \% \mathrm{Cl}), \text { mean } \\
\text { duration of lifetime lactation } \\
\text { (mos): } \\
\text { 1-3: } 0.90(0.87 \text { to } 0.93) \\
\text { 4-6: } 0.92(0.87 \text { to } 0.98) \\
\text { 7-9: } 0.93(0.86 \text { to } 0.99) \\
\text { 10-12: } 1.00(0.91 \text { to } 1.11) \\
>12: 1.06 \text { (0.99 to } 1.14) \\
\text { RR (95\% Cl), effect of } \\
\text { combination obesity and } \\
\text { lactation history (ever vs. } \\
\text { never) on incident HTN } \\
\text { (reference }=\text { BMl <23.05 } \\
\text { and }+ \text { lactation) } \\
\text { BMI <23.05/no lactation: } \\
\text { 1.06 (1.02 to } 1.11) \\
\text { BMI } \geq 23.05 / l a c t a t i o n: \\
1.65(1.58 \text { to } 1.72) \\
\text { BMI } \geq 23.05 / \text { no lactation: } \\
1.85(1.75 \text { to } 1.90)\end{array}$ & $\begin{array}{l}\text { Age, obesity, } \\
\text { smoking, alcohol } \\
\text { drinking, exercise, } \\
\text { number of } \\
\text { children, and age } \\
\text { at first pregnancy }\end{array}$ \\
\hline
\end{tabular}




\begin{tabular}{|c|c|c|c|c|c|c|}
\hline $\begin{array}{l}\text { First } \\
\text { Author, } \\
\text { Year } \\
\text { ROB }\end{array}$ & $\begin{array}{l}\text { Study } \\
\text { Description } \\
\text { N } \\
\text { Participants }\end{array}$ & Population & $\begin{array}{l}\text { Definition } \\
\text { of Exposure }\end{array}$ & $\begin{array}{l}\text { Outcome } \\
\text { Definition } \\
\text { Timing of } \\
\text { Followup }\end{array}$ & Results & $\begin{array}{l}\text { Confounders } \\
\text { Adjusted for }\end{array}$ \\
\hline $\begin{array}{l}\text { Lupton, } \\
2013^{153} \\
\text { Medium }\end{array}$ & $\begin{array}{l}\text { Nested } \\
\text { case- control } \\
\text { study of } \\
\text { women }>45 \\
\text { yrs } \\
\text { participating } \\
\text { in an } \\
\text { Australian } \\
\text { population- } \\
\text { based cohort } \\
\text { study } \\
\text { without HTN } \\
\text { during } \\
\text { pregnancy } \\
74,785 \\
\text { (64,199 } \\
\text { parous } \\
\text { women) }\end{array}$ & $\begin{array}{l}\text { \% of cases in } \\
\text { each age } \\
\text { category: } \\
45 \text { to <54: } 35 \\
54 \text { to <64: } 33 \\
\geq 64: 32 \\
\% \text { of cases } \\
\text { who were } \\
\text { current or } \\
\text { former } \\
\text { smokers: } 35\end{array}$ & $\begin{array}{l}\text { Self-reported } \\
\text { total lifetime } \\
\text { BF duration } \\
\text { (and } \\
\text { average BF } \\
\text { duration per } \\
\text { child) }\end{array}$ & $\begin{array}{l}\text { Self- } \\
\text { reported } \\
\text { current } \\
\text { treatment } \\
\text { of HTN } \\
\text { (onset } \\
\text { after age } \\
\text { of first } \\
\text { birth) } \\
\text { NA } \\
\text { (recruited } \\
\text { from 2006 } \\
\text { to 2009) }\end{array}$ & 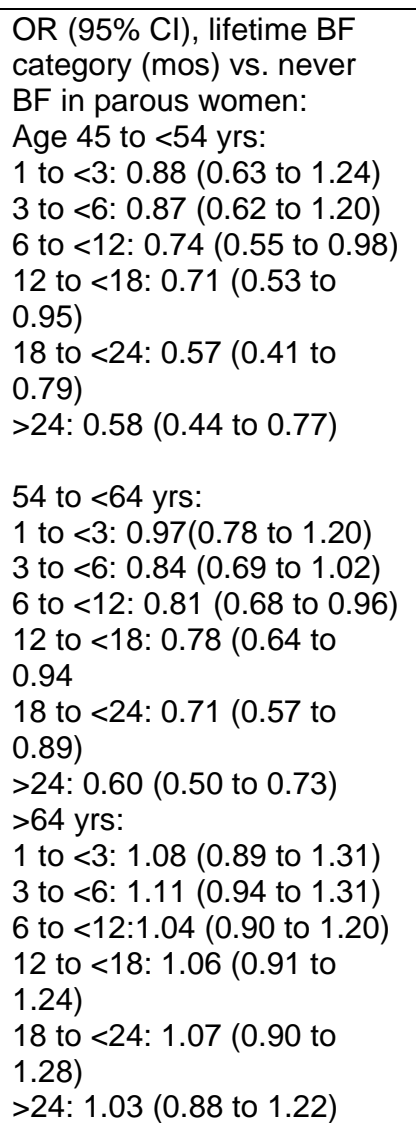 & $\begin{array}{l}\text { Country of origin } \\
\text { (Australia or } \\
\text { other), income } \\
\text { level, BMI, } \\
\text { smoking status, } \\
\text { alcohol } \\
\text { consumption, } \\
\text { physical activity, } \\
\text { family history of } \\
\text { HTN, history of } \\
\text { OC use, and } \\
\text { history of HRT use }\end{array}$ \\
\hline $\begin{array}{l}\text { First } \\
\text { Author, } \\
\text { Year } \\
\text { ROB }\end{array}$ & $\begin{array}{l}\text { Study } \\
\text { Description } \\
\mathrm{N} \\
\text { Participants }\end{array}$ & Population & $\begin{array}{l}\text { Definition of } \\
\text { Exposure }\end{array}$ & $\begin{array}{l}\text { Outcome } \\
\text { Definition } \\
\text { Timing of } \\
\text { Followup }\end{array}$ & Results & $\begin{array}{l}\text { Confounders } \\
\text { Adjusted for }\end{array}$ \\
\hline $\begin{array}{l}\text { Natland } \\
\text { Fagerha } \\
\text { ug, } \\
2013^{156} \\
\text { Medium }\end{array}$ & $\begin{array}{l}\text { Norwegian } \\
\text { population- } \\
\text { based cohort } \\
\text { enrolling } \\
\text { women ages } \\
30 \text { to } 85 \text { who } \\
\text { attended a } \\
\text { health } \\
\text { survey } \\
\text { followup in } \\
1995-1997^{a} \\
21,889 \\
(15,000 \\
\text { parous } \\
\text { women) }\end{array}$ & $\begin{array}{l}\text { Age: } \\
\text { Ever } \\
\text { lactated: } \\
52.1(14.1) \\
\text { Never } \\
\text { lactated: } \\
52.5 \text { (12.5) } \\
\text { Nulliparous: } \\
55.9 \text { (16.8) } \\
\text { \% nonwhite: } \\
\text { NR } \\
\text { \% current or } \\
\text { former } \\
\text { smokers: } \\
51.2 \\
\text { \% post- } \\
\text { menopausal } \\
\text { : NR }\end{array}$ & $\begin{array}{l}\text { Self-reported } \\
\text { lactation } \\
\text { history, ever } \\
\text { and lifetime } \\
\text { lactation } \\
\text { (defined as } \\
\text { sum of } \\
\text { lactation } \\
\text { duration for all } \\
\text { births) }\end{array}$ & $\begin{array}{l}\text { Death } \\
\text { from CVD } \\
\text { (MI or } \\
\text { stroke) } \\
\text { defined by } \\
\text { ICD 9: } \\
\text { 390-459 } \\
\text { and ICD- } \\
\text { 10: 100- } \\
99 \\
\text { Median } \\
14.5 \text { yrs } \\
\text { after } \\
\text { enrollment }\end{array}$ & $\begin{array}{l}\text { HR }(95 \% \mathrm{Cl}) \text { : never vs. } \\
\text { ever lactation in parous } \\
\text { women } \\
<65 \text { yrs: } \\
2.86(1.51 \text { to } 5.39) \\
45-64 \text { yrs: } \\
3.15((1.66 \text { to } 5.00) \\
>65 \text { yrs: } \\
1.11 \text { (0.77 to } 1.69) \\
\text { HR ( } 95 \% \mathrm{Cl}) \text { by lifetime } \\
\text { lactation duration in parous } \\
\text { women <65 yrs: } \\
\text { Never vs. >24 mos: } \\
2.77(1.28 \text { vs. } 5.99) \\
7-12 \text { mos vs. }>24 \text { mos: } \\
0.55(0.27 \text { to } 1.09) \\
\text { HR ( } 95 \% \text { Cl), lifetime } \\
\text { lactation duration in parous } \\
\text { women } 45 \text { to } 64 \text { yrs: }\end{array}$ & $\begin{array}{l}\text { Age, smoking } \\
\text { status, physical } \\
\text { activity, education, } \\
\text { marital status, } \\
\text { parity }\end{array}$ \\
\hline
\end{tabular}




\begin{tabular}{|c|c|c|c|c|c|c|}
\hline $\begin{array}{l}\text { First } \\
\text { Author, } \\
\text { Year } \\
\text { ROB }\end{array}$ & $\begin{array}{l}\text { Study } \\
\text { Description } \\
\text { N } \\
\text { Participants }\end{array}$ & Population & $\begin{array}{l}\text { Definition } \\
\text { of Exposure }\end{array}$ & $\begin{array}{l}\begin{array}{l}\text { Outcome } \\
\text { Definition }\end{array} \\
\text { Timing of } \\
\text { Followup } \\
\end{array}$ & Results & $\begin{array}{l}\text { Confounders } \\
\text { Adjusted for }\end{array}$ \\
\hline & & $\begin{array}{l}\text { \% with type } \\
2 \text { diabetes: } \\
2.9 \%\end{array}$ & & & $\begin{array}{l}\text { Never vs. >24 mos: } \\
3.03 \text { (1.38 to } 6.70) \\
7-12 \text { mos vs. }>24 \text { mos: } \\
0.45(0.21 \text { to } 0.97)\end{array}$ & \\
\hline $\begin{array}{l}\text { Parikh, } \\
2016^{155} \\
\text { Medium }\end{array}$ & $\begin{array}{l}\text { U.S. women } \\
\text { participating } \\
\text { in the WHI } \\
\text { observationa } \\
\text { I cohort } \\
\text { study who } \\
\text { had no prior } \\
\text { history of } \\
\text { CHD } \\
72,982\end{array}$ & $\begin{array}{l}\text { Mean age } \\
\text { (SD): } 63.2 \\
(7.3) \\
\% \text { nonwhite: } \\
14 \\
\% \\
\text { postmeno- } \\
\text { pausal: } 100 \\
\text { \% using } \\
\text { HRT: } 30 \\
\text { \% current or } \\
\text { former } \\
\text { smokers: } 49 \\
\text { \% with type } \\
2 \text { diabetes: } \\
4.7\end{array}$ & $\begin{array}{l}\text { Self-reported } \\
\text { lifetime BF } \\
\text { duration } \\
\text { assessed at } \\
\text { WHI } \\
\text { enrollment }\end{array}$ & $\begin{array}{l}\text { Physician- } \\
\text { adjudicate } \\
\mathrm{d} \text { fatal and } \\
\text { nonfatal } \\
\text { CHD }^{\mathrm{b}} \\
\text { Median } 12 \\
\text { yrs }\end{array}$ & $\begin{array}{l}\mathrm{HR}(95 \% \mathrm{Cl}), \mathrm{BF} \text { for at } \\
\text { least } 1 \mathrm{mo} \text { vs. <1 mo: } \\
0.90(0.85-0.96)\end{array}$ & $\begin{array}{l}\text { Menstrual } \\
\text { irregularity, age at } \\
\text { first birth, still } \\
\text { births, } \\
\text { miscarriages, age, } \\
\text { medication use for } \\
\text { high cholesterol, } \\
\text { medication use for } \\
\text { HTN, log of } \\
\text { systolic blood } \\
\text { pressure, current } \\
\text { smoker, and } \\
\text { diabetes mellitus }\end{array}$ \\
\hline
\end{tabular}




\begin{tabular}{|c|c|c|c|c|c|c|}
\hline $\begin{array}{l}\text { First } \\
\text { Author, } \\
\text { Year } \\
\text { ROB }\end{array}$ & $\begin{array}{l}\text { Study } \\
\text { Description } \\
\mathrm{N} \\
\text { Participants }\end{array}$ & Population & $\begin{array}{l}\text { Definition of } \\
\text { Exposure }\end{array}$ & $\begin{array}{l}\text { Outcome } \\
\text { Definition } \\
\text { Timing of } \\
\text { Followup }\end{array}$ & Results & $\begin{array}{l}\text { Confounders } \\
\text { Adjusted for }\end{array}$ \\
\hline $\begin{array}{l}\text { Schwarz, } \\
2009^{4} \\
\text { Medium }\end{array}$ & $\begin{array}{l}\text { Cohort study } \\
\text { (WHI) } \\
\text { enrolling } \\
\text { generally } \\
\text { healthy post- } \\
\text { menopausal } \\
\text { women from } \\
\text { the United } \\
\text { States who } \\
\text { reported at } \\
\text { least } 1 \text { live } \\
\text { birth } \\
139,681\end{array}$ & $\begin{array}{l}\text { Mean age: } \\
63-64 \text { (SD } \\
\text { NR) } \\
\text { \% nonwhite; } \\
\text { 14-19 } \\
\text { \% with } \\
\text { GDM: NR }\end{array}$ & $\begin{array}{l}\text { Self-reported } \\
\text { lifetime BF } \\
\text { duration } \\
\text { assessed at } \\
\text { WHI } \\
\text { enrollment } \\
\text { Median } 7.9 \text { yrs }\end{array}$ & $\begin{array}{l}\text { Self- } \\
\text { reported } \\
\text { HTN and } \\
\text { history of } \\
\text { CVD }^{c} \text { prior } \\
\text { to } \\
\text { enrollment; } \\
\text { incident } \\
\text { CVDd over } \\
7.9 \text { yrs of } \\
\text { WHI } \\
\text { (validated } \\
\text { by } \\
\text { physician } \\
\text { adjudicatio } \\
\text { n) }\end{array}$ & 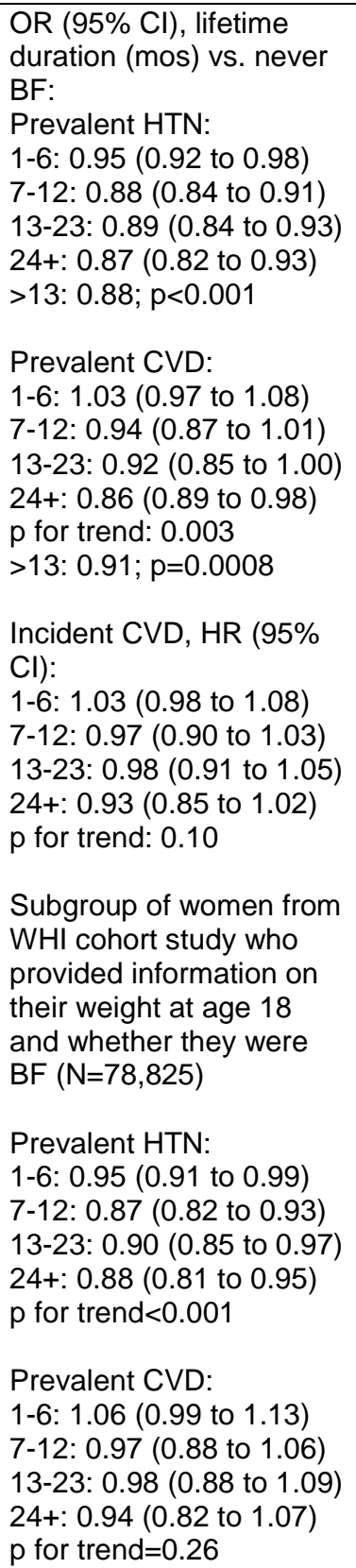 & $\begin{array}{l}\text { Age; race; parity; } \\
\text { age at } \\
\text { menopause; } \\
\text { education; } \\
\text { income; family } \\
\text { history (of } \\
\text { diabetes mellitus; } \\
\text { Ml or stroke); } \\
\text { physical activity; } \\
\text { energy, } \\
\text { cholesterol, fat, } \\
\text { fiber, and sodium } \\
\text { intakes; tobacco } \\
\text { history; HRT use, } \\
\text { aspirin use; and } \\
\text { MVI use }\end{array}$ \\
\hline
\end{tabular}




\begin{tabular}{|c|c|c|c|c|c|c|}
\hline $\begin{array}{l}\text { First } \\
\text { Author, } \\
\text { Year } \\
\text { ROB }\end{array}$ & $\begin{array}{l}\text { Study } \\
\text { Description } \\
\text { N } \\
\text { Participants }\end{array}$ & Population & $\begin{array}{l}\text { Definition of } \\
\text { Exposure }\end{array}$ & $\begin{array}{l}\text { Outcome } \\
\text { Definition } \\
\text { Timing of } \\
\text { Followup }\end{array}$ & Results & $\begin{array}{l}\text { Confounders } \\
\text { Adjusted for }\end{array}$ \\
\hline $\begin{array}{l}\text { Stuebe, } \\
2009^{6} \\
\text { High }\end{array}$ & $\begin{array}{l}\text { Participants } \\
\text { from the } \\
\text { Nurses' } \\
\text { Health Study } \\
\text { I who were } \\
\text { parous with } \\
\text { no history of } \\
\text { MI, angina, } \\
\text { or CABG } \\
\text { before } 1986 \\
89,326\end{array}$ & $\begin{array}{l}\text { Age: } 30-55 \\
\text { yrs at } \\
\text { enrollment } \\
\text { (mean NR) } \\
\text { \% nonwhite: } \\
\text { NR } \\
\text { \% with type } \\
2 \text { diabetes: } \\
1.7-2.1 \\
\% \text { with } \\
\text { HTN: } 18.5 \\
\text { to } 23.3 \\
\text { \% past or } \\
\text { current } \\
\text { smoker: } \\
60.4 \text { to } 44.1\end{array}$ & $\begin{array}{l}\text { Self-reported } \\
\text { total duration } \\
\text { of lactation for } \\
\text { all } \\
\text { pregnancies } \\
\text { (via } \\
\text { questionnaire } \\
\text { in 1986) }\end{array}$ & $\begin{array}{l}\text { Incident } \\
\text { CHD } \\
\text { (nonfatal } \\
\text { MI }^{\mathrm{e}} \text { and } \\
\text { mortality } \\
\text { due to } \\
\text { CHD) } \\
\text { confirmed } \\
\text { by medical } \\
\text { records } \\
\text { (from 1986 } \\
\text { to } 2002)^{f} \\
16 \text { yrs }\end{array}$ & $\begin{array}{l}\text { HR }(95 \% \mathrm{Cl}) \text {, lifetime BF } \\
\text { duration (mos) vs. never: } \\
<3: 1.01(0.91 \text { to } 1.11) \\
\text { 3-6: } 1.0(0.88 \text { to } 1.14) \\
6-111.02(0.88 \text { to } 1.18) \\
\text { 11-23: } 0.93(0.8 \text { to } 1.07) \\
>23: 0.77(0.62 \text { to } 0.94) \\
\text { p for trend: } 0.02\end{array}$ & $\begin{array}{l}\text { Age; parity; history } \\
\text { of stillbirth; BMI at } \\
\text { age } 18 \text { yrs; } \\
\text { birthweight of } \\
\text { subject; parental } \\
\text { history of MI } \\
\text { before age } 60 \text { yrs; } \\
\text { diet quintile; } \\
\text { physical activity; } \\
\text { smoking; } \\
\text { menopausal } \\
\text { status; and use of } \\
\text { aspirin, alcohol, } \\
\text { MVI, and HRT }\end{array}$ \\
\hline $\begin{array}{l}\text { Stuebe, } \\
2011^{5} \\
\text { Medium }\end{array}$ & $\begin{array}{l}\text { Participants } \\
\text { from the } \\
\text { Nurses' } \\
\text { Health Study } \\
\text { II without } \\
\text { HTN, }{ }^{9} \text { self- } \\
\text { reported } \\
\text { diabetes, } \\
\text { CVD, } \\
\text { hyperlipidemi } \\
\text { a, or cancer } \\
\text { (1991-2005) } \\
55,636\end{array}$ & $\begin{array}{l}\text { Age: } 25-42 \\
\text { yrs at } \\
\text { enrollment } \\
\text { (mean=35.7 } \\
\text { ) in 1989) } \\
\text { \% nonwhite: } \\
6.2 \\
\text { \% current or } \\
\text { former } \\
\text { smokers: } \\
32.3\end{array}$ & $\begin{array}{l}\text { Self-reported } \\
\text { total duration } \\
\text { and exclusive } \\
\text { duration of } \\
\text { lactation (for } \\
\text { first } 4 \text { children) }\end{array}$ & $\begin{array}{l}\text { Incident } \\
\text { self- } \\
\text { reported } \\
\text { HTN } \\
\text { (excluding } \\
\text { pregnancy) } \\
\text { NA: Cross- } \\
\text { sectional } \\
\text { data from } \\
\text { cohort } \\
\text { study }\end{array}$ & 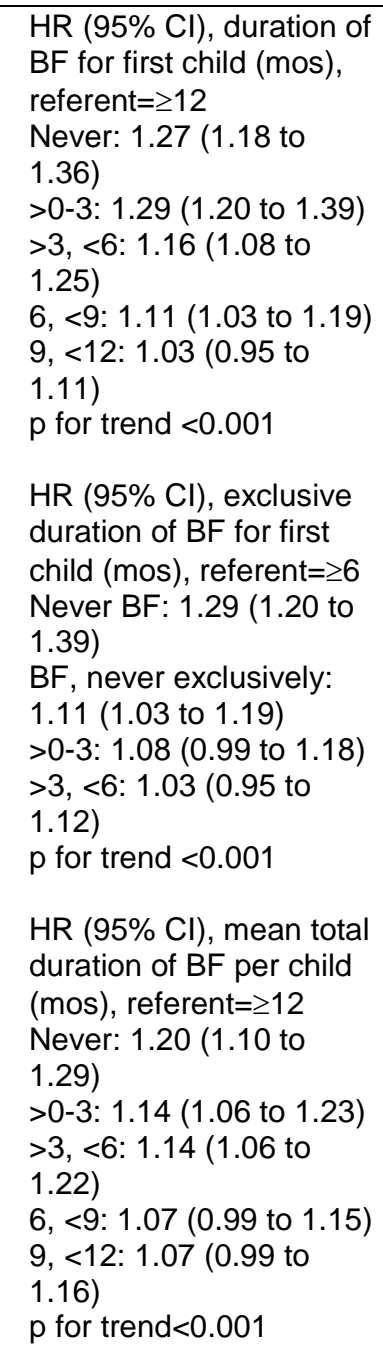 & $\begin{array}{l}\text { Age and inverse } \\
\text { probability weights } \\
\text { (derived from } \\
\text { models including } \\
\text { maternal BMI at } \\
\text { age 18, year of } \\
\text { first birth, self- } \\
\text { reported history of } \\
\text { preeclampsia, } \\
\text { gestational HTN, } \\
\text { gestational } \\
\text { diabetes, birth of } \\
\text { an infant at <37 } \\
\text { wks, birth of an } \\
\text { infant weighing } \\
<2,500 \text { g, } \\
\text { miscarriage or } \\
\text { stillbirth at }>12 \\
\text { wks, smoking } \\
\text { status, vigorous } \\
\text { physical activity, } \\
\text { alcohol } \\
\text { consumption, } \\
\text { DASH diet score } \\
\text { quintile, family } \\
\text { history of HTN, } \\
\text { current OC use, } \\
\text { current } \\
\text { nonnarcotic } \\
\text { analgesic use, } \\
\text { and self-reported } \\
\text { race) }\end{array}$ \\
\hline
\end{tabular}


women who breastfed for less than 13 months (OR, 0.91; $\mathrm{p}=0.008) .{ }^{4}$ The same study also reported on incident CVD (after WHI enrollment); over an average of 7.9 years of followup there was no significant difference in CVD incidence between women based on lifetime breastfeeding duration in adjusted models (Table 22). Finally, an analysis of participants from the Nurses' Health Study 1 who were parous with no history of CVD prior to enrollment $(\mathrm{N}=89,326)$ found that women who had breastfed for greater than 23 months had lower rates of incident MI (defined as fatal or nonfatal MI) over 16 years of followup (HR, 0.77; 95\% CI, 0.62 to 0.94). ${ }^{6}$ Women who breastfed for shorter durations (<23 months) did not have a significantly different rate of MI compared with women who never breastfed (Table 22).

One Norwegian population-based cohort study ( $\mathrm{N}=15,000$ parous women) compared incident CVD mortality among women ages 30 to 85 years at enrollment over approximately 14 years of followup. ${ }^{156}$ Parous women younger than 65 at enrollment who had never lactated had higher CVD mortality than women who lactated 24 months or more (HR 2.77; 95\% CI, 1.28 to 5.99). No clear associations were observed among women 65 years of age or older at cohort enrollment (Table 22).

\section{Type 2 Diabetes}

\section{Background}

Observational studies suggest that lactation has a beneficial effect on glucose and lipid metabolism that persists long after weaning. ${ }^{151}$ For women with gestational diabetes, evidence suggests that lactation is associated with improved pancreatic beta-cell function and glucose levels. ${ }^{157}$ Studies have reported that mothers who do not breastfeed (or breastfeed for shorter durations) have less visceral fat than mothers who breastfed for at least 3 months. Thus, it is plausible that lactation could reduce the risk of the development of type 2 diabetes. Commonly considered confounders in studies of relationship between maternal type 2 diabetes and breastfeeding are parity, BMI, diet, physical activity, family history of diabetes, and smoking status.

\section{Methods}

We included both case-control and cohort studies that examined the association between breastfeeding and incidence of type 2 diabetes, in addition to recent systematic reviews (published within the past 5 years). We excluded measures of glucose metabolism that could not be used to establish a diagnosis of diabetes (e.g., changes in mean levels of fasting blood glucose in women without known diabetes) and other laboratory markers of abnormal glucose metabolism (e.g., measures of insulin levels). We included studies enrolling women with a history of gestational diabetes that reported on the incidence of type 2 diabetes.

\section{Systematic Reviews}

One systematic review rated low ROB examined the association between breastfeeding and maternal risk of subsequent type 2 diabetes (Table 23). ${ }^{158}$ The authors identified six prospective cohort studies (described in 5 publications) that evaluated the association between breastfeeding and incident type 2 diabetes. Three studies were set in the United States, and one each was set in Germany, China, and Australia. One study focused on women with gestational diabetes (N=304), and the others focused on an unselected sample of women with no diabetes at enrollment (sample sizes ranged from 1,829 to 83,585 participants). All studies defined exposure as duration 
of breastfeeding, and most (5 of 6) reported on multiple categories of breastfeeding exposure defined in months or years of breastfeeding. All studies controlled for a range of known confounders; however, specific measures varied across studies; most studies adjusted for age, smoking, alcohol, BMI, physical activity, and family history of diabetes, and some had further adjusted for income, education, and parity. Systematic review authors assessed the ROB of individual studies using the Newcastle-Ottawa Scale, rating it on a 0 to 9-point scale (with higher values indicating better quality); four studies were rated a 6 , one was rated a 7 , and one was rated an $8 .{ }^{158}$

Table 23. Breastfeeding and type 2 diabetes: Summary of published systematic review

\begin{tabular}{|c|c|c|c|c|c|c|c|c|}
\hline $\begin{array}{l}\text { First } \\
\text { Author, } \\
\text { Year }\end{array}$ & $\begin{array}{l}\text { Number of } \\
\text { Studies (N } \\
\text { Participants) } \\
\text { Study } \\
\text { Design }\end{array}$ & $\begin{array}{l}\text { Search Date } \\
\text { (Databases) }\end{array}$ & $\begin{array}{l}\text { Study } \\
\text { Characteristics }\end{array}$ & $\begin{array}{l}\text { Definition } \\
\text { of } \\
\text { Exposure }\end{array}$ & $\begin{array}{l}\text { Definition } \\
\text { of } \\
\text { Outcome }\end{array}$ & $\begin{array}{l}\text { Timing of } \\
\text { Followup }\end{array}$ & Results & ROB \\
\hline $\begin{array}{l}\text { Aune, } \\
2014^{158}\end{array}$ & $\begin{array}{l}6(273,961) \\
\text { prospective } \\
\text { cohort studies }\end{array}$ & $\begin{array}{l}\text { Inception } \\
\text { through } \\
\text { September } \\
2013 \\
\text { (PubMed, } \\
\text { Embase, } \\
\text { Ovid) }\end{array}$ & $\begin{array}{l}\text { Three studies } \\
\text { set in the U.S. } \\
\text { and one each in } \\
\text { Germany, } \\
\text { China, and } \\
\text { Australia; } \\
\text { studies enrolled } \\
\text { women between } \\
1989 \text { and } 2008 \\
\text { One focused on } \\
\text { women with } \\
\text { GDM; the } \\
\text { others focused } \\
\text { on women } \\
\text { without known } \\
\text { diabetes }\end{array}$ & $\begin{array}{l}\text { Measures } \\
\text { of BF } \\
\text { duration } \\
\text { (lifetime } \\
\text { and per- } \\
\text { child } \\
\text { categories } \\
\text { ) }\end{array}$ & $\begin{array}{l}\text { NR: any } \\
\text { measure } \\
\text { of type } 2 \\
\text { diabetes } \\
\text { appears } \\
\text { eligible }\end{array}$ & $\begin{array}{l}\text { Unclear; } \\
\text { studies } \\
\text { assessed } \\
\text { lifetime } \\
\text { duration of } \\
\text { BF }\end{array}$ & $\begin{array}{l}\text { Pooled RR } \\
\text { (95\% CI): } \\
\text { highest vs. } \\
\text { lowest BF } \\
\text { duration: } \\
0.68(0.57- \\
0.82) \\
\text { 3-mo } \\
\text { increase in } \\
\text { BF } \\
\text { duration } \\
\text { per child: } \\
0.89(0.77- \\
1.04) \\
1-y e a r \\
\text { increase in } \\
\text { total BF } \\
\text { duration: } \\
0.91(0.86- \\
0.96)\end{array}$ & Low \\
\hline
\end{tabular}

The review authors conducted meta-analyses using random effects models to calculate summary risk ratios (RRs) for different durations of breastfeeding. ${ }^{158}$ Authors first pooled studies based on comparisons of the highest versus lowest breastfeeding duration reported by each study; all included a comparison of "no breastfeeding," longest duration included $>3$ months (1 study), $\geq 6$ months ( 2 studies), $>23$ months' total duration ( 2 studies), and $\geq 4$ years ( study). The pooled RR for high versus low breastfeeding (6 studies, 273,961 participants) was 0.68 (95\% CI, 0.57 to $\left.0.82 ; I^{2} 74.7 \%\right)$. To explore heterogeneity, authors conducted sensitivity analyses excluding individual studies. When excluding results from the Nurses' Health Study I, ${ }^{6}$ the pooled RR (5 studies) was 0.62 (95\% CI, 0.56 to 0.69$)$ and heterogeneity was lower $\left(I^{2} 0 \%\right)$. Authors also reported on subgroup and meta-regression analyses and reported no significant heterogeneity between subgroups of studies based on country setting, number of cases, study quality scores, or extent to which individual studies adjusted for confounding factors. There was no evidence of publication bias with Egger's test, $\mathrm{p}=0.27$. 
Authors also conducted pooled analyses that examined the dose-response of different levels of breastfeeding duration across four studies (by computing study-specific linear trends across categories of breastfeeding duration); the summary RR was 0.91 (95\% CI, 0.86 to 0.96; $I^{2}$ 80.9\%) per 12 months' increase in lifetime duration of breastfeeding, and 0.89 (95\% CI, 0.77 to 1.04; $I^{2} 92.9 \%$ ) per 3 months' increase in duration of breastfeeding per child. ${ }^{158}$ No further sensitivity analyses were conducted, presumably because of the small number of studies available.

\section{Individual Studies}

\section{Characteristics}

We identified five cohort studies not included in the systematic review described above (Table 24). ${ }^{4,159-163}$ Two were limited to women with gestational diabetes, ${ }^{159,160,163}$ two included women who had no history of diabetes prior to delivery, ${ }^{4,162}$ and one reported on subgroups of women with and without gestational diabetes. ${ }^{161}$

Table 24. Breastfeeding and type 2 diabetes: Summary of individual studies

\begin{tabular}{|c|c|c|c|c|c|c|}
\hline $\begin{array}{l}\text { First } \\
\text { Author, } \\
\text { Year } \\
\text { ROB }\end{array}$ & $\begin{array}{l}\text { Study } \\
\text { Description } \\
\text { N } \\
\text { Participants }\end{array}$ & Population & $\begin{array}{l}\text { Definition of } \\
\text { Exposure }\end{array}$ & $\begin{array}{l}\text { Definition } \\
\text { of Type } 2 \\
\text { Diabetes } \\
\text { Timing of } \\
\text { Followup } \\
\end{array}$ & Results & $\begin{array}{l}\text { Confounders } \\
\text { Adjusted for }\end{array}$ \\
\hline $\begin{array}{l}\text { Chamberlai } \\
\text { n, } 2016^{159} \\
\text { High }\end{array}$ & $\begin{array}{l}\text { Cohort study } \\
\text { of women } \\
\text { with GDM } \\
\text { who gave } \\
\text { birth at a } \\
\text { regional } \\
\text { Australian } \\
\text { hospital from } \\
2004-2010 \\
289\end{array}$ & $\begin{array}{l}\text { Age } \\
\text { category } \\
\text { (y), \%: } \\
<25: 9 \\
\text { 25-29: } 20 \\
\text { 30-34: } 28 \\
35+: 44 \\
\% \\
\text { nonwhite: } \\
32^{a}\end{array}$ & $\begin{array}{l}\text { BF status at } \\
\text { hospital } \\
\text { discharge, } \\
\text { defined as } \\
\text { fully BF, } \\
\text { partially BF, or } \\
\text { no BF }\end{array}$ & $\begin{array}{l}\text { Positive } \\
\text { OGTT } \\
7 \text { yrs } \\
\text { postpartu } \\
\text { m }\end{array}$ & $\begin{array}{l}\text { HR }(95 \% \mathrm{Cl}) \\
\text { Partial vs. full BF (ref): } \\
2.34 \text { (1.23 to } 4.47) \\
\text { No vs. full BF (ref): } \\
\text { HR } 1.33 \text { (0.40 to } 4.37)\end{array}$ & None \\
\hline
\end{tabular}




\begin{tabular}{|c|c|c|c|c|c|c|}
\hline $\begin{array}{l}\text { First } \\
\text { Author, } \\
\text { Year } \\
\text { ROB }\end{array}$ & $\begin{array}{l}\text { Study } \\
\text { Description } \\
\text { N } \\
\text { Participants }\end{array}$ & Population & $\begin{array}{l}\text { Definition of } \\
\text { Exposure }\end{array}$ & $\begin{array}{l}\text { Definition } \\
\text { of Type } 2 \\
\text { Diabetes } \\
\text { Timing of } \\
\text { Followup }\end{array}$ & Results & $\begin{array}{l}\text { Confounders } \\
\text { Adjusted for }\end{array}$ \\
\hline $\begin{array}{l}\text { Gunderson, } \\
2012^{163} ; \\
\text { Gunderson, } \\
2015^{160} \\
\text { Medium }\end{array}$ & $\begin{array}{l}\text { Cohort study } \\
\text { of women } \\
\text { with GDM } \\
\text { who gave } \\
\text { birth at } \\
\text { Kaiser } \\
\text { Northern CA } \\
\text { hospitals } \\
\text { from 2008- } \\
2011 \\
959\end{array}$ & $\begin{array}{l}\text { Mean age: } \\
\text { 33-34 (SD } \\
\text { NR) } \\
\% \\
\text { nonwhite: } \\
77 \\
\text { \% with } \\
\text { GDM: } 100^{c}\end{array}$ & $\begin{array}{l}\text { BF duration/ } \\
\text { intensity } \\
\text { assessed } \\
\text { prospectively } \\
\text { via feeding } \\
\text { diaries, phone } \\
\text { calls, in- } \\
\text { person exams, } \\
\text { and monthly } \\
\text { mailed } \\
\text { questionnaires } \\
\text { Intensity } \\
\text { represents } \\
\text { cumulative } \\
\text { amount of } \\
\text { formula and } \\
\text { breast milk fed } \\
\text { since delivery } \\
\text { and the } \\
\text { intensity for } \\
\text { the past } 7 \\
\text { days } \\
\text { (measured at } \\
6-9 \text { wks after } \\
\text { delivery) }\end{array}$ & $\begin{array}{l}\text { Positive } \\
\text { OGTT or } \\
\text { diagnosis } \\
\text { obtained } \\
\text { from } \\
\text { EMRs }^{\text {b }} \\
2 \text { yrs post- } \\
\text { partum }\end{array}$ & $\begin{array}{l}\text { Adjusted HR }(95 \% \mathrm{Cl}) \text {, BF } \\
\text { group compared with } \\
\text { exclusive formula feeding: } \\
\text { Exclusive lactation: } \\
0.47(0.25 \text { to } 0.91) \\
\text { Mostly lactation: } \\
0.53(0.31 \text { to } 0.91) \\
\text { Mostly formula: } \\
0.65 \text { ( } 0.37 \text { to } 1.13) \\
\text { p for trend=0.017 } \\
\text { Adjusted HR (95\% Cl), BF } \\
\text { duration group compared } \\
\text { with } 0-2 \text { mos duration: } \\
>2-5 \text { mos: } \\
0.43(0.23 \text { to } 0.82) \\
>5-10 \text { mos: } \\
0.50(0.25 \text { to } 0.99) \\
>10 \text { mos: } \\
0.55 \text { ( } 0.31 \text { to } 1.01) \\
p \text { for trend=0.007 }\end{array}$ & $\begin{array}{l}\text { Age, } \\
\text { race/ethnicity, } \\
\text { education, } \\
\text { prepregnancy } \\
\text { BMI, GDM } \\
\text { treatment, sum of } \\
\text { prenatal oral } \\
\text { glucose tolerance } \\
\text { test Z score, } \\
\text { gestational age at } \\
\text { GDM diagnosis, } \\
\text { subsequent birth } \\
\text { (0 vs. 1) during 2- } \\
\text { yr followup, large } \\
\text { for gestational age } \\
\text { vs. not large for } \\
\text { gestational age } \\
\text { (reference), } \\
\text { hospital stay >3 } \\
\text { days, and } \\
\text { neonatal intensive } \\
\text { care unit } \\
\text { admission }\end{array}$ \\
\hline
\end{tabular}




\begin{tabular}{|c|c|c|c|c|c|c|}
\hline $\begin{array}{l}\text { First } \\
\text { Author, } \\
\text { Year } \\
\text { ROB }\end{array}$ & $\begin{array}{l}\text { Study } \\
\text { Description } \\
\text { N } \\
\text { Participants }\end{array}$ & Population & $\begin{array}{l}\text { Definition of } \\
\text { Exposure }\end{array}$ & $\begin{array}{l}\text { Definition } \\
\text { of Type } 2 \\
\text { Diabetes } \\
\text { Timing of } \\
\text { Followup }\end{array}$ & Results & $\begin{array}{l}\text { Confounders } \\
\text { Adjusted for }\end{array}$ \\
\hline $\begin{array}{l}\text { Martens, } \\
2016^{161} \\
\text { Medium }\end{array}$ & $\begin{array}{l}\text { Cohort study } \\
\text { of women } \\
\text { who gave } \\
\text { birth in } \\
\text { Manitoba } \\
\text { Canada } \\
\text { (1987-2011), } \\
\text { without } \\
\text { diabetes } \\
\text { before } \\
\text { pregnancy } \\
\text { 180,107 } \\
\text { women } \\
\text { (334,553 } \\
\text { deliveries) }\end{array}$ & $\begin{array}{l}\text { Mean age } \\
\text { (at } \\
\text { delivery): } \\
26-28 \text { (6) }\end{array}$ & $\begin{array}{l}\text { BF initiation } \\
\text { before hospital } \\
\text { discharge } \\
\text { (from hospital } \\
\text { administrative } \\
\text { database, } \\
\text { recorded by } \\
\text { nurses) }\end{array}$ & $\begin{array}{l}\text { One } \\
\text { hospital- } \\
\text { ization or } \\
\text { two } \\
\text { outpatient } \\
\text { visits with } \\
\text { a diabetes } \\
\text { diagnosis } \\
\text { code in a } \\
\text { 3- year } \\
\text { period } \\
\text { (using ICD } \\
\text { codes) } \\
\text { Maximum } \\
\text { of } 24 \text { yrs }\end{array}$ & $\begin{array}{l}\text { Adjusted HR ( } 95 \% \mathrm{Cl}) \text { for } \\
\text { incident DM, BF vs. not-BF: } \\
\text { With GDM: } \\
\text { First Nations: } \\
0.82 \text { (073 to } 0.92) \\
\text { Non-First Nations: } \\
0.78 \text { (0.69 to } 0.82) \\
\text { Without GDM: } \\
\text { First Nations: } \\
0.89 \text { (0.81 to 0.98) } \\
\text { Non-First Nations: } \\
0.73 \text { (0.68 to } 0.79)\end{array}$ & $\begin{array}{l}\text { Age at birth of } \\
\text { child, parity, } \\
\text { rurality, income } \\
\text { quintile, year of } \\
\text { delivery }\end{array}$ \\
\hline $\begin{array}{l}\text { Schwarz, } \\
2009^{4} \\
\text { Medium }\end{array}$ & $\begin{array}{l}\text { Cohort study } \\
\text { (WHI) } \\
\text { enrolling } \\
\text { generally } \\
\text { healthy, } \\
\text { postmeno- } \\
\text { pausal } \\
\text { women from } \\
\text { the U.S. } \\
\text { starting in } \\
\text { 1994 who } \\
\text { reported at } \\
\text { least one } \\
\text { live birth } \\
139,681\end{array}$ & $\begin{array}{l}\text { Mean age: } \\
63-64 \text { (SD } \\
\text { NR) } \\
\% \\
\text { nonwhite: } \\
14-19 \\
\text { \% with } \\
\text { GDM: NR }\end{array}$ & $\begin{array}{l}\text { Self-reported } \\
\text { lifetime BF } \\
\text { duration } \\
\text { assessed at } \\
\text { WHI } \\
\text { enrollment }\end{array}$ & 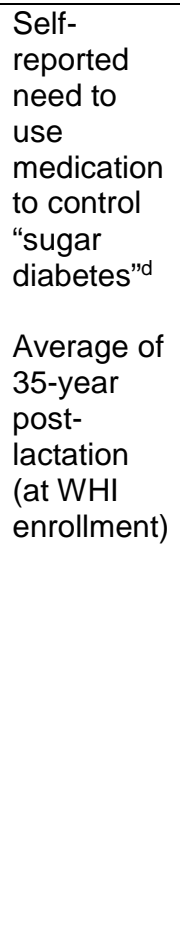 & $\begin{array}{l}\text { OR }(95 \% \mathrm{Cl}) \text {, lifetime } \\
\text { duration (mos) vs. never } \\
\text { BF: } \\
\text { 1-6: } 0.92(0.85 \text { to } 0.99) \\
7-12: 0.87(0.78 \text { to } 0.97) \\
\text { 13-23: } 0.74(0.65 \text { to } 0.84) \\
24+: 0.89(0.77 \text { to } 1.02) \\
\text { p for trend<0.0001 } \\
\geq 13: 0.80 \text { (NR); p<0.001 } \\
\text { Subgroup of women from } \\
\text { WHI cohort study who } \\
\text { provided information on } \\
\text { their weight at age } 18 \text { and } \\
\text { whether they were BF } \\
\text { (N=78,825): } \\
\text { OR (95\% Cl), lifetime } \\
\text { duration (mos) vs. never } \\
\text { BF: } \\
\text { 1-6: } 0.94(0.84 \text { to } 1.04) \\
7-12: 0.91(0.78 \text { to } 1.06) \\
13-23: 0.69(0.58 \text { to } 0.84) \\
24+: 0.99(0.82 \text { to } 1.19) \\
\text { p for trend=0.02 }\end{array}$ & $\begin{array}{l}\text { Age; race; parity; } \\
\text { age at } \\
\text { menopause; } \\
\text { education; } \\
\text { income; family } \\
\text { history (of } \\
\text { diabetes mellitus, } \\
\text { Ml or stroke); } \\
\text { physical activity; } \\
\text { energy, } \\
\text { cholesterol, fat, } \\
\text { fiber, and sodium } \\
\text { intakes; tobacco } \\
\text { history; HRT use; } \\
\text { aspirin use; and } \\
\text { MVI use }\end{array}$ \\
\hline
\end{tabular}




\begin{tabular}{|c|c|c|c|c|c|c|}
\hline $\begin{array}{l}\text { First } \\
\text { Author, } \\
\text { Year } \\
\text { ROB }\end{array}$ & $\begin{array}{l}\text { Study } \\
\text { Description } \\
\text { N } \\
\text { Participants }\end{array}$ & Population & $\begin{array}{l}\text { Definition of } \\
\text { Exposure }\end{array}$ & $\begin{array}{l}\text { Definition } \\
\text { of Type } 2 \\
\text { Diabetes }\end{array}$ & Results & $\begin{array}{l}\text { Confounders } \\
\text { Adjusted for }\end{array}$ \\
\hline $\begin{array}{l}\text { Zong, } \\
2016^{162} \\
\text { High }\end{array}$ & $\begin{array}{l}\text { Cohort study } \\
\text { enrolling } \\
\text { parous } \\
\text { women } \\
\text { without } \\
\text { diabetes } \\
\text { before } \\
\text { delivery who } \\
\text { completed } \\
\text { the } \\
\text { NHANES } \\
\text { survey } \\
(1999-2006) \\
4,779\end{array}$ & $\begin{array}{l}\text { Mean age } \\
46-51 \text { at } \\
\text { enrollment } \\
\text { (SD NR) } \\
\% \\
\text { nonwhite: } \\
27-34 \\
\text { \% with } \\
\text { GDM: NR }\end{array}$ & 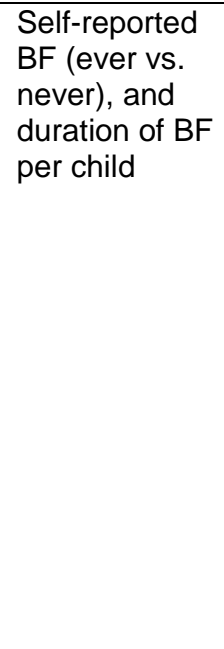 & $\begin{array}{l}\text { Self-report } \\
\text { physician- } \\
\text { diagnosed } \\
\text { diabetes, } \\
\text { current } \\
\text { use of } \\
\text { diabetes } \\
\text { medicatio } \\
\text { n or } \\
\text { HbA1c } \\
\geq 6.5 \% \\
\text { NA: } \\
\text { Cross- } \\
\text { sectional } \\
\text { data from } \\
\text { cohort } \\
\text { study }\end{array}$ & $\begin{array}{l}\text { OR }(95 \% \mathrm{Cl}) \text {, ever vs. } \\
\text { never } \mathrm{BF}: \\
0.75(0.58 \text { to } 0.97) \\
\text { OR ( } 95 \% \mathrm{Cl}) \text {, by } \mathrm{N} \text { children } \\
\text { BF for } \geq 1 \mathrm{mo}: \\
1-2 \text { vs. } 0: \\
0.84 \text { ( } 0.63 \text { to } 1.13) \\
3+\text { vs. } 0: \\
0.61(0.41 \text { to } 0.89)\end{array}$ & $\begin{array}{l}\text { Age, survey yrs, } \\
\text { ethnicity, country } \\
\text { of birth, education, } \\
\text { family history of } \\
\text { chronic disease, } \\
\text { smoking, alcohol, } \\
\text { physical activity, } \\
\text { and BMI }\end{array}$ \\
\hline $\begin{array}{l}\text { a Refers to } \\
\text { Aboriginal } \\
\text { b Per author } \\
\text { OGTT, ran } \\
\text { care from o } \\
{ }^{c} \text { To be incl } \\
9 \text { wks postp } \\
\text { d Per authol } \\
\text { diabetes we }\end{array}$ & $\begin{array}{l}\text { tage of populati } \\
\text { orres Strait Islan } \\
\text { a from health pl } \\
\text { lucose, or } \mathrm{HbA} \text { - } \\
\text { facilities. } \\
\text { women identifie } \\
\text { n. } \\
\text { dication use was } \\
\text { teligible to parti }\end{array}$ & $\begin{array}{l}\text { n that was ind } \\
\text { der. } \\
\text { ns were also us } \\
\text { c); self-report c } \\
\text { d with gestatio } \\
\text { validated on eI } \\
\text { ipate in the W. }\end{array}$ & $\begin{array}{l}\text { genous, defined as } \\
\text { diabetes with hea } \\
\text { al diabetes during } \\
\text { ollment by nurse } \\
\text { II. }\end{array}$ & $\begin{array}{l}\text { elf-identificati } \\
\text { tht diabetes-bas } \\
\text { h care provide } \\
\text { regnancy had } \\
\text { xamination of }\end{array}$ & $\begin{array}{l}\text { n as Aboriginal, Torres Strait Isl } \\
\text { d clinical laboratory testing (fast } \\
\text { diagnosis was allowed for wome } \\
\text { have a normal blood sugar mea } \\
\text { edication bottles. Women with }\end{array}$ & $\begin{array}{l}\text { nder, or } \\
\text { ing, 2-hour } \\
\text { n receiving } \\
\text { urement 6- } \\
\text { pe } 1\end{array}$ \\
\hline \multicolumn{7}{|c|}{$\begin{array}{l}\text { BF = breastfeeding; BMI = body mass index; } \mathrm{CA}=\text { California; } \mathrm{CI}=\text { confidence interval; } \mathrm{DM}=\text { diabetes mellitus; } \mathrm{EMR}= \\
\text { electronic medical record; GDM = gestational diabetes mellitus; } \mathrm{HR}=\text { hazard ratio; } \mathrm{HRT}=\text { hormone replacement therapy; ICD = } \\
\text { International Classification of Diseases; } \mathrm{MI}=\text { myocardial infarction; MVI = multivitamin; } \mathrm{N}=\text { number; NHANES = National } \\
\text { Health and Nutrition Examination Survey; NR = not reported; OGTT = oral glucose tolerance test; OR = odds ratio; } \mathrm{ROB}=\text { risk } \\
\text { of bias; SD = standard deviation; WHI = Women’s Health Initiative. }\end{array}$} \\
\hline $\begin{array}{l}\text { In th } \\
\text { two incl } \\
160,163 \text { an } \\
\text { set in the } \\
\text { based on } \\
\text { record fo } \\
\text { administ } \\
\text { codes). }{ }^{16} \\
\text { studies. } \\
\text { diaries, } \mathrm{t} \\
\text { categoriz } \\
\text { formula, } \\
\text { measure } \\
\text { and follo } \\
\text { and the c } \\
\text { Thre } \\
\text { diabetes. }\end{array}$ & $\begin{array}{l}\text { ee studies re } \\
\text { l women giv } \\
\text { ee enrolled u } \\
\text { ited States, }{ }^{1 \epsilon} \\
\text { oral glucose } \\
\text { agnoses mad } \\
\text { e databases } \\
\text { certainment } \\
\text { study measu } \\
\text { hone intervi } \\
\text { oreastfeeding } \\
\text { tlly formula, } \\
2 \text { years. }{ }^{160} \mathrm{~T} \\
\text { I women ove } \\
\text { followed w } \\
\text { idies reporte } \\
\text {, } 162 \text { Two stu }\end{array}$ & $\begin{array}{l}\text { porting on i } \\
\text { ng birth du } \\
\text { omen who } \\
\text { 0, } 163 \text { Austral } \\
\text { olerance te } \\
\text { e on other } \\
\text { o determin } \\
\text { of breastfe } \\
\text { red breastfe } \\
\text { ws, in-pers } \\
\text { exposure b } \\
\text { mostly lact } \\
\text { le two othe } \\
\text { r a longer ti } \\
\text { men for ur } \\
\text { on incider } \\
\text { dies were st }\end{array}$ & $\begin{array}{l}\text { 1cident diabet } \\
\text { ing a similar t } \\
\text { ave birth bet } \\
\text { a, }{ }^{159} \text { and Cana } \\
\text { t: }{ }^{159,160,163} \text { on } \\
\text { iteria (e.g., he } \\
\text { incident diab } \\
\text { ding exposure } \\
\text { eding duratior } \\
\text { n exams, and } \\
\text { ased on interv } \\
\text { tion, and exc } \\
\text { studies meas } \\
\text { ne frame; one } \\
\text { to } 24 \text { years. } \\
\text { diabetes amo } \\
\text { in the Unitec }\end{array}$ & $\begin{array}{l}\text { s among w } \\
\text { me frame ( } \\
\text { een } 1987 \text { a } \\
\text { la. }{ }^{161} \text { Two } \\
\text { of these a } \\
\text { moglobin } \\
\text { tes (using } \\
\text { and length } \\
\text { and exclus } \\
\text { monthly m } \\
\text { ews at } 6 \text { to } \\
\text { isive lactat } \\
\text { red breastf } \\
\text { followed } \mathrm{n}\end{array}$ & $\begin{array}{l}\text { omen with gestational dia } \\
\text { pproximately } 2004 \text { to } 20 \\
\text { ad } 2011 .{ }^{161} \text { One study ea } \\
\text { neasured incident type } 2 \\
\text { so searched an electronic } \\
1 \mathrm{c}){ }^{160}, 163 \text { The third relie } \\
\text { ospital and outpatient di } \\
\text { of followup differed acro } \\
\text { vity prospectively (via fe } \\
\text { iled questionnaires) and } \\
9 \text { weeks postpartum (exc } \\
\text { on); incident diabetes wa } \\
\text { oding status at hospital } \\
\text { omen for } 7 \text { years postpar }\end{array}$ & $\begin{array}{l}\text { betes, } \\
11),{ }^{159}, \\
\text { h was } \\
\text { diabetes } \\
\text { medical } \\
\text { d on } \\
\text { gnosis } \\
\text { ed } \\
\text { eding } \\
\text { usive } \\
\text { ischarge } \\
\text { um }^{159}\end{array}$ \\
\hline
\end{tabular}


the two studies set in the United States, one enrolled parous women participating in the WHI (an average of 35 years postlactation), ${ }^{4}$ and the other enrolled women participating in the National Health and Nutrition Examination Survey (NHANES) survey (1999-2006) with a mean age of 46 to 51 years. ${ }^{162}$ Both assessed breastfeeding exposure based on self-reported lifetime breastfeeding duration. In the WHI, diabetes status was measured based on self-reported need to take medication for "sugar diabetes"; although the study did not make a distinction between whether women had been treated for type 1 or type 2 diabetes, women with type 1 diabetes were not eligible to participate in the WHI. ${ }^{164}$ The study enrolling women participating in the NHANES study ascertained diabetes status based on self-report, use of medications for diabetes, or measured hemoglobin A1c greater than 6.5 percent. ${ }^{162}$ The study set in Canada relied on administrative databases to categorize breastfeeding status at discharge and incident diabetes. ${ }^{161}$

Two studies were rated high $\mathrm{ROB}^{159,162}$ (primarily due to selection bias and no adjustment for potential confounding factors), and the other studies were rated medium ROB. The two studies enrolling women who had gestational diabetes followed women for a relatively short period of time (2 to 7 years postpartum), which may be inadequate followup to determine a difference in the incidence of type 2 diabetes. ${ }^{159,160}$

\section{Results}

Despite differences in study design, breastfeeding definition, and outcome ascertainment, all studies found a lower incidence of diabetes among women who initiated breastfeeding or breastfed for longer durations or increased intensity (Table 24). Results were consistent with the findings of the published systematic review described above. ${ }^{158}$

In the three studies reporting outcomes among women with gestational diabetes, two followed women for 2 years postpartum and categorized breastfeeding exposure based on duration and exclusivity. ${ }^{159,160}$ One of these followed women prospectively and found a lower rate of incident type 2 diabetes among women who reported exclusive breastfeeding at 6 to 9 weeks postpartum than women who exclusively formula fed (HR, 0.47; 95\% CI, 0.24 to 0.93). ${ }^{160}$ Women who mostly lactated or mostly formula fed had a lower incidence of type 2 diabetes than women who exclusively formula fed, but the difference was not statistically significant (Table 24). The second study ascertained breastfeeding exposure via hospital discharge records (as fully, partially, or no breastfeeding); compared with women who fully breastfed, women who partially breastfed at discharge had a higher rate of incident type 2 diabetes over 2 years (HR 2.34; 95\% CI, 1.23 to 4.47); however, there was no significant difference in incident type 2 diabetes between women not breastfeeding and those fully breastfeeding at discharge (HR 1.33; 95\% CI, 0.40 to 4.37$).{ }^{159}$ One Canadian study categorized women as breastfeeding or not breastfeeding at hospital discharge and reported outcomes separately for women with gestational diabetes who were First Nations or Non-First Nations; incident diabetes was measured over a variable time frame postpartum depending on year of enrollment (to a maximum followup of 24 years). ${ }^{161}$ Incident diabetes was lower among women breastfeeding at discharge than those not breastfeeding in both First Nations women (HR 0.82; 95\% CI, 0.73 to 0.92) and Non-First Nations women (HR 0.78; 95\% CI, 0.69 to 0.82). ${ }^{161}$

Of the three studies enrolling women without known gestational diabetes, two reported outcomes based on lifetime duration of breastfeeding. ${ }^{4,162}$ The study enrolling women participating in the WHI found that increasing duration of breastfeeding was associated with lower prevalence of diabetes ( $\mathrm{p}$ for trend $<0001$ ); ${ }^{4}$ women reporting a cumulative lifetime duration of lactation equal to or greater than 13 months were less likely to have diabetes than 
those who did not breastfeed $(\mathrm{OR}=0.80, \mathrm{p}<0.001) .{ }^{4}$ Similarly, the study enrolling women participating in the NHANES survey found a lower rate of diabetes among women who breasted one or two infants for at least 1 month each (OR, 0.83; 95\% CI, 0.61 to 1.13) and those who breastfed three or more infants for at least 1 month each (OR, 0.63; 95\% CI, 0.44 to 0.91) than women who did not breastfeed. ${ }^{162}$ Finally, in the Canadian study (described above), there was a lower rate of incident diabetes among both First Nations and Non-First Nations women who breastfed at discharge than those who were not breastfeeding at discharge. ${ }^{161}$

\section{Fracture}

\section{Background}

Pregnancy and lactation lead to changes in bone metabolism. During lactation, the body can meet the burden of demand of calcium by increasing resorption from maternal bones. However, such losses typically reverse themselves over time. ${ }^{165}$ Additionally, increased weight during gestation and consequent bone strengthening (and expansion) in response to greater loads on the maternal skeleton may also serve to counteract the effects of loss of bone mass. ${ }^{166}$ This review focuses on the long-term and clinically relevant implications of lactation by evaluating the risk of fractures rather than the risk of loss in bone mass. Other variables that could influence the relationship between lactation and fractures include age, hormone replacement therapy, physical activity, parity, and BMI. ${ }^{165}$

\section{Methods}

We included all studies that examined the link between breastfeeding and fracture (casecontrol or cohort studies). Although recent systematic reviews (published within the last 5 years) were eligible, we did not find one that was relevant to the scope of this review rated low or unclear risk of bias. We excluded studies with surrogate measures of fracture (e.g., fracture risk score or index), bone turnover markers, or with measures of osteoporosis only.

\section{Individual Studies}

\section{Characteristics}

We included a total of six case-control studies ${ }^{167-172}$ and five cohort studies ${ }^{165,173-176}$ that examined the risk of fractures in relation to a history of breastfeeding (Table 25). The casecontrol studies included 1,609 cases with hip, forearm, or vertebral fractures and 2,967 controls. The five cohort studies (constructed from cross-sectional data in two cases) ${ }^{173,174}$ included 97,150 participants, with most $(92,980)$ from a single study. ${ }^{176}$ Mean ages of enrolled women ranged from 58 to 75 . Five studies were conducted in the United States; one study each was conducted in Australia, Greece, Hong Kong, Spain, South Korea, and Sweden. Four studies were rated high ROB and the rest were rated medium. Two cohort studies did not report the number of cases; the rate of fractures in the other two studies was reported as 15.6 percent in one study ${ }^{175}$ and 1.2 percent in a second study. ${ }^{173} \mathrm{~A}$ fifth cohort study reported the rate of incident hip fractures as $1.27 \%$ over the followup period of 7.9 years. ${ }^{176}$ 
Table 25. Breastfeeding and fractures: Summary of individual studies

Author, Year Study Description

ROB

$\mathrm{N}$ of Participants

Exposure(s)

$\begin{array}{lll}\begin{array}{l}\text { Definition of } \\ \text { Outcome(s) }\end{array} & \text { Results } & \begin{array}{l}\text { Confounders } \\ \text { Adjusted for }\end{array}\end{array}$

\begin{tabular}{ll}
\hline Alderman, 1986 $^{167}$ & $\begin{array}{l}\text { Case-control study of } \\
\text { women between 50 and } \\
\text { Medium }\end{array}$ \\
& 74 yrs of age in King \\
County, Washington; \\
cases seeking orthopedic \\
care, controls from general \\
population (door-to-door \\
recruitment) \\
\\
Cases: 355 women who \\
sought care from \\
orthopedists between \\
1976 and 1980; controls: \\
562 unmatched women \\
from the same county
\end{tabular}

Timing of

Followup

Hip or forearm

Age range:

Duration of

lactation: sum of

fractures

OR $(95 \% \mathrm{Cl})$ for duration

Age, relative weight,

the number of mos

of lactation

associated with

NA: Case-

of lactation versus 0 mos

and exogenous

each birth,

categorized as 0,1

to 12,13 to 24 , or

more than 24 mos $^{a}$

1-12 mos: 1.2 (0.73 to

estrogen use

control

1.95)

13-24 mos: 0.7 (0.34 to

1.29)

$>24$ mos: 0.8 (0.38 to

1.51)

52 unmatched women 


\begin{tabular}{|c|c|c|c|c|c|c|}
\hline $\begin{array}{l}\text { First Author, Year } \\
\text { ROB }\end{array}$ & $\begin{array}{l}\text { Study Description } \\
\text { N of Participants }\end{array}$ & Population & $\begin{array}{l}\text { Definition of } \\
\text { Exposure(s) }\end{array}$ & $\begin{array}{l}\text { Definition of } \\
\text { Outcome(s) } \\
\text { Timing of } \\
\text { Followup }\end{array}$ & Results & $\begin{array}{l}\text { Confounders } \\
\text { Adjusted for }\end{array}$ \\
\hline $\begin{array}{l}\text { Chan, } 1996^{168} \\
\text { Medium }^{b}\end{array}$ & $\begin{array}{l}\text { Case-control study of } \\
\text { Chinese women ages 70- } \\
79 \text { yrs who were living in } \\
\text { three housing blocks } \\
\text { under a Geriatric Priority } \\
\text { Housing Scheme in } \\
\text { Shatin, Hong Kong } \\
\text { Cases: } 144 \text { women with } \\
\text { one or more definite } \\
\text { fractures; controls: } 163 \\
\text { women with no fractures }\end{array}$ & $\begin{array}{l}\text { Mean age: } \\
75 \text { yrs }\end{array}$ & $\begin{array}{l}\text { Duration of } \\
\text { lactation: } \\
\text { categorized as } 0,1 \\
\text { to }<24 \text {, or } \geq 24 \text { mos }\end{array}$ & $\begin{array}{l}\text { Diagnosis of } \\
\text { vertebral } \\
\text { fracture (when } \\
\text { any of the } 3 \\
\text { vertebral ratios } \\
\text { [anterior to } \\
\text { posterior, } \\
\text { middle to } \\
\text { posterior and } \\
\text { posterior to } \\
\text { posterior } \\
\text { ratios] } \\
\text { compare with } \\
\text { the vertebral } \\
\text { bodies above } \\
\text { and below the } \\
\text { vertebra of } \\
\text { interest) was } 3 \\
\text { SD or more } \\
\text { away from the } \\
\text { mean } \\
\text { NA: Case- } \\
\text { control }\end{array}$ & $\begin{array}{l}\text { OR }(95 \% \mathrm{Cl}) \text { for duration } \\
\text { of lactation vs. } 0 \text { mos } \\
1 \text { to <24 mos: } 0.7 \text { ( } 0.4 \text { to } \\
1.3) \\
\geq 24 \text { mos: } 0.6 \text { (0.3 to } 1.0)\end{array}$ & Age \\
\hline
\end{tabular}




\begin{tabular}{|c|c|c|c|c|c|c|}
\hline $\begin{array}{l}\text { First Author, Year } \\
\text { ROB }\end{array}$ & $\begin{array}{l}\text { Study Description } \\
\text { N of Participants }\end{array}$ & Population & $\begin{array}{l}\text { Definition of } \\
\text { Exposure(s) }\end{array}$ & $\begin{array}{l}\text { Definition of } \\
\text { Outcome(s) } \\
\text { Timing of } \\
\text { Followup }\end{array}$ & Results & $\begin{array}{l}\text { Confounders } \\
\text { Adjusted for }\end{array}$ \\
\hline $\begin{array}{l}\text { Crandall, } 2017^{176} \\
\text { Medium }\end{array}$ & $\begin{array}{l}\text { Cohort study, using data } \\
\text { from the WHI observational } \\
\text { study, of } 92,980 \\
\text { postmenopausal } \\
\text { participants ages } 50-79 \text { yrs } \\
92,980\end{array}$ & $\begin{array}{l}\text { Mean age } \\
\text { (SD): } 64 \\
(7.4) \text { yrs }\end{array}$ & $\begin{array}{l}\text { Average duration of } \\
\text { BF per child, } \\
\text { categorized as } 0,1- \\
6,7-12,13-23 \text {, and } \\
\geq 24 \text { mos }\end{array}$ & $\begin{array}{l}\text { Physician- } \\
\text { adjudicated hip } \\
\text { fractures } \\
\text { Self-reported } \\
\text { incident clinical } \\
\text { fractures } \\
\text { Within } 7.9 \text { yrs }\end{array}$ & $\begin{array}{l}\text { Incident hip fractures: HR } \\
(95 \% \mathrm{Cl}) \text { for average } \\
\text { duration of BF vs. } 0 \text { mos } \\
1-6 \text { mos: } 0.85 \text { ( } 0.71 \text { to } \\
1.01) \\
7-12 \text { mos: } 0.77 \text { ( } 0.60 \text { to } \\
0.99) \\
13-23 \text { mos: } 0.880 .68 \text { to } \\
1.15) \\
\geq 24 \text { mos: } 0.92 \text { ( } 0.67 \text { to } \\
1.27) \\
\text { Incident clinical fractures: } \\
\text { HR (95\% CI) for average } \\
\text { duration of BF vs. } 0 \text { mos } \\
1-6 \text { mos: } 0.96 \text { ( } 0.84 \text { to } \\
1.10) \\
7-12 \text { mos: } 0.92 \text { ( } 0.76 \text { to } \\
1.11) \\
13-23 \text { mos: } 0.93 \text { (0.76 to } \\
1.15) \\
\geq 24 \text { mos: } 1.12 \text { (0.88 to } \\
1.42)\end{array}$ & $\begin{array}{l}\text { Age; race-ethnicity; } \\
\text { education; smoking } \\
\text { status; alcohol } \\
\text { consumption; } \\
\text { hysterectomy; } \\
\text { bilateral } \\
\text { oophorectomy; years } \\
\text { since menopause; } \\
\text { previous fracture age } \\
55 \text { yrs or older; } \\
\text { parental history of } \\
\text { fracture; number of } \\
\text { falls in the past } 12 \\
\text { mos; estrogen use, } \\
\text { route of delivery, and } \\
\text { recent use; use of } \\
\text { prescription } \\
\text { osteoporosis } \\
\text { medication, } \\
\text { aromatase inhibitors, } \\
\text { tamoxifen, } \\
\text { antidepressants, } \\
\text { corticosteroids, } \\
\text { proton pump } \\
\text { inhibitors, } \\
\text { antiepileptics, } \\
\text { antineoplastics, and } \\
\text { thiazolidinedione; } \\
\text { BMl; total energy } \\
\text { expenditure per week } \\
\text { from physical } \\
\text { activities; total } \\
\text { calcium intake; total } \\
\text { vitamin D intake at } \\
\text { baseline; and weight } \\
\text { change since age } 35 \\
\text { yrs }\end{array}$ \\
\hline
\end{tabular}




\begin{tabular}{|c|c|c|c|c|c|c|}
\hline $\begin{array}{l}\text { First Author, Year } \\
\text { ROB }\end{array}$ & $\begin{array}{l}\text { Study Description } \\
\text { N of Participants }\end{array}$ & $\begin{array}{l}\text { Populatio } \\
\text { n }\end{array}$ & $\begin{array}{l}\text { Definition of } \\
\text { Exposure(s) }\end{array}$ & $\begin{array}{l}\text { Definition of } \\
\text { Outcome(s) } \\
\text { Timing of } \\
\text { Followup }\end{array}$ & Results & $\begin{array}{l}\text { Confounders } \\
\text { Adjusted for }\end{array}$ \\
\hline $\begin{array}{l}\text { Cumming, } 1993^{170} \\
\text { Medium }^{\mathrm{b}}\end{array}$ & $\begin{array}{l}\text { Case-control study of } \\
\text { women ages } 65 \text { yrs or older } \\
\text { living in a defined region in } \\
\text { Sydney, Australia, during } \\
\text { 1990-1991 } \\
\text { Cases: } 174 \text { women were } \\
\text { from } 12 \text { hospitals; controls: } \\
137 \text { selected using an area } \\
\text { probability sampling } \\
\text { method, with additional } \\
\text { sampling from nursing } \\
\text { homes }\end{array}$ & $\begin{array}{l}\text { Mean age } \\
\text { NR }\end{array}$ & $\begin{array}{l}\text { Ever BF vs. never } \\
\text { BF } \\
\text { Average duration of } \\
\text { BF per child, } \\
\text { categorized as } 0 \text {, } \\
0.5-3,3-6,6-9 \text {, and } \\
>9 \text { mos }\end{array}$ & $\begin{array}{l}\text { Hip fractures } \\
\text { presenting to } \\
\text { the hospitals } \\
\text { NA: Case- } \\
\text { control }\end{array}$ & $\begin{array}{l}\text { OR ( } 95 \% \mathrm{Cl}) \text { for ever BF } \\
\text { vs. never } \mathrm{BF} \\
0.55(0.10 \text { to } 2.90) \\
\text { OR ( } 95 \% \mathrm{Cl}) \text { for average } \\
\text { duration of BF vs. never } \\
\text { BF } \\
0.5-3 \text { mos: } 0.64 \text { ( } 0.13 \text { to } \\
3.06) \\
3-6 \text { mos: } 0.79 \text { ( } 0.18 \text { to } \\
3.51) \\
6-9 \text { mos: } 0.41 \text { ( } 0.09 \text { to } \\
1.82) \\
>9 \text { mos: } 0.24 \text { (0.04 to } \\
1.53)\end{array}$ & $\begin{array}{l}\text { Age, BMI, history of } \\
\text { HRT use, current } \\
\text { slate of psychotropic } \\
\text { medications, current } \\
\text { smoking status, } \\
\text { current dairy product } \\
\text { consumption, score } \\
\text { on mental state } \\
\text { questionnaire, current } \\
\text { physical activity } \\
\text { (number of hours of } \\
\text { work in the house or } \\
\text { garden per week), } \\
\text { and health status } \\
\text { (number of self- } \\
\text { reported illnesses) }\end{array}$ \\
\hline $\begin{array}{l}\text { Hoffman, } 1993^{169} \\
\text { Medium }^{\mathrm{b}}\end{array}$ & $\begin{array}{l}\text { Case-control study of } \\
\text { women ages } 45 \text { or older } \\
\text { in New York and } \\
\text { Philadelphia between } \\
1987-89 \\
174 \text { cases, with } \\
\text { radiologically confirmed } \\
\text { first hip fracture, from } 30 \\
\text { hospitals in New York } \\
\text { and Philadelphia (103 } \\
\text { parous); } 174 \text { controls } \\
\text { from general surgical } \\
\text { and orthopedic services } \\
\text { during the same time } \\
\text { period and were } \\
\text { frequency-matched to } \\
\text { cases by age and } \\
\text { hospital (123 parous) }\end{array}$ & $\begin{array}{l}71 \% \text { over age } \\
75\end{array}$ & $\begin{array}{l}\text { Ever BF vs. never } \\
\text { BF } \\
\text { Lactated } \leq 12 \text { mos } \\
\text { or }>12 \text { mos }\end{array}$ & $\begin{array}{l}\text { Radiologically } \\
\text { confirmed first } \\
\text { hip fracture } \\
\text { NA: Case- } \\
\text { control }\end{array}$ & $\begin{array}{l}\text { OR ( } 95 \% \mathrm{Cl} \text { for ever vs. } \\
\text { never BF } \\
\text { All women: } 0.87 \text { ( } 0.47 \text { to } \\
1.61) \\
\text { Parous women: } 0.66 \text { ( } 0.41 \\
\text { to } 1.05) \\
\text { OR ( } 95 \% \text { Cl) for duration } \\
\text { of BF vs. never BF } \\
\text { All women } \\
>12 \text { mos: } 0.64 \text { ( } 0.32 \text { to } \\
1.29) \\
\leq 12 \text { mos: } 0.67 \text { ( } 0.39 \text { to } \\
1.13) \\
\text { Parous women } \\
>12 \text { mos: } 1.08 \text { ( } 0.45 \text { to } \\
2.60) \\
\leq 12 \text { mos: } 0.80 \text { ( } 0.42 \text { to } \\
1.55)\end{array}$ & $\begin{array}{l}\text { Hospital of } \\
\text { recruitment, age } \\
\text { group, and age for } \\
\text { models that included } \\
\text { all women; } \\
\text { additionally, adjusted } \\
\text { for number of live } \\
\text { births for models } \\
\text { restricted to parous } \\
\text { women }\end{array}$ \\
\hline
\end{tabular}




\begin{tabular}{|c|c|c|c|c|c|c|}
\hline $\begin{array}{l}\text { First Author, Year } \\
\text { ROB }\end{array}$ & $\begin{array}{l}\text { Study Description } \\
\text { N of Participants }\end{array}$ & Population & $\begin{array}{l}\text { Definition of } \\
\text { Exposure(s) }\end{array}$ & $\begin{array}{l}\text { Definition of } \\
\text { Outcome(s) } \\
\text { Timing of } \\
\text { Followup }\end{array}$ & Results & $\begin{array}{l}\text { Confounders } \\
\text { Adjusted for }\end{array}$ \\
\hline $\begin{array}{l}\text { Hwang, 2016 } \\
\text { High }\end{array}$ & $\begin{array}{l}\text { Cohorts constructed } \\
\text { from the KHANES, a } \\
\text { nationwide, population- } \\
\text { based, cross-sectional } \\
\text { survey } \\
1,222\end{array}$ & $\begin{array}{l}\text { Mean age } \\
(S D): 63(8.8) \\
\text { yrs }\end{array}$ & $\begin{array}{l}\text { BF for } 7-18 \text { mos, } \\
19-36 \text { mos, and } \geq 37 \\
\text { mos vs. } 0-6 \text { mos }\end{array}$ & $\begin{array}{l}\text { Self-reported } \\
\text { lumbar spine } \\
\text { fracture or hip } \\
\text { fracture } \\
\text { NA: Cross- } \\
\text { sectional data } \\
\text { from cohort } \\
\text { study }\end{array}$ & $\begin{array}{l}\mathrm{p}=0.026 \text { for trend of } \\
\text { positive correlation } \\
\text { between BF duration and } \\
\text { lumbar spine fractures } \\
\mathrm{p}=0.322 \text { for trend of } \\
\text { positive correlation } \\
\text { between BF duration and } \\
\text { hip fractures }\end{array}$ & None \\
\hline $\begin{array}{l}\text { Kreiger, } 1982^{171} \\
\text { High }^{b}\end{array}$ & $\begin{array}{l}\text { Case-control study of } \\
\text { women between } 45 \text { and } \\
74 \text { yrs of age in } \\
\text { Connecticut recruited } \\
\text { from 1977-79 } \\
\text { Cases: } 98 \text { women with } \\
\text { hip fractures; controls: } \\
83 \text { trauma cases from } \\
\text { the same hospitals }\end{array}$ & $\begin{array}{l}\text { Age range: } \\
45-74 \text { yrs }\end{array}$ & $\begin{array}{l}\text { BF (12 mos } \\
\text { increase) }\end{array}$ & $\begin{array}{l}\text { First hip } \\
\text { fracture } \\
\text { confirmed by } \\
\text { radiography } \\
\text { identified from } \\
\text { hospital record } \\
\text { NA: Case- } \\
\text { control }\end{array}$ & $\begin{array}{l}\text { OR, } 0.50(95 \% \mathrm{Cl}, 0.20 \text { to } \\
0.90)\end{array}$ & $\begin{array}{l}\text { Age, Quetelet index, } \\
\text { bilateral ovariectomy, } \\
\text { and estrogen } \\
\text { replacement therapy }\end{array}$ \\
\hline $\begin{array}{l}\text { Lambrinoudaki, } \\
2015^{174} \\
\text { High }\end{array}$ & $\begin{array}{l}\text { Cohort constructed from } \\
\text { cross-sectional survey } \\
\text { of all postmenopausal } \\
\text { women who presented } \\
\text { in a menopause clinic } \\
\text { between August } 2007 \\
\text { and July } 2013 \text { in Greece } \\
454\end{array}$ & $\begin{array}{l}\text { Mean age } \\
(S D): 57(7.1) \\
\text { yrs }\end{array}$ & Months of lactation & $\begin{array}{l}\text { Radiologically } \\
\text { assessed } \\
\text { vertebral } \\
\text { fracture } \\
\text { NA: Cross- } \\
\text { sectional data } \\
\text { from cohort } \\
\text { study }\end{array}$ & $\begin{array}{l}\text { OR }(95 \% \mathrm{Cl}) \text { for } \\
\text { presenting with at least } \\
\text { one vertical fracture } \\
1.03(0.99 \text { to } 1.06) \\
p=0.127\end{array}$ & None \\
\hline
\end{tabular}




\begin{tabular}{|c|c|c|c|c|c|c|}
\hline $\begin{array}{l}\text { First Author, } \\
\text { Year } \\
\text { ROB }\end{array}$ & $\begin{array}{l}\text { Study Description } \\
\text { N of Participants }\end{array}$ & Population & $\begin{array}{l}\text { Definition of } \\
\text { Exposure(s) }\end{array}$ & $\begin{array}{l}\text { Definition of } \\
\text { Outcome(s) } \\
\text { Timing of } \\
\text { Followup }\end{array}$ & Results & $\begin{array}{l}\text { Confounders } \\
\text { Adjusted for }\end{array}$ \\
\hline $\begin{array}{l}\text { Michaelsson, } \\
2001^{172} \\
\text { Medium }^{b}\end{array}$ & $\begin{array}{l}\text { Case-control study of } \\
\text { Swedish } \\
\text { postmenopausal } \\
\text { women ages } 50-81 \text { yrs } \\
\text { between October } 1993 \\
\text { and February } 1995 \\
\\
\text { Cases: } 664 \text { women with } \\
\text { hip fractures; controls: } \\
1,848 \text { women randomly } \\
\text { selected from the } \\
\text { population register }\end{array}$ & $\begin{array}{l}\text { Age range: } \\
50-81 \text { yrs, }\end{array}$ & $\begin{array}{l}\text { BF (3 mos } \\
\text { increase) } \\
\text { Total months of BF, } \\
\text { categorized as } 6-10 \\
\text { mos } 11-16 \text { mos } \\
>16 \text { mos vs. } 1-5 \\
\text { mos }\end{array}$ & $\begin{array}{l}\text { Hip fracture } \\
\text { based on clinical } \\
\text { or register } \\
\text { records } \\
\text { NA: Case-control }\end{array}$ & $\begin{array}{l}\text { OR }(95 \% \mathrm{Cl}) \text { for BF (3 } \\
\text { mos increase) } \\
0.98(0.95 \text { to } 1.01) \\
\text { OR ( } 95 \% \mathrm{Cl}) \text { for total } \\
\text { months BF vs. } 1-5 \text { mos } \\
6-10 \text { mos: } 0.87 \text { (0.68 to } \\
1.11) \\
11-16 \text { mos: } 0.86 \text { (0.66 to } \\
1.12) \\
>16 \text { mos: } 0.86 \text { (0.67 to } \\
1.12)\end{array}$ & $\begin{array}{l}\text { Age, HRT use, OC } \\
\text { use, and BMl }\end{array}$ \\
\hline $\begin{array}{l}\text { Mori, } 2015^{165} \\
\text { High }\end{array}$ & $\begin{array}{l}\text { Cohort study of pre- or } \\
\text { early perimenopausal } \\
\text { women from the Study } \\
\text { of Women's Health } \\
\text { Across the Nation } \\
\text { (United States): ages } \\
42-52 \text { yrs } \\
2,239\end{array}$ & $\begin{array}{l}\text { Age range: } \\
42-52 \text { yrs } \\
\text { Median age } \\
\text { (IQR): } \\
46(44-48) \text { yrs }\end{array}$ & $\begin{array}{l}\text { Accumulated length } \\
\text { of lactation before } \\
\text { age } 42\end{array}$ & $\begin{array}{l}\text { Self-reported } \\
\text { nondigital } \\
\text { noncraniofacial } \\
\text { fractures after } \\
\text { age } 42 \text { ( } 95 \% \text { of } \\
\text { them confirmed } \\
\text { by medical } \\
\text { records) } \\
\text { Median } 15.7 \text { yrs }\end{array}$ & $\begin{array}{l}\mathrm{HR}, 0.97(95 \% \mathrm{Cl}, 0.92 \text { to } \\
1.02) \text { per every additional } \\
6 \text { mos of lactation }(p=N S)\end{array}$ & $\begin{array}{l}\text { Race/ethnicity, BMI, } \\
\text { smoking status, } \\
\text { smoking pack-years, } \\
\text { alcohol consumption, } \\
\text { physical activity, } \\
\text { employment status, } \\
\text { diabetes, } \\
\text { hyperthyroidism, } \\
\text { supplementary } \\
\text { calcium, } \\
\text { supplementary } \\
\text { vitamin D, use of sex } \\
\text { steroid hormone } \\
\text { pill/patch injection, } \\
\text { birth control pills, } \\
\text { depo-provera } \\
\text { injection, oral } \\
\text { corticosteroids, } \\
\text { proton pump } \\
\text { inhibitors, other bone- } \\
\text { adverse medications, } \\
\text { study site }\end{array}$ \\
\hline
\end{tabular}




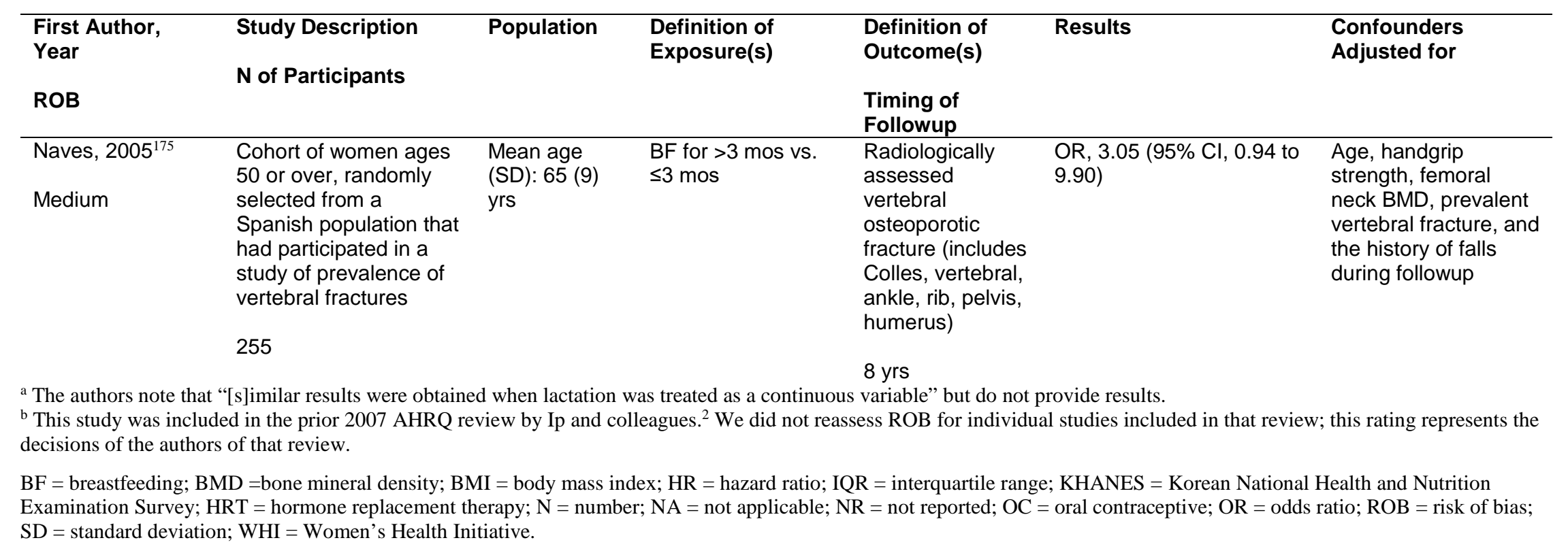


All except two studies reported on hip, vertebral, or forearm fractures identified from hospital or clinical records (with or without radiography confirmations). One study relied on recall for fracture outcomes. A second relied on adjudication by physicians to confirm hip fractures and recall for nonhip fractures. ${ }^{176}$ Of the six case-control studies, two identified matched or unmatched general population controls living in the same area ${ }^{167,172}$ and three studies used hospital controls. ${ }^{169-171}$ In the sixth study, ${ }^{168}$ women with vertebral fractures (classified according to the radiological diagnoses) were defined as definite or doubtful cases, and the remaining enrolled subjects without fractures were classified as control subjects; our analysis is restricted to comparisons of definite cases versus controls. Assessments of breastfeeding history were based on subjects' long-term recalls in all studies.

Studies defined exposures as ever versus never breastfeeding, total breastfeeding, and duration of breastfeeding per child. For studies reporting on duration of breastfeeding, results were reported as categorical variables with cut points that varied by study or as a continuous variable. No study reported on exclusive breastfeeding.

\section{Results}

With the exception of two high ROB studies, ${ }^{171,173}$ no study reported a statistically significant association between breastfeeding and fracture. The majority of studies reported lower odds of fractures with greater breastfeeding duration, but the results were generally not statistically significant. We rated the SOE as low for no association.

\section{Postpartum Depression}

\section{Background}

According to 2012 data from the PRAMS, nearly 12 percent of postpartum women reported a history of postpartum depressive symptoms. ${ }^{177}$ Women with postpartum depression are at increased risk of maternal suicide, infanticide, and impaired maternal sensitivity and attachment with the infant. ${ }^{178-181}$ Women with depression are also less likely to engage in enriching interactions (e.g., reading, singing) with their children. ${ }^{182}$ Prevention of postpartum depression is thus a major public health priority. Breastfeeding is thought to affect depression risk through the hormone oxytocin, which is implicated in maternal bonding, and through modulation of the hypothalamic pituitary adrenal axis.

Elucidating the relationship between breastfeeding and postpartum depression is challenging, because women with depression may have difficulty initiating and sustaining breastfeeding, and women who experience breastfeeding difficulties may develop depression. Some of the potential confounders thought to be important in studies of depressive symptoms and feeding practices include prior depression history, marital status, employment status, pregnancy intention, and intention to breastfeed.

\section{Methods}

We included both case-control and cohort studies that examined the link between breastfeeding and postpartum depression, in addition to recent systematic reviews (published within the past 5 years). All methods of assessment of depression were included (e.g., the Edinburgh Postnatal Depression Scale and other questionnaires). The prior AHRQ systematic review $^{2}$ was also limited to studies that had at least 100 nursing mothers; for this update, all 
newly included individual studies had a sample size greater than 100 (i.e., we did not exclude any studies because of inadequate sample size).

\section{Systematic Reviews}

A recent systematic review ${ }^{183}$ identified 48 articles on associations among breastfeeding, prenatal, and postpartum depression (Table 26). The review included studies from 12 very highincome countries (Australia, Canada, Norway, Finland, Iceland, Italy, Japan, Portugal, Sweden, United Arab Emirates, United Kingdom, and United States) and 7 other countries (Brazil, Barbados, China, Congo, Mexico, Pakistan, and Turkey). No exclusion criteria were based on study design. 2, 184

The authors explored the relationship between depression and breastfeeding and, in doing so, accounted for the temporal relationship in an effort to address causal direction. ${ }^{183}$ They reported associations between postpartum depression and breastfeeding in 18 included studies; shorter breastfeeding duration was associated with higher rates of depressive symptoms. However, the causal direction of this association was unclear. In longitudinal studies that measure depression symptoms and breastfeeding status over time, the authors reported that three studies in very highincome countries found no association between early breastfeeding cessation or bottle-feeding and subsequent depression; two studies found that breastfeeding pain or low breastfeeding selfefficacy predicts depression. The authors concluded that breastfeeding difficulties predict postpartum depression.

Notably, they also concluded that depression symptoms during pregnancy and postpartum depression are associated with early cessation of lactation. Given the potential confounding between these variables, authors reported mixed results in studies that include prenatal and postpartum assessment of maternal mood as well as longitudinal assessment of breastfeeding outcomes. Among seven studies that measured the association between prenatal depression and breastfeeding duration, two found no association, and five found an inverse association. Shorter breastfeeding duration was associated with postpartum depression in four studies but not in three other studies. The authors concluded that approaches are needed to identify and assist women with prenatal depression or early breastfeeding problems to improve both breastfeeding outcomes and maternal mood. 
Table 26. Breastfeeding and postpartum depression: Summary of published systematic review

\begin{tabular}{|c|c|c|c|c|c|c|c|c|}
\hline $\begin{array}{l}\text { Author, } \\
\text { Year }\end{array}$ & $\begin{array}{l}\text { Number of } \\
\text { Studies (N } \\
\text { Participants } \\
\text { Study } \\
\text { Design }\end{array}$ & $\begin{array}{l}\text { Search Date } \\
\text { (Databases) }\end{array}$ & $\begin{array}{l}\text { Study } \\
\text { Characteristics }\end{array}$ & $\begin{array}{l}\text { Definition of } \\
\text { Exposure }\end{array}$ & $\begin{array}{l}\text { Definition } \\
\text { of } \\
\text { Outcome }\end{array}$ & $\begin{array}{l}\text { Timing of } \\
\text { Followup }\end{array}$ & Results & ROB \\
\hline $\begin{array}{l}\text { Dias, } \\
2015^{183}\end{array}$ & $\begin{array}{l}48(71,245) ; \\
\text { cohort } \\
\text { studies }\end{array}$ & $\begin{array}{l}1980 \\
\text { through } \\
\text { December } \\
2013 \\
\text { (MEDLINE, } \\
\text { Web of } \\
\text { Science, } \\
\text { PsycINFO) }\end{array}$ & $\begin{array}{l}\text { Most studies } \\
\text { were conducted } \\
\text { in the U.S. } \\
\text { ( } n=15) \text { and U.K. } \\
\text { ( } n=6) \text {, with } \\
\text { others in } \\
\text { Australia, Brazil, } \\
\text { Canada, } \\
\text { Barbados, } \\
\text { Norway, } \\
\text { Pakistan, } \\
\text { Turkey, China, } \\
\text { Congo, Finland, } \\
\text { Iceland, Italy, } \\
\text { Japan, Mexico, } \\
\text { Portugal, } \\
\text { Sweden, and } \\
\text { the UAE }\end{array}$ & $\begin{array}{l}\text { BF exposure defined } \\
\text { according to different } \\
\text { criteria: Labbok and } \\
\text { Krasovec criteria } \\
(n=5) \text {, WHO criteria } \\
(n=1) \text {, as a } \\
\text { dichotomous variable } \\
(n=10) \text {, as a 3- or 4- } \\
\text { group variable }(n=5) \text {, } \\
\text { with the inclusion of } \\
\text { solids ( } n=2) \text {, exclusive } \\
\text { BF status ( } n=7) \text {, and } \\
\text { not specified ( } n=13) \text {; } \\
\text { duration was } \\
\text { assessed at different } \\
\text { time points: before } \\
\text { the } 6-m o \text { public } \\
\text { recommendations } \\
(n=26) \text { and at } 6 \text { mos } \\
(n=12)\end{array}$ & $\begin{array}{l}\text { Pregnancy } \\
\text { depression, } \\
\text { PPD (any } \\
\text { measure) }\end{array}$ & $\begin{array}{l}\text { Three } \\
\text { studies } \\
\text { assessed } \\
\text { depression } \\
\text { only during } \\
\text { pregnancy, } \\
35 \\
\text { measured } \\
\text { symptoms } \\
\text { postpartum, } \\
\text { and nine } \\
\text { assessed } \\
\text { depression } \\
\text { both during } \\
\text { pregnancy } \\
\text { and post- } \\
\text { partum; } \\
\text { postpartum } \\
\text { followup } \\
\text { ranged } \\
\text { from } 1 \text { wk } \\
\text { to } 2 \text { yrs }\end{array}$ & $\begin{array}{l}\text { No meta-analyses: authors } \\
\text { conclude BF duration is } \\
\text { associated with PPD; PPD } \\
\text { predicts and is predicted by } \\
\text { BF cessation in several } \\
\text { studies; pregnancy } \\
\text { depression and PPD are } \\
\text { associated with shorter BF } \\
\text { duration, BF may mediate the } \\
\text { association between } \\
\text { pregnancy and PPD; } \\
\text { pregnancy depression } \\
\text { predicts shorter BF duration } \\
\text { and that may increase } \\
\text { depressive symptoms during } \\
\text { postpartum }\end{array}$ & Unclear \\
\hline
\end{tabular}

$\mathrm{BF}$ = breastfeeding; $\mathrm{N}$ = number; $\mathrm{ROB}$ = risk of bias; $\mathrm{PPD}=$ postpartum depression; $\mathrm{UAE}$ = United Arab Emirates; U.K. = United Kingdom; WHO = World Health Organization; wks $=$ weeks. 


\section{Individual Studies}

\section{Characteristics}

We included 14 individual prospective cohort studies not included in the systematic review described above (Table 27); 13 were newly identified, and 1 study was included in the prior 2007 review. ${ }^{184}$ Sample size ranged from 119 to 14,541 participants. Studies generally included women in their 20s and 30s. Eleven studies described the proportion of enrolled women with prior depression; of these, 2 excluded women who had a previous or current history of depression, ${ }^{185,186}$ and others enrolled a minority of women with previous or current depression (5 to $45 \%$ of participants). Three studies did not describe whether women had a prior history of depression. ${ }^{184,187,188}$ Three studies were conducted in the United States; ${ }^{189-191} 2$ in Canada; ${ }^{192,} 193$ 2 in the United Kingdom; ${ }^{184,}, 194$ and 1 each in Europe, ${ }^{195}$ Australia, ${ }^{196}$ Denmark, ${ }^{197}$ Hong Kong, ${ }^{198}$ Spain, ${ }^{199}$ Sweden, ${ }^{200}$ and the United Arab Emerites. ${ }^{201}$ In general, studies followed women over time (6 weeks to 8 months postpartum) and measured both depression symptoms and breastfeeding duration/exclusivity. Studies varied in terms of whether they focused on any versus exclusive breastfeeding measures (Table 27); 7 studies compared women who were not breastfeeding with those who were currently breastfeeding. Two studies compared women who had never breastfed, women who had initiated but stopped, and women who were still breastfeeding, ${ }^{197,}, 198$ and 5 studies used other comparisons: women who did or did not initiate breastfeeding, ${ }^{188,194,195}$ women who were exclusively breastfeeding, mixed feeding, or formula feeding, ${ }^{185,}, 194$ and women who were predominantly breastfeeding or predominantly formula feeding at 6 months. ${ }^{189}$

Thirteen studies evaluated outcomes using the Edinburgh Postnatal Depression Scale (EPDS). In nine studies, a threshold score was used to identify women with depression symptoms (thresholds ranged from $\geq 10$ to $\geq 13$ across studies); one study used a threshold score $\geq 10$ and a positive answer to question 8, "I have felt sad or miserable," 195 and three studies analyzed EPDS scores as continuous variables. ${ }^{185,189,198}$ Two studies used other instruments in addition to the EPDS, one assessed depression using the Mini International Neuro-psychiatric interview to establish a diagnosis of depression among women with an EPDS $\geq 10,{ }^{201}$ and another assessed self-reported history of postpartum depression diagnosis or treatment. ${ }^{196}$

Of the 13 new studies, we rated 4 as medium ROB and the remainder as high ROB. Common methodological limitations included lack of information on the temporality of breastfeeding cessation and onset of depression symptoms, recall bias, lack of adjustment for potential confounding factors, and high rate of missing data. 
Table 27. Breastfeeding and postpartum depression: Summary of individual studies

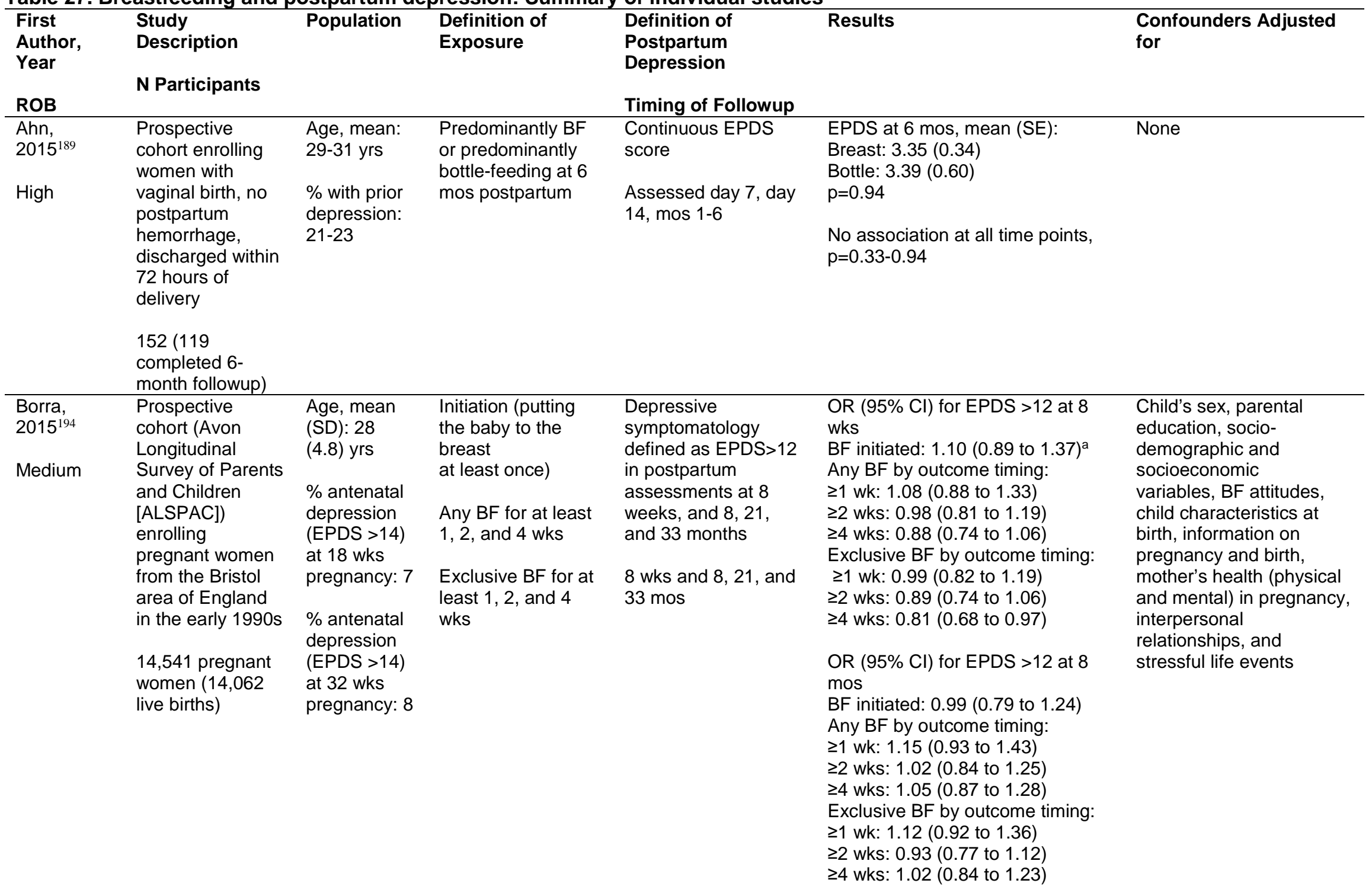




\begin{tabular}{|c|c|c|c|c|c|c|}
\hline $\begin{array}{l}\text { First } \\
\text { Author, } \\
\text { Year } \\
\text { ROB } \\
\end{array}$ & $\begin{array}{l}\text { Study } \\
\text { Description } \\
\text { N Participants }\end{array}$ & Population & $\begin{array}{l}\text { Definition of } \\
\text { Exposure }\end{array}$ & $\begin{array}{l}\text { Definition of } \\
\text { Postpartum } \\
\text { Depression } \\
\text { Timing of Followup }\end{array}$ & Results & $\begin{array}{l}\text { Confounders Adjusted } \\
\text { for }\end{array}$ \\
\hline $\begin{array}{l}\text { Chojenta, } \\
2016^{196} \\
\text { High }\end{array}$ & $\begin{array}{l}\text { Population cohort } \\
\text { study of } \\
\text { Australian women } \\
\text { born 1973-1978 } \\
\text { with one or more } \\
\text { children born } \\
\text { before } 2009 \\
5,219\end{array}$ & $\begin{array}{l}\text { Age } 31-36 \\
\text { yrs at } \\
\text { assessment } \\
\text { of pregnancy } \\
\text { history } \\
\text { \% with prior } \\
\text { PPD: } 26\end{array}$ & $\begin{array}{l}\text { Any BF }<6 \text { months } \\
\text { vs. } \geq 6 \text { for each } \\
\text { child born }\end{array}$ & $\begin{array}{l}\text { Retrospective self- } \\
\text { report for each child } \\
\text { born: "Were you } \\
\text { diagnosed or treated } \\
\text { for postnatal } \\
\text { depression?" } \\
\text { NA (age } 31-36 \text { yrs at } \\
\text { cohort enrollment) }\end{array}$ & $\begin{array}{l}\text { OR }(95 \% \mathrm{Cl}) \text { of PPD for } \\
\text { pregnancy followed by } \mathrm{BF}<6 \text { mos } \\
\text { vs. } \geq 6 \text { : } \\
1.29(1.12 \text { to } 1.50)\end{array}$ & $\begin{array}{l}\text { Pregnancy complications, } \\
\text { social support, proportion } \\
\text { of stressful life events, } \\
\text { SF-36 mental health } \\
\text { index, SF-36 general } \\
\text { health index, income } \\
\text { stress, education, partner } \\
\text { status, mean stress, LOT- } \\
\text { R optimism, history of } \\
\text { pregnancy, birth and } \\
\text { postpartum events, } \\
\text { history of self-harm, } \\
\text { violent relationship, } \\
\text { depression, anxiety, } \\
\text { tobacco use }\end{array}$ \\
\hline $\begin{array}{l}\text { Davey, } \\
2011^{192} \\
\text { Medium }\end{array}$ & $\begin{array}{l}\text { Secondary } \\
\text { analysis of RCT } \\
\text { enrolling } \\
\text { pregnant women } \\
2,015 \text { (1,403 } \\
\text { completed 8- } \\
\text { week followup) }\end{array}$ & $\begin{array}{l}\text { Age: } \% \\
<25: 18 \\
\geq 25: 82 \\
\text { \% with prior } \\
\text { depression: } \\
\text { No PPD: } 20 \\
\text { Subclinical } \\
\text { PPD: } 37 \\
\text { Major PPD: } \\
45\end{array}$ & $\begin{array}{l}\text { Any BF at } 8 \text { wks } \\
\text { postpartum (yes or } \\
\text { no) }\end{array}$ & $\begin{array}{l}\text { EPDS score, } \\
\text { categorized by score } \\
\text { indicating risk of } \\
\text { PPD: } \\
\text { None: }<10 \\
\text { Subclinical: } 10-12 \\
\text { Major: } 13+ \\
8 \text { wks postpartum }\end{array}$ & $\begin{array}{l}\text { BF status at } 8 \text { wks was not } \\
\text { associated with subclinical PPD } \\
\text { (OR not reported; p>0.05) } \\
\text { Not BF at } 8 \text { wks was associated } \\
\text { with an increased risk of major } \\
\text { PPD, OR (95\% CI): } 2.12 \text { (1.21 to } \\
3.70) \\
\text { No information on temporality of } \\
\text { depression symptom onset and BF } \\
\text { cessation }\end{array}$ & $\begin{array}{l}\text { History of depression, } \\
\text { country where mother } \\
\text { was born, depressive } \\
\text { symptoms during } \\
\text { pregnancy }\end{array}$ \\
\hline
\end{tabular}




\begin{tabular}{|c|c|c|c|c|c|c|}
\hline $\begin{array}{l}\text { First } \\
\text { Author, } \\
\text { Year } \\
\text { ROB }\end{array}$ & $\begin{array}{l}\text { Study } \\
\text { Description } \\
\text { N Participants }\end{array}$ & Population & $\begin{array}{l}\text { Definition of } \\
\text { Exposure }\end{array}$ & $\begin{array}{l}\text { Definition of } \\
\text { Postpartum } \\
\text { Depression } \\
\text { Timing of Followup }\end{array}$ & Results & $\begin{array}{l}\text { Confounders Adjusted } \\
\text { for }\end{array}$ \\
\hline $\begin{array}{l}\text { Fiala, } \\
2017^{195} \\
\text { High }\end{array}$ & $\begin{array}{l}\text { Prospective } \\
\text { cohort study } \\
7,589 \text { (3,233 } \\
\text { included in } \\
\text { analysis) }\end{array}$ & $\begin{array}{l}\text { Age, mean } \\
\text { (SD): } 26 \\
(4.8) \text { yrs } \\
\text { \% with prior } \\
\text { depression: } \\
5.2\end{array}$ & Any BF vs. no BF & $\begin{array}{l}\text { EPDS score } \geq 10 \text { and } \\
\text { positive answer to } \\
\text { question number } 8 \text {, "I } \\
\text { have felt sad or } \\
\text { miserable" } \\
6 \text { wks and } 6 \text { mos } \\
\text { postpartum }\end{array}$ & $\begin{array}{l}\text { Multivariate-adjusted OR were } \\
\text { reported for association between } \\
\text { not BF and PPD } \\
\text { OR of PPD (95\% Cl); p value } \\
6 \text { wks: } 1.2(0.8-1.9) ; 0.39 \\
6 \text { mos: } 1.5(1.0-2.3) ; 0.07\end{array}$ & $\begin{array}{l}\text { Personal history of } \\
\text { depression, mother or } \\
\text { father of expectant mother } \\
\text { had history of depression, } \\
\text { unintentional pregnancy, } \\
\text { mother unhappy about } \\
\text { being pregnant, } \\
\text { primiparity, gender of } \\
\text { child, preterm birth, C- } \\
\text { section, ICU, mother }<18 \\
\text { yrs, education, family } \\
\text { savings, mother living } \\
\text { alone, psychosocial } \\
\text { stressors }\end{array}$ \\
\hline
\end{tabular}




\begin{tabular}{|c|c|c|c|c|c|c|}
\hline $\begin{array}{l}\text { First } \\
\text { Author, } \\
\text { Year } \\
\text { ROB }\end{array}$ & $\begin{array}{l}\text { Study } \\
\text { Description } \\
\text { N Participants }\end{array}$ & Population & $\begin{array}{l}\text { Definition of } \\
\text { Exposure }\end{array}$ & $\begin{array}{l}\text { Definition of } \\
\text { Postpartum } \\
\text { Depression } \\
\text { Timing of Followup }\end{array}$ & Results & $\begin{array}{l}\text { Confounders Adjusted } \\
\text { for }\end{array}$ \\
\hline $\begin{array}{l}\text { Hamdan, } \\
2012^{201} \\
\text { High }\end{array}$ & $\begin{array}{l}\text { Prospective } \\
\text { cohort study of } \\
\text { women recruited } \\
\text { during pregnancy } \\
\text { in UAE } \\
137\end{array}$ & $\begin{array}{l}\text { Age, } \%: \\
<30: 74 \\
30+: 26 \\
\\
\text { Prior } \\
\text { depression: } \\
\text { NR }\end{array}$ & $\begin{array}{l}\text { Any BF vs. no BF, } \\
2 \text { and } 4 \text { mos } \\
\text { postpartum }\end{array}$ & $\begin{array}{l}\text { PPD diagnosis: } \\
\text { women with } \\
\text { EPDS } \geq 10 \text { were } \\
\text { assessed using the } \\
\text { Mini International } \\
\text { Neuro-psychiatric } \\
\text { interview } \\
2 \text { and } 4 \text { mos } \\
\text { postpartum }\end{array}$ & $\begin{array}{l}\text { Unadjusted results were reported } \\
\text { for cross-sectional and longitudinal } \\
\text { associations between BF status } \\
\text { and PPD. } N(\%) \\
\text { BF at } 2 \text { mos } \times \text { PPD at } 2 \text { mos: } \\
\text { Any breast: } 11 \text { (9) } \\
\text { No breast: } 3(17.7) \\
\text { p=0.38 } \\
\text { PPD at } 2 \text { mos } \times \text { BF at } 4 \text { mos: } \\
\text { Any breast: } 8 \text { (7) } \\
\text { No breast: } 6 \text { (31.6) } \\
\text { p<0.01 } \\
\text { BF at } 2 \text { mos } \times \text { PPD at } 4 \text { mos: } \\
\text { Any breast: } 6 \text { (5.1) } \\
\text { No breast: } 3(17.7) \\
\text { p=0.05 } \\
\text { BF at } 4 \text { mos } \times \text { PPD at } 4 \text { mos: } \\
\text { Any breast: } 4(3.3) \\
\text { No breast: } 5 \text { (26.3) } \\
p<0.01\end{array}$ & $\begin{array}{l}\text { Multiple confounders } \\
\text { measured, no adjusted } \\
\text { results reported }\end{array}$ \\
\hline
\end{tabular}




\begin{tabular}{|c|c|c|c|c|c|c|}
\hline $\begin{array}{l}\text { First } \\
\text { Author, } \\
\text { Year } \\
\text { ROB }\end{array}$ & $\begin{array}{l}\text { Study } \\
\text { Description } \\
\text { N Participants }\end{array}$ & Population & $\begin{array}{l}\text { Definition of } \\
\text { Exposure }\end{array}$ & $\begin{array}{l}\text { Definition of } \\
\text { Postpartum } \\
\text { Depression } \\
\text { Timing of Followup }\end{array}$ & Results & $\begin{array}{l}\text { Confounders Adjusted } \\
\text { for }\end{array}$ \\
\hline $\begin{array}{l}\text { Illiadis, } \\
2015^{200} \\
\text { High }\end{array}$ & $\begin{array}{l}\text { Longitudinal } \\
\text { cohort study } \\
\text { among Swedish- } \\
\text { speaking women } \\
\text { age 18+ enrolled } \\
\text { during pregnancy } \\
\\
365 \text { enrolled } \\
181 \text { included in } \\
\text { analysis }\end{array}$ & $\begin{array}{l}\text { Age, \%: } \\
20-34: 73 \\
\geq 35: 27 \\
\text { \% with prior } \\
\text { depression: } \\
27\end{array}$ & $\begin{array}{l}\text { Any BF vs. no BF, } \\
6 \text { wks postpartum }\end{array}$ & $\begin{array}{l}\text { Depressive } \\
\text { symptoms, defined as } \\
\text { EPDS } \geq 10 \\
\text { Healthy women: no } \\
\text { EPDS } \geq 10 \\
\text { Symptoms, but not } \\
\text { PPD: Depression } \\
\text { symptoms } \\
\text { before/during } \\
\text { pregnancy, but } \\
\text { EPDS }<10 \text { postpartum } \\
\text { PPD: EPDS } \geq 10 \\
6 \text { wks postpartum }\end{array}$ & $\begin{array}{l}\text { Association with BF status at } 6 \\
\text { wks postpartum: } \\
\text { EPDS } \geq 10, N(\%) \text { : } \\
\text { Any BF: } 21(15.4) \\
\text { No BF: } 11(25) \\
\text { History of depression symptoms, } \\
\text { but not postpartum, N (\%) } \\
\text { Any BF: } 37(27.2) \\
\text { No BF: } 15(34.0) \\
\text { No depression symptoms, N (\%) } \\
\text { Any BF: } 78(57) \\
\text { No BF: } 11(40) \\
\text { p=0.14 }\end{array}$ & None \\
\hline $\begin{array}{l}\text { Lau, } \\
2006^{198} \\
\text { High }\end{array}$ & $\begin{array}{l}\text { Prospective } \\
\text { cohort enrolling } \\
\text { Chinese women } \\
\text { in Hong Kong } \\
\text { with no personal } \\
\text { or family } \\
\text { psychiatric history } \\
\text { 2,178 enrolled } \\
\text { (598 completed } \\
6 \text {-wk followup) }\end{array}$ & $\begin{array}{l}\text { Age, \%: } \\
\text { >25: } 89 \\
\\
\text { Prenatal } \\
\text { depression: } \\
\text { EPDS score, } \\
\text { mean (SD) } \\
18 \text { wks: } \\
8.28(4.63) \\
32 \text { wks: } \\
7.55(4.79)\end{array}$ & $\begin{array}{l}\text { Ever BF vs. never } \\
\text { BF } \\
\text { Among those who } \\
\text { initiated, duration } \\
\leq 3 \text { wks vs. }>3 \text { wks }\end{array}$ & $\begin{array}{l}\text { Continuous EPDS } \\
\text { score } \\
6 \text { wks postpartum }\end{array}$ & $\begin{array}{l}\text { Unadjusted EPDS score at } 6 \text { wks, } \\
\text { mean (SD) } \\
\text { Did not initiate BF: } 7.13(5.30) \\
\text { Initiated BF: } 7.25(4.90 \\
p=0.854 \\
\text { BF, } \leq 3 \text { wks: } 7.21(4.55) \\
\text { BF, }>3 \text { wks: } 7.26(5.01) \\
p=0.943 \\
\text { Odds of BF outcome, by EPDS } \\
\text { score at } 6 \text { wks, OR (95\% Cl) } \\
\text { Ever BF vs. never BF: } \\
1.00(0.96,1.05) \\
\text { BF >3 wks vs. } \leq 3 \text { wks: } \\
0.95(0.87,1.03)\end{array}$ & $\begin{array}{l}\text { Age, educational level, } \geq 1 \\
\text { year in Hong Kong, } \\
\text { married, length of } \\
\text { marriage } \geq 1 \text { yr, housewife } \\
\text { or part-time work, family } \\
\text { income }<\$ 5 \mathrm{~K} \text {, private } \\
\text { housing, size of residence } \\
\geq 500 \mathrm{ft}^{2} \text {, living with } \\
\text { parents, living with } \\
\text { parents-in-law }\end{array}$ \\
\hline
\end{tabular}




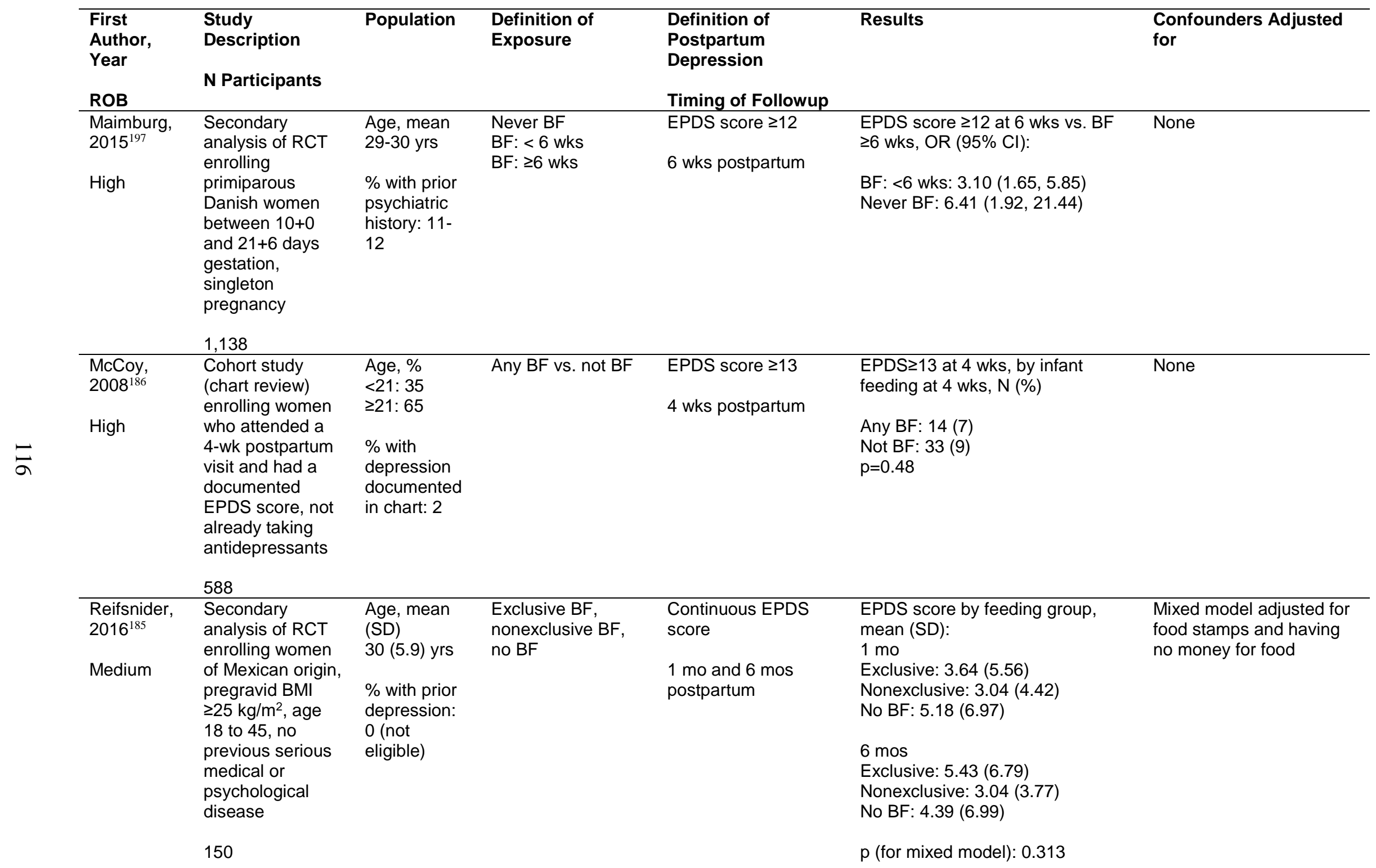




\begin{tabular}{|c|c|c|c|c|c|c|}
\hline $\begin{array}{l}\text { First } \\
\text { Author, } \\
\text { Year } \\
\text { ROB }\end{array}$ & $\begin{array}{l}\text { Study } \\
\text { Description } \\
\text { N Participants }\end{array}$ & Population & $\begin{array}{l}\text { Definition of } \\
\text { Exposure }\end{array}$ & $\begin{array}{l}\text { Definition of } \\
\text { Postpartum } \\
\text { Depression } \\
\text { Timing of Followup }\end{array}$ & Results & $\begin{array}{l}\text { Confounders Adjusted } \\
\text { for }\end{array}$ \\
\hline $\begin{array}{l}\text { Sword, } \\
2011^{188} \\
\text { Medium }\end{array}$ & $\begin{array}{l}\text { Prospective } \\
\text { cohort study, } \\
\text { enrolling } \\
\text { Canadian } \\
\text { women, >16 yrs } \\
\text { delivering } \\
\text { singleton at >37 } \\
\text { wks gestation } \\
2,560\end{array}$ & $\begin{array}{l}\text { Age, mean } \\
\text { (SD): } \\
31 \text { (5.3) yrs } \\
\text { \% with prior } \\
\text { depression: } \\
\text { NR }\end{array}$ & $\begin{array}{l}\text { BF initiated vs. not } \\
\text { initiated }\end{array}$ & $\begin{array}{l}\text { EPDS } \geq 12 \\
6 \text { wks postpartum }\end{array}$ & $\begin{array}{l}\text { EPDS } \geq 12 \text { at } 6 \text { wks postpartum } \\
\text { (BF initiated vs. not initiated), } \\
\text { OR }(95 \% \mathrm{Cl}): \\
2.07(1.02 \text { to } 4.21) \\
p=0.0449\end{array}$ & $\begin{array}{l}\text { Maternal age <25 yrs, first } \\
\text { pregnancy, MacArthur } \\
\text { SES score, number of } \\
\text { unmet learning needs, } \\
\text { maternal hospital } \\
\text { readmission, health since } \\
\text { delivery, SF-12 mental } \\
\text { component score, SF-12 } \\
\text { physical component } \\
\text { score, urinary } \\
\text { incontinence, mode of } \\
\text { delivery, low social } \\
\text { support, exhaustion/ } \\
\text { extreme fatigue, previous } \\
\text { depression, household } \\
\text { income, country of birth }\end{array}$ \\
\hline $\begin{array}{l}\text { Trevino- } \\
\text { Juarez, } \\
2016^{187} \\
\text { Medium }\end{array}$ & $\begin{array}{l}\text { Prospective } \\
\text { cohort enrolling } \\
\text { Spanish-speaking } \\
\text { primiparas in } \\
\text { Madrid with } \\
\text { healthy singleton } \\
\text { infants (36-42 } \\
\text { wks gestation) } \\
\text { not admitted to } \\
\text { NICU }\end{array}$ & $\begin{array}{l}\text { Age, mean } \\
\text { (SD): } \\
32 \text { (5.3) yrs } \\
\text { \% with prior } \\
\text { depression: } \\
\text { NR }\end{array}$ & $\begin{array}{l}\text { Any BF vs. artificial } \\
\text { milk feeding }\end{array}$ & $\begin{array}{l}\text { EPDS } \geq 11 \\
6 \mathrm{wks} \text { and } 6 \mathrm{mos}\end{array}$ & $\begin{array}{l}\text { EPDS } \geq 11 \text { at } 6 \text { wks, N (\%) } \\
\text { Any BF: } 35(13.2) \\
\text { No BF: } 7(16.7) \\
p=0.538 \\
\text { At } 6 \text { mos, } N(\%) \\
\text { Any BF: } 11(6.1) \\
\text { No BF: } 3(2.3) \\
p=0.118\end{array}$ & $\begin{array}{l}\text { Infant sex, mode of birth, } \\
\text { "baby does not sleep } \\
\text { well," "baby does not eat } \\
\text { well," use of emergency } \\
\text { medical services for } \\
\text { baby's health, mother's } \\
\text { employment status, } \\
\text { socioeconomic level, age } \\
\text { at childbearing }\end{array}$ \\
\hline
\end{tabular}




\begin{tabular}{|c|c|c|c|c|c|c|}
\hline $\begin{array}{l}\text { First } \\
\text { Author, } \\
\text { Year } \\
\text { ROB }\end{array}$ & $\begin{array}{l}\text { Study } \\
\text { Description } \\
\text { N Participants }\end{array}$ & Population & $\begin{array}{l}\text { Definition of } \\
\text { Exposure }\end{array}$ & $\begin{array}{l}\text { Definition of } \\
\text { Postpartum } \\
\text { Depression } \\
\text { Timing of Followup }\end{array}$ & Results & $\begin{array}{l}\text { Confounders Adjusted } \\
\text { for }\end{array}$ \\
\hline $\begin{array}{l}\text { Warner, } \\
\text { 1996 }^{184} \\
\text { Medium }\end{array}$ & $\begin{array}{l}\text { Prospective } \\
\text { cohort recruited } \\
\text { from postnatal } \\
\text { wards, followed } \\
\text { up with home } \\
\text { interview 6-8 wks } \\
\text { postpartum } \\
2,375\end{array}$ & $\begin{array}{l}\text { Mean age } \\
\text { (range): } \\
28 \text { (15 to 46) } \\
\text { \% with prior } \\
\text { depression: } \\
\text { NR }\end{array}$ & $\begin{array}{l}\text { Not BF vs. BF at } \\
\text { home interview }\end{array}$ & $\begin{array}{l}\text { EPDS } \geq 13 \\
6-8 \text { wks postpartum }\end{array}$ & $\begin{array}{l}\text { OR }(95 \% \mathrm{Cl}), \mathrm{EPDS} \geq 13 \text { at } 6 \text { wks, } \\
\text { not } \mathrm{BF} \text { vs. BF: } \\
1.52(1.12 \text { to } 2.06)\end{array}$ & $\begin{array}{l}\text { Unplanned pregnancy, } \\
\text { maternal unemployment, } \\
\text { head of household } \\
\text { unemployed }\end{array}$ \\
\hline $\begin{array}{l}\text { a Authors al } \\
\text { In subgroup } \\
\text { pregnancy. } \\
\text { b This study } \\
\text { decisions of }\end{array}$ & $\begin{array}{l}\text { sessed outcomes at } 2 \\
\text { yses, authors note the } \\
\text { included in the prior } \\
\text { authors of that review }\end{array}$ & $\begin{array}{l}\text { and } 33 \text { months } p \\
\text { ssociation betwe } \\
07 \text { AHRQ revie }\end{array}$ & $\begin{array}{l}\text { tpartum and concluc } \\
\text { breastfeeding and } \\
\text { by Ip and colleague }\end{array}$ & $\begin{array}{l}\text { the association betwe } \\
\text { ssion is mediated by wl } \\
\text { te did not reassess ROB }\end{array}$ & $\begin{array}{l}\text { reastfeeding and postpartum depressio } \\
\text { ler women had planned to breastfeed ar } \\
\text { r individual studies included in that rev }\end{array}$ & $\begin{array}{l}\text { s weak at both timepoints. } \\
\text { depression during } \\
\text { v; this rating represents the }\end{array}$ \\
\hline
\end{tabular}




\section{Results}

Thirteen studies measured postpartum depression using the EPDS scale; however, studies varied in terms of how scores were analyzed and interpreted (Table 27). Overall, results were mixed.

Nine studies reported on the association between breastfeeding and EPDS scores above and below a set threshold. ${ }^{184,186-188,192,194,197,200,201}$ Only one study verified EPDS scores using a diagnostic instrument. ${ }^{201}$ Enrolled women $(\mathrm{N}=137)$ completed the EPDS and reported on breastfeeding status at 2 and 4 months postpartum; women with an EPDS $\geq 10$ underwent further assessment with the Mini International Neuro-psychiatric interview to establish a diagnosis of depression. In unadjusted analyses, feeding status at 2 months was not associated with postpartum depression diagnosis; however, postpartum depression at 2 months predicted not breastfeeding at 4 months ( $<<0.01)$, and not breastfeeding at 2 months predicted postpartum depression at 4 months $(\mathrm{p}=0.05){ }^{201}$

Three studies accounted for prior history of depression or depressive symptoms in the analysis and found inconsistent results. ${ }^{192,195,200}$ One of these analyzed data separately for women with and without a prior history of depression symptoms; enrolled women $(\mathrm{N}=181)$ reported data on breastfeeding status at 6 weeks (any vs. none) and were grouped by prior history of depression symptoms (none, prepregnancy, or prenatal depression, but EPDS < 10 postpartum; and EPDS $\geq 10$ postpartum, regardless of previous history). Breastfeeding status at 6 weeks did not differ significantly by depression symptom history $(p=0.14) .{ }^{200}$ The second study was a secondary analysis of women enrolled in an RCT of an unsuccessful prenatal support intervention to reduce postpartum depression. ${ }^{192}$ At 8 weeks postpartum, current breastfeeding status (any breastfeeding vs. no breastfeeding) was not associated with subclinical postpartum depression (EPDS scores 10-12); however, in a prediction model adjusting for history of depression, country where the mother was born, and depressive symptoms during pregnancy, not breastfeeding at 8 weeks was associated with a higher risk of major postpartum depression (EPDS $\geq 13$, adjusted OR, 2.12; 95\% CI, 1.21 to 3.70). ${ }^{192}$ The third study found that a history of depression was associated with prenatal depression and depression at 6 weeks but not 6 months; not breastfeeding was not associated with postnatal depression (EPDS $\geq 10$ and a positive response to question 8; OR at 6 weeks 1.2, 95\% CI, 0.8 to 1.9; at 6 months, OR 1.5, 95\% CI, 1.0 to 2.3). ${ }^{195}$ Six additional studies did not specifically account for depression symptoms before or during pregnancy. ${ }^{186-188,194,197,202}$ Three found an association between breastfeeding status at 6 to 8 weeks postpartum and three did not. ${ }^{186,187,194}$ Of those that found an association, one focused on nulliparous women enrolled in an RCT $(\mathrm{N}=1,138)$ evaluating a prenatal care education and support intervention ("Ready for Child”); postpartum depression was assessed at 6 weeks postpartum and categorized as EPDS score $\geq 12$. In the whole sample, 1 percent of participants had never breastfed, and 8 percent had stopped breastfeeding at 6 weeks postpartum. In unadjusted analyses, compared with women who were breastfeeding at 6 weeks, never breastfeeding was associated with increased risk of depression (OR, 6.41; 95\% CI, 1.93 to 21.44) as was cessation of breastfeeding by 6 weeks (OR, 3.10; 95\% CI, 1.65 to 5.85). ${ }^{197}$ The second study $(\mathrm{N}=2,560)$ followed women from birth through 6 weeks postpartum; never initiating breastfeeding was associated with an increased risk of postpartum depression (EPDS $\geq 12$ ) at 6 weeks compared with initiating breastfeeding (adjusted OR, 2.07; 95\% CI, 1.02 to 4.21, $\mathrm{p}=0.0449) .{ }^{188}$ The third study that found an association enrolled women $(\mathrm{N}=2,375) 6$ to 8 weeks postpartum and assessed both current feeding status and EPDS. In models adjusted for unplanned 
pregnancy, maternal employment, and head of household employment, not breastfeeding was associated with an increased risk of EPDS $\geq 13$ compared with breastfeeding (adjusted OR, 1.52; 95\% CI, 1.12 to 2.06). ${ }^{202}$ The three studies that found no difference in depression scores and breastfeeding status measured outcomes at a similar time point (4 to 8 weeks postpartum). In one ( $\mathrm{N}=551)$ unadjusted analyses showed no association between current breastfeeding (at 4 weeks) and EPDS scores $\geq 13$ (7\% vs. 9\%, respectively; $\mathrm{p}=0.48) .{ }^{186}$ The second study $(\mathrm{N}=364)$ breastfeeding was categorized as any breastfeeding or no breastfeeding and collected at 6 weeks and 6 months postpartum; in unadjusted analyses, there were no associations between infant feeding status and EPDS $\geq 11$ at 6 weeks or 6 months. ${ }^{187}$ The third study found no association between various definitions of breastfeeding (e.g., initiation, any, or exclusive breastfeeding for 1, 2, and 4 or more weeks) and EPDS >12 at both 8 weeks and 8 months. ${ }^{194}$

Three studies assessed the relationship between breastfeeding status and mean EPDS scores; all three found no association between breastfeeding status and depressive symptoms. ${ }^{185,189,198}$ One $(\mathrm{N}=119)$ followed participants who had an uncomplicated vaginal birth and measured EPDS scores at multiple time points (7 days, 14 days, and monthly from 1 to 6 months); there was no association between breastfeeding status at 6 months (classified as predominantly breastfeeding vs. predominantly bottle-feeding) and EPDS scores at any time point (p-value range: 0.33 to 0.94). ${ }^{189}$ The second enrolled pregnant women $(\mathrm{N}=2,178)$ who had no personal or family psychiatric history; at 6 weeks postpartum, there was no association between EPDS scores and breastfeeding initiation and noninitiation $(\mathrm{p}=0.85)$ and no difference in scores among those who breastfed for 3 weeks or less and those who breastfed for longer than 3 weeks $(p=0.94){ }^{198}$ The third study enrolled Mexican-American participants ( $\mathrm{N}=150)$ from an RCT focused on preventing childhood obesity; there was no significant associations were found between feeding status (categorized as exclusive breastfeeding, nonexclusive breastfeeding, or not breastfeeding) and EPDS score or EPDS score trajectory (at 1 and 6 months). ${ }^{185}$

Finally, one Australian population cohort study (the Australian Longitudinal Study on Women's Health) assessed self-reported breastfeeding and postpartum depression for each birth (at ages 31 to 36 years). ${ }^{196}$ Postpartum depression was ascertained with the question, "Were you diagnosed or treated for postnatal depression?"; breastfeeding was categorized as less than 6 months vs. more than 6 months or more for each child. The authors found a modest association between breastfeeding less than 6 months and self-reported diagnosis or treatment of postpartum depression (adjusted OR, 1.29; 95\% CI, 1.12 to 1.50). Interpretation of this result is limited by lack of information on the temporal sequence of depression and breastfeeding cessation. ${ }^{196}$

\section{Return to Prepregnancy Weight or Postpartum Weight Change}

\section{Background}

The relationship between breastfeeding and weight retention is not clearly understood; a host of related factors influence weight, making it difficult to isolate the effect of breastfeeding on weight change. These factors include prepregnancy weight, parity, activity level, nutritional intake, socioeconomic status, and ethnicity, among others. ${ }^{203}$ Unmeasured confounding could also arise from an underlying propensity that may influence both the decision to breastfeed and maintain other health behaviors ${ }^{204}$ that might influence weight changes. 


\section{Methods}

Our eligibility criteria follow that of the previous systematic review; ${ }^{2}$ we limited our review to prospective cohort studies conducted in developed countries that directly compared weight changes of nonlactating women with weight changes of lactating women and for whom the exclusivity or the amount of breastfeeding was clear. Studies of the relationship between postpartum weight change and breastfeeding needed to control for subjects' gestational weight gain or prepregnancy weight and have at least 3 months postpartum followup to be included. The previous review ${ }^{2}$ also restricted inclusion to studies with at least 50 women per feeding group in the final analyses (e.g., lactating vs. nonlactating); however, all eligible studies for the update met this sample size criterion (in other words, we did not exclude studies because of inadequate sample size). For each included publication that reported multiple time points, we focused on the furthest time point available. Although recent systematic reviews (published within the last 5 years) were eligible, we did not find one that was relevant to the scope of this review.

\section{Individual Studies}

\section{Characteristics}

In total, the evidence base includes 47,655 women in 16 studies (described in 19 publications), predominantly from the United States (exceptions include studies or analyses from Canada, ${ }^{205}$ Taiwan, ${ }^{206}$ Sweden, ${ }^{207}$ Australia, ${ }^{208}$ and Norway ${ }^{203}$ ). Of these, 7 cohort studies that examined postpartum weight changes in relation to exclusive breastfeeding were included in the prior 2007 AHRQ review; ${ }^{205,207,209-215}$ short- $^{212,215}$ and long-term ${ }^{207}$ outcomes were reported for the Stockholm Pregnancy and Women's Nutrition Study (SPAWN) in three publications. The searches for this update review identified 10 additional relevant publications. ${ }^{203,204,206,208,216-221}$ Of these, 2 reported on the same cohort from the Infant Feeding Practices Study II (IFPSII), ${ }^{216,}$ ${ }^{220}$ and one, from the WHO Multicentre Growth Reference Study (MGRS), reported analyses from Norway and the United States. ${ }^{203}$ Despite the substantial breadth of this evidence base, its heterogeneity precluded meta-analysis.

Studies varied in their definition of exposure, using duration, duration and intensity, or duration and exclusivity. Studies also varied in how they reported outcomes. All reported a change in weight, but the time points that marked the beginning and the end of the measurement varied. Start times for outcome measurement included a fixed time point (e.g., 1989), before the index pregnancy, at the first prenatal visit, at the point of the highest pregnancy weight, within 1 to 2 days of delivery, or at 14 days after delivery. Stop times for outcome measurement were similarly varied, ranging from a fixed time point (e.g., 1993), just before a second pregnancy, and at a postpartum date ranging from 6 months to 15 years after the index delivery. In addition to the heterogeneity in PICOTS, this database spans publication of results starting in 1989. This period of time also covers secular changes in breastfeeding practices and women's weight.

We present results below by definition of exposure first and then by the time frame of the weight change outcome. Table 28 summarizes characteristics and results for individual studies. 
Table 28. Breastfeeding and postpartum weight change: Summary of individual studies

\begin{tabular}{|c|c|c|c|c|c|c|}
\hline $\begin{array}{l}\text { First Author, } \\
\text { Year } \\
\text { ROB }\end{array}$ & $\begin{array}{l}\text { Study Description } \\
\text { Number of } \\
\text { Participants }\end{array}$ & Population & $\begin{array}{l}\text { Definition of } \\
\text { Exposure(s) }\end{array}$ & $\begin{array}{l}\text { Definition of } \\
\text { Outcome(s) (Start } \\
\text { to End of } \\
\text { Followup) }\end{array}$ & Results & $\begin{array}{l}\text { Confounders } \\
\text { Adjusted for }\end{array}$ \\
\hline $\begin{array}{l}\text { Brewer, 1989214 } \\
\text { Medium }^{\mathrm{a}}\end{array}$ & $\begin{array}{l}\text { Pregnant women } \\
\text { recruited through mail } \\
\text { and telephone } \\
\text { contacts before } \\
\text { delivery with } \\
\text { assistance from a local } \\
\text { woman's hospital in } \\
\text { Louisiana } \\
56\end{array}$ & $\begin{array}{l}\text { Prepregnancy weight, } \\
\text { kg (SD) } \\
\text { Exclusive BF: } 59.8 \\
\text { (13.1) } \\
\text { Formula feed: } 54.9 \\
(6.0) \\
\text { Mixed feed: } 57.3 \\
(7.5)\end{array}$ & $\begin{array}{l}\text { Exclusive } \\
\text { formula feed or mixed } \\
\text { feed at } 6 \text { mos }\end{array}$ & $\begin{array}{l}\text { Change in weight } \\
\text { and weight-height } \\
\text { index from 1-2 days } \\
\text { to } 6 \text { mos postpartum }\end{array}$ & $\begin{array}{l}\text { No statistically } \\
\text { significant } \\
\text { differences } \\
\text { between groups }\end{array}$ & $\begin{array}{l}\text { Age, parity, } \\
\text { prepregnancy weight, } \\
\text { socioeconomic data, } \\
\text { energy intake, energy } \\
\text { expenditure exclusive } \\
\text { of lactation (physical } \\
\text { activity) }\end{array}$ \\
\hline $\begin{array}{l}\text { Endres, } 2015^{219} \\
\text { Medium }\end{array}$ & $\begin{array}{l}\text { Women ages 18-40 } \\
\text { with a live birth at } 20 \\
\text { wks of gestation or } \\
\text { longer from a 5-site } \\
\text { prospective cohort } \\
\text { NICHD study } \\
774\end{array}$ & $\begin{array}{l}\text { Prepregnancy weight, } \\
\text { lbs (SD) } \\
161.5(46.2)\end{array}$ & $\begin{array}{l}\text { Partial or exclusive } \\
\text { BF at } 6 \text { mos vs. no } \\
\text { BF at } 6 \text { mos }\end{array}$ & $\begin{array}{l}\text { Weight retention of } \\
20 \text { lbs or more from } \\
\text { prepregnancy } \\
\text { weight } \\
\text { to } 1 \text { yr postpartum }\end{array}$ & $\begin{array}{l}\text { OR }(95 \% \mathrm{Cl}): \\
0.46(0.24 \text { to } 0.87)\end{array}$ & $\begin{array}{l}\text { Age, race, type of } \\
\text { insurance, marital } \\
\text { status, poverty level, } \\
\text { GWG, prepregnancy } \\
\text { BMI, BF, working } \\
\text { outside the home, and } \\
\text { hours of nightly sleep }\end{array}$ \\
\hline $\begin{array}{l}\text { Haiek, } 2001^{205} \\
\text { Medium }^{\mathrm{a}}\end{array}$ & $\begin{array}{l}\text { Mothers recruited at } \\
\text { the immunization } \\
\text { clinics } \\
\text { while waiting for their } \\
\text { child's visit in } 2 \text { public } \\
\text { health clinics in } \\
\text { Montreal, Canada } \\
236\end{array}$ & $\begin{array}{l}\text { Mean prepregnancy } \\
\text { BMI (SD): } 22.5 \text { (3.4) }\end{array}$ & $\begin{array}{l}\text { Mixed-feeding (i.e., } \\
\text { average daily intake } \\
\text { of formula and breast } \\
\text { milk of }>4 \text { oz) or } \\
\text { predominantly bottle- } \\
\text { feeding (i.e., } \\
\text { exclusive bottle- } \\
\text { feeding or average } \\
\text { daily intake of breast } \\
\text { milk of } 4 \text { oz or less) } \\
\text { vs. predominantly BF } \\
\text { (i.e., exclusive BF or } \\
\text { average daily intake } \\
\text { of formula of } 4 \text { oz or } \\
\text { less) (time-varying } \\
\text { measurement of } \\
\text { exposure) }\end{array}$ & $\begin{array}{l}\text { Average monthly } \\
\text { rate of weight } \\
\text { change from } \\
\text { delivery to up to } 9 \\
\text { mos }\end{array}$ & $\begin{array}{l}\text { No statistically } \\
\text { significant } \\
\text { differences }\end{array}$ & $\begin{array}{l}\text { GWG, postpartum } \\
\text { smoking, infant's solid } \\
\text { intake, maternal place } \\
\text { of birth }\end{array}$ \\
\hline
\end{tabular}




\begin{tabular}{|c|c|c|c|c|c|c|}
\hline $\begin{array}{l}\text { First Author, } \\
\text { Year } \\
\text { ROB }\end{array}$ & $\begin{array}{l}\text { Study Description } \\
\text { Number of } \\
\text { Participants }\end{array}$ & Population & $\begin{array}{l}\text { Definition of } \\
\text { Exposure(s) }\end{array}$ & $\begin{array}{l}\text { Definition of } \\
\text { Outcome(s) (Start } \\
\text { to End of } \\
\text { Followup) }\end{array}$ & Results & $\begin{array}{l}\text { Confounders } \\
\text { Adjusted for }\end{array}$ \\
\hline $\begin{array}{l}\text { Janney, } 1997^{211} \\
\text { Medium }^{\mathrm{a}}\end{array}$ & $\begin{array}{l}\text { Pregnant nulliparous } \\
\text { and primiparous } \\
\text { women ages } 20-40 \text { yrs } \\
\text { in their third trimester } \\
\text { recruited from birthing } \\
\text { education classes and } \\
\text { obstetric practices in } \\
\text { the Ann Arbor, } \\
\text { Michigan, area } \\
110\end{array}$ & $\begin{array}{l}\text { Prepregnancy mean } \\
\text { BMI (SD): } 22.2 \text { (3.4) }\end{array}$ & $\begin{array}{l}\text { Fully } B F,{ }^{b} \text { partially } B F \text {, } \\
\text { bottle-feeding }\end{array}$ & $\begin{array}{l}\text { Weight change from } \\
\text { prepregnancy } \\
\text { weight to weight at } \\
18 \text { mos (time } \\
\text { varying) }\end{array}$ & $\begin{array}{l}\text { Duration of } \\
\text { lactation practice } \\
\text { was a significant } \\
\text { predictor of } \\
\text { postpartum weight } \\
\text { retention over time } \\
(p<0.001)\end{array}$ & $\begin{array}{l}\text { GWG, months since } \\
\text { parturition, marital } \\
\text { status, age }\end{array}$ \\
\hline $\begin{array}{l}\text { Jarlenski, } \\
2014 ;{ }^{216} \text { Sharma, } \\
2014^{220} \\
\text { Medium }\end{array}$ & $\begin{array}{l}\text { Infant Feeding } \\
\text { Practices Study II } \\
\text { (IFPSII) following } \\
\text { women from last } \\
\text { trimester through } 12 \\
\text { mos; sampled from a } \\
\text { national consumer } \\
\text { opinion panel }{ }^{216} \\
\text { Women who had no } \\
\text { additional births after } \\
\text { participating in the } \\
\text { IFPSII were followed } \\
\text { through } 6 \text { yrs } \\
\\
2,102^{216}(726 \text { followed } \\
\text { through } 6 \text { yrs })^{220}\end{array}$ & $\begin{array}{l}\text { Original cohort }{ }^{216} \\
\text { Exclusive BF (\%): } \\
\text { Underweight: } 2.8 \\
\text { Healthy weight: } 46.4 \\
\text { Overweight: } 28.6 \\
\text { Obese: } 22.3 \\
\text { Nonexclusive or no } \\
\text { BF (\%): } \\
\text { Underweight: } 2.0 \\
\text { Healthy weight: } 44.0 \\
\text { Overweight: } 29.5 \\
\text { Obese: } 24.5 \\
\text { Followup cohort's } \\
\text { prepregnancy BMI } \\
\text { by adherence } \\
\text { categories } 220 \\
\text { Never initiated BF } \\
\text { (\%) } \\
\text { Normal: } 15.8 \\
\text { Overweight: } 16.2 \\
\text { Obese: } 23.7\end{array}$ & $\begin{array}{l}\text { Exclusive BF for } \geq 3 \\
\text { mos vs. nonexclusive } \\
\text { BF for } \geq 3 \text { mos or } \\
\text { never } \mathrm{BF}^{216} \\
\text { (1) Initiated BF, did } \\
\text { not exclusively BF for } \\
\geq 4 \text { mos, (2) adhered } \\
\text { to exclusivity for } \geq 4 \\
\text { mos, BF duration }<12 \\
\text { mos, ( } 3 \text { ) adhered to } \\
\text { exclusivity for } \geq 4 \text { mos, } \\
\text { duration } \geq 12 \text { mos vs. } \\
\text { never BF } 220\end{array}$ & $\begin{array}{l}\text { Postpartum weight } \\
\text { loss (highest } \\
\text { pregnancy weight to } \\
\text { her postpregnancy } \\
\text { weight) at } 12 \text { mos } \\
\text { postpartum }{ }^{216} \\
\text { Return to } \\
\text { prepregnancy BMI } \\
\text { category at } 12 \text { mos } \\
\text { postpartum }{ }^{216} \\
\text { Return to } \\
\text { prepregnancy } \\
\text { weight at } 12 \text { mos } \\
\text { postpartum } \\
\\
\text { Weight change from } \\
\text { prepregnancy to } 6 \\
\text { yrs postpartum }\end{array}$ & $\begin{array}{l}\text { Postpartum weight } \\
\text { loss } 216 \\
\text { Difference in weight } \\
\text { loss of } \\
-3.2 \text { pounds ( } 95 \% \\
\mathrm{Cl}, 1.7 \text { to } 4.7 \\
\text { pounds, p<0.05) } \\
\text { Return to } \\
\text { prepregnancy } \\
\mathrm{BMI}^{216} \\
6.0 \text { percentage } \\
\text { point increase } \\
(95 \% \mathrm{Cl}, 2.3 \text { to } \\
9.7), \mathrm{p}<0.01 \\
\\
\text { Return to } \\
\text { prepregnancy } \\
\text { weight }{ }^{216} \\
6.1 \text { percentage- } \\
\text { point increase } \\
(95 \% \mathrm{Cl}, 1.0 \text { to } \\
11.3), \mathrm{p}<0.05\end{array}$ & $\begin{array}{l}\text { Cohort matched } \\
\text { based on propensity } \\
\text { score that included } \\
\text { the following } \\
\text { covariates: maternal } \\
\text { age, race/ethnicity, } \\
\text { parity, education, } \\
\text { WIC enrollment, } \\
\text { prepregnancy } \\
\text { obesity, prenatal } \\
\text { insurance coverage, } \\
\text { postpartum smoking, } \\
\text { C-section, infant in } \\
\text { ICU postpartum, and } \\
\text { BF support } 216 \\
\text { Maternal age, race or } \\
\text { ethnicity, education, } \\
\text { poverty income ratio, } \\
\text { marital status, parity, } \\
\text { GWG, smoking } \\
\text { status, physical } \\
\text { activity } 220\end{array}$ \\
\hline
\end{tabular}




\begin{tabular}{|c|c|c|c|c|c|c|}
\hline $\begin{array}{l}\text { First Author, } \\
\text { Year } \\
\text { ROB }\end{array}$ & $\begin{array}{l}\text { Study Description } \\
\text { Number of } \\
\text { Participants }\end{array}$ & Population & $\begin{array}{l}\text { Definition of } \\
\text { Exposure(s) }\end{array}$ & $\begin{array}{l}\text { Definition of } \\
\text { Outcome(s) (Start } \\
\text { to End of Followup) }\end{array}$ & Results & $\begin{array}{l}\text { Confounders } \\
\text { Adjusted for }\end{array}$ \\
\hline $\begin{array}{l}\text { Jarlenski, } \\
20144^{216} \text { Sharma, } \\
2014^{220} \\
\text { (continued) }\end{array}$ & & $\begin{array}{l}\text { Initiated BF, did not } \\
\text { exclusively BF for } \geq 4 \\
\text { mos (\%) } \\
\text { Normal: } 55.4 \\
\text { Overweight: } 60.8 \\
\text { Obese: } 60.8 \\
\text { Adhered to } \\
\text { exclusivity for } \geq 4 \\
\text { mos, BF duration } \\
<12 \text { mos (\%) } \\
\text { Normal: } 8.9 \\
\text { Overweight: } 5.9 \\
\text { Obese: } 5.4 \\
\text { Adhered to } \\
\text { exclusivity for } \geq 4 \\
\text { mos, BF duration } \\
<12 \text { mos (\%) } \\
\text { Normal: } 19.9 \\
\text { Overweight: } 17.2 \\
\text { Obese: } 10.2^{\mathrm{c}}\end{array}$ & & & $\begin{array}{l}\text { No statistically } \\
\text { significant } \\
\text { differences for any } \\
\text { category of } \\
\text { prepregnancy } \\
\text { weight by any } \\
\text { category of } \\
\text { adherence on } \\
\text { weight retention, } \\
\text { with the exception } \\
\text { of the most } \\
\text { adherent obese } \\
\text { women }(\mathrm{N}=19 \text {, } \\
\text { mean change in } \mathrm{kg} \text { : } \\
-8.0[95 \% \mathrm{Cl}, \\
-15.4 \text { to }-0.7]]^{220}\end{array}$ & \\
\hline $\begin{array}{l}\text { Lyu, } 2009^{206} \\
\text { Medium }\end{array}$ & $\begin{array}{l}\text { Women over } 20 \text { yrs old } \\
\text { with Han ethnicity, } \\
\text { living in North Taiwan } \\
\text { for more than } 10 \text { yrs } \\
\text { and being less than } 20 \\
\text { wks pregnant with } \\
\text { singleton gestations } \\
130 \text { at } 6 \text { mos, } 122 \text { at } \\
12 \text { mos }\end{array}$ & $\begin{array}{l}\text { Mean prepregnancy } \\
\text { weight: } 53.4 \mathrm{~kg} \\
(\mathrm{SD}=7.97)\end{array}$ & $\begin{array}{l}\text { BF duration in } \\
\text { months }\end{array}$ & $\begin{array}{l}\text { Weight retention } \\
\text { from prepregnancy } \\
\text { to } 12 \text { mos } \\
\text { postpartum }\end{array}$ & $\begin{array}{l}\text { Regression } \\
\text { coefficient in } \\
\text { multiple models is } \\
\text { not statistically } \\
\text { significant }\end{array}$ & $\begin{array}{l}\text { Model with the most } \\
\text { variables includes } \\
\text { age, socioeconomic } \\
\text { status, BF, parity, } \\
\text { physical activity, } \\
\text { prepregnancy BMI, } \\
\text { GWG, 1-mo } \\
\text { postpartum energy } \\
\text { intakes, 6-mo weight } \\
\text { retention, postpartum } \\
\text { average energy } \\
\text { intakes and energy } \\
\text { consumption }\end{array}$ \\
\hline
\end{tabular}




\begin{tabular}{|c|c|c|c|c|c|c|}
\hline $\begin{array}{l}\text { First Author, } \\
\text { Year } \\
\text { ROB }\end{array}$ & $\begin{array}{l}\text { Study Description } \\
\text { Number of } \\
\text { Participants }\end{array}$ & Population & $\begin{array}{l}\text { Definition of } \\
\text { Exposure(s) }\end{array}$ & $\begin{array}{l}\text { Definition of } \\
\text { Outcome(s) (Start } \\
\text { to End of Followup) }\end{array}$ & Results & $\begin{array}{l}\text { Confounders } \\
\text { Adjusted for }\end{array}$ \\
\hline $\begin{array}{l}\mathrm{Ng}, 2014^{208} \\
\text { High }\end{array}$ & $\begin{array}{l}\text { Women who planned } \\
\text { to give birth at one of } \\
\text { three participating } \\
\text { hospitals in Australia } \\
\text { and were over age } 16 \\
\text { (Environments for } \\
\text { Healthy Living cohort) } \\
1,316\end{array}$ & $\begin{array}{l}\text { Prepregnancy BMI } \\
\text { (\%) } \\
\text { Underweight: } 8.3 \\
\text { Normal weight: } 52.4 \\
\text { Overweight: } 21.4 \\
\text { Obese: } 17.9\end{array}$ & $\begin{array}{l}\text { BF for at least } 3 \text { mos } \\
\text { vs. BF for }<3 \text { mos or } \\
\text { no BF }\end{array}$ & $\begin{array}{l}\text { High postpartum } \\
\text { weight retention from } \\
\text { prepregnancy to } 12 \\
\text { mos (within the top } \\
\text { quintile) }\end{array}$ & $\begin{array}{l}\text { Adjusted OR: } 0.673 \\
(95 \% \mathrm{Cl}, 0.471 \text { to } \\
0.961), p=0.03\end{array}$ & $\begin{array}{l}\text { Maternal age, } \\
\text { employment and } \\
\text { education, alcohol } \\
\text { intake and smoking } \\
\text { during pregnancy, } \\
\text { nonmedical drug use, } \\
\text { primaparity, } \\
\text { preexisting } \\
\text { hypertension, marital } \\
\text { status, paternal } \\
\text { employment and } \\
\text { education, household } \\
\text { ownership, number of } \\
\text { children under age } \\
\text { 16, and year of } \\
\text { recruitment }\end{array}$ \\
\hline $\begin{array}{l}\text { Ohlin, 1990;212 } \\
\text { Ohlin, 1996; }{ }^{215} \\
\text { Linne, 2003 } \\
\text { Medium } \\
\text { and high } \\
\text { 207a }\end{array}$ & $\begin{array}{l}\text { Stockholm Pregnancy } \\
\text { and Women's } \\
\text { Nutrition Study } \\
\text { (SPAWN) } \\
1,423 \text { followed to } 1 \mathrm{yr} \\
\text { postpartum }{ }^{212,215} \text { and } \\
563 \text { followed to } 15 \mathrm{yrs} \\
\text { postpartum } 207\end{array}$ & $\begin{array}{l}\text { Prepregnancy BMI: } \\
21.5\end{array}$ & $\begin{array}{l}\text { Lactation score } \\
\text { (scale 0-48): } \\
\text { Every mo with full } \\
\text { lactation } \\
\text { was given } 4 \text { points, } \\
\text { and every } \\
\text { mo with mixed } \\
\text { feeding was given } 2 \\
\text { points }\end{array}$ & $\begin{array}{l}\text { Weight change from } \\
\text { prepregnancy to } 1 \\
\text { yr }^{212,215} \text { or } 15 \text { yrs }{ }^{207} \\
\text { postpartum }\end{array}$ & $\begin{array}{l}\text { Correlation } \\
\text { between weight } \\
\text { change through } 1 \\
\text { yr postpartum and } \\
\text { lactation score, } \\
r=-0.09, p<0.01 \text {, } \\
\text { multiple stepwise } \\
\text { regression analysis } \\
\text { had a regression } \\
\text { coefficient of }-0.04 \text {, } \\
\text { p<0.001212, 215 } \\
\text { Those women who } \\
\text { became overweight } \\
\text { had lower lactation } \\
\text { scores than women } \\
\text { who remained } \\
\text { normal weight at } 15 \\
\text { yrs followup ( } 21.7 \\
\text { vs. } 24.0, p<0.05 \text { for } \\
\text { t test) }\end{array}$ & $\begin{array}{l}\text { GWG, age, } \\
\text { prepregnancy BMI, } \\
\text { and parity }{ }^{212,215} \\
\text { None }^{207}\end{array}$ \\
\hline
\end{tabular}




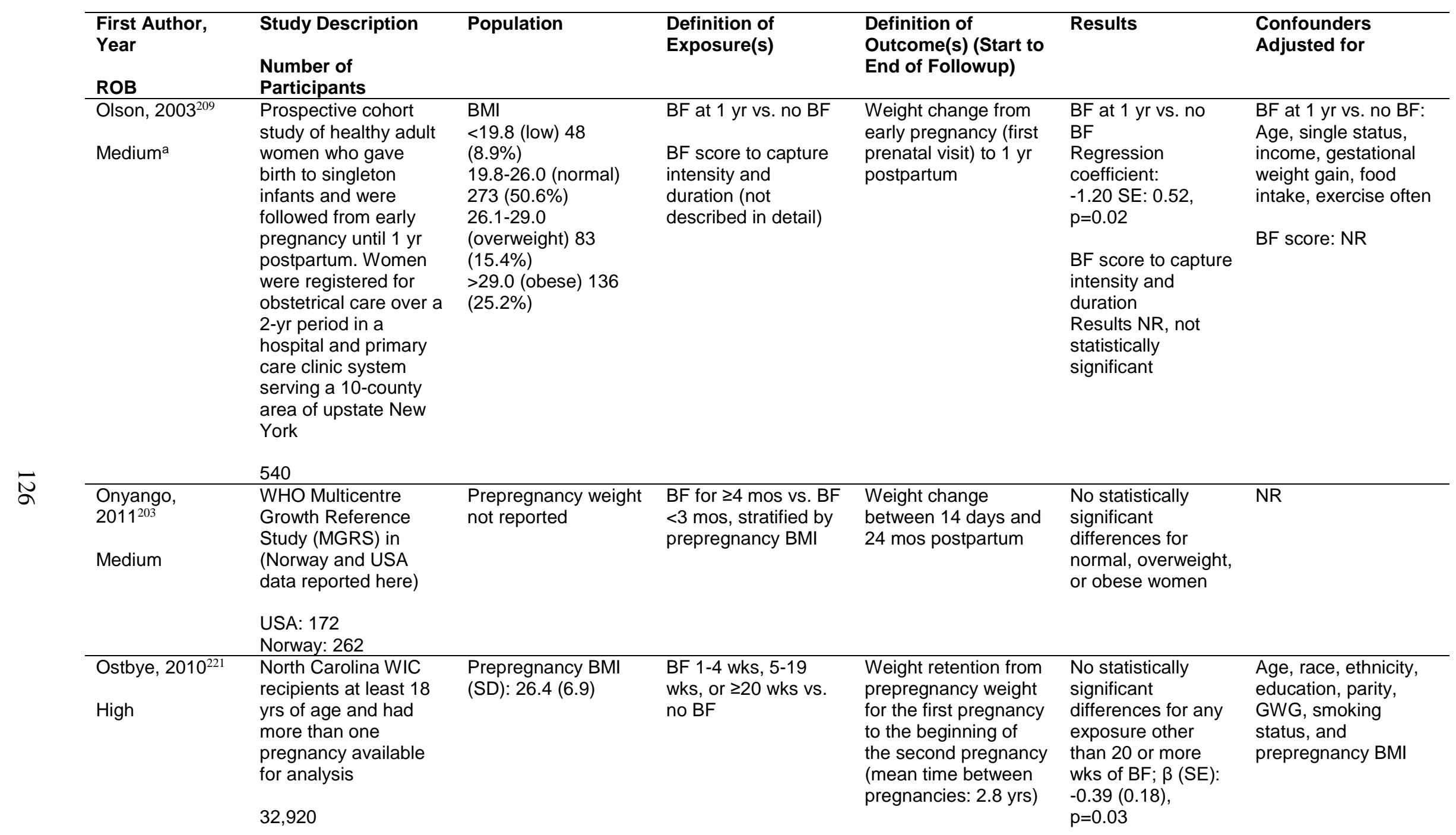




\begin{tabular}{|c|c|c|c|c|c|c|}
\hline $\begin{array}{l}\text { First Author, } \\
\text { Year } \\
\text { ROB }\end{array}$ & $\begin{array}{l}\text { Study Description } \\
\text { Number of } \\
\text { Participants }\end{array}$ & Population & $\begin{array}{l}\text { Definition of } \\
\text { Exposure(s) }\end{array}$ & $\begin{array}{l}\text { Definition of } \\
\text { Outcome(s) (Start to } \\
\text { End of Followup) }\end{array}$ & Results & $\begin{array}{l}\text { Confounders } \\
\text { Adjusted for }\end{array}$ \\
\hline $\begin{array}{l}\text { Palmer, } 2015^{217} \\
\text { High }\end{array}$ & $\begin{array}{l}\text { Women from the } \\
\text { Black Women's } \\
\text { Health Study who } \\
\text { gave birth for the first } \\
\text { time from } 1995 \text { to } \\
2003 \\
3,147\end{array}$ & $\begin{array}{l}\text { Mean prepregnancy } \\
\text { BMI (SD) } \\
\text { No BF: } 27.5(6.8) \\
\text { <3 mos: } 27.5(7.0) \\
\text { 3-5 mos: } 26.2(5.6) \\
\text { 6-11 mos: } 25.2(5.0) \\
\geq 12 \text { mos: } 25.4(5.0)\end{array}$ & $\begin{array}{l}\text { Duration of } B F(<3, \\
4-7,8-11, \text { and } \geq 12 \\
\text { mos) vs. no BF }\end{array}$ & $\begin{array}{l}\text { Weight change from } \\
\text { baseline (year of first } \\
\text { birth) to an average of } \\
4 \text { and } 8 \text { yrs } \\
\text { postpartum }\end{array}$ & $\begin{array}{l}\text { No differences by } \\
\text { duration of lactation }\end{array}$ & $\begin{array}{l}\text { Age, GWG, preterm } \\
\text { birth, vigorous } \\
\text { physical activity, } \\
\text { dietary pattern, years } \\
\text { of education, number } \\
\text { of additional births } \\
\text { during followup, and } \\
\text { mean duration of } \\
\text { lactation for the } \\
\text { additional births } \\
\text { during followup }\end{array}$ \\
\hline $\begin{array}{l}\text { Sicheiri, } 2003^{210} \\
\text { Medium }^{\mathrm{a}}\end{array}$ & $\begin{array}{l}\text { Nurse's Health Study } \\
\text { II, with analysis } \\
\text { restricted to women } \\
\text { who were ages } 24 \text { to } \\
40 \text { yrs at baseline } \\
\text { (1989), who had a } \\
\text { history of no more } \\
\text { than one past full-term } \\
\text { pregnancy at } \\
\text { baseline, gave birth to } \\
\text { one child between } \\
\text { 1990 and } 1991 \text { but } \\
\text { had no other } \\
\text { pregnancies during } \\
\text { the followup } \\
\text { Nulliparous: } \\
\text { 1,538; } \\
\text { Primiparous: } \\
2,810\end{array}$ & $\begin{array}{l}\text { BMI > } 25 \mathrm{~kg} / \mathrm{m}^{2} \text { in } \\
1989(\%) \\
\text { Never: } 27.8 \\
<3 \text { mos:18.4 } \\
4-7 \text { mos:15.8 } \\
8-11 \text { mos:17.9 } \\
>12 \text { mos:10.7 }\end{array}$ & $\begin{array}{l}\text { Exclusive } \mathrm{BF}^{\mathrm{d}} \text { vs. no } \\
\text { BF, stratified by } \\
\text { parity and BMl } \\
\text { Duration of exclusive } \\
\text { BF }(<3,4-7,8-11 \text {, } \\
\text { and } \geq 12 \text { mos })^{d} \text { vs. no } \\
\text { BF }\end{array}$ & $\begin{array}{l}\text { Weight change from } \\
\text { baseline } \\
\text { (prepregnancy, 1989) } \\
\text { to 1-2 yrs postpartum } \\
\text { (1993) }\end{array}$ & $\begin{array}{l}\text { Exclusive BF was } \\
\text { associated with a } \\
\text { weight gain of } \\
\text { approximately } 1 \mathrm{~kg} \\
\text { (statistically } \\
\text { significantly greater } \\
\text { only for nulliparous } \\
\text { women with a } \\
\text { baseline BMl }<25 \\
\text { who BF } 1-3 \text { and } 4-7 \\
\text { mos ( } p<0.05 \text { ) and } \\
\text { for primiparous } \\
\text { women with a } \\
\text { baseline BMI } \geq 25 \\
\text { who BF } \geq 12 \text { mos } \\
\text { ( } p=0.04 \text { ) when } \\
\text { compared with } \\
\text { women who did not } \\
\text { BF } \\
\text { Duration of } \\
\text { lactation was } \\
\text { unrelated to the } \\
\text { magnitude of } \\
\text { weight change } \\
\text { ( } p>0.40 \text { for all } \\
\text { comparisons) }\end{array}$ & $\begin{array}{l}\text { Exclusive BF: Age, } \\
\text { physical activity, BMI, } \\
\text { GWG } \\
\text { Duration: NR }\end{array}$ \\
\hline
\end{tabular}




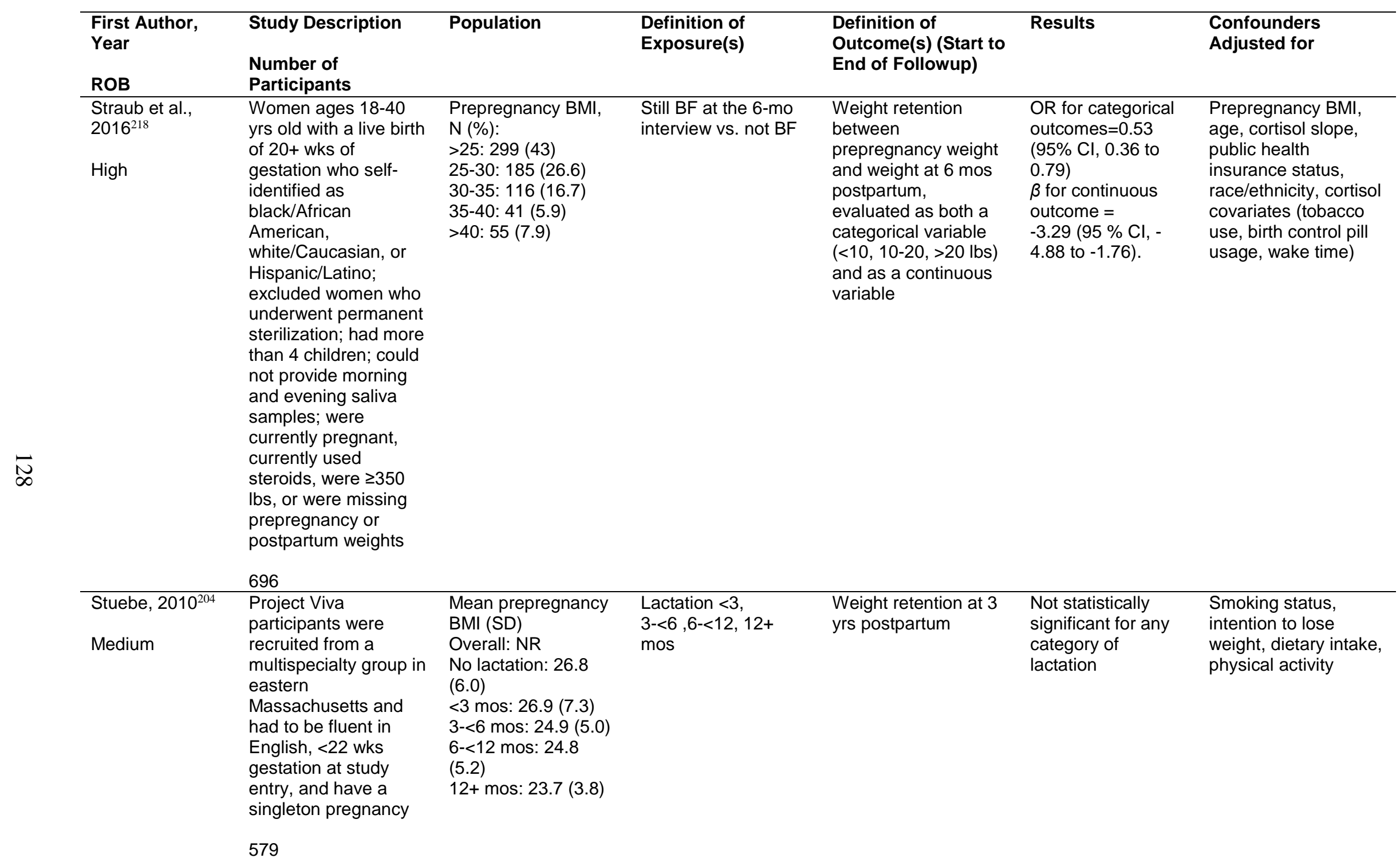




\begin{tabular}{|c|c|c|c|c|c|c|}
\hline $\begin{array}{l}\text { First Author, } \\
\text { Year } \\
\text { ROB }\end{array}$ & $\begin{array}{l}\text { Study Description } \\
\text { Number of } \\
\text { Participants }\end{array}$ & Population & $\begin{array}{l}\text { Definition of } \\
\text { Exposure(s) }\end{array}$ & $\begin{array}{l}\text { Definition of } \\
\text { Outcome(s) (Start to } \\
\text { End of Followup) }\end{array}$ & Results & $\begin{array}{l}\text { Confounders } \\
\text { Adjusted for }\end{array}$ \\
\hline $\begin{array}{l}\text { Walker, 2004 } \\
\text { Medium }^{\mathrm{a}}\end{array}$ & $\begin{array}{l}\text { Austin New Mothers } \\
\text { Study } \\
\text { (ANMS), a } \\
\text { longitudinal study of a } \\
\text { low-income, tri-ethnic } \\
\text { sample of } \\
\text { postpartum women } \\
\text { that incorporated } \\
\text { serial assessment of } \\
\text { weight and behavioral } \\
\text { and psychosocial } \\
\text { variables }\end{array}$ & $\begin{array}{l}\text { Mean prepregnancy } \\
\text { BMI (SD) } \\
\text { White: } 24.23 \pm 5.78 \\
\text { African American: } \\
25.39 \pm 5.40 \\
\text { Hispanic: } 26.75 \pm \\
6.19^{222}\end{array}$ & $\begin{array}{l}\text { Partial BF or full } \\
\text { bottle-feeding vs. full } \\
\text { (exclusive BF) (time- } \\
\text { varying } \\
\text { measurement of } \\
\text { exposure) }\end{array}$ & $\begin{array}{l}\text { Postpartum BMI over } \\
\text { time (delivery to } 12 \\
\text { mos) }\end{array}$ & $\begin{array}{l}\text { Infant feeding } \\
\text { method was not } \\
\text { associated with } \\
\text { postpartum BMI } \\
(p=0.140)\end{array}$ & $\begin{array}{l}\text { Ethnicity, maternal } \\
\text { education, parity, } \\
\text { smoking, physical } \\
\text { activity, time of weight } \\
\text { measurement, } \\
\text { interaction of ethnicity } \\
\text { and time, prepregnant } \\
\text { BMI, GWG, energy } \\
\text { intakes, fat intake, } \\
\text { contraception, } \\
\text { emotional eating, } \\
\text { depressive symptoms }\end{array}$ \\
\hline
\end{tabular}

a This study was included in the prior 2007 AHRQ review by Ip and colleagues. ${ }^{2}$ We did not reassess ROB for individual studies included in that review; this rating represents the decisions of the authors of that review.

${ }^{\mathrm{b}}$ Fully BF was defined as providing at least two-thirds of the needed energy intake per kilogram of the infant's weight in breast milk.

c Underweight is defined is $<18.5 \mathrm{~kg} / \mathrm{m}^{2}$. Healthy or normal weight is defined as $18.5-24.9 \mathrm{~kg} / \mathrm{m}^{2}$. Overweight is defined as $25-29.9 \mathrm{~kg} / \mathrm{m}^{2}$. Obese is defined as $\geq 30 \mathrm{~kg} / \mathrm{m}^{2}$.

${ }^{\mathrm{d}}$ Introduction of daily formula/milk was assumed to represent the end of exclusive BF period.

ANMS = Austin New Mothers Study; BF = breastfeeding; BMI = body mass index; CI = confidence interval; GWG = gestational weight gain; ICU = intensive care unit; IFPSII = Infant Feeding Practices Study II; kg = kilogram; lbs = pounds; MGRS = Multicentre Growth Reference Study; N = number; NICHD = ; NR = not reported; OR = odds ratio; ROB = risk of bias; SD = standard deviation; SE =standard error; SPAWN = Stockholm Pregnancy and Women’s Nutrition Study; WHO = World Health Organization; WIC = Special Supplemental Nutrition Program for Women, Infants and Children; wks = weeks. 


\section{Results}

\section{Duration}

Ten studies defined breastfeeding in terms of duration, ${ }^{203,204,206,208,209,213,217-219,} 221$ but their measurement of outcomes varied. Three of 10 studies reported weight change between an early postpartum period (1 to 14 days after delivery) to later in the postpartum period (12 to 24 months). ${ }^{203,208,213}$ Only 1 of these 3 studies, set in Australia, reported statistically significant differences. Breastfeeding for at least 3 months predicted lower odds of having postpartum weight retention from prepregnancy to 12 months within the top quintile, when compared with breastfeeding for less than 3 months or not breastfeeding. ${ }^{208}$ One of 10 studies reported on weight change between early pregnancy (first prenatal visit) and 1 year postpartum and found a greater and statistically significant weight reduction. ${ }^{209}$ The remaining 6 of 10 studies reported on weight change from prepregnancy weight to postpartum weight measured at time points ranging from 6 months to 8 years postpartum. ${ }^{204,206,217-219,221}$ Of these, 3 reported no statistically significant differences, ${ }^{204,206,217}$ and 1 reported a difference only for the group with the longest period of exposure (20 weeks or more). ${ }^{221}$ Two studies, set in the United States, reported differences by exposure. Specifically, 1 study reported a lower risk of weight retention of 20 pounds or more among those who partially or exclusively breastfed for 6 months or more when compared with those who did not, ${ }^{219}$ and a second reported lower weight retention among those who were still breastfeeding at 6 months postpartum compared with those who were not. ${ }^{218}$

\section{Duration and Intensity}

Four studies, reported in six publications, reported on duration and intensity. ${ }^{205,207,209,211,212 \text {, }}$ ${ }^{215}$ Of these, two publications reported on 1 year postpartum outcomes ${ }^{212,215}$ and a third reported on 15-year outcomes from the Swedish SPAWN Study. ${ }^{207}$ One study, evaluating weight change from immediately after delivery to 9 months postpartum, found no difference. A second, evaluating weight change from the first prenatal visit to 1 year postpartum, also found no differences. ${ }^{209}$ The two studies that evaluated weight change from a prepregnancy measure to weight at 1 year, ${ }^{212,} 21518$ months, ${ }^{211}$ and 15 years ${ }^{207}$ all reported statistically significant differences favoring greater intensity of breastfeeding.

\section{Duration and Exclusivity}

Four publications reported on duration and exclusivity of breastfeeding from three studies ${ }^{210}$, 214, 216, 220 (2 publications reported on the Infant Feeding Practices Study II but chose different weight change measures). ${ }^{216,220}$ Of the three studies, one study that evaluated weight change from the highest pregnancy weight to 12 months postpartum reported a difference in postpartum weight loss return to prepregnancy BMI and BMI category among those who were exclusively breastfeeding for 3 or more months compared with those who did not. ${ }^{216}$ Another publication using a subset of participants from the same study, but evaluating change in weight from a prepregnancy measure to 6 years postpartum, found benefit only for obese women who were most adherent to breastfeeding guidelines in terms of duration and exclusivity. ${ }^{20}$ A second study that evaluated change in weight and weight-height index from 1 to 2 days to 6 months postpartum found no statistically significant differences, ${ }^{214}$ and a third that stratified results by BMI, parity, and intensity generally found no differences in weight from a prepregnancy measurement to weight at 1 to 2 years postpartum, with some exceptions (i.e., higher weight retention in underweight nulliparous women who breastfed exclusively for up to 7 months vs. 
those who did not; obese primiparous women who breastfed exclusively $\geq 12$ months vs. those who did not). ${ }^{210}$ 


\section{Chapter 4. Discussion}

\section{Key Findings and Strength of Evidence}

For this report, we conducted a systematic review to evaluate the evidence for community, workplace, and health care system-based programs and policies aimed at supporting and promoting breastfeeding. In addition, we also updated the evidence on the association between breastfeeding and maternal health. Below, we summarize the main findings by each Key Question (KQ), including giving the strength of evidence (SOE) for the bodies of evidence pertaining to the effectiveness and harms of programs and policies on initiation, duration, and exclusivity of breastfeeding (KQ 1) and association between breastfeeding and maternal health (KQ 2).

\section{Effectiveness and Harms of Breastfeeding Programs and Policies}

The 40 studies that met our inclusion criteria evaluated a range of strategies to improve rates of breastfeeding initiation and duration. We categorized interventions primarily based on intervention type and delivery setting: Baby-Friendly Hospital Initiative (BFHI), non-BFHI health care system-based interventions, Women, Infants and Children (WIC)-based interventions, and community-based interventions. No included studies assessed workplace interventions or potential harms of interventions. Results are discussed below by intervention category.

\section{Baby-Friendly Hospital Initiative Interventions}

Results from 12 included studies assessing BFHI interventions support the effectiveness of BFHI for improving rates of breastfeeding initiation and duration. Table 29 summarizes key findings and SOE. For breastfeeding initiation, evidence from 9 cohort studies $(1,227,532$ women) comparing women giving birth in BFHI-certified (or accredited) hospitals with noncertified hospitals supports the effectiveness of BFHI (low SOE); although the included studies consistently found higher rates of initiation at BFHI hospitals, results were imprecise and the magnitude of benefit varied across studies. For breastfeeding duration, evidence from 1 large randomized controlled trial (RCT) (Promotion of Breastfeeding Intervention Trial [PROBIT], $\mathrm{N}=17,046$ women) and 8 cohort studies ( $\mathrm{N}=62,834$ women) supports the effectiveness of BFHI for increasing rates of breastfeeding duration (moderate SOE). In the PROBIT trial, women in the intervention group had significantly higher rates of exclusive breastfeeding and lower rates of weaning across multiple time points (1 to 12 months postpartum). Although the 8 observational studies were mostly consistent in finding benefit, results were imprecise and the magnitude of benefit varied. One cohort study compared rates of breastfeeding at 6 months among women discharged from hospitals that differed in the number of BFHI steps implemented; low SOE supports the conclusion that implementation of four or more BFHI steps is associated with lower rates of weaning than implementation of fewer than four steps. 
Table 29. Summary of key findings and strength of evidence: Studies assessing BFHI

\begin{tabular}{|c|c|c|c|}
\hline $\begin{array}{l}\text { Breastfeeding Outcome } \\
\text { Intervention Versus } \\
\text { Comparator }\end{array}$ & $\begin{array}{l}\mathbf{N} \\
\text { Studies; } \\
\mathrm{N} \\
\text { Subjects } \\
\text { Study } \\
\text { Limitation } \\
\mathrm{S}\end{array}$ & Outcome and Results & $\begin{array}{l}\text { Strength } \\
\text { of } \\
\text { Evidence }\end{array}$ \\
\hline $\begin{array}{l}\text { Initiation } \\
\text { BFHI certified/accredited } \\
\text { vs. no BFHI status }\end{array}$ & $\begin{array}{l}9 \\
\text { cohorts; } \\
55,57,58,60-64 \\
1,227,532 \\
\text { Medium }\end{array}$ & $\begin{array}{l}\text { Any BF initiation ( } k=6) \text { : higher rates of BF at discharge among } \\
\text { BFHI-accredited hospitals than control hospitals (by } 0.5 \% \text { to } \\
10 \% \text { ); differences between groups were not statistically } \\
\text { significant in } 4 \text { studies } \\
\text { Exclusive BF initiation ( } k=5 \text { ): significantly higher rates of } \\
\text { exclusive BF at discharge among BFHI-accredited hospitals than } \\
\text { control hospitals; magnitude varied, ranging from } 3 \text { to } 56 \%\end{array}$ & $\begin{array}{l}\text { Low for } \\
\text { benefit } \\
\text { (consisten } \\
\text { t, } \\
\text { imprecise) }\end{array}$ \\
\hline $\begin{array}{l}\text { Duration } \\
\text { BFHI vs. no BFHI } \\
\text { intervention (evidence } \\
\text { from RCTs) }\end{array}$ & $\begin{array}{l}1 \mathrm{RCT} ; 52 \\
53 \text { 17,046 }\end{array}$ & $\begin{array}{l}\text { One RCT found significantly higher rates of exclusive BF among } \\
\text { women at BFHI hospitals at } 3 \text { mos ( } 43 \% \text { vs. } 6 \% \text {; } p<0.001) \text { and } 6 \\
\text { mos postpartum ( } 7.9 \% \text { vs. } 0.6 \% ; p=0.01) \text {, and lower odds of } \\
\text { weaning (from any BF) at } 3,6,9 \text {, and } 12 \text { mos postpartum than } \\
\text { women in control hospitals }\end{array}$ & $\begin{array}{l}\text { Moderate } \\
\text { for benefit } \\
\text { (consisten } \\
\text { t, } \\
\text { imprecise) }\end{array}$ \\
\hline $\begin{array}{l}\text { Duration } \\
\text { BFHI certified/accredited } \\
\text { vs. no BFHI status } \\
\text { (evidence from } \\
\text { observational studies) }\end{array}$ & $\begin{array}{l}8 \\
\text { cohorts; } 54- \\
56,58,60,61, \\
63,64 \\
136,983 \\
\text { Medium }\end{array}$ & $\begin{array}{l}\text { Any BF duration ( } \mathrm{k}=8 \text { cohort studies): higher rates of BF } 1 \text { to } 12 \\
\text { mos postpartum among women at BFHI hospitals (by } \\
\text { approximately } 0.6 \% \text { to } 15 \% \text { ) than women at control hospitals; } \\
\text { one study found slightly higher BF rates at } 1 \text { mo among women } \\
\text { in control hospitals than BFHI hospitals (by } 0.4 \% \text { to } 7 \% \text { ) } \\
\text { Exclusive BF duration ( } \mathrm{k}=5 \text { cohort studies): higher rates of } \\
\text { exclusive BF over } 1 \text { to } 2 \text { mos among infants born in BFHI } \\
\text { hospitals than control hospitals (by approximately } 4 \% \text { to } 25 \% \text { ) }\end{array}$ & \\
\hline $\begin{array}{l}\text { Duration } \\
\text { Six or more BFHI steps } \\
\text { vs. fewer than six steps }\end{array}$ & $\begin{array}{l}1 \text { cohort; } 56 \\
1,417 \\
\text { Medium }\end{array}$ & $\begin{array}{l}\text { Significantly higher odds of weaning at or before } 8 \text { wks } \\
\text { postpartum among women giving birth in hospitals practicing } \leq \\
\text { four BFHI steps than women giving birth in hospitals practicing } \\
\text { six BFHI steps (ORs ranged from } 2.08 \text { and 3.13); no difference } \\
\text { between women exposed to five vs. six steps }\end{array}$ & $\begin{array}{l}\text { Low for } \\
\text { benefit } \\
\text { (consisten } \\
\text { t }^{\mathrm{a}} \text { precise) }\end{array}$ \\
\hline
\end{tabular}

BF = breastfeeding; BFHI = Baby-Friendly Hospital Initiative; HV = home visits; $\mathrm{k}=$ number of studies; $\mathrm{N}=$ number; OR = odds ratio; $\mathrm{RCT}$ = randomized controlled trial; $\mathrm{SOE}=$ strength of evidence.

\section{Other (Non-BFHI) Health Care System-Based Interventions}

Fifteen studies (described in 16 publications) assessed the effectiveness of other (non-BFHI) health care system-based interventions. ${ }^{68,70-85,223}$ Studies assessed a variety of intervention types; the majority focused on health care provider education or training related to breastfeeding, with or without additional services offered (e.g., breastfeeding groups, home visits). Table 30 presents key findings and SOE conclusions. Overall, the evidence supports the effectiveness of three intervention types for improving the duration of exclusive breastfeeding: modified-BFHI policy implementation in outpatient settings (e.g., development of a breastfeeding policy, staff training, outcome assessment, and quality improvement initiatives), continuous nursing care during the perinatal period (the same nurse provides routine perinatal care to the mother and infant), and health care provider education combined with a series of home visits (low SOE). In addition, the evidence suggests that health care provider education and training alone (without additional breastfeeding support services) are not effective in improving rates of breastfeeding 
initiation (low SOE). Because of methodological limitations and imprecise and inconsistent findings, we rated the SOE as insufficient for other intervention types.

Table 30. Summary of key findings and strength of evidence: Non-BFHI health care system-based interventions

\begin{tabular}{|c|c|c|c|}
\hline $\begin{array}{l}\text { Breastfeeding Outcome } \\
\text { Intervention Versus } \\
\text { Comparator }\end{array}$ & $\begin{array}{l}\text { N Studies; N } \\
\text { Subjects } \\
\text { Study } \\
\text { Limitations }\end{array}$ & Outcome and Results & Strength of Evidence \\
\hline $\begin{array}{l}\text { Initiation } \\
\text { Education/staff training } \\
\text { related to BF alone vs. } \\
\text { usual practice }\end{array}$ & $\begin{array}{l}4\left(2 \mathrm{RCTS}^{70,71,75}\right. \\
\left.2 \mathrm{NRCTs}^{73,78}\right) \\
1,532^{\mathrm{a}} \\
\text { Medium }\end{array}$ & $\begin{array}{l}\text { No significant difference between } \\
\text { intervention and control groups in } \\
\text { rates of any or exclusive BF } \\
\text { initiation }\end{array}$ & $\begin{array}{l}\text { Low for no benefit } \\
\text { (consistent, imprecise) }\end{array}$ \\
\hline $\begin{array}{l}\text { Initiation } \\
\text { Education and staff } \\
\text { training plus additional } \\
\text { individual services vs. } \\
\text { usual care }\end{array}$ & $\begin{array}{l}4\left(2 \text { RCTs, }{ }^{74,77} 1\right. \\
\text { NRCT, }{ }^{68} 1 \text { pre- } \\
\left.\text { post study }{ }^{82}\right) ; \\
34,018 \\
\text { Medium }\end{array}$ & $\begin{array}{l}\text { Inconsistent findings across four } \\
\text { studies assessing heterogeneous } \\
\text { interventions }\end{array}$ & $\begin{array}{l}\text { Insufficient (inconsistent, } \\
\text { imprecise) }\end{array}$ \\
\hline $\begin{array}{l}\text { Duration } \\
\text { Education and staff } \\
\text { training related to BF } \\
\text { only vs. usual practice }\end{array}$ & $\begin{array}{l}3 \text { (2 RCTs, }^{70,71,79} \\
\left.1 \mathrm{NRCT}^{73}\right) \\
1,526^{\mathrm{a}} \\
\text { Medium }\end{array}$ & $\begin{array}{l}\text { Inconsistent findings across three } \\
\text { studies for duration of any and } \\
\text { exclusive BF }\end{array}$ & $\begin{array}{l}\text { Insufficient (inconsistent, } \\
\text { imprecise) }\end{array}$ \\
\hline $\begin{array}{l}\text { Duration } \\
\text { Education and staff } \\
\text { training plus additional }\end{array}$ & $\begin{array}{l}\text { 4 RCTs; }{ }^{74,76,77,84} \\
21,253 \\
\text { Medium }\end{array}$ & $\begin{array}{l}\text { Two RCTs assessing staff } \\
\text { education combined with a series } \\
\text { of postpartum HVs found improved } \\
\text { rates of any BF duration }\end{array}$ & $\begin{array}{l}\text { Staff education plus HVs: } \\
\text { Low for benefit (consistent, } \\
\text { precise) }\end{array}$ \\
\hline
\end{tabular}

individual services vs.

usual care

Two RCTs assessing staff education combined with different clinic-based patient education strategies found no significant difference between groups

\begin{tabular}{ll}
\hline Duration & $\begin{array}{l}\text { 1 NRCT; } 81 \text { 3,948 } \\
\text { Medium }\end{array}$ \\
Adaptation of the BFHI &
\end{tabular}

for integration into routine primary care (maternal and child health centers) vs. usual care

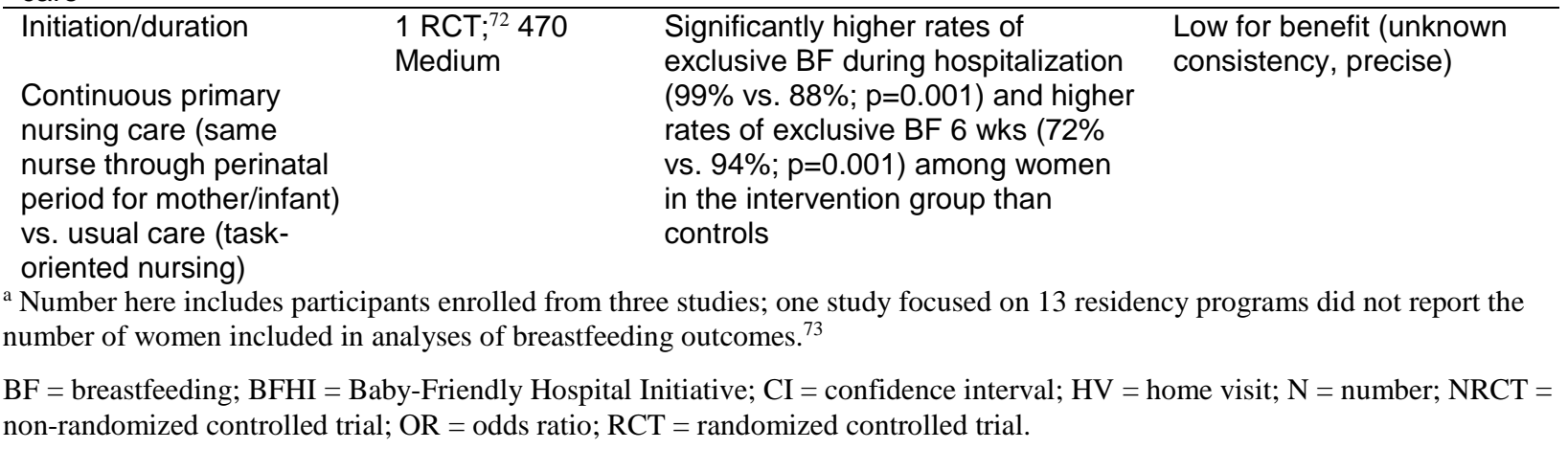

Staff education plus clinicbased education/support: Insufficient (inconsistent, imprecise)

Low for benefit (unknown consistency, precise) group than controls at 6 mos (OR 1.33; $95 \% \mathrm{Cl}, 1.03$ to 1.72); no difference between groups in rates of any BF at 5 or 12 mos

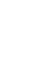




\section{WIC-Based Interventions}

Eight included studies assessed changes in breastfeeding rates associated with a WIC program or policy; ${ }^{86-93}$ studies enrolled women from diverse States and assessed heterogeneous interventions; key findings and SOE assessments are shown in Table 31. Few studies assessed the same intervention type. Low SOE supports the effectiveness of WIC-based peer-support programs for improving rates of any breastfeeding initiation and duration from 6 weeks to 6 months postpartum. We found insufficient evidence (primarily due to unknown consistency and imprecision) to make a conclusion on the benefit of other WIC programs or policies for improving breastfeeding outcomes, including policy changes related to WIC food packages, provision of different types of breast pumps (electric vs. manual), cash incentives, and peersupport programs targeted at fathers.

Table 31. Summary of key findings and strength of evidence: WIC-based interventions

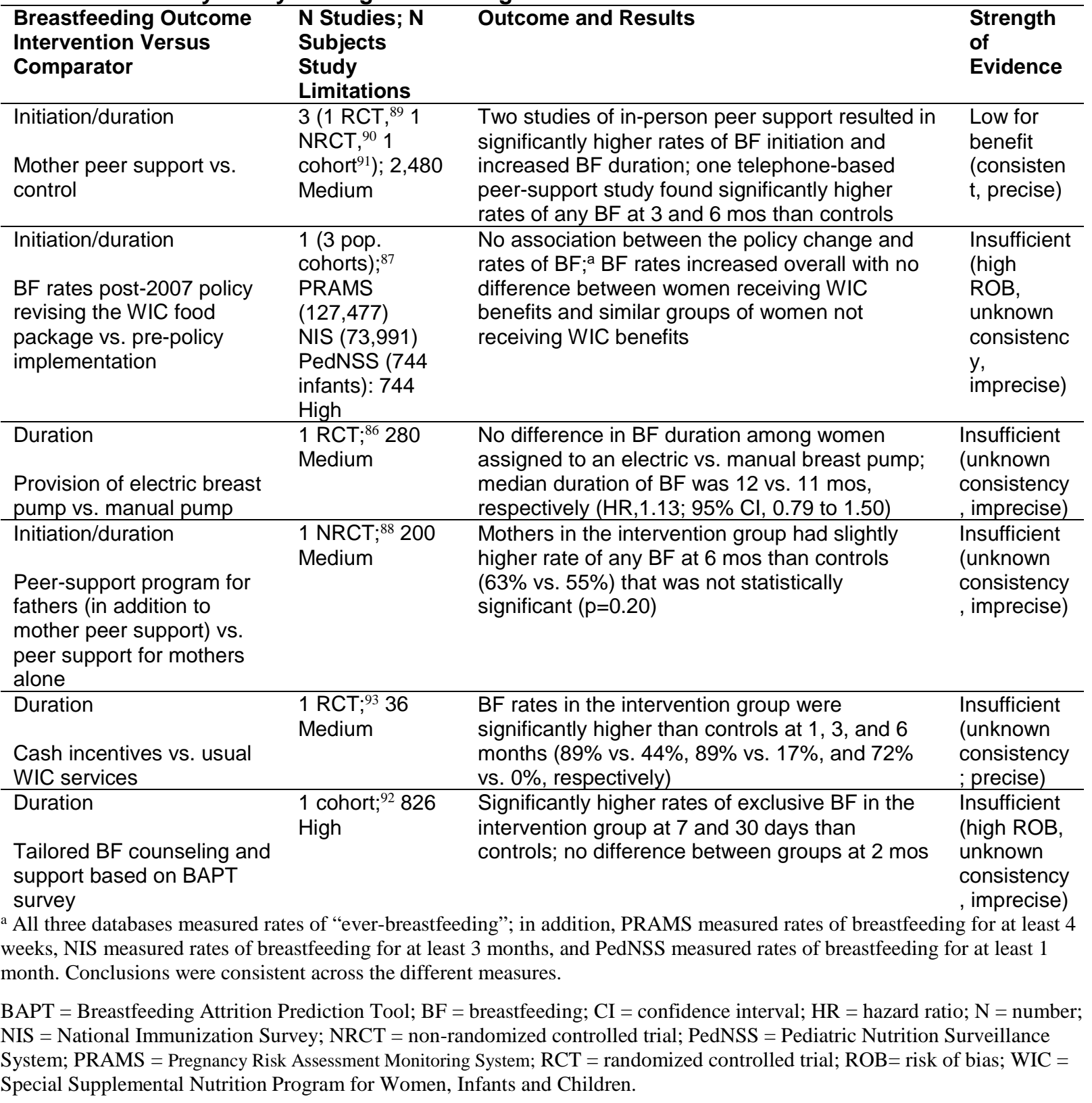




\section{Community-Based Interventions}

Five included studies (described in five publications) assessed the effectiveness of a community-based intervention; ${ }^{94-100}$ key findings and SOE assessments are shown in Table 32. No studies assessed the same intervention type, which limited our ability to make conclusions on the SOE for most intervention types. Low SOE supports the benefit of community-based interventions that provide mothers with peer support (via home visits). In addition, access to a community-based breastfeeding drop-in center among women receiving early home-based breastfeeding support does not increase breastfeeding duration (low SOE).

Table 32. Summary of key findings and strength of evidence: Community-based interventions

\begin{tabular}{|c|c|c|c|}
\hline $\begin{array}{l}\text { Breastfeeding Outcome } \\
\text { Intervention Versus } \\
\text { Comparator }\end{array}$ & $\begin{array}{l}\text { N Studies; N } \\
\text { Subjects } \\
\text { Study } \\
\text { Limitations }\end{array}$ & Outcome and Results & $\begin{array}{l}\text { Strength of } \\
\text { Evidence }\end{array}$ \\
\hline Initiation/duration & $\begin{array}{l}1 \text { NRCT; } 94,99 \\
5,094\end{array}$ & $\begin{array}{l}\text { No significant difference in rates of exclusive BF at } \\
\text { discharge, } 3 \text { and } 6 \text { mos, or rates of any BF at } 5 \text { and }\end{array}$ & $\begin{array}{l}\text { Insufficient } \\
\text { (unknown }\end{array}$ \\
\hline $\begin{array}{l}\text { Community-based policy aimed } \\
\text { at promoting BF in nonhospital- } \\
\text { based health and community } \\
\text { centers vs. no intervention }\end{array}$ & Medium & 12 mos between groups & $\begin{array}{l}\text { consistency, } \\
\text { imprecise) }\end{array}$ \\
\hline Duration & $\begin{array}{l}1 \mathrm{RCT} ; 95,100 \\
9,675\end{array}$ & $\begin{array}{l}\text { No difference between groups in rates of any BF at } \\
3,4 \text {, or } 5 \text { mos. }\end{array}$ & $\begin{array}{l}\text { Low for no } \\
\text { benefit }\end{array}$ \\
\hline $\begin{array}{l}\text { Access to community-based BF } \\
\text { drop-in centers (plus early BF } \\
\text { support) vs. early BF support } \\
\text { alone vs. usual care }\end{array}$ & Low & & $\begin{array}{l}\text { (unknown } \\
\text { consistency, } \\
\text { precise) }\end{array}$ \\
\hline Duration & $\begin{array}{l}1 \mathrm{RCT} ; 96 \\
130\end{array}$ & $\begin{array}{l}\text { Significantly higher rates of exclusive BF at } 3 \text { mos } \\
\text { among intervention groups (50\% to } 67 \% \text { ) than }\end{array}$ & $\begin{array}{l}\text { Low for benefit } \\
\text { (unknown }\end{array}$ \\
\hline $\begin{array}{l}\text { Community-based peer support } \\
\text { vs. usual care }\end{array}$ & Low & $\begin{array}{l}\text { control group }(12 \%), p<0.001 \text {; rates of any BF were } \\
\text { significantly longer in intervention groups } \\
\text { (combined) than in the control group at } 3 \text { mos (but } \\
\text { not } 6 \text { mos) }\end{array}$ & $\begin{array}{l}\text { consistency, } \\
\text { precise) }\end{array}$ \\
\hline $\begin{array}{l}\text { Duration } \\
\text { Peer-led BF support class vs. } \\
\text { Nurse-led BF support class }\end{array}$ & $\begin{array}{l}\text { 1 cohort; } 98 \\
109 \\
\text { High }\end{array}$ & $\begin{array}{l}\text { No significant difference between groups in rates of } \\
\text { any BF at } 1 \text { and } 6 \text { mos postpartum }\end{array}$ & $\begin{array}{l}\text { Insufficient } \\
\text { (high ROB, } \\
\text { unknown } \\
\text { consistency, } \\
\text { imprecise) }\end{array}$ \\
\hline $\begin{array}{l}\text { Duration } \\
\text { Integrated postpartum program } \\
\text { (BF education and support, } \\
\text { maternal/infant health care) vs. } \\
\text { usual care }\end{array}$ & $\begin{array}{l}1 \text { NRCT; } 97 \\
392 \\
\text { High }\end{array}$ & $\begin{array}{l}\text { Significantly higher rates of exclusive BF at } 6 \text { mos } \\
\text { among the intervention group than control group } \\
\text { ( } 74 \% \text { vs. } 10 \% ; p=0.001 \text { ) }\end{array}$ & $\begin{array}{l}\text { Insufficient } \\
\text { (high ROB, } \\
\text { unknown } \\
\text { consistency, } \\
\text { precise) }\end{array}$ \\
\hline
\end{tabular}

BF = breastfeeding; $\mathrm{N}$ = number; $\mathrm{NRCT}$ = nonrandomized controlled trial; $\mathrm{ROB}$ = risk of bias; $\mathrm{RCT}$ = randomized controlled trial.

\section{Effectiveness and Harms of Breastfeeding Programs and Policies for Subpopulations of Women}

Few studies reported on subgroups of women. Of the four included studies reporting on subgroups of women, two focused on BFHI and reported on differences by education status, and two focused on WIC interventions and reported on differences relevant to race/ethnicity. Table 33 summarizes our conclusions below by intervention type. Low SOE supports the conclusion that BFHI effectiveness may vary among women who differ by education status. For WIC interventions, we found insufficient evidence to make a conclusion on whether benefit of 
telephone peer support varies by subgroups of women based on language spoken (Spanish only vs. English) or whether benefit of tailored breastfeeding counseling intervention varies by race/ethnicity, primarily due to unknown consistency (and inconsistency across time points) and imprecision.

Table 33. Summary of key findings and strength of evidence: KQ 1 studies reporting on subgroups

\begin{tabular}{|c|c|c|c|}
\hline $\begin{array}{l}\text { Breastfeeding Outcome } \\
\text { Intervention Versus } \\
\text { Comparator }\end{array}$ & $\begin{array}{l}\text { N Studies; } \\
\text { N Subjects } \\
\text { Study } \\
\text { Limitations }\end{array}$ & Outcome and Results & $\begin{array}{l}\text { Strength of } \\
\text { Evidence }\end{array}$ \\
\hline $\begin{array}{l}\text { Initiation (subgroups: } \\
\text { education status) } \\
\text { BFHI certified/accredited vs. } \\
\text { no BFHI status }\end{array}$ & $\begin{array}{l}2 \text { cohort; } 54,55 \\
27,341 \\
\text { Medium }\end{array}$ & $\begin{array}{l}\text { Higher rates of BF initiation found among women with } \\
\text { lower education ( } \leq 12 \text { yrs) at BFHI hospitals compared } \\
\text { with control hospitals, but no difference in rates among } \\
\text { women with higher education ( } \geq 13 \text { yrs) }\end{array}$ & $\begin{array}{l}\text { Low } \\
\text { (consistent, } \\
\text { imprecise) }\end{array}$ \\
\hline $\begin{array}{l}\text { Duration (subgroups: } \\
\text { education status) } \\
\text { BFHI certified/accredited vs. } \\
\text { no BFHI status }\end{array}$ & $\begin{array}{l}2 \text { cohort; } 54,55 \\
27,341 \\
\text { Medium }\end{array}$ & Two studies found mixed results. & $\begin{array}{l}\text { Insufficient } \\
\text { (inconsistent, } \\
\text { imprecise) }\end{array}$ \\
\hline $\begin{array}{l}\text { Initiation/duration } \\
\text { (subgroups: language } \\
\text { spoken) } \\
\text { Mother peer support vs. } \\
\text { control }\end{array}$ & $\begin{array}{l}1 \mathrm{RCT} ; 89 \\
1948 \\
\text { Medium }\end{array}$ & $\begin{array}{l}\text { One RCT of telephone peer support found mixed } \\
\text { results for subgroups of women defined by language } \\
\text { (English-speaking vs. Spanish-speaking only) }\end{array}$ & $\begin{array}{l}\text { Insufficient } \\
\text { (unknown } \\
\text { consistency, } \\
\text { imprecise) }\end{array}$ \\
\hline $\begin{array}{l}\text { Duration (subgroups: } \\
\text { race/ethnicity) } \\
\text { Tailored BF counseling and } \\
\text { support based on BAPT } \\
\text { survey }\end{array}$ & $\begin{array}{l}1 \text { cohort; } 92 \\
826 \\
\text { High }\end{array}$ & $\begin{array}{l}\text { Significantly higher rates of exclusive BF among non- } \\
\text { Hispanic black and Hispanic women in the intervention } \\
\text { group than controls at } 1 \text { and } 2 \text { mos; no significant } \\
\text { difference in exclusive BF rates among white women at } \\
\text { any time point }\end{array}$ & $\begin{array}{l}\text { Insufficient } \\
\text { (high ROB, } \\
\text { unknown } \\
\text { consistency, } \\
\text { precise) }\end{array}$ \\
\hline
\end{tabular}

\section{Effect of Intervention Characteristics on Breastfeeding Outcomes}

This KQ focused on the extent to which intervention-related characteristics (e.g., type of breast pump provided - manual or electric, delivery personnel) influence the initiation, duration, and exclusivity of breastfeeding. We found no evidence to address this KQ.

\section{Maternal Health Outcomes Associated With Breastfeeding}

Table 34 summarizes our key findings related to KQ 2, including evidence for subpopulations of women, by outcome. Low SOE supports the conclusion that ever breastfeeding, as well as longer durations of breastfeeding, may be associated with a reduced risk of developing (any) breast cancer, luminal breast cancer, or triple-negative breast cancer. 
Table 34. Summary of key findings and strength of evidence: Maternal health outcomes

\begin{tabular}{|c|c|c|c|}
\hline $\begin{array}{l}\text { Maternal Health } \\
\text { Outcome }\end{array}$ & $\begin{array}{l}\text { N Studies; N Subjects } \\
\text { Study Limitations }\end{array}$ & Outcome and Results ${ }^{a}$ & $\begin{array}{l}\text { Strength of } \\
\text { Evidence }\end{array}$ \\
\hline Breast cancer & $\begin{array}{l}1 \text { SR of } 98 \text { cohort/case- } \\
\text { control studies; }{ }^{1} \mathrm{NR}^{\mathrm{b}} \\
19 \text { cohort/case-control } \\
\text { studies; }^{108-126} 256,891 \\
\text { women } \\
\text { Medium }\end{array}$ & $\begin{array}{l}\text { Consistent association in one SR ( } 98 \\
\text { observational studies) between ever BF and } \\
\text { lower rates of breast cancer compared with } \\
\text { never BF (pooled OR, 0.78, 95\% Cl, } 0.74 \text { to } \\
0.82 \text { ); longer durations of BF was also } \\
\text { associated with significantly lower rates of } \\
\text { breast cancer than never BF. Results of } \\
\text { individual studies were generally consistent in } \\
\text { direction of effect (although results were } \\
\text { imprecise); magnitude varied significantly across } \\
\text { all studies and pooled results were associated } \\
\text { with significant heterogeneity, only partially } \\
\text { explained by subgroup analyses. }\end{array}$ & $\begin{array}{l}\text { Low for } \\
\text { beneficial } \\
\text { association } \\
\text { (consistent, } \\
\text { imprecise) }\end{array}$ \\
\hline $\begin{array}{l}\text { Breast cancer: } \\
\text { BRCA1/2 carriers }\end{array}$ & $\begin{array}{l}1 \text { case-control study; }{ }^{129} \\
5,708 \text { women } \\
\text { Medium }\end{array}$ & $\begin{array}{l}\text { Unclear association between BF and breast } \\
\text { cancer among BRCA carriers. }\end{array}$ & $\begin{array}{l}\text { Insufficient } \\
\text { (unknown } \\
\text { consistency, } \\
\text { imprecise) }\end{array}$ \\
\hline $\begin{array}{l}\text { Breast cancer: In } \\
\text { situ }\end{array}$ & $\begin{array}{l}\text { 3 cohort/case-control } \\
\text { studies;122, 125, } 13267,234 \\
\text { women } \\
\text { Medium }\end{array}$ & $\begin{array}{l}\text { Unclear association between BF and breast } \\
\text { cancer in situ. }\end{array}$ & $\begin{array}{l}\text { Insufficient } \\
\text { (inconsistent, } \\
\text { imprecise) }\end{array}$ \\
\hline $\begin{array}{l}\text { Breast cancer: } \\
\text { Hormone receptor } \\
\text { subtypes }\end{array}$ & $\begin{array}{l}1 \text { SR of } 11 \text { cohort/case- } \\
\text { control studies; } 107 \\
169,879 \text { women for } \\
\text { luminal, } 14,266 \text { women } \\
\text { for HER2, and } 176,430 \\
\text { women for triple- } \\
\text { negative analyses } \\
7 \text { cohort/case-control } \\
\text { studies; }{ }^{110,117,121,133-136} \\
592,558 \text { women } \\
\text { Medium }\end{array}$ & $\begin{array}{l}\text { Consistent association between ever BF or } \\
\text { longer duration of BF and lower rates of luminal } \\
\text { and triple negative breast cancer (although } \\
\text { magnitude of association varies); for HER2, } \\
\text { pooled estimates show unclear association } \\
\text { between BF and lower rates of breast cancer } \\
\text { (results are imprecise and pooled estimate is not } \\
\text { statistically significant). }\end{array}$ & $\begin{array}{l}\text { Low for } \\
\text { beneficial } \\
\text { association } \\
\text { (luminal, } \\
\text { triple- } \\
\text { negative; } \\
\text { consistent, } \\
\text { imprecise); } \\
\text { insufficient } \\
\text { (HER2, } \\
\text { inconsistent, } \\
\text { imprecise) }\end{array}$ \\
\hline $\begin{array}{l}\text { Breast cancer: } \\
\text { Mortality }\end{array}$ & $\begin{array}{l}1 \text { cohort study; } 137 \\
250,470 \text { parous women } \\
\text { Medium }\end{array}$ & $\begin{array}{l}\text { Unclear association; one study found no } \\
\text { significant association between BF and breast } \\
\text { cancer mortality (HR,1.01; } 95 \% \mathrm{Cl}, 0.79 \text { to } 1.29) \text {. }\end{array}$ & $\begin{array}{l}\text { Insufficient } \\
\text { (unknown } \\
\text { consistency, } \\
\text { imprecise) }\end{array}$ \\
\hline Ovarian cancer & $\begin{array}{l}1 \text { SR of } 41 \text { cohort/case- } \\
\text { control studies; }{ }^{1} \text { NR }^{c} \\
9 \text { cohort/case-control } \\
\text { studies; }{ }^{140-149} 42,611 \\
\text { women } \\
\text { Medium }\end{array}$ & $\begin{array}{l}\text { Consistent association between ever BF and } \\
\text { longer durations of BF and lower risk of ovarian } \\
\text { cancer; magnitude of association varies across } \\
\text { studies by BF exposure definition. }\end{array}$ & $\begin{array}{l}\text { Moderate for } \\
\text { beneficial } \\
\text { association } \\
\text { (inconsistent, } \\
\text { precise) }\end{array}$ \\
\hline Hypertension & $\begin{array}{l}5 \text { cohort studies; ;, 5, 152, } \\
\text { 153, } 224441,989 \text { women } \\
\text { Medium }\end{array}$ & $\begin{array}{l}\text { Consistent association between longer duration } \\
\text { of BF ( }>6-12 \text { mos) and lower rates of HTN; } \\
\text { magnitude of association varies by BF exposure } \\
\text { comparisons and study design. }\end{array}$ & $\begin{array}{l}\text { Low for } \\
\text { beneficial } \\
\text { association } \\
\text { (consistent, } \\
\text { imprecise) }\end{array}$ \\
\hline CVD & $\begin{array}{l}3 \text { cohort studies; } 4,6,155 \\
301,989 \text { women } \\
\text { Medium }\end{array}$ & $\begin{array}{l}\text { Unclear association between BF and CVD; three } \\
\text { studies conclude an association between longer } \\
\text { BF duration and lower CVD rates, each using a } \\
\text { different composite outcome; magnitude of } \\
\text { association varies by exposure comparisons, } \\
\text { age at cohort enrolment, and study design. }\end{array}$ & $\begin{array}{l}\text { Insufficient } \\
\text { (unknown } \\
\text { consistency, } \\
\text { imprecise) }\end{array}$ \\
\hline
\end{tabular}




\begin{tabular}{|c|c|c|c|}
\hline Intervention type & $\begin{array}{l}\text { N Studies; N Subjects } \\
\text { Study Limitations }\end{array}$ & Outcome and Results & $\begin{array}{l}\text { Strength of } \\
\text { Evidence }\end{array}$ \\
\hline CVD mortality & $\begin{array}{l}1 \text { cohort study: } 156 \\
15,000 \text { women } \\
\text { Medium }\end{array}$ & $\begin{array}{l}\text { Unclear association between BF and CVD } \\
\text { mortality. One study found mixed results: parous } \\
\text { women } \leq 65 \text { yrs at enrollment who had never BF } \\
\text { had higher CVD mortality over } 14 \text { yrs of followup } \\
\text { than women who BF } \geq 24 \text { mos (HR, } 2.77 ; 95 \% \\
\mathrm{Cl}, 1.28 \text { to } 5.99 \text { ). No clear associations were } \\
\text { observed among women } \leq 65 \text { yrs at enrollment. }\end{array}$ & $\begin{array}{l}\text { Insufficient } \\
\text { (unknown } \\
\text { consistency, } \\
\text { imprecise) }\end{array}$ \\
\hline Type 2 diabetes & $\begin{array}{l}1 \text { SR of } 6 \text { cohort } \\
\text { studies; }{ }^{: 58} 273,961 \\
\text { women } \\
5 \text { cohort studies; } 4,159-163 \\
325,815 \text { women } \\
\text { Medium }\end{array}$ & $\begin{array}{l}\text { Consistent association between ever BF and } \\
\text { longer durations of BF and lower rates of type } 2 \\
\text { diabetes (among women with and without } \\
\text { gestational diabetes); magnitude of association } \\
\text { varies by BF exposure duration and study } \\
\text { design. }\end{array}$ & $\begin{array}{l}\text { Low for } \\
\text { beneficial } \\
\text { association } \\
\text { (consistent, } \\
\text { imprecise) }\end{array}$ \\
\hline Fractures & $\begin{array}{l}11 \text { cohort/case-control } \\
\text { studies); }{ }^{165,167-176} \\
\text { 101,726 women } \\
\text { Medium }\end{array}$ & $\begin{array}{l}\text { Consistent lack of association between BF and } \\
\text { fractures. Magnitude varies by exposure and } \\
\text { outcome measure, but only } 1 \text { high ROB study } \\
\text { reported statistically significant differences. }\end{array}$ & $\begin{array}{l}\text { Low for no } \\
\text { association } \\
\text { (consistent, } \\
\text { imprecise) }\end{array}$ \\
\hline $\begin{array}{l}\text { Postpartum } \\
\text { depression }\end{array}$ & $\begin{array}{l}\text { 1 SR of } 48 \text { cohort } \\
\text { studies; } 183 \text { 71,245 } \\
\text { women } \\
\text { 14 cohort studies; } 184-189 \text {, } \\
\text { 192, 195-198, 200, 201, 225 } \\
\text { 39,372 women } \\
\text { Medium }\end{array}$ & $\begin{array}{l}\text { Unclear association between BF and postpartum } \\
\text { depression. Magnitude of association and } \\
\text { direction of effect unclear; studies are } \\
\text { heterogeneous in design and results } \\
\text { inconsistent. }\end{array}$ & $\begin{array}{l}\text { Insufficient } \\
\text { (unknown } \\
\text { consistency, } \\
\text { imprecise) }\end{array}$ \\
\hline $\begin{array}{l}\text { Postpartum weight } \\
\text { change }\end{array}$ & $\begin{array}{l}16 \text { cohort studies; }{ }^{203-221} \\
47,655 \text { women } \\
\text { Medium }\end{array}$ & $\begin{array}{l}\text { Unclear association between BF and postpartum } \\
\text { weight change. Magnitude of postpartum weight } \\
\text { change varies by BF exposure and outcome } \\
\text { measure. }\end{array}$ & $\begin{array}{l}\text { Insufficient } \\
\text { (inconsistent, } \\
\text { imprecise) }\end{array}$ \\
\hline \multicolumn{4}{|c|}{$\begin{array}{l}{ }^{\text {a }} \text { We marked outcomes as indirect for long-term maternal health outcomes primarily due to uncertainty of the relative } \\
\text { contribution of breastfeeding to risk (given that many other potential factors also contribute to outcomes such as hypertension, } \\
\text { fracture, and breast cancer); for short-term maternal health outcomes (e.g., postpartum depression) there is uncertainty in the } \\
\text { direction of effect between breastfeeding and health outcomes. } \\
{ }^{b} \text { Per authors, there were } 52 \text { studies with }>1,500 \text { women, } 31 \text { studies with } 500-1,499 \text { women, and } 15 \text { studies with }<500 \text { women. } \\
\text { Exact number of participants is unclear. } \\
\text { c Per authors, there were } 22 \text { studies with }>1,500 \text { women, } 12 \text { studies with } 500-1,499 \text { women, and } 7 \text { studies with }<500 \text { women. }\end{array}$} \\
\hline
\end{tabular}

$\mathrm{BF}$ = breastfeeding; $\mathrm{CI}$ = confidence interval; $\mathrm{CVD}$ = cardiovascular disease; HER2 = human epidermal growth factor receptor 2; $\mathrm{HR}=$ hazard ratio; HTN = hypertension; $\mathrm{N}$ = number; $\mathrm{NR}=$ not reported; OR = odds ratio; $\mathrm{ROB}=$ risk of bias; $\mathrm{SR}=$ systematic review.

We rated the evidence as insufficient (primarily due to unknown consistency) for the association between breastfeeding and subtypes of breast cancer defined by tumor behavior (i.e., in situ breast cancer) or hormone receptor status, breast cancer among BRCA1/2 mutation carriers, and mortality due to breast cancer. Low SOE supports the association between ever breastfeeding, as well as longer versus shorter durations of breastfeeding, and a reduced risk of developing epithelial ovarian cancer.

For both hypertension and type 2 diabetes, studies consistently found an association between longer duration of breastfeeding and lower rates of hypertension and type 2 diabetes (low SOE for both outcomes). Because of heterogeneous outcome measures and imprecision, we were not 
able to make a conclusion on the association between breastfeeding and other cardiovascular outcomes.

Eleven studies reported on the association between breastfeeding using different measures (e.g., ever vs. never and duration per child) and hip, vertebral, and forearm fracture risk. Apart from two studies (rated high ROB), no study reported a statistically significant association between breastfeeding and fracture. We rated the SOE as low for no association.

Because of significant heterogeneity in study design, breastfeeding exposure definitions, outcomes, and inconsistency in results, we found insufficient evidence on whether breastfeeding is associated with postpartum depression or postpartum weight change. For postpartum depression, current evidence does not establish the direction of relationship between breastfeeding and higher or lower rates of postpartum depression.

\section{Deficiencies in Methods}

Many included studies for KQ 1 have methodological limitations. Studies often did not adequately describe the flow of participants or facilities; this was particularly true of those that randomized or assigned interventions at the hospital or other systems level. Many studies do not adequately describe or define breastfeeding outcome measures. Among studies that described breastfeeding outcome measures, studies vary in terms of definitions used (particularly for breastfeeding exclusivity) and also measure initiation rates using different criteria (e.g., selfreported initiation of any breastfeeding, breastfeeding status at hospital discharge). Selection bias is also a concern for observational studies. Overall attrition was high in several studies, and many did not conduct an intention-to-treat analysis (i.e., they analyzed only completers). In general, authors did not describe the potential for contamination (from other concurrent programs and policies to promote and support breastfeeding). None of the included studies assessed potential harms of interventions. We found no eligible studies of workplace breastfeeding interventions. Studies describing workplace breastfeeding interventions identified in our literature searches did not have an eligible comparator (i.e., no concurrent control group or, for pre-post studies, no multiple pre- and post-outcome measures). For KQ 2, the evidence base comprises observational studies only (cohorts and case-controls). For outcomes such as depression and weight gain, the direction of causality is unclear and the evidence does not offer definitive guidance. For other outcomes (e.g., cardiovascular disease), confounding and selection bias pose threats to causal inference, and existing studies cannot rule out residual confounding. For most outcomes, the vast majority of studies rely on self-report to categorize breastfeeding exposure. In studies that enroll women decades after giving birth, differential recall (particularly for outcomes where women are aware of the purported benefits of breastfeeding) can result in misclassification of the duration of exposure.

\section{Findings in Relation to What Is Already Known}

For KQ 1, our findings related to the benefit of BFHI for improving breastfeeding initiation and duration support continued efforts to implement this policy. Because of heterogeneity in study design, country setting, and outcome measures, we were not able to pool results. The absolute difference in rates of breastfeeding initiation and duration vary by setting and are likely influenced by a range of factors such as intervention fidelity, social factors, and others. Although our scope is narrower (in terms of eligible country setting and study design), our conclusions are consistent with a recent narrative review ${ }^{226}$ focused on BFHI; the authors conclude that adherence to the BFHI Ten Steps has a positive influence on breastfeeding outcomes. In terms of 
other health care interventions, staff training alone (without other breastfeeding support components) did not lead to improved breastfeeding outcomes. However, health care interventions that pair staff education with other services, such as a series of home visits, lead to improved rates of exclusive breastfeeding duration.

For other intervention types, our results show that WIC programs providing in-person or telephone peer support improve breastfeeding outcomes. We also identified evidence on a range of other WIC programs (e.g., cash incentives, provision of different types of breast pumps, and changes in food package policies); however, primarily as a result of unknown consistency and imprecision, we had insufficient evidence to draw a conclusion regarding the benefit of these interventions. We identified no eligible studies assessing workplace breastfeeding interventions; other reviews have highlighted the lack of controlled trials of workplace interventions for promoting breastfeeding in employed women. ${ }^{26,226}$ We looked for both trials and observational studies with a control group and still found no eligible studies. The absence of evidence on this topic precludes us from commenting on the effectiveness of workplace breastfeeding interventions. In 2012, the Affordable Care Act required large employers to provide reasonable break time and a private place for expressing breastmilk and mandated insurance coverage of lactation support services and equipment without cost-sharing for new health insurance policies. Without adequate time to express breastmilk in the workplace, working mothers would face significant barriers to breastfeeding. Future studies (as noted below) could address whether certain workplace interventions are more effective than others in improving breastfeeding duration among working mothers.

Our conclusions related to the maternal benefits of breastfeeding (KQ 2) suggest that breastfeeding is associated with lower rates of breast cancer, ovarian cancer, hypertension, and type 2 diabetes. The potential to improve maternal health could be highlighted as a rationale for improving rate of breastfeeding by health care and public health practitioners. For cardiometabolic outcomes, it has been hypothesized that lactation "resets" maternal metabolism after pregnancy, thereby reducing cardiovascular disease risk. ${ }^{151}$ Our conclusions related to hypertension and type 2 diabetes support this hypothesis. Results of our current review are, in general, consistent with those in previous reviews with respect to conclusions about the limitations of the evidence base. As was the case in 2007, we are not able to draw a conclusion about the association between breastfeeding and postpartum weight change or postpartum depression (due to study limitations and imprecise and inconsistent results). For this review, we added two additional maternal health outcomes: hypertension and cardiovascular disease. We concluded that low SOE supports the association between breastfeeding and hypertension; however, primarily as a result of heterogeneity in outcome measures and study limitations, we concluded that evidence was insufficient to reach a conclusion about cardiovascular disease.

Overall, given the benefit associated with health care system-level interventions, WIC peersupport programs for improving breastfeeding outcomes, and the maternal health benefits associated with breastfeeding, our results support continued efforts to ensure that mothers have adequate breastfeeding support. This support includes provisions contained in the Affordable Care $\mathrm{Act}^{227}$ that aim to support breastfeeding, such as requirements of insurers to provide coverage of breastfeeding supplies and support services and workplace policies supporting breastfeeding. 


\section{Applicability}

For KQ 1, several factors may limit the applicability of findings. Although most studies enrolled women of a similar age (20s to 30s), many had other specific inclusion criteria related to parity or health status (of the mother, infant, or both); a few enrolled only women who intended to breastfeed or were undecided. ${ }^{52,89}$ In general, the findings are applicable to healthy women who have access to routine maternity care and gave birth to healthy infants. Interventions focused on critically ill infants were beyond the scope of this review. Factors most likely to limit the applicability of the evidence include country setting, variation in usual maternity care practices (including other policies and practices to support breastfeeding), and (potentially) socioeconomic factors. For example, usual maternity care in one Danish study included at least one home visit within the first 5 weeks of birth, ${ }^{76}$ which may reflect a higher level of support than other country settings. Other studies evaluated interventions in resource-poor settings, such as one intervention that enrolled mothers from neighborhoods in Santiago, Chile, with a high rate of poverty. ${ }^{97}$ In terms of subgroups of women, limited data from the United States suggest that the magnitude of benefit for BFHI policies may be greater among women with less education than women with more education. ${ }^{54,55}$ Although the PROBIT trial, conducted in the Republic of Belarus, demonstrated benefit for increasing breastfeeding duration, results may not be applicable to the United States. Only women who initiated breastfeeding were enrolled in PROBIT. At the time of enrollment, for example, mothers in Belarus often stayed in the hospital close to 1 week postpartum and infant formula was costly (up to 20\% of an average yearly salary). ${ }^{52}$ The benefit demonstrated in PROBIT for BFHI may represent a best-case scenario.

For KQ 2, similar concerns about applicability apply. However, for KQ 2, we did limit to studies conducted in very high income countries (to be consistent with the 2007 AHRQ review). One limitation of the evidence related to time frame of enrollment. Many observational studies (including data from WHI participants ${ }^{4}$ ) enrolled women who breastfed decades ago. In 1970, only 26.5 percent of women initiated breastfeeding, ${ }^{228}$ compared with more than 80 percent of women today. Because of these secular changes, confounders of the association between breastfeeding and maternal health have changed over time, and evidence on the association between breastfeeding from older cohorts of women may or may not reflect the strength of association for women currently breastfeeding. Women who chose to breastfeed when breastfeeding rates in the United States were lower could be different in ways that affect risk of adverse maternal health outcomes.

\section{Limitations of the Review Process}

For KQ 1, we looked for and included a broad range of interventions to promote and support breastfeeding. At the same time, we specifically excluded primary care-relevant interventions delivered to individual women (to avoid duplicating a recent review conducted for the U.S. Preventive Services Task Force). ${ }^{37}$ The studies that met our inclusion criteria assessed a variety of different intervention types but did not fully span all interventions potentially relevant to policymakers. Because of our inclusion criteria, we may have excluded some interventions that could be considered systems level or community based. The breadth of our eligibility criteria was also a limitation in terms of the evidence synthesis; included studies may have been categorized in different ways. We chose to focus on intervention type and setting because these may be important factors for decisionmakers who plan to implement breastfeeding programs and policies. Despite our broad intervention inclusion criteria, we identified no eligible studies 
assessing workplace interventions (primarily due to no control group of women who did not receive the intervention).

Publication bias and selective reporting of outcomes are potential limitations. Although we searched for unpublished trials and unpublished outcomes, we did not find direct evidence of either of these biases. However, the majority of included studies are not trials (and were published before trial registries became available); these factors limit our certainty about the potential for publication bias. Finally, for this review, we excluded non-English-language studies based largely on limitations of time and resources.

For KQ 2, we chose to include recent, relevant systematic reviews in our evidence synthesis. Although this approach may improve efficiency, it has limitations. Some included systematic reviews do not fully report details related to methods (particularly ROB assessment). In addition, reporting of study characteristics was often lacking. Among the included systematic reviews that conducted meta-analyses, strategies to assess clinical and statistical heterogeneity were not always clearly described.

The scope of outcomes evaluated for KQ 2 was limited to an update of the 2007 review, ${ }^{2}$ with the addition of hypertension and cardiovascular disease outcomes. These outcomes represent common conditions that contribute significantly to burden of disease in very high income countries. However, studies have linked lactation with other maternal health outcomes, including reduced risk of endometrial cancer, multiple sclerosis, ${ }^{229}$ endometriosis, ${ }^{230}$ rheumatoid arthritis, ${ }^{231}$ and short-interval pregnancy. ${ }^{232,233}$ These outcomes and others may be of interest for future systematic reviews.

\section{Limitations of the Evidence Base}

For KQ 1, we found no evidence on certain types of interventions (e.g., workplace and school-based interventions), limited evidence for subgroups of women, and no included studies reported on potential harms of interventions. Studies used various definitions of breastfeeding initiation and exclusivity, which may limit the comparability of findings. In addition, as a result of the heterogeneity across studies, we were not able to assess whether certain characteristics of interventions have a greater influence on breastfeeding initiation, duration, and exclusivity. We were also not able to determine whether heterogeneity within some categories of interventions such as BFHI is due to study design, differences in outcome measures, fidelity of implementation, or country setting (given that variation exists across all these factors).

For KQ 2, although we found a large volume of evidence supporting the association between breastfeeding and improved maternal health, methodological limitations specific to observational study designs limit the ability to determine the magnitude of the effect that lactation has on maternal health. Although a growing literature documents protective associations between lifetime lactation and improved maternal health, these findings do not establish that breastfeeding prevents poor maternal health. Several other factors may be at work. First, women in very high income countries who choose to breastfeed and succeed in doing so are typically better educated, wealthier, and more likely to engage in other beneficial health behaviors. ${ }^{234,235}$ Moreover, it is plausible that, rather than breastfeeding preventing poor maternal health, poor maternal health may prevent the initiation or continuation of breastfeeding. This is of particular concern for studies of postpartum depression, in that preexisting depression or anxiety may disrupt breastfeeding, leading to early weaning; ${ }^{4, ~ 155, ~ 236, ~} 237$ moreover, there is evidence that early breastfeeding difficulties are associated with depressive symptoms. ${ }^{238}$ Similarly, emerging evidence similarly suggests that women who are insulin resistant are more likely to experience 
breastfeeding difficulties, ${ }^{239}$ potentially leading to shorter breastfeeding durations. In this context, rather than breastfeeding preventing cardiometabolic disease, early weaning may be a marker for maternal risk. ${ }^{240}$ One limitation of the evidence is related to time frame of enrollment. Many observational studies (including data from Women's Health Initiative participants ${ }^{4}$ ) enrolled women who breastfed decades ago. In 1970, only 26.5 percent of women initiated breastfeeding $^{228}$ compared with more than 80 percent of women today. Because of these secular changes, confounders of the association between breastfeeding and maternal health have changed over time, and evidence on the association between breastfeeding from older cohorts of women may or may not reflect the strength of association for women currently breastfeeding. Women who chose to breastfeed when breastfeeding rates in the United States were lower could be different in ways that affect risk of adverse maternal health outcomes.

\section{Future Research Needs}

For KQ 1, future research should assess the benefit of workplace, school-based, and other community-based interventions for improving rates of breastfeeding. Studies are also needed to inform context-specific types of professional and material support (e.g., double-electric vs. manual breast pumps) that enable women to achieve their infant feeding goals. Authors of future studies should more clearly describe characteristics of usual care and other breastfeeding support services are available. For studies conducted in the United States, future research could address whether certain interventions are more effective for groups of women who differ by socioeconomic factors in order to assess the consistency of current evidence suggesting a difference by education status. Study designs with a concurrent control group (e.g., trials or prospective cohort studies) would be helpful in reducing bias and informing the benefit of breastfeeding programs or policies implemented in a wide range of settings, particularly workplace programs. Methods to address selection bias, confounding, and measurement bias should also be considered in future research related to breastfeeding programs and policies. In addition, future studies assessing breastfeeding interventions should collect outcomes related to potential harms of interventions. In addition, studies are needed to compare types of support, such as manual versus electric pumps or interventions delivered by International Board Certified Lactation Consultants vs. Certified Lactation Consultants, to tailor support to the needs of each woman.

For KQ 2, observational studies will likely remain the major source of evidence on the association between breastfeeding and maternal health. Long-term followup of prospectively assessed breastfeeding duration may eliminate or significantly reduce potential of recall bias. Use of standardized breastfeeding definitions and clear reporting of how participants were selected would help minimize bias. In terms of analyses, authors should adequately address known confounders, such as breastfeeding intention, birth complications, diet, physical activity, tobacco use, mental health, and social support and clearly report a rationale for why certain factors were chosen. Future studies might also consider the extent to which adverse lactation outcomes, like adverse pregnancy outcomes, ${ }^{241}$ may be a window to maternal health.

More generally, standardized definitions of breastfeeding, as well as consistent methods of collecting these data, are needed to facilitate future systematic reviews and meta-analyses.

\section{Conclusions}

The body of evidence for breastfeeding programs and policies was diverse in terms of interventions and settings. Current evidence supports the effectiveness of BFHI for improving 
rates of breastfeeding initiation and duration; however, evidence from one large RCT (PROBIT) has limited applicability, and observational studies do not clearly establish the magnitude of benefit. For U.S. women enrolled in WIC, low SOE supports peer-support interventions for improving breastfeeding outcomes. The identified associations between breastfeeding and improved maternal health outcomes are supported by evidence from observational studies, which cannot determine cause and effect relationships. 


\section{References}

1. Chowdhury R, Sinha B, Sankar MJ, et al. Breastfeeding and maternal health outcomes: a systematic review and metaanalysis. Acta Paediatr. 2015

Dec;104(467):96-113. doi: 10.1111/apa.13102. PMID: 26172878.

2. Ip S, Chung M, Raman G, et al. Breastfeeding and maternal and infant health outcomes in developed countries. Evid Rep Technol Assess (Full Rep). 2007 Apr(153):1-186. PMID: 17764214.

3. Victora CG, Bahl R, Barros AJ, et al. Breastfeeding in the 21st century: epidemiology, mechanisms, and lifelong effect. Lancet. 2016 Jan 30;387(10017):47590. doi: 10.1016/s0140-6736(15)01024-7. PMID: 26869575.

4. Schwarz EB, Ray RM, Stuebe AM, et al. Duration of lactation and risk factors for maternal cardiovascular disease. Obstet Gynecol. 2009 May;113(5):974-82. doi: 10.1097/01.AOG.0000346884.67796.ca. PMID: 19384111.

5. Stuebe AM, Schwarz EB, Grewen K, et al. Duration of lactation and incidence of maternal hypertension: a longitudinal cohort study. Am J Epidemiol. 2011 Nov 15;174(10):1147-58. doi: 10.1093/aje/kwr227. PMID: 21997568.

6. Stuebe AM, Michels KB, Willett WC, et al. Duration of lactation and incidence of myocardial infarction in middle to late adulthood. Am J Obstet Gynecol. 2009 Feb;200(2):138.e1-8. doi:

10.1016/j.ajog.2008.10.001. PMID: 19110223.

7. Schwarz EB, McClure CK, Tepper PG, et al. Lactation and maternal measures of subclinical cardiovascular disease. Obstet Gynecol. 2010 Jan;115(1):41-8. doi: 10.1097/AOG.0b013e3181c5512a. PMID: 20027032.

8. Centers for Disease Control and Prevention. Breastfeeding report cards. Atlanta, GA: Centers for Disease Control and Prevention; 2017.

http://www.cdc.gov/breastfeeding/data/repor tcard.htm. Accessed on November 282017.
9.

Office of Disease Prevention and Health Promotion. Maternal, Infant, and Child Health. Washington, DC: Office of Disease Prevention and Health Promotion; 2016. https://www.healthypeople.gov/2020/topicsobjectives/topic/maternal-infant-and-childhealth/objectives. Accessed on September 8 2016.

10. Stuebe AM, Horton BJ, Chetwynd E, et al. Prevalence and risk factors for early, undesired weaning attributed to lactation dysfunction. J Womens Health (Larchmt). 2014 May;23(5):404-12. doi: 10.1089/jwh.2013.4506. PMID: 24655291.

11. Centers for Disease Control and Prevention. Progress in increasing breastfeeding and reducing racial/ethnic differences-United States, 2000-2008 Births. Atlanta, GA: Centers for Disease Control and Prevention; 2013.

www.cdc.gov/breastfeeding/resources/breast feeding-trends.htm. Accessed on January 3 2017.

12. Centers for Disease Control and Prevention. Breastfeeding among U.S. Children Born 2002-2012, CDC National Immunization Surveys. Atlanta, GA: Centers for Disease Control and Prevention; 2016. www.cdc.gov/breastfeeding/data/nis data/in dex.htm.

13. Ahluwalia IB, Li R, Morrow B. Breastfeeding practices: does method of delivery matter? Matern Child Health J. 2012 Dec;16 Suppl 2:231-7. doi: 10.1007/s10995-012-1093-9. PMID: 22926268.

14. Skafida V. The relative importance of social class and maternal education for breastfeeding initiation. Public Health Nutr. 2009 Dec;12(12):2285-92. doi: 10.1017/s1368980009004947. PMID: 19243673.

15. McKinney CO, Hahn-Holbrook J, ChaseLansdale PL, et al. Racial and ethnic differences in breastfeeding. Pediatrics. 2016 Aug;138(2). doi: 10.1542/peds.20152388. PMID: 27405771. 
16. World Health Organization. BFHI Section 1. Background and Implementation. Sweden: World Health Organization, UNICEF; 2009. http://www.who.int/nutrition/publications/in fantfeeding/bfhi_trainingcourse_s1/en/.

Accessed on February 192018.

17. World Health Organization. International Code of Marketing Breast-milk Substitutes. Geneva: World Health Organization; 1981.

18. Baby-Friendly USA. Guidelines and evaluation criteria for facilities seeking baby-friendly designation. Baby-Friendly USA. Sandwich, MA: 2010.

19. Baby-Friendly USA. The Gold Standard of Care. Albany, NY: Baby-Friendly USA; 2012. Accessed on January 32017.

20. Texas Department of State Health Services. Texas 10 Step Program. Austin, TX: Texas Department of State Health Services Texas Ten Step Program; 1999.

http://texastenstep.org/. Accessed on January 32017.

21. North Carolina Nutrition Services Branch. North Carolina Maternity Center Breastfeeding-Friendly Designation Program Raleigh, NC: N.C. Department of Health and Human Services; 2016. www.nutritionnc.com/breastfeeding/breastfe eding-friendly.htm. Accessed on January 3 2017.

22. Centers for Disease Control and Prevention. Maternity Practices in Infant Nutrition and Care (mPINC) Survey. Atlanta, GA: Centers for Disease Control and Prevention; 2016. www.cdc.gov/breastfeeding/data/mpinc/. Accessed on January 32017.

23. Baby-Friendly USA. Baby-Friendly Hospital Initiative. The Ten Steps to Successful Breastfeeding. Albany, NY: Baby-Friendly USA; 2010. www.babyfriendlyusa.org/about-us/babyfriendly-hospital-initiative/the-ten-steps. Accessed on January 32017.

24. Health Resources and Services Administration. Women's Preventive Services Guidelines. Rockville, MD: Health Resources and Services Administration; 2016. www.hrsa.gov/womensguidelines/. Accessed on January 32017.
25. U.S. Department of Agriculture. Women, Infants and Children (WIC). About WICWIC at a Glance. Washington, DC: U.S. Department of Agriculture; n.d. Accessed on February 192018.

26. Abdulwadud OA, Snow ME. Interventions in the workplace to support breastfeeding for women in employment. Cochrane Database Syst Rev. 2012 Oct 17;10:Cd006177. doi: 10.1002/14651858.CD006177.pub3. PMID: 23076920.

27. U.S. Department of Labor. Wage and hour division (WHD): Break time for nursing mothers. Washington, DC: U.S. Department of Labor. www.dol.gov/whd/nursingmothers/.

28. American Academy of Pediatrics. Breastfeeding and the use of human milk. Pediatrics. 2012 Mar;129(3):e827-41. doi: 10.1542/peds.2011-3552. PMID: 22371471.

29. American College of Obstetricians and Gynecologists. Optimizing support for breastfeeding as part of obstetric practice. Committee Opinion No. 658. Obstet Gynecol Annu. 2016;127:e86-92.

30. World Health Organization. Health topics. Breastfeeding. Switzerland: World Health Organization; 2017. Accessed on January 3 2017.

31. World Health Organization, UNICEF. Protecting, promoting and supporting breastfeeding: the special role of maternity services. Geneva: World Health Organization; 1989.

32. American Academy of Family Physicians. Breastfeeding (policy statement): American Academy of Family Physicians [serial on the Internet]; 2012.

33. Philipp BL, Merewood A, Miller LW, et al. Baby-friendly hospital initiative improves breastfeeding initiation rates in a US hospital setting. Pediatrics. 2001 Sep;108(3):677-81. PMID: 11533335.

34. Cohen R, Mrtek MB. The impact of two corporate lactation programs on the incidence and duration of breast-feeding by employed mothers. Am J Health Promot. 1994 Jul-Aug;8(6):436-41. PMID: 10147273. 
35. Forster D, McLachlan H, Lumley J, et al. Two mid-pregnancy interventions to increase the initiation and duration of breastfeeding: a randomized controlled trial. Birth. 2004 Sep;31(3):176-82. doi: 10.1111/j.0730-7659.2004.00302.x. PMID: 15330879.

36. Bibbins-Domingo K, Grossman DC, Curry SJ, et al. Primary care interventions to support breastfeeding: US Preventive Services Task Force recommendation statement. JAMA. 2016 Oct 25;316(16):1688-93. doi: 10.1001/jama.2016.14697. PMID: 27784102.

37. Patnode CD, Henninger ML, Senger CA, et al. Primary care interventions to support breastfeeding: updated evidence report and systematic review for the US Preventive Services Task Force. JAMA. 2016 Oct 25;316(16):1694-705. doi: 10.1001/jama.2016.8882. PMID: 27784101.

38. Wang L, Li J, Shi Z. Association between breastfeeding and endometrial cancer risk: evidence from a systematic review and meta-analysis. Nutrients. $2015 \mathrm{Jul}$ 14;7(7):5697-711. doi: 10.3390/nu7075248. PMID: 26184301.

39. Chen H, Wang J, Zhou W, et al. Breastfeeding and risk of rheumatoid arthritis: a systematic review and metaanalysis. J Rheumatol. 2015 Sep;42(9):1563-9. doi: 10.3899/jrheum.150195. PMID: 26178286.

40. Zhou Y, Chen J, Li Q, et al. Association between breastfeeding and breast cancer risk: evidence from a meta-analysis. Breastfeed Med. 2015 Apr;10(3):175-82. doi: 10.1089/bfm.2014.0141. PMID: 25785349.

41. United Nations Development Programme (UNDP). Human Development Report 2015: Work for Human Development. New York, NY: UNDP; 2015. www.hdr.undp.org/en/2015-report.

42. National Statistics, Republic of China (Taiwan). What is the human development index (HDI)? How is relevant data queried? Taiwan City, Taiwan, R. O. C.; n.d. https://eng.stat.gov.tw/ct.asp?xItem=31068 \&ctNode $=5710 \& m p=5$. Accessed on March 62017.
43. Programme UND. Human Development Report 2015: Work for Human Development. Table 1: Human Development Index and its components. United Nations Development Programme; n.d. New York, NY: 2015. http://hdr.undp.org/en/composite/HDI.

44. Sterne JA, Hernan MA, Reeves BC, et al. ROBINS-I: a tool for assessing risk of bias in non-randomised studies of interventions. BMJ. 2016 Oct 12;355:i4919. doi: 10.1136/bmj.i4919. PMID: 27733354.

45. Higgins JPT, Green S. Cochrane Handbook for Systematic Reviews of Interventions. The Cochrane Collaboration.; 2011. www.handbook.cochrane.org. Accessed on January 102017.

46. Agency for Healthcare Research and Quality. Methods Guide for Effectiveness and Comparative Effectiveness Reviews. AHRQ Publication No. 10(14)-EHC063-EF. Rockville, MD: Agency for Healthcare Research and Quality; January 2014. www.effectivehealthcare.ahrq.gov/methodsg uide.cfm.

47. Whiting P, Savovic J, Higgins JP, et al. ROBIS: A new tool to assess risk of bias in systematic reviews was developed. J Clin Epidemiol. 2016 Jan;69:225-34. doi: 10.1016/j.jclinepi.2015.06.005. PMID: 26092286.

48. Cornell JE, Mulrow CD, Localio R, et al. Random-effects meta-analysis of inconsistent effects: a time for change. Ann Intern Med. 2014 Feb 18;160(4):267-70. doi: 10.7326/m13-2886. PMID: 24727843.

49. Berkman ND, Lohr KN, Ansari MT, et al. Grading the strength of a body of evidence when assessing health care interventions: an EPC update. J Clin Epidemiol. 2015

Nov;68(11):1312-24. doi: 10.1016/j.jclinepi.2014.11.023. PMID: 25721570.

50. Atkins D, Chang SM, Gartlehner G, et al. Assessing applicability when comparing medical interventions: AHRQ and the Effective Health Care Program. J Clin Epidemiol. 2011 Nov;64(11):1198-207. doi: 10.1016/j.jclinepi.2010.11.021. PMID: 21463926. 
51. Ryan AS, Zhou WJ, Acosta A. Breastfeeding continues to increase into the new millennium. Pediatrics. 2002

Dec;110(6):1103-9. doi: DOI 10.1542/peds.110.6.1103. PMID: WOS:000179549200024.

52. Kramer MS, Chalmers B, Hodnett ED, et al. Promotion of Breastfeeding Intervention Trial (PROBIT): a randomized trial in the Republic of Belarus. JAMA. 2001 Jan 2431;285(4):413-20. PMID: 11242425.

53. Yang S, Platt RW, Dahhou M, et al. Do population-based interventions widen or narrow socioeconomic inequalities? The case of breastfeeding promotion. Int $\mathrm{J}$ Epidemiol. 2014 Aug;43(4):1284-92. doi: 10.1093/ije/dyu051. PMID: 24639438.

54. Hawkins SS, Stern AD, Baum CF, et al. Evaluating the impact of the Baby-Friendly Hospital Initiative on breast-feeding rates: a multi-state analysis. Public Health Nutr. 2015 Feb;18(2):189-97. doi: 10.1017/s1368980014000238. PMID: 24625787.

55. Hawkins SS, Stern AD, Baum CF, et al. Compliance with the Baby-Friendly Hospital Initiative and impact on breastfeeding rates. Arch Dis Child Fetal Neonatal Ed. 2014 Mar;99(2):F138-43. doi: 10.1136/archdischild-2013-304842. PMID: 24277661.

56. Tarrant M, Wu KM, Fong DY, et al. Impact of baby-friendly hospital practices on breastfeeding in Hong Kong. Birth. 2011 Sep;38(3):238-45. doi: 10.1111/j.1523536X.2011.00483.x. PMID: 21884232.

57. Mydlilova A, Sipek A, Vignerova J. Breastfeeding rates in baby-friendly and non-baby-friendly hospitals in the Czech Republic from 2000 to 2006. J Hum Lact. 2009 Feb;25(1):73-8. doi: 10.1177/0890334408325820. PMID: 19020285.

58. Abolyan LV. The breastfeeding support and promotion in Baby-Friendly Maternity Hospitals and Not-as-Yet Baby-Friendly Hospitals in Russia. Breastfeed Med. 2006 Summer;1(2):71-8. doi: 10.1089/bfm.2006.1.71. PMID: 17661566.
59. Bosnjak AP, Batinica M, Hegedus-Jungvirth $\mathrm{M}$, et al. The effect of baby friendly hospital initiative and postnatal support on breastfeeding rates--Croatian experience. Coll Antropol. 2004 Jun;28(1):235-43. PMID: 15636080.

60. Gau ML. Evaluation of a lactation intervention program to encourage breastfeeding: a longitudinal study. Int J Nurs Stud. 2004 May;41(4):425-35. doi: 10.1016/j.ijnurstu.2003.11.002. PMID: 15050853.

61. Bartington S, Griffiths LJ, Tate AR, et al. Are breastfeeding rates higher among mothers delivering in Baby Friendly accredited maternity units in the UK? Int $\mathrm{J}$ Epidemiol. 2006 Oct;35(5):1178-86. doi: 10.1093/ije/dyl155. PMID: 16926214.

62. Broadfoot M, Britten J, Tappin DM, et al. The Baby Friendly Hospital Initiative and breast feeding rates in Scotland. Arch Dis Child Fetal Neonatal Ed. 2005

Mar;90(2):F114-6. doi: 10.1136/adc.2003.041558. PMID: 15724033.

63. Venancio SI, Saldiva SR, Escuder MM, et al. The Baby-Friendly Hospital Initiative shows positive effects on breastfeeding indicators in Brazil. J Epidemiol Community Health. 2012 Oct;66(10):914-8. doi: 10.1136/jech-2011-200332. PMID: 22080818.

64. Weng DR, Hsu CS, Gau ML, et al. Analysis of the outcomes at baby-friendly hospitals: appraisal in Taiwan. Kaohsiung J Med Sci. 2003 Jan;19(1):19-28. doi: 10.1016/s1607551x(09)70443-7. PMID: 12693722.

65. Yotebieng M, Labbok M, Soeters HM, et al. Ten Steps to Successful Breastfeeding programme to promote early initiation and exclusive breastfeeding in DR Congo: a cluster-randomised controlled trial. Lancet Glob Health. 2015 Sep;3(9):e546-55. doi: 10.1016/s2214-109x(15)00012-1. PMID: 26246225.

66. Osband YB, Altman RL, Patrick PA, et al. Breastfeeding education and support services offered to pediatric residents in the US. Acad Pediatr. 2011 Jan-Feb;11(1):75-9. doi: 10.1016/j.acap.2010.11.002. PMID: 21272827. 
67. Abu Naser AA, Ghbn N, Khoudary R. Relation of nitrate contamination of groundwater with methaemoglobin level among infants in Gaza. East Mediterr Health J. 2007 Sep-Oct;13(5):994-1004. PMID: 18290391.

68. Hannula LS, Kaunonen ME, Puukka PJ. A study to promote breast feeding in the Helsinki Metropolitan area in Finland. Midwifery. 2014 Jun;30(6):696-704. doi: 10.1016/j.midw.2013.10.005. PMID: 24210842.

69. Minkovitz C, Strobino D, Hughart N, et al. Early effects of the healthy steps for young children program. Arch Pediatr Adolesc Med. 2001 Apr;155(4):470-9. PMID: 11296075.

70. Ekstrom A, Abrahamsson H, Eriksson RM, et al. Women's use of nipple shields-their influence on breastfeeding duration after a process-oriented education for health professionals. Breastfeed Med. 2014 Nov;9(9):458-66. doi: 10.1089/bfm.2014.0026. PMID: 25188544.

71. Ekstrom A, Kylberg E, Nissen E. A processoriented breastfeeding training program for healthcare professionals to promote breastfeeding: an intervention study. Breastfeed Med. 2012 Apr;7(2):85-92. doi: 10.1089/bfm.2010.0084. PMID: 22168946.

72. Wan H, Hu S, Thobaben M, et al. Continuous primary nursing care increases satisfaction with nursing care and reduces postpartum problems for hospitalized pregnant women. Contemp Nurse. 2011 Feb;37(2):149-59. doi: 10.5172/conu.2011.37.2.149. PMID: 21692586.

73. Feldman-Winter L, Barone L, Milcarek B, et al. Residency curriculum improves breastfeeding care. Pediatrics. 2010 Aug;126(2):289-97. doi: 10.1542/peds.2009-3250. PMID: 20603262.

74. Hoddinott P, Britten J, Prescott GJ, et al. Effectiveness of policy to provide breastfeeding groups (BIG) for pregnant and breastfeeding mothers in primary care: cluster randomised controlled trial. BMJ. 2009 Jan 30;338:a3026. doi: 10.1136/bmj.a3026. PMID: 19181729.
75. Senarath U, Fernando DN, Rodrigo I. Effect of training for care providers on practice of essential newborn care in hospitals in Sri Lanka. J Obstet Gynecol Neonatal Nurs. 2007 Nov-Dec;36(6):531-41. doi: 10.1111/j.1552-6909.2007.00183.x. PMID: 17973696.

76. Kronborg H, Vaeth M, Olsen J, et al. Effect of early postnatal breastfeeding support: a cluster-randomized community based trial. Acta Paediatr. 2007 Jul;96(7):1064-70. doi: 10.1111/j.1651-2227.2007.00341.x. PMID: 17524018.

77. Kools EJ, Thijs C, Kester AD, et al. A breast-feeding promotion and support program a randomized trial in The Netherlands. Prev Med. 2005 Jan;40(1):6070. doi: 10.1016/j.ypmed.2004.05.013. PMID: 15530582.

78. Martens PJ. Does breastfeeding education affect nursing staff beliefs, exclusive breastfeeding rates, and Baby-Friendly Hospital Initiative compliance? The experience of a small, rural Canadian hospital. J Hum Lact. 2000 Nov;16(4):30918. PMID: 11155609.

79. Taddei JA, Westphal MF, Venancio S, et al. Breastfeeding training for health professionals and resultant changes in breastfeeding duration. Sao Paulo Med J. 2000 Nov 09;118(6):185-91. PMID: 11120550.

80. Madden JM, Soumerai SB, Lieu TA, et al. Effects on breastfeeding of changes in maternity length-of-stay policy in a large health maintenance organization. Pediatrics. 2003 Mar;111(3):519-24. PMID: 12612230.

81. Bærug A, Langsrud Ø, Løland BF, et al. Effectiveness of baby-friendly community health services on exclusive breastfeeding and maternal satisfaction: a pragmatic trial. Matern Child Nutr. 2016;12(3):428-39. doi: 10.1111/mcn.12273. PMID: 116415662.

82. Wagner CL, Hulsey TC, Southgate WM, et al. Breastfeeding rates at an urban medical university after initiation of an educational program. South Med J. 2002 Aug;95(8):90913. PMID: 12190230. 
83. Nilsson IMS, Strandberg-Larsen K, Knight $\mathrm{CH}$, et al. Focused breastfeeding counselling improves short- and long-term success in an early-discharge setting: A cluster-

randomized study. Matern Child Nutr.

2017;13(4):n/a-n/a. doi:

10.1111/mcn.12432. PMID: 125350139.

Language: English. Entry Date: 20171006.

Revision Date: 20171006. Publication Type: Article. Journal Subset: Allied Health.

84. Coutinho SB, de Lira PI, de Carvalho Lima $\mathrm{M}$, et al. Comparison of the effect of two systems for the promotion of exclusive breastfeeding. Lancet. 2005 Sep 2430;366(9491):1094-100. doi:

10.1016/s0140-6736(05)67421-1. PMID: 16182897.

85. Barcellos NT, Fuchs SC, Mondini LG, et al. Human T lymphotropic virus type I/II infection: prevalence and risk factors in individuals testing for HIV in counseling centers from Southern Brazil. Sex Transm Dis. 2006 May;33(5):302-6. doi: 10.1097/01.olq.0000194598.47821.b6. PMID: 16505751.

86. Hayes DK, Prince CB, Espinueva V, et al. Comparison of manual and electric breast pumps among WIC women returning to work or school in Hawaii. Breastfeed Med. 2008 Mar;3(1):3-10. doi: 10.1089/bfm.2007.0022. PMID: 18333763.

87. Joyce T, Reeder J. Changes in breastfeeding among WIC participants following implementation of the new food package. Matern Child Health J. 2015 Apr;19(4):86876. doi: 10.1007/s10995-014-1588-7. PMID: 25095768.

88. Lovera D, Sanderson M, Bogle ML, et al. Evaluation of a breastfeeding peer support program for fathers of Hispanic participants in a Texas special supplemental nutrition program for women, infants, and children. J Am Diet Assoc. 2010 Nov;110(11):1696702. doi: 10.1016/j.jada.2010.08.001. PMID: 21034883.

89. Reeder JA, Joyce T, Sibley K, et al. Telephone peer counseling of breastfeeding among WIC participants: a randomized controlled trial. Pediatrics. 2014 Sep;134(3):e700-9. doi: 10.1542/peds.20134146. PMID: 25092936.
90. Schafer E, Vogel MK, Viegas S, et al. Volunteer peer counselors increase breastfeeding duration among rural lowincome women. Birth. 1998 Jun;25(2):1016. PMID: 9668744.

91. Shaw E, Kaczorowski J. The effect of a peer counseling program on breastfeeding initiation and longevity in a low-income rural population. J Hum Lact. 1999 Mar;15(1):19-25. PMID: 10578771.

92. Edmunds LS, Lee FF, Eldridge JD, et al. Outcome evaluation of the You Can Do It Initiative to Promote Exclusive Breastfeeding Among Women Enrolled in the New York State WIC Program by Race/Ethnicity. J Nutr Educ Behav. 2017;49:S162-S8.e1. doi: 10.1016/j.jneb.2017.05.350. PMID: 123721522.

93. Washio Y, Humphreys M, Colchado E, et al. Incentive-based intervention to maintain breastfeeding among low-income Puerto Rican mothers. Pediatrics. 2017 Mar;139(3). doi: 10.1542/peds.2016-3119. PMID: 28167511.

94. Cattaneo A, Bettinelli M, Chapin E, et al. Effectiveness of the baby friendly community initiative in Italy: a nonrandomised controlled study. BMJ Open. 2016;6(5). doi: 10.1136/bmjopen-2015010232. PMID: CN-01159959.

95. McLachlan HL, Forster DA, Amir LH, et al. Supporting breastfeeding In Local Communities (SILC) in Victoria, Australia: a cluster randomised controlled trial. BMJ Open. 2016 Feb 01;6(2):e008292. doi: 10.1136/bmjopen-2015-008292. PMID: 26832427.

96. Morrow AL, Guerrero ML, Shults J, et al. Efficacy of home-based peer counselling to promote exclusive breastfeeding: a randomised controlled trial. Lancet. 1999 Apr 10;353(9160):1226-31. doi: 10.1016/s0140-6736(98)08037-4. PMID: 10217083.

97. Alvarado R, Zepeda A, Rivero S, et al. Integrated maternal and infant health care in the postpartum period in a poor neighborhood in Santiago, Chile. Stud Fam Plann. 1999 Jun;30(2):133-41. PMID: 16617547. 
98. Rempel LA, Moore KC. Peer-led prenatal breast-feeding education: a viable alternative to nurse-led education. Midwifery. 2012

Feb;28(1):73-9. doi: 10.1016/j.midw.2010.11.005. PMID: 21236530 .

99. Macaluso A, Bettinelli ME, Chapin EM, et al. A controlled study on baby-friendly communities in Italy: methods and baseline data. Breastfeed Med. 2013 Apr;8:198-204. doi: 10.1089/bfm.2012.0130. PMID: 23398142

100. Cramer RL, McLachlan HL, Shafiei T, et al. Implementation and evaluation of community-based drop-in centres for breastfeeding support in Victoria, Australia. International Breastfeeding Journal. 2017;12(46).

101. Siegel RL, Miller KD, Jemal A. Cancer statistics, 2018. CA Cancer J Clin. 2018 Jan;68(1):7-30. doi: 10.3322/caac.21442. PMID: 29313949.

102. American Cancer Society. Cancer Facts \& Figures 2017. Atlanta, GA: American Cancer Society; 2017.

103. Thulier D. Weighing the Facts: A Systematic Review of Expected Patterns of Weight Loss in Full-Term, Breastfed Infants. J Hum Lact. 2016 Feb;32(1):28-34. doi: 10.1177/0890334415597681. PMID: 26253288.

104. Bernier MO, Plu-Bureau G, Bossard N, et al. Breastfeeding and risk of breast cancer: $a$ metaanalysis of published studies. Hum Reprod Update. 2000 Jul-Aug;6(4):374-86. PMID: 10972524.

105. Lipworth L, Bailey LR, Trichopoulos D. History of breast-feeding in relation to breast cancer risk: a review of the epidemiologic literature. J Natl Cancer Inst. 2000 Feb 16;92(4):302-12. PMID: 10675379.

106. Breast cancer and breastfeeding: collaborative reanalysis of individual data from 47 epidemiological studies in 30 countries, including 50302 women with breast cancer and 96973 women without the disease. Lancet. 2002 Jul 20;360(9328):18795. doi: 10.1016/s0140-6736(02)09454-0. PMID: 12133652.
107. Lambertini M, Santoro L, Del Mastro L, et al. Reproductive behaviors and risk of developing breast cancer according to tumor subtype: a systematic review and metaanalysis of epidemiological studies. Cancer Treat Rev. 2016 Sep;49:65-76. doi: 10.1016/j.ctrv.2016.07.006. PMID: 27529149.

108. Stendell-Hollis NR, Thompson PA, Thomson CA, et al. Investigating the association of lactation history and postmenopausal breast cancer risk in the Women's Health Initiative. Nutr Cancer. 2013;65(7):969-81. doi: 10.1080/01635581.2013.815787. PMID: 24127779.

109. Sugawara Y, Kakizaki M, Nagai M, et al. Lactation pattern and the risk for hormonerelated female cancer in Japan: the Ohsaki Cohort Study. Eur J Cancer Prev. 2013 Mar;22(2):187-92. doi: 10.1097/CEJ.0b013e3283564610. PMID: 23358107.

110. Tamimi RM, Spiegelman D, Smith-Warner SA, et al. Population Attributable Risk of Modifiable and Nonmodifiable Breast Cancer Risk Factors in Postmenopausal Breast Cancer. Am J Epidemiol. 2016 Dec 15;184(12):884-93. doi: 10.1093/aje/kww145. PMID: 27923781.

111. Al-Amri FA, Saeedi MY, Al-Tahan FM, et al. Breast cancer correlates in a cohort of breast screening program participants in Riyadh, KSA. J Egypt Natl Canc Inst. 2015 Jun;27(2):77-82. doi: 10.1016/j.jnci.2015.04.002. PMID: 25935858.

112. Al-Qutub ST, Al-Raddadi RM, Bin Sadiq BM, et al. Potential breast cancer risk factors among Saudi women aged 19-50 years in Jeddah: a case-control study. J Egypt Public Health Assoc. 2013 Dec;88(3):165-70. doi: 10.1097/01.EPX.0000435728.60811.bd. PMID: 24374950.

113. Beaber EF, Holt VL, Malone KE, et al. Reproductive factors, age at maximum height, and risk of three histologic types of breast cancer. Cancer Epidemiol Biomarkers Prev. 2008 Dec;17(12):3427-34. doi: 10.1158/1055-9965.epi-08-0641. PMID: 19064558. 
114. Castello A, Martin M, Ruiz A, et al. Lower breast cancer risk among women following the World Cancer Research Fund and American Institute for Cancer Research Lifestyle Recommendations: EpiGEICAM Case-Control Study. PLoS One. 2015;10(5):e0126096. doi: 10.1371/journal.pone.0126096. PMID: 25978407.

115. Dalamaga M, Karmaniolas K, Papadavid E, et al. Elevated serum visfatin/nicotinamide phosphoribosyl-transferase levels are associated with risk of postmenopausal breast cancer independently from adiponectin, leptin, and anthropometric and metabolic parameters. Menopause. 2011 Nov;18(11):1198-204. doi: 10.1097/gme.0b013e31821e21f5. PMID: 21712732.

116. Hadji P, Gottschalk M, Ziller V, et al. Bone mass and the risk of breast cancer: the influence of cumulative exposure to oestrogen and reproductive correlates. Results of the Marburg breast cancer and osteoporosis trial (MABOT). Maturitas. 2007 Mar 20;56(3):312-21. doi: 10.1016/j.maturitas.2006.09.005. PMID: 17049767.

117. Holm J, Eriksson L, Ploner A, et al. Assessment of Breast Cancer Risk Factors Reveals Subtype Heterogeneity. Cancer Res. 2017 Jul 01;77(13):3708-17. doi: 10.1158/0008-5472.can-16-2574. PMID: 28512241.

118. Kruk J. Association between vegetable, fruit and carbohydrate intake and breast cancer risk in relation to physical activity. Asian Pac J Cancer Prev. 2014;15(11):4429-36. PMID: 24969864.

119. Lee E, Ma H, McKean-Cowdin R, et al. Effect of reproductive factors and oral contraceptives on breast cancer risk in BRCA1/2 mutation carriers and noncarriers: results from a population-based study. Cancer Epidemiol Biomarkers Prev. 2008 Nov;17(11):3170-8. doi: 10.1158/10559965.epi-08-0396. PMID: 18990759.
120. Lumachi F, Frigo AC, Basso U, et al. Estrogen therapy and risk of breast cancer in postmenopausal women: a case-control study and results of a multivariate analysis. Menopause. 2010 May-Jun;17(3):524-8. doi: 10.1097/gme.0b013e3181ca0c74. PMID: 20130492.

121. Ma H, Bernstein L, Ross RK, et al. Hormone-related risk factors for breast cancer in women under age 50 years by estrogen and progesterone receptor status: results from a case-control and a case-case comparison. Breast Cancer Res. 2006;8(4):R39. doi: 10.1186/bcr1514. PMID: 16846528.

122. Phillips LS, Millikan RC, Schroeder JC, et al. Reproductive and hormonal risk factors for ductal carcinoma in situ of the breast. Cancer Epidemiol Biomarkers Prev. 2009 May;18(5):1507-14. doi: 10.1158/10559965.epi-08-0967. PMID: 19423528.

123. Pieta B, Opala T, Wilczak M, et al. Past obstetric history and risk of malignant breast neoplasms. Eur J Gynaecol Oncol. 2008;29(4):374-7. PMID: 18714573.

124. Press DJ, Pharoah P. Risk factors for breast cancer: a reanalysis of two case-control studies from 1926 and 1931. Epidemiology. 2010 Jul;21(4):566-72. doi: 10.1097/EDE.0b013e3181e08eb3. PMID: 20498604.

125. Ruszczyk M, Zirpoli G, Kumar S, et al. Breast cancer risk factor associations differ for pure versus invasive carcinoma with an in situ component in case-control and casecase analyses. Cancer Causes Control. 2016 Feb;27(2):183-98. doi: 10.1007/s10552-0150696-z. PMID: 26621543.

126. Ge I, Rudolph A, Shivappa N, et al. Dietary inflammation potential and postmenopausal breast cancer risk in a German case-control study. Breast. 2015 Aug;24(4):491-6. doi: 10.1016/j.breast.2015.04.012. PMID: 25987487.

127. Guedes KM, Guimaraes AM, Bastos Ade S, et al. Stomatognathic evaluation at five years of age in children born premature and at term. BMC Pediatr. 2015 Mar 29;15:27. doi: 10.1186/s12887-015-0343-6. PMID: 25880084. 
128. Li DP, Du C, Zhang ZM, et al.

Breastfeeding and ovarian cancer risk: a systematic review and meta-analysis of 40 epidemiological studies. Asian Pac J Cancer Prev. 2014;15(12):4829-37. PMID: 24998548.

129. Kotsopoulos J, Lubinski J, Lynch HT, et al. Oophorectomy after menopause and the risk of breast cancer in BRCA1 and BRCA2 mutation carriers. Cancer Epidemiol Biomarkers Prev. 2012 Jul;21(7):1089-96. doi: 10.1158/1055-9965.epi-12-0201. PMID: 22564871.

130. Jernstrom H, Lubinski J, Lynch HT, et al. Breast-feeding and the risk of breast cancer in BRCA1 and BRCA2 mutation carriers. J Natl Cancer Inst. 2004 Jul 21;96(14):10948. doi: 10.1093/jnci/djh211. PMID: 15265971.

131. Kotsopoulos J, Lubinski J, Salmena L, et al. Breastfeeding and the risk of breast cancer in BRCA1 and BRCA2 mutation carriers. Breast Cancer Res. 2012 Mar 09;14(2):R42. doi: 10.1186/bcr3138. PMID: 22405187.

132. Kabat GC, Kim MY, Woods NF, et al. Reproductive and menstrual factors and risk of ductal carcinoma in situ of the breast in a cohort of postmenopausal women. Cancer Causes Control. 2011 Oct;22(10):1415-24. doi: 10.1007/s10552-011-9814-8. PMID: 21750889.

133. Ritte R, Tikk K, Lukanova A, et al. Reproductive factors and risk of hormone receptor positive and negative breast cancer: a cohort study. BMC Cancer. 2013 Dec 09;13:584. doi: 10.1186/1471-2407-13-584. PMID: 24321460.

134. Warner ET, Tamimi RM, Boggs DA, et al. Estrogen receptor positive tumors: do reproductive factors explain differences in incidence between black and white women? Cancer Causes Control. 2013 Apr;24(4):731-9. doi: 10.1007/s10552-0130153-9. PMID: 23380944.

135. Ma H, Ursin G, Xu X, et al. Reproductive factors and the risk of triple-negative breast cancer in white women and AfricanAmerican women: a pooled analysis. Breast Cancer Res. 2017 Jan 13;19(1):6. doi: 10.1186/s13058-016-0799-9. PMID: 28086982.
136. Atkinson RL, El-Zein R, Valero V, et al. Epidemiological risk factors associated with inflammatory breast cancer subtypes.

Cancer Causes Control. 2016 Mar;27(3):359-66. doi: 10.1007/s10552015-0712-3. PMID: 26797453.

137. Merritt MA, Riboli E, Murphy N, et al. Reproductive factors and risk of mortality in the European Prospective Investigation into Cancer and Nutrition; a cohort study. BMC Med. 2015 Oct 30;13:252. doi: 10.1186/s12916-015-0484-3. PMID: 26515238.

138. Luan NN, Wu QJ, Gong TT, et al. Breastfeeding and ovarian cancer risk: a meta-analysis of epidemiologic studies. Am J Clin Nutr. 2013 Oct;98(4):1020-31. doi: 10.3945/ajcn.113.062794. PMID: 23966430.

139. Sung HK, Ma SH, Choi JY, et al. The Effect of Breastfeeding Duration and Parity on the Risk of Epithelial Ovarian Cancer: A Systematic Review and Meta-analysis. J Prev Med Public Health. 2016 Nov;49(6):349-66. doi: 10.3961/jpmph.16.066. PMID: 27951628.

140. John EM, Whittemore AS, Harris R, et al. Characteristics relating to ovarian cancer risk: collaborative analysis of seven U.S. case-control studies. Epithelial ovarian cancer in black women. Collaborative Ovarian Cancer Group. J Natl Cancer Inst. 1993 Jan 20;85(2):142-7. PMID: 8418303.

141. Risch HA, Marrett LD, Howe GR. Parity, contraception, infertility, and the risk of epithelial ovarian cancer. Am J Epidemiol. 1994 Oct 01;140(7):585-97. PMID: 7942759.

142. West RO. Epidemiologic study of malignancies of the ovaries. Cancer. 1966 Jul;19(7):1001-7. PMID: 5939299.

143. Wynder EL, Dodo H, Barber HR. Epidemiology of cancer of the ovary. Cancer. 1969 Feb;23(2):352-70. PMID: 5764976.

144. Cook LS, Pestak CR, Leung AC, et al. Combined oral contraceptive use before the first birth and epithelial ovarian cancer risk. Br J Cancer. 2017 Jan 17;116(2):265-9. doi: 10.1038/bjc.2016.400. PMID: 27959890. 
145. Gay GM, Lim JS, Chay WY, et al. Reproductive factors, adiposity, breastfeeding and their associations with ovarian cancer in an Asian cohort. Cancer Causes Control. 2015 Nov;26(11):1561-73. doi: 10.1007/s10552-015-0649-6. PMID: 26342607.

146. Gierach GL, Modugno F, Ness RB. Gender of offspring and maternal ovarian cancer risk. Gynecol Oncol. 2006 Jun;101(3):47680. doi: 10.1016/j.ygyno.2005.11.008. PMID: 16364411.

147. Jordan SJ, Green AC, Whiteman DC, et al. Serous ovarian, fallopian tube and primary peritoneal cancers: a comparative epidemiological analysis. Int J Cancer. 2008 Apr 01;122(7):1598-603. doi: 10.1002/ijc.23287. PMID: 18058817.

148. Nagle CM, Olsen CM, Webb PM, et al. Endometrioid and clear cell ovarian cancers: a comparative analysis of risk factors. Eur J Cancer. 2008 Nov;44(16):2477-84. doi: 10.1016/j.ejca.2008.07.009. PMID: 18707869.

149. Kotsopoulos J, Lubinski J, Gronwald J, et al. Factors influencing ovulation and the risk of ovarian cancer in BRCA1 and BRCA2 mutation carriers. Int J Cancer. 2015 Sep 01;137(5):1136-46. doi: 10.1002/ijc.29386. PMID: 25482078.

150. Jonas W, Nissen E, Ransjo-Arvidson AB, et al. Short- and long-term decrease of blood pressure in women during breastfeeding. Breastfeed Med. 2008 Jun;3(2):103-9. doi: 10.1089/bfm.2007.0031. PMID: 18563998.

151. Stuebe AM, Rich-Edwards JW. The reset hypothesis: lactation and maternal metabolism. Am J Perinatol. 2009 Jan;26(1):81-8. doi: 10.1055/s-00281103034. PMID: 19031350.

152. Lee SY, Kim MT, Jee SH, et al. Does longterm lactation protect premenopausal women against hypertension risk? A Korean women's cohort study. Prev Med. 2005 Aug;41(2):433-8. doi: 10.1016/j.ypmed.2004.11.025. PMID: 15917038.
153. Lupton SJ, Chiu CL, Lujic S, et al. Association between parity and breastfeeding with maternal high blood pressure. Am J Obstet Gynecol. 2013 Jun;208(6):454.e1-7. doi: 10.1016/j.ajog.2013.02.014. PMID: 23395924.

154. Choi SR, Kim YM, Cho MS, et al. Association between duration of breast feeding and metabolic syndrome: The Korean National Health and Nutrition Examination Surveys. J Womens Health (Larchmt). 2017 Apr;26(4):361-7. doi: 10.1089/jwh.2016.6036. PMID: 28072915.

155. Parikh NI, Jeppson RP, Berger JS, et al. Reproductive risk factors and coronary heart disease in the Women's Health Initiative Observational Study. Circulation. 2016 May 31;133(22):2149-58. doi: 10.1161/circulationaha.115.017854. PMID: 27143682.

156. Natland Fagerhaug T, Forsmo S, Jacobsen $\mathrm{GW}$, et al. A prospective population-based cohort study of lactation and cardiovascular disease mortality: the HUNT study. BMC Public Health. 2013 Nov 13;13:1070. doi: 10.1186/1471-2458-13-1070. PMID: 24219620.

157. Much D, Beyerlein A, Rossbauer M, et al. Beneficial effects of breastfeeding in women with gestational diabetes mellitus. Mol Metab. 2014 Jun;3(3):284-92. doi: 10.1016/j.molmet.2014.01.002. PMID: 24749058.

158. Aune D, Norat T, Romundstad P, et al. Breastfeeding and the maternal risk of type 2 diabetes: a systematic review and doseresponse meta-analysis of cohort studies. Nutr Metab Cardiovasc Dis. 2014 Feb;24(2):107-15. doi: 10.1016/j.numecd.2013.10.028. PMID: 24439841.

159. Chamberlain CR, Oldenburg B, Wilson AN, et al. Type 2 diabetes after gestational diabetes: greater than fourfold risk among Indigenous compared with non-Indigenous Australian women. Diabetes Metab Res Rev. 2016 Feb;32(2):217-27. doi: 10.1002/dmrr.2715. PMID: 26385131. 
160. Gunderson EP, Hurston SR, Ning X, et al. Lactation and progression to Type 2 Diabetes Mellitus after Gestational Diabetes Mellitus: a prospective cohort study. Ann Intern Med. 2015 Dec 15;163(12):889-98. doi: 10.7326/m15-0807. PMID: 26595611.

161. Martens PJ, Shafer LA, Dean HJ, et al. Breastfeeding initiation associated with reduced incidence of diabetes in mothers and offspring. Obstet Gynecol. 2016 Nov;128(5):1095-104. doi: 10.1097/aog.0000000000001689. PMID: 27741196.

162. Zong G, Grandjean P, Wang X, et al. Lactation history, serum concentrations of persistent organic pollutants, and maternal risk of diabetes. Environ Res. 2016 Oct;150:282-8. doi: 10.1016/j.envres.2016.06.023. PMID: 27336232.

163. Gunderson EP, Hedderson MM, Chiang V, et al. Lactation intensity and postpartum maternal glucose tolerance and insulin resistance in women with recent GDM: the SWIFT cohort. Diabetes Care. 2012 Jan;35(1):50-6. doi: 10.2337/dc11-1409. PMID: 22011407.

164. The Women's Health Initiative Study. Design of the women's health initiative clinical trial and observational study. Control Clin Trials. 1998;19(1):61-109.

165. Mori T, Ishii S, Greendale GA, et al. Parity, lactation, bone strength, and 16-year fracture risk in adult women: findings from the Study of Women's Health Across the Nation (SWAN). Bone. 2015 Apr;73:160-6. doi: 10.1016/j.bone.2014.12.013. PMID: 25528102.

166. Wiklund PK, Xu L, Wang Q, et al. Lactation is associated with greater maternal bone size and bone strength later in life. Osteoporos Int. 2012 Jul;23(7):1939-45. doi: 10.1007/s00198-011-1790-z. PMID: 21927916.

167. Alderman BW, Weiss NS, Daling JR, et al. Reproductive history and postmenopausal risk of hip and forearm fracture. Am J Epidemiol. 1986 Aug;124(2):262-7. PMID: 3728442.
168. Chan HH, Lau EM, Woo J, et al. Dietary calcium intake, physical activity and the risk of vertebral fracture in Chinese. Osteoporos Int. 1996;6(3):228-32. PMID: 8783297.

169. Hoffman S, Grisso JA, Kelsey JL, et al. Parity, lactation and hip fracture. Osteoporos Int. 1993 Jul;3(4):171-6. PMID: 8338971.

170. Cumming RG, Klineberg RJ. Breastfeeding and other reproductive factors and the risk of hip fractures in elderly women. Int J Epidemiol. 1993 Aug;22(4):684-91. PMID: 8225744.

171. Kreiger N, Kelsey JL, Holford TR, et al. An epidemiologic study of hip fracture in postmenopausal women. Am J Epidemiol. 1982 Jul;116(1):141-8. PMID: 7102649.

172. Michaelsson K, Baron JA, Farahmand BY, et al. Influence of parity and lactation on hip fracture risk. Am J Epidemiol. 2001 Jun 15;153(12):1166-72. PMID: 11415951.

173. Hwang IR, Choi YK, Lee WK, et al. Association between prolonged breastfeeding and bone mineral density and osteoporosis in postmenopausal women: KNHANES 2010-2011. Osteoporos Int. 2016 Jan;27(1):257-65. doi: 10.1007/s00198-015-3292-x. PMID: 26373982.

174. Lambrinoudaki I, Flokatoula M, Armeni E, et al. Vertebral fracture prevalence among Greek healthy middle-aged postmenopausal women: association with demographics, anthropometric parameters, and bone mineral density. Spine J. 2015 Jan 01;15(1):86-94. doi: 10.1016/j.spinee.2014.07.021. PMID: 25106754.

175. Naves M, Diaz-Lopez JB, Gomez C, et al. Determinants of incidence of osteoporotic fractures in the female Spanish population older than 50. Osteoporos Int. 2005 Dec;16(12):2013-7. doi: 10.1007/s00198005-1983-4. PMID: 16091836.

176. Crandall CJ, Liu J, Cauley J, et al. Associations of parity, breastfeeding, and fractures in the Women's Health Observational Study. Obstet Gynecol. 2017 Jul;130(1):171-80. doi: 10.1097/aog.0000000000002096. PMID: 28594759. 
177. Ko JY, Rockhill KM, Tong VT, et al. Trends in postpartum depressive symptoms 27 States, 2004, 2008, and 2012. MMWR Morb Mortal Wkly Rep. 2017 Feb 17;66(6):153-8. doi: 10.15585/mmwr.mm6606a1. PMID: 28207685.

178. Lindahl V, Pearson JL, Colpe L. Prevalence of suicidality during pregnancy and the postpartum. Arch Womens Ment Health. 2005 Jun;8(2):77-87. doi: 10.1007/s00737005-0080-1. PMID: 15883651.

179. Campbell SB, Brownell CA, Hungerford A, et al. The course of maternal depressive symptoms and maternal sensitivity as predictors of attachment security at 36 months. Dev Psychopathol. 2004 Spring;16(2):231-52. PMID: 15487594.

180. McLearn KT, Minkovitz CS, Strobino DM, et al. The timing of maternal depressive symptoms and mothers' parenting practices with young children: implications for pediatric practice. Pediatrics. 2006 Jul;118(1):e174-82. doi: 10.1542/peds.20051551. PMID: 16818531.

181. Paulson JF, Dauber S, Leiferman JA. Individual and combined effects of postpartum depression in mothers and fathers on parenting behavior. Pediatrics. 2006 Aug;118(2):659-68. doi: 10.1542/peds.2005-2948. PMID: 16882821.

182. Pries AM, Huffman SL, Adhikary I, et al. Promotion and prelacteal feeding of breastmilk substitutes among mothers in Kathmandu Valley, Nepal. Matern Child Nutr. 2016 Apr;12 Suppl 2:8-21. doi: 10.1111/mcn.12205. PMID: 27061953.

183. Dias CC, Figueiredo B. Breastfeeding and depression: a systematic review of the literature. J Affect Disord. 2015 Jan 15;171:142-54. doi: 10.1016/j.jad.2014.09.022. PMID: 25305429.

184. Warner R, Appleby L, Whitton A, et al. Demographic and obstetric risk factors for postnatal psychiatric morbidity. Br J Psychiatry. 1996 May;168(5):607-11. PMID: 8733800.
185. Reifsnider E, Flowers J, Todd M, et al. The relationship among breastfeeding, postpartum depression, and postpartum weight in Mexican American Women. J Obstet Gynecol Neonatal Nurs.

2016;45(6):760-71. doi: 10.1016/j.jogn.2016.05.009. PMID: 119339849.

186. McCoy SJ, Beal JM, Saunders B, et al. Risk factors for postpartum depression: a retrospective investigation. J Reprod Med. 2008 Mar;53(3):166-70. PMID: 18441719.

187. Trivino-Juarez JM, Nieto-Pereda B, Romero-Ayuso D, et al. Quality of life of mothers at the sixth week and sixth month post partum and type of infant feeding. Midwifery. 2016 Mar;34:230-8. doi: 10.1016/j.midw.2015.11.003. PMID: 26621376.

188. Sword W, Landy CK, Thabane L, et al. Is mode of delivery associated with postpartum depression at 6 weeks: a prospective cohort study. BJOG. 2011 Jul;118(8):966-77. doi: 10.1111/j.1471-0528.2011.02950.x. PMID: 21489126.

189. Ahn S, Corwin EJ. The association between breastfeeding, the stress response, inflammation, and postpartum depression during the postpartum period: prospective cohort study. Int J Nurs Stud. 2015 Oct;52(10):1582-90. doi: 10.1016/j.ijnurstu.2015.05.017. PMID: 26143358.

190. Otokpa AO, Lawoyin TO, Asuzu MC. HIV/AIDS-related knowledge and misconceptions among women attending government-owned antenatal clinics in Gwagwalada Area Council of Abuja, Nigeria. Afr J Reprod Health. 2013 Mar;17(1):114-21. PMID: 24069740.

191. Grover S, Gupta S, Mehra A, et al. Comorbidity, knowledge and attitude towards sex among patients with Dhat syndrome: a retrospective study. Asian J Psychiatr. 2015 Oct;17:50-5. doi: 10.1016/j.ajp.2015.07.002. PMID: 26259894. 
192. Davey HL, Tough SC, Adair CE, et al. Risk factors for sub-clinical and major postpartum depression among a community cohort of Canadian women. Matern Child Health J. 2011 Oct;15(7):866-75. doi: 10.1007/s10995-008-0314-8. PMID: 18256913.

193. Guilkey DK, Popkin BM, Flieger W, et al. Changes in breast-feeding in the Philippines 1973-1983. Soc Sci Med. 1990;31(12):136575. PMID: 2287963.

194. Borra C, Iacovou M, Sevilla A. New evidence on breastfeeding and postpartum depression: the importance of understanding women's intentions. Matern Child Health J. 2015 Apr;19(4):897-907. doi: 10.1007/s10995-014-1591-z. PMID: 25138629.

195. Fiala A, Svancara J, Klanova J, et al. Sociodemographic and delivery risk factors for developing postpartum depression in a sample of 3233 mothers from the Czech ELSPAC study. BMC Psychiatry. 2017 Mar 21;17(1):104. doi: 10.1186/s12888-0171261-y. PMID: 28327118.

196. Chojenta CL, Lucke JC, Forder PM, et al. Maternal health factors as risks for postnatal depression: a prospective longitudinal study. PLoS One. 2016;11(1):e0147246. doi: 10.1371/journal.pone.0147246. PMID: 26785131.

197. Maimburg RD, Vaeth M. Postpartum depression among first-time mothers results from a parallel randomised trial. Sex Reprod Healthc. 2015 Jun;6(2):95-100. doi: 10.1016/j.srhc.2015.01.003. PMID: 25998877.

198. Lau Y, Chan KS. Perinatal depressive symptoms, sociodemographic correlates, and breast-feeding among Chinese women. J Perinat Neonatal Nurs. 2009 OctDec;23(4):335-45. doi: 10.1097/JPN.0b013e3181bbbea9. PMID: 19915417.

199. Holmberg KS, Peterson UM, Oscarsson MG. A two-decade perspective on mothers' experiences and feelings related to breastfeeding initiation in Sweden. Sex Reprod Healthc. 2014 Oct;5(3):125-30. doi: 10.1016/j.srhc.2014.04.001. PMID: 25200973.
200. Iliadis SI, Comasco E, Sylven S, et al. Prenatal and postpartum evening salivary cortisol levels in association with peripartum depressive symptoms. PLoS One. 2015;10(8):e0135471. doi: 10.1371/journal.pone.0135471. PMID: 26322643.

201. Hamdan A, Tamim H. The relationship between postpartum depression and breastfeeding. Int J Psychiatry Med. 2012;43(3):243-59. PMID: 22978082.

202. De Palma G, Capilla A, Nadal I, et al. Interplay between human leukocyte antigen genes and the microbial colonization process of the newborn intestine. Curr Issues Mol Biol. 2010;12(1):1-10. PMID: 19478349.

203. Onyango AW, Nommsen-Rivers L, Siyam A, et al. Post-partum weight change patterns in the WHO Multicentre Growth Reference Study. Matern Child Nutr. 2011 Jul;7(3):228-40. doi: 10.1111/j.17408709.2010.00295.x. PMID: 21338469.

204. Stuebe AM, Kleinman K, Gillman MW, et al. Duration of lactation and maternal metabolism at 3 years postpartum. J Womens Health (Larchmt). 2010 May;19(5):941-50. doi: 10.1089/jwh.2009.1660. PMID: 20459331.

205. Haiek LN, Kramer MS, Ciampi A, et al. Postpartum weight loss and infant feeding. J Am Board Fam Pract. 2001 MarApr;14(2):85-94. PMID: 11314929.

206. Lyu LC, Lo CC, Chen HF, et al. A prospective study of dietary intakes and influential factors from pregnancy to postpartum on maternal weight retention in Taipei, Taiwan. Br J Nutr. 2009

Dec;102(12):1828-37. doi: 10.1017/s0007114509991243. PMID: 19678967.

207. Linne Y, Dye L, Barkeling B, et al. Weight development over time in parous women-the SPAWN study--15 years follow-up. Int J Obes Relat Metab Disord. 2003

Dec;27(12):1516-22. doi: 10.1038/sj.ijo.0802441. PMID: 14634683. 
208. Ng SK, Cameron CM, Hills AP, et al. Socioeconomic disparities in prepregnancy BMI and impact on maternal and neonatal outcomes and postpartum weight retention: the EFHL longitudinal birth cohort study. BMC Pregnancy Childbirth. 2014 Sep 08;14:314. doi: 10.1186/1471-2393-14-314. PMID: 25201481.

209. Olson CM, Strawderman MS, Hinton PS, et al. Gestational weight gain and postpartum behaviors associated with weight change from early pregnancy to 1 y postpartum. Int J Obes Relat Metab Disord. 2003

Jan;27(1):117-27. doi:

10.1038/sj.ijo.0802156. PMID: 12532163.

210. Sichieri R, Field AE, Rich-Edwards J, et al. Prospective assessment of exclusive breastfeeding in relation to weight change in women. Int J Obes Relat Metab Disord. 2003 Jul;27(7):815-20. doi: 10.1038/sj.ijo.0802285. PMID: 12821967.

211. Janney CA, Zhang D, Sowers M. Lactation and weight retention. Am J Clin Nutr. 1997 Nov;66(5):1116-24. PMID: 9356528.

212. Ohlin A, Rossner S. Maternal body weight development after pregnancy. Int J Obes. 1990 Feb;14(2):159-73. PMID: 2341224.

213. Walker L, Freeland-Graves JH, Milani T, et al. Weight and behavioral and psychosocial factors among ethnically diverse, lowincome women after childbirth: II. Trends and correlates. Women Health. 2004;40(2):19-34. doi: 10.1300/J013v40n02_02. PMID: 15778136.

214. Brewer MM, Bates MR, Vannoy LP. Postpartum changes in maternal weight and body fat depots in lactating vs nonlactating women. Am J Clin Nutr. 1989 Feb;49(2):259-65. PMID: 2916446.

215. Ohlin A, Rossner S. Factors related to body weight changes during and after pregnancy: the Stockholm Pregnancy and Weight Development Study. Obes Res. 1996 May;4(3):271-6. PMID: 8732961.

216. Jarlenski MP, Bennett WL, Bleich SN, et al. Effects of breastfeeding on postpartum weight loss among U.S. women. Prev Med. 2014 Dec;69:146-50. doi: 10.1016/j.ypmed.2014.09.018. PMID: 25284261.
217. Palmer JR, Kipping-Ruane K, Wise LA, et al. Lactation in relation to long-term maternal weight gain in African-American Women. Am J Epidemiol. 2015 Jun 15;181(12):932-9. doi: 10.1093/aje/kwv027. PMID: 25944887.

218. Straub H, Simon C, Plunkett B, et al. Evidence for a complex relationship among weight retention, cortisol and breastfeeding in postpartum women. Matern Child Health J. 2016;20(7):1375-83. doi: 10.1007/s10995016-1934-z. PMID: 116170909.

219. Endres LK, Straub H, McKinney C, et al. Postpartum weight retention risk factors and relationship to obesity at 1 year. Obstet Gynecol. 2015 Jan;125(1):144-52. doi: 10.1097/aog.0000000000000565. PMID: 25560116.

220. Sharma AJ, Dee DL, Harden SM. Adherence to breastfeeding guidelines and maternal weight 6 years after delivery. Pediatrics. 2014 Sep;134 Suppl 1:S42-9. doi: 10.1542/peds.2014-0646H. PMID: 25183755.

221. Ostbye T, Krause KM, Swamy GK, et al. Effect of breastfeeding on weight retention from one pregnancy to the next: results from the North Carolina WIC program. Prev Med. 2010 Nov;51(5):368-72. doi: 10.1016/j.ypmed.2010.07.017. PMID: 20655944.

222. Walker LO, Freeland-Graves JH, Milani T, et al. Weight and behavioral and psychosocial factors among ethnically diverse, low-income women after childbirth: I. Methods and context. Women Health. 2004;40(2):1-17. doi: 10.1300/J013v40n02_01. PMID: 15778135.

223. Walsh A, Moseley J, Jackson W. The effects of an infant-feeding classroom activity on the breast-feeding knowledge and intentions of adolescents. J Sch Nurs. 2008 Jun;24(3):164-9. doi: 10.1622/10598405(2008)024[0164:teoaic]2.0.co;2. PMID: 18557676.

224. Aboud FE, Moore AC, Akhter S. Effectiveness of a community-based responsive feeding programme in rural Bangladesh: a cluster randomized field trial. Matern Child Nutr. 2008 Oct;4(4):275-86. doi: 10.1111/j.1740-8709.2008.00146.x. PMID: 18811792. 
225. Banks E, Killpack S, Furman L. Lowincome inner-city fathers and breastfeeding-where's the program for us? Breastfeed Med. 2013 Dec;8(6):507-8. doi: 10.1089/bfm.2012.0147. PMID: 23560474.

226. Perez-Escamilla R, Martinez JL, SeguraPerez S. Impact of the Baby-friendly Hospital Initiative on breastfeeding and child health outcomes: a systematic review. Matern Child Nutr. 2016 Jul;12(3):402-17. doi: 10.1111/mcn.12294. PMID: 26924775.

227. U.S. Government. Patient protection and affordable care act, Pub. Law 111-148, as amended by the Health Care and Education Reconciliation Act (HCERA).

228. Grummer-Strawn LM, Shealy KR. Progress in protecting, promoting, and supporting breastfeeding: 1984-2009. Breastfeed Med. 2009 Oct;4 Suppl 1:S31-9. doi: 10.1089/bfm.2009.0049. PMID: 19827921.

229. Langer-Gould A, Smith JB, Hellwig K, et al. Breastfeeding, ovulatory years, and risk of multiple sclerosis. Neurology. 2017 Aug 08;89(6):563-9. doi: 10.1212/wnl.0000000000004207. PMID: 28701499.

230. Farland LV, Eliassen AH, Tamimi RM, et al. History of breast feeding and risk of incident endometriosis: prospective cohort study. BMJ. 2017 Aug 29;358:j3778. doi: 10.1136/bmj.j3778. PMID: 28851765.

231. Karlson EW, Mandl LA, Hankinson SE, et al. Do breast-feeding and other reproductive factors influence future risk of rheumatoid arthritis? Results from the Nurses' Health Study. Arthritis Rheum. 2004 Nov;50(11):3458-67. doi: 10.1002/art.20621. PMID: 15529351.

232. Kennedy KI, Visness CM. Contraceptive efficacy of lactational amenorrhoea. Lancet. 1992 Jan 25;339(8787):227-30. PMID: 1346183.

233. Van der Wijden C, Manion C. Lactational amenorrhoea method for family planning. Cochrane Database Syst Rev. 2015 Oct 12(10):Cd001329. doi: 10.1002/14651858.CD001329.pub2. PMID: 26457821.
234. Beck LF, Morrow B, Lipscomb LE, et al. Prevalence of selected maternal behaviors and experiences, Pregnancy Risk Assessment Monitoring System (PRAMS), 1999. MMWR Surveill Summ. 2002;51(2):1-27.

235. Pesa JA, Shelton MM. Health-enhancing behaviors correlated with breastfeeding among a national sample of mothers. Public Health Nurs. 1999;16(2):120-4.

236. Paul IM, Downs DS, Schaefer EW, et al. Postpartum anxiety and maternal-infant health outcomes. Pediatrics. 2013 Apr;131(4):e1218-24. doi: 10.1542/peds.2012-2147. PMID: 23460682.

237. Dennis CL, McQueen K. The relationship between infant-feeding outcomes and postpartum depression: a qualitative systematic review. Pediatrics. 2009 Apr;123(4):e736-51. doi: 10.1542/peds.2008-1629. PMID: 19336362.

238. Watkins S, Meltzer-Brody S, Zolnoun D, et al. Early breastfeeding experiences and postpartum depression. Obstet Gynecol. 2011 Aug;118(2 Pt 1):214-21. doi: 10.1097/AOG.0b013e3182260a2d. PMID: 21734617.

239. Nommsen-Rivers LA. Does insulin explain the relation between maternal obesity and poor lactation outcomes? An overview of the literature. Adv Nutr. 2016 Mar;7(2):40714. doi: 10.3945/an.115.011007. PMID: 26980825.

240. Stuebe AM. Does breastfeeding prevent the metabolic syndrome, or does the metabolic syndrome prevent breastfeeding? Semin Perinatol. 2015 Jun;39(4):290-5. doi: 10.1053/j.semperi.2015.05.008. PMID: 26187772.

241. Saade GR. Pregnancy as a window to future health. Obstet Gynecol. 2009

Nov;114(5):958-60. doi: 10.1097/AOG.0b013e3181bf5588. PMID: WOS:000271293500001. 


\section{Appendix A. Literature Search Strategies and Yields Breastfeeding Original Searches KQ 1}

FINAL

\section{PubMed}

Searched February 8, 2017

Results:

RCTs and other study designs: 2,632 (Line 14)

2,632 imported, no duplicates

SRs: 193 (Line 19)

144 imported, 49 duplicates

\section{PubMed Search Strategy}

\begin{tabular}{|c|c|c|}
\hline Search & Query & $\begin{array}{l}\text { Items } \\
\text { Found }\end{array}$ \\
\hline \#1 & $\begin{array}{l}\text { Search "Infant Nutritional Physiological Phenomena"[Mesh] OR "Milk, Human"[Mesh] OR } \\
\text { "Breast Feeding"[Mesh] OR "breast feeding"[All Fields] OR "human milk"[All Fields] OR } \\
\text { (human[tiab] AND milk[tiab]) OR "breast milk"[All Fields] OR breastmilk OR breastfeed* } \\
\text { OR (breast[tiab] AND fed[tiab]) OR breastfed OR "Lactation"[Mesh] OR lactating OR } \\
\text { lactation }\end{array}$ & 130104 \\
\hline \#2 & $\begin{array}{l}\text { Search (((Absenteeism[Mesh] OR "Affordable Care Act" OR ACA[tiab] OR "Baby friendly } \\
\text { Hospital Initiative" OR BFHI OR (break*[tiab] AND (express* AND milk)) OR "Breast Milk } \\
\text { Expression"[Mesh] OR "breast pump"[All Fields] OR "Child Day Care Centers"[Mesh] OR } \\
\text { "Employment"[Mesh] OR (employ* AND (polic* OR program*)) OR "Health Knowledge, } \\
\text { Attitudes, Practice"[Mesh] OR "Health Promotion"[Mesh] OR "Insurance Benefits"[Mesh] } \\
\text { OR ("Iactation consultant" OR "lactation consultants") OR "Maternal Health } \\
\text { Services"[Mesh] OR "Mothers/psychology"[Majr] OR "Nurseries, Hospital"[Mesh] OR } \\
\text { "Occupational Health Services"[Mesh] OR "Parental Leave"[Mesh] OR "Program } \\
\text { Evaluation"[Mesh] OR "Salaries and Fringe Benefits"[Mesh] OR "Social Support"[Mesh] } \\
\text { OR "Women, Working"[Mesh]))) }\end{array}$ & 460877 \\
\hline \#3 & $\begin{array}{l}\text { Search ("baby friendly"[All Fields] OR "hospital practices"[All Fields] OR "Ten Steps"[All } \\
\text { Fields] OR Counseling[Mesh] OR WIC OR "Women, Infants, and Children Program" OR } \\
\text { SNAP OR "Food Stamps"[All Fields] OR "Food Assistance"[Mesh] OR "Food } \\
\text { assistance"[All Fields] OR "Health Education"[Mesh] OR "House Calls"[Mesh] OR } \\
\text { "Organizational Policy"[Mesh] OR "Patient Education as Topic"[Mesh] OR "Promotion of } \\
\text { Breastfeeding Intervention Trial"[All Fields] OR PROBIT[All Fields] OR "Postnatal } \\
\text { Care"[Mesh] OR "Social Support"[Mesh] OR 'Ten Steps to Successful Breastfeeding'[All } \\
\text { Fields] OR "Workplace"[Mesh]) }\end{array}$ & 281167 \\
\hline \#4 & $\begin{array}{l}\text { Search ((("schools"[MeSH] OR "schools"[All Fields] OR "school"[All Fields] OR } \\
\text { "universities"[MeSH] OR "universities"[All Fields] OR "university"[All Fields]) AND } \\
\text { ("wellness programmes"[All Fields] OR "health promotion"[MeSH Terms] OR ("health"[All } \\
\text { Fields] AND "promotion"[All Fields] OR "health promotion"[All Fields]) OR ("wellness"[All } \\
\text { Fields] AND "programs"[All Fields]) OR "wellness programs"[All Fields]) OR ("school health } \\
\text { services"[MeSH] OR ("school"[All Fields] AND "health"[All Fields] AND "services"[All } \\
\text { Fields]) OR "school health services"[All Fields])) OR (("workplace"[MeSH] OR } \\
\text { "workplace"[All Fields]) AND ("wellness programmes"[All Fields] OR "health } \\
\text { promotion"[MeSH] OR ("health"[All Fields] AND "promotion"[All Fields]) OR "health } \\
\text { promotion"[All Fields] OR ("wellness"[All Fields] AND "programs"[All Fields]) OR "wellness } \\
\text { programs"[All Fields] OR programs[All Fields]))) }\end{array}$ & 175566 \\
\hline \#5 & Search (\#2 OR \#3 OR \#4) & 732183 \\
\hline \#6 & Search (\#1 AND \#5) & 12024 \\
\hline
\end{tabular}




\begin{tabular}{|c|c|c|}
\hline Search & Query & $\begin{array}{l}\text { Items } \\
\text { Found }\end{array}$ \\
\hline \#7 & $\begin{array}{l}\text { Search ((((randomized[title/abstract] OR randomised[title/abstract]) AND } \\
\text { controlled[title/abstract] AND trial[title/abstract]) OR (controlled[title/abstract] AND } \\
\text { trial[title/abstract]) OR "controlled clinical trial"[publication type] OR "Randomized } \\
\text { Controlled Trial"[Publication Type] OR "Single-Blind Method"[MeSH] OR "Double-Blind } \\
\text { Method"[MeSH] OR "Random Allocation"[MeSH])) }\end{array}$ & 662215 \\
\hline$\# 8$ & Search (\#6 AND \#7) & 820 \\
\hline$\# 9$ & $\begin{array}{l}\text { Search ("Cohort Studies"[Mesh] OR "Epidemiologic Studies"[Mesh] OR "Follow-up } \\
\text { Studies"[Mesh] OR “prospective cohort" OR "prospective studies"[MeSH] OR } \\
\text { (prospective*[All Fields] AND cohort[All Fields] AND (study[All Fields] OR studies[All } \\
\text { Fields]))) }\end{array}$ & 1979868 \\
\hline$\# 10$ & Search (\#6 AND \#9) & 2248 \\
\hline$\# 11$ & Search (\#8 OR \#10) & 2849 \\
\hline$\# 12$ & Search (\#8 OR \#10) Filters: Publication date from 1980/01/01 to 2017/12/31 & 2836 \\
\hline$\# 13$ & Search (\#8 OR \#10) Filters: Publication date from 1980/01/01 to 2017/12/31; Humans & 2774 \\
\hline$\# 14$ & $\begin{array}{l}\text { Search (\#8 OR \#10) Filters: Publication date from 1980/01/01 to 2017/12/31; Humans; } \\
\text { English }\end{array}$ & 2632 \\
\hline \#15 & $\begin{array}{l}\text { Search ((("systematic review"[ti] OR "meta-analysis"[pt] OR "meta-analysis"[ti] OR } \\
\text { "systematic literature review"[ti] OR "this systematic review"[tw] OR ("systematic } \\
\text { review"[tiab] AND review[pt]) OR meta synthesis[ti] OR "meta synthesis"[ti] OR "cochrane } \\
\text { database syst rev"[ta]))) }\end{array}$ & 148009 \\
\hline$\# 16$ & Search (\#6 AND \#15) & 218 \\
\hline$\# 17$ & Search (\#6 AND \#15) Filters: Publication date from 1980/01/01 to 2017/12/31 & 218 \\
\hline$\# 18$ & Search (\#6 AND \#15) Filters: Publication date from 1980/01/01 to 2017/12/31; Humans & 200 \\
\hline \#19 & $\begin{array}{l}\text { Search (\#6 AND \#15) Filters: Publication date from 1980/01/01 to 2017/12/31; Humans; } \\
\text { English }\end{array}$ & 193 \\
\hline
\end{tabular}




\section{CINAHL}

Searched February 8, 2017

Excluded Medline citations

Results: 79

79 imported, no duplicates

Limiters/Expanders Results

S1 MH "Infant Nutritional Physiological Phenomena" OR MH "Milk, Human" OR MH Search modes - 29,283

"Breast Feeding" OR "breast feeding" OR "human milk" OR (human AND milk) Boolean/Phrase

OR "breast milk" OR breastmilk OR breastfeed* OR (breast AND fed) OR

breastfed OR MH "Lactation" OR lactating OR lactation

S2 (((MH "Absenteeism" OR "Affordable Care Act" OR ACA OR "Baby friendly Search modes -

Hospital Initiative" OR BFHI OR (break* AND (express* AND milk)) OR MH Boolean/Phrase

"Breast Milk Expression" OR "breast pump" OR MH "Child Day Care Centers"

OR MH "Employment" OR (employ* AND (polic* OR program)) OR MH "Health

Knowledge, Attitudes, Practice" OR MH "Health Promotion" OR MH "Insurance

Benefits" OR ("lactation consultant" OR "lactation consultants") OR MH

"Maternal Health Services" OR "Mothers/psychology" OR MH "Nurseries,

Hospital" OR MH "Occupational Health Services" OR MH "Parental Leave" OR

$\mathrm{MH}$ "Program Evaluation" OR MH "Salaries and Fringe Benefits" OR MH "Social

Support" OR MH "Women, Working")))

S3 "baby friendly" OR "hospital practices" OR "Ten Steps" OR MH Counseling OR Search modes -

WIC OR "Women, Infants, and Children Program" OR SNAP OR "Food Stamps" Boolean/Phrase

OR MH "Food Assistance" OR "Food assistance" OR MH "Health Education"

OR MH "House Calls" OR MH "Organizational Policy" OR MH "Patient

Education as Topic" OR "Promotion of Breastfeeding Intervention Trial" OR

PROBIT OR MH "Postnatal Care" OR MH "Social Support" OR "Ten Steps to

Successful Breastfeeding" OR MH "Workplace"

S4 ((MH "schools" OR "schools"[All Fields] OR "school"[All Fields] OR MH

"universities" OR "universities"[All Fields] OR "university"[All Fields]) AND

Search modes -

Boolean/Phrase

("wellness programmes"[All Fields] OR MH "health promotion" OR ("health"[All

Fields] AND "promotion"[All Fields] OR "health promotion"[All Fields]) OR

("wellness"[All Fields] AND "programs"[All Fields]) OR "wellness programs"[All

Fields]) OR (MH "school health services" OR ("school"[All Fields] AND

"health"[All Fields] AND "services"[All Fields]) OR "school health services"[All

Fields])) OR ((MH "workplace" OR "workplace"[All Fields]) AND ("wellness

programmes"[All Fields] OR MH "health promotion" OR ("health"[All Fields] AND

"promotion"[All Fields]) OR "health promotion"[All Fields] OR ("wellness"[All

Fields] AND "programs"[All Fields]) OR "wellness programs"[All Fields] OR programs[All Fields]))

\begin{tabular}{|c|c|c|c|}
\hline S5 & S2 OR S3 OR S4 & $\begin{array}{l}\text { Search modes - } \\
\text { Boolean/Phrase }\end{array}$ & 194,378 \\
\hline & S1 AND S5 & $\begin{array}{l}\text { Search modes - } \\
\text { Boolean/Phrase }\end{array}$ & 4,156 \\
\hline S7 & $\begin{array}{l}\text { (randomized OR randomised) AND controlled AND trial) OR (controlled AND } \\
\text { trial) OR "controlled clinical trial" OR "Randomized Controlled Trial" OR MH } \\
\text { "Single-Blind Method" OR MH "Double-Blind Method" OR MH "Random } \\
\text { Allocation" }\end{array}$ & $\begin{array}{l}\text { Search modes - } \\
\text { Boolean/Phrase }\end{array}$ & 112,810 \\
\hline S9 & $\begin{array}{l}\text { MH "Cohort Studies" OR MH "Epidemiologic Studies" OR MH "Follow-up } \\
\text { Studies" OR "prospective cohort" OR MH "prospective studies" OR } \\
\text { (prospective* AND cohort AND (study OR studies)) }\end{array}$ & $\begin{array}{l}\text { Search modes - } \\
\text { Boolean/Phrase }\end{array}$ & 306,067 \\
\hline \multicolumn{2}{|r|}{ S10 S6 AND S9 } & $\begin{array}{l}\text { Search modes - } \\
\text { Boolean/Phrase }\end{array}$ & 307 \\
\hline \multicolumn{2}{|c|}{ S11 S8 OR S10 } & $\begin{array}{l}\text { Search modes - } \\
\text { Boolean/Phrase }\end{array}$ & 450 \\
\hline
\end{tabular}




\begin{tabular}{ll}
\hline$\# \quad$ Query & Limiters/Expanders Results \\
\hline S12 S11 & Limiters - Published 79 \\
& Date: 19800101- \\
& 20171231; English \\
& Language; Exclude \\
& MEDLINE records \\
& Search modes - \\
& Boolean/Phrase \\
\hline
\end{tabular}

\section{Cochrane Library}

Total results: 1,611; 1,602 imported, 9 duplicates

Cochrane Reviews: 774; all imported, no duplicates

Other Reviews: 6; all imported, no duplicates

Trials: 810; 801 imported, 9 duplicates

Methods Studies: 2; all imported, no duplicates

Technology Assessments: 1; all imported, no duplicates

Economic Evaluations: 13; all imported, no duplicates

Cochrane Groups: 5; all imported, no duplicates

\begin{tabular}{|c|c|c|}
\hline ID & Search & Hits \\
\hline \#1 & $\begin{array}{l}\text { [mh "Infant Nutritional Physiological Phenomena"] or [mh "Milk, Human"] or [mh "Breast Feeding"] or } \\
\text { "breast feeding" or "human milk" or (human and milk) or "breast milk" or breastmilk or breastfeed* or } \\
\text { (breast and fed) or breastfed or [mh Lactation] or lactating or lactation }\end{array}$ & 9220 \\
\hline \#2 & $\begin{array}{l}\text { [mh Absenteeism] or "Affordable Care Act" or ACA or "Baby friendly Hospital Initiative" or BFHI or } \\
\text { (break* and (express* and milk)) or [mh "Breast Milk Expression"] or "breast pump" or [mh "Child } \\
\text { Day Care Centers"] or [mh Employment] or (employ* and (polic* or program*)) or [mh "Health } \\
\text { Knowledge, Attitudes, Practice"] or [mh "Health Promotion"] or [mh "Insurance Benefits"] or } \\
\text { ("lactation consultant" or "lactation consultants") or [mh "Maternal Health Services"] or [mh } \\
\text { Mothers/psychology] or [mh "Nurseries, Hospital"] or [mh "Occupational Health Services"] or [mh } \\
\text { "Parental Leave"] or [mh "Program Evaluation"] or [mh "Salaries and Fringe Benefits"] or [mh "Social } \\
\text { Support"] or [mh "Women, Working"] }\end{array}$ & 26093 \\
\hline \#3 & $\begin{array}{l}\text { "baby friendly" or "hospital practices" or "Ten Steps" or [mh Counseling] or WIC or "Women, Infants, } \\
\text { and Children Program" or SNAP or "Food Stamps" or [mh "Food Assistance"] or "Food assistance" } \\
\text { or [mh "Health Education"] or [mh "House Calls"] or [mh "Organizational Policy"] or [mh "Patient } \\
\text { Education as Topic"] or "Promotion of Breastfeeding Intervention Trial" or PROBIT or [mh "Postnatal } \\
\text { Care"] or [mh "Social Support"] or "Ten Steps to Successful Breastfeeding" or [mh Workplace] }\end{array}$ & 19417 \\
\hline \#4 & $\begin{array}{l}(([m h \text { schools] or "schools" or "school" or [mh universities] or "universities" or "university") and } \\
\text { ("wellness programmes" or [mh "health promotion"] or ("health" and "promotion") or "health } \\
\text { promotion" or ("wellness" and "programs") or "wellness programs")) or ([mh "school health services"] } \\
\text { or ("school" and "health" and "services") or "school health services")) or (([mh workplace] or } \\
\text { "workplace") and ("wellness programmes" or [mh "health promotion"] or ("health" and "promotion") or } \\
\text { "health promotion" or ("wellness" and "programs") or "wellness programs" or programs)) }\end{array}$ & 10257 \\
\hline$\# 5$ & $\# 2$ or \#3 or \#4 & 40711 \\
\hline \#6 & $\# 1$ and \#5 & 1919 \\
\hline$\# 7$ & $\begin{array}{l}\text { ((controlled:ti or controlled:ab) and (trial:ti or trial:ab)) or "controlled clinical trial" or "randomized } \\
\text { controlled trial":pt or "randomized controlled trial as topic":pt or "single-blind method":pt or "double- } \\
\text { blind method":pt or "random allocation":pt }\end{array}$ & 618067 \\
\hline \#8 & $\# 6$ and \#7 & 1552 \\
\hline$\# 9$ & $\begin{array}{l}\text { [mh "Cohort Studies"] or [mh "Epidemiologic Studies"] or [mh "Follow-up Studies"] or "prospective } \\
\text { cohort" or [mh "prospective studies"] or (prospective* and cohort and (study or studies)) }\end{array}$ & 146506 \\
\hline$\# 10$ & $\# 6$ and \#9 & 521 \\
\hline$\# 11$ & \#8 or \#10 Publication Year from 1980 to 2017 & 1611 \\
\hline
\end{tabular}


Breastfeeding Original Searches KQ 2

FINAL

PubMed

Searched February 2, 2017

Results:

Case control studies and other study types: 6,660 (Line 10)

6,659 imported, 1 duplicate

SRs: 660 (Line 12)

508 imported, 152 duplicates

\begin{tabular}{|c|c|c|}
\hline Search & Query & $\begin{array}{l}\text { Items } \\
\text { found }\end{array}$ \\
\hline$\overline{\# 1}$ & $\begin{array}{l}\text { Search ("Infant Nutritional Physiological Phenomena"[Mesh] OR "Milk, Human"[Mesh] OR } \\
\text { "Breast Feeding"[Mesh] OR "breast feeding"[All Fields] OR "human milk"[All Fields] OR } \\
\text { (human[tiab] AND milk[tiab]) OR "breast milk"[All Fields] OR breastmilk OR breastfeed* OR } \\
\text { (breast[tiab] AND fed[tiab]) OR breastfed OR “Lactation"[Mesh] OR lactating OR lactation) }\end{array}$ & 130094 \\
\hline \#2 & Search ("HIV Infections"[Mesh] OR HIV OR "Fatty Acids"[Majr] OR "Amino Acids"[Majr]) & 936134 \\
\hline \#3 & Search (\#1 NOT \#2) & 120404 \\
\hline \#4 & $\begin{array}{l}\text { Search ("Case-Control Studies"[MeSH] OR "Cohort Studies"[MeSH] OR "Epidemiologic } \\
\text { Studies"[MeSH] OR "Cross-Sectional Studies"[MeSH] OR "Organizational Case } \\
\text { Studies"[MeSH] OR "Cross-Over Studies"[MeSH] OR "Follow-Up Studies"[MeSH] OR } \\
\text { "Seroepidemiologic Studies"[MeSH] OR "Evaluation Studies"[Publication Type] OR } \\
\text { "observational study" OR "observational studies" OR "Comparative Study"[MeSH] OR } \\
\text { "prospective studies"[MeSH] OR (prospective*[All Fields] AND cohort[All Fields] AND (study[All } \\
\text { Fields] OR studies[All Fields]) OR "Longitudinal Studies" OR cohort*) }\end{array}$ & 2384206 \\
\hline$\# 5$ & Search (\#3 AND \#4) & 14133 \\
\hline \#6 & $\begin{array}{l}\text { Search (Addresses[pt] OR Autobiography[pt] OR Bibliography[pt] OR Biography[pt] OR "Case } \\
\text { Reports"[pt] OR Congresses[pt] OR "Consensus Development Conference"[pt] OR } \\
\text { "Consensus Development Conference, NIH"[pt] OR Dictionary[pt] OR Directory[pt] OR } \\
\text { Editorial[pt] OR Festschrift[pt] OR “Government Publications"[pt] OR Interview[pt] OR } \\
\text { Lectures[pt] OR "Legal Cases"[pt] OR Legislation[pt] OR Letter[pt] OR News[pt] OR } \\
\text { "Newspaper Article"[pt] OR Overall[pt] OR "Patient Education Handout"[pt] OR "Periodical } \\
\text { Index"[pt]) }\end{array}$ & 3486139 \\
\hline$\# 7$ & Search (\#5 NOT \#6) & 13859 \\
\hline \#8 & Search (\#5 NOT \#6) Filters: Publication date from 2005/01/11 to 2017/12/31 & 8356 \\
\hline$\# 9$ & Search (\#5 NOT \#6) Filters: Publication date from 2005/01/11 to 2017/12/31; Humans & 7084 \\
\hline$\# 10$ & Search (\#5 NOT \#6) Filters: Publication date from 2005/01/11 to 2017/12/31; Humans; English & 6660 \\
\hline \#11 & $\begin{array}{l}\text { Search ("systematic review"[ti] OR "meta-analysis"[pt] OR "meta-analysis"[ti] OR "systematic } \\
\text { literature review"[ti] OR "this systematic review"[tw] OR ("systematic review"[tiab] AND } \\
\text { review[pt]) OR meta synthesis[ti] OR "meta synthesis"[ti] OR "cochrane database syst rev"[ta]) } \\
\text { Filters: Publication date from 2005/01/11 to 2017/12/31; Humans; English }\end{array}$ & 97194 \\
\hline \#12 & $\begin{array}{l}\text { Search (\#3 AND \#11) Filters: Publication date from 2005/01/11 to 2017/12/31; Humans; } \\
\text { English }\end{array}$ & 660 \\
\hline
\end{tabular}




\section{CINAHL}

Searched February 2, 2017

Results: 304

304 imported

\begin{tabular}{|c|c|c|c|}
\hline$\#$ & Query & Limiters/Expanders & Results \\
\hline S1 & $\begin{array}{l}\text { S1 MH "Infant Nutritional Physiological Phenomena" OR MH "Milk, } \\
\text { Human" OR MH "Breast Feeding" OR "breast feeding" OR "human } \\
\text { milk" OR (human AND milk) OR "breast milk" OR breastmilk OR } \\
\text { breastfeed* OR (breast AND fed) OR breastfed OR MH "Lactation" } \\
\text { OR lactating OR lactation }\end{array}$ & $\begin{array}{l}\text { Search modes - } \\
\text { Boolean/Phrase }\end{array}$ & 29,240 \\
\hline S2 & $\begin{array}{l}\text { MH "HIV Infections" OR HIV OR MH "Fatty Acids" OR MH "Amino } \\
\text { Acids" }\end{array}$ & $\begin{array}{l}\text { Search modes - } \\
\text { Boolean/Phrase }\end{array}$ & 94,304 \\
\hline S3 & S1 NOT S2 & $\begin{array}{l}\text { Search modes - } \\
\text { Boolean/Phrase }\end{array}$ & 27,700 \\
\hline S4 & $\begin{array}{l}\text { MH "Case-Control Studies" OR MH "Cohort Studies" OR MH } \\
\text { "Epidemiologic Studies" OR MH "Cross-Sectional Studies" OR MH } \\
\text { "Organizational Case Studies" OR MH "Cross-Over Studies" OR } \\
\text { MH "Follow-Up Studies" OR MH "Seroepidemiologic Studies" OR } \\
\text { "Evaluation Studies" OR "observational study" OR "observational } \\
\text { studies" OR MH "Comparative Study" OR MH "prospective studies" } \\
\text { OR (prospective* AND cohort AND (study OR studies)) OR } \\
\text { "Longitudinal Studies" OR cohort* }\end{array}$ & $\begin{array}{l}\text { Search modes - } \\
\text { Boolean/Phrase }\end{array}$ & 367,212 \\
\hline S5 & S3 AND S4 & $\begin{array}{l}\text { Search modes - } \\
\text { Boolean/Phrase }\end{array}$ & 3,524 \\
\hline S6 & S5 & $\begin{array}{l}\text { Limiters - Published Date: } \\
\text { 20050301-20171231; } \\
\text { English Language; Exclude } \\
\text { MEDLINE records; Human } \\
\text { Search modes - } \\
\text { Boolean/Phrase }\end{array}$ & 304 \\
\hline
\end{tabular}




\section{Cochrane Library}

Searched February 2, 2017

Results:

Cochrane Reviews: 572

Other Reviews: 29

Trials: 818

Methods Studies: 1

Technology Assessments: 2

Economic Evaluations: 13

Cochrane Groups: 4

\section{1,428 imported, 11 duplicates}

\begin{tabular}{lll}
\hline ID & Search & Hits \\
\hline$\# 1$ & [mh "Infant Nutritional Physiological Phenomena"] or [mh "Milk, Human"] or [mh "Breast & 9214 \\
& $\begin{array}{l}\text { Feeding"] or "breast feeding" or "human milk" or (human and milk) or "breast milk" or } \\
\text { breastmilk or breastfeed* or (breast and fed) or breastfed or [mh Lactation] or lactating or }\end{array}$ \\
& lactation & 52102 \\
\hline$\# 2$ & [mh "HIV Infections"] or HIV or [mh "Fatty Acids"] or [mh "Amino Acids"] & 7659 \\
\hline$\# 3$ & \#1 not \#2 & 202770 \\
& [mh "Case-Control Studies"] or [mh "Cohort Studies"] or [mh "Epidemiologic Studies"] or \\
& $\begin{array}{l}\text { [mh "Cross-Sectional Studies"] or [mh "Organizational Case Studies"] or [mh "Cross-Over } \\
\text { Studies"] or [mh "Follow-Up Studies"] or [mh "Seroepidemiologic Studies"] or "Evaluation }\end{array}$ \\
& $\begin{array}{l}\text { Studies" or "observational study" or "observational studies" or [mh "Comparative Study"] or } \\
\text { [mh "prospective studies"] or (prospective* and cohort and (study or studies)) or }\end{array}$ \\
\hline "Longitudinal Studies" or cohort* \\
\hline \#5 & \#3 and \#4 Publication Year from 2005 to 2017 & 1439 \\
\hline
\end{tabular}




\section{Breastfeeding Update Searches KQ 1}

\section{PubMed}

September 2, 2016, to October 12, 2017

Results:

RCTs and other study designs: 129 (Line 14)

129 imported, no duplicates

SRs: 15 (Line 19)

10 imported, 5 duplicates

\begin{tabular}{|c|c|c|}
\hline Search & Query & Items found \\
\hline$\# 1$ & $\begin{array}{l}\text { Search "Infant Nutritional Physiological Phenomena"[Mesh] OR "Milk, Human"[Mesh] OR "Breast } \\
\text { Feeding"[Mesh] OR "breast feeding"[All Fields] OR "human milk"[All Fields] OR (human[tiab] AND } \\
\text { milk[tiab]) OR "breast milk"[All Fields] OR breastmilk OR breastfeed* OR (breast[tiab] AND } \\
\text { fed[tiab]) OR breastfed OR "Lactation"[Mesh] OR lactating OR lactation }\end{array}$ & 134155 \\
\hline$\# 2$ & $\begin{array}{l}\text { Search (((Absenteeism[Mesh] OR "Affordable Care Act" OR ACA[tiab] OR "Baby friendly Hospital } \\
\text { Initiative" OR BFHI OR (break*[tiab] AND (express* AND milk)) OR "Breast Milk Expression"[Mesh] } \\
\text { OR "breast pump"[All Fields] OR "Child Day Care Centers"[Mesh] OR "Employment"[Mesh] OR } \\
\text { (employ* AND (polic* OR program*)) OR "Health Knowledge, Attitudes, Practice"[Mesh] OR } \\
\text { "Health Promotion"[Mesh] OR "Insurance Benefits"[Mesh] OR ("lactation consultant" OR "lactation } \\
\text { consultants") OR "Maternal Health Services"[Mesh] OR "Mothers/psychology"[Majr] OR } \\
\text { "Nurseries, Hospital"[Mesh] OR "Occupational Health Services"[Mesh] OR "Parental Leave"[Mesh] } \\
\text { OR "Program Evaluation"[Mesh] OR "Salaries and Fringe Benefits"[Mesh] OR "Social } \\
\text { Support"[Mesh] OR "Women, Working"[Mesh]))) }\end{array}$ & 479351 \\
\hline \#3 & $\begin{array}{l}\text { Search (("baby friendly"[All Fields] OR "hospital practices"[All Fields] OR "Ten Steps"[All Fields] } \\
\text { OR Counseling[Mesh] OR WIC OR "Women, Infants, and Children Program" OR SNAP OR "Food } \\
\text { Stamps"[All Fields] OR "Food Assistance"[Mesh] OR "Food assistance"[All Fields] OR "Health } \\
\text { Education"[Mesh] OR "House Calls"[Mesh] OR "Organizational Policy"[Mesh] OR "Patient } \\
\text { Education as Topic"[Mesh] OR "Promotion of Breastfeeding Intervention Trial"[All Fields] OR } \\
\text { PROBIT[All Fields] OR "Postnatal Care"[Mesh] OR "Social Support"[Mesh] OR 'Ten Steps to } \\
\text { Successful Breastfeeding'[All Fields] OR "Workplace"[Mesh])) }\end{array}$ & $\underline{289089}$ \\
\hline \#4 & $\begin{array}{l}\text { Search ((("schools"[MeSH] OR "schools"[All Fields] OR "school"[All Fields] OR } \\
\text { "universities"[MeSH] OR "universities"[All Fields] OR "university"[All Fields]) AND ("wellness } \\
\text { programmes"[All Fields] OR "health promotion"[MeSH Terms] OR ("health"[All Fields] AND } \\
\text { "promotion"[All Fields] OR "health promotion"[All Fields]) OR ("wellness"[All Fields] AND } \\
\text { "programs"[All Fields]) OR "wellness programs"[All Fields]) OR ("school health services"[MeSH] } \\
\text { OR ("school"[All Fields] AND "health"[All Fields] AND "services"[All Fields]) OR "school health } \\
\text { services"[All Fields])) OR (("workplace"[MeSH] OR "workplace"[All Fields]) AND ("wellness } \\
\text { programmes"[All Fields] OR "health promotion"[MeSH] OR ("health"[All Fields] AND "promotion"[All } \\
\text { Fields]) OR "health promotion"[All Fields] OR ("wellness"[All Fields] AND "programs"[All Fields]) } \\
\text { OR "wellness programs"[All Fields] OR programs[All Fields]))) }\end{array}$ & $\underline{191440}$ \\
\hline \#5 & Search (\#2 OR \#3 OR \#4) & $\underline{765688}$ \\
\hline$\underline{\underline{\# 6}}$ & Search (\#1 AND \#5) & 12574 \\
\hline$\overline{\# 7}$ & $\begin{array}{l}\text { Search ((((randomized[title/abstract] OR randomised[title/abstract]) AND controlled[title/abstract] } \\
\text { AND trial[title/abstract]) OR (controlled[title/abstract] AND trial[title/abstract]) OR "controlled clinical } \\
\text { trial"[publication type] OR "Randomized Controlled Trial"[Publication Type] OR "Single-Blind } \\
\text { Method"[MeSH] OR "Double-Blind Method"[MeSH] OR "Random Allocation"[MeSH])) }\end{array}$ & $\underline{687174}$ \\
\hline$\#$ & Search (\#6 AND \#7) & 892 \\
\hline$\underline{\# 9}$ & $\begin{array}{l}\text { Search ("Cohort Studies"[Mesh] OR "Epidemiologic Studies"[Mesh] OR "Follow-up Studies"[Mesh] } \\
\text { OR "prospective cohort" OR "prospective studies"[MeSH] OR (prospective*[All Fields] AND } \\
\text { cohort[All Fields] AND (study[All Fields] OR studies[All Fields])) }\end{array}$ & 2085344 \\
\hline$\# 10$ & Search (\#6 AND \#9) & $\underline{2400}$ \\
\hline$\# 11$ & Search (\#8 OR \#10) & $\underline{3055}$ \\
\hline \#12 & Search (\#8 OR \#10) Filters: Publication date from 2016/09/02 & 169 \\
\hline$\# 13$ & Search (\#8 OR \#10) Filters: Publication date from 2016/09/02; Humans & 131 \\
\hline$\# 14$ & Search (\#8 OR \#10) Filters: Publication date from 2016/09/02; Humans; English & 129 \\
\hline
\end{tabular}




\begin{tabular}{lll}
\hline Search & Query & Items found \\
\hline$\# 15$ & $\begin{array}{l}\text { Search ("systematic review"[ti] OR "meta-analysis"[pt] OR "meta-analysis"[ti] OR "systematic } \\
\text { literature review"[ti] OR "this systematic revieww"[tw] OR ("systematic review"[tiab] AND review[pt]) }\end{array}$ & $\underline{164355}$ \\
& OR meta synthesis[t] OR "meta synthesis"[ti] OR "cochrane database syst rev"[ta]) & \\
\hline$\# 16$ & Search (\#6 AND \#15) & $\underline{241}$ \\
\hline$\# 17$ & Search (\#6 AND \#15) Filters: Publication date from 2016/09/02 & $\underline{25}$ \\
\hline$\# 18$ & Search (\#6 AND \#15) Filters: Publication date from 2016/09/02; Humans & $\underline{\underline{15}}$ \\
\hline$\# 19$ & Search (\#6 AND \#15) Filters: Publication date from 2016/09/02; Humans; English & $\underline{\mathbf{1 5}}$ \\
\hline
\end{tabular}

\section{CINAHL \\ KQ 1}

Searched October 12, 2017

Limited to September 2016 to present

Excluded Medline citations

Results: 37

37 imported, no duplicates

\begin{tabular}{|c|c|c|c|}
\hline \# & Query & Limiters/Expanders & Results \\
\hline S1 & $\begin{array}{l}\text { MH "Infant Nutritional Physiological Phenomena" OR MH } \\
\text { "Milk, Human" OR MH "Breast Feeding" OR "breast feeding" } \\
\text { OR "human milk" OR (human AND milk) OR "breast milk" } \\
\text { OR breastmilk OR breastfeed* OR (breast AND fed) OR } \\
\text { breastfed OR MH "Lactation" OR lactating OR lactation }\end{array}$ & $\begin{array}{l}\text { Search modes - } \\
\text { Boolean/Phrase }\end{array}$ & 31,657 \\
\hline S2 & $\begin{array}{l}((\text { MH Absenteeism OR "Affordable Care Act" OR ACA OR } \\
\text { "Baby friendly Hospital Initiative" OR BFHI OR (break* AND } \\
\text { (express* AND milk)) OR MH "Breast Milk Expression" OR } \\
\text { "breast pump" OR MH "Child Day Care Centers" OR MH } \\
\text { "Employment" OR (employ* AND (polic* OR program*)) OR } \\
\text { MH "Health Knowledge, Attitudes, Practice" OR MH "Health } \\
\text { Promotion" OR MH "Insurance Benefits" OR ("lactation } \\
\text { consultant" OR "lactation consultants") OR MH "Maternal } \\
\text { Health Services" OR "Mothers/psychology" OR MH } \\
\text { "Nurseries, Hospital" OR MH "Occupational Health } \\
\text { Services" OR MH "Parental Leave" OR MH "Program } \\
\text { Evaluation" OR MH "Salaries and Fringe Benefits" OR MH } \\
\text { "Social Support" OR MH "Women, Working"))) }\end{array}$ & $\begin{array}{l}\text { Search modes - } \\
\text { Boolean/Phrase }\end{array}$ & 159,128 \\
\hline S3 & $\begin{array}{l}\text { "baby friendly" OR "hospital practices" OR "Ten Steps" OR } \\
\text { MH Counseling OR WIC OR "Women, Infants, and Children } \\
\text { Program" OR SNAP OR "Food Stamps" OR MH "Food } \\
\text { Assistance" OR "Food assistance" OR MH "Health } \\
\text { Education" OR MH "House Calls" OR MH "Organizational } \\
\text { Policy" OR MH "Patient Education as Topic" OR "Promotion } \\
\text { of Breastfeeding Intervention Trial" OR PROBIT OR MH } \\
\text { "Postnatal Care" OR MH "Social Support" OR "Ten Steps to } \\
\text { Successful Breastfeeding" OR MH "Workplace" }\end{array}$ & $\begin{array}{l}\text { Search modes - } \\
\text { Boolean/Phrase }\end{array}$ & 49,828 \\
\hline
\end{tabular}




\begin{tabular}{|c|c|c|c|}
\hline \# & Query & Limiters/Expanders & Results \\
\hline$\overline{\mathrm{S} 4}$ & $\begin{array}{l}\text { ((MH "schools" OR "schools"[All Fields] OR "school"[All } \\
\text { Fields] OR MH “universities" OR "universities"[All Fields] OR } \\
\text { "university"[All Fields]) AND ("wellness programmes"[All } \\
\text { Fields] OR MH "health promotion" OR ("health"[All Fields] } \\
\text { AND "promotion"[All Fields] OR "health promotion"[All } \\
\text { Fields]) OR ("wellness"[All Fields] AND "programs"[All } \\
\text { Fields]) OR "wellness programs"[All Fields]) OR (MH } \\
\text { "school health services" OR ("school"[All Fields] AND } \\
\text { "health"[All Fields] AND "services"[All Fields]) OR "school } \\
\text { health services"[All Fields])) OR ((MH “workplace" OR } \\
\text { "workplace"[All Fields]) AND ("wellness programmes"[All } \\
\text { Fields] OR MH "health promotion" OR ("health"[All Fields] } \\
\text { AND "promotion"[All Fields]) OR "health promotion"[All } \\
\text { Fields] OR ("wellness"[All Fields] AND "programs"[All } \\
\text { Fields]) OR "wellness programs"[All Fields] OR programs[All } \\
\text { Fields])) }\end{array}$ & $\begin{array}{l}\text { Search modes - } \\
\text { Boolean/Phrase }\end{array}$ & 8,254 \\
\hline S5 & S2 OR S3 OR S4 & $\begin{array}{l}\text { Search modes - } \\
\text { Boolean/Phrase }\end{array}$ & 206,321 \\
\hline S6 & S1 AND S5 & $\begin{array}{l}\text { Search modes - } \\
\text { Boolean/Phrase }\end{array}$ & 4,497 \\
\hline S7 & $\begin{array}{l}\text { (randomized OR randomised) AND controlled AND trial) OR } \\
\text { (controlled AND trial) OR "controlled clinical trial" OR } \\
\text { "Randomized Controlled Trial" OR MH "Single-Blind } \\
\text { Method" OR MH "Double-Blind Method" OR MH "Random } \\
\text { Allocation" }\end{array}$ & $\begin{array}{l}\text { Search modes - } \\
\text { Boolean/Phrase }\end{array}$ & 128,349 \\
\hline S8 & S6 AND S7 & $\begin{array}{l}\text { Search modes - } \\
\text { Boolean/Phrase }\end{array}$ & 211 \\
\hline S9 & $\begin{array}{l}\text { MH "Cohort Studies" OR MH "Epidemiologic Studies" OR } \\
\text { MH “Follow-up Studies" OR "prospective cohort" OR MH } \\
\text { "prospective studies" OR (prospective* AND cohort AND } \\
\text { (study OR studies)) }\end{array}$ & $\begin{array}{l}\text { Search modes - } \\
\text { Boolean/Phrase }\end{array}$ & 329,266 \\
\hline S10 & S6 AND S9 & $\begin{array}{l}\text { Search modes - } \\
\text { Boolean/Phrase }\end{array}$ & 344 \\
\hline S11 & S8 OR S10 & $\begin{array}{l}\text { Limiters - Published } \\
\text { Date: 20160901- } \\
\text { 20171231; English } \\
\text { Language; Exclude } \\
\text { MEDLINE records } \\
\text { Search modes - } \\
\text { Boolean/Phrase }\end{array}$ & 37 \\
\hline
\end{tabular}




\section{KQ 1}

\section{Cochrane Library}

Total results: 123; all imported, no duplicates

Cochrane Reviews: 101; all imported, no duplicates

Other Reviews: 0;

Trials: 21; all imported, no duplicates

Methods Studies: 0

Technology Assessments: 0

Economic Evaluations: 0

Cochrane Groups: 1; all imported, no duplicates

\begin{tabular}{|c|c|c|}
\hline ID & Search & Hits \\
\hline \#1 & $\begin{array}{l}\text { [mh "Infant Nutritional Physiological Phenomena"] or [mh "Milk, Human"] or [mh "Breast } \\
\text { Feeding"] or "breast feeding" or "human milk" or (human and milk) or "breast milk" or breastmilk } \\
\text { or breastfeed* or (breast and fed) or breastfed or [mh Lactation] or lactating or lactation }\end{array}$ & 10043 \\
\hline \#2 & $\begin{array}{l}\text { [mh Absenteeism] or "Affordable Care Act" or ACA or "Baby friendly Hospital Initiative" or BFHI } \\
\text { or (break* and (express* and milk)) or [mh "Breast Milk Expression"] or "breast pump" or [mh } \\
\text { "Child Day Care Centers"] or [mh Employment] or (employ* and (polic* or program*)) or [mh } \\
\text { "Health Knowledge, Attitudes, Practice"] or [mh "Health Promotion"] or [mh "Insurance Benefits"] } \\
\text { or ("lactation consultant" or "lactation consultants") or [mh "Maternal Health Services"] or [mh } \\
\text { Mothers/psychology] or [mh "Nurseries, Hospital"] or [mh "Occupational Health Services"] or [mh } \\
\text { "Parental Leave"] or [mh "Program Evaluation"] or [mh "Salaries and Fringe Benefits"] or [mh } \\
\text { "Social Support"] or [mh "Women, Working"] }\end{array}$ & 27683 \\
\hline \#3 & $\begin{array}{l}\text { "baby friendly" or "hospital practices" or "Ten Steps" or [mh Counseling] or WIC or "Women, } \\
\text { Infants, and Children Program" or SNAP or "Food Stamps" or [mh "Food Assistance"] or "Food } \\
\text { assistance" or [mh "Health Education"] or [mh "House Calls"] or [mh "Organizational Policy"] or } \\
\text { [mh "Patient Education as Topic"] or "Promotion of Breastfeeding Intervention Trial" or PROBIT } \\
\text { or [mh "Postnatal Care"] or [mh "Social Support"] or "Ten Steps to Successful Breastfeeding" or } \\
\text { [mh Workplace] }\end{array}$ & 20423 \\
\hline \#4 & $\begin{array}{l}\text { ((([mh schools] or "schools" or "school" or [mh universities] or "universities" or "university") and } \\
\text { ("wellness programmes" or [mh "health promotion"] or ("health" and "promotion") or "health } \\
\text { promotion" or ("wellness" and "programs") or "wellness programs")) or ([mh "school health } \\
\text { services"] or ("school" and "health" and "services") or "school health services")) or (([mh } \\
\text { workplace] or "workplace") and ("wellness programmes" or [mh "health promotion"] or ("health" } \\
\text { and "promotion") or "health promotion" or ("wellness" and "programs") or "wellness programs" or } \\
\text { programs)) }\end{array}$ & 11032 \\
\hline$\# 5$ & $\# 2$ or \#3 or \#4 & 43283 \\
\hline$\# 6$ & $\# 1$ and \#5 & 2075 \\
\hline \#7 & $\begin{array}{l}(\text { controlled:ti or controlled:ab) and (trial:ti or trial:ab)) or "controlled clinical trial" or "randomized } \\
\text { controlled trial":pt or "randomized controlled trial as topic":pt or "single-blind method":pt or } \\
\text { "double-blind method":pt or "random allocation":pt }\end{array}$ & 695356 \\
\hline$\# 8$ & $\# 6$ and \#7 & 1701 \\
\hline \#9 & $\begin{array}{l}\text { [mh "Cohort Studies"] or [mh "Epidemiologic Studies"] or [mh "Follow-up Studies"] or } \\
\text { "prospective cohort" or [mh "prospective studies"] or (prospective* and cohort and (study or } \\
\text { studies)) }\end{array}$ & 154536 \\
\hline$\# 10$ & \#6 and \#9 Publication Year from 2016 to 2017 & 123 \\
\hline
\end{tabular}




\section{Breastfeeding Update Searches KQ 2}

\section{PubMed}

KQ 2

Searched October 12, 2017

Results:

Case control studies and other study types: 405 (Line 10)

315 imported, 90 duplicates

SRs: 53 (Line 12)

32 imported, 21 duplicates

\begin{tabular}{|c|c|c|}
\hline Search & Query & $\begin{array}{l}\text { Items } \\
\text { found }\end{array}$ \\
\hline$\# 1$ & $\begin{array}{l}\text { Search (("Infant Nutritional Physiological Phenomena"[Mesh] OR "Milk, Human"[Mesh] OR } \\
\text { "Breast Feeding"[Mesh] OR "breast feeding"[All Fields] OR "human milk"[All Fields] OR } \\
\text { (human[tiab] AND milk[tiab]) OR "breast milk"[All Fields] OR breastmilk OR breastfeed* OR } \\
\text { (breast[tiab] AND fed[tiab]) OR breastfed OR "Lactation"[Mesh] OR lactating OR lactation)) }\end{array}$ & $\underline{134155}$ \\
\hline$\# 2$ & Search ("HIV Infections"[Mesh] OR HIV OR "Fatty Acids"[Majr] OR "Amino Acids"[Majr]) & $\underline{957218}$ \\
\hline$\overline{\# 3}$ & Search (\#1 NOT \#2) & 124091 \\
\hline \#4 & $\begin{array}{l}\text { Search ("Case-Control Studies"[MeSH] OR "Cohort Studies"[MeSH] OR "Epidemiologic } \\
\text { Studies"[MeSH] OR "Cross-Sectional Studies"[MeSH] OR "Organizational Case Studies"[MeSH] } \\
\text { OR "Cross-Over Studies"[MeSH] OR "Follow-Up Studies"[MeSH] OR "Seroepidemiologic } \\
\text { Studies"[MeSH] OR "Evaluation Studies"[Publication Type] OR "observational study" OR } \\
\text { "observational studies" OR "Comparative Study"[MeSH] OR "prospective studies"[MeSH] OR } \\
\text { (prospective*[All Fields] AND cohort[All Fields] AND (study[All Fields] OR studies[All Fields]) OR } \\
\text { "Longitudinal Studies" OR cohort*) }\end{array}$ & 2513165 \\
\hline$\underline{\underline{\# 5}}$ & Search (\#3 AND \#4) & 14952 \\
\hline$\underline{\# 6}$ & $\begin{array}{l}\text { Search (Addresses[pt] OR Autobiography[pt] OR Bibliography[pt] OR Biography[pt] OR "Case } \\
\text { Reports"[pt] OR Congresses[pt] OR "Consensus Development Conference"[pt] OR "Consensus } \\
\text { Development Conference, NIH"[pt] OR Dictionary[pt] OR Directory[pt] OR Editorial[pt] OR } \\
\text { Festschrift[pt] OR "Government Publications"[pt] OR Interview[pt] OR Lectures[pt] OR "Legal } \\
\text { Cases"[pt] OR Legislation[pt] OR Letter[pt] OR News[pt] OR "Newspaper Article"[pt] OR } \\
\text { Overall[pt] OR "Patient Education Handout"[pt] OR "Periodical Index"[pt]) }\end{array}$ & 3567609 \\
\hline$\underline{\# 7}$ & Search (\#5 NOT \#6) & 14672 \\
\hline$\underline{\underline{\# 8}}$ & Search (\#5 NOT \#6) Filters: Publication date from 2016/09/02 & 822 \\
\hline$\overline{\underline{\# 9}}$ & Search (\#5 NOT \#6) Filters: Publication date from 2016/09/02; Humans & 421 \\
\hline$\# 10$ & Search (\#5 NOT \#6) Filters: Publication date from 2016/09/02; Humans; English & 405 \\
\hline \#11 & $\begin{array}{l}\text { Search ("systematic review"[ti] OR "meta-analysis"[pt] OR "meta-analysis"[ti] OR "systematic } \\
\text { literature review"[ti] OR "this systematic review"[tw] OR ("systematic review"[tiab] AND review[pt]) } \\
\text { OR meta synthesis[ti] OR "meta synthesis"[ti] OR "cochrane database syst rev"[ta]) Filters: } \\
\text { Publication date from 2016/09/02; Humans; English }\end{array}$ & 9081 \\
\hline$\# 12$ & Search (\#3 AND \#11) Filters: Publication date from 2016/09/02; Humans; English & $\underline{53}$ \\
\hline
\end{tabular}




\section{CINAHL}

KQ 2

Searched October 12, 2017

Results: 177

177 imported

\begin{tabular}{|c|c|c|c|}
\hline \# & Query & Limiters/Expanders & Results \\
\hline S1 & $\begin{array}{l}\text { MH "Infant Nutritional Physiological Phenomena" OR MH "Milk, } \\
\text { Human" OR MH "Breast Feeding" OR "breast feeding" OR "human } \\
\text { milk" OR (human AND milk) OR "breast milk" OR breastmilk OR } \\
\text { breastfeed* OR (breast AND fed) OR breastfed OR MH "Lactation" } \\
\text { OR lactating OR lactation }\end{array}$ & $\begin{array}{l}\text { Search modes - } \\
\text { Boolean/Phrase }\end{array}$ & 31,679 \\
\hline S2 & $\begin{array}{l}\text { MH "HIV Infections" OR HIV OR MH "Fatty Acids" OR MH “Amino } \\
\text { Acids" }\end{array}$ & $\begin{array}{l}\text { Search modes - } \\
\text { Boolean/Phrase }\end{array}$ & 99,823 \\
\hline S3 & S1 NOT S2 & $\begin{array}{l}\text { Search modes - } \\
\text { Boolean/Phrase }\end{array}$ & 30,014 \\
\hline S4 & $\begin{array}{l}\text { MH "Case-Control Studies" OR MH "Cohort Studies" OR MH } \\
\text { "Epidemiologic Studies" OR MH "Cross-Sectional Studies" OR MH } \\
\text { "Organizational Case Studies" OR MH "Cross-Over Studies" OR } \\
\text { MH "Follow-Up Studies" OR MH "Seroepidemiologic Studies" OR } \\
\text { "Evaluation Studies" OR "observational study" OR "observational } \\
\text { studies" OR MH "Comparative Study" OR MH "prospective studies" } \\
\text { OR (prospective* AND cohort AND (study OR studies)) OR } \\
\text { "Longitudinal Studies" OR cohort* }\end{array}$ & $\begin{array}{l}\text { Search modes - } \\
\text { Boolean/Phrase }\end{array}$ & 403,999 \\
\hline S5 & S3 AND S4 & $\begin{array}{l}\text { Limiters - English } \\
\text { Language } \\
\text { Search modes - } \\
\text { Boolean/Phrase }\end{array}$ & 3,886 \\
\hline S6 & S5 & \multicolumn{2}{|c|}{$\begin{array}{l}\text { Limiters - Published Date: } 177 \\
\text { 20160901-20171231; } \\
\text { English Language; } \\
\text { Exclude MEDLINE } \\
\text { records; Human } \\
\text { Search modes - } \\
\text { Boolean/Phrase }\end{array}$} \\
\hline
\end{tabular}




\section{Cochrane Library \\ KQ 2}

Searched October 12, 2017

Results: Total: 332

Cochrane Reviews: 175; 118 imported, 58 duplicates

Other Reviews: 0

Trials: 156; 131 imported, 25 duplicates

Methods Studies: 0

Technology Assessments: 0

Economic Evaluations: 0

Cochrane Groups: 1; 1 imported, no duplicates

250 imported, 83 duplicates

\begin{tabular}{|c|c|c|}
\hline ID & Search & Hits \\
\hline$\overline{\# 1}$ & $\begin{array}{l}\text { [mh "Infant Nutritional Physiological Phenomena"] or [mh "Milk, Human"] or [mh "Breast Feeding"] } \\
\text { or "breast feeding" or "human milk" or (human and milk) or "breast milk" or breastmilk or } \\
\text { breastfeed* or (breast and fed) or breastfed or [mh Lactation] or lactating or lactation }\end{array}$ & 10043 \\
\hline \#2 & [mh "HIV Infections"] or HIV or [mh "Fatty Acids"] or [mh "Amino Acids"] & 54286 \\
\hline \#3 & $\# 1$ not \#2 & 8401 \\
\hline$\overline{\# 4}$ & $\begin{array}{l}\text { [mh "Case-Control Studies"] or [mh "Cohort Studies"] or [mh "Epidemiologic Studies"] or [mh } \\
\text { "Cross-Sectional Studies"] or [mh "Organizational Case Studies"] or [mh "Cross-Over Studies"] or } \\
\text { [mh "Follow-Up Studies"] or [mh "Seroepidemiologic Studies"] or "Evaluation Studies" or } \\
\text { "observational study" or "observational studies" or [mh "Comparative Study"] or [mh "prospective } \\
\text { studies"] or (prospective* and cohort and (study or studies)) or "Longitudinal Studies" or cohort* }\end{array}$ & 219736 \\
\hline$\# 5$ & \#3 and \#4 Publication Year from 2005 to 2017 & 332 \\
\hline
\end{tabular}




\section{Appendix B. PRISMA}

\section{Figure B-1. Summary of evidence and search selection}

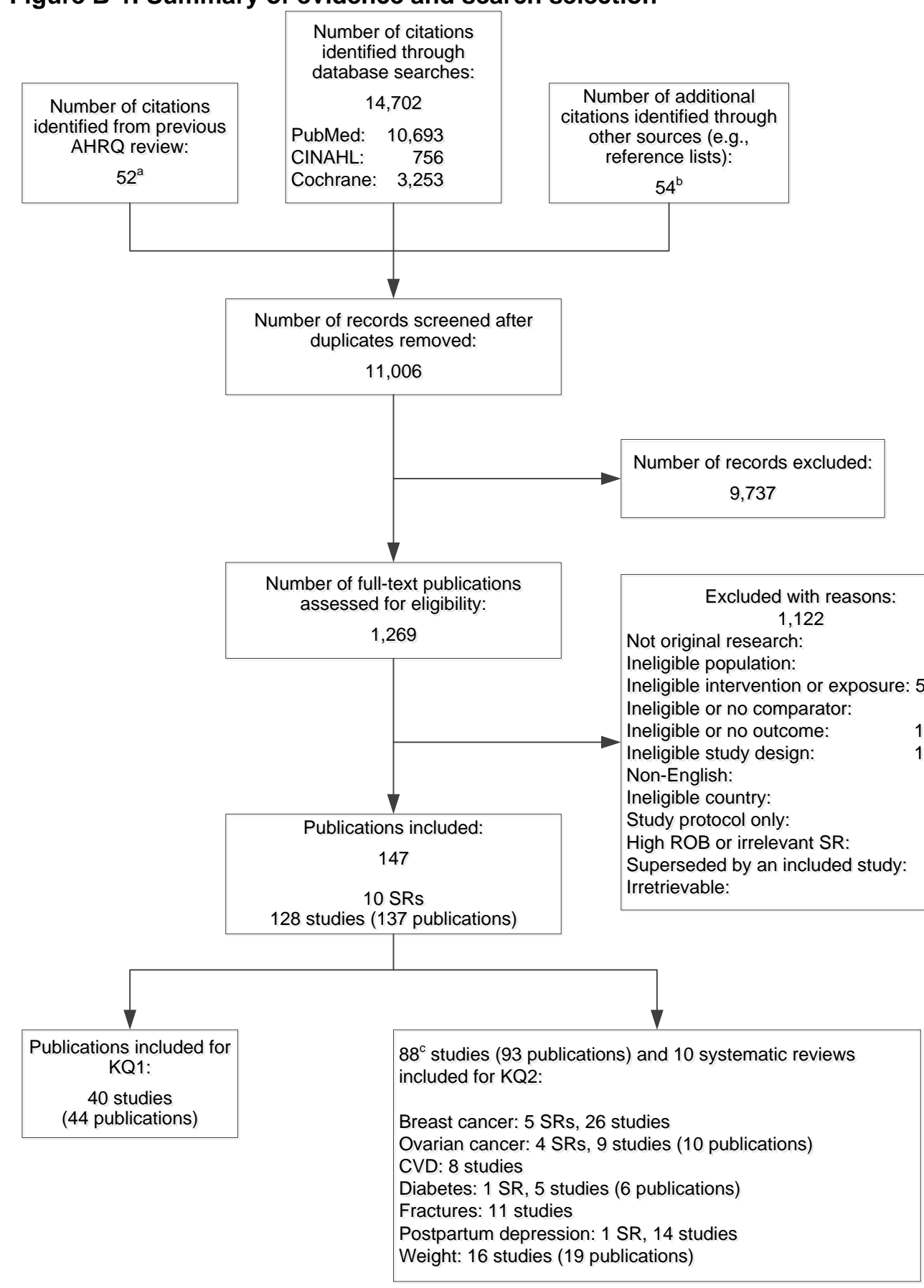

a This represents the number of studies relevant to maternal health only (not infant health). ${ }^{1}$

b Sources include reference lists of reviews and included studies, ClinicalTrials.gov, the World Health Organization International Clinical Trials Registry Platform, and suggestions from expert reviewers and public commentators.

c 1 systematic review and 1 individual study cover two eligible outcomes each: (breast and ovarian cancer, ${ }^{2}$ CVD and diabetes, ${ }^{3}$ respectively)

AHRQ = Agency for Healthcare Research and Quality; CVD = cardiovascular disease; KQ = Key Question; RCT = randomized controlled trial; $\mathrm{ROB}=$ risk of bias; $\mathrm{SR}=$ systematic review. 


\section{Appendix C. Relevance and Risk of Bias Assessments}

Table C-1. Relevance assessment for systematic reviews evaluating an eligible KQ 1 outcome

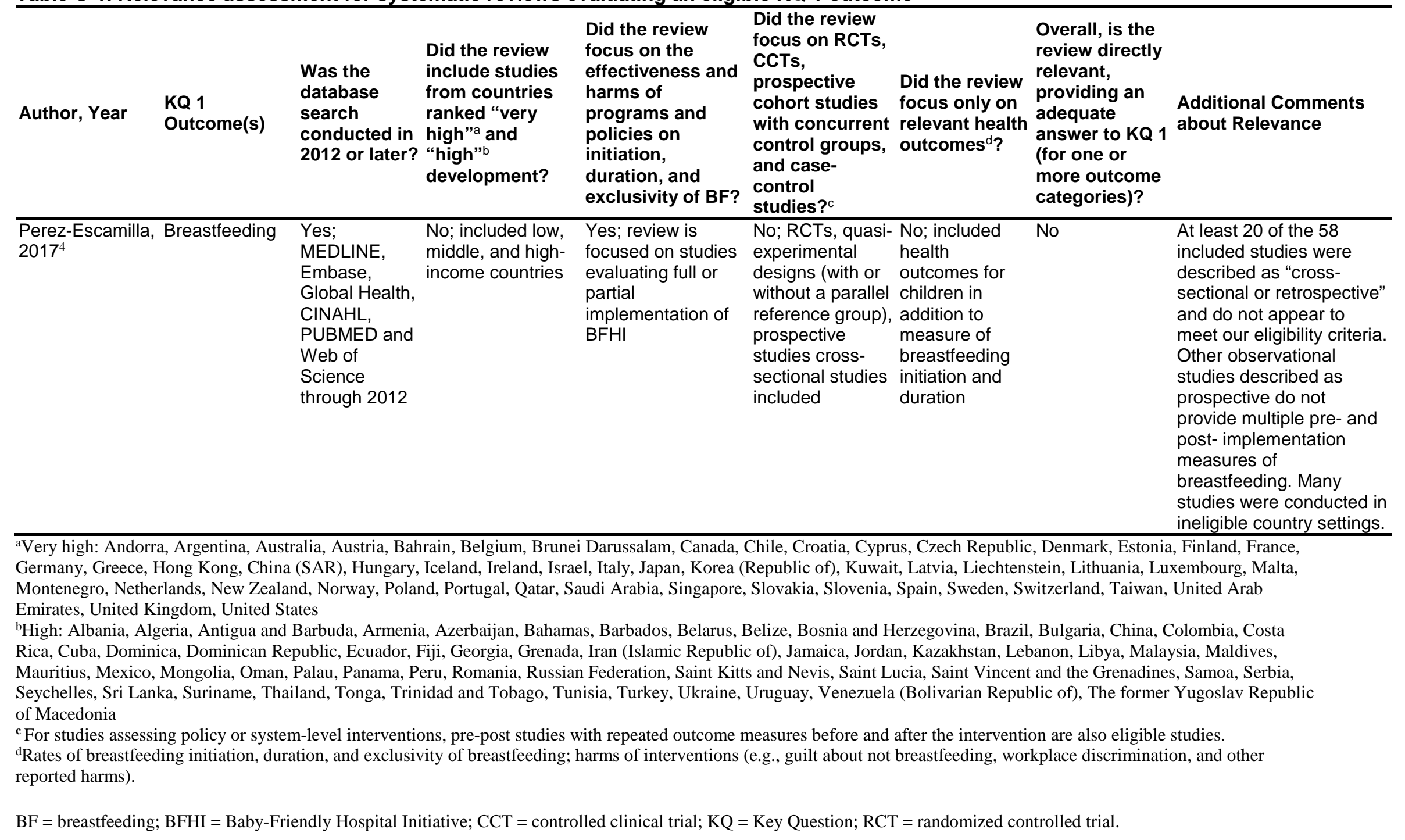


Table C-2. KQ 1 risk of bias assessment: Randomized controlled trials, part 1

\begin{tabular}{|c|c|c|c|c|c|c|c|}
\hline Author, Year & $\begin{array}{l}\text { Overall } \\
\text { Risk of } \\
\text { Bias Rating }\end{array}$ & $\begin{array}{l}\text { Overall Rationale for Risk of } \\
\text { Bias Rating }\end{array}$ & $\begin{array}{l}\text { 1. Was method } \\
\text { of randomi- } \\
\text { zation } \\
\text { adequate? }\end{array}$ & $\begin{array}{l}\text { 2. Was } \\
\text { allocation } \\
\text { concealment } \\
\text { adequate? }\end{array}$ & $\begin{array}{l}\text { 3. Were } \\
\text { group } \\
\text { characteris- } \\
\text { tics balanced } \\
\text { at baseline? }\end{array}$ & $\begin{array}{l}\text { Bias arising } \\
\text { from } \\
\text { randomiza- } \\
\text { tion or } \\
\text { selection? }\end{array}$ & Comments \\
\hline Coutinho, $2005^{5}$ & Low & NA & Yes & Yes & Yes & Low & NA \\
\hline $\begin{array}{l}\text { Ekstrom, 2012; } \\
\text { Ekstrom, 2014 }\end{array}$ & $\begin{array}{l}\text { Some } \\
\text { concerns }\end{array}$ & $\begin{array}{l}\text { See individual domains. Authors } \\
\text { report on a number of outcomes } \\
\text { and subgroups, but the } \\
\text { denominators for calculations are } \\
\text { not always apparent. }\end{array}$ & No information & No information & Probably yes & $\begin{array}{l}\text { Some } \\
\text { concerns }\end{array}$ & $\begin{array}{l}\text { Unclear what randomization } \\
\text { methods were used and } \\
\text { whether they were adequate } \\
\text { given the small sample size. }\end{array}$ \\
\hline Hayes, $2008^{8}$ & $\begin{array}{l}\text { Some } \\
\text { concerns }\end{array}$ & $\begin{array}{l}\text { No clear definition of BF reported, } \\
\text { inadequate reporting on design } \\
\text { elements and differences at } \\
\text { baseline raise concerns about the } \\
\text { success of randomization. }\end{array}$ & No Information & No Information & No Information & Uncertain & $\begin{array}{l}\text { No information on how groups } \\
\text { were randomized and there are } \\
\text { several differences between } \\
\text { arms. }\end{array}$ \\
\hline Hoddinott, $2009^{9}$ & $\begin{array}{l}\text { Some } \\
\text { concerns }\end{array}$ & $\begin{array}{l}\text { Potential for co-interventions to } \\
\text { explain the results. }\end{array}$ & Yes & Probably yes & No & Uncertain & $\begin{array}{l}\text { No information on how groups } \\
\text { were randomized and there are } \\
\text { several differences between } \\
\text { arms. }\end{array}$ \\
\hline Kools, $2005^{10}$ & $\begin{array}{l}\text { Some } \\
\text { concerns }\end{array}$ & $\begin{array}{l}\text { Potential for co-interventions to } \\
\text { explain the results. }\end{array}$ & Probably no & Probably no & Probably yes & $\begin{array}{l}\text { Some } \\
\text { concerns }\end{array}$ & $\begin{array}{l}\text { Coin flip for randomization, and } \\
\text { no evidence of allocation } \\
\text { concealment, but baseline } \\
\text { characteristics similar. }\end{array}$ \\
\hline $\begin{array}{l}\text { Kramer, 2001:11 } \\
\text { Yang, 2014 }\end{array}$ & $\begin{array}{l}\text { Some } \\
\text { concerns }\end{array}$ & $\begin{array}{l}\text { See individual domains; bias } \\
\text { related to missing data and } \\
\text { potential deviations from intended } \\
\text { intervention. }{ }^{11} \\
\text { Differential attrition, failure to } \\
\text { address missing data, outcomes } \\
\text { not prespecified. }{ }^{12}\end{array}$ & $\begin{array}{l}\text { Yes; }{ }^{11} \text { Probably } \\
\text { yes }^{12}\end{array}$ & $\begin{array}{l}\text { NA:; }{ }^{11} \text { Probably } \\
\text { yes }^{12}\end{array}$ & Yes & Low & $\begin{array}{l}\text { Sites (clusters) were stratified } \\
\text { based on important } \\
\text { characteristics (e.g., urban vs. } \\
\text { rural, etc.) prior to } \\
\text { randomization. Final } \\
\text { determination of group } \\
\text { assignment (after individual } \\
\text { sites were randomized) was } \\
\text { based on a coin toss. }\end{array}$ \\
\hline Kronborg, $2007^{13}$ & $\begin{array}{l}\text { Some } \\
\text { concerns }\end{array}$ & $\begin{array}{l}\text { No blinding; fidelity and sources of } \\
\text { potential contamination are } \\
\text { unclear; risk of measurement bias } \\
\text { (home-visitors assessed and } \\
\text { recorded outcomes, unclear how } \\
\text { exclusive BF was defined and } \\
\text { measured). }\end{array}$ & Probably yes & Probably no & Yes & Low & $\begin{array}{l}\text { Maternal characteristics were } \\
\text { balanced at baseline (shown in } \\
\text { online appendix). }\end{array}$ \\
\hline
\end{tabular}




\begin{tabular}{|c|c|c|c|c|c|c|c|}
\hline Author, Year & $\begin{array}{l}\text { Overall } \\
\text { Risk of } \\
\text { Bias Rating }\end{array}$ & $\begin{array}{l}\text { Overall Rationale for Risk of } \\
\text { Bias Rating }\end{array}$ & $\begin{array}{l}\text { 1. Was method } \\
\text { of randomi- } \\
\text { zation } \\
\text { adequate? }\end{array}$ & $\begin{array}{l}\text { 2. Was } \\
\text { allocation } \\
\text { concealment } \\
\text { adequate? }\end{array}$ & $\begin{array}{l}\text { 3. Were } \\
\text { group } \\
\text { characteris- } \\
\text { tics balanced } \\
\text { at baseline? }\end{array}$ & $\begin{array}{l}\text { Bias arising } \\
\text { from } \\
\text { randomiza- } \\
\text { tion or } \\
\text { selection? }\end{array}$ & Comments \\
\hline $\begin{array}{l}\text { MacLachlan, } \\
2016 ; ;^{14} \\
\text { Cramer, } 2017^{15}\end{array}$ & $\begin{array}{l}\text { Some } \\
\text { concerns }\end{array}$ & $\begin{array}{l}\text { Differences between arms, } \\
\text { differential and high overall } \\
\text { attrition, and failure to address } \\
\text { missing data. }\end{array}$ & Probably yes & Probably yes & Probably no & $\begin{array}{l}\text { Some } \\
\text { concerns }\end{array}$ & $\begin{array}{l}\text { Allocation of clusters took place } \\
\text { at a state-wide forum; an } \\
\text { audience member chose } \\
\text { opaque envelopes with cluster } \\
\text { assignments. The proportion of } \\
\text { Australian-born women was } \\
\text { unequal across the arms. }\end{array}$ \\
\hline Morrow, $1999^{16}$ & Low & NA & Yes & No information & Yes & Low & NA \\
\hline Nilsson, $2017^{17}$ & $\begin{array}{l}\text { Some } \\
\text { Concerns }\end{array}$ & $\begin{array}{l}\text { High overall attrition and unknown } \\
\text { fidelity/possible contamination are } \\
\text { sources of potential bias. }\end{array}$ & Yes & NA & Yes & Low & NA \\
\hline Reeder, $2014^{18}$ & $\begin{array}{l}\text { Some } \\
\text { concerns }\end{array}$ & $\begin{array}{l}\text { Risk of selection bias; moderate } \\
\text { rates of missing data ( } 21 \% \text { overall) } \\
\text { and unclear handling of missing } \\
\text { data. }\end{array}$ & No information & No information & Yes & $\begin{array}{l}\text { Some } \\
\text { concerns }\end{array}$ & $\begin{array}{l}\text { Of } 36 \text { Oregon LWAs, } 6 \\
\text { expressed interest in the study } \\
\text { ( } 5 \text { were selected). Only women } \\
\text { who indicated an intention to } \\
\text { breastfeed or were undecided } \\
\text { on their first WIC appointment } \\
\text { were invited to participate. }\end{array}$ \\
\hline
\end{tabular}




\begin{tabular}{|c|c|c|c|c|c|c|c|}
\hline Author, Year & $\begin{array}{l}\text { Overall } \\
\text { Risk of } \\
\text { Bias Rating }\end{array}$ & $\begin{array}{l}\text { Overall Rationale for Risk of } \\
\text { Bias Rating }\end{array}$ & $\begin{array}{l}\text { 1. Was method } \\
\text { of randomi- } \\
\text { zation } \\
\text { adequate? }\end{array}$ & $\begin{array}{l}\text { 2. Was } \\
\text { allocation } \\
\text { concealment } \\
\text { adequate? }\end{array}$ & $\begin{array}{l}\text { 3. Were } \\
\text { group } \\
\text { characteris- } \\
\text { tics balanced } \\
\text { at baseline? }\end{array}$ & $\begin{array}{l}\text { Bias arising } \\
\text { from } \\
\text { randomiza- } \\
\text { tion or } \\
\text { selection? }\end{array}$ & Comments \\
\hline Senarath, $2007^{19}$ & High & $\begin{array}{l}\text { High risk of selection bias (no } \\
\text { description of randomization and } \\
\text { women in intervention and control } \\
\text { hospitals differed in ways that } \\
\text { could affect rates of BF) and } \\
\text { measurement bias. }\end{array}$ & No Information & No Information & No & High & $\begin{array}{l}\text { Group characteristics differed } \\
\text { by ethnicity, employment status, } \\
\text { and parity; there also appear to } \\
\text { be important baseline } \\
\text { differences in hospital practices } \\
\text { across intervention and control } \\
\text { sites. }\end{array}$ \\
\hline Taddei, $2000^{20}$ & $\begin{array}{l}\text { Some } \\
\text { concerns }\end{array}$ & $\begin{array}{l}\text { Unclear randomization and group } \\
\text { selection; attrition is } 16-19 \% \\
\text { overall, with no comment on } \\
\text { differential attrition and no } \\
\text { methods used to address missing } \\
\text { data. }\end{array}$ & No information & Probably yes & No information & Uncertain & $\begin{array}{l}\text { Randomization and allocation } \\
\text { concealment not described; } \\
\text { groups of women were similar } \\
\text { at baseline, but there is no } \\
\text { description on whether } \\
\text { hospitals or staff were similar } \\
\text { (intervention focuses on staff } \\
\text { training). }\end{array}$ \\
\hline Wan, $2011^{21}$ & $\begin{array}{l}\text { Some } \\
\text { concerns }\end{array}$ & $\begin{array}{l}\text { Unclear whether outcome } \\
\text { assessors were blinded; fidelity to } \\
\text { intervention not assessed. }\end{array}$ & No information & No information & Yes & Low & $\mathrm{NA}$ \\
\hline Washio, $2017^{22}$ & $\begin{array}{l}\text { Some } \\
\text { concerns }\end{array}$ & $\begin{array}{l}\text { No blinding of participants or staff; } \\
\text { women in the control group also } \\
\text { received a small financial incentive } \\
\text { to participate which may have } \\
\text { affected reports of BF. }\end{array}$ & Yes & No information & Yes & Low & NA \\
\hline
\end{tabular}

$\mathrm{BF}$ = breastfeeding; KQ = Key Question; LWAs = local WIC agencies; NA = not applicable. 
Table C-3. KQ 1 risk of bias assessment: Randomized controlled trials, part 2

\begin{tabular}{|c|c|c|c|c|c|c|c|}
\hline Author, Year & $\begin{array}{l}\text { 4. What was the } \\
\text { overall attrition and } \\
\text { attrition by group? }\end{array}$ & $\begin{array}{l}\text { 5. Did the study } \\
\text { have low } \\
\text { attrition? }\end{array}$ & $\begin{array}{l}\text { 6. Are the } \\
\text { proportion of } \\
\text { participants and } \\
\text { reasons for } \\
\text { missing data } \\
\text { similar across } \\
\text { interventions? }\end{array}$ & $\begin{array}{l}\text { 7. For BF } \\
\text { outcomes, was } \\
\text { ITT analysis } \\
\text { used? }\end{array}$ & $\begin{array}{l}\text { 8. Were appropriate } \\
\text { statistical methods } \\
\text { used to account for } \\
\text { missing data? }\end{array}$ & $\begin{array}{l}\text { Bias arising } \\
\text { from missing } \\
\text { outcome data? }\end{array}$ & Comments \\
\hline Coutinho, $2005^{5}$ & $14 / 175$ vs. $6 / 175=5 \%$ & Yes & Yes & Yes & Probably no & Low & $\begin{array}{l}\text { Analysis appears to be } \\
\text { focused on completers; } \\
\text { given low rate of missing } \\
\text { data, this publication was } \\
\text { not downgraded based } \\
\text { on lack of statistical } \\
\text { methods to account for } \\
\text { missing data. }\end{array}$ \\
\hline $\begin{array}{l}\text { Ekstrom, 2012;6 } \\
\text { Ekstrom, 2014 }\end{array}$ & $\begin{array}{l}\text { 3 days: } \\
\text { G1: } 17 \%(100-172 / 206) \\
\text { G2: } 9 \%(100-148 / 162) \\
\text { G3: } 7 \%(100-160 / 172) \\
3 \text { months: } \\
\text { G1: } 16 \%(100-145 / 172) \\
\text { G2: } 22 \%(100-126 / 148) \\
\text { G3: } 17 \%(100-132 / 160) \\
\text { 9 months: } \\
\text { G1: } 24 \%(100-131 / 172) \\
\text { G2: } 22 \%(100-116 / 148) \\
\text { G3: } 22 \%(100-125 / 160)\end{array}$ & Probably no & Probably yes & Probably yes & Probably no & Some concerns & $\begin{array}{l}\text { It seems as if consent } \\
\text { happened after } \\
\text { randomization at } 3 \text { days } \\
\text { postpartum; attrition } \\
\text { calculations are based } \\
\text { on the women who } \\
\text { responded to the first } \\
\text { questionnaire/signed } \\
\text { consent. Authors } \\
\text { reported that there were } \\
\text { no significant differences } \\
\text { between women who } \\
\text { answered all } 3 \\
\text { questionnaires and those } \\
\text { who only answered the } \\
\text { first. Authors do not } \\
\text { specify ITT analyses, but } \\
\text { given cluster RCT } \\
\text { design, it seems likely. }\end{array}$ \\
\hline Hayes, $2008^{8}$ & $\mathrm{NR}$ & Yes & No information & Yes & Yes & Low & NA \\
\hline Hoddinott, $2009^{9}$ & $\mathrm{NA}$ & Yes & NA & NA & NA & Low & NA \\
\hline
\end{tabular}




\begin{tabular}{|c|c|c|c|c|c|c|c|}
\hline Author, Year & $\begin{array}{l}\text { 4. What was the } \\
\text { overall attrition } \\
\text { and attrition by } \\
\text { group? }\end{array}$ & $\begin{array}{l}\text { 5. Did the study } \\
\text { have low attrition? }\end{array}$ & $\begin{array}{l}\text { 6. Are the } \\
\text { proportion of } \\
\text { participants and } \\
\text { reasons for } \\
\text { missing data } \\
\text { similar across } \\
\text { interventions? }\end{array}$ & $\begin{array}{l}\text { 7. For BF } \\
\text { outcomes, was } \\
\text { ITT analysis } \\
\text { used? }\end{array}$ & $\begin{array}{l}\text { 8. Were } \\
\text { appropriate } \\
\text { statistical } \\
\text { methods used to } \\
\text { account for } \\
\text { missing data? }\end{array}$ & $\begin{array}{l}\text { Bias arising } \\
\text { from missing } \\
\text { outcome } \\
\text { data? }\end{array}$ & Comments \\
\hline Kools, $2005^{10}$ & $\begin{array}{l}\text { T1 (1 month } \\
\text { postpartum) } \\
\text { G1: } 91 \%(371 / 408) \\
\text { G2: 88\% (330/373) } \\
\text { T2 (3 months } \\
\text { postpartum) } \\
\text { G1: } 90 \%(368 / 408) \\
\text { G2: } 88 \%(330 / 373) \\
\text { T3 }(6 \text { months } \\
\text { postpartum) } \\
\text { G1: } 89 \%(364 / 408) \\
\text { G2: } 86 \%(319 / 373) \\
\end{array}$ & Yes & No information & No & NA & Low & NA \\
\hline $\begin{array}{l}\text { Kramer, 2001;11 } \\
\text { Yang, 2014 }\end{array}$ & $\begin{array}{l}\text { Sites: } 6 \%(16 / 17 \\
\text { vs. 15/17 sites) } \\
\text { Women: } \\
\text { (8547//8865)- } \\
\text { (7895/8930)=8\% }\end{array}$ & Yes & No & $\begin{array}{l}\text { Probably yes } ; 11 \\
\text { No }^{12}\end{array}$ & No information & $\begin{array}{l}\text { Some } \\
\text { concerns }\end{array}$ & $\begin{array}{l}\text { One site (in the } \\
\text { intervention group) was } \\
\text { excluded owing to } \\
\text { falsification of outcome } \\
\text { data; this accounts for } \\
\text { most of attrition. } \\
\text { Analyses did not account } \\
\text { for missing data. }\end{array}$ \\
\hline Kronborg, $2007^{13}$ & $0 \%$ & Yes & Yes & Yes & NA & Low & $\begin{array}{l}\text { No attrition for clusters } \\
\text { (municipalities or health } \\
\text { visitors). For mothers, } \\
9 \% \text { who were potentially } \\
\text { eligible were not enrolled } \\
\text { (declined or did not } \\
\text { breast feed); of those } \\
\text { enrolled, only } 2 \text { women } \\
\text { had missing information } \\
\text { on breastfeeding status } \\
\text { and were not enrolled in } \\
\text { the final analysis. }\end{array}$ \\
\hline
\end{tabular}




\begin{tabular}{|c|c|c|c|c|c|c|c|}
\hline Author, Year & $\begin{array}{l}\text { 4. What was the } \\
\text { overall attrition and } \\
\text { attrition by group? }\end{array}$ & $\begin{array}{l}\text { 5. Did the study } \\
\text { have low } \\
\text { attrition? }\end{array}$ & $\begin{array}{l}\text { 6. Are the } \\
\text { proportion of } \\
\text { participants and } \\
\text { reasons for } \\
\text { missing data } \\
\text { similar across } \\
\text { interventions? }\end{array}$ & $\begin{array}{l}\text { 7. For BF } \\
\text { outcomes, was } \\
\text { ITT analysis } \\
\text { used? }\end{array}$ & $\begin{array}{l}\text { 8. Were appropriate } \\
\text { statistical methods } \\
\text { used to account for } \\
\text { missing data? }\end{array}$ & $\begin{array}{l}\text { Bias arising } \\
\text { from missing } \\
\text { outcome data? }\end{array}$ & Comments \\
\hline $\begin{array}{l}\text { MacLachlan, } \\
2016 ; ;^{14} \\
\text { Cramer, } 2017^{15}\end{array}$ & $\begin{array}{l}\text { G1: Comparison arm: } \\
30.0 \%([3,449- \\
2,414] / 3,449) \\
\text { G2: Home visit arm: } \\
31.6 \%([3,335- \\
2,281] / 23,335) \\
\text { G3: Home visit plus } \\
\text { drop-in center arm: } \\
\text { 18.9\% }([2,891- \\
2,344] / 2,891)\end{array}$ & No & No information & No & No & Some concerns & $\begin{array}{l}\text { Relatively high overall } \\
\text { attrition, and differential } \\
\text { attrition, no controls for } \\
\text { loss to followup. }\end{array}$ \\
\hline Morrow, $1999^{16}$ & $\begin{array}{l}\text { G1: } 2 / 44=4.5 \% \\
\text { G2: } 2 / 52=3.8 \% \\
\text { G3: } 1 / 34=2.9 \%\end{array}$ & Yes & Yes & Yes & Yes & Low & NA \\
\hline Nilsson, $2017^{17}$ & $\begin{array}{l}\text { For women who agreed } \\
\text { to participate, attrition } \\
\text { was } 31 \% \text { at } 1 \text { month } \\
\text { and } 44 \% \text { at } 6 \text { months. }\end{array}$ & No & No information & Yes & Yes & Some concerns & $\begin{array}{l}\text { Overall attrition is high, } \\
\text { however authors } \\
\text { conducted an ITT } \\
\text { analysis using imputation } \\
\text { of missing data. }\end{array}$ \\
\hline
\end{tabular}




\begin{tabular}{|c|c|c|c|c|c|c|c|}
\hline Author, Year & $\begin{array}{l}\text { 4. What was the } \\
\text { overall attrition and } \\
\text { attrition by group? }\end{array}$ & $\begin{array}{l}5 . \text { Did the study } \\
\text { have low } \\
\text { attrition? }\end{array}$ & $\begin{array}{l}\text { 6. Are the } \\
\text { proportion of } \\
\text { participants and } \\
\text { reasons for } \\
\text { missing data } \\
\text { similar across } \\
\text { interventions? }\end{array}$ & $\begin{array}{l}\text { 7. For BF } \\
\text { outcomes, was } \\
\text { ITT analysis } \\
\text { used? }\end{array}$ & $\begin{array}{l}\text { 8. Were appropriate } \\
\text { statistical methods } \\
\text { used to account for } \\
\text { missing data? }\end{array}$ & $\begin{array}{l}\text { Bias arising } \\
\text { from missing } \\
\text { outcome data? }\end{array}$ & Comments \\
\hline Reeder, $2014^{18}$ & $\begin{array}{l}\text { G1: } 25 \% \\
\text { G2: } 14 \%\end{array}$ & No & No information & No & No information & Some concerns & $\begin{array}{l}\text { Women from one local } \\
\text { WIC agency ( } n=179 \text { ) } \\
\text { were excluded mid-study } \\
\text { when a peer counselor } \\
\text { left and could not be } \\
\text { replaced. These women } \\
\text { are not accounted for in } \\
\text { attrition calculations. } \\
\text { Attrition as noted in the } \\
\text { flow diagram is low; } \\
\text { however, authors state } \\
\text { later that up to } 20 \% \text { of } \\
\text { participants were missing } \\
\text { data on BF exclusivity. } \\
\text { Unclear whether main } \\
\text { analyses account for } \\
\text { missing data. }\end{array}$ \\
\hline $\begin{array}{l}\text { Senarath, } \\
2007^{19}\end{array}$ & Unclear & Probably yes & No information & Yes & NA & Some concerns & $\begin{array}{l}\text { The number of mother- } \\
\text { newborn pairs enrolled } \\
\text { was based on sample } \\
\text { size calculations; how } \\
\text { sample was selected is } \\
\text { not clear. Authors note } \\
\text { that }<1 \% \text { of sample } \\
\text { refused to be } \\
\text { interviewed. }\end{array}$ \\
\hline
\end{tabular}




\begin{tabular}{|c|c|c|c|c|c|c|c|}
\hline Author, Year & $\begin{array}{l}\text { 4. What was the } \\
\text { overall attrition and } \\
\text { attrition by group? }\end{array}$ & $\begin{array}{l}\text { 5. Did the study } \\
\text { have low } \\
\text { attrition? }\end{array}$ & $\begin{array}{l}\text { 6. Are the } \\
\text { proportion of } \\
\text { participants and } \\
\text { reasons for } \\
\text { missing data } \\
\text { similar across } \\
\text { interventions? }\end{array}$ & $\begin{array}{l}\text { 7. For BF } \\
\text { outcomes, was } \\
\text { ITT analysis } \\
\text { used? }\end{array}$ & $\begin{array}{l}\text { 8. Were appropriate } \\
\text { statistical methods } \\
\text { used to account for } \\
\text { missing data? }\end{array}$ & $\begin{array}{l}\text { Bias arising } \\
\text { from missing } \\
\text { outcome data? }\end{array}$ & Comments \\
\hline Taddei, $2000^{20}$ & NR & Probably yes & No information & Yes & No & Some concerns & $\begin{array}{l}\text { Rates of attrition are not } \\
\text { described, however, } \\
\text { authors make a case fol } \\
\text { why attrition is likely to } \\
\text { be similar in } \\
\text { intervention/control } \\
\text { hospitals. }\end{array}$ \\
\hline Wan, $2011^{21}$ & $0 \%$ & Yes & $\mathrm{NA}$ & NA & $\mathrm{NA}$ & Low & NA \\
\hline Washio, $2017^{22}$ & $3 \%$ & Yes & $\mathrm{NA}$ & Yes & $\mathrm{NA}$ & Low & $\begin{array}{l}\text { No blinding of } \\
\text { participants or staff. }\end{array}$ \\
\hline
\end{tabular}

$\mathrm{BF}$ = breastfeeding; $\mathrm{G}$ = group; ITT = intent-to-treat; KQ = Key Question; NA = not applicable; NR = not reported; RCT = randomized controlled trial; $\mathrm{T}$ = treatment; WIC = Women, Infants, and Children. 
Table C-4. KQ 1 risk of bias assessment: Randomized controlled trials, part 3

\begin{tabular}{|c|c|c|c|c|c|c|c|}
\hline Author, Year & $\begin{array}{l}\text { 9. Were } \\
\text { participants } \\
\text { unaware of their } \\
\text { intervention } \\
\text { status? }\end{array}$ & $\begin{array}{l}\text { 10. Were the trial } \\
\text { personnel and } \\
\text { clinicians unaware } \\
\text { of the intervention } \\
\text { status of } \\
\text { participants? }\end{array}$ & $\begin{array}{l}\text { 11. Were } \\
\text { outcome } \\
\text { assessors } \\
\text { unaware of the } \\
\text { intervention } \\
\text { status of } \\
\text { participants? }\end{array}$ & $\begin{array}{l}\text { 12. Was } \\
\text { intervention } \\
\text { fidelity } \\
\text { adequate? }\end{array}$ & $\begin{array}{l}\text { 13. Were cross- } \\
\text { overs or } \\
\text { contamination } \\
\text { minimal such } \\
\text { that it would not } \\
\text { raise concern } \\
\text { for bias? }\end{array}$ & $\begin{array}{l}\text { Bias arising } \\
\text { from departures } \\
\text { from intended } \\
\text { interventions? }\end{array}$ & Comments \\
\hline $\begin{array}{l}\text { Coutinho, } \\
2005^{5}\end{array}$ & Probably no & Probably no & Probably yes & No information & Probably yes & Some concerns & $\begin{array}{l}\text { Participants and clinicians } \\
\text { unlikely to be blinded based on } \\
\text { intervention type (systems- } \\
\text { level, home visiting } \\
\text { intervention); per authors, } \\
\text { outcome assessors were } \\
\text { blinded from intervention status. } \\
\text { Fidelity to intervention unclear. }\end{array}$ \\
\hline $\begin{array}{l}\text { Ekstrom, } \\
2012 ; 6 \\
\text { Ekstrom, } \\
2014^{7}\end{array}$ & Yes & NA & Probably yes & No information & No information & Some concerns & $\begin{array}{l}\text { The midwife in the hospital } \\
\text { likely knew of the intervention } \\
\text { status of the hospital; the child } \\
\text { health nurse from the } \\
\text { community health center may } \\
\text { have been blinded to } \\
\text { intervention status. }\end{array}$ \\
\hline Hayes, $2008^{8}$ & No & No & No information & No information & No information & Uncertain & None \\
\hline $\begin{array}{l}\text { Hoddinott, } \\
2009^{9}\end{array}$ & No information & No & Yes & No information & No & Some concerns & $\begin{array}{l}\text { Both arms had some BF } \\
\text { certified hospitals, and results } \\
\text { were different in these } \\
\text { hospitals, unclear how the } \\
\text { results overall were influenced } \\
\text { by the potential competing } \\
\text { intervention. }\end{array}$ \\
\hline Kools, $2005^{10}$ & No & No & No information & Probably no & Probably no & Some concerns & $\begin{array}{l}\text { Potential for secular changes in } \\
\text { both regions, on participant and } \\
\text { organizational behavior. }\end{array}$ \\
\hline
\end{tabular}




\begin{tabular}{|c|c|c|c|c|c|c|c|}
\hline Author, Year & $\begin{array}{l}\text { 9. Were } \\
\text { participants } \\
\text { unaware of their } \\
\text { intervention } \\
\text { status? }\end{array}$ & $\begin{array}{l}\text { 10. Were the trial } \\
\text { personnel and } \\
\text { clinicians unaware } \\
\text { of the intervention } \\
\text { status of } \\
\text { participants? }\end{array}$ & $\begin{array}{l}\text { 11. Were } \\
\text { outcome } \\
\text { assessors } \\
\text { unaware of the } \\
\text { intervention } \\
\text { status of } \\
\text { participants? }\end{array}$ & $\begin{array}{l}\text { 12. Was } \\
\text { intervention } \\
\text { fidelity } \\
\text { adequate? }\end{array}$ & $\begin{array}{l}\text { 13. Were cross- } \\
\text { overs or } \\
\text { contamination } \\
\text { minimal such } \\
\text { that it would not } \\
\text { raise concern } \\
\text { for bias? }\end{array}$ & $\begin{array}{l}\text { Bias arising } \\
\text { from departures } \\
\text { from intended } \\
\text { interventions? }\end{array}$ & Comments \\
\hline $\begin{array}{l}\text { Kramer, } \\
2001 ; 11 \\
\text { Yang, 2014 }\end{array}$ & $\begin{array}{l}\text { Probably no; }{ }^{11} \\
\mathrm{No}^{12}\end{array}$ & No & $\begin{array}{l}\text { Probably no; }{ }^{11} \\
\text { No information }{ }^{12}\end{array}$ & $\begin{array}{l}\text { Probably yes; }{ }^{11} \\
\text { No information }^{12}\end{array}$ & No information & Some concerns & $\begin{array}{l}\text { Participants, health } \\
\text { professionals not blinded owing } \\
\text { to the nature of the intervention. } \\
\text { Given that one intervention site } \\
\text { falsified outcome data and was } \\
\text { excluded, outcome assessors } \\
\text { were unlikely to be blinded. } \\
\text { Insufficient information on } \\
\text { intervention fidelity. }\end{array}$ \\
\hline $\begin{array}{l}\text { Kronborg, } \\
2007^{13}\end{array}$ & No information & No & No & No information & Probably yes & Some concerns & $\begin{array}{l}\text { No blinding of participants, staff } \\
\text { or outcome assessors; home- } \\
\text { visiting nurses assessed } \\
\text { outcomes during mother } \\
\text { interviews. Fidelity and potential } \\
\text { contamination from other } \\
\text { programs/polices unclear. }\end{array}$ \\
\hline $\begin{array}{l}\text { MacLachlan, } \\
2016 ; 14 \\
\text { Cramer, } \\
2017^{15}\end{array}$ & No & No & Yes & no & $\begin{array}{l}\text { Uncertain } \\
\text { because no } \\
\text { information }\end{array}$ & Some concerns & $\begin{array}{l}\text { The drop-in aspect of the home } \\
\text { visit+drop-in intervention did not } \\
\text { succeed in terms of } \\
\text { implementation, and were } \\
\text { "poorly attended, with one } \\
\text { attendance per session on } \\
\text { average, and in two, peer } \\
\text { supporters to staff the drop-in } \\
\text { centers could not be recruited." } \\
\text { As a result the two active } \\
\text { intervention arms are likely not } \\
\text { different from one another. }\end{array}$ \\
\hline
\end{tabular}




\begin{tabular}{|c|c|c|c|c|c|c|c|}
\hline Author, Year & $\begin{array}{l}\text { 9. Were } \\
\text { participants } \\
\text { unaware of their } \\
\text { intervention } \\
\text { status? }\end{array}$ & $\begin{array}{l}\text { 10. Were the trial } \\
\text { personnel and } \\
\text { clinicians unaware } \\
\text { of the intervention } \\
\text { status of } \\
\text { participants? }\end{array}$ & $\begin{array}{l}11 . \text { Were } \\
\text { outcome } \\
\text { assessors } \\
\text { unaware of the } \\
\text { intervention } \\
\text { status of } \\
\text { participants? }\end{array}$ & $\begin{array}{l}\text { 12. Was } \\
\text { intervention } \\
\text { fidelity } \\
\text { adequate? }\end{array}$ & $\begin{array}{l}\text { 13. Were cross- } \\
\text { overs or } \\
\text { contamination } \\
\text { minimal such } \\
\text { that it would not } \\
\text { raise concern } \\
\text { for bias? }\end{array}$ & $\begin{array}{l}\text { Bias arising } \\
\text { from departures } \\
\text { from intended } \\
\text { interventions? }\end{array}$ & Comments \\
\hline $\begin{array}{l}\text { Morrow, } \\
199^{16}\end{array}$ & No & No & No & No information & Probably no & Low & NA \\
\hline $\begin{array}{l}\text { Nilsson, } \\
2017^{17}\end{array}$ & Probably yes & No & No information & No information & Probably yes & Some concerns & $\begin{array}{l}\text { Mothers were not blinded } \\
\text { (owing to the nature of the } \\
\text { intervention); however, they } \\
\text { were not informed whether thei } \\
\text { facility was randomized to the } \\
\text { intervention or usual care. } \\
\text { Health care providers at } \\
\text { reference facilities were not } \\
\text { informed about the content of } \\
\text { the intervention (providers at } \\
\text { intervention facilities } \\
\text { participated in development of } \\
\text { intervention). No assessment o } \\
\text { intervention fidelity. }\end{array}$ \\
\hline $\begin{array}{l}\text { Reeder, } \\
2014^{18}\end{array}$ & No & No & No information & Probably no & Probably yes & Some concerns & $\begin{array}{l}\text { Fidelity was a concern, leading } \\
\text { to no difference in contacts } \\
\text { among those in the high and } \\
\text { low frequency groups. These } \\
\text { groups were analyzed together. } \\
\text { No assessment was made of } \\
\text { the type/quality of support } \\
\text { provided to women and } \\
\text { whether this differed across } \\
\text { peer counselors. No description } \\
\text { of the type of BF } \\
\text { support/services received } \\
\text { outside of WIC. }\end{array}$ \\
\hline
\end{tabular}




\begin{tabular}{|c|c|c|c|c|c|c|c|}
\hline Author, Year & $\begin{array}{l}\text { 9. Were } \\
\text { participants } \\
\text { unaware of their } \\
\text { intervention } \\
\text { status? }\end{array}$ & $\begin{array}{l}\text { 10. Were the trial } \\
\text { personnel and } \\
\text { clinicians unaware } \\
\text { of the intervention } \\
\text { status of } \\
\text { participants? }\end{array}$ & $\begin{array}{l}\text { 11. Were } \\
\text { outcome } \\
\text { assessors } \\
\text { unaware of the } \\
\text { intervention } \\
\text { status of } \\
\text { participants? }\end{array}$ & $\begin{array}{l}\text { 12. Was } \\
\text { intervention } \\
\text { fidelity } \\
\text { adequate? }\end{array}$ & $\begin{array}{l}\text { 13. Were cross- } \\
\text { overs or } \\
\text { contamination } \\
\text { minimal such } \\
\text { that it would not } \\
\text { raise concern } \\
\text { for bias? }\end{array}$ & $\begin{array}{l}\text { Bias arising } \\
\text { from departures } \\
\text { from intended } \\
\text { interventions? }\end{array}$ & Comments \\
\hline $\begin{array}{l}\text { Senarath, } \\
2007^{19}\end{array}$ & NA & NA & No information & No information & Probably yes & Some concerns & $\begin{array}{l}\text { Owing to design, contamination } \\
\text { is not a major concern. Staff } \\
\text { would be aware of intervention } \\
\text { status (intervention focuses on } \\
\text { staff training); unclear if } \\
\text { outcome assessors were aware } \\
\text { of status; fidelity to training } \\
\text { intervention is not discussed } \\
\text { but may not be a major } \\
\text { concern. }\end{array}$ \\
\hline Taddei, $2000^{20}$ & NA & NA & No information & Probably yes & Probably yes & Some concerns & $\begin{array}{l}\text { Owing to cluster-randomization, } \\
\text { contamination and crossovers } \\
\text { are not a concern. Intervention } \\
\text { is focused on staff training, so } \\
\text { blinding of staff/participants is } \\
\text { not a major concern. Unclear } \\
\text { whether outcome assessors } \\
\text { were aware of intervention } \\
\text { status. }\end{array}$ \\
\hline Wan, $2011^{21}$ & No & No & No information & No information & Probably yes & Some concerns & $\begin{array}{l}\text { Fidelity was not described; } \\
\text { unclear if outcome assessors } \\
\text { were blinded. }\end{array}$ \\
\hline $\begin{array}{l}\text { Washio, } \\
2017^{22}\end{array}$ & No & No & No & No information & No information & Some concerns & $\begin{array}{l}\text { No blinding of participants or } \\
\text { staff; control group also } \\
\text { received some compensation } \\
\text { for participation ( } \$ 25 \text { per visit, } \\
\text { maximum of } \$ 100) \text { which may } \\
\text { have led to increased rates of } \\
\text { BF among controls. }\end{array}$ \\
\hline
\end{tabular}

BF = breastfeeding; KQ = Key Question; NA = not applicable; WIC = Women, Infants, and Children. 
Table C-5. KQ 1 risk of bias assessment: Randomized controlled trials, part 4

\begin{tabular}{|c|c|c|c|c|}
\hline Author, Year & $\begin{array}{l}\text { 14. Were BF outcomes } \\
\text { (e.g., duration, initiation, } \\
\text { exclusivity) adequately } \\
\text { described, prespecified, } \\
\text { valid, and reliable? }\end{array}$ & $\begin{array}{l}\text { 15. Were similar } \\
\text { techniques used } \\
\text { among groups to } \\
\text { ascertain BF } \\
\text { outcomes? }\end{array}$ & $\begin{array}{l}\text { Bias arising from } \\
\text { measurement of } \\
\text { breastfeeding } \\
\text { outcomes? }\end{array}$ & Comments \\
\hline Coutinho, $2005^{5}$ & Yes & Yes & Low & NA \\
\hline $\begin{array}{l}\text { Ekstrom, 2012; } \\
\text { Ekstrom, 20147 }\end{array}$ & Probably yes & Yes & Some concerns & $\begin{array}{l}\text { Authors provided definitions of outcomes, but it was not always clear } \\
\text { what the denominators were in their calculations for the different } \\
\text { outcomes. }\end{array}$ \\
\hline Hayes, $2008^{8}$ & No & Yes & Some concerns & BF measurement not clear \\
\hline Hoddinott, $2009^{9}$ & Yes & Yes & Low & NA \\
\hline Kools, $2005^{10}$ & Yes & Yes & Low & NA \\
\hline $\begin{array}{l}\text { Kramer, 2001;11 } \\
\text { Yang, 2014 }\end{array}$ & $\begin{array}{l}\text { Yes; }{ }^{11} \\
\mathrm{No}^{12}\end{array}$ & $\begin{array}{l}\text { Probably yes; }{ }^{11} \\
\text { Yes }^{12}\end{array}$ & $\begin{array}{l}\text { Low; }{ }^{11} \\
\text { Uncertain } \\
\text { because no } \\
\text { information }^{12} \\
\end{array}$ & $\begin{array}{l}\text { Yang uses discontinuation of exclusive BF (i.e., introducing any foods } \\
\text { other than breast milk) before } 3 \text { months and discontinuation of BF to } \\
\text { any degree (weaning) before } 12 \text { months. Kramer uses other outcomes. } \\
\text { Neither describes which outcomes were prespecified. }\end{array}$ \\
\hline Kronborg, $2007^{13}$ & Probably yes & Probably yes & Some concerns & $\begin{array}{l}\text { Outcome was exclusive breastfeeding, but little details are given in } \\
\text { terms of how this was defined and operationalized. }\end{array}$ \\
\hline $\begin{array}{l}\text { MacLachlan, } \\
2016:{ }^{14} \\
\text { Cramer, } 2017^{15}\end{array}$ & Yes & Yes & Low & The measure was "any breastfeeding." \\
\hline Morrow, $1999^{16}$ & Yes & Yes & Low & NA \\
\hline Nilsson, $2017^{17}$ & Probably yes & Yes & Low & NA \\
\hline Reeder, $2014^{18}$ & Probably yes & Probably yes & Some concerns & $\begin{array}{l}\text { Outcomes assessed during regular WIC appointments (which did not } \\
\text { differ by groups). Authors note lower rates of missing data among } \\
\text { intervention participants than controls and theorize that having peer } \\
\text { support may have led to women staying engaged in WIC for longer } \\
\text { periods of time and/or keeping appointments. }\end{array}$ \\
\hline Senarath, $2007^{19}$ & No information & Yes & Some concerns & $\begin{array}{l}\text { Authors note that "breastfeeding" was a practice that was measured, } \\
\text { but do not indicate the type of measure/question used. Results include } \\
\text { BF within the first half hour and exclusive BF (no note about how } \\
\text { exclusivity was defined). }\end{array}$ \\
\hline Taddei, $2000^{20}$ & Probably yes & Yes & Low & $\begin{array}{l}\text { A random sample of interviews on feeding practices were repeated by } \\
\text { a second field supervisor to check for reliability. }\end{array}$ \\
\hline Wan, 2011 & Yes & Probably yes & Low & NA \\
\hline Washio, $2017^{22}$ & Yes & Probably yes & Low & NA \\
\hline
\end{tabular}

$\mathrm{BF}$ = breastfeeding; $\mathrm{KQ}=$ Key Question; $\mathrm{NA}$ = not applicable; WIC = Women, Infants, and Children. 
Table C-6. KQ 1 risk of bias assessment: Randomized controlled trials, part 5

\begin{tabular}{|c|c|c|c|}
\hline Author, Year & $\begin{array}{l}\text { 16. Is the reported effect estimate unlikely } \\
\text { to be selected, on the basis of the results, } \\
\text { from multiple outcomes measurements } \\
\text { within the domain, multiple analyses, or } \\
\text { different subgroups?* }\end{array}$ & $\begin{array}{l}\text { Bias arising from } \\
\text { selection of reported } \\
\text { results? }\end{array}$ & Comments \\
\hline Coutinho, $2005^{5}$ & Yes & Low & NA \\
\hline Ekstrom, 2012; ${ }^{6}$ Ekstrom, $2014^{7}$ & Probably yes & Low & NA \\
\hline Hayes, $2008^{8}$ & No & Low & NA \\
\hline Hoddinott, $2009^{9}$ & No & Low & NA \\
\hline Kools, $2005^{10}$ & Yes & Low & NA \\
\hline $\begin{array}{l}\text { Kramer, 2001:11 } \\
\text { Yang, 2014 }\end{array}$ & $\begin{array}{l}\text { Yes: }^{11} \\
\text { Probably yes }^{12}\end{array}$ & $\begin{array}{l}\text { Low:; }^{11} \\
\text { Some concerns }\end{array}$ & $\begin{array}{l}\text { Unclear whether the timing of the reported outcome } \\
\text { was determined a priori or was the result of selective } \\
\text { reporting. }\end{array}$ \\
\hline Kronborg, $2007^{13}$ & Probably no & Low & NA \\
\hline $\begin{array}{l}\text { MacLachlan, 2016; } \\
\text { Cramer, 2017 }\end{array}$ & Low & Low & NA \\
\hline Morrow, 1999916 & No & Low & NA \\
\hline Nilsson, $2017^{17}$ & Yes & Low & NA \\
\hline Reeder, 2014 ${ }^{18}$ & Probably yes & Low & NA \\
\hline Senarath, $2007^{19}$ & Probably yes & Low & NA \\
\hline Taddei, $2000^{20}$ & Yes & Low & NA \\
\hline Wan, 2011 & Probably yes & Low & NA \\
\hline Washio, $2017^{22}$ & Yes & Low & NA \\
\hline
\end{tabular}

KQ = Key Question; NA = not applicable. 
Table C-7. KQ 1 risk of bias assessment: Nonrandomized trials and observational studies, part 1

\begin{tabular}{|c|c|c|c|c|c|c|c|c|}
\hline Author, Year & $\begin{array}{l}\text { Overall } \\
\text { Risk of } \\
\text { Bias } \\
\text { Rating }\end{array}$ & $\begin{array}{l}\text { Overall Rationale for } \\
\text { Risk of Bias Rating }\end{array}$ & $\begin{array}{l}\text { 1. Was } \\
\text { method of } \\
\text { selection } \\
\text { unrelated to } \\
\text { intervention/ } \\
\text { outcome? }\end{array}$ & $\begin{array}{l}\text { 2. Were post- } \\
\text { intervention } \\
\text { variables that } \\
\text { influenced } \\
\text { selection } \\
\text { related to } \\
\text { intervention/ } \\
\text { outcome? }\end{array}$ & $\begin{array}{l}\text { 3. Do start of } \\
\text { followup and } \\
\text { start of } \\
\text { intervention } \\
\text { coincide? }\end{array}$ & $\begin{array}{l}\text { 4. Were } \\
\text { adjustment } \\
\text { techniques } \\
\text { used to } \\
\text { correct for } \\
\text { presence of } \\
\text { selection } \\
\text { biases? }\end{array}$ & $\begin{array}{l}\text { Bias arising } \\
\text { from } \\
\text { selection? }\end{array}$ & Comments \\
\hline Abolyan, $2006^{23}$ & $\begin{array}{l}\text { Some } \\
\text { concerns }\end{array}$ & $\begin{array}{l}\text { Although selection bias } \\
\text { is a concern, baseline } \\
\text { characteristics of women } \\
\text { were very similar and } \\
\text { unlikely to influence } \\
\text { results. For data on BF } \\
\text { prevalence over time, } \\
\text { determining intervention } \\
\text { status is a potential risk } \\
\text { of bias. Some hospitals } \\
\text { may have started } \\
\text { changing maternity } \\
\text { practice before } \\
\text { becoming BF certified. }\end{array}$ & Probably yes & NA & NA & $\mathrm{NA}$ & $\begin{array}{l}\text { Some } \\
\text { concerns }\end{array}$ & $\begin{array}{l}\text { This comparison of BFHI } \\
\text { certification status and BF } \\
\text { initiation rates obtains } \\
\text { information in two ways: } \\
\text { survey of mothers giving } \\
\text { birth in hospitals, and use } \\
\text { of administrative data to } \\
\text { compare changes in BF } \\
\text { practices over time. } \\
\text { Selection is based only on } \\
\text { where women give birth } \\
\text { (and hence whether they } \\
\text { were exposed to the } \\
\text { intervention vs. control } \\
\text { hospital maternity } \\
\text { practice). Characteristics } \\
\text { of women are very } \\
\text { similar, other control } \\
\text { group women having a } \\
\text { slightly higher income. }\end{array}$ \\
\hline
\end{tabular}




\begin{tabular}{|c|c|c|c|c|c|c|c|c|}
\hline Author, Year & $\begin{array}{l}\text { Overall } \\
\text { Risk of } \\
\text { Bias } \\
\text { Rating }\end{array}$ & $\begin{array}{l}\text { Overall Rationale for } \\
\text { Risk of Bias Rating }\end{array}$ & $\begin{array}{l}\text { 1. Was } \\
\text { method of } \\
\text { selection } \\
\text { unrelated to } \\
\text { intervention/ } \\
\text { outcome? }\end{array}$ & $\begin{array}{l}\text { 2. Were post- } \\
\text { intervention } \\
\text { variables that } \\
\text { influenced } \\
\text { selection } \\
\text { related to } \\
\text { intervention/ } \\
\text { outcome? }\end{array}$ & $\begin{array}{l}\text { 3. Do start of } \\
\text { followup and } \\
\text { start of } \\
\text { intervention } \\
\text { coincide? }\end{array}$ & $\begin{array}{l}\text { 4. Were } \\
\text { adjustment } \\
\text { techniques } \\
\text { used to } \\
\text { correct for } \\
\text { presence of } \\
\text { selection } \\
\text { biases? }\end{array}$ & $\begin{array}{l}\text { Bias arising } \\
\text { from } \\
\text { selection? }\end{array}$ & Comments \\
\hline$\overline{\text { Alvarado, } 1999^{24}}$ & High & $\begin{array}{l}\text { Selection bias, } \\
\text { confounding and } \\
\text { measurement bias are a } \\
\text { concern and are not } \\
\text { adequately addressed in } \\
\text { analyses. }\end{array}$ & Probably yes & NA & Yes & NA & $\begin{array}{l}\text { Some } \\
\text { concerns }\end{array}$ & $\begin{array}{l}\text { Participants were } \\
\text { selected based on where } \\
\text { they lived; a neighboring } \\
\text { community was selected } \\
\text { as the control. Pregnant } \\
\text { women were invited into } \\
\text { the intervention group and } \\
\text { control women were } \\
\text { selected based on public } \\
\text { clinic records. There were } \\
\text { differences between } \\
\text { intervention and control } \\
\text { women at baseline. }\end{array}$ \\
\hline Bærug, 2016²5 & $\begin{array}{l}\text { Some } \\
\text { concerns }\end{array}$ & $\begin{array}{l}\text { Potential for bias arising } \\
\text { from high refusal rate } \\
\text { overall, although the } \\
\text { attrition rate does not } \\
\text { differ between arms. }\end{array}$ & Yes & NA & Yes & NA & Low & NA \\
\hline $\begin{array}{l}\text { Bartington, } \\
2006^{26}\end{array}$ & $\begin{array}{l}\text { Some } \\
\text { concerns }\end{array}$ & $\begin{array}{l}\text { Potential for selection } \\
\text { bias and confounding. }\end{array}$ & Probably yes & NA & NA & NA & Low & NA \\
\hline Bosnjak, 2004 ${ }^{27}$ & High & $\begin{array}{l}\text { standard definitions were } \\
\text { not used, the study relied } \\
\text { on recall in the pre- } \\
\text { intervention period and } \\
\text { in the first phase of the } \\
\text { intervention. }\end{array}$ & NA & NA & Yes & No & Low & NA \\
\hline
\end{tabular}




\begin{tabular}{|c|c|c|c|c|c|c|c|c|}
\hline Author, Year & $\begin{array}{l}\text { Overall } \\
\text { Risk of } \\
\text { Bias } \\
\text { Rating }\end{array}$ & $\begin{array}{l}\text { Overall Rationale for } \\
\text { Risk of Bias Rating }\end{array}$ & $\begin{array}{l}\text { 1. Was } \\
\text { method of } \\
\text { selection } \\
\text { unrelated to } \\
\text { intervention/ } \\
\text { outcome? }\end{array}$ & $\begin{array}{l}\text { 2. Were post- } \\
\text { intervention } \\
\text { variables that } \\
\text { influenced } \\
\text { selection } \\
\text { related to } \\
\text { intervention/ } \\
\text { outcome? }\end{array}$ & $\begin{array}{l}\text { 3. Do start of } \\
\text { followup and } \\
\text { start of } \\
\text { intervention } \\
\text { coincide? }\end{array}$ & $\begin{array}{l}\text { 4. Were } \\
\text { adjustment } \\
\text { techniques } \\
\text { used to } \\
\text { correct for } \\
\text { presence of } \\
\text { selection } \\
\text { biases? }\end{array}$ & $\begin{array}{l}\text { Bias arising } \\
\text { from } \\
\text { selection? }\end{array}$ & Comments \\
\hline $\begin{array}{l}\text { Broadfoot, } \\
2005^{28}\end{array}$ & $\begin{array}{l}\text { Some } \\
\text { concerns }\end{array}$ & $\begin{array}{l}\text { Potential for selection } \\
\text { bias and confounding; up } \\
\text { to } 17 \% \text { (or more) records } \\
\text { had missing data on } \\
\text { important confounders or } \\
\text { outcomes. }\end{array}$ & Probably yes & NA & NA & NA & Low & NA \\
\hline $\begin{array}{l}\text { Edmunds, } \\
2017^{29}\end{array}$ & High & $\begin{array}{l}\text { High risk of selection } \\
\text { bias and bias due to } \\
\text { unmeasured } \\
\text { confounding. }\end{array}$ & Probably no & $\begin{array}{l}\text { Related to } \\
\text { outcome }\end{array}$ & NA & NA & $\begin{array}{l}\text { Some } \\
\text { concerns }\end{array}$ & $\begin{array}{l}\text { Of } 96 \text { NYS WIC local } \\
\text { agencies invited, } 31 \\
\text { responded and } 12 \text { were } \\
\text { chosen to participate. } \\
\text { Only women who planned } \\
\text { to BF or were considering } \\
\text { BF were eligible. }\end{array}$ \\
\hline $\begin{array}{l}\text { Feldman-Winter, } \\
2010^{30}\end{array}$ & $\begin{array}{l}\text { Some } \\
\text { concerns }\end{array}$ & $\begin{array}{l}\text { Potential for } \\
\text { contamination, but the } \\
\text { effect would be to reduce } \\
\text { the difference between } \\
\text { arms. }\end{array}$ & Probably yes & NA & Yes & NA & Low & NA \\
\hline Gau, 2004 & High & $\begin{array}{l}\text { Differential attrition, } \\
\text { unclear whether there } \\
\text { was a potential for } \\
\text { confounding in the } \\
\text { design, outcomes poorly } \\
\text { defined (BF duration did } \\
\text { not account for } \\
\text { exclusivity). }\end{array}$ & Yes & NA & Yes & NA & Low & NA \\
\hline
\end{tabular}




\begin{tabular}{|c|c|c|c|c|c|c|c|c|}
\hline Author, Year & $\begin{array}{l}\text { Overall } \\
\text { Risk of } \\
\text { Bias } \\
\text { Rating }\end{array}$ & $\begin{array}{l}\text { Overall Rationale for } \\
\text { Risk of Bias Rating }\end{array}$ & $\begin{array}{l}\text { 1. Was } \\
\text { method of } \\
\text { selection } \\
\text { unrelated to } \\
\text { intervention/ } \\
\text { outcome? }\end{array}$ & $\begin{array}{l}\text { 2. Were post- } \\
\text { intervention } \\
\text { variables that } \\
\text { influenced } \\
\text { selection } \\
\text { related to } \\
\text { intervention/ } \\
\text { outcome? }\end{array}$ & $\begin{array}{l}\text { 3. Do start of } \\
\text { followup and } \\
\text { start of } \\
\text { intervention } \\
\text { coincide? }\end{array}$ & $\begin{array}{l}\text { 4. Were } \\
\text { adjustment } \\
\text { techniques } \\
\text { used to } \\
\text { correct for } \\
\text { presence of } \\
\text { selection } \\
\text { biases? }\end{array}$ & $\begin{array}{l}\text { Bias arising } \\
\text { from } \\
\text { selection? }\end{array}$ & Comments \\
\hline Hannula, $2014^{32}$ & High & $\begin{array}{l}\text { Potential for selection } \\
\text { bias and confounding, } \\
\text { high and differential } \\
\text { attrition. }\end{array}$ & Probably no & $\begin{array}{l}\text { Related to } \\
\text { intervention }\end{array}$ & No & No & High & $\begin{array}{l}\text { The sample is limited to } \\
\text { the women who agreed to } \\
\text { participate, so high } \\
\text { attrition between } \\
\text { experiencing the } \\
\text { intervention and } \\
\text { participating in the study } \\
\text { could potentially be } \\
\text { associated with } \\
\text { postintervention variables } \\
\text { and result in a selection } \\
\text { bias. Start of the } \\
\text { intervention precedes } \\
\text { start of followup. }\end{array}$ \\
\hline Hawkins, $2014^{33}$ & $\begin{array}{l}\text { Some } \\
\text { concerns }\end{array}$ & $\begin{array}{l}\text { Poor compliance within } \\
\text { BFHI accredited } \\
\text { hospitals coupled with } \\
\text { adoption of some BF } \\
\text { initiatives in } \\
\text { nonaccredited facilities } \\
\text { make the distinction } \\
\text { between study arms } \\
\text { weak; absence of effects } \\
\text { could potentially be } \\
\text { attributed to poor } \\
\text { intervention definition } \\
\text { and contamination/co- } \\
\text { interventions }\end{array}$ & Yes & NA & Yes & NA & Low & NA \\
\hline
\end{tabular}




\begin{tabular}{|c|c|c|c|c|c|c|c|c|}
\hline Author, Year & $\begin{array}{l}\text { Overall } \\
\text { Risk of } \\
\text { Bias } \\
\text { Rating }\end{array}$ & $\begin{array}{l}\text { Overall Rationale for } \\
\text { Risk of Bias Rating }\end{array}$ & $\begin{array}{l}\text { 1. Was } \\
\text { method of } \\
\text { selection } \\
\text { unrelated to } \\
\text { intervention/ } \\
\text { outcome? }\end{array}$ & $\begin{array}{l}\text { 2. Were post- } \\
\text { intervention } \\
\text { variables that } \\
\text { influenced } \\
\text { selection } \\
\text { related to } \\
\text { intervention/ } \\
\text { outcome? }\end{array}$ & $\begin{array}{l}\text { 3. Do start of } \\
\text { followup and } \\
\text { start of } \\
\text { intervention } \\
\text { coincide? }\end{array}$ & $\begin{array}{l}\text { 4. Were } \\
\text { adjustment } \\
\text { techniques } \\
\text { used to } \\
\text { correct for } \\
\text { presence of } \\
\text { selection } \\
\text { biases? }\end{array}$ & $\begin{array}{l}\text { Bias arising } \\
\text { from } \\
\text { selection? }\end{array}$ & Comments \\
\hline Hawkins, $2014^{34}$ & $\begin{array}{l}\text { Some } \\
\text { concerns }\end{array}$ & $\begin{array}{l}\text { Poor compliance within } \\
\text { BFHI accredited } \\
\text { hospitals coupled with } \\
\text { adoption of some BF } \\
\text { initiatives in } \\
\text { nonaccredited facilities } \\
\text { make the distinction } \\
\text { between study arms } \\
\text { weak; absence of effects } \\
\text { could potentially be } \\
\text { attributed to poor } \\
\text { intervention definition } \\
\text { and contamination/co- } \\
\text { interventions. }\end{array}$ & Yes & NA & Yes & NA & Low & NA \\
\hline Joyce, $2015^{35}$ & High & $\begin{array}{l}\text { Potential for selection } \\
\text { bias and measurement } \\
\text { bias. Inadequate } \\
\text { adjustment for } \\
\text { confounding variables. } \\
\text { Data sources used were } \\
\text { not designed to measure } \\
\text { BF rates and extent of } \\
\text { missing data is unclear. }\end{array}$ & Probably no & Yes & $\mathrm{NA}$ & No & $\begin{array}{l}\text { Some } \\
\text { concerns }\end{array}$ & $\begin{array}{l}\text { Authors use three } \\
\text { separate population } \\
\text { databases to contrast } \\
\text { trends in BF pre- and } \\
\text { post-WIC policy changes; } \\
\text { two have a non-WIC } \\
\text { control group. Adjustment } \\
\text { for variables is limited. } \\
\text { Some women may have } \\
\text { applied for WIC post- } \\
\text { intervention due to } \\
\text { nonformula benefits. }\end{array}$ \\
\hline
\end{tabular}




\begin{tabular}{|c|c|c|c|c|c|c|c|c|}
\hline Author, Year & $\begin{array}{l}\text { Overall } \\
\text { Risk of } \\
\text { Bias } \\
\text { Rating }\end{array}$ & $\begin{array}{l}\text { Overall Rationale for } \\
\text { Risk of Bias Rating }\end{array}$ & $\begin{array}{l}\text { 1. Was } \\
\text { method of } \\
\text { selection } \\
\text { unrelated to } \\
\text { intervention/ } \\
\text { outcome? }\end{array}$ & $\begin{array}{l}\text { 2. Were post- } \\
\text { intervention } \\
\text { variables that } \\
\text { influenced } \\
\text { selection } \\
\text { related to } \\
\text { intervention/ } \\
\text { outcome? }\end{array}$ & $\begin{array}{l}\text { 3. Do start of } \\
\text { followup and } \\
\text { start of } \\
\text { intervention } \\
\text { coincide? }\end{array}$ & $\begin{array}{l}\text { 4. Were } \\
\text { adjustment } \\
\text { techniques } \\
\text { used to } \\
\text { correct for } \\
\text { presence of } \\
\text { selection } \\
\text { biases? }\end{array}$ & $\begin{array}{l}\text { Bias arising } \\
\text { from } \\
\text { selection? }\end{array}$ & Comments \\
\hline Lovera, $2010^{36}$ & $\begin{array}{l}\text { Some } \\
\text { concerns }\end{array}$ & $\begin{array}{l}\text { See individual domains. } \\
\text { Selection into the study } \\
\text { groups is not clear. The } \\
\text { response rate on the part } \\
\text { of the fathers was poor, } \\
\text { but outcome data were } \\
\text { probably from the } \\
\text { mother. There was no } \\
\text { description of } \\
\text { intervention departures, } \\
\text { but given the response } \\
\text { rate from the fathers, } \\
\text { there is a concern about } \\
\text { intervention fidelity and } \\
\text { participation (which } \\
\text { could bias the result } \\
\text { toward the null). }\end{array}$ & Probably yes & NA & Yes & NA & Low & NA \\
\hline Madden, $2003^{37}$ & $\begin{array}{l}\text { Some } \\
\text { concerns }\end{array}$ & $\begin{array}{l}\text { Pre-post data, unclear if } \\
\text { other secular changes or } \\
\text { cointerventions were } \\
\text { occurring at the same } \\
\text { time, unclear how study } \\
\text { was implemented, study } \\
\text { excluded complex } \\
\text { deliveries, study used } \\
\text { but did not provide } \\
\text { information on validation } \\
\text { of automation to identify } \\
\text { outcome }\end{array}$ & Probably yes & NA & NA & NA & Low & $\begin{array}{l}\text { Pre-post study looking at } \\
\text { a policy intervention } \\
\text { related to length of } \\
\text { hospital stay. }\end{array}$ \\
\hline
\end{tabular}




\begin{tabular}{|c|c|c|c|c|c|c|c|c|}
\hline Author, Year & $\begin{array}{l}\text { Overall } \\
\text { Risk of } \\
\text { Bias } \\
\text { Rating }\end{array}$ & $\begin{array}{l}\text { Overall Rationale for } \\
\text { Risk of Bias Rating }\end{array}$ & $\begin{array}{l}\text { 1. Was } \\
\text { method of } \\
\text { selection } \\
\text { unrelated to } \\
\text { intervention/ } \\
\text { outcome? }\end{array}$ & $\begin{array}{l}\text { 2. Were post- } \\
\text { intervention } \\
\text { variables that } \\
\text { influenced } \\
\text { selection } \\
\text { related to } \\
\text { intervention/ } \\
\text { outcome? }\end{array}$ & $\begin{array}{l}\text { 3. Do start of } \\
\text { followup and } \\
\text { start of } \\
\text { intervention } \\
\text { coincide? }\end{array}$ & $\begin{array}{l}\text { 4. Were } \\
\text { adjustment } \\
\text { techniques } \\
\text { used to } \\
\text { correct for } \\
\text { presence of } \\
\text { selection } \\
\text { biases? }\end{array}$ & $\begin{array}{l}\text { Bias arising } \\
\text { from } \\
\text { selection? }\end{array}$ & Comments \\
\hline $\begin{array}{l}\text { Macaluso, } \\
2013 ;{ }^{38} \\
\text { Cattaneo, } \\
2016^{39}\end{array}$ & $\begin{array}{l}\text { Some } \\
\text { concerns }\end{array}$ & $\begin{array}{l}\text { Potential for selection } \\
\text { bias (at cluster level) and } \\
\text { confounding. }\end{array}$ & Probably yes & NA & Probably no & Probably yes & $\begin{array}{l}\text { Some } \\
\text { concerns }\end{array}$ & $\begin{array}{l}\text { Intervention steps were } \\
\text { implemented in a phased } \\
\text { way; the early intervention } \\
\text { group may have been } \\
\text { different from the late } \\
\text { intervention group, } \\
\text { leading to risk of selection } \\
\text { bias (the early } \\
\text { intervention group had } \\
\text { already implemented } \\
\text { some intervention steps } \\
\text { before the start of the } \\
\text { study). }\end{array}$ \\
\hline Martens, $2000^{40}$ & High & $\begin{array}{l}\text { Baseline differences not } \\
\text { adjusted in models }\end{array}$ & Probably yes & $\mathrm{NA}$ & Yes & $\mathrm{NA}$ & Low & NA \\
\hline $\begin{array}{l}\text { Mydlilova, } \\
2009^{41}\end{array}$ & $\begin{array}{l}\text { Some } \\
\text { concerns }\end{array}$ & $\begin{array}{l}\text { The authors did attempt } \\
\text { to evaluate the presence } \\
\text { of perinatal centers and } \\
\text { neonatal intermediate } \\
\text { care units. The } \\
\text { percentage of missing } \\
\text { newborn reports is very } \\
\text { low but it is unclear how } \\
\text { unbiased these reports } \\
\text { are between non BFHI } \\
\text { and BFHI hospitals. } \\
\text { Does having BFHI } \\
\text { certification result in } \\
\text { inflated reported BF } \\
\text { rates. }\end{array}$ & Yes & NA & Yes & NA & Low & $\mathrm{NA}$ \\
\hline
\end{tabular}




\begin{tabular}{|c|c|c|c|c|c|c|c|c|}
\hline Author, Year & $\begin{array}{l}\text { Overall } \\
\text { Risk of } \\
\text { Bias } \\
\text { Rating }\end{array}$ & $\begin{array}{l}\text { Overall Rationale for } \\
\text { Risk of Bias Rating }\end{array}$ & $\begin{array}{l}\text { 1. Was } \\
\text { method of } \\
\text { selection } \\
\text { unrelated to } \\
\text { intervention/ } \\
\text { outcome? }\end{array}$ & $\begin{array}{l}\text { 2. Were post- } \\
\text { intervention } \\
\text { variables that } \\
\text { influenced } \\
\text { selection } \\
\text { related to } \\
\text { intervention/ } \\
\text { outcome? } \\
\end{array}$ & $\begin{array}{l}\text { 3. Do start of } \\
\text { followup and } \\
\text { start of } \\
\text { intervention } \\
\text { coincide? }\end{array}$ & $\begin{array}{l}\text { 4. Were } \\
\text { adjustment } \\
\text { techniques } \\
\text { used to } \\
\text { correct for } \\
\text { presence of } \\
\text { selection } \\
\text { biases? } \\
\end{array}$ & $\begin{array}{l}\text { Bias arising } \\
\text { from } \\
\text { selection? }\end{array}$ & Comments \\
\hline Rempel, $2010^{42}$ & High & $\begin{array}{l}\text { See individual domains. } \\
\text { Selection into the study } \\
\text { groups is not clear, nor is } \\
\text { the pattern of missing } \\
\text { data due to attendees } \\
\text { not completing some or } \\
\text { all of the study surveys } \\
\text { at different points in time. }\end{array}$ & Probably yes & NA & Yes & NA & Low & NA \\
\hline Schafer, $1998^{43}$ & High & $\begin{array}{l}\text { High and differential } \\
\text { attrition between arms, } \\
\text { weak measure of } \\
\text { outcomes. }\end{array}$ & Yes & NA & Yes & NA & Low & NA \\
\hline Shaw, $1999^{44}$ & $\begin{array}{l}\text { Some } \\
\text { concerns }\end{array}$ & $\begin{array}{l}\text { Baseline differences, not } \\
\text { fully adjusted in models }\end{array}$ & Probably no & $\begin{array}{l}\text { Related to } \\
\text { outcome }\end{array}$ & Yes & No & $\begin{array}{l}\text { Some } \\
\text { concerns }\end{array}$ & $\begin{array}{l}\text { Women needed to have } \\
\text { been seen at least for } \\
\text { prenatal care to be } \\
\text { eligible for the study, this } \\
\text { may have resulted in a } \\
\text { cohort of women who are } \\
\text { different from those who } \\
\text { did not get seen before } \\
\text { pregnancy. }\end{array}$ \\
\hline Tarrant, $2011^{45}$ & $\begin{array}{l}\text { Some } \\
\text { concerns }\end{array}$ & $\begin{array}{l}\text { Despite the } \\
\text { observational nature of } \\
\text { this study and the lack of } \\
\text { information on how BFHI } \\
\text { is implemented in Hong } \\
\text { Kong hospitals, this was } \\
\text { a fairly well done study } \\
\text { with minimal missing } \\
\text { data and an appropriate } \\
\text { analytic approach. }\end{array}$ & Yes & NA & Yes & NA & Low & NA \\
\hline
\end{tabular}




\begin{tabular}{|c|c|c|c|c|c|c|c|c|}
\hline Author, Year & $\begin{array}{l}\text { Overall } \\
\text { Risk of } \\
\text { Bias } \\
\text { Rating }\end{array}$ & $\begin{array}{l}\text { Overall Rationale for } \\
\text { Risk of Bias Rating }\end{array}$ & $\begin{array}{l}\text { 1. Was } \\
\text { method of } \\
\text { selection } \\
\text { unrelated to } \\
\text { intervention/ } \\
\text { outcome? }\end{array}$ & $\begin{array}{l}\text { 2. Were post- } \\
\text { intervention } \\
\text { variables that } \\
\text { influenced } \\
\text { selection } \\
\text { related to } \\
\text { intervention/ } \\
\text { outcome? }\end{array}$ & $\begin{array}{l}\text { 3. Do start of } \\
\text { followup and } \\
\text { start of } \\
\text { intervention } \\
\text { coincide? }\end{array}$ & $\begin{array}{l}\text { 4. Were } \\
\text { adjustment } \\
\text { techniques } \\
\text { used to } \\
\text { correct for } \\
\text { presence of } \\
\text { selection } \\
\text { biases? }\end{array}$ & $\begin{array}{l}\text { Bias arising } \\
\text { from } \\
\text { selection? }\end{array}$ & Comments \\
\hline $\begin{array}{l}\text { Venancio, } \\
2011^{46}\end{array}$ & $\begin{array}{l}\text { Some } \\
\text { concerns }\end{array}$ & $\begin{array}{l}\text { Potential for selection } \\
\text { bias and measurement } \\
\text { bias. }\end{array}$ & Probably no & $\begin{array}{l}\text { Related to } \\
\text { outcome and } \\
\text { intervention }\end{array}$ & Yes & NA & $\begin{array}{l}\text { Some } \\
\text { concerns }\end{array}$ & $\begin{array}{l}\text { There is no discussion of } \\
\text { whether women who had } \\
\text { a strong intention to } \\
\text { breastfeed might choose } \\
\text { to give birth at a BFHI } \\
\text { certified hospital, nor was } \\
\text { there any discussion of } \\
\text { whether regions with } \\
\text { higher BF rates might be } \\
\text { more inclined to pursue } \\
\text { BFHI certification. }\end{array}$ \\
\hline Wagner, $2002^{47}$ & $\begin{array}{l}\text { Some } \\
\text { concerns }\end{array}$ & $\begin{array}{l}\text { No description of } \\
\text { maternal characteristics } \\
\text { or adjustment for } \\
\text { potential confounders in } \\
\text { analyses. Extent of } \\
\text { missing data and } \\
\text { adherence are not } \\
\text { reported. }\end{array}$ & Yes & NA & NA & $\mathrm{NA}$ & Low & NA \\
\hline Weng, $2003^{48}$ & High & $\begin{array}{l}\text { High risk of selection } \\
\text { bias, no description of } \\
\text { participant } \\
\text { characteristics or } \\
\text { adjustment for } \\
\text { confounding factors, and } \\
\text { potential for } \\
\text { measurement bias (no } \\
\text { description of how BF } \\
\text { outcomes were defined } \\
\text { or assessed). }\end{array}$ & Probably no & $\begin{array}{l}\text { Related to } \\
\text { outcome and } \\
\text { intervention }\end{array}$ & Yes & NA & High & $\begin{array}{l}\text { High risk of selection bias } \\
\text { in relationship to the } \\
\text { hospitals sampled; } \\
\text { unclear whether the } \\
\text { sample that applied for } \\
\text { BFHI is different than } \\
\text { those that did not apply. } \\
\text { There are clear } \\
\text { differences in hospital } \\
\text { practices by region which } \\
\text { were not accounted for in } \\
\text { the analyses. }\end{array}$ \\
\hline
\end{tabular}

BF = breastfeeding; BFHI = Baby Friendly Hospital Initiative; KQ = Key Question; NA = not applicable; NYS = New York State; RCT = randomized controlled trial; WIC = Women, Infants, and Children. 
Table C-8. KQ 1 risk of bias assessment: Nonrandomized trials and observational studies, part 2

\begin{tabular}{|c|c|c|c|c|c|c|c|c|}
\hline Author, Year & $\begin{array}{l}5 . \text { Is } \\
\text { confounding } \\
\text { of the } \\
\text { intervention } \\
\text { effect } \\
\text { unlikely? }\end{array}$ & $\begin{array}{l}\text { 5a. Did the } \\
\text { authors use an } \\
\text { appropriate } \\
\text { analysis to } \\
\text { adjust for } \\
\text { confounders? }\end{array}$ & $\begin{array}{l}\text { 5b. Were } \\
\text { confounding } \\
\text { domains that } \\
\text { were } \\
\text { controlled for } \\
\text { measured } \\
\text { validly and } \\
\text { reliably? }\end{array}$ & $\begin{array}{l}5 c . \text { Did the } \\
\text { authors avoid } \\
\text { adjusting for } \\
\text { post- } \\
\text { intervention } \\
\text { variables? }\end{array}$ & $\begin{array}{l}\text { 5d. Were } \\
\text { participants } \\
\text { analyzed } \\
\text { according to } \\
\text { their initial } \\
\text { intervention } \\
\text { group } \\
\text { throughout } \\
\text { followup? }\end{array}$ & $\begin{array}{l}\text { 5e. Were } \\
\text { intervention } \\
\text { discontinuations } \\
\text { or switches } \\
\text { unlikely to be } \\
\text { related to factors } \\
\text { prognostic for } \\
\text { the outcome? }\end{array}$ & $\begin{array}{l}\text { Bias arising } \\
\text { from } \\
\text { confounding? }\end{array}$ & Comments \\
\hline Abolyan, $2006^{23}$ & Probably no & Probably no & NA & Yes & NA & Yes & Some concerns & $\begin{array}{l}\text { Women giving birth in } \\
\text { intervention and control } \\
\text { hospitals (in 2004) who } \\
\text { were surveyed had very } \\
\text { similar baseline } \\
\text { characteristics; for } \\
\text { comparisons of BF rates } \\
\text { over time, changes are } \\
\text { given for both intervention } \\
\text { and control (non-BFHI) } \\
\text { hospitals which takes into } \\
\text { account contamination } \\
\text { from other policy (or } \\
\text { cultural) changes in BF } \\
\text { initiation rates. }\end{array}$ \\
\hline $\begin{array}{l}\text { Alvarado, } \\
1999^{24}\end{array}$ & No & No & NA & Yes & Yes & Yes & High & $\begin{array}{l}\text { Baseline characteristics of } \\
\text { women differed in } \\
\text { important ways (women in } \\
\text { control group were less } \\
\text { likely to be primiparous) } \\
\text { and do not appear to have } \\
\text { been addressed in } \\
\text { analyses. }\end{array}$ \\
\hline Bærug, $2016^{25}$ & Yes & NA & NA & NA & NA & NA & Low & $\begin{array}{l}\text { Clusters assigned by } \\
\text { investigators. }\end{array}$ \\
\hline
\end{tabular}




\begin{tabular}{|c|c|c|c|c|c|c|c|c|}
\hline Author, Year & $\begin{array}{l}\text { 5. Is } \\
\text { confounding } \\
\text { of the } \\
\text { intervention } \\
\text { effect } \\
\text { unlikely? }\end{array}$ & $\begin{array}{l}5 a \text {. Did the } \\
\text { authors use an } \\
\text { appropriate } \\
\text { analysis to } \\
\text { adjust for } \\
\text { confounders? }\end{array}$ & $\begin{array}{l}\text { 5b. Were } \\
\text { confounding } \\
\text { domains that } \\
\text { were } \\
\text { controlled for } \\
\text { measured } \\
\text { validly and } \\
\text { reliably? }\end{array}$ & $\begin{array}{l}5 c . \text { Did the } \\
\text { authors avoid } \\
\text { adjusting for } \\
\text { post- } \\
\text { intervention } \\
\text { variables? }\end{array}$ & $\begin{array}{l}\text { 5d. Were } \\
\text { participants } \\
\text { analyzed } \\
\text { according to } \\
\text { their initial } \\
\text { intervention } \\
\text { group } \\
\text { throughout } \\
\text { followup? }\end{array}$ & $\begin{array}{l}\text { 5e. Were } \\
\text { intervention } \\
\text { discontinuations } \\
\text { or switches } \\
\text { unlikely to be } \\
\text { related to factors } \\
\text { prognostic for } \\
\text { the outcome? }\end{array}$ & $\begin{array}{l}\text { Bias arising } \\
\text { from } \\
\text { confounding? }\end{array}$ & Comments \\
\hline $\begin{array}{l}\text { Bartington, } \\
2006^{26}\end{array}$ & Probably no & Probably no & Probably yes & Yes & Yes & Yes & Some concerns & $\begin{array}{l}\text { Individual level } \\
\text { confounders were } \\
\text { addressed. Women living } \\
\text { in communities where } \\
\text { BFHI was adopted may } \\
\text { be different in other } \\
\text { factors from women in } \\
\text { communities were BFHI } \\
\text { was not implemented yet. }\end{array}$ \\
\hline Bosnjak, 2004²7 & $\mathrm{NA}$ & NA & NA & NA & NA & NA & NA & $\begin{array}{l}\text { Single arm study, so } \\
\text { confounding not } \\
\text { applicable. }\end{array}$ \\
\hline $\begin{array}{l}\text { Broadfoot, } \\
2005^{28}\end{array}$ & Probably no & Probably no & Probably yes & Yes & Yes & Yes & Some concerns & $\begin{array}{l}\text { Potential for confounding; } \\
\text { those adjusted for were } \\
\text { limited to Carstaris } \\
\text { deprivation category, } \\
\text { mother's age, hospital } \\
\text { size, and year of birth. }\end{array}$ \\
\hline $\begin{array}{l}\text { Edmunds, } \\
2017^{29}\end{array}$ & No & No & Probably no & Yes & Yes & No & High & $\begin{array}{l}\text { Authors compare } \\
\text { outcomes among women } \\
\text { who agreed to participate } \\
\text { and those who declined to } \\
\text { participate in the study; it } \\
\text { is likely that those who did } \\
\text { not participate may have } \\
\text { been less likely to } \\
\text { continue BF. Few factors } \\
\text { were included in } \\
\text { regression analyses aside } \\
\text { from age, race, parity and } \\
\text { number of nutrition } \\
\text { education sessions. }\end{array}$ \\
\hline
\end{tabular}




\begin{tabular}{|c|c|c|c|c|c|c|c|c|}
\hline Author, Year & $\begin{array}{l}5 . \text { Is } \\
\text { confounding } \\
\text { of the } \\
\text { intervention } \\
\text { effect } \\
\text { unlikely? }\end{array}$ & $\begin{array}{l}5 a \text {. Did the } \\
\text { authors use an } \\
\text { appropriate } \\
\text { analysis to } \\
\text { adjust for } \\
\text { confounders? }\end{array}$ & $\begin{array}{l}\text { 5b. Were } \\
\text { confounding } \\
\text { domains that } \\
\text { were } \\
\text { controlled for } \\
\text { measured } \\
\text { validly and } \\
\text { reliably? }\end{array}$ & $\begin{array}{l}5 c . \text { Did the } \\
\text { authors avoid } \\
\text { adjusting for } \\
\text { post- } \\
\text { intervention } \\
\text { variables? }\end{array}$ & $\begin{array}{l}\text { 5d. Were } \\
\text { participants } \\
\text { analyzed } \\
\text { according to } \\
\text { their initial } \\
\text { intervention } \\
\text { group } \\
\text { throughout } \\
\text { followup? }\end{array}$ & $\begin{array}{l}\text { 5e. Were } \\
\text { intervention } \\
\text { discontinuations } \\
\text { or switches } \\
\text { unlikely to be } \\
\text { related to factors } \\
\text { prognostic for } \\
\text { the outcome? }\end{array}$ & $\begin{array}{l}\text { Bias arising } \\
\text { from } \\
\text { confounding? }\end{array}$ & Comments \\
\hline $\begin{array}{l}\text { Feldman- } \\
\text { Winter, } 2010^{30}\end{array}$ & Probably yes & NA & NA & NA & NA & NA & Low & NA \\
\hline Gau, 2004 & No information & NA & NA & NA & NA & NA & $\begin{array}{l}\text { Uncertain } \\
\text { because no } \\
\text { information }\end{array}$ & $\begin{array}{l}\text { Unclear how hospitals } \\
\text { self-selected into } \\
\text { intervention and control } \\
\text { groups. }\end{array}$ \\
\hline $\begin{array}{l}\text { Hannula, } \\
2014^{32}\end{array}$ & No & No & NA & Yes & Yes & NA & High & $\begin{array}{l}\text { Groups of participants in } \\
\text { intervention clusters } \\
\text { appear to have different } \\
\text { characteristics, } \\
\text { suggesting that their } \\
\text { choice of delivery } \\
\text { institution (and therefore } \\
\text { intervention group) could } \\
\text { potentially have been } \\
\text { influenced by underlying } \\
\text { traits that also influence } \\
\text { the outcome. }\end{array}$ \\
\hline $\begin{array}{l}\text { Hawkins, } \\
2014^{33}\end{array}$ & No & Yes & Probably yes & Yes & Yes & NA & Low & NA \\
\hline $\begin{array}{l}\text { Hawkins, } \\
2014^{34}\end{array}$ & No & Yes & Probably yes & Yes & Yes & NA & Low & NA \\
\hline Joyce, $2015^{35}$ & Probably no & No & Probably yes & NA & Yes & Yes & High & $\begin{array}{l}\text { Unlikely that authors were } \\
\text { able to adjust for all } \\
\text { important confounding } \\
\text { domains given limitations } \\
\text { of population databases } \\
\text { (which were designed for } \\
\text { different purposes). }\end{array}$ \\
\hline
\end{tabular}




\begin{tabular}{|c|c|c|c|c|c|c|c|c|}
\hline Author, Year & $\begin{array}{l}5 . \text { Is } \\
\text { confounding } \\
\text { of the } \\
\text { intervention } \\
\text { effect } \\
\text { unlikely? }\end{array}$ & $\begin{array}{l}5 \text { a. Did the } \\
\text { authors use an } \\
\text { appropriate } \\
\text { analysis to } \\
\text { adjust for } \\
\text { confounders? }\end{array}$ & $\begin{array}{l}\text { 5b. Were } \\
\text { confounding } \\
\text { domains that } \\
\text { were } \\
\text { controlled for } \\
\text { measured } \\
\text { validly and } \\
\text { reliably? }\end{array}$ & $\begin{array}{l}\text { 5c. Did the } \\
\text { authors avoid } \\
\text { adjusting for } \\
\text { post- } \\
\text { intervention } \\
\text { variables? }\end{array}$ & $\begin{array}{l}\text { 5d. Were } \\
\text { participants } \\
\text { analyzed } \\
\text { according to } \\
\text { their initial } \\
\text { intervention } \\
\text { group } \\
\text { throughout } \\
\text { followup? }\end{array}$ & $\begin{array}{l}\text { 5e. Were } \\
\text { intervention } \\
\text { discontinuations } \\
\text { or switches } \\
\text { unlikely to be } \\
\text { related to factors } \\
\text { prognostic for } \\
\text { the outcome? }\end{array}$ & $\begin{array}{l}\text { Bias arising } \\
\text { from } \\
\text { confounding? }\end{array}$ & Comments \\
\hline Lovera, $2010^{36}$ & Probably no & Probably yes & Yes & Yes & Yes & Probably yes & Low & $\begin{array}{l}\text { Authors reported that } \\
\text { none of the potential } \\
\text { confounders they } \\
\text { evaluated changed the } \\
\text { overall effect estimate } \\
\text { more than } 10 \% .\end{array}$ \\
\hline $\begin{array}{l}\text { Macaluso, } \\
2013 ; 38 \\
\text { Cattaneo, } \\
2016^{39}\end{array}$ & Probably no & Probably yes & Probably yes & Yes & Yes & NA & Some concerns & $\begin{array}{l}\text { Authors adjust for } \\
\text { individual level } \\
\text { confounders; unclear } \\
\text { whether there are } \\
\text { population level } \\
\text { differences between } \\
\text { intervention and control } \\
\text { communities that may } \\
\text { affect outcomes. }\end{array}$ \\
\hline Madden, $2003^{37}$ & Probably yes & NA & NA & NA & NA & $\overline{N A}$ & Some concerns & $\begin{array}{l}\text { Complex deliveries (23\% } \\
\text { were excluded, including } \\
\text { mothers with C-sections } \\
\text { and more than } 4 \text { nights } \\
\text { postpartum LOS or } \\
\text { unequal mother/infant } \\
\text { LOS. This is a systems- } \\
\text { level intervention that } \\
\text { relies on administrative } \\
\text { data. Mothers were not } \\
\text { selected for participation } \\
\text { by staff (or self-selected } \\
\text { based on individual } \\
\text { factors) based on factors } \\
\text { associated with BF. }\end{array}$ \\
\hline
\end{tabular}




\begin{tabular}{|c|c|c|c|c|c|c|c|c|}
\hline Author, Year & $\begin{array}{l}5 . \text { Is } \\
\text { confounding } \\
\text { of the } \\
\text { intervention } \\
\text { effect } \\
\text { unlikely? }\end{array}$ & $\begin{array}{l}5 \text { a. Did the } \\
\text { authors use an } \\
\text { appropriate } \\
\text { analysis to } \\
\text { adjust for } \\
\text { confounders? }\end{array}$ & $\begin{array}{l}\text { 5b. Were } \\
\text { confounding } \\
\text { domains that } \\
\text { were } \\
\text { controlled for } \\
\text { measured } \\
\text { validly and } \\
\text { reliably? }\end{array}$ & $\begin{array}{l}5 c . \text { Did the } \\
\text { authors avoid } \\
\text { adjusting for } \\
\text { post- } \\
\text { intervention } \\
\text { variables? }\end{array}$ & $\begin{array}{l}\text { 5d. Were } \\
\text { participants } \\
\text { analyzed } \\
\text { according to } \\
\text { their initial } \\
\text { intervention } \\
\text { group } \\
\text { throughout } \\
\text { followup? }\end{array}$ & $\begin{array}{l}\text { ee. Were } \\
\text { intervention } \\
\text { discontinuation } \\
\text { s or switches } \\
\text { unlikely to be } \\
\text { related to } \\
\text { factors } \\
\text { prognostic for } \\
\text { the outcome? }\end{array}$ & $\begin{array}{l}\text { Bias arising } \\
\text { from } \\
\text { confounding? }\end{array}$ & Comments \\
\hline Martens, $2000^{40}$ & No & No & Yes & Yes & Yes & NA & High & $\begin{array}{l}\text { Significant differences at } \\
\text { baseline between groups, } \\
\text { suggesting that some } \\
\text { underlying factors may } \\
\text { influence the selection of } \\
\text { hospital and the outcome. }\end{array}$ \\
\hline $\begin{array}{l}\text { Mydlilova, } \\
2009^{41}\end{array}$ & Probably no & Probably no & NA & Probably yes & Yes & $\overline{N A}$ & Some concerns & $\begin{array}{l}\text { Authors did look at effect } \\
\text { of intervention on BF } \\
\text { rates stratified by } \\
\text { perinatal center and } \\
\text { neonatal intermediate } \\
\text { care unit status, but there } \\
\text { was no other attempt to } \\
\text { consider potential } \\
\text { confounders between the } \\
\text { non BFHI and BFHI } \\
\text { hospitals. Note that } \\
\text { question 5e does not } \\
\text { make sense in the context } \\
\text { of this study design. }\end{array}$ \\
\hline Rempel, $2010^{42}$ & Probably no & No & NA & Yes & Yes & Probably no & Some concerns & $\begin{array}{l}\text { Authors do not provide } \\
\text { adjusted results; it is } \\
\text { unlikely that a participant } \\
\text { would have attended } \\
\text { more than one of the } \\
\text { prenatal classes or had } \\
\text { access to PLCs (if in the } \\
\text { NLC group). }\end{array}$ \\
\hline
\end{tabular}




\begin{tabular}{|c|c|c|c|c|c|c|c|c|}
\hline Author, Year & $\begin{array}{l}\text { 5. Is } \\
\text { confounding of } \\
\text { the intervention } \\
\text { effect unlikely? }\end{array}$ & $\begin{array}{l}5 \text { a. Did the } \\
\text { authors use an } \\
\text { appropriate } \\
\text { analysis to } \\
\text { adjust for } \\
\text { confounders? }\end{array}$ & $\begin{array}{l}\text { 5b. Were } \\
\text { confounding } \\
\text { domains that } \\
\text { were controlled } \\
\text { for measured } \\
\text { validly and } \\
\text { reliably? }\end{array}$ & $\begin{array}{l}\text { 5c. Did the } \\
\text { authors avoid } \\
\text { adjusting for } \\
\text { post- } \\
\text { intervention } \\
\text { variables? }\end{array}$ & $\begin{array}{l}\text { 5d. Were } \\
\text { participants } \\
\text { analyzed } \\
\text { according to } \\
\text { their initial } \\
\text { intervention } \\
\text { group } \\
\text { throughout } \\
\text { followup? }\end{array}$ & $\begin{array}{l}\text { 5e. Were } \\
\text { intervention } \\
\text { discontinuation } \\
\text { s or switches } \\
\text { unlikely to be } \\
\text { related to } \\
\text { factors } \\
\text { prognostic for } \\
\text { the outcome? }\end{array}$ & $\begin{array}{l}\text { Bias arising } \\
\text { from } \\
\text { confounding? }\end{array}$ & Comments \\
\hline Schafer, $1998^{43}$ & No & No information & No information & No & Yes & NA & $\begin{array}{l}\text { Uncertain } \\
\text { because no } \\
\text { information }\end{array}$ & $\begin{array}{l}\text { No information on } \\
\text { important baseline } \\
\text { characteristics for } \\
\text { the intervention } \\
\text { arms, so cannot } \\
\text { judge the potential } \\
\text { for confounding } \\
\text { fully. }\end{array}$ \\
\hline Shaw, 199944 & No & No & Probably yes & Yes & Yes & NA & Some concerns & $\begin{array}{l}\text { Analysis did not } \\
\text { adjust for the } \\
\text { variables that } \\
\text { were different } \\
\text { between groups } \\
\text { at baseline (i.e., } \\
\text { age, marital } \\
\text { status, Medicaid } \\
\text { status), all which } \\
\text { are associated } \\
\text { with breastfeeding } \\
\text { rates. Baseline } \\
\text { differences could } \\
\text { have masked a } \\
\text { greater difference } \\
\text { in outcomes } \\
\text { between the } \\
\text { groups. }\end{array}$ \\
\hline
\end{tabular}




\begin{tabular}{|c|c|c|c|c|c|c|c|c|}
\hline Author, Year & $\begin{array}{l}5 . \text { Is } \\
\text { confounding } \\
\text { of the } \\
\text { intervention } \\
\text { effect } \\
\text { unlikely? }\end{array}$ & $\begin{array}{l}5 a \text {. Did the } \\
\text { authors use an } \\
\text { appropriate } \\
\text { analysis to } \\
\text { adjust for } \\
\text { confounders? }\end{array}$ & $\begin{array}{l}\text { 5b. Were } \\
\text { confounding } \\
\text { domains that } \\
\text { were } \\
\text { controlled for } \\
\text { measured } \\
\text { validly and } \\
\text { reliably? }\end{array}$ & $\begin{array}{l}5 c . \text { Did the } \\
\text { authors avoid } \\
\text { adjusting for } \\
\text { post- } \\
\text { intervention } \\
\text { variables? }\end{array}$ & $\begin{array}{l}\text { 5d. Were } \\
\text { participants } \\
\text { analyzed } \\
\text { according to } \\
\text { their initial } \\
\text { intervention } \\
\text { group } \\
\text { throughout } \\
\text { follow-up? }\end{array}$ & $\begin{array}{l}\text { 5e. Were } \\
\text { intervention } \\
\text { discontinuation } \\
\text { s or switches } \\
\text { unlikely to be } \\
\text { related to } \\
\text { factors } \\
\text { prognostic for } \\
\text { the outcome? }\end{array}$ & $\begin{array}{l}\text { Bias arising } \\
\text { from } \\
\text { confounding? }\end{array}$ & Comments \\
\hline Tarrant, $2011^{45}$ & Probably yes & NA & NA & NA & $\mathrm{NA}$ & NA & Low & $\begin{array}{l}\text { Authors presented } \\
\text { multiple models, adjusted } \\
\text { for a number of factors } \\
\text { related to the intervention } \\
\text { and to the outcome and } \\
\text { adequately interpreted the } \\
\text { results. The one potential } \\
\text { confounder that was not } \\
\text { adjusted for was hospital } \\
\text { (there were 4); however, } \\
\text { adjustment for BFHI steps } \\
\text { would probably account } \\
\text { for most of the potential } \\
\text { confounding from hospital } \\
\text { site. }\end{array}$ \\
\hline $\begin{array}{l}\text { Venancio, } \\
2011^{46}\end{array}$ & No & Probably yes & Probably yes & Yes & Yes & Probably yes & Some concerns & $\begin{array}{l}\text { Maternal education and } \\
\text { age are both associated } \\
\text { with several outcomes of } \\
\text { interest, and these are } \\
\text { dichotomized, rather than } \\
\text { measured in narrower } \\
\text { categories, raising } \\
\text { concerns regarding } \\
\text { residual confounding. } \\
\text { Maternal BF intention, a } \\
\text { critical potential } \\
\text { confounder, is not } \\
\text { measured. }\end{array}$ \\
\hline
\end{tabular}




\begin{tabular}{|c|c|c|c|c|c|c|c|c|}
\hline Author, Year & $\begin{array}{l}\text { 5. Is } \\
\text { confounding } \\
\text { of the } \\
\text { intervention } \\
\text { effect } \\
\text { unlikely? }\end{array}$ & $\begin{array}{l}\text { 5a. Did the } \\
\text { authors use an } \\
\text { appropriate } \\
\text { analysis to } \\
\text { adjust for } \\
\text { confounders? }\end{array}$ & $\begin{array}{l}\text { 5b. Were } \\
\text { confounding } \\
\text { domains that } \\
\text { were } \\
\text { controlled for } \\
\text { measured } \\
\text { validly and } \\
\text { reliably? }\end{array}$ & $\begin{array}{l}5 c . \text { Did the } \\
\text { authors avoid } \\
\text { adjusting for } \\
\text { post- } \\
\text { intervention } \\
\text { variables? }\end{array}$ & $\begin{array}{l}\text { 5d. Were } \\
\text { participants } \\
\text { analyzed } \\
\text { according to } \\
\text { their initial } \\
\text { intervention } \\
\text { group } \\
\text { throughout } \\
\text { followup? }\end{array}$ & $\begin{array}{l}\text { 5e. Were } \\
\text { intervention } \\
\text { discontinuation } \\
\text { s or switches } \\
\text { unlikely to be } \\
\text { related to } \\
\text { factors } \\
\text { prognostic for } \\
\text { the outcome? }\end{array}$ & $\begin{array}{l}\text { Bias arising } \\
\text { from } \\
\text { confounding? }\end{array}$ & Comments \\
\hline Wagner, 200247 & Probably no & No & $\mathrm{NA}$ & Yes & Yes & Yes & Some concerns & $\begin{array}{l}\text { Pre- and post-intervention } \\
\text { variables were compared } \\
\text { without any adjustment for } \\
\text { possible confounders. }\end{array}$ \\
\hline Weng, $2003^{48}$ & No & No & $\mathrm{NA}$ & NA & Yes & $\mathrm{NA}$ & High & $\begin{array}{l}\text { No sociodemographic } \\
\text { data are provided on the } \\
\text { mothers surveyed, nor is } \\
\text { any adjustment for } \\
\text { confounding performed } \\
\text { (based on participant or } \\
\text { hospital characteristics). }\end{array}$ \\
\hline
\end{tabular}


Table C-9. KQ 1 risk of bias assessment: Nonrandomized trials and observational studies, part 3

\begin{tabular}{|c|c|c|c|c|c|}
\hline Author, Year & $\begin{array}{l}\text { 6. Is intervention } \\
\text { status well- } \\
\text { defined? }\end{array}$ & $\begin{array}{l}\text { 7. Was information } \\
\text { on intervention } \\
\text { status recorded at } \\
\text { the time of } \\
\text { intervention? }\end{array}$ & $\begin{array}{l}\text { 8. Was } \\
\text { classification of } \\
\text { intervention } \\
\text { status unaffected } \\
\text { by knowledge of } \\
\text { the outcome or } \\
\text { risk of the } \\
\text { outcome? }\end{array}$ & $\begin{array}{l}\text { Bias arising from } \\
\text { measurement of } \\
\text { interventions? }\end{array}$ & Comments \\
\hline Abolyan, $2006^{23}$ & Probably yes & Yes & Yes & Some concerns & $\begin{array}{l}\text { For measurement of BF prevalence in intervention/control } \\
\text { groups over time, it is not clear how BFHI "status" coincided } \\
\text { with changes in actual care delivered across hospitals. Some } \\
\text { women giving birth in control hospitals (each year) may have } \\
\text { had exposure to BFHI practices. }\end{array}$ \\
\hline $\begin{array}{l}\text { Alvarado, } \\
1999^{24}\end{array}$ & Probably yes & Yes & Yes & Low & NA \\
\hline Bærug, $2016^{25}$ & Yes & Yes & Yes & Low & NA \\
\hline $\begin{array}{l}\text { Bartington, } \\
2006^{26}\end{array}$ & Yes & Yes & Probably yes & Low & NA \\
\hline Bosnjak, $2004^{27}$ & no & No & No & High & $\begin{array}{l}\text { "Standard WHO breastfeeding definitions were not used. It is } \\
\text { possible that each doctor may have used his/her own } \\
\text { definitions and recall period, especially in the research period } \\
\text { without intervention and the first phase of the intervention } \\
\text { period." }\end{array}$ \\
\hline $\begin{array}{l}\text { Broadfoot, } \\
2005^{28}\end{array}$ & Yes & Yes & Probably yes & Low & NA \\
\hline $\begin{array}{l}\text { Edmunds, } \\
2017^{29}\end{array}$ & Yes & Yes & Probably yes & Some concerns & $\begin{array}{l}\text { Unclear whether other, similar interventions were available } \\
\text { across WIC agencies. }\end{array}$ \\
\hline $\begin{array}{l}\text { Feldman- } \\
\text { Winter, } 2010^{30}\end{array}$ & Yes & Yes & Yes & Low & NA \\
\hline Gau, $2004^{31}$ & Yes & Yes & Yes & Low & NA \\
\hline $\begin{array}{l}\text { Hannula, } \\
2014^{32}\end{array}$ & Yes & Yes & Yes & Low & None \\
\hline $\begin{array}{l}\text { Hawkins, } \\
2014^{33}\end{array}$ & Probably no & No information & Yes & Some concerns & $\begin{array}{l}\text { The definition of the intervention rests on accreditation, rather } \\
\text { than the number of practices followed in accredited hospitals. } \\
\text { As a result, accredited hospitals with low compliance and } \\
\text { nonaccredited hospitals with some BF practices cannot be } \\
\text { easily distinguished. }\end{array}$ \\
\hline
\end{tabular}




\begin{tabular}{|c|c|c|c|c|c|}
\hline Author, Year & $\begin{array}{l}\text { 6. Is intervention } \\
\text { status well- } \\
\text { defined? }\end{array}$ & $\begin{array}{l}\text { 7. Was information } \\
\text { on intervention } \\
\text { status recorded at } \\
\text { the time of } \\
\text { intervention? }\end{array}$ & $\begin{array}{l}\text { 8. Was } \\
\text { classification of } \\
\text { intervention } \\
\text { status unaffected } \\
\text { by knowledge of } \\
\text { the outcome or } \\
\text { risk of the } \\
\text { outcome? }\end{array}$ & $\begin{array}{l}\text { Bias arising from } \\
\text { measurement of } \\
\text { interventions? }\end{array}$ & Comments \\
\hline $\begin{array}{l}\text { Hawkins, } \\
2014^{34}\end{array}$ & Probably no & No information & Yes & Some concerns & $\begin{array}{l}\text { The definition of the intervention rests on accreditation, rather } \\
\text { than the number of practices followed in accredited hospitals. } \\
\text { As a result, accredited hospitals with low compliance and } \\
\text { nonaccredited hospitals with some BF practices cannot be } \\
\text { easily distinguished. }\end{array}$ \\
\hline Joyce, $2015^{35}$ & Yes & Probably yes & NA & Low & Intervention is a change in WIC policy. \\
\hline Lovera, $2010^{36}$ & Yes & Probably yes & Yes & Low & NA \\
\hline $\begin{array}{l}\text { Macaluso, } \\
2013 ; 38 \\
\text { Cattaneo, } \\
2016^{39}\end{array}$ & Yes & Yes & Yes & Low & NA \\
\hline Madden, $2003^{37}$ & Probably yes & Probably yes & Yes & Some concerns & $\begin{array}{l}\text { Intervention is a policy change; women were analyzed in a } \\
\text { time-series before, during and after the intervention. There is } \\
\text { clear timing regarding the policy change, however, details of } \\
\text { implementation are not clear. }\end{array}$ \\
\hline Martens, $2000^{40}$ & Yes & Yes & Yes & Low & NA \\
\hline $\begin{array}{l}\text { Mydlilova, } \\
2009^{41}\end{array}$ & Yes & Yes & Yes & Low & NA \\
\hline Rempel, $2010^{42}$ & Yes & Yes & Yes & Low & $\begin{array}{l}\text { The difference between the two intervention groups is relatively } \\
\text { clear (the selection into them is not). }\end{array}$ \\
\hline Shaw, 199944 & Yes & Yes & Yes & Low & NA \\
\hline Schafer, $1998^{43}$ & Yes & Yes & Yes & Low & NA \\
\hline Tarrant, $2011^{45}$ & Probably no & Probably yes & Yes & Some concerns & $\begin{array}{l}\text { Authors noted that data on exposure to the BFHI steps were } \\
\text { available on the participants' hospital records (except for step } \\
10 \text {, available through self-report at } 1 \text { month). However, no } \\
\text { further details were available about how BFHI was } \\
\text { implemented at the hospitals or why certain steps may or may } \\
\text { not have been implemented, either at the hospital or the } \\
\text { participant level. It seems as though implementation of BFHI in } \\
\text { Hong Kong has not been done in any official capacity. }\end{array}$ \\
\hline
\end{tabular}




\begin{tabular}{|c|c|c|c|c|c|}
\hline Author, Year & $\begin{array}{l}\text { 6. Is intervention } \\
\text { status well- } \\
\text { defined? }\end{array}$ & $\begin{array}{l}\text { 7. Was information } \\
\text { on intervention } \\
\text { status recorded at } \\
\text { the time of } \\
\text { intervention? }\end{array}$ & $\begin{array}{l}\text { 8. Was } \\
\text { classification of } \\
\text { intervention } \\
\text { status unaffected } \\
\text { by knowledge of } \\
\text { the outcome or } \\
\text { risk of the } \\
\text { outcome? }\end{array}$ & $\begin{array}{l}\text { Bias arising from } \\
\text { measurement of } \\
\text { interventions? }\end{array}$ & Comments \\
\hline $\begin{array}{l}\text { Venancio, } \\
2011^{46}\end{array}$ & Yes & Yes & Yes & Low & NA \\
\hline Wagner, 2002 ${ }^{47}$ & Yes & Yes & Yes & Low & NA \\
\hline Weng, $2003^{48}$ & Probably yes & Probably yes & Probably yes & Low & NA \\
\hline
\end{tabular}


Table C-10. KQ 1 risk of bias assessment: Nonrandomized trials and observational studies, part 4

\begin{tabular}{|c|c|c|c|c|c|c|c|}
\hline Author, Year & $\begin{array}{l}\text { 9. Were } \\
\text { outcome data } \\
\text { available for } \\
\text { all, or nearly } \\
\text { all, } \\
\text { participants? }\end{array}$ & $\begin{array}{l}\text { 10. Were few } \\
\text { or no } \\
\text { participants } \\
\text { excluded } \\
\text { because of } \\
\text { missing data } \\
\text { on } \\
\text { intervention } \\
\text { status? }\end{array}$ & $\begin{array}{l}\text { 11. Were few } \\
\text { or no } \\
\text { participants } \\
\text { excluded due } \\
\text { to missing } \\
\text { data on other } \\
\text { variables? }\end{array}$ & $\begin{array}{l}\text { 12. Was the } \\
\text { proportion of } \\
\text { participants and } \\
\text { reasons for } \\
\text { missing data } \\
\text { similar across } \\
\text { intervention } \\
\text { groups? }\end{array}$ & $\begin{array}{l}\text { 13. Were } \\
\text { appropriate } \\
\text { statistical methods } \\
\text { used to account } \\
\text { for missing data or } \\
\text { assess robustness } \\
\text { to presence of } \\
\text { missing data? }\end{array}$ & $\begin{array}{l}\text { Bias Arising } \\
\text { from Missing } \\
\text { Data }\end{array}$ & Comments \\
\hline $\begin{array}{l}\text { Abolyan, } \\
2006^{23}\end{array}$ & Probably yes & Probably yes & Probably yes & Yes & NA & Low & $\begin{array}{l}\text { For administrative data on BF initiation, } \\
\text { rates of missing data are likely very low. } \\
\text { For survey of women giving birth in } 2004 \text {, } \\
\text { survey completion rates were high (95\% } \\
\text { and } 89 \% \text {, respectively, in intervention } \\
\text { and control groups). }\end{array}$ \\
\hline $\begin{array}{l}\text { Alvarado, } \\
1999^{24}\end{array}$ & Probably yes & Probably yes & Probably yes & No & No & Some concerns & $\begin{array}{l}\text { Unclear rate of missing data for control } \\
\text { sites; authors state that } 20 \text { (of 200) } \\
\text { women had no information (but } \\
\text { information was available for their infant). } \\
\text { At intervention sites, missing data } \\
\text { appears low but is not clearly reported by } \\
\text { outcome. }\end{array}$ \\
\hline $\begin{array}{l}\text { Bærug, } \\
2016^{25}\end{array}$ & No & Yes & No & Yes & Yes & Some concerns & $\begin{array}{l}\text { Although authors used an ITT analysis } \\
\text { and retained all the clusters (only 18/27 } \\
\text { completed the intervention), a large } \\
\text { proportion of participants refused to } \\
\text { participate in the survey ( } 740.3 \% \text { in the } \\
\text { intervention arm and } 43.5 \% \text { in the control } \\
\text { arm). }\end{array}$ \\
\hline $\begin{array}{l}\text { Bartington, } \\
2006^{26}\end{array}$ & Probably yes & Probably yes & Probably yes & No information & No information & Low & $\begin{array}{l}\text { Missing data is unclear, however, due to } \\
\text { population level databases information on } \\
\text { most births appear to be accounted for. }\end{array}$ \\
\hline $\begin{array}{l}\text { Bosnjak, } \\
2004^{27}\end{array}$ & Probably yes & No information & No information & NA & No & $\begin{array}{l}\text { Uncertain } \\
\text { because no } \\
\text { information } \\
\end{array}$ & Sample consisted of $90.3 \%$ of newborns. \\
\hline $\begin{array}{l}\text { Broadfoot, } \\
2005^{28}\end{array}$ & No & Yes & Probably yes & No information & No information & Some concerns & $\begin{array}{l}\text { Feeding status was missing on a small } \\
\text { number of records }(2.4-3.2 \%) ; \text { Carstairs } \\
\text { deprivation category was missing on } \\
17.4 \% \text { of records. }\end{array}$ \\
\hline
\end{tabular}




\begin{tabular}{|c|c|c|c|c|c|c|c|}
\hline Author, Year & $\begin{array}{l}\text { 9. Were } \\
\text { outcome data } \\
\text { available for } \\
\text { all, or nearly } \\
\text { all, } \\
\text { participants? }\end{array}$ & $\begin{array}{l}\text { 10. Were few } \\
\text { or no } \\
\text { participants } \\
\text { excluded } \\
\text { because of } \\
\text { missing data } \\
\text { on } \\
\text { intervention } \\
\text { status? }\end{array}$ & $\begin{array}{l}\text { 11. Were few } \\
\text { or no } \\
\text { participants } \\
\text { excluded due } \\
\text { to missing } \\
\text { data on other } \\
\text { variables? }\end{array}$ & $\begin{array}{l}\text { 12. Was the } \\
\text { proportion of } \\
\text { participants and } \\
\text { reasons for } \\
\text { missing data } \\
\text { similar across } \\
\text { intervention } \\
\text { groups? }\end{array}$ & $\begin{array}{l}\text { 13. Were } \\
\text { appropriate } \\
\text { statistical methods } \\
\text { used to account } \\
\text { for missing data or } \\
\text { assess robustness } \\
\text { to presence of } \\
\text { missing data? }\end{array}$ & $\begin{array}{l}\text { Bias Arising } \\
\text { from Missing } \\
\text { Data }\end{array}$ & Comments \\
\hline $\begin{array}{l}\text { Macaluso, } \\
2013 ;{ }^{38} \text { Cattan } \\
\text { eo, } 2016^{39}\end{array}$ & Yes & Yes & Yes & Yes & No & Some concerns & $\begin{array}{l}\text { Authors report no significant differences } \\
\text { by intervention and control groups for } \\
\text { missing data; missing data at } 12 \text { months } \\
\text { ranged from } 9-20 \% \text { across community } \\
\text { clusters. No methods appear to be used } \\
\text { to account for missing data. }\end{array}$ \\
\hline $\begin{array}{l}\text { Edmunds, } \\
2017^{29}\end{array}$ & Probably yes & Yes & Yes & Yes & No & Some concerns & $\begin{array}{l}\text { Attrition ranged from } 13-15 \% \text {; only } \\
\text { completers analysis was performed. }\end{array}$ \\
\hline $\begin{array}{l}\text { Feldman- } \\
\text { Winter, } \\
2010^{30} \\
\end{array}$ & No information & No information & No information & No information & NA & $\begin{array}{l}\text { Uncertain } \\
\text { because no } \\
\text { information } \\
\end{array}$ & None \\
\hline Gau, 2004 ${ }^{31}$ & Probably no & No information & No information & No & NA & High & $\begin{array}{l}\text { Two of seven control hospitals dropped } \\
\text { out because birth rates were so low that } \\
\text { their caseloads went to zero eventually. } \\
\text { This raises a larger question of whether } \\
\text { the intervention and control hospitals } \\
\text { were truly similar. Also, the text seems to } \\
\text { suggest that a minimum threshold of } \\
\text { cases was necessary to compile the data } \\
\text { "Fifty cases were randomly sampled } \\
\text { every month from each hospital, and all } \\
\text { the cases were collected if the number } \\
\text { was less than } 50 . " \text { Very little information } \\
\text { on dropouts. }\end{array}$ \\
\hline $\begin{array}{l}\text { Hannula, } \\
2014^{32}\end{array}$ & No & No & No information & No & No & High & $\begin{array}{l}\text { High and differential attrition between } \\
\text { arms, coupled with baseline differences } \\
\text { in characteristics suggest the potential for } \\
\text { bias due to missing data. }\end{array}$ \\
\hline $\begin{array}{l}\text { Hawkins, } \\
2014^{33}\end{array}$ & No information & No information & No information & No information & No information & $\begin{array}{l}\text { Uncertain } \\
\text { because no } \\
\text { information }\end{array}$ & $\begin{array}{l}\text { Study does not disclose what proportion } \\
\text { of PRAMS participants completed } \\
\text { surveys. }\end{array}$ \\
\hline
\end{tabular}




\begin{tabular}{|c|c|c|c|c|c|c|c|}
\hline Author, Year & $\begin{array}{l}\text { 9. Were } \\
\text { outcome data } \\
\text { available for } \\
\text { all, or nearly } \\
\text { all, } \\
\text { participants? }\end{array}$ & $\begin{array}{l}\text { 10. Were few } \\
\text { or no } \\
\text { participants } \\
\text { excluded } \\
\text { because of } \\
\text { missing data } \\
\text { on } \\
\text { intervention } \\
\text { status? }\end{array}$ & $\begin{array}{l}\text { 11. Were few } \\
\text { or no } \\
\text { participants } \\
\text { excluded due } \\
\text { to missing } \\
\text { data on other } \\
\text { variables? }\end{array}$ & $\begin{array}{l}\text { 12. Was the } \\
\text { proportion of } \\
\text { participants and } \\
\text { reasons for } \\
\text { missing data } \\
\text { similar across } \\
\text { intervention } \\
\text { groups? }\end{array}$ & $\begin{array}{l}\text { 13. Were } \\
\text { appropriate } \\
\text { statistical methods } \\
\text { used to account } \\
\text { for missing data or } \\
\text { assess robustness } \\
\text { to presence of } \\
\text { missing data? }\end{array}$ & $\begin{array}{l}\text { Bias Arising } \\
\text { from Missing } \\
\text { Data }\end{array}$ & Comments \\
\hline $\begin{array}{l}\text { Hawkins, } \\
2014^{34}\end{array}$ & No information & No information & No information & No information & No information & $\begin{array}{l}\text { Uncertain } \\
\text { because no } \\
\text { information }\end{array}$ & $\begin{array}{l}\text { Study does not disclose what proportion } \\
\text { of PRAMS participants completed } \\
\text { surveys. }\end{array}$ \\
\hline Joyce, $2015^{35}$ & No information & No information & No information & No information & No information & $\begin{array}{l}\text { Uncertain } \\
\text { because no } \\
\text { information } \\
\end{array}$ & None \\
\hline $\begin{array}{l}\text { Lovera, } \\
2010^{36}\end{array}$ & Probably yes & Yes & Probably yes & No information & No information & Some concerns & $\begin{array}{l}\text { The outcome data and the confounder } \\
\text { data came from the mothers' responses. } \\
\text { Though it does not appear that any data } \\
\text { (other than baseline characteristics) } \\
\text { came from the father, it is important to } \\
\text { note that only } 53 \% \text { of the intervention and } \\
50 \% \text { of the control group responded to } \\
\text { paternal interviews. }\end{array}$ \\
\hline $\begin{array}{l}\text { Madden, } \\
2003^{37}\end{array}$ & Probably yes & Yes & Yes & No information & NA & Some concerns & $\begin{array}{l}\text { Per authors, they were able to categorize } \\
\text { all but } 0.5 \% \text { of infants based on feeding } \\
\text { patters (breastfeeding exposure). No } \\
\text { other mention is made of missing data or } \\
\text { other exclusions. Not clear if missing data } \\
\text { is different for pre- vs. post-comparison. }\end{array}$ \\
\hline $\begin{array}{l}\text { Martens, } \\
2000^{40} \\
\end{array}$ & Yes & Yes & Yes & NA & NA & Low & NA \\
\hline $\begin{array}{l}\text { Mydlilova, } \\
2009^{41}\end{array}$ & Yes & No & Probably yes & Probably yes & Probably yes & Low & $\begin{array}{l}\text { Newborn reports were only missing for } \\
1.5 \% \text { of infants; authors didn't describe } \\
\text { missingness by BFHI status. The rate of } \\
\text { missing data is so small that any } \\
\text { differential missingness is unlikely to } \\
\text { impact the findings. }\end{array}$ \\
\hline
\end{tabular}




\begin{tabular}{|c|c|c|c|c|c|c|c|}
\hline Author, Year & $\begin{array}{l}\text { 9. Were } \\
\text { outcome data } \\
\text { available for } \\
\text { all, or nearly } \\
\text { all, } \\
\text { participants? }\end{array}$ & $\begin{array}{l}\text { 10. Were few } \\
\text { or no } \\
\text { participants } \\
\text { excluded } \\
\text { because of } \\
\text { missing data } \\
\text { on } \\
\text { intervention } \\
\text { status? }\end{array}$ & $\begin{array}{l}\text { 11. Were few } \\
\text { or no } \\
\text { participants } \\
\text { excluded due } \\
\text { to missing } \\
\text { data on other } \\
\text { variables? }\end{array}$ & $\begin{array}{l}\text { 12. Was the } \\
\text { proportion of } \\
\text { participants and } \\
\text { reasons for } \\
\text { missing data } \\
\text { similar across } \\
\text { intervention } \\
\text { groups? }\end{array}$ & $\begin{array}{l}\text { 13. Were } \\
\text { appropriate } \\
\text { statistical methods } \\
\text { used to account } \\
\text { for missing data or } \\
\text { assess robustness } \\
\text { to presence of } \\
\text { missing data? }\end{array}$ & $\begin{array}{l}\text { Bias Arising } \\
\text { from Missing } \\
\text { Data }\end{array}$ & Comments \\
\hline $\begin{array}{l}\text { Rempel, } \\
2010^{42}\end{array}$ & No & Probably no & Probably no & No information & No & High & $\begin{array}{l}\text { Authors report that } 54 \text { PLC and } 55 \text { NLC } \\
\text { attendees completed at least one study } \\
\text { survey. It is not clear how many } \\
\text { attendees did not complete at least one } \\
\text { survey. The authors provided the number } \\
\text { of participants completing each survey in } \\
\text { table } 2 \text {, but it seems to be inconsistent } \\
\text { with some of the results text. It's clear } \\
\text { that there is a lot of missing data due to } \\
\text { attendees not completing surveys at each } \\
\text { timepoint, but it's not clear how it tracks } \\
\text { over the course of each attendee (e.g., is } \\
\text { there anyone who completed all surveys). } \\
\text { According to Table } 2 \text {, only } 24 \text { PLC and } \\
23 \text { NLC participants completed the } 6- \\
\text { month survey. According to text in the } \\
\text { second column on page } 77,5 \text { PLC and } 7 \\
\text { NLC mothers stopped BF between } 1 \text { and } \\
6 \text { months. However, there is no way to } \\
\text { tell what the denominators are for these } \\
\text { two results. There are fewer mothers who } \\
\text { completed the } 6 \text {-month survey than the } \\
1 \text {-month survey and we have no idea } \\
\text { what the overlap between those groups } \\
\text { are. According to text in the second } \\
\text { column on page } 77,49 \% \text { of the } 35 \text { NLC } \\
\text { mothers and } 61 \% \text { of the } 31 \text { PLC mothers } \\
\text { for whom duration data were available } \\
\text { were still BF at six months. It's not clear } \\
\text { where the denominators of } 31 \text { (PLC) and } \\
35 \text { (NLC) came from! }\end{array}$ \\
\hline
\end{tabular}




\begin{tabular}{|c|c|c|c|c|c|c|c|}
\hline Author, Year & $\begin{array}{l}\text { 9. Were } \\
\text { outcome data } \\
\text { available for } \\
\text { all, or nearly } \\
\text { all, } \\
\text { participants? }\end{array}$ & $\begin{array}{l}\text { 10. Were few } \\
\text { or no } \\
\text { participants } \\
\text { excluded } \\
\text { because of } \\
\text { missing data } \\
\text { on } \\
\text { intervention } \\
\text { status? }\end{array}$ & $\begin{array}{l}\text { 11. Were few } \\
\text { or no } \\
\text { participants } \\
\text { excluded due } \\
\text { to missing } \\
\text { data on other } \\
\text { variables? }\end{array}$ & $\begin{array}{l}\text { 12. Was the } \\
\text { proportion of } \\
\text { participants and } \\
\text { reasons for } \\
\text { missing data } \\
\text { similar across } \\
\text { intervention } \\
\text { groups? }\end{array}$ & $\begin{array}{l}\text { 13. Were } \\
\text { appropriate } \\
\text { statistical methods } \\
\text { used to account } \\
\text { for missing data or } \\
\text { assess robustness } \\
\text { to presence of } \\
\text { missing data? }\end{array}$ & $\begin{array}{l}\text { Bias Arising } \\
\text { from Missing } \\
\text { Data }\end{array}$ & Comments \\
\hline $\begin{array}{l}\text { Schafer, } \\
1998^{43}\end{array}$ & No & No & No & No information & No & High & $\begin{array}{l}\text { Nearly one-half ( } 71 / 143) \text { participants in } \\
\text { the intervention arm did not respond, the } \\
\text { analysis could not account for such a } \\
\text { high attrition. }\end{array}$ \\
\hline Shaw, 199944 & No & Yes & Yes & No information & NA & Low & $\begin{array}{l}\text { Low attrition rate: for the OR analysis, the } \\
\text { minimum sample appears to be } 287 \text { of } \\
293 \text { (98\%), so the failure to adjust for } \\
\text { missing data probably does not have a } \\
\text { big impact. }\end{array}$ \\
\hline $\begin{array}{l}\text { Tarrant, } \\
2011^{45}\end{array}$ & Probably yes & Probably yes & Probably yes & No information & Probably yes & Low & $\begin{array}{l}1242 / 1417(88 \%) \text { of mother-infant pairs } \\
\text { recruited were analyzed. Of the } 31 \\
\text { participants lost-to-followup, } 21(68 \%) \\
\text { had provided enough data to analyze the } \\
\text { main study outcome. Authors performed } \\
\text { a sensitivity analysis of the } 31 \\
\text { participants lost to followup and ultimately } \\
\text { included them in analyses. }\end{array}$ \\
\hline $\begin{array}{l}\text { Venancio, } \\
2011^{46}\end{array}$ & Yes & Yes & No information & No information & No information & $\begin{array}{l}\text { Uncertain } \\
\text { because no } \\
\text { information } \\
\end{array}$ & $\begin{array}{l}\text { No information is provided on missing } \\
\text { data. }\end{array}$ \\
\hline $\begin{array}{l}\text { Wagner, } \\
2002^{47}\end{array}$ & No information & Probably yes & No information & NA & No information & Some concerns & $\begin{array}{l}\text { No description of exclusions for missing } \\
\text { data from the perinatal database. }\end{array}$ \\
\hline Weng, $2003^{48}$ & Probably yes & Probably no & NA & No information & No & Some concerns & $\begin{array}{l}\text { No information provided on differences } \\
\text { between those missing at } 1 \text { month and } \\
\text { the full sample, and no information } \\
\text { provided on the number of women } \\
\text { approached for survey vs. the number } \\
\text { who provided data. }\end{array}$ \\
\hline
\end{tabular}

BF = breastfeeding; BFHI = Baby Friendly Hospital Initiative; ITT = intent-to-treat; KQ = Key Question; NA = not applicable; NLC = nurse-led class; OR = odds ratio; PLC = peer-led class; PRAMS = Pregnancy Risk Assessment Monitoring System. 
Table C-11. KQ 1 risk of bias assessment: Nonrandomized trials and observational studies, part 5

14a. Were these

deviations from 15 . Were

14. Were there intended important

Author, Year

no or minimal intervention

16. Was the

deviations from unbalanced

balanced

the intended

between groups across

implemented

16a. Did the

intervention?

between grour

intervention

successfully

study measure Bias arising

have affected

most

adherence with

the outcome?

Abolyan, 2006 $23 \quad$ Probably yes NA

groups?

participants?

the defined

from departures Comments
from intended

No information NA

intervention?

interventions

NA

Low

Alvarado, $1999^{24}$

No information

NA

Yes

NA
NA

A

No in

$\begin{array}{lll}\text { No information } & \text { Yes } & \text { NA } \\ \text { Probably yes } & \text { NA } & \text { NA }\end{array}$

NA

A

\begin{tabular}{ll} 
Low & major factor. \\
\hline Some concerns & $\begin{array}{l}\text { Exposure is defined as giving } \\
\text { birth in a BFHI accredited facility; } \\
\text { unclear to what extent hospitals } \\
\text { not accredited may have been }\end{array}$
\end{tabular}

Exposure is defined as giving
birth in a BFHI accredited facility;
unclear to what extent hospitals

not accredited may have been

offering similar

\begin{tabular}{|c|c|c|c|c|c|c|}
\hline Bosnjak, 200427 & No information & NA & NA & No information & No information & $\begin{array}{l}\text { Uncertain } \\
\text { because no } \\
\text { information }\end{array}$ \\
\hline Broadfoot, $2005^{28}$ & No information & $\overline{N A}$ & Probably yes & $\overline{N A}$ & NA & Some concerns \\
\hline
\end{tabular}
of adherence.

policies/programs. None

information

No formal assessment of fidelity

Study looks at a systems-level intervention; responses from

more women giving birth in BFcertified hospitals received recommended practices (e.g.,

BF education, rooming-in) which could be considered a measure or description of other community or health care interventions to support or promote breastfeeding. 


\begin{tabular}{|c|c|c|c|c|c|c|c|}
\hline Author, Year & $\begin{array}{l}\text { 14. Were there } \\
\text { no or minimal } \\
\text { deviations from } \\
\text { the intended } \\
\text { intervention? }\end{array}$ & $\begin{array}{l}\text { 14a. Were these } \\
\text { deviations from } \\
\text { intended } \\
\text { intervention } \\
\text { unbalanced } \\
\text { between groups } \\
\text { and likely to } \\
\text { have affected } \\
\text { the outcome? }\end{array}$ & $\begin{array}{l}\text { 15. Were } \\
\text { important } \\
\text { cointerventions } \\
\text { balanced } \\
\text { across } \\
\text { intervention } \\
\text { groups? }\end{array}$ & $\begin{array}{l}\text { 16. Was the } \\
\text { intervention } \\
\text { implemented } \\
\text { successfully for } \\
\text { most } \\
\text { participants? }\end{array}$ & $\begin{array}{l}\text { 16a. Did the } \\
\text { study measure } \\
\text { adherence with } \\
\text { the defined } \\
\text { intervention? }\end{array}$ & $\begin{array}{l}\text { Bias arising } \\
\text { from departures } \\
\text { from intended } \\
\text { interventions }\end{array}$ & Comments \\
\hline Edmunds, $2017^{29}$ & No information & $\mathrm{NA}$ & NA & No information & No & $\begin{array}{l}\text { Uncertain } \\
\text { because no } \\
\text { information }\end{array}$ & $\begin{array}{l}\text { No assessment or description of } \\
\text { fidelity. }\end{array}$ \\
\hline Feldman-Winter, $2010^{30}$ & Probably no & NA & Probably no & No information & No & Some concerns & $\begin{array}{l}\text { Potential for contamination } \\
\text { between arms. }\end{array}$ \\
\hline $\mathrm{Gau}, 2004^{31}$ & Yes & No information & No information & Probably no & No & Some concerns & $\begin{array}{l}\text { The structure of hospitals } \\
\text { prevented implementation of } \\
\text { early tactile contact and rooming } \\
\text { in. }\end{array}$ \\
\hline Hannula, 2014 & Probably no & Probably no & Probably no & No information & No & Some concerns & $\begin{array}{l}\text { The co-interventions varied by } \\
\text { arm but details of the control } \\
\text { group are not described "support } \\
\text { and care practices used in the } \\
\text { intervention and control group } \\
\text { differed from each other." }\end{array}$ \\
\hline Hawkins, $2014^{33}$ & Probably no & Probably yes & Probably no & No information & Yes & Some concerns & $\begin{array}{l}\text { Study notes the potential for } \\
\text { contamination between arms; } \\
\text { nonaccredited facilities had BF } \\
\text { practices. }\end{array}$ \\
\hline Hawkins, $2014^{34}$ & Probably no & Probably yes & Probably no & No information & No information & Some concerns & $\begin{array}{l}\text { "At least half of the mothers from } \\
\text { Maine reported experiencing } 6-7 \\
\text { breastfeeding practices } \\
\text { consistent with the BFHI, } \\
\text { regardless of the birth facility's } \\
\text { BFHI status." }\end{array}$ \\
\hline Joyce, $2015^{35}$ & Probably yes & $\mathrm{NA}$ & No information & NA & NA & Some concerns & $\begin{array}{l}\text { Unclear whether implementation } \\
\text { of WIC policy was consistent } \\
\text { during timepoints measured. }\end{array}$ \\
\hline Lovera, $2010^{36}$ & No information & No information & No information & No information & No information & $\begin{array}{l}\text { Uncertain } \\
\text { because no } \\
\text { information }\end{array}$ & None \\
\hline
\end{tabular}




\begin{tabular}{|c|c|c|c|c|c|c|c|}
\hline Author, Year & $\begin{array}{l}\text { 14. Were there } \\
\text { no or minimal } \\
\text { deviations from } \\
\text { the intended } \\
\text { intervention? }\end{array}$ & $\begin{array}{l}\text { 14a. Were these } \\
\text { deviations from } \\
\text { intended } \\
\text { intervention } \\
\text { unbalanced } \\
\text { between groups } \\
\text { and likely to } \\
\text { have affected } \\
\text { the outcome? }\end{array}$ & $\begin{array}{l}\text { 15. Were } \\
\text { important } \\
\text { cointerventions } \\
\text { balanced } \\
\text { across } \\
\text { intervention } \\
\text { groups? }\end{array}$ & $\begin{array}{l}\text { 16. Was the } \\
\text { intervention } \\
\text { implemented } \\
\text { successfully for } \\
\text { most } \\
\text { participants? }\end{array}$ & $\begin{array}{l}\text { 16a. Did the } \\
\text { study measure } \\
\text { adherence with } \\
\text { the defined } \\
\text { intervention? }\end{array}$ & $\begin{array}{l}\text { Bias arising } \\
\text { from departures } \\
\text { from intended } \\
\text { interventions }\end{array}$ & Comments \\
\hline $\begin{array}{l}\text { Macaluso, 2013;38 } \\
\text { Cattaneo, } 2016^{39}\end{array}$ & Probably yes & NA & Probably yes & NA & NA & Some concerns & $\begin{array}{l}\text { No formal assessment of fidelity } \\
\text { or detailed description of what } \\
\text { other community or health care } \\
\text { interventions to promote } \\
\text { breastfeeding were available } \\
\text { (aside from BFHI). }\end{array}$ \\
\hline Madden, $2003^{37}$ & Probably yes & NA & No information & NA & NA & Low & $\begin{array}{l}\text { Intervention is a policy change; } \\
\text { there is no information on } \\
\text { whether the policy was amended } \\
\text { or changed after implemented. } \\
\text { Theoretically, there could be } \\
\text { other breastfeeding } \\
\text { interventions/ policy changes } \\
\text { occurring in the post- } \\
\text { intervention time period (but no } \\
\text { specific co-intervention). }\end{array}$ \\
\hline Martens, $2000^{40}$ & Yes & NA & No information & No information & No & Low & NA \\
\hline Mydlilova, 200941 & No information & No information & NA & No information & No information & $\begin{array}{l}\text { Uncertain } \\
\text { because no } \\
\text { information }\end{array}$ & $\begin{array}{l}\text { Authors do not report on the } \\
\text { extent of BFHI status at the Baby } \\
\text { Friendly hospitals other than to } \\
\text { note that over time, a hospital } \\
\text { may change from non BFHI to } \\
\text { BFHI. }\end{array}$ \\
\hline Rempel, $2010^{42}$ & No information & No information & No information & No information & No information & $\begin{array}{l}\text { Uncertain } \\
\text { because no } \\
\text { information }\end{array}$ & None \\
\hline Schafer, $1998^{43}$ & Yes & NA & No information & No information & No & Low & NA \\
\hline Shaw, $1999^{44}$ & Yes & NA & No information & Yes & Yes & Low & NA \\
\hline Tarrant, $2011^{45}$ & Probably yes & NA & Probably yes & No information & No information & Some concerns & $\begin{array}{l}\text { See bias in intervention } \\
\text { measurement domain. }\end{array}$ \\
\hline
\end{tabular}




\begin{tabular}{|c|c|c|c|c|c|c|c|}
\hline Author, Year & $\begin{array}{l}\text { 14. Were there } \\
\text { no or minimal } \\
\text { deviations from } \\
\text { the intended } \\
\text { intervention? }\end{array}$ & $\begin{array}{l}\text { 14a. Were these } \\
\text { deviations from } \\
\text { intended } \\
\text { intervention } \\
\text { unbalanced } \\
\text { between groups } \\
\text { and likely to } \\
\text { have affected } \\
\text { the outcome? }\end{array}$ & $\begin{array}{l}\text { 15. Were } \\
\text { important } \\
\text { cointerventions } \\
\text { balanced } \\
\text { across } \\
\text { intervention } \\
\text { groups? }\end{array}$ & $\begin{array}{l}\text { 16. Was the } \\
\text { intervention } \\
\text { implemented } \\
\text { successfully for } \\
\text { most } \\
\text { participants? }\end{array}$ & $\begin{array}{l}\text { 16a. Did the } \\
\text { study measure } \\
\text { adherence with } \\
\text { the defined } \\
\text { intervention? }\end{array}$ & $\begin{array}{l}\text { Bias arising } \\
\text { from departures } \\
\text { from intended } \\
\text { interventions }\end{array}$ & Comments \\
\hline Venancio, $2011^{46}$ & Probably yes & No information & No information & Probably no & NA & $\begin{array}{l}\text { Uncertain } \\
\text { because no } \\
\text { information }\end{array}$ & $\begin{array}{l}\text { No description on compliance } \\
\text { with BFHI steps for intervention } \\
\text { group. }\end{array}$ \\
\hline Wagner, $2002^{47}$ & Probably yes & NA & No information & No information & No & Some concerns & $\begin{array}{l}\text { No description of attendance, } \\
\text { adherence or practice-change } \\
\text { following intervention. } \\
\text { Participation in patient BF } \\
\text { groups was low }(<10 \%) \text { but } \\
\text { unclear whether this was a } \\
\text { change from pre-intervention. }\end{array}$ \\
\hline Weng, $2003^{48}$ & Probably yes & $\mathrm{NA}$ & No information & NA & Yes & Some concerns & $\begin{array}{l}\text { Co-interventions are not } \\
\text { discussed, although the authors } \\
\text { note in the discussion that "some } \\
\text { hospitals refused to accept on- } \\
\text { the-job training and had no } \\
\text { intention to improve their } \\
\text { services; these hospitals failed } \\
\text { the evaluation." This suggests } \\
\text { that failing BFHI evaluation may } \\
\text { reflect staff resistance to } \\
\text { breastfeeding promotion, which } \\
\text { in turn may lower BF rates. }\end{array}$ \\
\hline
\end{tabular}

$\overline{\mathrm{BF}}$ = breastfeeding; BFHI = Baby Friendly Hospital Initiative; KQ = Key Question; NA = not applicable; WIC = Women, Infants, and Children. 
Table C-12. KQ 1 risk of bias assessment: Nonrandomized trials and observational studies, part 6

17. Was

measurement of 18. Were

methods of

Author, Year breastfeeding

benefit

to have been outcome

influenced by

assessment

knowledge of the

across

received?

groups?

\section{Bias Arising}

from

Measurement of Comments

Breastfeeding

Outcomes

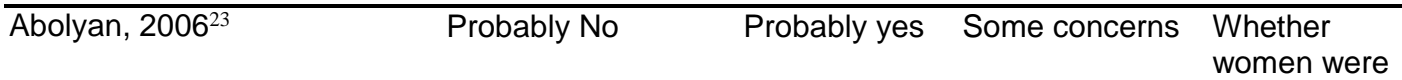

19. Is the reported effect

estimate unlikely to be

selected, on the basis of Bias Arising

the results, from from

multiple outcomes Selection of Comments

measurements within Reported

the domain, multiple Results

analyses, or different

subgroups?

aware of BFHI

status in their

hospital is not

clear; however,

it is unlikely that

women or

hospital staff

were blinded.

Unclear

whether

outcome

assessors of BF

prevalence data

over time were

blinded. 


\begin{tabular}{|c|c|c|c|c|c|c|c|}
\hline Author, Year & $\begin{array}{l}\text { 17. Was } \\
\text { measurement of } \\
\text { breastfeeding } \\
\text { outcomes unlikely } \\
\text { to have been } \\
\text { influenced by } \\
\text { knowledge of the } \\
\text { intervention } \\
\text { received? }\end{array}$ & $\begin{array}{l}\text { 18. Were } \\
\text { methods of } \\
\text { benefit } \\
\text { outcome } \\
\text { assessment } \\
\text { comparable } \\
\text { across } \\
\text { groups? }\end{array}$ & $\begin{array}{l}\text { Bias Arising } \\
\text { from } \\
\text { Measurement of } \\
\text { Breastfeeding } \\
\text { Outcomes }\end{array}$ & Comments & $\begin{array}{l}\text { 19. Is the reported effect } \\
\text { estimate unlikely to be } \\
\text { selected, on the basis of } \\
\text { the results, from } \\
\text { multiple outcomes } \\
\text { measurements within } \\
\text { the domain, multiple } \\
\text { analyses, or different } \\
\text { subgroups? }\end{array}$ & $\begin{array}{l}\text { Bias Arising } \\
\text { from } \\
\text { Selection of } \\
\text { Reported } \\
\text { Results }\end{array}$ & Comments \\
\hline Alvarado, $1999^{24}$ & No information & No & High & $\begin{array}{l}\text { Owing to } \\
\text { different } \\
\text { methods of } \\
\text { obtaining } \\
\text { outcomes at } \\
\text { intervention and } \\
\text { control sites, } \\
\text { measurement } \\
\text { bias is a } \\
\text { concern. } \\
\text { Women } \\
\text { receiving care } \\
\text { at control sites } \\
\text { had more } \\
\text { frequent } \\
\text { interactions with } \\
\text { staff and } \\
\text { outcome } \\
\text { assessors do } \\
\text { not appear to } \\
\text { have been } \\
\text { blinded. }\end{array}$ & Probably yes & Low & NA \\
\hline Bærug, 2016 & No & Yes & Low & NA & No & Low & NA \\
\hline Bartington, $2006^{26}$ & Yes & Yes & Low & NA & Probably yes & Low & NA \\
\hline
\end{tabular}




\begin{tabular}{|c|c|c|c|c|c|c|c|}
\hline Author, Year & $\begin{array}{l}\text { 17. Was } \\
\text { measurement of } \\
\text { breastfeeding } \\
\text { outcomes unlikely } \\
\text { to have been } \\
\text { influenced by } \\
\text { knowledge of the } \\
\text { intervention } \\
\text { received? }\end{array}$ & $\begin{array}{l}\text { 18. Were } \\
\text { methods of } \\
\text { benefit } \\
\text { outcome } \\
\text { assessment } \\
\text { comparable } \\
\text { across } \\
\text { groups? }\end{array}$ & $\begin{array}{l}\text { Bias Arising } \\
\text { from } \\
\text { Measurement of } \\
\text { Breastfeeding } \\
\text { Outcomes }\end{array}$ & Comments & $\begin{array}{l}\text { 19. Is the reported effect } \\
\text { estimate unlikely to be } \\
\text { selected, on the basis of } \\
\text { the results, from } \\
\text { multiple outcomes } \\
\text { measurements within } \\
\text { the domain, multiple } \\
\text { analyses, or different } \\
\text { subgroups? }\end{array}$ & $\begin{array}{l}\text { Bias Arising } \\
\text { from } \\
\text { Selection of } \\
\text { Reported } \\
\text { Results }\end{array}$ & Comments \\
\hline Bosnjak, 2004²7 & Yes & $\mathrm{NA}$ & High & $\begin{array}{l}\text { "Standard WHO } \\
\text { breastfeeding } \\
\text { definitions were } \\
\text { not used. It is } \\
\text { possible that } \\
\text { each doctor may } \\
\text { have used } \\
\text { his/her own } \\
\text { definitions and } \\
\text { recall period, } \\
\text { especially in the } \\
\text { research period } \\
\text { without } \\
\text { intervention and } \\
\text { the first phase of } \\
\text { the intervention } \\
\text { period." }\end{array}$ & No information & $\begin{array}{l}\text { Uncertain } \\
\text { because no } \\
\text { information }\end{array}$ & None \\
\hline Broadfoot, $2005^{28}$ & Yes & Yes & Low & NA & Probably yes & Low & $\mathrm{NA}$ \\
\hline Edmunds, $2017^{29}$ & Probably no & Probably yes & Some concerns & $\begin{array}{l}\text { No description of } \\
\text { blinding. }\end{array}$ & Yes & Low & $\overline{N A}$ \\
\hline Feldman-Winter, $2010^{30}$ & No & Yes & Low & NA & No & Low & $\mathrm{NA}$ \\
\hline Gau, 2004 & no & Yes & Low & NA & No & Low & $\overline{N A}$ \\
\hline Hannula, 2014 ${ }^{32}$ & Yes & Yes & Low & NA & no & Low & $\mathrm{NA}$ \\
\hline Hawkins, $2014^{33}$ & Yes & Yes & Low & NA & No & Low & $\mathrm{NA}$ \\
\hline Hawkins, 2014 ${ }^{34}$ & Yes & Yes & Low & NA & No & Low & $\mathrm{NA}$ \\
\hline Joyce, $2015^{35}$ & Probably yes & Probably yes & Low & NA & Probably yes & Low & $\mathrm{NA}$ \\
\hline Lovera, $2010^{36}$ & Probably yes & Yes & Low & NA & Probably yes & Low & NA \\
\hline
\end{tabular}




\begin{tabular}{|c|c|c|c|c|c|c|c|}
\hline Author, Year & $\begin{array}{l}\text { 17. Was } \\
\text { measurement of } \\
\text { breastfeeding } \\
\text { outcomes unlikely } \\
\text { to have been } \\
\text { influenced by } \\
\text { knowledge of the } \\
\text { intervention } \\
\text { received? } \\
\end{array}$ & $\begin{array}{l}\text { 18. Were } \\
\text { methods of } \\
\text { benefit } \\
\text { outcome } \\
\text { assessment } \\
\text { comparable } \\
\text { across } \\
\text { groups? }\end{array}$ & $\begin{array}{l}\text { Bias Arising } \\
\text { from } \\
\text { Measurement of } \\
\text { Breastfeeding } \\
\text { Outcomes }\end{array}$ & Comments & $\begin{array}{l}\text { 19. Is the reported effect } \\
\text { estimate unlikely to be } \\
\text { selected, on the basis of } \\
\text { the results, from } \\
\text { multiple outcomes } \\
\text { measurements within } \\
\text { the domain, multiple } \\
\text { analyses, or different } \\
\text { subgroups? }\end{array}$ & $\begin{array}{l}\text { Bias Arising } \\
\text { from } \\
\text { Selection of } \\
\text { Reported } \\
\text { Results } \\
\end{array}$ & Comments \\
\hline $\begin{array}{l}\text { Macaluso, 2013;38 } \\
\text { Cattaneo, } 2016^{39} \\
\end{array}$ & Probably yes & Probably yes & Low & NA & Probably yes & Low & NA \\
\hline Madden, $2003^{37}$ & Yes & Probably yes & Some concerns & $\begin{array}{l}\text { From } \\
\text { automated } \\
\text { medical } \\
\text { records, using } \\
\text { text-search } \\
\text { algorithms, } \\
\text { unclear how } \\
\text { outcomes were } \\
\text { validated. } \\
\end{array}$ & No & Low & NA \\
\hline Martens, $2000^{40}$ & Yes & No information & Some concerns & $\begin{array}{l}\text { Outcomes in } \\
\text { the control } \\
\text { group in the } \\
\text { second time } \\
\text { point look very } \\
\text { different from } \\
\text { the first time } \\
\text { point and the } \\
\text { author notes } \\
\text { the potential for } \\
\text { error in the } \\
\text { small sample } \\
\text { size. Unclear } \\
\text { whether } \\
\text { outcomes are } \\
\text { recorded the } \\
\text { same way in } \\
\text { both units. }\end{array}$ & No & Low & NA \\
\hline
\end{tabular}




\begin{tabular}{|c|c|c|c|c|c|c|c|}
\hline Author, Year & $\begin{array}{l}\text { 17. Was } \\
\text { measurement of } \\
\text { breastfeeding } \\
\text { outcomes unlikely } \\
\text { to have been } \\
\text { influenced by } \\
\text { knowledge of the } \\
\text { intervention } \\
\text { received? }\end{array}$ & $\begin{array}{l}\text { 18. Were } \\
\text { methods of } \\
\text { benefit } \\
\text { outcome } \\
\text { assessment } \\
\text { comparable } \\
\text { across } \\
\text { groups? }\end{array}$ & $\begin{array}{l}\text { Bias Arising } \\
\text { from } \\
\text { Measurement of } \\
\text { Breastfeeding } \\
\text { Outcomes }\end{array}$ & Comments & $\begin{array}{l}\text { 19. Is the reported effect } \\
\text { estimate unlikely to be } \\
\text { selected, on the basis of } \\
\text { the results, from } \\
\text { multiple outcomes } \\
\text { measurements within } \\
\text { the domain, multiple } \\
\text { analyses, or different } \\
\text { subgroups? }\end{array}$ & $\begin{array}{l}\text { Bias Arising } \\
\text { from } \\
\text { Selection of } \\
\text { Reported } \\
\text { Results }\end{array}$ & Comments \\
\hline Mydlilova, $2009^{41}$ & Probably no & Yes & Some concerns & $\begin{array}{l}\text { Newborn } \\
\text { reports are } \\
\text { required of all } \\
\text { hospitals but } \\
\text { there is no } \\
\text { discussion of } \\
\text { whether the } \\
\text { reports at BFHI } \\
\text { hospitals are } \\
\text { reported more } \\
\text { positively } \\
\text { because of their } \\
\text { BFHI } \\
\text { certification. } \\
\end{array}$ & Probably yes & Low & NA \\
\hline Rempel, 2010 & Probably yes & Probably yes & Low & NA & Probably yes & Low & NA \\
\hline Schafer, $1998^{43}$ & No & Yes & High & $\begin{array}{l}\text { Outcomes } \\
\text { based on self- } \\
\text { report at } 12 \\
\text { weeks, and any } \\
\text { BF qualified as } \\
\text { the outcome }\end{array}$ & No & Low & NA \\
\hline Shaw, 199944 & No & Yes & Low & NA & No & Low & NA \\
\hline Tarrant, 2011 & Probably yes & Yes & Low & NA & Probably yes & Low & NA \\
\hline Venancio, $2011^{46}$ & Probably yes & Probably yes & Some concerns & $\begin{array}{l}\text { Data collection } \\
\text { was not } \\
\text { blinded, and } \\
\text { social } \\
\text { desirability bias } \\
\text { may have } \\
\text { affected } \\
\text { responses. }\end{array}$ & Probably no & Low & NA \\
\hline
\end{tabular}




\begin{tabular}{|c|c|c|c|c|c|c|c|}
\hline Author, Year & $\begin{array}{l}\text { 17. Was } \\
\text { measurement of } \\
\text { breastfeeding } \\
\text { outcomes unlikely } \\
\text { to have been } \\
\text { influenced by } \\
\text { knowledge of the } \\
\text { intervention } \\
\text { received? }\end{array}$ & $\begin{array}{l}\text { 18. Were } \\
\text { methods of } \\
\text { benefit } \\
\text { outcome } \\
\text { assessment } \\
\text { comparable } \\
\text { across } \\
\text { groups? }\end{array}$ & $\begin{array}{l}\text { Bias Arising } \\
\text { from } \\
\text { Measurement o } \\
\text { Breastfeeding } \\
\text { Outcomes }\end{array}$ & Comments & $\begin{array}{l}\text { 19. Is the reported effect } \\
\text { estimate unlikely to be } \\
\text { selected, on the basis of } \\
\text { the results, from } \\
\text { multiple outcomes } \\
\text { measurements within } \\
\text { the domain, multiple } \\
\text { analyses, or different } \\
\text { subgroups? }\end{array}$ & $\begin{array}{l}\text { Bias Arising } \\
\text { from } \\
\text { Selection of } \\
\text { Reported } \\
\text { Results }\end{array}$ & Comments \\
\hline Wagner, $2002^{47}$ & No information & Probably yes & Some concerns & $\begin{array}{l}\text { Unclear } \\
\text { whether } \\
\text { documentation } \\
\text { or data } \\
\text { abstraction for } \\
\text { the perinatal } \\
\text { database may } \\
\text { have changed } \\
\text { after the } \\
\text { intervention. }\end{array}$ & Probably yes & Low & NA \\
\hline Weng, $2003^{48}$ & No information & No information & Some concerns & $\begin{array}{l}\text { No information } \\
\text { is provided on } \\
\text { how BF } \\
\text { outcomes were } \\
\text { defined or } \\
\text { assessed, or } \\
\text { whether } \\
\text { outcome } \\
\text { assessors were } \\
\text { blinded. }\end{array}$ & Probably no & Low & NA \\
\hline
\end{tabular}


Table C-13. Relevance assessment for systematic reviews evaluating an eligible KQ 2 outcome

\begin{tabular}{|c|c|c|c|c|c|c|c|c|}
\hline Author, Year & $\begin{array}{l}\text { KQ } 2 \\
\text { Outcome(s) }\end{array}$ & $\begin{array}{l}\text { Was the } \\
\text { database } \\
\text { search } \\
\text { conducted } \\
\text { in } 2012 \text { or } \\
\text { later? }\end{array}$ & $\begin{array}{l}\text { Did the review } \\
\text { include only } \\
\text { studies from } \\
\text { countries ranked } \\
\text { "very high" } \\
\text { developmenta? }\end{array}$ & $\begin{array}{l}\text { Did the review } \\
\text { focus on benefits } \\
\text { and harms of } \\
\text { maternal exposure } \\
\text { to breastfeeding } \\
\text { (or different } \\
\text { intensities/ } \\
\text { duration of BF)? }\end{array}$ & $\begin{array}{l}\text { Did the review } \\
\text { focus on RCTs, } \\
\text { CCTs, cohort } \\
\text { studies, and } \\
\text { case-control } \\
\text { studies? }\end{array}$ & $\begin{array}{l}\text { Did the review } \\
\text { focus only on } \\
\text { relevant health } \\
\text { outcomes }^{\text {b }}\end{array}$ & $\begin{array}{l}\text { Overall, is the } \\
\text { review directly } \\
\text { relevant, } \\
\text { providing an } \\
\text { adequate } \\
\text { answer to KQ } 2 \\
\text { (for one or } \\
\text { more outcome } \\
\text { categories)? }\end{array}$ & $\begin{array}{l}\text { Additional } \\
\text { Comments about } \\
\text { Relevance }\end{array}$ \\
\hline $\begin{array}{l}\text { Anothaisintawee, } \\
2013^{49}\end{array}$ & Breast cancer & $\begin{array}{l}\text { No; last } \\
\text { search date } \\
\text { was January } \\
2011 .\end{array}$ & NA & NA & NA & NA & No & $\begin{array}{l}\text { In addition to } \\
\text { outdated search, only } \\
39 / 69(57 \%) \text { were } \\
\text { from very highly } \\
\text { developed countries, } \\
\text { and this review } \\
\text { primarily relies on } \\
\text { older SRs to identify } \\
\text { relevant studies. }\end{array}$ \\
\hline
\end{tabular}




\begin{tabular}{|c|c|c|c|c|c|c|c|c|}
\hline Author, Year & $\begin{array}{l}\text { KQ } 2 \\
\text { Outcome(s) }\end{array}$ & $\begin{array}{l}\text { Was the } \\
\text { database } \\
\text { search } \\
\text { conducted } \\
\text { in } 2012 \text { or } \\
\text { later? }\end{array}$ & $\begin{array}{l}\text { Did the review } \\
\text { include only } \\
\text { studies from } \\
\text { countries ranked } \\
\text { "very high" } \\
\text { development }{ }^{\text {a? }}\end{array}$ & $\begin{array}{l}\text { Did the review } \\
\text { focus on benefits } \\
\text { and harms of } \\
\text { maternal exposure } \\
\text { to breastfeeding } \\
\text { (or different } \\
\text { intensities/ } \\
\text { duration of BF)? }\end{array}$ & $\begin{array}{l}\text { Did the review } \\
\text { focus on RCTs, } \\
\text { CCTs, cohort } \\
\text { studies, and } \\
\text { case-control } \\
\text { studies? }\end{array}$ & $\begin{array}{l}\text { Did the review } \\
\text { focus only on } \\
\text { relevant health } \\
\text { outcomes }^{\text {b? }}\end{array}$ & $\begin{array}{l}\text { Overall, is the } \\
\text { review directly } \\
\text { relevant, } \\
\text { providing an } \\
\text { adequate } \\
\text { answer to KQ } 2 \\
\text { (for one or } \\
\text { more outcome } \\
\text { categories)? }\end{array}$ & $\begin{array}{l}\text { Additional } \\
\text { Comments about } \\
\text { Relevance }\end{array}$ \\
\hline Aune, $2014^{50}$ & $\begin{array}{l}\text { Type } 2 \\
\text { diabetes }\end{array}$ & $\begin{array}{l}\text { Yes; } \\
\text { PubMed, } \\
\text { Embase and } \\
\text { Ovid } \\
\text { searched } \\
\text { through } \\
\text { September } \\
2013\end{array}$ & $\begin{array}{l}\text { No. 83\% (5/6) } \\
\text { articles are from } \\
\text { U.S., Germany, or } \\
\text { Australia, and one } \\
\text { is from China. The } \\
\text { subgroup analysis } \\
\text { also includes the } \\
\text { Chinese study. }\end{array}$ & $\begin{array}{l}\text { Yes; includes } \\
\text { highest vs. lowest } \\
\text { level of exposure } \\
\text { and duration of } \\
\text { breastfeeding }\end{array}$ & $\begin{array}{l}\text { Includes cohorts } \\
\text { and case control } \\
\text { studies }\end{array}$ & $\begin{array}{l}\text { Yes; type } 2 \\
\text { diabetes }\end{array}$ & Yes & $\begin{array}{l}\text { This study includes } \\
\text { the three studies in } \\
\text { Jager, and then an } \\
\text { additional three. It } \\
\text { doesn't include the } \\
\text { German data from } \\
\text { Jager because those } \\
\text { data came out after } \\
\text { the publication of the } \\
\text { Aune study; it also } \\
\text { excludes cross- } \\
\text { sectional studies, but } \\
\text { it does include } \\
\text { prospective cohort, } \\
\text { case-cohort or nested } \\
\text { case control design. } \\
\text { That could possibly } \\
\text { explain the } \\
\text { discrepancy. Also, } \\
\text { one of the included } \\
\text { studies was entirely } \\
\text { in women in } \\
\text { gestational diabetes, } \\
\text { but we haven't } \\
\text { excluded such } \\
\text { populations for type } 2 \\
\text { either. }\end{array}$ \\
\hline
\end{tabular}




\begin{tabular}{|c|c|c|c|c|c|c|c|c|}
\hline Author, Year & $\begin{array}{l}\text { KQ } 2 \\
\text { Outcome(s) }\end{array}$ & $\begin{array}{l}\text { Was the } \\
\text { database } \\
\text { search } \\
\text { conducted } \\
\text { in } 2012 \text { or } \\
\text { later? }\end{array}$ & $\begin{array}{l}\text { Did the review } \\
\text { include only } \\
\text { studies from } \\
\text { countries ranked } \\
\text { "very high" } \\
\text { development? }\end{array}$ & $\begin{array}{l}\text { Did the review } \\
\text { focus on benefits } \\
\text { and harms of } \\
\text { maternal } \\
\text { exposure to } \\
\text { breastfeeding (or } \\
\text { different } \\
\text { intensities/ } \\
\text { duration of BF)? }\end{array}$ & $\begin{array}{l}\text { Did the review } \\
\text { focus on RCTs, } \\
\text { CCTs, cohort } \\
\text { studies, and } \\
\text { case-control } \\
\text { studies? }\end{array}$ & $\begin{array}{l}\text { Did the review } \\
\text { focus only on } \\
\text { relevant } \\
\text { health } \\
\text { outcomes }^{b} \text { ? }\end{array}$ & $\begin{array}{l}\text { Overall, is the } \\
\text { review directly } \\
\text { relevant, } \\
\text { providing an } \\
\text { adequate } \\
\text { answer to KQ } \\
2 \text { (for one or } \\
\text { more outcome } \\
\text { categories)? }\end{array}$ & $\begin{array}{l}\text { Additional } \\
\text { Comments about } \\
\text { Relevance }\end{array}$ \\
\hline Chowdhury, $2015^{2}$ & $\begin{array}{l}\text { Ovarian and } \\
\text { breast cancer }\end{array}$ & $\begin{array}{l}\text { Yes; } \\
\text { PubMed, } \\
\text { Cochrane, } \\
\text { CABI } \\
\text { searched } \\
\text { through } \\
\text { February } \\
2015\end{array}$ & $\begin{array}{l}\text { No; ovarian CA: } \\
88 \%(35 / 40) \\
\text { according to Table } \\
\text { A3 or } 85 \%(35 / 41) \\
\text { according to Table } \\
2 \text { were from HIC; } \\
1 \text { of the } 41 \\
\text { references is an } \\
\text { SR they } \\
\text { updated. } \\
\text { Breast CA: } 73 \% \\
\text { (72 of } 98 \text { ) were } \\
\text { HIC. } \\
\text { Analyses } \\
\text { presented for HIC } \\
\text { and LIC strata for } \\
\text { both outcomes. }\end{array}$ & $\begin{array}{l}\text { Yes; includes ever } \\
\text { vs. never and } \\
\text { breastfeeding } \\
\text { duration (never, <6 } \\
\text { months, } 6-12 \\
\text { months, }>12 \\
\text { months) }\end{array}$ & $\begin{array}{l}\text { Yes: cohort and } \\
\text { case-control }\end{array}$ & $\begin{array}{l}\text { Yes: Ovarian } \\
\text { and breast } \\
\text { carcinoma (no } \\
\text { other details } \\
\text { provided) }\end{array}$ & $\begin{array}{l}\text { Yes, though } \\
\text { includes some } \\
\text { ineligible } \\
\text { countries }\end{array}$ & $\begin{array}{l}\text { Breast cancer: } 34 \text { of } \\
98 \text { included studies } \\
\text { were previously } \\
\text { included in at least } \\
\text { one of the other } \\
\text { identified SRs } \\
\text { included in the } 2007 \\
\text { AHRQ report. } \\
\text { Ovarian cancer: } 40 \\
\text { of } 41 \text { included } \\
\text { studies are included } \\
\text { by other identified } \\
\text { SRs. }\end{array}$ \\
\hline
\end{tabular}




\begin{tabular}{|c|c|c|c|c|c|c|c|c|}
\hline Author, Year & $\begin{array}{l}\text { KQ } 2 \\
\text { Outcome(s) }\end{array}$ & $\begin{array}{l}\text { Was the } \\
\text { database } \\
\text { search } \\
\text { conducted } \\
\text { in } 2012 \text { or } \\
\text { later? }\end{array}$ & $\begin{array}{l}\text { Did the review } \\
\text { include only } \\
\text { studies from } \\
\text { countries ranked } \\
\text { "very high" } \\
\text { development"? }\end{array}$ & $\begin{array}{l}\text { Did the review } \\
\text { focus on benefits } \\
\text { and harms of } \\
\text { maternal exposure } \\
\text { to breastfeeding } \\
\text { (or different } \\
\text { intensities/ } \\
\text { duration of BF)? }\end{array}$ & $\begin{array}{l}\text { Did the review } \\
\text { focus on RCTs, } \\
\text { CCTs, cohort } \\
\text { studies, and } \\
\text { case-control } \\
\text { studies? }\end{array}$ & $\begin{array}{l}\text { Did the review } \\
\text { focus only on } \\
\text { relevant health } \\
\text { outcomes }^{\text {b? }}\end{array}$ & $\begin{array}{l}\text { Overall, is the } \\
\text { review directly } \\
\text { relevant, } \\
\text { providing an } \\
\text { adequate } \\
\text { answer to KQ } 2 \\
\text { (for one or } \\
\text { more outcome } \\
\text { categories)? }\end{array}$ & $\begin{array}{l}\text { Additional } \\
\text { Comments about } \\
\text { Relevance }\end{array}$ \\
\hline Dias, $2015^{52}$ & Depression & $\begin{array}{l}\text { Yes; Medline, } \\
\text { Web of } \\
\text { Knowledge } \\
\text { and } \\
\text { PsyclnFO } \\
\text { searched } \\
\text { from } 1980 \text { to } \\
\text { Dec } 2013\end{array}$ & $\begin{array}{l}\text { No; } 28 \% \text { (5 of } 18 \\
\text { relevant studies in } \\
\text { Table 2) are from } \\
\text { wrong country } \\
\text { setting and } 40 \% \text { of } \\
\text { studies from Table } \\
3 \text { (7 of 18). }\end{array}$ & $\begin{array}{l}\text { Yes; this review is } \\
\text { also broader and } \\
\text { includes studies } \\
\text { assessing the } \\
\text { association } \\
\text { between } \\
\text { depression during } \\
\text { pregnancy and } \\
\text { rates of BF as well } \\
\text { as other questions } \\
\text { (see comments). }\end{array}$ & $\begin{array}{l}\text { Not well } \\
\text { described; } \\
\text { methods note } \\
\text { "associative } \\
\text { studies" and } \\
\text { "prospective } \\
\text { studies" were } \\
\text { included } \\
\text { regardless of the } \\
\text { study design." }\end{array}$ & $\begin{array}{l}\text { Yes; however, } \\
\text { measures of } \\
\text { "depressive } \\
\text { symptoms" } \\
\text { were also } \\
\text { included (in } \\
\text { addition to } \\
\text { changes in } \\
\text { EPDS scores). } \\
\text { Not clear how } \\
\text { many studies } \\
\text { look at } \\
\text { validated } \\
\text { measures of } \\
\text { depression. }\end{array}$ & $\begin{array}{l}\text { Yes; however, } \\
\text { scope is broader } \\
\text { than our KQ and } \\
\text { presentation of } \\
\text { data my limit } \\
\text { usefulness in } \\
\text { the data } \\
\text { synthesis. }\end{array}$ & $\begin{array}{l}\text { Review includes } \\
\text { studies that address } \\
\text { various questions } \\
\text { related to depression } \\
\text { and breastfeeding; } \\
\text { only a subset of the } \\
\text { results is relevant to } \\
\text { our KQ. }\end{array}$ \\
\hline Feng, $2014^{53}$ & $\begin{array}{l}\text { Ovarian } \\
\text { cancer }\end{array}$ & $\begin{array}{l}\text { Yes; Medline } \\
\text { and Embase } \\
\text { through } \\
\text { December } \\
2012\end{array}$ & $\begin{array}{l}\text { No; } 79 \%(15 / 19) \\
\text { conducted in } \\
\text { developed } \\
\text { countries } \\
\text { No subgroup } \\
\text { analyses by } \\
\text { country setting }\end{array}$ & $\begin{array}{l}\text { Yes; includes ever } \\
\text { (as defined by the } \\
\text { study) vs never and } \\
\text { duration (dose- } \\
\text { response by month) }\end{array}$ & $\begin{array}{l}\text { Yes: cohort and } \\
\text { case-control } \\
\text { (both hospital- } \\
\text { and population- } \\
\text { based) }\end{array}$ & $\begin{array}{l}\text { Yes: Ovarian } \\
\text { cancer } \\
\text { incidence (no } \\
\text { other details } \\
\text { provided) }\end{array}$ & $\begin{array}{l}\text { Yes, though } \\
\text { includes some } \\
\text { ineligible } \\
\text { countries }\end{array}$ & $\begin{array}{l}\text { Quality of included } \\
\text { articles was } \\
\text { assessed according } \\
\text { to Newcastle-Ottawa } \\
\text { Scale. Most studies } \\
\text { were rated high } \\
\text { quality (NOS } 7 \text { or } 8 \text {; } \\
\text { no } 9 \text { ratings) but } \\
\text { there were a handful } \\
\text { of low quality } \\
\text { (NOS=6). }\end{array}$ \\
\hline
\end{tabular}




\begin{tabular}{|c|c|c|c|c|c|c|c|c|}
\hline Author, Year & $\begin{array}{l}\text { KQ } 2 \\
\text { Outcome(s) }\end{array}$ & $\begin{array}{l}\text { Was the } \\
\text { database } \\
\text { search } \\
\text { conducted } \\
\text { in } 2012 \text { or } \\
\text { later? }\end{array}$ & $\begin{array}{l}\text { Did the review } \\
\text { include only } \\
\text { studies from } \\
\text { countries ranked } \\
\text { "very high" } \\
\text { development }{ }^{\mathrm{a}} \text { ? }\end{array}$ & $\begin{array}{l}\text { Did the review } \\
\text { focus on benefits } \\
\text { and harms of } \\
\text { maternal exposure } \\
\text { to breastfeeding } \\
\text { (or different } \\
\text { intensities/ } \\
\text { duration of BF)? }\end{array}$ & $\begin{array}{l}\text { Did the review } \\
\text { focus on RCTs, } \\
\text { CCTs, cohort } \\
\text { studies, and } \\
\text { case-control } \\
\text { studies? }\end{array}$ & $\begin{array}{l}\text { Did the review } \\
\text { focus only on } \\
\text { relevant health } \\
\text { outcomes }^{\text {b? }}\end{array}$ & $\begin{array}{l}\text { Overall, is the } \\
\text { review directly } \\
\text { relevant, } \\
\text { providing an } \\
\text { adequate } \\
\text { answer to KQ } 2 \\
\text { (for one or } \\
\text { more outcome } \\
\text { categories)? }\end{array}$ & $\begin{array}{l}\text { Additional } \\
\text { Comments about } \\
\text { Relevance }\end{array}$ \\
\hline $\mathrm{He}, 2015^{54}$ & PPWR & $\begin{array}{l}\text { Yes; Medline, } \\
\text { Embase, } \\
\text { Cochrane } \\
\text { searched } \\
\text { through Oct } \\
2014\end{array}$ & $\begin{array}{l}\text { No; } 27 \% \text { ( } 3 \text { of } 11 \\
\text { included studies) } \\
\text { were from an } \\
\text { ineligible country } \\
\text { setting. }\end{array}$ & $\begin{array}{l}\text { Yes; meta-analysis } \\
\text { includes } 2 \\
\text { subgroups: } \\
\text { exclusive BF vs. } \\
\text { formula-feeding and } \\
\text { "mixed-feeding" vs. } \\
\text { formula-feeding } \\
\text { (and an overall } \\
\text { summary effect that } \\
\text { includes all studies, } \\
\text { i.e., any BF vs. no } \\
\text { BF presumably). }\end{array}$ & $\begin{array}{l}\text { RCTs and cohort } \\
\text { studies were } \\
\text { included in meta- } \\
\text { analysis but } \\
\text { other study } \\
\text { designs were } \\
\text { identified (see } \\
\text { notes). }\end{array}$ & $\begin{array}{l}\text { Yes; however, } \\
\text { outcomes had } \\
\text { to have data on } \\
\text { weight change } \\
\text { or weight } \\
\text { retention, report } \\
\text { both mean and } \\
\text { SD and include } \\
\text { a measurement } \\
\text { of weight } \\
\text { (rather than } \\
\text { self-reported } \\
\text { weight). }\end{array}$ & $\begin{array}{l}\text { Yes; however, } \\
\text { relatively few } \\
\text { relevant studies } \\
\text { reporting on } \\
\text { weight change } \\
\text { appear to be } \\
\text { included in the } \\
\text { quantitative } \\
\text { analysis. }\end{array}$ & $\begin{array}{l}\text { Authors note } 26 \\
\text { studies met inclusion } \\
\text { criteria but } 15 \text { were } \\
\text { "not eligible for the } \\
\text { meta-analyses"; } \\
\text { these are listed in an } \\
\text { online supplement.c } \\
\text { Three of } 11 \text { studies in } \\
\text { the analysis are from } \\
\text { a noneligible country; } \\
\text { there is a sensitivity } \\
\text { analysis that includes } \\
\text { U.S. studies only (7). }\end{array}$ \\
\hline Islami, $2015^{55}$ & Breast cancer & $\begin{array}{l}\text { Yes: PubMed } \\
\text { and Scopus } \\
\text { through } \\
8 / 2014\end{array}$ & $\begin{array}{l}\text { No; } 79 \% \text { (15 of 19) } \\
\text { case-control } \\
\text { studies set in } \\
\text { highly developed } \\
\text { countries and } \\
100 \%(8 / 8) \\
\text { prospective cohort } \\
\text { studies set in } \\
\text { highly developed } \\
\text { countries. }\end{array}$ & $\begin{array}{l}\text { Yes; included } \\
\text { measures of } \\
\text { ever/never, and } \\
\text { various durations; } \\
\text { in some cases, } \\
\text { studies of shorter } \\
\text { vs. longer duration } \\
\text { were combined with } \\
\text { studies reporting } \\
\text { ever/never. }\end{array}$ & $\begin{array}{l}\text { Yes; prospective } \\
\text { cohort and case- } \\
\text { control studies }\end{array}$ & $\begin{array}{l}\text { Yes: review is } \\
\text { limited to } \\
\text { association } \\
\text { between BF } \\
\text { and specific } \\
\text { cancer } \\
\text { subtypes } \\
\text { defined by } \\
\text { hormone } \\
\text { receptor status } \\
\text { (e.g., luminal, } \\
\text { nonluminal, } \\
\text { triple negative). }\end{array}$ & $\begin{array}{l}\text { Yes; though } \\
\text { includes some } \\
\text { ineligible } \\
\text { countries. }\end{array}$ & $\begin{array}{l}\text { Review is focused on } \\
\text { a subset of the BF } \\
\text { literature (studies } \\
\text { reporting subtypes of } \\
\text { cancer by BF status). }\end{array}$ \\
\hline
\end{tabular}




\begin{tabular}{|c|c|c|c|c|c|c|c|c|}
\hline Author, Year & $\begin{array}{l}\text { KQ } 2 \\
\text { Outcome(s) }\end{array}$ & $\begin{array}{l}\text { Was the } \\
\text { database } \\
\text { search } \\
\text { conducted } \\
\text { in } 2012 \text { or } \\
\text { later? }\end{array}$ & $\begin{array}{l}\text { Did the review } \\
\text { include only } \\
\text { studies from } \\
\text { countries ranked } \\
\text { "very high" } \\
\text { development }{ }^{a} \text { ? }\end{array}$ & $\begin{array}{l}\text { Did the review } \\
\text { focus on benefits } \\
\text { and harms of } \\
\text { maternal exposure } \\
\text { to breastfeeding } \\
\text { (or different } \\
\text { intensities/ } \\
\text { duration of BF)? }\end{array}$ & $\begin{array}{l}\text { Did the review } \\
\text { focus on RCTs, } \\
\text { CCTs, cohort } \\
\text { studies, and } \\
\text { case-control } \\
\text { studies? }\end{array}$ & $\begin{array}{l}\text { Did the review } \\
\text { focus only on } \\
\text { relevant health } \\
\text { outcomes }^{\text {b? }}\end{array}$ & $\begin{array}{l}\text { Overall, is the } \\
\text { review directly } \\
\text { relevant, } \\
\text { providing an } \\
\text { adequate } \\
\text { answer to KQ } 2 \\
\text { (for one or } \\
\text { more outcome } \\
\text { categories)? }\end{array}$ & $\begin{array}{l}\text { Additional } \\
\text { Comments about } \\
\text { Relevance }\end{array}$ \\
\hline Jager, $2014^{56}$ & $\begin{array}{l}\text { Type } 2 \\
\text { diabetes }\end{array}$ & $\begin{array}{l}\text { Yes; PubMed } \\
\text { and Web of } \\
\text { Science } \\
\text { searched } \\
\text { through } \\
\text { March } 2014 \\
\end{array}$ & $\begin{array}{l}\text { No; } 75 \% \text { (3/4 } \\
\text { articles) from U.S. } \\
\text { (2 articles, } 1 \text { study) } \\
\text { and Germany } \\
\text { (Germany data are } \\
\text { not from an article } \\
\text { but are from the } \\
\text { other part of the } \\
\text { article), } 1 \text { article } \\
\text { from China; some } \\
\text { subgroup analyses } \\
\text { exclude the } \\
\text { Shanghai study } \\
\text { and so are in very } \\
\text { high developed. }\end{array}$ & $\begin{array}{l}\text { Yes; includes both } \\
\text { yes/no and duration } \\
\text { of breastfeeding }\end{array}$ & $\begin{array}{l}\text { Not very clear, } \\
\text { but appears to } \\
\text { have included } \\
\text { prospective } \\
\text { cohort studies } \\
\text { only. }\end{array}$ & $\begin{array}{l}\text { Yes; type } 2 \\
\text { diabetes }\end{array}$ & Yes & $\begin{array}{l}\text { Although the article } \\
\text { doesn't say so } \\
\text { explicitly, the } \\
\text { PRISMA figure } \\
\text { makes clear that they } \\
\text { excluded noncohort } \\
\text { studies. }\end{array}$ \\
\hline Jiang, $2017^{57}$ & $\begin{array}{l}\text { Osteoporotic } \\
\text { fractures }\end{array}$ & Yes & No & Yes & Yes & Yes & Yes & $\begin{array}{l}3 \text { of } 12 \text { included } \\
\text { studies are not from } \\
\text { very highly developed } \\
\text { countries ( } 2 \text { China, } 1 \\
\text { Mexico) but subgroup } \\
\text { analysis is presented } \\
\text { in supplemental } \\
\text { material. }\end{array}$ \\
\hline $\begin{array}{l}\text { Lambertini, } 2016^{58,} \\
59\end{array}$ & Breast cancer & $\begin{array}{l}\text { Yes: PubMed } \\
\text { and Embase } \\
\text { through } \\
\text { 10/2014 }\end{array}$ & $\begin{array}{l}\text { No; } 91 \% \text { (10 of 11) } \\
\text { were from the U.S. } \\
\text { and Japan (1 } \\
\text { study was from } \\
\text { China). Separate } \\
\text { analyses } \\
\text { performed for } \\
\text { studies set in U.S. } \\
\text { and Asia. }\end{array}$ & $\begin{array}{l}\text { Yes; focused on } \\
\text { measures of } \\
\text { ever/never }\end{array}$ & $\begin{array}{l}\text { Yes: cohort and } \\
\text { case control } \\
\text { studies were } \\
\text { included (and } 2 \\
\text { pooled analyses } \\
\text { of cohort/ case- } \\
\text { control studies). }\end{array}$ & $\begin{array}{l}\text { Yes; Breast } \\
\text { cancer } \\
\text { subtypes } \\
\text { defined by } \\
\text { hormone } \\
\text { receptor status } \\
\text { (e.g., Luminal, } \\
\text { HER2, triple } \\
\text { negative). }\end{array}$ & $\begin{array}{l}\text { Yes; though } \\
\text { includes one } \\
\text { study from } \\
\text { China and a few } \\
\text { pooled } \\
\text { analyses. }\end{array}$ & None \\
\hline
\end{tabular}




\begin{tabular}{|c|c|c|c|c|c|c|c|c|}
\hline Author, Year & $\begin{array}{l}\text { KQ } 2 \\
\text { Outcome(s) }\end{array}$ & $\begin{array}{l}\text { Was the } \\
\text { database } \\
\text { search } \\
\text { conducted } \\
\text { in } 2012 \text { or } \\
\text { later? }\end{array}$ & $\begin{array}{l}\text { Did the review } \\
\text { include only } \\
\text { studies from } \\
\text { countries ranked } \\
\text { "very high" } \\
\text { development }{ }^{a} \text { ? }\end{array}$ & $\begin{array}{l}\text { Did the review } \\
\text { focus on benefits } \\
\text { and harms of } \\
\text { maternal exposure } \\
\text { to breastfeeding } \\
\text { (or different } \\
\text { intensities/ } \\
\text { duration of BF)? }\end{array}$ & $\begin{array}{l}\text { Did the review } \\
\text { focus on RCTs, } \\
\text { CCTs, cohort } \\
\text { studies, and } \\
\text { case-control } \\
\text { studies? }\end{array}$ & $\begin{array}{l}\text { Did the review } \\
\text { focus only on } \\
\text { relevant health } \\
\text { outcomes }^{\text {b? }}\end{array}$ & $\begin{array}{l}\text { Overall, is the } \\
\text { review directly } \\
\text { relevant, } \\
\text { providing an } \\
\text { adequate } \\
\text { answer to KQ } 2 \\
\text { (for one or } \\
\text { more outcome } \\
\text { categories)? }\end{array}$ & $\begin{array}{l}\text { Additional } \\
\text { Comments about } \\
\text { Relevance }\end{array}$ \\
\hline $\mathrm{Li}, 2014^{60}$ & $\begin{array}{l}\text { Ovarian } \\
\text { cancer }\end{array}$ & $\begin{array}{l}\text { Yes; Medline } \\
\text { and Embase } \\
\text { through } \\
\text { March } 2013\end{array}$ & $\begin{array}{l}\text { No; 83\% (33/40) } \\
\text { conducted in } \\
\text { developed } \\
\text { countries } \\
\text { Subgroup } \\
\text { analyses } \\
\text { presented for } \\
\text { North American } \\
\text { (19), Asian (10), } \\
\text { European (9), } \\
\text { Australian (2) } \\
\text { populations }\end{array}$ & $\begin{array}{l}\text { Yes; includes ever } \\
\text { vs never; total } \\
\text { breastfeeding } \\
\text { duration in months } \\
(<6 ; 6-12 ;>12 ; \\
>24) \text {, and average } \\
\text { breastfeeding } \\
\text { duration in months } \\
(<6 ; 6-12 ;>12)\end{array}$ & $\begin{array}{l}\text { Yes: cohort and } \\
\text { case-control } \\
\text { (both hospital- } \\
\text { and population- } \\
\text { based) }\end{array}$ & $\begin{array}{l}\text { Yes: Ovarian } \\
\text { cancer } \\
\text { confirmed by } \\
\text { histology } \\
\text { Subgroup } \\
\text { analyses } \\
\text { presented for } \\
\text { invasive and } \\
\text { borderline } \\
\text { ovarian tumors, } \\
\text { and histologic } \\
\text { subtype } \\
\text { (serous, } \\
\text { mucinous, } \\
\text { endometrioid/ } \\
\text { clear cell). }\end{array}$ & $\begin{array}{l}\text { Yes, though } \\
\text { includes some } \\
\text { ineligible } \\
\text { countries }\end{array}$ & $\begin{array}{l}\text { Quality of included } \\
\text { articles was } \\
\text { assessed according } \\
\text { to Newcastle-Ottawa } \\
\text { Scale. Approximately } \\
2 / 3 \text { of studies were } \\
\text { considered high } \\
\text { quality, but only } 12 \\
\text { studies had a NOS } \\
\text { score of } 7 \text {; remaining } \\
\text { NOS scores ranged } \\
\text { from } 4 \text { to } 6 \text {. }\end{array}$ \\
\hline Luan, $2013^{51}$ & $\begin{array}{l}\text { Ovarian } \\
\text { cancer }\end{array}$ & $\begin{array}{l}\text { Yes; Medline } \\
\text { through } \\
\text { December } \\
2012\end{array}$ & $\begin{array}{l}\text { No; 86\% (30/35) } \\
\text { conducted in } \\
\text { developed } \\
\text { countries } \\
\text { Subgroup } \\
\text { analyses } \\
\text { presented for } \\
\text { Asian, American, } \\
\text { and European } \\
\text { study populations. }\end{array}$ & $\begin{array}{l}\text { Yes; includes } \\
\text { measures of ever } \\
\text { vs. never and total } \\
\text { duration of } \\
\text { breastfeeding } \\
\text { (longest compared } \\
\text { with shortest } \\
\text { categories). }\end{array}$ & $\begin{array}{l}\text { Yes: cohort and } \\
\text { case-control }\end{array}$ & $\begin{array}{l}\text { Yes: Incident } \\
\text { epithelial } \\
\text { ovarian cancer } \\
\text { Subgroup } \\
\text { analyses } \\
\text { presented for } \\
\text { invasive and } \\
\text { borderline } \\
\text { tumors and by } \\
\text { histology } \\
\text { (serous, } \\
\text { mucinous, } \\
\text { endometrioid, } \\
\text { clear cell) }\end{array}$ & $\begin{array}{l}\text { Yes, though } \\
\text { includes some } \\
\text { ineligible } \\
\text { countries. }\end{array}$ & $\begin{array}{l}\text { No quality } \\
\text { assessment; relied } \\
\text { on numerous } \\
\text { subgroup and } \\
\text { sensitivity analyses. }\end{array}$ \\
\hline
\end{tabular}




\begin{tabular}{|c|c|c|c|c|c|c|c|c|}
\hline Author, Year & $\begin{array}{l}\text { KQ } 2 \\
\text { Outcome(s) }\end{array}$ & $\begin{array}{l}\text { Was the } \\
\text { database } \\
\text { search } \\
\text { conducted } \\
\text { in } 2012 \text { or } \\
\text { later? }\end{array}$ & $\begin{array}{l}\text { Did the review } \\
\text { include only } \\
\text { studies from } \\
\text { countries ranked } \\
\text { "very high" } \\
\text { development }{ }^{a} \text { ? }\end{array}$ & $\begin{array}{l}\text { Did the review } \\
\text { focus on benefits } \\
\text { and harms of } \\
\text { maternal exposure } \\
\text { to breastfeeding } \\
\text { (or different } \\
\text { intensities/ } \\
\text { duration of BF)? }\end{array}$ & $\begin{array}{l}\text { Did the review } \\
\text { focus on RCTs, } \\
\text { CCTs, cohort } \\
\text { studies, and } \\
\text { case-control } \\
\text { studies? }\end{array}$ & $\begin{array}{l}\text { Did the review } \\
\text { focus only on } \\
\text { relevant health } \\
\text { outcomes }^{\text {b? }}\end{array}$ & $\begin{array}{l}\text { Overall, is the } \\
\text { review directly } \\
\text { relevant, } \\
\text { providing an } \\
\text { adequate } \\
\text { answer to KQ } 2 \\
\text { (for one or } \\
\text { more outcome } \\
\text { categories)? }\end{array}$ & $\begin{array}{l}\text { Additional } \\
\text { Comments about } \\
\text { Relevance }\end{array}$ \\
\hline Neville, $2014^{61}$ & $\begin{array}{l}\text { Postpartum } \\
\text { weight change }\end{array}$ & $\begin{array}{l}\text { Yes; } 8 \\
\text { databases } \\
\text { through June } \\
2012\end{array}$ & $\begin{array}{l}\text { Most likely; criteria } \\
\text { state studies } \\
\text { conducted in } \\
\text { "developing } \\
\text { countries" were } \\
\text { excluded. } 32 \\
\text { included studies } \\
\text { were from U.S., } \\
\text { UK, Europe, } \\
\text { Japan, } \\
\text { Taiwan/Hong } \\
\text { Kong, Canada and } \\
\text { Australia. }\end{array}$ & $\begin{array}{l}\text { Yes; results } \\
\text { reported by } \\
\text { exposure (including } \\
\text { separately for } \\
\text { studies reporting on } \\
\text { measures of } \\
\text { ever/never, vs. both } \\
\text { intensity and } \\
\text { duration of BF). }\end{array}$ & $\begin{array}{l}\text { Yes; obs. studies } \\
\text { were included } \\
\text { (categorized as } \\
\text { prospective vs. } \\
\text { retrospective); } \\
\text { case-control } \\
\text { studies appear to } \\
\text { be excluded. }\end{array}$ & $\begin{array}{l}\text { Yes; review } \\
\text { included any } \\
\text { measure of } \\
\text { postpartum } \\
\text { weight change } \\
\text { (measured and } \\
\text { self-reported) } \\
\text { but also other } \\
\text { measures of } \\
\text { body mass we } \\
\text { would exclude } \\
\text { (i.e., lean body } \\
\text { mass, fat-free } \\
\text { mass). } \\
\end{array}$ & $\begin{array}{l}\text { Yes; however, } \\
\text { presentation of } \\
\text { data my limit } \\
\text { usefulness in } \\
\text { the evidence } \\
\text { synthesis (see } \\
\text { comments). }\end{array}$ & $\begin{array}{l}\text { No meta-analyses } \\
\text { were performed. } \\
\text { Main table is a } \\
\text { summary and does } \\
\text { not include results of } \\
\text { weight change } \\
\text { measures. Results } \\
\text { are in an online } \\
\text { appendix; however, } \\
\text { data presentation is } \\
\text { difficult to interpret. }\end{array}$ \\
\hline Pan, $2013^{62}$ & Breast cancer & $\begin{array}{l}\text { Yes; PubMed } \\
\text { searched } \\
\text { through } \\
2 / 2013\end{array}$ & $\begin{array}{l}\text { Yes; all } 3 \text { included } \\
\text { studies enrolled } \\
\text { women from highly } \\
\text { developed } \\
\text { countries. }\end{array}$ & $\begin{array}{l}\text { Yes; ever/never } \\
\text { and longer vs. } \\
\text { shorter duration of } \\
\text { BF }\end{array}$ & $\begin{array}{l}\text { Yes; cohort } \\
\text { studies (including } \\
\text { pooled data from } \\
\text { a consortium of } \\
\text { observational } \\
\text { studies) and } \\
\text { case-control } \\
\text { studies. }\end{array}$ & $\begin{array}{l}\text { Yes: Breast } \\
\text { cancer (no } \\
\text { other details } \\
\text { provided) }\end{array}$ & Yes & $\begin{array}{l}\text { Only } 3 \text { included } \\
\text { studies included ( } 2 \text { of } \\
\text { those studies include } \\
\text { women from various } \\
\text { eligible, countries; } \\
\text { this may not be } \\
\text { surprising since } \\
\text { genetic testing is } \\
\text { likely more often } \\
\text { done in developed } \\
\text { countries. }\end{array}$ \\
\hline Sung, $2016^{63}$ & $\begin{array}{l}\text { Ovarian } \\
\text { cancer }\end{array}$ & Yes & Yes & Yes & Yes & Yes & Yes & None \\
\hline
\end{tabular}




\begin{tabular}{|c|c|c|c|c|c|c|c|c|}
\hline Author, Year & $\begin{array}{l}\text { KQ } 2 \\
\text { Outcome(s) }\end{array}$ & $\begin{array}{l}\text { Was the } \\
\text { database } \\
\text { search } \\
\text { conducted } \\
\text { in } 2012 \text { or } \\
\text { later? }\end{array}$ & $\begin{array}{l}\text { Did the review } \\
\text { include only } \\
\text { studies from } \\
\text { countries ranked } \\
\text { "very high" } \\
\text { development"? }\end{array}$ & $\begin{array}{l}\text { Did the review } \\
\text { focus on benefits } \\
\text { and harms of } \\
\text { maternal exposure } \\
\text { to breastfeeding } \\
\text { (or different } \\
\text { intensities/ } \\
\text { duration of BF)? }\end{array}$ & $\begin{array}{l}\text { Did the review } \\
\text { focus on RCTs, } \\
\text { CCTs, cohort } \\
\text { studies, and } \\
\text { case-control } \\
\text { studies? }\end{array}$ & $\begin{array}{l}\text { Did the review } \\
\text { focus only on } \\
\text { relevant health } \\
\text { outcomes }^{\text {b? }}\end{array}$ & $\begin{array}{l}\text { Overall, is the } \\
\text { review directly } \\
\text { relevant, } \\
\text { providing an } \\
\text { adequate } \\
\text { answer to KQ } 2 \\
\text { (for one or } \\
\text { more outcome } \\
\text { categories)? }\end{array}$ & $\begin{array}{l}\text { Additional } \\
\text { Comments about } \\
\text { Relevance }\end{array}$ \\
\hline $\begin{array}{l}\text { Unar-Munguia, } \\
2017^{64}\end{array}$ & Breast cancer & Yes & No & Yes & Yes & Yes & No & $\begin{array}{l}28(43 \%) \text { of included } \\
\text { studies were from } \\
\text { Asia, } 4(6 \%) \text { were } \\
\text { from Africa, and } 5 \\
(8 \%) \text { were from Latin } \\
\text { America. There is no } \\
\text { list that provides } \\
\text { country but it's likely } \\
\text { that a majority of the } \\
\text { included studies are } \\
\text { not from very highly } \\
\text { development } \\
\text { countries. }\end{array}$ \\
\hline Zhou, $2015^{65}$ & Breast cancer & $\begin{array}{l}\text { Yes; PubMed } \\
\text { searched } \\
\text { from 1/2008 } \\
\text { through } \\
7 / 2014\end{array}$ & $\begin{array}{l}\text { No; } 19 \% \text { (5 of } 27 \\
\text { studies) were from } \\
\text { highly developed } \\
\text { countries; most } \\
\text { were from Middle } \\
\text { Eastern or } \\
\text { Western Asian } \\
\text { countries. }\end{array}$ & $\begin{array}{l}\text { Yes; included } \\
\text { Ever/Never and } \\
\text { Longest/shortest } \\
\text { duration (of total or } \\
\text { average duration) } \\
\text { comparisons. }\end{array}$ & $\begin{array}{l}\text { Yes: cohort and } \\
\text { case-control, } \\
\text { though one of } \\
\text { the included } \\
\text { studies is } \\
\text { classified as } \\
\text { cross-sectional. }\end{array}$ & $\begin{array}{l}\text { Yes; Breast } \\
\text { Cancer (no } \\
\text { other details } \\
\text { provided) }\end{array}$ & $\begin{array}{l}\text { No; a large } \\
\text { majority of the } \\
\text { studies are from } \\
\text { countries that } \\
\text { would not be } \\
\text { eligible for our } \\
\text { review. }\end{array}$ & $\begin{array}{l}\text { Consider hand- } \\
\text { searching this review } \\
\text { to ensure that we've } \\
\text { captured the included } \\
\text { studies that would } \\
\text { meet our country } \\
\text { eligibility criteria. }\end{array}$ \\
\hline
\end{tabular}

\section{No subgroup \\ analysis by \\ country setting.}

a Very high: Andorra, Argentina, Australia, Austria, Bahrain, Belgium, Brunei Darussalam, Canada, Chile, Croatia, Cyprus, Czech Republic, Denmark, Estonia, Finland, France,

Germany, Greece, Hong Kong, China (SAR), Hungary, Iceland, Ireland, Israel, Italy, Japan, Korea (Republic of), Kuwait, Latvia, Liechtenstein, Lithuania, Luxembourg, Malta,

Montenegro, Netherlands, New Zealand, Norway, Poland, Portugal, Qatar, Saudi Arabia, Singapore, Slovakia, Slovenia, Spain, Sweden, Switzerland, Taiwan, United Arab

Emirates, United Kingdom, United States

bostpartum depression, breast cancer, ovarian cancer, osteoporosis, cardiovascular (CVD) outcomes [e.g., stroke, myocardial infarction (MI)], postpartum weight change, type 2

diabetes, hypertension

${ }^{\mathrm{C}}$ Reasons for not being included in meta-analyses include no reporting of PPWR and no reporting of a standard deviation. Not clear whether authors tried to calculate these

measures when individual studies reported other measures of weight change. 
${ }^{\mathrm{d}}$ Reasons we did not formally consider the relevance of Chowdhury for other outcomes we included: (1) Osteoporosis: Six studies (two from LMICs) countries were included.

Only BMD was an included outcome (not fracture incidence). Chowdhury pooled four studies reporting on femoral neck and distal radius BMD. No description of study design, timing of BMD measurement, etc. At least one from HIC is a case-control study of BMD and BF (these were excluded by 2007 review). (2) Diabetes: No new studies; describes results from Aune et. al. (3) Postpartum Depression: No new studies; describes results from Dias et. al. (4) Postpartum weight change: Describes results from Neville et. al and five additional studies (no meta-analysis).

eHigh Income Country (HIC) setting defined according to 2014 World Bank data (add citation from Choudhry).

AHRQ = Agency for Healthcare Research and Quality; BF = breastfeeding; CA = cancer; CABI = Centre for Agriculture and Biosciences International; CCT = controlled clinical trial; CVD = cardiovascular; EPDS = Edinburgh Postnatal Depression Scale; HER2 = human epidermal growth factor receptor 2; HIC = High Income Country; KQ = Key Question; LIC = Low Income Country; MI = myocardial infarction; NA = not applicable; NOS = Newcastle-Ottawa Scale; obs = observational; PPWR = postpartum weigh retention; PRISMA = Preferred Reporting Items for Systematic Reviews and Meta- Analyses; RCT = randomized controlled trial; SD = standard deviation; SR = systematic review; UK = United Kingdom; U.S.= United States. 
Table C-14. KQ 2 risk of bias assessment: Systematic reviews, part 1

\begin{tabular}{|c|c|c|c|c|c|c|c|c|c|}
\hline $\begin{array}{l}\text { Author, Year } \\
\text { Health } \\
\text { Outcome }\end{array}$ & $\begin{array}{l}\text { Overall Risk } \\
\text { of Bias } \\
\text { Rating }\end{array}$ & $\begin{array}{l}\text { Overall } \\
\text { Rationale for } \\
\text { Risk of Bias } \\
\text { Rating }\end{array}$ & $\begin{array}{l}1.1 \text { Did the } \\
\text { review } \\
\text { adhere to } \\
\text { pre-defined } \\
\text { objectives } \\
\text { and } \\
\text { eligibility } \\
\text { criteria? }\end{array}$ & $\begin{array}{l}1.2 \text { Were the } \\
\text { eligibility } \\
\text { criteria } \\
\text { appropriate } \\
\text { for the review } \\
\text { question? }\end{array}$ & $\begin{array}{l}1.3 \text { Were } \\
\text { eligibility } \\
\text { criteria } \\
\text { unambi- } \\
\text { guous? }\end{array}$ & $\begin{array}{l}\text { 1.4 Were all } \\
\text { restrictions in } \\
\text { eligibility criteria } \\
\text { based on study } \\
\text { characteristics } \\
\text { appropriate (e.g., } \\
\text { date, sample } \\
\text { size, study } \\
\text { quality, } \\
\text { outcomes } \\
\text { measured)? }\end{array}$ & $\begin{array}{l}1.5 \text { Were any } \\
\text { restrictions in } \\
\text { eligibility } \\
\text { criteria based } \\
\text { on sources of } \\
\text { information } \\
\text { appropriate } \\
\text { (e.g., } \\
\text { publication } \\
\text { status or } \\
\text { format, } \\
\text { language, } \\
\text { availability of } \\
\text { data)? }\end{array}$ & $\begin{array}{l}\text { Concerns } \\
\text { Regarding } \\
\text { Specifi- } \\
\text { Cation of } \\
\text { Study } \\
\text { Eligibility } \\
\text { Criteria }\end{array}$ & $\begin{array}{l}\text { Rationale for } \\
\text { Concern }\end{array}$ \\
\hline $\begin{array}{l}\text { Aune, } 2014^{50} \\
\text { Diabetes }\end{array}$ & Low & $\begin{array}{l}\text { Although no grey } \\
\text { literature or } \\
\text { unpublished } \\
\text { studies were } \\
\text { included, the } \\
\text { potential for bias } \\
\text { from other } \\
\text { sources is low. }\end{array}$ & Yes & Yes & Yes & Yes & Yes & Low & NA \\
\hline
\end{tabular}




\begin{tabular}{|c|c|c|c|c|c|c|c|c|c|}
\hline $\begin{array}{l}\text { Author, Year } \\
\text { Health } \\
\text { Outcome }\end{array}$ & $\begin{array}{l}\text { Overall Risk } \\
\text { of Bias } \\
\text { Rating }\end{array}$ & $\begin{array}{l}\text { Overall } \\
\text { Rationale for } \\
\text { Risk of Bias } \\
\text { Rating }\end{array}$ & $\begin{array}{l}1.1 \text { Did the } \\
\text { review } \\
\text { adhere to } \\
\text { pre-defined } \\
\text { objectives } \\
\text { and } \\
\text { eligibility } \\
\text { criteria? }\end{array}$ & $\begin{array}{l}1.2 \text { Were the } \\
\text { eligibility } \\
\text { criteria } \\
\text { appropriate } \\
\text { for the review } \\
\text { question? }\end{array}$ & $\begin{array}{l}1.3 \text { Were } \\
\text { eligibility } \\
\text { criteria } \\
\text { unambi- } \\
\text { guous? }\end{array}$ & $\begin{array}{l}1.4 \text { Were all } \\
\text { restrictions in } \\
\text { eligibility criteria } \\
\text { based on study } \\
\text { characteristics } \\
\text { appropriate (e.g., } \\
\text { date, sample } \\
\text { size, study } \\
\text { quality, } \\
\text { outcomes } \\
\text { measured)? }\end{array}$ & $\begin{array}{l}\text { 1.5 Were any } \\
\text { restrictions in } \\
\text { eligibility } \\
\text { criteria based } \\
\text { on sources of } \\
\text { information } \\
\text { appropriate } \\
\text { (e.g., } \\
\text { publication } \\
\text { status or } \\
\text { format, } \\
\text { language, } \\
\text { availability of } \\
\text { data)? }\end{array}$ & $\begin{array}{l}\text { Concerns } \\
\text { Regarding } \\
\text { Specifi- } \\
\text { Cation of } \\
\text { Study } \\
\text { Eligibility } \\
\text { Criteria }\end{array}$ & $\begin{array}{l}\text { Rationale for } \\
\text { Concern }\end{array}$ \\
\hline $\begin{array}{l}\text { Chowdhury, } \\
2015^{2} \\
\text { Breast and } \\
\text { ovarian cancer }\end{array}$ & Unclear & $\begin{array}{l}\text { Little description } \\
\text { of individual } \\
\text { studies is } \\
\text { provided; no } \\
\text { other methods } \\
\text { were used to } \\
\text { identify } \\
\text { unpublished } \\
\text { articles; analyses } \\
\text { (funnel plot) } \\
\text { showed evidence } \\
\text { of publication } \\
\text { bias for breast } \\
\text { cancer. Unclear } \\
\text { whether this is a } \\
\text { potential bias for } \\
\text { the group of } \\
\text { studies relevant } \\
\text { for our review. } \\
\text { Whether data } \\
\text { abstraction and } \\
\text { ROB were dually } \\
\text { assessed is not } \\
\text { clear. }\end{array}$ & Yes & Yes & $\begin{array}{l}\text { Probably } \\
\text { yes }\end{array}$ & Probably yes & Yes & Low & $\begin{array}{l}\text { Excluded non- } \\
\text { English studies; } \\
\text { authors note that } \\
\text { RCTs were } \\
\text { eligible but do } \\
\text { not explain } \\
\text { whether they } \\
\text { would have } \\
\text { included RCTs } \\
\text { assessing BF } \\
\text { interventions } \\
\text { that report on } \\
\text { health } \\
\text { outcomes. All } \\
\text { included studies } \\
\text { appear to be } \\
\text { observational. }\end{array}$ \\
\hline
\end{tabular}




\begin{tabular}{|c|c|c|c|c|c|c|c|c|c|}
\hline $\begin{array}{l}\text { Author, Year } \\
\text { Health } \\
\text { Outcome }\end{array}$ & $\begin{array}{l}\text { Overall Risk } \\
\text { of Bias } \\
\text { Rating }\end{array}$ & $\begin{array}{l}\text { Overall } \\
\text { Rationale for } \\
\text { Risk of Bias } \\
\text { Rating }\end{array}$ & $\begin{array}{l}1.1 \text { Did the } \\
\text { review } \\
\text { adhere to } \\
\text { pre-defined } \\
\text { objectives } \\
\text { and } \\
\text { eligibility } \\
\text { criteria? }\end{array}$ & $\begin{array}{l}1.2 \text { Were the } \\
\text { eligibility } \\
\text { criteria } \\
\text { appropriate } \\
\text { for the review } \\
\text { question? }\end{array}$ & $\begin{array}{l}1.3 \text { Were } \\
\text { eligibility } \\
\text { criteria } \\
\text { unambi- } \\
\text { guous? }\end{array}$ & $\begin{array}{l}1.4 \text { Were all } \\
\text { restrictions in } \\
\text { eligibility criteria } \\
\text { based on study } \\
\text { characteristics } \\
\text { appropriate (e.g., } \\
\text { date, sample } \\
\text { size, study } \\
\text { quality, } \\
\text { outcomes } \\
\text { measured)? }\end{array}$ & $\begin{array}{l}1.5 \text { Were any } \\
\text { restrictions in } \\
\text { eligibility } \\
\text { criteria based } \\
\text { on sources of } \\
\text { information } \\
\text { appropriate } \\
\text { (e.g., } \\
\text { publication } \\
\text { status or } \\
\text { format, } \\
\text { language, } \\
\text { availability of } \\
\text { data)? }\end{array}$ & $\begin{array}{l}\text { Concerns } \\
\text { Regarding } \\
\text { Specifi- } \\
\text { Cation of } \\
\text { Study } \\
\text { Eligibility } \\
\text { Criteria }\end{array}$ & $\begin{array}{l}\text { Rationale for } \\
\text { Concern }\end{array}$ \\
\hline $\begin{array}{l}\text { Dias, } 2015^{52} \\
\text { Postpartum } \\
\text { depression }\end{array}$ & Unclear & $\begin{array}{l}\text { See individual } \\
\text { domains; no } \\
\text { assessment for } \\
\text { included studies. } \\
\text { Characteristics of } \\
\text { studies are not } \\
\text { well desribed and } \\
\text { syntheses } \\
\text { focuses on } \\
\text { number of } \\
\text { studies that found } \\
\text { a postiive } \\
\text { association } \\
\text { grouped by study } \\
\text { design. }\end{array}$ & Yes & Yes & Yes & Probably yes & Probably yes & Low & NA \\
\hline
\end{tabular}




\begin{tabular}{|c|c|c|c|c|c|c|c|c|c|}
\hline $\begin{array}{l}\text { Author, Year } \\
\text { Health } \\
\text { Outcome }\end{array}$ & $\begin{array}{l}\text { Overall Risk } \\
\text { of Bias } \\
\text { Rating }\end{array}$ & $\begin{array}{l}\text { Overall } \\
\text { Rationale for } \\
\text { Risk of Bias } \\
\text { Rating }\end{array}$ & $\begin{array}{l}1.1 \text { Did the } \\
\text { review } \\
\text { adhere to } \\
\text { pre-defined } \\
\text { objectives } \\
\text { and } \\
\text { eligibility } \\
\text { criteria? }\end{array}$ & $\begin{array}{l}1.2 \text { Were the } \\
\text { eligibility } \\
\text { criteria } \\
\text { appropriate } \\
\text { for the review } \\
\text { question? }\end{array}$ & $\begin{array}{l}1.3 \text { Were } \\
\text { eligibility } \\
\text { criteria } \\
\text { unambi- } \\
\text { guous? }\end{array}$ & $\begin{array}{l}1.4 \text { Were all } \\
\text { restrictions in } \\
\text { eligibility criteria } \\
\text { based on study } \\
\text { characteristics } \\
\text { appropriate (e.g., } \\
\text { date, sample } \\
\text { size, study } \\
\text { quality, } \\
\text { outcomes } \\
\text { measured)? }\end{array}$ & $\begin{array}{l}\text { 1.5 Were any } \\
\text { restrictions in } \\
\text { eligibility } \\
\text { criteria based } \\
\text { on sources of } \\
\text { information } \\
\text { appropriate } \\
\text { (e.g., } \\
\text { publication } \\
\text { status or } \\
\text { format, } \\
\text { language, } \\
\text { availability of } \\
\text { data)? }\end{array}$ & $\begin{array}{l}\text { Concerns } \\
\text { Regarding } \\
\text { Specifi- } \\
\text { Cation of } \\
\text { Study } \\
\text { Eligibility } \\
\text { Criteria }\end{array}$ & $\begin{array}{l}\text { Rationale for } \\
\text { Concern }\end{array}$ \\
\hline $\begin{array}{l}\text { Feng, } 2014^{53} \\
\text { Ovarian } \\
\text { cancer }\end{array}$ & High & $\begin{array}{l}\text { See individual } \\
\text { domains. Authors } \\
\text { strongly } \\
\text { suggested that } \\
\text { their findings } \\
\text { were supportive } \\
\text { of multiple } \\
\text { breastfeeding } \\
\text { guidelines due to } \\
\text { their dose- } \\
\text { response } \\
\text { analysis; authors } \\
\text { acknowledged } \\
\text { the substantial } \\
\text { heterogeneity but } \\
\text { said they didn't } \\
\text { enough data to } \\
\text { explore further, } \\
\text { which is not true } \\
\text { based on the } \\
\text { study } \\
\text { characteristics } \\
\text { they provided. }\end{array}$ & Yes & Yes & Yes & Probably yes & Probably no & Unclear & $\begin{array}{l}\text { Authors note } \\
\text { that studies had } \\
\text { to report a } \\
\text { measure of } \\
\text { effect (ORs) and } \\
95 \% \text { Cl: unclear } \\
\text { if some relevant } \\
\text { studies might } \\
\text { have been } \\
\text { excluded due to } \\
\text { availability of } \\
\text { data (e.g., no Cl } \\
\text { reported) and } \\
\text { not clear } \\
\text { whether authors } \\
\text { attempted to } \\
\text { calculate } \\
\text { measures (or } \\
\text { obtained from } \\
\text { authors of } \\
\text { individual } \\
\text { studies). Limited } \\
\text { to English- } \\
\text { language } \\
\text { studies. }\end{array}$ \\
\hline
\end{tabular}




\begin{tabular}{|c|c|c|c|c|c|c|c|c|c|}
\hline $\begin{array}{l}\text { Author, Year } \\
\text { Health } \\
\text { Outcome }\end{array}$ & $\begin{array}{l}\text { Overall Risk } \\
\text { of Bias } \\
\text { Rating }\end{array}$ & $\begin{array}{l}\text { Overall } \\
\text { Rationale for } \\
\text { Risk of Bias } \\
\text { Rating }\end{array}$ & $\begin{array}{l}1.1 \text { Did the } \\
\text { review } \\
\text { adhere to } \\
\text { pre-defined } \\
\text { objectives } \\
\text { and } \\
\text { eligibility } \\
\text { criteria? }\end{array}$ & $\begin{array}{l}1.2 \text { Were the } \\
\text { eligibility } \\
\text { criteria } \\
\text { appropriate } \\
\text { for the review } \\
\text { question? }\end{array}$ & $\begin{array}{l}1.3 \text { Were } \\
\text { eligibility } \\
\text { criteria } \\
\text { unambi- } \\
\text { guous? }\end{array}$ & $\begin{array}{l}\text { 1.4 Were all } \\
\text { restrictions in } \\
\text { eligibility criteria } \\
\text { based on study } \\
\text { characteristics } \\
\text { appropriate (e.g., } \\
\text { date, sample } \\
\text { size, study } \\
\text { quality, } \\
\text { outcomes } \\
\text { measured)? }\end{array}$ & $\begin{array}{l}1.5 \text { Were any } \\
\text { restrictions in } \\
\text { eligibility } \\
\text { criteria based } \\
\text { on sources of } \\
\text { information } \\
\text { appropriate } \\
\text { (e.g., } \\
\text { publication } \\
\text { status or } \\
\text { format, } \\
\text { language, } \\
\text { availability of } \\
\text { data)? }\end{array}$ & $\begin{array}{l}\text { Concerns } \\
\text { Regarding } \\
\text { Specifi- } \\
\text { Cation of } \\
\text { Study } \\
\text { Eligibility } \\
\text { Criteria }\end{array}$ & $\begin{array}{l}\text { Rationale for } \\
\text { Concern }\end{array}$ \\
\hline $\begin{array}{l}\text { He, } 2015^{54} \\
\text { Postpartum } \\
\text { weight change }\end{array}$ & Unclear & $\begin{array}{l}\text { See domain } \\
\text { specific } \\
\text { comments; no } \\
\text { dual assessment } \\
\text { of data } \\
\text { abstraction. } \\
\text { Unclear whether } \\
\text { results of studies } \\
\text { not included in } \\
\text { quanitative } \\
\text { synthesis support } \\
\text { consculstions } \\
\text { based on meta- } \\
\text { analyses. Pooled } \\
\text { studies had } \\
\text { substantial } \\
\text { heterogeneity } \\
\text { that was oly } \\
\text { partially explored. } \\
\text { Inadequate } \\
\text { description of } \\
\text { study } \\
\text { characteristics. }\end{array}$ & Yes & Probably yes & Yes & Probably yes & Probably yes & Low & $\begin{array}{l}\text { Eligibility criteria } \\
\text { is well defined; } \\
\text { authors make } \\
\text { restrictions on } \\
\text { studies that } \\
\text { could be } \\
\text { included in } \\
\text { quantitative } \\
\text { analyses based } \\
\text { on reporting of } \\
\text { appropriate data, } \\
\text { but summarize } \\
\text { other relevant } \\
\text { studies in an } \\
\text { appendix (those } \\
\text { that could not be } \\
\text { included in } \\
\text { quantitative } \\
\text { synthesis). }\end{array}$ \\
\hline
\end{tabular}




\begin{tabular}{|c|c|c|c|c|c|c|c|c|c|}
\hline $\begin{array}{l}\text { Author, Year } \\
\text { Health } \\
\text { Outcome }\end{array}$ & $\begin{array}{l}\text { Overall Risk } \\
\text { of Bias } \\
\text { Rating }\end{array}$ & $\begin{array}{l}\text { Overall } \\
\text { Rationale for } \\
\text { Risk of Bias } \\
\text { Rating }\end{array}$ & $\begin{array}{l}1.1 \text { Did the } \\
\text { review } \\
\text { adhere to } \\
\text { pre-defined } \\
\text { objectives } \\
\text { and } \\
\text { eligibility } \\
\text { criteria? }\end{array}$ & $\begin{array}{l}1.2 \text { Were the } \\
\text { eligibility } \\
\text { criteria } \\
\text { appropriate } \\
\text { for the review } \\
\text { question? }\end{array}$ & $\begin{array}{l}1.3 \text { Were } \\
\text { eligibility } \\
\text { criteria } \\
\text { unambi- } \\
\text { guous? }\end{array}$ & $\begin{array}{l}\text { 1.4 Were all } \\
\text { restrictions in } \\
\text { eligibility criteria } \\
\text { based on study } \\
\text { characteristics } \\
\text { appropriate (e.g., } \\
\text { date, sample } \\
\text { size, study } \\
\text { quality, } \\
\text { outcomes } \\
\text { measured)? }\end{array}$ & $\begin{array}{l}1.5 \text { Were any } \\
\text { restrictions in } \\
\text { eligibility } \\
\text { criteria based } \\
\text { on sources of } \\
\text { information } \\
\text { appropriate } \\
\text { (e.g., } \\
\text { publication } \\
\text { status or } \\
\text { format, } \\
\text { language, } \\
\text { availability of } \\
\text { data)? }\end{array}$ & $\begin{array}{l}\text { Concerns } \\
\text { Regarding } \\
\text { Specifi- } \\
\text { Cation of } \\
\text { Study } \\
\text { Eligibility } \\
\text { Criteria }\end{array}$ & $\begin{array}{l}\text { Rationale for } \\
\text { Concern }\end{array}$ \\
\hline $\begin{array}{l}\text { Islami, } 2015^{55} \\
\text { Breast cancer }\end{array}$ & High & $\begin{array}{l}\text { See individual } \\
\text { domains; } \\
\text { unpublished } \\
\text { reports were } \\
\text { ineligible, unclear } \\
\text { whether literature } \\
\text { review and data } \\
\text { abstraction were } \\
\text { dually reviewed. } \\
\text { No ROB of } \\
\text { individual studies } \\
\text { was described. } \\
\text { However, some } \\
\text { potential bias } \\
\text { (confounding) is } \\
\text { addressed by the } \\
\text { evidence } \\
\text { synthesis. } \\
\text { Authors may } \\
\text { have over- } \\
\text { interpreted the } \\
\text { importance of } \\
\text { their findings } \\
\text { given the results } \\
\text { and the } \\
\text { limitations. }\end{array}$ & Yes & Yes & Yes & Probably yes & Probably no & Unclear & $\begin{array}{l}\text { Authors state } \\
\text { that abstracts } \\
\text { and unpublished } \\
\text { studies were } \\
\text { ineligible; some } \\
\text { relevant } \\
\text { measures of BF } \\
\text { duration were } \\
\text { also excluded } \\
\text { (e.g., duration of } \\
\text { BF per child). } \\
\text { Non-English } \\
\text { studies were } \\
\text { excluded. }\end{array}$ \\
\hline
\end{tabular}




\begin{tabular}{|c|c|c|c|c|c|c|c|c|c|}
\hline $\begin{array}{l}\text { Author, Year } \\
\text { Health } \\
\text { Outcome }\end{array}$ & $\begin{array}{l}\text { Overall Risk } \\
\text { of Bias } \\
\text { Rating }\end{array}$ & $\begin{array}{l}\text { Overall } \\
\text { Rationale for } \\
\text { Risk of Bias } \\
\text { Rating }\end{array}$ & $\begin{array}{l}1.1 \text { Did the } \\
\text { review } \\
\text { adhere to } \\
\text { pre-defined } \\
\text { objectives } \\
\text { and } \\
\text { eligibility } \\
\text { criteria? }\end{array}$ & $\begin{array}{l}1.2 \text { Were the } \\
\text { eligibility } \\
\text { criteria } \\
\text { appropriate } \\
\text { for the review } \\
\text { question? }\end{array}$ & $\begin{array}{l}1.3 \text { Were } \\
\text { eligibility } \\
\text { criteria } \\
\text { unambi- } \\
\text { guous? }\end{array}$ & $\begin{array}{l}\text { 1.4 Were all } \\
\text { restrictions in } \\
\text { eligibility criteria } \\
\text { based on study } \\
\text { characteristics } \\
\text { appropriate (e.g., } \\
\text { date, sample } \\
\text { size, study } \\
\text { quality, } \\
\text { outcomes } \\
\text { measured)? }\end{array}$ & $\begin{array}{l}1.5 \text { Were any } \\
\text { restrictions in } \\
\text { eligibility } \\
\text { criteria based } \\
\text { on sources of } \\
\text { information } \\
\text { appropriate } \\
\text { (e.g., } \\
\text { publication } \\
\text { status or } \\
\text { format, } \\
\text { language, } \\
\text { availability of } \\
\text { data)? }\end{array}$ & $\begin{array}{l}\text { Concerns } \\
\text { Regarding } \\
\text { Specifi- } \\
\text { Cation of } \\
\text { Study } \\
\text { Eligibility } \\
\text { Criteria }\end{array}$ & $\begin{array}{l}\text { Rationale for } \\
\text { Concern }\end{array}$ \\
\hline $\begin{array}{l}\text { Jager, } 2014^{56} \\
\text { Diabetes }\end{array}$ & High & $\begin{array}{l}\text { No grey literature } \\
\text { or unpublished } \\
\text { studies were } \\
\text { included and } \\
\text { authors required } \\
\text { studies to report } \\
\text { Cls, leading to a } \\
\text { risk of publication } \\
\text { bias (which the } \\
\text { review found } \\
\text { evidence of). }\end{array}$ & Yes & Yes & Yes & Yes & Probably no & Unclear & $\begin{array}{l}\text { Authors } \\
\text { restricted } \\
\text { eligibility to } \\
\text { studies reporting } \\
\text { a RR and 95\% } \\
\text { Cl; unclear if this } \\
\text { meant that they } \\
\text { would have } \\
\text { excluded } \\
\text { otherwise } \\
\text { eligible studies } \\
\text { that reported a } \\
\text { different } \\
\text { measure of } \\
\text { diabetes risk } \\
\text { associated with } \\
\text { BF (e.g., OR). }\end{array}$ \\
\hline
\end{tabular}




\begin{tabular}{|c|c|c|c|c|c|c|c|c|c|}
\hline $\begin{array}{l}\text { Author, Year } \\
\text { Health } \\
\text { Outcome }\end{array}$ & $\begin{array}{l}\text { Overall Risk } \\
\text { of Bias } \\
\text { Rating }\end{array}$ & $\begin{array}{l}\text { Overall } \\
\text { Rationale for } \\
\text { Risk of Bias } \\
\text { Rating }\end{array}$ & $\begin{array}{l}1.1 \text { Did the } \\
\text { review } \\
\text { adhere to } \\
\text { pre-defined } \\
\text { objectives } \\
\text { and } \\
\text { eligibility } \\
\text { criteria? }\end{array}$ & $\begin{array}{l}1.2 \text { Were the } \\
\text { eligibility } \\
\text { criteria } \\
\text { appropriate } \\
\text { for the review } \\
\text { question? }\end{array}$ & $\begin{array}{l}1.3 \text { Were } \\
\text { eligibility } \\
\text { criteria } \\
\text { unambi- } \\
\text { guous? }\end{array}$ & $\begin{array}{l}\text { 1.4 Were all } \\
\text { restrictions in } \\
\text { eligibility criteria } \\
\text { based on study } \\
\text { characteristics } \\
\text { appropriate (e.g., } \\
\text { date, sample } \\
\text { size, study } \\
\text { quality, } \\
\text { outcomes } \\
\text { measured)? }\end{array}$ & $\begin{array}{l}1.5 \text { Were any } \\
\text { restrictions in } \\
\text { eligibility } \\
\text { criteria based } \\
\text { on sources of } \\
\text { information } \\
\text { appropriate } \\
\text { (e.g., } \\
\text { publication } \\
\text { status or } \\
\text { format, } \\
\text { language, } \\
\text { availability of } \\
\text { data)? }\end{array}$ & $\begin{array}{l}\text { Concerns } \\
\text { Regarding } \\
\text { Specifi- } \\
\text { Cation of } \\
\text { Study } \\
\text { Eligibility } \\
\text { Criteria }\end{array}$ & $\begin{array}{l}\text { Rationale for } \\
\text { Concern }\end{array}$ \\
\hline $\begin{array}{l}\text { Jiang, } 2017^{57} \\
\text { Osteoporotic } \\
\text { fractures }\end{array}$ & High & $\begin{array}{l}\text { Exclusion of } \\
\text { studies without } \\
\text { effect sizes } \\
\text { reporting, and no } \\
\text { consideration of } \\
\text { bias in primary } \\
\text { studies. }\end{array}$ & Probably yes & Probably no & Yes & Probably no & Probably yes & High & $\begin{array}{l}\text { The review } \\
\text { restricted } \\
\text { inclusion to } \\
\text { studies that } \\
\text { provided an } \\
\text { effect size. } \\
\text { Studies with the } \\
\text { same underlying } \\
\text { information, i.e., } \\
\text { event rates and } \\
\text { total sample } \\
\text { size, for which } \\
\text { effect sizes } \\
\text { could be } \\
\text { calculated, were } \\
\text { excluded. This } \\
\text { suggests a } \\
\text { potential for } \\
\text { excluding } \\
\text { relevant studies. }\end{array}$ \\
\hline
\end{tabular}




\begin{tabular}{|c|c|c|c|c|c|c|c|c|c|}
\hline $\begin{array}{l}\text { Author, Year } \\
\text { Health } \\
\text { Outcome }\end{array}$ & $\begin{array}{l}\text { Overall Risk } \\
\text { of Bias } \\
\text { Rating }\end{array}$ & $\begin{array}{l}\text { Overall } \\
\text { Rationale for } \\
\text { Risk of Bias } \\
\text { Rating }\end{array}$ & $\begin{array}{l}1.1 \text { Did the } \\
\text { review } \\
\text { adhere to } \\
\text { pre-defined } \\
\text { objectives } \\
\text { and } \\
\text { eligibility } \\
\text { criteria? }\end{array}$ & $\begin{array}{l}1.2 \text { Were the } \\
\text { eligibility } \\
\text { criteria } \\
\text { appropriate } \\
\text { for the review } \\
\text { question? }\end{array}$ & $\begin{array}{l}1.3 \text { Were } \\
\text { eligibility } \\
\text { criteria } \\
\text { unambi- } \\
\text { guous? }\end{array}$ & $\begin{array}{l}\text { 1.4 Were all } \\
\text { restrictions in } \\
\text { eligibility criteria } \\
\text { based on study } \\
\text { characteristics } \\
\text { appropriate (e.g., } \\
\text { date, sample } \\
\text { size, study } \\
\text { quality, } \\
\text { outcomes } \\
\text { measured)? }\end{array}$ & $\begin{array}{l}1.5 \text { Were any } \\
\text { restrictions in } \\
\text { eligibility } \\
\text { criteria based } \\
\text { on sources of } \\
\text { information } \\
\text { appropriate } \\
\text { (e.g., } \\
\text { publication } \\
\text { status or } \\
\text { format, } \\
\text { language, } \\
\text { availability of } \\
\text { data)? }\end{array}$ & $\begin{array}{l}\text { Concerns } \\
\text { Regarding } \\
\text { Specifi- } \\
\text { Cation of } \\
\text { Study } \\
\text { Eligibility } \\
\text { Criteria }\end{array}$ & $\begin{array}{l}\text { Rationale for } \\
\text { Concern }\end{array}$ \\
\hline $\begin{array}{l}\text { Lambertini, } \\
2016^{58} \\
\text { Breast cancer }\end{array}$ & Unclear & $\begin{array}{l}\text { Unclear reporting } \\
\text { of whether } \\
\text { literature review } \\
\text { and data } \\
\text { abstraction were } \\
\text { dually reviewed. } \\
\text { No ROB } \\
\text { assessment of } \\
\text { individual studies. } \\
\text { Exclusion of } \\
\text { studies that do } \\
\text { not report an OR } \\
\text { (or give sufficient } \\
\text { information to } \\
\text { calculate one) } \\
\text { may lead to } \\
\text { exclusion of } \\
\text { some relevant } \\
\text { studies. Analyses } \\
\text { are appropriate; } \\
\text { however, more } \\
\text { may have been } \\
\text { done to address } \\
\text { substantial } \\
\text { heterogeneity } \\
\text { across studies. }\end{array}$ & Yes & Yes & Yes & Probably yes & Probably yes & Unclear & $\begin{array}{l}\text { Authors specify } \\
\text { that OR had to } \\
\text { be reported (or } \\
\text { computed from } \\
\text { available data) } \\
\text { for study to meet } \\
\text { inclusion criteria. } \\
\text { Unclear how } \\
\text { many potentially } \\
\text { relevant articles } \\
\text { were excluded } \\
\text { due to reporting } \\
\text { of a different } \\
\text { measure of } \\
\text { association } \\
\text { and/or no } \\
\text { measure of } \\
\text { variance. } \\
\text { English- } \\
\text { language only } \\
\text { restriction. }\end{array}$ \\
\hline
\end{tabular}




\begin{tabular}{|c|c|c|c|c|c|c|c|c|c|}
\hline $\begin{array}{l}\text { Author, Year } \\
\text { Health } \\
\text { Outcome }\end{array}$ & $\begin{array}{l}\text { Overall Risk } \\
\text { of Bias } \\
\text { Rating }\end{array}$ & $\begin{array}{l}\text { Overall } \\
\text { Rationale for } \\
\text { Risk of Bias } \\
\text { Rating }\end{array}$ & $\begin{array}{l}1.1 \text { Did the } \\
\text { review } \\
\text { adhere to } \\
\text { pre-defined } \\
\text { objectives } \\
\text { and } \\
\text { eligibility } \\
\text { criteria? }\end{array}$ & $\begin{array}{l}1.2 \text { Were the } \\
\text { eligibility } \\
\text { criteria } \\
\text { appropriate } \\
\text { for the review } \\
\text { question? }\end{array}$ & $\begin{array}{l}1.3 \text { Were } \\
\text { eligibility } \\
\text { criteria } \\
\text { unambi- } \\
\text { guous? }\end{array}$ & $\begin{array}{l}1.4 \text { Were all } \\
\text { restrictions in } \\
\text { eligibility criteria } \\
\text { based on study } \\
\text { characteristics } \\
\text { appropriate (e.g., } \\
\text { date, sample } \\
\text { size, study } \\
\text { quality, } \\
\text { outcomes } \\
\text { measured)? }\end{array}$ & $\begin{array}{l}\text { 1.5 Were any } \\
\text { restrictions in } \\
\text { eligibility } \\
\text { criteria based } \\
\text { on sources of } \\
\text { information } \\
\text { appropriate } \\
\text { (e.g., } \\
\text { publication } \\
\text { status or } \\
\text { format, } \\
\text { language, } \\
\text { availability of } \\
\text { data)? }\end{array}$ & $\begin{array}{l}\text { Concerns } \\
\text { Regarding } \\
\text { Specifi- } \\
\text { Cation of } \\
\text { Study } \\
\text { Eligibility } \\
\text { Criteria }\end{array}$ & $\begin{array}{l}\text { Rationale for } \\
\text { Concern }\end{array}$ \\
\hline $\begin{array}{l}\mathrm{Li}, 2014^{60} \\
\text { Ovarian } \\
\text { cancer }\end{array}$ & Unclear & $\begin{array}{l}\text { Unclear for } \\
\text { several domains } \\
\text { (including issues } \\
\text { related to study } \\
\text { selection and } \\
\text { data collection). } \\
\text { Evidence } \\
\text { synthesis } \\
\text { appears overall } \\
\text { well done (aside } \\
\text { from sensitivity } \\
\text { analyses } \\
\text { involving fixed } \\
\text { effects), though } \\
\text { substantial } \\
\text { heterogeneity } \\
\text { remained. }\end{array}$ & Yes & Yes & Yes & Probably yes & Probably yes & Unclear & $\begin{array}{l}\text { Limited to } \\
\text { studies reporting } \\
\text { a RR/OR and } \\
\text { measure of } \\
\text { variation; } \\
\text { however, } \\
\text { authors note that } \\
\text { they also } \\
\text { attempted to } \\
\text { calculate } \\
\text { measures when } \\
\text { data was } \\
\text { available. } \\
\text { Unclear how } \\
\text { may potentially } \\
\text { relevant studies } \\
\text { were excluded } \\
\text { due to } \\
\text { availability of } \\
\text { data. Limited to } \\
\text { English studies. }\end{array}$ \\
\hline
\end{tabular}




\begin{tabular}{|c|c|c|c|c|c|c|c|c|c|}
\hline $\begin{array}{l}\text { Author, Year } \\
\text { Health } \\
\text { Outcome }\end{array}$ & $\begin{array}{l}\text { Overall Risk } \\
\text { of Bias } \\
\text { Rating }\end{array}$ & $\begin{array}{l}\text { Overall } \\
\text { Rationale for } \\
\text { Risk of Bias } \\
\text { Rating }\end{array}$ & $\begin{array}{l}1.1 \text { Did the } \\
\text { review } \\
\text { adhere to } \\
\text { pre-defined } \\
\text { objectives } \\
\text { and } \\
\text { eligibility } \\
\text { criteria? }\end{array}$ & $\begin{array}{l}1.2 \text { Were the } \\
\text { eligibility } \\
\text { criteria } \\
\text { appropriate } \\
\text { for the review } \\
\text { question? }\end{array}$ & $\begin{array}{l}1.3 \text { Were } \\
\text { eligibility } \\
\text { criteria } \\
\text { unambi- } \\
\text { guous? }\end{array}$ & $\begin{array}{l}\text { 1.4 Were all } \\
\text { restrictions in } \\
\text { eligibility criteria } \\
\text { based on study } \\
\text { characteristics } \\
\text { appropriate (e.g., } \\
\text { date, sample } \\
\text { size, study } \\
\text { quality, } \\
\text { outcomes } \\
\text { measured)? }\end{array}$ & $\begin{array}{l}1.5 \text { Were any } \\
\text { restrictions in } \\
\text { eligibility } \\
\text { criteria based } \\
\text { on sources of } \\
\text { information } \\
\text { appropriate } \\
\text { (e.g., } \\
\text { publication } \\
\text { status or } \\
\text { format, } \\
\text { language, } \\
\text { availability of } \\
\text { data)? }\end{array}$ & $\begin{array}{l}\text { Concerns } \\
\text { Regarding } \\
\text { Specifi- } \\
\text { Cation of } \\
\text { Study } \\
\text { Eligibility } \\
\text { Criteria }\end{array}$ & $\begin{array}{l}\text { Rationale for } \\
\text { Concern }\end{array}$ \\
\hline $\begin{array}{l}\text { Luan, } 2013^{51} \\
\text { Ovarian } \\
\text { cancer }\end{array}$ & Low & $\begin{array}{l}\text { Although only } \\
\text { one database } \\
\text { was searched, } \\
\text { authors searched } \\
\text { references of all } \\
\text { included studies } \\
\text { to identify } \\
\text { publications that } \\
\text { may have been } \\
\text { missed in their } \\
\text { Medline search. } \\
\text { No ROB of } \\
\text { individual studies } \\
\text { was conducted, } \\
\text { however } \\
\text { analyses address } \\
\text { a number of } \\
\text { factors related to } \\
\text { potential bias in } \\
\text { this literature } \\
\text { (e.g., known } \\
\text { confounders, } \\
\text { aspects of study } \\
\text { design). }\end{array}$ & Yes & Yes & Yes & Probably yes & Probably yes & Low & $\begin{array}{l}\text { Authors note } \\
\text { that studies had } \\
\text { to report a } \\
\text { measure of } \\
\text { effect and Cl; } \\
\text { however, unlike } \\
\text { other studies, } \\
\text { they at least } \\
\text { note that any } \\
\text { measure would } \\
\text { be eligible (HR, } \\
\text { RR and OR) and } \\
\text { note they } \\
\text { attempted to } \\
\text { calculate } \\
\text { measures when } \\
\text { data was } \\
\text { available. } \\
\text { English- } \\
\text { language only } \\
\text { restriction. }\end{array}$ \\
\hline
\end{tabular}




\begin{tabular}{|c|c|c|c|c|c|c|c|c|c|}
\hline $\begin{array}{l}\text { Author, Year } \\
\text { Health } \\
\text { Outcome }\end{array}$ & $\begin{array}{l}\text { Overall Risk } \\
\text { of Bias } \\
\text { Rating }\end{array}$ & $\begin{array}{l}\text { Overall } \\
\text { Rationale for } \\
\text { Risk of Bias } \\
\text { Rating }\end{array}$ & $\begin{array}{l}1.1 \text { Did the } \\
\text { review } \\
\text { adhere to } \\
\text { pre-defined } \\
\text { objectives } \\
\text { and } \\
\text { eligibility } \\
\text { criteria? }\end{array}$ & $\begin{array}{l}1.2 \text { Were the } \\
\text { eligibility } \\
\text { criteria } \\
\text { appropriate } \\
\text { for the review } \\
\text { question? }\end{array}$ & $\begin{array}{l}1.3 \text { Were } \\
\text { eligibility } \\
\text { criteria } \\
\text { unambi- } \\
\text { guous? }\end{array}$ & $\begin{array}{l}\text { 1.4 Were all } \\
\text { restrictions in } \\
\text { eligibility criteria } \\
\text { based on study } \\
\text { characteristics } \\
\text { appropriate (e.g., } \\
\text { date, sample } \\
\text { size, study } \\
\text { quality, } \\
\text { outcomes } \\
\text { measured)? }\end{array}$ & $\begin{array}{l}\text { 1.5 Were any } \\
\text { restrictions in } \\
\text { eligibility } \\
\text { criteria based } \\
\text { on sources of } \\
\text { information } \\
\text { appropriate } \\
\text { (e.g., } \\
\text { publication } \\
\text { status or } \\
\text { format, } \\
\text { language, } \\
\text { availability of } \\
\text { data)? }\end{array}$ & $\begin{array}{l}\text { Concerns } \\
\text { Regarding } \\
\text { Specifi- } \\
\text { Cation of } \\
\text { Study } \\
\text { Eligibility } \\
\text { Criteria }\end{array}$ & $\begin{array}{l}\text { Rationale for } \\
\text { Concern }\end{array}$ \\
\hline $\begin{array}{l}\text { Neville, } 2014^{61} \\
\text { Postpartum } \\
\text { weight change }\end{array}$ & High & $\begin{array}{l}\text { See individual } \\
\text { domains; no dual } \\
\text { review of } \\
\text { abstracts/full } \\
\text { texts or ROB } \\
\text { assessment for } \\
\text { included studies. } \\
\text { Characteristics of } \\
\text { studies are not } \\
\text { well desribed and } \\
\text { syntheses } \\
\text { focuses on } \\
\text { number of } \\
\text { studies that found } \\
\text { a postiive } \\
\text { association } \\
\text { grouped by study } \\
\text { design and } \\
\text { outcome. }\end{array}$ & Yes & Yes & Yes & Yes & Probably yes & Low & $\begin{array}{l}\text { No specific } \\
\text { restrictions on } \\
\text { eligibility were } \\
\text { reported based } \\
\text { on sources of } \\
\text { information, } \\
\text { language or } \\
\text { availability of } \\
\text { data. }\end{array}$ \\
\hline
\end{tabular}




\begin{tabular}{|c|c|c|c|c|c|c|c|c|c|}
\hline $\begin{array}{l}\text { Author, Year } \\
\text { Health } \\
\text { Outcome }\end{array}$ & $\begin{array}{l}\text { Overall Risk } \\
\text { of Bias } \\
\text { Rating }\end{array}$ & $\begin{array}{l}\text { Overall } \\
\text { Rationale for } \\
\text { Risk of Bias } \\
\text { Rating }\end{array}$ & $\begin{array}{l}\text { 1.1 Did the } \\
\text { review } \\
\text { adhere to } \\
\text { pre-defined } \\
\text { objectives } \\
\text { and } \\
\text { eligibility } \\
\text { criteria? }\end{array}$ & $\begin{array}{l}1.2 \text { Were the } \\
\text { eligibility } \\
\text { criteria } \\
\text { appropriate } \\
\text { for the review } \\
\text { question? }\end{array}$ & $\begin{array}{l}1.3 \text { Were } \\
\text { eligibility } \\
\text { criteria } \\
\text { unambi- } \\
\text { guous? }\end{array}$ & $\begin{array}{l}\text { 1.4 Were all } \\
\text { restrictions in } \\
\text { eligibility criteria } \\
\text { based on study } \\
\text { characteristics } \\
\text { appropriate (e.g., } \\
\text { date, sample } \\
\text { size, study } \\
\text { quality, } \\
\text { outcomes } \\
\text { measured)? }\end{array}$ & $\begin{array}{l}1.5 \text { Were any } \\
\text { restrictions in } \\
\text { eligibility } \\
\text { criteria based } \\
\text { on sources of } \\
\text { information } \\
\text { appropriate } \\
\text { (e.g., } \\
\text { publication } \\
\text { status or } \\
\text { format, } \\
\text { language, } \\
\text { availability of } \\
\text { data)? }\end{array}$ & $\begin{array}{l}\text { Concerns } \\
\text { Regarding } \\
\text { Specifi- } \\
\text { Cation of } \\
\text { Study } \\
\text { Eligibility } \\
\text { Criteria }\end{array}$ & $\begin{array}{l}\text { Rationale for } \\
\text { Concern }\end{array}$ \\
\hline $\begin{array}{l}\text { Pan, } 2013^{62} \\
\text { Breast cancer }\end{array}$ & High & $\begin{array}{l}\text { See individual } \\
\text { domains; unclear } \\
\text { ratings for } \\
\text { multiple } \\
\text { categories and } \\
\text { high risk of bias } \\
\text { for data } \\
\text { collection. } \\
\text { Unclear whether } \\
\text { literature review } \\
\text { was dually } \\
\text { assessed. No } \\
\text { ROB of individual } \\
\text { studies. } \\
\text { Exclusion of } \\
\text { studies based on } \\
\text { specific type of } \\
\text { outcome } \\
\text { measure reported } \\
\text { may bias study } \\
\text { selection. Meta- } \\
\text { analyses } \\
\text { conducted on } \\
\text { only } 3 \text { studies } \\
\text { with limited } \\
\text { assessment of } \\
\text { heterogeneity. }\end{array}$ & Yes & Yes & Yes & Probably yes & Probably yes & Unclear & $\begin{array}{l}\text { Authors specify } \\
\text { that included } \\
\text { studies had to } \\
\text { report a RR and } \\
\text { corresponding } \\
95 \% \mathrm{Cl} \text {; no note } \\
\text { regarding } \\
\text { whether they } \\
\text { attempted to } \\
\text { calculate this } \\
\text { with sufficient } \\
\text { data was } \\
\text { available. } \\
\text { English- } \\
\text { language only } \\
\text { restriction. }\end{array}$ \\
\hline
\end{tabular}




\begin{tabular}{|c|c|c|c|c|c|c|c|c|c|}
\hline $\begin{array}{l}\text { Author, Year } \\
\text { Health } \\
\text { Outcome }\end{array}$ & $\begin{array}{l}\text { Overall Risk } \\
\text { of Bias } \\
\text { Rating }\end{array}$ & $\begin{array}{l}\text { Overall } \\
\text { Rationale for } \\
\text { Risk of Bias } \\
\text { Rating }\end{array}$ & $\begin{array}{l}1.1 \text { Did the } \\
\text { review } \\
\text { adhere to } \\
\text { pre-defined } \\
\text { objectives } \\
\text { and } \\
\text { eligibility } \\
\text { criteria? }\end{array}$ & $\begin{array}{l}1.2 \text { Were the } \\
\text { eligibility } \\
\text { criteria } \\
\text { appropriate } \\
\text { for the review } \\
\text { question? }\end{array}$ & $\begin{array}{l}1.3 \text { Were } \\
\text { eligibility } \\
\text { criteria } \\
\text { unambi- } \\
\text { guous? }\end{array}$ & $\begin{array}{l}\text { 1.4 Were all } \\
\text { restrictions in } \\
\text { eligibility criteria } \\
\text { based on study } \\
\text { characteristics } \\
\text { appropriate (e.g., } \\
\text { date, sample } \\
\text { size, study } \\
\text { quality, } \\
\text { outcomes } \\
\text { measured)? }\end{array}$ & $\begin{array}{l}1.5 \text { Were any } \\
\text { restrictions in } \\
\text { eligibility } \\
\text { criteria based } \\
\text { on sources of } \\
\text { information } \\
\text { appropriate } \\
\text { (e.g., } \\
\text { publication } \\
\text { status or } \\
\text { format, } \\
\text { language, } \\
\text { availability of } \\
\text { data)? }\end{array}$ & $\begin{array}{l}\text { Concerns } \\
\text { Regarding } \\
\text { Specifi- } \\
\text { Cation of } \\
\text { Study } \\
\text { Eligibility } \\
\text { Criteria }\end{array}$ & $\begin{array}{l}\text { Rationale for } \\
\text { Concern }\end{array}$ \\
\hline $\begin{array}{l}\text { Sung, } 2016^{63} \\
\text { Ovarian } \\
\text { cancer }\end{array}$ & Unclear & $\begin{array}{l}\text { See individual } \\
\text { domains for } \\
\text { details. Briefly, } \\
\text { study selection } \\
\text { methods were } \\
\text { not reported, } \\
\text { there was } \\
\text { substantial } \\
\text { heterogeneity } \\
\text { that was } \\
\text { unexplained, and } \\
\text { several } \\
\text { opportunities for } \\
\text { additional } \\
\text { analyses were } \\
\text { not taken. }\end{array}$ & Yes & Yes & Yes & No & Yes & Low & NA \\
\hline
\end{tabular}

bias; RR = relative risk. 
Table C-15. KQ 2 risk of bias assessment: Systematic reviews, part 2

\begin{tabular}{|c|c|c|c|c|c|c|c|}
\hline $\begin{array}{l}\text { Author, Year } \\
\text { Health } \\
\text { Outcome }\end{array}$ & $\begin{array}{l}2.1 \text { Did the search } \\
\text { include an } \\
\text { appropriate range of } \\
\text { databases/ } \\
\text { electronic } \\
\text { sources for } \\
\text { published and } \\
\text { unpublished } \\
\text { reports? }\end{array}$ & $\begin{array}{l}2.2 \text { Were } \\
\text { methods } \\
\text { additional to } \\
\text { database } \\
\text { searching } \\
\text { used to } \\
\text { identify } \\
\text { relevant } \\
\text { reports? }\end{array}$ & $\begin{array}{l}2.3 \text { Were the } \\
\text { terms and } \\
\text { structure of the } \\
\text { search strategy } \\
\text { likely to retrieve } \\
\text { as many eligible } \\
\text { studies as } \\
\text { possible? }\end{array}$ & $\begin{array}{l}2.4 \text { Were } \\
\text { restrictions } \\
\text { based on date, } \\
\text { publication } \\
\text { format, or } \\
\text { language } \\
\text { appropriate? }\end{array}$ & $\begin{array}{l}2.5 \text { Were efforts } \\
\text { made to minimize } \\
\text { error in selection } \\
\text { of studies? }\end{array}$ & $\begin{array}{l}\text { Concerns } \\
\text { Regarding } \\
\text { Methods } \\
\text { Used to } \\
\text { Identify } \\
\text { and/or Select } \\
\text { Studies }\end{array}$ & Rationale for Concern \\
\hline $\begin{array}{l}\text { Aune, } 2014^{50} \\
\text { Diabetes }\end{array}$ & Probably yes & Yes & Yes & Yes & No information & Unclear & $\begin{array}{l}\text { Authors searched references of } \\
\text { included articles to identify further } \\
\text { studies but did not look for } \\
\text { unpublished (grey) literature. } \\
\text { Methods unclear whether abstracts } \\
\text { and full-texts were dually reviewed. }\end{array}$ \\
\hline $\begin{array}{l}\text { Chowdhury, } \\
2015^{2} \\
\text { Breast and } \\
\text { ovarian cancer }\end{array}$ & Probably yes & Yes & Probably yes & Yes & Yes & Low & $\begin{array}{l}\text { Authors searched multiple } \\
\text { databases and reference lists of } \\
\text { relevant articles; I considered this } \\
\text { sufficient. }\end{array}$ \\
\hline $\begin{array}{l}\text { Dias, } 2015^{52} \\
\text { Postpartum } \\
\text { depression }\end{array}$ & Probably yes & No information & Probably yes & Yes & Yes & Low & $\begin{array}{l}\text { Multiple databases were searched } \\
\text { and review of abstracts and full- } \\
\text { texts was conducted dually; unclear } \\
\text { if grey/unpublished literature was } \\
\text { searched. Only a brief description of } \\
\text { search terms/strategy is provided. }\end{array}$ \\
\hline $\begin{array}{l}\text { Feng, } 2014^{53} \\
\text { Ovarian cancer }\end{array}$ & Yes & Yes & Probably yes & Probably no & No information & Unclear & $\begin{array}{l}\text { As noted in Domain \#1, unclear } \\
\text { whether restrictions were made } \\
\text { during study selection based on } \\
\text { outcome measures. Unclear } \\
\text { whether literature was dually } \\
\text { reviewed. Authors searched } \\
\text { conference proceedings and } \\
\text { reference lists of included studies. }\end{array}$ \\
\hline
\end{tabular}




\begin{tabular}{|c|c|c|c|c|c|c|c|}
\hline $\begin{array}{l}\text { Author, Year } \\
\text { Health } \\
\text { Outcome }\end{array}$ & $\begin{array}{l}2.1 \text { Did the search } \\
\text { include an } \\
\text { appropriate range of } \\
\text { databases } \\
\text { electronic } \\
\text { sources for } \\
\text { published and } \\
\text { unpublished } \\
\text { reports? }\end{array}$ & $\begin{array}{l}2.2 \text { Were } \\
\text { methods } \\
\text { additional to } \\
\text { database } \\
\text { searching } \\
\text { used to } \\
\text { identify } \\
\text { relevant } \\
\text { reports? }\end{array}$ & $\begin{array}{l}2.3 \text { Were the } \\
\text { terms and } \\
\text { structure of the } \\
\text { search strategy } \\
\text { likely to retrieve } \\
\text { as many eligible } \\
\text { studies as } \\
\text { possible? }\end{array}$ & $\begin{array}{l}2.4 \text { Were } \\
\text { restrictions } \\
\text { based on date, } \\
\text { publication } \\
\text { format, or } \\
\text { language } \\
\text { appropriate? }\end{array}$ & $\begin{array}{l}2.5 \text { Were efforts } \\
\text { made to minimize } \\
\text { error in selection } \\
\text { of studies? }\end{array}$ & $\begin{array}{l}\text { Concerns } \\
\text { Regarding } \\
\text { Methods } \\
\text { Used to } \\
\text { Identify } \\
\text { and/or Select } \\
\text { Studies }\end{array}$ & Rationale for Concern \\
\hline $\begin{array}{l}\text { He, } 2015^{54} \\
\text { Postpartum } \\
\text { weight change }\end{array}$ & Probably yes & Yes & Probably yes & Probably yes & Yes & Unclear & $\begin{array}{l}\text { In addition to databases, authors } \\
\text { assessed included studies to } \\
\text { identify relevant citations missed in } \\
\text { database searches. Dual review of } \\
\text { abstract and full-texts was } \\
\text { conducted. Methods state that } \\
\text { RCTs were eligible but there is no } \\
\text { note of whether studies of weight } \\
\text { loss interventions were eligible. }\end{array}$ \\
\hline $\begin{array}{l}\text { Islami, } 2015^{55} \\
\text { Breast cancer }\end{array}$ & Probably yes & Yes & Probably yes & Probably no & Probably yes & Unclear & $\begin{array}{l}\text { Searches were not designed to } \\
\text { identify unpublished reports } \\
\text { (unpublished abstracts/reports were } \\
\text { not eligible); authors did look at } \\
\text { references from relevant studies to } \\
\text { identify articles database searches } \\
\text { may have missed. Methods note } \\
\text { that three authors "independently" } \\
\text { performed the search and evaluated } \\
\text { articles (unclear if each article was } \\
\text { dually reviewed). }\end{array}$ \\
\hline $\begin{array}{l}\text { Jager, } 2014^{56} \\
\text { Diabetes }\end{array}$ & Probably no & Yes & Yes & Yes & Yes & Unclear & $\begin{array}{l}\text { No grey literature search; this, in } \\
\text { addition to requirement that studies } \\
\text { report Cls could elevate publication } \\
\text { bias. }\end{array}$ \\
\hline $\begin{array}{l}\text { Jiang, } 2017^{57} \\
\text { Osteoporotic } \\
\text { fractures }\end{array}$ & Yes & Yes & Yes & Yes & Probably no & High & $\begin{array}{l}\text { Studies without reported effect sizes } \\
\text { were excluded (studies with relevant } \\
\text { data that allowed calculation of } \\
\text { effect size data were potentially } \\
\text { excluded). }\end{array}$ \\
\hline
\end{tabular}




\begin{tabular}{|c|c|c|c|c|c|c|c|}
\hline $\begin{array}{l}\text { Author, Year } \\
\text { Health } \\
\text { Outcome }\end{array}$ & $\begin{array}{l}2.1 \text { Did the search } \\
\text { include an } \\
\text { appropriate range of } \\
\text { databases/ } \\
\text { electronic } \\
\text { sources for } \\
\text { published and } \\
\text { unpublished } \\
\text { reports? }\end{array}$ & $\begin{array}{l}2.2 \text { Were } \\
\text { methods } \\
\text { additional to } \\
\text { database } \\
\text { searching } \\
\text { used to } \\
\text { identify } \\
\text { relevant } \\
\text { reports? }\end{array}$ & $\begin{array}{l}2.3 \text { Were the } \\
\text { terms and } \\
\text { structure of the } \\
\text { search strategy } \\
\text { likely to retrieve } \\
\text { as many eligible } \\
\text { studies as } \\
\text { possible? }\end{array}$ & $\begin{array}{l}2.4 \text { Were } \\
\text { restrictions } \\
\text { based on date, } \\
\text { publication } \\
\text { format, or } \\
\text { language } \\
\text { appropriate? }\end{array}$ & $\begin{array}{l}2.5 \text { Were efforts } \\
\text { made to minimize } \\
\text { error in selection } \\
\text { of studies? }\end{array}$ & $\begin{array}{l}\text { Concerns } \\
\text { Regarding } \\
\text { Methods } \\
\text { Used to } \\
\text { Identify } \\
\text { and/or Select } \\
\text { Studies }\end{array}$ & Rationale for Concern \\
\hline $\begin{array}{l}\text { Lambertini, } \\
2016^{58} \\
\text { Breast cancer }\end{array}$ & Probably yes & Yes & Probably yes & Probably yes & No information & Unclear & $\begin{array}{l}\text { Unclear whether literature review } \\
\text { was conducted dually or by one of } \\
\text { two authors (methods are } \\
\text { ambiguous). Inclusion criteria based } \\
\text { on type of outcome measure } \\
\text { available may exclude some } \\
\text { potentially relevant studies. } \\
\text { Restricted to English-language } \\
\text { publications. }\end{array}$ \\
\hline $\begin{array}{l}\text { Li, } 2014^{60} \\
\text { Ovarian cancer }\end{array}$ & Yes & Yes & Probably yes & Probably yes & No information & Unclear & $\begin{array}{l}\text { No information on whether literature } \\
\text { review was dually assessed; as } \\
\text { noted in Domain } \# 1 \text {, studies were } \\
\text { excluded if they did not report an } \\
\text { OR/RR and } 95 \% \mathrm{Cl} \text { (or data } \\
\text { sufficient to calculate these). } \\
\text { Authors searched reference lists of } \\
\text { included studies. }\end{array}$ \\
\hline $\begin{array}{l}\text { Luan, } 2013^{51} \\
\text { Ovarian cancer }\end{array}$ & Probably no & Yes & Probably yes & Probably yes & Probably yes & Unclear & $\begin{array}{l}\text { Only searched MEDLINE, but did } \\
\text { search the reference lists of all } \\
\text { included studies for additional } \\
\text { publications; no unpublished } \\
\text { sources were evaluated; unclear } \\
\text { whether dual or singular screening } \\
\text { of titles/abstracts (full-text review } \\
\text { was dual). }\end{array}$ \\
\hline $\begin{array}{l}\text { Neville, } 2014^{61} \\
\text { Postpartum } \\
\text { weight change }\end{array}$ & Probably yes & Yes & Probably yes & Probably yes & Probably no & Unclear & $\begin{array}{l}\text { Multiple databases were searched; } \\
\text { authors also reviewed additional } \\
\text { relevant citations from articles } \\
\text { retrieved via database searches. } \\
\text { One reviewer screened all } \\
\text { abstracts/full-texts, and a second } \\
\text { reviewer was consulted for } \\
\text { questions about eligibility. }\end{array}$ \\
\hline
\end{tabular}




\begin{tabular}{|c|c|c|c|c|c|c|c|}
\hline $\begin{array}{l}\text { Author, Year } \\
\text { Health } \\
\text { Outcome }\end{array}$ & $\begin{array}{l}2.1 \text { Did the search } \\
\text { include an } \\
\text { appropriate range of } \\
\text { databases/ } \\
\text { electronic } \\
\text { sources for } \\
\text { published and } \\
\text { unpublished } \\
\text { reports? }\end{array}$ & $\begin{array}{l}2.2 \text { Were } \\
\text { methods } \\
\text { additional to } \\
\text { database } \\
\text { searching } \\
\text { used to } \\
\text { identify } \\
\text { relevant } \\
\text { reports? }\end{array}$ & $\begin{array}{l}2.3 \text { Were the } \\
\text { terms and } \\
\text { structure of the } \\
\text { search strategy } \\
\text { likely to retrieve } \\
\text { as many eligible } \\
\text { studies as } \\
\text { possible? }\end{array}$ & $\begin{array}{l}2.4 \text { Were } \\
\text { restrictions } \\
\text { based on date, } \\
\text { publication } \\
\text { format, or } \\
\text { language } \\
\text { appropriate? }\end{array}$ & $\begin{array}{l}2.5 \text { Were efforts } \\
\text { made to minimize } \\
\text { error in selection } \\
\text { of studies? }\end{array}$ & $\begin{array}{l}\text { Concerns } \\
\text { Regarding } \\
\text { Methods } \\
\text { Used to } \\
\text { Identify } \\
\text { and/or Select } \\
\text { Studies }\end{array}$ & Rationale for Concern \\
\hline $\begin{array}{l}\text { Pan, } 2013^{62} \\
\text { Breast cancer }\end{array}$ & Probably no & Yes & Probably yes & Probably yes & No information & Unclear & $\begin{array}{l}\text { Only one database was searched. } \\
\text { Unclear whether literature review } \\
\text { was conducted dually or by one of } \\
\text { two authors (methods are } \\
\text { ambiguous). Inclusion criteria based } \\
\text { on type of outcome measure } \\
\text { available (RR and } 95 \% \mathrm{Cl} \text { ) may } \\
\text { exclude some potentially relevant } \\
\text { studies. Searched reference lists of } \\
\text { included studies and reported that } \\
\text { they did not identify any } \\
\text { unpublished data (though didn't } \\
\text { describe their search methods). }\end{array}$ \\
\hline $\begin{array}{l}\text { Sung, } 2016^{63} \\
\text { Ovarian cancer }\end{array}$ & Probably yes & Yes & Probably yes & Yes & No information & Unclear & $\begin{array}{l}450 \text { of } 489 \text { articles identified for the } \\
\text { breastfeeding analysis were } \\
\text { excluded at title/abstract review, } \\
\text { which is a very high rate of } \\
\text { exclusion. Authors did not describe } \\
\text { whether title/abstract or full-text } \\
\text { review was dual. }\end{array}$ \\
\hline
\end{tabular}


Table C-16. KQ 2 risk of bias assessment: Systematic reviews, part 3

\subsection{Were}

\begin{tabular}{|c|c|c|c|c|c|c|c|}
\hline $\begin{array}{l}\text { Author, Year } \\
\text { Health } \\
\text { Outcome }\end{array}$ & $\begin{array}{l}3.1 \text { Were } \\
\text { efforts made } \\
\text { to minimize } \\
\text { error in data } \\
\text { collection? }\end{array}$ & $\begin{array}{l}\text { sufficient study } \\
\text { characteristics } \\
\text { available for } \\
\text { both review } \\
\text { authors and } \\
\text { readers to be } \\
\text { able to interpret } \\
\text { the results? }\end{array}$ & $\begin{array}{l}3.3 \text { Were all } \\
\text { relevant } \\
\text { study } \\
\text { results } \\
\text { collected for } \\
\text { use in the } \\
\text { synthesis? }\end{array}$ & $\begin{array}{l}3.4 \text { Was risk of } \\
\text { bias (or } \\
\text { methodological } \\
\text { quality) formally } \\
\text { assessed using } \\
\text { appropriate } \\
\text { criteria? }\end{array}$ & $\begin{array}{l}3.5 \text { Were efforts } \\
\text { made to minimize } \\
\text { error in risk of bias } \\
\text { assessment? }\end{array}$ & $\begin{array}{l}\text { Concerns } \\
\text { Regarding } \\
\text { Methods Used } \\
\text { to Collect Data } \\
\text { and Appraise } \\
\text { Studies }\end{array}$ & Rationale for Concern \\
\hline $\begin{array}{l}\text { Aune, } 2014^{50} \\
\text { Diabetes }\end{array}$ & Yes & Yes & Yes & Yes & No information & Unclear & $\begin{array}{l}\text { Methods state that two authors } \\
\text { independently abstracted data from } \\
\text { studies, but it is unclear whether risk } \\
\text { of bias of included studies was dually } \\
\text { assessed. }\end{array}$ \\
\hline $\begin{array}{l}\text { Chowdhury, } \\
2015^{2} \\
\text { Breast and } \\
\text { ovarian cancer }\end{array}$ & No information & Probably no & Probably yes & Yes & No information & Unclear & $\begin{array}{l}\text { Unclear whether data abstraction was } \\
\text { checked by a second author. Very few } \\
\text { study characteristics were } \\
\text { presented/described, and only then } \\
\text { on an aggregate level, probably due } \\
\text { to the scope of the review (i.e., } \\
\text { multiple outcomes). Authors cited the } \\
\text { ACROBAT-NRSI tool as a measure of } \\
\text { study quality, explaining that study } \\
\text { quality was used in subgroup } \\
\text { analyses; no additional details were } \\
\text { provided, including whether the } \\
\text { assessment was performed dually. }\end{array}$ \\
\hline $\begin{array}{l}\text { Dias, } 2015^{52} \\
\text { Postpartum } \\
\text { depression }\end{array}$ & Probably yes & Probably no & Probably yes & No & No information & Unclear & $\begin{array}{l}\text { No sample characteristics were } \\
\text { reported, effect sizes were not } \\
\text { reported, and risk of bias was only } \\
\text { determined based on two criteria: (1) } \\
\text { women should be clearly identified as } \\
\text { postpartum women, and (2) studies } \\
\text { should identify the outcome } \\
\text { measurements. }\end{array}$ \\
\hline $\begin{array}{l}\text { Feng, } 2014^{53} \\
\text { Ovarian } \\
\text { cancer }\end{array}$ & No information & Probably yes & Probably yes & Yes & Yes & Unclear & $\begin{array}{l}\text { Unclear whether data abstractions for } \\
\text { each article were reviewed by two } \\
\text { authors. Description of database } \\
\text { searches is limited. }\end{array}$ \\
\hline
\end{tabular}




\subsection{Were}

sufficient study $\quad 3.3$ Were all $\mathbf{3 . 4}$ Was risk of

Author, Year

3.1 Were

efforts made available for

Health

to minimize

both review

relevant

bias (or

error in data authors and

study

methodological

Outcome

collection?

collected for assessed using

readers to be use in the appropriate

synthesis? criteria?

the results?

He, 2015 5 No information Probably no

Probably yes Yes

Yes Yes

Concerns

made to minimize Methods Used

error in risk of bias to Collect Data

assessment? and Appraise

Rationale for Concern

Postpartum

weight change

Islami, $2015^{55}$ Probably yes Probably no Probably yes Probably no

No information

High

Breast cancer

@ీ

Jager, 2014 ${ }^{56}$ No information Yes

Yes

Diabetes

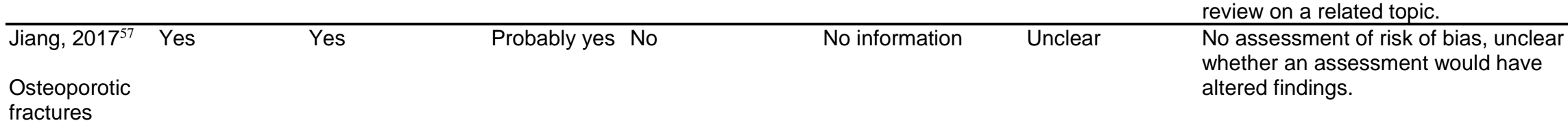

Unclear whether data abstraction was dually reviewed. Authors assessed study quality using predefined criteria; however, tool appears to be

developed by authors and may not address all potential risks of bias.

There is limited description of study characteristics.

Unclear whether data abstractions for each article were reviewed by two authors. ROB assessment for individual studies was not mentioned in the methods or results.

Unclear whether data abstraction and risk of bias assessment was dually reviewed. Authors rate risk of bias and show assessments in an appendix; questions appear appropriate. Tool used was adapted from another existing systematic altered findings. 


\begin{tabular}{|c|c|c|c|c|c|c|c|}
\hline $\begin{array}{l}\text { Author, Year } \\
\text { Health } \\
\text { Outcome }\end{array}$ & $\begin{array}{l}3.1 \text { Were } \\
\text { efforts made } \\
\text { to minimize } \\
\text { error in data } \\
\text { collection? }\end{array}$ & $\begin{array}{l}3.2 \text { Were } \\
\text { sufficient study } \\
\text { characteristics } \\
\text { available for } \\
\text { both review } \\
\text { authors and } \\
\text { readers to be } \\
\text { able to interpret } \\
\text { the results? }\end{array}$ & $\begin{array}{l}3.3 \text { Were all } \\
\text { relevant } \\
\text { study } \\
\text { results } \\
\text { collected for } \\
\text { use in the } \\
\text { synthesis? }\end{array}$ & $\begin{array}{l}3.4 \text { Was risk of } \\
\text { bias (or } \\
\text { methodological } \\
\text { quality) formally } \\
\text { assessed using } \\
\text { appropriate } \\
\text { criteria? }\end{array}$ & $\begin{array}{l}3.5 \text { Were efforts } \\
\text { made to minimize } \\
\text { error in risk of bias } \\
\text { assessment? }\end{array}$ & $\begin{array}{l}\text { Concerns } \\
\text { Regarding } \\
\text { Methods Used } \\
\text { to Collect Data } \\
\text { and Appraise } \\
\text { Studies }\end{array}$ & Rationale for Concern \\
\hline $\begin{array}{l}\text { Lambertini, } \\
2016^{58} \\
\text { Breast cancer }\end{array}$ & Probably yes & Probably no & Probably yes & Probably no & No information & High & $\begin{array}{l}\text { Unclear how data extraction } \\
\text { discrepancies were resolved. } \\
\text { Insufficient information is provided for } \\
\text { study characteristics in terms of how } \\
\text { BF exposure was measured and then } \\
\text { classified by authors in ever/never } \\
\text { analyses. Authors do not appear to } \\
\text { have conducted a ROB assessment } \\
\text { of included studies. }\end{array}$ \\
\hline $\begin{array}{l}\mathrm{Li}, 2014^{60} \\
\text { Ovarian } \\
\text { cancer }\end{array}$ & Yes & Probably yes & Probably yes & Yes & Probably yes & Unclear & $\begin{array}{l}\text { Study characteristics are limited. } \\
\text { There was dual data abstraction and } \\
\text { ROB assessment, though it is unclear } \\
\text { how ROB discrepancies were } \\
\text { reconciled. }\end{array}$ \\
\hline $\begin{array}{l}\text { Luan, } 2013^{51} \\
\text { Ovarian } \\
\text { cancer }\end{array}$ & Probably yes & Probably no & Probably yes & No & NA & High & $\begin{array}{l}\text { Methods suggest data abstraction } \\
\text { was dually reviewed but description is } \\
\text { ambiguous. There are very few study } \\
\text { characteristics described. Authors do } \\
\text { not describe the definition of } \\
\text { ever/never breastfed nor explain } \\
\text { about the role of parity in the } \\
\text { analyses. Authors did not formally } \\
\text { assess ROB of included studies. } \\
\text { However, some characteristics } \\
\text { abstracted (and analyses performed) } \\
\text { do address issues of confounding. }\end{array}$ \\
\hline
\end{tabular}




\begin{tabular}{|c|c|c|c|c|c|c|c|}
\hline $\begin{array}{l}\text { Author, Year } \\
\text { Health } \\
\text { Outcome }\end{array}$ & $\begin{array}{l}3.1 \text { Were } \\
\text { efforts made } \\
\text { to minimize } \\
\text { error in data } \\
\text { collection? }\end{array}$ & $\begin{array}{l}3.2 \text { Were } \\
\text { sufficient study } \\
\text { characteristics } \\
\text { available for } \\
\text { both review } \\
\text { authors and } \\
\text { readers to be } \\
\text { able to interpret } \\
\text { the results? }\end{array}$ & $\begin{array}{l}3.3 \text { Were all } \\
\text { relevant } \\
\text { study } \\
\text { results } \\
\text { collected for } \\
\text { use in the } \\
\text { synthesis? }\end{array}$ & $\begin{array}{l}3.4 \text { Was risk of } \\
\text { bias (or } \\
\text { methodological } \\
\text { quality) formally } \\
\text { assessed using } \\
\text { appropriate } \\
\text { criteria? }\end{array}$ & $\begin{array}{l}3.5 \text { Were efforts } \\
\text { made to minimize } \\
\text { error in risk of bias } \\
\text { assessment? }\end{array}$ & $\begin{array}{l}\text { Concerns } \\
\text { Regarding } \\
\text { Methods Used } \\
\text { to Collect Data } \\
\text { and Appraise } \\
\text { Studies }\end{array}$ & Rationale for Concern \\
\hline $\begin{array}{l}\text { Neville, } 2014^{61} \\
\text { Postpartum } \\
\text { weight change }\end{array}$ & No information & Probably no & Probably no & Probably no & Probably no & High & $\begin{array}{l}\text { No formal risk of bias/ quality } \\
\text { assessment of included studies is } \\
\text { described in the methods or tables. A } \\
\text { few (brief) remarks are made in the } \\
\text { discussion regarding study rigor and } \\
\text { heterogeneity of outcome measures. } \\
\text { Unclear whether data abstraction was } \\
\text { dually reviewed. Minimal study } \\
\text { characteristics were described; } \\
\text { unclear whether all relevant results } \\
\text { were collected. Results reporting is } \\
\text { minimal. }\end{array}$ \\
\hline $\begin{array}{l}\text { Pan, } 2013^{62} \\
\text { Breast cancer }\end{array}$ & Probably yes & Probably no & Probably yes & Probably no & No information & High & $\begin{array}{l}\text { Data abstraction likely dually reviewed } \\
\text { but methods are ambiguous (state } \\
\text { that two authors independently } \\
\text { abstracted data and differences were } \\
\text { addressed by consensus). Study } \\
\text { characteristics are limited (e.g., no } \\
\text { country setting described). No ROB of } \\
\text { individual studies performed. }\end{array}$ \\
\hline Sung, $2016^{63}$ & Yes & Probably yes & Probably yes & Probably yes & Yes & Low & $\begin{array}{l}\text { Dual data extraction and dual quality } \\
\text { assessment using the NOS. }\end{array}$ \\
\hline
\end{tabular}

cancer

ACROBAT-NRSI = A Cochrane Risk of Bias Assessment Tool for Non-Randomized Studies of Interventions; BF = breastfeeding; KQ = Key Question; NOS = Newcastle-Ottawa Scale; ROB = risk of bias. 
Table C-17. KQ 2 risk of bias assessment: Systematic reviews, part 4

4.3 Was the

synthesis

\section{appropriate}

appropriat

Author,

4.1 Did the 4.2 Were all given the

4.4 Was

4.5 Were the

nature and between-study

Year

pre-defined

synthesis analyses

similarity in

variation

4.6 Were

Year

include all reported or

\section{e.g. as}

the research (heterogeneity)

primary

studies that departures

Outcome

it should?

departures
explained?

minimal or

through funnel

study designs addressed in the plot or

and outcomes synthesis?

plot or

primary

studies

minimal or

Concerns

regarding the

analyses?

addressed in

synthesis and

across

the synthesis?

Aune, $2014^{50}$ Probably yes Yes

Aune, 2014
Diabetes
Chowdhu

Yes

Yes

Yes

Yes

Low

NA

Chowdhury, Probably yes Yes

Breast and

ovarian

cancer

Probably yes Probably yes

Probably yes

Probably yes

Probably yes Probably yes Probably yes Probably yes Unclear

(1)




\begin{tabular}{|c|c|c|c|c|c|c|c|c|}
\hline $\begin{array}{l}\text { Author, } \\
\text { Year } \\
\text { Health } \\
\text { Outcome }\end{array}$ & $\begin{array}{l}4.1 \text { Did the } \\
\text { synthesis } \\
\text { include all } \\
\text { studies that } \\
\text { it should? }\end{array}$ & $\begin{array}{l}4.2 \text { Were all } \\
\text { pre-defined } \\
\text { analyses } \\
\text { reported or } \\
\text { departures } \\
\text { explained? }\end{array}$ & $\begin{array}{l}4.3 \text { Was the } \\
\text { synthesis } \\
\text { appropriate } \\
\text { given the } \\
\text { nature and } \\
\text { similarity in } \\
\text { the research } \\
\text { questions, } \\
\text { study designs } \\
\text { and outcomes } \\
\text { across } \\
\text { included } \\
\text { studies? }\end{array}$ & $\begin{array}{l}4.4 \text { Was } \\
\text { between-study } \\
\text { variation } \\
\text { (heterogeneity) } \\
\text { minimal or } \\
\text { addressed in the } \\
\text { synthesis? }\end{array}$ & $\begin{array}{l}4.5 \text { Were the } \\
\text { findings robust, } \\
\text { e.g. as } \\
\text { demonstrated } \\
\text { through funnel } \\
\text { plot or } \\
\text { sensitivity } \\
\text { analyses? }\end{array}$ & $\begin{array}{l}4.6 \text { Were } \\
\text { biases in } \\
\text { primary } \\
\text { studies } \\
\text { minimal or } \\
\text { addressed in } \\
\text { the synthesis? }\end{array}$ & $\begin{array}{l}\text { Concerns } \\
\text { Regarding the } \\
\text { Synthesis and } \\
\text { Findings }\end{array}$ & Rationale for Concern \\
\hline $\begin{array}{l}\text { Dias, } 2015^{52} \\
\text { Postpartum } \\
\text { depression }\end{array}$ & Probably yes & Yes & Probably yes & No information & No information & No information & Unclear & $\begin{array}{l}\text { Evidence synthesis focuses on a } \\
\text { range of questions related to } \\
\text { breastfeeding and post-partum } \\
\text { depression. No quantitative } \\
\text { synthesis was performed and } \\
\text { results (including outcome } \\
\text { measures) appear } \\
\text { heterogeneous. Criteria used to } \\
\text { group outcomes is not reported. }\end{array}$ \\
\hline $\begin{array}{l}\text { Feng, } 2014^{53} \\
\text { Ovarian } \\
\text { cancer }\end{array}$ & Probably yes & Probably yes & Probably yes & Probably no & No information & Probably yes & High & $\begin{array}{l}\text { Authors describe I-squared in } \\
\text { random effects meta-analysis, but } \\
\text { do not adequately address the } \\
\text { substantial between-study } \\
\text { variation in the synthesis. They } \\
\text { report fixed effects meta-analyses } \\
\text { as a way to assess the } \\
\text { robustness of their findings which } \\
\text { does not seem appropriate, } \\
\text { especially given the substantial } \\
\text { heterogeneity. Quality of included } \\
\text { articles was assessed according } \\
\text { to Newcastle-Ottawa Scale; most } \\
\text { studies were rated high quality } \\
\text { (NOS } 7 \text { or } 8 \text {; no } 9 \text { ratings) but } \\
\text { there were a handful of low } \\
\text { quality (NOS=6) and no subgroup } \\
\text { analysis done by rating. There } \\
\text { was no evidence of publication } \\
\text { bias as reported by the authors. }\end{array}$ \\
\hline
\end{tabular}




\begin{tabular}{|c|c|c|c|c|c|c|c|c|}
\hline $\begin{array}{l}\text { Author, } \\
\text { Year } \\
\text { Health } \\
\text { Outcome }\end{array}$ & $\begin{array}{l}4.1 \text { Did the } \\
\text { synthesis } \\
\text { include all } \\
\text { studies that } \\
\text { it should? }\end{array}$ & $\begin{array}{l}4.2 \text { Were all } \\
\text { pre-defined } \\
\text { analyses } \\
\text { reported or } \\
\text { departures } \\
\text { explained? }\end{array}$ & $\begin{array}{l}4.3 \text { Was the } \\
\text { synthesis } \\
\text { appropriate } \\
\text { given the } \\
\text { nature and } \\
\text { similarity in } \\
\text { the research } \\
\text { questions, } \\
\text { study designs } \\
\text { and outcomes } \\
\text { across } \\
\text { included } \\
\text { studies? }\end{array}$ & $\begin{array}{l}4.4 \text { Was } \\
\text { between-study } \\
\text { variation } \\
\text { (heterogeneity) } \\
\text { minimal or } \\
\text { addressed in the } \\
\text { synthesis? }\end{array}$ & $\begin{array}{l}4.5 \text { Were the } \\
\text { findings robust, } \\
\text { e.g. as } \\
\text { demonstrated } \\
\text { through funnel } \\
\text { plot or } \\
\text { sensitivity } \\
\text { analyses? }\end{array}$ & $\begin{array}{l}4.6 \text { Were } \\
\text { biases in } \\
\text { primary } \\
\text { studies } \\
\text { minimal or } \\
\text { addressed in } \\
\text { the synthesis? }\end{array}$ & $\begin{array}{l}\text { Concerns } \\
\text { Regarding the } \\
\text { Synthesis and } \\
\text { Findings }\end{array}$ & Rationale for Concern \\
\hline $\begin{array}{l}\text { He, } 2015^{54} \\
\text { Postpartum } \\
\text { weight } \\
\text { change }\end{array}$ & Probably yes & Probably yes & Probably yes & Probably no & Probably no & Probably yes & Unclear & $\begin{array}{l}\text { Some relevant studies were } \\
\text { identified but not included in the } \\
\text { quantitative synthesis for various } \\
\text { reasons (e.g., inadequate } \\
\text { outcome reporting). Reasons are } \\
\text { described in text and studies are } \\
\text { noted in an Appendix. Not clear } \\
\text { whether results of these studies } \\
\text { were congruent with the meta- } \\
\text { analyses. Analyses had } \\
\text { significant heterogeneity across } \\
\text { studies, only partially addressed } \\
\text { by subgroup analyses. No } \\
\text { assessment of publication bias. }\end{array}$ \\
\hline $\begin{array}{l}\text { Islami, } \\
2015^{55} \\
\text { Breast } \\
\text { cancer }\end{array}$ & Probably yes & Probably yes & Probably yes & Probably yes & Probably yes & No information & Unclear & $\begin{array}{l}\text { Authors address heterogeneity } \\
\text { primarily through subgroup } \\
\text { analyses (and focus on } \\
\text { differences in study design); } \\
\text { publication bias was addressed } \\
\text { via funnel plot (even though } \\
\text { unpublished studies were not } \\
\text { eligible). No formal ROB of } \\
\text { individual studies was described. } \\
\text { Authors do address some } \\
\text { potential bias by performing } \\
\text { subgroups based on whether } \\
\text { estimates from individual studies } \\
\text { were adjusted for known } \\
\text { confounders. }\end{array}$ \\
\hline
\end{tabular}




\begin{tabular}{|c|c|c|c|c|c|c|c|c|}
\hline $\begin{array}{l}\text { Author, } \\
\text { Year } \\
\text { Health } \\
\text { Outcome }\end{array}$ & $\begin{array}{l}4.1 \text { Did the } \\
\text { synthesis } \\
\text { include all } \\
\text { studies that } \\
\text { it should? }\end{array}$ & $\begin{array}{l}4.2 \text { Were all } \\
\text { pre-defined } \\
\text { analyses } \\
\text { reported or } \\
\text { departures } \\
\text { explained? }\end{array}$ & $\begin{array}{l}4.3 \text { Was the } \\
\text { synthesis } \\
\text { appropriate } \\
\text { given the } \\
\text { nature and } \\
\text { similarity in } \\
\text { the research } \\
\text { questions, } \\
\text { study designs } \\
\text { and outcomes } \\
\text { across } \\
\text { included } \\
\text { studies? }\end{array}$ & $\begin{array}{l}4.4 \text { Was } \\
\text { between-study } \\
\text { variation } \\
\text { (heterogeneity) } \\
\text { minimal or } \\
\text { addressed in the } \\
\text { synthesis? }\end{array}$ & $\begin{array}{l}4.5 \text { Were the } \\
\text { findings robust, } \\
\text { e.g. as } \\
\text { demonstrated } \\
\text { through funnel } \\
\text { plot or } \\
\text { sensitivity } \\
\text { analyses? }\end{array}$ & $\begin{array}{l}4.6 \text { Were } \\
\text { biases in } \\
\text { primary } \\
\text { studies } \\
\text { minimal or } \\
\text { addressed in } \\
\text { the synthesis? }\end{array}$ & $\begin{array}{l}\text { Concerns } \\
\text { Regarding the } \\
\text { Synthesis and } \\
\text { Findings }\end{array}$ & Rationale for Concern \\
\hline $\begin{array}{l}\text { Jager, } 2014^{56} \\
\text { Diabetes }\end{array}$ & $\begin{array}{l}\text { Probably } \\
\text { yes }\end{array}$ & Yes & Probably yes & Probably yes & Yes & Yes & Low & $\begin{array}{l}\text { Meta-analyses included few (3 } \\
\text { studies); however, heterogeneity } \\
\text { across studies which was } \\
\text { addressed in the text and I- } \\
\text { squared was reported. }\end{array}$ \\
\hline $\begin{array}{l}\text { Jiang, } 2017^{57} \\
\text { Osteoporotic } \\
\text { fractures }\end{array}$ & Yes & No information & Yes & Yes & Probably yes & no information & Unclear & $\begin{array}{l}\text { No assessment of risk of bias, } \\
\text { unclear whether an assessment } \\
\text { would have altered findings. }\end{array}$ \\
\hline $\begin{array}{l}\text { Lambertini, } \\
2016^{58} \\
\text { Breast } \\
\text { cancer }\end{array}$ & Probably yes & Probably yes & Probably yes & Probably no & Probably yes & Probably yes & Unclear & $\begin{array}{l}\text { Authors report a clear description } \\
\text { of analyses and conduct } \\
\text { subgroup analyses to address } \\
\text { heterogeneity. However, there } \\
\text { was substantial heterogeneity } \\
\text { even among the subgroups, } \\
\text { some of which were defined by } \\
\text { correlated variables. }\end{array}$ \\
\hline $\begin{array}{l}\text { Li, } 2014^{60} \\
\text { Ovarian } \\
\text { cancer }\end{array}$ & Probably yes & Probably yes & Probably yes & Probably yes & Probably yes & Probably yes & Low & $\begin{array}{l}\text { Although authors conducted } \\
\text { several subgroup analyses and } \\
\text { meta-regression to address } \\
\text { heterogeneity (based on } \\
\text { important study and population } \\
\text { characteristics), there was } \\
\text { residual heterogeneity within } \\
\text { subgroups. Publication bias was } \\
\text { also addressed. }\end{array}$ \\
\hline
\end{tabular}




\begin{tabular}{|c|c|c|c|c|c|c|c|c|}
\hline $\begin{array}{l}\text { Author, } \\
\text { Year } \\
\text { Health } \\
\text { Outcome }\end{array}$ & $\begin{array}{l}\text { 4.1 Did the } \\
\text { synthesis } \\
\text { include all } \\
\text { studies that } \\
\text { it should? }\end{array}$ & $\begin{array}{l}4.2 \text { Were all } \\
\text { pre-defined } \\
\text { analyses } \\
\text { reported or } \\
\text { departures } \\
\text { explained? }\end{array}$ & $\begin{array}{l}4.3 \text { Was the } \\
\text { synthesis } \\
\text { appropriate } \\
\text { given the } \\
\text { nature and } \\
\text { similarity in } \\
\text { the research } \\
\text { questions, } \\
\text { study designs } \\
\text { and outcomes } \\
\text { across } \\
\text { included } \\
\text { studies? }\end{array}$ & $\begin{array}{l}4.4 \text { Was } \\
\text { between-study } \\
\text { variation } \\
\text { (heterogeneity) } \\
\text { minimal or } \\
\text { addressed in the } \\
\text { synthesis? }\end{array}$ & $\begin{array}{l}4.5 \text { Were the } \\
\text { findings robust, } \\
\text { e.g. as } \\
\text { demonstrated } \\
\text { through funnel } \\
\text { plot or } \\
\text { sensitivity } \\
\text { analyses? }\end{array}$ & $\begin{array}{l}4.6 \text { Were } \\
\text { biases in } \\
\text { primary } \\
\text { studies } \\
\text { minimal or } \\
\text { addressed in } \\
\text { the synthesis? }\end{array}$ & $\begin{array}{l}\text { Concerns } \\
\text { Regarding the } \\
\text { Synthesis and } \\
\text { Findings }\end{array}$ & Rationale for Concern \\
\hline $\begin{array}{l}\text { Luan, } 2013^{51} \\
\text { Ovarian } \\
\text { cancer }\end{array}$ & Probably yes & Probably yes & Probably yes & Probably yes & Probably yes & Probably yes & Low & $\begin{array}{l}\text { Multiple subgroup analyses } \\
\text { performed to address potential } \\
\text { heterogeneity, in addition to } \\
\text { meta-regression. Authors also } \\
\text { conducted analyses removing } \\
\text { one study at a time. Publication } \\
\text { bias was addressed. There was } \\
\text { residual heterogeneity within } \\
\text { subgroups. }\end{array}$ \\
\hline $\begin{array}{l}\text { Neville, } \\
2014^{61} \\
\text { Postpartum } \\
\text { weight } \\
\text { change }\end{array}$ & Probably yes & No information & Probably no & Probably no & No information & No information & High & $\begin{array}{l}\text { No meta-analyses; results are } \\
\text { described by study design and } \\
\text { outcome timing of measure (in } \\
\text { relation to postpartum period). } \\
\text { Unclear whether different study } \\
\text { designs that measured the same } \\
\text { weight outcome found similar } \\
\text { results. Synthesis primarily } \\
\text { focuses on number of studies that } \\
\text { had statistically significant results. } \\
\text { No formal assessment of } \\
\text { heterogeneity per specific } \\
\text { outcomes or publication bias. } \\
\end{array}$ \\
\hline $\begin{array}{l}\text { Pan, } 2013^{62} \\
\text { Breast } \\
\text { cancer }\end{array}$ & Probably yes & Probably yes & Probably yes & Probably no & Probably yes & Probably no & Unclear & $\begin{array}{l}\text { Small number of studies (three) } \\
\text { were pooled, which may not be } \\
\text { appropriate given heterogeneity; } \\
\text { analyses seem appropriate and } \\
\text { well described, but very limited } \\
\text { attention to heterogeneity. }\end{array}$ \\
\hline
\end{tabular}




\begin{tabular}{|c|c|c|c|c|c|c|c|c|}
\hline $\begin{array}{l}\text { Author, } \\
\text { Year } \\
\text { Health } \\
\text { Outcome }\end{array}$ & $\begin{array}{l}\text { 4.1 Did the } \\
\text { synthesis } \\
\text { include all } \\
\text { studies that } \\
\text { it should? }\end{array}$ & $\begin{array}{l}4.2 \text { Were all } \\
\text { pre-defined } \\
\text { analyses } \\
\text { reported or } \\
\text { departures } \\
\text { explained? }\end{array}$ & $\begin{array}{l}4.3 \text { Was the } \\
\text { synthesis } \\
\text { appropriate } \\
\text { given the } \\
\text { nature and } \\
\text { similarity in } \\
\text { the research } \\
\text { questions, } \\
\text { study designs } \\
\text { and outcomes } \\
\text { across } \\
\text { included } \\
\text { studies? }\end{array}$ & $\begin{array}{l}4.4 \text { Was } \\
\text { between-study } \\
\text { variation } \\
\text { (heterogeneity) } \\
\text { minimal or } \\
\text { addressed in the } \\
\text { synthesis? }\end{array}$ & $\begin{array}{l}4.5 \text { Were the } \\
\text { findings robust, } \\
\text { e.g. as } \\
\text { demonstrated } \\
\text { through funnel } \\
\text { plot or } \\
\text { sensitivity } \\
\text { analyses? }\end{array}$ & $\begin{array}{l}4.6 \text { Were } \\
\text { biases in } \\
\text { primary } \\
\text { studies } \\
\text { minimal or } \\
\text { addressed in } \\
\text { the synthesis? }\end{array}$ & $\begin{array}{l}\text { Concerns } \\
\text { Regarding the } \\
\text { Synthesis and } \\
\text { Findings }\end{array}$ & Rationale for Concern \\
\hline $\begin{array}{l}\text { Sung, } 2016^{63} \\
\text { Ovarian } \\
\text { cancer }\end{array}$ & Probably yes & Yes & Probably yes & Probably no & Probably yes & Probably no & Unclear & $\begin{array}{l}\text { Substantial heterogeneity of } \\
\text { results, even after subgroup } \\
\text { analysis was performed; } 73 \% \text { of } \\
\text { the included studies were rated } \\
\text { "low-quality" according to the } \\
\text { NOS, and even though authors } \\
\text { performed subgroup analysis by } \\
\text { quality, they did not go further. } \\
\text { While most studies did adjust for } \\
\text { a number of confounders, not all } \\
\text { did. Additionally, authors do not } \\
\text { report on the types of case- } \\
\text { control studies (i.e., population- or } \\
\text { hospital-based). }\end{array}$ \\
\hline
\end{tabular}


Table C-18. KQ 2 risk of bias assessment: Systematic reviews, part 5

\begin{tabular}{|c|c|c|c|}
\hline $\begin{array}{l}\text { Author, Year } \\
\text { Health Outcome }\end{array}$ & $\begin{array}{l}\text { A. Did the interpretation of findings } \\
\text { address all of the concerns } \\
\text { identified in Domains } 1 \text { to } 4 \text { ? }\end{array}$ & $\begin{array}{l}\text { B. Was the relevance of identified } \\
\text { studies to the review's research } \\
\text { question appropriately } \\
\text { considered? }\end{array}$ & $\begin{array}{l}\text { C. Did the reviewers avoid } \\
\text { emphasizing results on the basis } \\
\text { of their statistical significance? }\end{array}$ \\
\hline $\begin{array}{l}\text { Aune, } 2014^{50} \\
\text { Diabetes }\end{array}$ & Probably yes & Yes & Yes \\
\hline $\begin{array}{l}\text { Chowdhury, } 2015^{2} \\
\text { Breast and ovarian cancer }\end{array}$ & Probably yes & Yes & Probably yes \\
\hline $\begin{array}{l}\text { Dias, } 2015^{52} \\
\text { Postpartum depression } \\
\end{array}$ & Probably no & Yes & No \\
\hline $\begin{array}{l}\text { Feng, } 2014^{53} \\
\text { Ovarian cancer }\end{array}$ & Probably no & Yes & Probably no \\
\hline $\begin{array}{l}\mathrm{He}, 2015^{54} \\
\text { Postpartum weight change }\end{array}$ & Probably yes & Yes & Yes \\
\hline $\begin{array}{l}\text { Islami, } 2015^{55} \\
\text { Breast cancer } \\
\end{array}$ & Probably yes & Yes & Probably no \\
\hline $\begin{array}{l}\text { Jager, } 2014^{56} \\
\text { Diabetes }\end{array}$ & Probably yes & Yes & Yes \\
\hline $\begin{array}{l}\text { Jiang, } 2017^{57} \\
\text { Osteoporotic Fractures }\end{array}$ & No & Yes & No \\
\hline $\begin{array}{l}\text { Lambertini, } 2016^{58} \\
\text { Breast cancer }\end{array}$ & Probably no & Yes & Probably yes \\
\hline $\begin{array}{l}\mathrm{Li}, 2014^{60} \\
\text { Ovarian cancer }\end{array}$ & Probably yes & Yes & Probably no \\
\hline $\begin{array}{l}\text { Luan, } 2013^{51} \\
\text { Ovarian cancer }\end{array}$ & Probably yes & Yes & Yes \\
\hline $\begin{array}{l}\text { Neville, } 2014^{61} \\
\text { Postpartum weight change }\end{array}$ & No & Yes & No \\
\hline $\begin{array}{l}\text { Pan, } 2013^{62} \\
\text { Breast cancer }\end{array}$ & Probably no & Yes & Probably yes \\
\hline $\begin{array}{l}\text { Sung, } 2016^{63} \\
\text { Ovarian cancer }\end{array}$ & No & Yes & Probably yes \\
\hline
\end{tabular}

KQ = Key Question. 
Table C-19. KQ 2 risk of bias assessment: Breast cancer, part 1

\begin{tabular}{|c|c|c|c|c|c|c|c|c|c|}
\hline $\begin{array}{l}\text { Author, } \\
\text { Year }\end{array}$ & $\begin{array}{l}\text { Overall } \\
\text { Risk of } \\
\text { Bias } \\
\text { Rating }\end{array}$ & $\begin{array}{l}\text { Overall Rationale for Risk } \\
\text { of Bias Rating }\end{array}$ & $\begin{array}{l}\text { 1. Was } \\
\text { method of } \\
\text { selection } \\
\text { unrelated to } \\
\text { exposurel } \\
\text { outcome? }\end{array}$ & $\begin{array}{l}\text { 1a. Were } \\
\text { post- } \\
\text { exposure } \\
\text { variables that } \\
\text { influenced } \\
\text { selection } \\
\text { related to } \\
\text { exposurel } \\
\text { outcome? }\end{array}$ & $\begin{array}{l}\text { 2. Do start of } \\
\text { followup and } \\
\text { start of } \\
\text { exposure } \\
\text { coincide? }\end{array}$ & $\begin{array}{l}\text { 3. Were } \\
\text { adjustment } \\
\text { techniques } \\
\text { used to } \\
\text { correct for } \\
\text { presence of } \\
\text { selection } \\
\text { biases? }\end{array}$ & $\begin{array}{l}\text { 4. Were the } \\
\text { controls sampled } \\
\text { from the } \\
\text { population that } \\
\text { gave rise to the } \\
\text { cases, or using } \\
\text { another method } \\
\text { that avoids } \\
\text { selection bias? }\end{array}$ & $\begin{array}{l}\text { Bias arising } \\
\text { from } \\
\text { selection? }\end{array}$ & Comments \\
\hline $\begin{array}{l}\text { Al-Amri, } \\
2015^{66}\end{array}$ & High & $\begin{array}{l}\text { High risk of measurement } \\
\text { bias (exposure status and } \\
\text { confounding variables); } \\
\text { unclear analysis regarding } \\
\text { adjustment for potential } \\
\text { confounders. Women } \\
\text { (cases and controls) are } \\
\text { relatively young and } \\
\text { duration may not be } \\
\text { sufficient to determine a } \\
\text { difference in breast cancer } \\
\text { outcomes. }\end{array}$ & $\overline{N A}$ & NA & NA & NA & Yes & Low & NA \\
\hline $\begin{array}{l}\text { Al-Qutub, } \\
2013^{67}\end{array}$ & High & $\begin{array}{l}\text { High risk for measurement } \\
\text { bias for exposure (recall } \\
\text { bias); lack of adjustment for } \\
\text { any confounders in } \\
\text { analyses, dissimilarity } \\
\text { between cases and controls } \\
\text { at baseline not accounted } \\
\text { for in analyses. }\end{array}$ & NA & $\overline{N A}$ & $\overline{N A}$ & $\overline{N A}$ & Probably no & $\begin{array}{l}\text { Some } \\
\text { concerns }\end{array}$ & $\begin{array}{l}\text { Controls were recruited } \\
\text { from communities and } \\
\text { from the same hospitals } \\
\text { as cases, but little } \\
\text { additional information is } \\
\text { provided about control } \\
\text { selection. }\end{array}$ \\
\hline $\begin{array}{l}\text { Atkinson, } \\
2016^{68}\end{array}$ & High & $\begin{array}{l}\text { High risk of selection bias } \\
\text { (dissimilar criteria used to } \\
\text { select cases and controls); } \\
\text { risk of recall bias for BF } \\
\text { exposure and confounding } \\
\text { (differences between groups } \\
\text { at baseline not fully } \\
\text { addressed in analyses). }\end{array}$ & NA & $\overline{N A}$ & NA & NA & No & High & $\begin{array}{l}\text { Although cases and } \\
\text { controls were recruited } \\
\text { from the same clinical } \\
\text { setting, they were } \\
\text { recruited for two different } \\
\text { studies with different } \\
\text { racial eligibility criteria- } \\
\text { controls were only } \\
\text { Caucasian and cases } \\
\text { were not restricted by } \\
\text { race/ethnicity. }\end{array}$ \\
\hline
\end{tabular}




\begin{tabular}{|c|c|c|c|c|c|c|c|c|c|}
\hline Author, Year & $\begin{array}{l}\text { Overall } \\
\text { Risk of } \\
\text { Bias } \\
\text { Rating }\end{array}$ & $\begin{array}{l}\text { Overall Rationale for Risk } \\
\text { of Bias Rating }\end{array}$ & $\begin{array}{l}\text { 1. Was } \\
\text { method of } \\
\text { selection } \\
\text { unrelated to } \\
\text { exposure/ou } \\
\text { tcome? }\end{array}$ & $\begin{array}{l}\text { 1a. Were } \\
\text { post- } \\
\text { exposure } \\
\text { variables that } \\
\text { influenced } \\
\text { selection } \\
\text { related to } \\
\text { exposurel } \\
\text { outcome? } \\
\end{array}$ & $\begin{array}{l}\text { 2. Do start of } \\
\text { followup and } \\
\text { start of } \\
\text { exposure } \\
\text { coincide? }\end{array}$ & $\begin{array}{l}\text { 3. Were } \\
\text { adjustment } \\
\text { techniques } \\
\text { used to } \\
\text { correct for } \\
\text { presence of } \\
\text { selection } \\
\text { biases? }\end{array}$ & $\begin{array}{l}\text { 4. Were the } \\
\text { controls sampled } \\
\text { from the } \\
\text { population that } \\
\text { gave rise to the } \\
\text { cases, or using } \\
\text { another method } \\
\text { that avoids } \\
\text { selection bias? }\end{array}$ & $\begin{array}{l}\text { Bias arising } \\
\text { from } \\
\text { selection? }\end{array}$ & Comments \\
\hline Beaber, 200869 & $\begin{array}{l}\text { Some } \\
\text { concerns }\end{array}$ & $\begin{array}{l}\text { Breastfeeding history was } \\
\text { assessed after diagnosis of } \\
\text { breast cancer among } \\
\text { cases, resulting in concern } \\
\text { for differential recall bias. }\end{array}$ & NA & NA & NA & NA & Yes & Low & NA \\
\hline $\begin{array}{l}\text { Castello, } \\
2015^{70}\end{array}$ & $\begin{array}{l}\text { Some } \\
\text { concerns }\end{array}$ & $\begin{array}{l}\text { Risk of recall bias in } \\
\text { breastfeeding } \\
\text { measurement, lack of } \\
\text { adequate adjustment for } \\
\text { confounders (and some } \\
\text { adjustment for } \\
\text { postexposure variables). }\end{array}$ & NA & NA & $\mathrm{NA}$ & $\mathrm{NA}$ & Probably yes & Low & $\begin{array}{l}\text { Controls were selected } \\
\text { from cases' in-law } \\
\text { relatives, friends, } \\
\text { neighbors, or work } \\
\text { colleagues residing in } \\
\text { the same town and } \\
\text { were matched on age. } \\
\text { No other eligibility } \\
\text { criteria are listed for } \\
\text { controls. }\end{array}$ \\
\hline $\begin{array}{l}\text { Dalamaga, } \\
2011^{71}\end{array}$ & High & $\begin{array}{l}\text { Risk of selection bias; } \\
\text { analyses did not adjust for } \\
\text { potential confounders; risk } \\
\text { of recall bias (including } \\
\text { differential recall bias). }\end{array}$ & NA & NA & NA & NA & Probably yes & $\begin{array}{l}\text { Some } \\
\text { concerns }\end{array}$ & $\begin{array}{l}\text { Cases and controls } \\
\text { were selected from the } \\
\text { same hospital; controls } \\
\text { were women from } \\
\text { outpatient clinics who } \\
\text { came for an annual } \\
\text { checkup and had a } \\
\text { negative mammogram. }\end{array}$ \\
\hline Ge, $2015^{72}$ & High & $\begin{array}{l}\text { Risk of recall bias and lack } \\
\text { of precision in } \\
\text { breastfeeding } \\
\text { measurement, lack of } \\
\text { adjustment for any } \\
\text { potential confounders. }\end{array}$ & NA & NA & NA & NA & Probably yes & Low & NA \\
\hline
\end{tabular}




\begin{tabular}{|c|c|c|c|c|c|c|c|c|c|}
\hline Author, Year & $\begin{array}{l}\text { Overall } \\
\text { Risk of } \\
\text { Bias } \\
\text { Rating }\end{array}$ & $\begin{array}{l}\text { Overall Rationale for } \\
\text { Risk of Bias Rating }\end{array}$ & $\begin{array}{l}\text { 1. Was } \\
\text { method of } \\
\text { selection } \\
\text { unrelated to } \\
\text { exposure/out } \\
\text { come? }\end{array}$ & $\begin{array}{l}\text { 1a. Were } \\
\text { post- } \\
\text { exposure } \\
\text { variables that } \\
\text { influenced } \\
\text { selection } \\
\text { related to } \\
\text { exposurel } \\
\text { outcome? } \\
\end{array}$ & $\begin{array}{l}\text { 2. Do start of } \\
\text { followup and } \\
\text { start of } \\
\text { exposure } \\
\text { coincide? }\end{array}$ & $\begin{array}{l}\text { 3. Were } \\
\text { adjustment } \\
\text { techniques } \\
\text { used to } \\
\text { correct for } \\
\text { presence of } \\
\text { selection } \\
\text { biases? }\end{array}$ & $\begin{array}{l}\text { 4. Were the } \\
\text { controls sampled } \\
\text { from the } \\
\text { population that } \\
\text { gave rise to the } \\
\text { cases, or using } \\
\text { another method } \\
\text { that avoids } \\
\text { selection bias? }\end{array}$ & $\begin{array}{l}\text { Bias arising } \\
\text { from } \\
\text { selection? }\end{array}$ & Comments \\
\hline Hadji, $2007^{73}$ & High & $\begin{array}{l}\text { Risk of recall bias, } \\
\text { ascertainment of breast } \\
\text { cancer outcome not } \\
\text { described, and lack of } \\
\text { adjustment for potential } \\
\text { confounders. }\end{array}$ & NA & NA & NA & NA & Probably yes & Low & NA \\
\hline Holm, $2017^{74}$ & $\begin{array}{l}\text { Some } \\
\text { concerns }\end{array}$ & $\begin{array}{l}\text { Potential selection bias; } \\
\text { cases were recruited from } \\
\text { two cohort studies of } \\
\text { different time periods } \\
\text { while controls came from } \\
\text { a mammography cohort } \\
\text { from one-time period. BF } \\
\text { exposure broadly defined } \\
\text { and reported many years } \\
\text { after the exposure. }\end{array}$ & NA & NA & $\mathrm{NA}$ & $\mathrm{NA}$ & Probably no & $\begin{array}{l}\text { Some } \\
\text { concerns }\end{array}$ & $\begin{array}{l}\text { Controls were sampled } \\
\text { from a mammography } \\
\text { screening cohort from } \\
2001-2008 \text { while some } \\
\text { cases were sampled } \\
\text { from a cohort from } \\
2011-2013 \text {; geographic } \\
\text { regions overlapped. }\end{array}$ \\
\hline Kabat, 2011 & $\begin{array}{l}\text { Some } \\
\text { concerns }\end{array}$ & $\begin{array}{l}\text { Risk of inception bias, } \\
\text { residual confounding, and } \\
\text { recall bias. }\end{array}$ & Probably no & NA & No & $\overline{N A}$ & $\overline{N A}$ & $\begin{array}{l}\text { Some } \\
\text { concerns }\end{array}$ & $\begin{array}{l}\text { Potential inception } \\
\text { bias; women were } \\
\text { postmenopausal at } \\
\text { enrollment and } \\
\text { generally healthy. } \\
\end{array}$ \\
\hline $\begin{array}{l}\text { Kotsopoulos, } \\
2012^{76}\end{array}$ & $\begin{array}{l}\text { Some } \\
\text { concerns }\end{array}$ & $\begin{array}{l}\text { Risk of selection bias, } \\
\text { residual confounding and } \\
\text { recall bias (breastfeeding } \\
\text { duration). }\end{array}$ & NA & NA & NA & NA & Probably yes & Low & $\begin{array}{l}\text { Although controls were } \\
\text { likely sampled from } \\
\text { different populations, } \\
\text { they were matched to } \\
\text { cases on several key } \\
\text { variables and selected } \\
\text { from similar countries } \\
\text { and timepoints. }\end{array}$ \\
\hline
\end{tabular}




\begin{tabular}{|c|c|c|c|c|c|c|c|c|c|}
\hline $\begin{array}{l}\text { Author, } \\
\text { Year }\end{array}$ & $\begin{array}{l}\text { Overall } \\
\text { Risk of } \\
\text { Bias } \\
\text { Rating }\end{array}$ & $\begin{array}{l}\text { Overall Rationale for Risk } \\
\text { of Bias Rating }\end{array}$ & $\begin{array}{l}\text { 1. Was } \\
\text { method of } \\
\text { selection } \\
\text { unrelated to } \\
\text { exposure/ou } \\
\text { tcome? }\end{array}$ & $\begin{array}{l}\text { 1a. Were } \\
\text { post- } \\
\text { exposure } \\
\text { variables that } \\
\text { influenced } \\
\text { selection } \\
\text { related to } \\
\text { exposurel } \\
\text { outcome? }\end{array}$ & $\begin{array}{l}\text { 2. Do start of } \\
\text { followup and } \\
\text { start of } \\
\text { exposure } \\
\text { coincide? }\end{array}$ & $\begin{array}{l}\text { 3. Were } \\
\text { adjustment } \\
\text { techniques } \\
\text { used to } \\
\text { correct for } \\
\text { presence of } \\
\text { selection } \\
\text { biases? }\end{array}$ & $\begin{array}{l}\text { 4. Were the } \\
\text { controls sampled } \\
\text { from the } \\
\text { population that } \\
\text { gave rise to the } \\
\text { cases, or using } \\
\text { another method } \\
\text { that avoids } \\
\text { selection bias? }\end{array}$ & $\begin{array}{l}\text { Bias arising } \\
\text { from } \\
\text { selection? }\end{array}$ & Comments \\
\hline Kruk, $2014^{77}$ & High & $\begin{array}{l}\text { Risk of selection bias, } \\
\text { recall bias (including } \\
\text { differential recall bias on } \\
\text { BF exposure measures) } \\
\text { and lack of adjustment for } \\
\text { potential confounders. }\end{array}$ & NA & NA & NA & NA & Probably no & High & $\begin{array}{l}\text { Controls were recruited } \\
\text { from a variety of clinical } \\
\text { settings (similar geographic } \\
\text { setting as controls), } \\
\text { including those visiting } \\
\text { ambulatory clinics for } \\
\text { "health controlling" or a } \\
\text { cold, from hospitals being } \\
\text { treated for fractures, CVD, } \\
\text { back pain, and other } \\
\text { diseases. Response rates } \\
\text { for questionnaires sent in } \\
\text { both cases and controls } \\
\text { was relatively low at } 74 \% \\
\text { and } 69 \% \text { respectively. }\end{array}$ \\
\hline Lee, $2008^{78}$ & $\begin{array}{l}\text { Some } \\
\text { concerns }\end{array}$ & $\begin{array}{l}\text { Recall bias and testing for } \\
\text { numerous effect modifiers } \\
\text { are concerns. }\end{array}$ & NA & NA & NA & NA & Yes & Low & NA \\
\hline $\begin{array}{l}\text { Lumachi, } \\
2010^{79}\end{array}$ & High & $\begin{array}{l}\text { Breastfeeding history was } \\
\text { assessed after diagnosis of } \\
\text { breast cancer among } \\
\text { cases, resulting in concern } \\
\text { for differential recall bias. } \\
\text { Analyses did not adjust for } \\
\text { any potential confounders. }\end{array}$ & NA & NA & NA & NA & Probably no & $\begin{array}{l}\text { Some } \\
\text { concerns }\end{array}$ & $\begin{array}{l}\text { Controls were women who } \\
\text { had undergone } \\
\text { mammograms twice. } \\
\text { Depending on screening } \\
\text { guidelines in the source } \\
\text { population, this could have } \\
\text { selected for women who } \\
\text { were more likely to follow } \\
\text { medical recommendations, } \\
\text { and thus more likely to } \\
\text { breastfeed, than the } \\
\text { general population. }\end{array}$ \\
\hline
\end{tabular}




\begin{tabular}{|c|c|c|c|c|c|c|c|c|c|}
\hline $\begin{array}{l}\text { Author, } \\
\text { Year }\end{array}$ & $\begin{array}{l}\text { Overall } \\
\text { Risk of } \\
\text { Bias } \\
\text { Rating }\end{array}$ & $\begin{array}{l}\text { Overall Rationale for Risk } \\
\text { of Bias Rating }\end{array}$ & $\begin{array}{l}\text { 1. Was } \\
\text { method of } \\
\text { selection } \\
\text { unrelated to } \\
\text { exposurelou } \\
\text { tcome? }\end{array}$ & $\begin{array}{l}\text { 1a. Were } \\
\text { post- } \\
\text { exposure } \\
\text { variables that } \\
\text { influenced } \\
\text { selection } \\
\text { related to } \\
\text { exposurel } \\
\text { outcome? }\end{array}$ & $\begin{array}{l}\text { 2. Do start of } \\
\text { followup and } \\
\text { start of } \\
\text { exposure } \\
\text { coincide? }\end{array}$ & $\begin{array}{l}\text { 3. Were } \\
\text { adjustment } \\
\text { techniques } \\
\text { used to } \\
\text { correct for } \\
\text { presence of } \\
\text { selection } \\
\text { biases? }\end{array}$ & $\begin{array}{l}\text { 4. Were the } \\
\text { controls sampled } \\
\text { from the } \\
\text { population that } \\
\text { gave rise to the } \\
\text { cases, or using } \\
\text { another method } \\
\text { that avoids } \\
\text { selection bias? }\end{array}$ & $\begin{array}{l}\text { Bias arising } \\
\text { from } \\
\text { selection? }\end{array}$ & Comments \\
\hline Ma, 2006 & $\begin{array}{l}\text { Some } \\
\text { concerns }\end{array}$ & $\begin{array}{l}\text { Risk of selection bias, } \\
\text { residual confounding and } \\
\text { recall bias (breastfeeding } \\
\text { duration). }\end{array}$ & NA & $\mathrm{NA}$ & NA & $\overline{N A}$ & Probably yes & Low & NA \\
\hline Ma, $2017^{81}$ & $\begin{array}{l}\text { Some } \\
\text { concerns }\end{array}$ & $\begin{array}{l}\text { Investigators pooled data } \\
\text { from } 3 \text { studies that did not } \\
\text { occur at the same time. } \\
\text { Potential confounding or } \\
\text { recall bias. }\end{array}$ & NA & $\overline{N A}$ & NA & $\overline{N A}$ & Probably yes & $\begin{array}{l}\text { Some } \\
\text { concerns }\end{array}$ & $\begin{array}{l}\text { Study pooled women from } \\
\text { three different population- } \\
\text { based studies. Two studies } \\
\text { shared controls from the } \\
\text { same time and geographic } \\
\text { area. The third study } \\
\text { recruited neighborhood } \\
\text { controls and was } \\
\text { conducted after the other } \\
\text { two studies. }\end{array}$ \\
\hline $\begin{array}{l}\text { Merritt, } \\
2015^{82}\end{array}$ & $\begin{array}{l}\text { Some } \\
\text { concerns }\end{array}$ & $\begin{array}{l}\text { Risk of selection bias, } \\
\text { measurement bias, and } \\
\text { residual confounding. }\end{array}$ & Probably no & Probably no & Probably no & $\overline{N A}$ & NA & $\begin{array}{l}\text { Some } \\
\text { concerns }\end{array}$ & $\begin{array}{l}\text { Only those with complete } \\
\text { data on lifestyle and } \\
\text { breastfeeding were } \\
\text { included from the larger } \\
\text { cohort (women who BF } \\
\text { may have been more likely } \\
\text { to complete expose data). }\end{array}$ \\
\hline $\begin{array}{l}\text { Phillips, } \\
2009^{83}\end{array}$ & $\begin{array}{l}\text { Some } \\
\text { concerns }\end{array}$ & $\begin{array}{l}\text { Recall bias with respect to } \\
\text { breastfeeding history is a } \\
\text { concern. }\end{array}$ & NA & NA & NA & NA & Probably yes & Low & NA \\
\hline
\end{tabular}




\begin{tabular}{|c|c|c|c|c|c|c|c|c|c|}
\hline Author, Year & $\begin{array}{l}\text { Overall } \\
\text { Risk of } \\
\text { Bias } \\
\text { Rating }\end{array}$ & $\begin{array}{l}\text { Overall Rationale for Risk } \\
\text { of Bias Rating }\end{array}$ & $\begin{array}{l}\text { 1. Was } \\
\text { method of } \\
\text { selection } \\
\text { unrelated to } \\
\text { exposurelou } \\
\text { tcome? }\end{array}$ & $\begin{array}{l}\text { 1a. Were } \\
\text { post- } \\
\text { exposure } \\
\text { variables that } \\
\text { influenced } \\
\text { selection } \\
\text { related to } \\
\text { exposurel } \\
\text { outcome? }\end{array}$ & $\begin{array}{l}\text { 2. Do start of } \\
\text { followup and } \\
\text { start of } \\
\text { exposure } \\
\text { coincide? }\end{array}$ & $\begin{array}{l}\text { 3. Were } \\
\text { adjustment } \\
\text { techniques } \\
\text { used to } \\
\text { correct for } \\
\text { presence of } \\
\text { selection } \\
\text { biases? }\end{array}$ & $\begin{array}{l}\text { 4. Were the } \\
\text { controls sampled } \\
\text { from the } \\
\text { population that } \\
\text { gave rise to the } \\
\text { cases, or using } \\
\text { another method } \\
\text { that avoids } \\
\text { selection bias? }\end{array}$ & $\begin{array}{l}\text { Bias arising } \\
\text { from } \\
\text { selection? }\end{array}$ & Comments \\
\hline Pieta, $2008^{84}$ & $\begin{array}{l}\text { Some } \\
\text { concerns }\end{array}$ & $\begin{array}{l}\text { Risk of recall bias and } \\
\text { selection bias, particularly } \\
\text { with respect to differences } \\
\text { in ages of cases and } \\
\text { controls. }\end{array}$ & NA & NA & NA & NA & Probably no & $\begin{array}{l}\text { Some } \\
\text { concerns }\end{array}$ & $\begin{array}{l}\text { Controls were women who } \\
\text { had had a breast exam and } \\
\text { normal mammogram. They } \\
\text { were younger than cases, } \\
\text { and it was unclear whether } \\
\text { cases were similarly } \\
\text { screened and then } \\
\text { diagnosed, or referred from } \\
\text { a different clinic/patient } \\
\text { population. Moreover, } \\
\text { secular trends in } \\
\text { breastfeeding rates may be } \\
\text { driving differences by age, } \\
\text { rather than association with } \\
\text { cancer risk. }\end{array}$ \\
\hline Press, $2010^{85}$ & High & $\begin{array}{l}\text { Cases and controls } \\
\text { sampled from different } \\
\text { populations, no adjustment } \\
\text { for potential confounders; } \\
\text { potential recall bias and } \\
\text { measurement bias (for BF } \\
\text { exposure). }\end{array}$ & NA & NA & NA & NA & No & $\begin{array}{l}\text { Some } \\
\text { concerns }\end{array}$ & $\begin{array}{l}\text { Controls sampled from } \\
\text { women admitted to the } \\
\text { hospital for another } \\
\text { disease, or applying for } \\
\text { outpatient care. }\end{array}$ \\
\hline Ritte, $2013^{86}$ & $\begin{array}{l}\text { Some } \\
\text { concerns }\end{array}$ & $\begin{array}{l}\text { Risk of recall bias for } \\
\text { breastfeeding exposure, } \\
\text { residual confounding, and } \\
\text { adjusted analyses included } \\
\text { several post-exposure } \\
\text { variables. }\end{array}$ & Yes & NA & Probably no & NA & NA & $\begin{array}{l}\text { Some } \\
\text { concerns }\end{array}$ & $\begin{array}{l}\text { Women were recruited into } \\
\text { the cohort between the } \\
\text { ages of } 25 \text { and } 70 \text { years; } \\
\text { for most women, this would } \\
\text { have been after } \\
\text { breastfeeding exposure. }\end{array}$ \\
\hline
\end{tabular}




\begin{tabular}{|c|c|c|c|c|c|c|c|c|c|}
\hline $\begin{array}{l}\text { Author, } \\
\text { Year }\end{array}$ & $\begin{array}{l}\text { Overall } \\
\text { Risk of } \\
\text { Bias } \\
\text { Rating }\end{array}$ & $\begin{array}{l}\text { Overall Rationale for Risk } \\
\text { of Bias Rating }\end{array}$ & $\begin{array}{l}\text { 1. Was } \\
\text { method of } \\
\text { selection } \\
\text { unrelated to } \\
\text { exposure/ou } \\
\text { tcome? }\end{array}$ & $\begin{array}{l}\text { 1a. Were } \\
\text { post- } \\
\text { exposure } \\
\text { variables that } \\
\text { influenced } \\
\text { selection } \\
\text { related to } \\
\text { exposurel } \\
\text { outcome? } \\
\end{array}$ & $\begin{array}{l}\text { 2. Do start of } \\
\text { followup and } \\
\text { start of } \\
\text { exposure } \\
\text { coincide? }\end{array}$ & $\begin{array}{l}\text { 3. Were } \\
\text { adjustment } \\
\text { techniques } \\
\text { used to } \\
\text { correct for } \\
\text { presence of } \\
\text { selection } \\
\text { biases? }\end{array}$ & $\begin{array}{l}\text { 4. Were the } \\
\text { controls sampled } \\
\text { from the } \\
\text { population that } \\
\text { gave rise to the } \\
\text { cases, or using } \\
\text { another method } \\
\text { that avoids } \\
\text { selection bias? }\end{array}$ & $\begin{array}{l}\text { Bias arising } \\
\text { from } \\
\text { selection? }\end{array}$ & Comments \\
\hline $\begin{array}{l}\text { Ruszczyk, } \\
2016^{87}\end{array}$ & $\begin{array}{l}\text { Some } \\
\text { concerns }\end{array}$ & $\begin{array}{l}\text { Risk of recall bias for } \\
\text { breastfeeding exposure, } \\
\text { unclear amount of missing } \\
\text { data, risk of residual } \\
\text { confounding. }\end{array}$ & NA & NA & NA & NA & Probably yes & $\begin{array}{l}\text { Some } \\
\text { concerns }\end{array}$ & $\begin{array}{l}\text { Some controls recruited } \\
\text { with random digit dialing } \\
\text { of residential telephone } \\
\text { numbers. However, some } \\
\text { recruited through } \\
\text { community-based efforts } \\
\text { that were not well } \\
\text { described. } \\
\end{array}$ \\
\hline $\begin{array}{l}\text { Stendell- } \\
\text { Hollis, } \\
2013^{88}\end{array}$ & $\begin{array}{l}\text { Some } \\
\text { concerns }\end{array}$ & $\begin{array}{l}\text { Risk of residual } \\
\text { confounding and some } \\
\text { post-exposure variables } \\
\text { were included in the } \\
\text { adjusted analyses. }\end{array}$ & Yes & NA & No & NA & NA & Low & NA \\
\hline $\begin{array}{l}\text { Sugawara, } \\
2013^{89}\end{array}$ & $\begin{array}{l}\text { Some } \\
\text { concerns }\end{array}$ & $\begin{array}{l}\text { Risk of inception bias, } \\
\text { residual confounding, and } \\
\text { measurement bias. }\end{array}$ & Probably no & Probably yes & No & No & NA & $\begin{array}{l}\text { Some } \\
\text { concerns }\end{array}$ & $\begin{array}{l}\text { Potential inception bias; } \\
\text { women were generally } \\
50-60 \text { years old at cohort } \\
\text { enrollment and only those } \\
\text { who were cancer-free } \\
\text { were followed. }\end{array}$ \\
\hline $\begin{array}{l}\text { Tamimi, } \\
\text { 2016 }\end{array}$ & $\begin{array}{l}\text { Some } \\
\text { concerns }\end{array}$ & $\begin{array}{l}\text { Potential selection bias, } \\
\text { recall bias, and } \\
\text { confounding bias; } \\
\text { moderate missing data for } \\
\text { a number of covariates. }\end{array}$ & Yes & NA & Probably no & NA & NA & Low & NA \\
\hline $\begin{array}{l}\text { Warner, } \\
2013^{91}\end{array}$ & $\begin{array}{l}\text { Some } \\
\text { concerns }\end{array}$ & $\begin{array}{l}\text { Risk of selection bias and } \\
\text { residual confounding; } \\
\text { some post-exposure } \\
\text { variables were included in } \\
\text { adjusted analyses. }\end{array}$ & Yes & Probably yes & No & Probably yes & NA & $\begin{array}{l}\text { Some } \\
\text { concerns }\end{array}$ & $\begin{array}{l}\text { Healthy user bias in } \\
\text { participants from Nurses } \\
\text { Health Study may lead to } \\
\text { selection bias. }\end{array}$ \\
\hline
\end{tabular}

$\mathrm{BF}$ = breastfeeding; $\mathrm{CVD}$ = cardiovascular disease; $\mathrm{KQ}$ = Key Question; $\mathrm{NA}$ = not applicable. 
Table C-20. KQ 2 risk of bias assessment: Breast cancer, part 2

\begin{tabular}{|c|c|c|c|c|c|c|c|c|}
\hline $\begin{array}{l}\text { Author, } \\
\text { Year }\end{array}$ & $\begin{array}{l}\text { 5. Is } \\
\text { confounding of } \\
\text { the exposure } \\
\text { effect unlikely? }\end{array}$ & $\begin{array}{l}5 a \text {. Did the } \\
\text { authors use an } \\
\text { appropriate } \\
\text { analysis to } \\
\text { adjust for } \\
\text { confounders? }\end{array}$ & $\begin{array}{l}\text { 5b. Were } \\
\text { confounding } \\
\text { domains that } \\
\text { were controlled } \\
\text { for measured } \\
\text { validly and } \\
\text { reliably? }\end{array}$ & $\begin{array}{l}5 c . \text { Did the } \\
\text { authors } \\
\text { avoid } \\
\text { adjusting for } \\
\text { post- } \\
\text { exposure } \\
\text { variables? }\end{array}$ & $\begin{array}{l}\text { 5d. Were } \\
\text { participants } \\
\text { analyzed } \\
\text { according to their } \\
\text { initial exposure } \\
\text { group throughout } \\
\text { followup? }\end{array}$ & $\begin{array}{l}\text { 5e. Were } \\
\text { exposure } \\
\text { discontinuations } \\
\text { or switches } \\
\text { unlikely to be } \\
\text { related to factors } \\
\text { prognostic for the } \\
\text { outcome? }\end{array}$ & $\begin{array}{l}\text { Bias arising } \\
\text { from } \\
\text { confounding? }\end{array}$ & Comments \\
\hline $\begin{array}{l}\text { Al-Amri, } \\
2015^{66}\end{array}$ & Probably no & Probably yes & No information & No & Probably no & NA & Some concerns & $\begin{array}{l}\text { Baseline differences were } \\
\text { adjusted for during statistical } \\
\text { analyses, however statistical } \\
\text { analyses were not well described } \\
\text { Validity/reliability of measurement } \\
\text { of confounders was also not well } \\
\text { described; results potentially } \\
\text { adjusted for post-exposure } \\
\text { variables (HRT use). }\end{array}$ \\
\hline $\begin{array}{l}\text { Al-Qutub, } \\
2013^{67}\end{array}$ & No & No & NA & Yes & NA & NA & High & $\begin{array}{l}\text { The analysis of BF and cancer } \\
\text { outcomes did not adjust for any } \\
\text { confounders. Groups are } \\
\text { dissimilar in regards to parity and } \\
\text { other factors at baseline. }\end{array}$ \\
\hline $\begin{array}{l}\text { Atkinson, } \\
2016^{68}\end{array}$ & Probably no & Probably yes & No information & No & NA & NA & Some concerns & $\begin{array}{l}\text { Race/ethnicity is a likely } \\
\text { confounder and was not adjusted } \\
\text { for; validity of covariate measures } \\
\text { unclear. Some potential post- } \\
\text { exposure variables were } \\
\text { considered in analyses (BMI, } \\
\text { smoking status). }\end{array}$ \\
\hline $\begin{array}{l}\text { Beaber, } \\
2008^{69}\end{array}$ & Probably no & Probably yes & Probably yes & Probably yes & Yes & Yes & Low & NA \\
\hline $\begin{array}{l}\text { Castello, } \\
2015^{70}\end{array}$ & Probably no & Probably no & Probably yes & No & NA & NA & Some concerns & $\begin{array}{l}\text { Adjusted analyses included some } \\
\text { factors that are post exposure } \\
\text { (total calorie intake, smoking, } \\
\text { history of breast problems). } \\
\text { History of breast problems is } \\
\text { unclear in terms of clinical } \\
\text { significance; could indicate BF } \\
\text { problems or factors related to } \\
\text { cancer. }\end{array}$ \\
\hline
\end{tabular}




\begin{tabular}{|c|c|c|c|c|c|c|c|c|}
\hline Author, Year & $\begin{array}{l}\text { 5. Is } \\
\text { confounding of } \\
\text { the exposure } \\
\text { effect unlikely? }\end{array}$ & $\begin{array}{l}5 a \text {. Did the } \\
\text { authors use an } \\
\text { appropriate } \\
\text { analysis to } \\
\text { adjust for } \\
\text { confounders? }\end{array}$ & $\begin{array}{l}\text { 5b. Were } \\
\text { confounding } \\
\text { domains that } \\
\text { were controlled } \\
\text { for measured } \\
\text { validly and } \\
\text { reliably? }\end{array}$ & $\begin{array}{l}5 c . \text { Did the } \\
\text { authors } \\
\text { avoid } \\
\text { adjusting for } \\
\text { post- } \\
\text { exposure } \\
\text { variables? }\end{array}$ & $\begin{array}{l}\text { 5d. Were } \\
\text { participants } \\
\text { analyzed } \\
\text { according to their } \\
\text { initial exposure } \\
\text { group throughout } \\
\text { followup? }\end{array}$ & $\begin{array}{l}\text { 5e. Were } \\
\text { exposure } \\
\text { discontinuations } \\
\text { or switches } \\
\text { unlikely to be } \\
\text { related to factors } \\
\text { prognostic for } \\
\text { the outcome? }\end{array}$ & $\begin{array}{l}\text { Bias arising } \\
\text { from } \\
\text { confounding? }\end{array}$ & Comments \\
\hline $\begin{array}{l}\text { Dalamaga, } \\
2011^{71}\end{array}$ & Probably no & No & NA & NA & NA & NA & High & $\begin{array}{l}\text { Main analyses look at association } \\
\text { between serum vistatin and } \\
\text { breast cancer; analysis reporting } \\
\text { on association between BF } \\
\text { exposure and cancer is not } \\
\text { adjusted for potential } \\
\text { confounders. }\end{array}$ \\
\hline $\mathrm{Ge}, 2015^{72}$ & Probably no & No & NA & NA & NA & NA & High & $\begin{array}{l}\text { The analysis of BF and cancer } \\
\text { outcomes did not adjust for } \\
\text { potential confounders. }\end{array}$ \\
\hline Hadji, $2007^{73}$ & No & No & NA & NA & NA & NA & High & $\begin{array}{l}\text { Analysis did not adjust for any } \\
\text { potential confounders; authors } \\
\text { report a multiple linear regression } \\
\text { analysis but provide no details or } \\
\text { quantitative results. }\end{array}$ \\
\hline Holm, $2017^{74}$ & Probably no & Yes & Probably yes & Yes & NA & NA & Some concerns & $\begin{array}{l}\text { Analysis controlled for multiple } \\
\text { factors, but residual confounding } \\
\text { may be of concern. }\end{array}$ \\
\hline Kabat, $2011^{75}$ & Probably no & Probably no & Probably yes & No & NA & $\overline{N A}$ & Some concerns & $\begin{array}{l}\text { Analysis controlled for multiple } \\
\text { factors, but residual confounding } \\
\text { may be of concern. }\end{array}$ \\
\hline $\begin{array}{l}\text { Kotsopoulos, } \\
2012^{76}\end{array}$ & Probably no & Probably yes & Probably yes & Probably yes & NA & $\mathrm{NA}$ & Some concerns & $\begin{array}{l}\text { Analysis controlled for multiple } \\
\text { confounders, but residual } \\
\text { confounding may be of concern. }\end{array}$ \\
\hline Kruk, 201477 & Probably no & No & NA & NA & NA & NA & High & $\begin{array}{l}\text { The analysis of BF and cancer } \\
\text { outcomes did not adjust for any } \\
\text { potential confounders. }\end{array}$ \\
\hline Lee, $2008^{78}$ & Probably no & Probably yes & Probably yes & Probably no & Yes & Yes & Low & NA \\
\hline
\end{tabular}




\begin{tabular}{|c|c|c|c|c|c|c|c|c|}
\hline Author, Year & $\begin{array}{l}\text { 5. Is } \\
\text { confounding } \\
\text { of the } \\
\text { exposure } \\
\text { effect } \\
\text { unlikely? }\end{array}$ & $\begin{array}{l}\text { 5a. Did the } \\
\text { authors use an } \\
\text { appropriate } \\
\text { analysis to } \\
\text { adjust for } \\
\text { confounders? }\end{array}$ & $\begin{array}{l}\text { 5b. Were } \\
\text { confounding } \\
\text { domains that } \\
\text { were controlled } \\
\text { for measured } \\
\text { validly and } \\
\text { reliably? }\end{array}$ & $\begin{array}{l}5 c . \text { Did the } \\
\text { authors avoid } \\
\text { adjusting for } \\
\text { post- } \\
\text { exposure } \\
\text { variables? }\end{array}$ & $\begin{array}{l}5 \mathrm{~d} \text {. Were } \\
\text { participants } \\
\text { analyzed } \\
\text { according to } \\
\text { their initial } \\
\text { exposure group } \\
\text { throughout } \\
\text { followup? }\end{array}$ & $\begin{array}{l}\text { 5e. Were exposure } \\
\text { discontinuations } \\
\text { or switches } \\
\text { unlikely to be } \\
\text { related to factors } \\
\text { prognostic for the } \\
\text { outcome? }\end{array}$ & $\begin{array}{l}\text { Bias arising } \\
\text { from } \\
\text { confounding? }\end{array}$ & Comments \\
\hline $\begin{array}{l}\text { Lumachi, } \\
2010^{79}\end{array}$ & No & No & NA & NA & NA & NA & High & $\begin{array}{l}\text { Analysis did adjust for any } \\
\text { potential confounders. }\end{array}$ \\
\hline Ma, $2006^{80}$ & Probably no & Probably no & Probably yes & No & NA & NA & Some concerns & $\begin{array}{l}\text { Some adjustment was made for } \\
\text { variables related to exposure } \\
\text { and outcome (BMI). }\end{array}$ \\
\hline Ma, $2017^{81}$ & Probably no & Yes & Probably yes & Yes & NA & NA & Some concerns & $\begin{array}{l}\text { Investigators included a } \\
\text { substantial number of potential } \\
\text { confounders in the models but } \\
\text { did not quantitatively assess } \\
\text { confounding; there is a potential } \\
\text { for over-adjustment or residual } \\
\text { confounding. }\end{array}$ \\
\hline Merritt, $2015^{82}$ & Probably no & No & Probably yes & No & NA & NA & Some concerns & $\begin{array}{l}\text { Analyses adjusted for multiple } \\
\text { potential confounders; some } \\
\text { (BMI) were likely related to both } \\
\text { exposure and outcome. }\end{array}$ \\
\hline Phillips, $2009^{83}$ & Probably no & Probably yes & Probably yes & Probably yes & Probably yes & NA & Low & $\begin{array}{l}\text { No confounders met the } 10 \% \\
\text { threshold for inclusion, so only } \\
\text { adjusted for age and race. }\end{array}$ \\
\hline Pieta, $2008^{84}$ & No & No & NA & Probably no & Probably yes & $\mathrm{NA}$ & High & $\begin{array}{l}\text { Only unadjusted odds ratios for } \\
\text { breastfeeding are presented. }\end{array}$ \\
\hline Press, $2010^{85}$ & No & No & NA & NA & Yes & Yes & High & $\begin{array}{l}\text { Analyses do not adjust for any } \\
\text { potential confounding factors. }\end{array}$ \\
\hline Ritte, $2013^{86}$ & Probably no & Yes & Probably yes & No & Probably yes & NA & Some concerns & $\begin{array}{l}\text { Adjusted results included post- } \\
\text { exposure variables, including } \\
\text { BMl (at enrollment), HRT use, } \\
\text { smoking and alcohol } \\
\text { consumption, and others. }\end{array}$ \\
\hline
\end{tabular}




\begin{tabular}{|c|c|c|c|c|c|c|c|c|}
\hline Author, Year & $\begin{array}{l}\text { 5. Is } \\
\text { confounding } \\
\text { of the } \\
\text { exposure } \\
\text { effect } \\
\text { unlikely? }\end{array}$ & $\begin{array}{l}\text { 5a. Did the } \\
\text { authors use an } \\
\text { appropriate } \\
\text { analysis to } \\
\text { adjust for } \\
\text { confounders? }\end{array}$ & $\begin{array}{l}\text { 5b. Were } \\
\text { confounding } \\
\text { domains that } \\
\text { were controlled } \\
\text { for measured } \\
\text { validly and } \\
\text { reliably? }\end{array}$ & $\begin{array}{l}\text { 5c. Did the } \\
\text { authors avoid } \\
\text { adjusting for } \\
\text { post- } \\
\text { exposure } \\
\text { variables? }\end{array}$ & $\begin{array}{l}\text { 5d. Were } \\
\text { participants } \\
\text { analyzed } \\
\text { according to } \\
\text { their initial } \\
\text { exposure group } \\
\text { throughout } \\
\text { followup? }\end{array}$ & $\begin{array}{l}\text { 5e. Were exposure } \\
\text { discontinuations } \\
\text { or switches } \\
\text { unlikely to be } \\
\text { related to factors } \\
\text { prognostic for the } \\
\text { outcome? }\end{array}$ & $\begin{array}{l}\text { Bias arising } \\
\text { from } \\
\text { confounding? }\end{array}$ & Comments \\
\hline $\begin{array}{l}\text { Ruszczyk, } \\
2016^{87}\end{array}$ & Probably no & Probably yes & Probably yes & Probably yes & NA & $\mathrm{NA}$ & Low & $\mathrm{NA}$ \\
\hline $\begin{array}{l}\text { Stendell-Hollis, } \\
2013^{88}\end{array}$ & Probably no & Probably yes & Probably yes & No & Probably yes & NA & Some concerns & $\begin{array}{l}\text { Analysis adjusted for some post- } \\
\text { exposure variables, including } \\
\text { BMI, smoking status, and use of } \\
\text { HRT. }\end{array}$ \\
\hline $\begin{array}{l}\text { Sugawara, } \\
2013^{89}\end{array}$ & Probably no & Probably yes & Probably yes & No & Yes & $\mathrm{NA}$ & Some concerns & $\begin{array}{l}\text { Analysis adjusted for BMl, } \\
\text { number of deliveries, and other } \\
\text { health behaviors potentially } \\
\text { associated with both exposure } \\
\text { and outcome. }\end{array}$ \\
\hline $\begin{array}{l}\text { Tamimi, } \\
2016^{90}\end{array}$ & Probably no & Probably no & Probably no & Probably yes & Probably yes & $\mathrm{NA}$ & Some concerns & $\begin{array}{l}\text { Multiple analyses adjusted for a } \\
\text { number of potential confounders; } \\
\text { the specific confounders varied } \\
\text { by model. Data were self- } \\
\text { reported via surveys. }\end{array}$ \\
\hline $\begin{array}{l}\text { Warner, } \\
2013^{91}\end{array}$ & Probably no & Probably yes & Probably yes & No & Yes & $\mathrm{NA}$ & Some concerns & $\begin{array}{l}\text { Models included some post- } \\
\text { exposure covariates (alcohol } \\
\text { consumption, postmenopausal } \\
\text { HRT, weight change since age } \\
\text { 18). }\end{array}$ \\
\hline
\end{tabular}

$\overline{\mathrm{BF}}$ = breastfeeding; $\mathrm{BMI}=$ body mass index; $\mathrm{HRT}$ = hormone replacement therapy; $\mathrm{KQ}=$ Key Question; $\mathrm{NA}$ = not applicable. 
Table C-21. KQ 2 risk of bias assessment: Breast cancer, part 3

\begin{tabular}{|c|c|c|c|c|c|}
\hline Author, Year & $\begin{array}{l}\text { 6. Is exposure } \\
\text { status well- } \\
\text { defined? }\end{array}$ & $\begin{array}{l}\text { 7. Was information on } \\
\text { exposure status recorded } \\
\text { at the time of exposure? }\end{array}$ & $\begin{array}{l}\text { 8. Was classification of } \\
\text { exposure status unaffected by } \\
\text { knowledge of the outcome or } \\
\text { risk of the outcome? }\end{array}$ & $\begin{array}{l}\text { Bias arising from } \\
\text { measurement of } \\
\text { exposures? }\end{array}$ & Comments \\
\hline
\end{tabular}

No High

Measurement of BF exposure is not well described (and there was no assessment of duration/intensity). Potential for recall bias (and differential recall bias among cases).

Al-Qutub, 2013 ${ }^{67} \quad$ No

Risk of recall bias due to amount of time

between BF and data collection. BF measure not well-defined (greater than or less than 12 months of lifetime BF).

Atkinson, 201668

Some concerns

Risk of recall bias due to amount of time between breastfeeding and data collection. BF measure (ever/never) does not take into account duration or intensity.

\begin{tabular}{lllll}
\hline Beaber, 200869 & Probably yes & No & No & Some concerns
\end{tabular}

BF history was assessed after diagnosis of breast cancer among cases, resulting in concern for differential recall bias.

\begin{tabular}{|c|c|c|c|c|}
\hline Castello, $2015^{70}$ & Yes & No & No & Some concerns \\
\hline Dalamaga, $2011^{71}$ & No & No & No & High \\
\hline
\end{tabular}

$\mathrm{Ge}, 2015^{72}$

Probably yes

Some concerns

\begin{tabular}{lllll}
\hline Hadji, $2007^{73}$ & Probably yes & No & Probably no & Some concerns \\
& & & & Some concerns
\end{tabular}
Risk of recall bias due to amount of time between breastfeeding and data collection.

No description of how BF exposure status was measured; potential for recall bias and differential recall among cases vs. controls. Risk of recall bias due to length of time between breastfeeding and data collection. Breastfeeding measure not well-defined (ever vs. never).

Risk of recall bias due to length of time between breastfeeding and data collection for poorly defined analysis of BF duration.

Potential for recall bias, though exposure is broadly defined. BF exposure was measured decades later in most women. 


\section{Author, Year \\ 6. Is exposure 7. Was information on \\ status well- \\ defined? \\ exposure status recorded \\ at the time of exposure? \\ 8. Was classification of \\ exposure status unaffected by \\ knowledge of the outcome or \\ risk of the outcome? \\ Bias arising from \\ measurement of \\ exposures?}

\begin{tabular}{lllll}
\hline Kabat, 2011 & Probably yes & No & Probably yes & High \\
\hline Kotsopoulos, $2012^{76}$ & Yes & No & Probably no & Some concerns \\
\hline Kruk, 201477 & Probably no & No & No & Some concerns
\end{tabular}

Lee, $2008^{78} \quad$ Probably yes No

No

No

No

Probably yes

Yes

No

No

No
Lumachi, $2010^{7}$
No

No

Probably yes

Probably no

Yes

No
Risk of recall bias; BF exposure was

measured decades later in most women. Risk

of recall bias for BF duration.

Recall bias may be present due to the length of time between exposure and assessment.

Risk of recall bias due to amount of time

between breastfeeding and data collection. BF measure not well-defined $(0$, less than 6

months, greater than 6 months).

BF history was assessed after diagnosis of breast cancer among cases, resulting in concern for differential recall bias.

BF history was assessed after diagnosis of breast cancer among cases, resulting in

concern for differential recall bias.

Potential recall bias (BF duration), and differential recall among cases.

Potential for recall bias since exposure occurred long before the outcome; information ascertained after the outcome occurred.

Ever/never BF is likely to be accurate but the duration of BF may have been more difficult to accurately recall.

\begin{tabular}{|c|c|c|c|c|c|}
\hline Merritt, $2015^{82}$ & Yes & No & Yes & Some concerns & $\begin{array}{l}\text { Risk of recall bias for some of the participants } \\
\text { who were older at baseline due to amount of } \\
\text { time between BF and data collection. }\end{array}$ \\
\hline Phillips, $2009^{83}$ & No & No & No & High & $\begin{array}{l}\text { BF history was assessed after diagnosis of } \\
\text { breast cancer among cases, resulting in } \\
\text { concern for differential recall bias. } \\
\text { Furthermore, assessment was limited to "ever } \\
\text { vs. never." }\end{array}$ \\
\hline
\end{tabular}




\section{Author, Year \\ 6. Is exposure 7. Was information on \\ status well- \\ 7. Was information on \\ 8. Was classification of \\ defined? \\ at the time of exposure? \\ exposure status unaffected by \\ Bias arising from \\ measurement of \\ risk of the outcome? \\ exposures? \\ Comments}

Pieta, 2008

No

No

No

High

BF history was assessed after diagnosis of breast cancer among cases, resulting in

concern for differential recall bias. Details

regarding how breastfeeding was assessed were not provided.

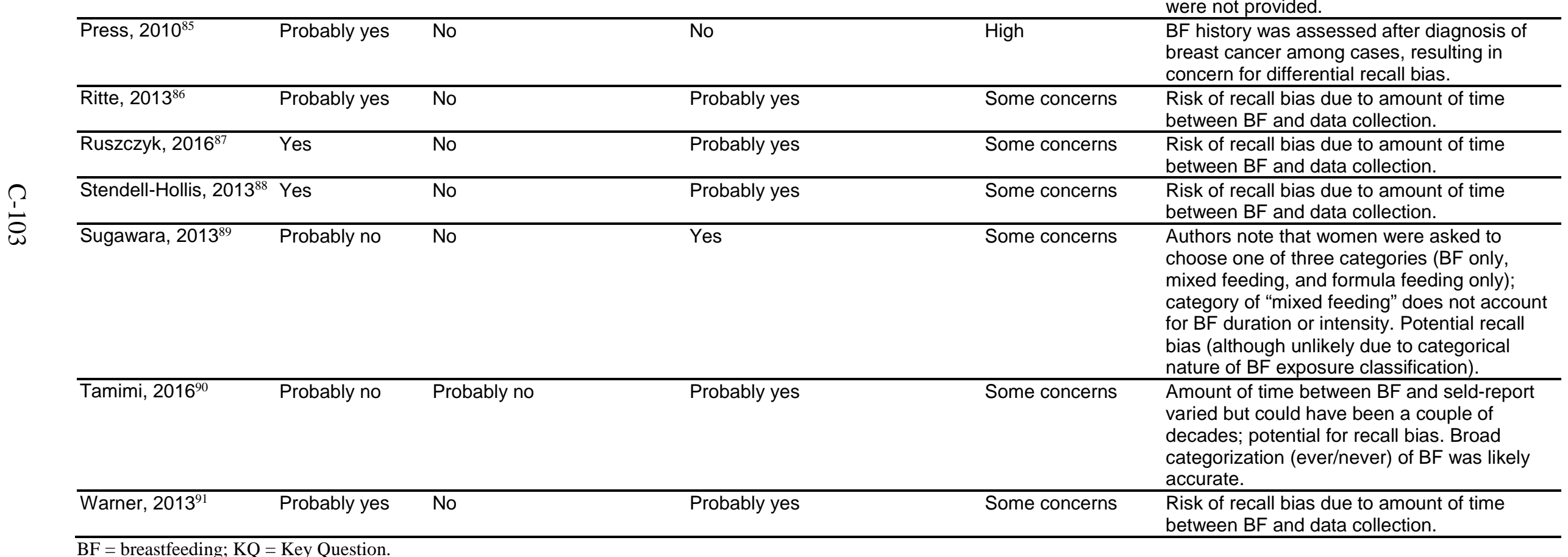

$\mathrm{BF}$ = breastfeeding; KQ = Key Question. 
Table C-22. KQ 2 risk of bias assessment: Breast cancer, part 4

\begin{tabular}{|c|c|c|c|c|c|c|c|}
\hline Author, Year & $\begin{array}{l}\text { 9. Were } \\
\text { outcome data } \\
\text { available for } \\
\text { all, or nearly } \\
\text { all, } \\
\text { participants? }\end{array}$ & $\begin{array}{l}\text { 10. Were few or } \\
\text { no participants } \\
\text { excluded } \\
\text { because of } \\
\text { missing data on } \\
\text { exposure } \\
\text { status? }\end{array}$ & $\begin{array}{l}\text { 11. Were few } \\
\text { or no } \\
\text { participants } \\
\text { excluded due } \\
\text { to missing } \\
\text { data on other } \\
\text { variables? }\end{array}$ & $\begin{array}{l}\text { 12. Was the } \\
\text { proportion of } \\
\text { participants and } \\
\text { reasons for } \\
\text { missing data } \\
\text { similar across } \\
\text { exposure } \\
\text { groups? }\end{array}$ & $\begin{array}{l}\text { 13. Were appropriate } \\
\text { statistical methods } \\
\text { used to account for } \\
\text { missing data or } \\
\text { assess robustness } \\
\text { to presence of } \\
\text { missing data? }\end{array}$ & $\begin{array}{l}\text { Bias Arising } \\
\text { From Missing } \\
\text { Data }\end{array}$ & Comments \\
\hline $\begin{array}{l}\text { Al-Amri, } \\
2015^{66}\end{array}$ & Probably yes & Probably yes & Probably yes & NA & NA & Low & NA \\
\hline $\begin{array}{l}\text { Al-Qutub, } \\
2013^{67}\end{array}$ & No information & No information & No information & No information & No information & $\begin{array}{l}\text { Uncertain } \\
\text { because no } \\
\text { information }\end{array}$ & None \\
\hline $\begin{array}{l}\text { Atkinson, } \\
2016^{68}\end{array}$ & No information & No information & No information & No information & No information & $\begin{array}{l}\text { Uncertain } \\
\text { because no } \\
\text { information }\end{array}$ & None \\
\hline Beaber, $2008^{69}$ & Yes & Probably yes & Probably yes & Probably yes & $\mathrm{NA}$ & Low & NA \\
\hline $\begin{array}{l}\text { Castello, } \\
2015^{70}\end{array}$ & Yes & Probably yes & Probably yes & No information & Yes & Some concerns & $\begin{array}{l}\text { Number of participants with missing } \\
\text { data unclear; however, analyses do } \\
\text { include imputation for missing data } \\
\text { specific to some variables in } \\
\text { analyses. }\end{array}$ \\
\hline $\begin{array}{l}\text { Dalamaga, } \\
2011^{71}\end{array}$ & Yes & Probably yes & Probably yes & No information & NA & Low & NA \\
\hline Ge, $2015^{72}$ & Yes & No information & No information & No information & No information & $\begin{array}{l}\text { Uncertain } \\
\text { because no } \\
\text { information }\end{array}$ & $\begin{array}{l}\text { Authors note "participants with } \\
\text { missing values in any of the } \\
\text { adjustment variables were excluded", } \\
\text { but the rates of missing values are not } \\
\text { reported. }\end{array}$ \\
\hline Hadji, $2007^{73}$ & No information & No information & No information & No information & No information & $\begin{array}{l}\text { Uncertain } \\
\text { because no } \\
\text { information }\end{array}$ & $\begin{array}{l}\text { Number of participants with missing } \\
\text { data unclear. }\end{array}$ \\
\hline Holm, $2017^{74}$ & Yes & Probably yes & Probably yes & No information & No information & Some concerns & $\begin{array}{l}\text { No details were provided about extent } \\
\text { of missing data or how the analytic } \\
\text { approach dealt with missing data. } \\
\text { Over } 99 \% \text { of parous participants } \\
\text { reported breastfeeding status. }\end{array}$ \\
\hline
\end{tabular}




\begin{tabular}{|c|c|c|c|c|c|c|c|}
\hline Author, Year & $\begin{array}{l}\text { 9. Were } \\
\text { outcome data } \\
\text { available for } \\
\text { all, or nearly } \\
\text { all, } \\
\text { participants? }\end{array}$ & $\begin{array}{l}\text { 10. Were few or } \\
\text { no participants } \\
\text { excluded } \\
\text { because of } \\
\text { missing data on } \\
\text { exposure } \\
\text { status? }\end{array}$ & $\begin{array}{l}\text { 11. Were few } \\
\text { or no } \\
\text { participants } \\
\text { excluded due } \\
\text { to missing } \\
\text { data on other } \\
\text { variables? }\end{array}$ & $\begin{array}{l}\text { 12. Was the } \\
\text { proportion of } \\
\text { participants and } \\
\text { reasons for } \\
\text { missing data } \\
\text { similar across } \\
\text { exposure } \\
\text { groups? }\end{array}$ & $\begin{array}{l}\text { 13. Were appropriate } \\
\text { statistical methods } \\
\text { used to account for } \\
\text { missing data or } \\
\text { assess robustness } \\
\text { to presence of } \\
\text { missing data? }\end{array}$ & $\begin{array}{l}\text { Bias Arising } \\
\text { From Missing } \\
\text { Data }\end{array}$ & Comments \\
\hline Kabat, $2011^{75}$ & Yes & Yes & Yes & No information & No information & Low & NA \\
\hline $\begin{array}{l}\text { Kotsopoulos, } \\
2012^{76}\end{array}$ & Probably yes & Probably yes & Probably yes & No information & No information & Low & NA \\
\hline Kruk, 2014 ${ }^{77}$ & Probably yes & No information & Yes & No information & No information & $\begin{array}{l}\text { Uncertain } \\
\text { because no } \\
\text { information }\end{array}$ & NA \\
\hline Lee, $2008^{78}$ & Probably yes & Probably yes & Probably yes & Probably yes & NA & Low & NA \\
\hline $\begin{array}{l}\text { Lumachi, } \\
2010^{79}\end{array}$ & Yes & No information & No information & No information & No information & $\begin{array}{l}\text { Uncertain } \\
\text { because no } \\
\text { information } \\
\end{array}$ & $\begin{array}{l}\text { Information on missing data not } \\
\text { provided. }\end{array}$ \\
\hline Ma, $2006^{80}$ & Probably yes & Probably yes & Probably no & No information & Probably yes & Some concerns & $\begin{array}{l}\text { Extent of missing data unclear; } \\
\text { analyses appear to impute values for } \\
\text { some variables. }\end{array}$ \\
\hline Ma, $2017^{81}$ & Yes & Yes & Yes & Probably yes & Probably no & Low & $\begin{array}{l}\text { Very few participants were excluded } \\
\text { due to missing data, but a majority of } \\
\text { those missing data were cases. } \\
\text { Investigators used a complete-case } \\
\text { analysis rather than employing } \\
\text { methods like multiple imputation. }\end{array}$ \\
\hline Merritt, $2015^{82}$ & Yes & Yes & Yes & NA & Probably no & Low & NA \\
\hline Phillips, $2009^{83}$ & Yes & Probably yes & Probably yes & No information & No information & Low & NA \\
\hline Pieta, $2008^{84}$ & Yes & No information & No information & No information & NA & $\begin{array}{l}\text { Uncertain } \\
\text { because no } \\
\text { information }\end{array}$ & $\begin{array}{l}\text { No information on missing data } \\
\text { provided. }\end{array}$ \\
\hline Press, $2010^{85}$ & Yes & No & NA & No information & No & Some concerns & $\begin{array}{l}\text { Missing exposure data for }>10 \% \text { of } \\
\text { control births in United States study. }\end{array}$ \\
\hline Ritte, $2013^{86}$ & Yes & Yes & Yes & No information & No information & Low & $\mathrm{NA}$ \\
\hline
\end{tabular}




\begin{tabular}{|c|c|c|c|c|c|c|c|}
\hline Author, Year & $\begin{array}{l}\text { 9. Were } \\
\text { outcome data } \\
\text { available for } \\
\text { all, or nearly } \\
\text { all, } \\
\text { participants? }\end{array}$ & $\begin{array}{l}\text { 10. Were few or } \\
\text { no participants } \\
\text { excluded } \\
\text { because of } \\
\text { missing data on } \\
\text { exposure } \\
\text { status? }\end{array}$ & $\begin{array}{l}\text { 11. Were few } \\
\text { or no } \\
\text { participants } \\
\text { excluded due } \\
\text { to missing } \\
\text { data on other } \\
\text { variables? }\end{array}$ & $\begin{array}{l}\text { 12. Was the } \\
\text { proportion of } \\
\text { participants and } \\
\text { reasons for } \\
\text { missing data } \\
\text { similar across } \\
\text { exposure } \\
\text { groups? }\end{array}$ & $\begin{array}{l}\text { 13. Were appropriate } \\
\text { statistical methods } \\
\text { used to account for } \\
\text { missing data or } \\
\text { assess robustness } \\
\text { to presence of } \\
\text { missing data? }\end{array}$ & $\begin{array}{l}\text { Bias Arising } \\
\text { From Missing } \\
\text { Data }\end{array}$ & Comments \\
\hline $\begin{array}{l}\text { Ruszczyk, } \\
2016^{87}\end{array}$ & No information & Probably yes & No information & No information & No information & $\begin{array}{l}\text { Uncertain } \\
\text { because no } \\
\text { information }\end{array}$ & $\begin{array}{l}\text { Amount of missing data is unclear; } \\
\text { discussion notes that up to } 30 \% \\
\text { otherwise eligible cases were } \\
\text { excluded due to missing data on } \\
\text { histology. }\end{array}$ \\
\hline $\begin{array}{l}\text { Stendell-Hollis } \\
2013^{88}\end{array}$ & No information & No information & No information & No information & Probably no & $\begin{array}{l}\text { Uncertain } \\
\text { because no } \\
\text { information }\end{array}$ & $\begin{array}{l}\text { Analysis was limited to those with } \\
\text { "known duration of lactation" but the } \\
\text { number of participants missing on that } \\
\text { variable is not reported. }\end{array}$ \\
\hline $\begin{array}{l}\text { Sugawara, } \\
2013^{89}\end{array}$ & Yes & Yes & Yes & No information & Probably no & Low & NA \\
\hline $\begin{array}{l}\text { Tamimi, } \\
2016^{90}\end{array}$ & Probably yes & Probably no & Probably no & No information & Probably no & Some concerns & $\begin{array}{l}\text { More than } 99 \% \text { of breast cancer } \\
\text { cases were confirmed; ER status was } \\
\text { only defined for } 79 \% \text { of cases. Over } \\
13 \% \text { of parous participants were } \\
\text { missing total breastfeeding duration. } \\
\text { Several covariates were moderately } \\
\text { missing data ( }>10 \% \text { missing). Authors } \\
\text { used a missing covariate indicator } \\
\text { method in analyses. }\end{array}$ \\
\hline $\begin{array}{l}\text { Warner, } \\
2013^{91}\end{array}$ & Probably yes & No information & No information & No information & No information & $\begin{array}{l}\text { Uncertain } \\
\text { because no } \\
\text { information }\end{array}$ & None \\
\hline
\end{tabular}

ER = estrogen receptor; KQ = Key Question; NA = not applicable. 
Table C-23. KQ 2 risk of bias assessment: Breast cancer, part 5

\begin{tabular}{|c|c|c|c|c|c|c|c|c|}
\hline Author, Year & $\begin{array}{l}\text { 14. Was } \\
\text { measurement of } \\
\text { outcomes } \\
\text { unlikely to have } \\
\text { been influenced } \\
\text { by knowledge of } \\
\text { the exposure } \\
\text { received? }\end{array}$ & $\begin{array}{l}\text { 15. Were } \\
\text { methods of } \\
\text { outcome } \\
\text { assessment } \\
\text { comparable } \\
\text { across } \\
\text { groups? }\end{array}$ & $\begin{array}{l}\text { 16. Was the } \\
\text { duration of } \\
\text { followup } \\
\text { adequate to } \\
\text { assess } \\
\text { outcomes? }\end{array}$ & $\begin{array}{l}\text { Bias Arising } \\
\text { From } \\
\text { Measurement } \\
\text { of Outcome }\end{array}$ & Comments & $\begin{array}{l}\text { effect estimate } \\
\text { unlikely to be } \\
\text { selected, on the } \\
\text { basis of the results, } \\
\text { from multiple } \\
\text { outcomes } \\
\text { measurements } \\
\text { within the domain, } \\
\text { multiple analyses, } \\
\text { or different } \\
\text { subgroups?* }\end{array}$ & $\begin{array}{l}\text { Bias Arising } \\
\text { From } \\
\text { Selection of } \\
\text { Reported } \\
\text { Results }\end{array}$ & Comments \\
\hline Al-Amri, $2015^{66}$ & Yes & NA & Probably no & Some concerns & $\begin{array}{l}\text { Mean (and median) } \\
\text { age of sample is } 48- \\
49 ; \text { this may not be } \\
\text { sufficient duration to } \\
\text { detect a difference in } \\
\text { breast cancer } \\
\text { outcomes. }\end{array}$ & Probably yes & Low & NA \\
\hline Al-Qutub, $2013^{67}$ & Probably yes & Probably yes & Probably no & Some concerns & $\begin{array}{l}\text { Mean age of cases } \\
\text { and controls ( } 40 \\
\text { years) may not be } \\
\text { sufficient to determine } \\
\text { risk of breast cancer. }\end{array}$ & Probably yes & Low & NA \\
\hline Atkinson, $2016^{68}$ & Yes & Probably yes & Yes & Low & NA & Probably yes & Low & NA \\
\hline Beaber, 2008 ${ }^{69}$ & Probably yes & Probably yes & NA & Low & NA & Probably yes & Low & $\mathrm{NA}$ \\
\hline Castello, $2015^{70}$ & Probably yes & Probably yes & Probably yes & Some concerns & $\begin{array}{l}\text { Age of women at } \\
\text { cohort enrollment } \\
\text { unclear, close to } 50 \% \\
\text { are classified as pre- } \\
\text { menopausal. Duration } \\
\text { may not be adequate } \\
\text { to fully assess BF } \\
\text { outcomes. }\end{array}$ & Probably yes & Low & NA \\
\hline
\end{tabular}




\begin{tabular}{|c|c|c|c|c|c|c|c|c|}
\hline Author, Year & $\begin{array}{l}\text { 14. Was } \\
\text { measurement of } \\
\text { outcomes } \\
\text { unlikely to have } \\
\text { been influenced } \\
\text { by knowledge of } \\
\text { the exposure } \\
\text { received? }\end{array}$ & $\begin{array}{l}\text { 15. Were } \\
\text { methods of } \\
\text { outcome } \\
\text { assessment } \\
\text { comparable } \\
\text { across } \\
\text { groups? }\end{array}$ & $\begin{array}{l}\text { 16. Was the } \\
\text { duration of } \\
\text { followup } \\
\text { adequate to } \\
\text { assess } \\
\text { outcomes? }\end{array}$ & $\begin{array}{l}\text { Bias Arising } \\
\text { From } \\
\text { Measurement } \\
\text { of Outcome }\end{array}$ & Comments & $\begin{array}{l}\text { 17. Is the reported } \\
\text { effect estimate } \\
\text { unlikely to be } \\
\text { selected, on the } \\
\text { basis of the results, } \\
\text { from multiple } \\
\text { outcomes } \\
\text { measurements } \\
\text { within the domain, } \\
\text { multiple analyses, } \\
\text { or different } \\
\text { subgroups?* }\end{array}$ & $\begin{array}{l}\text { Bias Arising } \\
\text { From } \\
\text { Selection of } \\
\text { Reported } \\
\text { Results }\end{array}$ & Comments \\
\hline Dalamaga, $2011^{71}$ & Probably yes & NA & Probably yes & Some concerns & $\begin{array}{l}\text { Unclear if outcome } \\
\text { assessors were } \\
\text { blinded. }\end{array}$ & $\begin{array}{l}\text { Uncertain because } \\
\text { no information }\end{array}$ & $\begin{array}{l}\text { Uncertain } \\
\text { because no } \\
\text { information }\end{array}$ & $\begin{array}{l}\text { Main outcome is } \\
\text { not relationship } \\
\text { between BF and } \\
\text { cancer; unclear } \\
\text { whether authors } \\
\text { considered or } \\
\text { obtained } \\
\text { additional } \\
\text { information (or } \\
\text { conducted other } \\
\text { analyses) related } \\
\text { to the association } \\
\text { between BF and } \\
\text { cancer. }\end{array}$ \\
\hline$\overline{\mathrm{Ge}, 2015^{72}}$ & Probably yes & Probably yes & Probably yes & Some concerns & $\begin{array}{l}\text { Enrolled cases were } \\
\text { between } 50 \text { and } 74 \\
\text { years of age; unclear } \\
\text { how many women } \\
\text { were in their } 50 \text { s vs. } \\
\text { older. Unclear } \\
\text { whether duration is } \\
\text { adequate across both } \\
\text { cases and controls to } \\
\text { assess outcome. }\end{array}$ & Probably yes & Low & NA \\
\hline Hadji, $2007^{73}$ & Probably yes & No information & Probably yes & Some concerns & $\begin{array}{l}\text { Ascertainment of } \\
\text { breast cancer status } \\
\text { was not described. }\end{array}$ & Probably yes & Low & NA \\
\hline
\end{tabular}




\begin{tabular}{|c|c|c|c|c|c|c|c|c|}
\hline Author, Year & $\begin{array}{l}\text { 14. Was } \\
\text { measurement of } \\
\text { outcomes } \\
\text { unlikely to have } \\
\text { been influenced } \\
\text { by knowledge of } \\
\text { the exposure } \\
\text { received? }\end{array}$ & $\begin{array}{l}\text { 15. Were } \\
\text { methods of } \\
\text { outcome } \\
\text { assessment } \\
\text { comparable } \\
\text { across } \\
\text { groups? }\end{array}$ & $\begin{array}{l}\text { 16. Was the } \\
\text { duration of } \\
\text { followup } \\
\text { adequate to } \\
\text { assess } \\
\text { outcomes? }\end{array}$ & $\begin{array}{l}\text { Bias Arising } \\
\text { From } \\
\text { Measurement } \\
\text { of Outcome }\end{array}$ & Comments & $\begin{array}{l}\text { 17. Is the reported } \\
\text { effect estimate } \\
\text { unlikely to be } \\
\text { selected, on the } \\
\text { basis of the results, } \\
\text { from multiple } \\
\text { outcomes } \\
\text { measurements } \\
\text { within the domain, } \\
\text { multiple analyses, } \\
\text { or different } \\
\text { subgroups?* }\end{array}$ & $\begin{array}{l}\text { Bias Arising } \\
\text { From } \\
\text { Selection of } \\
\text { Reported } \\
\text { Results }\end{array}$ & Comments \\
\hline Holm, $2017^{74}$ & Yes & Probably yes & Yes & Low & NA & Probably yes & Low & NA \\
\hline Kabat, $2011^{75}$ & Yes & Yes & Probably yes & Low & NA & Yes & Low & NA \\
\hline Kotsopoulos, $2012^{76}$ & Yes & Probably yes & Probably yes & Low & NA & Yes & Low & NA \\
\hline Kruk, $2014^{77}$ & Yes & Probably yes & Yes & Low & NA & Probably yes & Low & NA \\
\hline Lee, $2008^{78}$ & Probably yes & Probably yes & $\mathrm{NA}$ & Low & NA & Probably no & $\begin{array}{l}\text { Some } \\
\text { concerns }\end{array}$ & $\begin{array}{l}\text { Multiple effect } \\
\text { modifiers were } \\
\text { tested with no } \\
\text { correction for } \\
\text { multiple testing. }\end{array}$ \\
\hline Lumachi, $2010^{79}$ & Yes & Yes & NA & Low & NA & Probably yes & Low & $\begin{array}{l}\text { The association } \\
\text { between BF and } \\
\text { cancer was not } \\
\text { the primary } \\
\text { outcome of the } \\
\text { study, and } \\
\text { adjusted odds } \\
\text { ratios were not } \\
\text { reported. }\end{array}$ \\
\hline Ma, $2006^{80}$ & No & Probably yes & NA & Low & NA & Probably yes & Low & NA \\
\hline $\mathrm{Ma}, 2017^{81}$ & Yes & Probably yes & Yes & Low & $\overline{N A}$ & Yes & Low & NA \\
\hline
\end{tabular}




\begin{tabular}{|c|c|c|c|c|c|c|c|c|}
\hline Author, Year & $\begin{array}{l}\text { 14. Was } \\
\text { measurement of } \\
\text { outcomes } \\
\text { unlikely to have } \\
\text { been influenced } \\
\text { by knowledge of } \\
\text { the exposure } \\
\text { received? }\end{array}$ & $\begin{array}{l}\text { 15. Were } \\
\text { methods of } \\
\text { outcome } \\
\text { assessment } \\
\text { comparable } \\
\text { across } \\
\text { groups? }\end{array}$ & $\begin{array}{l}\text { 16. Was the } \\
\text { duration of } \\
\text { followup } \\
\text { adequate to } \\
\text { assess } \\
\text { outcomes? }\end{array}$ & $\begin{array}{l}\text { Bias Arising } \\
\text { From } \\
\text { Measurement } \\
\text { of Outcome }\end{array}$ & Comments & $\begin{array}{l}\text { 17. Is the reported } \\
\text { effect estimate } \\
\text { unlikely to be } \\
\text { selected, on the } \\
\text { basis of the results, } \\
\text { from multiple } \\
\text { outcomes } \\
\text { measurements } \\
\text { within the domain, } \\
\text { multiple analyses, } \\
\text { or different } \\
\text { subgroups?* }\end{array}$ & $\begin{array}{l}\text { Bias Arising } \\
\text { From } \\
\text { Selection of } \\
\text { Reported } \\
\text { Results }\end{array}$ & Comments \\
\hline Merritt, $2015^{82}$ & Yes & Yes & Probably yes & Some concerns & $\begin{array}{l}\text { Outcome assessment } \\
\text { varied by country; } \\
\text { duration of outcome } \\
\text { assessment may not } \\
\text { be sufficient for } \\
\text { younger women. }\end{array}$ & Probably yes & Low & NA \\
\hline Phillips, $2009^{83}$ & Probably yes & Probably yes & $\mathrm{NA}$ & Low & NA & Probably yes & Low & NA \\
\hline Pieta, $2008^{84}$ & Yes & Probably yes & $\mathrm{NA}$ & Some concerns & $\begin{array}{l}\text { Control participants } \\
\text { were considerably } \\
\text { younger than cases, } \\
\text { resulting in } \\
\text { (essentially) } \\
\text { differential followup } \\
\text { time-controls might } \\
\text { go on to get breast } \\
\text { cancer if they were } \\
\text { followed into the } \\
\text { Postmenopausal } \\
\text { period. }\end{array}$ & Probably no & $\begin{array}{l}\text { Some } \\
\text { concerns }\end{array}$ & $\begin{array}{l}\text { Authors report an } \\
\text { increased risk of } \\
\text { breast cancer with } \\
\text { "breast problems } \\
\text { needing } \\
\text { intervention in the } \\
\text { puerperium" - } \\
\text { unclear what data } \\
\text { were collected } \\
\text { and to what extent } \\
\text { this finding reflects } \\
\text { multiple testing. } \\
\end{array}$ \\
\hline Press, $2010^{85}$ & Yes & Yes & NA & Low & NA & Probably yes & Low & NA \\
\hline Ritte, $2013^{86}$ & Yes & Yes & Yes & Low & NA & Probably yes & Low & NA \\
\hline Ruszczyk, $2016^{87}$ & Yes & NA & Probably no & Some concerns & $\begin{array}{l}\text { Mean age of } \\
\text { participants was in the } \\
50 \text { s, which may not } \\
\text { be adequate to } \\
\text { assess outcomes. }\end{array}$ & Probably yes & Low & $\mathrm{NA}$ \\
\hline
\end{tabular}




\begin{tabular}{|c|c|c|c|c|c|c|c|c|}
\hline Author, Year & $\begin{array}{l}\text { 14. Was } \\
\text { measurement of } \\
\text { outcomes } \\
\text { unlikely to have } \\
\text { been influenced } \\
\text { by knowledge of } \\
\text { the exposure } \\
\text { received? }\end{array}$ & $\begin{array}{l}\text { 15. Were } \\
\text { methods of } \\
\text { outcome } \\
\text { assessment } \\
\text { comparable } \\
\text { across } \\
\text { groups? }\end{array}$ & $\begin{array}{l}\text { 16. Was the } \\
\text { duration of } \\
\text { followup } \\
\text { adequate to } \\
\text { assess } \\
\text { outcomes? }\end{array}$ & $\begin{array}{l}\text { Bias Arising } \\
\text { From } \\
\text { Measurement } \\
\text { of Outcomes }\end{array}$ & Comments & $\begin{array}{l}\text { 17. Is the reported } \\
\text { effect estimate } \\
\text { unlikely to be } \\
\text { selected, on the } \\
\text { basis of the results, } \\
\text { from multiple } \\
\text { outcomes } \\
\text { measurements } \\
\text { within the domain, } \\
\text { multiple analyses, } \\
\text { or different } \\
\text { subgroups?* }\end{array}$ & $\begin{array}{l}\text { Bias Arising } \\
\text { From } \\
\text { Selection of } \\
\text { Reported } \\
\text { Results }\end{array}$ & Comments \\
\hline $\begin{array}{l}\text { Stendell-Hollis, } \\
2013^{88}\end{array}$ & Yes & Yes & Yes & Low & NA & Probably yes & Low & NA \\
\hline Sugawara, $2013^{89}$ & Yes & Yes & Probably yes & Some concerns & $\begin{array}{l}\text { Unclear whether } 10 \\
\text { years sufficient } \\
\text { duration to measure } \\
\text { outcomes among all } \\
\text { women (age varied } \\
\text { from } 50-60 \text { s at } \\
\text { enrolment). }\end{array}$ & Probably yes & Low & NA \\
\hline Tamimi, $2016^{90}$ & Yes & Yes & Yes & Low & NA & Probably yes & Low & NA \\
\hline Warner, 2013 & Yes & Yes & Probably no & Some concerns & $\begin{array}{l}\text { Twelve years may not } \\
\text { be sufficient duration } \\
\text { to assess outcomes } \\
\text { among younger } \\
\text { women (age at } \\
\text { enrollment ranged } \\
\text { from } 39 \text { to } 40 \text { years). }\end{array}$ & Probably yes & Low & NA \\
\hline
\end{tabular}


Table C-24. KQ 2 risk of bias assessment: Ovarian cancer, part 1

\begin{tabular}{|c|c|c|c|c|c|c|c|c|c|}
\hline Author, Year & $\begin{array}{l}\text { Overall } \\
\text { Risk of } \\
\text { Bias } \\
\text { Rating }\end{array}$ & $\begin{array}{l}\text { Overall Rationale } \\
\text { for Risk of Bias } \\
\text { Rating }\end{array}$ & $\begin{array}{l}\text { 1. Was } \\
\text { method of } \\
\text { selection } \\
\text { unrelated to } \\
\text { exposurel } \\
\text { outcome? }\end{array}$ & $\begin{array}{l}\text { 1a. Were } \\
\text { post- } \\
\text { exposure } \\
\text { variables } \\
\text { that } \\
\text { influenced } \\
\text { selection } \\
\text { related to } \\
\text { exposurel } \\
\text { outcome? }\end{array}$ & $\begin{array}{l}\text { 2. Do start } \\
\text { of followup } \\
\text { and start of } \\
\text { exposure } \\
\text { coincide? }\end{array}$ & $\begin{array}{l}\text { 3. Were } \\
\text { adjustment } \\
\text { techniques } \\
\text { used to } \\
\text { correct for } \\
\text { presence of } \\
\text { selection } \\
\text { biases? }\end{array}$ & $\begin{array}{l}\text { 4. Were the } \\
\text { controls sampled } \\
\text { from the } \\
\text { population that } \\
\text { gave rise to the } \\
\text { cases, or using } \\
\text { another method } \\
\text { that avoids } \\
\text { selection bias? }\end{array}$ & $\begin{array}{l}\text { Bias arising } \\
\text { from } \\
\text { selection? }\end{array}$ & Comments \\
\hline Cook, $2017^{92}$ & $\begin{array}{l}\text { Some } \\
\text { concerns }\end{array}$ & $\begin{array}{l}\text { Potential } \\
\text { confounding and } \\
\text { recall bias as well } \\
\text { as potential } \\
\text { selection bias } \\
\text { related to } \\
\text { ascertainment of } \\
\text { controls. }\end{array}$ & NA & NA & NA & NA & Probably no & $\begin{array}{l}\text { Some } \\
\text { concerns }\end{array}$ & $\begin{array}{l}\text { Controls were } \\
\text { identified from } \\
\text { provincial health } \\
\text { rosters and a } \\
\text { mammography } \\
\text { screening } \\
\text { program. These } \\
\text { two groups may } \\
\text { only represent a } \\
\text { portion of the } \\
\text { source population; } \\
\text { the provincial } \\
\text { health rosters only } \\
\text { cover people with } \\
\text { public insurance. }\end{array}$ \\
\hline
\end{tabular}




\begin{tabular}{|c|c|c|c|c|c|c|c|c|c|}
\hline Author, Year & $\begin{array}{l}\text { Overall } \\
\text { Risk of } \\
\text { Bias } \\
\text { Rating }\end{array}$ & $\begin{array}{l}\text { Overall Rationale } \\
\text { for Risk of Bias } \\
\text { Rating }\end{array}$ & $\begin{array}{l}\text { 1. Was } \\
\text { method of } \\
\text { selection } \\
\text { unrelated to } \\
\text { exposurel } \\
\text { outcome? }\end{array}$ & $\begin{array}{l}\text { 1a. Were } \\
\text { post- } \\
\text { exposure } \\
\text { variables } \\
\text { that } \\
\text { influenced } \\
\text { selection } \\
\text { related to } \\
\text { exposurel } \\
\text { outcome? }\end{array}$ & $\begin{array}{l}\text { 2. Do start } \\
\text { of followup } \\
\text { and start of } \\
\text { exposure } \\
\text { coincide? }\end{array}$ & $\begin{array}{l}\text { 3. Were } \\
\text { adjustment } \\
\text { techniques } \\
\text { used to } \\
\text { correct for } \\
\text { presence of } \\
\text { selection } \\
\text { biases? }\end{array}$ & $\begin{array}{l}\text { 4. Were the } \\
\text { controls sampled } \\
\text { from the } \\
\text { population that } \\
\text { gave rise to the } \\
\text { cases, or using } \\
\text { another method } \\
\text { that avoids } \\
\text { selection bias? }\end{array}$ & $\begin{array}{l}\text { Bias arising } \\
\text { from } \\
\text { selection? }\end{array}$ & Comments \\
\hline Gay, $2015^{93}$ & $\begin{array}{l}\text { Some } \\
\text { concerns }\end{array}$ & $\begin{array}{l}\text { Potential for recall } \\
\text { bias and } \\
\text { confounding (as } \\
\text { noted in individual } \\
\text { domains). }\end{array}$ & Probably yes & Probably yes & No & No & NA & $\begin{array}{l}\text { Some } \\
\text { concerns }\end{array}$ & $\begin{array}{l}\text { Postmenopausal } \\
\text { women interested } \\
\text { in breast cancer } \\
\text { screening } \\
\text { comprise the } \\
\text { cohort and were } \\
\text { then followed for } \\
\text { an average of } 17 \\
\text { years. Women } \\
\text { who choose to } \\
\text { enter a breast } \\
\text { cancer screening } \\
\text { trial may reflect a } \\
\text { higher than } \\
\text { average risk pool } \\
\text { for ovarian } \\
\text { cancer, especially } \\
\text { if family history of } \\
\text { breast or ovarian } \\
\text { cancer is present. }\end{array}$ \\
\hline Gierach, $2006^{94}$ & $\begin{array}{l}\text { Some } \\
\text { concerns }\end{array}$ & $\begin{array}{l}\text { Potential recall } \\
\text { bias and poor } \\
\text { definition of } \\
\text { exposure. }\end{array}$ & NA & NA & NA & NA & Yes & Low & NA \\
\hline Jordan, $2008^{95}$ & $\begin{array}{l}\text { Some } \\
\text { concerns }\end{array}$ & $\begin{array}{l}\text { Potential bias } \\
\text { could arise from } \\
\text { recall bias, } \\
\text { residual } \\
\text { confounding, and } \\
\text { missing data (not } \\
\text { completely at } \\
\text { random). }\end{array}$ & NA & NA & NA & NA & Yes & Low & NA \\
\hline
\end{tabular}




\begin{tabular}{|c|c|c|c|c|c|c|c|c|c|}
\hline Author, Year & $\begin{array}{l}\text { Overall } \\
\text { Risk of } \\
\text { Bias } \\
\text { Rating }\end{array}$ & $\begin{array}{l}\text { Overall Rationale } \\
\text { for Risk of Bias } \\
\text { Rating }\end{array}$ & $\begin{array}{l}\text { 1. Was } \\
\text { method of } \\
\text { selection } \\
\text { unrelated to } \\
\text { exposurel } \\
\text { outcome? }\end{array}$ & $\begin{array}{l}\text { 1a. Were } \\
\text { post- } \\
\text { exposure } \\
\text { variables } \\
\text { that } \\
\text { influenced } \\
\text { selection } \\
\text { related to } \\
\text { exposurel } \\
\text { outcome? }\end{array}$ & $\begin{array}{l}\text { 2. Do start } \\
\text { of followup } \\
\text { and start of } \\
\text { exposure } \\
\text { coincide? }\end{array}$ & $\begin{array}{l}\text { 3. Were } \\
\text { adjustment } \\
\text { techniques } \\
\text { used to } \\
\text { correct for } \\
\text { presence of } \\
\text { selection } \\
\text { biases? }\end{array}$ & $\begin{array}{l}\text { 4. Were the } \\
\text { controls sampled } \\
\text { from the } \\
\text { population that } \\
\text { gave rise to the } \\
\text { cases, or using } \\
\text { another method } \\
\text { that avoids } \\
\text { selection bias? }\end{array}$ & $\begin{array}{l}\text { Bias arising } \\
\text { from } \\
\text { selection? }\end{array}$ & Comments \\
\hline $\begin{array}{l}\text { Kotsopoulos, } \\
2015^{96}\end{array}$ & High & $\begin{array}{l}\text { High risk of } \\
\text { selection bias, } \\
\text { recall bias (for } \\
\text { exposure status), } \\
\text { and bias related } \\
\text { to outcome } \\
\text { assessment. }\end{array}$ & No & Probably yes & No & No & Yes & High & $\begin{array}{l}\text { The average age } \\
\text { of cases at } \\
\text { diagnosis is } \\
\text { several years } \\
\text { younger than the } \\
\text { average age at } \\
\text { interview/ } \\
\text { enrollment in } \\
\text { study, suggesting } \\
\text { that cases are } \\
\text { prevalent. } \\
\text { BRCA1/2 } \\
\text { mutation carriers } \\
\text { are at higher than } \\
\text { average risk of } \\
\text { ovarian cancer to } \\
\text { begin with. }\end{array}$ \\
\hline Nagle, $2008^{97}$ & $\begin{array}{l}\text { Some } \\
\text { concerns }\end{array}$ & $\begin{array}{l}\text { Potential bias } \\
\text { could arise from } \\
\text { recall bias, } \\
\text { residual } \\
\text { confounding, and } \\
\text { missing data (not } \\
\text { completely at } \\
\text { random). } \\
\end{array}$ & NA & NA & NA & NA & Yes & Low & NA \\
\hline
\end{tabular}

BRCA = breast cancer gene; KQ = Key Question; NA = not applicable. 
Table C-25. KQ 2 risk of bias assessment: Ovarian cancer, part 2

\begin{tabular}{|c|c|c|c|c|c|c|c|c|}
\hline Author, Year & $\begin{array}{l}5 . \text { Is } \\
\text { confounding } \\
\text { of the } \\
\text { exposure } \\
\text { effect } \\
\text { unlikely? }\end{array}$ & $\begin{array}{l}5 a \text {. Did the } \\
\text { authors use an } \\
\text { appropriate } \\
\text { analysis to } \\
\text { adjust for } \\
\text { confounders? }\end{array}$ & $\begin{array}{l}\text { 5b. Were } \\
\text { confounding } \\
\text { domains that } \\
\text { were } \\
\text { controlled for } \\
\text { measured } \\
\text { validly and } \\
\text { reliably? }\end{array}$ & $\begin{array}{l}\text { 5c. Did the } \\
\text { authors } \\
\text { avoid } \\
\text { adjusting for } \\
\text { post- } \\
\text { exposure } \\
\text { variables? }\end{array}$ & $\begin{array}{l}\text { 5d. Were } \\
\text { participants } \\
\text { analyzed } \\
\text { according to } \\
\text { their initial } \\
\text { exposure } \\
\text { group } \\
\text { throughout } \\
\text { followup? }\end{array}$ & $\begin{array}{l}\text { 5e. Were exposure } \\
\text { discontinuations } \\
\text { or switches } \\
\text { unlikely to be } \\
\text { related to factors } \\
\text { prognostic for the } \\
\text { outcome? }\end{array}$ & $\begin{array}{l}\text { Bias arising } \\
\text { from } \\
\text { confounding? }\end{array}$ & Comments \\
\hline Cook, 201792 & No & No & NA & NA & Yes & NA & High & $\begin{array}{l}\text { Authors only provide } \\
\text { unadjusted frequencies of } \\
\text { BF exposure by outcome } \\
\text { status. }\end{array}$ \\
\hline Gay, $2015^{93}$ & Probably no & Probably yes & No & Probably yes & Yes & NA & Some concerns & $\begin{array}{l}\text { Authors did not adjust for a } \\
\text { couple of key risk factors } \\
\text { for ovarian cancer (oral } \\
\text { contraceptive use, tubal } \\
\text { ligation). Data were self- } \\
\text { reported at the beginning } \\
\text { of followup; some } \\
\text { information (e.g., BMI) may } \\
\text { have changed over the } \\
\text { course of followup. }\end{array}$ \\
\hline
\end{tabular}




\begin{tabular}{|c|c|c|c|c|c|c|c|c|}
\hline Author, Year & $\begin{array}{l}\text { 5. Is } \\
\text { confounding } \\
\text { of the } \\
\text { exposure } \\
\text { effect } \\
\text { unlikely? }\end{array}$ & $\begin{array}{l}5 \text { a. Did the } \\
\text { authors use an } \\
\text { appropriate } \\
\text { analysis to } \\
\text { adjust for } \\
\text { confounders? }\end{array}$ & $\begin{array}{l}\text { 5b. Were } \\
\text { confounding } \\
\text { domains that } \\
\text { were } \\
\text { controlled for } \\
\text { measured } \\
\text { validly and } \\
\text { reliably? }\end{array}$ & $\begin{array}{l}5 c . \text { Did the } \\
\text { authors } \\
\text { avoid } \\
\text { adjusting for } \\
\text { post- } \\
\text { exposure } \\
\text { variables? }\end{array}$ & $\begin{array}{l}\text { 5d. Were } \\
\text { participants } \\
\text { analyzed } \\
\text { according to } \\
\text { their initial } \\
\text { exposure } \\
\text { group } \\
\text { throughout } \\
\text { followup? }\end{array}$ & $\begin{array}{l}\text { 5e. Were exposure } \\
\text { discontinuations } \\
\text { or switches } \\
\text { unlikely to be } \\
\text { related to factors } \\
\text { prognostic for the } \\
\text { outcome? }\end{array}$ & $\begin{array}{l}\text { Bias arising } \\
\text { from } \\
\text { confounding? }\end{array}$ & Comments \\
\hline Jordan, $2008^{95}$ & Probably no & Probably yes & Probably yes & Probably yes & NA & NA & Some concerns & $\begin{array}{l}\text { Authors adjusted for a } \\
\text { number of ovarian cancer } \\
\text { risk factors that are related } \\
\text { (but not affected) by BF; } \\
\text { some residual confounding } \\
\text { may remain. Other } \\
\text { potential confounders, } \\
\text { though not described, did } \\
\text { not change the effect } \\
\text { estimate by more than 10\% } \\
\text { and were not included in } \\
\text { final adjusted models. } \\
\text { Analyses of breastfeeding } \\
\text { were appropriately } \\
\text { restricted to women who } \\
\text { had at least one live birth. }\end{array}$ \\
\hline $\begin{array}{l}\text { Kotsopoulos, } \\
2015^{96}\end{array}$ & Probably no & Probably yes & Probably yes & Probably yes & Yes & Yes & Some concerns & $\begin{array}{l}\text { Analysis of BF was not } \\
\text { restricted to parous women } \\
\text { but models were adjusted } \\
\text { for parity; methods are } \\
\text { unclear. }\end{array}$ \\
\hline Nagle, $2008^{97}$ & Probably no & Probably yes & Probably yes & Probably yes & NA & NA & Some concerns & $\begin{array}{l}\text { Authors adjusted for a } \\
\text { number of ovarian cancer } \\
\text { risk factors that are related } \\
\text { (but not affected) by BF; } \\
\text { some residual confounding } \\
\text { may remain. }\end{array}$ \\
\hline
\end{tabular}

$\overline{\mathrm{BF}}$ = breastfeeding; BMI = body mass index; KQ = Key Question; NA = not applicable. 


\section{Table C-26. KQ 2 risk of bias assessment: Ovarian cancer, part 3}

\begin{tabular}{|c|c|c|c|c|c|}
\hline Author, Year & $\begin{array}{l}\text { 6. Is exposure status } \\
\text { well-defined? }\end{array}$ & $\begin{array}{l}\text { 7. Was information on } \\
\text { exposure status recorded } \\
\text { at the time of exposure? }\end{array}$ & $\begin{array}{l}\text { 8. Was classification } \\
\text { of exposure status } \\
\text { unaffected by } \\
\text { knowledge of the } \\
\text { outcome or risk of the } \\
\text { outcome? }\end{array}$ & $\begin{array}{l}\text { Bias arising from } \\
\text { measurement of } \\
\text { exposures? }\end{array}$ & Comments \\
\hline Cook, $2017^{92}$ & Probably no & No & Probably no & Some concerns & $\begin{array}{l}\text { Potential for recall bias since } \\
\text { exposure occurred long before } \\
\text { the outcome. BF was only } \\
\text { measured by ever/never and a } \\
\text { dichotomous duration variable; } \\
\text { though the variables do not } \\
\text { provide details of BF history, } \\
\text { they are likely to be reported } \\
\text { relatively accurately. }\end{array}$ \\
\hline Gay, $2015^{93}$ & Probably yes & No & Probably yes & Low & $\begin{array}{l}\text { Recall bias regarding the } \\
\text { ever/never status is likely to be } \\
\text { minimal; exposure data was } \\
\text { collected prior to the outcome } \\
\text { occurring. }\end{array}$ \\
\hline Gierach, $2006^{94}$ & No & No & No & Some concerns & $\begin{array}{l}\text { BF status was only defined as } \\
\text { ever/never (no additional } \\
\text { information) and was collected at } \\
\text { the time of outcome (case or } \\
\text { control), possibly decades after } \\
\text { the exposure happened. Recall } \\
\text { bias is not likely to be a big issue } \\
\text { since the exposure is broadly } \\
\text { defined. }\end{array}$ \\
\hline Jordan, $2008^{95}$ & Probably yes & No & No & Some concerns & $\begin{array}{l}\text { There is unlikely to be recall bias } \\
\text { associated with an ever/never } \\
\text { BF status, but there may be } \\
\text { some recall bias associated with } \\
\text { duration of breastfeeding; } \\
\text { investigators suggested that the } \\
\text { question was asked for each live } \\
\text { birth which may support more } \\
\text { accurate recall. }\end{array}$ \\
\hline
\end{tabular}




\begin{tabular}{|c|c|c|c|c|c|}
\hline Author, Year & $\begin{array}{l}\text { 6. Is exposure status } \\
\text { well-defined? }\end{array}$ & $\begin{array}{l}\text { 7. Was information on } \\
\text { exposure status recorded } \\
\text { at the time of exposure? }\end{array}$ & $\begin{array}{l}\text { 8. Was classification } \\
\text { of exposure status } \\
\text { unaffected by } \\
\text { knowledge of the } \\
\text { outcome or risk of the } \\
\text { outcome? }\end{array}$ & $\begin{array}{l}\text { Bias arising from } \\
\text { measurement of } \\
\text { exposures? }\end{array}$ & Comments \\
\hline Kotsopoulos, $2015^{96}$ & Probably yes & No & No & High & $\begin{array}{l}\text { Women suspected/knew they } \\
\text { were BRCA1/2 mutation carriers } \\
\text { at the time of study enrollment } \\
\text { (and thus, that they were at } \\
\text { higher risk of ovarian cancer). } \\
\text { Recall bias is also a concern in } \\
\text { this study as BF exposure was, } \\
\text { for the most part, years prior to } \\
\text { the interview. Ever/never status } \\
\text { of BF is not as susceptible to } \\
\text { recall bias, but duration may be } \\
\text { more so. }\end{array}$ \\
\hline Nagle, $2008^{97}$ & Yes & No & No & Low & $\begin{array}{l}\text { Recall bias regarding the } \\
\text { ever/never exposure status is } \\
\text { likely to be minimal. }\end{array}$ \\
\hline
\end{tabular}

$\mathrm{BF}=$ breastfeeding; $\mathrm{BRCA}=$ breast cancer gene; $\mathrm{KQ}=$ Key Question. 
Table C-27. KQ 2 risk of bias assessment: Ovarian cancer, part 4

\begin{tabular}{|c|c|c|c|c|c|c|c|}
\hline Author, Year & $\begin{array}{l}\text { 9. Were } \\
\text { outcome data } \\
\text { available for } \\
\text { all, or nearly } \\
\text { all, } \\
\text { participants? }\end{array}$ & $\begin{array}{l}\text { 10. Were few or } \\
\text { no participants } \\
\text { excluded } \\
\text { because of } \\
\text { missing data on } \\
\text { exposure } \\
\text { status? }\end{array}$ & $\begin{array}{l}\text { 11. Were few } \\
\text { or no } \\
\text { participants } \\
\text { excluded due } \\
\text { to missing } \\
\text { data on other } \\
\text { variables? }\end{array}$ & $\begin{array}{l}\text { 12. Was the } \\
\text { proportion of } \\
\text { participants and } \\
\text { reasons for } \\
\text { missing data } \\
\text { similar across } \\
\text { exposure } \\
\text { groups? }\end{array}$ & $\begin{array}{l}\text { 13. Were } \\
\text { appropriate } \\
\text { statistical methods } \\
\text { used to account } \\
\text { for missing data or } \\
\text { assess robustness } \\
\text { to presence of } \\
\text { missing data? }\end{array}$ & $\begin{array}{l}\text { Bias Arising } \\
\text { From Missing } \\
\text { Data }\end{array}$ & Comments \\
\hline Cook, 201792 & Yes & Yes & Yes & Probably yes & No & Low & NA \\
\hline Gay, 201593 & Yes & Yes & Probably yes & No information & No information & Low & NA \\
\hline Gierach, 2006 ${ }^{94}$ & Yes & Yes & Yes & Probably yes & No & Some concerns & $\begin{array}{l}\text { Complete case analysis was } \\
\text { performed (i.e., no imputation); only } \\
1 \% \text { of women were excluded due to } \\
\text { missing offspring gender or } \\
\text { demographic data. }\end{array}$ \\
\hline Jordan, $2008^{95}$ & Probably yes & Probably yes & Probably yes & Probably yes & Probably no & Some concerns & $\begin{array}{l}\text { Though investigators only performed } \\
\text { a complete case analysis, the amount } \\
\text { of missing data for the exposure and } \\
\text { confounders is minimal. }\end{array}$ \\
\hline $\begin{array}{l}\text { Kotsopoulos, } \\
2015^{96}\end{array}$ & Yes & Probably no & Probably yes & Probably yes & Probably no & Some concerns & $\begin{array}{l}\text { Approximately } 10 \% \text { of participants } \\
\text { were missing BF status and a similar } \\
\text { amount were missing tubal ligation } \\
\text { status; a complete case analysis was } \\
\text { performed rather than multiple } \\
\text { imputation. }\end{array}$ \\
\hline Nagle, $2008^{97}$ & Probably yes & Probably yes & Probably yes & Probably yes & Probably no & Some concerns & $\begin{array}{l}\text { Though investigators only performed } \\
\text { a complete case analysis, the amount } \\
\text { of missing data for the exposure and } \\
\text { confounders is minimal. }\end{array}$ \\
\hline
\end{tabular}




\section{Table C-28. KQ 2 risk of bias assessment: Ovarian cancer, part 5}

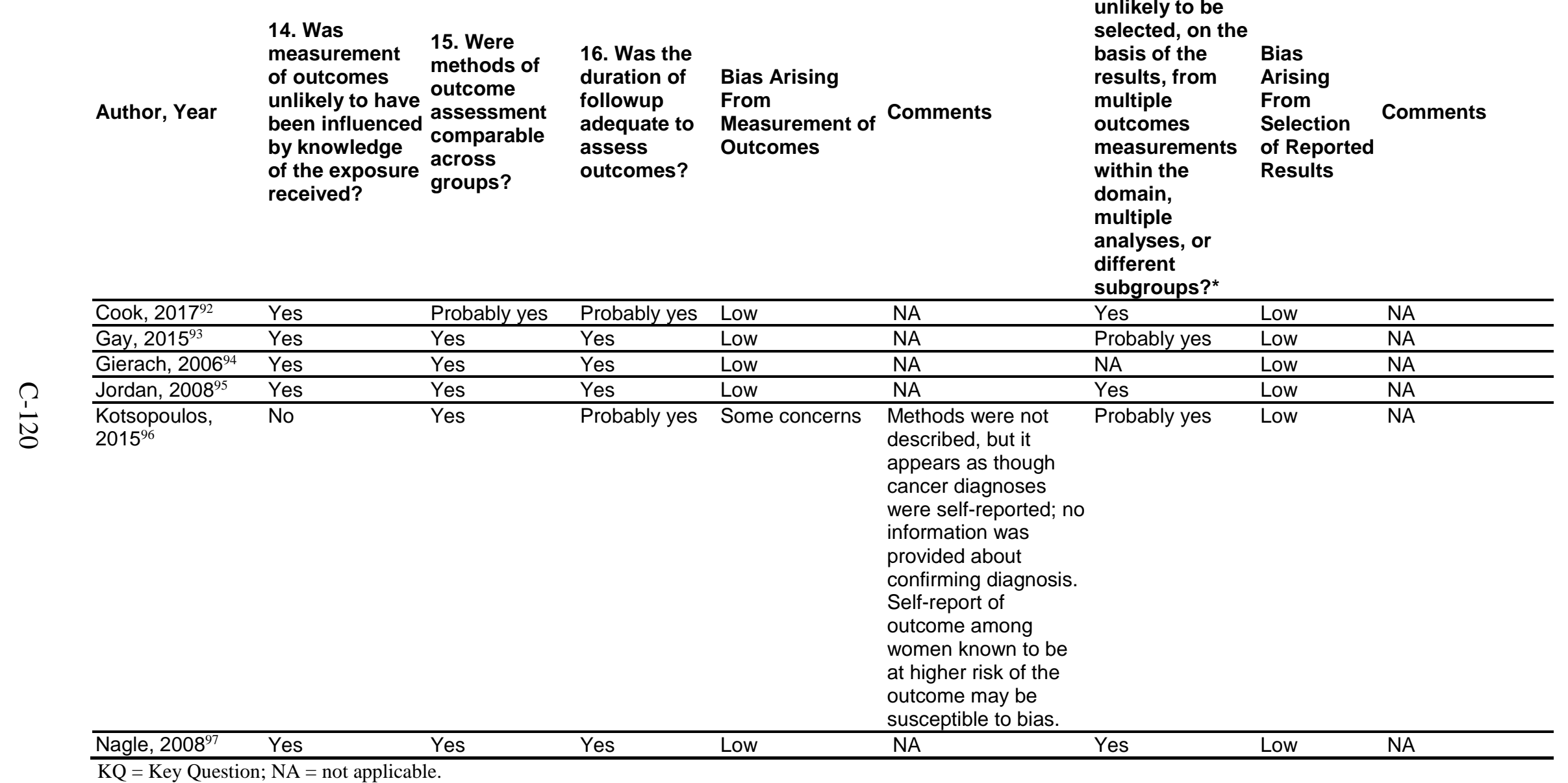

\section{Is the}

reported effect

estimate

unlikely to be

selected, on the

measurement 15 . Were

of outcomes methods

Comments

Arising

multiple

within the Results

different 
Table C-29. KQ 2 risk of bias assessment: Cardiovascular disease, part 1

\begin{tabular}{|c|c|c|c|c|c|c|c|c|c|}
\hline Author, Year & $\begin{array}{l}\text { Overall } \\
\text { Risk of } \\
\text { Bias Rating }\end{array}$ & $\begin{array}{l}\text { Overall Rationale } \\
\text { for Risk of Bias } \\
\text { Rating }\end{array}$ & $\begin{array}{l}\text { 1. Was } \\
\text { method of } \\
\text { selection } \\
\text { unrelated to } \\
\text { exposurel } \\
\text { outcome? }\end{array}$ & $\begin{array}{l}\text { 1a. Were } \\
\text { post- } \\
\text { exposure } \\
\text { variables } \\
\text { that } \\
\text { influenced } \\
\text { selection } \\
\text { related to } \\
\text { exposurel } \\
\text { outcome? }\end{array}$ & $\begin{array}{l}\text { 2. Do start } \\
\text { of followup } \\
\text { and start of } \\
\text { exposure } \\
\text { coincide? }\end{array}$ & $\begin{array}{l}\text { 3. Were } \\
\text { adjustment } \\
\text { techniques } \\
\text { used to } \\
\text { correct for } \\
\text { presence of } \\
\text { selection } \\
\text { biases? }\end{array}$ & $\begin{array}{l}\text { 4. Were the } \\
\text { controls } \\
\text { sampled from } \\
\text { the population } \\
\text { that gave rise to } \\
\text { the cases, or } \\
\text { using another } \\
\text { method that } \\
\text { avoids selection } \\
\text { bias? }\end{array}$ & $\begin{array}{l}\text { Bias arising } \\
\text { from } \\
\text { selection? }\end{array}$ & Comments \\
\hline Choi, $2017^{98}$ & High & $\begin{array}{l}\text { Multiple concerns } \\
\text { across all ROB } \\
\text { domain, including } \\
\text { potential for } \\
\text { selection bias, } \\
\text { measurement bias, } \\
\text { attrition bias, and } \\
\text { bias arising from } \\
\text { selection of } \\
\text { reported outcomes. }\end{array}$ & Probably yes & NA & No & No & NA & $\begin{array}{l}\text { Some } \\
\text { concerns }\end{array}$ & $\begin{array}{l}\text { Participants were } \\
\text { selected from the } \\
\text { Korean National } \\
\text { Health and Nutrition } \\
\text { survey if they had a } \\
\text { prior history of } \\
\text { pregnancy (but were } \\
\text { not currently pregnant) } \\
\text { and data on } \\
\text { breastfeeding } \\
\text { exposure. }\end{array}$ \\
\hline Lee, $2005^{99}$ & $\begin{array}{l}\text { Some } \\
\text { concerns }\end{array}$ & $\begin{array}{l}\text { Potential for } \\
\text { confounding, } \\
\text { selection bias, and } \\
\text { recall bias }\end{array}$ & Probably yes & No & No & No & Yes & $\begin{array}{l}\text { Some } \\
\text { concerns }\end{array}$ & $\begin{array}{l}\text { Data collection of } \\
\text { exposure information } \\
\text { follows data collection } \\
\text { on outcomes. Because } \\
\text { the study excludes } \\
\text { those with high BP at } \\
\text { baseline, which } \\
\text { merges } 1992 \text { and } \\
1994 \text { data, it is } \\
\text { possible that some } \\
\text { outcome data were } \\
\text { inappropriately } \\
\text { excluded. }\end{array}$ \\
\hline $\begin{array}{l}\text { Lupton, } \\
2013^{100}\end{array}$ & $\begin{array}{l}\text { Some } \\
\text { concerns }\end{array}$ & $\begin{array}{l}\text { Potential for recall } \\
\text { bias and } \\
\text { confounding. }\end{array}$ & NA & NA & NA & $\mathrm{NA}$ & Yes & Low & NA \\
\hline
\end{tabular}




\begin{tabular}{|c|c|c|c|c|c|c|c|c|c|}
\hline Author, Year & $\begin{array}{l}\text { Overall } \\
\text { Risk of } \\
\text { Bias Rating }\end{array}$ & $\begin{array}{l}\text { Overall Rationale } \\
\text { for Risk of Bias } \\
\text { Rating }\end{array}$ & $\begin{array}{l}\text { 1. Was } \\
\text { method of } \\
\text { selection } \\
\text { unrelated to } \\
\text { exposurel } \\
\text { outcome? }\end{array}$ & $\begin{array}{l}\text { 1a. Were } \\
\text { post- } \\
\text { exposure } \\
\text { variables } \\
\text { that } \\
\text { influenced } \\
\text { selection } \\
\text { related to } \\
\text { exposurel } \\
\text { outcome? }\end{array}$ & $\begin{array}{l}\text { 2. Do start } \\
\text { of followup } \\
\text { and start of } \\
\text { exposure } \\
\text { coincide? }\end{array}$ & $\begin{array}{l}\text { 3. Were } \\
\text { adjustment } \\
\text { techniques } \\
\text { used to } \\
\text { correct for } \\
\text { presence of } \\
\text { selection } \\
\text { biases? }\end{array}$ & $\begin{array}{l}\text { 4. Were the } \\
\text { controls } \\
\text { sampled from } \\
\text { the population } \\
\text { that gave rise to } \\
\text { the cases, or } \\
\text { using another } \\
\text { method that } \\
\text { avoids selection } \\
\text { bias? }\end{array}$ & $\begin{array}{l}\text { Bias arising } \\
\text { from } \\
\text { selection? }\end{array}$ & Comments \\
\hline $\begin{array}{l}\text { Natland } \\
\text { Fagerhaug, } \\
2013^{101}\end{array}$ & $\begin{array}{l}\text { Some } \\
\text { concerns }\end{array}$ & $\begin{array}{l}\text { Very low rate of } \\
\text { "never } \\
\text { breastfeeding," } \\
\text { suggesting unique } \\
\text { factors that might } \\
\text { have led to this } \\
\text { group of women } \\
\text { not initiating } \\
\text { breastfeeding. } \\
\text { Recall bias is also } \\
\text { a concern. }\end{array}$ & Yes & Probably yes & No & No & NA & $\begin{array}{l}\text { Some } \\
\text { concerns }\end{array}$ & $\begin{array}{l}\text { Concern for immortal } \\
\text { person-time bias- } \\
\text { limited to individuals } \\
\text { who were alive at } \\
\text { enrollment, which } \\
\text { occurred remote from } \\
\text { breastfeeding, } \\
\text { especially among } \\
\text { older participants. }\end{array}$ \\
\hline Parikh, $2016^{102}$ & $\begin{array}{l}\text { Some } \\
\text { concerns }\end{array}$ & $\begin{array}{l}\text { Potential for recall } \\
\text { and attrition bias, } \\
\text { immortal person } \\
\text { time bias, and } \\
\text { confounding. }\end{array}$ & Probably no & Probably yes & No & NA & NA & $\begin{array}{l}\text { Some } \\
\text { concerns }\end{array}$ & $\begin{array}{l}\text { Women were enrolled } \\
\text { after menopause, and } \\
\text { women with prevalent } \\
\text { CHD were excluded. } \\
\text { Thus, to be eligible for } \\
\text { the observational } \\
\text { cohort study women } \\
\text { had to have had a } \\
\text { period of disease-free } \\
\text { survival that creates } \\
\text { immortal person-time } \\
\text { bias. }\end{array}$ \\
\hline $\begin{array}{l}\text { Schwarz, } \\
2009^{3}\end{array}$ & $\begin{array}{l}\text { Some } \\
\text { concerns }\end{array}$ & $\begin{array}{l}\text { Potential for } \\
\text { immortal person } \\
\text { time bias, } \\
\text { confounding, recall } \\
\text { bias and } \\
\text { measurement bias } \\
\text { (of prevalent HTN } \\
\text { and CVD at WHI } \\
\text { enrollment). }\end{array}$ & Probably no & Probably yes & No & Probably yes & NA & $\begin{array}{l}\text { Some } \\
\text { concerns }\end{array}$ & $\begin{array}{l}\text { Concern for immortal } \\
\text { person-time bias - } \\
\text { limited to individuals } \\
\text { who were alive at } \\
\text { enrollment, which } \\
\text { occurred remote from } \\
\text { breastfeeding, } \\
\text { especially among } \\
\text { older participants. }\end{array}$ \\
\hline
\end{tabular}




\begin{tabular}{|c|c|c|c|c|c|c|c|c|c|}
\hline Author, Year & $\begin{array}{l}\text { Overall } \\
\text { Risk of } \\
\text { Bias Rating }\end{array}$ & $\begin{array}{l}\text { Overall Rationale } \\
\text { for Risk of Bias } \\
\text { Rating }\end{array}$ & $\begin{array}{l}\text { 1. Was } \\
\text { method of } \\
\text { selection } \\
\text { unrelated to } \\
\text { exposurel } \\
\text { outcome? }\end{array}$ & $\begin{array}{l}\text { 1a. Were } \\
\text { post- } \\
\text { exposure } \\
\text { variables } \\
\text { that } \\
\text { influenced } \\
\text { selection } \\
\text { related to } \\
\text { exposurel } \\
\text { outcome? }\end{array}$ & $\begin{array}{l}\text { 2. Do start } \\
\text { of followup } \\
\text { and start of } \\
\text { exposure } \\
\text { coincide? }\end{array}$ & $\begin{array}{l}\text { 3. Were } \\
\text { adjustment } \\
\text { techniques } \\
\text { used to } \\
\text { correct for } \\
\text { presence of } \\
\text { selection } \\
\text { biases? }\end{array}$ & $\begin{array}{l}\text { 4. Were the } \\
\text { controls } \\
\text { sampled from } \\
\text { the population } \\
\text { that gave rise to } \\
\text { the cases, or } \\
\text { using another } \\
\text { method that } \\
\text { avoids selection } \\
\text { bias? }\end{array}$ & $\begin{array}{l}\text { Bias arising } \\
\text { from } \\
\text { selection? }\end{array}$ & Comments \\
\hline $\begin{array}{l}\text { Stuebe, } \\
2009^{103}\end{array}$ & High & $\begin{array}{l}\text { Potential for } \\
\text { selection bias, } \\
\text { confounding, recall } \\
\text { bias, attrition. }\end{array}$ & Probably no & Yes & No & No & Yes & High & $\begin{array}{l}\text { Risk of selection bias } \\
\text { from a healthy cohort } \\
\text { of nurses. The study } \\
\text { excluded participants } \\
\text { with cardiovascular } \\
\text { issues or events (MI, } \\
\text { angina, CABG) } \\
\text { between the start of } \\
\text { the study in } 1976 \text { and } \\
\text { the collection of } \\
\text { lactation history in } \\
\text { 1986, which was the } \\
\text { starting point for } \\
\text { evaluating } \\
\text { cardiovascular } \\
\text { outcomes. }\end{array}$ \\
\hline $\begin{array}{l}\text { Stuebe, } \\
2011^{104}\end{array}$ & $\begin{array}{l}\text { Some } \\
\text { concerns }\end{array}$ & $\begin{array}{l}\text { Potential for recall } \\
\text { and attrition bias. }\end{array}$ & Probably no & Yes & No & Yes & Yes & $\begin{array}{l}\text { Some } \\
\text { concerns }\end{array}$ & $\begin{array}{l}\text { Risk of selection bias } \\
\text { from a healthy cohort } \\
\text { of nurses. The study } \\
\text { excluded participants } \\
\text { with hypertension, } \\
\text { elevated BP, and other } \\
\text { conditions between } \\
\text { the start of the study in } \\
1989 \text { and the } \\
\text { collection of dietary } \\
\text { history in } 1991 .\end{array}$ \\
\hline
\end{tabular}

myocardial infarction; NA = not applicable; ROB = risk of bias; WHI = Women's Health Initiative. 
Table C-30. KQ 2 risk of bias assessment: Cardiovascular disease, part 2

\begin{tabular}{|c|c|c|c|c|c|c|c|c|}
\hline Author, Year & $\begin{array}{l}\text { 5. Is } \\
\text { confoundin } \\
\text { g of the } \\
\text { exposure } \\
\text { effect } \\
\text { unlikely? }\end{array}$ & $\begin{array}{l}5 \text { a. Did the } \\
\text { authors use an } \\
\text { appropriate } \\
\text { analysis to } \\
\text { adjust for } \\
\text { confounders? }\end{array}$ & $\begin{array}{l}\text { 5b. Were } \\
\text { confounding } \\
\text { domains that } \\
\text { were } \\
\text { controlled for } \\
\text { measured } \\
\text { validly and } \\
\text { reliably? }\end{array}$ & $\begin{array}{l}\text { 5c. Did the } \\
\text { authors } \\
\text { avoid } \\
\text { adjusting for } \\
\text { post- } \\
\text { exposure } \\
\text { variables? }\end{array}$ & $\begin{array}{l}\text { 5d. Were } \\
\text { participants } \\
\text { analyzed } \\
\text { according to } \\
\text { their initial } \\
\text { exposure } \\
\text { group } \\
\text { throughout } \\
\text { followup? }\end{array}$ & $\begin{array}{l}\text { 5e. Were exposure } \\
\text { discontinuations } \\
\text { or switches } \\
\text { unlikely to be } \\
\text { related to factors } \\
\text { prognostic for the } \\
\text { outcome? }\end{array}$ & $\begin{array}{l}\text { Bias arising } \\
\text { from } \\
\text { confounding? }\end{array}$ & Comments \\
\hline Choi, $2017^{98}$ & No & Probably yes & Probably yes & No & NA & Probably yes & Some concerns & $\begin{array}{l}\text { Postexposure variables } \\
\text { associated with BF and } \\
\text { metabolic outcomes were } \\
\text { controlled for in analyses } \\
\text { (e.g., BMI). }\end{array}$ \\
\hline Lee, $2005^{99}$ & No & Yes & Yes & No & Yes & NA & Some concerns & $\begin{array}{l}\text { Authors adjust for potential } \\
\text { confounders, but include } \\
\text { parity which can be a } \\
\text { collider variable. }\end{array}$ \\
\hline $\begin{array}{l}\text { Lupton, } \\
2013^{100}\end{array}$ & No & Yes & Probably no & Yes & Yes & NA & Some concerns & $\begin{array}{l}\text { The authors adjusted for } \\
\text { lifestyle variables at the } \\
\text { time of enrollment, which } \\
\text { was, by definition, after the } \\
\text { exposure. This is of } \\
\text { particular concern for body } \\
\text { mass index, which may be } \\
\text { a mediator of any } \\
\text { association between } \\
\text { breastfeeding and } \\
\text { subsequent hypertension. }\end{array}$ \\
\hline $\begin{array}{l}\text { Natland } \\
\text { Fagerhaug, } \\
2013^{101}\end{array}$ & Probably no & Probably yes & Probably yes & Probably no & Yes & NA & Some concerns & $\begin{array}{l}\text { Worry about residual } \\
\text { confound by perinatal } \\
\text { events that led moms not } \\
\text { to breastfeed. Only } 3 \% \text { of } \\
\text { population had "never } \\
\text { breastfed," suggesting it is } \\
\text { highly atypical for women } \\
\text { not to initiate BF in this } \\
\text { population. Some } \\
\text { confounding variables e.g. } \\
\text { (smoking status- current, } \\
\text { former or "ever") may have } \\
\text { reflected post exposure } \\
\text { health behavior. }\end{array}$ \\
\hline
\end{tabular}




\begin{tabular}{|c|c|c|c|c|c|c|c|c|}
\hline Author, Year & $\begin{array}{l}\text { 5. Is } \\
\text { confoundin } \\
\text { g of the } \\
\text { exposure } \\
\text { effect } \\
\text { unlikely? }\end{array}$ & $\begin{array}{l}5 a \text {. Did the } \\
\text { authors use an } \\
\text { appropriate } \\
\text { analysis to } \\
\text { adjust for } \\
\text { confounders? }\end{array}$ & $\begin{array}{l}\text { 5b. Were } \\
\text { confounding } \\
\text { domains that } \\
\text { were } \\
\text { controlled for } \\
\text { measured } \\
\text { validly and } \\
\text { reliably? }\end{array}$ & $\begin{array}{l}5 c . \text { Did the } \\
\text { authors } \\
\text { avoid } \\
\text { adjusting for } \\
\text { post- } \\
\text { exposure } \\
\text { variables? }\end{array}$ & $\begin{array}{l}\text { 5d. Were } \\
\text { participants } \\
\text { analyzed } \\
\text { according to } \\
\text { their initial } \\
\text { exposure } \\
\text { group } \\
\text { throughout } \\
\text { followup? }\end{array}$ & $\begin{array}{l}\text { 5e. Were exposure } \\
\text { discontinuations } \\
\text { or switches } \\
\text { unlikely to be } \\
\text { related to factors } \\
\text { prognostic for the } \\
\text { outcome? }\end{array}$ & $\begin{array}{l}\text { Bias arising } \\
\text { from } \\
\text { confounding? }\end{array}$ & Comments \\
\hline $\begin{array}{l}\text { Parikh, } \\
2016^{102}\end{array}$ & No & Yes & Yes & Probably no & Yes & NA & Some concerns & $\begin{array}{l}\text { Models adjusted for CVD } \\
\text { risk factors (hypertension, } \\
\text { diabetes, BMI) that may } \\
\text { mediate associations } \\
\text { between breastfeeding } \\
\text { history and disease } \\
\text { outcome. }\end{array}$ \\
\hline $\begin{array}{l}\text { Schwarz, } \\
2009^{3}\end{array}$ & Probably no & Probably yes & Probably yes & Probably yes & Yes & NA & Some concerns & $\begin{array}{l}\text { Authors adjust for lifestyle } \\
\text { (diet) which is both pre and } \\
\text { post exposure (but } \\
\text { measured post exposure). }\end{array}$ \\
\hline $\begin{array}{l}\text { Stuebe, } \\
2009^{103}\end{array}$ & No & Yes & Yes & No & No & NA & High & $\begin{array}{l}\text { Although the study controls } \\
\text { for potential confounders, } \\
\text { the likelihood of a healthy } \\
\text { user effect likely results in } \\
\text { unmeasured confounding. } \\
\text { The study also adjusts for } \\
\text { supplement use, which } \\
\text { could potentially be a post- } \\
\text { exposure variable that } \\
\text { arises from breastfeeding } \\
\text { and could influence } \\
\text { cardiovascular outcomes. }\end{array}$ \\
\hline
\end{tabular}




\begin{tabular}{|c|c|c|c|c|c|c|c|c|}
\hline Author, Year & $\begin{array}{l}\text { 5. Is } \\
\text { confoundin } \\
\text { g of the } \\
\text { exposure } \\
\text { effect } \\
\text { unlikely? }\end{array}$ & $\begin{array}{l}\text { 5a. Did the } \\
\text { authors use an } \\
\text { appropriate } \\
\text { analysis to } \\
\text { adjust for } \\
\text { confounders? }\end{array}$ & $\begin{array}{l}\text { 5b. Were } \\
\text { confounding } \\
\text { domains that } \\
\text { were } \\
\text { controlled for } \\
\text { measured } \\
\text { validly and } \\
\text { reliably? }\end{array}$ & $\begin{array}{l}5 c . \text { Did the } \\
\text { authors } \\
\text { avoid } \\
\text { adjusting for } \\
\text { post- } \\
\text { exposure } \\
\text { variables? }\end{array}$ & $\begin{array}{l}\text { 5d. Were } \\
\text { participants } \\
\text { analyzed } \\
\text { according to } \\
\text { their initial } \\
\text { exposure } \\
\text { group } \\
\text { throughout } \\
\text { followup? }\end{array}$ & $\begin{array}{l}\text { 5e. Were exposure } \\
\text { discontinuations } \\
\text { or switches } \\
\text { unlikely to be } \\
\text { related to factors } \\
\text { prognostic for the } \\
\text { outcome? }\end{array}$ & $\begin{array}{l}\text { Bias arising } \\
\text { from } \\
\text { confounding? }\end{array}$ & Comments \\
\hline $\begin{array}{l}\text { Stuebe, } \\
2011^{104}\end{array}$ & No & Yes & Yes & Yes & Yes & NA & Some concerns & $\begin{array}{l}\text { Although the study controls } \\
\text { for potential confounders, } \\
\text { the likelihood of a healthy } \\
\text { user effect likely results in } \\
\text { unmeasured confounding. } \\
\text { The study did use inverse } \\
\text { probability weights in the } \\
\text { analysis. To address the } \\
\text { risk of bias from parity as a } \\
\text { collider, the authors limited } \\
\text { analysis to the first child } \\
\text { and incident hypertension. }\end{array}$ \\
\hline
\end{tabular}


Table C-31. KQ 2 risk of bias assessment: Cardiovascular disease, part 3

\begin{tabular}{|c|c|c|c|c|c|}
\hline Author, Year & $\begin{array}{l}\text { 6. Is exposure } \\
\text { status well- } \\
\text { defined? }\end{array}$ & $\begin{array}{l}\text { 7. Was information } \\
\text { on exposure status } \\
\text { recorded at the time } \\
\text { of exposure? }\end{array}$ & $\begin{array}{l}\text { 8. Was classification of } \\
\text { exposure status } \\
\text { unaffected by } \\
\text { knowledge of the } \\
\text { outcome or risk of the } \\
\text { outcome? }\end{array}$ & $\begin{array}{l}\text { Bias arising from } \\
\text { measurement of } \\
\text { exposures? }\end{array}$ & Comments \\
\hline Choi, $2017^{98}$ & Probably yes & No & Probably yes & Some concerns & $\begin{array}{l}\text { BF duration was measured by participant self- } \\
\text { report; women varied in age and recall bias is a } \\
\text { potential concern. Exposure is based on months of } \\
\text { total BF and does not take into consideration } \\
\text { intensity or BF duration per child. }\end{array}$ \\
\hline Lee, $2005^{99}$ & No & No & Probably no & Some concerns & Potential recall bias. \\
\hline Lupton, $2013^{100}$ & No & No & Probably no & Some concerns & $\begin{array}{l}\text { Potential recall bias. To the extent that there is } \\
\text { some evidence for validity of duration data for BF } \\
\text { this addresses some concerns. }\end{array}$ \\
\hline $\begin{array}{l}\text { Natland Fagerhaug, } \\
2013^{101}\end{array}$ & Probably yes & No & Probably yes & Some concerns & Potential recall bias. \\
\hline Parikh, 2016 & No & No & Probably no & Some concerns & $\begin{array}{l}\text { Potential recall bias, variable categorized as } \\
\text { yes/no, but yes is } 1 \text { or more months. To the extent } \\
\text { that there is some evidence for validity of duration } \\
\text { data for BF this addresses some concerns. }\end{array}$ \\
\hline Schwarz, $2009^{3}$ & Probably yes & No & Probably no & Some concerns & $\begin{array}{l}\text { Potential for recall bias (and differential recall bias) } \\
\text { given that exposure and outcomes were assessed } \\
\text { remote from BF status. }\end{array}$ \\
\hline Stuebe, $2009^{103}$ & No & No & Probably no & Some concerns & $\begin{array}{l}\text { Potential recall bias from 10-year lag between } \\
\text { baseline and collection of lactation history, some } \\
\text { births occurred prior to 1976, and so total recall } \\
\text { period was likely longer; one study (Primoslow) } \\
\text { suggests no difference between recall and } \\
\text { prospective recording for duration of BF. }\end{array}$ \\
\hline Stuebe, $2011^{104}$ & No & No & Probably no & Some concerns & $\begin{array}{l}\text { Potential recall bias from 10-year lag between } \\
\text { baseline and collection of lactation history, some } \\
\text { births occurred prior to 1976, and so total recall } \\
\text { period was likely longer; one study (Primoslow) } \\
\text { suggests no difference between recall and } \\
\text { prospective recording for duration of BF. Extent of } \\
\text { bias for yes/no outcome unclear and could } \\
\text { potentially be high risk of bias for that exposure } \\
\text { variable because of the risk of misclassification. }\end{array}$ \\
\hline
\end{tabular}


Table C-32. KQ 2 risk of bias assessment: Cardiovascular disease, part 4

\begin{tabular}{|c|c|c|c|c|c|c|c|}
\hline Author, Year & $\begin{array}{l}\text { 9. Were } \\
\text { outcome data } \\
\text { available for } \\
\text { all, or nearly } \\
\text { all, } \\
\text { participants? }\end{array}$ & $\begin{array}{l}\text { 10. Were few } \\
\text { or no } \\
\text { participants } \\
\text { excluded } \\
\text { because of } \\
\text { missing data } \\
\text { on exposure } \\
\text { status? } \\
\end{array}$ & $\begin{array}{l}\text { 11. Were few } \\
\text { or no } \\
\text { participants } \\
\text { excluded due } \\
\text { to missing } \\
\text { data on other } \\
\text { variables? }\end{array}$ & $\begin{array}{l}\text { 12. Was the } \\
\text { proportion of } \\
\text { participants and } \\
\text { reasons for } \\
\text { missing data } \\
\text { similar across } \\
\text { exposure } \\
\text { groups? }\end{array}$ & $\begin{array}{l}\text { 13. Were } \\
\text { appropriate } \\
\text { statistical methods } \\
\text { used to account } \\
\text { for missing data or } \\
\text { assess robustness } \\
\text { to presence of } \\
\text { missing data? }\end{array}$ & $\begin{array}{l}\text { Bias Arising } \\
\text { From Missing } \\
\text { Data }\end{array}$ & Comments \\
\hline Choi, $2017^{98}$ & Probably yes & Probably no & Probably no & No information & No & Some concerns & $\begin{array}{l}\text { Approximately } 19 \% \text { of the sample } \\
(\mathrm{n}=881) \text { were excluded due to } \\
\text { incomplete analytic data; unclear } \\
\text { whether this was primarily data on } \\
\text { exposure or other outcomes. }\end{array}$ \\
\hline Lee, $2005^{99}$ & Yes & Yes & Yes & No information & No & Low & Total excluded for missing data=0.3\%. \\
\hline $\begin{array}{l}\text { Lupton, } \\
2013^{100}\end{array}$ & No & No & No & No information & No & Some concerns & Missing data: $8.7 \%$ \\
\hline $\begin{array}{l}\text { Natland } \\
\text { Fagerhaug, } \\
2013^{101} \\
\end{array}$ & Probably yes & Probably yes & No information & No information & No information & Low & NA \\
\hline Parikh, 2016 & No & No & No & No information & No & Some concerns & $\begin{array}{l}\text { Missing data }>18.9 \% \text {, possibly up to } \\
22.1 \% \text {. }\end{array}$ \\
\hline $\begin{array}{l}\text { Schwarz, } \\
2009^{3}\end{array}$ & Probably yes & Probably yes & No & No information & No & Some concerns & $\begin{array}{l}\text { A minority of participants were } \\
\text { excluded due to no information on } \\
\text { parity }(\mathrm{N}=973) \text { or duration of lactation } \\
(\mathrm{N}=1,705) .\end{array}$ \\
\hline $\begin{array}{l}\text { Stuebe, } \\
2009^{103}\end{array}$ & No & No & No & No information & No & Some concerns & $\begin{array}{l}17 \% \text { of the sample excluded for } \\
\text { missing exposure data. }\end{array}$ \\
\hline $\begin{array}{l}\text { Stuebe, } \\
2011^{104}\end{array}$ & No & No & No & No information & No & Some concerns & $\begin{array}{l}\text { Missing data ranges from } 5.5 \% \text { to } \\
13.9 \% \text {. }\end{array}$ \\
\hline
\end{tabular}


Table C-33. KQ 2 risk of bias assessment: Cardiovascular disease, part 5

\begin{tabular}{|c|c|c|c|c|c|c|c|c|}
\hline Author, Year & $\begin{array}{l}\text { 14. Was } \\
\text { measurement } \\
\text { of outcomes } \\
\text { unlikely to } \\
\text { have been } \\
\text { influenced by } \\
\text { knowledge of } \\
\text { the exposure } \\
\text { received? }\end{array}$ & $\begin{array}{l}\text { 15. Were } \\
\text { methods of } \\
\text { outcome } \\
\text { assessment } \\
\text { comparable } \\
\text { across } \\
\text { groups? }\end{array}$ & $\begin{array}{l}\text { 16. Was the } \\
\text { duration of } \\
\text { followup } \\
\text { adequate to } \\
\text { assess } \\
\text { outcomes? }\end{array}$ & $\begin{array}{l}\text { Bias Arising } \\
\text { From } \\
\text { Measurement } \\
\text { of Outcomes }\end{array}$ & Comments & $\begin{array}{l}\text { estimate unlikely } \\
\text { to be selected, on } \\
\text { the basis of the } \\
\text { results, from } \\
\text { multiple } \\
\text { outcomes } \\
\text { measurements } \\
\text { within the } \\
\text { domain, multiple } \\
\text { analyses, or } \\
\text { different } \\
\text { subgroups?* }\end{array}$ & $\begin{array}{l}\text { Bias } \\
\text { Arising } \\
\text { From } \\
\text { Selection of } \\
\text { Reported } \\
\text { Results } \\
\end{array}$ & Comments \\
\hline Choi, $2017^{98}$ & Probably yes & Yes & Probably no & Some concerns & $\begin{array}{l}\text { Followup after BF likely } \\
\text { varies by age; women } \\
19 \text { to } 40 \text { years were } \\
\text { eligible (mean age: } 39 \\
\text { to } 40 \text { years). Strength } \\
\text { of association for } \\
\text { younger versus older } \\
\text { women may vary } \\
\text { based on several } \\
\text { factors. }\end{array}$ & Probably no & $\begin{array}{l}\text { Some } \\
\text { concerns }\end{array}$ & $\begin{array}{l}\text { Authors report } \\
\text { history of type } 2 \\
\text { DM diagnosis in } \\
\text { the description of } \\
\text { characteristics, } \\
\text { but only report } \\
\text { mean blood } \\
\text { sugar levels in } \\
\text { adjusted } \\
\text { analyses. }\end{array}$ \\
\hline Lee, $2005^{99}$ & Probably no & Yes & Yes & Low & $\begin{array}{l}\text { Outcomes based on } \\
\text { self-report, but authors } \\
\text { report that validation of } \\
\text { self-report has been } \\
\text { done ( } 94 \% \text { sensitivity, } \\
85 \% \text { specificity) }\end{array}$ & Probably no & Low & NA \\
\hline Lupton, 2013 & Probably yes & Yes & Yes & Low & NA & Probably yes & Low & NA \\
\hline $\begin{array}{l}\text { Natland Fagerhaug, } \\
2013^{101}\end{array}$ & Probably yes & Yes & Yes & Low & $\mathrm{NA}$ & Probably yes & Low & $\mathrm{NA}$ \\
\hline Parikh, 2016 & Yes & Yes & Yes & Low & NA & Yes & Low & NA \\
\hline
\end{tabular}

17. Is the

estimate unlikely

16. Was the

equate to

assess of Outcomes

within the Results

(n), or

different

varies by age; women

to 40 years). Strength 


\section{Was}

measurement 15 . Were

of outcomes methods of 16. Was the

unlikely to outcome

have been assessment

influenced by comparable

knowledge of across

the exposure groups?

received? duration of Bias Arising

\section{ollowup From}

adequate to Measurement

assess

of Outcomes

17. Is the

reported effect

estimate unlikely

to be selected, on

the basis of the Bias

results, from Arising

multiple From

Selection of Comments

measurements Reported

within the Results

domain, multiple

analyses, or

different

subgroups?*

\begin{tabular}{|c|c|c|c|c|c|c|c|c|}
\hline Schwarz, $2009^{3}$ & Probably no & Yes & Probably yes & Some concerns & $\begin{array}{l}\text { Outcomes primarily } \\
\text { based on self-report } \\
\text { (for prevalent HTN and } \\
\text { CVD at enrollment); } \\
\text { incident CVD during } \\
\text { WHI study period was } \\
\text { verified using standard } \\
\text { protocols. }\end{array}$ & Probably no & Low & NA \\
\hline Stuebe, $2009^{103}$ & Probably no & Yes & Yes & Some concerns & $\begin{array}{l}\text { Some outcomes } \\
\text { (angina, CABG) based } \\
\text { on self-report. }\end{array}$ & Probably no & Low & NA \\
\hline Stuebe, $2011^{104}$ & Probably no & Yes & Yes & Low & $\begin{array}{l}\text { Outcomes based on } \\
\text { self-report, but authors } \\
\text { report that validation of } \\
\text { self-report has been } \\
\text { done ( } 94 \% \text { sensitivity, } \\
85 \% \text { specificity). }\end{array}$ & Probably no & Low & NA \\
\hline
\end{tabular}

BF = breastfeeding; CABG = coronary artery bypass grafting; CVD = cardiovascular disease; DM = diabetes mellitus; HTN = hypertension; KQ = Key Question; NA = not applicable; WHI = Women's Health Initiative. 
Table C-34. KQ 2 risk of bias assessment: Diabetes, part 1

\begin{tabular}{|c|c|c|c|c|c|c|c|c|c|}
\hline Author, Year & $\begin{array}{l}\text { Overall } \\
\text { Risk of } \\
\text { Bias Rating }\end{array}$ & $\begin{array}{l}\text { Overall Rationale } \\
\text { for Risk of Bias } \\
\text { Rating }\end{array}$ & $\begin{array}{l}\text { 1. Was } \\
\text { method of } \\
\text { selection } \\
\text { unrelated } \\
\text { to } \\
\text { exposurel } \\
\text { outcome? }\end{array}$ & $\begin{array}{l}\text { 1a. Were } \\
\text { post- } \\
\text { exposure } \\
\text { variables } \\
\text { that } \\
\text { influenced } \\
\text { selection } \\
\text { related to } \\
\text { exposurel } \\
\text { outcome? }\end{array}$ & $\begin{array}{l}\text { 2. Do start } \\
\text { of followup } \\
\text { and start of } \\
\text { exposure } \\
\text { coincide? }\end{array}$ & $\begin{array}{l}\text { 3. Were } \\
\text { adjustment } \\
\text { techniques } \\
\text { used to } \\
\text { correct for } \\
\text { presence of } \\
\text { selection } \\
\text { biases? }\end{array}$ & $\begin{array}{l}\text { 4. Were the } \\
\text { controls } \\
\text { sampled from } \\
\text { the population } \\
\text { that gave rise to } \\
\text { the cases, or } \\
\text { using another } \\
\text { method that } \\
\text { avoids selection } \\
\text { bias? }\end{array}$ & $\begin{array}{l}\text { Bias arising } \\
\text { from } \\
\text { selection? }\end{array}$ & Comments \\
\hline $\begin{array}{l}\text { Chamberlain, } \\
2016^{105}\end{array}$ & High & $\begin{array}{l}\text { Risk of selection } \\
\text { bias due to method } \\
\text { of outcome } \\
\text { assessment } \\
\text { (selection of } \\
\text { women with } \\
\text { postpartum lab } \\
\text { testing } \\
\text { documented); high } \\
\text { risk of } \\
\text { measurement bias } \\
\text { for exposure status } \\
\text { (BF status } \\
\text { determined at } \\
\text { hospital discharge } \\
\text { only) and high risk } \\
\text { of bias due to } \\
\text { confounding. }\end{array}$ & Probably no & Probably no & Yes & No & NA & $\begin{array}{l}\text { Some } \\
\text { concerns }\end{array}$ & $\begin{array}{l}\text { Only women who had } \\
\text { postpartum laboratory } \\
\text { assessments were } \\
\text { included in the analysis; } \\
\text { women without lab } \\
\text { testing may have been } \\
\text { less concerned about } \\
\text { type } 2 \text { diabetes and } \\
\text { could have deferred } \\
\text { testing (or testing may } \\
\text { not have been } \\
\text { recommended). }\end{array}$ \\
\hline
\end{tabular}




\begin{tabular}{|c|c|c|c|c|c|c|c|c|c|}
\hline Author, Year & $\begin{array}{l}\text { Overall } \\
\text { Risk of } \\
\text { Bias Rating }\end{array}$ & $\begin{array}{l}\text { Overall Rationale } \\
\text { for Risk of Bias } \\
\text { Rating }\end{array}$ & $\begin{array}{l}\text { 1. Was } \\
\text { method of } \\
\text { selection } \\
\text { unrelated } \\
\text { to } \\
\text { exposurel } \\
\text { outcome? }\end{array}$ & $\begin{array}{l}\text { 1a. Were } \\
\text { post- } \\
\text { exposure } \\
\text { variables } \\
\text { that } \\
\text { influenced } \\
\text { selection } \\
\text { related to } \\
\text { exposurel } \\
\text { outcome? }\end{array}$ & $\begin{array}{l}\text { 2. Do start } \\
\text { of followup } \\
\text { and start of } \\
\text { exposure } \\
\text { coincide? }\end{array}$ & $\begin{array}{l}\text { 3. Were } \\
\text { adjustment } \\
\text { techniques } \\
\text { used to } \\
\text { correct for } \\
\text { presence of } \\
\text { selection } \\
\text { biases? }\end{array}$ & $\begin{array}{l}\text { 4. Were the } \\
\text { controls } \\
\text { sampled from } \\
\text { the population } \\
\text { that gave rise to } \\
\text { the cases, or } \\
\text { using another } \\
\text { method that } \\
\text { avoids selection } \\
\text { bias? }\end{array}$ & $\begin{array}{l}\text { Bias arising } \\
\text { from } \\
\text { selection? }\end{array}$ & Comments \\
\hline $\begin{array}{l}\text { Gunderson, } \\
\text { 2012 } \\
\text { Gunder; } \\
\text { 2015 } \\
\end{array}$ & $\begin{array}{l}\text { Some } \\
\text { concerns }\end{array}$ & $\begin{array}{l}\text { Lack of adjustment } \\
\text { for important } \\
\text { confounders, } \\
\text { including baseline } \\
\text { metabolic status } \\
\text { and SES, raises } \\
\text { concern about } \\
\text { residual } \\
\text { confounding; } \\
\text { duration ( } 2 \text { years) } \\
\text { may not be } \\
\text { sufficient to assess } \\
\text { incident type } 2 \\
\text { diabetes among } \\
\text { younger women. }\end{array}$ & No & Probably yes & No & No & NA & $\begin{array}{l}\text { Some } \\
\text { concerns }\end{array}$ & $\begin{array}{l}\text { Women were screened } \\
\text { during pregnancy, but } \\
\text { enrolled based on BF } \\
\text { status at } 4-6 \text { weeks } \\
\text { after delivery; women } \\
\text { who were mixed } \\
\text { feeding at } 4-6 \text { weeks } \\
\text { were ineligible. Thus, } \\
\text { feeding behavior (the } \\
\text { exposure) was an } \\
\text { exclusion criteria for the } \\
\text { study. Women who had } \\
\text { stopped BF by } 3 \text { weeks, } \\
\text { potentially due to failure } \\
\text { of lactogenesis, were } \\
\text { classified as formula } \\
\text { feeding. }\end{array}$ \\
\hline $\begin{array}{l}\text { Martens, } \\
2016^{108}\end{array}$ & $\begin{array}{l}\text { Some } \\
\text { Concerns }\end{array}$ & $\begin{array}{l}\text { Risk of } \\
\text { measurement bias } \\
\text { and bias due to } \\
\text { unmeasured } \\
\text { confounding. }\end{array}$ & $\begin{array}{l}\text { Probably } \\
\text { yes }\end{array}$ & NA & Yes & NA & NA & Low & NA \\
\hline
\end{tabular}




\begin{tabular}{|c|c|c|c|c|c|c|c|c|c|}
\hline Author, Year & $\begin{array}{l}\text { Overall } \\
\text { Risk of } \\
\text { Bias Rating }\end{array}$ & $\begin{array}{l}\text { Overall Rationale } \\
\text { for Risk of Bias } \\
\text { Rating }\end{array}$ & $\begin{array}{l}\text { 1. Was } \\
\text { method of } \\
\text { selection } \\
\text { unrelated } \\
\text { to } \\
\text { exposurel } \\
\text { outcome? }\end{array}$ & $\begin{array}{l}\text { 1a. Were } \\
\text { post- } \\
\text { exposure } \\
\text { variables } \\
\text { that } \\
\text { influenced } \\
\text { selection } \\
\text { related to } \\
\text { exposurel } \\
\text { outcome? }\end{array}$ & $\begin{array}{l}\text { 2. Do start } \\
\text { of followup } \\
\text { and start of } \\
\text { exposure } \\
\text { coincide? }\end{array}$ & $\begin{array}{l}\text { 3. Were } \\
\text { adjustment } \\
\text { techniques } \\
\text { used to } \\
\text { correct for } \\
\text { presence of } \\
\text { selection } \\
\text { biases? }\end{array}$ & $\begin{array}{l}\text { 4. Were the } \\
\text { controls } \\
\text { sampled from } \\
\text { the population } \\
\text { that gave rise to } \\
\text { the cases, or } \\
\text { using another } \\
\text { method that } \\
\text { avoids selection } \\
\text { bias? }\end{array}$ & $\begin{array}{l}\text { Bias arising } \\
\text { from } \\
\text { selection? }\end{array}$ & Comments \\
\hline Schwarz, $2009^{3}$ & $\begin{array}{l}\text { Some } \\
\text { concerns }\end{array}$ & $\begin{array}{l}\text { Potential for } \\
\text { immortal person- } \\
\text { time bias, } \\
\text { confounding, recall } \\
\text { bias, and } \\
\text { measurement bias } \\
\text { (of prevalent HTN } \\
\text { and CVD at WHI } \\
\text { enrollment). } \\
\end{array}$ & Probably no & Probably yes & No & Probably yes & NA & $\begin{array}{l}\text { Some } \\
\text { concerns }\end{array}$ & $\begin{array}{l}\text { Concern for immortal } \\
\text { person-time bias limited } \\
\text { to individuals who were } \\
\text { alive at enrollment, } \\
\text { which occurred remote } \\
\text { from breastfeeding, } \\
\text { especially among older } \\
\text { participants. }\end{array}$ \\
\hline Zong, 2016 & High & $\begin{array}{l}\text { High risk of } \\
\text { selection bias and } \\
\text { measurement of } \\
\text { exposure. }\end{array}$ & Probably no & Probably yes & No & No & $\overline{N A}$ & High & $\begin{array}{l}\text { Participants were } \\
\text { selected from the } \\
\text { NHANES survey if they } \\
\text { had completed a } \\
\text { medical exam, had a } \\
\text { pregnancy resulting in a } \\
\text { live birth, were not } \\
\text { currently pregnant or } \\
\text { BF, or had prevalent } \\
\text { cardiovascular disease } \\
\text { or cancer. Both } \\
\text { cardiovascular disease } \\
\text { and cancer may be } \\
\text { associated with BF } \\
\text { duration (exposure) and } \\
\text { diabetes (outcome). }\end{array}$ \\
\hline
\end{tabular}

socioeconomic status. 
Table C-35. KQ 2 risk of bias assessment: Diabetes, part 2

\begin{tabular}{|c|c|c|c|c|c|c|c|c|}
\hline Author, Year & $\begin{array}{l}\text { 5. Is } \\
\text { confounding } \\
\text { of the } \\
\text { exposure } \\
\text { effect } \\
\text { unlikely? }\end{array}$ & $\begin{array}{l}5 a . \text { Did the } \\
\text { authors use an } \\
\text { appropriate } \\
\text { analysis to } \\
\text { adjust for } \\
\text { confounders? }\end{array}$ & $\begin{array}{l}\text { 5b. Were } \\
\text { confounding } \\
\text { domains that } \\
\text { were } \\
\text { controlled for } \\
\text { measured } \\
\text { validly and } \\
\text { reliably? }\end{array}$ & $\begin{array}{l}\text { 5c. Did the } \\
\text { authors } \\
\text { avoid } \\
\text { adjusting } \\
\text { for post- } \\
\text { exposure } \\
\text { variables? }\end{array}$ & $\begin{array}{l}\text { 5d. Were } \\
\text { participants } \\
\text { analyzed } \\
\text { according to } \\
\text { their initial } \\
\text { exposure group } \\
\text { throughout } \\
\text { followup? }\end{array}$ & $\begin{array}{l}\text { 5e. Were } \\
\text { exposure } \\
\text { discontinuations } \\
\text { or switches } \\
\text { unlikely to be } \\
\text { related to } \\
\text { factors } \\
\text { prognostic for } \\
\text { the outcome? }\end{array}$ & $\begin{array}{l}\text { Bias arising } \\
\text { from } \\
\text { confounding? }\end{array}$ & Comments \\
\hline $\begin{array}{l}\text { Chamberlain, } \\
2016^{105}\end{array}$ & No & No & NA & Yes & Yes & Yes & High & $\begin{array}{l}\text { Breastfeeding at discharge is } \\
\text { primary handled as a } \\
\text { confounder for increased } \\
\text { rates of type } 2 \text { diabetes in } \\
\text { this population; authors do } \\
\text { not appear to have } \\
\text { addressed confounding in the } \\
\text { analysis of type } 2 \text { diabetes } \\
\text { incidence by breastfeeding } \\
\text { status (e.g., obesity). }\end{array}$ \\
\hline $\begin{array}{l}\text { Gunderson, } \\
2012^{106} ; \\
\text { Gunderson, } \\
2015^{107}\end{array}$ & No & Probably no & Probably yes & No & Yes & NA & Some concerns & $\begin{array}{l}\text { No adjustment for some } \\
\text { important confounding factors } \\
\text { related to SES (e.g., use of } \\
\text { WIC). Unclear how the } \\
\text { confounders included in the } \\
\text { adjusted analyses were } \\
\text { selected. }\end{array}$ \\
\hline
\end{tabular}




\begin{tabular}{|c|c|c|c|c|c|c|c|c|}
\hline Author, Year & $\begin{array}{l}\text { 5. Is } \\
\text { confounding of } \\
\text { the exposure } \\
\text { effect unlikely? }\end{array}$ & $\begin{array}{l}5 a \text {. Did the } \\
\text { authors use an } \\
\text { appropriate } \\
\text { analysis to } \\
\text { adjust for } \\
\text { confounders? }\end{array}$ & $\begin{array}{l}\text { 5b. Were } \\
\text { confounding } \\
\text { domains that } \\
\text { were controlled } \\
\text { for measured } \\
\text { validly and } \\
\text { reliably? }\end{array}$ & $\begin{array}{l}\text { 5c. Did the } \\
\text { authors avoid } \\
\text { adjusting for } \\
\text { post-exposure } \\
\text { variables? }\end{array}$ & $\begin{array}{l}\text { 5d. Were } \\
\text { participants } \\
\text { analyzed } \\
\text { according to } \\
\text { their initial } \\
\text { exposure group } \\
\text { throughout } \\
\text { followup? }\end{array}$ & $\begin{array}{l}\text { 5e. Were } \\
\text { exposure } \\
\text { discontinuation } \\
\text { s or switches } \\
\text { unlikely to be } \\
\text { related to } \\
\text { factors } \\
\text { prognostic for } \\
\text { the outcome? }\end{array}$ & $\begin{array}{l}\text { Bias arising } \\
\text { from } \\
\text { confounding? }\end{array}$ & Comments \\
\hline Martens, $2016^{108}$ & Probably no & Probably yes & Probably yes & Yes & NA & Probably yes & Some concerns & $\begin{array}{l}\text { Some potentially } \\
\text { confounding } \\
\text { factors were not } \\
\text { considered in the } \\
\text { analyses, such as } \\
\text { pre-pregnancy } \\
\text { BMl. }\end{array}$ \\
\hline Schwarz, $2009^{3}$ & Probably no & Probably yes & Probably yes & Probably yes & Yes & NA & Some concerns & $\begin{array}{l}\text { Authors adjusted } \\
\text { for lifestyle (diet), } \\
\text { which is both pre- } \\
\text { and postexposure } \\
\text { (but measured } \\
\text { postexposure). } \\
\end{array}$ \\
\hline Zong, 2016 & No & Probably yes & Probably yes & No & NA & Probably yes & Some concerns & $\begin{array}{l}\text { Exposure and } \\
\text { outcome data } \\
\text { collected at same } \\
\text { time (exposure } \\
\text { based on recall); } \\
\text { domains that } \\
\text { were adjusted for } \\
\text { may reflect } \\
\text { postexposure } \\
\text { variables } \\
\text { associated with } \\
\text { both BF and } \\
\text { diabetes (e.g., } \\
\text { SES, other health } \\
\text { behaviors). }\end{array}$ \\
\hline
\end{tabular}


Table C-36. KQ 2 risk of bias assessment: Diabetes, part 3

\begin{tabular}{|c|c|c|c|c|c|}
\hline Author, Year & $\begin{array}{l}\text { 6. Is exposure status } \\
\text { well-defined? }\end{array}$ & $\begin{array}{l}\text { 7. Was information on } \\
\text { exposure status recorded } \\
\text { at the time of exposure? }\end{array}$ & $\begin{array}{l}\text { 8. Was classification } \\
\text { of exposure status } \\
\text { unaffected by } \\
\text { knowledge of the } \\
\text { outcome or risk of the } \\
\text { outcome? }\end{array}$ & $\begin{array}{l}\text { Bias arising from } \\
\text { measurement of } \\
\text { exposures? }\end{array}$ & Comments \\
\hline $\begin{array}{l}\text { Chamberlain, } \\
2016^{105}\end{array}$ & No & Probably no & Yes & High & $\begin{array}{l}\text { Exposure status is measured } \\
\text { and defined only in terms of BF } \\
\text { status at hospital discharge; } \\
\text { whether women who were fully } \\
\text { or partially breastfeeding } \\
\text { continued with their recorded } \\
\text { behavior is unclear. }\end{array}$ \\
\hline $\begin{array}{l}\text { Gunderson, 2012106; } \\
\text { Gunderson, } 2015^{107}\end{array}$ & Yes & Yes & Probably yes & Low & NA \\
\hline Martens, 2016 & Probably no & Yes & Yes & Some concerns & $\begin{array}{l}\text { Exposure is defined as BF } \\
\text { initiation only (at time of hospital } \\
\text { discharge). A small\% of mothers } \\
\text { may initiate BF after discharge; } \\
\text { among those who did initiate, } \\
\text { duration and intensity of BF was } \\
\text { not considered. }\end{array}$ \\
\hline Schwarz, $2009^{3}$ & Probably yes & No & Probably no & Some concerns & $\begin{array}{l}\text { Potential for recall bias (and } \\
\text { differential recall bias) given that } \\
\text { exposure and outcomes were } \\
\text { assessed remote from BF status. }\end{array}$ \\
\hline Zong, 2016 & Probably no & No & Probably yes & High & $\begin{array}{l}\text { BF exposure was measured by } \\
\text { asking whether participants had } \\
\text { ever breastfed then whether they } \\
\text { breastfed for more than one } \\
\text { month per child. No assessment } \\
\text { of BF intensity and no } \\
\text { information on duration for longer } \\
\text { than one month per child. }\end{array}$ \\
\hline
\end{tabular}


Table C-37. KQ 2 risk of bias assessment: Diabetes, part 4

\begin{tabular}{|c|c|c|c|c|c|c|c|}
\hline Author, Year & $\begin{array}{l}\text { 9. Were outcome } \\
\text { data available for } \\
\text { all, or nearly all, } \\
\text { participants? }\end{array}$ & $\begin{array}{l}\text { 10. Were few or } \\
\text { no participants } \\
\text { excluded } \\
\text { because of } \\
\text { missing data on } \\
\text { exposure } \\
\text { status? }\end{array}$ & $\begin{array}{l}\text { 11. Were few } \\
\text { or no } \\
\text { participants } \\
\text { excluded due } \\
\text { to missing } \\
\text { data on other } \\
\text { variables? }\end{array}$ & $\begin{array}{l}\text { 12. Was the } \\
\text { proportion } \\
\text { of } \\
\text { participants } \\
\text { and reasons } \\
\text { for missing } \\
\text { data similar } \\
\text { across } \\
\text { exposure } \\
\text { groups? }\end{array}$ & $\begin{array}{l}\text { 13. Were } \\
\text { appropriate } \\
\text { statistical } \\
\text { methods used } \\
\text { to account for } \\
\text { missing data or } \\
\text { assess } \\
\text { robustness to } \\
\text { presence of } \\
\text { missing data? }\end{array}$ & $\begin{array}{l}\text { Bias Arising From } \\
\text { Missing Data }\end{array}$ & Comments \\
\hline $\begin{array}{l}\text { Chamberlain, } \\
2016^{105}\end{array}$ & Probably no & Probably no & NA & $\begin{array}{l}\text { No } \\
\text { information }\end{array}$ & No & High & $\begin{array}{l}\text { Authors report on association between BF } \\
\text { and type } 2 \text { diabetes only in the subsample } \\
\text { of women who had a chart review ( } 289 \\
\text { births out of } 483 \text { births with a postpartum } \\
\text { screening test). This is less than the size } \\
\text { noted by authors in the methods section for } \\
\text { detecting a } 10 \% \text { difference in diabetes } \\
\text { among indigenous and nonindigenous } \\
\text { women. }\end{array}$ \\
\hline $\begin{array}{l}\text { Gunderson, } \\
\text { 2012 } \\
\text { Gunderson, } \\
2015^{107}\end{array}$ & Probably yes & Probably yes & Yes & $\begin{array}{l}\text { No } \\
\text { information }\end{array}$ & No information & Low & NA \\
\hline Martens, $2016^{108}$ & Probably yes & No information & Probably yes & $\begin{array}{l}\text { No } \\
\text { information }\end{array}$ & NA & Some concerns & $\begin{array}{l}\text { Approximately } 4 \% \text { of total deliveries were } \\
\text { excluded due to no information on } \\
\text { prepregnancy diabetes or breastfeeding } \\
\text { data. Degree of missing outcome data is } \\
\text { unclear; measurement was based on } \\
\text { hospitalization and outpatient visit codes } \\
\text { only. }\end{array}$ \\
\hline Schwarz, $2009^{3}$ & Probably yes & Probably yes & No & $\begin{array}{l}\text { No } \\
\text { information }\end{array}$ & No & Some concerns & $\begin{array}{l}\text { A minority of participants were excluded due } \\
\text { to no information on parity }(\mathrm{N}=973) \text { or } \\
\text { duration of lactation }(\mathrm{N}=1,705) \text {. }\end{array}$ \\
\hline Zong, 2016 & Probably no & Probably no & Probably no & $\begin{array}{l}\text { No } \\
\text { information }\end{array}$ & No & High & $\begin{array}{l}4,779 \text { women were ultimately included (of } \\
10,701 \text { women older than } 20 \text { potentially } \\
\text { eligible); women were excluded due to } \\
\text { incomplete data }(n=919) \text {, currently pregnant } \\
\text { or breastfeeding ( } n=800 \text { ), prevalent CVD or } \\
\text { cancer }(n=1,211) \text {, and other reasons. }\end{array}$ \\
\hline
\end{tabular}


Table C-38. KQ 2 risk of bias assessment: Diabetes, part 5

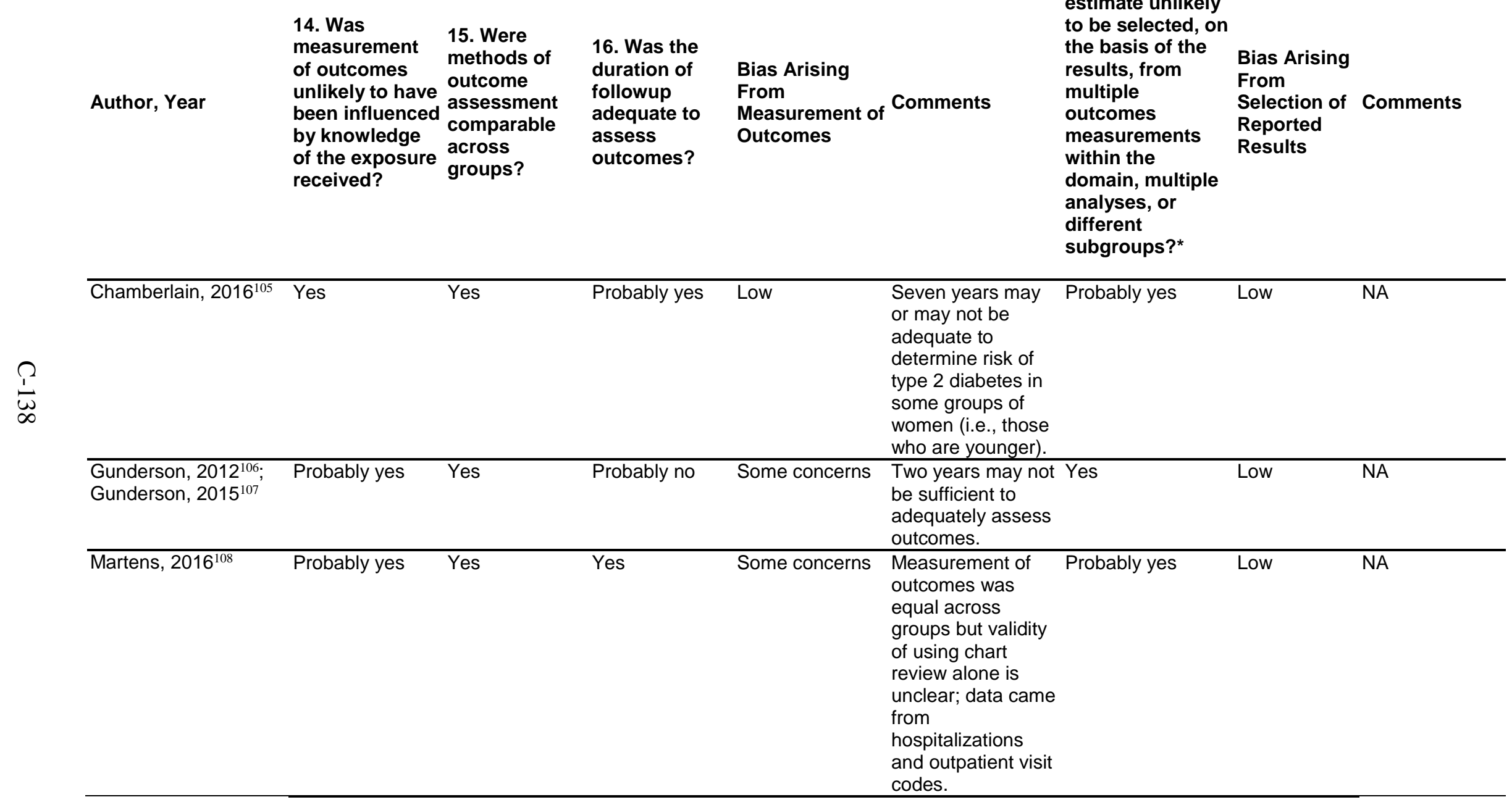

17. Is the

reported effect

14. Was

duration of assessment comparable

across

From

multiple

within the

analyses, or

different

subgroups? 


\section{Is the}

reported effect

14. Was

measurement 15. Were

methods of

outcome

Author, Year

unlikely to have assessment

by knowled comparable

of the exposure across

received?

groups?
16. Was the

duration of

followup

adequate to

assess

outcomes?
Bias Arising

\section{From}

Measurement of Comments

Outcomes

estimate unlikely

to be selected, on

the basis of the

results, from

Bias Arising

multiple

From

outcomes

Selection of Comments

Reported

within the

domain, multiple

analyses, or

different

subgroups?*

\begin{tabular}{|c|c|c|c|c|c|c|c|}
\hline Schwarz, $2009^{3}$ & Probably no & Yes & Probably yes & Some concerns & $\begin{array}{l}\text { Outcomes primarily } \text { Probably no } \\
\text { based on self-report } \\
\text { (for prevalent HTN } \\
\text { and CVD at } \\
\text { enrollment); incident } \\
\text { CVD during WHI } \\
\text { study period was } \\
\text { verified using } \\
\text { standard protocols. }\end{array}$ & Low & NA \\
\hline Zong, $2016^{109}$ & Probably yes & Yes & Probably yes & Some concerns & $\begin{array}{l}\text { Duration of followup Probably yes } \\
\text { after BF exposure } \\
\text { likely varies by age; } \\
\text { women } 20 \text { years } \\
\text { and older were } \\
\text { eligible. Older } \\
\text { women are likely } \\
\text { different from } \\
\text { younger women not } \\
\text { only in terms of } \\
\text { parity but also other } \\
\text { factors related to the } \\
\text { exposure and } \\
\text { outcome. }\end{array}$ & Low & NA \\
\hline
\end{tabular}

$\overline{\mathrm{BF}}$ = breastfeeding; CVD = cardiovascular disease; HTN = hypertension; KQ = Key Question; NA = not applicable; WHI = Women’s Health Initiative. 
Table C-39. KQ 2 risk of bias assessment: Fracture, part 1

\begin{tabular}{|c|c|c|c|c|c|c|c|c|c|}
\hline Author, Year & $\begin{array}{l}\text { Overall } \\
\text { Risk of } \\
\text { Bias } \\
\text { Rating }\end{array}$ & $\begin{array}{l}\text { Overall Rationale for } \\
\text { Risk of Bias Rating }\end{array}$ & $\begin{array}{l}\text { 1. Was } \\
\text { method of } \\
\text { selection } \\
\text { unrelated to } \\
\text { exposure } \\
\text { loutcome? }\end{array}$ & $\begin{array}{l}\text { 1a. Were } \\
\text { post- } \\
\text { exposure } \\
\text { variables that } \\
\text { influenced } \\
\text { selection } \\
\text { related to } \\
\text { exposure } \\
\text { loutcome? }\end{array}$ & $\begin{array}{l}\text { 2. Do start } \\
\text { of } \\
\text { followup } \\
\text { and start } \\
\text { of } \\
\text { exposure } \\
\text { coincide? }\end{array}$ & $\begin{array}{l}\text { 3. Were } \\
\text { adjustment } \\
\text { techniques } \\
\text { used to } \\
\text { correct for } \\
\text { presence of } \\
\text { selection } \\
\text { biases? }\end{array}$ & $\begin{array}{l}\text { 4. Were the } \\
\text { controls sampled } \\
\text { from the } \\
\text { population that } \\
\text { gave rise to the } \\
\text { cases, or using } \\
\text { another method } \\
\text { that avoids } \\
\text { selection bias? }\end{array}$ & $\begin{array}{l}\text { Bias } \\
\text { arising } \\
\text { from } \\
\text { selection? }\end{array}$ & Comments \\
\hline $\begin{array}{l}\text { Crandall, } \\
2017^{110}\end{array}$ & $\begin{array}{l}\text { Some } \\
\text { Concerns }\end{array}$ & $\begin{array}{l}\text { Some concerns arising } \\
\text { from recall bias of } \\
\text { exposure and from recall } \\
\text { of nonhip fractures. }\end{array}$ & Yes & Probably no & Yes & NA & NA & Low & NA \\
\hline Hwang, $2016^{111}$ & High & $\begin{array}{l}\text { Potential risk of recall bias } \\
\text { leading to } \\
\text { misclassification, high } \\
\text { attrition rate. }\end{array}$ & Probably no & Yes & No & No & NA & $\begin{array}{l}\text { Some } \\
\text { concerns }\end{array}$ & $\begin{array}{l}\text { Some concern that those } \\
\text { with poor health (including } \\
\text { those with fractures) who } \\
\text { did not remain in the study } \\
\text { were selectively excluded. }\end{array}$ \\
\hline $\begin{array}{l}\text { Lambrinoudaki, } \\
2015^{112}\end{array}$ & High & $\begin{array}{l}\text { Potential risk of recall bias } \\
\text { leading to } \\
\text { misclassification, risk of } \\
\text { confounding. }\end{array}$ & Probably yes & NA & No & NA & NA & Low & NA \\
\hline Mori, $2015^{113}$ & High & $\begin{array}{l}\text { Potential risk of recall bias } \\
\text { leading to } \\
\text { misclassification of } \\
\text { exposure, some risk of } \\
\text { recall bias from fracture } \\
\text { outcomes (confirmed in } \\
67 \% \text { of cases), fracture } \\
\text { outcomes are not specific } \\
\text { to or completely } \\
\text { representative of low } \\
\text { trauma. }\end{array}$ & Probably yes & NA & No & NA & NA & Low & $\begin{array}{l}\text { Although start of followup } \\
\text { and start of exposure do not } \\
\text { coincide, followup occurs in } \\
\text { a period succeeding } \\
\text { exposure, and so there's no } \\
\text { risk of selection into the } \\
\text { study for followup based on } \\
\text { outcome status. }\end{array}$ \\
\hline Naves, $2005^{114}$ & $\begin{array}{l}\text { Some } \\
\text { concerns }\end{array}$ & $\begin{array}{l}\text { Potential risk of recall bias } \\
\text { leading to } \\
\text { misclassification. Amount } \\
\text { of missing data is unclear. }\end{array}$ & No & NA & No & NA & NA & Low & $\begin{array}{l}\text { Although start of followup } \\
\text { and start of exposure do not } \\
\text { coincide, followup occurs in } \\
\text { a period succeeding } \\
\text { exposure, and so there's no } \\
\text { risk of selection into the } \\
\text { study for followup based on } \\
\text { outcome status. }\end{array}$ \\
\hline
\end{tabular}

KQ = Key Question; NA = not applicable. 
Table C-40. KQ 2 risk of bias assessment: Fracture, part 2

\begin{tabular}{|c|c|c|c|c|c|c|c|c|}
\hline Author, Year & $\begin{array}{l}\text { 5. Is } \\
\text { confounding } \\
\text { of the } \\
\text { exposure } \\
\text { effect } \\
\text { unlikely? }\end{array}$ & $\begin{array}{l}5 \text { a. Did the } \\
\text { authors use an } \\
\text { appropriate } \\
\text { analysis to } \\
\text { adjust for } \\
\text { confounders? }\end{array}$ & $\begin{array}{l}\text { 5b. Were } \\
\text { confounding } \\
\text { domains that } \\
\text { were } \\
\text { controlled for } \\
\text { measured } \\
\text { validly and } \\
\text { reliably? }\end{array}$ & $\begin{array}{l}5 c \text {. Did the } \\
\text { authors } \\
\text { avoid } \\
\text { adjusting for } \\
\text { post- } \\
\text { exposure } \\
\text { variables? }\end{array}$ & $\begin{array}{l}\text { 5d. Were } \\
\text { participants } \\
\text { analyzed } \\
\text { according to } \\
\text { their initial } \\
\text { exposure } \\
\text { group } \\
\text { throughout } \\
\text { followup? }\end{array}$ & $\begin{array}{l}\text { 5e. Were } \\
\text { exposure } \\
\text { discontinuation } \\
\text { s or switches } \\
\text { unlikely to be } \\
\text { related to } \\
\text { factors } \\
\text { prognostic for } \\
\text { the outcome? }\end{array}$ & $\begin{array}{l}\text { Bias arising } \\
\text { from } \\
\text { confounding? }\end{array}$ & Comments \\
\hline Crandall, $2017^{110}$ & No & Yes & Yes & Yes & Yes & NA & Low & NA \\
\hline Hwang, 2016 & No & Yes & Probably yes & No & Yes & Probably yes & $\begin{array}{l}\text { Some } \\
\text { concerns }\end{array}$ & $\begin{array}{l}\text { There's some adjustment } \\
\text { for variables (reproductive } \\
\text { status, medication status) } \\
\text { that could have arisen from } \\
\text { factors common to } \\
\text { exposure and those } \\
\text { variables, that also predict } \\
\text { outcomes }\end{array}$ \\
\hline $\begin{array}{l}\text { Lambrinoudaki, } \\
2015^{112}\end{array}$ & No & No & $\mathrm{NA}$ & Yes & Yes & NA & 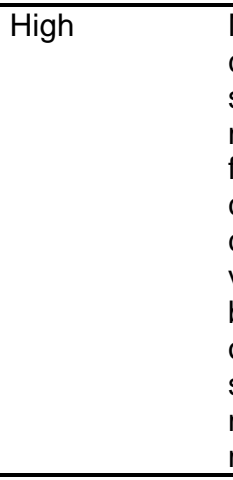 & $\begin{array}{l}\text { No controls for } \\
\text { confounding. With a cross- } \\
\text { sectional design and } \\
\text { measurement of prevalent } \\
\text { fractures, there's a risk of } \\
\text { confounding arising from } \\
\text { common prognostic } \\
\text { variables that influenced } \\
\text { both exposure and } \\
\text { outcome (e.g., health } \\
\text { status or other issues that } \\
\text { may have led to early } \\
\text { menopause). }\end{array}$ \\
\hline Mori, $2015^{113}$ & No & Yes & Probably yes & No & Yes & Probably yes & $\begin{array}{l}\text { Some } \\
\text { concerns }\end{array}$ & $\begin{array}{l}\text { Some adjustment for } \\
\text { variables (health, } \\
\text { medication status) that } \\
\text { could have arisen from } \\
\text { factors common to BF } \\
\text { exposure and those } \\
\text { variables, that also predict } \\
\text { outcomes. }\end{array}$ \\
\hline Naves, $2005^{114}$ & Probably yes & NA & Yes & Yes & Yes & NA & Low & NA \\
\hline
\end{tabular}

$\mathrm{BF}$ = breastfeeding; $\mathrm{KQ}=$ Key Question; $\mathrm{NA}=$ not applicable. 
Table C-41. KQ 2 risk of bias assessment: Fracture, part 3

\begin{tabular}{|c|c|c|c|c|c|}
\hline Author, Year & $\begin{array}{l}\text { 6. Is exposure status } \\
\text { well-defined? }\end{array}$ & $\begin{array}{l}\text { 7. Was } \\
\text { information on } \\
\text { exposure status } \\
\text { recorded at the } \\
\text { time of } \\
\text { exposure? }\end{array}$ & $\begin{array}{l}\text { 8. Was classification of } \\
\text { exposure status } \\
\text { unaffected by } \\
\text { knowledge of the } \\
\text { outcome or risk of the } \\
\text { outcome? }\end{array}$ & $\begin{array}{l}\text { Bias arising from } \\
\text { measurement of } \\
\text { exposures? }\end{array}$ & Comments \\
\hline Crandall, $2017^{110}$ & Probably yes & No & Yes & Some concerns & $\begin{array}{l}\text { Some recall bias likely present due } \\
\text { to length of time between exposure } \\
\text { and } B F \text { self-report (women were } \\
\text { between } 50-79 \text { ). }\end{array}$ \\
\hline Hwang, 2016 & No & No & Probably no & High & $\begin{array}{l}\text { Duration of lactation is collected } \\
\text { retrospectively over a potentially } \\
\text { long period of recall, and did not } \\
\text { account for intensity of BF. }\end{array}$ \\
\hline $\begin{array}{l}\text { Lambrinoudaki, } \\
2015^{112}\end{array}$ & No & No & Probably no & High & $\begin{array}{l}\text { Duration of lactation is collected } \\
\text { retrospectively over a potentially } \\
\text { long period of recall, and did not } \\
\text { account for intensity of BF. }\end{array}$ \\
\hline Mori, 2015'113 & No & No & Probably no & High & $\begin{array}{l}\text { Duration of lactation is collected } \\
\text { retrospectively over a potentially } \\
\text { long period of recall, and did not } \\
\text { account for intensity of BF. }\end{array}$ \\
\hline Naves, 2005 & Yes & No & Yes & Some concerns & $\begin{array}{l}\text { BF information was collected } \\
\text { retrospectively (at age } 50 \text { or older) } \\
\text { which may introduce recall bias. }\end{array}$ \\
\hline
\end{tabular}

$\overline{\mathrm{BF}}$ = breastfeeding; KQ = Key Question. 
Table C-42. KQ 2 risk of bias assessment: Fracture, part 4

\begin{tabular}{|c|c|c|c|c|c|c|c|}
\hline Author, Year & $\begin{array}{l}\text { 9. Were } \\
\text { outcome data } \\
\text { available for } \\
\text { all, or nearly } \\
\text { all, } \\
\text { participants? }\end{array}$ & $\begin{array}{l}\text { 10. Were few } \\
\text { or no } \\
\text { participants } \\
\text { excluded } \\
\text { because of } \\
\text { missing data } \\
\text { on exposure } \\
\text { status? }\end{array}$ & $\begin{array}{l}\text { 11. Were few } \\
\text { or no } \\
\text { participants } \\
\text { excluded due } \\
\text { to missing } \\
\text { data on other } \\
\text { variables? }\end{array}$ & $\begin{array}{l}\text { 12. Was the } \\
\text { proportion of } \\
\text { participants } \\
\text { and reasons for } \\
\text { missing data } \\
\text { similar across } \\
\text { exposure } \\
\text { groups? }\end{array}$ & $\begin{array}{l}\text { 13. Were } \\
\text { appropriate } \\
\text { statistical methods } \\
\text { used to account } \\
\text { for missing data or } \\
\text { assess robustness } \\
\text { to presence of } \\
\text { missing data? }\end{array}$ & $\begin{array}{l}\text { Bias Arising } \\
\text { From Missing } \\
\text { Data }\end{array}$ & Comments \\
\hline $\begin{array}{l}\text { Crandall, } \\
2017^{110}\end{array}$ & Probably yes & Probably yes & Probably no & No information & No & Low & $\begin{array}{l}\text { The proportion excluded due to } \\
\text { missing outcome or exposure data is } \\
\text { very low }(>1 \%) \text {. }\end{array}$ \\
\hline Hwang, 2016 111 & No & No & No & No information & Yes & High & $\begin{array}{l}\text { Only } 1,222 \text { of } 2,105 \text { women in the } \\
\text { original sample were retained, } \\
\text { suggesting a high attrition owing to } \\
\text { missing data (although the details of } \\
\text { reasons for exclusion are not clearly } \\
\text { specified). }\end{array}$ \\
\hline $\begin{array}{l}\text { Lambrinoudaki, } \\
2015^{112}\end{array}$ & No information & No information & No information & No information & No information & $\begin{array}{l}\text { Uncertain } \\
\text { because no } \\
\text { information }\end{array}$ & None \\
\hline Mori, $2015^{113}$ & Yes & Yes & Yes & No information & Yes & Low & NA \\
\hline Naves, $2005^{114}$ & Probably yes & No information & No information & No information & No & $\begin{array}{l}\text { Uncertain } \\
\text { because no } \\
\text { information }\end{array}$ & $\begin{array}{l}17 \% \text { of the sample dropped out } \\
\text { (208/250) for the fracture analysis, } \\
\text { differences by exposure not specified, } \\
\text { leading to some uncertainty. }\end{array}$ \\
\hline
\end{tabular}




\section{Table C-43. KQ 2 risk of bias assessment: Fracture, part 5}

\begin{tabular}{|c|c|c|c|c|c|c|c|c|}
\hline Author, Year & $\begin{array}{l}\text { 14. Was } \\
\text { measurement } \\
\text { of outcomes } \\
\text { unlikely to have } \\
\text { been influenced } \\
\text { by knowledge } \\
\text { of the exposure } \\
\text { received? }\end{array}$ & $\begin{array}{l}\text { 15. Were } \\
\text { methods of } \\
\text { outcome } \\
\text { assessment } \\
\text { comparable } \\
\text { across } \\
\text { groups? }\end{array}$ & $\begin{array}{l}\text { 16. Was the } \\
\text { duration of } \\
\text { followup } \\
\text { adequate to } \\
\text { assess } \\
\text { outcomes? }\end{array}$ & $\begin{array}{l}\text { Bias Arising } \\
\text { From } \\
\text { Measurement of } \\
\text { Outcomes }\end{array}$ & Comments & $\begin{array}{l}\text { to be selected, on } \\
\text { the basis of the } \\
\text { results, from } \\
\text { multiple } \\
\text { outcomes } \\
\text { measurements } \\
\text { within the } \\
\text { domain, multiple } \\
\text { analyses, or } \\
\text { different } \\
\text { subgroups?* }\end{array}$ & $\begin{array}{l}\text { Bias Arising } \\
\text { From } \\
\text { Selection of } \\
\text { Reported } \\
\text { Results }\end{array}$ & Comments \\
\hline Crandall, $2017^{110}$ & Yes & Yes & Yes & Low & $\begin{array}{l}\text { Self-report included for } \\
\text { nonhip fractures, so likely } \\
\text { to undercount vertebral } \\
\text { fractures, but there's no } \\
\text { reason to think that the } \\
\text { risk of underreporting } \\
\text { would vary by exposure } \\
\text { status. }\end{array}$ & Yes & Low & NA \\
\hline Hwang, 2016 ${ }^{111}$ & Yes & Yes & Yes & Low & NA & Yes & Low & NA \\
\hline $\begin{array}{l}\text { Lambrinoudaki, } \\
2015^{112}\end{array}$ & Yes & Yes & Yes & Low & NA & Yes & Low & NA \\
\hline Mori, 2015 & Yes & Yes & Yes & High & $\begin{array}{l}\text { Outcome measures not } \\
\text { specific to or entirely } \\
\text { representative of low } \\
\text { trauma/osteoporosis (they } \\
\text { include "nondigital, } \\
\text { noncranio-facial" fractures } \\
\text { and exclude nonclinical } \\
\text { vertebral fractures. }\end{array}$ & Yes & Low & NA \\
\hline
\end{tabular}

$\mathrm{KQ}=$ Key Question; NA = not applicable. 
Table C-44. KQ 2 risk of bias assessment: Postpartum depression, part 1

\begin{tabular}{|c|c|c|c|c|c|c|c|c|c|}
\hline Author, Year & $\begin{array}{l}\text { Overall } \\
\text { Risk of } \\
\text { Bias Rating }\end{array}$ & $\begin{array}{l}\text { Overall Rationale } \\
\text { for Risk of Bias } \\
\text { Rating }\end{array}$ & $\begin{array}{l}\text { 1. Was } \\
\text { method of } \\
\text { selection } \\
\text { unrelated to } \\
\text { exposurel } \\
\text { outcome? }\end{array}$ & $\begin{array}{l}\text { 1a. Were } \\
\text { post- } \\
\text { exposure } \\
\text { variables } \\
\text { that } \\
\text { influenced } \\
\text { selection } \\
\text { related to } \\
\text { exposurel } \\
\text { outcome? }\end{array}$ & $\begin{array}{l}\text { 2. Do start } \\
\text { of followup } \\
\text { and start of } \\
\text { exposure } \\
\text { coincide? }\end{array}$ & $\begin{array}{l}\text { 3. Were } \\
\text { adjustment } \\
\text { techniques } \\
\text { used to } \\
\text { correct for } \\
\text { presence of } \\
\text { selection } \\
\text { biases? }\end{array}$ & $\begin{array}{l}\text { 4. Were the } \\
\text { controls } \\
\text { sampled from } \\
\text { the } \\
\text { population } \\
\text { that gave rise } \\
\text { to the cases, } \\
\text { or using } \\
\text { another } \\
\text { method that } \\
\text { avoids } \\
\text { selection } \\
\text { bias? }\end{array}$ & $\begin{array}{l} \\
\text { Bias arising } \\
\text { from } \\
\text { selection? }\end{array}$ & Comments \\
\hline Ahn, $2015^{115}$ & High & $\begin{array}{l}\text { High risk of bias } \\
\text { due to confounding } \\
\text { and measurement } \\
\text { bias. The outcome } \\
\text { (depression scores } \\
\text { at } 7 \text { days to } 6 \\
\text { months) was } \\
\text { assessed before } \\
\text { the exposure, } \\
\text { defined as } \\
\text { predominant breast } \\
\text { vs. bottle feeding at } \\
6 \text { months. No } \\
\text { adjusted results } \\
\text { were presented, } \\
\text { despite differences } \\
\text { in depression risk } \\
\text { factors at baseline. }\end{array}$ & No & Yes & Yes & No & NA & $\begin{array}{l}\text { Some } \\
\text { concerns }\end{array}$ & $\begin{array}{l}\text { Limited to } \\
\text { uncomplicated vaginal } \\
\text { birth, limiting } \\
\text { generalizability }\end{array}$ \\
\hline Borra, 2015 & $\begin{array}{l}\text { Some } \\
\text { concerns }\end{array}$ & $\begin{array}{l}\text { High attrition, } \\
\text { potential for } \\
\text { confounding } \\
\end{array}$ & Yes & NA & Yes & NA & NA & Low & NA \\
\hline Chojenta $^{117}$ & High & $\begin{array}{l}\text { High risk of recall } \\
\text { bias, for both } \\
\text { exposure and } \\
\text { outcome. } \\
\text { Measurement of } \\
\text { BF duration may } \\
\text { lead to } \\
\text { misclassification. }\end{array}$ & Yes & NA & Yes & NA & NA & Low & NA \\
\hline
\end{tabular}




\begin{tabular}{|c|c|c|c|c|c|c|c|c|c|}
\hline Author, Year & $\begin{array}{l}\text { Overall } \\
\text { Risk of } \\
\text { Bias Rating }\end{array}$ & $\begin{array}{l}\text { Overall Rationale } \\
\text { for Risk of Bias } \\
\text { Rating }\end{array}$ & $\begin{array}{l}\text { 1. Was } \\
\text { method of } \\
\text { selection } \\
\text { unrelated to } \\
\text { exposurel } \\
\text { outcome? }\end{array}$ & $\begin{array}{l}\text { 1a. Were } \\
\text { post- } \\
\text { exposure } \\
\text { variables } \\
\text { that } \\
\text { influenced } \\
\text { selection } \\
\text { related to } \\
\text { exposurel } \\
\text { outcome? }\end{array}$ & $\begin{array}{l}\text { 2. Do start } \\
\text { of followup } \\
\text { and start of } \\
\text { exposure } \\
\text { coincide? }\end{array}$ & $\begin{array}{l}\text { 3. Were } \\
\text { adjustment } \\
\text { techniques } \\
\text { used to } \\
\text { correct for } \\
\text { presence of } \\
\text { selection } \\
\text { biases? }\end{array}$ & $\begin{array}{l}\text { 4. Were the } \\
\text { controls } \\
\text { sampled from } \\
\text { the } \\
\text { population } \\
\text { that gave rise } \\
\text { to the cases, } \\
\text { or using } \\
\text { another } \\
\text { method that } \\
\text { avoids } \\
\text { selection } \\
\text { bias? }\end{array}$ & $\begin{array}{l}\text { Bias arising } \\
\text { from } \\
\text { selection? }\end{array}$ & Comments \\
\hline Davey, $2011^{118}$ & $\begin{array}{l}\text { Some } \\
\text { concerns }\end{array}$ & $\begin{array}{l}\text { Substantial loss to } \\
\text { followup and lack } \\
\text { of temporality with } \\
\text { respect to stopping } \\
\text { BF and onset of } \\
\text { depression } \\
\text { symptoms. }\end{array}$ & Yes & $\mathrm{NA}$ & Yes & NA & NA & Low & $\mathrm{NA}$ \\
\hline Fiala, $2017^{119}$ & High & $\begin{array}{l}\text { Unclear definition } \\
\text { of exposure and } \\
\text { unvalidated } \\
\text { measurement of } \\
\text { outcomes, }>50 \% \\
\text { loss to followup. }\end{array}$ & Yes & NA & Yes & NA & NA & Low & $\mathrm{NA}$ \\
\hline $\begin{array}{l}\text { Hamdan, } \\
2012^{120}\end{array}$ & High & $\begin{array}{l}\text { No adjustment for } \\
\text { confounding. }\end{array}$ & Yes & NA & Yes & $\mathrm{NA}$ & NA & Low & $\mathrm{NA}$ \\
\hline
\end{tabular}




\begin{tabular}{|c|c|c|c|c|c|c|c|c|c|}
\hline Author, Year & $\begin{array}{l}\text { Overall } \\
\text { Risk of } \\
\text { Bias Rating }\end{array}$ & $\begin{array}{l}\text { Overall Rationale } \\
\text { for Risk of Bias } \\
\text { Rating }\end{array}$ & $\begin{array}{l}\text { 1. Was } \\
\text { method of } \\
\text { selection } \\
\text { unrelated to } \\
\text { exposurel } \\
\text { outcome? }\end{array}$ & $\begin{array}{l}\text { 1a. Were } \\
\text { post- } \\
\text { exposure } \\
\text { variables } \\
\text { that } \\
\text { influenced } \\
\text { selection } \\
\text { related to } \\
\text { exposurel } \\
\text { outcome? }\end{array}$ & $\begin{array}{l}\text { 2. Do start } \\
\text { of followup } \\
\text { and start of } \\
\text { exposure } \\
\text { coincide? }\end{array}$ & $\begin{array}{l}\text { 3. Were } \\
\text { adjustment } \\
\text { techniques } \\
\text { used to } \\
\text { correct for } \\
\text { presence of } \\
\text { selection } \\
\text { biases? }\end{array}$ & $\begin{array}{l}\text { 4. Were the } \\
\text { controls } \\
\text { sampled from } \\
\text { the } \\
\text { population } \\
\text { that gave rise } \\
\text { to the cases, } \\
\text { or using } \\
\text { another } \\
\text { method that } \\
\text { avoids } \\
\text { selection } \\
\text { bias? }\end{array}$ & $\begin{array}{l}\text { Bias arising } \\
\text { from } \\
\text { selection? }\end{array}$ & Comments \\
\hline Illiadis, $2015^{59}$ & High & $\begin{array}{l}\text { High risk of } \\
\text { selection bias, high } \\
\text { rate of } \\
\text { attrition/missing } \\
\text { data, no } \\
\text { adjustment for } \\
\text { potential } \\
\text { confounders; } \\
\text { unclear temporal } \\
\text { sequence of BF } \\
\text { exposure and } \\
\text { depression. }\end{array}$ & Yes & NA & Yes & NA & NA & Low & NA \\
\hline Lau, 2006 & High & $\begin{array}{l}\text { Risk of selection } \\
\text { bias due to high } \\
\text { attrition; only } 38 \% \\
\text { of the sample } \\
\text { returned } \\
\text { postpartum } \\
\text { questionnaires. } \\
\text { Women who chose } \\
\text { not to complete the } \\
\text { followup survey } \\
\text { may differ in } \\
\text { exposure and/or } \\
\text { outcome. }\end{array}$ & Yes & NA & Yes & NA & NA & Low & $\mathrm{NA}$ \\
\hline
\end{tabular}




\begin{tabular}{|c|c|c|c|c|c|c|c|c|c|}
\hline Author, Year & $\begin{array}{l}\text { Overall } \\
\text { Risk of } \\
\text { Bias Rating }\end{array}$ & $\begin{array}{l}\text { Overall Rationale } \\
\text { for Risk of Bias } \\
\text { Rating }\end{array}$ & $\begin{array}{l}\text { 1. Was } \\
\text { method of } \\
\text { selection } \\
\text { unrelated to } \\
\text { exposurel } \\
\text { outcome? }\end{array}$ & $\begin{array}{l}\text { 1a. Were } \\
\text { post- } \\
\text { exposure } \\
\text { variables } \\
\text { that } \\
\text { influenced } \\
\text { selection } \\
\text { related to } \\
\text { exposurel } \\
\text { outcome? }\end{array}$ & $\begin{array}{l}\text { 2. Do start } \\
\text { of followup } \\
\text { and start of } \\
\text { exposure } \\
\text { coincide? }\end{array}$ & $\begin{array}{l}\text { 3. Were } \\
\text { adjustment } \\
\text { techniques } \\
\text { used to } \\
\text { correct for } \\
\text { presence of } \\
\text { selection } \\
\text { biases? }\end{array}$ & $\begin{array}{l}\text { 4. Were the } \\
\text { controls } \\
\text { sampled from } \\
\text { the } \\
\text { population } \\
\text { that gave rise } \\
\text { to the cases, } \\
\text { or using } \\
\text { another } \\
\text { method that } \\
\text { avoids } \\
\text { selection } \\
\text { bias? }\end{array}$ & $\begin{array}{l}\text { Bias arising } \\
\text { from } \\
\text { selection? }\end{array}$ & Comments \\
\hline $\begin{array}{l}\text { Maimburg, } \\
2015^{122}\end{array}$ & High & $\begin{array}{l}\text { The analysis only } \\
\text { controlled for food } \\
\text { insecurity and did } \\
\text { not assess or } \\
\text { control for history } \\
\text { of depression; } \\
\text { unclear whether } \\
\text { mothers who were } \\
\text { more predisposed } \\
\text { to depression were } \\
\text { also more likely to } \\
\text { stop BF. }\end{array}$ & Yes & $\mathrm{NA}$ & Yes & $\mathrm{NA}$ & NA & $\begin{array}{l}\text { Some } \\
\text { concerns }\end{array}$ & $\begin{array}{l}\text { Sample comprised of } \\
\text { participants in an RCT, } \\
\text { in which about half of } \\
\text { women approached } \\
\text { agreed to participate. } \\
\text { This may limit } \\
\text { generalizability. }\end{array}$ \\
\hline
\end{tabular}




\begin{tabular}{|c|c|c|c|c|c|c|c|c|c|}
\hline Author, Year & $\begin{array}{l}\text { Overall } \\
\text { Risk of } \\
\text { Bias Rating }\end{array}$ & $\begin{array}{l}\text { Overall Rationale } \\
\text { for Risk of Bias } \\
\text { Rating }\end{array}$ & $\begin{array}{l}\text { 1. Was } \\
\text { method of } \\
\text { selection } \\
\text { unrelated to } \\
\text { exposurel } \\
\text { outcome? }\end{array}$ & $\begin{array}{l}\text { 1a. Were } \\
\text { post- } \\
\text { exposure } \\
\text { variables } \\
\text { that } \\
\text { influenced } \\
\text { selection } \\
\text { related to } \\
\text { exposurel } \\
\text { outcome? }\end{array}$ & $\begin{array}{l}\text { 2. Do start } \\
\text { of followup } \\
\text { and start of } \\
\text { exposure } \\
\text { coincide? }\end{array}$ & $\begin{array}{l}\text { 3. Were } \\
\text { adjustment } \\
\text { techniques } \\
\text { used to } \\
\text { correct for } \\
\text { presence of } \\
\text { selection } \\
\text { biases? }\end{array}$ & $\begin{array}{l}\text { 4. Were the } \\
\text { controls } \\
\text { sampled from } \\
\text { the } \\
\text { population } \\
\text { that gave rise } \\
\text { to the cases, } \\
\text { or using } \\
\text { another } \\
\text { method that } \\
\text { avoids } \\
\text { selection } \\
\text { bias? }\end{array}$ & $\begin{array}{l}\text { Bias arising } \\
\text { from } \\
\text { selection? }\end{array}$ & Comments \\
\hline $\begin{array}{l}\text { McCoy, } \\
2008^{123}\end{array}$ & High & $\begin{array}{l}\text { Risk of selection } \\
\text { bias due to high } \\
\text { attrition; only } 38 \% \\
\text { of the sample } \\
\text { returned } \\
\text { postpartum } \\
\text { questionnaires. } \\
\text { Women who chose } \\
\text { not to complete the } \\
\text { mailed followup } \\
\text { survey may differ in } \\
\text { exposure and/or } \\
\text { outcome. No } \\
\text { adjustment for } \\
\text { potential } \\
\text { confounders. } \\
\text { Duration (4 weeks) } \\
\text { may be insufficient } \\
\text { to detect outcome. }\end{array}$ & No & Yes & No & No & NA & High & $\begin{array}{l}\text { Approximately } 25 \% \text { of } \\
\text { the eligible sample did } \\
\text { not return for PP visit } \\
\text { and only half of the } \\
\text { target population of } \\
\text { women had EPDS } \\
\text { screeners in their } \\
\text { medical charts, even } \\
\text { though it is supposed } \\
\text { to be routinely } \\
\text { conducted. Authors list } \\
\text { potential reasons for } \\
\text { the large number of } \\
\text { missing screeners, all } \\
\text { of which may have } \\
\text { biased the sample. }\end{array}$ \\
\hline
\end{tabular}




\begin{tabular}{|c|c|c|c|c|c|c|c|c|c|}
\hline Author, Year & $\begin{array}{l}\text { Overall } \\
\text { Risk of } \\
\text { Bias Rating }\end{array}$ & $\begin{array}{l}\text { Overall Rationale } \\
\text { for Risk of Bias } \\
\text { Rating }\end{array}$ & $\begin{array}{l}\text { 1. Was } \\
\text { method of } \\
\text { selection } \\
\text { unrelated to } \\
\text { exposurel } \\
\text { outcome? }\end{array}$ & $\begin{array}{l}\text { 1a. Were } \\
\text { post- } \\
\text { exposure } \\
\text { variables } \\
\text { that } \\
\text { influenced } \\
\text { selection } \\
\text { related to } \\
\text { exposurel } \\
\text { outcome? }\end{array}$ & $\begin{array}{l}\text { 2. Do start } \\
\text { of followup } \\
\text { and start of } \\
\text { exposure } \\
\text { coincide? }\end{array}$ & $\begin{array}{l}\text { 3. Were } \\
\text { adjustment } \\
\text { techniques } \\
\text { used to } \\
\text { correct for } \\
\text { presence of } \\
\text { selection } \\
\text { biases? }\end{array}$ & $\begin{array}{l}\text { 4. Were the } \\
\text { controls } \\
\text { sampled from } \\
\text { the } \\
\text { population } \\
\text { that gave rise } \\
\text { to the cases, } \\
\text { or using } \\
\text { another } \\
\text { method that } \\
\text { avoids } \\
\text { selection } \\
\text { bias? }\end{array}$ & $\begin{array}{l}\text { Bias arising } \\
\text { from } \\
\text { selection? }\end{array}$ & Comments \\
\hline $\begin{array}{l}\text { Reifsnider, } \\
2016^{124}\end{array}$ & $\begin{array}{l}\text { Some } \\
\text { concerns }\end{array}$ & $\begin{array}{l}\text { Risk of bias due to } \\
\text { confounding (not } \\
\text { addressed by } \\
\text { analyses) and } \\
\text { potential for } \\
\text { selected outcome } \\
\text { reporting. }\end{array}$ & Yes & NA & Yes & NA & NA & Low & NA \\
\hline Sword, $2012^{125}$ & $\begin{array}{l}\text { Some } \\
\text { concerns }\end{array}$ & $\begin{array}{l}\text { Risk of bias due to } \\
\text { confounding, } \\
\text { adjustment for } \\
\text { potential mediators } \\
\text { in the association } \\
\text { between BF and } \\
\text { depression. }\end{array}$ & Yes & NA & Yes & NA & NA & Low & NA \\
\hline $\begin{array}{l}\text { Trivino-Juarez, } \\
2016^{126}\end{array}$ & $\begin{array}{l}\text { Some } \\
\text { concerns }\end{array}$ & $\begin{array}{l}\text { Missing information } \\
\text { on loss to followup, } \\
\text { no statistical } \\
\text { adjustment for } \\
\text { attrition, unclear } \\
\text { adjustment } \\
\text { methods for } \\
\text { potential } \\
\text { confounding. }\end{array}$ & Yes & NA & Yes & NA & NA & Low & NA \\
\hline
\end{tabular}

BF = breastfeeding; EPDS = Edinburgh Postnatal Depression Scale; KQ = Key Question; NA = not applicable; PP = postpartum; RCT = randomized controlled trial. 
Table C-45. KQ 2 risk of bias assessment: Postpartum depression, part 2

\begin{tabular}{|c|c|c|c|c|c|c|c|c|}
\hline Author, Year & $\begin{array}{l}5 . \text { Is } \\
\text { confounding } \\
\text { of the } \\
\text { exposure } \\
\text { effect } \\
\text { unlikely? }\end{array}$ & $\begin{array}{l}\text { 5a. Did the } \\
\text { authors use an } \\
\text { appropriate } \\
\text { analysis to } \\
\text { adjust for } \\
\text { confounders? }\end{array}$ & $\begin{array}{l}\text { 5b. Were } \\
\text { confounding } \\
\text { domains that } \\
\text { were } \\
\text { controlled for } \\
\text { measured } \\
\text { validly and } \\
\text { reliably? }\end{array}$ & $\begin{array}{l}5 c . \text { Did the } \\
\text { authors } \\
\text { avoid } \\
\text { adjusting for } \\
\text { post- } \\
\text { exposure } \\
\text { variables? }\end{array}$ & $\begin{array}{l}\text { 5d. Were } \\
\text { participants } \\
\text { analyzed } \\
\text { according to } \\
\text { their initial } \\
\text { exposure } \\
\text { group } \\
\text { throughout } \\
\text { followup? }\end{array}$ & $\begin{array}{l}\text { 5e. Were exposure } \\
\text { discontinuations } \\
\text { or switches } \\
\text { unlikely to be } \\
\text { related to factors } \\
\text { prognostic for the } \\
\text { outcome? }\end{array}$ & $\begin{array}{l}\text { Bias arising } \\
\text { from } \\
\text { confounding? }\end{array}$ & Comments \\
\hline Ahn, $2015^{115}$ & No & No & NA & NA & NA & NA & High & $\begin{array}{l}\text { The authors measured the } \\
\text { association between } \\
\text { feeding status at } 6 \text { months } \\
\text { and mood; in table 2, they } \\
\text { present mean EPDS } \\
\text { scores at multiple times } \\
\text { from } 7 \text { days to } 6 \text { months } \\
\text { postpartum according to } \\
\text { feeding status at } 6 \text { months. } \\
\text { These data suggest that } \\
\text { mood does not predict } \\
\text { feeding behavior, but does } \\
\text { not address the stated } \\
\text { objective of the study, to } \\
\text { test if feeding behavior } \\
\text { affects mood. Moreover, no } \\
\text { data are presented with } \\
\text { adjustment for pregnancy } \\
\text { mood or consideration of } \\
\text { timing of or reasons for } \\
\text { discontinuing BF. }\end{array}$ \\
\hline Borra, 2015 & Probably no & No information & No information & Yes & Yes & NA & Some concerns & $\begin{array}{l}\text { Model C adjusts for } \\
\text { "antenatal depression" } \\
\text { scores according to the } \\
\text { text, but Table } 6 \text { in the } \\
\text { appendix says that these } \\
\text { antenatal measures came } \\
\text { from } 18 \text { and } 33 \text { months of } \\
\text { pregnancy. It is possible } \\
\text { that Table } 6 \text { contains a } \\
\text { typo, but otherwise, the } \\
\text { potential for confounding is } \\
\text { high. }\end{array}$ \\
\hline
\end{tabular}




\begin{tabular}{|c|c|c|c|c|c|c|c|c|}
\hline Author, Year & $\begin{array}{l}\text { 5. Is } \\
\text { confounding } \\
\text { of the } \\
\text { exposure } \\
\text { effect } \\
\text { unlikely? }\end{array}$ & $\begin{array}{l}5 a . \text { Did the } \\
\text { authors use an } \\
\text { appropriate } \\
\text { analysis to } \\
\text { adjust for } \\
\text { confounders? }\end{array}$ & $\begin{array}{l}\text { 5b. Were } \\
\text { confounding } \\
\text { domains that } \\
\text { were } \\
\text { controlled for } \\
\text { measured } \\
\text { validly and } \\
\text { reliably? }\end{array}$ & $\begin{array}{l}5 c \text {. Did the } \\
\text { authors } \\
\text { avoid } \\
\text { adjusting for } \\
\text { post- } \\
\text { exposure } \\
\text { variables? }\end{array}$ & $\begin{array}{l}\text { 5d. Were } \\
\text { participants } \\
\text { analyzed } \\
\text { according to } \\
\text { their initial } \\
\text { exposure } \\
\text { group } \\
\text { throughout } \\
\text { followup? }\end{array}$ & $\begin{array}{l}\text { 5e. Were exposure } \\
\text { discontinuations } \\
\text { or switches } \\
\text { unlikely to be } \\
\text { related to factors } \\
\text { prognostic for the } \\
\text { outcome? }\end{array}$ & $\begin{array}{l}\text { Bias arising } \\
\text { from } \\
\text { confounding? }\end{array}$ & Comments \\
\hline $\begin{array}{l}\text { Chojenta, } \\
2016^{117}\end{array}$ & No & Probably yes & Probably no & Yes & Yes & NA & Some concerns & $\begin{array}{l}\text { It looks like PND was only } \\
\text { assessed at the 5-year } \\
\text { follow up for each child, } \\
\text { and asks mothers to recall } \\
\text { "were you diagnosed or } \\
\text { treated for postnatal } \\
\text { depression." This does not } \\
\text { seem like it would be a } \\
\text { valid measure considering } \\
\text { the potentially long recall } \\
\text { time. Also, this only } \\
\text { measures diagnosis/ } \\
\text { treatment, and more } \\
\text { women may have } \\
\text { experienced PND than } \\
\text { were actually diagnosed, } \\
\text { so access to care plays a } \\
\text { part here as well which } \\
\text { may be a confounder } \\
\text { (women with more access } \\
\text { to care may have more } \\
\text { assistance BF and more } \\
\text { screening for PND). }\end{array}$ \\
\hline
\end{tabular}




\begin{tabular}{|c|c|c|c|c|c|c|c|c|}
\hline Author, Year & $\begin{array}{l}\text { 5. Is } \\
\text { confounding } \\
\text { of the } \\
\text { exposure } \\
\text { effect } \\
\text { unlikely? }\end{array}$ & $\begin{array}{l}5 a \text {. Did the } \\
\text { authors use an } \\
\text { appropriate } \\
\text { analysis to } \\
\text { adjust for } \\
\text { confounders? }\end{array}$ & $\begin{array}{l}\text { 5b. Were } \\
\text { confounding } \\
\text { domains that } \\
\text { were } \\
\text { controlled for } \\
\text { measured } \\
\text { validly and } \\
\text { reliably? }\end{array}$ & $\begin{array}{l}5 c . \text { Did the } \\
\text { authors } \\
\text { avoid } \\
\text { adjusting for } \\
\text { post- } \\
\text { exposure } \\
\text { variables? }\end{array}$ & $\begin{array}{l}\text { 5d. Were } \\
\text { participants } \\
\text { analyzed } \\
\text { according to } \\
\text { their initial } \\
\text { exposure } \\
\text { group } \\
\text { throughout } \\
\text { followup? }\end{array}$ & $\begin{array}{l}\text { 5e. Were exposure } \\
\text { discontinuations } \\
\text { or switches } \\
\text { unlikely to be } \\
\text { related to factors } \\
\text { prognostic for the } \\
\text { outcome? }\end{array}$ & $\begin{array}{l}\text { Bias arising } \\
\text { from } \\
\text { confounding? }\end{array}$ & Comments \\
\hline $\begin{array}{l}\text { Davey, } \\
2011^{118}\end{array}$ & No & Probably yes & Yes & Probably yes & No & No & High & $\begin{array}{l}\text { Depression is measured } \\
\text { during pregnancy and then } \\
\text { at } 8 \text { weeks postpartum, } \\
\text { and BF is measured at } 8 \\
\text { weeks postpartum. Data } \\
\text { regarding the temporality of } \\
\text { weaning and onset of } \\
\text { depression symptoms is } \\
\text { not provided, so it is not } \\
\text { possible to determine } \\
\text { whether women stopped } \\
\text { BF and became } \\
\text { depressed, or became } \\
\text { depressed and stopped } \\
\text { BF. In the final model for } \\
\text { clinical depression } \\
\text { symptoms, no confounders } \\
\text { that occurred after the } \\
\text { exposure were included, } \\
\text { but multiple measures at } 8 \\
\text { weeks were tested for } \\
\text { inclusion in the model. }\end{array}$ \\
\hline
\end{tabular}




\begin{tabular}{|c|c|c|c|c|c|c|c|c|}
\hline Author, Year & $\begin{array}{l}\text { 5. Is } \\
\text { confounding } \\
\text { of the } \\
\text { exposure } \\
\text { effect } \\
\text { unlikely? }\end{array}$ & $\begin{array}{l}5 a . \text { Did the } \\
\text { authors use an } \\
\text { appropriate } \\
\text { analysis to } \\
\text { adjust for } \\
\text { confounders? }\end{array}$ & $\begin{array}{l}\text { 5b. Were } \\
\text { confounding } \\
\text { domains that } \\
\text { were } \\
\text { controlled for } \\
\text { measured } \\
\text { validly and } \\
\text { reliably? }\end{array}$ & $\begin{array}{l}5 c . \text { Did the } \\
\text { authors } \\
\text { avoid } \\
\text { adjusting for } \\
\text { post- } \\
\text { exposure } \\
\text { variables? }\end{array}$ & $\begin{array}{l}\text { 5d. Were } \\
\text { participants } \\
\text { analyzed } \\
\text { according to } \\
\text { their initial } \\
\text { exposure } \\
\text { group } \\
\text { throughout } \\
\text { followup? }\end{array}$ & $\begin{array}{l}\text { 5e. Were exposure } \\
\text { discontinuations } \\
\text { or switches } \\
\text { unlikely to be } \\
\text { related to factors } \\
\text { prognostic for the } \\
\text { outcome? }\end{array}$ & $\begin{array}{l}\text { Bias arising } \\
\text { from } \\
\text { confounding? }\end{array}$ & Comments \\
\hline Fiala, $2017^{119}$ & No & Probably no & Probably yes & No & Probably yes & No information & Some concerns & $\begin{array}{l}\text { BF exposure is poorly } \\
\text { defined and it is unclear } \\
\text { when it was measured; no } \\
\text { information is provided on } \\
\text { which variables were } \\
\text { included in the adjusted } \\
\text { analyses. }\end{array}$ \\
\hline $\begin{array}{l}\text { Hamdan, } \\
2012^{120}\end{array}$ & No & No & NA & NA & No & No & High & $\begin{array}{l}\text { Women with depression } \\
\text { during pregnancy were } \\
\text { less likely to breastfeed, } \\
\text { but the authors did not } \\
\text { adjust/account for } \\
\text { pregnancy depression or } \\
\text { anxiety in their analyses. } \\
\text { Moreover, although they } \\
\text { report that women who } \\
\text { were depressed at } 2 \\
\text { months were less likely to } \\
\text { breastfeed at } 4 \text { months, } \\
\text { they do not adjust for 2- } \\
\text { month depression in their } \\
\text { 4-month results. It is highly } \\
\text { plausible that depression } \\
\text { symptoms cause women to } \\
\text { change exposure groups } \\
\text { (e.g., stop BF) but this is } \\
\text { not accounted for in the } \\
\text { analysis. }\end{array}$ \\
\hline
\end{tabular}




\begin{tabular}{|c|c|c|c|c|c|c|c|c|}
\hline Author, Year & $\begin{array}{l}\text { 5. Is } \\
\text { confounding } \\
\text { of the } \\
\text { exposure } \\
\text { effect } \\
\text { unlikely? }\end{array}$ & $\begin{array}{l}5 a \text {. Did the } \\
\text { authors use an } \\
\text { appropriate } \\
\text { analysis to } \\
\text { adjust for } \\
\text { confounders? }\end{array}$ & $\begin{array}{l}\text { 5b. Were } \\
\text { confounding } \\
\text { domains that } \\
\text { were } \\
\text { controlled for } \\
\text { measured } \\
\text { validly and } \\
\text { reliably? }\end{array}$ & $\begin{array}{l}5 c . \text { Did the } \\
\text { authors } \\
\text { avoid } \\
\text { adjusting for } \\
\text { post- } \\
\text { exposure } \\
\text { variables? }\end{array}$ & $\begin{array}{l}\text { 5d. Were } \\
\text { participants } \\
\text { analyzed } \\
\text { according to } \\
\text { their initial } \\
\text { exposure } \\
\text { group } \\
\text { throughout } \\
\text { followup? }\end{array}$ & $\begin{array}{l}\text { 5e. Were exposure } \\
\text { discontinuations } \\
\text { or switches } \\
\text { unlikely to be } \\
\text { related to factors } \\
\text { prognostic for the } \\
\text { outcome? }\end{array}$ & $\begin{array}{l}\text { Bias arising } \\
\text { from } \\
\text { confounding? }\end{array}$ & Comments \\
\hline Illiadis, $2015^{59}$ & No & No & Probably yes & NA & No & No & High & $\begin{array}{l}\text { No adjustment for } \\
\text { covariates in association } \\
\text { between depression and } \\
\text { BF status; temporal } \\
\text { sequence of BF cessation } \\
\text { and depression not known } \\
\text { for PPD group. }\end{array}$ \\
\hline Lau, 2006 & No & No & No & NA & NA & NA & High & $\begin{array}{l}\text { Multivariate adjusted } \\
\text { results were not presented. }\end{array}$ \\
\hline $\begin{array}{l}\text { Maimburg, } \\
2015^{122}\end{array}$ & No & Probably no & Yes & Yes & Yes & NA & High & $\begin{array}{l}\text { The analysis only } \\
\text { controlled for intervention } \\
\text { arm, and did not assess or } \\
\text { control for history of } \\
\text { depression before or } \\
\text { during pregnancy. }\end{array}$ \\
\hline $\begin{array}{l}\text { McCoy, } \\
2008^{123}\end{array}$ & No & No & NA & NA & Probably yes & NA & High & $\begin{array}{l}\text { Although they assessed } \\
\text { prior history of depression, } \\
\text { they did not include it (or } \\
\text { any other variables) in a } \\
\text { model with BF because BF } \\
\text { did not predict PPD } \\
\text { individually. }\end{array}$ \\
\hline
\end{tabular}




\begin{tabular}{|c|c|c|c|c|c|c|c|c|}
\hline Author, Year & $\begin{array}{l}5 . \text { Is } \\
\text { confounding } \\
\text { of the } \\
\text { exposure } \\
\text { effect } \\
\text { unlikely? }\end{array}$ & $\begin{array}{l}5 a \text {. Did the } \\
\text { authors use an } \\
\text { appropriate } \\
\text { analysis to } \\
\text { adjust for } \\
\text { confounders? }\end{array}$ & $\begin{array}{l}\text { 5b. Were } \\
\text { confounding } \\
\text { domains that } \\
\text { were } \\
\text { controlled for } \\
\text { measured } \\
\text { validly and } \\
\text { reliably? }\end{array}$ & $\begin{array}{l}5 c . \text { Did the } \\
\text { authors } \\
\text { avoid } \\
\text { adjusting for } \\
\text { post- } \\
\text { exposure } \\
\text { variables? }\end{array}$ & $\begin{array}{l}\text { 5d. Were } \\
\text { participants } \\
\text { analyzed } \\
\text { according to } \\
\text { their initial } \\
\text { exposure } \\
\text { group } \\
\text { throughout } \\
\text { followup? }\end{array}$ & $\begin{array}{l}\text { 5e. Were exposure } \\
\text { discontinuations } \\
\text { or switches } \\
\text { unlikely to be } \\
\text { related to factors } \\
\text { prognostic for the } \\
\text { outcome? }\end{array}$ & $\begin{array}{l}\text { Bias arising } \\
\text { from } \\
\text { confounding? }\end{array}$ & Comments \\
\hline $\begin{array}{l}\text { Reifsnider, } \\
2016^{124}\end{array}$ & No & Probably no & Yes & Probably yes & Probably no & Probably no & High & $\begin{array}{l}\text { The analysis only } \\
\text { controlled for food } \\
\text { insecurity (the main } \\
\text { outcome of the analysis } \\
\text { and parent RCT was } \\
\text { weight), and did not assess } \\
\text { or control for history of } \\
\text { depression before or } \\
\text { during pregnancy. There is } \\
\text { no way to tell whether } \\
\text { mothers who were more } \\
\text { depressed were also more } \\
\text { likely to stop BF. }\end{array}$ \\
\hline $\begin{array}{l}\text { Sword, } \\
2012^{125}\end{array}$ & No & Probably no & Yes & Probably yes & Yes & NA & Some concerns & $\begin{array}{l}\text { As exposure was whether } \\
\text { BF was initiated, concerns } \\
\text { regarding changing } \\
\text { exposure category due to } \\
\text { depression are lessened. } \\
\text { However, it appears that } \\
\text { confounders were only } \\
\text { included in the model if } \\
\text { they independently } \\
\text { predicted the outcome, w/o } \\
\text { consideration of whether } \\
\text { they materially changed } \\
\text { effect estimates for } \\
\text { exposures of interest. }\end{array}$ \\
\hline $\begin{array}{l}\text { Trivino- } \\
\text { Juarez, } \\
2016^{126}\end{array}$ & No & Probably yes & Probably yes & Yes & NA & NA & Low & $\begin{array}{l}\text { The way they describe } \\
\text { confounders is confusing } \\
\text { so I am not confident about } \\
\text { this rating. }\end{array}$ \\
\hline
\end{tabular}
randomized controlled trial. 
Table C-46. KQ 2 risk of bias assessment: Postpartum depression, part 3

\begin{tabular}{|c|c|c|c|c|c|}
\hline Author, Year & $\begin{array}{l}\text { 6. Is exposure } \\
\text { status well- } \\
\text { defined? }\end{array}$ & $\begin{array}{l}\text { 7. Was information } \\
\text { on exposure status } \\
\text { recorded at the time } \\
\text { of exposure? }\end{array}$ & $\begin{array}{l}\text { 8. Was classification of } \\
\text { exposure status unaffected by } \\
\text { knowledge of the outcome or } \\
\text { risk of the outcome? }\end{array}$ & $\begin{array}{l}\text { Bias arising from } \\
\text { measurement of } \\
\text { exposures? }\end{array}$ & Comments \\
\hline Ahn, $2015^{115}$ & Probably no & Yes & No information & Some concerns & $\begin{array}{l}\text { Timing of stopping breastfeeding / } \\
\text { introducing formula was not considered } \\
\text { in the analysis of EPDS scores. }\end{array}$ \\
\hline Borra, $2015^{116}$ & Probably yes & Probably yes & Probably yes & Some concerns & $\begin{array}{l}\text { Potential for recall bias for duration of BF } \\
\text { because BF status was collected at } 8 \\
\text { weeks and } 8,18 \text {, and } 24 \text { months. Other } \\
\text { outcomes (initiation; BF at } 1,2 \text {, and } 4 \\
\text { weeks; and exclusivity at those times) } \\
\text { are likely to have less recall bias if } \\
\text { women responded at the } 8 \text {-week survey. }\end{array}$ \\
\hline Chojenta, $2016^{117}$ & Probably yes & No & Probably yes & Some concerns & $\begin{array}{l}\text { Breastfeeding was assessed annually for } \\
\text { each child born during the year, and the } \\
\text { measure is imprecise (BF<6m: someone } \\
\text { BF for } 5.5 \text { months is very different from } \\
\text { someone who did not initiate). }\end{array}$ \\
\hline Davey, $2011^{118}$ & No & No & No information & Some concerns & $\begin{array}{l}\text { Because timing of weaning was not } \\
\text { collected, the temporal order of } \\
\text { depression symptoms and breastfeeding } \\
\text { cessation is not known. }\end{array}$ \\
\hline Fiala, $2017^{119}$ & No & No information & No information & High & $\begin{array}{l}\text { It is not clear whether the exposure is } \\
\text { never breastfeeding vs ever } \\
\text { breastfeeding or not breastfeeding vs } \\
\text { breastfeeding at } 6 \text { weeks or } 6 \text { months. }\end{array}$ \\
\hline Hamdan, 2012 ${ }^{120}$ & Probably yes & Probably yes & Probably yes & Low & $\mathrm{NA}$ \\
\hline Illiadis, $2015^{59}$ & No & Yes & No & Some concerns & $\begin{array}{l}\text { Unclear whether breastfeeding classified } \\
\text { as exclusive (yes) vs. nonexclusive or } \\
\text { not at all (no), or whether yes/no } \\
\text { references to any current breastfeeding. }\end{array}$ \\
\hline Lau, $2006^{121}$ & No & Yes & No & Some concerns & $\begin{array}{l}\text { Validity of obstetric chart review for } \\
\text { breastfeeding status is not clear. }\end{array}$ \\
\hline Maimburg, $2015^{122}$ & Probably yes & Yes & Probably yes & Some concerns & $\begin{array}{l}\text { Breastfeeding was assessed at the } \\
\text { same time as EPDS. }\end{array}$ \\
\hline
\end{tabular}




\begin{tabular}{|c|c|c|c|c|c|}
\hline Author, Year & $\begin{array}{l}\text { 6. Is exposure } \\
\text { status well- } \\
\text { defined? }\end{array}$ & $\begin{array}{l}\text { 7. Was information } \\
\text { on exposure status } \\
\text { recorded at the time } \\
\text { of exposure? }\end{array}$ & $\begin{array}{l}\text { 8. Was classification of } \\
\text { exposure status unaffected by } \\
\text { knowledge of the outcome or } \\
\text { risk of the outcome? }\end{array}$ & $\begin{array}{l}\text { Bias arising from } \\
\text { measurement of } \\
\text { exposures? }\end{array}$ & Comments \\
\hline McCoy, $2008^{123}$ & Probably yes & Yes & Probably yes & Some concerns & $\begin{array}{l}\text { Breastfeeding was assessed at the } \\
\text { same time as EPDS. }\end{array}$ \\
\hline Reifsnider, $2016^{124}$ & Yes & Yes & Probably yes & Low & $\mathrm{NA}$ \\
\hline Sword, $2012^{125}$ & Yes & No & No information & Some concerns & $\begin{array}{l}\text { It is odd that breastfeeding initiation was } \\
\text { not assessed during the postpartum } \\
\text { stay, and raises concerns that infant } \\
\text { illness / other complications that might } \\
\text { predispose to depression confound the } \\
\text { observed associations. It is also difficult } \\
\text { to interpret the results in the absence of } \\
\text { any information on the proportion of } \\
\text { women who did not breastfeed - based } \\
\text { on the large confidence intervals } \\
\text { reported, this was likely a small number } \\
\text { of women. }\end{array}$ \\
\hline $\begin{array}{l}\text { Trivino-Juarez, } \\
2016^{126}\end{array}$ & Yes & Yes & Probably yes & Low & NA \\
\hline
\end{tabular}

BF = breastfeeding; EPDS = Edinburgh Postnatal Depression Scale; KQ = Key Question; NA = not applicable. 
Table C-47. KQ 2 risk of bias assessment: Postpartum depression, part 4

\begin{tabular}{|c|c|c|c|c|c|c|c|}
\hline Author, Year & $\begin{array}{l}\text { 9. Were } \\
\text { outcome data } \\
\text { available for } \\
\text { all, or nearly } \\
\text { all, } \\
\text { participants? }\end{array}$ & $\begin{array}{l}\text { 10. Were few } \\
\text { or no } \\
\text { participants } \\
\text { excluded } \\
\text { because of } \\
\text { missing data } \\
\text { on exposure } \\
\text { status? }\end{array}$ & $\begin{array}{l}\text { 11. Were few } \\
\text { or no } \\
\text { participants } \\
\text { excluded due } \\
\text { to missing } \\
\text { data on other } \\
\text { variables? }\end{array}$ & $\begin{array}{l}\text { 12. Was the } \\
\text { proportion of } \\
\text { participants } \\
\text { and reasons for } \\
\text { missing data } \\
\text { similar across } \\
\text { exposure } \\
\text { groups? }\end{array}$ & $\begin{array}{l}\text { 13. Were } \\
\text { appropriate } \\
\text { statistical methods } \\
\text { used to account } \\
\text { for missing data or } \\
\text { assess robustness } \\
\text { to presence of } \\
\text { missing data? }\end{array}$ & $\begin{array}{l}\text { Bias Arising } \\
\text { From Missing } \\
\text { Data }\end{array}$ & Comments \\
\hline Ahn, $2015^{115}$ & No & No & No & No information & No & High & $\begin{array}{l}20 \% \text { of participants did not complete } \\
\text { the } 6 \text {-month visit, and no information } \\
\text { is provided on differences between } \\
\text { the baseline population and the } \\
\text { women who completed the } 6 \text {-month } \\
\text { visit. Furthermore, information on } \\
\text { missing data points are not provided. }\end{array}$ \\
\hline Borra, $2015^{116}$ & No & No & Yes & No information & NA & Some concerns & $\begin{array}{l}\text { Proportion of missingness in analyses } \\
\text { ranges from } 58 \%(8,172 \text { in the fully } \\
\text { adjusted model) to } 63 \% \text { ( } 8,805 \text { in the } \\
\text { model with the least adjustment) } \\
\text { using the } 1 \text {-year denominator of } \\
13,988) \text {. }\end{array}$ \\
\hline $\begin{array}{l}\text { Chojenta, } \\
2016^{117}\end{array}$ & Yes & Yes & Probably yes & Yes & NA & Low & NA \\
\hline Davey, $2011^{118}$ & No & No & No & No information & No & Some concerns & $\begin{array}{l}30 \% \text { of women did not complete the } \\
8 \text {-week evaluation, and data are not } \\
\text { provided regarding differences in } \\
\text { pregnancy depression/anxiety or } \\
\text { infant feeding intention. }\end{array}$ \\
\hline Fiala, $2017^{119}$ & No & No & No & No information & No information & High & $\begin{array}{l}\text { Only } 3,233(42 \%) \text { of } 7589 \text { completed } \\
\text { questionnaires at all three time points } \\
\text { and were included in the analysis. }\end{array}$ \\
\hline $\begin{array}{l}\text { Hamdan, } \\
2012^{120}\end{array}$ & No information & No information & No information & No information & No information & $\begin{array}{l}\text { Uncertain } \\
\text { because no } \\
\text { information }\end{array}$ & $\begin{array}{l}\text { No information reported on loss to } \\
\text { followup. }\end{array}$ \\
\hline Illiadis, $2015^{59}$ & No & No & No & No information & No & High & $\begin{array}{l}181 / 365 \text { women were included in the } \\
\text { analytic sample. }\end{array}$ \\
\hline Lau, 2006 & Probably yes & Yes & No information & No information & No & Some concerns & $\begin{array}{l}\text { Complete case analysis limited to } \\
\text { charts that had no missing data; no } \\
\text { information provided on the number of } \\
\text { charts w/incomplete data. }\end{array}$ \\
\hline
\end{tabular}




\begin{tabular}{|c|c|c|c|c|c|c|c|}
\hline Author, Year & $\begin{array}{l}\text { 9. Were } \\
\text { outcome data } \\
\text { available for } \\
\text { all, or nearly } \\
\text { all, } \\
\text { participants? }\end{array}$ & $\begin{array}{l}\text { 10. Were few } \\
\text { or no } \\
\text { participants } \\
\text { excluded } \\
\text { because of } \\
\text { missing data } \\
\text { on exposure } \\
\text { status? } \\
\end{array}$ & $\begin{array}{l}\text { 11. Were few } \\
\text { or no } \\
\text { participants } \\
\text { excluded due } \\
\text { to missing } \\
\text { data on other } \\
\text { variables? }\end{array}$ & $\begin{array}{l}\text { 12. Was the } \\
\text { proportion of } \\
\text { participants } \\
\text { and reasons for } \\
\text { missing data } \\
\text { similar across } \\
\text { exposure } \\
\text { groups? }\end{array}$ & $\begin{array}{l}\text { 13. Were } \\
\text { appropriate } \\
\text { statistical methods } \\
\text { used to account } \\
\text { for missing data or } \\
\text { assess robustness } \\
\text { to presence of } \\
\text { missing data? }\end{array}$ & $\begin{array}{l}\text { Bias Arising } \\
\text { From Missing } \\
\text { Data }\end{array}$ & Comments \\
\hline $\begin{array}{l}\text { Maimburg, } \\
2015^{122}\end{array}$ & Yes & Yes & Yes & No information & No information & Low & NA \\
\hline $\begin{array}{l}\text { McCoy, } \\
2008^{123} \\
\end{array}$ & Yes & Yes & Probably yes & Probably yes & Probably no & Low & NA \\
\hline $\begin{array}{l}\text { Reifsnider, } \\
2016^{124}\end{array}$ & No information & No information & No information & No information & No information & $\begin{array}{l}\text { Uncertain } \\
\text { because no } \\
\text { information }\end{array}$ & None \\
\hline Sword, $2012^{125}$ & No & No & No & No information & No & Some concerns & $\begin{array}{l}\text { Substantial LTFU, with no information } \\
\text { on whether there was differential } \\
\text { LTFU by exposure or outcome group }\end{array}$ \\
\hline $\begin{array}{l}\text { Trivino-Juarez, } \\
2016^{126}\end{array}$ & Yes & Probably yes & Probably yes & No information & No information & Low & NA \\
\hline
\end{tabular}


Table C-48. KQ 2 risk of bias assessment: Postpartum depression, part 5

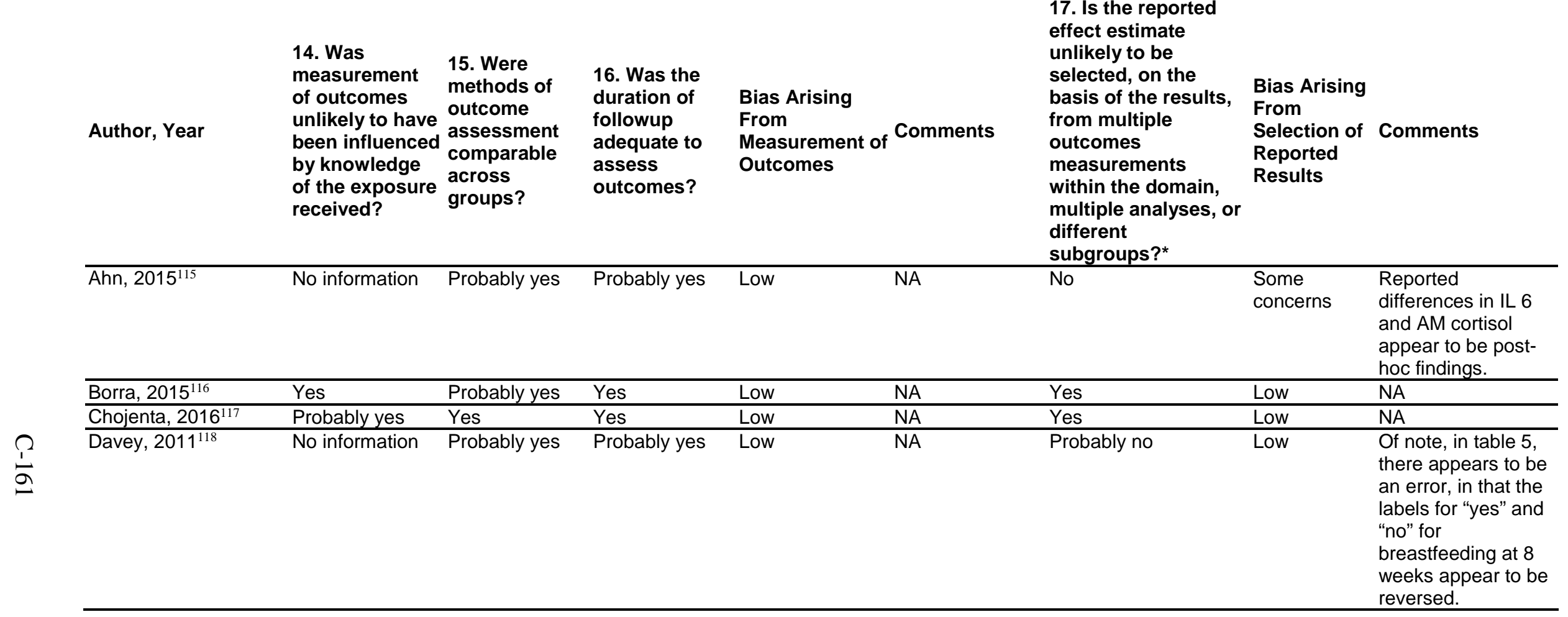




\begin{tabular}{|c|c|c|c|c|c|c|c|c|}
\hline Author, Year & $\begin{array}{l}\text { 14. Was } \\
\text { measurement } \\
\text { of outcomes } \\
\text { unlikely to have } \\
\text { been influenced } \\
\text { by knowledge } \\
\text { of the exposure } \\
\text { received? }\end{array}$ & $\begin{array}{l}\text { 15. Were } \\
\text { methods of } \\
\text { outcome } \\
\text { assessment } \\
\text { comparable } \\
\text { across } \\
\text { groups? }\end{array}$ & $\begin{array}{l}\text { 16. Was the } \\
\text { duration of } \\
\text { followup } \\
\text { adequate to } \\
\text { assess } \\
\text { outcomes? }\end{array}$ & $\begin{array}{l}\text { Bias Arising } \\
\text { From } \\
\text { Measurement of } \\
\text { Outcomes }\end{array}$ & Comments & $\begin{array}{l}\text { 17. Is the reported } \\
\text { effect estimate } \\
\text { unlikely to be } \\
\text { selected, on the } \\
\text { basis of the results, } \\
\text { from multiple } \\
\text { outcomes } \\
\text { measurements } \\
\text { within the domain, } \\
\text { multiple analyses, or } \\
\text { different } \\
\text { subgroups?* }\end{array}$ & $\begin{array}{l}\text { Bias Arising } \\
\text { From } \\
\text { Selection of } \\
\text { Reported } \\
\text { Results }\end{array}$ & Comments \\
\hline Fiala, $2017^{119}$ & Probably yes & Yes & Yes & High & $\begin{array}{l}\text { Investigators } \\
\text { modified the } \\
\text { EPDS and did } \\
\text { not provide } \\
\text { information on } \\
\text { reliability/validity } \\
\text { of this method: } \\
\text { "we conditioned } \\
\text { the } \\
\text { determination of } \\
\text { depressive } \\
\text { symptoms on a } \\
\text { positive answer } \\
\text { to question } \\
\text { number eight, } \\
\text { which refers to } \\
\text { mood problems } \\
\text { (a score of at } \\
\text { least two means } \\
\text { the participants } \\
\text { have felt sad or } \\
\text { miserable at } \\
\text { least quite } \\
\text { often). This } \\
\text { condition was } \\
\text { added to a } \\
\text { threshold of ten } \\
\text { points among } \\
\text { the original ten } \\
\text { questions." }\end{array}$ & Probably yes & Low & NA \\
\hline
\end{tabular}




\begin{tabular}{|c|c|c|c|c|c|c|c|c|}
\hline Author, Year & $\begin{array}{l}\text { 14. Was } \\
\text { measurement } \\
\text { of outcomes } \\
\text { unlikely to have } \\
\text { been influenced } \\
\text { by knowledge } \\
\text { of the exposure } \\
\text { received? }\end{array}$ & $\begin{array}{l}\text { 15. Were } \\
\text { methods of } \\
\text { outcome } \\
\text { assessment } \\
\text { comparable } \\
\text { across } \\
\text { groups? }\end{array}$ & $\begin{array}{l}\text { 16. Was the } \\
\text { duration of } \\
\text { followup } \\
\text { adequate to } \\
\text { assess } \\
\text { outcomes? }\end{array}$ & $\begin{array}{l}\text { Bias Arising } \\
\text { From } \\
\text { Measurement of } \\
\text { Outcomes }\end{array}$ & Comments & $\begin{array}{l}\text { 17. Is the reported } \\
\text { effect estimate } \\
\text { unlikely to be } \\
\text { selected, on the } \\
\text { basis of the results, } \\
\text { from multiple } \\
\text { outcomes } \\
\text { measurements } \\
\text { within the domain, } \\
\text { multiple analyses, or } \\
\text { different } \\
\text { subgroups?* }\end{array}$ & $\begin{array}{l}\text { Bias Arising } \\
\text { From } \\
\text { Selection of } \\
\text { Reported } \\
\text { Results }\end{array}$ & Comments \\
\hline Hamdan, $2012^{120}$ & No information & Probably yes & Probably yes & $\begin{array}{l}\text { Uncertain } \\
\text { because no } \\
\text { information }\end{array}$ & $\begin{array}{l}\text { Unclear whether } \\
\text { individuals } \\
\text { performing the } \\
\text { MINI were aware } \\
\text { of breastfeeding } \\
\text { status. }\end{array}$ & No & $\begin{array}{l}\text { Some } \\
\text { concerns }\end{array}$ & $\begin{array}{l}\text { No adjusted } \\
\text { analysis is } \\
\text { performed, and } \\
\text { authors emphasize } \\
\text { the hypothesized } \\
\text { association between } \\
\text { breastfeeding and } \\
\text { lower depression } \\
\text { risk, with far less } \\
\text { emphasis on } \\
\text { depression and } \\
\text { lower breastfeeding } \\
\text { likelihood. }\end{array}$ \\
\hline Illiadis, $2015^{59}$ & No information & Probably yes & Probably yes & Low & NA & NA & Uncertain & $\begin{array}{l}\text { Association } \\
\text { between } \\
\text { breastfeeding \& } \\
\text { depression was not } \\
\text { the goal of the } \\
\text { paper; we are } \\
\text { extracting data from } \\
\text { the table to look at } \\
\text { the association. }\end{array}$ \\
\hline Lau, 2006 ${ }^{121}$ & No & Yes & No & Some concerns & $\begin{array}{l}\text { Four weeks } \\
\text { postpartum may } \\
\text { be insufficient } \\
\text { followup for a } \\
\text { depression } \\
\text { assessment. }\end{array}$ & Probably no & Low & NA \\
\hline
\end{tabular}




\begin{tabular}{|c|c|c|c|c|c|c|c|c|}
\hline Author, Year & $\begin{array}{l}\text { 14. Was } \\
\text { measurement } \\
\text { of outcomes } \\
\text { unlikely to have } \\
\text { been influenced } \\
\text { by knowledge } \\
\text { of the exposure } \\
\text { received? }\end{array}$ & $\begin{array}{l}\text { 15. Were } \\
\text { methods of } \\
\text { outcome } \\
\text { assessment } \\
\text { comparable } \\
\text { across } \\
\text { groups? }\end{array}$ & $\begin{array}{l}\text { 16. Was the } \\
\text { duration of } \\
\text { followup } \\
\text { adequate to } \\
\text { assess } \\
\text { outcomes? }\end{array}$ & $\begin{array}{l}\text { Bias Arising } \\
\text { From } \\
\text { Measurement of } \\
\text { Outcomes }\end{array}$ & Comments & $\begin{array}{l}\text { 17. Is the reported } \\
\text { effect estimate } \\
\text { unlikely to be } \\
\text { selected, on the } \\
\text { basis of the results, } \\
\text { from multiple } \\
\text { outcomes } \\
\text { measurements } \\
\text { within the domain, } \\
\text { multiple analyses, or } \\
\text { different } \\
\text { subgroups?* }\end{array}$ & $\begin{array}{l}\text { Bias Arising } \\
\text { From } \\
\text { Selection of } \\
\text { Reported } \\
\text { Results }\end{array}$ & Comments \\
\hline Maimburg, 2015 & Probably yes & Yes & No & Some concerns & $\begin{array}{l}\text { EPDS should } \\
\text { have been } \\
\text { assessed after } 6 \\
\text { weeks in order to } \\
\text { reflect causality } \\
\text { from } \\
\text { breastfeeding. }\end{array}$ & Yes & Low & $\mathrm{NA}$ \\
\hline McCoy, 2008 ${ }^{123}$ & Probably yes & Yes & No & Some concerns & $\begin{array}{l}\text { EPDS should } \\
\text { have been } \\
\text { assessed after } 4 \\
\text { weeks in order to } \\
\text { reflect causality } \\
\text { from } \\
\text { breastfeeding. }\end{array}$ & Yes & Low & NA \\
\hline Reifsnider, 2016 & Probably yes & Yes & Yes & Low & NA & Yes & Low & NA \\
\hline Sword, $2012^{125}$ & Probably no & Probably yes & Probably yes & Low & NA & Yes & High & $\begin{array}{l}\text { The authors report } \\
\text { that not } \\
\text { breastfeeding was a } \\
\text { significant predictor } \\
\text { of depression } \\
\text { status, but the } \\
\text { confidence interval } \\
\text { for this effect } \\
\text { estimate crosses } 1 \\
\text { and the p value } \\
\text { exceeds the a priori } \\
\text { alpha of } 0.05 \text {. }\end{array}$ \\
\hline
\end{tabular}




\begin{tabular}{|c|c|c|c|c|c|c|c|}
\hline Author, Year & $\begin{array}{l}\text { 14. Was } \\
\text { measurement } \\
\text { of outcomes } \\
\text { unlikely to have } \\
\text { been influenced } \\
\text { by knowledge } \\
\text { of the exposure } \\
\text { received? }\end{array}$ & $\begin{array}{l}\text { 15. Were } \\
\text { methods of } \\
\text { outcome } \\
\text { assessment } \\
\text { comparable } \\
\text { across } \\
\text { groups? }\end{array}$ & $\begin{array}{l}\text { 16. Was the } \\
\text { duration of } \\
\text { followup } \\
\text { adequate to } \\
\text { assess } \\
\text { outcomes? }\end{array}$ & $\begin{array}{l}\text { Bias Arising } \\
\text { From } \\
\text { Measurement of Comments } \\
\text { Outcomes }\end{array}$ & $\begin{array}{l}\text { 17. Is the reported } \\
\text { effect estimate } \\
\text { unlikely to be } \\
\text { selected, on the } \\
\text { basis of the results, } \\
\text { from multiple } \\
\text { outcomes } \\
\text { measurements } \\
\text { within the domain, } \\
\text { multiple analyses, or } \\
\text { different } \\
\text { subgroups?* }\end{array}$ & $\begin{array}{l}\text { Bias Arising } \\
\text { From } \\
\text { Selection of } \\
\text { Reported } \\
\text { Results }\end{array}$ & Comments \\
\hline Trivino-Juarez, $2016^{126}$ & No information & Yes & Yes & $\begin{array}{l}\text { Uncertain } \\
\text { because no } \\
\text { information }\end{array}$ & Yes & Low & NA \\
\hline
\end{tabular}

AM = morning; EPDS = Edinburgh Postnatal Depression Scale; IL = inflammatory cytokines; KQ = Key Question; MINI = Mini International Neuro-psychiatric Interview; NA = not applicable. 
Table C-49. KQ 2 risk of bias assessment: Postpartum weight change, part 1

\begin{tabular}{|c|c|c|c|c|c|c|c|c|c|}
\hline Author, Year & $\begin{array}{l}\text { Overall } \\
\text { Risk of } \\
\text { Bias Rating }\end{array}$ & $\begin{array}{l}\text { Overall Rationale for } \\
\text { Risk of Bias Rating }\end{array}$ & $\begin{array}{l}\text { 1. Was } \\
\text { method of } \\
\text { selection } \\
\text { unrelated } \\
\text { to } \\
\text { exposurel } \\
\text { outcome? }\end{array}$ & $\begin{array}{l}\text { 1a. Were } \\
\text { post- } \\
\text { exposure } \\
\text { variables } \\
\text { that } \\
\text { influenced } \\
\text { selection } \\
\text { related to } \\
\text { exposurel } \\
\text { outcome? }\end{array}$ & $\begin{array}{l}\text { 2. Do start } \\
\text { of followup } \\
\text { and start of } \\
\text { exposure } \\
\text { coincide? }\end{array}$ & $\begin{array}{l}\text { 3. Were } \\
\text { adjustment } \\
\text { techniques } \\
\text { used to } \\
\text { correct for } \\
\text { presence of } \\
\text { selection } \\
\text { biases? }\end{array}$ & $\begin{array}{l}\text { 4. Were the } \\
\text { controls sampled } \\
\text { from the } \\
\text { population that } \\
\text { gave rise to the } \\
\text { cases, or using } \\
\text { another method } \\
\text { that avoids } \\
\text { selection bias? }\end{array}$ & $\begin{array}{l}\text { Bias arising } \\
\text { from } \\
\text { selection? }\end{array}$ & Comments \\
\hline $\begin{array}{l}\text { Endres, } \\
2014^{127}\end{array}$ & $\begin{array}{l}\text { Some } \\
\text { concerns }\end{array}$ & $\begin{array}{l}\text { Potential for selection } \\
\text { bias, attrition and } \\
\text { residual confounding. }\end{array}$ & No & Probably yes & Yes & No & NA & $\begin{array}{l}\text { Some } \\
\text { concerns }\end{array}$ & $\begin{array}{l}\text { Selected into sample } \\
\text { on the basis of having } \\
\text { prepregnancy weight, } \\
\text { to the extent that such } \\
\text { variables are } \\
\text { correlated with BF or } \\
\text { eventual weight, } \\
\text { potential for selection. }\end{array}$ \\
\hline $\begin{array}{l}\text { Jarlenski, } \\
2014: 128 \\
\text { Sharma, } \\
2014^{129}\end{array}$ & $\begin{array}{l}\text { Some } \\
\text { concerns }\end{array}$ & $\begin{array}{l}\text { Substantial loss to } \\
\text { followup, however } \\
\text { sensitivity analyses } \\
\text { conducted; potential } \\
\text { for residual } \\
\text { confounding. }\end{array}$ & Yes & NA & Yes & $\begin{array}{l}\text { Yes; }{ }^{128} \\
\mathrm{No}^{129}\end{array}$ & NA & Low & NA \\
\hline Lyu, $2009^{130}$ & $\begin{array}{l}\text { Some } \\
\text { concerns }\end{array}$ & $\begin{array}{l}\text { Potential for } \\
\text { confounding, self- } \\
\text { reported weight. }\end{array}$ & Yes & NA & Yes & No & NA & Low & NA \\
\hline $\mathrm{Ng}, 2014^{131}$ & High & $\begin{array}{l}\text { Potential for attrition } \\
\text { and residual } \\
\text { confounding. }\end{array}$ & Yes & NA & Yes & No & NA & $\begin{array}{l}\text { Some } \\
\text { concerns }\end{array}$ & $\begin{array}{l}\text { Selected into sample } \\
\text { on the basis of having } \\
\text { prepregnancy weight, } \\
\text { to the extent that such } \\
\text { variables are } \\
\text { correlated with BF or } \\
\text { eventual weight, } \\
\text { potential for selection. }\end{array}$ \\
\hline $\begin{array}{l}\text { Onyango, } \\
2011^{132} \\
\end{array}$ & $\begin{array}{l}\text { Some } \\
\text { concerns }\end{array}$ & $\begin{array}{l}\text { Potential for residual } \\
\text { confounding. }\end{array}$ & Yes & NA & Yes & No & NA & Low & NA \\
\hline $\begin{array}{l}\text { Ostbye, } \\
2010^{133}\end{array}$ & High & $\begin{array}{l}\text { Potential for bias from } \\
\text { attrition and selection } \\
\text { bias. }\end{array}$ & Yes & NA & $\begin{array}{l}\text { Probably } \\
\text { yes }\end{array}$ & Probably yes & NA & Low & NA \\
\hline
\end{tabular}




\begin{tabular}{|c|c|c|c|c|c|c|c|c|c|}
\hline Author, Year & $\begin{array}{l}\text { Overall } \\
\text { Risk of } \\
\text { Bias Rating }\end{array}$ & $\begin{array}{l}\text { Overall Rationale for } \\
\text { Risk of Bias Rating }\end{array}$ & $\begin{array}{l}\text { 1. Was } \\
\text { method of } \\
\text { selection } \\
\text { unrelated } \\
\text { to } \\
\text { exposurel } \\
\text { outcome? }\end{array}$ & $\begin{array}{l}\text { 1a. Were } \\
\text { post- } \\
\text { exposure } \\
\text { variables } \\
\text { that } \\
\text { influenced } \\
\text { selection } \\
\text { related to } \\
\text { exposurel } \\
\text { outcome? }\end{array}$ & $\begin{array}{l}\text { 2. Do start } \\
\text { of followup } \\
\text { and start of } \\
\text { exposure } \\
\text { coincide? }\end{array}$ & $\begin{array}{l}\text { 3. Were } \\
\text { adjustment } \\
\text { techniques } \\
\text { used to } \\
\text { correct for } \\
\text { presence of } \\
\text { selection } \\
\text { biases? }\end{array}$ & $\begin{array}{l}\text { 4. Were the } \\
\text { controls sampled } \\
\text { from the } \\
\text { population that } \\
\text { gave rise to the } \\
\text { cases, or using } \\
\text { another method } \\
\text { that avoids } \\
\text { selection bias? }\end{array}$ & $\begin{array}{l}\text { Bias arising } \\
\text { from } \\
\text { selection? }\end{array}$ & Comments \\
\hline $\begin{array}{l}\text { Palmer, } \\
2015^{134}\end{array}$ & High & $\begin{array}{l}\text { Potential for } \\
\text { confounding, self- } \\
\text { reported weight, } \\
\text { attrition }\end{array}$ & Yes & NA & Yes & No & $\mathrm{NA}$ & $\begin{array}{l}\begin{array}{l}\text { Some } \\
\text { concerns }\end{array}\end{array}$ & $\begin{array}{l}\text { Selected into sample } \\
\text { on the basis of having } \\
\text { prepregnancy weight, } \\
\text { to the extent that such } \\
\text { variables are } \\
\text { correlated with BF or } \\
\text { eventual weight, } \\
\text { potential for selection. }\end{array}$ \\
\hline Straub, 2016 & High & $\begin{array}{l}\text { Potential for selection } \\
\text { bias, confounding, } \\
\text { and attrition bias }\end{array}$ & No & Probably yes & Yes & No & $\overline{N A}$ & $\begin{array}{l}\begin{array}{l}\text { Some } \\
\text { concerns }\end{array}\end{array}$ & $\begin{array}{l}\text { Selected into sample } \\
\text { on the basis of having } \\
\text { salivary samples and } \\
\text { not missing } \\
\text { prepregnancy weights, } \\
\text { to the extent that such } \\
\text { variables are } \\
\text { correlated with BF or } \\
\text { eventual weight, } \\
\text { potential for selection. }\end{array}$ \\
\hline
\end{tabular}




\begin{tabular}{|c|c|c|c|c|c|c|c|c|c|}
\hline Author, Year & $\begin{array}{l}\text { Overall } \\
\text { Risk of } \\
\text { Bias Rating }\end{array}$ & $\begin{array}{l}\text { Overall Rationale for } \\
\text { Risk of Bias Rating }\end{array}$ & $\begin{array}{l}\text { 1. Was } \\
\text { method of } \\
\text { selection } \\
\text { unrelated } \\
\text { to } \\
\text { exposurel } \\
\text { outcome? }\end{array}$ & $\begin{array}{l}\text { 1a. Were } \\
\text { post- } \\
\text { exposure } \\
\text { variables } \\
\text { that } \\
\text { influenced } \\
\text { selection } \\
\text { related to } \\
\text { exposurel } \\
\text { outcome? }\end{array}$ & $\begin{array}{l}\text { 2. Do start } \\
\text { of followup } \\
\text { and start of } \\
\text { exposure } \\
\text { coincide? }\end{array}$ & $\begin{array}{l}\text { 3. Were } \\
\text { adjustment } \\
\text { techniques } \\
\text { used to } \\
\text { correct for } \\
\text { presence of } \\
\text { selection } \\
\text { biases? }\end{array}$ & $\begin{array}{l}\text { 4. Were the } \\
\text { controls sampled } \\
\text { from the } \\
\text { population that } \\
\text { gave rise to the } \\
\text { cases, or using } \\
\text { another method } \\
\text { that avoids } \\
\text { selection bias? }\end{array}$ & $\begin{array}{l}\text { Bias arising } \\
\text { from } \\
\text { selection? }\end{array}$ & Comments \\
\hline $\begin{array}{l}\text { Stuebe, } \\
2010^{136}\end{array}$ & $\begin{array}{l}\text { Some } \\
\text { concerns }\end{array}$ & $\begin{array}{l}\text { Potential for residual } \\
\text { confounding and } \\
\text { attrition bias. }\end{array}$ & No & Probably yes & Yes & No & NA & $\begin{array}{l}\text { Some } \\
\text { concerns }\end{array}$ & $\begin{array}{l}\text { Risk of selection bias: } \\
\text { "women who } \\
\text { recertified prior to } 20 \\
\text { weeks and were still } \\
\text { breastfeeding at the } \\
\text { time were not included } \\
\text { in the analysis, since } \\
\text { an accurate duration } \\
\text { of breastfeeding could } \\
\text { not be reasonably } \\
\text { ascertained". To the } \\
\text { extent that these early } \\
\text { recertifiers had } \\
\text { different weight } \\
\text { retention outcomes, } \\
\text { the results can be } \\
\text { biased, but not clear in } \\
\text { which direction the } \\
\text { bias could operate. }\end{array}$ \\
\hline
\end{tabular}


Table C-50. KQ 2 risk of bias assessment: Postpartum weight change, part 2

\begin{tabular}{|c|c|c|c|c|c|c|c|c|}
\hline Author, Year & $\begin{array}{l}\text { 5. Is confounding } \\
\text { of the exposure } \\
\text { effect unlikely? }\end{array}$ & $\begin{array}{l}5 a \text {. Did the } \\
\text { authors use an } \\
\text { appropriate } \\
\text { analysis to } \\
\text { adjust for } \\
\text { confounders? }\end{array}$ & $\begin{array}{l}\text { 5b. Were } \\
\text { confounding } \\
\text { domains that } \\
\text { were controlled } \\
\text { for measured } \\
\text { validly and } \\
\text { reliably? }\end{array}$ & $\begin{array}{l}5 c . \text { Did the } \\
\text { authors avoid } \\
\text { adjusting for } \\
\text { post-exposure } \\
\text { variables? }\end{array}$ & $\begin{array}{l}\text { 5d. Were } \\
\text { participants } \\
\text { analyzed } \\
\text { according to } \\
\text { their initial } \\
\text { exposure group } \\
\text { throughout } \\
\text { followup? }\end{array}$ & $\begin{array}{l}\text { 5e. Were } \\
\text { exposure } \\
\text { discontinuations } \\
\text { or switches } \\
\text { unlikely to be } \\
\text { related to factors } \\
\text { prognostic for } \\
\text { the outcome? }\end{array}$ & $\begin{array}{l}\text { Bias arising from } \\
\text { confounding? }\end{array}$ & Comments \\
\hline Endres, $2014^{127}$ & No & Probably yes & Probably yes & Yes & Yes & NA & Some concerns & $\begin{array}{l}\text { Potential for } \\
\text { residual } \\
\text { confounding. }\end{array}$ \\
\hline $\begin{array}{l}\text { Jarlenski, 2014; }{ }^{128} \\
\text { Sharma, 2014 }\end{array}$ & No & Probably yes & Probably yes & Yes & Yes & NA & $\begin{array}{l}\text { Low; }^{128} \\
\text { Some concerns } \\
\end{array}$ & $\begin{array}{l}\text { Conducted } \\
\text { propensity score } \\
\text { analysis; potential } \\
\text { for residual } \\
\text { confounding. }\end{array}$ \\
\hline Lyu, 2009 & No & Probably no & Probably yes & No & Yes & NA & Some concerns & $\begin{array}{l}\text { One of the models } \\
\text { adjusted for } \\
\text { postexposure } \\
\text { variables of 1- } \\
\text { month energy } \\
\text { intake, but other } \\
\text { models did not. }\end{array}$ \\
\hline $\mathrm{Ng}, 2014^{131}$ & No & Probably yes & Probably yes & Yes & Yes & NA & Some concerns & $\begin{array}{l}\text { Potential for } \\
\text { residual } \\
\text { confounding. }\end{array}$ \\
\hline Onyango, $2011^{132}$ & No & Probably yes & Probably yes & Yes & Yes & NA & Some concerns & $\begin{array}{l}\text { Potential for } \\
\text { residual } \\
\text { confounding. }\end{array}$ \\
\hline Ostbye, $2010^{133}$ & No & Probably yes & Probably yes & Yes & Yes & NA & Some concerns & $\begin{array}{l}\text { Potential for } \\
\text { residual } \\
\text { confounding. }\end{array}$ \\
\hline Palmer, $2015^{134}$ & No & Probably yes & Probably yes & No & Yes & NA & High & $\begin{array}{l}\text { Adjusted for } \\
\text { postexposure } \\
\text { variables of } \\
\text { additional births } \\
\text { and subsequent } \\
\text { lactation. }\end{array}$ \\
\hline
\end{tabular}




\begin{tabular}{|c|c|c|c|c|c|c|c|c|}
\hline Author, Year & $\begin{array}{l}\text { 5. Is confounding } \\
\text { of the exposure } \\
\text { effect unlikely? }\end{array}$ & $\begin{array}{l}5 \text { a. Did the } \\
\text { authors use an } \\
\text { appropriate } \\
\text { analysis to } \\
\text { adjust for } \\
\text { confounders? }\end{array}$ & $\begin{array}{l}\text { 5b. Were } \\
\text { confounding } \\
\text { domains that } \\
\text { were controlled } \\
\text { for measured } \\
\text { validly and } \\
\text { reliably? }\end{array}$ & $\begin{array}{l}5 c . \text { Did the } \\
\text { authors avoid } \\
\text { adjusting for } \\
\text { post-exposure } \\
\text { variables? }\end{array}$ & $\begin{array}{l}\text { 5d. Were } \\
\text { participants } \\
\text { analyzed } \\
\text { according to } \\
\text { their initial } \\
\text { exposure group } \\
\text { throughout } \\
\text { followup? }\end{array}$ & $\begin{array}{l}\text { 5e. Were } \\
\text { exposure } \\
\text { discontinuations } \\
\text { or switches } \\
\text { unlikely to be } \\
\text { related to factors } \\
\text { prognostic for } \\
\text { the outcome? }\end{array}$ & $\begin{array}{l}\text { Bias arising from } \\
\text { confounding? }\end{array}$ & Comments \\
\hline Straub, $2016^{135}$ & No & Probably no & Probably yes & Yes & Yes & $\mathrm{NA}$ & Some concerns & $\begin{array}{l}\text { Potential for } \\
\text { residual } \\
\text { confounding, did } \\
\text { not control for } \\
\text { prior pregnancies. }\end{array}$ \\
\hline Stuebe, $2010^{136}$ & No & Probably yes & Probably yes & Yes & Yes & NA & Some concerns & $\begin{array}{l}\text { Potential for } \\
\text { residual } \\
\text { confounding. }\end{array}$ \\
\hline
\end{tabular}


Table C-51. KQ 2 risk of bias assessment: Postpartum weight change, part 3

\begin{tabular}{|c|c|c|c|c|c|}
\hline Author, Year & $\begin{array}{l}\text { 6. Is exposure status well- } \\
\text { defined? }\end{array}$ & $\begin{array}{l}\text { 7. Was information on } \\
\text { exposure status recorded } \\
\text { at the time of exposure? }\end{array}$ & $\begin{array}{l}\text { 8. Was classification of } \\
\text { exposure status } \\
\text { unaffected by knowledge } \\
\text { of the outcome or risk of } \\
\text { the outcome? }\end{array}$ & $\begin{array}{l}\text { Bias arising from } \\
\text { measurement of } \\
\text { exposures? }\end{array}$ & Comments \\
\hline
\end{tabular}

\begin{tabular}{llll}
\hline Endres, 2014 127 & Yes & Yes & Yes \\
\hline
\end{tabular}

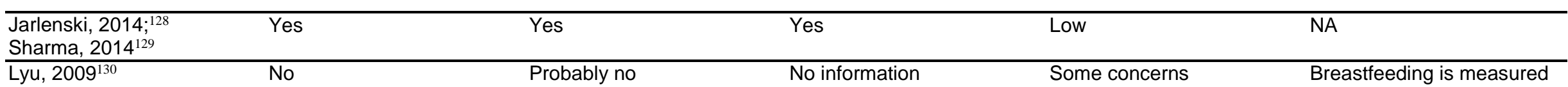

in number of months.

Because the analysis also

includes BF>6 months in a

correlation analysis of

weight retention at 6

months, it's not clear

whether the BF exposure

variable was appropriately

cut off to include months of

BF that occurred before the

6-month weight retention

variable only or whether it

included all months of BF

duration.

\begin{tabular}{|c|c|c|c|c|c|}
\hline $\mathrm{Ng}, 2014^{131}$ & No & No & Probably yes & Some concerns & Recall \\
\hline Onyango, $2011^{132}$ & Yes & Yes & Yes & Low & NA \\
\hline Ostbye, $2010^{133}$ & Yes & Yes & Yes & Low & NA \\
\hline Palmer, $2015^{134}$ & Yes & No & Probably yes & Some concerns & Recall bias \\
\hline Straub, $2016^{135}$ & Yes & Yes & Yes & Low & NA \\
\hline Stuebe, $2010^{136}$ & Yes & Yes & Yes & Low & NA \\
\hline
\end{tabular}

$\mathrm{BF}$ = breastfeeding; KQ = Key Question; NA = not applicable. 
Table C-52. KQ 2 risk of bias assessment: Postpartum weight change, part 4

\begin{tabular}{|c|c|c|c|c|c|c|c|}
\hline Author, Year & $\begin{array}{l}\text { 9. Were outcome } \\
\text { data available for } \\
\text { all, or nearly all, } \\
\text { participants? }\end{array}$ & $\begin{array}{l}\text { 10. Were few or } \\
\text { no participants } \\
\text { excluded because } \\
\text { of missing data } \\
\text { on exposure } \\
\text { status? }\end{array}$ & $\begin{array}{l}\text { 11. Were few or } \\
\text { no participants } \\
\text { excluded due to } \\
\text { missing data on } \\
\text { other variables? }\end{array}$ & $\begin{array}{l}\text { 12. Was the } \\
\text { proportion of } \\
\text { participants and } \\
\text { reasons for } \\
\text { missing data } \\
\text { similar across } \\
\text { exposure } \\
\text { groups? }\end{array}$ & $\begin{array}{l}\text { 13. Were } \\
\text { appropriate } \\
\text { statistical } \\
\text { methods used to } \\
\text { account for } \\
\text { missing data or } \\
\text { assess } \\
\text { robustness to } \\
\text { presence of } \\
\text { missing data? }\end{array}$ & $\begin{array}{l}\text { Bias Arising } \\
\text { From Missing } \\
\text { Data }\end{array}$ & Comments \\
\hline Endres, 2014 & No & Yes & Yes & No information & Yes & Some concerns & $\begin{array}{l}\text { Only } 774 \text { of } 2,510 \\
\text { women had } \\
\text { baseline and } \\
\text { followup weight } \\
\text { information. The } \\
\text { authors note that } \\
\text { they "we compared } \\
\text { demographic } \\
\text { characteristics } \\
\text { between women } \\
\text { with and without a } \\
\text { prepregnancy BMI } \\
\text { and found no } \\
\text { statistically } \\
\text { significant } \\
\text { differences." } \\
\text { Nonetheless, this } \\
\text { high rate of } \\
\text { exclusion could } \\
\text { result in biased } \\
\text { results. }\end{array}$ \\
\hline
\end{tabular}




\begin{tabular}{|c|c|c|c|c|c|c|c|}
\hline Author, Year & $\begin{array}{l}\text { 9. Were outcome } \\
\text { data available for } \\
\text { all, or nearly all, } \\
\text { participants? }\end{array}$ & $\begin{array}{l}\text { 10. Were few or } \\
\text { no participants } \\
\text { excluded because } \\
\text { of missing data } \\
\text { on exposure } \\
\text { status? }\end{array}$ & $\begin{array}{l}\text { 11. Were few or } \\
\text { no participants } \\
\text { excluded due to } \\
\text { missing data on } \\
\text { other variables? }\end{array}$ & $\begin{array}{l}\text { 12. Was the } \\
\text { proportion of } \\
\text { participants and } \\
\text { reasons for } \\
\text { missing data } \\
\text { similar across } \\
\text { exposure } \\
\text { groups? }\end{array}$ & $\begin{array}{l}\text { 13. Were } \\
\text { appropriate } \\
\text { statistical } \\
\text { methods used to } \\
\text { account for } \\
\text { missing data or } \\
\text { assess } \\
\text { robustness to } \\
\text { presence of } \\
\text { missing data? }\end{array}$ & $\begin{array}{l}\text { Bias Arising } \\
\text { From Missing } \\
\text { Data }\end{array}$ & Comments \\
\hline $\begin{array}{l}\text { Jarlenski, 2014;:128 } \\
\text { Sharma, 2014 }\end{array}$ & No & $\begin{array}{l}\text { Probably no; }{ }^{128} \\
\text { no }^{129}\end{array}$ & $\begin{array}{l}\text { Probably no; }{ }^{128} \\
\text { no }^{129}\end{array}$ & No information & $\begin{array}{l}\text { Probably yes; }{ }^{128} \\
\text { no }^{129}\end{array}$ & Some concerns & $\begin{array}{l}\text { The study } \\
\text { evaluated baseline } \\
\text { characteristics } \\
\text { between those with } \\
\text { and without data } \\
(15 \%) \text { and found } \\
\text { differences; } \\
\text { implications of } \\
\text { missing data were } \\
\text { not provided in } \\
\text { detail. Response } \\
\text { rate was } 65 \% \text { at } 12 \\
\text { months. }\end{array}$ \\
\hline
\end{tabular}




\begin{tabular}{|c|c|c|c|c|c|c|c|}
\hline Author, Year & $\begin{array}{l}\text { 9. Were outcome } \\
\text { data available for } \\
\text { all, or nearly all, } \\
\text { participants? }\end{array}$ & $\begin{array}{l}\text { 10. Were few or } \\
\text { no participants } \\
\text { excluded because } \\
\text { of missing data } \\
\text { on exposure } \\
\text { status? }\end{array}$ & $\begin{array}{l}\text { 11. Were few or } \\
\text { no participants } \\
\text { excluded due to } \\
\text { missing data on } \\
\text { other variables? }\end{array}$ & $\begin{array}{l}\text { 12. Was the } \\
\text { proportion of } \\
\text { participants and } \\
\text { reasons for } \\
\text { missing data } \\
\text { similar across } \\
\text { exposure } \\
\text { groups? }\end{array}$ & $\begin{array}{l}\text { 13. Were } \\
\text { appropriate } \\
\text { statistical } \\
\text { methods used to } \\
\text { account for } \\
\text { missing data or } \\
\text { assess } \\
\text { robustness to } \\
\text { presence of } \\
\text { missing data? }\end{array}$ & $\begin{array}{l}\text { Bias Arising } \\
\text { From Missing } \\
\text { Data }\end{array}$ & Comments \\
\hline Lyu, 2009130 & Yes & No information & No information & No information & No & Some concerns & $\begin{array}{l}130 \text { of } 151 \\
\text { available at } 6 \\
\text { months and } 122 \text { at } \\
12 \text { months, but no } \\
\text { analysis of } \\
\text { implications of } \\
\text { missing data. }\end{array}$ \\
\hline $\mathrm{Ng}, 2014^{131}$ & No & Probably no & Probably no & No information & No & High & $\begin{array}{l}\text { Analysis on } 1,213 \\
\text { of } 2,254 \text { women, } \\
\text { no sensitivity } \\
\text { analysis. }\end{array}$ \\
\hline Onyango, $2011^{132}$ & Yes & No information & Probably no & No information & No & Some concerns & $\begin{array}{l}\text { Analysis of } \\
172 / 208 \text { in the U.S. } \\
\text { and } 262 / 300 \text { in } \\
\text { Norway, but no } \\
\text { analysis of } \\
\text { implications of } \\
\text { missing data. }\end{array}$ \\
\hline Ostbye, $2010^{133}$ & No & No & No & No information & No & High & $\begin{array}{l}\text { Analysis on } \\
32,920 / 70,353 \\
(47 \%) ; \text { no analysis } \\
\text { of implications of } \\
\text { missing data. }\end{array}$ \\
\hline Palmer, $2015^{134}$ & No & No & Yes & No information & No & High & $\begin{array}{l}\text { Data available for } \\
\text { only } 70 \% \text { of the } \\
\text { sample, others are } \\
\text { missing exposure } \\
\text { or outcome data, } \\
\text { no analysis of the } \\
\text { implications of } \\
\text { missing data. }\end{array}$ \\
\hline
\end{tabular}




\begin{tabular}{|c|c|c|c|c|c|c|c|}
\hline Author, Year & $\begin{array}{l}\text { 9. Were outcome } \\
\text { data available for } \\
\text { all, or nearly all, } \\
\text { participants? }\end{array}$ & $\begin{array}{l}\text { 10. Were few or } \\
\text { no participants } \\
\text { excluded because } \\
\text { of missing data } \\
\text { on exposure } \\
\text { status? }\end{array}$ & $\begin{array}{l}\text { 11. Were few or } \\
\text { no participants } \\
\text { excluded due to } \\
\text { missing data on } \\
\text { other variables? }\end{array}$ & $\begin{array}{l}\text { 12. Was the } \\
\text { proportion of } \\
\text { participants and } \\
\text { reasons for } \\
\text { missing data } \\
\text { similar across } \\
\text { exposure } \\
\text { groups? }\end{array}$ & $\begin{array}{l}\text { 13. Were } \\
\text { appropriate } \\
\text { statistical } \\
\text { methods used to } \\
\text { account for } \\
\text { missing data or } \\
\text { assess } \\
\text { robustness to } \\
\text { presence of } \\
\text { missing data? }\end{array}$ & $\begin{array}{l}\text { Bias Arising } \\
\text { From Missing } \\
\text { Data }\end{array}$ & Comments \\
\hline Straub, $2016^{135}$ & No & Yes & No & No information & No & High & $\begin{array}{l}\text { Only women who } \\
\text { returned morning } \\
\text { and evening } \\
\text { salivary samples } \\
\text { were included; } \\
\text { women were then } \\
\text { excluded if there } \\
\text { were data errors } \\
\text { (n=81), if they were } \\
\text { currently pregnant } \\
\text { (n=20) or using } \\
\text { steroids ( } \mathrm{n}=5 \text { ), or if } \\
\text { they lacked pre- } \\
\text { pregnancy or } \\
\text { postpartum weights } \\
\text { (n=349). } \\
\text { Additionally, one } \\
\text { woman was } \\
\text { excluded for } \\
\text { weighing more } \\
\text { than } 350 \text { pounds } \\
\text { (due to scale } \\
\text { limitations). } \\
\text { Authors do not } \\
\text { comment on the } \\
\text { missing data that } \\
\text { resulted in } \\
\text { selection of } \\
\text { patients for the } \\
\text { analysis. }\end{array}$ \\
\hline
\end{tabular}




\begin{tabular}{|c|c|c|c|c|c|c|c|}
\hline Author, Year & $\begin{array}{l}\text { 9. Were outcome } \\
\text { data available for } \\
\text { all, or nearly all, } \\
\text { participants? }\end{array}$ & $\begin{array}{l}\text { 10. Were few or } \\
\text { no participants } \\
\text { excluded because } \\
\text { of missing data } \\
\text { on exposure } \\
\text { status? }\end{array}$ & $\begin{array}{l}\text { 11. Were few or } \\
\text { no participants } \\
\text { excluded due to } \\
\text { missing data on } \\
\text { other variables? }\end{array}$ & $\begin{array}{l}\text { 12. Was the } \\
\text { proportion of } \\
\text { participants and } \\
\text { reasons for } \\
\text { missing data } \\
\text { similar across } \\
\text { exposure } \\
\text { groups? }\end{array}$ & $\begin{array}{l}\text { 13. Were } \\
\text { appropriate } \\
\text { statistical } \\
\text { methods used to } \\
\text { account for } \\
\text { missing data or } \\
\text { assess } \\
\text { robustness to } \\
\text { presence of } \\
\text { missing data? }\end{array}$ & $\begin{array}{l}\text { Bias Arising From } \\
\text { Missing Data }\end{array}$ & Comments \\
\hline Stuebe, $2010^{136}$ & Yes & Yes & Yes & No information & Probably no & Some concerns & $\begin{array}{l}557 \text { of } 611 \text { eligible } \\
\text { with blood samples } \\
\text { included in the } \\
\text { analysis ( } 91 \%) \text {, but } \\
\text { no analysis of the } \\
\text { implications of } \\
\text { missing data. }\end{array}$ \\
\hline
\end{tabular}




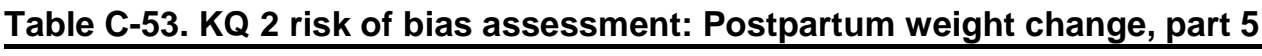

\begin{tabular}{|c|c|c|c|c|c|c|c|c|}
\hline Author, Year & $\begin{array}{l}\text { 14. Was } \\
\text { measurement of } \\
\text { outcomes } \\
\text { unlikely to have } \\
\text { been influenced } \\
\text { by knowledge of } \\
\text { the exposure } \\
\text { received? }\end{array}$ & $\begin{array}{l}15 . \text { Were } \\
\text { methods of } \\
\text { outcome } \\
\text { assessment } \\
\text { comparable } \\
\text { across groups? }\end{array}$ & $\begin{array}{l}\text { 16. Was the } \\
\text { duration of } \\
\text { followup } \\
\text { adequate to } \\
\text { assess } \\
\text { outcomes? }\end{array}$ & $\begin{array}{l}\text { Bias Arising } \\
\text { From } \\
\text { Measurement of } \\
\text { Outcomes }\end{array}$ & Comments & $\begin{array}{l}\text { reported effect } \\
\text { estimate unlikely } \\
\text { to be selected, } \\
\text { on the basis of } \\
\text { the results, from } \\
\text { multiple } \\
\text { outcomes } \\
\text { measurements } \\
\text { within the } \\
\text { domain, multiple } \\
\text { analyses, or } \\
\text { different } \\
\text { subgroups?* }\end{array}$ & $\begin{array}{l}\text { Bias Arising } \\
\text { From Selection } \\
\text { of Reported } \\
\text { Results }\end{array}$ & Comments \\
\hline Endres, 2014 & Yes & Yes & Yes & Low & NA & Probably yes & Low & NA \\
\hline $\begin{array}{l}\text { Jarlenski, 2014; }{ }^{128} \\
\text { Sharma, } 2014^{129} \\
\end{array}$ & Probably no & Yes & Yes & Some concerns & $\begin{array}{l}\text { Self-reported } \\
\text { weight }\end{array}$ & Probably yes & Low & NA \\
\hline Lyu, $2009^{130}$ & Probably no & Yes & Yes & Some concerns & $\begin{array}{l}\text { Self-reported } \\
\text { weight }\end{array}$ & Probably yes & Low & NA \\
\hline $\mathrm{Ng}, 2014^{131}$ & Probably no & Yes & Yes & Some concerns & $\begin{array}{l}\text { Self-reported } \\
\text { weight }\end{array}$ & Probably yes & Low & NA \\
\hline Onyango, $2011^{132}$ & Yes & Yes & Yes & Low & NA & Probably yes & Low & NA \\
\hline Ostbye, $2010^{133}$ & Probably no & Yes & Yes & Some concerns & $\begin{array}{l}\text { Self-reported } \\
\text { weight }\end{array}$ & Probably yes & Low & NA \\
\hline Palmer, 2015 & Probably no & Yes & Yes & Low & $\begin{array}{l}\text { Although weight } \\
\text { was self-reported, } \\
\text { authors noted that } \\
\text { "In a validation } \\
\text { study conducted } \\
\text { among } 115 \\
\text { participants, self- } \\
\text { reported height } \\
\text { and weight were } \\
\text { highly correlated } \\
\text { with technician- } \\
\text { measured height } \\
\text { and weight." }\end{array}$ & Probably yes & Low & NA \\
\hline Straub, 2016 ${ }^{135}$ & Yes & Yes & Yes & Low & NA & Probably yes & Low & NA \\
\hline Stuebe, $2010^{136}$ & No information & Yes & Yes & Low & NA & Probably yes & Low & NA \\
\hline
\end{tabular}

$\mathrm{KQ}=$ Key Question; NA = not applicable. 


\section{Appendix D. Strength of Evidence Tables}

Table D-1. KQ 1a/b: Studies assessing Baby Friendly Hospital Initiative

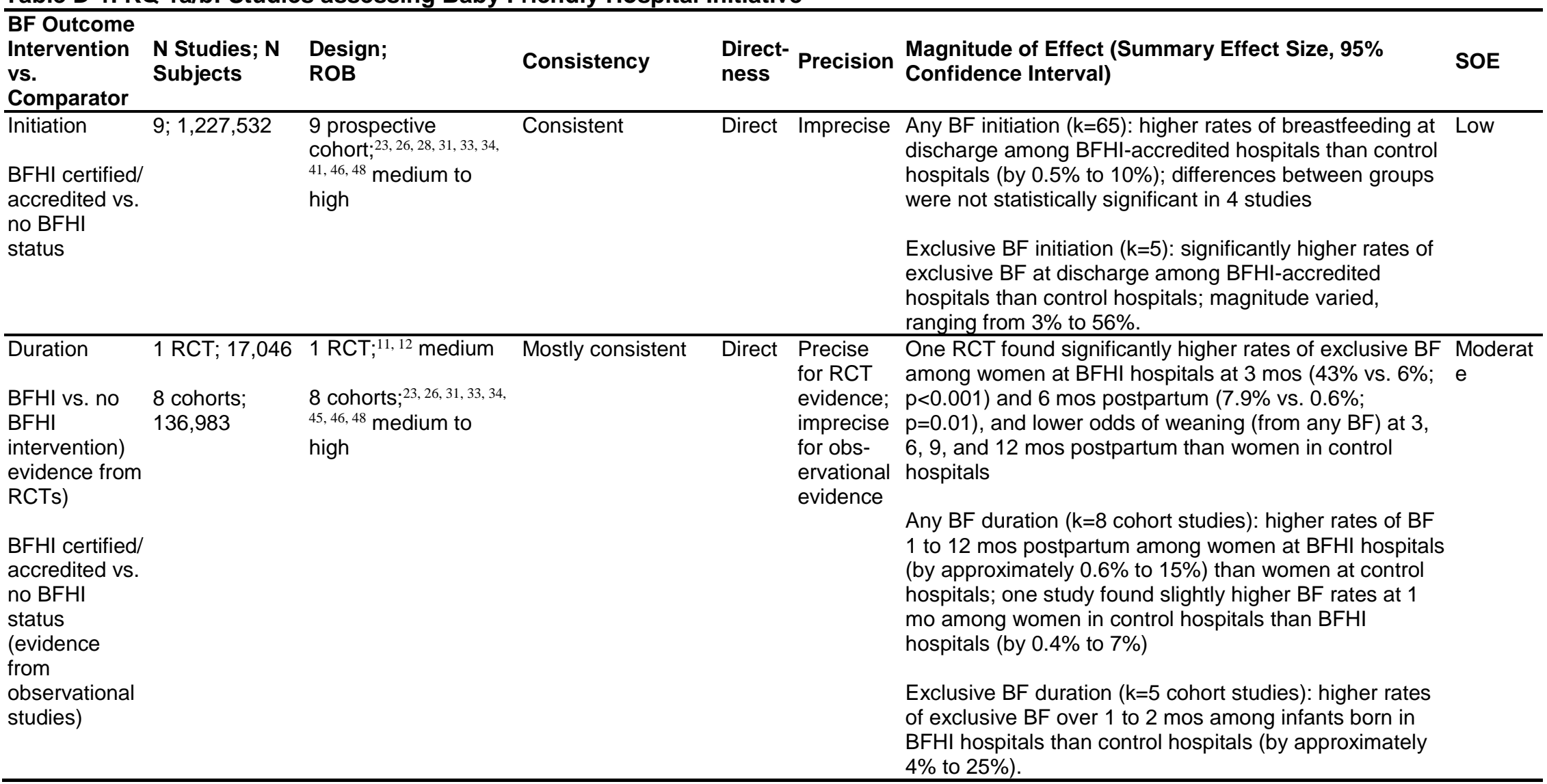




\begin{tabular}{|c|c|c|c|c|c|c|c|}
\hline $\begin{array}{l}\text { Intervention vs. } \\
\text { Comparison } \\
\text { Outcome }\end{array}$ & $\begin{array}{l}\text { N Studies; } \\
\text { N Subjects }\end{array}$ & $\begin{array}{l}\text { Design; } \\
\text { ROB }\end{array}$ & Consistency & $\begin{array}{l}\text { Direct- } \\
\text { ness }\end{array}$ & Precision & $\begin{array}{l}\text { Magnitude of Effect (Summary Effect Size, 95\% } \\
\text { Confidence Interval) }\end{array}$ & SOE \\
\hline $\begin{array}{l}\text { Initiation } \\
\text { (subgroups: } \\
\text { education status) } \\
\text { BFHI certified/ } \\
\text { accredited vs. no } \\
\text { BFHI status }\end{array}$ & $2 ; 27,341$ & $\begin{array}{l}2 \text { prospective } \\
\text { cohorts; }^{33,34} \\
\text { medium }\end{array}$ & Consistent & Direct & Imprecise & $\begin{array}{l}\text { Both studies found higher rates of BF initiation } \\
\text { among women with lower education ( } \leq 12 \text { years) at } \\
\text { BFHI hospitals compared with nonaccredited } \\
\text { hospitals, but no difference in rates among women } \\
\text { with higher education ( } \geq 13 \text { years). }\end{array}$ & Low \\
\hline $\begin{array}{l}\text { Duration } \\
\text { (subgroups: } \\
\text { education status) } \\
\text { BFHI certified/ } \\
\text { accredited vs. no } \\
\text { BFHI status }\end{array}$ & $2 ; 27,341$ & $\begin{array}{l}2 \text { prospective } \\
\text { cohorts; } 33,34 \\
\text { medium }\end{array}$ & Inconsistent & Direct & Imprecise & $\begin{array}{l}\text { One study enrolling women from five U.S. States } \\
\text { found significantly increased rates of exclusive BF } \\
\text { for } \geq 4 \text { weeks among women with lower } \\
\text { educational attainment }(p=0.02) \text {, but no difference } \\
\text { for women with higher education; no difference } \\
\text { was seen for rates of any BF for } \geq 4 \text { weeks by } \\
\text { education. The study evaluating women giving } \\
\text { birth in Maine hospitals found no difference in } \\
\text { rates of exclusive or any breastfeeding for } \geq 4 \\
\text { weeks postpartum among women who differed by } \\
\text { education status. }\end{array}$ & Insufficient \\
\hline $\begin{array}{l}\text { Duration } \\
\text { Six or more BFHI } \\
\text { steps vs. less } \\
\text { than six steps }\end{array}$ & $1 ; 1,417$ & $\begin{array}{l}1 \text { prospective } \\
\text { cohort; } 45 \text { medium }\end{array}$ & Unknown $^{\mathrm{a}}$ & Direct & Precise & $\begin{array}{l}\text { Women giving birth in hospitals practicing } \leq 4 \text { BFHI } \\
\text { steps had higher odds of weaning at or before } 8 \\
\text { weeks postpartum than women giving birth in } \\
\text { facilities practicing six BFHI steps (ORs ranged } \\
\text { from } 2.08 \text { and } 3.13 \text { ); no difference between } \\
\text { women exposed to five vs. six steps. }\end{array}$ & Low \\
\hline
\end{tabular}

${ }^{a}$ Although only one study compared groups of women based on number of BFHI practiced by hospitals, we considered evidence on duration from studies comparing BFHI accreditation with nonaccredited hospitals that reported on duration.

$\mathrm{BF}=$ breastfeeding; $\mathrm{BFHI}=$ Baby Friendly Hospital Initiative; $\mathrm{KQ}=$ Key Question; $\mathrm{N}=$ number; $\mathrm{OR}=$ odds ratio; $\mathrm{RCT}=$ randomized controlled trial; $\mathrm{ROB}=$ risk of bias; $\mathrm{SOE}=$ strength of evidence; U.S.= United States 
Table D-2. KQ 1a/b: Studies assessing Non-Baby Friendly Hospital Initiative health care system-based interventions

\begin{tabular}{|c|c|c|c|c|c|c|c|}
\hline $\begin{array}{l}\text { Intervention vs. } \\
\text { Comparison } \\
\text { (Outcome) }\end{array}$ & $\begin{array}{l}\text { N Studies; N } \\
\text { Subjects }\end{array}$ & $\begin{array}{l}\text { Design; } \\
\text { ROB }\end{array}$ & Consistency & $\begin{array}{l}\text { Direct- } \\
\text { ness }\end{array}$ & Precision & $\begin{array}{l}\text { Magnitude of Effect (Summary Effect Size, } \\
95 \% \text { Confidence Interval) }\end{array}$ & SOE \\
\hline $\begin{array}{l}\text { Initiation } \\
\text { Education/ staff } \\
\text { training related to } \\
\text { BF alone vs. } \\
\text { usual practice } \\
\end{array}$ & $4 ; 1,532^{a}$ & $\begin{array}{l}2 \text { RCTs; } ;, 7,19 \\
\text { medium } \\
2 \text { NRCT; } 30,40 \\
\text { medium to high }\end{array}$ & Consistent & Direct & Imprecise & $\begin{array}{l}\text { Three studies assessing maternity staff or } \\
\text { nursing education/training found no benefit for } \\
\text { rates of BF initiation. One NRCT assessing a } \\
\text { residency BF curriculum found similar increases } \\
\text { in BF initiation rates at intervention and control } \\
\text { programs. }\end{array}$ & Low \\
\hline $\begin{array}{l}\text { Initiation } \\
\text { Education and } \\
\text { staff training plus } \\
\text { additional } \\
\text { individual } \\
\text { services vs. usual } \\
\text { care }\end{array}$ & $4 ; 34,018$ & $\begin{array}{l}2 \text { RCTs; } 9,10 \\
\text { medium } \\
1 \text { NRCT; } ; 2 \text { high } \\
1 \text { pre-post } \\
\text { study; }^{47} \text { medium }\end{array}$ & Inconsistent & Direct & Imprecise & $\begin{array}{l}\text { Two RCTs assessed interventions combining } \\
\text { staff training plus additional BF support services } \\
\text { (expanding access to BF groups, and provision } \\
\text { of additional HVs); neither found benefit. Two } \\
\text { studies assessed staff training additional } \\
\text { individual-level BF counseling and support } \\
\text { services and both found significant benefit in } \\
\text { favor of the intervention. }\end{array}$ & Insufficient \\
\hline $\begin{array}{l}\text { Duration } \\
\text { Education and } \\
\text { staff training } \\
\text { related to BF only } \\
\text { vs. usual practice }\end{array}$ & $3 ; 1,526^{a}$ & $\begin{array}{l}2 \text { RCTs; } 6,7,20 \\
\text { medium } \\
1 \text { NRCT; } 30 \\
\text { medium }\end{array}$ & Inconsistent & Direct & Imprecise & $\begin{array}{l}\text { Two RCTs assessing maternity staff } \\
\text { education/training found inconsistent results: } \\
\text { one found similar durations of any BF in } \\
\text { intervention and control groups ( } 7.5,7.1 \text {, and } \\
7.0 \text { months; } p=N S \text { ) and higher exclusive BF } \\
\text { duration among the intervention vs. one control } \\
\text { group ( } 3.9 \text { vs. } 3.2 \text { months; } p=0.002 \text { ) but not the } \\
\text { other ( } 3.9 \text { vs. } 3.6 \text { months; } p=N S) \text {; the second } \\
\text { RCT found significantly lower rates of weaning } \\
\text { in the intervention group (exclusive BF, full BF } \\
\text { and any BF) than controls). One NRCT of a BF } \\
\text { residency curriculum found higher pre-post } \\
\text { increases in any BF at intervention vs. control } \\
\text { programs at } 6 \text { months. (3.4\% vs. 1.6\%) and } \\
\text { exclusive BF at } 6 \text { months. (6.7\% vs. -5.4\%); no } \\
\text { measure of various provided for difference } \\
\text { between groups. }\end{array}$ & Insufficient \\
\hline
\end{tabular}




\begin{tabular}{|c|c|c|c|c|c|c|c|}
\hline $\begin{array}{l}\text { Intervention vs. } \\
\text { Comparison } \\
\text { (Outcome) }\end{array}$ & $\begin{array}{l}\text { N Studies; N } \\
\text { Subjects }\end{array}$ & $\begin{array}{l}\text { Design; } \\
\text { ROB }\end{array}$ & Consistency & $\begin{array}{l}\text { Direct- } \\
\text { ness }\end{array}$ & Precision & $\begin{array}{l}\text { Magnitude of Effect (Summary Effect Size, } \\
\text { 95\% Confidence Interval) }\end{array}$ & SOE \\
\hline $\begin{array}{l}\text { Duration } \\
\text { Education and } \\
\text { staff training plus } \\
\text { additional } \\
\text { individual } \\
\text { services vs. usual } \\
\text { care }\end{array}$ & $4 ; 21,253$ & $\begin{array}{l}4 \text { RCTs; } 5,9,10,13 \\
\text { medium }\end{array}$ & Inconsistent & Direct & Imprecise & $\begin{array}{l}\text { One RCT found no difference in rates of any or } \\
\text { exclusive BF at } 3 \text { months between the } \\
\text { intervention (health care provider training, } \\
\text { increased individual support: counseling, free } \\
\text { access to lactation consultants) and usual care; } \\
\text { one cluster RCT assessing a policy to provide } \\
\text { BF groups found no difference in rates of any } \\
\text { BF among intervention and control practices } 6 \\
\text { to } 8 \text { weeks and } 8 \text { to } 9 \text { months postpartum. } \\
\text { Two RCTs assessing staff education combined } \\
\text { with expanded or new home visits found } \\
\text { consistent benefit; both provided staff training, } \\
\text { one provided an additional } 1-3 \mathrm{HVs} \text { for BF } \\
\text { support and found a lower rate of BF cessation } \\
\text { rate HR, 0.86; } 95 \% \mathrm{Cl}, 0.75 \text { to } 0.99 \text { ), the other } \\
\text { compared staff training plus } 10 \mathrm{HVs} \text { with staff } \\
\text { training alone and found higher rates of any BF } \\
\text { and exclusive BF over } 6 \text { months postpartum. }\end{array}$ & $\begin{array}{l}\text { Low } \\
\text { (interventions } \\
\text { combining } \\
\text { staff training } \\
\text { and home } \\
\text { visits) } \\
\text { Insufficient } \\
\text { (other } \\
\text { intervention } \\
\text { types) }\end{array}$ \\
\hline $\begin{array}{l}\text { Duration } \\
\text { BFHI adaptation } \\
\text { and } \\
\text { implementation in } \\
\text { antenatal and } \\
\text { child health } \\
\text { (outpatient) } \\
\text { practices vs. } \\
\text { usual care }\end{array}$ & $1 ; 3,948$ & $\begin{array}{l}1 \mathrm{NRCT} ;{ }^{25} \\
\text { medium }\end{array}$ & Unknown & Direct & Precise & $\begin{array}{l}\text { No difference between the intervention and } \\
\text { control group in rates of any } \mathrm{BF} \text { at } 6 \text { months } \\
(\mathrm{OR}, 1.24 ; 95 \% \mathrm{Cl}, 0.99 \text { to } 1.54) \text { or at } 12 \\
\text { months }(\mathrm{p}=0.43) \text {. Rates of exclusive } \mathrm{BF} \text { were } \\
\text { higher in the intervention group than controls at } \\
5 \text { months (OR, } 1.39 ; 95 \% \mathrm{Cl}, 1.09 \text { to } 1.77) \text { and } \\
\text { at } 6 \text { months (OR, } 1.33 ; 95 \% \mathrm{Cl}, 1.03 \text { to } 1.72) \text {. }\end{array}$ & Low \\
\hline
\end{tabular}




\begin{tabular}{|c|c|c|c|c|c|c|}
\hline $\begin{array}{l}\text { Intervention vs. } \\
\text { Comparison } \\
\text { (Outcome) }\end{array}$ & $\begin{array}{l}\text { N Studies; N } \\
\text { Subjects }\end{array}$ & $\begin{array}{l}\text { Design; } \\
\text { ROB }\end{array}$ & $\begin{array}{l}\text { Direct- } \\
\text { ness }\end{array}$ & Precision & $\begin{array}{l}\text { Magnitude of Effect (Summary Effect Size, } \\
95 \% \text { Confidence Interval) }\end{array}$ & SOE \\
\hline $\begin{array}{l}\text { Initiation/ } \\
\text { Duration } \\
\text { Continuous } \\
\text { primary nursing } \\
\text { care (same nurse } \\
\text { through perinatal } \\
\text { period for } \\
\text { mother/infant) vs. } \\
\text { usual care (task } \\
\text { oriented nursing) } \\
\end{array}$ & $1 ; 470$ & $1 \mathrm{RCT} ;{ }^{21}$ medium Unknown & Direct & Precise & $\begin{array}{l}\text { Women in the intervention group had higher } \\
\text { rates of exclusive BF during hospitalization than } \\
\text { women in the control group ( } 99 \% \text { vs. } 88 \% \text {; } \\
p=0.001 \text { ) and higher rates of exclusive BF } 6 \\
\text { weeks than women in the control group ( } 72 \% \\
\text { vs. } 94 \% ; p=0.001) \text {. }\end{array}$ & Low \\
\hline
\end{tabular}


Table D-3. KQ 1a/b: Studies assessing Women, Infants and Children-based interventions

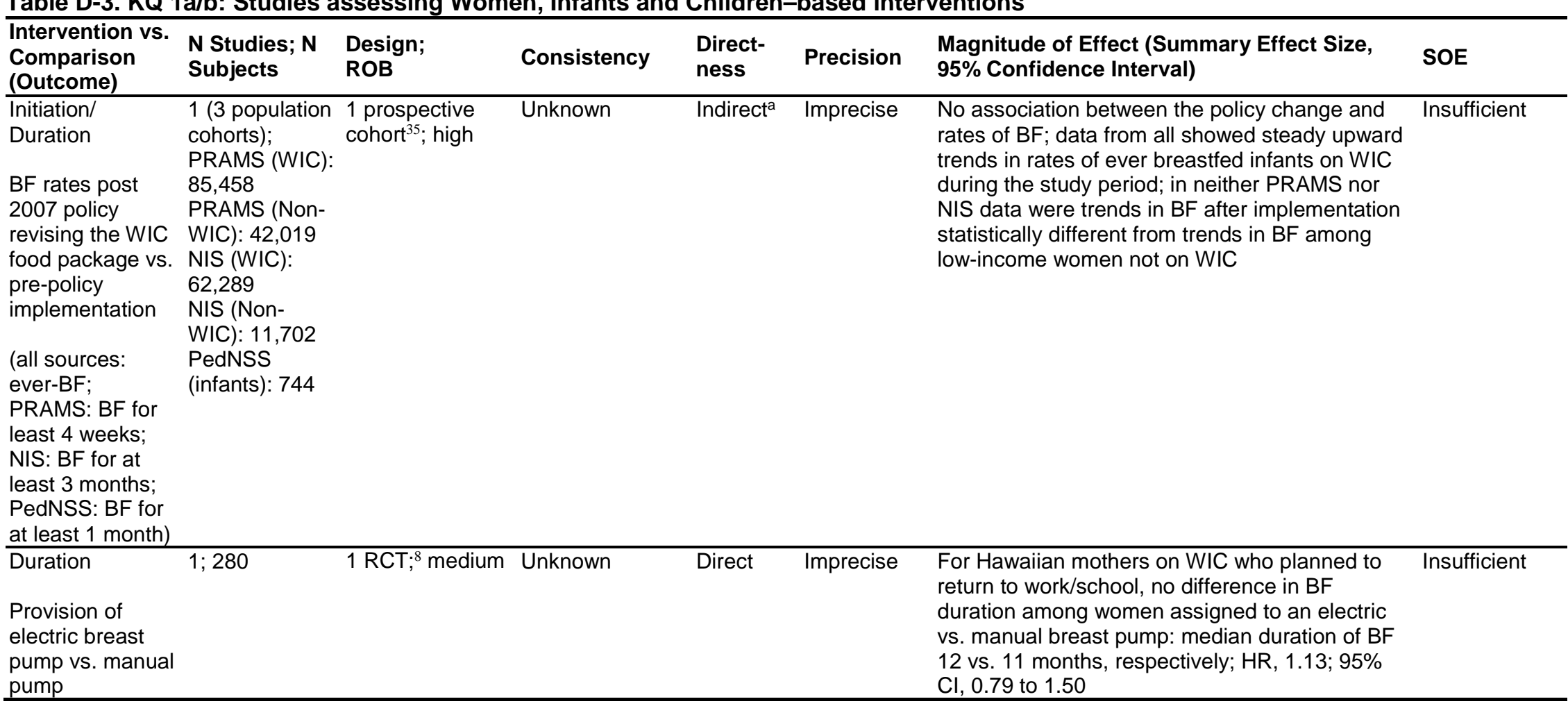




\begin{tabular}{|c|c|c|c|c|c|c|c|}
\hline $\begin{array}{l}\text { Intervention vs. } \\
\text { Comparison } \\
\text { (Outcome) }\end{array}$ & $\begin{array}{l}\text { N Studies; N } \\
\text { Subjects }\end{array}$ & $\begin{array}{l}\text { Design; } \\
\text { ROB }\end{array}$ & Consistency & $\begin{array}{l}\text { Direct- } \\
\text { ness }\end{array}$ & Precision & $\begin{array}{l}\text { Magnitude of Effect (Summary Effect Size, } \\
95 \% \text { Confidence Interval) }\end{array}$ & SOE \\
\hline $\begin{array}{l}\text { Initiation/ } \\
\text { Duration } \\
\text { Mother peer } \\
\text { support vs. } \\
\text { control }\end{array}$ & $3 ; 2,480$ & $\begin{array}{l}1 \mathrm{RCT} ;{ }^{18} \text { medium } \\
1 \mathrm{NRCT} ;{ }^{43} \text { high } \\
1 \text { cohort; }{ }^{44} \\
\text { medium }\end{array}$ & 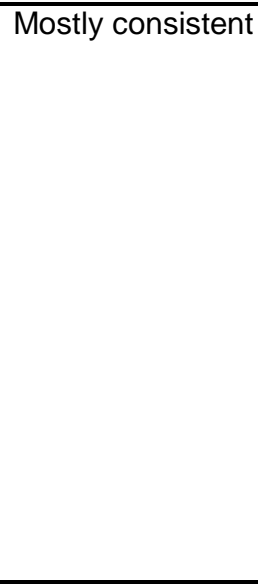 & Direct & Precise & $\begin{array}{l}\text { Two studies of in-person peer support for } \\
\text { mothers found significant benefit; in one, women } \\
\text { in the intervention group had a higher rate of BF } \\
\text { initiation than controls ( } 82 \% \text { vs. } 31 \%) \text { and a } \\
\text { higher rate of any BF at } 12 \text { weeks ( } 43 \% \text { vs. } 0 \% \text {; } \\
\text { p<0.001). The other RCT found higher rates of } \\
\text { BF at hospital discharge in the intervention group } \\
\text { than controls ( } 44 \% \text { vs. } 26 \%, p<0.01 \text { ) and higher } \\
\text { rates of any BF at } 6 \text { weeks ( } 26 \% \text { vs. } 13 \% \text {, } \\
\text { p<0.01). One RCT comparing telephone BF } \\
\text { support, there was no difference between groups } \\
\text { in BF initiation rates and rates of exclusive BF } \\
\text { rates at } 3 \text { and } 6 \text { months postpartum; rates of any } \\
\text { BF were higher in the intervention group than } \\
\text { controls at } 3 \text { and } 6 \text { months (RR, } 1.18 ; 95 \% \mathrm{Cl} \text {, } \\
1.03 \text { to } 1.34 \text { ) }\end{array}$ & Low \\
\hline $\begin{array}{l}\text { Initiation/ } \\
\text { Duration } \\
\text { (subgroups: } \\
\text { language } \\
\text { spoken) } \\
\text { Mother peer } \\
\text { support vs. } \\
\text { control }\end{array}$ & $1 ; 1,948$ & $1 \mathrm{RCT} ;{ }^{18}$ Medium & Unknown & Direct & Imprecise & $\begin{array}{l}\text { One RCT (telephone peer support) reported on } \\
\text { subgroups of women defined by language } \\
\text { (English-speaking vs. Spanish-speaking only), } \\
\text { results for any BF at } 3 \text { months were significant for } \\
\text { both groups; at } 6 \text { months, benefit for any BF was } \\
\text { significant only for the Spanish-speaking } \\
\text { subgroup. There was no significant difference in } \\
\text { rates of exclusive BF in either subgroup at } 3 \text { or } 6 \\
\text { months. }\end{array}$ & Insufficient \\
\hline
\end{tabular}




\begin{tabular}{|c|c|c|c|c|c|c|c|}
\hline $\begin{array}{l}\text { Intervention vs. } \\
\text { Comparison } \\
\text { (Outcome) }\end{array}$ & $\begin{array}{l}\text { N Studies; N } \\
\text { Subjects }\end{array}$ & $\begin{array}{l}\text { Design; } \\
\text { ROB }\end{array}$ & Consistency & $\begin{array}{l}\text { Direct- } \\
\text { ness }\end{array}$ & Precision & $\begin{array}{l}\text { Magnitude of Effect (Summary Effect Size, } \\
95 \% \text { Confidence Interval) }\end{array}$ & SOE \\
\hline $\begin{array}{l}\text { Initiation/ } \\
\text { Duration } \\
\text { Peer support } \\
\text { program for } \\
\text { fathers (in } \\
\text { addition to } \\
\text { mother peer } \\
\text { support) vs. peer } \\
\text { support for } \\
\text { mothers alone }\end{array}$ & $1 ; 200$ & $\begin{array}{l}1 \mathrm{NRCT} ;{ }^{36} \\
\text { medium }\end{array}$ & Unknown & Direct & Imprecise & $\begin{array}{l}\text { Mothers in the intervention group had slightly } \\
\text { higher rate of any BF at } 6 \text { months, but the } \\
\text { difference was not statistically significant ( } 63 \% \\
\text { vs. } 55 \% ; p=0.20) \text {. }\end{array}$ & Insufficient \\
\hline $\begin{array}{l}\text { Duration } \\
\text { Cash incentives } \\
\text { vs. usual WIC } \\
\text { services }\end{array}$ & $1: 36$ & $1 \mathrm{RCT} ; 22$ Medium & Unknown & Direct & Precise & $\begin{array}{l}\text { Breastfeeding rates in the intervention group } \\
\text { were significantly higher than controls at } 1,3 \text {, and } \\
6 \text { months ( } 89 \% \text { vs. } 44 \%, 89 \% \text { vs. } 17 \% \text {, and } 72 \% \\
\text { vs. } 0 \% \text {, respectively) }\end{array}$ & Insufficient \\
\hline $\begin{array}{l}\text { Duration } \\
\text { Tailored BF } \\
\text { counseling and } \\
\text { support based on } \\
\text { BAPT survey } \\
\end{array}$ & $1 ; 826$ & 1 cohort; ${ }^{29}$ High & Unknown & Direct & Imprecise & $\begin{array}{l}\text { Significantly higher rates of exclusive BF in the } \\
\text { intervention group at } 7 \text { and } 30 \text { days than controls; } \\
\text { no difference between groups at } 2 \text { months }\end{array}$ & Insufficient \\
\hline $\begin{array}{l}\text { Duration } \\
\text { (subgroups: } \\
\text { race/ethnicity) } \\
\text { Tailored BF } \\
\text { counseling and } \\
\text { support based on } \\
\text { BAPT survey }\end{array}$ & $1 ; 826$ & 1 cohort; ${ }^{29}$ High & Unknown & Direct & Imprecise & $\begin{array}{l}\text { Significantly higher rates of exclusive BF among } \\
\text { non-Hispanic black and Hispanic women in the } \\
\text { intervention group than controls at } 30 \text { and } 60 \\
\text { days; no significant difference in exclusive BF } \\
\text { rates among white women at any timepoint }\end{array}$ & Insufficient \\
\hline
\end{tabular}

${ }^{a}$ Databases not designed to collect information on breastfeeding status; unclear to what extent data reflects true rate of breastfeeding in women enrolled in WIC and those not enrolled who are similar in socioeconomic status.

BAPT = Breastfeeding Attrition Prediction Tool; BF = breastfeeding; CI = confidence interval; HR = hazard ratio; HV = home visit; KQ = Key Question; N = number; NIS = National Immunization Survey; NRCT = nonrandomized controlled trial; PedNSS = Pediatric Nutrition Surveillance System; PRAMS = Pregnancy Risk Assessment Monitoring System; RCT = randomized controlled trial; ROB = risk of bias; RR = risk ratio; SOE = strength of evidence; U.S.= United States; WIC = Special Supplemental Nutrition Program for Women, Infants, and Children. 
Table D-4. KQ 1a/b: Studies assessing community-based interventions

\begin{tabular}{|c|c|c|c|c|c|c|c|}
\hline $\begin{array}{l}\text { Intervention vs. } \\
\text { Comparison } \\
\text { (Outcome) }\end{array}$ & $\begin{array}{l}\text { N Studies; N } \\
\text { Subjects }\end{array}$ & $\begin{array}{l}\text { Design; } \\
\text { ROB }\end{array}$ & Consistency & $\begin{array}{l}\text { Direct- } \\
\text { ness }\end{array}$ & Precision & $\begin{array}{l}\text { Magnitude of Effect (Summary Effect Size, } \\
95 \% \text { Confidence Interval) }\end{array}$ & SOE \\
\hline $\begin{array}{l}\text { Initiation/ Duration } \\
\text { Community-based } \\
\text { policy aimed at } \\
\text { promoting BF in } \\
\text { nonhospital-based } \\
\text { health and } \\
\text { community centers } \\
\text { vs. no intervention }\end{array}$ & $1 ; 5,094$ & $\begin{array}{l}1 \mathrm{NRCT} ; 38,39 \\
\text { medium }\end{array}$ & Unknown & Direct & Imprecise & $\begin{array}{l}\text { The rate of exclusive BF at discharge, } 3 \text { and } 6 \\
\text { months, and of any BF at } 5 \text { and } 12 \text { months } \\
\text { increased during the study period in both groups } \\
\text { (with no significant differences between groups). }\end{array}$ & Insufficient \\
\hline $\begin{array}{l}\text { Duration } \\
\text { Access to } \\
\text { community-based } \\
\text { BF drop-in centers } \\
\text { (plus early BF } \\
\text { support) vs. early } \\
\text { BF support alone vs. } \\
\text { usual care }\end{array}$ & $1 ; 9,675$ & $\begin{array}{l}\text { 1 RCT; } 14,15 \\
\text { low }\end{array}$ & Unknown & Direct & Precise & $\begin{array}{l}\text { No difference between groups in rates of any } \\
\text { breast milk feeding at } 3,4 \text {, or } 5 \text { months. }\end{array}$ & Low \\
\hline $\begin{array}{l}\text { Duration } \\
\text { Community-based } \\
\text { peer support vs. } \\
\text { usual care }\end{array}$ & $1 ; 130$ & 1 RCT; 16 low & Unknown & Direct & Precise & $\begin{array}{l}\text { More women in the intervention groups were } \\
\text { exclusively BF than controls at } 3 \text { months } \\
\text { postpartum ( } 67 \% \text { of the } 6 \text {-visit group, } 50 \% \text { of } \\
\text { the } 3 \text {-visit group, vs. } 12 \% \text { of controls; } p<0.001 \text { ); } \\
\text { rates of any BF were significantly longer in } \\
\text { intervention groups (combined) than in the } \\
\text { control group at } 3 \text { months, but not } 6 \text { months. }\end{array}$ & Low \\
\hline $\begin{array}{l}\text { Duration } \\
\text { Peer-led BF support } \\
\text { class vs. nurse-led } \\
\text { BF support class }\end{array}$ & $1 ; 109$ & $\begin{array}{l}1 \\
\text { prospective } \\
\text { cohort; } 42 \\
\text { high }\end{array}$ & Unknown & Direct & Imprecise & $\begin{array}{l}\text { No significant difference between groups in } \\
\text { rates of any breastfeeding at } 1 \text { and } 6 \text { months } \\
\text { postpartum. }\end{array}$ & Insufficient \\
\hline $\begin{array}{l}\text { Duration } \\
\text { Integrated } \\
\text { postpartum program } \\
\text { (BF education and } \\
\text { support, maternal/ } \\
\text { infant health care) } \\
\text { vs. usual care }\end{array}$ & $1 ; 392$ & $\begin{array}{l}1 \mathrm{NRCT} ;{ }^{24} \\
\text { high }\end{array}$ & Unknown & Direct & Precise & $\begin{array}{l}\text { Significantly higher rates of exclusive BF at } 6 \\
\text { mos among the intervention group than control } \\
\text { group ( } 74 \% \text { vs. } 10 \% ; p=0.001)\end{array}$ & Insufficient \\
\hline
\end{tabular}

vs. usual care

BF = breastfeeding; KQ = Key Question; N = number; NRCT = nonrandomized controlled trial; RCT = randomized controlled trial; SOE = strength of evidence. 
Table D-5. SOE for KQ 2 summarized by outcome

\begin{tabular}{|c|c|c|c|c|c|c|c|}
\hline Outcome & $\begin{array}{l}\text { N Studies; N } \\
\text { Subjects }\end{array}$ & $\begin{array}{l}\text { Design; } \\
\text { Risk of Bias }\end{array}$ & Consistency & Directness $^{a}$ & Precision & $\begin{array}{l}\text { Magnitude of effect (Summary Effect } \\
\text { Size, } 95 \% \text { Confidence Interval) }\end{array}$ & SOE \\
\hline Breast cancer & $\begin{array}{l}1 \text { SR of } 98 \text { studies; }{ }^{2} \\
\text { NR }^{\mathrm{b}} \\
19 \text { individual } \\
\text { studies; } 66,67,69-74,77-80 \text {, } \\
\text { 83-85, } 87-90256,891 \\
\text { women }\end{array}$ & $\begin{array}{l}\text { Cohort and case- } \\
\text { control; high to } \\
\text { medium }\end{array}$ & $\begin{array}{l}\text { Mostly } \\
\text { consistent }\end{array}$ & Indirect & Imprecise & $\begin{array}{l}\text { In one SR (k=98), ever BF was } \\
\text { associated with lower rates of breast } \\
\text { cancer compared with never BF (pooled } \\
\text { OR 0.78, 95\% CI 0.74 to 0.82); women } \\
\text { who BF for longer durations also had } \\
\text { significantly lower rates of breast cancer } \\
\text { than women who did not BF. Results of } \\
\text { individual studies were generally } \\
\text { consistent in direction of effect (although } \\
\text { results were imprecise); magnitude varied } \\
\text { significantly across all studies and pooled } \\
\text { results were associated with significant } \\
\text { heterogeneity, only partially explained by } \\
\text { subgroup analyses. }\end{array}$ & Low \\
\hline $\begin{array}{l}\text { Breast } \\
\text { Cancer: } \\
\text { BRCA1/2 } \\
\text { Carriers }\end{array}$ & $1 ; 76$ 5,708 women & $\begin{array}{l}\text { Case-control; } \\
\text { medium }\end{array}$ & Unknown & Indirect & Imprecise & $\begin{array}{l}\text { Association between BF duration and } \\
\text { lower rates of breast cancer among } \\
B R C A 1 \text { mutation carriers ( } p \text { for } \\
\text { trend<0.0001), but not } B R C A 2 \text { mutation } \\
\text { carriers ( } p \text { for trend }=0.68) \text {. }\end{array}$ & Insufficient \\
\hline $\begin{array}{l}\text { Breast cancer: } \\
\text { In situ }\end{array}$ & $\begin{array}{l}3 ; 75,83,8767,234 \\
\text { women }\end{array}$ & $\begin{array}{l}1 \text { cohort and } 2 \\
\text { case-control; } \\
\text { medium }\end{array}$ & Inconsistent & Indirect & Imprecise & $\begin{array}{l}\text { One case-control study found no } \\
\text { association between BF and DCIS and } \\
\text { the other found no association between } \\
\text { BF duration and diagnosis of DCIS or } \\
\text { invasive ductal carcinoma; one cohort } \\
\text { study found no association between BF } \\
\text { duration and incident DCIS. }\end{array}$ & Insufficient \\
\hline $\begin{array}{l}\text { Breast cancer: } \\
\text { Hormone } \\
\text { receptor } \\
\text { subtypes }\end{array}$ & $\begin{array}{l}\text { 1 SR of } 11 \text { studies; }{ }^{58} \\
169,879 \text { women for } \\
\text { luminal, 14,266 } \\
\text { women for HER2, } \\
\text { and } 176,430 \text { women } \\
\text { for triple-negative } \\
\text { analyses } \\
7 \text { individual studies; } 68 \text {, } \\
74,80,81,86,90,91592,558 \\
\text { women }\end{array}$ & $\begin{array}{l}\text { Cohort and case- } \\
\text { control; high to } \\
\text { medium }^{c}\end{array}$ & $\begin{array}{l}\text { Luminal, triple- } \\
\text { negative: } \\
\text { consistent } \\
\text { HER2: } \\
\text { inconsistent }\end{array}$ & Indirect & Imprecise & $\begin{array}{l}\text { Consistent association between ever BF } \\
\text { or longer duration of BF and lower rates } \\
\text { of luminal and triple- negative breast } \\
\text { cancer (although magnitude of } \\
\text { association varies); for HER2, pooled } \\
\text { estimates show unclear association } \\
\text { between BF and lower rates of breast } \\
\text { cancer (results are imprecise and pooled } \\
\text { estimate is not statistically significant) }\end{array}$ & $\begin{array}{l}\text { Low } \\
\text { (luminal, } \\
\text { triple- } \\
\text { negative; } \\
\text { inconsistent, } \\
\text { consistent, } \\
\text { imprecise); } \\
\text { Insufficient } \\
\text { (HER2, } \\
\text { inconsistent, } \\
\text { imprecise) } \\
\end{array}$ \\
\hline $\begin{array}{l}\text { Breast } \\
\text { Cancer: } \\
\text { Mortality }\end{array}$ & $\begin{array}{l}1 ; 82250,470 \text { parous } \\
\text { women }\end{array}$ & Cohort; medium & Unknown & Indirect & Imprecise & $\mathrm{HR}=1.01(95 \% \mathrm{Cl}: 0.79$ to 1.29$)$ & Insufficient \\
\hline
\end{tabular}




\begin{tabular}{|c|c|c|c|c|c|c|c|}
\hline Outcome & $\begin{array}{l}\text { N Studies; N } \\
\text { Subjects }\end{array}$ & $\begin{array}{l}\text { Design; } \\
\text { Risk of Bias }\end{array}$ & Consistency & Directness $^{a}$ & Precision & $\begin{array}{l}\text { Magnitude of effect (Summary Effect } \\
\text { Size, } 95 \% \text { Confidence Interval) }\end{array}$ & SOE \\
\hline $\begin{array}{l}\text { Ovarian } \\
\text { cancer }\end{array}$ & $\begin{array}{l}1 \text { SR of } 41 \text { studies; }^{2} \\
\text { NR }^{\mathrm{e}} \\
9 \text { individual studies; }{ }^{92-} \\
97,137-14042,611 \\
\text { women }\end{array}$ & $\begin{array}{l}\text { Cohort and case- } \\
\text { control; high to } \\
\text { medium }\end{array}$ & Consistent & Indirect & Precise & $\begin{array}{l}\text { Results from included systematic review: } \\
\text { Ever breastfed } \\
\text { Pooled OR }=0.70 \text { ( } 95 \% \mathrm{Cl}: 0.64 \text { to } 0.77) \text {, } \\
\mathrm{I}^{2}=70 \\
<6 \text { months } \\
\text { Pooled OR }=0.83(95 \% \mathrm{Cl}: 0.78 \text { to } 0.89) \text {, } \\
\mathrm{I}^{2}=3 \\
6-12 \text { months } \\
\text { Pooled OR }=0.72(95 \% \mathrm{Cl}: 0.66 \text { to } 0.78) \text {, } \\
\mathrm{I}^{2}=22 \\
>12 \text { months } \\
\text { Pooled OR }=0.63(95 \% \mathrm{Cl}: 0.56 \text { to } 0.71) \text {, } \\
\mathrm{I}^{2}=52 \text {. }\end{array}$ & Moderate \\
\hline Hypertension & $\begin{array}{l}5 ; 3,99,100,104,141 \\
441,989 \text { women }\end{array}$ & $\begin{array}{l}\text { Cohort; medium } \\
\text { to high }\end{array}$ & Consistent & Indirect & Imprecise & $\begin{array}{l}\text { Consistent association between longer } \\
\text { duration of BF ( }>6-12 \text { months) and lower } \\
\text { rates HTN; magnitude of association } \\
\text { varies by BF exposure comparisons and } \\
\text { study design. }\end{array}$ & Low \\
\hline CVD & $\begin{array}{l}3 ; 3,102,103301,989 \\
\text { women }\end{array}$ & Cohort; medium & Unknown & Indirect & Imprecise & $\begin{array}{l}\text { Three studies conclude an association } \\
\text { between longer BF duration and lower } \\
\text { CVD rates, each using a different } \\
\text { composite outcome; magnitude of } \\
\text { association varies by exposure } \\
\text { comparisons, age at cohort enrolment, } \\
\text { and study design }\end{array}$ & Insufficient \\
\hline CVD mortality & $1 ; 10115,000$ & Cohort; medium & Unknown & Indirect & Imprecise & $\begin{array}{l}\text { Parous women } \leq 65 \text { years at enrollment } \\
\text { who had never lactated had higher CVD } \\
\text { mortality over } 14 \text { years of followup than } \\
\text { women who lactated } \geq 24 \text { months (HR } \\
2.77 ; 95 \% \mathrm{Cl}, 1.28 \text { to } 5.99 \text { ). No clear } \\
\text { associations were observed among } \\
\text { women } \leq 65 \text { years at enrollment. }\end{array}$ & Insufficient \\
\hline $\begin{array}{l}\text { Type } 2 \\
\text { diabetes }\end{array}$ & $\begin{array}{l}1 \text { SR of } 6 \text { studies; } 50 \\
273,961 \text { women } \\
5 \text { individual studies; } \\
105-109325,815 \text { women }\end{array}$ & Cohort: medium & Consistent & Indirect & Imprecise & $\begin{array}{l}\text { Consistent association between ever BF } \\
\text { and longer durations of BF and lower } \\
\text { rates of type } 2 \text { diabetes (among women } \\
\text { with and without gestational diabetes); } \\
\text { magnitude of association varies by BF } \\
\text { exposure duration and study design. }\end{array}$ & Low \\
\hline
\end{tabular}




\begin{tabular}{|c|c|c|c|c|c|c|c|}
\hline Outcome & $\begin{array}{l}\text { N Studies; N } \\
\text { Subjects }\end{array}$ & $\begin{array}{l}\text { Design; } \\
\text { Risk of Bias }\end{array}$ & Consistency & Directness $^{a}$ & Precision & $\begin{array}{l}\text { Magnitude of effect (Summary } \\
\text { Effect Size, 95\% Confidence } \\
\text { Interval) }\end{array}$ & SOE \\
\hline Fractures & $\begin{array}{l}11 ;, 110-114,142-147 \\
101,726 \text { women }\end{array}$ & $\begin{array}{l}6 \text { case-control and } 5 \\
\text { cohort studies; } \\
\text { medium to high }\end{array}$ & Consistent & Indirect & Imprecise & $\begin{array}{l}\text { Magnitude varies by exposure and } \\
\text { outcome measure }\end{array}$ & Low \\
\hline $\begin{array}{l}\text { Postpartum } \\
\text { depression }\end{array}$ & $\begin{array}{l}\text { 1 SR of } 48 \text { studies; }{ }^{52} \\
71,245 \text { women } \\
14 \text { individual } \\
\text { studies; } 59,115,117-126,148 \text {, } \\
14939,372 \text { women }\end{array}$ & $\begin{array}{l}\text { Cohort; high to } \\
\text { medium }\end{array}$ & Inconsistent & Indirect & Imprecise & $\begin{array}{l}\text { Magnitude of association and } \\
\text { direction of effect unclear; studies are } \\
\text { heterogeneous in design and results } \\
\text { inconsistent. }\end{array}$ & Insufficient \\
\hline $\begin{array}{l}\text { Postpartum } \\
\text { weight change }\end{array}$ & $\begin{array}{l}\text { 16;'127-136, 150-158 47,655 } \\
\text { women }\end{array}$ & $\begin{array}{l}\text { Prospective cohort } \\
\text { studies; medium }\end{array}$ & Inconsistent & Indirect & Imprecise & $\begin{array}{l}\text { Magnitude varies by exposure and } \\
\text { outcome measure }\end{array}$ & Insufficient \\
\hline
\end{tabular}

a We marked outcomes as indirect for long-term maternal health outcomes primarily due to uncertainty of the relative contribution of breastfeeding to risk (given that many other potential factors also contribute to outcomes such as hypertension, fracture and breast cancer); for short-term maternal health outcomes (e.g., postpartum depression) there is uncertainty in the direction of effect between breastfeeding and health outcomes.

b Per authors, there were 52 studies with $>1,500$ women, 31 studies with 500-1,499 women, and 15 studies with $<500$ women. Exact number of participants is unclear.

c The systematic review was rated unclear risk of bias and did not provide quality ratings for the included studies. Of the four additional studies, 3 were rated medium risk of bias and one was rated high risk of bias.

$\stackrel{\mathrm{d}}{\mathrm{d}}$ Results from 4 additional individual studies were generally consistent in direction and magnitude of effect.

$\sim \quad$ e There were 22 studies with $>1,500$ women, 12 studies with 500-1,499 women, and 7 studies with $<500$ women.

$\mathrm{BF}=$ breastfeeding; $B R C A=$ breast cancer gene; $\mathrm{CI}=$ confidence interval; $\mathrm{CVD}=$ cardiovascular disease; DCIS = ductal carcinoma in situ; HER2 = human epidermal growth factor receptor 2; HR = hazard ratio; HTN = hypertension; KQ = Key Question; N = number; NR = not reported; OR = odds ratio; $\mathrm{SOE}=$ strength of evidence; SR = systematic review. 


\section{Appendix E. Excluded Studies}

Exclusion codes:

$\mathrm{X} 1$ : Not original research

X2: Wrong population

$\mathrm{X} 3$ : Wrong exposure or intervention

$\mathrm{X} 4$ : Wrong or no comparator

X5: Wrong or no outcome

X6: Wrong study design

X7: Non-English

X8: Wrong country

X9: Study protocol only

X10: Superseded by an included systematic review

$\mathrm{X} 11$ : Irrelevant or high risk of bias systematic review

1. Independent study module for lactation consultants. J Hum Lact. 2012;28(2):251-3. doi: 10.1177/0890334412436722. PMID: 104555631. Exclusion Code: X1.

2. 04 'Who Loves Their Wives and Children More?': Vietnamese Fathers' Support Improves Early and Exclusive Breastfeeding. J Nutr Educ Behav. 2012;44(4S1):S14-S. PMID: 108143853. Exclusion Code: X9.

3. Counselling sessions increased duration of exclusive breastfeeding: a randomized clinical trial with adolescent mothers and grandmothers. Essentially MIDIRS. 2014;5(10):47-. PMID: 103915666. Exclusion Code: X3.

4. Research evidence. Community Practitioner. 2014;87(10):12-. PMID: 103806569. Exclusion Code: X2.

5. Evaluation of the Healthy Start Voucher Scheme in UK: a natural experiment using the Growing Up in Scotland record linkage study and the Infant Feeding Survey (Project record). Health Technology Assessment Database: Health Technology Assessment; 2015. Exclusion Code: X6.

7. Abbass-Dick J, Xie F, Koroluk J, et al. The Development and piloting of an eHealth breastfeeding resource targeting fathers and partners as co-parents. Midwifery. 2017;50:139-47. doi: 10.1016/j.midw.2017.04.004. PMID: 123257423. Exclusion Code: X3.

8. Abdulwadud OA, Snow ME. Interventions in the workplace to support breastfeeding for women in employment. Cochrane Database Syst Rev. 2012 Oct 17;10:Cd006177. doi: 10.1002/14651858.CD006177.pub3. PMID: 23076920. Exclusion Code: X5.

9. Abrahams RR, MacKay-Dunn MH, Nevmerjitskaia V, et al. An evaluation of rooming-in among substance-exposed newborns in British Columbia. J Obstet Gynaecol Can. 2010 Sep;32(9):866-71. PMID: 21050520. Exclusion Code: X3.

10. Adams IK, Okoli CT, Dulin Keita A, et al. Breastfeeding Practices among Native Hawaiians and Pacific Islanders. J Obes. 2016;2016:2489021. doi: 10.1155/2016/2489021. PMID: 27774314. Exclusion Code: X3.

6. Cochrane Pregnancy and Childbirth Group. About The Cochrane Collaboration: John Wiley \& Sons, Ltd; 2016. Exclusion Code: $\mathrm{X} 1$.

\footnotetext{
Adedinsewo DA, Fleming AS, Steiner M, et al. Maternal anxiety and breastfeeding: findings from the MAVAN (Maternal Adversity, Vulnerability and Neurodevelopment) Study. J Hum Lact. 2014 Feb;30(1):102-9. doi: 10.1177/0890334413504244. PMID: 24065719. Exclusion Code: X3.
} 
12. Adolfsson A, Linden K, Sparud-Lundin C, et al. A web-based support for pregnant women and new mothers with type 1 diabetes mellitus in Sweden (MODIABWeb): study protocol for a randomized controlled trial. Trials. 2014 Dec 29;15:513. doi: 10.1186/1745-6215-15-513. PMID: 25543854. Exclusion Code: X3.

13. Agampodi SB, Agampodi TC. Effect of low cost public health staff training on exclusive breastfeeding. Indian J Pediatr. 2008 Nov;75(11):1115-9. doi: 10.1007/s12098008-0185-4. PMID: 18810343. Exclusion Code: X6.

14. Aghdas K, Talat K, Sepideh B. Effect of immediate and continuous mother-infant skin-to-skin contact on breastfeeding selfefficacy of primiparous women: a randomised control trial. Women Birth. 2014 Mar;27(1):37-40. doi: 10.1016/j.wombi.2013.09.004. PMID: 24216342. Exclusion Code: X3.

15. Ahlqvist-Bjorkroth S, Vaarno J, Junttila N, et al. Initiation and exclusivity of breastfeeding: association with mothers' and fathers' prenatal and postnatal depression and marital distress. Acta Obstet Gynecol Scand. 2016 Apr;95(4):396-404. doi: 10.1111/aogs.12857. PMID: 26826608. Exclusion Code: X3.

16. Ahluwalia IB, Tessaro I, Grummer-Strawn LM, et al. Georgia's breastfeeding promotion program for low-income women. Pediatrics. 2000 Jun;105(6):E85. PMID: 10835098. Exclusion Code: X6.

17. Ahmed A, Ouzzani M. Development and assessment of an interactive web-based breastfeeding monitoring system (LACTOR). Matern Child Health J. 2013 Jul;17(5):809-15. doi: 10.1007/s10995-0121074-z. PMID: 22791207. Exclusion Code: X3.

18. Ahmed AH, Roumani AM, Szucs K, et al. The Effect of Interactive Web-Based Monitoring on Breastfeeding Exclusivity, Intensity, and Duration in Healthy, Term Infants After Hospital Discharge. J Obstet Gynecol Neonatal Nurs. 2016 MarApr;45(2):143-54. doi: 10.1016/j.jogn.2015.12.001. PMID: 26779838. Exclusion Code: X3.
19. Ahnfeldt AM, Stanchev H, Jorgensen HL, et al. Age and weight at final discharge from an early discharge programme for stable but tube-fed preterm infants. Acta Paediatr. 2015 Apr;104(4):377-83. doi: 10.1111/apa.12917. PMID: 25545824. Exclusion Code: X3.

20. Ajmera V, Terrault N, VanWagner L, et al. Longer lactation duration is associated with decreased non-alcoholic fatty liver disease in the cardia cohort study. Hepatology. Conference: 67th annual meeting of the american association for the study of liver diseases: the liver meeting 2016. United states. Conference start: 20161111. Conference end: 20161115; 2017. p. 590a. Exclusion Code: X5.

21. Akhter M, Inoue M, Kurahashi N, et al. Reproductive factors, exogenous female hormone use and colorectal cancer risk: the Japan Public Health Center-based Prospective Study. Eur J Cancer Prev. 2008 Nov;17(6):515-24. doi: 10.1097/CEJ.0b013e3282f521f8. PMID: 18941373. Exclusion Code: X5.

22. Akman I, Kuscu MK, Yurdakul Z, et al. Breastfeeding duration and postpartum psychological adjustment: role of maternal attachment styles. J Paediatr Child Health. 2008 Jun;44(6):369-73. doi: 10.1111/j.14401754.2008.01336.x. PMID: 18476931. Exclusion Code: X5.

23. Aksu H, Kucuk M, Duzgun G. The effect of postnatal breastfeeding education/support offered at home 3 days after delivery on breastfeeding duration and knowledge: a randomized trial. J Matern Fetal Neonatal Med. 2011 Feb;24(2):354-61. doi: 10.3109/14767058.2010.497569. PMID: 20608806. Exclusion Code: X3.

24. Al Mamun A, O'Callaghan MJ, Williams $\mathrm{GM}$, et al. Breastfeeding is protective to diabetes risk in young adults: a longitudinal study. Acta Diabetol. 2015 Oct;52(5):83744. doi: 10.1007/s00592-014-0690-z. PMID: 25539880. Exclusion Code: X5.

25. Albernaz E, Araujo CL, Tomasi E, et al. Influence of breastfeeding support on the tendencies of breastfeeding rates in the city of Pelotas (RS), Brazil, from 1982 to 2004. J Pediatr (Rio J). 2008 Nov-Dec;84(6):560-4. doi: doi:10.2223/JPED.1823. PMID: 18923797. Exclusion Code: X3. 
26. Albernaz E, Victora CG, Haisma H, et al. Lactation counseling increases breastfeeding duration but not breast milk intake as measured by isotopic methods. J Nutr. 2003 Jan;133(1):205-10. PMID: 12514291. Exclusion Code: X3.

27. Alex MR, MacLellan-Peters J. The Relationship Among Skin-to-Skin Contact, Breastfeeding, and Mother-Infant Interaction: Implications for Nursing. JOGNN: Journal of Obstetric, Gynecologic \& Neonatal Nursing. 2013;42:S88-9. doi: 10.1111/1552-6909.12181. PMID: 104179300. Exclusion Code: X4.

28. Alexander GR, Hulsey TC, Foley K, et al. An assessment of the use and impact of ancillary prenatal care services to Medicaid women in managed care. Matern Child Health J. 1997 Sep;1(3):139-49. PMID: 10728237. Exclusion Code: X3.

29. Allen J, Hector D. Benefits of breastfeeding. N S W Public Health Bull. 2005 MarApr;16(3-4):42-6. PMID: 16106271. Exclusion Code: X6.

30. Almeida H, Venancio SI, Sanches MT, et al. The impact of kangaroo care on exclusive breastfeeding in low birth weight newborns. J Pediatr (Rio J). 2010 May-Jun;86(3):2503. doi: doi:10.2223/JPED.1974. PMID: 20424798. Exclusion Code: X4.

31. Alshaikh B, Kostecky L, Blachly N, et al. Effect of a Quality Improvement Project to Use Exclusive Mother's Own Milk on Rate of Necrotizing Enterocolitis in Preterm Infants. Breastfeed Med. 2015

Sep;10(7):355-61. doi: 10.1089/bfm.2015.0042. PMID: 26230909. Exclusion Code: X3.

32. Ambrosone CB, Zirpoli G, Ruszczyk M, et al. Parity and breastfeeding among AfricanAmerican women: differential effects on breast cancer risk by estrogen receptor status in the Women's Circle of Health Study.

Cancer Causes Control. 2014

Feb;25(2):259-65. doi: 10.1007/s10552-0130323-9. PMID: 24249438. Exclusion Code: $\mathrm{X} 10$.
33. Amir LH, Cullinane M, Garland SM, et al. The role of micro-organisms (Staphylococcus aureus and Candida albicans) in the pathogenesis of breast pain and infection in lactating women: study protocol. BMC Pregnancy Childbirth. 2011 Jul 22;11:54. doi: 10.1186/1471-2393-1154. PMID: 21777483. Exclusion Code: X3.

34. Amissah EA, Kancherla V, Ko YA, et al. Validation Study of Maternal Recall on Breastfeeding Duration 6 Years After Childbirth. J Hum Lact. 2017 May;33(2):390-400. doi: 10.1177/0890334417691506. PMID: 28418809. Exclusion Code: X3.

35. Andaya E, Bonuck K, Barnett J, et al. Perceptions of primary care-based breastfeeding promotion interventions: qualitative analysis of randomized controlled trial participant interviews. Breastfeed Med. 2012 Dec;7(6):417-22. doi: 10.1089/bfm.2011.0151. PMID: 22621223. Exclusion Code: X5.

36. Anderson AK, Damio G, Chapman DJ, et al. Differential response to an exclusive breastfeeding peer counseling intervention: the role of ethnicity. J Hum Lact. 2007 Feb;23(1):16-23. doi: 10.1177/0890334406297182. PMID: 17293547. Exclusion Code: X3.

37. Anderson AK, Damio G, Young S, et al. A randomized trial assessing the efficacy of peer counseling on exclusive breastfeeding in a predominantly Latina low-income community. Arch Pediatr Adolesc Med. 2005 Sep;159(9):836-41. doi: 10.1001/archpedi.159.9.836. PMID: 16143742. Exclusion Code: X3.

38. Anderson KN, Schwab RB, Martinez ME. Reproductive risk factors and breast cancer subtypes: a review of the literature. Breast Cancer Res Treat. 2014 Feb;144(1):1-10. doi: 10.1007/s10549-014-2852-7. PMID: 24477977. Exclusion Code: X1.

39. Andrieu N, Goldgar DE, Easton DF, et al. Pregnancies, breast-feeding, and breast cancer risk in the International BRCA1/2 Carrier Cohort Study (IBCCS). J Natl Cancer Inst. 2006 Apr 19;98(8):535-44. doi: 10.1093/jnci/djj132. PMID: 16622123. Exclusion Code: X10. 
40. Annagur A, Annagur BB, Sahin A, et al. Is maternal depressive symptomatology effective on success of exclusive breastfeeding during postpartum 6 weeks? Breastfeed Med. 2013 Feb;8(1):53-7. doi: 10.1089/bfm.2012.0036. PMID: 23039400. Exclusion Code: X3.

41. Anothaisintawee T, Wiratkapun C, Lerdsitthichai $\mathrm{P}$, et al. Risk factors of breast cancer: a systematic review and metaanalysis. Asia Pac J Public Health. 2013 Sep;25(5):368-87. doi: 10.1177/1010539513488795. PMID: 23709491. Exclusion Code: X11.

42. Antonakou A, Papoutsis D, Panou I, et al. Role of exclusive breastfeeding in energy balance and weight loss during the first six months postpartum. Clin Exp Obstet Gynecol. 2013;40(4):485-8. PMID: 24597239. Exclusion Code: X4.

43. Aragon M, Chhoa E, Dayan R, et al. Perspectives of expectant women and health care providers on birth plans. J Obstet Gynaecol Can. 2013 Nov;35(11):979-85. PMID: 24246397. Exclusion Code: X3.

44. Arikpo D, Edet ES, Chibuzor MT, et al. Educational interventions for improving complementary feeding practices. Cochrane Database of Systematic Reviews: John Wiley \& Sons, Ltd; 2015. Exclusion Code: X3.

45. Arlotti JP, Cottrell BH, Lee SH, et al. Breastfeeding among low-income women with and without peer support. J Community Health Nurs. 1998;15(3):163-78. doi: 10.1207/s15327655jchn1503_4. PMID: 9747023. Exclusion Code: X3.

46. Armenta RF, Kritz-Silverstein D, Wingard D, et al. Association of breastfeeding with postmenopausal visceral adiposity among three racial/ethnic groups. Obesity (Silver Spring). 2015 Feb;23(2):475-80. doi: 10.1002/oby.20956. PMID: 25522135. Exclusion Code: X5.

47. Arora A, Manohar N, Hayen A, et al. Determinants of breastfeeding initiation among mothers in Sydney, Australia: findings from a birth cohort study. International Breastfeeding Journal. 2017;12:1-10. doi: 10.1186/s13006-0170130-0. PMID: 125191819. Exclusion Code: X3.
48. Artieta-Pinedo I, Paz-Pascual C, Grandes G, et al. Antenatal education and breastfeeding in a cohort of primiparas. J Adv Nurs. 2013 Jul;69(7):1607-17. doi: 10.1111/jan.12022. PMID: 23013265. Exclusion Code: X3.

49. Ashman AM, Brown LJ, Collins CE, et al. Factors Associated with Effective Nutrition Interventions for Pregnant Indigenous Women: A Systematic Review. J Acad Nutr Diet. 2017 Aug;117(8):1222-53.e2. doi: 10.1016/j.jand.2017.03.012. PMID: 28476322. Exclusion Code: X3.

50. Asiodu I, Waters C, Dailey D, et al. Infant Feeding Decision-Making and the Influences of Social Support Persons Among First-Time African American Mothers. Matern Child Health J. 2017;21(4):863-72. doi: 10.1007/s10995016-2167-x. PMID: 122278901. Exclusion Code: X3.

51. Askelsdottir B, Lam-de Jonge W, Edman G, et al. Home care after early discharge: impact on healthy mothers and newborns. Midwifery. 2013 Aug;29(8):927-34. doi: 10.1016/j.midw.2012.11.001. PMID: 23434021. Exclusion Code: X6.

52. Avoa A, Fischer PR. The influence of perinatal instruction about breast-feeding on neonatal weight loss. Pediatrics. 1990 Aug;86(2):313-5. PMID: 2371108. Exclusion Code: X5.

53. Ayton J, van der Mei I, Wills K, et al. Cumulative risks and cessation of exclusive breast feeding: Australian cross-sectional survey. Arch Dis Child. 2015

Sep;100(9):863-8. doi: 10.1136/archdischild-2014-307833. PMID: 26056146. Exclusion Code: X3.

54. Bai DL, Fong DY, Tarrant M. Factors associated with breastfeeding duration and exclusivity in mothers returning to paid employment postpartum. Matern Child Health J. 2015 May;19(5):990-9. doi: 10.1007/s10995-014-1596-7. PMID: 25095769. Exclusion Code: X3.

55. Bai DL, Wu KM, Tarrant M. Association between intrapartum interventions and breastfeeding duration. J Midwifery Womens Health. 2013 Jan-Feb;58(1):25-32. doi: 10.1111/j.1542-2011.2012.00254.x. PMID: 23317341. Exclusion Code: X3. 
56. Bajaj H, Ye C, Hanley AJ, et al. Prior lactation reduces future diabetic risk through sustained postweaning effects on insulin sensitivity. Am J Physiol Endocrinol Metab. 2017 Mar 01;312(3):E215-e23. doi: 10.1152/ajpendo.00403.2016. PMID: 27965206. Exclusion Code: X5.

57. Baker JL, Gamborg M, Heitmann BL, et al. Breastfeeding reduces postpartum weight retention. Am J Clin Nutr. 2008

Dec;88(6):1543-51. doi: 10.3945/ajcn.2008.26379. PMID: 19064514. Exclusion Code: X10.

58. Balkam JA, Cadwell K, Fein SB. Effect of components of a workplace lactation program on breastfeeding duration among employees of a public-sector employer. Matern Child Health J. 2011 Jul;15(5):67783. doi: 10.1007/s10995-010-0620-9. PMID: 20552261. Exclusion Code: X4.

59. Ball HL, Ward-Platt MP, Howel D, et al. Randomised trial of sidecar crib use on breastfeeding duration (NECOT). Arch Dis Child. 2011 Jul;96(7):630-4. doi: 10.1136/adc.2010.205344. PMID: 21474481. Exclusion Code: X3.

60. Balogun OO, O'Sullivan EJ, McFadden A, et al. Interventions for promoting the initiation of breastfeeding. Cochrane Database Syst Rev. 2016 Nov 09;11:Cd001688. doi: 10.1002/14651858.CD001688.pub3. PMID: 27827515. Exclusion Code: X3.

61. Banderali G, Riva E, Scaglioni S, et al. Monitoring breastfeeding rates in Italy. Acta Paediatr Suppl. 2003 Sep;91(441):6-8. PMID: 14599035. Exclusion Code: X6.

62. Bao PP, Shu XO, Gao YT, et al. Association of hormone-related characteristics and breast cancer risk by estrogen receptor/progesterone receptor status in the shanghai breast cancer study. Am J Epidemiol. 2011 Sep 15;174(6):661-71. doi: 10.1093/aje/kwr145. PMID: 21768404. Exclusion Code: X8.

63. Barlow A, Varipatis-Baker E, Speakman K, et al. Home-visiting intervention to improve child care among American Indian adolescent mothers: a randomized trial. Arch Pediatr Adolesc Med; 2006. p. 1101-7. Exclusion Code: X5.
64. Barlow J, Smailagic N, Bennett C, et al. Individual and group based parenting programmes for improving psychosocial outcomes for teenage parents and their children. Cochrane Database of Systematic Reviews: John Wiley \& Sons, Ltd; 2011. Exclusion Code: X3.

65. Barnett GC, Shah M, Redman K, et al. Risk factors for the incidence of breast cancer: do they affect survival from the disease? J Clin Oncol. 2008 Jul 10;26(20):3310-6. doi: 10.1200/jco.2006.10.3168. PMID: 18612147. Exclusion Code: X3.

66. Barros F, Halpern R, Victora C, et al. [Promotion of breast-feeding in urban localities of southern Brazil: a randomized intervention study]. Rev Saude Publica; 1994. p. 277-83. Exclusion Code: X7.

67. Barros FC, Semer TC, Tonioli Filho S, et al. The impact of lactation centres on breastfeeding patterns, morbidity and growth: a birth cohort study. Acta Paediatr. 1995 Nov;84(11):1221-6. PMID: 8580615. Exclusion Code: X3.

68. Bartick MC, Jegier BJ, Green BD, et al. Disparities in Breastfeeding: Impact on Maternal and Child Health Outcomes and Costs. J Pediatr. 2017 Feb;181:49-55.e6. doi: 10.1016/j.jpeds.2016.10.028. PMID: 27837954. Exclusion Code: X6.

69. Bartick MC, Stuebe AM, Schwarz EB, et al. Cost analysis of maternal disease associated with suboptimal breastfeeding. Obstet Gynecol. 2013 Jul;122(1):111-9. doi: 10.1097/AOG.0b013e318297a047. PMID: 23743465. Exclusion Code: X3.

70. Bartu A, Sharp J, Ludlow J, et al. Postnatal home visiting for illicit drug-using mothers and their infants: a randomised controlled trial. Aust N Z J Obstet Gynaecol. 2006 Oct;46(5):419-26. doi: 10.1111/j.1479828X.2006.00628.x. PMID: 16953857. Exclusion Code: X3.

71. Bascom EM, Napolitano MA. Breastfeeding Duration and Primary Reasons for Breastfeeding Cessation among Women with Postpartum Depressive Symptoms. J Hum Lact. 2016 May;32(2):282-91. doi: 10.1177/0890334415619908. PMID: 26644420. Exclusion Code: X5. 
72. Bassani DG, Arora P, Wazny K, et al. Financial incentives and coverage of child health interventions: a systematic review and meta-analysis. BMC Public Health. 2013;13 Suppl 3:S30. doi: 10.1186/14712458-13-s3-s30. PMID: 24564520.

Exclusion Code: X5.

73. Batal M, Boulghaurjian C. Breastfeeding initiation and duration in Lebanon: are the hospitals "mother friendly"? J Pediatr Nurs. 2005 Feb;20(1):53-9. doi: 10.1016/j.pedn.2004.09.004. PMID: 15834361. Exclusion Code: X3.

74. Batan M, Li R, Scanlon K. Association of child care providers breastfeeding support with breastfeeding duration at 6 months. Matern Child Health J. 2013 May;17(4):708-13. doi: 10.1007/s10995012-1050-7. PMID: 22706997. Exclusion Code: X3.

75. Battersby C, Santhakumaran S, Upton M, et al. The impact of a regional care bundle on maternal breast milk use in preterm infants: outcomes of the East of England quality improvement programme. Arch Dis Child Fetal Neonatal Ed. 2014 Sep;99(5):F395401. doi: 10.1136/archdischild-2013305475. PMID: 24876197. Exclusion Code: X5.

76. Beake S, McCourt C, Rowan C, et al. Evaluation of the use of health care assistants to support disadvantaged women breastfeeding in the community. Matern Child Nutr. 2005 Jan;1(1):32-43. doi: 10.1111/j.1740-8709.2004.00007.x. PMID: 16881877. Exclusion Code: X6.

77. Beake S, Pellowe C, Dykes F, et al. A systematic review of structured compared with non-structured breastfeeding programmes to support the initiation and duration of exclusive and any breastfeeding in acute and primary health care settings. Matern Child Nutr. 2012 Apr;8(2):141-61. doi: 10.1111/j.1740-8709.2011.00381.x. PMID: 22188596. Exclusion Code: X6.

78. Benjamin Neelon SE, Stroo M, Mayhew M, et al. Correlation between maternal and infant cortisol varies by breastfeeding status. Infant Behav Dev. 2015 Aug;40:252-8. doi: 10.1016/j.infbeh.2015.06.005. PMID: 26196472. Exclusion Code: X5.
79. Benjamin Neelon SE, Taveras EM, Ostbye $\mathrm{T}$, et al. Preventing obesity in infants and toddlers in child care: results from a pilot randomized controlled trial. Matern Child Health J. 2014 Jul;18(5):1246-57. doi: 10.1007/s10995-013-1359-x. PMID: 24065371. Exclusion Code: X3.

80. Bennett C, Macdonald GM, Dennis J, et al. Home-based support for disadvantaged adult mothers. Cochrane Database Syst Rev. 2007 Jul 18(3):Cd003759. doi: 10.1002/14651858.CD003759.pub2. PMID: 17636732. Exclusion Code: X1.

81. Bennett C, Macdonald GM, Dennis J, et al. WITHDRAWN: Home-based support for disadvantaged adult mothers. Cochrane Database Syst Rev. 2008 Jan 23(1):Cd003759. doi: 10.1002/14651858.CD003759.pub3. PMID: 18254033. Exclusion Code: X1.

82. Bensley RJ, Brusk JJ, Anderson JV, et al. wichealth.org: impact of a stages of changebased Internet nutrition education program. $\mathrm{J}$ Nutr Educ Behav. 2006 Jul-Aug;38(4):2229. doi: 10.1016/j.jneb.2006.03.008. PMID: 16785091. Exclusion Code: X5.

83. Bentley JP, Bond D, de Vroome M, et al. Factors Associated with Recurrent Infant Feeding Practices in Subsequent Births. J Hum Lact. 2016 Nov;32(4):721-9. doi: 10.1177/0890334416663188. PMID: 27542971. Exclusion Code: X3.

84. Bergink V, Lambregtse-van den Berg MP, Koorengevel KM, et al. First-onset psychosis occurring in the postpartum period: a prospective cohort study. J Clin Psychiatry. 2011 Nov;72(11):1531-7. doi: 10.4088/JCP.10m06648. PMID: 21903022. Exclusion Code: X5.

85. Bernard-Bonnin AC, Stachtchenko S, Girard G, et al. Hospital practices and breastfeeding duration: a meta-analysis of controlled trials. Birth. 1989 Jun;16(2):64-6. PMID: 2547392. Exclusion Code: X3.

86. Berry DC, Neal M, Hall EG, et al. Rationale, design, and methodology for the optimizing outcomes in women with gestational diabetes mellitus and their infants study. BMC Pregnancy Childbirth. 2013 Oct 10;13:184. doi: 10.1186/14712393-13-184. PMID: 24112417. Exclusion Code: X3. 
87. Besevic J, Gunter MJ, Fortner RT, et al. Reproductive factors and epithelial ovarian cancer survival in the EPIC cohort study. $\mathrm{Br}$ J Cancer. 2015 Dec 01;113(11):1622-31. doi: 10.1038/bjc.2015.377. PMID: 26554655. Exclusion Code: X6.

88. Bevan G, Brown M. Interventions in exclusive breastfeeding: a systematic review. Br J Nurs. 2014 Jan 23-Feb 12;23(2):86-9. doi: 10.12968/bjon.2014.23.2.86. PMID: 24464112. Exclusion Code: X3.

89. Bhutta ZA, Ahmed T, Black RE, et al. What works? Interventions for maternal and child undernutrition and survival. Lancet. 2008 Feb 02;371(9610):417-40. doi: 10.1016/s0140-6736(07)61693-6. PMID: 18206226. Exclusion Code: X3.

90. Bica OC, Giugliani ER. Influence of counseling sessions on the prevalence of breastfeeding in the first year of life: a randomized clinical trial with adolescent mothers and grandmothers. Birth. 2014 Mar;41(1):39-45. doi: 10.1111/birt.12097. PMID: 24654636. Exclusion Code: X3.

91. Bjornerem A, Ghasem-Zadeh A, Wang X, et al. Irreversible Deterioration of Cortical and Trabecular Microstructure Associated With Breastfeeding. J Bone Miner Res. 2017 Apr;32(4):681-7. doi: 10.1002/jbmr.3018. PMID: 27736021. Exclusion Code: X5.

92. Black MM, Cutts DB, Frank DA, et al. Special Supplemental Nutrition Program for Women, Infants, and Children participation and infants' growth and health: a multisite surveillance study. Pediatrics. 2004 Jul;114(1):169-76. PMID: 15231924. Exclusion Code: X6.

93. Black MM, Papas MA, Bentley ME, et al. Overweight adolescent African-American mothers gain weight in spite of intentions to lose weight. J Am Diet Assoc. 2006 Jan;106(1):80-7. doi: 10.1016/j.jada.2005.09.049. PMID: 16390669. Exclusion Code: X5.

94. Black MM, Siegel EH, Abel Y, et al. Home and videotape intervention delays early complementary feeding among adolescent mothers. Pediatrics. 2001 May;107(5):E67. PMID: 11331717. Exclusion Code: X3.
95. Blaine RE, Davison KK, Hesketh K, et al. Child Care Provider Adherence to Infant and Toddler Feeding Recommendations: Findings from the Baby Nutrition and Physical Activity Self-Assessment for Child Care (Baby NAP SACC) Study. Child Obes. 2015 Jun;11(3):304-13. doi: 10.1089/chi.2014.0099. PMID: 25918873. Exclusion Code: X2.

96. Bliss MC, Wilkie J, Acredolo C, et al. The effect of discharge pack formula and breast pumps on breastfeeding duration and choice of infant feeding method. Birth. 1997 Jun;24(2):90-7. PMID: 9271974. Exclusion Code: X3.

97. Bobrow KL, Quigley MA, Green J, et al. Persistent effects of women's parity and breastfeeding patterns on their body mass index: results from the Million Women Study. Int J Obes (Lond). 2013 May;37(5):712-7. doi: 10.1038/ijo.2012.76. PMID: 22777544. Exclusion Code: X5.

98. Bogaerts AF, Van den Bergh BR, Witters I, et al. Anxiety during early pregnancy predicts postpartum weight retention in obese mothers. Obesity (Silver Spring). 2013 Sep;21(9):1942-9. doi: 10.1002/oby.20352. PMID: 23408496. Exclusion Code: X6.

99. Boghossian NS, Yeung EH, Lipsky LM, et al. Dietary patterns in association with postpartum weight retention. Am J Clin Nutr. 2013 Jun;97(6):1338-45. doi: 10.3945/ajcn.112.048702. PMID: 23576044. Exclusion Code: X3.

100. Bonet M, Marchand L, Kaminski M, et al. Breastfeeding duration, social and occupational characteristics of mothers in the French 'EDEN mother-child' cohort. Matern Child Health J. 2013 May;17(4):714-22. doi: 10.1007/s10995012-1053-4. PMID: 22729659. Exclusion Code: X3.

101. Bonuck K, Stuebe A, Barnett J, et al. Effect of primary care intervention on breastfeeding duration and intensity. Am J Public Health. 2014 Feb;104 Suppl 1:S11927. doi: 10.2105/ajph.2013.301360. PMID: 24354834. Exclusion Code: X3. 
102. Bonuck KA, Trombley M, Freeman K, et al. Randomized, controlled trial of a prenatal and postnatal lactation consultant intervention on duration and intensity of breastfeeding up to 12 months. Pediatrics. 2005 Dec;116(6):1413-26. doi: 10.1542/peds.2005-0435. PMID: 16322166. Exclusion Code: X3.

103. Bortolini GA, Vitolo MR. The impact of systematic dietary counseling during the first year of life on prevalence rates of anemia and iron deficiency at 12-16 months. J Pediatr (Rio J). 2012 Jan-Feb;88(1):33-9. doi: doi:10.2223/JPED.2156. PMID: 22159301. Exclusion Code: X3.

104. Bosire R, Betz B, Aluisio A, et al. High Rates of Exclusive Breastfeeding in Both Arms of a Peer Counseling Study Promoting EBF Among HIV-Infected Kenyan Women. Breastfeed Med. 2016 Mar;11(2):56-63. doi: 10.1089/bfm.2015.0071. PMID: 26885769. Exclusion Code: X3.

105. Boulvain M, Perneger TV, Othenin-Girard $\mathrm{V}$, et al. Home-based versus hospital-based postnatal care: a randomised trial. BJOG. 2004 Aug;111(8):807-13. doi: 10.1111/j.1471-0528.2004.00227.x. PMID: 15270928. Exclusion Code: X3.

106. Brandao DS, Venancio SI, Giugliani ER. Association between the Brazilian Breastfeeding Network implementation and breastfeeding indicators. J Pediatr (Rio J). 2015 Mar-Apr;91(2):143-51. doi: 10.1016/j.jped.2014.06.009. PMID: 25449792. Exclusion Code: X6.

107. Brandhagen M, Lissner L, Brantsaeter AL, et al. Breast-feeding in relation to weight retention up to 36 months postpartum in the Norwegian Mother and Child Cohort Study: modification by socio-economic status? Public Health Nutr. 2014 Jul;17(7):1514-23. doi: 10.1017/s1368980013001869. PMID: 23915637. Exclusion Code: X10.

108. Brasileiro A, Possobon RF, Carrascoza K, et al. [The impact of breastfeeding promotion in women with formal employment]. Cad Saude Publica; 2010. p. 1705-13. Exclusion Code: X7.
109. Braun ML, Giugliani ER, Soares ME, et al. Evaluation of the impact of the babyfriendly hospital initiative on rates of breastfeeding. Am J Public Health. 2003 Aug;93(8):1277-9. PMID: 12893612. Exclusion Code: X6.

110. Brent NB, Redd B, Dworetz A, et al. Breastfeeding in a low-income population. Program to increase incidence and duration. Arch Pediatr Adolesc Med. 1995 Jul;149(7):798-803. PMID: 7795772. Exclusion Code: X3.

111. Briere CE, McGrath JM, Cong X, et al. Direct-Breastfeeding Premature Infants in the Neonatal Intensive Care Unit. J Hum Lact. 2015 Aug;31(3):386-92. doi: 10.1177/0890334415581798. PMID: 25900843. Exclusion Code: X3.

112. Britton C, McCormick FM, Renfrew MJ, et al. Support for breastfeeding mothers. Cochrane Database Syst Rev. 2007 Jan 24(1):Cd001141. doi: 10.1002/14651858.CD001141.pub3. PMID: 17253455. Exclusion Code: X6.

113. Britz SE, McDermott KC, Pierce CB, et al. Changes in maternal weight 5-10 years after a first delivery. Womens Health (Lond). 2012 Sep;8(5):513-9. doi: 10.2217/whe.12.35. PMID: 22934725. Exclusion Code: X5.

114. Brodribb W, Kruske S, Miller YD. Babyfriendly hospital accreditation, in-hospital care practices, and breastfeeding. Pediatrics. 2013 Apr;131(4):685-92. doi: 10.1542/peds.2012-2556. PMID: 23478863. Exclusion Code: X6.

115. Brooks JD, Boice JD, Jr., Stovall M, et al. Reproductive status at first diagnosis influences risk of radiation-induced second primary contralateral breast cancer in the WECARE study. Int J Radiat Oncol Biol Phys. 2012 Nov 15;84(4):917-24. doi: 10.1016/j.ijrobp.2012.01.047. PMID: 22483700. Exclusion Code: X3.

116. Brown A, Rance J, Bennett P. Understanding the relationship between breastfeeding and postnatal depression: the role of pain and physical difficulties. J Adv Nurs. 2016 Feb;72(2):273-82. doi: 10.1111/jan.12832. PMID: 26494433. Exclusion Code: X6. 
117. Brown A, Rance J, Warren L. Body image concerns during pregnancy are associated with a shorter breast feeding duration. Midwifery. 2015 Jan;31(1):80-9. doi: 10.1016/j.midw.2014.06.003. PMID: 25151278. Exclusion Code: X3.

118. Brown HC, Smith HJ, Mori R, et al. Giving women their own case notes to carry during pregnancy. Cochrane Database Syst Rev. 2015 Oct 14(10):Cd002856. doi: 10.1002/14651858.CD002856.pub3. PMID: 26465209. Exclusion Code: X5.

119. Brown S, Small R, Argus B, et al. Early postnatal discharge from hospital for healthy mothers and term infants. Cochrane Database of Systematic Reviews: John Wiley \& Sons, Ltd; 2002. Exclusion Code: X3.

120. Brownell EA, Hagadorn JI, Lussier MM, et al. Optimal periods of exclusive breastfeeding associated with any breastfeeding duration through one year. J Pediatr. 2015 Mar;166(3):566-70.e1. doi: 10.1016/j.jpeds.2014.11.015. PMID: 25524314. Exclusion Code: X3.

121. Bruce N, Griffioen A. Usefulness of a nonexperimental study design in the evaluation of service developments for infant feeding in a general hospital. Soc Sci Med. 1995 Apr;40(8):1109-16. PMID: 7597464. Exclusion Code: X6.

122. Bunik M, Krebs NF, Beaty B, et al. Breastfeeding and WIC enrollment in the Nurse Family Partnership Program. Breastfeed Med. 2009 Sep;4(3):145-9. doi: 10.1089/bfm.2008.0140. PMID: 19243262. Exclusion Code: X3.

123. Burdette AM, Pilkauskas NV. Maternal religious involvement and breastfeeding initiation and duration. Am J Public Health. 2012 Oct;102(10):1865-8. doi: 10.2105/ajph.2012.300737. PMID: 22897559. Exclusion Code: X3.

124. Burton P, Kennedy K, Ahluwalia JS, et al. Randomized trial comparing the effectiveness of 2 electric breast pumps in the NICU. J Hum Lact. 2013

Aug;29(3):412-9. doi: 10.1177/0890334413490995. PMID: 23776081. Exclusion Code: X5.
125. Butt S, Borgquist S, Anagnostaki L, et al. Breastfeeding in relation to risk of different breast cancer characteristics. BMC Res Notes. 2014 Apr 07;7:216. doi: 10.1186/1756-0500-7-216. PMID: 24708573. Exclusion Code: X10.

126. Butt Z, Haider SF, Arif S, et al. Breast cancer risk factors: a comparison between pre-menopausal and post-menopausal women. J Pak Med Assoc. 2012 Feb;62(2):120-4. PMID: 22755371. Exclusion Code: X8.

127. Byrne JP, Crowther CA, Moss JR. A randomised controlled trial comparing birthing centre care with delivery suite care in Adelaide, Australia. Aust N Z J Obstet Gynaecol. 2000 Aug;40(3):268-74. PMID: 11065032. Exclusion Code: X3.

128. Caldeira A, Fagundes G, Aguiar G. [Educational intervention on breastfeeding promotion to the Family Health Program team]. Rev Saude Publica; 2008. p. 102733. Exclusion Code: X7.

129. Caldeira AP, Goncalves E. Assessment of the impact of implementing the BabyFriendly Hospital Initiative. J Pediatr (Rio J). 2007 Mar-Apr;83(2):127-32. doi: doi:10.2223/JPED.1596. PMID: 17342252. Exclusion Code: X6.

130. Cambonie G, Rey V, Sabarros S, et al. Early postpartum discharge and breastfeeding: an observational study from France. Pediatr Int. 2010 Apr;52(2):180-6. doi: 10.1111/j.1442200X.2009.02942.x. PMID: 19674353. Exclusion Code: X3.

131. Cameron S, Taylor R, Gray A, et al. Exclusive breastfeeding to six months: Results from a randomised controlled trial including lactation consultant support. FASEB J; 2013. Exclusion Code: X3.

132. Cameron SL, Heath AL, Gray AR, et al. Lactation Consultant Support from Late Pregnancy with an Educational Intervention at 4 Months of Age Delays the Introduction of Complementary Foods in a Randomized Controlled Trial. J Nutr. 2015

Jul;145(7):1481-90. doi: 10.3945/jn.114.202689. PMID: 25995280. Exclusion Code: X3. 
133. Campbell D, Scott KD, Klaus MH, et al. Female relatives or friends trained as labor doulas: outcomes at 6 to 8 weeks postpartum. Birth. 2007 Sep;34(3):220-7. doi: 10.1111/j.1523-536X.2007.00174.x. PMID: 17718872. Exclusion Code: X5.

134. Campbell LA, Wan J, Speck PM, et al. Women, Infant and Children (WIC) peer counselor contact with first time breastfeeding mothers. Public Health Nurs. 2014 Jan-Feb;31(1):3-9. doi:

10.1111/phn.12055. PMID: 24387771. Exclusion Code: X3.

135. Cao Y, Wang Z, Gu J, et al. Reproductive Factors but Not Hormonal Factors Associated with Thyroid Cancer Risk: A Systematic Review and Meta-Analysis. Biomed Res Int. 2015;2015:103515. doi: 10.1155/2015/103515. PMID: 26339585. Exclusion Code: X5.

136. Carfoot S, Williamson PR, Dickson R. A systematic review of randomised controlled trials evaluating the effect of mother/baby skin-to-skin care on successful breast feeding. Midwifery. 2003 Jun;19(2):148-55. PMID: 12809635. Exclusion Code: X3.

137. Carfoot S, Williamson PR, Dickson R. The value of a pilot study in breast-feeding research. Midwifery. 2004 Jun;20(2):18893. doi: 10.1016/j.midw.2003.11.002. PMID: 15177863. Exclusion Code: X3.

138. Carlsen EM, Kyhnaeb A, Renault KM, et al. Telephone-based support prolongs breastfeeding duration in obese women: a randomized trial. Am J Clin Nutr. 2013 Nov;98(5):1226-32. doi: 10.3945/ajen.113.059600. PMID: 24004897. Exclusion Code: X3.

139. Carneiro RM, Prebehalla L, Tedesco MB, et al. Lactation and bone turnover: a conundrum of marked bone loss in the setting of coupled bone turnover. J Clin Endocrinol Metab. 2010 Apr;95(4):1767-76. doi: 10.1210/jc.2009-1518. PMID: 20150580. Exclusion Code: X5.

140. Carvalho ML, Boccolini CS, Oliveira MI, et al. The baby-friendly hospital initiative and breastfeeding at birth in Brazil: a cross sectional study. Reprod Health. 2016 Oct 17;13(Suppl 3):119. doi: 10.1186/s12978016-0234-9. PMID: 27766969. Exclusion Code: X5.
141. Castrucci BC, Hoover KL, Lim S, et al. A comparison of breastfeeding rates in an urban birth cohort among women delivering infants at hospitals that employ and do not employ lactation consultants. J Public Health Manag Pract. 2006 NovDec;12(6):578-85. PMID: 17041307. Exclusion Code: X3.

142. Castrucci BC, Hoover KL, Lim S, et al. Availability of lactation counseling services influences breastfeeding among infants admitted to to neonatal intensive care units. Am J Health Promot. 2007 MayJun;21(5):410-5. PMID: 17515004. Exclusion Code: X3.

143. Catling CJ, Medley N, Foureur M, et al. Group versus conventional antenatal care for women. Cochrane Database Syst Rev. 2015 Feb 04(2):Cd007622. doi: 10.1002/14651858.CD007622.pub3. PMID: 25922865. Exclusion Code: X3.

144. Catling-Paull C, Coddington RL, Foureur MJ, et al. Publicly funded homebirth in Australia: a review of maternal and neonatal outcomes over 6 years. Med J Aust. 2013 Jun 17;198(11):616-20. PMID: 23919710. Exclusion Code: X6.

145. Cato K, Sylven SM, Lindback J, et al. Risk factors for exclusive breastfeeding lasting less than two months-Identifying women in need of targeted breastfeeding support. PLoS One. 2017;12(6):e0179402. doi: 10.1371/journal.pone.0179402. PMID: 28614419. Exclusion Code: X3.

146. Cattaneo A, Buzzetti R. Effect on rates of breast feeding of training for the baby friendly hospital initiative. BMJ. 2001 Dec 08;323(7325):1358-62. PMID: 11739226. Exclusion Code: X3.

147. Caulfield LE, Gross SM, Bentley ME, et al. WIC-based interventions to promote breastfeeding among African-American Women in Baltimore: effects on breastfeeding initiation and continuation. J Hum Lact. 1998 Mar;14(1):15-22. PMID: 9543954. Exclusion Code: X3. 
148. Ceccatto V, Cesa C, Kunradi Vieira FG, et al. Characteristics of newly diagnosed women with breast cancer: a comparison with the recommendations of the WCRF/AICR Second Report. Nutr Hosp. 2012 Nov-Dec;27(6):1973-80. doi: 10.3305/nh.2012.27.6.6006. PMID: 23588447. Exclusion Code: X2.

149. Chamberlain CR, Wilson AN, Amir LH, et al. Low rates of predominant breastfeeding in hospital after gestational diabetes, particularly among Indigenous women in Australia. Aust N Z J Public Health. 2017 Apr;41(2):144-50. doi: 10.1111/17536405.12629. PMID: 28110518. Exclusion Code: X3.

150. Chamberlain LB, McMahon M, Philipp BL, et al. Breast pump access in the inner city: a hospital-based initiative to provide breast pumps for low-income women. J Hum Lact. 2006 Feb;22(1):94-8. doi:

10.1177/0890334405284226. PMID: 16467290. Exclusion Code: X1.

151. Chan-Yip AM, Kramer MS. Promotion of breast-feeding in a Chinese community in Montreal. Can Med Assoc J. 1983 Nov 01;129(9):955-8. PMID: 6671183. Exclusion Code: X3.

152. Chapman D, Damio G, Young S, et al. Association of degree and timing of exposure to breastfeeding peer counseling services with breastfeeding duration. Adv Exp Med Biol. 2004;554:303-6. PMID: 15384587. Exclusion Code: X3.

153. Chapman DJ, Damio G, Perez-Escamilla R. Differential response to breastfeeding peer counseling within a low-income, predominantly Latina population. J Hum Lact. 2004 Nov;20(4):389-96. doi: 10.1177/0890334404269845. PMID: 15479657. Exclusion Code: X6.

154. Chapman DJ, Damio G, Young S, et al. Effectiveness of breastfeeding peer counseling in a low-income, predominantly Latina population: a randomized controlled trial. Arch Pediatr Adolesc Med. 2004 Sep;158(9):897-902. doi: 10.1001/archpedi.158.9.897. PMID: 15351756. Exclusion Code: X3.
155. Chapman DJ, Morel K, Anderson AK, et al. Breastfeeding peer counseling: from efficacy through scale-up. J Hum Lact. 2010 Aug;26(3):314-26. PMID: 20715336. Exclusion Code: X3.

156. Chapman DJ, Morel K, Bermudez-Millan A, et al. Breastfeeding education and support trial for overweight and obese women: a randomized trial. Pediatrics. 2013

Jan;131(1):e162-70. doi: 10.1542/peds.2012-0688. PMID: 23209111. Exclusion Code: X3.

157. Chatterji P, Brooks-Gunn J. WIC participation, breastfeeding practices, and well-child care among unmarried, lowincome mothers. Am J Public Health. 2004 Aug;94(8):1324-7. PMID: 15284035. Exclusion Code: X3.

158. Chaudron LH, Klein MH, Remington P, et al. Predictors, prodromes and incidence of postpartum depression. J Psychosom Obstet Gynaecol. 2001 Jun;22(2):103-12. PMID: 11446151. Exclusion Code: X10.

159. Chen M. Test a model of breast-feeding duration for Vietnamese mothers in Taiwan. Commun Nurs Res. 20052005

Spring;38:461-. PMID: 105740920. Exclusion Code: X5.

160. Chen YC, Chie WC, Kuo SC, et al. The association between infant feeding pattern and mother's quality of life in Taiwan. Qual Life Res. 2007 Oct;16(8):1281-8. doi: 10.1007/s11136-007-9233-1. PMID: 17616835. Exclusion Code: X5.

161. Chen YC, Wu YC, Chie WC. Effects of work-related factors on the breastfeeding behavior of working mothers in a Taiwanese semiconductor manufacturer: a crosssectional survey. BMC Public Health. 2006 Jun 21;6:160. doi: 10.1186/1471-2458-6160. PMID: 16787546. Exclusion Code: X4.

162. Chezem J, Friesen C, Montgomery P, et al. Lactation duration: influences of human milk replacements and formula samples on women planning postpartum employment. J Obstet Gynecol Neonatal Nurs. 1998 NovDec;27(6):646-51. PMID: 9836159. Exclusion Code: X3. 
163. Chiaffarino F, Pelucchi C, Negri E, et al. Breastfeeding and the risk of epithelial ovarian cancer in an Italian population. Gynecol Oncol. 2005 Aug;98(2):304-8. doi: 10.1016/j.ygyno.2005.05.006. PMID: 15975644. Exclusion Code: X10.

164. Chiasson MA, Findley SE, Sekhobo JP, et al. Changing WIC changes what children eat. Obesity (Silver Spring). 2013 Jul;21(7):1423-9. doi: 10.1002/oby.20295. PMID: 23703806. Exclusion Code: X6.

165. Chiasson MA, Scheinmann R, Hartel D, et al. Predictors of Obesity in a Cohort of Children Enrolled in WIC as Infants and Retained to 3 Years of Age. J Community Health. 2016 Feb;41(1):127-33. doi: 10.1007/s10900-015-0077-2. PMID: 26280211. Exclusion Code: X3.

166. Chiu CL, Lind JM. Past oral contraceptive use and self-reported high blood pressure in postmenopausal women. BMC Public Health. 2015 Jan 31;15:54. doi: 10.1186/s12889-015-1392-3. PMID: 25636949. Exclusion Code: X3.

167. Chiurco A, Montico M, Brovedani P, et al. An IBCLC in the Maternity Ward of a Mother and Child Hospital: A Pre- and PostIntervention Study. Int J Environ Res Public Health. 2015 Aug 20;12(8):9938-51. doi: 10.3390/ijerph120809938. PMID: 26308018. Exclusion Code: X6.

168. Cho GJ, Park HT, Shin JH, et al. The relationship between reproductive factors and metabolic syndrome in Korean postmenopausal women: Korea National Health and Nutrition Survey 2005. Menopause. 2009 Sep-Oct;16(5):998-1003. doi: 10.1097/gme.0b013e3181a03807. PMID: 19407665. Exclusion Code: X5.

169. Choi H, Yamashita T, Wada Y, et al. Predictors for exacerbation/improvement of postpartum depression--a focus on anxiety, the mothers' experiences of being cared for by their parents in childhood and borderline personality: a perspective study in Japan. J Affect Disord. 2013 Sep 05;150(2):507-12. doi: 10.1016/j.jad.2013.04.051. PMID: 23712025. Exclusion Code: X3.
170. Chong A, Biehle SN, Kooiman LY, et al. Postnatal Depression. Psychol Women Q. 2016;40(4):518-31. doi: 10.1177/0361684316658263. PMID: 119511603. Exclusion Code: X5.

171. Chow HW, Dong YH. Relationship between participation in leisure activities and constraints on Taiwanese breastfeeding mothers during leisure activities. BMC Public Health. 2013 Apr 30;13:410. doi: 10.1186/1471-2458-13-410. PMID: 23627993. Exclusion Code: X3.

172. Chuang $\mathrm{CH}$, Chang PJ, Chen $\mathrm{YC}$, et al. Maternal return to work and breastfeeding: a population-based cohort study. Int J Nurs Stud. 2010 Apr;47(4):461-74. doi: 10.1016/j.ijnurstu.2009.09.003. PMID: 19819449. Exclusion Code: X3.

173. Chumnijarakij T, Grossman RA, Onthuam Y, et al. A study of contraceptive choice and use in Bangkok Metropolis Health Clinics. Contraception. 1981 Sep;24(3):245-58. PMID: 7307522. Exclusion Code: X2.

174. Chung M, Raman G, Trikalinos T, et al. Clinical guidelines. Interventions in primary care to promote breastfeeding: an evidence review for the U.S. Preventive Services Task Force. Ann Intern Med. 2008;149(8):56552. PMID: 105711572. Exclusion Code: $\mathrm{X} 3$.

175. Chung S, Park SK, Sung H, et al. Association between chronological change of reproductive factors and breast cancer risk defined by hormone receptor status: results from the Seoul Breast Cancer Study. Breast Cancer Res Treat. 2013 Aug;140(3):557-65. doi: 10.1007/s10549013-2645-4. PMID: 23901017. Exclusion Code: X10.

176. Chwo M. Early kangaroo care for 34--36 week preterm infants: Effects on temperature, weight, behavior, and acuity: Case Western Reserve University (Health Sciences); 1999. Exclusion Code: X3.

177. Ciftci EK, Arikan D. The effect of training administered to working mothers on maternal anxiety levels and breastfeeding habits. J Clin Nurs. 2012 Aug;21(1516):2170-8. doi: 10.1111/j.13652702.2011.03957.x. PMID: 22151299. Exclusion Code: X3. 
178. Clifford H, Johnson NW, Brown C, et al. When can oral health education begin? Relative effectiveness of three oral health education strategies starting pre-partum. Community Dent Health. 2012 Jun;29(2):162-7. PMID: 22779378. Exclusion Code: X3.

179. Cloutier MM, Wiley J, Wang Z, et al. The Early Childhood Obesity Prevention Program (ECHO): an ecologically-based intervention delivered by home visitors for newborns and their mothers. BMC Public Health. 2015 Jun 24;15:584. doi: 10.1186/s12889-015-1897-9. PMID: 26104068. Exclusion Code: X6.

180. Coffield K. The benefits of phone support and home visits: an evaluation of the City of Kingston's Breastfeeding Support Service. Breastfeed Rev. 2008 Nov;16(3):17-21. PMID: 19133399. Exclusion Code: X4.

181. Cohen R, Mrtek MB, Mrtek RG. Comparison of maternal absenteeism and infant illness rates among breast-feeding and formula-feeding women in two corporations. Am J Health Promot. 1995 NovDec;10(2):148-53. PMID: 10160049. Exclusion Code: X3.

182. Cohen SS, Larson CO, Matthews CE, et al. Parity and breastfeeding in relation to obesity among black and white women in the southern community cohort study. J Womens Health (Larchmt). 2009 Sep;18(9):1323-32. doi: 10.1089/jwh.2008.1181. PMID: 19743905. Exclusion Code: X5.

183. Colchamiro R, Edwards RA, Nordstrom C, et al. Mobilizing Community Resources to Enhance Postdischarge Support for Breastfeeding in Massachusetts (USA): Results of a Catalyst Grant Approach. J Hum Lact. 2015 Nov;31(4):631-40. doi: 10.1177/0890334415597680. PMID: 26266946. Exclusion Code: X5.

184. Colson SD, Meek JH, Hawdon JM. Optimal positions for the release of primitive neonatal reflexes stimulating breastfeeding. Early Hum Dev. 2008 Jul;84(7):441-9. doi: 10.1016/j.earlhumdev.2007.12.003. PMID: 18243594. Exclusion Code: X3.
185. Conde-Agudelo A, Diaz-Rossello JL. Kangaroo mother care to reduce morbidity and mortality in low birthweight infants. Cochrane Database Syst Rev. 2014 Apr 22(4):Cd002771. doi: 10.1002/14651858.CD002771.pub3. PMID: 24752403. Exclusion Code: X3.

186. Conde-Agudelo A, Díaz-Rossello JL. Kangaroo mother care to reduce morbidity and mortality in low birthweight infants. Cochrane Database of Systematic Reviews: John Wiley \& Sons, Ltd; 2016. Exclusion Code: X5.

187. Condon L, Tiffany C, Symes N, et al. 'Cut out for breastfeeding': changing attitudes to breastfeeding. Community Pract. 2010 Apr;83(4):29-31. PMID: 20441098. Exclusion Code: X5.

188. Cooke M, Schmied V, Sheehan A. An exploration of the relationship between postnatal distress and maternal role attainment, breast feeding problems and breast feeding cessation in Australia. Midwifery. 2007 Mar;23(1):66-76. doi: 10.1016/j.midw.2005.12.003. PMID: 17011682. Exclusion Code: X3.

189. Cooklin AR, Donath SM, Amir LH. Maternal employment and breastfeeding: results from the longitudinal study of Australian children. Acta Paediatr. 2008 May;97(5):620-3. doi: 10.1111/j.16512227.2008.00740.x. PMID: 18394107. Exclusion Code: X3.

190. Cooklin AR, Rowe HJ, Fisher JR. Paid parental leave supports breastfeeding and mother-infant relationship: a prospective investigation of maternal postpartum employment. Aust N Z J Public Health. 2012 Jun;36(3):249-56. doi: 10.1111/j.17536405.2012.00846.x. PMID: 22672031. Exclusion Code: X3.

191. Cooper PJ, Murray L, Stein A. Psychosocial factors associated with the early termination of breast-feeding. J Psychosom Res. 1993;37(2):171-6. PMID: 8463993. Exclusion Code: X10.

192. Corriveau SK, Drake EE, Kellams AL, et al. Evaluation of an office protocol to increase exclusivity of breastfeeding. Pediatrics. 2013 May;131(5):942-50. doi: 10.1542/peds.2012-1310. PMID: 23545382. Exclusion Code: X6. 
193. Costa ML, Cecatti JG, Krupa FG, et al. Progestin-only contraception prevents bone loss in postpartum breastfeeding women. Contraception. 2012 Apr;85(4):374-80. doi: 10.1016/j.contraception.2011.08.015. PMID: 22036473. Exclusion Code: X4.

194. Costa ML, Krupa FG, Rehder PM, et al. Forearm bone mineral density changes during postpartum and the effects of breastfeeding, amenorrhea, body mass index and contraceptive use. Osteoporos Int. 2012 Jun;23(6):1691-8. doi: 10.1007/s00198-0111767-y. PMID: 21881967. Exclusion Code: X8.

195. Coutinho SB, Lira PI, Lima MC, et al. Promotion of exclusive breast-feeding at scale within routine health services: impact of breast-feeding counselling training for community health workers in Recife, Brazil. Public Health Nutr. 2014 Apr;17(4):948-55. doi: 10.1017/s1368980013001833. PMID: 23845723. Exclusion Code: X6.

196. Cox K, Giglia R, Zhao Y, et al. Factors associated with exclusive breastfeeding at hospital discharge in rural Western Australia. J Hum Lact. 2014 Nov;30(4):48897. doi: $10.1177 / 0890334414547274$. PMID: 25139681. Exclusion Code: X3.

197. Cramer DW, Hutchison GB, Welch WR, et al. Determinants of ovarian cancer risk. I. Reproductive experiences and family history. J Natl Cancer Inst. 1983 Oct;71(4):711-6. PMID: 6578366. Exclusion Code: X10.

198. Cronin-Fenton DP, Murray LJ, Whiteman DC, et al. Reproductive and sex hormonal factors and oesophageal and gastric junction adenocarcinoma: a pooled analysis. Eur J Cancer. 2010 Jul;46(11):2067-76. doi: 10.1016/j.ejca.2010.03.032. PMID: 20456945. Exclusion Code: X5.

199. Curro V, Lanni R, Scipione F, et al. Randomised controlled trial assessing the effectiveness of a booklet on the duration of breast feeding. Arch Dis Child. 1997 Jun;76(6):500-3; Discussion 3-4. PMID: 9245846. Exclusion Code: X3.
200. Curtis P, Woodhill R, Stapleton H. The peer-professional interface in a communitybased, breast feeding peer-support project. Midwifery. 2007 Jun;23(2):146-56. doi: 10.1016/j.midw.2006.04.003. PMID: 17055134. Exclusion Code: X2.

201. da Graca LC, Figueiredo Mdo C, Conceicao MT. Contributions of the nursing intervention in primary healthcare for the promotion of breastfeeding. Rev Lat Am Enfermagem. 2011 Mar-Apr;19(2):429-36. PMID: 21584392. Exclusion Code: X3.

202. da Silva CF, Nunes LM, Schwartz R, et al. Effect of a pro-breastfeeding intervention on the maintenance of breastfeeding for 2 years or more: randomized clinical trial with adolescent mothers and grandmothers. BMC Pregnancy Childbirth. 2016 May 03;16:97. doi: 10.1186/s12884-016-0878-z. PMID: 27141951. Exclusion Code: X3.

203. da Silva Mda C, Oliveira Assis AM, Pinheiro SM, et al. Breastfeeding and maternal weight changes during 24 months post-partum: a cohort study. Matern Child Nutr. 2015 Oct;11(4):780-91. doi: 10.1111/mcn.12071. PMID: 23941254. Exclusion Code: X8.

204. Dagher RK, McGovern PM, Dowd BE, et al. Postpartum depressive symptoms and the combined load of paid and unpaid work: a longitudinal analysis. Int Arch Occup Environ Health. 2011 Oct;84(7):735-43. doi: 10.1007/s00420-011-0626-7. PMID: 21373878. Exclusion Code: X3.

205. Dall'Oglio I, Salvatori G, Bonci E, et al. Breastfeeding promotion in neonatal intensive care unit: impact of a new program toward a BFHI for high-risk infants. Acta Paediatr. 2007 Nov;96(11):1626-31. doi: 10.1111/j.1651-2227.2007.00495.x. PMID: 17937687. Exclusion Code: X3.

206. Danforth KN, Tworoger SS, Hecht JL, et al. Breastfeeding and risk of ovarian cancer in two prospective cohorts. Cancer Causes Control. 2007 Jun;18(5):517-23. doi: 10.1007/s10552-007-0130-2. PMID: 17450440. Exclusion Code: X10. 
207. Daniels LA, Mallan KM, Battistutta D, et al. Evaluation of an intervention to promote protective infant feeding practices to prevent childhood obesity: outcomes of the

NOURISH RCT at 14 months of age and 6 months post the first of two intervention modules. Int J Obes (Lond). 2012

Oct;36(10):1292-8. doi:

10.1038/ijo.2012.96. PMID: 22710926.

Exclusion Code: X3.

208. Dashti M, Scott JA, Edwards CA, et al. Predictors of breastfeeding duration among women in Kuwait: results of a prospective cohort study. Nutrients. 2014 Feb 20;6(2):711-28. doi: 10.3390/nu6020711. PMID: 24561360. Exclusion Code: X3.

209. Davies B, Edwards N, Ploeg J, et al. Insights about the process and impact of implementing nursing guidelines on delivery of care in hospitals and community settings. BMC Health Serv Res. 2008 Feb 02;8:29. doi: 10.1186/1472-6963-8-29. PMID: 18241349. Exclusion Code: X6.

210. Davis SK, Stichler JF, Poeltler DM. Increasing exclusive breastfeeding rates in the well-baby population: an evidence-based change project. Nurs Womens Health. 2012 Dec;16(6):460-70. doi: 10.1111/j.1751486X.2012.01774.x. PMID: 23253573. Exclusion Code: X1.

211. de Fátima Buco Busto Moreno P, Trombini Schmidt K. BREAST-FEEDING AND FACTORS RELATED TO EARLY WEANING. Cogitare Enfermagem. 2014;19(3):531-7. PMID: 103900891. Exclusion Code: X3.

212. de Jersey SJ, Mallan K, Forster J, et al. A prospective study of breastfeeding intentions of healthy weight and overweight women as predictors of breastfeeding outcomes. Midwifery. 2017;53:20-7. doi: 10.1016/j.midw.2017.07.002. PMID: 124820840. Exclusion Code: X3.

213. de Oliveira LD, Giugliani ER, do Espirito Santo LC, et al. Effect of intervention to improve breastfeeding technique on the frequency of exclusive breastfeeding and lactation-related problems. J Hum Lact. 2006 Aug;22(3):315-21. doi: 10.1177/0890334406290221. PMID: 16885491. Exclusion Code: X3.
214. de Oliveira LD, Giugliani ER, Santo LC, et al. Impact of a strategy to prevent the introduction of non-breast milk and complementary foods during the first 6 months of life: a randomized clinical trial with adolescent mothers and grandmothers. Early Hum Dev. 2012 Jun;88(6):357-61. doi: 10.1016/j.earlhumdev.2011.09.010. PMID: 22001312. Exclusion Code: X3.

215. de Oliveira MI, Camacho LA, Tedstone AE. Extending breastfeeding duration through primary care: a systematic review of prenatal and postnatal interventions. J Hum Lact. 2001 Nov;17(4):326-43. PMID: 11847902. Exclusion Code: X3.

216. Declercq E, Labbok MH, Sakala C, et al. Hospital practices and women's likelihood of fulfilling their intention to exclusively breastfeed. Am J Public Health. 2009 May;99(5):929-35. doi: 10.2105/ajph.2008.135236. PMID: 19299680. Exclusion Code: X6.

217. Demirci JR, Bogen DL. An Ecological Momentary Assessment of Primiparous Women's Breastfeeding Behavior and Problems From Birth to 8 Weeks. J Hum Lact. 2017 May;33(2):285-95. doi: 10.1177/0890334417695206. PMID: 28418803. Exclusion Code: X3.

218. Dennis CL, Heaman M, Mossman M. Psychometric testing of the breastfeeding self-efficacy scale-short form among adolescents. J Adolesc Health. 2011 Sep;49(3):265-71. doi: 10.1016/j.jadohealth.2010.12.015. PMID: 21856518. Exclusion Code: X3.

219. Dennis CL, Hodnett E, Gallop R, et al. The effect of peer support on breast-feeding duration among primiparous women: a randomized controlled trial. CMAJ. 2002 Jan 08;166(1):21-8. PMID: 11800243. Exclusion Code: X3.

220. Dennis CL, Kingston D. A systematic review of telephone support for women during pregnancy and the early postpartum period. J Obstet Gynecol Neonatal Nurs. 2008 May-Jun;37(3):301-14. doi: 10.1111/j.1552-6909.2008.00235.x. PMID: 18507601. Exclusion Code: X3. 
221. Dennis CL, McQueen K. The relationship between infant-feeding outcomes and postpartum depression: a qualitative systematic review. Pediatrics. 2009 Apr;123(4):e736-51. doi: 10.1542/peds.2008-1629. PMID: 19336362. Exclusion Code: X6.

222. Dewey KG, Adu-Afarwuah S. Systematic review of the efficacy and effectiveness of complementary feeding interventions in developing countries. Matern Child Nutr. 2008 Apr;4 Suppl 1:24-85. doi: 10.1111/j.1740-8709.2007.00124.x. PMID: 18289157. Exclusion Code: X3.

223. Di Napoli A, Di Lallo D, Fortes C, et al. Home breastfeeding support by health professionals: findings of a randomized controlled trial in a population of Italian women. Acta Paediatr. 2004 Aug;93(8):1108-14. PMID: 15456204. Exclusion Code: X3.

224. Dias de Oliveira L, Justo Giugliani ER, Cordova do Espirito Santo L, et al. Counselling sessions increased duration of exclusive breastfeeding: a randomized clinical trial with adolescent mothers and grandmothers. Nutr J. 2014 Jul 17;13:73. doi: 10.1186/1475-2891-13-73. PMID: 25033743. Exclusion Code: X3.

225. DiGirolamo AM, Grummer-Strawn LM, Fein S. Maternity care practices: implications for breastfeeding. Birth. 2001 Jun;28(2):94-100. PMID: 11380380. Exclusion Code: X6.

226. Ding T, Wang DX, Qu Y, et al. Epidural labor analgesia is associated with a decreased risk of postpartum depression: a prospective cohort study. Anesth Analg. 2014 Aug;119(2):383-92. doi: 10.1213/ane.0000000000000107. PMID: 24797120. Exclusion Code: X8.

227. Dodd JM, Dowswell T, Crowther CA. Specialised antenatal clinics for women with a multiple pregnancy for improving maternal and infant outcomes. Cochrane Database Syst Rev. 2015 Nov 06(11):Cd005300. doi: 10.1002/14651858.CD005300.pub4. PMID: 26545291. Exclusion Code: X3.
228. Dogan L, Kalaylioglu Z, Karaman N, et al. Relationships between epidemiological features and tumor characteristics of breast cancer. Asian Pac J Cancer Prev. 2011;12(12):3375-80. PMID: 22471484. Exclusion Code: X8.

229. Dolgun G, Yuksel A. Assessing mandated breastfeeding education in Istanbul. MCN Am J Matern Child Nurs. 2010 SepOct;35(5):293-6. doi: 10.1097/NMC.0b013e3181e62802. PMID: 20706100. Exclusion Code: X6.

230. Domanico R, Davis DK, Coleman F, et al. Documenting the NICU design dilemma: comparative patient progress in open-ward and single family room units. J Perinatol. 2011 Apr;31(4):281-8. doi: 10.1038/jp.2010.120. PMID: 21072040. Exclusion Code: X3.

231. Donnelly A, Snowden HM, Renfrew MJ, et al. WITHDRAWN: Commercial hospital discharge packs for breastfeeding women. Cochrane Database Syst Rev. 2007 Jul 18(2):Cd002075. doi: 10.1002/14651858.CD002075.pub2. PMID: 17636696. Exclusion Code: X6.

232. Dorheim SK, Bondevik GT, Eberhard-Gran $\mathrm{M}$, et al. Sleep and depression in postpartum women: a population-based study. Sleep. 2009 Jul;32(7):847-55. PMID: 19639747. Exclusion Code: X6.

233. Duckett L, Henly SJ, Garvis M. Predicting breast-feeding duration during the postpartum hospitalization. West J Nurs Res. 1993 Apr;15(2):177-93; discussion 948. PMID: 8470374. Exclusion Code: X3.

234. Dujmovic M, Kresic G, Mandic ML, et al. Changes in dietary intake and body weight in lactating and non-lactating women: prospective study in northern coastal Croatia. Coll Antropol. 2014 Mar;38(1):17987. PMID: 24851615. Exclusion Code: $\mathrm{X} 10$.

235. Dumas L, Lepage M, Bystrova K, et al. Influence of skin-to-skin contact and rooming-in on early mother-infant interaction: a randomized controlled trial. Clin Nurs Res. 2013 Aug;22(3):310-36. doi: 10.1177/1054773812468316. PMID: 23291315. Exclusion Code: X3. 
236. Dumas L, Lepage M, Bystrova K, et al. Influence of Skin-to-Skin Contact and Rooming-In on Early Mother-Infant Interaction: A Randomized Controlled Trial. Clin Nurs Res. 2013;22(3):310-36. doi: 10.1177/1054773812468316. PMID: 104190843. Exclusion Code: X5.

237. Dumphy D, Thompson J, Clark M. A Breastfeeding Quality Improvement Project in Rural Primary Care. J Hum Lact. 2016;32(4):633-41. doi: 10.1177/0890334416662240. PMID: 118744603. Exclusion Code: X6.

238. Dungy CI, Christensen-Szalanski J, Losch $\mathrm{M}$, et al. Effect of discharge samples on duration of breast-feeding. Pediatrics. 1992 Aug;90(2 Pt 1):233-7. PMID: 1641288. Exclusion Code: X3.

239. Dungy CI, Losch ME, Russell D, et al. Hospital infant formula discharge packages. Do they affect the duration of breastfeeding? Arch Pediatr Adolesc Med. 1997 Jul;151(7):724-9. PMID: 9232049.

Exclusion Code: X3.

240. Durand M, Labarere J, Brunet E, et al. Evaluation of a training program for healthcare professionals about breastfeeding. Eur J Obstet Gynecol Reprod Biol. 2003 Feb 10;106(2):134-8. PMID: 12551778. Exclusion Code: X6.

241. Dursun N, Akin S, Dursun E, et al. Influence of duration of total breast-feeding on bone mineral density in a Turkish population: does the priority of risk factors differ from society to society? Osteoporos Int. 2006;17(5):651-5. doi: 10.1007/s00198-0050029-2. PMID: 16508701. Exclusion Code: X8.

242. Duyan Camurdan A, Ozkan S, Yuksel D, et al. The effect of the baby-friendly hospital initiative on long-term breast feeding. Int $\mathrm{J}$ Clin Pract. 2007 Aug;61(8):1251-5. doi: 10.1111/j.1742-1241.2007.00926.x. PMID: 17428268. Exclusion Code: X6.

243. Dweck N, Augustine M, Pandya D, et al. NICU lactation consultant increases percentage of outborn versus inborn babies receiving human milk. J Perinatol. 2008 Feb;28(2):136-40. doi: 10.1038/sj.jp.7211888. PMID: 18094704. Exclusion Code: X6.
244. Dyson L, McCormick F, Renfrew MJ. Interventions for promoting the initiation of breastfeeding. Cochrane Database Syst Rev. 2005 Apr 18(2):Cd001688. doi: 10.1002/14651858.CD001688.pub2. PMID: 15846621. Exclusion Code: X3.

245. Eastwood J, Ogbo FA, Hendry A, et al. The Impact of Antenatal Depression on Perinatal Outcomes in Australian Women. PLoS One. 2017;12(1):e0169907. doi: 10.1371/journal.pone.0169907. PMID: 28095461. Exclusion Code: X3.

246. Eastwood JG, Phung H, Barnett B. Postnatal depression and socio-demographic risk: factors associated with Edinburgh Depression Scale scores in a metropolitan area of New South Wales, Australia. Aust N Z J Psychiatry. 2011 Dec;45(12):1040-6. doi: 10.3109/00048674.2011.619160. PMID: 22017687. Exclusion Code: X6.

247. Edwards RA, Bickmore T, Jenkins L, et al. Use of an interactive computer agent to support breastfeeding. Matern Child Health J. 2013 Dec;17(10):1961-8. doi: 10.1007/s10995-013-1222-0. PMID: 23329167. Exclusion Code: X5.

248. Edwards RC, Thullen MJ, Korfmacher J, et al. Breastfeeding and complementary food: randomized trial of community doula home visiting. Pediatrics. 2013 Nov;132 Suppl 2:S160-6. doi: 10.1542/peds.2013-1021P. PMID: 24187119. Exclusion Code: X3.

249. Efrat MW, Esparza S, Mendelson SG, et al. The effect of lactation educators implementing a telephone-based intervention among low-income Hispanics: A randomised trial. Health Educ J. 2015;74(4):424-41. doi: 10.1177/0017896914542666. PMID: 103279390. Exclusion Code: X3.

250. Ehrlich SF, Hedderson MM, Quesenberry $\mathrm{CP}$, Jr., et al. Post-partum weight loss and glucose metabolism in women with gestational diabetes: the DEBI Study. Diabet Med. 2014 Jul;31(7):862-7. doi: 10.1111/dme.12425. PMID: 24597974. Exclusion Code: X6. 
251. Ekstrom A, Nissen E. A mother's feelings for her infant are strengthened by excellent breastfeeding counseling and continuity of care. Pediatrics. 2006 Aug;118(2):e309-14. doi: 10.1542/peds.2005-2064. PMID: 16882775. Exclusion Code: X5.

252. Ekstrom AC, Thorstensson S. Nurses and midwives professional support increases with improved attitudes - design and effects of a longitudinal randomized controlled process-oriented intervention. BMC Pregnancy Childbirth. 2015 Oct 26;15:275. doi: 10.1186/s12884-015-0712-z. PMID: 26503218. Exclusion Code: X3.

253. Elfzzani Z, Ojha S, Dorling J. Education of family members to support weaning to solids and nutrition in later infancy in termborn infants. Cochrane Database of Systematic Reviews: John Wiley \& Sons, Ltd; 2016. Exclusion Code: X3.

254. Elliott-Rudder M, Pilotto L, McIntyre E, et al. Motivational interviewing improves exclusive breastfeeding in an Australian randomised controlled trial. Acta Paediatr. 2014 Jan;103(1):e11-6. doi: 10.1111/apa.12434. PMID: 24117857. Exclusion Code: X3.

255. Emond A, Pollock J, Deave T, et al. An evaluation of the First Parent Health Visitor Scheme. Arch Dis Child. 2002 Mar;86(3):150-7. PMID: 11861228. Exclusion Code: X3.

256. Ericson J, Eriksson M, Hellstrom-Westas L, et al. The effectiveness of proactive telephone support provided to breastfeeding mothers of preterm infants: study protocol for a randomized controlled trial. BMC Pediatr. 2013 May 10;13:73. doi: 10.1186/1471-2431-13-73. PMID: 23663521. Exclusion Code: X3.

257. Escobar GJ, Braveman PA, Ackerson L, et al. A randomized comparison of home visits and hospital-based group follow-up visits after early postpartum discharge. Pediatrics. 2001 Sep;108(3):719-27. PMID: 11533342. Exclusion Code: X3.

258. Evans CJ, Lyons NB, Killien MG. The effect of infant formula samples on breastfeeding practice. J Obstet Gynecol Neonatal Nurs. 1986 Sep-Oct;15(5):401-5. PMID: 3639930. Exclusion Code: X3.
259. Fabian HM, Radestad IJ, Waldenstrom U. Childbirth and parenthood education classes in Sweden. Women's opinion and possible outcomes. Acta Obstet Gynecol Scand. 2005 May;84(5):436-43. doi: 10.1111/j.00016349.2005.00732.x. PMID: 15842207. Exclusion Code: X3.

260. Fahy K, Holschier J. Success or failure with breastfeeding. Aust J Adv Nurs. 1988 MarMay;5(3):12-8. PMID: 3415771. Exclusion Code: X3.

261. Fairbank L, O'Meara S, Renfrew MJ, et al. A systematic review to evaluate the effectiveness of interventions to promote the initiation of breastfeeding. Health Technol Assess. 2000;4(25):1-171. PMID: 11111103. Exclusion Code: X3.

262. Fallon A, Van der Putten D, Dring C, et al. Baby-led compared with scheduled (or mixed) breastfeeding for successful breastfeeding. Cochrane Database Syst Rev. 2014 Jul 31(7):Cd009067. doi: 10.1002/14651858.CD009067.pub2. PMID: 25080010. Exclusion Code: X3.

263. Fallon AB, Hegney D, O'Brien M, et al. An evaluation of a telephone-based postnatal support intervention for infant feeding in a regional Australian city. Birth. 2005 Dec;32(4):291-8. doi: 10.1111/j.07307659.2005.00386.x. PMID: 16336370. Exclusion Code: X6.

264. Fallon V, Groves R, Halford JC, et al. Postpartum Anxiety and Infant-Feeding Outcomes. J Hum Lact. 2016 Nov;32(4):740-58. doi: 10.1177/0890334416662241. PMID: 27565200. Exclusion Code: X5.

265. Fallon V, Komninou S, Bennett KM, et al. The emotional and practical experiences of formula-feeding mothers. Matern Child Nutr. 2017;13(4):n/a-n/a. doi: 10.1111/mcn.12392. PMID: 125350108. Exclusion Code: X3.

266. Feferbaum R. Interventions for promoting the initiation of breastfeeding. Sao Paulo Med J. 2014;132(1):68. doi: 10.1590/15163180.20141321t1. PMID: 24474084. Exclusion Code: X6. 
267. Fehring RJ, Schneider M, Bouchard T. Effectiveness of an Online Natural Family Planning Program for Breastfeeding Women. JOGNN: Journal of Obstetric, Gynecologic \& Neonatal Nursing. 2017;46(4):e129-e37. doi: 10.1016/j.jogn.2017.03.010. PMID: 123973636. Exclusion Code: X3.

268. Feldens CA, Ardenghi TM, Cruz LN, et al. Advising mothers about breastfeeding and weaning reduced pacifier use in the first year of life: a randomized trial. Community Dent Oral Epidemiol. 2013 Aug;41(4):31726. doi: 10.1111/cdoe.12030. PMID: 23240927. Exclusion Code: X5.

269. Feldman-Winter L, Grossman X, Palaniappan A, et al. Removal of industrysponsored formula sample packs from the hospital: does it make a difference? J Hum Lact. 2012 Aug;28(3):380-8. doi: 10.1177/0890334412444350. PMID: 22638306. Exclusion Code: X3.

270. Felice JP, Cassano PA, Rasmussen KM. Pumping human milk in the early postpartum period: its impact on long-term practices for feeding at the breast and exclusively feeding human milk in a longitudinal survey cohort. Am J Clin Nutr. 2016;103(5):1267-77. doi: 10.3945/ajcn.115.115733. PMID: 115169447. Exclusion Code: X4.

271. Feng LP, Chen HL, Shen MY. Breastfeeding and the risk of ovarian cancer: a meta-analysis. J Midwifery Womens Health. 2014 Jul-Aug;59(4):428-37. doi: 10.1111/jmwh.12085. PMID: 25066743. Exclusion Code: X11.

272. Fenger-Grøn J, Fenger-Grøn M, Blunck CH, et al. Low breastfeeding rates and body mass index in Danish children of women with gestational diabetes mellitus. International Breastfeeding Journal. 2015;10(1):1-12. doi: 10.1186/s13006-015-0051-8. PMID: 109993794. Exclusion Code: X5.

273. Fergusson DM, Horwood LJ, Shannon FT, et al. The Christchurch Child Development Study: a review of epidemiological findings. Paediatr Perinat Epidemiol. 1989 Jul;3(3):302-25. PMID: 2671961. Exclusion Code: X3.
274. Ferreira MU, Cardoso MA. Discontinuity indices of exclusive breastfeeding estimated by probit analysis of current status data. Int $\mathrm{J}$ Epidemiol. 1996 Apr;25(2):459-60. PMID: 9119576. Exclusion Code: X3.

275. Figueiredo B, Canario C, Field T. Breastfeeding is negatively affected by prenatal depression and reduces postpartum depression. Psychol Med. 2014

Apr;44(5):927-36. doi: 10.1017/s0033291713001530. PMID: 23822932. Exclusion Code: X10.

276. Finch M, Yoong SL, Thomson RJ, et al. A pragmatic randomised controlled trial of an implementation intervention to increase healthy eating and physical activitypromoting policies, and practices in centrebased childcare services: study protocol. BMJ Open. 2015 May 21;5(5):e006706. doi: 10.1136/bmjopen-2014-006706. PMID: 25998035. Exclusion Code: X3.

277. Flacking R, Dykes F, Ewald U. The influence of fathers' socioeconomic status and paternity leave on breastfeeding duration: a population-based cohort study. Scand J Public Health. 2010 Jun;38(4):33743. doi: $10.1177 / 1403494810362002$. PMID: 20147577. Exclusion Code: X3.

278. Flacking R, Ewald U, Wallin L. Positive effect of kangaroo mother care on long-term breastfeeding in very preterm infants. $\mathrm{J}$ Obstet Gynecol Neonatal Nurs. 2011 MarApr;40(2):190-7. doi: 10.1111/j.15526909.2011.01226.x. PMID: 21410756. Exclusion Code: X3.

279. Flaherman V, Beiler J, Cabana M, et al. Relationship of newborn weight loss to milk supply concern and anxiety: the impact on breastfeeding duration. Matern Child Nutr; 2016. p. 463-72. Exclusion Code: X5.

280. Flaherman VJ, Gay B, Scott C, et al. Randomised trial comparing hand expression with breast pumping for mothers of term newborns feeding poorly. Arch Dis Child Fetal Neonatal Ed. 2012

Jan;97(1):F18-23. doi: 10.1136/adc.2010.209213. PMID: 21747129. Exclusion Code: X3. 
281. Flores-Anton B, Temboury-Molina MC, Ares-Segura S, et al. Breastfeeding promotion plan in Madrid, Spain. J Hum Lact. 2012 Aug;28(3):363-9. doi: 10.1177/0890334412449516. PMID: 22689708. Exclusion Code: X5.

282. Flores-Quijano ME, Cordova A, ContrerasRamirez V, et al. Risk for postpartum depression, breastfeeding practices, and mammary gland permeability. J Hum Lact. 2008 Feb;24(1):50-7. doi: 10.1177/0890334407310587. PMID: 18281356. Exclusion Code: X5.

283. Forster D, McLachlan H, Lumley J. Factors associated with breastfeeding at six months postpartum in a group of Australian women. International breastfeeding journal; 2006. p. 18. Exclusion Code: X3.

284. Forster D, McLachlan H, Lumley J, et al. Two mid-pregnancy interventions to increase the initiation and duration of breastfeeding: a randomized controlled trial. Birth. 2004 Sep;31(3):176-82. doi: 10.1111/j.0730-7659.2004.00302.x. PMID: 15330879. Exclusion Code: X3.

285. Forster DA, Jacobs S, Amir LH, et al. Safety and efficacy of antenatal milk expressing for women with diabetes in pregnancy: protocol for a randomised controlled trial. BMJ Open. 2014 Oct 30;4(10):e006571. doi: 10.1136/bmjopen-2014-006571. PMID: 25358679. Exclusion Code: X3.

286. Forster DA, McLachlan HL, Davey MA, et al. Ringing Up about Breastfeeding: a randomised controlled trial exploring early telephone peer support for breastfeeding (RUBY) - trial protocol. BMC Pregnancy Childbirth. 2014 May 28;14:177. doi: 10.1186/1471-2393-14-177. PMID: 24886264. Exclusion Code: X6.

287. Francescon J, Haile ZT, Kling D, et al. Association Between WIC Enrollment and Exclusive Breastfeeding at 3 Months Postpartum Among Low-Income Mothers. J Am Osteopath Assoc. 2016 Dec 01;116(12):770-9. doi: 10.7556/jaoa.2016.152. PMID: 27893143. Exclusion Code: X3.
288. Frank DA, Wirtz SJ, Sorenson JR, et al. Commercial discharge packs and breastfeeding counseling: effects on infant-feeding practices in a randomized trial. Pediatrics. 1987 Dec;80(6):845-54. PMID: 3684395. Exclusion Code: X3.

289. Fraser A, Tilling K, Macdonald-Wallis C, et al. Associations of gestational weight gain with maternal body mass index, waist circumference, and blood pressure measured 16 y after pregnancy: the Avon Longitudinal Study of Parents and Children (ALSPAC). Am J Clin Nutr. 2011 Jun;93(6):1285-92. doi: 10.3945/ajcn.110.008326. PMID: 21471282. Exclusion Code: X3.

290. Frick KD, Pugh LC, Milligan RA. Costs related to promoting breastfeeding among urban low-income women. J Obstet Gynecol Neonatal Nurs. 2012 Jan-Feb;41(1):144-50. doi: 10.1111/j.1552-6909.2011.01316.x. PMID: 22151148. Exclusion Code: X5.

291. Friebel TM, Domchek SM, Rebbeck TR. Modifiers of cancer risk in BRCA1 and BRCA2 mutation carriers: systematic review and meta-analysis. J Natl Cancer Inst. 2014 Jun;106(6):dju091. doi: 10.1093/jnci/dju091. PMID: 24824314. Exclusion Code: X3.

292. Friedman S, Flidel-Rimon O, Lavie E, et al. The effect of prenatal consultation with a neonatologist on human milk feeding in preterm infants. Acta Paediatr. 2004 Jun;93(6):775-8. PMID: 15244226. Exclusion Code: X3.

293. Friel J. Short-term neonatal breastfeeding. Journal of human lactation : official journal of International Lactation Consultant Association; 1999. p. 97-8. Exclusion Code: X3.

294. Friesen CA, Hormuth LJ, Cardarelli TL. Community-Based Participatory Initiatives to Increase Breastfeeding Rates in Indiana. J Hum Lact. 2015 Nov;31(4):600-6. doi: 10.1177/0890334415599974. PMID: 26293654. Exclusion Code: X5. 
295. Friesen CA, Hormuth LJ, Petersen D, et al. Using Videoconferencing Technology to Provide Breastfeeding Support to LowIncome Women: Connecting Hospital-Based Lactation Consultants with Clients Receiving Care at a Community Health Center. J Hum Lact. 2015 Nov;31(4):595-9. doi: 10.1177/0890334415601088. PMID: 26297347. Exclusion Code: X3.

296. Froh EB, Spatz DL. Navigating Return to Work and Breastfeeding in a Hospital with a Comprehensive Employee Lactation Program. J Hum Lact. 2016 Nov;32(4):68994. doi: $10.1177 / 0890334416663475$. PMID: 27601461. Exclusion Code: X6.

297. Froozani MD, Permehzadeh K, Motlagh AR, et al. Effect of breastfeeding education on the feeding pattern and health of infants in their first 4 months in the Islamic Republic of Iran. Bull World Health Organ. 1999;77(5):381-5. PMID: 10361754. Exclusion Code: X3.

298. Fu IC, Fong DY, Heys M, et al. Professional breastfeeding support for first-time mothers: a multicentre cluster randomised controlled trial. BJOG. 2014 Dec;121(13):1673-83. doi: 10.1111/1471-0528.12884. PMID: 24861802. Exclusion Code: X3.

299. Furman L, Matthews L, Davis V, et al. Breast for Success: A CommunityAcademic Collaboration to Increase Breastfeeding Among High-Risk Mothers in Cleveland. Prog Community Health Partnersh. 2016;10(3):341-53. doi: 10.1353/cpr.2016.0041. PMID: 28230542. Exclusion Code: X4.

300. Gagnon AJ. Individual or group antenatal education for childbirth/parenthood. Cochrane Database Syst Rev. 2000(4):Cd002869. doi: 10.1002/14651858.cd002869. PMID: 11034780. Exclusion Code: X3.

301. Gagnon AJ, Dougherty G, Jimenez V, et al. Randomized trial of postpartum care after hospital discharge. Pediatrics. 2002 Jun;109(6):1074-80. PMID: 12042545. Exclusion Code: X3.
302. Gallagher LG, Davis LB, Ray RM, et al. Reproductive history and mortality from cardiovascular disease among women textile workers in Shanghai, China. Int J Epidemiol. 2011 Dec;40(6):1510-8. doi: 10.1093/ije/dyr134. PMID: 22158661. Exclusion Code: X8.

303. Gallegos D, Russell-Bennett R, Previte J, et al. Can a text message a week improve breastfeeding? BMC Pregnancy Childbirth; 2014. Exclusion Code: X3.

304. Gallo MV, Schell LM, DeCaprio AP, et al. Levels of persistent organic pollutant and their predictors among young adults. Chemosphere. 2011 May;83(10):1374-82. doi: 10.1016/j.chemosphere.2011.02.071. PMID: 21458024. Exclusion Code: X3.

305. Gammon MD, Neugut AI, Santella RM, et al. The Long Island Breast Cancer Study Project: description of a multi-institutional collaboration to identify environmental risk factors for breast cancer. Breast Cancer Res Treat. 2002 Jun;74(3):235-54. PMID: 12206514. Exclusion Code: X10.

306. Garcia-de-Leon-Gonzalez R, Oliver-Roig A, Hernandez-Martinez M, et al. Becoming baby-friendly in Spain: a qualityimprovement process. Acta Paediatr. 2011 Mar;100(3):445-50. doi: 10.1111/j.16512227.2010.02061.x. PMID: 20955351. Exclusion Code: X6.

307. Garcia-Fortea P, Gonzalez-Mesa E, Blasco $\mathrm{M}$, et al. Oxytocin administered during labor and breast-feeding: a retrospective cohort study. J Matern Fetal Neonatal Med. 2014 Oct;27(15):1598-603. doi: 10.3109/14767058.2013.871255. PMID: 24289796. Exclusion Code: X3.

308. Gardner H, Green K, Gardner A. Infant Feeding Practices of Emirati Women in the Rapidly Developing City of Abu Dhabi, United Arab Emirates. Int J Environ Res Public Health. 2015 Sep 02;12(9):10923-40. doi: 10.3390/ijerph120910923. PMID: 26404348. Exclusion Code: X3. 
309. Garmendia ML, Corvalan C, Araya M, et al. Effectiveness of a normative nutrition intervention (diet, physical activity and breastfeeding) on maternal nutrition and offspring growth: the Chilean maternal and infant nutrition cohort study (CHiMINCs). BMC Pregnancy Childbirth. 2015 Aug 18;15:175. doi: 10.1186/s12884-015-06051. PMID: 26283529. Exclusion Code: X3.

310. Gathwala G, Singh B, Singh J. Effect of Kangaroo Mother Care on physical growth, breastfeeding and its acceptability. Trop Doct. 2010 Oct;40(4):199-202. doi: 10.1258/td.2010.090513. PMID: 20667921. Exclusion Code: X3.

311. Gaudet MM, Press MF, Haile RW, et al. Risk factors by molecular subtypes of breast cancer across a population-based study of women 56 years or younger. Breast Cancer Res Treat. 2011 Nov;130(2):587-97. doi: 10.1007/s10549-011-1616-x. PMID: 21667121. Exclusion Code: X10.

312. Gavine A, MacGillivray S, Renfrew MJ, et al. Education and training of healthcare staff in the knowledge, attitudes and skills needed to work effectively with breastfeeding women: a systematic review. International Breastfeeding Journal. 2017;12:1-10. doi: 10.1186/s13006-016-0097-2. PMID: 122996483. Exclusion Code: X5.

313. Gercek E, Sarikaya Karabudak S, Ardic Celik N, et al. The relationship between breastfeeding self-efficacy and LATCH scores and affecting factors. J Clin Nurs. 2017 Apr;26(7-8):994-1004. doi: 10.1111/jocn.13423. PMID: 27272098. Exclusion Code: X3.

314. Ghavane S, Murki S, Subramanian S, et al. Kangaroo Mother Care in Kangaroo ward for improving the growth and breastfeeding outcomes when reaching term gestational age in very low birth weight infants. Acta Paediatr. 2012 Dec;101(12):e545-9. doi: 10.1111/apa.12023. PMID: 23016710. Exclusion Code: X3.

315. Gianni ML, Roggero P, Amato O, et al. Intervention for promoting breast milk use in neonatal intensive care unit: a pilot study. J Matern Fetal Neonatal Med. 2014 Mar;27(5):475-8. doi: 10.3109/14767058.2013.818971. PMID: 23796208. Exclusion Code: X6.
316. Giglia R, Binns C. The effectiveness of the internet in improving breastfeeding outcomes: a systematic review. J Hum Lact. 2014 May;30(2):156-60. doi: 10.1177/0890334414527165. PMID: 24646682. Exclusion Code: X3.

317. Giglia R, Cox K, Zhao Y, et al. Exclusive breastfeeding increased by an internet intervention. Breastfeed Med. 2015 JanFeb;10(1):20-5. doi: 10.1089/bfm.2014.0093. PMID: 25358119. Exclusion Code: X3.

318. Gijsbers B, Mesters I, Knottnerus J, et al. The success of an educational program to promote exclusive breastfeeding for 6 months in families with a history of asthma: A randomized controlled trial. Pediatr Asthma Allergy Immunol; 2006. p. 214-22. Exclusion Code: X3.

319. Gill SL, Reifsnider E, Lucke JF. Effects of support on the initiation and duration of breastfeeding. West J Nurs Res. 2007 Oct;29(6):708-23. doi: 10.1177/0193945906297376. PMID: 17557933. Exclusion Code: X3.

320. Giovannini M, Banderali G, Radaelli G, et al. Monitoring breastfeeding rates in Italy: national surveys 1995 and 1999. Acta Paediatr. 2003;92(3):357-63. PMID: 12725553. Exclusion Code: X3.

321. Girard LC, Cote SM, de Lauzon-Guillain B, et al. Factors Associated with Breastfeeding Initiation: A Comparison between France and French-Speaking Canada. PLoS One. 2016;11(11):e0166946. doi: 10.1371/journal.pone.0166946. PMID: 27902741. Exclusion Code: X3.

322. Girish M, Mujawar N, Gotmare P, et al. Impact and feasibility of breast crawl in a tertiary care hospital. J Perinatol. 2013 Apr;33(4):288-91. doi: 10.1038/jp.2012.109. PMID: 22918546. Exclusion Code: X3.

323. Glaser DB, Roberts KJ, Grosskopf NA, et al. An Evaluation of the Effectiveness of School-Based Breastfeeding Education. J Hum Lact. 2016 Feb;32(1):46-52. doi: 10.1177/0890334415595040. PMID: 26173810. Exclusion Code: X5. 
324. Glerean M, Furci A, Galich AM, et al. Bone and mineral metabolism in primiparous women and its relationship with breastfeeding: a longitudinal study. Medicina (B Aires). 2010;70(3):227-32. PMID: 20529771. Exclusion Code: X5.

325. Gluud C, Nikolova D, Klingenberg S. Cochrane Hepato-Biliary Group. About The Cochrane Collaboration: John Wiley \& Sons, Ltd; 2014. Exclusion Code: X1.

326. Gonzalez HF, Malpeli A, Mansur JL, et al. Changes in body composition in lactating adolescent mothers. Arch Latinoam Nutr. 2005 Sep;55(3):252-6. PMID: 16454051. Exclusion Code: X5.

327. Gonzalez-Jimenez E, Garcia PA, Aguilar $\mathrm{MJ}$, et al. Breastfeeding and the prevention of breast cancer: a retrospective review of clinical histories. J Clin Nurs. 2014 Sep;23(17-18):2397-403. doi: 10.1111/jocn.12368. PMID: 23937211. Exclusion Code: X6.

328. Gonzalez-Sistal A, Sanchez AB, Del Rio $\mathrm{MC}$, et al. Association between tumor size and immunohistochemical expression of $\mathrm{Ki}$ 67, p53 and BCL2 in a node-negative breast cancer population selected from a breast cancer screening program. Anticancer Res. 2014 Jan;34(1):269-73. PMID: 24403473. Exclusion Code: X4.

329. Goyal NK, Attanasio LB, Kozhimannil KB. Hospital care and early breastfeeding outcomes among late preterm, early-term, and term infants. Birth. 2014 Dec;41(4):3308. doi: 10.1111/birt.12135. PMID: 25294061. Exclusion Code: X6.

330. Graffy J, Taylor J, Williams A, et al. Randomised controlled trial of support from volunteer counsellors for mothers considering breast feeding. BMJ. 2004 Jan 03;328(7430):26. doi: 10.1136/bmj.328.7430.26. PMID: 14703543. Exclusion Code: X3.

331. Grassley JS, Sauls DJ. Evaluation of the supportive needs of adolescents during childbirth intrapartum nursing intervention on adolescents' childbirth satisfaction and breastfeeding rates. J Obstet Gynecol Neonatal Nurs. 2012 Jan-Feb;41(1):33-44. doi: 10.1111/j.1552-6909.2011.01310.x. PMID: 22150878. Exclusion Code: X6.
332. Green J, Roddam A, Pirie K, et al. Reproductive factors and risk of oesophageal and gastric cancer in the Million Women Study cohort. Br J Cancer. 2012 Jan 03;106(1):210-6. doi: 10.1038/bjc.2011.525. PMID: 22127287. Exclusion Code: X5.

333. Greene Z, O'Donnell CP, Walshe M. Oral stimulation for promoting oral feeding in preterm infants. Cochrane Database of Systematic Reviews: John Wiley \& Sons, Ltd; 2016. Exclusion Code: X3.

334. Greenwood K, Littlejohn P. Breastfeeding intentions and outcomes of adolescent mothers in the Starting Out program. Breastfeed Rev. 2002 Nov;10(3):19-23. PMID: 12592776. Exclusion Code: X4.

335. Greggi S, Parazzini F, Paratore MP, et al. Risk factors for ovarian cancer in central Italy. Gynecol Oncol. 2000 Oct;79(1):50-4. doi: 10.1006/gyno.2000.5909. PMID: 11006030. Exclusion Code: X10.

336. Gregory EF, Butz AM, Ghazarian SR, et al. Are unmet breastfeeding expectations associated with maternal depressive symptoms? Acad Pediatr. 2015 MayJun;15(3):319-25. doi: 10.1016/j.acap.2014.12.003. PMID: 25906701. Exclusion Code: X3.

337. Groer MW, Davis MW. Cytokines, infections, stress, and dysphoric moods in breastfeeders and formula feeders. J Obstet Gynecol Neonatal Nurs. 2006 SepOct;35(5):599-607. doi: 10.1111/j.15526909.2006.00083.x. PMID: 16958715. Exclusion Code: X5.

338. Groer MW, Jevitt CM, Sahebzamani F, et al. Breastfeeding status and maternal cardiovascular variables across the postpartum. J Womens Health (Larchmt). 2013 May;22(5):453-9. doi: 10.1089/jwh.2012.3981. PMID: 23659484. Exclusion Code: X5.

339. Gronwald J, Byrski T, Huzarski T, et al. Influence of selected lifestyle factors on breast and ovarian cancer risk in BRCA1 mutation carriers from Poland. Breast Cancer Res Treat. 2006 Jan;95(2):105-9. doi: 10.1007/s10549-005-9051-5. PMID: 16261399. Exclusion Code: X10. 
340. Gross SM, Caulfield LE, Bentley ME, et al. Counseling and motivational videotapes increase duration of breast-feeding in African-American WIC participants who initiate breast-feeding. J Am Diet Assoc. 1998 Feb;98(2):143-8. PMID: 12515413. Exclusion Code: X3.

341. Gross SM, Resnik AK, Cross-Barnet C, et al. The differential impact of WIC peer counseling programs on breastfeeding initiation across the state of Maryland. J Hum Lact. 2009 Nov;25(4):435-43. doi: 10.1177/0890334409342070. PMID: 19652195. Exclusion Code: X6.

342. Gross SM, Resnik AK, Nanda JP, et al. Early postpartum: a critical period in setting the path for breastfeeding success.

Breastfeed Med. 2011 Dec;6(6):407-12. doi: 10.1089/bfm.2010.0089. PMID: 21453122. Exclusion Code: X5.

343. Grossman LK, Harter C, Sachs L, et al. The effect of postpartum lactation counseling on the duration of breast-feeding in low-income women. Am J Dis Child. 1990 Apr;144(4):471-4. PMID: 2321612. Exclusion Code: X3.

344. Grubesic TH, Durbin KM. Community Rates of Breastfeeding Initiation. J Hum Lact. 2016 Nov;32(4):601-10. doi: 10.1177/0890334416651070. PMID: 27502514. Exclusion Code: X3.

345. Gryboski KL. Maternal and non-maternal time-allocation to infant care, and care during infant illness in rural Java, Indonesia. Soc Sci Med. 1996 Jul;43(2):209-19. PMID: 8844925. Exclusion Code: X3.

346. Gu Y, Zhu Y, Zhang Z, et al. Effectiveness of a theory-based breastfeeding promotion intervention on exclusive breastfeeding in China: A randomised controlled trial. Midwifery. 2016 Nov;42:93-9. doi: 10.1016/j.midw.2016.09.010. PMID: 27788416. Exclusion Code: X3.

347. Guendelman S, Goodman J, Kharrazi M, et al. Work-family balance after childbirth: the association between employer-offered leave characteristics and maternity leave duration. Matern Child Health J. 2014 Jan;18(1):2008. doi: 10.1007/s10995-013-1255-4. PMID: 23504130. Exclusion Code: X5.
348. Guendelman S, Kosa JL, Pearl M, et al. Juggling work and breastfeeding: effects of maternity leave and occupational characteristics. Pediatrics. 2009 Jan;123(1):e38-46. doi: 10.1542/peds.20082244. PMID: 19117845. Exclusion Code: $\mathrm{X} 4$.

349. Guerra GV, Cecatti JG, Souza JP, et al. Elective induction versus spontaneous labour in Latin America. Bull World Health Organ. 2011 Sep 01;89(9):657-65. doi: 10.2471/blt.08.061226. PMID: 21897486. Exclusion Code: X3.

350. Guise JM, Palda V, Westhoff C, et al. The effectiveness of primary care-based interventions to promote breastfeeding: systematic evidence review and metaanalysis for the US Preventive Services Task Force. Ann Fam Med. 2003 JulAug;1(2):70-8. PMID: 15040435. Exclusion Code: X3.

351. Gulmezoglu AM, Langer A, Piaggio G, et al. Cluster randomised trial of an active, multifaceted educational intervention based on the WHO Reproductive Health Library to improve obstetric practices. BJOG. 2007 Jan;114(1):16-23. doi: 10.1111/j.14710528.2006.01091.x. PMID: 17010115. Exclusion Code: X5.

352. Gunderson EP, Crites Y, Chiang V, et al. Influence of breastfeeding during the postpartum oral glucose tolerance test on plasma glucose and insulin. Obstet Gynecol. 2012 Jul;120(1):136-43. doi: 10.1097/AOG.0b013e31825b993d. PMID: 22914402. Exclusion Code: X4.

353. Gunderson EP, Hurston SR, Dewey KG, et al. The study of women, infant feeding and type 2 diabetes after GDM pregnancy and growth of their offspring (SWIFT Offspring study): prospective design, methodology and baseline characteristics. BMC Pregnancy Childbirth. 2015 Jul 17;15:150. doi: 10.1186/s12884-015-0587-z. PMID: 26177722. Exclusion Code: X9. 
354. Gunderson EP, Jacobs DR, Jr., Chiang V, et al. Duration of lactation and incidence of the metabolic syndrome in women of reproductive age according to gestational diabetes mellitus status: a 20-Year prospective study in CARDIA (Coronary Artery Risk Development in Young Adults). Diabetes. 2010 Feb;59(2):495-504. doi: 10.2337/db09-1197. PMID: 19959762. Exclusion Code: X5.

355. Gunderson EP, Kim C, Quesenberry CP, Jr., et al. Lactation intensity and fasting plasma lipids, lipoproteins, non-esterified free fatty acids, leptin and adiponectin in postpartum women with recent gestational diabetes mellitus: the SWIFT cohort. Metabolism. 2014 Jul;63(7):941-50. doi: 10.1016/j.metabol.2014.04.006. PMID: 24931281. Exclusion Code: X5.

356. Gunderson EP, Lewis CE, Wei GS, et al. Lactation and changes in maternal metabolic risk factors. Obstet Gynecol. 2007

Mar;109(3):729-38. doi: 10.1097/01.aog.0000252831.06695.03. PMID: 17329527. Exclusion Code: X5.

357. Gunderson EP, Matias SL, Hurston SR, et al. Study of Women, Infant Feeding, and Type 2 diabetes mellitus after GDM pregnancy (SWIFT), a prospective cohort study: methodology and design. BMC Public Health. 2011 Dec 23;11:952. doi: 10.1186/1471-2458-11-952. PMID: 22196129. Exclusion Code: X9.

358. Gunderson EP, Quesenberry CP, Jr., Ning $\mathrm{X}$, et al. Lactation Duration and Midlife Atherosclerosis. Obstet Gynecol. 2015 Aug;126(2):381-90. doi: 10.1097/aog.0000000000000919. PMID: 26241429. Exclusion Code: X5.

359. Gunn J, Lumley J, Chondros P, et al. Does an early postnatal check-up improve maternal health: results from a randomised trial in Australian general practice. $\mathrm{Br} \mathrm{J}$ Obstet Gynaecol. 1998 Sep;105(9):991-7. PMID: 9763051. Exclusion Code: X3.

360. Gunn TR, Thompson JM, Jackson H, et al. Does early hospital discharge with home support of families with preterm infants affect breastfeeding success? A randomized trial. Acta Paediatr. 2000 Nov;89(11):135863. PMID: 11106050. Exclusion Code: X3.
361. Gwinn ML, Lee NC, Rhodes PH, et al. Pregnancy, breast feeding, and oral contraceptives and the risk of epithelial ovarian cancer. J Clin Epidemiol. 1990;43(6):559-68. PMID: 2348208. Exclusion Code: X10.

362. Hadjisavvas A, Loizidou MA, Middleton N, et al. An investigation of breast cancer risk factors in Cyprus: a case control study. BMC Cancer. 2010 Aug 23;10:447. doi: 10.1186/1471-2407-10-447. PMID: 20727220. Exclusion Code: X10.

363. Haga SM, Ulleberg P, Slinning K, et al. A longitudinal study of postpartum depressive symptoms: multilevel growth curve analyses of emotion regulation strategies, breastfeeding self-efficacy, and social support. Arch Womens Ment Health. 2012 Jun;15(3):175-84. doi: 10.1007/s00737-0120274-2. PMID: 22451329. Exclusion Code: X3.

364. Hahn-Holbrook J, Haselton MG, Dunkel Schetter C, et al. Does breastfeeding offer protection against maternal depressive symptomatology?: A prospective study from pregnancy to 2 years after birth. Arch Womens Ment Health. 2013 Oct;16(5):41122. doi: 10.1007/s00737-013-0348-9. PMID: 23749095. Exclusion Code: X10.

365. Haider R, Islam A, Hamadani J, et al. Breast-feeding counselling in a diarrhoeal disease hospital. Bull World Health Organ. 1996;74(2):173-9. PMID: 8706233. Exclusion Code: X3.

366. Hake-Brooks SJ, Anderson GC. Kangaroo care and breastfeeding of mother-preterm infant dyads 0-18 months: a randomized, controlled trial. Neonatal Netw. 2008 MayJun;27(3):151-9. doi: 10.1891/07300832.27.3.151. PMID: 18557262. Exclusion Code: X3.

367. Hallowell SG, Rogowski JA, Spatz DL, et al. Factors associated with infant feeding of human milk at discharge from neonatal intensive care: Cross-sectional analysis of nurse survey and infant outcomes data. Int J Nurs Stud. 2016 Jan;53:190-203. doi: 10.1016/j.ijnurstu.2015.09.016. PMID: 26518107. Exclusion Code: X6. 
368. Hamad R, Rehkopf DH. Poverty, Pregnancy, and Birth Outcomes: A Study of the Earned Income Tax Credit. Paediatr Perinat Epidemiol. 2015 Sep;29(5):444-52. doi: 10.1111/ppe.12211. PMID: 26212041. Exclusion Code: X3.

369. Hamade H, Chaaya M, Saliba M, et al. Determinants of exclusive breastfeeding in an urban population of primiparas in Lebanon: a cross-sectional study. BMC Public Health. 2013 Jul 31;13:702. doi: 10.1186/1471-2458-13-702. PMID: 23902627. Exclusion Code: X3.

370. Hamdan A, Tamim H. Psychosocial risk and protective factors for postpartum depression in the United Arab Emirates. Arch Womens Ment Health. 2011 Apr;14(2):125-33. doi: 10.1007/s00737-010-0189-8. PMID: 21063891. Exclusion Code: X10.

371. Hamilton AE. Development of environmentally friendly messages to promote longer durations of breastfeeding for already breastfeeding mothers. Health Commun. 2015;30(3):231-40. doi: 10.1080/10410236.2013.840483. PMID: 24579567. Exclusion Code: X5.

372. Hammarberg K, Rowe HJ, Fisher JR. Early post-partum adjustment and admission to parenting services in Victoria, Australia after assisted conception. Hum Reprod. 2009 Nov;24(11):2801-9. doi: 10.1093/humrep/dep282. PMID: 19661124. Exclusion Code: X4.

373. Hanafi M, Shalaby S, Falatah N, et al. Impact of health education on knowledge of, attitude to and practice of breastfeeding among women attending primary health care centres in Almadinah Almunawwarah, Kingdom of Saudi Arabia: Controlled prepost study. Journal of Taibah University Medical Sciences; 2014. p. 187-93. Exclusion Code: X3.

374. Hannah P, Adams D, Lee A, et al. Links between early post-partum mood and postnatal depression. Br J Psychiatry. 1992 Jun;160:777-80. PMID: 1617360. Exclusion Code: X10.
375. Hannula L, Kaunonen M, Tarkka MT. A systematic review of professional support interventions for breastfeeding. J Clin Nurs. 2008 May;17(9):1132-43. doi: 10.1111/j.1365-2702.2007.02239.x. PMID: 18416790. Exclusion Code: X3.

376. Hansen MA, Overgaard K, Riis BJ, et al. Potential risk factors for development of postmenopausal osteoporosis--examined over a 12-year period. Osteoporos Int. 1991 Feb;1(2):95-102. PMID: 1790399. Exclusion Code: X5.

377. Harder T, Bergmann R, Kallischnigg G, et al. Duration of breastfeeding and risk of overweight: a meta-analysis. Am J Epidemiol. 2005 Sep 01;162(5):397-403. doi: 10.1093/aje/kwi222. PMID: 16076830. Exclusion Code: X5.

378. Hardy EE, Vichi AM, Sarmento RC, et al. Breastfeeding promotion: effect of an educational program in Brazil. Stud Fam Plann. 1982 Mar;13(3):79-86. PMID: 7147316. Exclusion Code: X3.

379. Haroon S, Das JK, Salam RA, et al. Breastfeeding promotion interventions and breastfeeding practices: a systematic review. BMC Public Health. 2013;13 Suppl 3:S20. doi: 10.1186/1471-2458-13-s3-s20. PMID: 24564836. Exclusion Code: X3.

380. Harris SJ, Farren MD, Janssen PA, et al. Single room maternity care: perinatal outcomes, economic costs, and physician preferences (Structured abstract). Journal of Obstetrics and Gynaecology Canada; 2004. p. 633-40. Exclusion Code: X3.

381. Harris SJ, Janssen PA, Saxell L, et al. Effect of a collaborative interdisciplinary maternity care program on perinatal outcomes. CMAJ. 2012 Nov 20;184(17):1885-92. doi: 10.1503/cmaj.111753. PMID: 22966055. Exclusion Code: X3.

382. Hart E. Duration of breastfeeding in women with varied prenatal preparation and postpartum followup. J Nurse Midwifery. 1981 Jan-Feb;26(1):38. PMID: 6907303. Exclusion Code: X3.

383. Hartge P, Schiffman MH, Hoover R, et al. A case-control study of epithelial ovarian cancer. Am J Obstet Gynecol. 1989 Jul;161(1):10-6. PMID: 2750791. Exclusion Code: X10. 
384. Hartley BM, O'Connor ME. Evaluation of the 'Best Start' breast-feeding education program. Arch Pediatr Adolesc Med. 1996 Aug;150(8):868-71. PMID: 8704896. Exclusion Code: X6.

385. Hassa H, Tanir HM, Senses T, et al. Related factors in bone mineral density of lumbal and femur in natural postmenopausal women. Arch Gynecol Obstet. 2005 Dec;273(2):86-9. doi: 10.1007/s00404-0050015-0. PMID: 16001199. Exclusion Code: $\mathrm{X} 8$.

386. Hasselmann MH, Werneck GL, Silva CV. Symptoms of postpartum depression and early interruption of exclusive breastfeeding in the first two months of life. Cad Saude Publica. 2008;24 Suppl 2:S341-52. PMID: 18670714. Exclusion Code: X3.

387. Hatton D, Harrison-Hohner J, Coste S, et al. Symptoms of postpartum depression and breastfeeding. Journal of human lactation : official journal of International Lactation Consultant Association; 2005. p. 444-9; quiz 50-4. Exclusion Code: X10.

388. Hauck YL, Dimmock JE. Evaluation of an information booklet on breastfeeding duration: a clinical trial. J Adv Nurs. 1994 Nov;20(5):836-43. PMID: 7745174. Exclusion Code: X3.

389. Hay AE, Campbell CMA. Volunteer counsellors for supporting breast feeding...Graffy J, Taylor J, Williams A et al. Randomised controlled trial of support from volunteer counsellors for mothers considering breast feeding. BMJ 2004;328:26. (3 January). BMJ: British Medical Journal (International Edition). 2004;328(7435):349-. doi: 10.1136/bmj.328.7435.349. PMID: 106732226. Exclusion Code: X1.

390. Hays NP, Mao M, Zhang L, et al. Infant feeding and health-related quality of life in healthy Chinese infants: results from a prospective, observational cohort study. Health Qual Life Outcomes. 2016 Aug 08;14(1):116. doi: 10.1186/s12955-0160518-3. PMID: 27502768. Exclusion Code: $\mathrm{X} 5$.
391. He X, Zhu M, Hu C, et al. Breast-feeding and postpartum weight retention: a systematic review and meta-analysis. Public Health Nutr. 2015 Dec;18(18):3308-16. doi: 10.1017/s1368980015000828. PMID: 25895506. Exclusion Code: X8.

392. Heads J. The Baby Friendly Hospital Initiative: a case study from NSW. N S W Public Health Bull. 2005 Mar-Apr;16(34):63-6. PMID: 16106276. Exclusion Code: $\mathrm{X} 5$.

393. Hector D, King L. Interventions to encourage and support breastfeeding. N S W Public Health Bull. 2005 Mar-Apr;16(34):56-61. PMID: 16106274. Exclusion Code: X6.

394. Hedges S, Simmes D, Martinez A, et al. A home visitation program welcomes home first-time moms and their infants. Home Healthc Nurse. 2005 May;23(5):286-9. PMID: 15891472. Exclusion Code: X3.

395. Helmerhorst F, Kulier R. Cochrane Fertility Regulation Group. About The Cochrane Collaboration: John Wiley \& Sons, Ltd; 2012. Exclusion Code: X1.

396. Henderson A, Stamp G, Pincombe J. Postpartum positioning and attachment education for increasing breastfeeding: a randomized trial. Birth. 2001 Dec;28(4):236-42. PMID: 11903211. Exclusion Code: X3.

397. Henderson JJ, Evans SF, Straton JA, et al. Impact of postnatal depression on breastfeeding duration. Birth. 2003 Sep;30(3):175-80. PMID: 12911800. Exclusion Code: X10.

398. Hendrick CE, Potter JE. Nativity, Country of Education, and Mexican-Origin Women's Breastfeeding Behaviors in the First 10 Months Postpartum. Birth: Issues in Perinatal Care. 2017;44(1):68-77. doi: 10.1111/birt.12261. PMID: 121269192. Exclusion Code: X3.

399. Henninger ML, Irving SA, Kauffman TL, et al. Predictors of Breastfeeding Initiation and Maintenance in an Integrated Healthcare Setting. J Hum Lact. 2017;33(2):256-66. doi: 10.1177/0890334417695202. PMID: 122841960. Exclusion Code: X3. 
400. Henriques A, Santos AC, Guimaraes JT, et al. Healthy excessive weight in Portuguese women 4 years after delivery of a liveborn. Prev Med. 2015 Jun;75:49-55. doi: 10.1016/j.ypmed.2015.03.009. PMID: 25770435. Exclusion Code: X5.

401. Heon M. Interventions infirmieres relatives a l'allaitement maternel de nouveau-nes prematures: Universite de Montreal (Canada); 2011. Exclusion Code: X7.

402. Heon M, Goulet C, Garofalo C, et al. Acceptability and feasibility of a breast milk expression education and support intervention in mothers of preterm infants. Adv Neonatal Care. 2014 Aug;14(4):E9e19. doi: 10.1097/anc.0000000000000113. PMID: 25075927. Exclusion Code: X3.

403. Héon M, Goulet C, Garofalo C, et al. An Intervention to Promote Breast Milk Production in Mothers of Preterm Infants. West J Nurs Res. 2016;38(5):529-52. doi: 10.1177/0193945914557501. PMID: 114575454. Exclusion Code: X3.

404. Heon M, Goulet C, Levy E, et al. Hindmilk: a head start in preterm nutrition (Structured abstract). Enferm Clin; 2009. p. 129-35. Exclusion Code: X3.

405. Herich LC, Cuttini M, Croci I, et al. Maternal Education Is Associated with Disparities in Breastfeeding at Time of Discharge but Not at Initiation of Enteral Feeding in the Neonatal Intensive Care Unit. J Pediatr. 2017 Mar;182:59-65.e7. doi: 10.1016/j.jpeds.2016.10.046. PMID: 27865429. Exclusion Code: X3.

406. Hewat RJ, Ellis DJ. Breastfeeding as a maternal-child team effort: women's perceptions. Health Care Women Int. 1984;5(5-6):437-52. doi: 10.1080/07399338409515671. PMID: 6571010. Exclusion Code: X3.

407. Hewat RJ, Ellis DJ. Similarities and differences between women who breastfeed for short and long duration. Midwifery. 1986 Mar;2(1):37-43. PMID: 3640983. Exclusion Code: X3.

408. Hoddinott P, Britten J, Pill R. Why do interventions work in some places and not others: a breastfeeding support group trial. Soc Sci Med. 2010 Mar;70(5):769-78. doi: 10.1016/j.socscimed.2009.10.067. PMID: 20005617. Exclusion Code: X6.
409. Hoddinott P, Craig L, MacLennan G, et al. The FEeding Support Team (FEST) randomised, controlled feasibility trial of proactive and reactive telephone support for breastfeeding women living in disadvantaged areas. BMJ Open; 2012. Exclusion Code: X3.

410. Hodnett E. Efficacy of home-based peer counseling to promote exclusive breastfeeding: a randomized controlled trial. J Pediatr. 1999 Nov;135(5):649-50. PMID: 10577054. Exclusion Code: X9.

411. Hodnett ED, Gates S, Hofmeyr GJ, et al. Continuous support for women during childbirth. Cochrane Database of Systematic Reviews: John Wiley \& Sons, Ltd; 2013. Exclusion Code: X3.

412. Hoedjes M, Berks D, Vogel I, et al. Effect of postpartum lifestyle interventions on weight loss, smoking cessation, and prevention of smoking relapse: a systematic review (Structured abstract). Obstet Gynecol Surv; 2010. p. 631-52. Exclusion Code: X3.

413. Hofmeyr GJ, Nikodem VC, Wolman WL, et al. Companionship to modify the clinical birth environment: effects on progress and perceptions of labour, and breastfeeding. $\mathrm{Br}$ J Obstet Gynaecol. 1991 Aug;98(8):756-64. PMID: 1911582. Exclusion Code: X3.

414. Holmes AV, McLeod AY, Thesing C, et al. Physician breastfeeding education leads to practice changes and improved clinical outcomes. Breastfeed Med. 2012

Dec;7(6):403-8. doi: 10.1089/bfm.2012.0028. PMID: 23046226. Exclusion Code: X4.

415. Homer CS, Leap N, Edwards N, et al. Midwifery continuity of carer in an area of high socio-economic disadvantage in London: A retrospective analysis of Albany Midwifery Practice outcomes using routine data (1997-2009). Midwifery. 2017 May;48:1-10. doi: 10.1016/j.midw.2017.02.009. PMID: 28284877. Exclusion Code: X3.

416. Hongo H, Green J, Nanishi K, et al. Development of the revised Japanese Maternal Breastfeeding Evaluation Scale, short version. Asia Pac J Clin Nutr. 2017 May;26(3):392-5. doi: 10.6133/apjcn.032016.08. PMID: 28429901. Exclusion Code: X3. 
417. Hongo H, Nanishi K, Shibanuma A, et al. Is baby-friendly breastfeeding support in maternity hospitals associated with breastfeeding satisfaction among Japanese mothers? Matern Child Health J. 2015 Jun;19(6):1252-62. doi: 10.1007/s10995014-1631-8. PMID: 25366103. Exclusion Code: X6.

418. Hopkinson J, Heird W. Maternal response to two electric breast pumps. Breastfeed Med. 2009 Mar;4(1):17-23. doi: 10.1089/bfm.2008.0133. PMID: 19196038. Exclusion Code: X3.

419. Hopkinson J, Konefal Gallagher M. Assignment to a hospital-based breastfeeding clinic and exclusive breastfeeding among immigrant Hispanic mothers: a randomized, controlled trial. J Hum Lact. 2009 Aug;25(3):287-96. doi: 10.1177/0890334409335482. PMID: 19436060. Exclusion Code: X3.

420. Horn J, Opdahl S, Engstrom MJ, et al. Reproductive history and the risk of molecular breast cancer subtypes in a prospective study of Norwegian women. Cancer Causes Control. 2014 Jul;25(7):8819. doi: 10.1007/s10552-014-0388-0. PMID: 24789514. Exclusion Code: X10.

421. Horner S, Simonelli AM, Schmidt H, et al. Setting the stage for successful oral feeding: the impact of implementing the SOFFI feeding program with medically fragile NICU infants. J Perinat Neonatal Nurs. 2014 Jan-Mar;28(1):59-68. doi: 10.1097/jpn.0000000000000003. PMID: 24476653. Exclusion Code: X6.

422. Horodynski MA, Olson B, Baker S, et al. Healthy babies through infant-centered feeding protocol: an intervention targeting early childhood obesity in vulnerable populations. BMC Public Health. 2011 Nov 15;11:868. doi: 10.1186/1471-2458-11-868. PMID: 22085421. Exclusion Code: X3.

423. Houghtaling B, Byker Shanks C, Jenkins M. Likelihood of Breastfeeding Within the USDA's Food and Nutrition Service Special Supplemental Nutrition Program for Women, Infants, and Children Population. J Hum Lact. 2017 Feb;33(1):83-97. doi: 10.1177/0890334416679619. PMID: 28135478. Exclusion Code: X3.
424. Houston M, Howie P, McNeilly A. Nursing mirror midwifery forum. 2. Breast-feeding. Nurs Mirror. 1983 Feb 09;156(6):i-ix. PMID: 6550366. Exclusion Code: X3.

425. Houston MJ, Howie PW. The importance of support for the breast feeding mother at home. Health Visit. 1981 Jun;54(6):243-4. PMID: 6909181. Exclusion Code: X1.

426. Howard C, Howard F, Lawrence R, et al. Office prenatal formula advertising and its effect on breast-feeding patterns. Obstet Gynecol. 2000 Feb;95(2):296-303. PMID: 10674597. Exclusion Code: X3.

427. Howell EA, Bodnar-Deren S, Balbierz A, et al. An intervention to extend breastfeeding among black and Latina mothers after delivery. Am J Obstet Gynecol. 2014 Mar;210(3):239.e1-5. doi: 10.1016/j.ajog.2013.11.028. PMID: 24262719. Exclusion Code: X3.

428. Hruschka DJ, Hagaman A. The physiological cost of reproduction for rich and poor across 65 countries. Am J Hum Biol. 2015 Sep-Oct;27(5):654-9. doi: 10.1002/ajhb.22707. PMID: 25809493. Exclusion Code: X8.

429. Huang MZ, Kuo SC, Avery MD, et al. Evaluating effects of a prenatal web-based breastfeeding education programme in Taiwan. J Clin Nurs. 2007 Aug;16(8):15719. doi: 10.1111/j.1365-2702.2006.01843.x. PMID: 17655546. Exclusion Code: X3.

430. Huang P, Ren J, Liu Y, et al. Factors affecting breastfeeding adherence among Chinese mothers: A multicenter study. Medicine (Baltimore). 2017 Sep;96(38):e7619. doi: 10.1097/md.0000000000007619. PMID: 28930818. Exclusion Code: X3.

431. Hughes A, Gallagher S, Hannigan A. A Cluster Analysis of Reported Sleeping Patterns of 9-Month Old Infants and the Association with Maternal Health: Results from a Population Based Cohort Study. Matern Child Health J. 2015

Aug;19(8):1881-9. doi: 10.1007/s10995015-1701-6. PMID: 25656722. Exclusion Code: X3. 
432. Hurley KM, Black MM, Merry BC, et al. Maternal mental health and infant dietary patterns in a statewide sample of Maryland WIC participants. Matern Child Nutr. 2015 Apr;11(2):229-39. doi: 10.1111/mcn.12004. PMID: 23167622. Exclusion Code: X3.

433. Hwang SS, Barfield WD, Smith RA, et al. Discharge timing, outpatient follow-up, and home care of late-preterm and early-term infants. Pediatrics. 2013 Jul;132(1):101-8. doi: 10.1542/peds.2012-3892. PMID: 23733794. Exclusion Code: X3.

434. Iacovou M, Sevilla A. Infant feeding: the effects of scheduled vs. on-demand feeding on mothers' wellbeing and children's cognitive development. Eur J Public Health. 2013 Feb;23(1):13-9. doi:

10.1093/eurpub/cks012. PMID: 22420982. Exclusion Code: X3.

435. Ickovics JR, Kershaw TS, Westdahl C, et al. Group prenatal care and perinatal outcomes: a randomized controlled trial. Obstet Gynecol. 2007 Aug;110(2 Pt 1):330-9. doi: 10.1097/01.aog.0000275284.24298.23. PMID: 17666608. Exclusion Code: X3.

436. Iheozor-Ejiofor Z, Worthington HV, Walsh $\mathrm{T}$, et al. Water fluoridation for the prevention of dental caries. Cochrane Database of Systematic Reviews: John Wiley \& Sons, Ltd; 2015. Exclusion Code: X3.

437. Iida M, Horiuchi S, Nagamori K. A comparison of midwife-led care versus obstetrician-led care for low-risk women in Japan. Women Birth. 2014 Sep;27(3):202-7. doi: 10.1016/j.wombi.2014.05.001. PMID: 24906495. Exclusion Code: X3.

438. Imdad A, Yakoob MY, Bhutta ZA. Effect of breastfeeding promotion interventions on breastfeeding rates, with special focus on developing countries. BMC Public Health. 2011 Apr 13;11 Suppl 3:S24. doi: 10.1186/1471-2458-11-s3-s24. PMID: 21501442. Exclusion Code: X3.

439. Imsiragic AS, Begic D, Sarajlic I, et al. Predictors of Exclusive Breastfeeding 6-9 Weeks After Delivery: a Prospective Cohort Study. Public Mental Health Perspective. Psychiatr Danub. 2016 Dec;28(4):395-403. PMID: 27855431. Exclusion Code: X8.
440. Inch S, Law S, Wallace L. Hands off! The Breastfeeding Best Start Project (2). Pract Midwife. 2003 Dec;6(11):24-5. PMID: 14694878. Exclusion Code: X5.

441. Ingram J, Johnson D, Condon L. The effects of Baby Friendly Initiative training on breastfeeding rates and the breastfeeding attitudes, knowledge and self-efficacy of community health-care staff. Prim Health Care Res Dev. 2011 Jul;12(3):266-75. doi: 10.1017/s1463423610000423. PMID: 21798124. Exclusion Code: X3.

442. Ingram J, Johnson D, Greenwood R. Breastfeeding in Bristol: teaching good positioning, and support from fathers and families. Midwifery. 2002 Jun;18(2):87-101. PMID: 12139907. Exclusion Code: X3.

443. Ingram J, Rosser J, Jackson D. Breastfeeding peer supporters and a community support group: evaluating their effectiveness. Matern Child Nutr. 2005 Apr;1(2):111-8. doi: 10.1111/j.17408709.2005.00005.x. PMID: 16881886. Exclusion Code: X3.

444. Ingram L, MacArthur C, Khan K, et al. Effect of antenatal peer support on breastfeeding initiation: a systematic review (Structured abstract). CMAJ; 2010. p. 173946. Exclusion Code: X6.

445. Ingram L, MacArthur C, Khan K, et al. Effect of antenatal peer support on breastfeeding initiation: a systematic review. CMAJ. 2010 Nov 09;182(16):1739-46. doi: 10.1503/cmaj.091729. PMID: 20940234.

Exclusion Code: X3.

446. Ip S, Chung M, Raman G, et al.

Breastfeeding and maternal and infant health outcomes in developed countries. Evid Rep Technol Assess (Full Rep). 2007 Apr(153):1-186. PMID: 17764214. Exclusion Code: X10.

447. Ip S, Chung M, Raman G, et al. A summary of the Agency for Healthcare Research and Quality's evidence report on breastfeeding in developed countries. Breastfeed Med. 2009 Oct;4 Suppl 1:S17-30. doi: 10.1089/bfm.2009.0050. PMID: 19827919. Exclusion Code: X6. 
448. Islam T, Matsuo K, Ito $\mathrm{H}$, et al.

Reproductive and hormonal risk factors for luminal, HER2-overexpressing, and triplenegative breast cancer in Japanese women. Ann Oncol. 2012 Sep;23(9):2435-41. doi: 10.1093/annonc/mdr613. PMID: 22328736. Exclusion Code: X10.

449. Islami F, Liu Y, Jemal A, et al. Breastfeeding and breast cancer risk by receptor status--a systematic review and meta-analysis. Ann Oncol. 2015 Dec;26(12):2398-407. doi: 10.1093/annonc/mdv379. PMID: 26504151. Exclusion Code: X11.

450. Iwasaki M, Otani T, Inoue M, et al. Role and impact of menstrual and reproductive factors on breast cancer risk in Japan. Eur J Cancer Prev. 2007 Apr;16(2):116-23. doi: 10.1097/01.cej.0000228410.14095.2d. PMID: 17297387. Exclusion Code: X10.

451. Iyer NP, Srinivasan R, Evans K, et al. Impact of an early weighing policy on neonatal hypernatraemic dehydration and breast feeding. Arch Dis Child. 2008 Apr;93(4):297-9. doi: 10.1136/adc.2006.108415. PMID: 17475691. Exclusion Code: X6.

452. Jaafar S, Ho J, Jahanfar S, et al. Effect of restricted pacifier use in breastfeeding term infants for increasing duration of breastfeeding. Cochrane database of systematic reviews (Online); 2016. Exclusion Code: X3.

453. Jaafar SH, Ho JJ, Jahanfar S, et al. Effect of restricted pacifier use in breastfeeding term infants for increasing duration of breastfeeding. Cochrane Database Syst Rev. 2016 Aug 30(8):Cd007202. doi: 10.1002/14651858.CD007202.pub4. PMID: 27572944. Exclusion Code: X3.

454. Jaafar SH, Ho JJ, Lee KS. Rooming-in for new mother and infant versus separate care for increasing the duration of breastfeeding. Cochrane Database Syst Rev. 2016 Aug 26(8):Cd006641. doi: 10.1002/14651858.CD006641.pub3. PMID: 27562563. Exclusion Code: X3.
455. Jaafar SH, Ho JJ, Lee KS. Rooming-in for new mother and infant versus separate care for increasing the duration of breastfeeding. Cochrane Database of Systematic Reviews: John Wiley \& Sons, Ltd; 2016. Exclusion Code: X3.

456. Jaafar SH, Jahanfar S, Angolkar M, et al. Effect of restricted pacifier use in breastfeeding term infants for increasing duration of breastfeeding. Cochrane Database Syst Rev. 2012 Jul 11(7):Cd007202. doi: 10.1002/14651858.CD007202.pub3. PMID: 22786506. Exclusion Code: X3.

457. Jaafar SH, Lee KS, Ho JJ. Separate care for new mother and infant versus rooming-in for increasing the duration of breastfeeding. Cochrane Database Syst Rev. 2012 Sep 12(9):Cd006641. doi: 10.1002/14651858.CD006641.pub2. PMID: 22972095. Exclusion Code: X3.

458. Jacknowitz A, Novillo D, Tiehen L. Special Supplemental Nutrition Program for Women, Infants, and Children and infant feeding practices. Pediatrics. 2007 Feb;119(2):281-9. doi: 10.1542/peds.20061486. PMID: 17272617. Exclusion Code: X3.

459. Jager S, Jacobs S, Kroger J, et al. Breastfeeding and maternal risk of type 2 diabetes: a prospective study and meta-analysis. Diabetologia. 2014 Jul;57(7):1355-65. doi: 10.1007/s00125-014-3247-3. PMID: 24789344. Exclusion Code: X11.

460. Jamieson DJ, Terrell ML, Aguocha NN, et al. Dietary exposure to brominated flame retardants and abnormal Pap test results. J Womens Health (Larchmt). 2011 Sep;20(9):1269-78. doi: 10.1089/jwh.2010.2275. PMID: 21797757. Exclusion Code: X3.

461. Jang G, Kim S. [Effects of breast-feeding education and support services on breastfeeding rates and infant's growth]. Journal of Korean Academy of Nursing; 2010. p. 277-86. Exclusion Code: X7.

462. Jang G, Kim S, Jeong K. [Effect of postpartum breast-feeding support by nurse on the breast-feeding prevalence]. Taehan Kanho Hakhoe chi; 2008. p. 172-9. Exclusion Code: X7. 
463. Janson S, Rydberg B. Early postpartum discharge and subsequent breastfeeding. Birth. 1998 Dec;25(4):222-5. PMID: 9892889. Exclusion Code: X6.

464. Janssen PA, Livingstone VH, Chang B, et al. Development and evaluation of a Chinese-language newborn feeding hotline: a prospective cohort study. BMC Pregnancy Childbirth. 2009 Jan 29;9:3. doi:

10.1186/1471-2393-9-3. PMID: 19178746.

Exclusion Code: X4.

465. Jarlenski M, McManus J, Diener-West M, et al. Association between support from a health professional and breastfeeding knowledge and practices among obese women: evidence from the Infant Practices Study II. Womens Health Issues. 2014 NovDec;24(6):641-8. doi: 10.1016/j.whi.2014.08.002. PMID: 25239780. Exclusion Code: X3.

466. Jenik AG, Vain NE, Gorestein AN, et al. Does the recommendation to use a pacifier influence the prevalence of breastfeeding? J Pediatr. 2009 Sep;155(3):350-4.e1. doi: 10.1016/j.jpeds.2009.03.038. PMID: 19464025. Exclusion Code: X3.

467. Jenner S. The influence of additional information, advice and support on the success of breast feeding in working class primiparas. Child Care Health Dev. 1988 Sep-Oct;14(5):319-28. PMID: 3228958. Exclusion Code: X3.

468. Jensen E. Participation in the Supplemental Nutrition Program for Women, Infants and Children (WIC) and breastfeeding: national, regional, and state level analyses. Matern Child Health J. 2012 Apr;16(3):624-31. doi: 10.1007/s10995-011-0796-7. PMID: 21505775. Exclusion Code: X3.

469. Jernstrom H, Lubinski J, Lynch HT, et al. Breast-feeding and the risk of breast cancer in BRCA1 and BRCA2 mutation carriers. J Natl Cancer Inst. 2004 Jul 21;96(14):10948. doi: 10.1093/jnci/djh211. PMID: 15265971. Exclusion Code: X10.

470. Jiang H, Li M, Wen LM, et al. Effect of short message service on infant feeding practice: findings from a community-based study in Shanghai, China. JAMA Pediatr. 2014 May;168(5):471-8. doi: 10.1001/jamapediatrics.2014.58. PMID: 24639004. Exclusion Code: X8.
471. Jiang X, Duan X, Wang J. A meta-analysis of breastfeeding and osteoporotic fracture risk in the females. Osteoporos Int. 2017;28(2):495-503. doi: 10.1007/s00198016-3753-x. PMID: 120966311. Language: English. Entry Date: 20170211. Revision Date: 20170211. Publication Type: Article. Journal Subset: Biomedical. Exclusion Code: X11.

472. Jirojwong S, Rossi D, Walker S, et al. What were the outcomes of home follow-up visits after postpartum hospital discharge? Aust J Adv Nurs. 2005 Sep-Nov;23(1):22-30. PMID: 16496814. Exclusion Code: X3.

473. Joham AE, Nanayakkara N, Ranasinha S, et al. Obesity, polycystic ovary syndrome and breastfeeding: an observational study. Acta Obstet Gynecol Scand. 2016 Apr;95(4):45866. doi: 10.1111/aogs.12850. PMID: 26782709. Exclusion Code: X5.

474. Johns HM, Amir LH, McLachlan HL, et al. Breast pump use amongst mothers of healthy term infants in Melbourne, Australia: A prospective cohort study. Midwifery. 2016;33:82-9. doi: 10.1016/j.midw.2015.10.009. PMID: 115685226. Exclusion Code: X3.

475. Johnson A, Kirk R, Rosenblum KL, et al. Enhancing breastfeeding rates among African American women: a systematic review of current psychosocial interventions. Breastfeed Med. 2015 JanFeb;10(1):45-62. doi: 10.1089/bfm.2014.0023. PMID: 25423601. Exclusion Code: X3.

476. Johnson DB, Lamson E, Schwartz R, et al. A Community Health Clinic BreastfeedingFriendly Pilot: What Can We Learn about the Policy Process? J Hum Lact. 2015

Nov;31(4):660-70. doi: 10.1177/0890334415579656. PMID: 25832650. Exclusion Code: X5.

477. Johnston BD, Huebner CE, Anderson ML, et al. Healthy steps in an integrated delivery system: child and parent outcomes at 30 months. Arch Pediatr Adolesc Med. 2006 Aug;160(8):793-800. doi: 10.1001/archpedi.160.8.793. PMID: 16894077. Exclusion Code: X3. 
478. Johnston JC, McNeil D, van der Lee G, et al. Piloting CenteringParenting in Two Alberta Public Health Well-Child Clinics. Public Health Nurs. 2017 May;34(3):22937. doi: 10.1111/phn.12287. PMID: 27501111. Exclusion Code: X3.

479. Jolly K, Ingram L, Freemantle N, et al. Effect of a peer support service on breastfeeding continuation in the UK: a randomised controlled trial. Midwifery. 2012 Dec;28(6):740-5. doi: 10.1016/j.midw.2011.08.005. PMID: 21944571. Exclusion Code: X3.

480. Jolly K, Ingram L, Khan KS, et al. Systematic review of peer support for breastfeeding continuation: metaregression analysis of the effect of setting, intensity, and timing. BMJ. 2012 Jan 25;344:d8287. doi: 10.1136/bmj.d8287. PMID: 22277543. Exclusion Code: X3.

481. Jones DA, West RR. Lactation nurse increases duration of breast feeding. Arch Dis Child. 1985 Aug;60(8):772-4. PMID: 4037866. Exclusion Code: X3.

482. Jones E, Jones P, Spencer A. Breastfeeding and returning to work. Pract Midwife. 2004 Dec;7(11):17-8, 20, 2. PMID: 15624531. Exclusion Code: X3.

483. Jones G, Scott F. Low bone mass in premenopausal parous women: identification and the effect of an information and bone density feedback program. J Clin Densitom. 1999 Summer;2(2):109-15. PMID: 10499969. Exclusion Code: X6.

484. Jones R, Jones L, Feary A-M. The Effects of Single-Family Rooms on Parenting Behavior and Maternal Psychological Factors. JOGNN: Journal of Obstetric, Gynecologic \& Neonatal Nursing. 2016;45(3):359-70. doi: 10.1016/j.jogn.2015.12.015. PMID: 116760196. Exclusion Code: X3.

485. Jonsdottir OH, Fewtrell MS, Gunnlaugsson $\mathrm{G}$, et al. Initiation of complementary feeding and duration of total breastfeeding: unlimited access to lactation consultants versus routine care at the well-baby clinics. Breastfeed Med. 2014 May;9(4):196-202. doi: 10.1089/bfm.2013.0094. PMID: 24621390. Exclusion Code: X6.
486. Jordan S, Murphy FA, Boucher C, et al. High dose versus low dose opioid epidural regimens for pain relief in labour. Cochrane Database of Systematic Reviews: John Wiley \& Sons, Ltd; 2016. Exclusion Code: X3.

487. Jordan SJ, Cushing-Haugen KL, Wicklund $\mathrm{KG}$, et al. Breast-feeding and risk of epithelial ovarian cancer. Cancer Causes Control. 2012 Jun;23(6):919-27. doi: 10.1007/s10552-012-9963-4. PMID: 22527170. Exclusion Code: X10.

488. Jordan SJ, Green AC, Whiteman DC, et al. Risk factors for benign, borderline and invasive mucinous ovarian tumors: epidemiological evidence of a neoplastic continuum? Gynecol Oncol. 2007 Nov;107(2):223-30. doi: 10.1016/j.ygyno.2007.06.006. PMID: 17662378. Exclusion Code: X10.

489. Jordan SJ, Na R, Johnatty SE, et al. Breastfeeding and Endometrial Cancer Risk: An Analysis From the Epidemiology of Endometrial Cancer Consortium. Obstet Gynecol. 2017 Jun;129(6):1059-67. doi: 10.1097/aog.0000000000002057. PMID: 28486362. Exclusion Code: X5.

490. Jordan SJ, Siskind V, A CG, et al. Breastfeeding and risk of epithelial ovarian cancer. Cancer Causes Control. 2010 Jan;21(1):109-16. doi: 10.1007/s10552-0099440-x. PMID: 19779839. Exclusion Code: $\mathrm{X} 10$.

491. Joshi A, Amadi C, Meza J, et al. Comparison of Socio-Demographic Characteristics of a Computer Based Breastfeeding Educational Intervention Among Rural Hispanic Women. J Community Health. 2015;40(5):993-1001. doi: 10.1007/s10900-015-0023-3. PMID: 109208162. Exclusion Code: X3.

492. Júnior WS, Martinez FE. Effect of intervention on the rates of breastfeeding of very low birth weight newborns. Neonatal Intensive Care. 2008;21(2):37-41. PMID: 105883235. Exclusion Code: X3.

493. Kac G, Benicio MH, Velasquez-Melendez $\mathrm{G}$, et al. Breastfeeding and postpartum weight retention in a cohort of Brazilian women. Am J Clin Nutr. 2004 Mar;79(3):487-93. PMID: 14985226. Exclusion Code: X8. 
494. Kacica MA, Kreiger L, Johnson GD.

Breastfeeding practices in New York State maternity hospitals: results from a statewide survey. Breastfeed Med. 2012 Dec;7(6):40916. doi: 10.1089/bfm.2011.0149. PMID: 22621224. Exclusion Code: X6.

495. Kacmar JE, Taylor JS, Nothnagle M, et al. Breastfeeding practices of resident physicians in Rhode Island. Med Health R I. 2006 Jul;89(7):230-1. PMID: 16925182. Exclusion Code: X3.

496. Kafatos AG, Tsitoura S, Pantelakis SN, et al. Maternal and infant health education in a rural Greek community. Hygie. 1991 Mar;10(1):32-7. PMID: 2040509. Exclusion Code: X3.

497. Kaikini KL, Hyrkas K. Mothers' Intentions to Breastfeed and Hospital Practices on Breastfeeding: A Longitudinal Study at 6 Months After Birth on Predictors of Breastfeeding in a Cohort of Mothers From a Large Northern New England Medical Center. JOGNN: Journal of Obstetric, Gynecologic \& Neonatal Nursing. 2014;43(Supp 1):S78-S. doi: 10.1111/15526909.12444. PMID: 103980260. Exclusion Code: X3.

498. Kair LR, Kenron D, Etheredge K, et al. Pacifier restriction and exclusive breastfeeding. Pediatrics. 2013 Apr;131(4):e1101-7. doi: 10.1542/peds.2012-2203. PMID: 23509161. Exclusion Code: X6.

499. Kang JS, Choi SY, Ryu EJ. Effects of a breastfeeding empowerment programme on Korean breastfeeding mothers: a quasiexperimental study. Int J Nurs Stud. 2008 Jan;45(1):14-23. doi: 10.1016/j.ijnurstu.2007.03.007. PMID: 17512527. Exclusion Code: X3.

500. Kang NM, Song Y, Hyun TH, et al. Evaluation of the breastfeeding intervention program in a Korean community health center. Int J Nurs Stud. 2005 May;42(4):409-13. doi: 10.1016/j.ijnurstu.2004.08.003. PMID: 15847903. Exclusion Code: X3.
501. Karanja N, Aickin M, Lutz T, et al. A community-based intervention to prevent obesity beginning at birth among American Indian children: study design and rationale for the PTOTS study. J Prim Prev. 2012 Aug;33(4):161-74. doi: 10.1007/s10935012-0278-8. PMID: 23001689. Exclusion Code: X6.

502. Karbandi S, Hosseini SM, Hosseini SA, et al. Evaluating the Effectiveness of Using a Progressive Muscle Relaxation Technique on the Self-Efficacy of Breastfeeding in Mothers With Preterm Infants. Journal of Nursing Research (Lippincott Williams \& Wilkins). 2017;25(4):283-8. doi: 10.1097/jnr.0000000000000217. PMID: 124470149. Exclusion Code: X3.

503. Kasemsarn P, Ngarmpiyasakul C, Phongpanich S, et al. Baby-friendly hospital: how to sustain? J Med Assoc Thai. 1995 Jul;78(7):362-8. PMID: 7658181. Exclusion Code: X6.

504. Kaunonen M, Hannula L, Tarkka MT. A systematic review of peer support interventions for breastfeeding. J Clin Nurs. 2012 Jul;21(13-14):1943-54. doi: 10.1111/j.1365-2702.2012.04071.x. PMID: 22672457. Exclusion Code: X3.

505. Kauppila A, Kyyronen P, Hinkula M, et al. Birth intervals and breast cancer risk. Br J Cancer. 2009 Oct 06;101(7):1213-7. doi: 10.1038/sj.bjc.6605300. PMID: 19738607. Exclusion Code: X3.

506. Kauppila A, Kyyronen P, Lehtinen M, et al. Dual effect of short interval between first and second birth on ductal breast cancer risk in Finland. Cancer Causes Control. 2012 Jan;23(1):187-93. doi: 10.1007/s10552-0119868-7. PMID: 22080277. Exclusion Code: X3.

507. Kawai M, Kakugawa Y, Nishino Y, et al. Reproductive factors and breast cancer risk in relation to hormone receptor and menopausal status in Japanese women. Cancer Sci. 2012 Oct;103(10):1861-70. doi: 10.1111/j.1349-7006.2012.02379.x. PMID: 22762156. Exclusion Code: X10.

508. Kearney L, Fulbrook P. The first 18 months: Parental choices regarding their infant's health care needs. Neonatal, Paediatric \& Child Health Nursing. 2014;17(3):17-22. PMID: 103779136. Exclusion Code: X3. 
509. Keith DR, Weaver BS, Vogel RL. The effect of music-based listening interventions on the volume, fat content, and caloric content of breast milk-produced by mothers of premature and critically ill infants. Adv Neonatal Care. 2012 Apr;12(2):112-9. doi: 10.1097/ANC.0b013e31824d9842. PMID: 22469966. Exclusion Code: X3.

510. Kellams AL, Gurka KK, Hornsby PP, et al. The Impact of a Prenatal Education Video on Rates of Breastfeeding Initiation and Exclusivity during the Newborn Hospital Stay in a Low-income Population. J Hum Lact. 2016 Feb;32(1):152-9. doi: 10.1177/0890334415599402. PMID: 26289058. Exclusion Code: X3.

511. Kemp L, Harris E, McMahon C, et al. Child and family outcomes of a long-term nurse home visitation programme: a randomised controlled trial. Arch Dis Child. 2011 Jun;96(6):533-40. doi: 10.1136/adc.2010.196279. PMID: 21429975. Exclusion Code: X3.

512. Kendal AP, Peterson A, Manning C, et al. Improving the health of infants on Medicaid by collocating special supplemental nutrition clinics with managed care provider sites. Am J Public Health. 2002 Mar;92(3):399403. PMID: 11867319. Exclusion Code: X3.

513. Kenyon S, Jolly K, Hemming K, et al. Lay support for pregnant women with social risk: a randomised controlled trial. BMJ open. 2016;6(3):e009203. doi: 10.1136/bmjopen2015-009203. PMID: CN-01259965. Exclusion Code: X3.

514. Kenyon S, Jolly K, Hemming K, et al. Lay support for pregnant women with social risk: a randomised controlled trial. BMJ Open. 2016 Mar 02;6(3):e009203. doi: 10.1136/bmjopen-2015-009203. PMID: 26936901. Exclusion Code: X3.

515. Kenyon S, Jolly K, Hemming K, et al. Evaluation of Lay Support in Pregnant women with Social risk (ELSIPS): a randomised controlled trial. BMC Pregnancy Childbirth. 2012 Feb 29;12:11. doi: 10.1186/1471-2393-12-11. PMID: 22375895. Exclusion Code: X3.
516. Kersting M, Dulon M. Assessment of breastfeeding promotion in hospitals and followup survey of mother-infant pairs in Germany: the SuSe Study. Public Health Nutr. 2002 Aug;5(4):547-52. doi: 10.1079/phn2001321. PMID: 12186663. Exclusion Code: X6.

517. Khresheh R, Suhaimat A, Jalamdeh F, et al. The effect of a postnatal education and support program on breastfeeding among primiparous women: a randomized controlled trial. Int J Nurs Stud. 2011 Sep;48(9):1058-65. doi: 10.1016/j.ijnurstu.2011.02.001. PMID: 21353220. Exclusion Code: X3.

518. Kim C. Maternal outcomes and follow-up after gestational diabetes mellitus. Diabet Med. 2014 Mar;31(3):292-301. doi: 10.1111/dme.12382. PMID: 24341443. Exclusion Code: X6.

519. Kim C, Berger DK, Chamany S. Recurrence of gestational diabetes mellitus: a systematic review. Diabetes Care. 2007

May;30(5):1314-9. doi: 10.2337/dc06-2517. PMID: 17290037. Exclusion Code: X5.

520. Kim Y. [Effects of a breast-feeding empowerment program on exclusive breastfeeding]. Journal of Korean Academy of Nursing; 2009. p. 279-87. Exclusion Code: $\mathrm{X} 7$.

521. Kim Y, Choi JY, Lee KM, et al. Dosedependent protective effect of breast-feeding against breast cancer among ever-lactated women in Korea. Eur J Cancer Prev. 2007 Apr;16(2):124-9. doi: 10.1097/01.cej.0000228400.07364.52. PMID: 17297388. Exclusion Code: X10.

522. Kind C, Schubiger G, Schwarz U, et al. Provision of supplementary fluids to breast fed infants and later breast feeding success. Adv Exp Med Biol. 2000;478:347-54. doi: 10.1007/0-306-46830-1_29. PMID: 11065084. Exclusion Code: X3.

523. Kirkan TS, Aydin N, Yazici E, et al. The depression in women in pregnancy and postpartum period: A follow-up study. Int J Soc Psychiatry. 2015 Jun;61(4):343-9. doi: 10.1177/0020764014543713. PMID: 25069455. Exclusion Code: X8. 
524. Kirkegaard H, Nohr EA, Rasmussen KM, et al. Maternal prepregnancy waist circumference and BMI in relation to gestational weight gain and breastfeeding behavior: the CARDIA study. Am J Clin Nutr. 2015 Aug;102(2):393-401. doi: 10.3945/ajcn.114.099184. PMID: 26135344. Exclusion Code: X5.

525. Kirkegaard H, Stovring H, Rasmussen KM, et al. How do pregnancy-related weight changes and breastfeeding relate to maternal weight and BMI-adjusted waist circumference 7 y after delivery? Results from a path analysis. Am J Clin Nutr. 2014 Feb;99(2):312-9. doi: 10.3945/ajcn.113.067405. PMID: 24335054. Exclusion Code: X5.

526. Kish MZ. Improving preterm infant outcomes: implementing an evidence-based oral feeding advancement protocol in the neonatal intensive care unit. Adv Neonatal Care. 2014 Oct;14(5):346-53. doi: 10.1097/anc.0000000000000099. PMID: 24979099. Exclusion Code: X3.

527. Kistin CJ, Barrero-Castillero A, Lewis S, et al. Maternal note-taking and infant care: a pilot randomised controlled trial. Arch Dis Child. 2012 Oct;97(10):916-8. doi: 10.1136/archdischild-2012-302289. PMID: 22806235. Exclusion Code: X3.

528. Kistin N, Abramson R, Dublin P. Effect of peer counselors on breastfeeding initiation, exclusivity, and duration among low-income urban women. J Hum Lact. 1994

Mar;10(1):11-5. PMID: 7619241. Exclusion Code: X3.

529. Kistin N, Benton D, Rao S, et al. Breastfeeding rates among black urban lowincome women: effect of prenatal education. Pediatrics. 1990 Nov;86(5):741-6. PMID: 2235229. Exclusion Code: X3.

530. Klingberg S, Brekke HK, Winkvist A, et al. Parity, weight change, and maternal risk of cardiovascular events. Am J Obstet Gynecol. 2017 Feb;216(2):172.e1-.e15. doi: 10.1016/j.ajog.2016.09.105. PMID: 27720863. Exclusion Code: X3.
531. Koehler S, Sichert-Hellert W, Kersting M. Measuring the effects of nutritional counseling on total infant diet in a randomized controlled intervention trial. J Pediatr Gastroenterol Nutr. 2007 Jul;45(1):106-13. doi: 10.1097/MPG.0b013e3180331e2a. PMID: 17592372. Exclusion Code: X3.

532. Kogan MD, Singh GK, Dee DL, et al. Multivariate analysis of state variation in breastfeeding rates in the United States. Am J Public Health. 2008 Oct;98(10):1872-80. doi: 10.2105/ajph.2007.127118. PMID: 18703441. Exclusion Code: X3.

533. Kola B. Small changes making big impact. Cogent medicine. Conference: 8th excellence in pediatrics conference 2016. United kingdom. Conference start: 20161208. Conference end: 20161210. 2016;3(1) (no pagination)doi: 10.1080/2331205X.2016.1265203. PMID: CN-01335024. Exclusion Code: X6.

534. Komara C, Simpson D, Teasdale C, et al. Intervening to promote early initiation of breastfeeding in the LDR. MCN Am J Matern Child Nurs. 2007 Mar-

Apr;32(2):117-21. doi: 10.1097/01.NMC.0000264293.72221.a6. PMID: 17356419. Exclusion Code: X4.

535. Kotsopoulos J, Lubinski J, Salmena L, et al. Breastfeeding and the risk of breast cancer in BRCA1 and BRCA2 mutation carriers. Breast Cancer Res. 2012 Mar 09;14(2):R42. doi: 10.1186/bcr3138. PMID: 22405187. Exclusion Code: X10.

536. Kramer MS, Chalmers B, Hodnett ED, et al. Promotion of breastfeeding intervention trial (PROBIT): a cluster-randomized trial in the Republic of Belarus. Design, follow-up, and data validation. Adv Exp Med Biol. 2000;478:327-45. PMID: 11065083. Exclusion Code: X9.

537. Kramer MS, Fombonne E, Igumnov S, et al. Effects of prolonged and exclusive breastfeeding on child behavior and maternal adjustment: evidence from a large, randomized trial. Pediatrics. 2008

Mar;121(3):e435-40. doi: 10.1542/peds.2007-1248. PMID: 18310164. Exclusion Code: X5. 
538. Kramer MS, Guo T, Platt RW, et al. Breastfeeding and infant growth: biology or bias? Pediatrics. 2002 Aug;110(2 Pt 1):3437. PMID: 12165588. Exclusion Code: X5.

539. Kramer MS, Kakuma R. Optimal duration of exclusive breastfeeding. Cochrane Database of Systematic Reviews: John Wiley \& Sons, Ltd; 2012. Exclusion Code: X8.

540. Kramer MS, Matush L, Vanilovich I, et al. A randomized breast-feeding promotion intervention did not reduce child obesity in Belarus. J Nutr. 2009 Feb;139(2):417s-21s. doi: 10.3945/jn.108.097675. PMID: 19106322. Exclusion Code: X5.

541. Krause KM, Lovelady CA, Peterson BL, et al. Effect of breast-feeding on weight retention at 3 and 6 months postpartum: data from the North Carolina WIC Programme. Public Health Nutr. 2010 Dec;13(12):201926. doi: $10.1017 / \mathrm{s} 1368980010001503$. PMID: 20519049. Exclusion Code: X10.

542. Kroke A. Prenatal and postnatal development of obesity: primary prevention trials and observational studies. Nestle Nutr Workshop Ser Pediatr Program. 2006;57:5162; discussion -5. doi: 10.1159/000091050. PMID: 16632959. Exclusion Code: X3.

543. Kronborg H, Maimburg RD, Vaeth M. Antenatal training to improve breast feeding: a randomised trial. Midwifery. 2012 Dec;28(6):784-90. doi: 10.1016/j.midw.2011.08.016. PMID: 22018394. Exclusion Code: X3.

544. Kronborg H, Vaeth M, Kristensen I. The effect of early postpartum home visits by health visitors: a natural experiment. Public Health Nurs. 2012 Jul-Aug;29(4):289-301. doi: 10.1111/j.1525-1446.2012.01019.x. PMID: 22765241. Exclusion Code: X3.

545. Kronborg H, Vaeth M, Olsen J, et al. Health visitors and breastfeeding support: influence of knowledge and self-efficacy. Eur J Public Health. 2008 Jun;18(3):283-8. doi: 10.1093/eurpub/ckm121. PMID: 18160390. Exclusion Code: X5.

546. Kruk J. Association of lifestyle and other risk factors with breast cancer according to menopausal status: a case-control study in the Region of Western Pomerania (Poland). Asian Pac J Cancer Prev. 2007 OctDec;8(4):513-24. PMID: 18260721. Exclusion Code: X10.
547. Kruske S, Schmied V, Cook M. The 'Earlybird' gets the breastmilk: findings from an evaluation of combined professional and peer support groups to improve breastfeeding duration in the first eight weeks after birth. Matern Child Nutr. 2007 Apr;3(2):108-19. doi: 10.1111/j.17408709.2007.00078.x. PMID: 17355443. Exclusion Code: X3.

548. Kullberg C, Selander J, Albin M, et al. Female white-collar workers remain at higher risk of breast cancer after adjustments for individual risk factors related to reproduction and lifestyle. Occup Environ Med. 2017 Sep;74(9):652-8. doi: 10.1136/oemed-2016-104043. PMID: 28456763. Exclusion Code: X3.

549. Kupratakul J, Taneepanichskul S, Voramongkol N, et al. A randomized controlled trial of knowledge sharing practice with empowerment strategies in pregnant women to improve exclusive breastfeeding during the first six months postpartum. J Med Assoc Thai. 2010 Sep;93(9):1009-18. PMID: 20873071. Exclusion Code: X3.

550. Kurta ML, Moysich KB, Weissfeld JL, et al. Use of fertility drugs and risk of ovarian cancer: results from a U.S.-based casecontrol study. Cancer Epidemiol Biomarkers Prev. 2012 Aug;21(8):1282-92. doi: 10.1158/1055-9965.epi-12-0426. PMID: 22707710. Exclusion Code: X3.

551. Kutlu R, Kara F, Durduran Y, et al. Assessment of effects of pre- and posttraining programme for healthcare professionals about breastfeeding. J Health Popul Nutr. 2007 Sep;25(3):382-6. PMID: 18330073. Exclusion Code: X6.

552. Kwan ML, Bernard PS, Kroenke CH, et al. Breastfeeding, PAM50 tumor subtype, and breast cancer prognosis and survival. J Natl Cancer Inst. 2015 Jul;107(7)doi: 10.1093/jnci/djv087. PMID: 25921910. Exclusion Code: X4.

553. Kwan ML, Kushi LH, Weltzien E, et al. Epidemiology of breast cancer subtypes in two prospective cohort studies of breast cancer survivors. Breast Cancer Res. 2009;11(3):R31. doi: 10.1186/bcr2261. PMID: 19463150. Exclusion Code: X6. 
554. Labarere J, Bellin V, Fourny M, et al. Assessment of a structured in-hospital educational intervention addressing breastfeeding: a prospective randomised open trial. BJOG. 2003 Sep;110(9):847-52. PMID: 14511968. Exclusion Code: X3.

555. Labarere J, Gelbert-Baudino N, Ayral AS, et al. Efficacy of breastfeeding support provided by trained clinicians during an early, routine, preventive visit: a prospective, randomized, open trial of 226 mother-infant pairs. Pediatrics. 2005 Feb;115(2):e139-46. doi: 10.1542/peds.2004-1362. PMID: 15687421. Exclusion Code: X3.

556. Labarere J, Gelbert-Baudino N, Laborde L, et al. CD-ROM-based program for breastfeeding mothers. Matern Child Nutr. 2011 Jul;7(3):263-72. doi: 10.1111/j.17408709.2009.00235.x. PMID: 21689269. Exclusion Code: X3.

557. Ladzani R, Steyn NP, Nel JH. An evaluation of the effectiveness of nutrition advisers in three rural areas of northern province. S Afr Med J. 2000 Aug;90(8):811-6. PMID: 11022632. Exclusion Code: X8.

558. Lakin A, Sutter MB, Magee S. Newborn well-child visits in the home setting: a pilot study in a family medicine residency. Fam Med. 2015 Mar;47(3):217-21. PMID: 25853533. Exclusion Code: X3.

559. Laliberte C, Dunn S, Pound C, et al. A Randomized Controlled Trial of Innovative Postpartum Care Model for Mother-Baby Dyads. PLoS One. 2016;11(2):e0148520. doi: 10.1371/journal.pone.0148520. PMID: 26871448. Exclusion Code: X3.

560. Lamontagne C, Hamelin AM, St-Pierre M. An assessment of the impact of breastfeeding clinic attendance on women's breastfeeding experiences. J Hum Lact. 2009 Feb;25(1):42-53. doi: 10.1177/0890334408324451. PMID: 18971504. Exclusion Code: X2.

561. Langellier BA, Chaparro MP, Wang MC, et al. The new food package and breastfeeding outcomes among women, infants, and children participants in Los Angeles County. Am J Public Health. 2014 Feb;104 Suppl 1:S112-8. doi: 10.2105/ajph.2013.301330. PMID: 24354843. Exclusion Code: X6.
562. Langer A, Campero L, Garcia C, et al. Effects of psychosocial support during labour and childbirth on breastfeeding, medical interventions, and mothers' wellbeing in a Mexican public hospital: a randomised clinical trial. Br J Obstet Gynaecol. 1998 Oct;105(10):1056-63. PMID: 9800927. Exclusion Code: X3.

563. Langseth H, Hankinson SE, Siemiatycki J, et al. Perineal use of talc and risk of ovarian cancer. J Epidemiol Community Health. 2008 Apr;62(4):358-60. doi: 10.1136/jech.2006.047894. PMID: 18339830. Exclusion Code: X3.

564. Largent JA, Capanu M, Bernstein L, et al. Reproductive history and risk of second primary breast cancer: the WECARE study. Cancer Epidemiol Biomarkers Prev. 2007 May;16(5):906-11. doi: 10.1158/10559965.epi-06-1003. PMID: 17507614. Exclusion Code: X4.

565. Lavender T, Baker L, Smyth R, et al. Breastfeeding expectations versus reality: a cluster randomised controlled trial. BJOG. 2005 Aug;112(8):1047-53. doi: 10.1111/j.1471-0528.2005.00644.x. PMID: 16045516. Exclusion Code: X3.

566. Lavender T, Richens Y, Milan SJ, et al. Telephone support for women during pregnancy and the first six weeks postpartum. Cochrane Database Syst Rev. 2013 Jul 18(7):Cd009338. doi: 10.1002/14651858.CD009338.pub2. PMID: 23881662. Exclusion Code: X3.

567. le Roux IM, Tomlinson M, Harwood JM, et al. Outcomes of home visits for pregnant mothers and their infants: a cluster randomized controlled trial. AIDS. 2013 Jun 01;27(9):1461-71. doi: 10.1097/QAD.0b013e3283601b53. PMID: 23435303. Exclusion Code: X8.

568. Lee Benitez Y, Parrilla-Rodriguez AM, Rios P. Effectiveness in the implantation of law 155 of 2002 ordering the designation of spaces for breastfeeding in government agencies. P R Health Sci J. 2005 Dec;24(4):297-301. PMID: 16570527. Exclusion Code: X5. 
569. Lee CC, Chiou ST, Chen LC, et al. Breastfeeding-Friendly Environmental Factors and Continuing Breastfeeding Until 6 Months Postpartum: 2008-2011 National Surveys in Taiwan. Birth. 2015 Sep;42(3):242-8. doi: 10.1111/birt.12170. PMID: 26095672. Exclusion Code: X6.

570. Lee CF, Hwang FM, Liou YM, et al. A preliminary study on the pattern of weight change from pregnancy to 6 months postpartum: a latent growth model approach. Int J Obes (Lond). 2011 Aug;35(8):1079-86. doi: 10.1038/ijo.2010.225. PMID: 21042322. Exclusion Code: X5.

571. Lee HC, Jegatheesan P, Gould JB, et al. Hospital-wide breastfeeding rates vs. breastmilk provision for very-low-birthweight infants. Acta Paediatr. 2013 Mar;102(3):268-72. doi: 10.1111/apa.12096. PMID: 23174012. Exclusion Code: X4.

572. Lee J, Oh M. Effects of interval between age at first pregnancy and age at diagnosis on breast cancer survival according to menopausal status: a register-based study in Korea. BMC Womens Health. 2014 Sep 18;14:113. doi: 10.1186/1472-6874-14-113. PMID: 25231360. Exclusion Code: X4.

573. Lee JS, Oh M. Reproductive factors and subtypes of breast cancer defined by estrogen receptor, progesterone receptor, and human epidermal growth factor receptor 2: a register-based study from Korea. Clin Breast Cancer. 2014 Dec;14(6):426-34. doi: 10.1016/j.clbc.2014.05.003. PMID: 25034438. Exclusion Code: X4.

574. Lee KH, Shu XO, Gao YT, et al. Breast cancer and urinary biomarkers of polycyclic aromatic hydrocarbon and oxidative stress in the Shanghai Women's Health Study. Cancer Epidemiol Biomarkers Prev. 2010 Mar;19(3):877-83. doi: 10.1158/10559965.epi-09-1098. PMID: 20160264. Exclusion Code: X8.

575. Lee SY, Kim MT, Kim SW, et al. Effect of lifetime lactation on breast cancer risk: a Korean women's cohort study. Int J Cancer. 2003 Jun 20;105(3):390-3. doi: 10.1002/ijc.11078. PMID: 12704674. Exclusion Code: X10.
576. Leite AJ, Puccini RF, Atalah AN, et al. Effectiveness of home-based peer counselling to promote breastfeeding in the northeast of Brazil: a randomized clinical trial. Acta Paediatr. 2005 Jun;94(6):741-6. doi: 10.1080/08035250410023854. PMID: 16188778. Exclusion Code: X3.

577. Lenell A, Friesen CA, Hormuth L. Breastfeeding Support in a Community Pharmacy: Improving Access through the Well Babies at Walgreens Program. J Hum Lact. 2015 Nov;31(4):577-81. doi: 10.1177/0890334415579418. PMID: 25829476. Exclusion Code: X5.

578. Lennon T, Willis E. Workplace Lactation Support in Milwaukee County 5 Years After the Affordable Care Act. J Hum Lact. 2017 Feb;33(1):214-9. doi: 10.1177/0890334416679617. PMID: 28135480. Exclusion Code: X3.

579. Leon DA, Ronalds G. Breast-feeding influences on later life--cardiovascular disease. Adv Exp Med Biol. 2009;639:15366. doi: 10.1007/978-1-4020-8749-3_13. PMID: 19227542. Exclusion Code: X6.

580. Leung BM, Giesbrecht GF, Letourneau N, et al. Perinatal nutrition in maternal mental health and child development: Birth of a pregnancy cohort. Early Hum Dev. 2016 Feb;93:1-7. doi: 10.1016/j.earlhumdev.2015.11.007. PMID: 26704573. Exclusion Code: X5.

581. Lewandowski AJ, Lamata P, Francis JM, et al. Breast Milk Consumption in Preterm Neonates and Cardiac Shape in Adulthood. Pediatrics. 2016;138(1):1-10. doi: 10.1542/peds.2016-0050. PMID: 116609667. Exclusion Code: X5.

582. Lewin LO, O'Connor ME.

"BreastfeedingBasics": web-based education that meets current knowledge competencies. J Hum Lact. 2012 Aug;28(3):407-13. doi: 10.1177/0890334411435990. PMID: 22550095. Exclusion Code: X5.

583. Lewin S, Munabi-Babigumira S, Glenton C, et al. Lay health workers in primary and community health care for maternal and child health and the management of infectious diseases. Cochrane Database Syst Rev. 2010 Mar 17(3):Cd004015. doi: 10.1002/14651858.CD004015.pub3. PMID: 20238326. Exclusion Code: X3. 
584. Lewin SA, Dick J, Pond P, et al. Lay health workers in primary and community health care. Cochrane Database Syst Rev. 2005 Jan 25(1):Cd004015. doi: 10.1002/14651858.CD004015.pub2. PMID: 15674924. Exclusion Code: X5.

585. Lewis N, Martinez LS, Freres DR, et al. Seeking cancer-related information from media and family/friends increases fruit and vegetable consumption among cancer patients. Health Commun. 2012;27(4):3808. doi: 10.1080/10410236.2011.586990. PMID: 21932985. Exclusion Code: X3.

586. Lewycka S, Mwansambo C, Rosato M, et al. Effect of women's groups and volunteer peer counselling on rates of mortality, morbidity, and health behaviours in mothers and children in rural Malawi (MaiMwana): a factorial, cluster-randomised controlled trial. Lancet. 2013 May 18;381(9879):1721-35. doi: 10.1016/s0140-6736(12)61959-x. PMID: 23683639. Exclusion Code: X8.

587. Li CI, Beaber EF, Tang MT, et al. Reproductive factors and risk of estrogen receptor positive, triple-negative, and HER2-neu overexpressing breast cancer among women 20-44 years of age. Breast Cancer Res Treat. 2013 Jan;137(2):579-87. doi: 10.1007/s10549-012-2365-1. PMID: 23224237. Exclusion Code: X10.

588. Li CM, Li R, Ashley CG, et al. Associations of hospital staff training and policies with early breastfeeding practices. J Hum Lact. 2014 Feb;30(1):88-96. doi: 10.1177/0890334413484551. PMID: 23603574. Exclusion Code: X3.

589. Li H, Sun X, Miller E, et al. BMI, reproductive factors, and breast cancer molecular subtypes: A case-control study and meta-analysis. J Epidemiol. 2017 Apr;27(4):143-51. doi: 10.1016/j.je.2016.05.002. PMID: 28142040. Exclusion Code: X8.

590. Lieu TA, Braveman PA, Escobar GJ, et al. A randomized comparison of home and clinic follow-up visits after early postpartum hospital discharge. Pediatrics. 2000 May;105(5):1058-65. PMID: 10790463. Exclusion Code: X3.
591. Lillehoj CJ, Dobson BL. Implementation of the baby-friendly hospital initiative steps in Iowa hospitals. J Obstet Gynecol Neonatal Nurs. 2012 Nov-Dec;41(6):717-27. doi: 10.1111/j.1552-6909.2012.01411.x. PMID: 23030657. Exclusion Code: X5.

592. Lilleston P, Nhim K, Rutledge G. An Evaluation of the CDC's Community-Based Breastfeeding Supplemental Cooperative Agreement: Reach, Strategies, Barriers, Facilitators, and Lessons Learned. J Hum Lact. 2015 Nov;31(4):614-22. doi: 10.1177/0890334415597904. PMID: 26261226. Exclusion Code: X5.

593. Lin CH, Kuo SC, Lin KC, et al. Evaluating effects of a prenatal breastfeeding education programme on women with caesarean delivery in Taiwan. J Clin Nurs. 2008 Nov;17(21):2838-45. doi: 10.1111/j.13652702.2008.02289.x. PMID: 18624780. Exclusion Code: X3.

594. Lin SS, Chien LY, Tai CJ, et al. Effectiveness of a prenatal education programme on breastfeeding outcomes in Taiwan. J Clin Nurs. 2008 Feb;17(3):296303. doi: $10.1111 / \mathrm{j} .1365-$ 2702.2006.01927.x. PMID: 17931376. Exclusion Code: X3.

595. Linares A, Wambach K, Rayens M, et al. Modeling the Influence of Early Skin-toSkin Contact on Exclusive Breastfeeding in a Sample of Hispanic Immigrant Women. Journal of Immigrant \& Minority Health. 2017;19(5):1027-34. doi: 10.1007/s10903016-0380-8. PMID: 124864533. Exclusion Code: X3.

596. Lindberg L. Trends in the relationship between breastfeeding and postpartum employment in the United States. Soc Biol. 1996 Fall-Winter;43(3-4):191-202. PMID: 9204696. Exclusion Code: X3.

597. Lipsky LM, Strawderman MS, Olson CM. Maternal weight change between 1 and 2 years postpartum: the importance of 1 year weight retention. Obesity (Silver Spring). 2012 Jul;20(7):1496-502. doi: 10.1038/oby.2012.41. PMID: 22334257. Exclusion Code: X5. 
598. Liu B, Beral V, Balkwill A. Childbearing, breastfeeding, other reproductive factors and the subsequent risk of hospitalization for gallbladder disease. Int J Epidemiol. 2009 Feb;38(1):312-8. doi: 10.1093/ije/dyn174. PMID: 18775873. Exclusion Code: X5.

599. Liu B, Jorm L, Banks E. Parity, breastfeeding, and the subsequent risk of maternal type 2 diabetes. Diabetes Care. 2010 Jun;33(6):1239-41. doi: 10.2337/dc100347. PMID: 20332359. Exclusion Code: $\mathrm{X} 10$.

600. Liu YT, Gao CM, Ding JH, et al. Physiological, reproductive factors and breast cancer risk in Jiangsu province of China. Asian Pac J Cancer Prev. 2011;12(3):787-90. PMID: 21627384. Exclusion Code: X8.

601. Loh NR, Kelleher CC, Long S, et al. Can we increase breast feeding rates? Ir Med J. 1997 Apr-May;90(3):100-1. PMID: 9183091. Exclusion Code: X3.

602. Long DG, Funk-Archuleta MA, Geiger CJ, et al. Peer counselor program increases breastfeeding rates in Utah Native American WIC population. J Hum Lact. 1995 Dec;11(4):279-84. PMID: 8634104. Exclusion Code: X6.

603. Loof-Johanson M, Brudin L, Sundquist M, et al. Hormone Use is Associated with Lymphovascular Invasion in Breast Cancer. Asian Pac J Cancer Prev. 2016;17(3):150712. PMID: 27039798. Exclusion Code: X4.

604. Lopes Sda S, Laignier MR, Primo CC, et al. Baby-friendly hospital initiative: evaluation of the ten steps to successful breastfeeding. Rev Paul Pediatr. 2013 Dec;31(4):488-93. doi: 10.1590/s0103-05822013000400011. PMID: 24473954. Exclusion Code: X6.

605. Lord SJ, Bernstein L, Johnson KA, et al. Breast cancer risk and hormone receptor status in older women by parity, age of first birth, and breastfeeding: a case-control study. Cancer Epidemiol Biomarkers Prev. 2008 Jul;17(7):1723-30. doi: 10.1158/10559965.epi-07-2824. PMID: 18628424.

Exclusion Code: X10.
606. Lumachi F, Ermani M, Marino F, et al. Relationship between oral contraceptive therapy and estrogen receptor status in patients with breast cancer. Anticancer Res. 2008 Jan-Feb;28(1b):491-3. PMID: 18383890. Exclusion Code: X4.

607. Lumbiganon P, Martis R, Laopaiboon M, et al. Antenatal breastfeeding education for increasing breastfeeding duration. Cochrane Database Syst Rev. 2011 Nov 09(11):Cd006425. doi: 10.1002/14651858.CD006425.pub2. PMID: 22071830. Exclusion Code: X3.

608. Lumbiganon P, Martis R, Laopaiboon M, et al. Antenatal breastfeeding education for increasing breastfeeding duration. Cochrane Database Syst Rev. 2012 Sep

12(9):Cd006425. doi: 10.1002/14651858.CD006425.pub3. PMID: 22972092. Exclusion Code: X3.

609. Lumbiganon P, Martis R, Laopaiboon M, et al. Antenatal breastfeeding education for increasing breastfeeding duration. Cochrane Database Syst Rev. 2016 Dec 06;12:Cd006425. doi: 10.1002/14651858.CD006425.pub4. PMID: 27922724. Exclusion Code: X3.

610. Lundquist J, Xu Z, Barfield W, et al. Do black-white racial disparities in breastfeeding persist in the military community? Matern Child Health J. 2015 Feb;19(2):419-27. doi: 10.1007/s10995-0141524-x. PMID: 24894729. Exclusion Code: X3.

611. Lussier MM, Brownell EA, Proulx TA, et al. Daily Breastmilk Volume in Mothers of Very Low Birth Weight Neonates: A Repeated-Measures Randomized Trial of Hand Expression Versus Electric Breast Pump Expression. Breastfeed Med. 2015 Jul-Aug;10(6):312-7. doi: 10.1089/bfm.2015.0014. PMID: 26204125. Exclusion Code: X5.

612. Lutter CK, Perez-Escamilla R, Segall A, et al. The effectiveness of a hospital-based program to promote exclusive breast-feeding among low-income women in Brazil. Am J Public Health. 1997 Apr;87(4):659-63. PMID: 9146449. Exclusion Code: X3. 
613. Lynch SA, Koch AM, Hislop TG, et al. Evaluating the effect of a breastfeeding consultant on the duration of breastfeeding. Can J Public Health. 1986 MayJun;77(3):190-5. PMID: 3742402.

Exclusion Code: X3.

614. Ma H, Bernstein L, Pike MC, et al. Reproductive factors and breast cancer risk according to joint estrogen and progesterone receptor status: a meta-analysis of epidemiological studies. Breast Cancer Res. 2006;8(4):R43. doi: 10.1186/bcr1525. PMID: 16859501. Exclusion Code: X6.

615. Ma H, Henderson KD, Sullivan-Halley J, et al. Pregnancy-related factors and the risk of breast carcinoma in situ and invasive breast cancer among postmenopausal women in the California Teachers Study cohort. Breast Cancer Res. 2010;12(3):R35. doi: 10.1186/bcr2589. PMID: 20565829. Exclusion Code: X10.

616. MacArthur C, Jolly K, Ingram L, et al. Antenatal peer support workers and initiation of breast feeding: cluster randomised controlled trial. BMJ. 2009 Jan 30;338:b131. doi: 10.1136/bmj.b131. PMID: 19181730. Exclusion Code: X3.

617. Machado MC, Assis KF, Oliveira Fde C, et al. Determinants of the exclusive breastfeeding abandonment: psychosocial factors. Rev Saude Publica. 2014 Dec;48(6):985-94. doi: 10.1590/s00348910.2014048005340. PMID: 26039402. Exclusion Code: X3.

618. Maehara K, Mori E, Iwata H, et al. Postpartum maternal function and parenting stress: Comparison by feeding methods. Int J Nurs Pract. 2017;23:n/a-n/a. doi: 10.1111/ijn.12549. PMID: 123692414. Exclusion Code: X5.

619. Magnusson BM, Thackeray CR, Van Wagenen SA, et al. Perceptions of Public Breastfeeding Images and Their Association With Breastfeeding Knowledge and Attitudes Among an Internet Panel of Men Ages 21-44 in the United States. J Hum Lact. 2017 Feb;33(1):157-64. doi: 10.1177/0890334416682002. PMID: 28061143. Exclusion Code: X3.
620. Malpeli A, Apezteguia M, Mansur JL, et al. Calcium supplementation, bone mineral density and bone mineral content. Predictors of bone mass changes in adolescent mothers during the 6-month postpartum period. Arch Latinoam Nutr. 2012 Mar;62(1):30-6. PMID: 23477205. Exclusion Code: X5.

621. Manant A, Dodgson JE.

CenteringPregnancy: an integrative literature review. J Midwifery Womens Health. 2011 Mar-Apr;56(2):94-102. doi: 10.1111/j.1542-2011.2010.00021.x. PMID: 21429072. Exclusion Code: X5.

622. Mandl KD, Brennan TA, Wise PH, et al. Maternal and infant health: effects of moderate reductions in postpartum length of stay. Arch Pediatr Adolesc Med. 1997 Sep;151(9):915-21. PMID: 9308869. Exclusion Code: X3.

623. Mannan I, Rahman SM, Sania A, et al. Can early postpartum home visits by trained community health workers improve breastfeeding of newborns? J Perinatol. 2008 Sep;28(9):632-40. doi: 10.1038/jp.2008.64. PMID: 18596714. Exclusion Code: X8.

624. Marín GM, Llana MI, López EA, et al. Randomized controlled trial of early skin-toskin contact: effects on the mother and the newborn. Acta paediatrica (Oslo, Norway : 1992); 2010. p. 1630-4. Exclusion Code: X3.

625. Martens PJ. What Do Kramer’s BabyFriendly Hospital Initiative PROBIT Studies Tell Us? A Review of a Decade of Research. J Hum Lact. 2012;28(3):335-42. doi: 10.1177/0890334412438264. PMID: 108138940. Exclusion Code: X6.

626. Martin J, MacDonald-Wicks L, Hure A, et al. Reducing postpartum weight retention and improving breastfeeding outcomes in overweight women: a pilot randomised controlled trial. Nutrients. 2015 Feb 25;7(3):1464-79. doi: 10.3390/nu7031464. PMID: 25723973. Exclusion Code: X3.

627. Martin JE, Hure AJ, Macdonald-Wicks L, et al. Predictors of post-partum weight retention in a prospective longitudinal study. Matern Child Nutr. 2014 Oct;10(4):496-509. doi: 10.1111/j.1740-8709.2012.00437.x. PMID: 22974518. Exclusion Code: X6. 
628. Martin RM, Patel R, Kramer MS, et al. Effects of promoting longer-term and exclusive breastfeeding on adiposity and insulin-like growth factor-I at age 11.5 years: a randomized trial. JAMA. 2013 Mar 13;309(10):1005-13. doi: 10.1001/jama.2013.167. PMID: 23483175. Exclusion Code: X5.

629. Martin-Iglesias S, del-Cura-Gonzalez I, Sanz-Cuesta T, et al. Effectiveness of an implementation strategy for a breastfeeding guideline in Primary Care: cluster randomised trial. BMC Fam Pract. 2011 Dec 30;12:144. doi: 10.1186/1471-2296-12-144. PMID: 22208800. Exclusion Code: X5.

630. Maslowsky J, Frost S, Hendrick C, et al. Effects of postpartum mobile phone-based education on maternal and infant health in Ecuador. Int J Gynaecol Obstet; 2016. p. 93-8. Exclusion Code: X3.

631. Matsushita H, Kurabayashi T, Tomita M, et al. The effect of multiple pregnancies on lumbar bone mineral density in Japanese women. Calcif Tissue Int. 2002 Jul;71(1):10-3. doi: 10.1007/s00223-0012100-0. PMID: 12200655. Exclusion Code: X5.

632. Mattar CN, Chong YS, Chan YS, et al. Simple antenatal preparation to improve breastfeeding practice: a randomized controlled trial. Obstet Gynecol. 2007 Jan;109(1):73-80. doi: 10.1097/01.aog.0000249613.15466.26. PMID: 17197590. Exclusion Code: X3.

633. Maycock B, Binns CW, Dhaliwal S, et al. Education and support for fathers improves breastfeeding rates: a randomized controlled trial. J Hum Lact. 2013 Nov;29(4):484-90. doi: 10.1177/0890334413484387. PMID: 23603573. Exclusion Code: X3.

634. Maycock BR, Scott JA, Hauck YL, et al. A study to prolong breastfeeding duration: design and rationale of the Parent Infant Feeding Initiative (PIFI) randomised controlled trial. BMC Pregnancy Childbirth. 2015 Aug 01;15:159. doi: 10.1186/s12884015-0601-5. PMID: 26231519. Exclusion Code: X6.
635. Mazzoni SE, Carter EB. Group prenatal care. Am J Obstet Gynecol. 2017 Jun;216(6):552-6. doi: 10.1016/j.ajog.2017.02.006. PMID: 28189608. Exclusion Code: X1.

636. McClure CK, Catov J, Ness R, et al. Maternal visceral adiposity by consistency of lactation. Matern Child Health J. 2012 Feb;16(2):316-21. doi: 10.1007/s10995-0110758-0. PMID: 21404071. Exclusion Code: X6.

637. McClure CK, Catov JM, Ness RB, et al. Lactation and maternal subclinical cardiovascular disease among premenopausal women. Am J Obstet Gynecol. 2012 Jul;207(1):46.e1-8. doi: 10.1016/j.ajog.2012.04.030. PMID: 22727348. Exclusion Code: X5.

638. McClure CK, Schwarz EB, Conroy MB, et al. Breastfeeding and subsequent maternal visceral adiposity. Obesity (Silver Spring). 2011 Nov;19(11):2205-13. doi: 10.1038/oby.2011.185. PMID: 21720436. Exclusion Code: X6.

639. McCoy SJ, Beal JM, Shipman SB, et al. Risk factors for postpartum depression: a retrospective investigation at 4-weeks postnatal and a review of the literature. $\mathrm{J}$ Am Osteopath Assoc. 2006 Apr;106(4):1938. PMID: 16627773. Exclusion Code: X10.

640. McDonald SJ, Henderson JJ, Faulkner S, et al. Effect of an extended midwifery postnatal support programme on the duration of breast feeding: a randomised controlled trial. Midwifery. 2010 Feb;26(1):88-100. doi: 10.1016/j.midw.2008.03.001. PMID: 18486287. Exclusion Code: X3.

641. McFadden A, Gavine A, Renfrew MJ, et al. Support for healthy breastfeeding mothers with healthy term babies. Cochrane Database Syst Rev. 2017 Feb 28;2:Cd001141. doi: 10.1002/14651858.CD001141.pub5. PMID: 28244064. Exclusion Code: X3.

642. McGovern P, Dagher RK, Rice HR, et al. A longitudinal analysis of total workload and women's health after childbirth. J Occup Environ Med. 2011 May;53(5):497-505. doi: 10.1097/JOM.0b013e318217197b. PMID: 21508869. Exclusion Code: X5. 
643. McInnes RJ, Chambers J. Infants admitted to neonatal units--interventions to improve breastfeeding outcomes: a systematic review 1990-2007. Matern Child Nutr. 2008 Oct;4(4):235-63. doi: 10.1111/j.17408709.2008.00150.x. PMID: 18811790. Exclusion Code: X6.

644. McInnes RJ, Love JG, Stone DH. Evaluation of a community-based intervention to increase breastfeeding prevalence. J Public Health Med. 2000 Jun;22(2):138-45. PMID: 10912550. Exclusion Code: X3.

645. McIsaac KE, Sellen DW, Lou W, et al. Prevalence and Characteristics Associated with Breastfeeding Initiation Among Canadian Inuit from the 2007-2008 Nunavut Inuit Child Health Survey. Matern Child Health J. 2015 Sep;19(9):2003-11. doi: 10.1007/s10995-015-1712-3. PMID: 25656726. Exclusion Code: X3.

646. McKeever P, Stevens B, Miller KL, et al. Home versus hospital breastfeeding support for newborns: a randomized controlled trial. Birth. 2002 Dec;29(4):258-65. PMID: 12431265. Exclusion Code: X3.

647. McKeever P, Stevens B, Miller K-L, et al. Home versus hospital breastfeeding support newborns: A randomized controlled trial. Birth (Berkeley, Calif.); 2002. p. 258-65. Exclusion Code: X3.

648. McKenzie F, Ellison-Loschmann L, Jeffreys $\mathrm{M}$, et al. Healthy lifestyle and risk of breast cancer for indigenous and non-indigenous women in New Zealand: a case control study. BMC Cancer. 2014 Jan 10;14:12. doi: 10.1186/1471-2407-14-12. PMID: 24410858. Exclusion Code: X10.

649. McLachlan HL, Forster DA, Amir LH, et al. Supporting breastfeeding In Local Communities (SILC): protocol for a cluster randomised controlled trial. BMC Pregnancy Childbirth. 2014 Oct 03;14:346. doi: 10.1186/1471-2393-14-346. PMID: 25281300. Exclusion Code: X6.

650. McLaughlin JR, Risch HA, Lubinski J, et al. Reproductive risk factors for ovarian cancer in carriers of BRCA1 or BRCA2 mutations: a case-control study. Lancet Oncol. 2007 Jan;8(1):26-34. doi: 10.1016/s14702045(06)70983-4. PMID: 17196508. Exclusion Code: X10.
651. McLennan JD, Kotelchuck M, Cho H. Prevalence, persistence, and correlates of depressive symptoms in a national sample of mothers of toddlers. J Am Acad Child Adolesc Psychiatry. 2001 Nov;40(11):131623. doi: 10.1097/00004583-20011100000012. PMID: 11699806. Exclusion Code: X6.

652. McQueen KA, Dennis CL, Stremler R, et al. A pilot randomized controlled trial of a breastfeeding self-efficacy intervention with primiparous mothers. J Obstet Gynecol Neonatal Nurs. 2011 Jan-Feb;40(1):35-46. doi: 10.1111/j.1552-6909.2010.01210.x. PMID: 21244493. Exclusion Code: X3.

653. Meah S. A breastfeeding intervention increased breast feeding and reduced GI tract infections and atopic eczema. Evidence Based Nursing. 2001:106-. PMID: 106950246. Exclusion Code: X1.

654. Meedya S, Fernandez R, Fahy K. Effect of educational and support interventions on long-term breastfeeding rates in primiparous women: a systematic review and metaanalysis. JBI Database of Systematic Reviews \& Implementation Reports. 2017;15(9):2307-32. doi: 10.11124/JBISRIR-2016-002955. PMID: 125339018. Exclusion Code: X Irretrievable.

655. Meerlo-Habing ZE, Kosters-Boes EA, Klip $\mathrm{H}$, et al. Early discharge with tube feeding at home for preterm infants is associated with longer duration of breast feeding. Arch Dis Child Fetal Neonatal Ed. 2009 Jul;94(4):F294-7. doi: 10.1136/adc.2008.146100. PMID: 19131433. Exclusion Code: X2.

656. Meglio GD, McDermott MP, Klein JD. A randomized controlled trial of telephone peer support's influence on breastfeeding duration in adolescent mothers. Breastfeed Med. 2010 Feb;5(1):41-7. doi: 10.1089/bfm.2009.0016. PMID: 20043705. Exclusion Code: X3.

657. Meier PP, Engstrom JL, Hurst NM, et al. A comparison of the efficiency, efficacy, comfort, and convenience of two hospitalgrade electric breast pumps for mothers of very low birthweight infants. Breastfeed Med. 2008 Sep;3(3):141-50. doi: 10.1089/bfm.2007.0021. PMID: 18778208. Exclusion Code: X5. 
658. Meier PP, Engstrom JL, Janes JE, et al. Breast pump suction patterns that mimic the human infant during breastfeeding: greater milk output in less time spent pumping for breast pump-dependent mothers with premature infants. J Perinatol. 2012 Feb;32(2):103-10. doi: 10.1038/jp.2011.64. PMID: 21818062. Exclusion Code: X3.

659. Meier PP, Engstrom JL, Rossman B. Breastfeeding peer counselors as direct lactation care providers in the neonatal intensive care unit. J Hum Lact. 2013 Aug;29(3):313-22. doi: 10.1177/0890334413482184. PMID: 23563112. Exclusion Code: X6.

660. Mejdoubi J, van den Heijkant SC, van Leerdam FJ, et al. Effects of nurse home visitation on cigarette smoking, pregnancy outcomes and breastfeeding: a randomized controlled trial. Midwifery. 2014 Jun;30(6):688-95. doi: 10.1016/j.midw.2013.08.006. PMID: 24041564. Exclusion Code: X3.

661. Mellin PS, Poplawski DT, Gole A, et al. Impact of a formal breastfeeding education program. MCN Am J Matern Child Nurs. 2011 Mar-Apr;36(2):82-8; quiz 9-90. doi: 10.1097/NMC.0b013e318205589e. PMID: 21150474. Exclusion Code: X6.

662. Mendez RO, Gallegos AC, Cabrera RM, et al. Bone mineral density changes in lactating adolescent mothers during the first postpartum year. Am J Hum Biol. 2013 Mar-Apr;25(2):222-4. doi: 10.1002/ajhb.22366. PMID: 24065361. Exclusion Code: X6.

663. Menezes MA, Garcia DC, de Melo EV, et al. Preterm newborns at Kangaroo Mother Care: a cohort follow-up from birth to six months. Rev Paul Pediatr. 2014 Jun;32(2):171-7. PMID: 25119747. Exclusion Code: X8.

664. Merewood A, Chamberlain LB, Cook JT, et al. The effect of peer counselors on breastfeeding rates in the neonatal intensive care unit: results of a randomized controlled trial. Arch Pediatr Adolesc Med. 2006 Jul;160(7):681-5. doi: 10.1001/archpedi.160.7.681. PMID: 16818832. Exclusion Code: X3.
665. Merewood A, Philipp BL, Chawla N, et al. The baby-friendly hospital initiative increases breastfeeding rates in a US neonatal intensive care unit. J Hum Lact. 2003 May;19(2):166-71. doi: 10.1177/0890334403252475. PMID: 12744533. Exclusion Code: X6.

666. Merten S, Dratva J, Ackermann-Liebrich U. Do baby-friendly hospitals influence breastfeeding duration on a national level? Pediatrics. 2005 Nov;116(5):e702-8. doi: 10.1542/peds.2005-0537. PMID: 16263985. Exclusion Code: X4.

667. Metallinos-Katsaras E, Brown L, Colchamiro R. Maternal WIC participation improves breastfeeding rates: a statewide analysis of WIC participants. Matern Child Health J. 2015 Jan;19(1):136-43. doi: 10.1007/s10995-014-1504-1. PMID: 24777674. Exclusion Code: X4.

668. Mettner J. The brain-boosting benefits of breastfeeding. Minn Med. 2006 Mar;89(3):20-1. PMID: 16669426. Exclusion Code: X1.

669. Meyer EC, Coll CT, Lester BM, et al. Family-based intervention improves maternal psychological well-being and feeding interaction of preterm infants. Pediatrics. 1994 Feb;93(2):241-6. PMID: 8121735. Exclusion Code: X3.

670. Mikami FCF, de Lourdes Brizot M, Tase TH, et al. Effect of Prenatal Counseling on Breastfeeding Rates in Mothers of Twins. JOGNN: Journal of Obstetric, Gynecologic \& Neonatal Nursing. 2017;46(2):229-37. doi: 10.1016/j.jogn.2016.10.005. PMID: 121618426. Exclusion Code: X3.

671. Mikiel-Kostyra K, Mazur J, Boltruszko I. Effect of early skin-to-skin contact after delivery on duration of breastfeeding: a prospective cohort study. Acta Paediatr. 2002;91(12):1301-6. PMID: 12578285. Exclusion Code: X3.

672. Milani HS, Amiri P, Mohseny M, et al. Postpartum home care and its effects on mothers' health: A clinical trial. J Res Med Sci. 2017:1-6. doi: 10.4103/jrms.JRMS_319_17. PMID: 125321308. Exclusion Code: X5. 
673. Miller J, Beharie MC, Taylor AM, et al. Parent Reports of Exclusive Breastfeeding After Attending a Combined Midwifery and Chiropractic Feeding Clinic in the United Kingdom: A Cross-Sectional Service Evaluation. J Evid Based Complementary Altern Med. 2016 Apr;21(2):85-91. doi: 10.1177/2156587215625399. PMID: 26763046. Exclusion Code: X3.

674. Millikan RC, Newman B, Tse CK, et al. Epidemiology of basal-like breast cancer.

Breast Cancer Res Treat. 2008 May;109(1):123-39. doi: 10.1007/s10549007-9632-6. PMID: 17578664. Exclusion Code: X10.

675. Milman N, Hvas AM, Bergholt T. Vitamin $D$ status during normal pregnancy and postpartum. A longitudinal study in 141 Danish women. J Perinat Med. 2011 Nov 19;40(1):57-61. doi: 10.1515/jpm.2011.120. PMID: 22098305. Exclusion Code: X5.

676. Minkovitz C, Strobino D, Hughart N, et al. Early effects of the healthy steps for young children program. Arch Pediatr Adolesc Med. 2001 Apr;155(4):470-9. PMID: 11296075. Exclusion Code: X3.

677. Miracle DJ, Meier PP, Bennett PA. Making my baby healthy: Changing the decision from formula to human milk feedings for very-low-birth-weight infants. Adv Exp Med Biol. 2004;554:317-9. PMID: 15384590. Exclusion Code: X3.

678. Mirghafourvand M, Mohammad-AlizadehCharandabi S, Ahmadpour P, et al. Breast Cancer Risk Based on the Gail Model and its Predictors in Iranian Women. Asian Pac J Cancer Prev. 2016;17(8):3741-5. PMID: 27644610. Exclusion Code: X8.

679. Mitchell-Box KM, Braun KL. Impact of male-partner-focused interventions on breastfeeding initiation, exclusivity, and continuation. J Hum Lact. 2013

Nov;29(4):473-9. doi: 10.1177/0890334413491833. PMID: 23792369. Exclusion Code: X3.

680. Mobarakeh ZS, Mirzaei K, Hatmi N, et al. Dietary habits contributing to breast cancer risk among Iranian women. Asian Pac J Cancer Prev. 2014;15(21):9543-7. PMID: 25422253. Exclusion Code: X8.
681. Modugno F, Moslehi R, Ness RB, et al. Reproductive factors and ovarian cancer risk in Jewish BRCA1 and BRCA2 mutation carriers (United States). Cancer Causes Control. 2003 Jun;14(5):439-46. PMID: 12946038. Exclusion Code: X10.

682. Modugno F, Ness RB, Wheeler JE. Reproductive risk factors for epithelial ovarian cancer according to histologic type and invasiveness. Ann Epidemiol. 2001 Nov;11(8):568-74. PMID: 11709277. Exclusion Code: X10.

683. Mohamad Yusuff AS, Tang L, Binns CW, et al. Breastfeeding and Postnatal Depression. J Hum Lact. 2016;32(2):277-81. doi: 10.1177/0890334415620788. PMID: 114575371. Exclusion Code: X8.

684. Mollborg P, Wennergren G, Norvenius SG, et al. Bed-sharing among six-month-old infants in western Sweden. Acta Paediatr. 2011 Feb;100(2):226-30. doi: 10.1111/j.1651-2227.2010.02008.x. PMID: 20840516. Exclusion Code: X5.

685. Moller UK, Vieth Streym S, Mosekilde L, et al. Changes in bone mineral density and body composition during pregnancy and postpartum. A controlled cohort study. Osteoporos Int. 2012 Apr;23(4):1213-23. doi: 10.1007/s00198-011-1654-6. PMID: 21607805. Exclusion Code: X5.

686. Monga D, Rai U, Kumari S. Breast feeding practices and maternal employment. Asia Oceania J Obstet Gynaecol. 1989 Dec;15(4):339-42. PMID: 2624576. Exclusion Code: X3.

687. Mongeon M, Allard R. [Controlled study of a regular telephone support program given by volunteers on the establishment of breastfeeding]. Canadian journal of public health $=$ Revue canadienne de santé publique; 1995. p. 124-7. Exclusion Code: X7.

688. Moore ER, Anderson GC, Bergman N. Early skin-to-skin contact for mothers and their healthy newborn infants. Cochrane Database Syst Rev. 2007 Jul 18(3):Cd003519. doi: 10.1002/14651858.CD003519.pub2. PMID: 17636727. Exclusion Code: X6. 
689. Moore ER, Anderson GC, Bergman N, et al. Early skin-to-skin contact for mothers and their healthy newborn infants. Cochrane Database Syst Rev. 2012 May

16(5):Cd003519. doi: 10.1002/14651858.CD003519.pub3. PMID: 22592691. Exclusion Code: X3.

690. Moore ER, Bergman N, Anderson GC, et al. Early skin-to-skin contact for mothers and their healthy newborn infants. Cochrane Database of Systematic Reviews: John Wiley \& Sons, Ltd; 2016. Exclusion Code: X3.

691. Moore Simas TA, Corvera S, Lee MM, et al. Understanding multifactorial influences on the continuum of maternal weight trajectories in pregnancy and early postpartum: study protocol, and participant baseline characteristics. BMC Pregnancy Childbirth. 2015 Mar 28;15:71. doi: 10.1186/s12884-015-0490-7. PMID: 25885002. Exclusion Code: X9.

692. Moorman PG, Calingaert B, Palmieri RT, et al. Hormonal risk factors for ovarian cancer in premenopausal and postmenopausal women. Am J Epidemiol. 2008 May 1;167(9):1059-69. doi: 10.1093/aje/kwn006. PMID: 18303003. Exclusion Code: X10.

693. Morales L, Alvarez-Garriga C, Matta J, et al. Factors associated with breast cancer in Puerto Rican women. J Epidemiol Glob Health. 2013 Dec;3(4):205-15. doi: 10.1016/j.jegh.2013.08.003. PMID: 24206792. Exclusion Code: X10.

694. Moran VH, Morgan H, Rothnie K, et al. Incentives to promote breastfeeding: a systematic review. Pediatrics. 2015 Mar;135(3):e687-702. doi: 10.1542/peds.2014-2221. PMID: 25647672. Exclusion Code: X3.

695. Mordini B, Bortoli E, Pagano R, et al. Correlations between welfare initiatives and breastfeeding rates: a 10-year follow-up study. Acta Paediatr. 2009 Jan;98(1):80-5. doi: 10.1111/j.1651-2227.2008.00986.x. PMID: 18710434. Exclusion Code: X6.

696. Morel K, Chapman D, Kyer N, et al. Peer counselors improve breastfeeding technique among low-income, obese women. FASEB J; 2010. Exclusion Code: X3.
697. Moreland JC, Lloyd L, Braun SB, et al. A new teaching model to prolong breastfeeding among Latinos. J Hum Lact. 2000 Nov;16(4):337-41. PMID: 11155611. Exclusion Code: X9.

698. Morgan H, Hoddinott P, Thomson G, et al. Benefits of Incentives for Breastfeeding and Smoking cessation in pregnancy (BIBS): a mixed-methods study to inform trial design. Health Technol Assess. 2015 Apr;19(30):1522, vii-viii. doi: 10.3310/hta19300. PMID: 25897655. Exclusion Code: X3.

699. Morrell CJ, Spiby H, Stewart P, et al. Costs and effectiveness of community postnatal support workers: randomised controlled trial. BMJ. 2000 Sep 09;321(7261):593-8. PMID: 10977833. Exclusion Code: X3.

700. Morrell CJ, Spiby H, Stewart P, et al. Costs and benefits of community postnatal support workers: a randomised controlled trial. Health Technol Assess. 2000;4(6):1-100. PMID: 10858637. Exclusion Code: X3.

701. Morrow AL, Guerrero ML. From bioactive substances to research on breast-feeding promotion. Adv Exp Med Biol. 2001;501:447-55. PMID: 11787715. Exclusion Code: X1.

702. Morse JM, Bottorff JL. Intending to breastfeed and work. J Obstet Gynecol Neonatal Nurs. 1989 Nov-Dec;18(6):493500. PMID: 2600687. Exclusion Code: X3.

703. Morton S, Kirkwood S, Thangaratinam S. Interventions to modify the progression to type 2 diabetes mellitus in women with gestational diabetes: a systematic review of literature. Curr Opin Obstet Gynecol. 2014 Dec;26(6):476-86. doi: 10.1097/gco.0000000000000127. PMID: 25333677. Exclusion Code: X3.

704. Mosack V, Shore ER. Screening for depression among pregnant and postpartum women. J Community Health Nurs. 2006 Spring;23(1):37-47. doi: 10.1207/s15327655jchn2301_4. PMID: 16445363. Exclusion Code: X3.

705. Mosley C, Whittle C, Hicks C. A pilot study to assess the viability of a randomised controlled trial of methods of supplementary feeding of breast-fed pre-term babies. Midwifery. 2001 Jun;17(2):150-7. doi: 10.1054/midw.2000.0244. PMID: 11399136. Exclusion Code: X3. 
706. Mottl-Santiago J, Walker C, Ewan J, et al. A hospital-based doula program and childbirth outcomes in an urban, multicultural setting. Matern Child Health J. 2008

May;12(3):372-7. doi: 10.1007/s10995-0070245-9. PMID: 17610053. Exclusion Code: $\mathrm{X} 4$.

707. Mourouti N, Papavagelis C, Psaltopoulou T, et al. Aims, design and methods of a casecontrol study for the assessment of the role of dietary habits, eating behaviors and environmental factors, on the development of breast cancer. Maturitas. 2013 Jan;74(1):31-6. doi: 10.1016/j.maturitas.2012.10.004. PMID: 23131812. Exclusion Code: X9.

708. Much D, Beyerlein A, Rossbauer M, et al. Beneficial effects of breastfeeding in women with gestational diabetes mellitus. Mol Metab. 2014 Jun;3(3):284-92. doi: 10.1016/j.molmet.2014.01.002. PMID: 24749058. Exclusion Code: X6.

709. Muhajarine N, Ng J, Bowen A, et al. Understanding the impact of the Canada Prenatal Nutrition Program: a quantitative evaluation. Can J Public Health. 2012 Mar 29;103(7 Suppl 1):eS26-31. PMID: 23618045. Exclusion Code: X4.

710. Muirhead PE, Butcher G, Rankin J, et al. The effect of a programme of organised and supervised peer support on the initiation and duration of breastfeeding: a randomised trial. Br J Gen Pract. 2006 Mar;56(524):1917. PMID: 16536959. Exclusion Code: X3.

711. Mukhopadhyay S, Lieberman ES, Puopolo KM, et al. Effect of early-onset sepsis evaluations on in-hospital breastfeeding practices among asymptomatic term neonates. Hosp Pediatr. 2015 Apr;5(4):20310. doi: 10.1542/hpeds.2014-0126. PMID: 25832975. Exclusion Code: X5.

712. Murphy L, Warner DD, Parks J, et al. A quality improvement project to improve the rate of early breast milk expression in mothers of preterm infants. J Hum Lact. 2014 Nov;30(4):398-401. doi: 10.1177/0890334414544124. PMID: 25063572. Exclusion Code: X3.

713. Murphy SP. The fitness of FITS. J Am Diet Assoc. 2010 Dec;110(12 Suppl):S8-10. doi: 10.1016/j.jada.2010.10.024. PMID: 21092771. Exclusion Code: X1.
714. Mustila T, Raitanen J, Keskinen P, et al. Pragmatic controlled trial to prevent childhood obesity in maternity and child health care clinics: pregnancy and infant weight outcomes (the VACOPP Study). BMC Pediatr. 2013 May 20;13:80. doi: 10.1186/1471-2431-13-80. PMID: 23688259. Exclusion Code: X3.

715. Nabulsi M, Hamadeh H, Tamim H, et al. A complex breastfeeding promotion and support intervention in a developing country: study protocol for a randomized clinical trial. BMC Public Health. 2014 Jan 15;14:36. doi: 10.1186/1471-2458-14-36. PMID: 24428951. Exclusion Code: X6.

716. Nagata C, Mizoue T, Tanaka K, et al. Breastfeeding and breast cancer risk: an evaluation based on a systematic review of epidemiologic evidence among the Japanese population. Jpn J Clin Oncol. 2012 Feb;42(2):124-30. doi: 10.1093/jjco/hyr182. PMID: 22210922. Exclusion Code: X6.

717. Nagle CM, Bain CJ, Green AC, et al. The influence of reproductive and hormonal factors on ovarian cancer survival. Int J Gynecol Cancer. 2008 May-Jun;18(3):40713. doi: 10.1111/j.1525-1438.2007.01031.x. PMID: 17645507. Exclusion Code: X4.

718. Narayanan I, Kumar H, Singhal PK, et al. Maternal participation in the care of the high risk infant: follow-up evaluation. Indian Pediatr. 1991 Feb;28(2):161-7. PMID: 2055631. Exclusion Code: X3.

719. Nascimento Souza MH, Aparecida Barbosa Nogueira JN, Domingues Sodré VR. MONITORING THE NUTRITIONAL AND HEALTH STATUS OF CHILDREN WHO ATTEND A COMMUNITY NURSERY. Journal of Nursing UFPE / Revista de Enfermagem UFPE. 2015;9(5):7862-8. doi: 10.5205/reuol.612157155-1-ED.0905201516. PMID: 109801893. Exclusion Code: X2.

720. Navarro JI, Sigulem DM, Ferraro AA, et al. The double task of preventing malnutrition and overweight: a quasi-experimental community-based trial. BMC Public Health. 2013 Mar 09;13:212. doi: 10.1186/14712458-13-212. PMID: 23496939. Exclusion Code: X5. 
721. Nayeri F, Dalili H, Shahzadeh Fazeli K, et al. Effects of Community-Based Newborn Care Intervention on Neonate Health Status in a District of Tehran (Iran). J Family Reprod Health. 2016 Jun;10(2):99-103. PMID: 27648100. Exclusion Code: X3.

722. Neifert M, Gray J, Gary N, et al. Effect of two types of hospital feeding gift packs on duration of breast-feeding among adolescent mothers. J Adolesc Health Care. 1988 Sep;9(5):411-3. PMID: 3049484. Exclusion Code: X3.

723. Nelson AL, Le MH, Musherraf Z, et al. Intermediate-term glucose tolerance in women with a history of gestational diabetes: natural history and potential associations with breastfeeding and contraception. Am J Obstet Gynecol. 2008 Jun;198(6):699.e1-7; discussion .e7-8. doi: 10.1016/j.ajog.2008.03.029. PMID: 18439553. Exclusion Code: X5.

724. Ness RB, Grisso JA, Cottreau C, et al. Factors related to inflammation of the ovarian epithelium and risk of ovarian cancer. Epidemiology. 2000 Mar;11(2):1117. PMID: 11021606. Exclusion Code: X10.

725. Neville CE, McKinley MC, Holmes VA, et al. The relationship between breastfeeding and postpartum weight change--a systematic review and critical evaluation. Int $\mathrm{J}$ Obes (Lond). 2014 Apr;38(4):577-90. doi: 10.1038/ijo.2013.132. PMID: 23892523. Exclusion Code: X11.

726. Neyzi O, Olgun P, Kutluay T, et al. An educational intervention on promotion of breast feeding. Paediatr Perinat Epidemiol. 1991 Jul;5(3):286-98. PMID: 1881839. Exclusion Code: X3.

727. Nichols C. A Simple Change in Process Results in Significant Improvement in the Rate of First Feeding at Breast. JOGNN: Journal of Obstetric, Gynecologic \& Neonatal Nursing. 2014;43(Supp 1):S40-1. doi: 10.1111/1552-6909.12413. PMID: 103980226. Exclusion Code: X3.
728. Nicklas JM, Miller LJ, Zera CA, et al. Factors associated with depressive symptoms in the early postpartum period among women with recent gestational diabetes mellitus. Matern Child Health J. 2013 Nov;17(9):1665-72. doi: 10.1007/s10995-012-1180-y. PMID: 23124798. Exclusion Code: X3.

729. Niela-Vilén H, Axelin A, Melender H-L, et al. Breastfeeding preterm infants - a randomized controlled trial of the effectiveness of an Internet-based peersupport group. J Adv Nurs. 2016;72(10):2495-507. doi: 10.1111/jan.12993. PMID: 117924135. Exclusion Code: X3.

730. Nikodem VC, Nolte AG, Wolman W, et al. Companionship by a lay labour supporter to modify the clinical birth environment: longterm effects on mother and child. Curationis. 1998 Mar;21(1):8-12. PMID: 9791343. Exclusion Code: X3.

731. Nishioka E, Haruna M, Ota E, et al. A prospective study of the relationship between breastfeeding and postpartum depressive symptoms appearing at 1-5 months after delivery. J Affect Disord. 2011 Oct;133(3):553-9. doi: 10.1016/j.jad.2011.04.027. PMID: 21705090. Exclusion Code: X10.

732. Noble S. Maternal employment and the initiation of breastfeeding. Acta Paediatr. 2001 Apr;90(4):423-8. PMID: 11332935. Exclusion Code: X3.

733. Noel-Weiss J, Rupp A, Cragg B, et al. Randomized controlled trial to determine effects of prenatal breastfeeding workshop on maternal breastfeeding self-efficacy and breastfeeding duration. J Obstet Gynecol Neonatal Nurs. 2006 Sep-Oct;35(5):616-24. doi: 10.1111/j.1552-6909.2006.00077.x. PMID: 16958717. Exclusion Code: X3.

734. Nommsen-Rivers LA. Does insulin explain the relation between maternal obesity and poor lactation outcomes? An overview of the literature. Adv Nutr. 2016 Mar;7(2):40714. doi: 10.3945/an.115.011007. PMID: 26980825. Exclusion Code: X6. 
735. Nommsen-Rivers LA, Mastergeorge AM, Hansen RL, et al. Doula care, early breastfeeding outcomes, and breastfeeding status at 6 weeks postpartum among lowincome primiparae. J Obstet Gynecol Neonatal Nurs. 2009 Mar-Apr;38(2):157-73. doi: 10.1111/j.1552-6909.2009.01005.x. PMID: 19323712. Exclusion Code: X3.

736. Nouer SS, Ware JL, Baldwin KM, et al. Changes in Breastfeeding Attitudes in a Metropolitan Community in Tennessee. J Hum Lact. 2015 Aug;31(3):519-29. doi: 10.1177/0890334415578648. PMID: 25829477. Exclusion Code: X3.

737. November L. Discussing breastfeeding: Turning an idea into an intervention. British Journal of School Nursing. 2014;9(5):23542. PMID: 107858997. Exclusion Code: $\mathrm{X} 1$.

738. Nunes LM, Giugliani ER, Santo LC, et al. Reduction of unnecessary intake of water and herbal teas on breast-fed infants: a randomized clinical trial with adolescent mothers and grandmothers. J Adolesc Health. 2011 Sep;49(3):258-64. doi: 10.1016/j.jadohealth.2010.12.009. PMID: 21856517. Exclusion Code: X3.

739. O'Brien K, Bracht M, Macdonell K, et al. A pilot cohort analytic study of Family Integrated Care in a Canadian neonatal intensive care unit. BMC Pregnancy Childbirth. 2013;13 Suppl 1:S12. doi: 10.1186/1471-2393-13-s1-s12. PMID: 23445639. Exclusion Code: X3.

740. O'Brien K, Bracht M, Robson K, et al. Evaluation of the Family Integrated Care model of neonatal intensive care: a cluster randomized controlled trial in Canada and Australia. BMC Pediatr. 2015 Dec 15;15:210. doi: 10.1186/s12887-015-05270. PMID: 26671340. Exclusion Code: X3.

741. Oddy WH, Smith GJ, Jacoby P. A possible strategy for developing a model to account for attrition bias in a longitudinal cohort to investigate associations between exclusive breastfeeding and overweight and obesity at 20 years. Ann Nutr Metab. 2014;65(23):234-5. doi: 10.1159/000360548. PMID: 25413663. Exclusion Code: X5.
742. Ogbuanu C, Glover S, Probst J, et al. Balancing work and family: effect of employment characteristics on breastfeeding. J Hum Lact. 2011 Aug;27(3):225-38; quiz 93-5. doi: 10.1177/0890334410394860. PMID: 21393503. Exclusion Code: X3.

743. Ogbuanu C, Glover S, Probst J, et al. The effect of maternity leave length and time of return to work on breastfeeding. Pediatrics. 2011 Jun;127(6):e1414-27. doi: 10.1542/peds.2010-0459. PMID: 21624878. Exclusion Code: X3.

744. Ogbuehi O. Commentary on Kaunonen M, Hannula L and Tarkka M-T (2012): a systematic review of peer support interventions for breastfeeding. Journal of Clinical Nursing 21, 1943-1954. J Clin Nurs. 2013 Jun;22(11-12):1786-7. doi: 10.1111/jocn.12023. PMID: 23574200. Exclusion Code: X1.

745. O'Hara MH, Frazier LM, Stembridge TW, et al. Physician-led, hospital-linked, birth care centers can decrease cesarean section rates without increasing rates of adverse events. Birth. 2013 Sep;40(3):155-63. doi: 10.1111/birt.12051. PMID: 24635500. Exclusion Code: X3.

746. O'Hara MW, Swain AM. Rates and risk of postpartum depression - a meta-analysis. Int Rev Psychiatry. 1996;8(1):37-54. Exclusion Code: X6.

747. Ojofeitimi EO. Mothers' awareness on benefits of breast-milk and cultural taboos during lactation. Soc Sci Med E. 1981 May;15(2):135-8. PMID: 7268475. Exclusion Code: X3.

748. Okamura C, Tsubono Y, Ito K, et al. Lactation and risk of endometrial cancer in Japan: a case-control study. Tohoku J Exp Med. 2006 Feb;208(2):109-15. PMID: 16434833. Exclusion Code: X5.

749. Okechukwu AA, Okpe EC, Okolo AA. Exclusive breastfeeding and postnatal changes in maternal anthropometry. Niger $\mathrm{J}$ Clin Pract. 2009 Dec;12(4):383-8. PMID: 20329677. Exclusion Code: X8. 
750. Oken E, Patel R, Guthrie LB, et al. Effects of an intervention to promote breastfeeding on maternal adiposity and blood pressure at 11.5 y postpartum: results from the Promotion of Breastfeeding Intervention Trial, a cluster-randomized controlled trial. Am J Clin Nutr. 2013 Oct;98(4):1048-56. doi: 10.3945/ajcn.113.065300. PMID: 23945719. Exclusion Code: X5.

751. Olenick PL. The effect of structured group prenatal education on breastfeeding confidence, duration and exclusivity to twelve weeks postpartum: Touro University International; 2006. Exclusion Code: X3.

752. Olson BH, Haider SJ, Vangjel L, et al. A quasi-experimental evaluation of a breastfeeding support program for low income women in Michigan. Matern Child Health J. 2010 Jan;14(1):86-93. doi: 10.1007/s10995-008-0430-5. PMID: 19082697. Exclusion Code: X3.

753. Olson CM, Baker IR, Demment MM, et al. The healthy start partnership: an approach to obesity prevention in young families. Fam Community Health. 2014 Jan-Mar;37(1):7485. doi: $10.1097 /$ fch.0000000000000016. PMID: 24297009. Exclusion Code: X6.

754. O'Reilly M, Avalos G, Dennedy MC, et al. Breast-feeding is associated with reduced postpartum maternal glucose intolerance after gestational diabetes. Ir Med J. 2012 May;105(5 Suppl):31-6. PMID: 22838108. Exclusion Code: X5.

755. Ortiz J, McGilligan K, Kelly P. Duration of breast milk expression among working mothers enrolled in an employer-sponsored lactation program. Pediatr Nurs. 2004 MarApr;30(2):111-9. PMID: 15185732. Exclusion Code: X4.

756. Owen CG, Whincup PH, Cook DG. Breastfeeding and cardiovascular risk factors and outcomes in later life: evidence from epidemiological studies. Proc Nutr Soc. 2011 Nov;70(4):478-84. doi: 10.1017/s0029665111000590. PMID: 21801475. Exclusion Code: X6.

757. Oza-Frank R, Bhatia A, Smith C. Impact of peer counselors on breastfeeding outcomes in a nondelivery NICU setting. Adv Neonatal Care. 2014 Aug;14(4):E1-8. doi: 10.1097/anc.0000000000000101. PMID: 25000101. Exclusion Code: X6.
758. Ozenoglu A, Sokulmez Kaya P, Asal Ulus C, et al. The Relationship of Knowledge and Breastfeeding Practice to Maternal BMI. Ecol Food Nutr. 2017 Mar-Apr;56(2):15270. doi: 10.1080/03670244.2016.1275604. PMID: 28112971. Exclusion Code: X8.

759. Ozmen V, Ozcinar B, Karanlik H, et al. Breast cancer risk factors in Turkish women--a University Hospital based nested case control study. World J Surg Oncol. 2009 Apr 08;7:37. doi: 10.1186/1477-78197-37. PMID: 19356229. Exclusion Code: $\mathrm{X} 8$.

760. Paes Pedras CT, Mezzacappa MA, da CostaPinto EA. Breastfeeding of very low-weight infants before and after implementation of the baby-friendly hospital initiative. J Trop Pediatr. 2012 Aug;58(4):324-6. doi: 10.1093/tropej/fmr075. PMID: 21940739. Exclusion Code: X3.

761. Page L, McCourt C, Beake S, et al. Clinical interventions and outcomes of One-to-One midwifery practice. J Public Health Med. 1999 Sep;21(3):243-8. PMID: 10528949. Exclusion Code: X3.

762. Palmer JR, Boggs DA, Wise LA, et al. Parity and lactation in relation to estrogen receptor negative breast cancer in African American women. Cancer Epidemiol Biomarkers Prev. 2011 Sep;20(9):1883-91. doi: 10.1158/1055-9965.epi-11-0465. PMID: 21846820. Exclusion Code: X6.

763. Palmer JR, Viscidi E, Troester MA, et al. Parity, lactation, and breast cancer subtypes in African American women: results from the AMBER Consortium. J Natl Cancer Inst. 2014 Oct;106(10)doi: 10.1093/jnci/dju237. PMID: 25224496. Exclusion Code: X10.

764. Pan H, He Z, Ling L, et al. Reproductive factors and breast cancer risk among BRCA1 or BRCA2 mutation carriers: results from ten studies. Cancer Epidemiol. 2014 Feb;38(1):1-8. doi: 10.1016/j.canep.2013.11.004. PMID: 24332935. Exclusion Code: X11.

765. Pandit N, Yeshwanth M, Albuquerque SI. Factors influencing initiation of breastfeeding in an urban set up. Indian Pediatr. 1994 Dec;31(12):1558-60. PMID: 7875823. Exclusion Code: X3. 
766. Papp LM. The longitudinal role of breastfeeding in mothers' and fathers' relationship quality trajectories. Breastfeed Med. 2012 Aug;7:241-7. doi: 10.1089/bfm.2011.0074. PMID: 22148926. Exclusion Code: X5.

767. Park B, Park S, Shin HR, et al. Population attributable risks of modifiable reproductive factors for breast and ovarian cancers in Korea. BMC Cancer. 2016 Jan 06;16:5. doi: 10.1186/s12885-015-2040-0. PMID: 26732868. Exclusion Code: X6.

768. Parker L, Krueger c, Sullivan S, et al. Effect of breast milk on hospital costs and length of stay among very low-birth-weight infants in the NICU (Provisional abstract). Adv Neonatal Care; 2012. p. 254-9. Exclusion Code: X3.

769. Parker LA, Sullivan S, Krueger C, et al. Effect of early breast milk expression on milk volume and timing of lactogenesis stage II among mothers of very low birth weight infants: a pilot study. J Perinatol. 2012 Mar;32(3):205-9. doi:

10.1038/jp.2011.78. PMID: 21904296. Exclusion Code: X3.

770. Parker LA, Sullivan S, Krueger C, et al. Association of timing of initiation of breastmilk expression on milk volume and timing of lactogenesis stage II among mothers of very low-birth-weight infants. Breastfeed Med. 2015 Mar;10(2):84-91. doi: 10.1089/bfm.2014.0089. PMID: 25659030. Exclusion Code: X5.

771. Parker M, Burnham L, Cook J, et al. 10 years after baby-friendly designation: breastfeeding rates continue to increase in a US neonatal intensive care unit. J Hum Lact. 2013 Aug;29(3):354-8. doi: 10.1177/0890334413489374. PMID: 23727629. Exclusion Code: X6.

772. Passanha A, Benicio MH, Venancio SI, et al. Influence of the support offered to breastfeeding by maternity hospitals. Rev Saude Publica. 2015;49doi: 10.1590/s00348910.2015049005354. PMID: 26759966. Exclusion Code: X6.
773. Pastor Climente IP, Morales Suarez Varela MM, Magraner Gil JF, et al. Gynecological characteristics related to breast cancer in pre and postmenopausal women. Clin Transl Oncol. 2006 Jun;8(6):416-22. PMID: 16790394. Exclusion Code: X4.

774. Pate B. A systematic review of the effectiveness of breastfeeding intervention delivery methods. J Obstet Gynecol Neonatal Nurs. 2009 Nov-Dec;38(6):64253. doi: 10.1111/j.1552-6909.2009.01068.x. PMID: 19930278. Exclusion Code: X3.

775. Patel R, Oken E, Bogdanovich N, et al. Cohort profile: The promotion of breastfeeding intervention trial (PROBIT). Int J Epidemiol. 2014 Jun;43(3):679-90. doi: 10.1093/ije/dyt003. PMID: 23471837. Exclusion Code: X1.

776. Patnode CD, Henninger ML, Senger CA, et al. Primary care interventions to support breastfeeding: updated evidence report and systematic review for the US Preventive Services Task Force. JAMA. 2016 Oct 25;316(16):1694-705. doi: 10.1001/jama.2016.8882. PMID: 27784101. Exclusion Code: X3.

777. Paul IM, Beiler JS, Schaefer EW, et al. A randomized trial of single home nursing visits vs office-based care after nursery/maternity discharge: the Nurses for Infants Through Teaching and Assessment After the Nursery (NITTANY) Study. Arch Pediatr Adolesc Med. 2012 Mar;166(3):263 70. doi: 10.1001/archpediatrics.2011.198. PMID: 22064874. Exclusion Code: X3.

778. Paul IM, Downs DS, Schaefer EW, et al. Postpartum anxiety and maternal-infant health outcomes. Pediatrics. 2013 Apr;131(4):e1218-24. doi: 10.1542/peds.2012-2147. PMID: 23460682. Exclusion Code: X3.

779. Pazandeh F, Huss R, Hirst J, et al. An evaluation of the quality of care for women with low risk pregnanacy: The use of evidence-based practice during labour and childbirth in four public hospitals in Tehran. Midwifery. 2015 Nov;31(11):1045-53. doi: 10.1016/j.midw.2015.07.003. PMID: 26292760. Exclusion Code: X8. 
780. Pearce MS, Birrell FN, Francis RM, et al. Lifecourse study of bone health at age 49-51 years: the Newcastle thousand families cohort study. J Epidemiol Community Health. 2005 Jun;59(6):475-80. doi: 10.1136/jech.2004.025999. PMID: 15911643. Exclusion Code: X3.

781. Pereira CR, Fonseca Vde M, Couto de Oliveira MI, et al. Assessment of factors that interfere on breastfeeding within the first hour of life. Rev Bras Epidemiol. 2013 Jun;16(2):525-34. doi: 10.1590/s1415790x2013000200026. PMID: 24142022. Exclusion Code: X3.

782. Perez A, Valdes V. Santiago Breastfeeding Promotion Program: preliminary results of an intervention study. Am J Obstet Gynecol. 1991 Dec;165(6 Pt 2):2039-44. PMID: 1755466. Exclusion Code: X6.

783. Perez-Blasco J, Viguer P, Rodrigo MF. Effects of a mindfulness-based intervention on psychological distress, well-being, and maternal self-efficacy in breast-feeding mothers: results of a pilot study. Arch Womens Ment Health. 2013 Jun;16(3):22736. doi: 10.1007/s00737-013-0337-z. PMID: 23512648. Exclusion Code: X3.

784. Perez-Escamilla R. Evidence-based breastfeeding promotion: A global scaling up model. Breastfeed Med; 2012. p. 571. Exclusion Code: X8.

785. Perez-Escamilla R, Hromi-Fiedler A, VegaLopez S, et al. Impact of peer nutrition education on dietary behaviors and health outcomes among Latinos: a systematic literature review. J Nutr Educ Behav. 2008 Jul-Aug;40(4):208-25. doi: 10.1016/j.jneb.2008.03.011. PMID: 18565462. Exclusion Code: X3.

786. Perez-Escamilla R, Martinez JL, SeguraPerez S. Impact of the Baby-friendly Hospital Initiative on breastfeeding and child health outcomes: a systematic review. Matern Child Nutr. 2016 Jul;12(3):402-17. doi: 10.1111/mcn.12294. PMID: 26924775. Exclusion Code: X6.

787. Perez-Escamilla R, Pollitt E, Lonnerdal B, et al. Infant feeding policies in maternity wards and their effect on breast-feeding success: an analytical overview. Am J Public Health. 1994 Jan;84(1):89-97. PMID: 8279619. Exclusion Code: X3.
788. Perez-Escamilla R, Segura-Millan S, Pollitt $E$, et al. Effect of the maternity ward system on the lactation success of low-income urban Mexican women. Early Hum Dev. 1992 Nov;31(1):25-40. PMID: 1486816. Exclusion Code: X3.

789. Perrine CG, Scanlon KS, Li R, et al. BabyFriendly hospital practices and meeting exclusive breastfeeding intention. Pediatrics. 2012 Jul;130(1):54-60. doi: 10.1542/peds.2011-3633. PMID: 22665406. Exclusion Code: X6.

790. Peterson KE, Sorensen G, Pearson M, et al. Design of an intervention addressing multiple levels of influence on dietary and activity patterns of low-income, postpartum women. Health Educ Res. 2002 Oct;17(5):531-40. PMID: 12408198. Exclusion Code: X5.

791. Petrova A, Ayers C, Stechna S, et al. Effectiveness of exclusive breastfeeding promotion in low-income mothers: a randomized controlled study. Breastfeed Med. 2009 Jun;4(2):63-9. doi: 10.1089/bfm.2008.0126. PMID: 19239405. Exclusion Code: X3.

792. Philipp BL, Malone KL, Cimo S, et al. Sustained breastfeeding rates at a US babyfriendly hospital. Pediatrics. 2003 Sep;112(3 Pt 1):e234-6. PMID: 12949318. Exclusion Code: X6.

793. Phillips KA, Milne RL, West DW, et al. Prediagnosis reproductive factors and allcause mortality for women with breast cancer in the breast cancer family registry. Cancer Epidemiol Biomarkers Prev. 2009 Jun;18(6):1792-7. doi: 10.1158/10559965.epi-08-1014. PMID: 19505912. Exclusion Code: X5.

794. Phillips RM, Merritt TA, Goldstein MR, et al. Prevention of postpartum smoking relapse in mothers of infants in the neonatal intensive care unit. J Perinatol. 2012 May;32(5):374-80. doi: 10.1038/jp.2011.106. PMID: 21836549. Exclusion Code: X3. 
795. Phipps AI, Malone KE, Porter PL, et al. Reproductive and hormonal risk factors for postmenopausal luminal, HER-2overexpressing, and triple-negative breast cancer. Cancer. 2008 Oct 01;113(7):1521-6. doi: 10.1002/cncr.23786. PMID: 18726992. Exclusion Code: X10.

796. Phoutthakeo P, Otsuka K, Ito C, et al. Crossborder promotion of formula milk in Lao People's Democratic Republic. J Paediatr Child Health. 2014 Jan;50(1):51-6. doi: 10.1111/jpc.12391. PMID: 24134830. Exclusion Code: X6.

797. Pietschnig B, Siklossy H, Gottling A, et al. Breastfeeding rates of VLBW infants-influence of professional breastfeeding support. Adv Exp Med Biol. 2000;478:42930. doi: 10.1007/0-306-46830-1_61. PMID: 11065116. Exclusion Code: X6.

798. Pincombe J, Baghurst P, Antoniou G, et al. Baby Friendly Hospital Initiative practices and breast feeding duration in a cohort of first-time mothers in Adelaide, Australia. Midwifery. 2008 Mar;24(1):55-61. doi: 10.1016/j.midw.2006.06.009. PMID: 17197061. Exclusion Code: X6.

799. Pineda RG, Foss J, Richards L, et al. Breastfeeding changes for VLBW infants in the NICU following staff education. Neonatal Netw. 2009 Sep-Oct;28(5):311-9. doi: 10.1891/0730-0832.28.5.311. PMID: 19720595. Exclusion Code: X6.

800. Pinelli J, Atkinson SA, Saigal S. Randomized trial of breastfeeding support in very low-birth-weight infants. Arch Pediatr Adolesc Med. 2001 May;155(5):548-53. PMID: 11343496. Exclusion Code: X3.

801. Pisacane A, Continisio GI, Aldinucci M, et al. A controlled trial of the father's role in breastfeeding promotion. Pediatrics. 2005 Oct;116(4):e494-8. doi: 10.1542/peds.20050479. PMID: 16199676. Exclusion Code: X3.

802. Pisacane A, Continisio P, Filosa C, et al. Use of baby carriers to increase breastfeeding duration among term infants: the effects of an educational intervention in Italy. Acta Paediatr. 2012 Oct;101(10):e4348. doi: 10.1111/j.1651-2227.2012.02758.x. PMID: 22734604. Exclusion Code: X3.
803. Pitonyak JS, Jessop AB, Pontiggia L, et al. Life Course Factors Associated with Initiation and Continuation of Exclusive Breastfeeding. Matern Child Health J. 2016 Feb;20(2):240-9. doi: 10.1007/s10995-0151823-x. PMID: 26496989. Exclusion Code: X6.

804. Pollard DL. The effect of self-regulation on breastfeeding duration in primiparous mothers: University of Pittsburgh; 1998. Exclusion Code: X3.

805. Pollard DL. Impact of a feeding log on breastfeeding duration and exclusivity. Matern Child Health J. 2011 Apr;15(3):395400. doi: 10.1007/s10995-010-0583-x. PMID: 20177755. Exclusion Code: X3.

806. Ponza M, Devaney B, Ziegler P, et al. Nutrient intakes and food choices of infants and toddlers participating in WIC. J Am Diet Assoc. 2004 Jan;104(1 Suppl 1):s71-9. doi: 10.1016/j.jada.2003.10.018. PMID: 14702021. Exclusion Code: X3.

807. Porteous R, Kaufman K, Rush J. The effect of individualized professional support on duration of breastfeeding: a randomized controlled trial. J Hum Lact. 2000 Nov;16(4):303-8. PMID: 11155608. Exclusion Code: X3.

808. Porto LA, Lora KJ, Soares JC, et al. Metabolic syndrome is an independent risk factor for breast cancer. Arch Gynecol Obstet. 2011 Nov;284(5):1271-6. doi: 10.1007/s00404-011-1837-6. PMID: 21249376. Exclusion Code: X8.

809. Pound C, Moreau K, Rohde K, et al. The impact of a breastfeeding support intervention on breastfeeding duration in jaundiced infants admitted to a tertiary care centre hospital: A randomized controlled trial. Paediatrics and Child Health (Canada); 2014. p. e94. Exclusion Code: X3.

810. Pound C, Ward N, Freuchet M, et al. Hospital Staff's Perceptions with Regards to the Baby-Friendly Initiative. J Hum Lact. 2016 Nov;32(4):648-57. doi: 10.1177/0890334416662630. PMID: 27565199. Exclusion Code: X5. 
811. Pound CM, Moreau K, Rohde K, et al. Lactation support and breastfeeding duration in jaundiced infants: a randomized controlled trial. PLoS One. 2015;10(3):e0119624. doi: 10.1371/journal.pone.0119624. PMID: 25747308. Exclusion Code: X3.

812. Pourhoseingholi MA, Mehrabi Y, AlaviMajd H, et al. Association between risk of breast cancer and fertility factors--a latent variable approach. Asian Pac J Cancer Prev. 2008 Apr-Jun;9(2):309-12. PMID:

18712981. Exclusion Code: X8.

813. Preer G, Pisegna JM, Cook JT, et al. Delaying the bath and in-hospital breastfeeding rates. Breastfeed Med. 2013 Dec;8(6):485-90. doi: 10.1089/bfm.2012.0158. PMID: 23635002. Exclusion Code: X6.

814. Prentice P, Acerini CL, Eleftheriou A, et al. Cohort Profile: the Cambridge Baby Growth Study (CBGS). Int J Epidemiol. 2016 Feb;45(1):35.a-g. doi: 10.1093/ije/dyv318. PMID: 26721600. Exclusion Code: X5.

815. Prime DK, Garbin CP, Hartmann PE, et al. Simultaneous breast expression in breastfeeding women is more efficacious than sequential breast expression. Breastfeed Med. 2012 Dec;7(6):442-7. doi:

10.1089/bfm.2011.0139. PMID: 23039397.

Exclusion Code: X5.

816. Provini LE, Corwin MJ, Geller NL, et al. Differences in Infant Care Practices and Smoking among Hispanic Mothers Living in the United States. J Pediatr. 2017

Mar;182:321-6.e1. doi:

10.1016/j.jpeds.2016.11.053. PMID:

27979582. Exclusion Code: X3.

817. Przybylik-Mazurek E, HubalewskaDydejczyk A, Fedorowicz A, et al. Factors connected with the female sex seem to play an important role in differentiated thyroid cancer. Gynecol Endocrinol. 2011 Nov;27(11):961-5. doi: 10.3109/09513590.2011.569606. PMID: 21675817. Exclusion Code: X5.

818. Pugh LC, Milligan RA. Nursing intervention to increase the duration of breastfeeding. Appl Nurs Res. 1998 Nov;11(4):190-4. PMID: 9852662. Exclusion Code: X3.
819. Pugh LC, Milligan RA, Frick KD, et al. Breastfeeding duration, costs, and benefits of a support program for low-income breastfeeding women. Birth. 2002 Jun;29(2):95-100. PMID: 12000411. Exclusion Code: X3.

820. Pugh LC, Serwint JR, Frick KD, et al. A randomized controlled community-based trial to improve breastfeeding rates among urban low-income mothers. Acad Pediatr. 2010 Jan-Feb;10(1):14-20. doi: 10.1016/j.acap.2009.07.005. PMID: 19854119. Exclusion Code: X3.

821. Pugin E, Valdes V, Labbok MH, et al. Does prenatal breastfeeding skills group education increase the effectiveness of a comprehensive breastfeeding promotion program? J Hum Lact. 1996 Mar;12(1):15-9. PMID: 8715233. Exclusion Code: X6.

822. Qi Y, Zhang Y, Fein S, et al. Maternal and breast pump factors associated with breast pump problems and injuries. J Hum Lact. 2014 Feb;30(1):62-72; quiz 110-2. doi: 10.1177/0890334413507499. PMID: 24166052. Exclusion Code: X4.

823. Qiu L, Xie X, Lee A, et al. Infants' first feeds in Hangzhou, PR China. Asia Pac J Clin Nutr. 2007;16 Suppl 1:458-61. PMID: 17392150. Exclusion Code: X3.

824. Quarles A, Williams PD, Hoyle DA, et al. Mothers' intention, age, education and the duration and management of breastfeeding. Matern Child Nurs J. 1994 JulSep;22(3):102-8. PMID: 7815846. Exclusion Code: X3.

825. Quigley MA. Re: “Duration of breastfeeding and risk of overweight: a meta-analysis”. Am J Epidemiol. 2006 May 01;163(9):8702; author reply 2-3. doi: 10.1093/aje/kwj134. PMID: 16554341. Exclusion Code: X1.

826. Quinlivan JA, Box H, Evans SF. Postnatal home visits in teenage mothers: a randomised controlled trial. Lancet. 2003 Mar 15;361(9361):893-900. doi: 10.1016/s0140-6736(03)12770-5. PMID: 12648967. Exclusion Code: X3.

827. Quinlivan JA, Evans SF. Teenage antenatal clinics may reduce the rate of preterm birth: a prospective study. BJOG. 2004 Jun;111(6):571-8. doi: 10.1111/j.14710528.2004.00146.x. PMID: 15198785. Exclusion Code: X3. 
828. Raat H, Struijk MK, Remmers T, et al. Primary prevention of overweight in preschool children, the BeeBOFT study (breastfeeding, breakfast daily, outside playing, few sweet drinks, less TV viewing): design of a cluster randomized controlled trial. BMC Public Health. 2013 Oct 19;13:974. doi: 10.1186/1471-2458-13-974. PMID: 24138805. Exclusion Code: X3.

829. Raeisi K, Shariat M, Nayeri F, et al. A single center study of the effects of trained fathers' participation in constant breastfeeding. Acta Med Iran. 2014;52(9):694-6. PMID: 25421843. Exclusion Code: X3.

830. Raghavan V, Bharti B, Kumar P, et al. First hour initiation of breastfeeding and exclusive breastfeeding at six weeks: prevalence and predictors in a tertiary care setting. Indian J Pediatr. 2014 Aug;81(8):743-50. doi: 10.1007/s12098013-1200-y. PMID: 24113879. Exclusion Code: X8.

831. Rahman A, Haq Z, Sikander S, et al. Using cognitive-behavioural techniques to improve exclusive breastfeeding in a low-literacy disadvantaged population. Matern Child Nutr. 2012;8(1):57-71. doi: 10.1111/j.17408709.2011.00362.x. PMID: 104610779.

Exclusion Code: X5.

832. Raisi Dehkordi Z, Raei M, Ghassab Shirazi $\mathrm{M}$, et al. Effect of Telephone Counseling on Continuity and Duration of Breastfeeding among Primiparus Women. HAYAT. 2012;18(2):9-. PMID: 104496551.

Exclusion Code: X7.

833. Raiskila S, Axelin A, Rapeli S, et al. Trends in care practices reflecting parental involvement in neonatal care. Early Hum Dev. 2014 Dec;90(12):863-7. doi: 10.1016/j.earlhumdev.2014.08.010. PMID: 25463833. Exclusion Code: X3.

834. Ramezani Tehrani F, Momenan AA, Khomami MB, et al. Does lactation protect mothers against metabolic syndrome? Findings from the Tehran Lipid and Glucose Study. J Obstet Gynaecol Res. 2014 Mar;40(3):736-42. PMID: 24738118. Exclusion Code: X8.
835. Rasmussen KM, Dieterich CM, Zelek ST, et al. Interventions to increase the duration of breastfeeding in obese mothers: the Bassett Improving Breastfeeding Study. Breastfeed Med. 2011 Apr;6(2):69-75. doi: 10.1089/bfm.2010.0014. PMID: 20958105. Exclusion Code: X3.

836. Ratnasari D, Paramashanti BA, Hadi H, et al. Family support and exclusive breastfeeding among Yogyakarta mothers in employment. Asia Pac J Clin Nutr. 2017 Jun;26(Suppl 1):S31-s5. doi: 10.6133/apjcn.062017.s8. PMID: 28625034. Exclusion Code: X3.

837. Ravn IH, Smith L, Smeby NA, et al. Effects of early mother-infant intervention on outcomes in mothers and moderately and late preterm infants at age 1 year: a randomized controlled trial. Infant Behav Dev. 2012 Feb;35(1):36-47. doi: 10.1016/j.infbeh.2011.09.006. PMID: 22024475. Exclusion Code: X3.

838. Rayfield S, Oakley L, Quigley MA. Association between breastfeeding support and breastfeeding rates in the UK: a comparison of late preterm and term infants. BMJ Open. 2015 Nov 13;5(11):e009144. doi: 10.1136/bmjopen-2015-009144. PMID: 26567257. Exclusion Code: X3.

839. Reat AM, Crixell SH, Friedman BJ, et al. Comparison of Food Intake Among Infants and Toddlers Participating in a South Central Texas WIC Program Reveals Some Improvements After WIC Package Changes. Matern Child Health J. 2015 Aug;19(8):1834-41. doi: 10.1007/s10995015-1697-y. PMID: 25656719. Exclusion Code: X6.

840. Redondo CM, Gago-Dominguez M, Ponte SM, et al. Breast feeding, parity and breast cancer subtypes in a Spanish cohort. PLoS One. 2012;7(7):e40543. doi: 10.1371/journal.pone.0040543. PMID: 22792365. Exclusion Code: X4.

841. Redsell SA, Edmonds B, Swift JA, et al. Systematic review of randomised controlled trials of interventions that aim to reduce the risk, either directly or indirectly, of overweight and obesity in infancy and early childhood. Matern Child Nutr. 2016 Jan;12(1):24-38. doi: 10.1111/mcn.12184. PMID: 25894857. Exclusion Code: X5. 
842. Reifsnider E, Eckhart D. Prenatal breastfeeding education: its effect on breastfeeding among WIC participants. J Hum Lact. 1997 Jun;13(2):121-5. PMID: 9233202. Exclusion Code: X3.

843. Reifsnider E, McCormick DP, Cullen KW, et al. A randomized controlled trial to prevent childhood obesity through early childhood feeding and parenting guidance: rationale and design of study. BMC Public Health. 2013 Sep 24;13:880. doi: 10.1186/1471-2458-13-880. PMID: 24063435. Exclusion Code: X1.

844. Relton C, Strong M, Renfrew MJ, et al. Cluster randomised controlled trial of a financial incentive for mothers to improve breast feeding in areas with low breastfeeding rates: the NOSH study protocol. BMJ Open. 2016 Apr 11;6(4):e010158. doi: 10.1136/bmjopen2015-010158. PMID: 27067889. Exclusion Code: X1.

845. Rempel LA, McCleary L. Effects of the implementation of a breastfeeding best practice guideline in a Canadian public health agency. Res Nurs Health. 2012 Oct;35(5):435-49. doi: 10.1002/nur.21495. PMID: 22736297. Exclusion Code: X6.

846. Renfrew MJ, Craig D, Dyson L, et al. Breastfeeding promotion for infants in neonatal units: a systematic review and economic analysis. Health Technol Assess. 2009 Aug;13(40):1-146, iii-iv. doi: 10.3310/hta13400. PMID: 19728934. Exclusion Code: X3.

847. Renfrew MJ, Dyson L, McCormick F, et al. Breastfeeding promotion for infants in neonatal units: a systematic review. Child Care Health Dev. 2010 Mar;36(2):165-78. doi: 10.1111/j.1365-2214.2009.01018.x. PMID: 19886907. Exclusion Code: X3.

848. Renfrew MJ, Lang S, Martin L, et al. Feeding schedules in hospitals for newborn infants. Cochrane Database Syst Rev. 2000(2):Cd000090. doi: 10.1002/14651858.cd000090. PMID: 10796125. Exclusion Code: X1.
849. Renfrew MJ, McCormick FM, Wade A, et al. Support for healthy breastfeeding mothers with healthy term babies. Cochrane Database Syst Rev. 2012 May

16(5):Cd001141. doi: 10.1002/14651858.CD001141.pub4. PMID: 22592675. Exclusion Code: X3.

850. Renfrew MJ, Spiby H, D'Souza L, et al. Rethinking research in breast-feeding: a critique of the evidence base identified in a systematic review of interventions to promote and support breast-feeding. Public Health Nutr. 2007 Jul;10(7):726-32. doi: 10.1017/s1368980007387405. PMID: 17381919. Exclusion Code: X5.

851. Retnakaran R, Qi Y, Sermer M, et al. The postpartum cardiovascular risk factor profile of women with isolated hyperglycemia at 1hour on the oral glucose tolerance test in pregnancy. Nutr Metab Cardiovasc Dis. 2011 Sep;21(9):706-12. doi: 10.1016/j.numecd.2011.02.010. PMID: 21703831. Exclusion Code: X3.

852. Reynolds D, Hennessy E, Polek E. Is breastfeeding in infancy predictive of child mental well-being and protective against obesity at 9 years of age? Child Care Health Dev. 2014 Nov;40(6):882-90. doi: 10.1111/cch.12126. PMID: 24354743. Exclusion Code: X4.

853. Riman T, Dickman PW, Nilsson S, et al. Risk factors for invasive epithelial ovarian cancer: results from a Swedish case-control study. Am J Epidemiol. 2002 Aug 15;156(4):363-73. PMID: 12181107. Exclusion Code: X10.

854. Ringo NA. Will golden hour approach of skin-to-skin contact increase breastfeeding exclusivity? Commun Nurs Res. 2013;46:573-. PMID: 107895852. Exclusion Code: X9.

855. Risch HA, Weiss NS, Lyon JL, et al. Events of reproductive life and the incidence of epithelial ovarian cancer. Am J Epidemiol. 1983 Feb;117(2):128-39. PMID: 6681935. Exclusion Code: X10.

856. Rishel PE, Sweeney P. Comparison of breastfeeding rates among women delivering infants in military treatment facilities with and without lactation consultants. Mil Med. 2005 May;170(5):435-8. PMID: 15974214. Exclusion Code: X3. 
857. Rito RV, Oliveira MI, Brito Ados S. Degree of compliance with the ten steps of the Breastfeeding Friendly Primary Care Initiative and its association with the prevalence of exclusive breastfeeding. $\mathrm{J}$ Pediatr (Rio J). 2013 Sep-Oct;89(5):477-84. doi: 10.1016/j.jped.2013.02.018. PMID: 23871498. Exclusion Code: X6.

858. Rivera-Pasquel M, Escobar-Zaragoza L, Gonzalez de Cosio T. Breastfeeding and maternal employment: results from three national nutritional surveys in Mexico. Matern Child Health J. 2015 May;19(5):1162-72. doi: 10.1007/s10995014-1622-9. PMID: 25366099. Exclusion Code: X6.

859. Riza E, Remoundos DD, Bakali E, et al. Anthropometric characteristics and mammographic parenchymal patterns in post-menopausal women: a populationbased study in Northern Greece. Cancer Causes Control. 2009 Mar;20(2):181-91. doi: 10.1007/s10552-008-9232-8. PMID: 18814046. Exclusion Code: X5.

860. Robling M, Bekkers MJ, Bell K, et al. Effectiveness of a nurse-led intensive homevisitation programme for first-time teenage mothers (Building Blocks): a pragmatic randomised controlled trial. Lancet. 2016 Jan 09;387(10014):146-55. doi: 10.1016/s0140-6736(15)00392-x. PMID: 26474809. Exclusion Code: X5.

861. Rogers SL, Hughes BA, Tomlinson JW, et al. Cortisol metabolism, postnatal depression and weight changes in the first 12 months postpartum. Clin Endocrinol (Oxf). 2016 Dec;85(6):881-90. doi: 10.1111/cen.13150. PMID: 27374760. Exclusion Code: X5.

862. Romaguera D, Vergnaud AC, Peeters PH, et al. Is concordance with World Cancer Research Fund/American Institute for Cancer Research guidelines for cancer prevention related to subsequent risk of cancer? Results from the EPIC study. Am J Clin Nutr. 2012 Jul;96(1):150-63. doi: 10.3945/ajcn.111.031674. PMID: 22592101. Exclusion Code: X3.

863. Rosen IM, Krueger MV, Carney LM, et al. Prenatal breastfeeding education and breastfeeding outcomes. MCN Am J Matern Child Nurs. 2008 Sep-Oct;33(5):315-9. doi: 10.1097/01.NMC.0000334900.22215.ec. PMID: 18758336. Exclusion Code: X6.
864. Rosenberg KD, Stull JD, Adler MR, et al. Impact of hospital policies on breastfeeding outcomes. Breastfeed Med. 2008

Jun;3(2):110-6. doi: 10.1089/bfm.2007.0039. PMID: 18563999. Exclusion Code: X4.

865. Rossiter JC. The effect of a culture-specific education program to promote breastfeeding among Vietnamese women in Sydney. Int J Nurs Stud. 1994 Aug;31(4):369-79. PMID: 7928125. Exclusion Code: X3.

866. Rousseaux J, Duhamel A, Turck D, et al. Breastfeeding shows a protective trend toward adolescents with higher abdominal adiposity. Obes Facts. 2014;7(5):289-301. doi: 10.1159/000368583. PMID: 25277836. Exclusion Code: X5.

867. Rowe-Murray HJ, Fisher JR. Baby friendly hospital practices: cesarean section is a persistent barrier to early initiation of breastfeeding. Birth. 2002 Jun;29(2):124-31. PMID: 12000413. Exclusion Code: X3.

868. Rozga MR, Kerver JM, Olson BH. Impact of peer counselling breast-feeding support programme protocols on any and exclusive breast-feeding discontinuation in lowincome women. Public Health Nutr. 2015 Feb;18(3):453-63. doi: 10.1017/s1368980014000603. PMID: 24809675. Exclusion Code: X4.

869. Rush D, Sloan NL, Leighton J, et al. The National WIC Evaluation: evaluation of the Special Supplemental Food Program for Women, Infants, and Children. V. Longitudinal study of pregnant women. Am J Clin Nutr. 1988 Aug;48(2 Suppl):439-83. PMID: 3400628. Exclusion Code: X6.

870. Russell BK, Aviles M, Brion LP. Relationship between perinatal counseling and incidence of breastfeeding in an innercity population. J Perinatol. 1999 OctNov;19(7):501-4. PMID: 10685299. Exclusion Code: X6.

871. Rutledge G, Ayers DR, MacGowan C, et al. An Overview of the CDC's CommunityBased Breastfeeding Supplemental Cooperative Agreement. J Hum Lact. 2015 Nov;31(4):571-6. doi: 10.1177/0890334415599779. PMID: 26303879. Exclusion Code: X3. 
872. Ryan AS, Zhou W. Lower breastfeeding rates persist among the Special Supplemental Nutrition Program for Women, Infants, and Children participants, 1978-2003. Pediatrics. 2006

Apr;117(4):1136-46. doi: 10.1542/peds.2005-1555. PMID: 16585308. Exclusion Code: X3.

873. Ryser FG. Breastfeeding attitudes, intention, and initiation in low-income women: the effect of the best start program. J Hum Lact. 2004 Aug;20(3):300-5. doi: 10.1177/0890334404266985. PMID: 15296584. Exclusion Code: X3.

874. Sadacharan R, Grossman X, Matlak S, et al. Hospital discharge bags and breastfeeding at 6 months: data from the infant feeding practices study II. J Hum Lact. 2014 Feb;30(1):73-9. doi: 10.1177/0890334413513653. PMID: 24305594. Exclusion Code: X3.

875. Sakkaki M, Khairkhah M. PROMOTION OF EXCLUSIVE BREASTFEEDING: TEACHING GOOD POSITIONING AND SUPPORT FROM FATHERS AND FAMILIES. Journal of Urmia Nursing \& Midwifery Faculty. 2013;10(6):824-31. PMID: 104138754. Exclusion Code: X7.

876. Salonen AH, Kaunonen M, Astedt-Kurki P, et al. Development of an internet-based intervention for parents of infants. J Adv Nurs. 2008 Oct;64(1):60-72. doi: 10.1111/j.1365-2648.2008.04759.x. PMID: 18808593. Exclusion Code: X5.

877. Samano R, Martinez-Rojano H, Godinez Martinez E, et al. Effects of breastfeeding on weight loss and recovery of pregestational weight in adolescent and adult mothers. Food Nutr Bull. 2013 Jun;34(2):123-30. PMID: 23964385. Exclusion Code: X8.

878. Sandy JM, Anisfeld E, Ramirez E. Effects of a prenatal intervention on breastfeeding initiation rates in a Latina immigrant sample. J Hum Lact. 2009 Nov;25(4):40411; quiz 58-9. doi: 10.1177/0890334409337308. PMID: 19487705. Exclusion Code: X3.

879. Santiago L, Bettiol H, Barbieri M, et al. [Promotion of breastfeeding: the importance of pediatricians with specific training]. $\mathrm{J}$ Pediatr (Rio J); 2003. p. 504-12. Exclusion Code: X3.
880. Santoro Junior W, Martinez FE. Effect of intervention on the rates of breastfeeding of very low birth weight newborns. J Pediatr (Rio J). 2007 Nov-Dec;83(6):541-6. doi: doi:10.2223/JPED.1724. PMID: 18074059. Exclusion Code: X3.

881. Saxton A, Fahy K, Skinner V, et al. Effects of immediate skin to skin contact and breastfeeding after birth on postpartum haemorrage (PPH) rates: A cohort study. Women \& Birth. 2013;26:S16-7. doi: 10.1016/j.wombi.2013.08.268. PMID: 104099308. Exclusion Code: X5.

882. Schafer E, Campo S, Colaizy T, et al. Influence of Experiences and Perceptions Related to Breastfeeding One's First Child on Breastfeeding Initiation of Second Child. Matern Child Health J. 2017;21(6):1288-96. doi: 10.1007/s10995-016-2228-1. PMID: 123203817. Exclusion Code: X3.

883. Schmidt ME, Chang-Claude J, Slanger T, et al. Physical activity and postmenopausal breast cancer: effect modification by other breast cancer risk factors. Methods Inf Med. 2009;48(5):444-50. doi: 10.3414/me9239. PMID: 19696953. Exclusion Code: X3.

884. Schmidt MI, Duncan BB, Castilhos C, et al. Lifestyle INtervention for Diabetes prevention After pregnancy (LINDABrasil): study protocol for a multicenter randomized controlled trial. BMC Pregnancy Childbirth. 2016 Mar 30;16:68. doi: 10.1186/s12884-016-0851-x. PMID: 27029489. Exclusion Code: X3.

885. Schnatz PF, Barker KG, Marakovits KA, et al. Effects of age at first pregnancy and breast-feeding on the development of postmenopausal osteoporosis. Menopause. 2010 Nov-Dec;17(6):1161-6. doi: 10.1097/gme.0b013e3181e0efb3. PMID: 20613669. Exclusion Code: X6.

886. Schneidrova D, Mullerova D, Janout V, et al. Impact of breast-feeding promotion on infant feeding in the Czech Republic. J Nutr Educ Behav. 2003 Sep-Oct;35(5):228-35. PMID: 14521822. Exclusion Code: X6.

887. Schrempp S, Ryan-Haddad A, Gait KA. Pharmacist counseling of pregnant or lactating women. J Am Pharm Assoc (Wash). 2001 Nov-Dec;41(6):887-90. PMID: 11765114. Exclusion Code: X3. 
888. Schroeder N, Rushovich B, Bartlett E, et al. Early Obesity Prevention: A Randomized Trial of a Practice-Based Intervention in 024-Month Infants. J Obes.

2015;2015:795859. doi:

10.1155/2015/795859. PMID: 26078877.

Exclusion Code: X3.

889. Schubiger G, Schwarz U, Tonz O. UNICEF/WHO baby-friendly hospital initiative: does the use of bottles and pacifiers in the neonatal nursery prevent successful breastfeeding? Neonatal Study Group. Eur J Pediatr. 1997 Nov;156(11):874-7. PMID: 9392404. Exclusion Code: X3.

890. Schultz DJ, Byker Shanks C, Houghtaling B. The Impact of the 2009 Special Supplemental Nutrition Program for Women, Infants, and Children Food Package Revisions on Participants: A Systematic Review. J Acad Nutr Diet. 2015 Nov;115(11):1832-46. doi: 10.1016/j.jand.2015.06.381. PMID: 26276067. Exclusion Code: X6.

891. Schwartz R, Ellings A, Baisden A, et al. Washington 'Steps' Up: A 10-Step Quality Improvement Initiative to Optimize Breastfeeding Support in Community Health Centers. J Hum Lact. 2015 Nov;31(4):651-9. doi: 10.1177/0890334415591147. PMID: 26124223. Exclusion Code: X5.

892. Schwartz R, Vigo A, de Oliveira LD, et al. The Effect of a Pro-Breastfeeding and Healthy Complementary Feeding Intervention Targeting Adolescent Mothers and Grandmothers on Growth and Prevalence of Overweight of Preschool Children. PLoS One. 2015;10(7):e0131884. doi: 10.1371/journal.pone.0131884. PMID: 26161657. Exclusion Code: X3.

893. Schwarz EB, Brown JS, Creasman JM, et al. Lactation and maternal risk of type 2 diabetes: a population-based study. Am J Med. 2010 Sep;123(9):863.e1-6. doi: 10.1016/j.amjmed.2010.03.016. PMID: 20800156. Exclusion Code: X10.

894. Schwarz EB, McClure CK, Tepper PG, et al. Lactation and maternal measures of subclinical cardiovascular disease. Obstet Gynecol. 2010 Jan;115(1):41-8. doi: 10.1097/AOG.0b013e3181c5512a. PMID: 20027032. Exclusion Code: X6.
895. Schy DS, Maglaya CF, Mendelson SG, et al. The effects of in-hospital lactation education on breastfeeding practice. J Hum Lact. 1996 Jun;12(2):117-22. PMID: 8932042. Exclusion Code: X3.

896. Sciacca JP, Dube DA, Phipps BL, et al. A breast feeding education and promotion program: effects on knowledge, attitudes, and support for breast feeding. J Community Health. 1995 Dec;20(6):473-90. PMID: 8568022. Exclusion Code: X3.

897. Sciacca JP, Phipps BL, Dube DA, et al. Influences on breast-feeding by lowerincome women: an incentive-based, partnersupported educational program. J Am Diet Assoc. $1995 \mathrm{Mar}$;95(3):323-8. doi: 10.1016/s0002-8223(95)00083-6. PMID: 7860944. Exclusion Code: X3.

898. Seguranyes G, Costa D, Fuentelsaz-Gallego C, et al. Efficacy of a videoconferencing intervention compared with standard postnatal care at primary care health centres in Catalonia. Midwifery. 2014 Jun;30(6):764-71. doi: 10.1016/j.midw.2013.08.004. PMID: 24016553. Exclusion Code: X3.

899. Seimyr L, Edhborg M, Lundh W, et al. In the shadow of maternal depressed mood: experiences of parenthood during the first year after childbirth. J Psychosom Obstet Gynaecol. 2004 Mar;25(1):23-34. PMID: 15376402. Exclusion Code: X10.

900. Serwint JR, Wilson ME, Vogelhut JW, et al. A randomized controlled trial of prenatal pediatric visits for urban, low-income families. Pediatrics. 1996 Dec;98(6 Pt 1):1069-75. PMID: 8951255. Exclusion Code: X3.

901. Shah MK, Austin KR. Do home visiting services received during pregnancy improve birth outcomes? Findings from Virginia PRAMS 2007-2008. Public Health Nurs. 2014 Sep-Oct;31(5):405-13. doi: 10.1111/phn.12103. PMID: 24438361. Exclusion Code: X3. 
902. Shakya P, Kunieda MK, Koyama M, et al. Effectiveness of community-based peer support for mothers to improve their breastfeeding practices: A systematic review and meta-analysis. PLoS One.

2017;12(5):e0177434. doi:

10.1371/journal.pone.0177434. PMID: 28510603. Exclusion Code: X3.

903. Shalofsky T. Telephone peer counselling of breastfeeding among WIC participants: a randomized controlled trial. MIDIRS Midwifery Digest. 2015;25(1):97-8. PMID: 107784093. Exclusion Code: X3.

904. Shantakumar S, Terry MB, Teitelbaum SL, et al. Reproductive factors and breast cancer risk among older women. Breast Cancer Res Treat. 2007 May;102(3):365-74. doi: 10.1007/s10549-006-9343-4. PMID: 17033925. Exclusion Code: X10.

905. Shapiro C. Shortened hospital stay for lowbirth-weight infants: nuts and bolts of a nursing intervention project. J Obstet Gynecol Neonatal Nurs. 1995 Jan;24(1):5662. PMID: 7714640. Exclusion Code: X5.

906. Sharma A. Efficacy of early skin-to-skin contact on the rate of exclusive breastfeeding in term neonates: a randomized controlled trial. African historical studies. 2016;16(3):790-7. doi: 10.4314/ahs.v16i3.20. PMID: CN01342313. Exclusion Code: X8.

907. Sharp D, Entwistle F. Why women stop breastfeeding in the early days. Pract Midwife. 2015 Oct;18(9):30-3. PMID: 26638652. Exclusion Code: X1.

908. Shema L, Ore L, Ben-Shachar M, et al. The association between breastfeeding and breast cancer occurrence among Israeli Jewish women: a case control study. J Cancer Res Clin Oncol. 2007 Aug;133(8):539-46. doi: 10.1007/s00432-007-0199-8. PMID: 17453241. Exclusion Code: X10.

909. Shiau SH. Randomized controlled trial of kangaroo care with fullterm infants: effects on maternal anxiety, breastmilk maturation, breast engorgement, and breastfeeding status: Case Western Reserve University (Health Sciences); 1997. Exclusion Code: X3.
910. Shinwell ES, Churgin Y, Shlomo M, et al. The effect of training nursery staff in breastfeeding guidance on the duration of breastfeeding in healthy term infants. Breastfeed Med. 2006 Winter;1(4):247-52. doi: 10.1089/bfm.2006.1.247. PMID: 17661605. Exclusion Code: X6.

911. Shorten A. Obstetric early discharge versus traditional hospital stay (Structured abstract). Aust Health Rev; 1995. p. 19-39. Exclusion Code: X5.

912. Sikorski J, Renfrew MJ. Support for breastfeeding mothers. Cochrane Database Syst Rev. 2000(2):Cd001141. doi: 10.1002/14651858.cd001141. PMID: 10796249. Exclusion Code: X1.

913. Sikorski J, Renfrew MJ, Pindoria S, et al. Support for breastfeeding mothers. Cochrane Database Syst Rev. 2002(1):Cd001141. doi: 10.1002/14651858.cd001141. PMID: 11869593. Exclusion Code: X1.

914. Sikorski J, Renfrew MJ, Pindoria S, et al. Support for breastfeeding mothers: a systematic review. Paediatr Perinat Epidemiol. 2003 Oct;17(4):407-17. PMID: 14629324. Exclusion Code: X3.

915. Silva SN, Cabral MN, Bezerra de Castro G, et al. Breast cancer risk and polymorphisms in genes involved in metabolism of estrogens (CYP17, HSD17beta1, COMT and MnSOD): possible protective role of MnSOD gene polymorphism Val/Ala and Ala/Ala in women that never breast fed. Oncol Rep. 2006 Oct;16(4):781-8. PMID: 16969494. Exclusion Code: X4.

916. Simonetti V, Palma E, Giglio A, et al. A structured telephonic counselling to promote the exclusive breastfeeding of healthy babies aged zero to six months: a pilot study. Int J Nurs Pract. 2012 Jun;18(3):289-94. doi: 10.1111/j.1440-172X.2012.02040.x. PMID: 22621300. Exclusion Code: X3.

917. Singh PJ, Guatee C, John R. Infant health care practices-a study in three communities. Med J Malaysia. 1981 Sep;36(3):166-70. PMID: 7329373. Exclusion Code: X3. 
918. Sinha B, Chowdhury R, Sankar MJ, et al. Interventions to improve breastfeeding outcomes: a systematic review and metaanalysis. Acta Paediatr. 2015

Dec;104(467):114-34. doi:

10.1111/apa.13127. PMID: 26183031.

Exclusion Code: X8.

919. Sioka C, Fotopoulos A, Georgiou A, et al. Age at menarche, age at menopause and duration of fertility as risk factors for osteoporosis. Climacteric. 2010

Feb;13(1):63-71. doi: 10.3109/13697130903075337. PMID: 19731122. Exclusion Code: X5.

920. Sipsma HL, Jones KL, Cole-Lewis H. Breastfeeding among adolescent mothers: a systematic review of interventions from high-income countries. J Hum Lact. 2015 May;31(2):221-9; quiz 321-2. doi: 10.1177/0890334414561264. PMID: 25480018. Exclusion Code: X3.

921. Sisk JE, Greer AL, Wojtowycz M, et al. Implementing evidence-based practice: evaluation of an opinion leader strategy to improve breast-feeding rates. Am J Obstet Gynecol. 2004 Feb;190(2):413-21. doi: 10.1016/j.ajog.2003.09.014. PMID: 14981383. Exclusion Code: X5.

922. Siskind V, Green A, Bain C, et al. Breastfeeding, menopause, and epithelial ovarian cancer. Epidemiology. 1997 Mar;8(2):188-91. PMID: 9229212. Exclusion Code: X10.

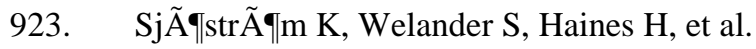
Comparison of breastfeeding in rural areas of Sweden and Australia - a cohort study. Women \& Birth. 2013;26(4):229-34. doi: 10.1016/j.wombi.2013.08.159. PMID: 104167614. Exclusion Code: X4.

924. Skafida V. Juggling work and motherhood: the impact of employment and maternity leave on breastfeeding duration: a survival analysis on Growing Up in Scotland data. Matern Child Health J. 2012 Feb;16(2):51927. doi: 10.1007/s10995-011-0743-7. PMID: 21274609. Exclusion Code: X3.

925. Skafida V. Change in breastfeeding patterns in Scotland between 2004 and 2011 and the role of health policy. Eur J Public Health. 2014 Dec;24(6):1033-41. doi: 10.1093/eurpub/cku029. PMID: 24642601. Exclusion Code: X6.
926. Skouteris H, Nagle C, Fowler M, et al. Interventions designed to promote exclusive breastfeeding in high-income countries: a systematic review. Breastfeed Med. 2014 Apr;9(3):113-27. doi: 10.1089/bfm.2013.0081. PMID: 24568270. Exclusion Code: X3.

927. Smirk CL, Bowman E, Doyle LW, et al. Home-based detoxification for neonatal abstinence syndrome reduces length of hospital admission without prolonging treatment. Acta Paediatr. 2014 Jun;103(6):601-4. doi: 10.1111/apa.12603. PMID: 24547949. Exclusion Code: X3.

928. Smith H, Embleto ND. Improving expressed breast milk (EBM) provision in the neonatal unit: A rapid and effective quality improvement (Ql) intervention. J Neonatal Nurs. 2013;19(4):149-53. doi: 10.1016/j.jnn.2013.02.001. PMID: 107792859. Exclusion Code: X6.

929. Smith HA, O'B Hourihane J, Kenny LC, et al. Early life factors associated with the exclusivity and duration of breast feeding in an Irish birth cohort study. MIDIRS Midwifery Digest. 2015;25(3):353-61. PMID: 109335115. Exclusion Code: X6.

930. Smithers LG, Kramer MS, Lynch JW. Effects of Breastfeeding on Obesity and Intelligence: Causal Insights From Different Study Designs. JAMA Pediatr. 2015 Aug;169(8):707-8. doi: 10.1001/jamapediatrics.2015.0175. PMID: 26053565. Exclusion Code: X5.

931. Soltani H, Sandall J. Organisation of maternity care and choices of mode of birth: a worldwide view. Midwifery. 2012 Apr;28(2):146-9. doi: 10.1016/j.midw.2012.01.009. PMID: 22365576. Exclusion Code: X3.

932. Sonchak L. The impact of WIC on breastfeeding initiation and gestational weight gain: Case study of South Carolina Medicaid mothers. Children \& Youth Services Review. 2017;79:115-25. doi: 10.1016/j.childyouth.2017.05.024. PMID: 124491427. Exclusion Code: X3. 
933. Song N, Choi JY, Sung H, et al. Heterogeneity of epidemiological factors by breast tumor subtypes in Korean women: a case-case study. Int J Cancer. 2014 Aug 01;135(3):669-81. doi: 10.1002/ijc.28685. PMID: 24916400. Exclusion Code: X6.

934. Sowers MR, Clark MK, Hollis B, et al. Radial bone mineral density in pre- and perimenopausal women: a prospective study of rates and risk factors for loss. J Bone Miner Res. 1992 Jun;7(6):647-57. doi: 10.1002/jbmr.5650070609. PMID: 1414483. Exclusion Code: X5.

935. Specker B, Binkley T. High parity is associated with increased bone size and strength. Osteoporos Int. 2005 Dec;16(12):1969-74. doi: 10.1007/s00198005-1978-1. PMID: 16091837. Exclusion Code: X5.

936. Spence AC, Campbell KJ, Crawford DA, et al. Mediators of improved child diet quality following a health promotion intervention: the Melbourne InFANT Program. Int J Behav Nutr Phys Act. 2014 Nov 04;11:137. doi: 10.1186/s12966-014-0137-5. PMID: 25366542. Exclusion Code: X2.

937. Spiby H, McCormick F, Wallace L, et al. A systematic review of education and evidence-based practice interventions with health professionals and breast feeding counsellors on duration of breast feeding. Midwifery. 2009 Feb;25(1):50-61. doi: 10.1016/j.midw.2007.01.006. PMID: 17418464. Exclusion Code: X6.

938. Spigelblatt L, Lainé-Ammara G, Arsenault $\mathrm{L}$, et al. [Influence of follow-up education of mothers about too early introduction of solid food to infants]. Pediatrie; 1991. p. 475-9. Exclusion Code: X7.

939. Spinelli MG, Endicott J, Goetz RR. Increased breastfeeding rates in black women after a treatment intervention. Breastfeed Med. 2013 Dec;8(6):479-84. doi: 10.1089/bfm.2013.0051. PMID: 23971683. Exclusion Code: X3.

940. Sprenger N, Odenwald H, Kukkonen AK, et al. FUT2-dependent breast milk oligosaccharides and allergy at 2 and 5 years of age in infants with high hereditary allergy risk. Eur J Nutr. 2017 Apr;56(3):1293-301. doi: 10.1007/s00394-016-1180-6. PMID: 26907090. Exclusion Code: X3.
941. Srinivas GL, Benson M, Worley S, et al. A clinic-based breastfeeding peer counselor intervention in an urban, low-income population: interaction with breastfeeding attitude. J Hum Lact. 2015 Feb;31(1):120-8. doi: 10.1177/0890334414548860. PMID: 25193602. Exclusion Code: X3.

942. Srinivasan A, Graves L, D'Souza V. Effectiveness of a 3-hour breastfeeding course for family physicians. Can Fam Physician. 2014 Dec;60(12):e601-6. PMID: 25642487. Exclusion Code: X3.

943. Srkalovic Imsiragic A, Begic D, Simicevic L, et al. Prediction of posttraumatic stress disorder symptomatology after childbirth A Croatian longitudinal study. Women Birth. 2017 Feb;30(1):e17-e23. doi: 10.1016/j.wombi.2016.06.007. PMID: 27421663. Exclusion Code: X5.

944. Staehelin K, Kurth E, Schindler C, et al. Predictors of early postpartum mental distress in mothers with midwifery home care--results from a nested case-control study. Swiss Med Wkly. 2013 Aug 27;143:w13862. doi: 10.4414/smw.2013.13862. PMID: 23986402. Exclusion Code: X5.

945. Stagg J, Ustianov J. Improving and Sustaining Breastfeeding Practices through a Statewide Learning Collaborative...Proceedings of the 2015 AWHONN Convention. JOGNN: Journal of Obstetric, Gynecologic \& Neonatal Nursing. 2015;44:S55-S. doi: 10.1111/15526909.12617. PMID: 109793662. Exclusion Code: X9.

946. Steel OCKO, Mowat DL, Scott HM, et al. A randomized trial of two public health nurse follow-up programs after early obstetrical discharge. An examination of breastfeeding rates, maternal confidence and utilization and costs of health services (Structured abstract). Canadian Journal of Public Health; 2003. p. 98-103. Exclusion Code: X3.

947. Steel O'Connor KO, Mowat DL, Scott HM, et al. A randomized trial of two public health nurse follow-up programs after early obstetrical discharge: an examination of breastfeeding rates, maternal confidence and utilization and costs of health services. Can J Public Health. 2003 Mar-Apr;94(2):98-103. PMID: 12675164. Exclusion Code: X3. 
948. Stevens B, Guerriere D, McKeever P, et al. Economics of home vs. hospital breastfeeding support for newborns. J Adv Nurs. 2006 Jan;53(2):233-43. doi: 10.1111/j.1365-2648.2006.03720.x. PMID: 16422722. Exclusion Code: X5.

949. Stevens RJ, Roddam AW, Green J, et al. Reproductive history and pancreatic cancer incidence and mortality in a cohort of postmenopausal women. Cancer Epidemiol Biomarkers Prev. 2009 May;18(5):1457-60. doi: 10.1158/1055-9965.epi-08-1134. PMID: 19423523. Exclusion Code: X5.

950. Stewart A. Paediatric Chiropractic and Infant Breastfeeding Difficulties: A Pilot Case Series Study involving 19 Cases. Chiropractic Journal of Australia. 2012;42(3):98-107. PMID: 104376157. Exclusion Code: X6.

951. Straoak Z, Feyereislova S, Cerna M, et al. Limited amount of formula may facilitate breastfeeding: Randomized, controlled trial to compare standard clinical practice versus limited supplemental feeding. PLoS One; 2016. Exclusion Code: X3.

952. Strauch J, Rohrer JE, Refaat A. Increased hospital documentation requirements may not increase breastfeeding among first-time mothers. J Eval Clin Pract. 2016 Apr;22(2):194-9. doi: 10.1111/jep.12453. PMID: 26471252. Exclusion Code: X3.

953. Stremler R, Hodnett E, Kenton L, et al. Effect of behavioural-educational intervention on sleep for primiparous women and their infants in early postpartum: multisite randomised controlled trial. BMJ.

2013 Mar 20;346:f1164. doi: 10.1136/bmj.f1164. PMID: 23516146. Exclusion Code: X3.

954. Stuart GS, Lesko CR, Stuebe AM, et al. A randomized trial of levonorgestrel intrauterine system insertion 6 to $48 \mathrm{~h}$ compared to 6 weeks after vaginal delivery; lessons learned. Contraception. 2015 Apr;91(4):284-8. doi: 10.1016/j.contraception.2014.12.009. PMID: 25553871. Exclusion Code: X3.
955. Stuebe AM, Rich-Edwards JW. The reset hypothesis: lactation and maternal metabolism. Am J Perinatol. 2009 Jan;26(1):81-8. doi: 10.1055/s-00281103034. PMID: 19031350. Exclusion Code: X6.

956. Stuebe AM, Rich-Edwards JW, Willett WC, et al. Duration of lactation and incidence of type 2 diabetes. JAMA. 2005 Nov 23;294(20):2601-10. doi: 10.1001/jama.294.20.2601. PMID: 16304074. Exclusion Code: X10.

957. Stuebe AM, Willett WC, Xue F, et al. Lactation and incidence of premenopausal breast cancer: a longitudinal study. Arch Intern Med. 2009 Aug 10;169(15):1364-71. doi: 10.1001/archinternmed.2009.231. PMID: 19667298. Exclusion Code: X10.

958. Su D, Pasalich M, Lee AH, et al. Ovarian cancer risk is reduced by prolonged lactation: a case-control study in southern China. Am J Clin Nutr. 2013 Feb;97(2):3549. doi: 10.3945/ajcn.112.044719. PMID: 23283498. Exclusion Code: X8.

959. Su LL, Chong YS, Chan YH, et al. Antenatal education and postnatal support strategies for improving rates of exclusive breast feeding: randomised controlled trial. BMJ. 2007 Sep 22;335(7620):596. doi: 10.1136/bmj.39279.656343.55. PMID: 17670909. Exclusion Code: X3.

960. Suchada J, Chutima S, Umaporn S, et al. Implementation of a Nutrition Program Reduced Post-Discharge Growth Restriction in Thai Very Low Birth Weight Preterm Infants. Nutrients. 2016;8(12):1-14. doi: 10.3390/nu8120820. PMID: 120544522. Exclusion Code: X3.

961. Susin LR, Giugliani ER. Inclusion of fathers in an intervention to promote breastfeeding: impact on breastfeeding rates. J Hum Lact. 2008 Nov;24(4):386-92; quiz 451-3. doi: 10.1177/0890334408323545. PMID: 18784322. Exclusion Code: X3.

962. Susin LR, Giugliani ER, Kummer SC, et al. Does parental breastfeeding knowledge increase breastfeeding rates? Birth. 1999 Sep;26(3):149-56. PMID: 10655814. Exclusion Code: X3. 
963. Symon A, Winter C, Inkster M, et al. Outcomes for births booked under an independent midwife and births in NHS maternity units: matched comparison study. BMJ. 2009 Jun 11;338:b2060. doi: 10.1136/bmj.b2060. PMID: 19520729. Exclusion Code: X3.

964. Tae-Hee K, Hae-Hyeog L, Junsik P, et al. Effects of early breastfeeding education on maintenance of breastfeeding practice: A prospective observational study. Open Journal of Nursing. 2013;3(2):209-13. doi: 10.4236/ojn.2013.32028. PMID: 104020461. Exclusion Code: X3.

965. Tahir NM, Al-Sadat N. Does telephone lactation counselling improve breastfeeding practices? A randomised controlled trial. Int J Nurs Stud. 2013 Jan;50(1):16-25. doi: 10.1016/j.ijnurstu.2012.09.006. PMID: 23084438. Exclusion Code: X3.

966. Tan HS, Tan MH, Chow KY, et al. Reproductive factors and lung cancer risk among women in the Singapore Breast Cancer Screening Project. Lung Cancer. 2015 Dec;90(3):499-508. doi: 10.1016/j.lungcan.2015.10.003. PMID: 26476714. Exclusion Code: X5.

967. Tang L, Lee AH, Binns CW. Factors associated with breastfeeding duration: a prospective cohort study in Sichuan Province, China. World J Pediatr. 2015 Aug;11(3):232-8. doi: 10.1007/s12519-0140520-y. PMID: 25410668. Exclusion Code: X3.

968. Tanner-Smith EE, Steinka-Fry KT, Lipsey MW. Effects of CenteringPregnancy group prenatal care on breastfeeding outcomes. J Midwifery Womens Health. 2013 JulAug;58(4):389-95. doi: 10.1111/jmwh.12008. PMID: 23855366. Exclusion Code: X3.

969. Tarrant M, Fong DY, Heys M, et al. Professional breastfeeding support to increase the exclusivity and duration of breastfeeding: a randomised controlled trial. Hong Kong Med J. 2014 Dec;20 Suppl 7:345. PMID: 25647824. Exclusion Code: X3.
970. Tarrant M, Lok KY, Fong DY, et al. Effect of a hospital policy of not accepting free infant formula on in-hospital formula supplementation rates and breast-feeding duration. Public Health Nutr. 2015 Oct;18(14):2689-99. doi: 10.1017/s1368980015000117. PMID: 25721766. Exclusion Code: X6.

971. Tarrant M, Lok KYW, Fong DYT, et al. Effect on Baby-Friendly Hospital Steps When Hospitals Implement a Policy to Pay for Infant Formula. J Hum Lact. 2016;32(2):238-49. doi: 10.1177/0890334415599399. PMID: 114575361. Exclusion Code: X6.

972. Tarrant RC, Sheridan-Pereira M, Younger $\mathrm{KM}$, et al. The positive role of breastfeeding on infant health during the first 6 weeks: findings from a prospective observational study based on maternal reports. Ir Med J. 2012 Mar;105(3):75-8. PMID: 22558812. Exclusion Code: X5.

973. Taveras EM, Capra AM, Braveman PA, et al. Clinician support and psychosocial risk factors associated with breastfeeding discontinuation. Pediatrics. 2003 Jul;112(1 Pt 1):108-15. PMID: 12837875. Exclusion Code: X3.

974. Tavoulari EF, Benetou V, Vlastarakos PV, et al. Factors affecting breast-feeding initiation in Greece: What is important? Midwifery. 2015 Feb;31(2):323-31. doi: 10.1016/j.midw.2014.10.006. PMID: 25467601. Exclusion Code: X3.

975. Taylor JS, Kacmar JE, Nothnagle M, et al. A systematic review of the literature associating breastfeeding with type 2 diabetes and gestational diabetes. J Am Coll Nutr. 2005 Oct;24(5):320-6. PMID: 16192255. Exclusion Code: X10.

976. Taylor NJ, Sahota P, Sargent J, et al. Using intervention mapping to develop a culturally appropriate intervention to prevent childhood obesity: the HAPPY (Healthy and Active Parenting Programme for Early Years) study. Int J Behav Nutr Phys Act. 2013 Dec 28;10:142. doi: 10.1186/14795868-10-142. PMID: 24373301. Exclusion Code: X6. 
977. Taylor R. Providing additional guidance and support to parents about sleep, diet and physical activity from birth to 2 years of age: The Prevention of Overweight in Infancy study. Obes Res Clin Pract; 2014. p. 102-3. Exclusion Code: X3.

978. Temple Newhook J, Newhook LA, Midodzi WK, et al. Determinants of Nonmedically Indicated In-Hospital Supplementation of Infants Whose Birthing Parents Intended to Exclusively Breastfeed. J Hum Lact. 2017 May;33(2):278-84. doi: 10.1177/0890334417695204. PMID: 28418804. Exclusion Code: X3.

979. Thome M, Alder EM, Ramel A. A population-based study of exclusive breastfeeding in Icelandic women: is there a relationship with depressive symptoms and parenting stress? Int J Nurs Stud. 2006 Jan;43(1):11-20. doi: 10.1016/j.ijnurstu.2004.10.009. PMID: 16326160. Exclusion Code: X6.

980. Thomson JL, Tussing-Humphreys LM, Goodman MH. Delta Healthy Sprouts: a randomized comparative effectiveness trial to promote maternal weight control and reduce childhood obesity in the Mississippi Delta. Contemp Clin Trials. 2014 May;38(1):82-91. doi: 10.1016/j.cct.2014.03.004. PMID: 24685997. Exclusion Code: X6.

981. Thorland W, Currie D, Wiegand E, et al. Status of Breastfeeding and Child Immunization Outcomes in Clients of the Nurse-Family Partnership. Matern Child Health J. 2017;21(3):439-45. doi: 10.1007/s10995-016-2231-6. PMID: 121919496. Exclusion Code: X4.

982. Thorstensson S, Nissen E, Ekstrom A. Professional Support in Pregnancy Influence Maternal Relation to and Feelings for the Baby after Cesarean Birth: An Intervention Study. Journal of Nursing and Care. 2012;1(4):112. Exclusion Code: X3.

983. Thukral A, Chawla D, Agarwal R, et al. Kangaroo mother care--an alternative to conventional care. Indian J Pediatr. 2008 May;75(5):497-503. doi: 10.1007/s12098008-0077-7. PMID: 18537012. Exclusion Code: X3.
984. Tipene-Leach D, Baddock S, Williams S, et al. Methodology and recruitment for a randomised controlled trial to evaluate the safety of wahakura for infant bedsharing. BMC Pediatr. 2014 Sep 28;14:240. doi: 10.1186/1471-2431-14-240. PMID: 25262145. Exclusion Code: X3.

985. Titus-Ernstoff L, Perez K, Cramer DW, et al. Menstrual and reproductive factors in relation to ovarian cancer risk. Br J Cancer. 2001 Mar 02;84(5):714-21. doi: 10.1054/bjoc.2000.1596. PMID: 11237375. Exclusion Code: X10.

986. Titus-Ernstoff L, Rees JR, Terry KL, et al. Breast-feeding the last born child and risk of ovarian cancer. Cancer Causes Control. 2010 Feb;21(2):201-7. doi: 10.1007/s10552009-9450-8. PMID: 19902367. Exclusion Code: X10.

987. Tohotoa J, Maycock B, Hauck YL, et al. Can father inclusive practice reduce paternal postnatal anxiety? A repeated measures cohort study using the Hospital Anxiety and Depression Scale. BMC Pregnancy Childbirth. 2012 Jul 31;12:75. doi: 10.1186/1471-2393-12-75. PMID: 22849509. Exclusion Code: X5.

988. Torio CM, Klassen AC, Curriero FC, et al. The modifying effect of social class on the relationship between body mass index and breast cancer incidence. Am J Public Health. 2010 Jan;100(1):146-51. doi: 10.2105/ajph.2007.126979. PMID: 19150917. Exclusion Code: X3.

989. Torowicz DL, Spatz DL, Seelhorst A. Human Milk and Breastfeeding in the Cardiac Center: A Prospective, Descriptive Study. Journal of Pediatric Healthcare. 2013;27(5):325-. doi: 10.1016/j.pedhc.2013.07.009. PMID: 104216482. Exclusion Code: X6.

990. Torris C, Thune I, Emaus A, et al. Duration of lactation, maternal metabolic profile, and body composition in the Norwegian EBBA I-study. Breastfeed Med. 2013 Feb;8(1):815. doi: 10.1089/bfm.2012.0048. PMID: 23057641. Exclusion Code: X5. 
991. Touzet S, Beissel A, Denis A, et al. Effectiveness of a nurse educational oral feeding programme on feeding outcomes in neonates: protocol for an interrupted time series design. BMJ Open. 2016 Apr 15;6(4):e010699. doi: 10.1136/bmjopen2015-010699. PMID: 27084282. Exclusion Code: X3.

992. Treuherz J, Cullinan TR, Saunders DI. Determinants of infant-feeding practice in East London. Hum Nutr Appl Nutr. 1982 Aug;36a(4):281-6. PMID: 7129961. Exclusion Code: X3.

993. Trotman G, Chhatre G, Darolia R, et al. The Effect of Centering Pregnancy versus Traditional Prenatal Care Models on Improved Adolescent Health Behaviors in the Perinatal Period. J Pediatr Adolesc Gynecol. 2015 Oct;28(5):395-401. doi: 10.1016/j.jpag.2014.12.003. PMID: 26233287. Exclusion Code: X3.

994. Trudnak TE, Arboleda E, Kirby RS, et al. Outcomes of Latina women in CenteringPregnancy group prenatal care compared with individual prenatal care. J Midwifery Womens Health. 2013 JulAug;58(4):396-403. doi: 10.1111/jmwh.12000. PMID: 23855970. Exclusion Code: X3.

995. Truijens SE, Meems M, Kuppens SM, et al. The HAPPY study (Holistic Approach to Pregnancy and the first Postpartum Year): design of a large prospective cohort study. BMC Pregnancy Childbirth. 2014 Sep 08;14:312. doi: 10.1186/1471-2393-14-312. PMID: 25201155. Exclusion Code: X3.

996. Tsai PJ, Nakashima L, Yamamoto J, et al. Postpartum follow-up rates before and after the postpartum follow-up initiative at Queen Emma Clinic. Hawaii Med J. 2011 Mar;70(3):56-9. PMID: 21365543. Exclusion Code: X3.

997. Tsai SY. Impact of a breastfeeding-friendly workplace on an employed mother's intention to continue breastfeeding after returning to work. Breastfeed Med. 2013 Apr;8:210-6. doi: 10.1089/bfm.2012.0119. PMID: 23390987. Exclusion Code: X3.
998. Tsai SY. Employee perception of breastfeeding-friendly support and benefits of breastfeeding as a predictor of intention to use breast-pumping breaks after returning to work among employed mothers. Breastfeed Med. 2014 Jan-Feb;9(1):16-23. doi: 10.1089/bfm.2013.0082. PMID: 24304034. Exclusion Code: X3.

999. Tsai TI, Huang SH, Lee SY. Maternal and Hospital Factors Associated with First-Time Mothers' Breastfeeding Practice: A Prospective Study. Breastfeed Med. 2015 Jul-Aug;10(6):334-40. doi: 10.1089/bfm.2015.0005. PMID: 26110594. Exclusion Code: X3.

1000. Tsvetov G, Levy S, Benbassat C, et al. Influence of number of deliveries and total breast-feeding time on bone mineral density in premenopausal and young postmenopausal women. Maturitas. 2014 Mar;77(3):249-54. doi: 10.1016/j.maturitas.2013.11.003. PMID: 24332872. Exclusion Code: X6.

1001. Tung KH, Goodman MT, Wu AH, et al. Reproductive factors and epithelial ovarian cancer risk by histologic type: a multiethnic case-control study. Am J Epidemiol. 2003 Oct 01;158(7):629-38. PMID: 14507598. Exclusion Code: X10.

1002. Tung KH, Wilkens LR, Wu AH, et al. Effect of anovulation factors on pre- and postmenopausal ovarian cancer risk: revisiting the incessant ovulation hypothesis. Am J Epidemiol. 2005 Feb 15;161(4):321-9. doi: 10.1093/aje/kwi046. PMID: 15692075. Exclusion Code: X10.

1003. Tuoni C, Scaramuzzo RT, Ghirri P, et al. Kangaroo Mother Care: four years of experience in very low birth weight and preterm infants. Minerva Pediatr. 2012 Aug;64(4):377-83. PMID: 22728609. Exclusion Code: X3.

1004. Turan JM, Say L. Community-based antenatal education in Istanbul, Turkey: effects on health behaviours. Health Policy Plan. 2003 Dec;18(4):391-8. PMID: 14654515. Exclusion Code: X3. 
1005. Turan V. Grand-grand multiparity (more than 10 deliveries) does not convey a risk for osteoporosis. Acta Obstet Gynecol Scand. 2011 Dec;90(12):1440-2. doi: 10.1111/j.1600-0412.2011.01250.x. PMID: 21797824. Exclusion Code: X3.

1006. Turkoz FP, Solak M, Petekkaya I, et al. Association between common risk factors and molecular subtypes in breast cancer patients. Breast. 2013 Jun;22(3):344-50. doi: 10.1016/j.breast.2012.08.005. PMID: 22981738. Exclusion Code: X4.

1007. Turnbull C, Osborn DA. Home visits during pregnancy and after birth for women with an alcohol or drug problem. Cochrane Database Syst Rev. 2012 Jan 18;1:Cd004456. doi: 10.1002/14651858.CD004456.pub3. PMID: 22258956. Exclusion Code: X5.

1008. Turner MJ, Layte R. Obesity levels in a national cohort of women 9 months after delivery. Am J Obstet Gynecol. 2013 Aug;209(2):124.e1-7. doi: 10.1016/j.ajog.2013.04.020. PMID: 23583210. Exclusion Code: X5.

1009. Tylleskar T, Jackson D, Meda N, et al. Exclusive breastfeeding promotion by peer counsellors in sub-Saharan Africa (PROMISE-EBF): a cluster-randomised trial. Lancet. 2011 Jul 30;378(9789):420-7. doi: 10.1016/s0140-6736(11)60738-1. PMID: 21752462. Exclusion Code: X8.

1010. Unar-Munguia M, Torres-Mejia G, Colchero MA, et al. Breastfeeding Mode and Risk of Breast Cancer: A Dose-Response MetaAnalysis. J Hum Lact. 2017 May;33(2):42234. doi: $10.1177 / 0890334416683676$. PMID: 28196329. Exclusion Code: X11.

1011. Ursin G, Bernstein L, Lord SJ, et al. Reproductive factors and subtypes of breast cancer defined by hormone receptor and histology. Br J Cancer. 2005 Aug 08;93(3):364-71. doi: 10.1038/sj.bjc.6602712. PMID: 16079783. Exclusion Code: X10.

1012. Uusi-Rasi K, Sievanen H, Pasanen M, et al. Association of physical activity and calcium intake with the maintenance of bone mass in premenopausal women. Osteoporos Int. 2002 Mar;13(3):211-7. PMID: 11991440. Exclusion Code: X5.
1013. Valdes V, Perez A, Labbok M, et al. The impact of a hospital and clinic-based breastfeeding promotion programme in a middle class urban environment. J Trop Pediatr. 1993 Jun;39(3):142-51. PMID: 8326533. Exclusion Code: X6.

1014. Valdes V, Pugin E, Schooley J, et al. Clinical support can make the difference in exclusive breastfeeding success among working women. J Trop Pediatr. 2000 Jun;46(3):149-54. PMID: 10893915. Exclusion Code: X3.

1015. van den Heuvel M, Hopkins J, Biscaro A, et al. A comparative analysis of early child health and development services and outcomes in countries with different redistributive policies. BMC Public Health. 2013 Nov 06;13:1049. doi: 10.1186/14712458-13-1049. PMID: 24195544. Exclusion Code: X6.

1016. van Rossem L, Vogel I, Steegers EA, et al. Breastfeeding patterns among ethnic minorities: the Generation R Study. J Epidemiol Community Health. 2010 Dec;64(12):1080-5. doi: 10.1136/jech.2009.095380. PMID: 19996356. Exclusion Code: X3.

1017. van Sleuwen BE, Engelberts AC, BoereBoonekamp MM, et al. Swaddling: a systematic review. Pediatrics. 2007 Oct;120(4):e1097-106. doi: 10.1542/peds.2006-2083. PMID: 17908730. Exclusion Code: X3.

1018. Vanky E, Nordskar J, Leithe H, et al. Breast size increment during pregnancy and breastfeeding in mothers with polycystic ovary syndrome: a follow-up study of a randomised controlled trial on metformin versus placebo. BJOG : an international journal of obstetrics and gynaecology; 2012. p. 1403-9. Exclusion Code: X3.

1019. Vari PM, Camburn J, Henly SJ. Professionally mediated peer support and early breastfeeding success. J Perinat Educ. 2000 Winter;9(1):22-30. doi: 10.1624/105812400x87473. PMID: 17273189. Exclusion Code: X4. 
1020. Vendittelli F, Riviere O, Crenn-Hebert C, et al. Do perinatal guidelines have an impact on obstetric practices? Rev Epidemiol Sante Publique. 2012 Oct;60(5):355-62. doi: 10.1016/j.respe.2012.03.002. PMID: 22981161. Exclusion Code: X3.

1021. Venkatesh KK, Castro VM, Perlis RH, et al. Impact of antidepressant treatment during pregnancy on obstetric outcomes among women previously treated for depression: an observational cohort study. J Perinatol. 2017;37(9):1003-9. doi: 10.1038/jp.2017.92. PMID: 125190327. Exclusion Code: X3.

1022. Vergnaud AC, Romaguera D, Peeters PH, et al. Adherence to the World Cancer Research Fund/American Institute for Cancer Research guidelines and risk of death in Europe: results from the European Prospective Investigation into Nutrition and Cancer cohort study1,4. Am J Clin Nutr. 2013 May;97(5):1107-20. doi: 10.3945/ajcn.112.049569. PMID: 23553166. Exclusion Code: X5.

1023. Viana Rêgo RM, Alves e. Souza ÂM, Assis da Rocha TN, et al. Paternity and breastfeeding: mediation of nurses. Acta Paulista de Enfermagem. 2016;29(4):37480. doi: 10.1590/1982-0194201600052. PMID: 119763304. Exclusion Code: X5.

1024. Vianna MN, Barbosa AP, Carvalhaes AS, et al. Music therapy may increase breastfeeding rates among mothers of premature newborns: a randomized controlled trial. J Pediatr (Rio J). 2011 MayJun 8;87(3):206-12. doi: doi:10.2223/JPED.2086. PMID: 21461451. Exclusion Code: X7.

1025. Victora CG, Bahl R, Barros AJ, et al. Breastfeeding in the 21st century: epidemiology, mechanisms, and lifelong effect. Lancet. 2016 Jan 30;387(10017):47590. doi: 10.1016/s0140-6736(15)01024-7. PMID: 26869575. Exclusion Code: X6.

1026. Vidas M, Folnegovic-Smalc V, Catipovic $\mathrm{M}$, et al. The application of autogenic training in counseling center for mother and child in order to promote breastfeeding. Coll Antropol. 2011 Sep;35(3):723-31. PMID: 22053548. Exclusion Code: X3.
1027. Vieira TO, Vieira GO, de Oliveira NF, et al. Duration of exclusive breastfeeding in a Brazilian population: new determinants in a cohort study. BMC Pregnancy Childbirth. 2014 May 26;14:175. doi: 10.1186/14712393-14-175. PMID: 24885939. Exclusion Code: X3.

1028. Vinter CA, Jensen DM, Ovesen P, et al. Postpartum weight retention and breastfeeding among obese women from the randomized controlled Lifestyle in Pregnancy (LiP) trial. Acta Obstet Gynecol Scand. 2014 Aug;93(8):794-801. doi: 10.1111/aogs.12429. PMID: 24834792. Exclusion Code: X6.

1029. Vitolo M, Bortolini G, Feldens C, et al. [Impacts of the 10 Steps to Healthy Feeding in Infants: a randomized field trial]. Cad Saude Publica; 2005. p. 1448-57. Exclusion Code: X7.

1030. Vítolo M, Louzada M, Rauber F, et al. [The impact of health workers' training on breastfeeding and complementary feeding practices]. Cad Saude Publica; 2014. p. 1695-707. Exclusion Code: X7.

1031. Vitolo MR, Bortolini GA, Campagnolo PD, et al. Maternal dietary counseling reduces consumption of energy-dense foods among infants: a randomized controlled trial. J Nutr Educ Behav. 2012 Mar-Apr;44(2):140-7. doi: 10.1016/j.jneb.2011.06.012. PMID: 22189004. Exclusion Code: X3.

1032. Vitonis AF, Titus-Ernstoff L, Cramer DW. Assessing ovarian cancer risk when considering elective oophorectomy at the time of hysterectomy. Obstet Gynecol. 2011 May;117(5):1042-50. doi: 10.1097/AOG.0b013e318212fcb7. PMID: 21471855. Exclusion Code: X10.

1033. Vittoz JP, Labarere J, Castell M, et al. Effect of a training program for maternity ward professionals on duration of breastfeeding. Birth. 2004 Dec;31(4):302-7. doi: 10.1111/j.0730-7659.2004.00323.x. PMID: 15566343. Exclusion Code: X6.

1034. von Au A, Klotzbuecher M, Uhlmann L, et al. Impact of reproductive factors on breast cancer subtypes in postmenopausal women: a retrospective single-center study. Arch Gynecol Obstet. 2017 Apr;295(4):971-8. doi: 10.1007/s00404-017-4298-8. PMID: 28176013. Exclusion Code: X6. 
1035. Von Luehrte B, Payne CS, Risola A, et al. Breastfeeding: Latching on With the Community. JOGNN: Journal of Obstetric, Gynecologic \& Neonatal Nursing. 2011;40:S106-7. doi: 10.1111/j.15526909.2011.01243_26.x. PMID: 104647035. Exclusion Code: X3.

1036. Vural F, Vural B. The effect of prenatal and postnatal education on exclusive breastfeeding rates. Minerva Pediatr. 2017 Feb;69(1):22-9. doi: 10.23736/s00264946.16.04183-9. PMID: 28102653. Exclusion Code: X3.

1037. Waite WM, Christakis D. The Impact of Mailed Samples of Infant Formula on Breastfeeding Rates. Breastfeed Med. 2016 Jan-Feb;11(1):21-5. doi: 10.1089/bfm.2015.0099. PMID: 26701801. Exclusion Code: X3.

1038. Waldenström U, Nilsson C. [No effect of birth center care on either duration or experience of breast feeding, but more complications]. Jordemodern; 1994. p. 17982. Exclusion Code: X7.

1039. Waldenstrom U, Nilsson CA. No effect of birth centre care on either duration or experience of breast feeding, but more complications: findings from a randomised controlled trial. Midwifery. 1994

Mar;10(1):8-17. PMID: 8159123. Exclusion Code: X3.

1040. Walker LO, Sterling BS, Kim M, et al. Trajectory of weight changes in the first 6 weeks postpartum. J Obstet Gynecol Neonatal Nurs. 2006 Jul-Aug;35(4):472-81. doi: 10.1111/j.1552-6909.2006.00062.x. PMID: 16881991. Exclusion Code: X3.

1041. Walkup JT, Barlow A, Mullany BC, et al. Randomized controlled trial of a paraprofessional-delivered in-home intervention for young reservation-based American Indian mothers. J Am Acad Child Adolesc Psychiatry. 2009 Jun;48(6):591601. doi: 10.1097/CHI.0b013e3181a0ab86. PMID: 19454915. Exclusion Code: X5.

1042. Wallace LM, Dunn OM, Law S, et al. A new approach to breastfeeding training. Pract Midwife. 2009 Jun;12(6):47-9. PMID: 19624065. Exclusion Code: X5.
1043. Wallin L, Eriksson M. Newborn Individual Development Care and Assessment Program (NIDCAP): a systematic review of the literature. Worldviews Evid Based Nurs. 2009;6(2):54-69. doi: 10.1111/j.17416787.2009.00150.x. PMID: 19413582. Exclusion Code: X3.

1044. Wambach KA, Aaronson L, Breedlove G, et al. A randomized controlled trial of breastfeeding support and education for adolescent mothers. West J Nurs Res. 2011 Jun;33(4):486-505. doi: 10.1177/0193945910380408. PMID: 20876551. Exclusion Code: X3.

1045. Wang L, Li J, Shi Z. Association between breastfeeding and endometrial cancer risk: evidence from a systematic review and meta-analysis. Nutrients. $2015 \mathrm{Jul}$ 14;7(7):5697-711. doi: 10.3390/nu7075248. PMID: 26184301. Exclusion Code: X5.

1046. Wardle K, De Domenico M, Li Ming W. Understanding infant feeding practices of new mothers: findings from the Healthy Beginnings Trial. Aust J Adv Nurs. 2014;32(1):6-15. PMID: 103910128. Exclusion Code: X4.

1047. Warner ET, Colditz GA, Palmer JR, et al. Reproductive factors and risk of premenopausal breast cancer by age at diagnosis: are there differences before and after age 40? Breast Cancer Res Treat. 2013 Nov;142(1):165-75. doi: 10.1007/s10549013-2721-9. PMID: 24136668. Exclusion Code: X10.

1048. Warren Andersen S, Trentham-Dietz A, Gangnon RE, et al. The associations between a polygenic score, reproductive and menstrual risk factors and breast cancer risk. Breast Cancer Res Treat. 2013 Jul;140(2):427-34. doi: 10.1007/s10549013-2646-3. PMID: 23893088. Exclusion Code: X6.

1049. Wataker H, Meberg A, Nestaas E. Neonatal family care for 24 hours per day: effects on maternal confidence and breast-feeding. J Perinat Neonatal Nurs. 2012 OctDec;26(4):336-42. doi: 10.1097/JPN.0b013e31826d928b. PMID: 23111722. Exclusion Code: X3. 
1050. Watkins S, Meltzer-Brody S, Zolnoun D, et al. Early breastfeeding experiences and postpartum depression. Obstet Gynecol. 2011 Aug;118(2 Pt 1):214-21. doi: 10.1097/AOG.0b013e3182260a2d. PMID: 21734617. Exclusion Code: X3.

1051. Watson J, McGuire W. Responsive versus scheduled feeding for preterm infants. Cochrane Database of Systematic Reviews: John Wiley \& Sons, Ltd; 2016. Exclusion Code: X3.

1052. Watt RG, McGlone P, Russell JJ, et al. The process of establishing, implementing and maintaining a social support infant feeding programme. Public Health Nutr. 2006 Sep;9(6):714-21. PMID: 16925876. Exclusion Code: X5.

1053. Watt RG, Tull KI, Hardy R, et al. Effectiveness of a social support intervention on infant feeding practices: randomised controlled trial. J Epidemiol Community Health. 2009 Feb;63(2):156-62. doi: 10.1136/jech.2008.077115. PMID: 19141661. Exclusion Code: X3.

1054. Watt TT, Appel L, Lopez V, et al. A Primary Care-Based Early Childhood Nutrition Intervention: Evaluation of a Pilot Program Serving Low-Income Hispanic Women. J Racial Ethn Health Disparities. 2015 Dec;2(4):537-47. doi: 10.1007/s40615015-0102-2. PMID: 26863560. Exclusion Code: X3.

1055. Webb MO, Ellerbee SM. Breastfeeding in Arkansas: the role of the Arkansas Department of Health. J Ark Med Soc. 1996 Sep;93(4):185-7. PMID: 8840748. Exclusion Code: X1.

1056. Webel AR, Okonsky J, Trompeta J, et al. A systematic review of the effectiveness of peer-based interventions on health-related behaviors in adults. Am J Public Health. 2010 Feb;100(2):247-53. doi: 10.2105/ajph.2008.149419. PMID: 20019321. Exclusion Code: X3.

1057. Weiderpass E, Sandin S, Inoue M, et al. Risk factors for epithelial ovarian cancer in Japan - results from the Japan Public Health Center-based Prospective Study cohort. Int J Oncol. 2012 Jan;40(1):21-30. doi: 10.3892/ijo.2011.1194. PMID: 21904774. Exclusion Code: X10.
1058. Wen L, Baur L, Simpson J, et al. Healthy beginnings trial: The journey from the beginning. Obes Res Clin Pract; 2013. p. e2. Exclusion Code: X3.

1059. Wen LM, Baur LA, Simpson JM, et al. Effectiveness of an early intervention on infant feeding practices and "tummy time": a randomized controlled trial. Arch Pediatr Adolesc Med. 2011 Aug;165(8):701-7. doi: 10.1001/archpediatrics.2011.115. PMID: 21810633. Exclusion Code: X3.

1060. Werner A, Uldbjerg N, Zachariae R, et al. Effect of self-hypnosis on duration of labor and maternal and neonatal outcomes: a randomized controlled trial. Acta Obstet Gynecol Scand. 2013 Jul;92(7):816-23. doi: 10.1111/aogs.12141. PMID: 23550694. Exclusion Code: X3.

1061. Westphal MF, Taddei JA, Venancio SI, et al. Breast-feeding training for health professionals and resultant institutional changes. Bull World Health Organ. 1995;73(4):461-8. PMID: 7554017. Exclusion Code: X5.

1062. Whipps MD. Education Attainment and Parity Explain the Relationship Between Maternal Age and Breastfeeding Duration in U.S. Mothers. J Hum Lact. 2017

Feb;33(1):220-4. doi: 10.1177/0890334416679385. PMID: 28135484. Exclusion Code: X3.

1063. Whitford HM, Wallis SK, Dowswell T, et al. Breastfeeding education and support for women with multiple pregnancies. Cochrane Database of Systematic Reviews: John Wiley \& Sons, Ltd; 2015. Exclusion Code: X3.

1064. Whitford HM, Wallis SK, Dowswell T, et al. Breastfeeding education and support for women with twins or higher order multiples. Cochrane Database Syst Rev. 2017 Feb 28;2:Cd012003. doi: 10.1002/14651858.CD012003.pub2. PMID: 28244065. Exclusion Code: X3.

1065. Whittemore AS, Harris R, Itnyre J. Characteristics relating to ovarian cancer risk: collaborative analysis of 12 US casecontrol studies. IV. The pathogenesis of epithelial ovarian cancer. Collaborative Ovarian Cancer Group. Am J Epidemiol. 1992 Nov 15;136(10):1212-20. PMID: 1476143. Exclusion Code: X10. 
1066. Wigertz A, Lonn S, Hall P, et al. Reproductive factors and risk of meningioma and glioma. Cancer Epidemiol Biomarkers Prev. 2008 Oct;17(10):2663-70. doi: 10.1158/1055-9965.epi-08-0406. PMID: 18843008. Exclusion Code: X5.

1067. Wiklund P, Xu L, Lyytikainen A, et al. Prolonged breast-feeding protects mothers from later-life obesity and related cardiometabolic disorders. Public Health Nutr. 2012 Jan;15(1):67-74. doi: 10.1017/s1368980011002102. PMID: 21859508. Exclusion Code: X5.

1068. Wiklund PK, Xu L, Wang Q, et al. Lactation is associated with greater maternal bone size and bone strength later in life. Osteoporos Int. 2012 Jul;23(7):1939-45. doi: 10.1007/s00198-011-1790-z. PMID: 21927916. Exclusion Code: X5.

1069. Wilcken N, Goodwin A, Alderman A, et al. Cochrane Breast Cancer Group. About The Cochrane Collaboration: John Wiley \& Sons, Ltd; 2016. Exclusion Code: X1.

1070. Wilde P, Wolf A, Fernandes M, et al. Foodpackage assignments and breastfeeding initiation before and after a change in the Special Supplemental Nutrition Program for Women, Infants, and Children. Am J Clin Nutr. 2012 Sep;96(3):560-6. doi: 10.3945/ajcn.112.037622. PMID: 22836028. Exclusion Code: X6.

1071. Wilhelm SL, Stepans MB, Hertzog M, et al. Motivational interviewing to promote sustained breastfeeding. J Obstet Gynecol Neonatal Nurs. 2006 May-Jun;35(3):340-8. doi: 10.1111/j.1552-6909.2006.00046.x. PMID: 16700683. Exclusion Code: X3.

1072. Wilkinson SA, van der Pligt P, Gibbons KS, et al. Trial for Reducing Weight Retention in New Mums: a randomised controlled trial evaluating a low intensity, postpartum weight management programme. J Hum Nutr Diet. 2015 Jan;28 Suppl 1:15-28. doi: 10.1111/jhn.12193. PMID: 24267102. Exclusion Code: X3.

1073. Witt AM, Smith S, Mason MJ, et al. Integrating routine lactation consultant support into a pediatric practice. Breastfeed Med. 2012 Feb;7(1):38-42. doi: 10.1089/bfm.2011.0003. PMID: 21657890. Exclusion Code: X3.
1074. Wojnar D. Maternal perceptions of early breastfeeding and feeding outcomes at 6 weeks. Commun Nurs Res. 20052005 Spring;38:262-. PMID: 105740760. Exclusion Code: X6.

1075. Wolfberg AJ, Michels KB, Shields W, et al. Dads as breastfeeding advocates: results from a randomized controlled trial of an educational intervention. Am J Obstet Gynecol. 2004 Sep;191(3):708-12. doi: 10.1016/j.ajog.2004.05.019. PMID: 15467529. Exclusion Code: X3.

1076. Wong EH, Nelson E, Choi KC, et al. Evaluation of a peer counselling programme to sustain breastfeeding practice in Hong Kong. Int Breastfeed J. 2007 Sep 20;2:12. doi: 10.1186/1746-4358-2-12. PMID: 17883851. Exclusion Code: X3.

1077. Wong KL, Fong DY, Lee IL, et al. Antenatal education to increase exclusive breastfeeding: a randomized controlled trial. Obstet Gynecol. 2014 Nov;124(5):961-8. doi: 10.1097/aog.0000000000000481. PMID: 25437725. Exclusion Code: X3.

1078. Woolhouse H, James J, Gartland D, et al. Maternal depressive symptoms at three months postpartum and breastfeeding rates at six months postpartum: implications for primary care in a prospective cohort study of primiparous women in Australia. Women and birth; 2016. p. 381-7. Exclusion Code: X3.

1079. Woolhouse H, James J, Gartland D, et al. Maternal depressive symptoms at three months postpartum and breastfeeding rates at six months postpartum: Implications for primary care in a prospective cohort study of primiparous women in Australia. Women Birth. 2016 Aug;29(4):381-7. doi: 10.1016/j.wombi.2016.05.008. PMID: 27450375. Exclusion Code: X4.

1080. Wouk K, Lara-Cinisomo S, Stuebe AM, et al. Clinical Interventions to Promote Breastfeeding by Latinas: A Meta-analysis. Pediatrics. 2016 Jan;137(1)doi: 10.1542/peds.2015-2423. PMID: 26668300. Exclusion Code: X3. 
1081. Wouk K, Tully KP, Labbok MH. Systematic Review of Evidence for Baby-Friendly Hospital Initiative Step 3. J Hum Lact. 2017 Feb;33(1):50-82. doi: 10.1177/0890334416679618. PMID: 28135481. Exclusion Code: X3.

1082. Wright A, Rice S, Wells S. Changing hospital practices to increase the duration of breastfeeding. Pediatrics. 1996 May;97(5):669-75. PMID: 8628605. Exclusion Code: X6.

1083. Xu F, Li Z, Binns C, et al. Does infant feeding method impact on maternal mental health? Breastfeed Med. 2014

May;9(4):215-21. doi:

10.1089/bfm.2013.0142. PMID: 24621349. Exclusion Code: X5.

1084. Ya Yi H, Jian Tao L, Meei Ling G, et al. The study of pacifier use in relation to infant sucking, maternal perception of milk supply and breastfeeding duration. Macau Journal of Nursing. 2011;10(2):16-21. PMID: 104564717. Exclusion Code: X4.

1085. Yalcin SS, Orun E. Breastfeeding status and maternal psychopathologies: in a longitudinal study. Arch Dis Child. 2011 Sep;96(9):900. doi: 10.1136/adc.2011.214007. PMID: 21482533. Exclusion Code: X3.

1086. Yang L, Jacobsen KH. A systematic review of the association between breastfeeding and breast cancer. J Womens Health (Larchmt). 2008 Dec;17(10):1635-45. doi: 10.1089/jwh.2008.0917. PMID: 19049358. Exclusion Code: X8.

1087. Yang X, Gao LL, Ip WY, et al. Predictors of breast feeding self-efficacy in the immediate postpartum period: A cross-sectional study. Midwifery. 2016 Oct;41:1-8. doi: 10.1016/j.midw.2016.07.011. PMID: 27479635. Exclusion Code: X3.

1088. Yanhong G, Yu Z, Zhihong Z, et al. Effectiveness of a theory-based breastfeeding promotion intervention on exclusive breastfeeding in China: A randomised controlled trial. Midwifery. 2016;42:93-9. doi: 10.1016/j.midw.2016.09.010. PMID: 119508382. Exclusion Code: X3.
1089. Yanicki S, Hasselback P, Sandilands M, et al. The safety of Canadian early discharge guidelines. Effects of discharge timing on readmission in the first year post-discharge and exclusive breastfeeding to four months. Can J Public Health. 2002 Jan-Feb;93(1):2630. PMID: 11925696. Exclusion Code: X3.

1090. Yazdani S, Iranpour Asli A, Salemi A, et al. Determination of clinical decision rule for estimation of bone mineral density in women. Med Princ Pract. 2011;20(5):41621. doi: 10.1159/000327661. PMID: 21757929. Exclusion Code: X6.

1091. Yeh C-H. Quasi-Experimental Longitudinal Cohort of the Perinatal Breastfeeding Program (PBP): Effects on Breastfeeding Outcomes in Taiwan: Case Western Reserve University; 2011. Exclusion Code: X6.

1092. Yehya N, Tamim H, Shamsedine L, et al. Validation of the Arabic Version of the Infant Feeding Intentions Scale Among Lebanese Women. J Hum Lact. 2017 May;33(2):383-9. doi: 10.1177/0890334416680790. PMID: 28099043. Exclusion Code: X3.

1093. Yen ML, Yen BL, Bai CH, et al. Risk factors for ovarian cancer in Taiwan: a casecontrol study in a low-incidence population. Gynecol Oncol. 2003 May;89(2):318-24. PMID: 12713998. Exclusion Code: X10.

1094. Yeo JH, Moon GN, Lee S-O. Effects of Self-breast Pumping in Primiparous Women after Cesarean Delivery. Korean Journal of Women Health Nursing. 2012;18(2):98-107. doi: 10.4069/kjwhn.2012.18.2.98. PMID: 104473332. Exclusion Code: X7.

1095. Yeo UH, Choi CJ, Choi WS, et al. Relationship between breast-feeding and bone mineral density among Korean women in the 2010 Korea National Health and Nutrition Examination Survey. J Bone Miner Metab. 2016 Jan;34(1):109-17. doi: 10.1007/s00774-015-0649-3. PMID: 25792237. Exclusion Code: X5.

1096. Yilmaz G, Caylan N, Karacan CD, et al. Effect of cup feeding and bottle feeding on breastfeeding in late preterm infants: a randomized controlled study. J Hum Lact. 2014 May;30(2):174-9. doi: 10.1177/0890334413517940. PMID: 24442532. Exclusion Code: X3. 
1097. Yoda T, Takahashi K, Yamauchi Y. Japanese trends in breastfeeding rate in baby-friendly hospitals between 2007 and 2010: a retrospective hospital-based surveillance study. BMC Pregnancy Childbirth. 2013 Nov 15;13:207. doi: 10.1186/1471-2393-13-207. PMID: 24229318. Exclusion Code: X4.

1098. Yonemoto N, Dowswell T, Nagai S, et al. Schedules for home visits in the early postpartum period. Cochrane Database Syst Rev. 2013 Jul 23(7):Cd009326. doi: 10.1002/14651858.CD009326.pub2. PMID: 23881661. Exclusion Code: X3.

1099. Yoo KY, Kim Y, Park SK, et al. Lifestyle, genetic susceptibility and future trends of breast cancer in Korea. Asian Pac J Cancer Prev. 2006 Oct-Dec;7(4):679-82. PMID: 17250452. Exclusion Code: X5.

1100. Yoon J, Park Y. [Effects of a breast feeding promotion program for working women]. Journal of Korean Academy of Nursing; 2008. p. 843-52. Exclusion Code: X7.

1101. Ystrom E. Breastfeeding cessation and symptoms of anxiety and depression: a longitudinal cohort study. BMC Pregnancy Childbirth. 2012 May 23;12:36. doi: 10.1186/1471-2393-12-36. PMID: 22621668. Exclusion Code: X5.

1102. Yumusakhuylu Y, Turgut ST, Icagasioglu A, et al. Bone mineral changes during pregnancy and lactation. Gynecol Endocrinol. 2013 Aug;29(8):763-6. doi: 10.3109/09513590.2013.801444. PMID: 23815509. Exclusion Code: X5.

1103. Zakarija-Grkovic I, Segvic O, Bozinovic T, et al. Hospital practices and breastfeeding rates before and after the UNICEF/WHO 20-hour course for maternity staff. J Hum Lact. 2012 Aug;28(3):389-99. doi: 10.1177/0890334412447079. PMID: 22674962. Exclusion Code: X6.

1104. Zakarija-Grković I, Šegvić O, Vučković Vukušić A, et al. Predictors of suboptimal breastfeeding: an opportunity for public health interventions. Eur J Public Health. 2016;26(2):282-9. doi: 10.1093/eurpub/ckv203. PMID: 114135426. Exclusion Code: X3.
1105. Zhan B, Liu X, Li F, et al. Breastfeeding and the incidence of endometrial cancer: a metaanalysis. Oncotarget. 2015 Nov 10;6(35):38398-409. doi: 10.18632/oncotarget.5049. PMID: 26384296. Exclusion Code: X8.

1106. Zhang BZ, Zhang HY, Liu HH, et al. Breastfeeding and maternal hypertension and diabetes: a population-based crosssectional study. Breastfeed Med. 2015 Apr;10(3):163-7. doi: 10.1089/bfm.2014.0116. PMID: 25785993. Exclusion Code: X6.

1107. Zhang J, Shi L, Chen DF, et al. Effectiveness of an educational intervention to improve child feeding practices and growth in rural China: updated results at 18 months of age. Matern Child Nutr. 2013 Jan;9(1):118-29. doi: 10.1111/j.17408709.2012.00447.x. PMID: 23020102. Exclusion Code: X3.

1108. Zhang M, Holman CD. Tubal ligation and survival of ovarian cancer patients. J Obstet Gynaecol Res. 2012 Jan;38(1):40-7. doi: 10.1111/j.1447-0756.2011.01683.x. PMID: 22070411. Exclusion Code: X4.

1109. Zhang Q, Liu LY, Wang F, et al. The changes in female physical and childbearing characteristics in China and potential association with risk of breast cancer. BMC Public Health. 2012 May 21;12:368. doi: 10.1186/1471-2458-12-368. PMID: 22612880. Exclusion Code: X5.

1110. Zhang Y, Carlton E, Fein SB. The association of prenatal media marketing exposure recall with breastfeeding intentions, initiation, and duration. J Hum Lact. 2013 Nov;29(4):500-9. doi: 10.1177/0890334413487256. PMID: 23686404. Exclusion Code: X6.

1111. Zhao J, Zhao Y, Binns CW, et al. Increased Calcium Supplementation Postpartum Is Associated with Breastfeeding among Chinese Mothers: Finding from Two Prospective Cohort Studies. Nutrients. 2016 Oct 09;8(10)doi: 10.3390/nu8100622. PMID: 27735835. Exclusion Code: X3. 
1112. Zhao Y, Munro-Kramer ML, Shi S, et al. A randomized controlled trial: effects of a prenatal depression intervention on perinatal outcomes among Chinese high-risk pregnant women with medically defined complications. Arch Womens Ment Health. 2017 Apr;20(2):333-44. doi: 10.1007/s00737-016-0712-7. PMID: 28058505. Exclusion Code: X3.

1113. Zhou Y, Chen J, Li Q, et al. Association between breastfeeding and breast cancer risk: evidence from a meta-analysis. Breastfeed Med. 2015 Apr;10(3):175-82. doi: 10.1089/bfm.2014.0141. PMID: 25785349. Exclusion Code: X11.

1114. Zhu B, Zhang J, Qiu L, et al. Breastfeeding Rates and Growth Charts--the Zhejiang Infant Feeding Trial. Int J Environ Res Public Health. 2015 Jun 30;12(7):7337-47. doi: 10.3390/ijerph120707337. PMID: 26133126. Exclusion Code: X3.

1115. Ziegler AG, Wallner M, Kaiser I, et al. Long-term protective effect of lactation on the development of type 2 diabetes in women with recent gestational diabetes mellitus. Diabetes. 2012 Dec;61(12):316771. doi: 10.2337/db12-0393. PMID: 23069624. Exclusion Code: X10.

1116. Zimmerman DR. You can make a difference: increasing breastfeeding rates in an inner-city clinic. J Hum Lact. 1999 Sep;15(3):217-20. doi: 10.1177/089033449901500311. PMID: 10578799. Exclusion Code: X6.
1117. Ziol-Guest KM, Hernandez DC. First- and second-trimester WIC participation is associated with lower rates of breastfeeding and early introduction of cow's milk during infancy. J Am Diet Assoc. 2010 May;110(5):702-9. doi: 10.1016/j.jada.2010.02.013. PMID: 20430131. Exclusion Code: X6.

1118. Zong H, Xu H, Geng Z, et al. Reproductive factors in relation to risk of brain tumors in women: an updated meta-analysis of 27 independent studies. Tumour Biol. 2014 Nov;35(11):11579-86. doi: 10.1007/s13277014-2448-1. PMID: 25135427. Exclusion Code: X5.

1119. Zsakai A, Mascie-Taylor N, Bodzsar EB. Relationship between some indicators of reproductive history, body fatness and the menopausal transition in Hungarian women. J Physiol Anthropol. 2015 Oct 22;34:35. doi: 10.1186/s40101-015-0076-0. PMID: 26494263. Exclusion Code: X3.

1120. Zucchetto A, Serraino D, Polesel J, et al. Hormone-related factors and gynecological conditions in relation to endometrial cancer risk. Eur J Cancer Prev. 2009 Aug;18(4):316-21. PMID: 19554665. Exclusion Code: X5.

1121. Zuppa AA, Alighieri G, Riccardi R, et al. Epidural Analgesia, Neonatal Care and Breastfeeding. Neonatal Intensive Care. 2015 Spring2015;28(2):41-4. PMID: 107782604. Exclusion Code: X3.

1122. Zyl Z, Maslin K, Dean T, et al. The accuracy of dietary recall of infant feeding and food allergen data. J Hum Nutr Diet. 2016;29(6):777-85. doi: 10.1111/jhn.12384. PMID: 119477822. Exclusion Code: X3. 


\section{Appendix F. Breast Cancer Evidence Tables}

Table F-1. Breastfeeding and breast cancer: Summary of individual studies

\begin{tabular}{|c|c|c|c|c|c|}
\hline $\begin{array}{l}\text { Author, Year } \\
\text { Study Design } \\
\text { Risk of Bias }\end{array}$ & $\begin{array}{l}\text { Description of Study }(\mathrm{N}) \\
\text { Description of Breast } \\
\text { Cancer Cases (N) }\end{array}$ & $\begin{array}{l}\text { Population } \\
\text { Characteristics }\end{array}$ & $\begin{array}{l}\text { Results: Ever } \\
\text { Breastfed }\end{array}$ & Results: Duration of Breastfeeding & $\begin{array}{l}\text { Confounders } \\
\text { Adjusted for }\end{array}$ \\
\hline $\begin{array}{l}\text { Al-Amri, } 2015^{66} \\
\text { Case-control } \\
\text { High }\end{array}$ & $\begin{array}{l}\text { Case-control study of } \\
\text { Saudi Arabian women } \\
\text { screening for a } \\
\text { mammogram; five age } \\
\text { comparable controls with } \\
\text { normal mammogram } \\
\text { results were selected from } \\
\text { the same mobile clinic as } \\
\text { cases (348) } \\
\text { Diagnosed during } \\
\text { mammogram screening } \\
\text { and confirmed by clinical } \\
\text { and pathological } \\
\text { examination (58) }\end{array}$ & $\begin{array}{l}\text { Mean age (SD): } \\
\text { Cases: } 49(7.1) \\
\text { Controls: } 49(6.9) \\
\text { Postmenopausal: } \\
\text { Cases: } 55 \% \\
\text { Control: } 38 \%\end{array}$ & $\begin{array}{l}\text { Cases: } 45(78 \%) \\
\text { Controls: } 273(94 \%) \\
\text { OR, } 0.30(95 \% \mathrm{Cl} 0.13 \\
\text { or 0.69), } p=0.004\end{array}$ & $\begin{array}{l}\text { Total duration of all breastfeeding periods for all } \\
\frac{\text { children }}{>2 \text { years cases: } 31(36 \%)} \\
>2 \text { years controls: } 231(80 \%) \\
>2 \text { years vs. } \leq 2 \text { years: OR, } 1.68(95 \% \mathrm{Cl}, 0.98 \text { to } \\
4.53), p=0.073\end{array}$ & $\begin{array}{l}\text { Ever breastfed } \\
\text { analysis: Age at } \\
\text { marriage, } \\
\text { menopausal age, } \\
\text { number of } \\
\text { pregnancies, } \\
\text { breastfeeding, } \\
\text { family history of } \\
\text { breast cancer } \\
\text { were controlled } \\
\text { for in adjusted } \\
\text { analysis } \\
\text { Duration } \\
\text { analysis: } \\
\text { Unadjusted }\end{array}$ \\
\hline $\begin{array}{l}\text { Al-Qutub, } \\
2013^{67} \\
\text { Case-control } \\
\text { High }\end{array}$ & $\begin{array}{l}\text { Case-control study of } \\
\text { Saudi women ages 19-50 } \\
\text { recruited at three } \\
\text { government hospitals in } \\
\text { Jeddah city, with controls } \\
\text { recruited from community } \\
\text { and hospital settings } \\
\text { (317) } \\
\text { Breast cancer diagnosis } \\
\text { during the previous } 2 \\
\text { years (151) }\end{array}$ & $\begin{array}{l}\text { Mean age (SD): } \\
\text { Cases: } 40(6.3) \\
\text { Controls: } 39(7.0) \\
\frac{\text { Use of exogenous }}{\text { hormones and/or }} \\
\text { contraception: } \\
\text { Cases: } 8 \% \\
\text { Controls: } 2 \% \\
\text { Current smokers: } \\
\text { Cases: } 6 \% \\
\text { Controls: } 13 \%\end{array}$ & NR & $\begin{array}{l}\text { Sum of breastfeeding duration in months for each } \\
\text { baby born to the participant } \\
\geq 12 \text { months Cases: } 81(54 \%) \\
\geq 12 \text { months Controls: } 112(68 \%) \\
\geq 12 \text { months: } \\
\text { OR, } 0.56 \text { ( } 95 \% \mathrm{Cl}, 0.35 \text { to } 0.88), p=0.01\end{array}$ & NR \\
\hline
\end{tabular}




\begin{tabular}{|c|c|c|c|c|c|}
\hline $\begin{array}{l}\text { Author, Year } \\
\text { Study Design } \\
\text { Risk of Bias }\end{array}$ & $\begin{array}{l}\text { Description of Study }(\mathrm{N}) \\
\text { Description of Breast } \\
\text { Cancer Cases }(\mathrm{N})\end{array}$ & $\begin{array}{l}\text { Population } \\
\text { Characteristics }\end{array}$ & $\begin{array}{l}\text { Results: Ever } \\
\text { Breastfed }\end{array}$ & Results: Duration of Breastfeeding & $\begin{array}{l}\text { Confounders } \\
\text { Adjusted for }\end{array}$ \\
\hline $\begin{array}{l}\text { Atkinson, } \\
2016^{68} \\
\text { Case-control } \\
\text { High }\end{array}$ & $\begin{array}{l}\text { Case-control study of } \\
\text { women at a Texas cancer } \\
\text { center with no prior } \\
\text { history of cancer except } \\
\text { for nonmelanoma skin } \\
\text { cancer or cervical cancer } \\
\text { in situ; controls underwent } \\
\text { routine mammography } \\
\text { screening at the cancer } \\
\text { center between 2005- } \\
2006 \text { (620) } \\
\text { Newly diagnosed } \\
\text { inflammatory breast } \\
\text { cancer at MD Anderson } \\
\text { cancer center between } \\
\text { 2004-2012 (224) }\end{array}$ & $\begin{array}{l}\frac{\text { Mean age (SD), }}{\text { range: }} \\
\text { Cases: } 51(\mathrm{NR}), \text { 23-80 } \\
\text { Controls: } 51 \text { (NR), 24-68 } \\
\text { Nonwhite: } \\
\text { Cases: } 23 \% \\
\text { Controls: } 0 \% \\
\text { Postmenopausal: } \\
\text { Cases: } 67 \% \\
\text { Controls: } 62 \% \\
\text { Ever smoker: } \\
\text { Cases: } 42 \% \\
\text { Controls: } 33 \%\end{array}$ & $\begin{array}{l}\text { Among parous } \\
\text { women: } \\
\text { Triple-negative } \\
\text { OR, } 0.30(95 \% \mathrm{Cl} \text {, } \\
0.15 \text { to } 0.62) \\
\text { HER2neu+ } \\
\text { OR, } 1.01(95 \% \mathrm{Cl} \text {, } \\
0.55 \text { to } 1.87) \\
\text { Luminal } \\
\text { OR, } 0.35(95 \% \mathrm{Cl} \text {, } \\
0.18 \text { to } 0.68)\end{array}$ & NR & $\begin{array}{l}\text { Age at } \\
\text { menarche, } \\
\text { menopausal } \\
\text { status, number } \\
\text { of children, age } \\
\text { at first } \\
\text { pregnancy, } \\
\text { breastfeeding } \\
\text { history, BMI, } \\
\text { smoking history, } \\
\text { breast cancer } \\
\text { family history }\end{array}$ \\
\hline $\begin{array}{l}\text { Beaber, } 2008^{69} \\
\text { Case-control } \\
\text { Medium }\end{array}$ & $\begin{array}{l}\text { Population-based case- } \\
\text { control study in the U.S., } \\
\text { with controls frequency } \\
\text { matched to cases on age } \\
\text { ( } 5 \text {-year age groups) and } \\
\text { reference year ( } 898 \\
\text { parous) } \\
\text { Ductal and lobular tumors; } \\
\text { based on histology review } \\
\text { by study pathologists } \\
\text { (when tissue available) or } \\
\text { review of pathology } \\
\text { reports by trained } \\
\text { abstractors (when not) } \\
\text { ( } 469 \text { parous) }\end{array}$ & $\begin{array}{l}\text { Mean age (SD): } \\
\text { NR } \\
\text { \% Nonwhite: } \\
\text { Cases: } 17 \% \\
\text { Controls: } 16 \% \\
\text { Current or prior HRT use: } \\
\text { Cases: } 74 \% \\
\text { Controls: } 74 \% \\
\text { Postmenopausal: } \\
\text { Cases: } 66 \% \\
\text { Controls: } 73 \%\end{array}$ & $\begin{array}{l}\text { Ever breastfed } \geq 1 \\
\text { month } \\
\text { Ductal } \\
\text { Cases: } 240 \\
\text { Controls: } 264 \\
\text { OR, } 0.7(95 \% \mathrm{Cl}, 0.5 \\
\text { to } 0.9), \mathrm{p}<0.05 \\
\text { Lobular } \\
\text { Cases: } 167 \\
\text { Controls: } 264 \\
\text { OR, } 0.9(95 \% \mathrm{Cl}, 0.7 \\
\text { to } 1.3), \mathrm{p}=\mathrm{NS}\end{array}$ & $\begin{array}{l}\text { Ductal } \\
<1 \text { month ( } 37 \text { exposed cases, } 27 \text { controls): OR, } 1.1 \\
\text { (95\% } \mathrm{Cl}, 0.6 \text { to } 1.9) \\
1.0-5.9 \text { months ( } 96 \text { exposed cases, } 112 \text { controls): OR, } \\
0.7(95 \% \mathrm{Cl}, 0.5 \text { to } 0.9) \\
6.0-11.9 \text { months ( } 61 \text { exposed cases, } 62 \text { controls): OR, } \\
0.8(95 \% \mathrm{Cl}, 0.5 \text { to } 1.2) \\
12.0-23.9 \text { months (58 exposed cases, } 56 \text { controls): } \\
\text { OR, } 0.8 \text { ( } 95 \% \mathrm{Cl}, 0.5 \text { to } 1.3) \\
\geq 24.0 \text { months ( } 25 \text { exposed cases, } 34 \text { controls): OR, } \\
0.6(95 \% \mathrm{Cl}, 0.3 \text { to } 1.0) \\
\text { p for trend }=0.43\end{array}$ & $\begin{array}{l}\text { Reference age, } \\
\text { Reference year, } \\
\text { number of live } \\
\text { births }\end{array}$ \\
\hline
\end{tabular}




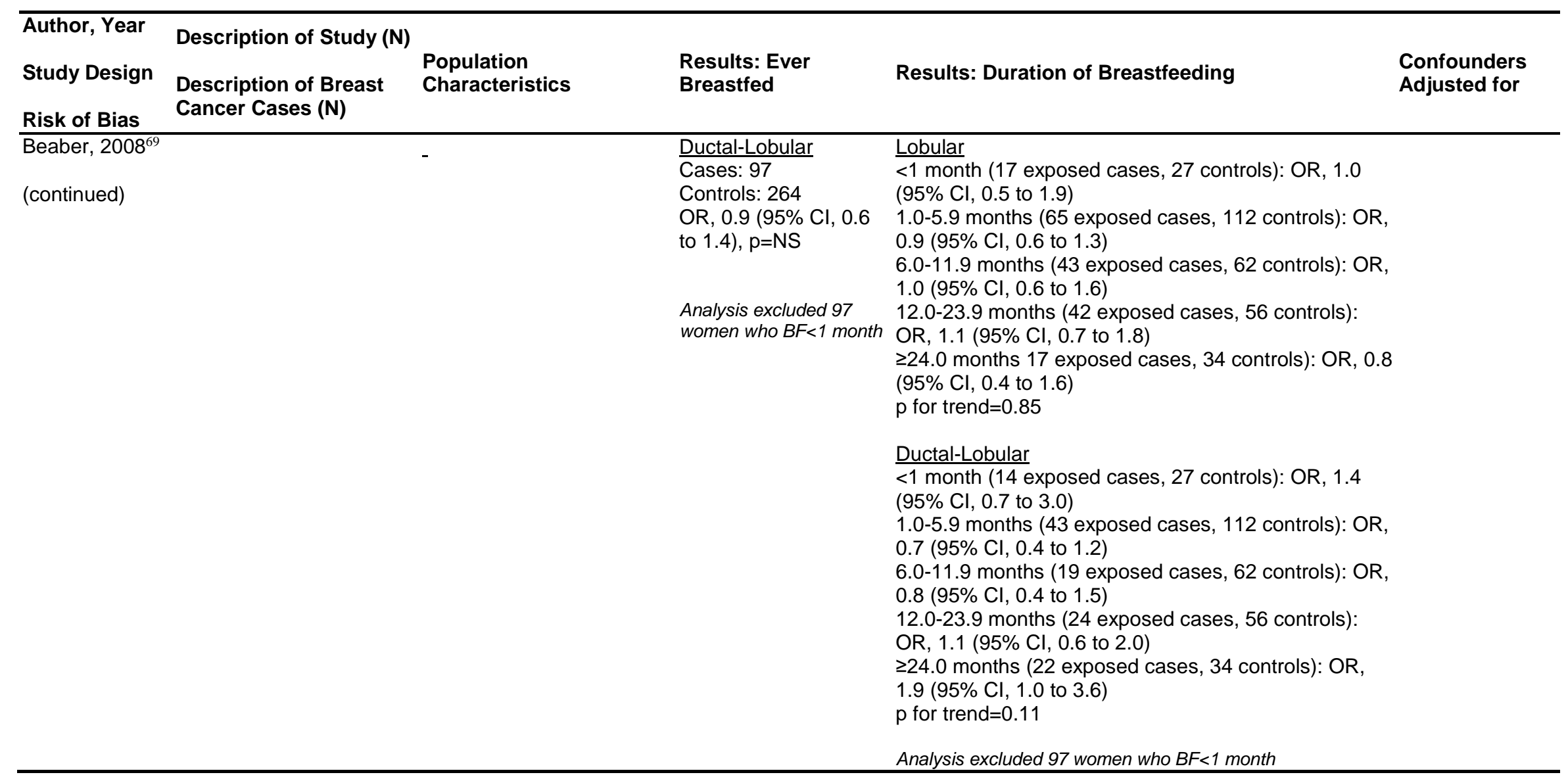




\begin{tabular}{|c|c|c|c|c|c|}
\hline $\begin{array}{l}\text { Author, Year } \\
\text { Study Design } \\
\text { Risk of Bias }\end{array}$ & $\begin{array}{l}\text { Description of Study }(\mathrm{N}) \\
\text { Description of Breast } \\
\text { Cancer Cases }(\mathrm{N})\end{array}$ & $\begin{array}{l}\text { Population } \\
\text { Characteristics }\end{array}$ & $\begin{array}{l}\text { Results: Ever } \\
\text { Breastfed }\end{array}$ & Results: Duration of Breastfeeding & $\begin{array}{l}\text { Confounders } \\
\text { Adjusted for }\end{array}$ \\
\hline $\begin{array}{l}\text { Castello, 201570 } \\
\text { Case-control } \\
\text { Medium }\end{array}$ & $\begin{array}{l}\text { Case-control study of } \\
\text { women diagnosed with } \\
\text { incident cases of breast } \\
\text { cancer in the oncology } \\
\text { departments of } 23 \\
\text { hospital members of the } \\
\text { Spanish Breast Cancer } \\
\text { Group located in } 9 \text { of } 17 \\
\text { regions in Spain; matched } \\
\text { healthy controls of similar } \\
\text { age }(+/-5 \text { years) were } \\
\text { selected from cases' in- } \\
\text { law relatives, neighbors, } \\
\text { or work colleagues } \\
\text { residing in same town } \\
(1,946) \\
\text { Incident breast cancer } \\
\text { diagnosed in oncology } \\
\text { department (973; sample } \\
\text { size for BF analysis } \\
\text { unclear) }\end{array}$ & $\begin{array}{l}\text { Mean age (SD): } \\
\text { NR } \\
\text { Postmenopausal: } \\
\text { Cases: } 43 \% \\
\text { Controls: } 47 \% \\
\text { Current or former smokers: } \\
\text { Cases: } 59 \% \\
\text { Controls: } 60 \%\end{array}$ & NR & $\begin{array}{l}\frac{\text { Cumulative BF duration }<6 \text { months, overall sample }}{\text { ( } \mathrm{n}=1,946 ; \text { OR is for lack of compliance with guideline }} \\
\frac{\text { to BF up to } 6 \text { months) }}{\text { Cases: } 394} \\
\text { Controls: } 386 \\
\text { OR, } 0.95 \text { ( } 95 \% \mathrm{Cl}, 0.70 \text { to } 1.27 \text { ) } \\
\text { Cumulative BF duration }<6 \text { months, premenopausal } \\
\frac{(\mathrm{n}=1,064)}{\text { Cases: } 217} \\
\text { Controls: } 210 \\
\text { OR, } 0.89 \text { ( } 95 \% \mathrm{Cl}, 0.61 \text { to } 1.30) \\
\text { Cumulative BF duration } \geq 6 \text { months, postmenopausal } \\
\text { Cases: } 177 \\
\text { Controls: } 176 \\
\text { OR, } 1.00 \text { ( } 95 \% \mathrm{Cl}, 0.69 \text { to } 1.45)\end{array}$ & $\begin{array}{l}\text { Total calorie } \\
\text { intake, smoking } \\
\text { habit, age at first } \\
\text { delivery, } \\
\text { education, history } \\
\text { of breast } \\
\text { problems, family } \\
\text { history of breast } \\
\text { cancer, } \\
\text { menopausal study } \\
\text { and composite } \\
\text { score derived } \\
\text { from adherence to } \\
\text { WCRF/AICR } \\
\text { recommendations } \\
\text { (excluding BF } \\
\text { recommendation) }\end{array}$ \\
\hline
\end{tabular}




\begin{tabular}{|c|c|c|c|c|c|}
\hline $\begin{array}{l}\text { Author, Year } \\
\text { Study Design } \\
\text { Risk of Bias }\end{array}$ & $\begin{array}{l}\text { Description of Study (N) } \\
\text { Description of Breast } \\
\text { Cancer Cases (N) }\end{array}$ & $\begin{array}{l}\text { Population } \\
\text { Characteristics }\end{array}$ & $\begin{array}{l}\text { Results: Ever } \\
\text { Breastfed }\end{array}$ & Results: Duration of Breastfeeding & $\begin{array}{l}\text { Confounders } \\
\text { Adjusted for }\end{array}$ \\
\hline $\begin{array}{l}\text { Dalamaga, } \\
2011^{71} \\
\text { Case-control } \\
\text { High }\end{array}$ & $\begin{array}{l}\text { Case-control study of } \\
\text { women at the Army Share } \\
\text { Fund Hospital, Veteran's } \\
\text { Hospital, with cases } \\
\text { admitted in the Internal } \\
\text { Medicine Department and } \\
\text { controls randomly } \\
\text { selected from women with } \\
\text { negative mammograms } \\
\text { and matched to cases } \\
\text { based on age and } \\
\text { proximity of the outpatient } \\
\text { visit to the case's time of } \\
\text { diagnosis (204) }\end{array}$ & $\begin{array}{l}\text { Mean age (SD), range: } \\
\text { Cases: } 62(8.2), \text { NR } \\
\text { Controls: } 63 \text { (8.9), NR } \\
\text { Postmenopausal: } \\
100 \% \\
\text { Current or prior HRT: } \\
\text { Cases: } 5 \% \\
\text { Controls: } 1 \% \\
\text { Current or former smokers: } \\
\text { Cases: } 38 \% \\
\text { Controls: } 27 \%\end{array}$ & NR & $\begin{array}{l}>6 \text { months breastfeeding: } \\
\text { Cases: } 45(44 \%) \\
\text { Controls: } 51(50 \%) \\
p=0.9\end{array}$ & $\begin{array}{l}\text { Analysis is } \\
\text { unadjusted }\end{array}$ \\
\hline & $\begin{array}{l}\text { Diagnosed with invasive } \\
\text { breast cancer between } \\
\text { October } 2003 \text { and } \\
\text { September } 2010 \text { (102) }\end{array}$ & & & & \\
\hline
\end{tabular}




\begin{tabular}{|c|c|c|c|c|c|}
\hline $\begin{array}{l}\text { Author, Year } \\
\text { Study Design } \\
\text { Risk of Bias } \\
\end{array}$ & $\begin{array}{l}\text { Description of Study (N) } \\
\text { Description of Breast } \\
\text { Cancer Cases (N) }\end{array}$ & $\begin{array}{l}\text { Population } \\
\text { Characteristics }\end{array}$ & $\begin{array}{l}\text { Results: Ever } \\
\text { Breastfed }\end{array}$ & Results: Duration of Breastfeeding & $\begin{array}{l}\text { Confounders } \\
\text { Adjusted for }\end{array}$ \\
\hline $\begin{array}{l}\text { Ge, } 2015^{72} \\
\text { Case-control } \\
\text { High }\end{array}$ & $\begin{array}{l}\text { Population-based case- } \\
\text { control study of } \\
\text { postmenopausal German } \\
\text { women ages } 50-74 \\
\text { recruited between 2001- } \\
2005 \text { ( } 8,399) ; \text { each case } \\
\text { was frequency matched } \\
\text { by birth year and study } \\
\text { region with } 2 \text { controls } \\
\text { drawn from random lists } \\
\text { provided by resident } \\
\text { registries }(8,399) \\
\\
\text { Diagnosed with } \\
\text { histologically confirmed } \\
\text { primary breast cancer } \\
(2,887)\end{array}$ & $\begin{array}{l}\text { Mean age (SD): } \\
\text { NR } \\
\text { Postmenopausal: } 100 \% \\
\text { Current or former smoker: } \\
\text { Cases: } 45 \% \\
\text { Controls: } 46 \%\end{array}$ & $\begin{array}{l}\text { Cases: } 63 \% \\
\text { Controls: } 67 \% \\
\\
\text { Article reports "Cases } \\
\text { had BF their children } \\
\text { less frequently", but } \\
\text { statistical tests NR }\end{array}$ & $\mathrm{NR}$ & $\mathrm{NR}$ \\
\hline $\begin{array}{l}\text { Hadji, } 2007^{73} \\
\text { Case-control } \\
\text { High }\end{array}$ & $\begin{array}{l}\text { Case-control study of } \\
\text { German women } \\
\text { consecutively recruited } \\
\text { from a university } \\
\text { gynecological oncology } \\
\text { and endocrinology clinic } \\
\text { for routine gynecological } \\
\text { checkup }(2,492) \\
\\
\text { Incident breast operation } \\
\text { (mean duration since } \\
\text { operation } 10 \pm 5 \text { days) } \\
\text { including a clear } \\
\text { histological diagnosis of } \\
\text { breast cancer (242) }\end{array}$ & $\begin{array}{l}\text { Mean age (SD), range: } 54 \\
(10.3), 22-88 \\
\frac{\text { Postmenopausal: Cases: }}{71 \%} \\
\text { Controls: } 67 \% \\
\text { On HRT: } \\
\text { Cases: } 29 \% \\
\text { Controls: } 42 \% \\
\text { Current smoker: } \\
\text { Cases: } 16 \% \\
\text { Controls: } 21 \%\end{array}$ & $\begin{array}{l}\text { Cases: } 69 \% \\
\text { Controls: } 52 \% \\
\text { p<0.001 } \\
\\
\text { Only unadjusted analysis } \\
\text { available for ever BF; } \\
\text { adjusted analysis } \\
\text { matched cases and } \\
\text { controls for BF. }\end{array}$ & $\begin{array}{l}\text { Multiple linear regression showed that women with } \\
\text { breast cancer had a significantly longer duration of } \\
\text { breastfeeding }(p<0.05)\end{array}$ & $\mathrm{NR}$ \\
\hline
\end{tabular}




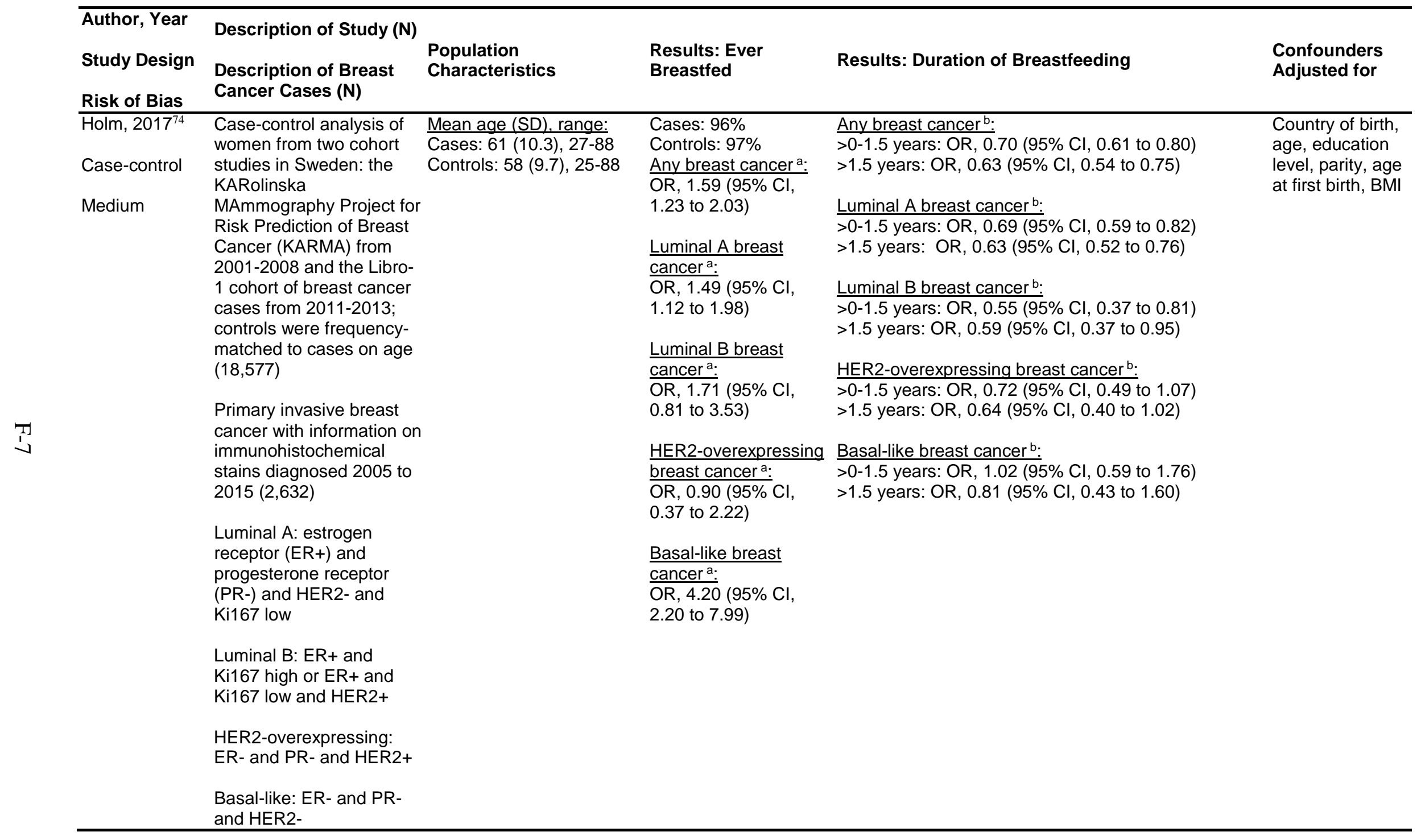




\begin{tabular}{|c|c|c|c|c|c|}
\hline $\begin{array}{l}\text { Author, Year } \\
\text { Study Design } \\
\text { Risk of Bias } \\
\end{array}$ & $\begin{array}{l}\text { Description of Study (N) } \\
\text { Description of Breast } \\
\text { Cancer Cases (N) }\end{array}$ & $\begin{array}{l}\text { Population } \\
\text { Characteristics }\end{array}$ & $\begin{array}{l}\text { Results: Ever } \\
\text { Breastfed }\end{array}$ & Results: Duration of Breastfeeding & $\begin{array}{l}\text { Confounders } \\
\text { Adjusted for }\end{array}$ \\
\hline $\begin{array}{l}\text { Kabat, } 2011^{75} \\
\text { Case-control } \\
\text { Medium }\end{array}$ & $\begin{array}{l}\text { Case-control analysis of } \\
\text { parous women ages 50- } \\
79 \text { from the Women's } \\
\text { Health Initiative Study, } \\
\text { recruited from } 40 \text { clinical } \\
\text { centers in the U.S.; } \\
\text { controls were not } \\
\text { matched with cases } \\
(63,396) \\
\text { Incident diagnosis of } \\
\text { ductal carcinoma in situ } \\
\text { breast cancer (664) }\end{array}$ & $\begin{array}{l}\text { Mean age (SD), range: } \\
\text { Cases: } 62(6.8), \text { NR } \\
\text { Controls: } 623 \text { (7.0), NR } \\
\text { Nonwhite: } \\
\text { Cases: } 17 \% \\
\text { Controls: } 18 \% \\
\text { Postmenopausal: } 100 \% \\
\text { Current or prior HRT: } \\
\text { Cases: } 59 \% \\
\text { Controls: } 52 \%\end{array}$ & NR & $\begin{array}{l}\mathrm{HR}(95 \% \mathrm{Cl}) \\
1-6 \text { months: } 1.05 \text { (0.86 to } 1.28) \\
7-12 \text { months: } 1.04(0.80 \text { to } 1.36) \\
>12 \text { months: } 1.01 \text { (0.80 to } 1.29) \\
\text { p for trend }=0.94\end{array}$ & $\begin{array}{l}\text { Age, education, } \\
\text { hormone } \\
\text { therapy, family } \\
\text { history of breast } \\
\text { cancer, history of } \\
\text { breast biopsy, } \\
\text { and } \\
\text { mammograms, } \\
\text { age at } \\
\text { menarche, age } \\
\text { at menopause }\end{array}$ \\
\hline $\begin{array}{l}\text { Kotsopoulos, } \\
2012^{76} \\
\text { Case-control } \\
\text { Medium }\end{array}$ & $\begin{array}{l}\text { Case-control study of } \\
\text { women who sought BRCA } \\
\text { mutation testing from one } \\
\text { of } 70 \text { participating centers } \\
\text { in } 12 \text { countries and were } \\
\text { confirmed as carriers of } \\
\text { deleterious mutations in } \\
\text { the BRCA1 or BRCA2 } \\
\text { genes, with controls } \\
\text { matched to cases on } \\
\text { mutation in the same } \\
\text { gene), year of birth (within } \\
1 \text { year), and country of } \\
\text { residence (5,708) } \\
\text { Diagnosis of invasive } \\
\text { breast cancer }(2,854)\end{array}$ & $\begin{array}{l}\text { Mean age (SD), range: } \\
\text { Cases: } 47(\mathrm{NR}), 21-85 \\
\text { Controls: } 47 \text { (NR), 18-86 } \\
\text { Postmenopausal: } \\
\text { Cases: } 14 \% \\
\text { Controls: } 7 \%\end{array}$ & NR & $\begin{array}{l}\text { Mean months breastfed } \\
\text { Cases: } 7.5(0-102) \\
\text { Controls: } 9.6 \text { (0-147) } \\
p<0.0001 \\
\text { BRCA1 Carriers: OR }(95 \% \mathrm{Cl}) \\
\leq 1 \text { year: } 0.81 \text { (0.66 to } 1.00), p=0.05 \\
1 \text { to } \leq 2 \text { years: } 0.65 \text { (0.50 to } 0.85), p=0.001 \\
2 \text { to } \leq 3 \text { years: } 0.51(0.35 \text { to } 0.75), p=0.0006 \\
>3 \text { years: } 0.45 \text { ( } 0.30 \text { to } 0.68), p=0.0002 \\
\text { BRCA2 Carriers: OR (95\% Cl) } \\
\leq 1 \text { year: } 1.03 \text { (0.76 to } 1.40), p=0.85 \\
1 \text { to } \leq 2 \text { years: } 1.04(0.70 \text { to } 1.53), p=0.86 \\
2 \text { to } \leq 3 \text { years: } 1.33(0.76 \text { to } 2.32), p=0.31 \\
>3 \text { years: } 1.02 \text { (0.56 to } 1.88), p=0.94\end{array}$ & $\begin{array}{l}\text { Age at } \\
\text { menarche, } \\
\text { parity, and oral } \\
\text { contraceptive } \\
\text { use }\end{array}$ \\
\hline
\end{tabular}




\begin{tabular}{|c|c|c|c|c|c|}
\hline $\begin{array}{l}\text { Author, Year } \\
\text { Study Design } \\
\text { Risk of Bias } \\
\end{array}$ & $\begin{array}{l}\text { Description of Study (N) } \\
\text { Description of Breast } \\
\text { Cancer Cases (N) }\end{array}$ & $\begin{array}{l}\text { Population } \\
\text { Characteristics }\end{array}$ & $\begin{array}{l}\text { Results: Ever } \\
\text { Breastfed }\end{array}$ & Results: Duration of Breastfeeding & $\begin{array}{l}\text { Confounders } \\
\text { Adjusted for }\end{array}$ \\
\hline $\begin{array}{l}\text { Kruk, 201477 } \\
\text { Case-control } \\
\text { High }\end{array}$ & $\begin{array}{l}\text { Case-control study of } \\
\text { women ages } 28-79 \\
\text { identified from the } \\
\text { Szczecin Regional } \\
\text { Cancer Registry in the } \\
\text { Region of Western } \\
\text { Pomerania; controls were } \\
\text { randomly recruited from } \\
\text { outpatient clinics and } \\
\text { frequency matched to } \\
\text { cases by age (5-year } \\
\text { interval) and residence } \\
\text { (urban, rural) }(1,943)\end{array}$ & $\begin{array}{l}\text { Mean age (SD), range: } \\
\text { Cases: } 55(9.7), N R \\
\text { Controls: } 55(9.5), \mathrm{NR}\end{array}$ & NR & $\begin{array}{l}\text { Compared with case subjects, controls reported a } \\
\text { longer duration of breastfeeding (P-value and values } \\
\text { by group NR) }\end{array}$ & $\begin{array}{l}\text { Analysis is } \\
\text { unadjusted }\end{array}$ \\
\hline & $\begin{array}{l}\text { Diagnosed with } \\
\text { histologically confirmed } \\
\text { invasive breast cancer } \\
\text { (858) }\end{array}$ & & & & \\
\hline
\end{tabular}




\begin{tabular}{|c|c|c|c|c|c|}
\hline $\begin{array}{l}\text { Author, Year } \\
\text { Study Design } \\
\text { Risk of Bias }\end{array}$ & $\begin{array}{l}\text { Description of Study (N) } \\
\text { Description of Breast } \\
\text { Cancer Cases (N) }\end{array}$ & $\begin{array}{l}\text { Population } \\
\text { Characteristics }\end{array}$ & $\begin{array}{l}\text { Results: Ever } \\
\text { Breastfed }\end{array}$ & Results: Duration of Breastfeeding & $\begin{array}{l}\text { Confounders } \\
\text { Adjusted for }\end{array}$ \\
\hline $\begin{array}{l}\text { Lee, } 2008^{78} \\
\text { Case-control } \\
\text { Medium }\end{array}$ & $\begin{array}{l}\text { Population-based case- } \\
\text { control study in the U.S., } \\
\text { with controls matched on } \\
\text { race and age (within } 5 \\
\text { years and ages } 20-49 \text { ) to } \\
\text { a subset of case patients } \\
\text { diagnosed between } \\
7 / 2000-3 / 2003 \text {, and met } \\
\text { the same eligibility criteria } \\
\text { as cases }(2,238) \\
\text { Histologically confirmed } \\
\text { first primary invasive } \\
\text { breast cancer identified } \\
\text { through the Los Angeles } \\
\text { County Cancer } \\
\text { Surveillance Program, a } \\
\text { population-based registry } \\
\text { sponsored by the NCl } \\
\text { Seer program }(1,794)\end{array}$ & $\begin{array}{l}\text { Mean age (SD): } \\
\text { Cases, BRCA carriers: } 41 \\
(6.4) \\
\text { Cases, BRCA noncarriers: } \\
43 \text { (5.1) } \\
\text { Controls: } 43 \text { (4.9) } \\
\text { \% Nonwhite: } 9 \% \\
\text { Current or prior HRT use: } \\
\text { NR } \\
\text { Postmenopausal: } 20 \%\end{array}$ & $\mathrm{NR}$ & $\begin{array}{l}\text { BRCA Carriers: OR ( } 95 \% \text { Cl) } \\
<1-6 \text { months ( } 22 \text { exposed cases, } 104 \text { controls): } 1.31 \\
(0.45 \text { to } 3.82) \\
7-23 \text { months ( } 16 \text { exposed cases, } 111 \text { controls): } 0.73 \\
(0.23 \text { to } 2.30) \\
\geq 24 \text { months }(11 \text { exposed cases, } 64 \text { controls): } 1.29 \\
(0.36 \text { to } 4.61) \\
\text { p for trend }=0.83 \\
\text { BRCA Non-carriers: OR ( } 95 \% \mathrm{Cl}) \\
<1-6 \text { months ( } 326 \text { exposed cases, } 104 \text { controls): } 0.66 \\
\text { (0.43 to } 1.02) \\
7-23 \text { months ( } 264 \text { exposed cases, } 104 \text { controls): } 0.52 \\
\text { (0.33 to } 0.81) \\
\geq 24 \text { months (147 exposed cases, } 104 \text { controls): } 0.49 \\
(0.29 \text { to } 0.81) \\
\text { p for trend=0.002 }\end{array}$ & $\begin{array}{l}\text { Age at reference } \\
\text { date, education, } \\
\text { family history of } \\
\text { breast or ovarian } \\
\text { cancer, race, } \\
\text { self-identified } \\
\text { Ashkenazi } \\
\text { Jewish origin, } \\
\text { number of full- } \\
\text { term } \\
\text { pregnancies, age } \\
\text { at first full-term } \\
\text { pregnancy }\end{array}$ \\
\hline
\end{tabular}




\begin{tabular}{|c|c|c|c|c|c|}
\hline $\begin{array}{l}\text { Author, Year } \\
\text { Study Design } \\
\text { Risk of Bias }\end{array}$ & $\begin{array}{l}\text { Description of Study (N) } \\
\text { Description of Breast } \\
\text { Cancer Cases (N) }\end{array}$ & $\begin{array}{l}\text { Population } \\
\text { Characteristics }\end{array}$ & $\begin{array}{l}\text { Results: Ever } \\
\text { Breastfed }\end{array}$ & Results: Duration of Breastfeeding & $\begin{array}{l}\text { Confounders } \\
\text { Adjusted for }\end{array}$ \\
\hline $\begin{array}{l}\text { Lumachi, } 2010 \\
79 \\
\text { Case-control } \\
\text { High }\end{array}$ & $\begin{array}{l}\text { Cases were identified by } \\
\text { retrospective review of } \\
404 \text { consecutive women } \\
\text { undergoing curative } \\
\text { surgery for BC. Women } \\
\text { were excluded who had a } \\
\text { history pf previous cancer, } \\
\text { BC onset during follow- } \\
\text { up, had used estrogen + } \\
\text { progestin therapy, or were } \\
\text { non-OC users. (238) } \\
\text { Randomly selected age- } \\
\text { matched healthy women } \\
\text { from the same region, } \\
\text { who had undergone } \\
\text { screening mammography } \\
\text { twice and were followed } \\
\text { up for } 2 \text { years. (255) }\end{array}$ & $\begin{array}{l}\text { Mean age (SD): } \\
\text { Cases: } 62(9.6) \\
\text { Controls: } 61(8.4) \\
\text { Postmenopausal: } 100 \% \\
\text { Current or prior HRT: } \\
\text { Cases: } 58 \% \\
\text { Controls: } 36 \% \\
\text { Current or former smokers: } \\
\text { Cases: } 18 \% \\
\text { Controls: } 18 \%\end{array}$ & $\begin{array}{l}\text { Cases: } 103(57 \%) \\
\text { Controls: } 145(70 \%) \\
\text { OR, } 1.82(95 \% \mathrm{Cl} \\
1.20 \text { to } 2.77), \mathrm{p}=0.006\end{array}$ & $\begin{array}{l}\text { Mean (SD) Months of breastfeeding } \\
\text { Cases: } 10.2(8.6) \\
\text { Controls: } 13.9(10.0) \\
\text { p<0.001 }\end{array}$ & $\begin{array}{l}\text { Bivariate } \\
\text { analyses } \\
\text { reported. } \\
\text { Multivariate } \\
\text { analysis } \\
\text { conducted with } \\
\text { years between } \\
\text { menarche and } \\
\text { menopause, BF, } \\
\text { OC and HRT } \\
\text { use, but only a } \\
\text { cumulative OR } \\
\text { (rather than BF } \\
\text { specific) was } \\
\text { reported: } 4.55 \\
\text { (95\% Cl, } 2.13 \text { to } \\
9.71) .\end{array}$ \\
\hline
\end{tabular}




\begin{tabular}{|c|c|c|c|c|c|}
\hline $\begin{array}{l}\text { Author, Year } \\
\text { Study Design } \\
\text { Risk of Bias }\end{array}$ & $\begin{array}{l}\text { Description of Study }(\mathrm{N}) \\
\text { Description of Breast } \\
\text { Cancer Cases (N) }\end{array}$ & $\begin{array}{l}\text { Population } \\
\text { Characteristics }\end{array}$ & $\begin{array}{l}\text { Results: Ever } \\
\text { Breastfed }\end{array}$ & Results: Duration of Breastfeeding & $\begin{array}{l}\text { Confounders } \\
\text { Adjusted for }\end{array}$ \\
\hline $\begin{array}{l}\text { Ma, 200680 } \\
\text { Case-control } \\
\text { Medium }\end{array}$ & $\begin{array}{l}\text { Case-control study among } \\
\text { white or African American } \\
\text { cases age } 20-49 \text { at time } \\
\text { of diagnosis identified } \\
\text { through LA Cancer } \\
\text { Surveillance Program } \\
\text { (CSP) and SEER registry, } \\
\text { and controls from the } \\
\text { same neighborhoods } \\
\text { matched on age and race } \\
\text { (within } 5 \text { years) }(2,165) \\
\text { Diagnosis of first primary } \\
\text { invasive ER and PR } \\
\text { breast cancer }(1,725)\end{array}$ & $\begin{array}{l}\text { Mean age (SD): } \\
\text { Cases (known receptor): } \\
423 \text { (5.4) } \\
\text { Cases } \\
\text { (borderline/undecided } \\
\text { receptor): } 43 \text { (5.2) } \\
\text { Cases (no info on } \\
\text { receptor): } 44 \text { (4.6) } \\
\text { Controls: } 43 \text { (4.9) } \\
\text { Nonwhite: } \\
\text { Cases (known receptor): } \\
12 \% \\
\text { Cases } \\
\text { (borderline/undecided } \\
\text { receptor): } 7 \% \\
\text { Cases (no info on } \\
\text { receptor): } 14 \% \\
\text { Controls: } 8 \%\end{array}$ & NR & $\begin{array}{l}\text { All participants: OR }(95 \% \mathrm{Cl}) \\
<1 \text { month: } 0.99(0.56 \text { to } 1.77) \\
1-6 \text { months: } 0.58(0.37 \text { to } 0.91) \\
7-23 \text { months: } 0.52 \text { (0.33 to } 0.82) \\
24+\text { months: } 0.51(0.30 \text { to } 0.86) \\
\text { p for trend }=0.001 \\
\\
\text { ER+PR+: OR }(95 \% \mathrm{Cl}) \\
<1 \text { month: } 1.01(0.53 \text { to } 1.90) \\
1-6 \text { months: } 0.57(0.34 \text { to } 0.94) \\
7-23 \text { months: } 0.52(0.31 \text { to } 0.87) \\
24+\text { months: } 0.49(0.27 \text { to } 0.87) \\
\text { p for trend }=0.002 \\
\text { ER-PR-: OR }(95 \% \mathrm{Cl}) \\
\text { <1 month: } 1.19(0.59 \text { to } 2.39) \\
1-6 \text { months: } 0.72(0.41 \text { to } 1.27) \\
7-23 \text { months: } 0.55(0.31 \text { to } 0.98) \\
24+\text { months: } 0.62(0.32 \text { to } 1.21) \\
\text { p for trend }=0.03\end{array}$ & $\begin{array}{l}\text { Race, age, } \\
\text { education, first- } \\
\text { degree breast } \\
\text { cancer family } \\
\text { history, age at } \\
\text { menarche, } \\
\text { gravidity, number } \\
\text { of full-term } \\
\text { pregnancies, } \\
\text { BMl } 1 \text { year } \\
\text { before reference } \\
\text { date, COC use, } \\
\text { average } \\
\text { alcoholic drinks } \\
\text { per week in } \\
\text { recent } 5 \text { years, } \\
\text { and a variable } \\
\text { combining } \\
\text { menopausal } \\
\text { status and } \\
\text { hormone therapy } \\
\text { usage. Age at } \\
\text { first full-term } \\
\text { pregnancy and } \\
\text { duration of BF } \\
\text { mutually } \\
\text { adjusted for each } \\
\text { other. }\end{array}$ \\
\hline
\end{tabular}




\begin{tabular}{|c|c|c|c|c|c|}
\hline $\begin{array}{l}\text { Author, Year } \\
\text { Study Design } \\
\text { Risk of Bias }\end{array}$ & $\begin{array}{l}\text { Description of Study (N) } \\
\text { Description of Breast } \\
\text { Cancer Cases (N) }\end{array}$ & $\begin{array}{l}\text { Population } \\
\text { Characteristics }\end{array}$ & $\begin{array}{l}\text { Results: Ever } \\
\text { Breastfed }\end{array}$ & Results: Duration of Breastfeeding & $\begin{array}{l}\text { Confounders } \\
\text { Adjusted for }\end{array}$ \\
\hline $\begin{array}{l}\text { Ma, } 2017^{81} \\
\text { Case-control } \\
\text { Medium }\end{array}$ & $\begin{array}{l}\text { Pooled analysis of women } \\
\text { from } 3 \text { population-based } \\
\text { studies of breast cancer, } \\
\text { predominantly in Los } \\
\text { Angeles: Women's } \\
\text { Contraceptive and } \\
\text { Reproductive Experiences } \\
\text { (CARE), Women's Breast } \\
\text { Carcinoma in situ (BCIS), } \\
\text { and Women's Learning } \\
\text { the Influence of Family } \\
\text { and Environment (LIFE). } \\
\text { Controls were frequency- } \\
\text { match to controls on age, } \\
\text { race, and geographic area } \\
\text { of residence (5,106) } \\
\text { Newly diagnosed in situ } \\
\text { and invasive breast } \\
\text { cancer; some were first } \\
\text { primary diagnoses and } \\
\text { were histologically } \\
\text { confirmed (2,658) } \\
\text { Triple-negative: ER-, PR-, } \\
\text { HER2- } \\
\text { Luminal A-like: ER+ } \\
\text { and/or PR+, HER2- } \\
\text { Luminal B-like: ER+ } \\
\text { and/or PR+, HER2+ } \\
\text { HER2-enriched: ER-, PR-, }\end{array}$ & $\begin{array}{l}\text { Mean age (SD), range } \\
\text { Cases: } \\
\text { Overall: } 47 \text { (8.1), 22-64 } \\
\text { CARE: } 49 \text { (8.6), 35-64 } \\
\text { BCIS: } 52 \text { (7.3), 35-64 } \\
\text { LIFE: } 43 \text { (5.4), 22-49 } \\
\text { Controls: } \\
\text { Overall: } 48 \text { (8.3), 24-64 } \\
\text { CARE: } 49 \text { (8.4), 35-64 } \\
\text { BCIS: NAc } \\
\text { LIFE: } 43 \text { (4.9), 24-49 } \\
\text { African-American Race } \\
\text { Cases: } \\
\text { Overall: } 26 \% \\
\text { CARE: 43\% } \\
\text { BCIS: 16\% } \\
\text { LIFE: } 11 \% \\
\text { Controls: } \\
\text { Overall: } 37 \% \\
\text { CARE: } 43 \% \\
\text { BCIS: NAc } \\
\text { LIFE: } 8 \% \\
\end{array}$ & $\begin{array}{l}\text { OR }(95 \% \mathrm{Cl}) \\
\text { Triple-negative: } 0.80 \\
\text { (0.63 to } 1.02) \\
\text { Luminal A-like: } 0.78 \\
\text { (0.65 to } 0.94) \\
\text { Luminal B-like: } 0.89 \\
\text { (0.65 to } 1.23 \text { ) } \\
\text { HER2-enriched: } 0.91 \\
\text { (0.63 to } 1.32 \text { ) }\end{array}$ & $\begin{array}{l}\text { Triple-negative: } \mathrm{OR}(95 \% \mathrm{Cl}) \\
<6 \text { months: } 0.96(0.74 \text { to } 1.26) \\
6-11 \text { months: } 0.55(0.37 \text { to } 0.82) \\
\geq 12 \text { months: } 0.69(0.50 \text { to } 0.96) \\
\text { p for trend }=0.006 \\
\text { Luminal A-like: } \mathrm{OR}(95 \% \mathrm{Cl}) \\
<6 \text { months: } 0.83(0.68 \text { to } 1.02) \\
6-11 \text { months: } 0.76 \text { (0.59 to } 0.99) \\
\geq 12 \text { months: } 0.71 \text { (0.56 to } 0.90) \\
\text { p for trend=0.004 } \\
\text { Luminal B-like: } \mathrm{OR}(95 \% \mathrm{Cl}) \\
<6 \text { months: } 0.99(0.70 \text { to } 1.41) \\
6-11 \text { months: } 0.70(0.44 \text { to } 1.12) \\
\geq 12 \text { months: } 0.85(0.56 \text { to } 1.30) \\
\text { p for trend }=0.28 \\
\\
\text { HER2-enriched: } \mathrm{OR}(95 \% \mathrm{Cl}) \\
<6 \text { months: } 0.68(0.43 \text { to } 1.07) \\
6-11 \text { months: } 1.28 \text { (0.78 to } 2.09) \\
\geq 12 \text { months: } 1.10(0.69 \text { to } 1.75) \\
\text { p for trend }=0.36\end{array}$ & $\begin{array}{l}\text { Sub-study } \\
\text { (CARE, BCIS, } \\
\text { LIFE), study site } \\
\text { (Los Angeles, } \\
\text { Detroit), race, } \\
\text { reference age, } \\
\text { education, first- } \\
\text { degree breast } \\
\text { cancer family } \\
\text { history, BMl, } \\
\text { menopausal } \\
\text { status, hormone } \\
\text { therapy use, } \\
\text { lifetime } \\
\text { recreational } \\
\text { physical activity, } \\
\text { alcohol intake, } \\
\text { smoking status, } \\
\text { age at } \\
\text { menarche, } \\
\text { completed } \\
\text { pregnancies, oral } \\
\text { contraceptive } \\
\text { use, age at first } \\
\text { completed } \\
\text { pregnancy }\end{array}$ \\
\hline
\end{tabular}




\begin{tabular}{|c|c|c|c|c|c|}
\hline $\begin{array}{l}\text { Author, Year } \\
\text { Study Design } \\
\text { Risk of Bias }\end{array}$ & $\begin{array}{l}\text { Description of Study (N) } \\
\text { Description of Breast } \\
\text { Cancer Cases (N) }\end{array}$ & $\begin{array}{l}\text { Population } \\
\text { Characteristics }\end{array}$ & $\begin{array}{l}\text { Results: Ever } \\
\text { Breastfed }\end{array}$ & Results: Duration of Breastfeeding & $\begin{array}{l}\text { Confounders } \\
\text { Adjusted for }\end{array}$ \\
\hline $\begin{array}{l}\text { Merritt, 201582 } \\
\text { Cohort } \\
\text { Medium }\end{array}$ & $\begin{array}{l}\text { Cohort study of women } \\
\text { from the European } \\
\text { Investigation into Cancer } \\
\text { and Nutrition (EPIC) } \\
\text { study, recruited from } 23 \\
\text { study centers in } 10 \\
\text { European countries } \\
\text { (Denmark, France, } \\
\text { Germany, Greece, Italy, } \\
\text { the Netherlands, Norway, } \\
\text { Spain, Sweden, United } \\
\text { Kingdom; inclusion criteria } \\
\text { varied slightly between } \\
\text { centers). } \\
\text { (212,041 included in } \\
\text { breast cancer mortality } \\
\text { analysis) } \\
\text { Cases were women with } \\
\text { breast cancer-specific } \\
\text { mortality; vital status was } \\
\text { collected via data linkages } \\
\text { with cancer registries, } \\
\text { boards of health, and } \\
\text { death indices (484) }\end{array}$ & $\begin{array}{l}\frac{\text { Mean age (SD): }}{50 \text { (9.6) }} \\
\text { Postmenopausal: } 46 \% \\
\text { Current smoker: } 20 \% \\
\\
\end{array}$ & $\begin{array}{l}\text { Among parous } \\
\text { women: } \\
\text { HR }(95 \% \mathrm{Cl}) \text { of breast } \\
\text { cancer mortality: } \\
1.01(0.79 \text { to } 1.29)\end{array}$ & $\begin{array}{l}\text { HR }(95 \% \mathrm{Cl}) \text { of breast cancer mortality: } \\
>1 \text { to } \leq 3 \text { months (102 exposed cases, } 41,583 \\
\text { controls): } 0.87 \text { ( } 0.62 \text { to } 1.21) \\
>3 \text { to } \leq 6 \text { months ( }(82 \text { exposed cases, } 43,445 \text { controls): } \\
0.68 \text { ( } 0.48 \text { to } 0.96) \\
>6 \text { to } \leq 12 \text { months }(101 \text { exposed cases, } 49,920 \\
\text { controls): } 0.69 \text { ( } 0.49 \text { to } 0.97) \\
>12 \text { to } \leq 18 \text { months ( } 63 \text { exposed cases, } 24,239 \\
\text { controls): } 0.88 \text { ( } 0.60 \text { to } 1.27) \\
>18 \text { months ( } 74 \text { exposed cases, } 29,149 \text { controls): } 0.94 \\
(0.65 \text { to } 1.37) \\
\text { p for trend }=0.35 \\
\text { BF info only available for first three and last full-term } \\
\text { pregnancies. BF duration calculated as sum of these } \\
\text { pregnancies. For women with > } 4 \text { full lerm pregnancies, } \\
\text { duration calculated as \# of pregnancies } x \text { mean duration of } \\
B F \text { per child. }\end{array}$ & $\begin{array}{l}\text { BMl, physical } \\
\text { activity, smoking, } \\
\text { education level, } \\
\text { menopausal } \\
\text { status }\end{array}$ \\
\hline
\end{tabular}




\begin{tabular}{|c|c|c|c|c|c|}
\hline $\begin{array}{l}\text { Author, Year } \\
\text { Study Design } \\
\text { Risk of Bias }\end{array}$ & $\begin{array}{l}\text { Description of Study (N) } \\
\text { Description of Breast } \\
\text { Cancer Cases (N) }\end{array}$ & $\begin{array}{l}\text { Population } \\
\text { Characteristics }\end{array}$ & $\begin{array}{l}\text { Results: Ever } \\
\text { Breastfed }\end{array}$ & Results: Duration of Breastfeeding & $\begin{array}{l}\text { Confounders } \\
\text { Adjusted for }\end{array}$ \\
\hline $\begin{array}{l}\text { Phillips, } 2009^{83} \\
\text { Case-control } \\
\text { Medium }\end{array}$ & $\begin{array}{l}\text { Population-based case- } \\
\text { control study of } \\
\text { Caucasian and African- } \\
\text { American women ages } \\
\text { 20-74; cases were } \\
\text { enrolled from the North } \\
\text { Carolina Central Cancer } \\
\text { Registry, and controls } \\
\text { from the Department of } \\
\text { Motor Vehicles and } \\
\text { Health Care Finance } \\
\text { Administration and } \\
\text { frequency-matched based } \\
\text { on race and 5-year age } \\
\text { intervals (4,276; } 904 \\
\text { DCIS, 3,372 IBC) } \\
\\
\text { First breast cancer } \\
\text { diagnoses (in situ or } \\
\text { invasive) (2254; } 446 \\
\text { DCIS, 1,808 IBC) }\end{array}$ & $\begin{array}{l}\text { Mean age (SD), range: } \\
\text { DCIS cases: } 55 \text { (11.1), 27- } \\
74 \\
\text { DCIS controls: } 55 \text { (10.3), } \\
22-74 \\
\text { IBC Phase } 1 \text { cases: } 51 \\
\text { (11.8), } 21-74 \\
\text { IBC Phase } 2 \text { cases: } 52 \\
\text { (11.3), } 24-74 \\
\text { IBC controls: } 52 \text { (11.5), 21- } \\
74 \\
\text { Nonwhite: } 39 \% \\
\text { Postmenopausal HRT: }\end{array}$ & $\begin{array}{l}\text { OR }(95 \% \mathrm{Cl}) \\
\frac{\mathrm{DCIS} A l l}{1.02(0.78 \text { to } 1.34)} \\
\frac{\mathrm{DCIS} \text { Comedo }}{0.82(0.57 \text { to } 1.20)} \\
\frac{\text { DCIS Non-comedo }}{1.02(0.72 \text { to } 1.42)} \\
\frac{\mathrm{IBC}}{0.77(0.67 \text { to } 0.89)}\end{array}$ & NR & $\begin{array}{l}\text { Age, race, and } \\
\text { frequency- } \\
\text { matching offset } \\
\text { terms }\end{array}$ \\
\hline
\end{tabular}




\begin{tabular}{|c|c|c|c|c|c|}
\hline $\begin{array}{l}\text { Author, Year } \\
\text { Study Design } \\
\text { Risk of Bias }\end{array}$ & $\begin{array}{l}\text { Description of Study }(\mathrm{N}) \\
\text { Description of Breast } \\
\text { Cancer Cases (N) }\end{array}$ & $\begin{array}{l}\text { Population } \\
\text { Characteristics }\end{array}$ & $\begin{array}{l}\text { Results: Ever } \\
\text { Breastfed }\end{array}$ & Results: Duration of Breastfeeding & $\begin{array}{l}\text { Confounders } \\
\text { Adjusted for }\end{array}$ \\
\hline $\begin{array}{l}\text { Pieta, } 2008^{84} \\
\text { Case-control } \\
\text { Medium }\end{array}$ & $\begin{array}{l}\text { Case-control study of } \\
\text { Polish women ages 35- } \\
70 ; \text { control women had no } \\
\text { changes in mammary } \\
\text { glands revealed by } \\
\text { examination and } \\
\text { mammography and/or } \\
\text { ultrasound imaging (555) } \\
\text { Malignant breast } \\
\text { neoplasms according to } \\
\text { pathological examination } \\
\text { of breast tissue from } \\
\text { biopsy or surgery (79) }\end{array}$ & $\begin{array}{l}\text { Mean age (SD), range: } \\
\text { Malignant cases: } 53(9.0), \\
32-73 \\
\text { Controls: } 48 \text { (7.96), 35-71 }\end{array}$ & NR & $\begin{array}{l}\text { Mean BF duration (months) } \\
\text { Cases with malignant neoplasms: } 8.3 \\
\text { Cases with benign neoplasms: } 6.3 \\
\text { Controls: } 6.8 \\
\text { p=NS } \\
\mathrm{BF} \geq 6 \text { months } \\
\mathrm{OR}, 1.65 \text { ( } 95 \% \mathrm{Cl}, 0.78 \text { to } 3.48 \text { ) } \\
\text { Cases are those with malignant neoplasms; unclear whether } \\
\text { those with benign neoplasms are considered controls in this } \\
\text { analysis }\end{array}$ & NR \\
\hline $\begin{array}{l}\text { Press, } 2010^{85} \\
\text { Case-control } \\
\text { High }\end{array}$ & $\begin{array}{l}\text { Reanalysis of a } 1926 \\
\text { case-control study in the } \\
\text { UK and a } 1931 \text { case- } \\
\text { control study in the U.S., } \\
\text { designed to replicate the } \\
\text { earlier study). In both } \\
\text { studies, cases were } \\
\text { women diagnosed with } \\
\text { breast cancer from area } \\
\text { hospitals and controls } \\
\text { were recruited from the } \\
\text { same hospitals }(2,263) \\
\text { Women diagnosed with } \\
\text { breast cancer }(1,187)\end{array}$ & $\begin{array}{l}\text { Postmenopausal: } \\
\text { UK cases: } 65 \% \\
\text { UK controls: } 65 \% \\
\text { U.S. cases: } 59 \% \\
\text { U.S. controls: } 59 \%\end{array}$ & NR & $\begin{array}{l}\text { UK: OR }(95 \% \mathrm{Cl}) \\
4-11 \text { months: } 1.05 \text { (0.84 to } 1.31) \\
12+\text { months: } 0.49 \text { (0.38 to } 0.64) \\
\text { U.S.: OR ( } 95 \% \mathrm{Cl}) \\
4-11 \text { months: } 0.91 \text { (0.78 to } 1.07) \\
12+\text { months: } 0.81 \text { (0.68 to } 0.96)\end{array}$ & NR \\
\hline
\end{tabular}




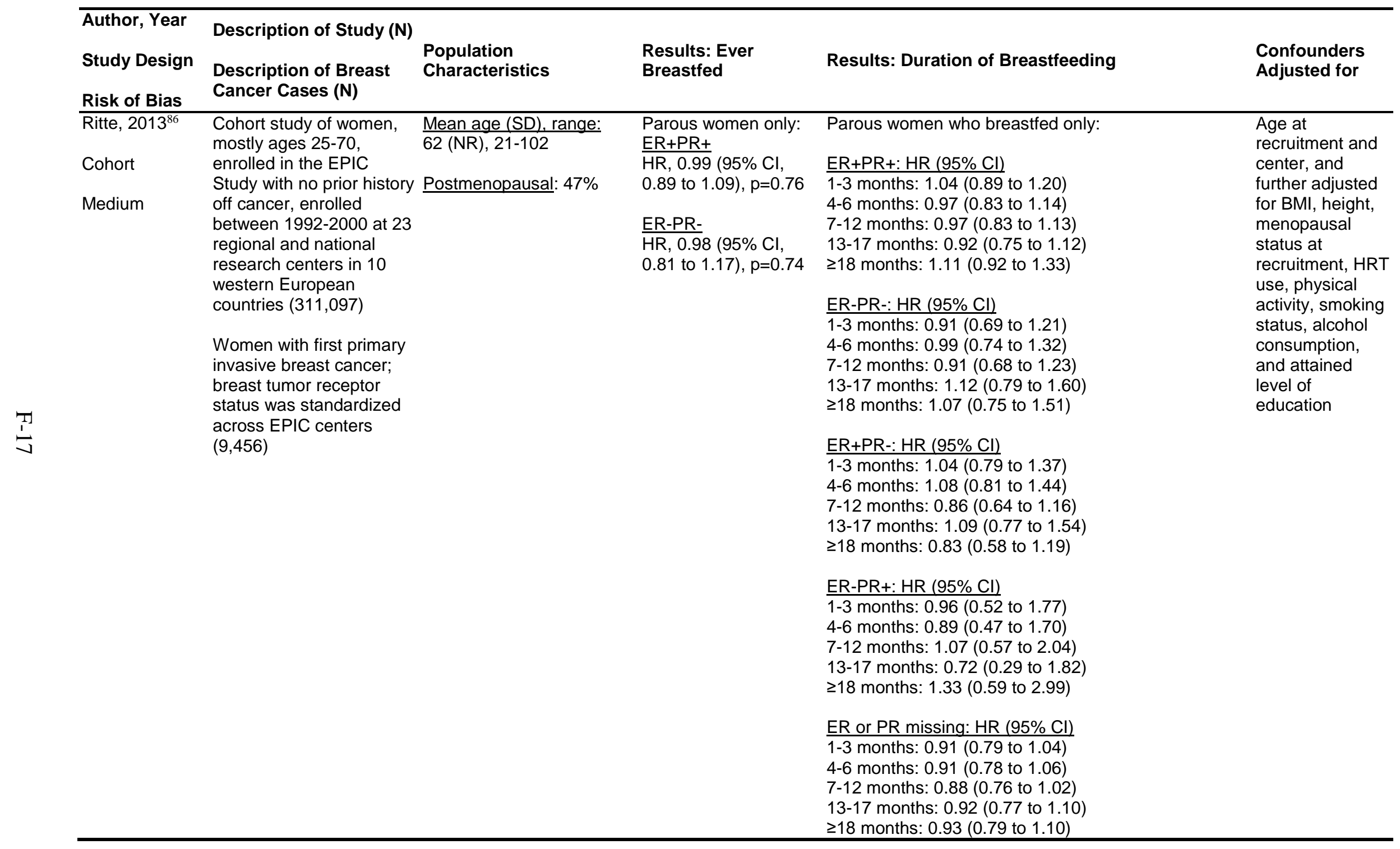




\begin{tabular}{|c|c|c|c|c|c|}
\hline $\begin{array}{l}\text { Author, Year } \\
\text { Study Design } \\
\text { Risk of Bias }\end{array}$ & $\begin{array}{l}\text { Description of Study (N) } \\
\text { Description of Breast } \\
\text { Cancer Cases (N) }\end{array}$ & $\begin{array}{l}\text { Population } \\
\text { Characteristics }\end{array}$ & $\begin{array}{l}\text { Results: Ever } \\
\text { Breastfed }\end{array}$ & Results: Duration of Breastfeeding & $\begin{array}{l}\text { Confounders } \\
\text { Adjusted for }\end{array}$ \\
\hline Ritte, $2013^{86}$ & & & & $\begin{array}{l}\text { ER+: } \mathrm{HR}(95 \% \mathrm{Cl}) \\
1-3 \text { months: } 1.01(0.90 \text { to } 1.14)\end{array}$ & \\
\hline Cohort & & & & $\begin{array}{l}\text { 4-6 months: } 0.89 \text { (0.77 to } 1.04) \\
7-12 \text { months: } 0.98(0.87-1.11)\end{array}$ & \\
\hline $\begin{array}{l}\text { Medium } \\
\text { (continued) }\end{array}$ & & & & $\begin{array}{l}\text { 13-17 months: } 0.91 \text { (0.81-1.03) } \\
\geq 18 \text { months: } 1.01 \text { (0.88-1.17) }\end{array}$ & \\
\hline & & & & $\begin{array}{l}\text { ER-: } \mathrm{HR}(95 \% \mathrm{Cl}) \\
\text { 1-3 months: } 0.88 \text { (0.69 to } 1.11) \\
\text { 4-6 months: } 1.06(0.78 \text { to } 1.42) \\
\text { 7-12 months: } 0.93(0.73 \text { to } 1.19) \\
\text { 13-17 months: } 0.92(0.72 \text { to } 1.18) \\
\geq 18 \text { months: } 1.10 \text { (0.83 to } 1.47)\end{array}$ & \\
\hline & & & & $\begin{array}{l}\text { PR+: } \mathrm{HR}(95 \% \mathrm{Cl}) \\
\text { 1-3 months: } 1.04 \text { (0.90 to } 1.20) \\
\text { 4-6 months: } 0.91(0.75 \text { to } 1.11) \\
\text { 7-12 months: } 0.97(0.83 \text { to } 1.13) \\
\text { 13-17 months: } 0.98(0.84 \text { to } 1.14) \\
\geq 18 \text { months: } 1.12(0.94 \text { to } 1.34)\end{array}$ & \\
\hline & & & & $\begin{array}{l}\text { PR-: } \mathrm{HR}(95 \% \mathrm{Cl}) \\
\text { 1-3 months: } 0.98(0.81 \text { to } 1.20) \\
\text { 4-6 months: } 1.13 \text { (0.88 to } 1.45) \\
\text { 7-12 months: } 1.05 \text { (0.86 to } 1.28) \\
\text { 13-17 months: } 0.90(0.73 \text { to } 1.11) \\
\geq 18 \text { months: } 0.96 \text { (0.75 to } 1.23)\end{array}$ & \\
\hline
\end{tabular}




\begin{tabular}{|c|c|c|c|c|c|}
\hline $\begin{array}{l}\text { Author, Year } \\
\text { Study Design } \\
\text { Risk of Bias }\end{array}$ & $\begin{array}{l}\text { Description of Study (N) } \\
\text { Description of Breast } \\
\text { Cancer Cases (N) }\end{array}$ & $\begin{array}{l}\text { Population } \\
\text { Characteristics }\end{array}$ & $\begin{array}{l}\text { Results: Ever } \\
\text { Breastfed }\end{array}$ & Results: Duration of Breastfeeding & $\begin{array}{l}\text { Confounders } \\
\text { Adjusted for }\end{array}$ \\
\hline $\begin{array}{l}\text { Ruszczyk, } \\
2016^{87} \\
\text { Case-control } \\
\text { Medium }\end{array}$ & $\begin{array}{l}\text { Case-control study of } \\
\text { African American and } \\
\text { white women } 20-75 \text { from } \\
12 \text { targeted NY hospitals } \\
\text { and the NJ State Cancer } \\
\text { Registry through rapid } \\
\text { case ascertainment; } \\
\text { controls were identified } \\
\text { through random digit } \\
\text { dialing and community- } \\
\text { based events, frequency } \\
\text { matched to cases by } \\
\text { telephone prefixes (1,912 } \\
\text { parous) } \\
\text { Primary, newly } \\
\text { diagnosed, histologically } \\
\text { confirmed breast cancer } \\
(642 \text { parous) }\end{array}$ & $\begin{array}{l}\text { Mean age (SD): } \\
\text { Pure IDC cases: } 51 \text { (9.9) } \\
\text { Mixed IDC/DCIS cases: } 51 \\
\text { (10.5) } \\
\text { Controls: } 50 \text { (9.4) } \\
\text { Nonwhite: } 44 \% \\
\frac{\text { Current/former smokers: }}{43 \%} \\
\text { Postmenopausal: } 47 \% \\
\frac{\text { Postmenopausal HRT use }}{\text { (among postmenopausal }} \\
\text { women): } 32 \%\end{array}$ & NR & $\begin{array}{l}\text { Among parous women: } \\
\text { Pure IDC Cases: OR }(95 \% \mathrm{Cl}) \\
0-12 \text { months }(28.0 \% \text { cases exposed, } 31.6 \% \text { controls): } \\
0.76(0.49 \text { to } 1.19) \\
>12 \text { months }(19.6 \% \text { cases exposed, } 25.3 \% \text { controls: } \\
0.61(0.37 \text { to } 1.02) \\
\text { p for trend }=0.07 \\
\text { Mixed IDC/DCIS Cases: OR }(95 \% \mathrm{Cl}) \\
0-12 \text { months }(34.3 \% \text { cases exposed, } 31.6 \% \text { controls): } \\
1.15(0.88 \text { to } 1.50) \\
>12 \text { months }(23.0 \% \text { cases exposed, } 25.3 \% \text { controls): } \\
0.94(0.70 \text { to } 1.27) \\
\text { p for trend }=0.49\end{array}$ & $\begin{array}{l}\text { Age, race, } \\
\text { birthplace, family } \\
\text { history, } \\
\text { composite } \\
\text { screening score, } \\
\text { education, OC } \\
\text { use, age at } \\
\text { menarche, parity } \\
\text { and menopausal } \\
\text { status }\end{array}$ \\
\hline
\end{tabular}




\begin{tabular}{|c|c|c|c|c|c|}
\hline $\begin{array}{l}\text { Author, Year } \\
\text { Study Design } \\
\text { Risk of Bias }\end{array}$ & $\begin{array}{l}\text { Description of Study }(\mathrm{N}) \\
\text { Description of Breast } \\
\text { Cancer Cases }(\mathrm{N})\end{array}$ & $\begin{array}{l}\text { Population } \\
\text { Characteristics }\end{array}$ & $\begin{array}{l}\text { Results: Ever } \\
\text { Breastfed }\end{array}$ & Results: Duration of Breastfeeding & $\begin{array}{l}\text { Confounders } \\
\text { Adjusted for }\end{array}$ \\
\hline $\begin{array}{l}\text { Stendell-Hollis, } \\
2013^{88} \\
\text { Cohort } \\
\text { Medium }\end{array}$ & $\begin{array}{l}\text { Cohort study of healthy } \\
\text { parous women in the } \\
\text { Women's Health Initiative } \\
\text { (WHI) Hormone Trial (HT) } \\
\text { and Observational Study } \\
\text { (OS). OS participants } \\
\text { were included in this } \\
\text { analysis if the woman was } \\
\text { post-hysterectomy at } \\
\text { enrollment and using the } \\
\text { same daily } 0.625 \mathrm{mg} \text { CEE } \\
\text { (conjugated equine } \\
\text { estrogen preparation) as } \\
\text { studied in the clinical trial, } \\
\text { or had an intact uterus } \\
\text { and was using the same } \\
\text { daily CEE/MPA (O.625 } \\
\text { CEE + } 2.5 \text { mg } \\
\text { medroxyprogesterone } \\
\text { acetate) combination as } \\
\text { women in the clinical trial; } \\
\text { if the woman had } \\
\text { previously used } \\
\text { postmenopausal } \\
\text { hormones but was not } \\
\text { currently using these } \\
\text { preparations, or if the } \\
\text { woman had never used } \\
\text { Postmenopausal } \\
\text { hormones (69,358) }\end{array}$ & $\begin{array}{l}\text { Nonwhite: } 15 \% \\
\text { Postmenopausal: } 100 \% \\
\text { Current or prior HRT: } 51 \% \\
\text { Current or former smokers: } \\
49 \% \\
\end{array}$ & $\begin{array}{l}\begin{array}{l}\text { Breastfed for } \geq 1 \\
\text { month: }\end{array} \\
\text { Hormone Trial: } \mathrm{HR} \\
\frac{\text { (95\% Cl) }}{\mathrm{CEE}: 0.72 \text { ( } 0.50 \text { to }} \\
\text { 1.06) } \\
\text { CEE Placebo: } 1.12 \\
\text { (0.80 to 1.57) } \\
\text { CEE/MPA: } 1.06 \text { (0.83 } \\
\text { to } 1.36) \\
\text { CEE/MPA Placebo: } \\
0.92 \text { (0.70 to } 1.21) \\
\text { Observational Study: } \\
\text { HR (95\% CI) } \\
\text { CEE: } 1.11 \text { (0.89 to } \\
\text { 1.39) } \\
\text { CEE/MPA: } 1.16 \text { (0.91 } \\
\text { to } 1.47) \\
\text { No prior HT: } 1.00 \\
\text { (0.86 to } 1.18) \\
\text { Prior HT: } 0.97 \text { ( } 0.78 \text { to } \\
1.22) \\
\text { P-values for trends } \\
\text { were all NS }\end{array}$ & $\begin{array}{l}\text { Cumulative lifetime months } \\
\text { Hormone Trial: HR ( } 95 \% \text { Cl) } \\
\text { CEE } \\
1-3 \text { months: } 0.70 \text { ( } 0.41 \text { to } 1.20) \\
\text { 4-12 months: } 0.78 \text { (0.48 to } 1.26 \\
13-23 \text { months: } 0.72 \text { (0.35 to } 1.46) \\
\geq 24 \text { months: } 0.64 \text { ( } 0.27 \text { to } 1.49) \\
\text { CEE Placebo } \\
1-3 \text { months: } 0.86 \text { ( } 0.52 \text { to } 1.42) \\
\text { 4-12 months: } 1.41 \text { (0.95 to } 2.08) \\
13-23 \text { months: } 1.15 \text { (0.64 to } 2.06) \\
\geq 24 \text { months: } 0.71 \text { ( } 0.30 \text { to } 1.64) \\
\text { CEE/MPA } \\
1-3 \text { months: } 1.13 \text { ( } 0.81 \text { to } 1.59) \\
\text { 4-12 months: } 1.02 \text { (0.75 to } 1.39) \\
13-23 \text { months: } 1.17(0.79 \text { to } 1.72) \\
\geq 24 \text { months: } 0.89 \text { ( } 0.54 \text { to } 1.45) \\
\text { CEE/MPA Placebo } \\
1-3 \text { months: } 0.87 \text { (0.59 to } 1.30) \\
\text { 4-12 months: } 1.00 \text { (0.71 to } 1.40) \\
13-23 \text { months: } 1.00 \text { (0.65 to } 1.54) \\
\geq 24 \text { months: } 0.70 \text { (0.40 to } 1.24)\end{array}$ & $\begin{array}{l}\text { HT: age, } \\
\text { race/ethnicity, } \\
\text { BMI, family } \\
\text { history of breast } \\
\text { cancer, age at } \\
\text { first birth, age at } \\
\text { menarche, and } \\
\text { participation in } \\
\text { WHI extension } \\
\text { study. } \\
\text { Observational } \\
\text { Study: age, } \\
\text { race/ethnicity, } \\
\text { BMI, smoking, } \\
\text { family history of } \\
\text { breast cancer, } \\
\text { number live } \\
\text { births, age at first } \\
\text { birth (except in } \\
\text { models for age } \\
\text { first breastfed), } \\
\text { years since } \\
\text { menopause, } \\
\text { duration of prior } \\
\text { HRT use, and } \\
\text { participation in } \\
\text { WHI extension } \\
\text { study. }\end{array}$ \\
\hline
\end{tabular}




\begin{tabular}{|c|c|c|c|c|c|}
\hline $\begin{array}{l}\text { Author, Year } \\
\text { Study Design } \\
\text { Risk of Bias }\end{array}$ & $\begin{array}{l}\text { Description of Study (N) } \\
\text { Description of Breast } \\
\text { Cancer Cases (N) }\end{array}$ & $\begin{array}{l}\text { Population } \\
\text { Characteristics }\end{array}$ & $\begin{array}{l}\text { Results: Ever } \\
\text { Breastfed }\end{array}$ & Results: Duration of Breastfeeding & $\begin{array}{l}\text { Confounders } \\
\text { Adjusted for }\end{array}$ \\
\hline $\begin{array}{l}\text { Stendell-Hollis, } \\
2013^{88} \\
\text { (continued) }\end{array}$ & $\begin{array}{l}\text { Invasive breast cancer, } \\
\text { verified by medical record } \\
\text { and pathology reports, } \\
\text { centrally reviewed by } \\
\text { study physicians (743) }\end{array}$ & & & $\begin{array}{l}\text { Observational Study: } \mathrm{HR}(95 \% \mathrm{Cl}) \\
\text { CEE } \\
\text { 1-3 months: } 1.08 \text { (0.80 to } 1.46) \\
\text { 4-12 months: } 1.15,(0.87 \text { to } 1.51) \\
\text { 13-23 months: } 1.19 \text { (0.81 to } 1.76) \\
\geq 24 \text { months: } 0.96 \text { (0.55 to } 1.68) \\
\text { CEE/MPA } \\
\text { 1-3 months: } 1.11 \text { (0.79 to } 1.55) \\
4-12 \text { months: } 1.06 \text { (0.79 to } 1.43) \\
13-23 \text { months: } 1.38 \text { (0.97 to } 1.97) \\
\geq 24 \text { months: } 1.32 \text { (0.84 to } 2.06) \\
\text { No Prior HT } \\
1-3 \text { months: } 0.99 \text { (0.79 to } 1.24) \\
4-12 \text { months: } 0.96(0.78 \text { to } 1.18) \\
13-23 \text { months: } 1.01 \text { (0.77 to } 1.32) \\
\geq 24 \text { months: } 1.22 \text { (0.90 to } 1.66) \\
\text { Prior HT } \\
1-3 \text { months: } 0.94 \text { (0.68 to } 1.29) \\
4-12 \text { months: } 0.92 \text { (0.69 to } 1.23) \\
13-23 \text { months: } 1.15 \text { (0.79 to } 1.67) \\
\geq 24 \text { months: } 1.05 \text { (0.64 to } 1.72)\end{array}$ & \\
\hline
\end{tabular}




\begin{tabular}{|c|c|c|c|c|c|}
\hline $\begin{array}{l}\text { Author, Year } \\
\text { Study Design } \\
\text { Risk of Bias }\end{array}$ & $\begin{array}{l}\text { Description of Study (N) } \\
\text { Description of Breast } \\
\text { Cancer Cases (N) }\end{array}$ & $\begin{array}{l}\text { Population } \\
\text { Characteristics }\end{array}$ & $\begin{array}{l}\text { Results: Ever } \\
\text { Breastfed }\end{array}$ & Results: Duration of Breastfeeding & $\begin{array}{l}\text { Confounders } \\
\text { Adjusted for }\end{array}$ \\
\hline $\begin{array}{l}\text { Sugawara, } \\
2013^{89} \\
\text { Cohort } \\
\text { Medium }\end{array}$ & $\begin{array}{l}\text { Analysis of data on } \\
\text { parous women ages } 40- \\
70 \text { years with no history of } \\
\text { cancer who were enrolled } \\
\text { in the Ohsaki National } \\
\text { Health Insurance }(\mathrm{NHI}) \\
\text { Cohort Study in } \\
\text { northeastern Japan } \\
(19,848) \\
\text { Incident breast cancer } \\
\text { cases ascertained from } \\
\text { the Miyagi Prefactural } \\
\text { Cancer Registry }(148)\end{array}$ & $\begin{array}{l}\text { Mean age (SD), range: } \\
\text { Overall: NR (NR), } 40-79 \\
\text { f Breastfeeding only: } 64 \\
\text { (8.4), NR } \\
\text { Mixed feeding: } 56 \text { (9.8), } \\
\text { NR } \\
\text { Formula feeding only: } 55 \\
\text { (9.3), NR } \\
\text { Postmenopausal: } 71 \% \\
\text { Current or prior use HRT: } \\
7 \%\end{array}$ & NR & $\begin{array}{l}\text { Duration NR } \\
\text { Exclusivity } \\
\text { Mixed feeding } \\
\mathrm{HR}=1.12 \text { ( } 95 \% \mathrm{Cl}, 0.92 \text { to } 1.37), \mathrm{p}=0.014 \\
\text { Formula feeding } \\
\mathrm{HR}=1.80 \text { ( } 95 \% \mathrm{Cl}, 1.14 \text { to } 2.86), \mathrm{p}=0.014\end{array}$ & $\begin{array}{l}\text { Age } \\
\text { (continuous), } \\
\text { BMl, family } \\
\text { history of cancer, } \\
\text { education, job } \\
\text { status, smoking } \\
\text { status, alcohol } \\
\text { consumption, } \\
\text { time spent } \\
\text { walking, total } \\
\text { calorie intake, } \\
\text { menopausal } \\
\text { status, age at } \\
\text { menarche, age } \\
\text { at first delivery, } \\
\text { number of } \\
\text { deliveries, } \\
\text { history of oral } \\
\text { contraceptive } \\
\text { drug use, history } \\
\text { of HRT use }\end{array}$ \\
\hline
\end{tabular}




\begin{tabular}{|c|c|c|c|c|c|}
\hline $\begin{array}{l}\text { Author, Year } \\
\text { Study Design } \\
\text { Risk of Bias }\end{array}$ & $\begin{array}{l}\text { Description of Study (N) } \\
\text { Description of Breast } \\
\text { Cancer Cases (N) }\end{array}$ & $\begin{array}{l}\text { Population } \\
\text { Characteristics }\end{array}$ & $\begin{array}{l}\text { Results: Ever } \\
\text { Breastfed }\end{array}$ & Results: Duration of Breastfeeding & $\begin{array}{l}\text { Confounders } \\
\text { Adjusted for }\end{array}$ \\
\hline $\begin{array}{l}\text { Tamimi, 201690 } \\
\text { Cohort } \\
\text { Medium }\end{array}$ & $\begin{array}{l}\text { Female registered nurses } \\
\text { between the ages of } 30 \\
\text { and } 55 \text { years enrolled in } \\
\text { the Nurses' Health Cohort } \\
\text { Study in } 1976 \text { and followed } \\
\text { up between } 1980 \text { and } 2010 \\
\text { through biennial } \\
\text { questionnaires (112,951 } \\
\text { postmenopausal women; } \\
2,424,778 \text { person-years) } \\
\text { Incident invasive breast } \\
\text { cancer identified through } \\
\text { self-report and confirmed } \\
\text { through review of medical } \\
\text { records (8,421 cases: } \\
5,376 \text { ER+ and } 1,270 \text { ER-) }\end{array}$ & $\begin{array}{l}\text { Mean age (SD), range: } \\
48 \text { (6.9), NR } \\
\text { Postmenopausal: 100\% } \\
\text { Current use HRT: 34\% } \\
\end{array}$ & $\begin{array}{l}\text { Among parous } \\
\text { women: } \\
\text { Invasive breast cancer } \\
\text { RR, } 1.05(95 \% \mathrm{Cl}, \\
1.00 \text { to } 1.10), \mathrm{p}=0.07 \text {; } \\
\text { PAR, } 1.6 \%(95 \% \mathrm{Cl} \text {, } \\
0.1 \% \text { to } 3.4 \%) \\
\\
\text { ER+ invasive breast } \\
\text { cancer } \\
\text { RR, } 0.96(95 \% \mathrm{Cl}, \\
0.91 \text { to } 1.02), \mathrm{p}=0.24 ; \\
\text { PAR, } 0(95 \% \mathrm{Cl}, 2.2 \% \\
\text { to } 2.2 \%) \\
\text { ER- invasive breast } \\
\text { Cancer } \\
\text { RR, } 1.07(95 \% \mathrm{Cl}, \\
0.94 \text { to } 1.21), \mathrm{p}=0.30 ; \\
\text { PAR, } 2.4(95 \% \mathrm{Cl}, \\
2.1 \% \text { to } 6.8 \%)\end{array}$ & $\mathrm{NR}$ & $\begin{array}{l}\text { Age in months, } \\
\text { calendar year, } \\
\text { age at } \\
\text { menarche, BMI } \\
\text { at age } 18 \text { years, } \\
\text { height in inches, } \\
\text { parity/age at first } \\
\text { birth, benign } \\
\text { breast disease } \\
\text { history, family } \\
\text { history of breast } \\
\text { cancer, age at } \\
\text { menopause, } \\
\text { weight change } \\
\text { since age 18 } \\
\text { years, } \\
\text { menopausal } \\
\text { hormone use, } \\
\text { alcohol } \\
\text { consumption, } \\
\text { physical activity }\end{array}$ \\
\hline
\end{tabular}




\begin{tabular}{|c|c|c|c|c|c|}
\hline $\begin{array}{l}\text { Author, Year } \\
\text { Study Design } \\
\text { Risk of Bias }\end{array}$ & $\begin{array}{l}\text { Description of Study (N) } \\
\text { Description of Breast } \\
\text { Cancer Cases (N) }\end{array}$ & $\begin{array}{l}\text { Population } \\
\text { Characteristics }\end{array}$ & $\begin{array}{l}\text { Results: Ever } \\
\text { Breastfed }\end{array}$ & Results: Duration of Breastfeeding & $\begin{array}{l}\text { Confounders } \\
\text { Adjusted for }\end{array}$ \\
\hline $\begin{array}{l}\text { Warner, } 2013^{91} \\
\text { Cohort } \\
\text { Medium }\end{array}$ & $\begin{array}{l}\text { Two cohort studies } \\
\text { contributed to a sample of } \\
\text { healthy women followed } \\
\text { to track breast cancer } \\
\text { incidence: 1) Nurse's } \\
\text { Health Study II, enrolling } \\
\text { registered nurses ages } \\
25-42 \text { in } 1989 \text { (followup } \\
\text { for this study began in } \\
\text { 1995, to synchronize with } \\
\text { BWHS cohort); } 2 \text { ) } \\
\text { Black Women's Health } \\
\text { Study (BWHS), enrolling } \\
\text { African-American women } \\
\text { ages 21-69 in 1995, from } \\
\text { communities in all regions } \\
\text { of the U.S.. Women were } \\
\text { excluded if they did not } \\
\text { identify as white or } \\
\text { African-American. } \\
\text { (BWHS: } 35,338 \\
\text { NHS II: 105,576) } \\
\text { Self-reported, invasive } \\
\text { ER+ breast cancer } \\
\text { diagnosis; pathology data } \\
\text { from hospital or cancer } \\
\text { registry records were } \\
\text { centrally reviewed by } \\
\text { study staff to confirm } \\
\text { diagnosis. ER status was } \\
\text { determined by } \\
\text { biochemical or } \\
\text { immunohistochemical } \\
\text { assays (1,506) }\end{array}$ & $\begin{array}{l}\text { Race } \\
\text { Black women: } 27 \% \\
\text { White women: } 73 \% \\
\text { Mean age (SD): } \\
\text { Black women: } 39.0 \text { (5.5) } \\
\text { White women: } 40.2 \text { (4.7) } \\
\text { Postmenopausal: } \\
\text { Black women: 9\% } \\
\text { White women: } 7 \% \\
\text { Current or past } \\
\text { Postmenopausal HRT use: } \\
\text { Black women: } 74 \% \\
\text { White women: } 91 \% \\
\end{array}$ & NR & $\begin{array}{l}\text { Among parous women: } \\
\text { HR (95\% Cl) } \\
<6 \text { months: } 0.85 \text { (0.70 to } 1.03) \\
\geq 6 \text { months: } 0.95 \text { (0.81 to } 1.10)\end{array}$ & $\begin{array}{l}\text { Age, time, age at } \\
\text { first birth, parity, } \\
\text { lactation, age at } \\
\text { menarche, } \\
\text { menopausal } \\
\text { status, age at } \\
\text { menopause, first } \\
\text { degree family } \\
\text { history, BMl at } \\
\text { age } 18, \text { weight } \\
\text { change since } \\
\text { age } 18 \text {, history of } \\
\text { benign breast } \\
\text { disease, alcohol } \\
\text { consumption, } \\
\text { OC use, and } \\
\text { Postmenopausal } \\
\text { hormone use }\end{array}$ \\
\hline
\end{tabular}

${ }^{a}$ Comparison was never breastfed compared with ever breastfed (referent).

${ }^{\mathrm{b}}$ Referent group is nulliparous women.

${ }^{c}$ The BCIS study shared controls from the CARE study. 
AICR = American Institute of Cancer Research; BC = breast cancer; BCIS = Women's Breast Carcinoma in situ; BF = breastfeeding; BMI = body mass index; BRCA = BrCa gene mutations; BWHS = Black Women's Health Study; and; CEE = conjugated equine estrogen; CI = confidence interval; COC = combined oral contraceptive; CSP = Cancer Surveillance Program; DCIS = ductal carcinoma in situ; EPIC = European Prospective Investigation into Cancer and Nutrition; ER = estrogen receptor; HR = hazard ratio; HRT = hormone replacement therapy; HT = hormone trial; IBC = inflammatory breast cancer; IDC = invasive ductal carcinoma; LA = Los Angeles; LIFE CARE = Women's Learning the Influence of Family and Environment Women's Contraceptive and Reproductive Experiences; MPA = medroxyprogesterone acetate; NCI = National Cancer Institute; NHI = National Health Insurance; NHS = National Health Service; NJ = New Jersey; NR = not reported; NS = not statistically significant; NY = New York; OC = oral contraceptive; OR = odds ratio; OS = observational study; PAR = population attributable risk; PR = progesterone receptor; $\mathrm{SD}=$ standard deviation; $\mathrm{SEER}=$ Surveillance, Epidemiology, and End Results; UK = United Kingdom; U.S. =United States; WCRF = World Cancer Research Fund; WHI = Women’s Health Initiative. 
Table F-2. Breastfeeding and breast cancer: Summary of results among subgroups (KQ 2b)

Author, Year

Study Design Description of Breast

Study Design

Cancer Outcome

\section{Risk of Bias}

Ma, $2017^{81}$

Case-contro

Incident in situ and

invasive breast cancer

categorized by ER, PR

and HER2 status

Medium

Triple-negative: ER-, PR-

HER2-

Luminal A-like: ER+ and/or PR+ plus HER2

Subgroup

Characteristics

Results: Ever Breastfed

Results: Duration of Breastfeeding

Mean age (SD), range:

Cases: 47 (8.1),

22-64

Controls: 48 (8.3), 24-

African-American

\section{Race:}

Cases: $26 \%$

Controls: $37 \%$

\section{Younger women $\left(20-44\right.$ years old): OR $(95 \% \mathrm{Cl})^{\mathrm{a}}$} Triple-negative: 0.75 (0.50 to 1.12) Luminal A-like: 0.70 (0.50 to 0.99)

Older women (45-64 years old): OR $(95 \% \mathrm{Cl})^{\mathrm{a}}$ Triple-negative: 0.85 (0.62 to 1.17 ) Luminal A-like: 0.83 (0.67 to 1.03):

White women: OR $(95 \% \mathrm{Cl})^{\mathrm{a}}$

Triple-negative: 0.97 (0.68 to 1.02)

Luminal A-like: 0.81 (0.64 to 1.02)

African-American women: OR $(95 \% \mathrm{Cl})^{\mathrm{a}}$

Triple-negative: 0.67 (0.47 to 0.96)

Luminal A-like: 0.78 (0.57 to 1.06)
Younger women $\left(20-44\right.$ years old): OR $(95 \% \mathrm{Cl})^{\mathrm{a}}$

Triple-negative

<6 months: 0.93 (0.60 to 1.44 )

6-11 months: 0.49 (0.27 to 0.89$)$

$\geq 12$ months: 0.60 (0.35 to 1.01$)$

$\mathrm{p}$ for trend $=0.02$

Luminal A-like

$<6$ months:0.72 (0.49 to 1.05$)$

6-11 months: 0.68 (0.44 to 1.07$)$

$\geq 12$ months: 0.68 (0.44 to 1.05 )

$p$ for trend $=0.12$

Older women $\left(45-64\right.$ years old): OR $(95 \% \mathrm{Cl})^{\mathrm{a}}$

Triple-negative

$<6$ months: 0.97 (0.67 to 1.39 )

6-11 months: 0.62 (0.35 to 1.10$)$

$\geq 12$ months: 0.68 (0.44 to 1.05 )

$p$ for trend $=0.17$

Luminal A-like

<6 months: 0.90 (0.70 to 1.16$)$

6-11 months: 0.84 (0.60 to 1.17)

$\geq 12$ months: 0.71 (0.53 to 0.96 )

$p$ for trend $=0.03$

White women: OR $(95 \% \mathrm{Cl})^{\mathrm{a}}$

Triple-negative

<6 months: 1.14 (0.78 to 1.38 )

6-11 months: 0.62 (0.37 to 1.05)

$\geq 12$ months: 0.88 (0.56 to 1.39 )

$\mathrm{p}$ for trend $=0.27$ 


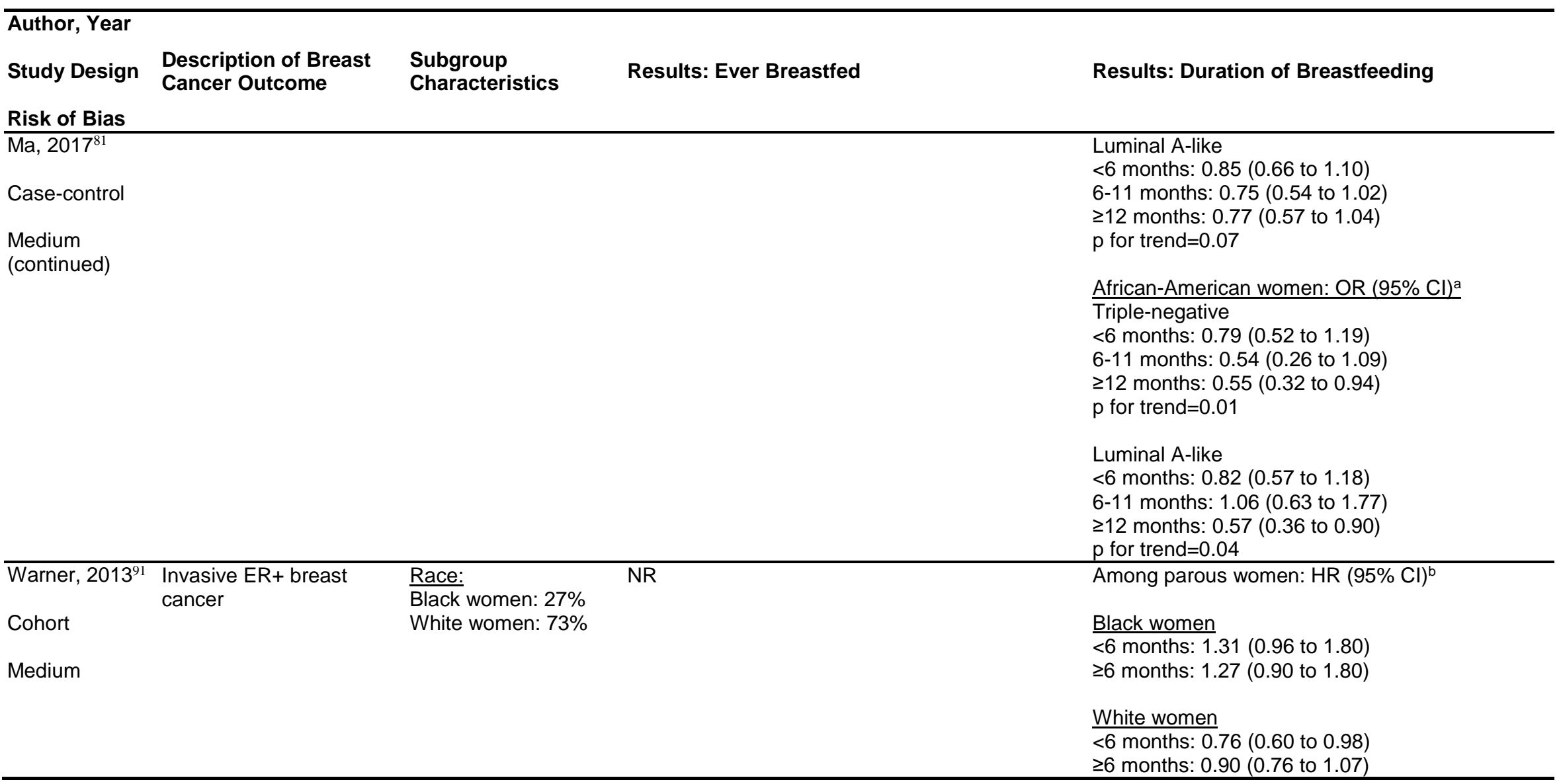

aConfounders adjusted for: sub-study, study site (Los Angeles, Detroit), race, reference age, education, first-degree breast cancer family history, BMI, menopausal status, hormone therapy use, lifetime recreational physical activity, alcohol intake, smoking status, age at menarche, completed pregnancies, oral contraceptive use, age at first completed pregnancy

${ }^{b}$ Confounders adjusted for: age, time, age at first birth, parity, lactation, age at menarche, menopausal status, age at menopause, first degree family history, BMI at age 18, weight change since age 18, history of benign breast disease, alcohol consumption, OC use, and Postmenopausal hormone use

BMI = body mass index; CI = confidence interval; ER = estrogen receptor; HER2 = human epidermal growth factor receptor 2; HR = hazard ratio; KQ = Key Question; NR = not reported; $\mathrm{OR}=$ odds ratio; $\mathrm{PR}=$ progesterone receptor; $\mathrm{SD}=$ standard deviation. 


\section{Appendix G. References for All Appendixes}

1. Ip S; Chung M; Raman G; Chew P; Magula N; DeVine D; Trikalinos T; Lau J. Breastfeeding and maternal and infant health outcomes in developed countries. Evid Rep Technol Assess (Full Rep). 2007 Apr(153):1-186. PMID: 17764214.

2. $\quad$ Chowdhury R; Sinha B; Sankar MJ; Taneja S; Bhandari N; Rollins N; Bahl R; Martines J. Breastfeeding and maternal health outcomes: a systematic review and meta-analysis. Acta Paediatr. 2015 Dec;104(467):96-113. doi: 10.1111/apa.13102. PMID: 26172878.

3. Schwarz EB; Ray RM; Stuebe AM; Allison MA; Ness RB; Freiberg MS; Cauley JA. Duration of lactation and risk factors for maternal cardiovascular disease. Obstet Gynecol. 2009 May;113(5):974-82. doi: 10.1097/01.AOG.0000346884.67796.ca. PMID: 19384111.

4. Perez-Escamilla R; Martinez JL; Segura-Perez S. Impact of the Baby-friendly Hospital Initiative on breastfeeding and child health outcomes: a systematic review. Matern Child Nutr. 2016 Jul;12(3):402-17. doi: 10.1111/mcn.12294. PMID: 26924775.

5. Coutinho SB; de Lira PI; de Carvalho Lima M; Ashworth A. Comparison of the effect of two systems for the promotion of exclusive breastfeeding. Lancet. 2005 Sep 2430;366(9491):1094-100. doi: 10.1016/s0140-6736(05)67421-1. PMID: 16182897.

6. Ekstrom A; Abrahamsson H; Eriksson RM; Martensson BL. Women's use of nipple shields-their influence on breastfeeding duration after a process-oriented education for health professionals. Breastfeed Med. 2014 Nov;9(9):458-66. doi: 10.1089/bfm.2014.0026. PMID: 25188544.

7. Ekstrom A; Kylberg E; Nissen E. A process-oriented breastfeeding training program for healthcare professionals to promote breastfeeding: an intervention study. Breastfeed Med. 2012 Apr;7(2):85-92. doi: 10.1089/bfm.2010.0084. PMID: 22168946.

8. $\quad$ Hayes DK; Prince CB; Espinueva V; Fuddy LJ; Li R; Grummer-Strawn LM. Comparison of manual and electric breast pumps among WIC women returning to work or school in Hawaii. Breastfeed Med. 2008 Mar;3(1):3-10. doi: 10.1089/bfm.2007.0022. PMID: 18333763.

9. Hoddinott P; Britten J; Prescott GJ; Tappin D; Ludbrook A; Godden DJ. Effectiveness of policy to provide breastfeeding groups (BIG) for pregnant and breastfeeding mothers in primary care: cluster randomised controlled trial. BMJ. 2009 Jan 30;338:a3026. doi: 10.1136/bmj.a3026. PMID: 19181729.

10. Kools EJ; Thijs C; Kester AD; van den Brandt PA; de Vries H. A breast-feeding promotion and support program a randomized trial in The Netherlands. Prev Med. 2005 Jan;40(1):60-70. doi: 10.1016/j.ypmed.2004.05.013. PMID: 15530582.

11. Kramer MS; Chalmers B; Hodnett ED; Sevkovskaya Z; Dzikovich I; Shapiro S; Collet JP; Vanilovich I; Mezen I; Ducruet T; Shishko G; Zubovich V; Mknuik D; Gluchanina E; Dombrovskiy V; Ustinovitch A; Kot T; Bogdanovich N; Ovchinikova L; Helsing E. Promotion of Breastfeeding Intervention Trial (PROBIT): a randomized trial in the Republic of Belarus. JAMA. 2001 Jan 24-31;285(4):413-20. PMID: 11242425.

12. Yang S; Platt RW; Dahhou M; Kramer MS. Do population-based interventions widen or narrow socioeconomic inequalities? The case of breastfeeding promotion. Int $\mathrm{J}$ Epidemiol. 2014 Aug;43(4):1284-92. doi: 10.1093/ije/dyu051. PMID: 24639438. 
13. Kronborg H; Vaeth M; Olsen J; Iversen L; Harder I. Effect of early postnatal breastfeeding support: a cluster-randomized community based trial. Acta Paediatr. 2007 Jul;96(7):1064-70. doi: 10.1111/j.1651-2227.2007.00341.x. PMID: 17524018.

14. McLachlan HL; Forster DA; Amir LH; Cullinane M; Shafiei T; Watson LF; Ridgway L; Cramer RL; Small R. Supporting breastfeeding In Local Communities (SILC) in Victoria, Australia: a cluster randomised controlled trial. BMJ Open. 2016 Feb 01;6(2):e008292. doi: 10.1136/bmjopen-2015-008292. PMID: 26832427.

15. Cramer RL; McLachlan HL; Shafiei T; Amir LH; Cullinane M; Small R; Forster DA. Implementation and evaluation of community-based drop-in centres for breastfeeding support in Victoria, Australia. International Breastfeeding Journal. 2017;12(46).

16. Morrow AL; Guerrero ML; Shults J; Calva JJ; Lutter C; Bravo J; Ruiz-Palacios G; Morrow RC; Butterfoss FD. Efficacy of home-based peer counselling to promote exclusive breastfeeding: a randomised controlled trial. Lancet. $1999 \mathrm{Apr}$ 10;353(9160):1226-31. doi: 10.1016/s0140-6736(98)08037-4. PMID: 10217083.

17. Nilsson IMS; Strandberg-Larsen K; Knight CH; Hansen AV; Kronborg H. Focused breastfeeding counselling improves short- and long-term success in an early-discharge setting: A cluster-randomized study. Matern Child Nutr. 2017;13(4):n/a-n/a. doi: 10.1111/mcn.12432. PMID: 125350139. Language: English. Entry Date: 20171006. Revision Date: 20171006. Publication Type: Article. Journal Subset: Allied Health.

18. Reeder JA; Joyce T; Sibley K; Arnold D; Altindag O. Telephone peer counseling of breastfeeding among WIC participants: a randomized controlled trial. Pediatrics. 2014 Sep;134(3):e700-9. doi: 10.1542/peds.2013-4146. PMID: 25092936.

19. Senarath U; Fernando DN; Rodrigo I. Effect of training for care providers on practice of essential newborn care in hospitals in Sri Lanka. J Obstet Gynecol Neonatal Nurs. 2007 Nov-Dec;36(6):531-41. doi: 10.1111/j.1552-6909.2007.00183.x. PMID: 17973696.

20. Taddei JA; Westphal MF; Venancio S; Bogus C; Souza S. Breastfeeding training for health professionals and resultant changes in breastfeeding duration. Sao Paulo Med J. 2000 Nov 09;118(6):185-91. PMID: 11120550.

21. Wan H; Hu S; Thobaben M; Hou Y; Yin T. Continuous primary nursing care increases satisfaction with nursing care and reduces postpartum problems for hospitalized pregnant women. Contemp Nurse. 2011 Feb;37(2):149-59. doi: 10.5172/conu.2011.37.2.149. PMID: 21692586.

22. Washio Y; Humphreys M; Colchado E; Sierra-Ortiz M; Zhang Z; Collins BN; Kilby LM; Chapman DJ; Higgins ST; Kirby KC. Incentive-based intervention to maintain breastfeeding among low-income Puerto Rican mothers. Pediatrics. 2017 Mar;139(3). doi: 10.1542/peds.2016-3119. PMID: 28167511.

23. Abolyan LV. The breastfeeding support and promotion in Baby-Friendly Maternity Hospitals and Not-as-Yet Baby-Friendly Hospitals in Russia. Breastfeed Med. 2006 Summer;1(2):71-8. doi: 10.1089/bfm.2006.1.71. PMID: 17661566.

24. Alvarado R; Zepeda A; Rivero S; Rico N; Lopez S; Diaz S. Integrated maternal and infant health care in the postpartum period in a poor neighborhood in Santiago, Chile. Stud Fam Plann. 1999 Jun;30(2):133-41. PMID: 16617547.

25. Bærug A; Langsrud Ø; Løland BF; Tufte E; Tylleskär T; Fretheim A. Effectiveness of baby-friendly community health services on exclusive breastfeeding and maternal satisfaction: a pragmatic trial. Matern Child Nutr. 2016;12(3):428-39. doi: 10.1111/mcn.12273. PMID: 116415662. 
26. Bartington S; Griffiths LJ; Tate AR; Dezateux C. Are breastfeeding rates higher among mothers delivering in Baby Friendly accredited maternity units in the UK? Int $\mathrm{J}$ Epidemiol. 2006 Oct;35(5):1178-86. doi: 10.1093/ije/dyl155. PMID: 16926214.

27. Bosnjak AP; Batinica M; Hegedus-Jungvirth M; Grguric J; Bozikov J. The effect of baby friendly hospital initiative and postnatal support on breastfeeding rates--Croatian experience. Coll Antropol. 2004 Jun;28(1):235-43. PMID: 15636080.

28. Broadfoot M; Britten J; Tappin DM; MacKenzie JM. The Baby Friendly Hospital Initiative and breast feeding rates in Scotland. Arch Dis Child Fetal Neonatal Ed. 2005 Mar;90(2):F114-6. doi: 10.1136/adc.2003.041558. PMID: 15724033.

29. Edmunds LS; Lee FF; Eldridge JD; Sekhobo JP. Outcome evaluation of the You Can Do It Initiative to Promote Exclusive Breastfeeding Among Women Enrolled in the New York State WIC Program by Race/Ethnicity. J Nutr Educ Behav. 2017;49:S162-S8.e1. doi: 10.1016/j.jneb.2017.05.350. PMID: 123721522.

30. Feldman-Winter L; Barone L; Milcarek B; Hunter K; Meek J; Morton J; Williams T; Naylor A; Lawrence RA. Residency curriculum improves breastfeeding care. Pediatrics. 2010 Aug;126(2):289-97. doi: 10.1542/peds.2009-3250. PMID: 20603262.

31. Gau ML. Evaluation of a lactation intervention program to encourage breastfeeding: a longitudinal study. Int J Nurs Stud. 2004 May;41(4):425-35. doi: 10.1016/j.ijnurstu.2003.11.002. PMID: 15050853.

32. Hannula LS; Kaunonen ME; Puukka PJ. A study to promote breast feeding in the Helsinki Metropolitan area in Finland. Midwifery. 2014 Jun;30(6):696-704. doi: 10.1016/j.midw.2013.10.005. PMID: 24210842.

33. Hawkins SS; Stern AD; Baum CF; Gillman MW. Evaluating the impact of the BabyFriendly Hospital Initiative on breast-feeding rates: a multi-state analysis. Public Health Nutr. 2015 Feb;18(2):189-97. doi: 10.1017/s1368980014000238. PMID: 24625787.

34. Hawkins SS; Stern AD; Baum CF; Gillman MW. Compliance with the Baby-Friendly Hospital Initiative and impact on breastfeeding rates. Arch Dis Child Fetal Neonatal Ed. 2014 Mar;99(2):F138-43. doi: 10.1136/archdischild-2013-304842. PMID: 24277661.

35. Joyce T; Reeder J. Changes in breastfeeding among WIC participants following implementation of the new food package. Matern Child Health J. 2015 Apr;19(4):868-76. doi: 10.1007/s10995-014-1588-7. PMID: 25095768.

36. Lovera D; Sanderson M; Bogle ML; Vela Acosta MS. Evaluation of a breastfeeding peer support program for fathers of Hispanic participants in a Texas special supplemental nutrition program for women, infants, and children. J Am Diet Assoc. 2010 Nov;110(11):1696-702. doi: 10.1016/j.jada.2010.08.001. PMID: 21034883.

37. Madden JM; Soumerai SB; Lieu TA; Mandl KD; Zhang F; Ross-Degnan D. Effects on breastfeeding of changes in maternity length-of-stay policy in a large health maintenance organization. Pediatrics. 2003 Mar;111(3):519-24. PMID: 12612230.

38. Macaluso A; Bettinelli ME; Chapin EM; Cordova do Espirito Santo L; Mascheroni R; Murante AM; Montico M; Cattaneo A. A controlled study on baby-friendly communities in Italy: methods and baseline data. Breastfeed Med. 2013 Apr;8:198-204. doi: 10.1089/bfm.2012.0130. PMID: 23398142.

39. Cattaneo A; Bettinelli M; Chapin E; Macaluso A; Cordova DESL; Murante A; Montico M. Effectiveness of the baby friendly community initiative in Italy: a non-randomised controlled study. BMJ Open. 2016;6(5). doi: 10.1136/bmjopen-2015-010232. PMID: CN-01159959. 
40. Martens PJ. Does breastfeeding education affect nursing staff beliefs, exclusive breastfeeding rates, and Baby-Friendly Hospital Initiative compliance? The experience of a small, rural Canadian hospital. J Hum Lact. 2000 Nov;16(4):309-18. PMID: 11155609.

41. Mydlilova A; Sipek A; Vignerova J. Breastfeeding rates in baby-friendly and non-babyfriendly hospitals in the Czech Republic from 2000 to 2006. J Hum Lact. 2009 Feb;25(1):73-8. doi: 10.1177/0890334408325820. PMID: 19020285.

42. Rempel LA; Moore KC. Peer-led prenatal breast-feeding education: a viable alternative to nurse-led education. Midwifery. 2012 Feb;28(1):73-9. doi: 10.1016/j.midw.2010.11.005. PMID: 21236530.

43. Schafer E; Vogel MK; Viegas S; Hausafus C. Volunteer peer counselors increase breastfeeding duration among rural low-income women. Birth. 1998 Jun;25(2):101-6. PMID: 9668744.

44. Shaw E; Kaczorowski J. The effect of a peer counseling program on breastfeeding initiation and longevity in a low-income rural population. J Hum Lact. 1999 Mar;15(1):19-25. PMID: 10578771.

45. Tarrant M; Wu KM; Fong DY; Lee IL; Wong EM; Sham A; Lam C; Dodgson JE. Impact of baby-friendly hospital practices on breastfeeding in Hong Kong. Birth. 2011 Sep;38(3):238-45. doi: 10.1111/j.1523-536X.2011.00483.x. PMID: 21884232.

46. Venancio SI; Saldiva SR; Escuder MM; Giugliani ER. The Baby-Friendly Hospital Initiative shows positive effects on breastfeeding indicators in Brazil. J Epidemiol Community Health. 2012 Oct;66(10):914-8. doi: 10.1136/jech-2011-200332. PMID: 22080818.

47. Wagner CL; Hulsey TC; Southgate WM; Annibale DJ. Breastfeeding rates at an urban medical university after initiation of an educational program. South Med J. 2002 Aug;95(8):909-13. PMID: 12190230.

48. Weng DR; Hsu CS; Gau ML; Chen CH; Li CY. Analysis of the outcomes at babyfriendly hospitals: appraisal in Taiwan. Kaohsiung J Med Sci. 2003 Jan;19(1):19-28. doi: 10.1016/s1607-551x(09)70443-7. PMID: 12693722.

49. Anothaisintawee T; Wiratkapun C; Lerdsitthichai P; Kasamesup V; Wongwaisayawan S; Srinakarin J; Hirunpat S; Woodtichartpreecha P; Boonlikit S; Teerawattananon Y; Thakkinstian A. Risk factors of breast cancer: a systematic review and meta-analysis. Asia Pac J Public Health. 2013 Sep;25(5):368-87. doi: 10.1177/1010539513488795. PMID: 23709491.

50. Aune D; Norat T; Romundstad P; Vatten LJ. Breastfeeding and the maternal risk of type 2 diabetes: a systematic review and dose-response meta-analysis of cohort studies. Nutr Metab Cardiovasc Dis. 2014 Feb;24(2):107-15. doi: 10.1016/j.numecd.2013.10.028. PMID: 24439841.

51. Luan NN; Wu QJ; Gong TT; Vogtmann E; Wang YL; Lin B. Breastfeeding and ovarian cancer risk: a meta-analysis of epidemiologic studies. Am J Clin Nutr. 2013 Oct;98(4):1020-31. doi: 10.3945/ajcn.113.062794. PMID: 23966430.

52. Dias CC; Figueiredo B. Breastfeeding and depression: a systematic review of the literature. J Affect Disord. 2015 Jan 15;171:142-54. doi: 10.1016/j.jad.2014.09.022. PMID: 25305429.

53. Feng LP; Chen HL; Shen MY. Breastfeeding and the risk of ovarian cancer: a metaanalysis. J Midwifery Womens Health. 2014 Jul-Aug;59(4):428-37. doi:

10.1111/jmwh.12085. PMID: 25066743. 
54. He X; Zhu M; Hu C; Tao X; Li Y; Wang Q; Liu Y. Breast-feeding and postpartum weight retention: a systematic review and meta-analysis. Public Health Nutr. 2015 Dec;18(18):3308-16. doi: 10.1017/s1368980015000828. PMID: 25895506.

55. Islami F; Liu Y; Jemal A; Zhou J; Weiderpass E; Colditz G; Boffetta P; Weiss M. Breastfeeding and breast cancer risk by receptor status--a systematic review and metaanalysis. Ann Oncol. 2015 Dec;26(12):2398-407. doi: 10.1093/annonc/mdv379. PMID: 26504151.

56. Jager S; Jacobs S; Kroger J; Fritsche A; Schienkiewitz A; Rubin D; Boeing H; Schulze MB. Breast-feeding and maternal risk of type 2 diabetes: a prospective study and metaanalysis. Diabetologia. 2014 Jul;57(7):1355-65. doi: 10.1007/s00125-014-3247-3. PMID: 24789344.

57. Jiang X; Duan X; Wang J. A meta-analysis of breastfeeding and osteoporotic fracture risk in the females. Osteoporos Int. 2017;28(2):495-503. doi: 10.1007/s00198-016-3753-X. PMID: 120966311. Language: English. Entry Date: 20170211. Revision Date: 20170211. Publication Type: Article. Journal Subset: Biomedical.

58. $\quad$ Lambertini M; Santoro L; Del Mastro L; Nguyen B; Livraghi L; Ugolini D; Peccatori FA; Azim HA, Jr. Reproductive behaviors and risk of developing breast cancer according to tumor subtype: a systematic review and meta-analysis of epidemiological studies. Cancer Treat Rev. 2016 Sep;49:65-76. doi: 10.1016/j.ctrv.2016.07.006. PMID: 27529149.

59. Iliadis SI; Comasco E; Sylven S; Hellgren C; Sundstrom Poromaa I; Skalkidou A. Prenatal and postpartum evening salivary cortisol levels in association with peripartum depressive symptoms. PLoS One. 2015;10(8):e0135471. doi:

10.1371/journal.pone.0135471. PMID: 26322643.

60. $\quad$ Li DP; Du C; Zhang ZM; Li GX; Yu ZF; Wang X; Li PF; Cheng C; Liu YP; Zhao YS. Breastfeeding and ovarian cancer risk: a systematic review and meta-analysis of 40 epidemiological studies. Asian Pac J Cancer Prev. 2014;15(12):4829-37. PMID: 24998548.

61. Neville CE; McKinley MC; Holmes VA; Spence D; Woodside JV. The relationship between breastfeeding and postpartum weight change--a systematic review and critical evaluation. Int J Obes (Lond). 2014 Apr;38(4):577-90. doi: 10.1038/ijo.2013.132. PMID: 23892523.

62. $\quad$ Pan H; He Z; Ling L; Ding Q; Chen L; Zha X; Zhou W; Liu X; Wang S. Reproductive factors and breast cancer risk among BRCA1 or BRCA2 mutation carriers: results from ten studies. Cancer Epidemiol. 2014 Feb;38(1):1-8. doi: 10.1016/j.canep.2013.11.004. PMID: 24332935.

63. Sung HK; Ma SH; Choi JY; Hwang Y; Ahn C; Kim BG; Kim YM; Kim JW; Kang S; Kim J; Kim TJ; Yoo KY; Kang D; Park S. The Effect of Breastfeeding Duration and Parity on the Risk of Epithelial Ovarian Cancer: A Systematic Review and Meta-analysis. J Prev Med Public Health. 2016 Nov;49(6):349-66. doi: 10.3961/jpmph.16.066. PMID: 27951628.

64. Unar-Munguia M; Torres-Mejia G; Colchero MA; Gonzalez de Cosio T. Breastfeeding Mode and Risk of Breast Cancer: A Dose-Response Meta-Analysis. J Hum Lact. 2017 May;33(2):422-34. doi: 10.1177/0890334416683676. PMID: 28196329. 
65. Zhou Y; Chen J; Li Q; Huang W; Lan H; Jiang H. Association between breastfeeding and breast cancer risk: evidence from a meta-analysis. Breastfeed Med. 2015 Apr;10(3):17582. doi: 10.1089/bfm.2014.0141. PMID: 25785349.

66. Al-Amri FA; Saeedi MY; Al-Tahan FM; Ali AM; Alomary SA; Arafa M; Ibrahim AK; Kassim KA. Breast cancer correlates in a cohort of breast screening program participants in Riyadh, KSA. J Egypt Natl Canc Inst. 2015 Jun;27(2):77-82. doi: 10.1016/j.jnci.2015.04.002. PMID: 25935858.

67. Al-Qutub ST; Al-Raddadi RM; Bin Sadiq BM; Sait W; Al-Gahmi A; Al-Amoudi S. Potential breast cancer risk factors among Saudi women aged 19-50 years in Jeddah: a case-control study. J Egypt Public Health Assoc. 2013 Dec;88(3):165-70. doi: 10.1097/01.EPX.0000435728.60811.bd. PMID: 24374950.

68. Atkinson RL; El-Zein R; Valero V; Lucci A; Bevers TB; Fouad T; Liao W; Ueno NT; Woodward WA; Brewster AM. Epidemiological risk factors associated with inflammatory breast cancer subtypes. Cancer Causes Control. 2016 Mar;27(3):359-66. doi: 10.1007/s10552-015-0712-3. PMID: 26797453.

69. Beaber EF; Holt VL; Malone KE; Porter PL; Daling JR; Li CI. Reproductive factors, age at maximum height, and risk of three histologic types of breast cancer. Cancer Epidemiol Biomarkers Prev. 2008 Dec;17(12):3427-34. doi: 10.1158/1055-9965.epi-08-0641. PMID: 19064558.

70. $\quad$ Castello A; Martin M; Ruiz A; Casas AM; Baena-Canada JM; Lope V; Antolin S; Sanchez P; Ramos M; Anton A; Munoz M; Bermejo B; De Juan-Ferre A; Jara C; Chacon JI; Jimeno MA; Rosado P; Diaz E; Guillem V; Lluch A; Carrasco E; Perez-Gomez B; Vioque J; Pollan M. Lower breast cancer risk among women following the World Cancer Research Fund and American Institute for Cancer Research Lifestyle Recommendations: EpiGEICAM Case-Control Study. PLoS One. 2015;10(5):e0126096. doi: 10.1371/journal.pone.0126096. PMID: 25978407.

71. Dalamaga M; Karmaniolas K; Papadavid E; Pelekanos N; Sotiropoulos G; Lekka A. Elevated serum visfatin/nicotinamide phosphoribosyl-transferase levels are associated with risk of postmenopausal breast cancer independently from adiponectin, leptin, and anthropometric and metabolic parameters. Menopause. 2011 Nov;18(11):1198-204. doi: 10.1097/gme.0b013e31821e21f5. PMID: 21712732.

72. Ge I; Rudolph A; Shivappa N; Flesch-Janys D; Hebert JR; Chang-Claude J. Dietary inflammation potential and postmenopausal breast cancer risk in a German case-control study. Breast. 2015 Aug;24(4):491-6. doi: 10.1016/j.breast.2015.04.012. PMID: 25987487.

73. Hadji P; Gottschalk M; Ziller V; Kalder M; Jackisch C; Wagner U. Bone mass and the risk of breast cancer: the influence of cumulative exposure to oestrogen and reproductive correlates. Results of the Marburg breast cancer and osteoporosis trial (MABOT). Maturitas. 2007 Mar 20;56(3):312-21. doi: 10.1016/j.maturitas.2006.09.005. PMID: 17049767.

74. Holm J; Eriksson L; Ploner A; Eriksson M; Rantalainen M; Li J; Hall P; Czene K. Assessment of Breast Cancer Risk Factors Reveals Subtype Heterogeneity. Cancer Res. 2017 Jul 01;77(13):3708-17. doi: 10.1158/0008-5472.can-16-2574. PMID: 28512241.

75. Kabat GC; Kim MY; Woods NF; Habel LA; Messina CR; Wactawski-Wende J; Stefanick ML; Chlebowski RT; Wassertheil-Smoller S; Rohan TE. Reproductive and menstrual factors and risk of ductal carcinoma in situ of the breast in a cohort of 
postmenopausal women. Cancer Causes Control. 2011 Oct;22(10):1415-24. doi: 10.1007/s10552-011-9814-8. PMID: 21750889.

76. $\quad$ Kotsopoulos J; Lubinski J; Lynch HT; Kim-Sing C; Neuhausen S; Demsky R; Foulkes WD; Ghadirian P; Tung N; Ainsworth P; Senter L; Karlan B; Eisen A; Eng C; Weitzel J; Gilchrist DM; Blum JL; Zakalik D; Singer C; Fallen T; Ginsburg O; Huzarski T; Sun P; Narod SA. Oophorectomy after menopause and the risk of breast cancer in BRCA1 and BRCA2 mutation carriers. Cancer Epidemiol Biomarkers Prev. 2012 Jul;21(7):1089-96. doi: 10.1158/1055-9965.epi-12-0201. PMID: 22564871.

77. Kruk J. Association between vegetable, fruit and carbohydrate intake and breast cancer risk in relation to physical activity. Asian Pac J Cancer Prev. 2014;15(11):4429-36. PMID: 24969864.

78. $\quad$ Lee E; Ma H; McKean-Cowdin R; Van Den Berg D; Bernstein L; Henderson BE; Ursin G. Effect of reproductive factors and oral contraceptives on breast cancer risk in BRCA1/2 mutation carriers and noncarriers: results from a population-based study. Cancer Epidemiol Biomarkers Prev. 2008 Nov;17(11):3170-8. doi: 10.1158/10559965.epi-08-0396. PMID: 18990759.

79. Lumachi F; Frigo AC; Basso U; Tombolan V; Ermani M. Estrogen therapy and risk of breast cancer in postmenopausal women: a case-control study and results of a multivariate analysis. Menopause. 2010 May-Jun;17(3):524-8. doi:

10.1097/gme.0b013e3181ca0c74. PMID: 20130492.

80. Ma H; Bernstein L; Ross RK; Ursin G. Hormone-related risk factors for breast cancer in women under age 50 years by estrogen and progesterone receptor status: results from a case-control and a case-case comparison. Breast Cancer Res. 2006;8(4):R39. doi: 10.1186/bcr1514. PMID: 16846528.

81. Ma H; Ursin G; Xu X; Lee E; Togawa K; Duan L; Lu Y; Malone KE; Marchbanks PA; McDonald JA; Simon MS; Folger SG; Sullivan-Halley J; Deapen DM; Press MF; Bernstein L. Reproductive factors and the risk of triple-negative breast cancer in white women and African-American women: a pooled analysis. Breast Cancer Res. 2017 Jan 13;19(1):6. doi: 10.1186/s13058-016-0799-9. PMID: 28086982.

82. Merritt MA; Riboli E; Murphy N; Kadi M; Tjonneland A; Olsen A; Overvad K; Dossus L; Dartois L; Clavel-Chapelon F; Fortner RT; Katzke VA; Boeing H; Trichopoulou A; Lagiou P; Trichopoulos D; Palli D; Sieri S; Tumino R; Sacerdote C; Panico S; Bueno-deMesquita HB; Peeters PH; Lund E; Nakamura A; Weiderpass E; Quiros JR; Agudo A; Molina-Montes E; Larranaga N; Dorronsoro M; Cirera L; Barricarte A; Olsson A; Butt S; Idahl A; Lundin E; Wareham NJ; Key TJ; Brennan P; Ferrari P; Wark PA; Norat T; Cross AJ; Gunter MJ. Reproductive factors and risk of mortality in the European Prospective Investigation into Cancer and Nutrition; a cohort study. BMC Med. 2015 Oct 30;13:252. doi: 10.1186/s12916-015-0484-3. PMID: 26515238.

83. Phillips LS; Millikan RC; Schroeder JC; Barnholtz-Sloan JS; Levine BJ. Reproductive and hormonal risk factors for ductal carcinoma in situ of the breast. Cancer Epidemiol Biomarkers Prev. 2009 May;18(5):1507-14. doi: 10.1158/1055-9965.epi-08-0967. PMID: 19423528.

84. $\quad$ Pieta B; Opala T; Wilczak M; Grodecka-Gazdecka S; Kramer L; Samulak D; Wieznowska-Maczynska K. Past obstetric history and risk of malignant breast neoplasms. Eur J Gynaecol Oncol. 2008;29(4):374-7. PMID: 18714573. 
85. Press DJ; Pharoah P. Risk factors for breast cancer: a reanalysis of two case-control studies from 1926 and 1931. Epidemiology. 2010 Jul;21(4):566-72. doi:

10.1097/EDE.0b013e3181e08eb3. PMID: 20498604.

86. Ritte R; Tikk K; Lukanova A; Tjonneland A; Olsen A; Overvad K; Dossus L; Fournier A; Clavel-Chapelon F; Grote V; Boeing H; Aleksandrova K; Trichopoulou A; Lagiou P; Trichopoulos D; Palli D; Berrino F; Mattiello A; Tumino R; Sacerdote C; Quiros JR; Buckland G; Molina-Montes E; Chirlaque MD; Ardanaz E; Amiano P; Bueno-deMesquita HB; van Gils CH; Peeters PH; Wareham N; Khaw KT; Key TJ; Travis RC; Weiderpass E; Dumeaux V; Lund E; Sund M; Andersson A; Romieu I; Rinaldi S; Vineis P; Merritt MA; Riboli E; Kaaks R. Reproductive factors and risk of hormone receptor positive and negative breast cancer: a cohort study. BMC Cancer. 2013 Dec 09;13:584. doi: 10.1186/1471-2407-13-584. PMID: 24321460.

87. $\quad$ Ruszczyk M; Zirpoli G; Kumar S; Bandera EV; Bovbjerg DH; Jandorf L; Khoury T; Hwang H; Ciupak G; Pawlish K; Schedin P; Masso-Welch P; Ambrosone CB; Hong CC. Breast cancer risk factor associations differ for pure versus invasive carcinoma with an in situ component in case-control and case-case analyses. Cancer Causes Control. 2016 Feb;27(2):183-98. doi: 10.1007/s10552-015-0696-z. PMID: 26621543.

88. Stendell-Hollis NR; Thompson PA; Thomson CA; O'Sullivan MJ; Ray RM; Chlebowski RT. Investigating the association of lactation history and postmenopausal breast cancer risk in the Women's Health Initiative. Nutr Cancer. 2013;65(7):969-81. doi: 10.1080/01635581.2013.815787. PMID: 24127779.

89. Sugawara Y; Kakizaki M; Nagai M; Tomata Y; Hoshi R; Watanabe I; Nishino Y; Kuriyama S; Tsuji I. Lactation pattern and the risk for hormone-related female cancer in Japan: the Ohsaki Cohort Study. Eur J Cancer Prev. 2013 Mar;22(2):187-92. doi: 10.1097/CEJ.0b013e3283564610. PMID: 23358107.

90. Tamimi RM; Spiegelman D; Smith-Warner SA; Wang M; Pazaris M; Willett WC; Eliassen AH; Hunter DJ. Population Attributable Risk of Modifiable and Nonmodifiable Breast Cancer Risk Factors in Postmenopausal Breast Cancer. Am J Epidemiol. 2016 Dec 15;184(12):884-93. doi: 10.1093/aje/kww145. PMID: 27923781.

91. Warner ET; Tamimi RM; Boggs DA; Rosner B; Rosenberg L; Colditz GA; Palmer JR. Estrogen receptor positive tumors: do reproductive factors explain differences in incidence between black and white women? Cancer Causes Control. 2013 Apr;24(4):7319. doi: 10.1007/s10552-013-0153-9. PMID: 23380944.

92. Cook LS; Pestak CR; Leung AC; Steed H; Nation J; Swenerton K; Gallagher R; Magliocco A; Kobel M; Brooks-Wilson A; Le N. Combined oral contraceptive use before the first birth and epithelial ovarian cancer risk. Br J Cancer. 2017 Jan 17;116(2):265-9. doi: 10.1038/bjc.2016.400. PMID: 27959890.

93. Gay GM; Lim JS; Chay WY; Chow KY; Tan MH; Lim WY. Reproductive factors, adiposity, breastfeeding and their associations with ovarian cancer in an Asian cohort. Cancer Causes Control. 2015 Nov;26(11):1561-73. doi: 10.1007/s10552-015-0649-6. PMID: 26342607.

94. Gierach GL; Modugno F; Ness RB. Gender of offspring and maternal ovarian cancer risk. Gynecol Oncol. 2006 Jun;101(3):476-80. doi: 10.1016/j.ygyno.2005.11.008. PMID: 16364411.

95. Jordan SJ; Green AC; Whiteman DC; Moore SP; Bain CJ; Gertig DM; Webb PM. Serous ovarian, fallopian tube and primary peritoneal cancers: a comparative epidemiological 
analysis. Int J Cancer. 2008 Apr 01;122(7):1598-603. doi: 10.1002/ijc.23287. PMID: 18058817.

96. Kotsopoulos J; Lubinski J; Gronwald J; Cybulski C; Demsky R; Neuhausen SL; KimSing C; Tung N; Friedman S; Senter L; Weitzel J; Karlan B; Moller P; Sun P; Narod SA. Factors influencing ovulation and the risk of ovarian cancer in BRCA1 and BRCA2 mutation carriers. Int J Cancer. 2015 Sep 01;137(5):1136-46. doi: 10.1002/ijc.29386. PMID: 25482078.

97. Nagle CM; Olsen CM; Webb PM; Jordan SJ; Whiteman DC; Green AC. Endometrioid and clear cell ovarian cancers: a comparative analysis of risk factors. Eur J Cancer. 2008 Nov;44(16):2477-84. doi: 10.1016/j.ejca.2008.07.009. PMID: 18707869.

98. Choi SR; Kim YM; Cho MS; Kim SH; Shim YS. Association between duration of breast feeding and metabolic syndrome: The Korean National Health and Nutrition Examination Surveys. J Womens Health (Larchmt). 2017 Apr;26(4):361-7. doi: 10.1089/jwh.2016.6036. PMID: 28072915.

99. Lee SY; Kim MT; Jee SH; Yang HP. Does long-term lactation protect premenopausal women against hypertension risk? A Korean women's cohort study. Prev Med. 2005 Aug;41(2):433-8. doi: 10.1016/j.ypmed.2004.11.025. PMID: 15917038.

100. Lupton SJ; Chiu CL; Lujic S; Hennessy A; Lind JM. Association between parity and breastfeeding with maternal high blood pressure. Am J Obstet Gynecol. 2013 Jun;208(6):454.e1-7. doi: 10.1016/j.ajog.2013.02.014. PMID: 23395924.

101. Natland Fagerhaug T; Forsmo S; Jacobsen GW; Midthjell K; Andersen LF; Ivar Lund Nilsen T. A prospective population-based cohort study of lactation and cardiovascular disease mortality: the HUNT study. BMC Public Health. 2013 Nov 13;13:1070. doi: 10.1186/1471-2458-13-1070. PMID: 24219620.

102. Parikh NI; Jeppson RP; Berger JS; Eaton CB; Kroenke CH; LeBlanc ES; Lewis CE; Loucks EB; Parker DR; Rillamas-Sun E; Ryckman KK; Waring ME; Schenken RS; Johnson KC; Edstedt-Bonamy AK; Allison MA; Howard BV. Reproductive risk factors and coronary heart disease in the Women's Health Initiative Observational Study. Circulation. 2016 May 31;133(22):2149-58. doi: 10.1161/circulationaha.115.017854. PMID: 27143682.

103. Stuebe AM; Michels KB; Willett WC; Manson JE; Rexrode K; Rich-Edwards JW. Duration of lactation and incidence of myocardial infarction in middle to late adulthood. Am J Obstet Gynecol. 2009 Feb;200(2):138.e1-8. doi: 10.1016/j.ajog.2008.10.001. PMID: 19110223.

104. Stuebe AM; Schwarz EB; Grewen K; Rich-Edwards JW; Michels KB; Foster EM; Curhan G; Forman J. Duration of lactation and incidence of maternal hypertension: a longitudinal cohort study. Am J Epidemiol. 2011 Nov 15;174(10):1147-58. doi: 10.1093/aje/kwr227. PMID: 21997568.

105. Chamberlain CR; Oldenburg B; Wilson AN; Eades SJ; O'Dea K; Oats JJ; Wolfe R. Type 2 diabetes after gestational diabetes: greater than fourfold risk among Indigenous compared with non-Indigenous Australian women. Diabetes Metab Res Rev. 2016 Feb;32(2):217-27. doi: 10.1002/dmrr.2715. PMID: 26385131.

106. Gunderson EP; Hedderson MM; Chiang V; Crites Y; Walton D; Azevedo RA; Fox G; Elmasian C; Young S; Salvador N; Lum M; Quesenberry CP; Lo JC; Sternfeld B; Ferrara A; Selby JV. Lactation intensity and postpartum maternal glucose tolerance and insulin 
resistance in women with recent GDM: the SWIFT cohort. Diabetes Care. 2012 Jan;35(1):50-6. doi: 10.2337/dc11-1409. PMID: 22011407.

107. Gunderson EP; Hurston SR; Ning X; Lo JC; Crites Y; Walton D; Dewey KG; Azevedo RA; Young S; Fox G; Elmasian CC; Salvador N; Lum M; Sternfeld B; Quesenberry CP, Jr. Lactation and progression to Type 2 Diabetes Mellitus after Gestational Diabetes Mellitus: a prospective cohort study. Ann Intern Med. 2015 Dec 15;163(12):889-98. doi: 10.7326/m15-0807. PMID: 26595611.

108. Martens PJ; Shafer LA; Dean HJ; Sellers EA; Yamamoto J; Ludwig S; Heaman M; Phillips-Beck W; Prior HJ; Morris M; McGavock J; Dart AB; Shen GX. Breastfeeding initiation associated with reduced incidence of diabetes in mothers and offspring. Obstet Gynecol. 2016 Nov;128(5):1095-104. doi: 10.1097/aog.0000000000001689. PMID: 27741196.

109. Zong G; Grandjean P; Wang X; Sun Q. Lactation history, serum concentrations of persistent organic pollutants, and maternal risk of diabetes. Environ Res. 2016 Oct;150:282-8. doi: 10.1016/j.envres.2016.06.023. PMID: 27336232.

110. Crandall CJ; Liu J; Cauley J; Newcomb PA; Manson JE; Vitolins MZ; Jacobson LT; Rykman KK; Stefanick ML. Associations of parity, breastfeeding, and fractures in the Women's Health Observational Study. Obstet Gynecol. 2017 Jul;130(1):171-80. doi: 10.1097/aog.0000000000002096. PMID: 28594759.

111. Hwang IR; Choi YK; Lee WK; Kim JG; Lee IK; Kim SW; Park KG. Association between prolonged breastfeeding and bone mineral density and osteoporosis in postmenopausal women: KNHANES 2010-2011. Osteoporos Int. 2016 Jan;27(1):257-65. doi: 10.1007/s00198-015-3292-x. PMID: 26373982.

112. Lambrinoudaki I; Flokatoula M; Armeni E; Pliatsika P; Augoulea A; Antoniou A; Alexandrou A; Creatsa M; Panoulis C; Dendrinos S; Papacharalambous X. Vertebral fracture prevalence among Greek healthy middle-aged postmenopausal women: association with demographics, anthropometric parameters, and bone mineral density. Spine J. 2015 Jan 01;15(1):86-94. doi: 10.1016/j.spinee.2014.07.021. PMID: 25106754.

113. Mori T; Ishii S; Greendale GA; Cauley JA; Ruppert K; Crandall CJ; Karlamangla AS. Parity, lactation, bone strength, and 16-year fracture risk in adult women: findings from the Study of Women's Health Across the Nation (SWAN). Bone. 2015 Apr;73:160-6. doi: 10.1016/j.bone.2014.12.013. PMID: 25528102.

114. Naves M; Diaz-Lopez JB; Gomez C; Rodriguez-Rebollar A; Cannata-Andia JB. Determinants of incidence of osteoporotic fractures in the female Spanish population older than 50. Osteoporos Int. 2005 Dec;16(12):2013-7. doi: 10.1007/s00198-005-19834. PMID: 16091836.

115. Ahn S; Corwin EJ. The association between breastfeeding, the stress response, inflammation, and postpartum depression during the postpartum period: prospective cohort study. Int J Nurs Stud. 2015 Oct;52(10):1582-90. doi: 10.1016/j.ijnurstu.2015.05.017. PMID: 26143358.

116. Borra C; Iacovou M; Sevilla A. New evidence on breastfeeding and postpartum depression: the importance of understanding women's intentions. Matern Child Health J. 2015 Apr;19(4):897-907. doi: 10.1007/s10995-014-1591-z. PMID: 25138629.

117. Chojenta CL; Lucke JC; Forder PM; Loxton DJ. Maternal health factors as risks for postnatal depression: a prospective longitudinal study. PLoS One. 2016;11(1):e0147246. doi: 10.1371/journal.pone.0147246. PMID: 26785131. 
118. Davey HL; Tough SC; Adair CE; Benzies KM. Risk factors for sub-clinical and major postpartum depression among a community cohort of Canadian women. Matern Child Health J. 2011 Oct;15(7):866-75. doi: 10.1007/s10995-008-0314-8. PMID: 18256913.

119. Fiala A; Svancara J; Klanova J; Kasparek T. Sociodemographic and delivery risk factors for developing postpartum depression in a sample of 3233 mothers from the Czech ELSPAC study. BMC Psychiatry. 2017 Mar 21;17(1):104. doi: 10.1186/s12888-0171261-y. PMID: 28327118.

120. Hamdan A; Tamim H. The relationship between postpartum depression and breastfeeding. Int J Psychiatry Med. 2012;43(3):243-59. PMID: 22978082.

121. Lau Y; Chan KS. Perinatal depressive symptoms, sociodemographic correlates, and breast-feeding among Chinese women. J Perinat Neonatal Nurs. 2009 OctDec;23(4):335-45. doi: 10.1097/JPN.0b013e3181bbbea9. PMID: 19915417.

122. Maimburg RD; Vaeth M. Postpartum depression among first-time mothers - results from a parallel randomised trial. Sex Reprod Healthc. 2015 Jun;6(2):95-100. doi: 10.1016/j.srhc.2015.01.003. PMID: 25998877.

123. McCoy SJ; Beal JM; Saunders B; Hill EN; Payton ME; Watson GH. Risk factors for postpartum depression: a retrospective investigation. J Reprod Med. 2008 Mar;53(3):16670. PMID: 18441719.

124. Reifsnider E; Flowers J; Todd M; Bever Babendure J; Moramarco M. The relationship among breastfeeding, postpartum depression, and postpartum weight in Mexican American Women. J Obstet Gynecol Neonatal Nurs. 2016;45(6):760-71. doi: 10.1016/j.jogn.2016.05.009. PMID: 119339849.

125. Sword W; Landy CK; Thabane L; Watt S; Krueger P; Farine D; Foster G. Is mode of delivery associated with postpartum depression at 6 weeks: a prospective cohort study. BJOG. 2011 Jul;118(8):966-77. doi: 10.1111/j.1471-0528.2011.02950.x. PMID: 21489126.

126. Trivino-Juarez JM; Nieto-Pereda B; Romero-Ayuso D; Arruti-Sevilla B; Aviles-Gamez B; Forjaz MJ; Oliver-Barrecheguren C; Mellizo-Diaz S; Soto-Lucia C; Pla-Mestre R. Quality of life of mothers at the sixth week and sixth month post partum and type of infant feeding. Midwifery. 2016 Mar;34:230-8. doi: 10.1016/j.midw.2015.11.003. PMID: 26621376.

127. Endres LK; Straub H; McKinney C; Plunkett B; Minkovitz CS; Schetter CD; Ramey S; Wang C; Hobel C; Raju T; Shalowitz MU. Postpartum weight retention risk factors and relationship to obesity at 1 year. Obstet Gynecol. 2015 Jan;125(1):144-52. doi: 10.1097/aog.0000000000000565. PMID: 25560116.

128. Jarlenski MP; Bennett WL; Bleich SN; Barry CL; Stuart EA. Effects of breastfeeding on postpartum weight loss among U.S. women. Prev Med. 2014 Dec;69:146-50. doi: 10.1016/j.ypmed.2014.09.018. PMID: 25284261.

129. Sharma AJ; Dee DL; Harden SM. Adherence to breastfeeding guidelines and maternal weight 6 years after delivery. Pediatrics. 2014 Sep;134 Suppl 1:S42-9. doi: 10.1542/peds.2014-0646H. PMID: 25183755.

130. Lyu LC; Lo CC; Chen HF; Wang CY; Liu DM. A prospective study of dietary intakes and influential factors from pregnancy to postpartum on maternal weight retention in Taipei, Taiwan. Br J Nutr. 2009 Dec;102(12):1828-37. doi: 10.1017/s0007114509991243. PMID: 19678967. 
131. Ng SK; Cameron CM; Hills AP; McClure RJ; Scuffham PA. Socioeconomic disparities in prepregnancy BMI and impact on maternal and neonatal outcomes and postpartum weight retention: the EFHL longitudinal birth cohort study. BMC Pregnancy Childbirth. 2014 Sep 08;14:314. doi: 10.1186/1471-2393-14-314. PMID: 25201481.

132. Onyango AW; Nommsen-Rivers L; Siyam A; Borghi E; de Onis M; Garza C; Lartey A; Baerug A; Bhandari N; Dewey KG; Araujo CL; Mohamed AJ; Van den Broeck J. Postpartum weight change patterns in the WHO Multicentre Growth Reference Study. Matern Child Nutr. 2011 Jul;7(3):228-40. doi: 10.1111/j.1740-8709.2010.00295.x. PMID: 21338469.

133. Ostbye T; Krause KM; Swamy GK; Lovelady CA. Effect of breastfeeding on weight retention from one pregnancy to the next: results from the North Carolina WIC program. Prev Med. 2010 Nov;51(5):368-72. doi: 10.1016/j.ypmed.2010.07.017. PMID: 20655944.

134. Palmer JR; Kipping-Ruane K; Wise LA; Yu J; Rosenberg L. Lactation in relation to long-term maternal weight gain in African-American Women. Am J Epidemiol. 2015 Jun 15;181(12):932-9. doi: 10.1093/aje/kwv027. PMID: 25944887.

135. Straub H; Simon C; Plunkett B; Endres L; Adam E; McKinney C; Hobel C; Thorp J; Raju T; Shalowitz M. Evidence for a complex relationship among weight retention, cortisol and breastfeeding in postpartum women. Matern Child Health J. 2016;20(7):1375-83. doi: 10.1007/s10995-016-1934-z. PMID: 116170909.

136. Stuebe AM; Kleinman K; Gillman MW; Rifas-Shiman SL; Gunderson EP; Rich-Edwards J. Duration of lactation and maternal metabolism at 3 years postpartum. J Womens Health (Larchmt). 2010 May;19(5):941-50. doi: 10.1089/jwh.2009.1660. PMID: 20459331.

137. John EM; Whittemore AS; Harris R; Itnyre J. Characteristics relating to ovarian cancer risk: collaborative analysis of seven U.S. case-control studies. Epithelial ovarian cancer in black women. Collaborative Ovarian Cancer Group. J Natl Cancer Inst. 1993 Jan 20;85(2):142-7. PMID: 8418303.

138. Risch HA; Marrett LD; Howe GR. Parity, contraception, infertility, and the risk of epithelial ovarian cancer. Am J Epidemiol. 1994 Oct 01;140(7):585-97. PMID: 7942759.

139. West RO. Epidemiologic study of malignancies of the ovaries. Cancer. 1966 Jul;19(7):1001-7. PMID: 5939299.

140. Wynder EL; Dodo H; Barber HR. Epidemiology of cancer of the ovary. Cancer. 1969 Feb;23(2):352-70. PMID: 5764976.

141. Aboud FE; Moore AC; Akhter S. Effectiveness of a community-based responsive feeding programme in rural Bangladesh: a cluster randomized field trial. Matern Child Nutr. 2008 Oct;4(4):275-86. doi: 10.1111/j.1740-8709.2008.00146.x. PMID: 18811792.

142. Alderman BW; Weiss NS; Daling JR; Ure CL; Ballard JH. Reproductive history and postmenopausal risk of hip and forearm fracture. Am J Epidemiol. 1986 Aug;124(2):2627. PMID: 3728442.

143. Chan HH; Lau EM; Woo J; Lin F; Sham A; Leung PC. Dietary calcium intake, physical activity and the risk of vertebral fracture in Chinese. Osteoporos Int. 1996;6(3):228-32. PMID: 8783297.

144. Hoffman S; Grisso JA; Kelsey JL; Gammon MD; O'Brien LA. Parity, lactation and hip fracture. Osteoporos Int. 1993 Jul;3(4):171-6. PMID: 8338971. 
145. Cumming RG; Klineberg RJ. Breastfeeding and other reproductive factors and the risk of hip fractures in elderly women. Int J Epidemiol. 1993 Aug;22(4):684-91. PMID: 8225744.

146. Kreiger N; Kelsey JL; Holford TR; O'Connor T. An epidemiologic study of hip fracture in postmenopausal women. Am J Epidemiol. 1982 Jul;116(1):141-8. PMID: 7102649.

147. Michaelsson K; Baron JA; Farahmand BY; Ljunghall S. Influence of parity and lactation on hip fracture risk. Am J Epidemiol. 2001 Jun 15;153(12):1166-72. PMID: 11415951.

148. Banks E; Killpack S; Furman L. Low-income inner-city fathers and breastfeeding-where's the program for us? Breastfeed Med. 2013 Dec;8(6):507-8. doi: 10.1089/bfm.2012.0147. PMID: 23560474.

149. Warner R; Appleby L; Whitton A; Faragher B. Demographic and obstetric risk factors for postnatal psychiatric morbidity. Br J Psychiatry. 1996 May;168(5):607-11. PMID: 8733800.

150. Brewer MM; Bates MR; Vannoy LP. Postpartum changes in maternal weight and body fat depots in lactating vs nonlactating women. Am J Clin Nutr. 1989 Feb;49(2):259-65. PMID: 2916446.

151. Haiek LN; Kramer MS; Ciampi A; Tirado R. Postpartum weight loss and infant feeding. J Am Board Fam Pract. 2001 Mar-Apr;14(2):85-94. PMID: 11314929.

152. Janney CA; Zhang D; Sowers M. Lactation and weight retention. Am J Clin Nutr. 1997 Nov;66(5):1116-24. PMID: 9356528.

153. Ohlin A; Rossner S. Maternal body weight development after pregnancy. Int J Obes. 1990 Feb;14(2):159-73. PMID: 2341224.

154. Ohlin A; Rossner S. Factors related to body weight changes during and after pregnancy: the Stockholm Pregnancy and Weight Development Study. Obes Res. 1996 May;4(3):271-6. PMID: 8732961.

155. Linne Y; Dye L; Barkeling B; Rossner S. Weight development over time in parous women--the SPAWN study--15 years follow-up. Int J Obes Relat Metab Disord. 2003 Dec;27(12):1516-22. doi: 10.1038/sj.ijo.0802441. PMID: 14634683.

156. Olson CM; Strawderman MS; Hinton PS; Pearson TA. Gestational weight gain and postpartum behaviors associated with weight change from early pregnancy to $1 \mathrm{y}$ postpartum. Int J Obes Relat Metab Disord. 2003 Jan;27(1):117-27. doi: 10.1038/sj.ijo.0802156. PMID: 12532163.

157. Sichieri R; Field AE; Rich-Edwards J; Willett WC. Prospective assessment of exclusive breastfeeding in relation to weight change in women. Int J Obes Relat Metab Disord. 2003 Jul;27(7):815-20. doi: 10.1038/sj.ijo.0802285. PMID: 12821967.

158. Walker L; Freeland-Graves JH; Milani T; George G; Hanss-Nuss H; Kim M; Sterling BS; Timmerman GM; Wilkinson S; Arheart KL; Stuifbergen A. Weight and behavioral and psychosocial factors among ethnically diverse, low-income women after childbirth: II. Trends and correlates. Women Health. 2004;40(2):19-34. doi: 10.1300/J013v40n02_02. PMID: 15778136.

159. Kotsopoulos J; Lubinski J; Salmena L; Lynch HT; Kim-Sing C; Foulkes WD; Ghadirian P; Neuhausen SL; Demsky R; Tung N; Ainsworth P; Senter L; Eisen A; Eng C; Singer C; Ginsburg O; Blum J; Huzarski T; Poll A; Sun P; Narod SA. Breastfeeding and the risk of breast cancer in BRCA1 and BRCA2 mutation carriers. Breast Cancer Res. 2012 Mar 09;14(2):R42. doi: 10.1186/bcr3138. PMID: 22405187. 